\title{
ESICM LIVES 2021: Part 1
}

\author{
Virtual 03-06 October 2021
}

Published: 11 October 2021

Best Oral Presentations 1

\section{0}

High-Protein Intake and Early Exercise in Adult Intensive Care Patients: A Randomized Controlled Phase III Study to Evaluate the Impact on Functional Outcomes

J. Azevedo ${ }^{1}$; H. Lima ${ }^{2}$;. F. Frota' ${ }^{1}$;. Nogueira' ${ }^{1}$ S. Souza ${ }^{3}$; E. Fernandes

'Intensive care unit, Hospital São Domingos, São Luís, Brazil; ${ }^{2}{ }^{2} \mathrm{Su}$, Hospital

São Domingos, São Luís, Brazil; ${ }^{3}$ Intensive care unit, Hospital São Domingos, Sao Luis, Brazil

Correspondence: J. Azevedo

Intensive Care Medicine Experimental 2020, 9(1): 000100

Introduction. The optimal integration between adequate protein intake and exercise in critically ill patients may have an impact on short and long-term outcomes, but this hypothesis has not been tested in studies with good methodology. In the given context, we conducted a prospective randomized controlled trial to evaluate the efficacy of high protein intake and early exercise versus guidelines recommended protein intake and routine physiotherapy on outcome of critically ill patients.

Methods. We randomized mechanically ventilated patients expected to stay in the intensive care unit (ICU) for at least 4 days. We used indirect calorimetry to determine energy expenditure and guide caloric provision to the patients randomized to the high protein and early exercise (HPE) group and the control group. Protein intakes were $2.0 \mathrm{~g} /$ $\mathrm{kg} /$ day and $1.4 \mathrm{~g} / \mathrm{kg} /$ day respectively; while the former was submitted to two daily sessions of cycle ergometry exercise, the latter received routine physiotherapy. We evaluated the primary outcome physical component summary (PCS) score at 3 and 6 months) and the secondary outcomes (handgrip strength at ICU discharge and ICU and hospital mortality).

Results. We analyzed 181 patients in the HPE (87) and control (94) group. There was no significant difference between groups in relation to the calories received. However, the amount of protein received by the HPE group was significantly higher than that received by the control group $(p<0.0001)$. The PCS score was significantly higher in the HPE group at 3 months $(p=0.01)$ and 6 months $(p=0.01)$. The hospital mortality was expressively higher in the control group $(p=0.006)$. We found an independent association between age and 3-month PCS and that between age and group and 6-month PCS.
Conclusion. This study showed that a high-protein intake and resistive exercise increase the survival rate and the physical quality of life of critically ill patients.

\section{0}

Fluid Management In Critically III Patients With Acute Kidney Injury: Role Of Noninvasive Assessment of Stroke Volume

H. Effat

${ }^{1}$ Critical Care Medicine, Cairo University, Cairo, Egypt

Correspondence: $\mathrm{H}$. Effat

Intensive Care Medicine Experimental 2020, 9(1): 000160

Introduction. Acute kidney injury (AKI) occurs in up to $50 \%$ of patients admitted to the intensive care units. Optimization of volume status is a challenging step in management of AKI patients.

Methods. This study was conducted on 40 critically ill patients with AKI. Patients were randomly divided into 2 groups: group A; volume status was optimized by CVP/DCVP and group B; volume status was optimized by passive leg raising (PLR) and fluid challenge induced stroke volume variation (SVV) using echocardiography. Both groups were compared regarding outcome.

Results. No significant difference in both groups regarding any of the baseline characteristics. Group A received more fluids compared to group B after $48 \mathrm{~h}(3.7 \pm 0.7$ VS $2.2 \pm 0.6 \mathrm{~L}$ respectively, $\mathrm{p}<0.001)$. This was associated with increased morbidity and mortality. In group $A$, the need for mechanical ventilation (MV) was higher ( $p$ 0.048), the duration of MV was longer ( $p$ 0.04) with larger number of patients failed weaning ( $p$ 0.036). The need for vasopressors was higher in group $A$ ( $p$ 0.019) with more patients in need for RRT compared to group $B$, but it didn't reach statistical significance ( $p$ 0.2). No difference between the two groups regarding the length of ICU stay $(12.10 \pm 11.83$ days in group $A$ versus $6.35 \pm 3.86$ group $B, P$ value 0.183 ). However, the mortality rate was higher in group $A(40 \%)$ compared to group $B(10 \%), p$ 0.028 .

Conclusion. The use of SVV for guiding fluid therapy in AKI patients was associated with less fluid intake, less morbidly and mortality compared to CVP/DCVP.

\section{Reference(s)}

1. Chew MS. Haemodynamic monitoring using echocardiography in the critically ill: a review. Cardiology research and practice. 2012;2012.

2. Rania Alhusseiny MD., Waleed Farouk MD. 


\section{9}

Outcomes, Costs and Healthcare Resource Utilization Related to Acute Kidney Injury Among Hospitalized Patients with COVID-19

R. Mackey ${ }^{\text {; J. Textoris }}{ }^{2}$; N. Rosenthal, ; ; LA. Carabuena, ; J. Kampf, ${ }^{3}$; A. Sanghani, ${ }^{3} ;$ T. Rodriguez, ${ }^{3}$; P. Mcpherson, ${ }^{3} ;$ J. Koyner ${ }^{4}$ ${ }^{1}$ Premier applied sciences, Premier, Inc., Charlotte, United States of America; ${ }^{2} \mathrm{Global}$ medical affairs, bioMérieux-Campus de l'étoile, Marcy-l'Étoile, France; ${ }^{3}$ Global medical affairs, bioMerieux Inc, Durham, United States of America; ${ }^{4}$ Medicine-nephrology, University of Chicago, Chicago, United States of America

Correspondence: R. Mackey

Intensive Care Medicine Experimental 2020, 9(1): 000169

Introduction. Acute kidney injury (AKI) in hospitalized COVID-19 patients is associated with higher mortality, but effects on healthcare resource utilization (HRU) and costs are unknown.

Objectives. To compare characteristics, clinical outcomes, HRU and costs for hospitalized US COVID-19 patients with and without AKI.

Methods. This retrospective observational study included patients aged $\geq 18$ years with COVID-19 (ICD-10-CM diagnosis code U07.1) discharged (index date) from US hospitals in the Premier Healthcare Database from April 1 to October 31, 2020. Exclusions were renal transplant or ESRD during index or prior 12 months, $\geq 2$ dialysis treatments during prior 12 months, or CKD stage 5 present on admission. AKI was defined by ICD-10-CM diagnosis code N17.\%. Charlson Comorbidity Index $(\mathrm{CCl})$ score and comorbidities were assessed at index and prior 12 months. Outcomes were in-hospital mortality, length of stay (LOS), and costs assessed during index and during 30-day follow-up, and during 30-day follow-up: dialysis and "new" dialysis (limited to patients with no dialysis during index hospitalization). Logistic and general linear regression models were adjusted for age, sex, race-ethnicity, $\mathrm{CCl}$, hospital characteristics (number of beds, teaching status, region, urban/rural), admission point of origin and type, medical vs. surgical MS-DRG, primary payer, and presence of CKD.

Results. Of 208,583 hospitalized COVID-19 patients, 62,553 (30.0\%) had $\mathrm{AKI}$, of whom 6,083 (9.7\%) had dialysis during index admission. AKI was more common among the $24.0 \%$ of patients with an ICU stay during index admission than the $76.0 \%$ without an ICU stay (AKI: $52.1 \%$ vs. $23.0 \%$ ). Patients with AKI were older, more likely to be male, black, have CKD, and have a higher mean $\mathrm{CCl}$ score $(p<0.0001$ for all). During index hospitalization, patients with AKI were more likely to have an ICU stay (41.6\% vs. $16.4 \%)$ and die (31.0\% vs. $6.0 \%)$, and had longer mean total hospitalization LOS (11.9 vs. 6.9 days) and ICU LOS than patients without AKI. Patients with $\mathrm{AKI}$ also had higher mean total hospitalization costs $(\$ 39,464$ vs. $\$ 17,325)$ and ICU costs during index hospitalization than patients without AKI. Patients with AKI were also more likely to have dialysis, new dialysis, $(0.4 \%$ vs. $0.1 \%)$, and longer mean readmission LOS (8.6 vs. 7.6 days) during 30-day follow-up than patients without AKI ( $p<0.0001$ for all). In adjusted models, AKI patients had higher adjusted odds ratios $(95 \% \mathrm{Cl})$ for ICU stay: $3.70(3.61-3.79)$ and death: 5.10 (4.95-5.25) during index admission, and for dialysis: 4.63 (3.64-5.88) and new dialysis: 2.69 (2.07-3.51) during 30-day followup. Compared to patients without AKI, patients with $\mathrm{AKI}$ had more than 1.5 times higher adjusted relative LOS $(95 \% \mathrm{Cl})$ for total index hospitalization LOS: 1.51 (1.50-1.53) and index hospitalization ICU LOS: 1.53 (1.51-1.56), and 7\% longer 30-day readmission LOS: 1.07 $(1.03-1.10)(p<0.0001$ for all). Finally, compared to patients without $\mathrm{AKI}$, patients with $\mathrm{AKI}$ had higher adjusted relative cost $(95 \% \mathrm{Cl})$ for total index hospitalization cost: 1.94 (1.92-1.95) and index hospitalization ICU cost: $1.74(1.71-1.78)$. Results persisted when additionally adjusted for ICU stay (yes/no).

Conclusion. Almost 1/3 of hospitalized US COVID-19 patients had AKI. Adusted for patient and hospital characteristics, AKI was associated with $>5$ times higher odds of death, $>1.5$ times longer total hospitalization LOS and ICU LOS, and almost doubled mean costs during index hospitalization. AKI patients also had higher adjusted odds of dialysis and longer LOS during 30-day follow-up.

\section{Reference(s)}

1. Kunutsor SK, Laukkanen JA. Renal complications in COVID-19: a systematic review and meta-analysis. Ann Med. 2020:1-9.

2. Joseph A, Zafrani L, Mabrouki A, Azoulay E, Darmon M. Acute kidney injury in patients with SARS-CoV-2 infection. Ann Intensive Care. 2020;10(1):117.

3. Nimkar A, Naaraayan A, Hasan A, et al. Incidence and Risk Factors for Acute Kidney Injury and its effect on Mortality in Patients Hospitalized from Covid-19. Mayo Clin Proc Innov Qual Outcomes. 2020.

4. Shao M, Li X, Liu F, Tian T, Luo J, Yang Y. Acute kidney injury is associated with severe infection and fatality in patients with COVID-19: A systematic review and meta-analysis of 40 studies and 24,527 patients. Pharmacol Res. 2020;161:105,107.

5. Robbins-Juarez SY, Qian L, King KL, et al. Outcomes for Patients With COVID-19 and Acute Kidney Injury: A Systematic Review and Meta-Analysis. Kidney Int Rep. 2020;5(8):1149-1160.

6. Hansrivijit P, Qian C, Boonpheng B, et al. Incidence of acute kidney injury and its association with mortality in patients with COVID-19: a metaanalysis. J Investig Med. 2020.

7. Nadim MK, Forni LG, Mehta RL, Connor MJ Jr, Liu KD, Ostermann M, Rimmelé T, Zarbock A, Bell S, Bihorac A, Cantaluppi V, Hoste E, Husain-Syed F, Germain MJ, Goldstein SL, Gupta S, Joannidis M, Kashani K, Koyner JL, Legrand M, Lumlertgul N, Mohan S, Pannu N, Peng Z, Perez-Fernandez XL, Pickkers P, Prowle J, Reis T, Srisawat N, Tolwani A, Vijayan A, Villa G, Yang L, Ronco C, Kellum JA. COVID-19-associated acute kidney injury: consensus report of the 25th Acute Disease Quality Initiative (ADQI) Workgroup. Nat Rev Nephrol. 2020 Dec;16(12):747-764.

8. Gupta S, Coca SG, Chan L, Melamed ML, Brenner SK, Hayek SS, Sutherland A, Puri S, Srivastava A, Leonberg-Yoo A, Shehata AM, Flythe JE, Rashidi A, Schenck EJ, Goyal N, Hedayati SS, Dy R, Bansal A, Athavale A, Nguyen HB, Vijayan A, Charytan DM, Schulze CE, Joo MJ, Friedman AN, Zhang J, Sosa MA, Judd E, Velez JCQ, Mallappallil M, Redfern RE, Bansal AD, Neyra JA, Liu KD, Renaghan AD, Christov M, Molnar MZ, Sharma S, Kamal O, Boateng JO, Short SAP, Admon AJ, Sise ME, Wang W, Parikh CR, Leaf DE; and the STOP-COVID Investigators. AKI Treated with Renal Replacement Therapy in Critically III Patients with COVID-19. J Am Soc Nephrol. 2021 Jan;32(1):161-176.

9. Silver SA, Beaubien-Souligny W, Shah PS, Harel S, Blum D, Kishibe T, Meraz-Munoz A, Wald R, Harel Z. The Prevalence of Acute Kidney Injury in Patients Hospitalized With COVID-19 Infection: A Systematic Review and Meta-analysis. Kidney Med. 2021 Jan-Feb;3(1):83-98.e1.

10. Chan L, Chaudhary K, Saha A, Chauhan K, Vaid A, Zhao S, Paranjpe I, Somani S, Richter F, Miotto R, Lala A, Kia A, Timsina P, Li L, Freeman R, Chen R, Narula J, Just AC, Horowitz C, Fayad Z, Cordon-Cardo C, Schadt E, Levin MA, Reich DL, Fuster V, Murphy B, He JC, Charney AW, Böttinger EP, Glicksberg BS, Coca SG, Nadkarni GN; Mount Sinai COVID Informatics Center (MSCIC). AKI in Hospitalized Patients with COVID-19. J Am Soc Nephrol. 2021 Jan;32(1):151-160.

11. N/A

\section{6}

Cardiorespiratory response to early reh $\$ 1$ In abilitation in critically ill adults: a secondary analysis of a randomised controlled trial

S. Eggmann 1; I. Irincheeva ${ }^{2}$; G. Luder'; ML. Verra, ${ }^{3}$; A. Moser²;

$\mathrm{CH}$. Bastiaenen, ${ }^{4}$; SM. Jakob ${ }^{5}$

${ }^{1}$ Physiotherapy, Inselspital, Bern, Switzerland; ${ }^{2}$ Ctu bern, University of Bern, Bern, Switzerland; ${ }^{3}$ Department of physiotherapy, Inselspital, Bern, Switzerland; ${ }^{4}$ Department of epidemiology. research line functioning, participation and rehabilitation caphri, Maastricht University, Maastricht, Netherlands; ${ }^{5}$ University clinic for intensive care medicine, Bern University Hospital, Bern, Switzerland

Correspondence: S. Eggmann

Intensive Care Medicine Experimental 2020, 9(1): 000176

Introduction. Early rehabilitation is indicated in critically ill adults to counter functional complications [1]. However, the physiological response to early physiotherapy is poorly understood and the appropriate type, intensity or duration unknown. 
Objectives. To explore cardiorespiratory trajectories from before, during and after physiotherapy and to investigate explanatory variables for changes during training and recovery.

Methods. This was a secondary analysis of a randomised controlled trial investigating two early rehabilitation programs, delivered by physiotherapists within $48 \mathrm{~h}$ of admission in mechanically ventilated adults $[2,3]$. Eligible participants were $\geq 18$ years old, expected to remain ventilated for $>72 \mathrm{~h}$ and had been independent before critical illness. Physiological data (2-min medians) were collected with standard ICU monitoring and indirect calorimetry, and their medians calculated for baseline (30 min before), training (during physiotherapy) and recovery (15 min after). We visualised physiological trajectories and quantified explanatory variables for changes with mixed-effects models.

Results. 716 sessions from 108 participants (67\% male; median age 67 years [IQR 19]; Sequential Organ Failure Assessment (SOFA) 8 [IQR 5] with a median of 3 [IQR 6] sessions per subject) were analysed. Overall, physiotherapy did not significantly affect heartrate (HR), mean arterial pressure (MAP), oxygen saturation (SpO2), oxygen consumption (VO2) and minute ventilation (MV) from baseline to training or recovery. However, there was a large range of variation within and across participants' sessions as well as across different types of rehabilitation interventions (Fig. 1). Clinically relevant changes (>10\%) occurred in more than 1 out of 4 sessions in MV, VO2 and MAP. Active patient participation increased VO2 by $23 \mathrm{ml} / \mathrm{min}(95 \%-\mathrm{Cl}: 13-34, \mathrm{p}<0.001)$ and $\mathrm{MV}$ by $0.7 \mathrm{I} / \mathrm{min}(0.4-1.0, \mathrm{p}<0.001)$, during training, but not recovery, when compared to passive participation. Other modifiable variables affecting training or recovery included session type, session duration, mobilisation level and daily medication (vasoactive support, sedatives), while non-modifiable variables were age, gender, body mass index and the daily SOFA.

Fig. 1 Average trajectories of median physiological values with standard errors across the seven defined intervention types
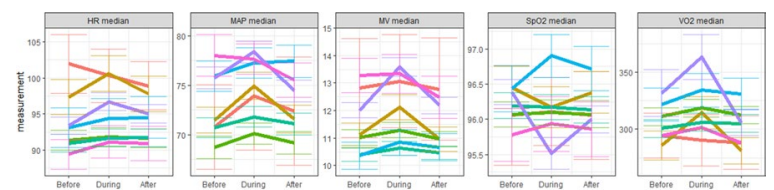

- eccins - exercise and res

Conclusion. Overall, early physiotherapy does not significantly affect physiological parameters, though variation is high, and clinically relevant changes occur in individual patients during training and recovery. Physiological parameters should therefore be closely monitored and rehabilitation interventions individually tailored. The identified explanatory variables guide clinicians' decision-making in delivering the optimal type, intensity and duration by enabling clinicians to prospectively estimate the cardiorespiratory response.

\section{Reference(s)}

1. Waldauf P, Jiroutková K, Krajčová A, Puthucheary Z, Duška F. Effects of Rehabilitation Interventions on Clinical Outcomes in Critically III Patients: Systematic Review and Meta-Analysis of Randomized Controlled Trials. Crit Care Med. 2020 Jul:48(7):1055-1065.

2. Eggmann S, Verra ML, Luder G, Takala J, Jakob SM. Effects of early, combined endurance and resistance training in mechanically ventilated, critically ill patients: A randomised controlled trial. PloS one. 2018; 13(11):e0207428.

3. PhD Grant from the Swiss Foundation for Physiotherapy Science

4. Eggmann S, Verra ML, Luder G, Takala J, Jakob SM. Effects of early, combined endurance and resistance training in mechanically ventilated, critically ill patients: a study protocol for a randomised controlled trial. Trials. 2016; 17:403.
000207

National Early Warning Score and the respiratory component: a prospective three-centre study with 18,899 general ward patients

J. Tirkkonen'; E. Loisa'; S. Hoppu²; A. Kallonen ${ }^{3}$

${ }^{1}$ Department of Intensive Care Medicine, Tampere University Hospital, Tampere, Finland; ${ }^{2}$ Department of emergency medical ser-

vices, TAYS, Tampere, Finland; ${ }^{3}$ Faculty of medicine and health technology, Tampere University, Tampere, Finland

Correspondence: J. Tirkkonen

Intensive Care Medicine Experimental 2020, 9(1): 000207

Introduction. The national early warning score (NEWS) has been widely implemented to enable early detection of patient deterioration in hospital wards, and to facilitate timely intensive care unit admissions if required.1 However, a recent systematic review concluded that most studies investigating early warning scores to date suffer from several methodological flaws (such as small sample sizes, missing vital signs data \& outcomes that have too long time horizons) and highquality research is warranted. 2

Objectives. To evaluate the ability of the national early warning score (NEWS) and its vital signs' components to discriminate short-term mortality among general ward patients. We further hypothesized that NEWS's respiratory parameters are more strongly associated with mortality.

Methods. We conducted a large, pragmatic prospective observational study in one university hospital and two regional hospitals in Finland treating patients from all medical and surgical specialties. Adult patients admitted to non-critical care wards of the three hospitals between 1.1.2019-31.12.2019 were included. The Medanets ${ }^{\circledR}$ mobile solution enabled reliable and comprehensive data capture during routine patient care on wards; the first measured vital signs datasets were included in the analyses. The area under receiver operator curves (AUROCs) were used to investigate associations with mortality. NEWS's respiratory components (respiratory rate, $\mathrm{SpO} 2$ ) and haemodynamic components (systolic blood pressure, heart rate) were further investigated separately and statistical AUROC comparisons were conducted with DeLong's test for two correlated ROC-curves. A p-value of $<0.05$ was considered statistically significant. The local Ethics Committee approved the study protocol (R20007R).

Results. After excluding 102/19,001 patients (0.53\%) with data on some vital signs missing, the final cohort consisted of 18,889 patients with full capture of all vital signs (and all NEWS components). Patients' median age was 70 years, $51 \%$ were male and $31 \%$ had a surgical reason for admission. The $24 \mathrm{~h}$ mortality was $0.36 \%$ and 30 -day mortality was $3.9 \%$. The NEWS discriminated $24 \mathrm{~h}$ non-survivors with excellent accuracy (AUROC 0.91, 95\% Cl 0.87-0.95) and 30-day non-survivors with acceptable accuracy $(0.75,0.73-0.77)$. The respiratory rate score alone discriminated $24 \mathrm{~h}$ non-survivors better $(0.78,0.72-0.84)$ as compared with $\mathrm{SpO} 2$ score $(0.66,0.59-0.73)$, systolic blood pressure score $(0.65,0.59-0.72)$ and heart rate score $(0.67,0.61-0.74)(p<0.01$ in all AUROC comparisons). As with the total NEWS, the discriminative accuracy of the individual score components decreased substantially for 30-day mortality.

Conclusion. NEWS discriminated general ward patients at risk for acute death with excellent statistical accuracy in this large pragmatic multicentre trial with a clinically relevant outcome measure. The respiratory rate component was especially strongly associated with shortterm mortality.

\section{Reference(s)}

1. Gerry S, Bonnici T, Birks J, Kirtley S, Virdee PS, Watkinson PJ, Collins GS. Early warning scores for detecting deterioration in adult hospital patients: systematic review and critical appraisal of methodology. BMJ 2020;369: m1501.

2. Smith GB, Prytherch DR, Meredith P, Schmidt PE, Featherstone PI. The ability of the National Early Warning Score (NEWS) to discriminate patients at risk of early cardiac arrest, unanticipated intensive care unit admission, and death. Resuscitation 2013;84:465-70.

3. None. 
Best Oral Presentations 2

\section{2}

Body mass index and mortality in COVID-19 and other diseases: a cohort study in $35,506 \mathrm{ICU}$ patients

E. Kooistra'; S. Brinkman, ${ }^{2}$; DVP. Van ${ }^{3}$; N. de Keizer ${ }^{2}$; D. Dongelmans ${ }^{2}$;

M. Kox'; P. Pickkers

${ }^{1}$ Intensive Care, Radboud University Medical Center, Nijmegen, Netherlands; ${ }^{2}$ Medical informatics, Amsterdam UMC, locatie AMC, Amsterdam, Netherlands; ${ }^{3}$ ntensive care, University Medical Center Groningen, Groningen, Netherlands

Correspondence: E. Kooistra

Intensive Care Medicine Experimental 2020, 9(1): 000282

Introduction. Obesity is a risk factor for severe COVID-19 and might play a role in its pathophysiology (1). It is unknown whether BMI is related to clinical outcome following ICU admission, as observed in various other categories of critically ill patients $(2,3)$.

Objectives. We investigated the relationship between $\mathrm{BMI}$ and in-hospital mortality in critically ill COVID-19 patients and in cohorts of ICU patients with non-SARS-CoV-2 viral pneumonia, bacterial pneumonia, and multiple trauma.

Methods. We performed a multicenter observational cohort study using data from 35.506 consecutive patients from 82 ICUs participating in the Dutch National Intensive Care Evaluation quality registry. Patient characteristics and clinical outcomes were compared between four cohorts and between BMI categories within cohorts. Adjusted analyses of the relationship between BMI and in-hospital mortality within each cohort were performed using multivariable logistic regression.

Results. COVID-19 patients were more likely male, had a higher BMI, lower $\mathrm{PaO} 2 / \mathrm{FiO} 2$ ratio, and were more likely mechanically ventilated during the first $24 \mathrm{~h}$ in the ICU compared to the other cohorts. COVID-19 patients had longer ICU and hospital length of stay, and higher in-hospital mortality. Odds ratios for in-hospital mortality for patients with $\mathrm{BMI} \geq 35 \mathrm{~kg} / \mathrm{m} 2$ compared with normal weight in the COVID-19, non-SARS-CoV-2 viral pneumonia, bacterial pneumonia, and trauma cohorts were 1.15 [0.79-1.67], 0.64 [0.43-0.95], 0.73 [0.61-0.87] and 0.81 [0.57-1.15], respectively (Table 1). Relative in-hospital mortality risks according to BMI in the four cohorts are depicted in Fig. 1.

Table 1 Odds ratios of in-hospital mortality of BMI categories in the multivariate logistic regression model, with BMI $18.5-25 \mathrm{~kg} / \mathrm{m} 2$ used as Reference category. Covariates used for this analyses included sex, age, medical history (chronic diagnoses), APACHE III acute physiology score (APS), vasoactive medication and mechanical ventilation and $\mathrm{PaO} 2 / \mathrm{FiO} 2$ ratio on $\mathrm{ICU}$ admission

\begin{tabular}{|l|l|l|l|l|}
\hline & & $\begin{array}{l}\text { non-SARS-CoV-2 } \\
\text { viral pneumonia } \\
(n=2940)\end{array}$ & $\begin{array}{l}\text { Bacterial } \\
\text { pneumonia } \\
(n=14250)\end{array}$ & $\begin{array}{l}\text { Trauma } \\
(n=15681)\end{array}$ \\
\hline COVID-19 $(\mathrm{n}=2635)$ & $1.50[0.95-2.37]$ & $1.88[1.57-2.25]$ & $1.23[0.86-1.78]$ \\
\hline BMI $18.5-25 \mathrm{~kg} / \mathrm{m} 2$ & 1.0 reference & 1.0 reference & 1.0 reference & 1.0 reference \\
\hline BMI $25-30 \mathrm{~kg} / \mathrm{m} 2$ & $0.95[0.75-1.21]$ & $0.78[0.61-0.99]$ & $0.78[0.70-0.86]$ & $0.9[0.78-1.03]$ \\
\hline BMI $30-35 \mathrm{~kg} / \mathrm{m} 2$ & $0.87[0.65-1.16]$ & $0.76[0.55-1.04]$ & $0.81[0.70-0.93]$ & $0.99[0.79-1.23]$ \\
\hline$B M I 235 \mathrm{~kg} / \mathrm{m} 2$ & $1.15[0.79-1.67]$ & $0.64[0.43-0.95]$ & $0.73[0.61-0.87]$ & $0.81[0.57-1.15]$ \\
\hline
\end{tabular}

Fig. 1 Relative in-hospital mortality risks according to BMI in the four cohorts. BMI of $25.0 \mathrm{~kg} / \mathrm{m}^{2}$ was used as Reference. Relative risks were adjusted for sex, age, chronic diagnosis, APACHE III acute physiology score (APS) and need for mechanical ventilation, use of vasoactive medication, and lowest $\mathrm{PaO} 2 / \mathrm{FiO}^{2}$-ratio in the first $24 \mathrm{~h}$ following ICU admission. The mortality risk decreases with higher $\mathrm{BMI}$ in the non-SARS-CoV-2 viral pneumonia, bacterial pneumonia, and trauma cohorts, but not in COVID-19 patients

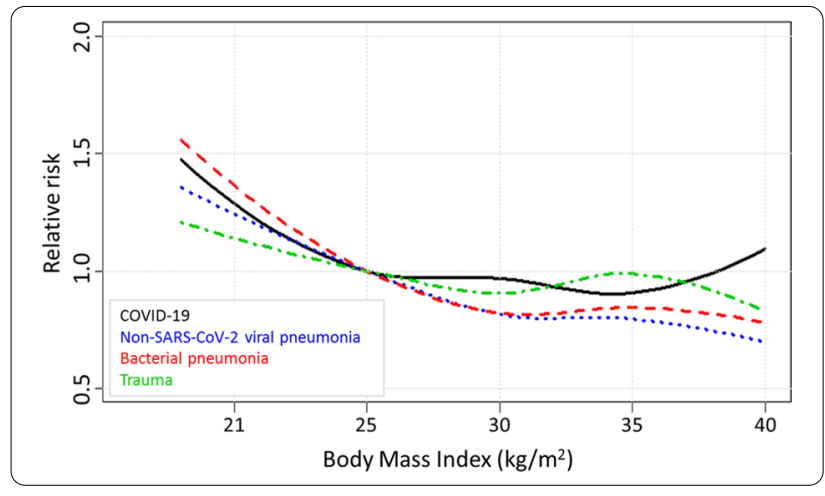

Conclusion. The obesity paradox, which is the inverse association between BMI and mortality in critically ill patients, is not present in ICU patients with COVID-19-related respiratory failure, in contrast to ICU patients suffering from non-SARS-CoV-2 viral and bacterial respiratory infections.

\section{Reference(s)}

1. Zhi G, Xin W, Ying W, Guohong X, Shuying L. "Obesity Paradox" in Acute Respiratory Distress Syndrome: Asystematic Review and Meta-Analysis. PLoS One. 2016;11(9):e0163677.

2. Pickkers P, de Keizer N, Dusseljee J, Weerheijm D, van der Hoeven $J G$, Peek N. Body mass index is associated with hospital mortality in critically ill patients: an observational cohort study. Crit Care Med. 2013:41(8):1878-83

3. Földi M, Farkas N, Kiss S, Zádori N, Váncsa S, Szakó L, et al. Obesity is a risk factor for developing critical condition in COVID-19 patients: A systematic review and meta-analysis. Obesity Reviews. 2020;21(10):e13095

4. None

\section{3}

Changes in Pupillary Dilation Velocity during delirium and septic shock in a general ICU cohort

S. Uhrenholt ${ }^{1}$; S. Linér, ${ }^{1}$; J. Stokholm, ${ }^{2}$; T. Christiensen, ${ }^{2}$; MH. Bestle

${ }^{1}$ Department of anaesthesiology and intensive care, Copenhagen University Hospital_North Zealand, Copenhagen, Denmark; ${ }^{2}$ Department of neurology, Copenhagen University Hospital—North Zealand, Copen-

hagen, Denmark

Correspondence: $\mathrm{S}$. Uhrenholt

Intensive Care Medicine Experimental 2020, 9(1): 001213

Introduction. Delirium and septic shock are common in the intensive care unit (ICU). Their pathophysiologies are poorly understood $(1,2)$ but autonomic dysfunction is likely involved. Pupillary light response (PLR) is a fast and non-invasive way to measure autonomic nervous system function(3).

Objectives. The aim was to determine if the dilation velocity in the pupillary light response (PLRdil.vel) could detect changes in the sympathetic autonomic nervous system in patients with either delirium or septic shock. Ambient light levels and noradrenaline infusions were examined for possible influence on the PLR measurements.

Methods. In this prospective observational cohort study, we included 100 adult patients acutely admitted to a mixed ICU at Copenhagen University Hospital-North Zealand, Denmark. The patients were followed for development of delirium and/or septic shock twice each day and until ICU discharge. PLRdil.vel was measured with a portable pupillometer, simultaneously delirium was assessed with the Confusion Assessment Method for the ICU (CAM-ICU) screening tool. Septic shock was determined by collection of medical records. Blood samples were collected each morning for noradrenaline analysis. 17 patients were excluded due to incomplete data, 83 remained for complete analysis. Main data analyses were done with linear mixed models. 
Results. Of the 83 patients, 28 experienced delirium and 30 were in septic shock. Septic shock slowed PLRdil.vel with $0.25 \mathrm{~mm} / \mathrm{sec}(95 \% \mathrm{Cl}$ $[-0.4 ;-0.1])$, but delirium did not $(95 \% \mathrm{Cl}[-0.1 ; 0.2])$. Noradrenaline infusions did not influence PLRdil.vel $(95 \% \mathrm{Cl}[-0.7 ; 0.3])$ and neither did ambient light levels (95\% Cl $[0.0 ; 0.0])$.

Conclusion. Septic shock slowed PLRdil.vel, but delirium and noradrenaline infusions did not. This indicates a loss of sympathetic drive on the pupillary light reflex which is possibly a surrogate marker for the loss of sympathetic tone on the blood vessels and a sign of autonomic dysregulation.

\section{Reference(s)}

1. Hall CA, Chilcott RP. Eyeing up the Future of the Pupillary Light Reflex in Neurodiagnostics. Diagnostics (Basel, Switzerland). 2018;8(1):1-20. 10. 3390/diagnostics8010019

2. Pun BT, Ely EW. The importance of diagnosing and managing ICU delirium. Chest. 2007:132(2):624-636. 10.1378/chest.06-1795

3. Singer M, Deutschman CS, Seymour CW, et al. The Third International Consensus Definitions for Sepsis and Septic Shock (Sepsis-3). JAMA. 2016;315(8):801-810. 10.1001/jama.2016.0287

4. Kaptajnløjtnant Harald Jensens og Hustrus Fond.

5. The research fund of Copenhagen University Hospital-North Zealand

\section{2}

Creatinine and Mitochondrial Metabolism in the Critically III: a metabolomics cohort study

H. Kobayashi' ; K. Amrein ${ }^{2}$; J. Lanky-Su ${ }^{3}$; K. Christopher'

${ }^{1}$ Division of renal medicine, Brigham and Women's Hospital, Boston, United States of America; '2Division of endocrinology and diabetology, Medical University of Graz, Graz, Austria; ${ }^{3}$ Channing division of network medicine, Brigham and Women's Hospital, Boston, United States of America

Correspondence: $\mathrm{H}$. Kobayashi

Intensive Care Medicine Experimental 2020, 9(1): 000392

Introduction. Animal evidence supports the hypothesis that uremic retention substances potentially impair mitochondrial function. Chronic kidney disease patients are noted to have decreased activity of mitochondrial enzymes required for the citric acid cycle and fatty acid oxidation.

Objectives. We hypothesized that an elevation of serum creatinine in the critically ill is associated with circulating metabolites that indicate impaired mitochondrial energy utilization.

Methods. We performed a post-hoc metabolomics study of the Correction of Vitamin D Deficiency in Critically III Patients (VITdALICU) trial conducted at a university hospital in Austria. The relative abundance of 659 metabolites in 1213 plasma samples from 444 patients at randomization (day 0 ), day 3 , and day 7 were analyzed. Exposure of interest was serum creatinine levels at each time point. For day 0 data, we performed a Student's t-test of individual metabolite abundance grouped according to median creatinine. For longitudinal data, we used multivariable linear mixed-effects models to find the linear association between the individual metabolites and serum creatinine levels at day 0,3 , and 7 adjusting for age, sex, baseline $25(\mathrm{OH}) \mathrm{D}$, an absolute increase in $25(\mathrm{OH}) \mathrm{D}$ at day 3 (intervention response), SAPS II, plasma day, admission diagnosis and patient identification as the random-intercept. To account for multiple comparisons, we utilized a conservative Bonferroni corrected P-value $<7.59 \times 10^{5}$.

Results. In the cohort, the median [IQR] of creatinine at day 0 was 1.1 $[0.8,1.8] \mathrm{mg} / \mathrm{dL}, 11 \%$ had AKI by day 7 and $5 \%$ had pre-existing EndStage Renal Disease requiring renal replacement therapy (Table 1). In plasma samples at day 0, 313 individual metabolites showed significant crude differences between subjects with or without or without creatinine $\geq 1.1 \mathrm{mg} / \mathrm{dL}$. In linear mixed-effects models for longitudinal data, 334 individual metabolites were significantly associated with increased serum creatinine. Three hundred metabolites had significant positive associations with elevated creatinine. These included modified nucleosides and other known uremic retention substances.
Importantly, multiple metabolites indicating incomplete mitochondrial fatty acid beta-oxidation were increased with elevations in creatine including the short- and medium-chain acylcarnitines, dicarboxylate fatty acids, branched-chain amino acids, and members of the pentose phosphate pathway (Summarized in Fig. 1). Further, 34 metabolites were significantly decreased with elevated creatinine including multiple members of acylcholine and lysophospholipid classes. Of the significant metabolites, 165 were not reported as known uremic retention substances. Metabolite abundance patterns remained the same with the removal of patients with End-Stage Renal Disease and when the analysis was limited to trial patients who received placebo.

\begin{tabular}{|c|c|c|c|c|}
\hline Characteristic & $\begin{array}{l}\text { Overall } \\
n=444\end{array}$ & $\begin{array}{c}\mathrm{Cr}<1.1 \\
\mathrm{mg} / \mathrm{dL} \\
\mathrm{n}=221\end{array}$ & $\begin{array}{c}\mathrm{Cr} \geq 1.1 \\
\mathrm{mg} / \mathrm{dL} \\
\mathrm{n}=223\end{array}$ & P-value \\
\hline Age, yr (mean (SD)) & $64.8(14.7)$ & $61.0(16.1)$ & $68.6(12.0)$ & $<0.001$ \\
\hline Female, $n(\%)$ & $155(35)$ & $94(43)$ & $61(27)$ & 0.001 \\
\hline $\begin{array}{l}\text { Charlson Comorbidity Index (median } \\
\text { [IQR]) }\end{array}$ & $3.0[1.0,4.3]$ & $2.0[1.0,3.0]$ & $4.0[2.0,5.0]$ & $<0.001$ \\
\hline Hemodialysis at baseline, $n(\%)$ & $22(5)$ & $3(1)$ & $19(9)$ & 0.001 \\
\hline SAPS \| (mean (SD)) & $33.5(15.4)$ & $31.8(16.0)$ & $35.2(14.7)$ & 0.023 \\
\hline ICU type, $n(\%)$ & & & & $<0.001$ \\
\hline Anesthesia, $n(\%)$ & $83(19)$ & $43(20)$ & $40(18)$ & \\
\hline Cardiac Surgery, $n(\%)$ & $130(29)$ & $35(16)$ & $95(43)$ & \\
\hline Medical, $n(\%)$ & $99(22)$ & $48(22)$ & $51(23)$ & \\
\hline Neurological, $n(\%)$ & $109(25)$ & $83(38)$ & $26(12)$ & \\
\hline Surgical, $n(\%)$ & $23(5)$ & $12(5)$ & $11(5)$ & \\
\hline 25(OH)D, ng/mL (mean (SD)) & $13.9(8.8)$ & $14.0(5.5)$ & $13.7(11.1)$ & 0.691 \\
\hline Vitamin D3 intervention, $n(\%)$ & $219(49)$ & $108(49)$ & $111(50)$ & 0.923 \\
\hline $\begin{array}{l}\text { Change in } 25(\mathrm{OH}) \mathrm{D} \text { Day } 0 \text { to Day } 3 \text {, } \\
\mathrm{ng} / \mathrm{mL} \\
\text { (median [IQR]) }\end{array}$ & $\begin{array}{l}3.1[0.0, \\
16.5]\end{array}$ & $\begin{array}{r}3.3[-0.2 \\
20.5]\end{array}$ & $\begin{aligned} & 2.85 {[0.0} \\
&12.1]\end{aligned}$ & 0.365 \\
\hline Serum Creatinine, $\mathrm{mg} / \mathrm{dL}$ (median [IQR]) & $1.1[0.8,1.8]$ & $0.8[0.6,0.9]$ & $1.8[1.4,2.3]$ & $<0.001$ \\
\hline $\begin{array}{l}\text { Serum procalcitonin, } \mathrm{ng} / \mathrm{mL} \text { (median } \\
\text { [IQR]) }\end{array}$ & $0.7[0.2,2.7]$ & $0.3[0.1,0.8]$ & $1.7[0.6,6.1]$ & $<0.001$ \\
\hline Acute kidney injury by day $7, \mathrm{n}(\%)$ & $50(11)$ & $24(11)$ & $26(12)$ & 0.768 \\
\hline 28-day mortality, n (\%) & $114(26)$ & $37(17)$ & $77(35)$ & $<0.001$ \\
\hline
\end{tabular}

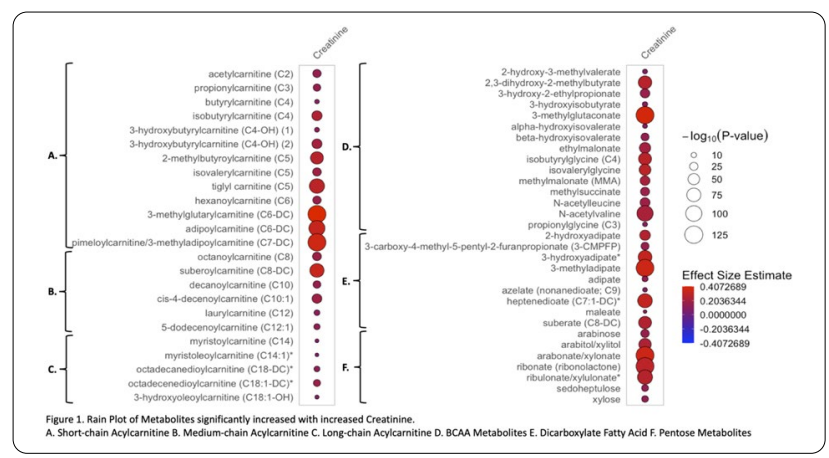

Conclusion. Our novel data show that elevated creatinine during critical illness is associated with circulating metabolites related to incomplete mitochondrial fatty acid beta-oxidation as well as known uremic retention substances. Such uremic retention substances may be important in impaired mitochondrial function via decreases in mitochondrial enzyme activities.

\section{Reference(s)}

1. Amrein K, Schnedl C, Holl A, et al. Effect of high-dose vitamin D3 on hospital length of stay in critically ill patients with vitamin D deficiency: the VITdAL-ICU randomized clinical trial. JAMA. 2014;312(15):1520-1530.

This work was supported by the National Institutes of Health [R01 GM115774]. 


\section{5}

A systematic review exploring physician approaches to conflict resolution in end-of-life decisions in the adult intensive care unit HK. Johal ${ }^{1}$; G. Birchley ${ }^{1}$; W. Orchard ${ }^{2}$; R. Huxtable ${ }^{1}$

${ }^{1}$ Centre for ethics in medicine, University of Bristol, Bristol, United Kingdom; ${ }^{2}$ Intensive care unit, Wexham Park Hospital, Slough, United Kingdom Correspondence: H.K. Johal

Intensive Care Medicine Experimental 2020, 9(1): 000365

Introduction. Conflict is unfortunately well-documented in the adult intensive care unit (AICU). In the context of end-of-life (EOL) decision-making (i.e. the withdrawal or withholding of life-sustaining treatment), conflict commonly occurs when a consensus cannot be reached between the healthcare team and the patient's family on the "best interests" of the critically ill, incapacitated patient. Whilst existing literature has identified potential routes for conflict resolution, it is less clear how these approaches are perceived and utilised by stakeholders in the EOL decision-making process.

Objectives. To explore conflict resolution strategies by systematically reviewing and synthesising the published evidence, which addresses the following research question: what does existing qualitative research reveal about physician approaches to addressing conflict arising in EOL decisions in the AICU?

Methods. Peer-reviewed qualitative studies (retrieved from MEDLINE, Project Muse, EMBASE, Web of Science, PsycINFO, CINAHL and LILACS) examining physician approaches to conflict resolution in the context of EOL decisions in the AICU setting were included. Two reviewers (HKJ and WO) independently screened for eligibility, and extracted data, from either all or $10 \%$ of the included studies. A third reviewer (GB) independently screened studies of uncertain eligibility. A narrative synthesis was undertaken to analyse the data, which involved: developing a preliminary synthesis of the findings of the included studies; exploring relationships in the data; and assessing the robustness of the synthesis. The quality of the included studies was assessed using the 2018 Mixed-Methods Assessment Tool (MMAT), and the GRADE-CERQual approach was used to assess the confidence in the findings. The protocol was registered on the PROSPERO database (\#CRD42021193769) [Johal et al., 2021].

Results. A total of 1101 records were screened. Seven studies from three countries (UK, USA, Canada) were included in our final synthesis. Physician approaches to resolving EOL conflicts in the AICU were organised into the following themes and subthemes:

(1) building a therapeutic relationship by treating the patient, building trust, gathering information about the patient, and assessing the family;

(2) determining the cause of conflict by identifying the nature of the conflict, differentiating stakeholders' views on the disputed decision, and distinguishing the emotional component of the conflict;

(3) co-ordinating an individualised response by involving others, and tailoring negotiations;

(4) balancing personal and professional values by maintaining professionalism, managing personal beliefs, and responding to ethical and legal uncertainty.

Conclusion. This is the first review, to our knowledge, to synthesise all the published evidence on physician approaches to EOL conflict resolution within the AICU. Our findings suggest both useful and objectionable strategies that physicians have employed, which may have implications for guiding ethical practice, and training healthcare professionals in how to approach conflict. Given the ongoing pressures on critical care services, we believe the findings will be of importance and of interest to all clinicians working in AICUs.

\section{Reference(s)}

1. This work was supported by the 'Balancing Best Interests in Health Care, Ethics and Law (BABEL)' Collaborative Award from the Wellcome Trust [209841/Z/17/Z]. The funding body had no role in the writing of this abstract. The work is based at the University of Bristol.

2. Johal H, Birchley G, Orchard W, Huxtable R. A systematic review exploring physician approaches to conflict resolution in end-of-life decisions in the adult intensive care unit. PROSPERO 2021 CRD42021193769 Available from: https://www.crd.york.ac.uk/prospero/display_record.php?ID= CRD42021193769
001113

Epidemiology and determinants of outcomes of Hospital-Acquired Blood Stream Infections in the ICU: the Eurobact II multinational cohort study

A. Tabah'; N. Buetti ; F. Barbier ${ }^{3}$; AT. Aslann ; S. Ruckly, ; Q. Staiquly, ; C. Dallongeville, ${ }^{5}$; N. Eurobact li National Coordinators'; N. Eurobact li Contributors $^{1}$; JF. Timsit ${ }^{6}$

${ }^{1}$ Intensive care unit, Redcliffe Hospital, Metro North Hospitals and Health Services, The University of Queensland, Brisbane, Australia; ${ }^{2}$ Inserm umr 1137, Paris Diderot University, Swiss National Science Foundation, Paris, France; ${ }^{3}$ Medical intensive care unit, CHR Orléans, Orléans, France; ${ }^{4}$ Department of internal medicine, Hacettepe University, Ankara, Turkey; ${ }^{5}$ Research, Outcomerea and ICUREsearch, Paris, France; ${ }^{6}$ Medical and infectious diseases icu, Bichat Hospital, Paris, France

Correspondence: A. Tabah

Intensive Care Medicine Experimental 2020, 9(1): 001113

Introduction. Blood stream infections represent a leading cause of hospital acquired complications and are associated with significant mortality, morbidity, and costs. Detailed knowledge of their characteristics is required to design intervention studies and performance improvement programs.

Objectives. To describe the epidemiology and determinants of outcomes of hospital-acquired blood stream infections (HA-BSI) in intensive care units (ICU) worldwide.

Methods. We conducted a prospective observational multinational multicenter cohort study of patients that were admitted to ICUs for the management of HA-BSI or that acquired HA-BSI during ICU admission. Primary outcome measure was mortality at day 28 after BSI acquisition.

Results. We included patients from 336 ICUs in 62 countries between September 2019 and February 2021. Eighty percent were mixed medical-surgical ICUs with a median of 14 [IQR 9; 20] beds. Thirteen percent reported having an ICU specialist as part of the ICU team, and $90 \%$ had a microbiology laboratory within the hospital. Hydrocortisone was used by $3 \%$ for patients with sepsis, $32 \%$ septic shock and $56 \%$ only if the patient required high dose vasoactive medications. At the time of submitting this abstract, data collection was completed and quality control ongoing. The preliminary dataset included 2559 patients with a median age of 64 [IQR 51; 73]. Seventy-four percent had at least 1 co-morbid condition and $8 \%$ an active cancer. HA-BSI were diagnosed before ICU admission for $21 \%$, within the first $48 \mathrm{~h}$ of ICU admission for $11 \%$ more than 2 days after ICU admission for $68 \%$. At the time of BSI $53 \%$ received vasopressors, $66 \%$ invasive mechanical ventilation and $20 \%$ dialysis. The median SOFA score was 8 [IQR 5;11]. Pathogens were predominantly gram-negative bacteria (59\%). Most common bacteria were Klebsiella pneumoniae (13\%), Staphylococcus aureus (9\%) and Acinetobacter baumannii (9\%). There was a significant proportion of difficult to treat resistance and pandrug resistant pathogens. Most common sources of infection were respiratory tract $(26 \%)$, catheter related (24\%), intra-abdominal (16\%) and primary bacteraemia (14\%). Source control was required in $56 \%$ of cases and was not achieved for $10 \%$ of cases. Up to $17 \%$ required more than one intervention. At day 7 , clinical resolution was achieved for $27 \%$, and improvement for $50 \%$. Clinical failure at day 7 was reported for $18 \%$ and $5 \%$ had died. Day 28 mortality was $37 \%$ in the whole cohort. At that time point $22 \%$ were still in the ICU and $21 \%$ still in hospital. A detailed analysis of the epidemiology, comprising a description and multivariable analysis of center, patient, pathogen, and management factors associated with mortality and morbidity will be provided.

Conclusion. HA-BSI is associated with significant morbidity and mortality as one month after the infection as $43 \%$ patients were still in hospital and $37 \%$ had died.

\section{Reference(s)}

1. A grant of 5000 Australian dollars has been awarded by the Norva Dahlia foundation specifically to facilitate patient inclusions in Australia.

2. A grant of 30000 euros has been awarded by the ESCMID for operational management of the study and statistical analysis. 
3. A grant of 25000 euros has been awarded by the ESICM Trials Group for operational management of the study and eCRF management.

4. A grant of 9400 Australian dollars has been awarded by the Redcliffe Hospital Private Practice Trust Fund to complete the study

\section{4}

Prevention of ventilator-associated pneumonia by noble metal coating of endotracheal tubes: A randomized, controlled, double-blind, multicenter, prospective pilot study

P. Damas ; B. Lambermont ${ }^{2}$; C. Legrain ${ }^{3}$; AF. Rousseau ${ }^{4}$; P. Demaret'; B. Misset ${ }^{6}$

${ }^{1}$ Chu De Liège, Liège, Belgium; ${ }^{2}$ Intensive care, University Hospital of Liège, Liège, Belgium; ${ }^{3}$ Intensive care, Clinical Chc Montlégia, Liège, Belgium; ${ }^{4}$ Intensive care, University Hospital of Liège, Liège, France;

${ }^{5}$ Department of anesthesia and intensive care., MontLegia HospitalGroupe Santé CHC, Liège, Belgium; ${ }^{6}$ Department of Intensive Care, CHU de Liège, Avenue de L'Hòpital, Liège, Belgium, Liège, Belgium Correspondence: P. Damas

Intensive Care Medicine Experimental 2020, 9(1): 001394

Introduction. Prevention of ventilator-associated pneumonia (VAP) in intensive care units (ICU) is currently based on several measures $[1,2]$. However, VAP still remains a significant clinical problem in mechanically ventilated ICU patients. Specifically, during the ongoing pandemic it has been demonstrated that VAP constitutes $50 \%$ of hospital-acquired infections in ICU treated COVID-19 patients [3].

An endotracheal tube (ETT) coated with a sub-micron layer of noble metals (gold, silver and palladium) firmly attached to the surface without significant release into the body (Bactiguard Infection Protection, BIP, Tullinge, Sweden) is available in Europe. The galvanic effect of noble metal alloys creates a microcurrent that reduces bacterial colonization and biofilm formation on the ETTs and thus reduces risk of subsequent respiratory infection.

Objectives. This study was done to test the potential benefit of antiinfective noble metal coating of ETTs for the prevention of VAP.

Methods. The study was a randomized, controlled, double-blind, multicenter prospective pilot study including adult patients in need of mechanical ventilation $>24 \mathrm{~h}$. Patients were randomly intubated with identical looking coated (noble metal alloy [NMA]-coated group) or non-coated (control group) ETTs. Primary endpoint was the incidence of VAP. Secondary endpoints were the proportion of antibiotic days during ICU stay and tracheal colonization by pathogenic bacteria.

Results. In total, 323 patients were enrolled; 168 in the NMA-coated group and 155 in the control group. No differences were observed with respect to age, sex, comorbidities, type of patients, cause of admission, SAPS II and SOFA score at admission.

VAP occurred in 11 patients (6.6\%) in the NMA-coated group and in 18 patients $(11.6 \%)$ in the control group $(p=0.11)$. VAP occurrence with respect to ventilation days was $10.5 \mathrm{VAP} / 1000 \mathrm{VD}$ in the NMA-coated group vs 22.4 VAP/1000 VD in the control group $(p=0.066)$. Using Cox $\mathrm{PH}$ regression analysis with adjustment for antibiotic use at admission, a statistically significant difference was noted when analyzing time to VAP suspicion $(p=0.009)$ or confirmed VAP $(p=0.022)$ (HR $0.41,95 \%$ Cl 0.19-0.88) (Fig. 1).

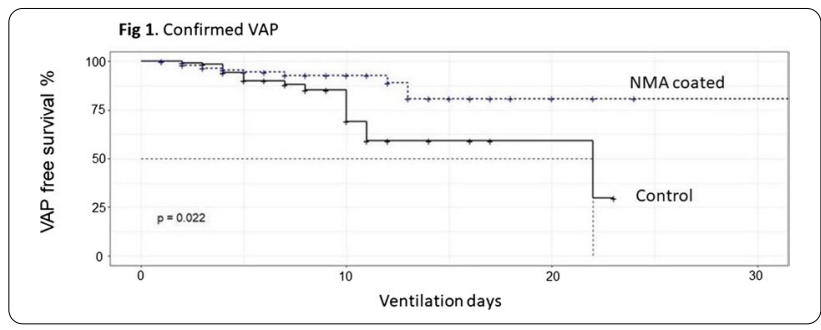

Conclusion. The study provided preliminary evidence to support the benefit of noble metal coating in the prevention of VAP. A larger confirmatory study would be valuable.
Reference(s)

1. Grasselli G, Scaravilli V, Mangioni D, Scudeller L, Alagna L, Bartoletti M, Bellani G, Biagioni E, Bonfanti P, Bottino N, Coloretti I, Cutuli SL, De Pascale G, Ferlicca D, Fior G, Forastieri A, Franzetti M, Greco M, Guzzardella A, Linguadoca S, Meschiari M, Messina A, Monti G, Morelli P, Muscatello A, Redaelli S, Stefanini F, Tonetti T, Antonelli M, Cecconi M, Foti G, Fumagalli R, Girardis M, Ranieri M, Viale P, Raviglione M, Pesenti A, Gori A, Bandera A, (2021) Hospital-acquired infections in critically-ill COVID-19 patients. Chest (online, in press)

2. Torres A, Niederman MS, Chastre J, Ewig S, Fernandez-Vandellos P, Hanberger H, Kollef M, Li Bassi G, Luna CM, Martin-Loeches I, Paiva JA, Read RC, Rigau D, Timsit JF, Welte T, Wunderink R, (2017) International ERS/ESICM/ESCMID/ALAT guidelines for the management of hospitalacquired pneumonia and ventilator-associated pneumonia: Guidelines for the management of hospital-acquired pneumonia (HAP)/ventilatorassociated pneumonia (VAP) of the European Respiratory Society (ERS), European Society of Intensive Care Medicine (ESICM), European Society of Clinical Microbiology and Infectious Diseases (ESCMID) and Asociación Latinoamericana del Tórax (ALAT). Eur Respir J 50:1,700,582

3. Kalil AC, Metersky ML, Klompas M, Muscedere J, Sweeney DA, Palmer LB, Napolitano LM, O'Grady NP, Bartlett JG, Carratalà J, El Solh AA, Ewig S, Fey PD, File TM, Jr., Restrepo MI, Roberts JA, Waterer GW, Cruse P, Knight SL, Brozek JL, (2016) Management of Adults With Hospital-acquired and Ventilator-associated Pneumonia: 2016 Clinical Practice Guidelines by the Infectious Diseases Society of America and the American Thoracic Society. Clin Infect Dis 63:e61-e111

4. Bactiguard $A B$, Sweden provided study tubes and funding for 2 nurses for 2 years.

Best Oral Presentations 3

\section{2}

ICU acquired weakness as a predictor of discharge destination in patients admitted to Intensive care with COVID 19 requiring mechanical ventilation

J. Weblin ; A. Harriman²; D. McWilliams ${ }^{3}$

${ }^{1}$ Physiotherapy, University Hospitals Birmingham NHS foundation

Trust, Birmingham, United Kingdom; ${ }^{2}$ Therapy services, Queen Elizabeth Hospital Birmingham, Birmingham, United Kingdom; ${ }^{3}$ Centre for care excellence, University Hospital Coventry \& Warwickshire, Coventry, United Kingdom

Correspondence: J. Weblin

Intensive Care Medicine Experimental 2020, 9(1): 000242

Introduction. Intensive care acquired weakness (ICU-AW) is a common complication in patients admitted with COVID-19 requiring mechanical ventilation (MV) (1). Previous studies have shown ICUAW to be associated with greater functional impairment in survivors of critical illness (2), necessitating the need for ongoing rehabilitation both within hospital and following discharge. Little is currently known regarding the impact of $I C U-A W$ on recovery and rehabilitation needs in survivors of COVID-19.

Objectives. To ascertain if the presence of ICU-AW at ICU discharge can predict hospital discharge destination in patients with COVID-19 requiring MV.

Methods. Adults admitted to a large UK ICU between January-March 2021 with a confirmed diagnosis of COVID 19, and MV $>24 \mathrm{~h}$ were included in analysis. Patients with known neurological insults i.e. CVA were excluded from the analysis. The presence of ICU-AW was assessed using the MRC sum score at ICU discharge, and classed as either 'significant' $(<48 / 60)$ or severe $(<36 / 60)$ (3). Hospital discharge destination was categorised as either home, home with Rehabilitation, or Inpatient Rehabilitation.

Results. 117 patients were analysed, of which 60 (51\%) presented with ICU-AW at ICU discharge. The development of persisting ICU-AW at ICU discharge was associated with longer periods of sedation (15 vs 6.5 days, $p<0.0001)$, longer duration of MV (27 vs 7 days, $p<0.0001)$ and longer lengths of stay in both ICU (31 vs 10 days, $p<0.0001$ ) and hospital (42 vs 19 days, $p<0.0001$ ). It was also noted patients were slower to mobilise (18 vs 7 days, $p<0.0001$ ), had lower mobility 
levels at both ICU (MMS 4 vs 5, p0.0001) and hospital discharge (MMS 6 vs 7, p0.0001). Patients with ICU-AW were significantly more likely to require ongoing rehabilitation either at home or as an inpatient $(87 \%$ vs $28 \%,<0.0001$ ). A sub-analysis (Table 2 ) demonstrated patients with severe ICU-AW were most likely to require in-patient rehabilitation $(12 / 28,43 \%)$.

Table 1 Pt demongraphics and outcomes

\begin{tabular}{llll}
\hline & MRC $<48(n=60)$ & MRC $\geq 48(n=57)$ & P value \\
\hline Gender (male) & $29(48 \%)$ & $42(74 \%)$ & 0.002 \\
Mean age (SD) & $56.4(9.87)$ & $53.4(10.98)$ & 0.14 \\
Mean APACHE II (SD) & $14.4(4.17)$ & $12.2(3.56)$ & 0.005 \\
Clinical frailty score & $2(1-3)$ & $2(2-3)$ & 0.79 \\
Sedation days & $15(7-25)$ & $6.5(1.75-10)$ & $<0.0001$ \\
Ventilation days & $27(11-37)$ & $7(3-17.75)$ & $<0.0001$ \\
Time to mobilise (days) & $18(9-29)$ & $7(2.5-11)$ & $<0.0001$ \\
MMS ICU discharge & $4(3-4)$ & $5(5-6)$ & 0.0001 \\
MMS hospital d/c & $\mathbf{6 ( 6 - 7 )}$ & $7(7-7)$ & 0.0001 \\
ICU LOS (days) & $\mathbf{3 1 ( 1 3 - 4 0 )}$ & $10(6.5-21)$ & $<0.0001$ \\
Hospital LOS (days) & $\mathbf{4 2 ( 2 8 - 5 8 )}$ & $19(9.5-31)$ & $<0.0001$ \\
Discharge destination & & & $<0.0001$ \\
Ongoing rehabilitation & $\mathbf{5 2 ( 8 7 \% )}$ & $\mathbf{1 6}(28 \%)$ & \\
\hline
\end{tabular}

Table 2 Discharge destination dependant on severity of ICU-AW

\begin{tabular}{lllll}
\hline & $\begin{array}{l}\text { MRC }<\mathbf{3 6} \\
(\mathbf{n}=\mathbf{2 8})\end{array}$ & $\begin{array}{l}\text { MRC }<\mathbf{4 8} \\
(\mathbf{n}=\mathbf{3 2})\end{array}$ & $\begin{array}{l}\text { MRC } \geq \mathbf{4 8} \\
(\mathbf{n}=\mathbf{5 7})\end{array}$ & p valve \\
\hline Home & $3(11 \%)$ & $5(16 \%)$ & $41(72 \%)$ & $<\mathbf{0 . 0 0 0 0 1}$ \\
$\begin{array}{l}\text { Home with } \\
\text { rehab }\end{array}$ & $13(46 \%)$ & $21(66 \%)$ & $15(26 \%)$ & \\
$\begin{array}{l}\text { Inpatient rehab } \\
\text { How }\end{array}$ & $12(43 \%)$ & $6(19 \%)$ & $1(2 \%)$ & \\
\hline
\end{tabular}

Conclusion. Patients admitted to ICU with a diagnosis of COVID-19 with ongoing ICU-AW at ICU discharge are significantly more likely to require ongoing rehabilitation either at home or as an inpatient than those without. Routine screening for the presence of ICU-AW with the MRC score at ICU discharge could therefore help manage patient expectations, allow pragmatic and early discharge planning, and provide an objective measure for rehab physicians to utilise and predict recovery trajectories in patients with COVID-19.

\section{Reference(s)}

1. McWilliams D, Weblin J, Hodson J, Veenith T, Whitehouse T, Snelson C. Rehabilitation Levels in Patients with COVID-19 Admitted to Intensive Care Requiring Invasive Ventilation. An Observational Study. Ann Am Thorac Soc. 2021 Jan;18(1):122-129

2. Jolley SE, Bunnell AE, Hough CL. ICU-Acquired Weakness. Chest. 2016 Nov; 150(5): 1129-1140.

3. Hermans G, Clerckx B, Vanhullebusch T, Segers J, Vanpee G, Robbeets $C$, et al. Interobserver agreement of Medical Research Council sumscore and handgrip strength in the intensive care unit. Muscle Nerve. 2012;45(1):18-25.

000370

Long-term application of Selective digestive decontamination in a mixed ICU: impact on nosocomial multi-resistant infection, antibiotic consumption and colistin- and tobramicyn colonization C. Sánchez Ramírez'; S. Hípola Escalada'; RE. Morales Sirgado';

MA. Hernández Viera'; M. Cabrera Santana'; C. Rodriguez Mata';
ME. Gallardo Santos ${ }^{1}$; CF. Lübbe Vázquez'; SM. Marrero Penichet; A. Padrón Mujica'; P. Saavedra-Santana ${ }^{3}$; S. Ruiz-Santana ${ }^{1}$

${ }^{1}$ Intensive care medicine, University Hospital of Gran Canaria Dr. Negrin, Las Palmas de Gran Canaria, Spain; ${ }^{2}$ Pharmacy department, University Hospital of Gran Canaria Dr. Negrin, Las Palmas de Gran Canaria, Spain; ${ }^{3}$ Mathematics and informatics deparment, University of Las Palmas:, Las Palmas de Gran Canar

ia, Spain

Correspondence: C. Sánchez Ramírez

Intensive Care Medicine Experimental 2020, 9(1): 000370

Introduction. Selective digestive decontamination (SDD) have been associated with reduced mortality and lower pulmonary tract nosocomial infection (NI) rates However, the effect SDD in areas where multidrug-resistant Gram-negative bacteria are endemic is of great interest. Objectives. To prospectively evaluate the impact of longterm SDD application on muli-resistant (MR) NI, antibiotic consumption and colistin- and tobramicyn colonization rates in a mixed ICU. Methods. This study was conducted in a 30-bed-medical-surgical ICU. All consecutive patients admitted to the ICU from October 1 , 2011 to June 30, 2020 expected to require tracheal intubation $>48 \mathrm{~h}$ were given SDD (SDD study group) with a 4-day course of intravenous cefotaxime, plus enteral colistin, tobramycin, nystatin in an oropharyngeal paste and in a digestive solution. Oropharyngeal and rectal swabs were obtained on admission and once weekly. We used ENVIN NI criteria. We compared all patients admitted to ICU with ICU $\mathrm{NI}$ from October 1, 2010 to September 30, 2011 (non-SDD group) to the SDD study group. A univariate and a multivariate logistic regression analysis were performed. For each one of the NI the incidences per 1000 days of exposure in each cohort and the corresponding relative risks were obtained using the Poisson regression. Statistical significance was $p \leq 0.05$. We analyzed colistin- and tobramycinresistant colonization and also antibiotic consumption as Defined antibiotics Daily Doses (DDD).

Results. Results are shown in Tables 1, 2 and 3.

\begin{tabular}{|c|c|c|c|}
\hline & \multicolumn{2}{|c|}{ Selective decontamination of the digestive tract } & \multirow[b]{2}{*}{$\mathrm{P}$} \\
\hline & $\begin{array}{c}\text { No } \\
N=138\end{array}$ & $\begin{array}{c}\text { Yes } \\
N=577\end{array}$ & \\
\hline Age, years & $60.0 \pm 14.8$ & $61.5 \pm 14.9$ & 0.286 \\
\hline APACHE II score & $21.2 \pm 7.9$ & $21.7 \pm 7.7$ & 0.507 \\
\hline Sex male & $94(68.1)$ & $374(64.9)$ & 0.024 \\
\hline Trauma patients & $19(13.8)$ & $63(10.9)$ & 0.349 \\
\hline Coronary arteria disease patient & $19(13.8)$ & $131(22.7)$ & 0.036 \\
\hline Emergency surgery & $34(24.6)$ & $135(23.4)$ & 0.202 \\
\hline Immunosupression & $3(2.2)$ & $2(0.3)$ & 0.052 \\
\hline Parenteral nutrition & $27(19.6)$ & $145(25.1)$ & 0.169 \\
\hline RRT & $41(29.7)$ & $224(38.8)$ & 0.094 \\
\hline Malnutrition & $15(10.9)$ & $56(9.7)$ & 0.188 \\
\hline Diabetes mellitus & $43(31.2)$ & $186(32.2)$ & 0.808 \\
\hline COPD & $15(10.9)$ & $96(16.6)$ & 0.093 \\
\hline Renal failure & $45(32.6)$ & $112(19.4)$ & $<0.001$ \\
\hline VAP & $68(49.3)$ & $194(33.6)$ & $<0.001$ \\
\hline CRB & $38(27.5)$ & $216(37.4)$ & 0.099 \\
\hline Secondary bacteremia & $38(27.5)$ & $150(26.0)$ & 0.835 \\
\hline Urinary infection & $29(21.0)$ & $158(27.4)$ & 0.050 \\
\hline ATB 48 hours before admission & $34(24.6)$ & $157(27.2)$ & 0.016 \\
\hline Death & $45(32.6)$ & $208(36.0)$ & 0.448 \\
\hline Acinetobacter baumannii & $14(10.1)$ & $6(1.0)$ & $<0.001$ \\
\hline MRSA & $4(2.9)$ & $9(1.6)$ & 0.290 \\
\hline ESBL & $43(31.2)$ & $122(21.1)$ & 0.012 \\
\hline MR Pseudomonas & $13(9.4)$ & $43(7.5)$ & 0.580 \\
\hline MR GNB & $13(9.4)$ & $23(4.0)$ & 0.009 \\
\hline Admission & & & 0.126 \\
\hline Medical & $106(76.8)$ & $408(70.9)$ & \\
\hline Scheduled surgery & $10(7.2)$ & $82(14.2)$ & \\
\hline Emergency surgery & $22(15.9)$ & $86(14.9)$ & \\
\hline Inflammatory response & & & 0.004 \\
\hline No sepsis & $2(1.4)$ & $23(4.0)$ & \\
\hline Sepsis & $23(16.7)$ & $151(26.2)$ & \\
\hline Septic shock & $113(81.9)$ & $403(45.6)$ & \\
\hline ICU days & $28.0(17.0-45.8)$ & $33.0(19.8-50.2)$ & 0.039 \\
\hline
\end{tabular}


Table 2. Multivariate logistic regression for SDD

\begin{tabular}{cccc}
\hline & P-value & BIC & Odd-Ratio $(95 \% \mathrm{Cl})$ \\
\hline VAP & 0.004 & 687.6 & $0.563(0.382,0.830)$ \\
Renal failure & 0.003 & 687.7 & $0.525(0.343,0.804)$ \\
Acinetobacter infections & $<.001$ & 699.4 & $0.114(0.042,0.310)$ \\
\hline
\end{tabular}

SDD: Selective Digestive Decontamination; VAP: ventilator associated pneumonia.

\begin{tabular}{|c|c|c|c|c|c|}
\hline & & \multicolumn{2}{|c|}{$S D D$} & \multirow[b]{2}{*}{$\mathbf{p}$} & \multirow{2}{*}{$\begin{array}{c}\text { Relative Risk } \\
\quad(95 \% \mathrm{Cl})\end{array}$} \\
\hline & & No & Yes & & \\
\hline VAP IMV & VAP/1000 days of MV & 10.31 & 3.82 & $<0.001$ & $\begin{array}{c}0.37 \\
(0.28-0.49)\end{array}$ \\
\hline Urinary infections & $\begin{array}{l}\text { Infections } / 1000 \text { days of urinary } \\
\text { catheter }\end{array}$ & 3.79 & 2.30 & 0.008 & $\begin{array}{c}0.607 \\
(0.419-0.880)\end{array}$ \\
\hline CRB & CRB/1000 days of CVC & 3.59 & 3.57 & 0.981 & $\begin{array}{c}0.995 \\
(0.665-1.490)\end{array}$ \\
\hline $\begin{array}{l}\text { Secondary } \\
\text { bacteremias }\end{array}$ & Bacteremias./1000 ICU days & 4.69 & 1.98 & $<0.001$ & $\begin{array}{c}0.422 \\
(0.302-0.590)\end{array}$ \\
\hline $\begin{array}{l}\text { Multiresistant } \\
\text { germs }\end{array}$ & Multiresistant germs/1000 ICU days & 9.59 & 2.49 & $<0.001$ & $\begin{array}{c}0.260 \\
(0.203-0.333)\end{array}$ \\
\hline
\end{tabular}

SDD: Selective Digestive Decontamination, VAP
rolated bacteromia; CVC: Central venous catteter

A total of 8325 patients were collected, of them 715 developed NI.There were no statistical significant differences between both groups in type of admission or demographic data. Patients with SDD had significantly less Extended Spectrum Betalactamase (ESBL), Gram Negative Bacteria Multirresistant (GNB-MR) and Acinetobacter spp infections. We had also a significant reduction in ventilator associated pneumonias (VAP), urinary, other secondary bacteremias and multiresistant (MR)NI rates, in SDD group versus non SDD. There was no infection by Clostridium difficile. Colistin- resistant colonization was $16,5 \%$ and tobramycin-resistant colonization was $25,7 \%$ of samples. There was a decrease on the DDD/100 ICU stays after SDD.

Conclusion. After long-term application of SDD a significant reduction of infections by ESBL, GNB-MR and Acinetobacter, was observed. A significant decrease of VAP, secondary bacteremias, urinary and MRNI rates was also shown. An antibiotic consumption reduction was found after SDD. Low rates of colistin and tobramycin-resistant colonization bacteria were observed.

\section{4}

Development of a vectorized-IL-7 immunotherapeutic to stimulate innate and adaptive immunity for the treatment of immunosuppression in sepsis patients

K. Lelu-Santolaria'; C. Dubois'; M. Baldazza'; A. Evlachev' ${ }^{1}$ N. Kehrer ${ }^{1}$. R. Brandely ${ }^{2}$;. Schlesinger'; N. Silvestre ${ }^{2}$; J. Unsinger ${ }^{3}$; R. Hotchkiss ${ }^{3}$; P. Martin ; G. Inchauspe ${ }^{4}$

1 Immunresq department, TRANSGENE, Lyon 69,007, France; ${ }^{2}$ Vectorology department, TRANSGENE, Illkirch-Graffenstaden 67,405, France; ${ }^{3}$ Department of anesthesiology, medicine and surgery, Washington University School of Medicine, St. Louis, United States of America; Immunresq department, TRANSGENE, Lyon, France

\section{Correspondence: G. INCHAUSPE}

Intensive Care Medicine Experimental 2020, 9(1): 000404

Introduction. Persistence of a late immunosuppressive state plays a role in sepsis patient morbidity and late mortality. Impairment of both innate and adaptative pathways are associated with poor prognosis pointing towards the need for immune-interventions targeting both arms of the immune system.
Objectives. Evaluate the well-established non-replicative Modified Vaccinia virus Ankara (MVA) vaccine platform and its attractive features i.e. capacity to stimulate innate immunity, provide long-lasting expression of encoded transgenes and target multiple organs, to restore immune-homeostasis in sepsis patients.

Methods. A recombinant MVA, MVA-hIL7-Fc, encoding a human-IL-7 fused to a human $\mathrm{Fc}$ was engineered and tested in vivo in naive and septic (single-hit cecal-ligation-puncture or CLP) mice in comparison with the soluble hIL7-Fc counterpart. hIL7-Fc pharmacokinetics, immune activities in spleens and lungs as well as survival studies were monitored following a single intravenous injection. B and T cells and their memory subpopulations, NK and NKT cells, as well as their activation status together with myeloid subpopulations were analysed by flow cytometry. Functionality of T cells was assessed by triple Intracellular Cytokine Staining (IFNg, IL-2, TNFa) following TCR stimulation. Candida albicans challenges are ongoing.

Results. IIn naïve mice, post-injection (p.i) of MVA-hIL7-Fc, circulating hIL7-Fc was detected from $6 \mathrm{~h}$ until day 5. In CLP mice levels of detected hIL-7-Fc at time of sacrifice (3 days p.i) were 10-90 fold higher than those observed p.i of the soluble hll7-Fc. Major immunological activities were observed at 3 and 9 days p.i, suggesting sustained activity overtime. Basically, all cell types were strongly increased (eg 2 and fivefold increase of splenic CD4 $+/ C D 8+T$ and NK cells respectively compared with untreated mice) as well as their activation status in the 2 tested organs (eg up to sixfold increase of lung activated $C D 4+/ C D 8+T$ cells in comparison with untreated mice). $T$ cell memory subpopulations and functional T cells were significantly increased compared with injection of soluble hIL7-Fc (upto 20 fold increase of splenic IFN-g producing CD8 T cells compared with IL7-Fc treated mice). Soluble IL7-Fc mainly displayed activities at day 3 p.i in spleen and no or very limited activities in lungs. Similar cell increase and activities as seen in naïve mice were globally detected in CLP mice treated with MVA-hIL7-Fc, notably improvement of splenic $\mathrm{T}$ cell functions (production of IFNg $\pm \mathrm{TNFa} \pm \mathrm{IL}-2$ ) and restoration of splenic lymphopenia. MVA-hIL7-Fc treated CLP mice showed better survival rate (96\%) while no survival benefit was seen p.i of the soluble hIL7-Fc which did not improve survival seen in untreated CLP mice (87\%).

Conclusion. This proof-of-concept study demonstrates the clinical relevance of the MVA-hIL7-Fc and support its use as a novel and highly effective immunotherapeutic in sepsis.

\section{9}

High prevalence of complicated grief among bereaved relatives after death of the patient in the ICU during the first wave of COVID-19

M. Van Mol' ; S. Wagener'; J. Rietjens ${ }^{2}$; C. Den Uil ${ }^{1}$

${ }^{1}$ Departement of intensive care, Erasmus University Medical Center, Rotterdam, Netherlands; ${ }^{2}$ Department of public health, Erasmus University Medical Center, Rotterdam, Netherlands

Correspondence: M. Van Mol

Intensive Care Medicine Experimental 2020, 9(1): 000409.

Introduction. During the COVID-19 pandemic, family-centered policies in the ICU were impacted by visiting restrictions, undesirable physical distance to the patient, and difficulties to involve the relatives directly [1]. This situation might increase psychological distress among bereaved relatives in the ICU, including complicated grief (CG). CG is characterized by extreme symptoms of grief, separation distress, intrusive thoughts, and feelings of meaninglessness for more than 6 months post-loss.

Objectives. This study aims to explore the prevalence of complicated grief and related psychological distress such as depression, anxiety and posttraumatic stress in bereaved relatives in the ICU.

Methods. This cross-sectional questionnaire study is part of the larger BRIC study in a Dutch ICU (48 beds, upscaling to 72 beds during the COVID-19 pandemic) [2]. Here, we report about the second cohort, which we stratified by the date of the patient's ICU death: September 2019-February 2020 (group 1; $n=114$, patients 
dying before the COVID pandemic) and March-May 2020 (group 2 , patients dying during the 1st COVID-19 wave; $n=43$ ). All first and second contact persons as reported in the medical file were approached by telephone for study participation $(n=269)$ of which 163 consented to participate (61\%). These relatives were invited to complete a survey measuring CG (Traumatic Grief Inventory-Self Report Version, 19 items; cut-off $>61$ ), anxiety and depression (Hospital Anxiety and Depression Scale, 14 items) and posttraumatic stress (Impact of Events Scale, 22 items).

Results. In total, 121 respondents completed the questionnaire at 6 months after death (crude response rate $=74 \%$, 86 relatives in group 1 and 35 in group 2). A follow-up questionnaire at 12 months was completed by 48 respondents. Most respondents were partners of the patient $(52 \%)$, were female $(71 \%)$ and had a mean age of 52 years (range $20-83$ ). The prevalence of CG was $1.3 \%$ and $48.6 \%$ in group 1 and 2, respectively, and $11.1 \%$ at follow-up. Means on psychological distress are presented in Table 1.

\begin{tabular}{|c|c|c|c|c|}
\hline & Group 1 & Group 2 & Follow-up & ANOVA \\
\hline Complicated grief & $37.4 \pm 8.8$ & $60.9 \pm 9.4$ & $39.0 \pm 14.7$ & $F=11.52 \quad p<.000$ \\
\hline Anxiety & $6.9 \pm 2.3$ & $9.2 \pm 1.9$ & $7.2 \pm 3.1$ & $F=9.43 \quad p<.000$ \\
\hline Depression & $9.4 \pm 2.4$ & $11.8 \pm 1.6$ & $9.1 \pm 2.7$ & $F=60.20 \quad p<.000$ \\
\hline Posttraumatic stress & $31.6 \pm 10.5$ & $56.24 \pm 11.2$ & $30.5 \pm 17.0$ & $F=44.57 p<.000$ \\
\hline
\end{tabular}

Assessment of communication with doctors on a scale from 0 to 10 was higher in group 1 (mean 6.2 \pm 1.4 ) compared to group 2 (mean $4.9 \pm 1.8$ ). The same trend was found on communication with nurses (mean $8.9 \pm 0.9$ versus $6.0 \pm 2.2$, respectively) and on support after the patient's death ( $8.1 \pm 0.9$ versus $5.5 \pm 1.9$, respectively).

Conclusion. The COVID-19 pandemic, with quarantine measures and subsequently changed family-centered ICU policies, had a negative impact on psychological distress in bereaved relatives with a high prevalence of complicated grief. In addition, communication and support, as a quality indicator of care, was perceived low.

\section{Reference(s)}

1. Jeitziner M, et al., End-of-life care during the COVID-19 pandemic-What makes the difference? Nurs Crit Care, 2021.

2. Van Mol M, et al., Developing and testing a nurse-led intervention to support bereavement in relatives in the intensive care (BRIC study): a protocol of a pre-post intervention study. BMC Pall Care, 2020. 19(1): p. 1-10

3. This work has been supported by a grant from Erasmus MC "Evidence Based Care by Nurses" (EBCN), project 2017-17,407, which had no role in the design of this study and has no role in its execution, analysis and interpretation of data, or publication of results.

\section{4}

The systemic inflammatory response induced by LPS administration is more pronounced in women than in men A. Jansen ${ }^{1}$; N. Bruse ${ }^{2}$; N. Waalders ${ }^{1}$; P. Pickkers ${ }^{1}$; M. Kox ${ }^{1}$

${ }^{1}$ Intensive care, Radboud University Medical Center, Nijmegen, Netherlands; ${ }^{2}$ Department of internal medicine, Radboud University Medical Center, Nijmegen, Netherlands

Correspondence: A. Jansen

Intensive Care Medicine Experimental 2020, 9(1): 000454.

Introduction. The sepsis induced inflammatory response is highly heterogeneous and comprises both hyperinflammatory and immunosuppressive phenotypes. Female patients have a lower incidence of sepsis and a better clinical outcome. A better understanding of the potential sex-specific differences in the immune response may facilitate personalized treatment approaches.

Objectives. To investigate whether sex affects the immune response and the development of endotoxin tolerance in a large cohort of healthy volunteers undergoing repeated experimental human endotoxemia.

Methods. Fifty-six male and 54 female healthy volunteers were included in this prospective experimental cohort study. On day 0 , subjects were intravenously challenged with $1 \mathrm{ng} / \mathrm{kg}$ bacterial lipopolysaccharide (LPS) to induce a transient systemic inflammatory response. This challenge was repeated on day 7 to determine the degree of endotoxin tolerance. Blood samples were serially obtained to construct plasma cytokine time-concentration curves. Areas under the time-concentration curve (AUCs) were calculated to provide an integral measure of the cytokine response. Temperature and reported flu-like symptoms were recorded every $30 \mathrm{~min}$.

Results. Median [interquartile range] age was 23 [21-25] years for males and 23 [21-24] years for females $(p=0.18)$, whereas BMI was 23.0 [20.8-25.1] and $23.6[21.9-25.7] \mathrm{kg} / \mathrm{m} 2$, respectively $(p=0.12)$. LPS administration resulted in a profound increase in plasma cytokines for both sexes on both days (Fig. 1). However, compared with males, females produced significantly higher levels of tumour necrosis factor (TNF)-a (41\% higher median AUC on day 0 in females), interleukin (IL)-6 $(+50 \%)$, interferon gamma induced protein (IP)-10 $(+47 \%)$, and IL-1 receptor antagonist (+112\%), but not IL-10 (4\% lower in females). Peak symptom scores were also higher in females than in males upon both LPS challenges (+33\%, Fig. 2). Nevertheless, the LPS-induced maximum increase in body temperature was not different between females and males $(-14 \%$, Fig. 2). Although a tolerant response was observed for all measured cytokines and for the symptom score upon the second LPS challenge (all $p<0.0001$ vs. first challenge), no differences in the degree of endotoxin tolerance between the sexes were observed (Figs. 1 and 2).

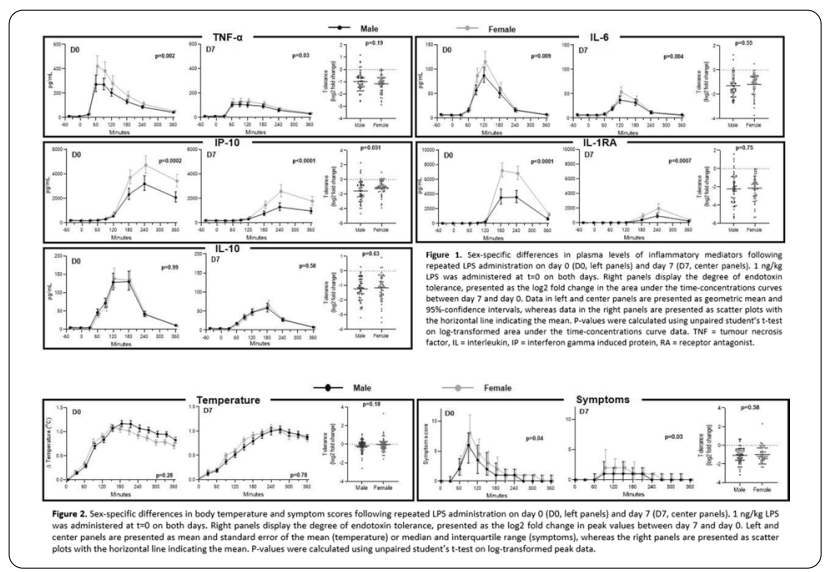

Conclusion. In a large cohort of healthy volunteers, we demonstrate that females mount a more pronounced proinflammatory cytokine response and report more flu-like symptoms following LPS administration than males, while levels of the anti-inflammatory cytokine IL-10 and the fever response were not different between the sexes. Furthermore, there were no differences in the development of endotoxin tolerance between men and women. These findings indicate sex-specific regulation of the innate immune response. Sex hormone profiles are currently being determined to assess whether these differences have a hormonal origin.

\section{Best Oral Presentations 4}

000711

ICU Care is Associated with Improved Survival in COVID-19 Patients Registered in the ISARIC WHO Clinical Characterisation Protocol: a Prospective, Multinational, Multicentre, Observational Study

ICG. Isaric Characterization Group ${ }^{1}$; LF. Reyes ${ }^{2}$

${ }^{1}$ Isaric, ISARIC, Oxford, United Kingdom; ${ }^{2}$ Critical Care Medicine-Infectious Disease Department, Universidad de la sabana, Bogotá, Colombia Correspondence: L.F. Reyes

Intensive Care Medicine Experimental 2020, 9(1): 000711

Introduction. Around one-third of hospitalized patients with COVID19 develop severe disease, requiring advanced oxygen support or 
admission to an intensive care unit (ICU). Due to the large number of patients with severe COVID-19, many were treated outside of the traditional walls of the ICU, and in many cases, by personnel who were not trained in critical care. The relative impact of caring for severe COVID-19 patients outside of the ICU and their clinical characteristics is unknown.

Objectives. To determine the impact of ICU admission on 28-day mortality in patients with severe COVID-19.

Methods. This was a multinational, multicentre, prospective cohort study embedded in the ISARIC WHO COVID-19 platform. Patients with confirmed rtPCR for SARS-CoV-2 that required hospital admission were registered prospectively. Severe COVID-19 patients were identified as those admitted to an ICU and/or those treated with one of the following treatments: invasive or non-invasive mechanical ventilation, high-flow nasal cannula, inotropes, and vasopressors. Descriptive statistics and logistic regressions were used to describe the clinical characteristics and compare clinical outcomes among patients admitted and not to the ICU.

Results. A total of 40,440 patients from 43 countries and six continents were included in this analysis. Severe COVID-19 patients were more frequently male (63\%), older adults (median [IQR], 67 years [55, $78]$ ), and with at least one comorbidity (70\%). The overall median (IQR) length of hospital stay was 10 days (5-19) and was longer in patients admitted to an ICU than in those that were cared for outside of ICU (12 [6-23] vs. 8 [4-15] days, $p<0.0001)$. The 28-day mortality was lower in ICU-admitted patients ( $31 \%$ vs. $39 \%, p<0.0001)$. Patients admitted to an ICU had a significantly lower probability of death than those who were not (adjusted OR 0.56 [95\% Cl 0.52-0.60]; $p<0.0001$ ).

Conclusion. Patients with severe COVID-19 admitted to an ICU had significantly lower 28-day mortality than those cared for outside of an ICU.

\section{Reference(s)}

1. This work was supported by the UK Foreign, Commonwealth and Development Office and Welcome [215091/Z/18/Z, the Bill \& Melinda Gates Foundation [OPP1209135], the Health Research Board of Ireland [CTN2014-012] and the Canadian Institutes of Health Research Coronavirus Rapid Research Funding Opportunity [OV2170359].

\section{8}

Clinical evaluation of a novel continuous absolute end-expiratory lung volume monitoring method based on the capnodynamic principle

JA. Sanchez Giralt ; M. Wallin²; M. Hallbäck ; T. Gerardo ${ }^{4}$; M. Sánchez Galindo ${ }^{5}$; B. Abad Santamaría ; MA. Pérez Lucendo ${ }^{1}$; A. Canabal Berlanga1; F. Suarez-Sipmann ${ }^{6}$

${ }^{1} \mathrm{ICU}$, Hospital de La Princesa, Madrid, Spain; ${ }^{2}$ Physiology and pharmacology, Karolinska Institute, Stockholm, Sweden; ${ }^{3}$ Maquet critical care, MAQUET Critical Care, Solna, Sweden; ${ }^{4}$ Anesteshia, Hospital Español de Rosario, Rosario, Argentina; ${ }^{5}$ TU, Hospital de La Princesa, Madrid, Spain; ${ }^{6}$ Intensive care medicine department, Hospital de La Princesa, Madrid, Spain

Correspondence: J.A. Sanchez Giralt

Intensive Care Medicine Experimental 2020, 9(1): 000858.

Introduction. The reduction in end-expiratory lung volume (EELV) during mechanical ventilation is closely related to the risk of an increased mechanical strain. However, measuring EELV at the bedside is not easy. One available clinical approximation to continuously monitor EELV is by means of electrical impedance tomography (EIT) that can track relative changes in EIT aeration. Capnodynamic EELV monitoring (EELVCO2) is a new method based on expired $\mathrm{CO} 2$ kinetics and the differential Fick equation that provides breath-by-breath absolute values of EELVCO2.

Objectives. To assess the ability of the capnodynamic method to continuously monitor changes in EELVCO2 in response to changes in PEEP by comparing it with simultaneous changes in EIT aeration.

Methods. ICU patients under passive mechanical ventilation and continuously monitored with EELVCO2 and EIT were submitted to the following protocol: Three incremental PEEP steps of $5 \mathrm{cmH} 2 \mathrm{O}$ starting at 5 or if poorly tolerated at $10 \mathrm{cmH} 2 \mathrm{O}$. This was followed by a recruitment maneuver (PEEP $20 \mathrm{cmH} 2 \mathrm{O}$ + Driving pressure $20 \mathrm{cmH} 2 \mathrm{O}$ ) and the same PEEP steps now in an inverse decremental order. EIT (Enlight 1800 ;Timpel ${ }^{\circledR}$ ) changes in aeration were expressed relative to the first incremental PEEP step in arbitrary units. The capnodynamic method is based on the generation of cyclic changes in alveolar $\mathrm{CO} 2$ concentration. These changes are induced by a modified breathing pattern applied by a Servo-i ventilator, consisting of the addition of a short expiratory pause to the last three consecutive breaths of a sequential repetitive series of 9 breaths needed to solve the capnodynamic equation. Correlation between variables was assessed by Pearson's correlation coefficient and concordance in the trending ability by the four-quadrant plot analysis.

Results. Data from 16 mixed ICU patients were included. Clinical characteristics of the patients at time of enrollment (mean \pm SD): $\mathrm{PaO} 2 / \mathrm{FIO} 2244 \pm 100 \mathrm{mmHg}, \mathrm{FlO} 20.47 \pm 0.2, \mathrm{PaO} 2102 \pm 29 \mathrm{mmHg}$, $\mathrm{PaCO} 242 \pm 8 \mathrm{mmHg}$, Compliance $42 \pm 8 \mathrm{ml} / \mathrm{cmH} 2 \mathrm{O}$, PEEP $7 \pm 2$ $\mathrm{cmH} 2 \mathrm{O}$, driving pressure $12 \pm 1 \mathrm{cmH} 2 \mathrm{O}, \mathrm{Vt} 435 \pm 63 \mathrm{ml}$. A total of 96 paired (EELVCO2 and EIT) measurements were included in the analysis. Incremental and decremental values of EELVCO2 and EIT aeration were significantly correlated $(r 2=0.627 \mathrm{p}<0.01)$. Concordance between methods of all paired values was $92 \%$ (Fig. 1). Concordance was better in the decremental PEEP steps (95\%) as compared with the incremental PEEP steps (90\%). Measured absolute EELVCO2 values at each decremental PEEP step were higher than its corresponding incremental PEEP steps with an absolute difference of $+79.3 \pm 198 \mathrm{ml}$ $(\mathrm{p}<0.05),+125.4 \pm 315 \mathrm{ml}(\mathrm{NS})$ and $+140.9 \pm 260 \mathrm{ml}(\mathrm{NS})$.

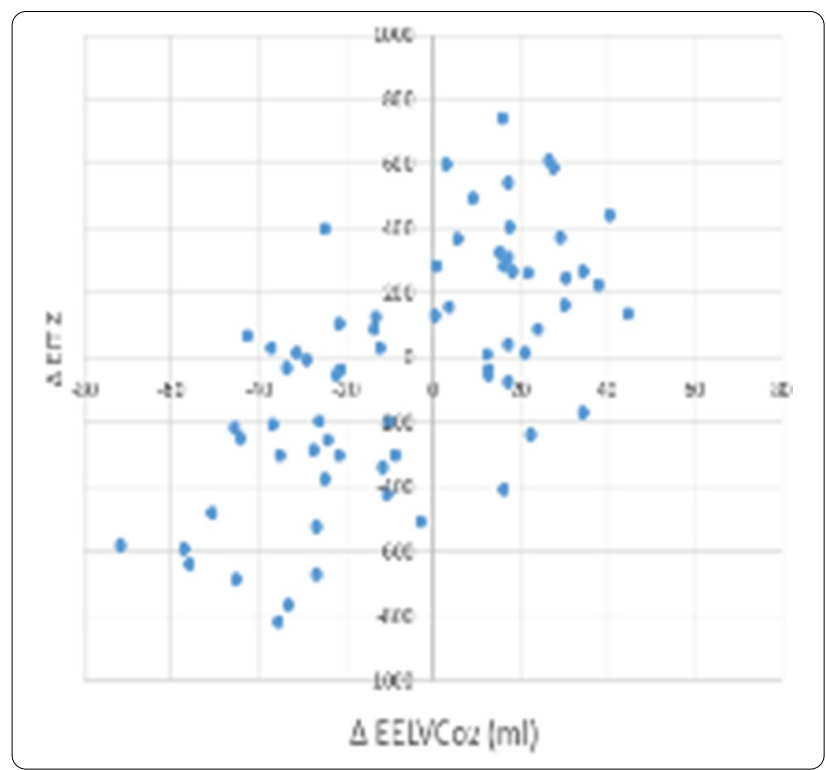

Conclusion. Capnodynamic EELVCO2 correlated well and had a high concordance with EIT changes in aeration. This is the first clinical method that provides absolute continuous breath-by-breath static volume values. If further validated in different ICU populations and lung conditions it can become a promising bedside monitoring option.

\section{Reference(s)}

1. 001170 Validation of a novel non-invasive continuous method to monitor end expiratory lung volume based on CO2 kinetics. JA. Sanchez Giralt, B. Abad Santamaría, M. Wallin, M. Hallbäck, M. Trigueros Genao, J. Lázaro Gonzalez, B. Muñoz Molina, G. Tusman, F. Suarez Sipmann. Intensive Care Medicine Experimental 2020, 8(Suppl 2):73 https://doi.org/10.1186/ s40635-020-00354-8

2. Öhman T, Sigmundsson TS, Hallbäck M, Suarez Sipmann F, Wallin M, Oldner A, Björne H, Hällsjö Sander C. Clinical and experimental validation 
of a capnodynamic method for end-expiratory lung volume assessment. Acta Anaesthesiol Scand. 2020 May;64(5):670-676. https://doi.org/10. 1111/aas.13552. Epub 2020 Jan 30. PMID: 31965563.

3. Grant PI20/01548 from the Fondo de Investigaciones Sanitarias. Instituto de salud Carlos III

\section{1}

Rehabilitation levels in patients admitted to intensive care with COVID-19: An observational cohort study

M. Crinage ${ }^{1}$; C. Woods ${ }^{1}$; L. Cawtheray'; D. McWilliams ${ }^{2}$

${ }^{1}$ Physiotherapy, University Hospital Coventry \& Warwickshire, Coventry, United Kingdom; ${ }^{2}$ Centre for care excellence, University Hospital Coventry \& Warwickshire, Coventry, United Kingdom

Correspondence: D. McWilliams

Intensive Care Medicine Experimental 2020, 9(1): 000871

Introduction. Patients with COVID-19 admitted to intensive care units (ICU's) often require prolonged periods of respiratory support, resulting in an increased risk of developing ICU acquired weakness [1]. Early and structured rehabilitation has been shown to be feasible in patients with a confirmed diagnosis of COVID-19, resulting in improved mobility status at the point of ICU discharge (Manchester mobility score of 5-step transfer) although this was limited to patients who were invasively ventilated and within a single centre [2]. More research is required to understand rehabilitation needs for patients admitted to ICU with COVID-19 in order to inform and improve planning of ICU rehabilitation services for future waves.

Objectives. 1 Describe the characteristics and demographics, level of rehabilitation and mobility status of patients admitted to ICU with a diagnosis of COVID-19. 2. To compare rehabilitation outcomes between invasively ventilated and non-ventilated patients

Methods. All adults admitted to ICU with a confirmed diagnosis of COVID-19, with a length of stay greater than 3 days, and surviving to ICU discharge were included in the analysis. Rehabilitation status was documented daily with Manchester Mobility Score (MMS) to measure time to first mobilise, defined as sitting on the edge of the bed or higher (MMS $\geq 2$ ), and highest level achieved by ICU discharge. Data was assessed for normality and compared using the student t-test or Mann-Whitney $U$ test as accordingly. Categorical varaiables were analysed using the Fisher Exact test.

Results. A total of 144 patients were admitted to ICU with a positive diagnosis of COVID-19 and surviving to ICU discharge. Patients spent an average of 15.2 days in ICU, with just under half (47\%) invasively ventilated. The mean time to first mobilisation was 9.3 days, with a median MMS of 4 (standing with assistance) at the point pf ICU discharge.

A sub analysis was performed to compare outcomes for patients depending on ventilation status (see Table 1). There was no significant difference in the rate of mobilisation for patients regarding ventilation status ( $88 \%$ vs $93 \%, p=0.384)$, although ventilated patients were slower to mobilise (15.7 vs 3.8 days, $\mathrm{P}<0.00001$ ), spent longer in ICU ( 22.9 vs 8.5 days, $p<0.00001)$ and had lower a MMS at the point of ICU discharge ( 2 vs 5, p <0.00001). Initial mobilisation was delivered a mean of 1 day before patients had weaned from mechanical ventilation, although rehabilitation delivery was inconsistent and delivered at a rate of 0.7 sessions per day (i.e. 2 out of 3 days).

Table 1-- comparison of outcomes for ventilated and non ventilated patients

\begin{tabular}{lllc}
\hline & $\begin{array}{l}\text { Ventilated } \\
(\mathbf{n}=68)\end{array}$ & $\begin{array}{l}\text { Non-venti- } \\
\text { lated (n=76) }\end{array}$ & $\mathbf{p}$ \\
\hline ICU length of stay (days) & 22.9 & 8.5 & $<0.00001$ \\
Ventilation days & 16.7 & 0 & - \\
Proportion Mobilised in ICU & $60(88 \%)$ & $71(93 \%)$ & 0.384 \\
Time to 1st Mobilisation (days) & 15.7 & 3.8 & $<0.00001$ \\
Median MMS ICU discharge & 2 & 5 & $<0.00001$ \\
Physio contacts / day & 1.3 & 1.0 & $<0.00001$ \\
$\begin{array}{l}\text { Physio rehab contacts / day } \\
\text { after day of 1st mobilisation }\end{array}$ & 0.7 & 0.7 & 1.00
\end{tabular}

All data is mean unless stated.

Conclusion. Early experience of the COVID-19 pandemic for ICU Physiotherapists at our trust illustrated high acuity of illness, with half of all admissions requiring prolonged periods of mechanical ventilation. Whilst the majority of patients were mobilised in the ICU, this was on average performed the day before patients had completely weaned from invasive ventilation. Rehabilitation delivery was inconsistent and the overall mobility status at ICU discharge was low, particularly for patients who were mechanically ventilated. Future research is needed to identify any modifiable causes for any delays in initiation of rehabilitation and inconsistency of delivery, such as potentially limited staff availability or a lack or clear guidance on rehabilitation.

\section{Reference(s) \\ 1. Stevens RD, et al. A framework for diagnosing and classifying intensive care unit-acquired weakness. Crit. Care Med. 2009;37:S299-S308. \\ 2. McWilliams D, et al. Rehabilitation levels in COVID-19 patients admitted to intensive care requiring invasive ventilation: An observational study. Annals ATS. 2021. 18(1):122-129. \\ 3. $\mathrm{n} / \mathrm{a}$}

\section{6}

Achieving Lung- and Diaphragm- Protective Ventilation in Patients with Acute Hypoxemic Respiratory Failure: The LANDMARK I Clinical Trial

J. Dianti ${ }^{1}$; S. Fard, ${ }^{1}$; J. Wong, ${ }^{1}$; CC. Timothy ${ }^{2}$; SL. Del ${ }^{1}$; E. Fan ${ }^{1}$; M. Amato ${ }^{3}$; J. Granton"; L. Burry ; D. Reid ${ }^{5}$; S. Keshavjee ; A. Slutsky'; L. Brochard"; ND. Ferguson, ${ }^{8}$; EC. Goligher ${ }^{8}$

${ }^{1}$ Interdepartmental Division of Critical Care Medicine, University of Toronto, Toronto, Canada; ${ }^{2}$ Department of mechanical and industrial engineering, University of Toronto, Toronto, Canada; ${ }^{3} \mathrm{Hospital}$ das clínicas da faculdade de medicina, Universidade de São Paulo, Sao Paulo, Brazil; ${ }^{4}$ Pharmacy, Mount Sinai Hospital, Toronto, Canada; ${ }^{5}$ Department of physical therapy, University of Toronto, Toronto, Canada; ${ }^{6}$ Department of surgery, University of Toronto, Toronto, Canada; ${ }^{7}$ Keenan research centre for biomedical science, Interdepartmental Division of Critical Care, Toronto, Canada; ${ }^{8}$ University health network, department of medicine, Interdepartmental Division of Critical Care Medicine, University of Toronto, Toronto, Canada

Correspondence: J. Diant

Intensive Care Medicine Experimental 2020, 9(1): 001146

Introduction. Spontaneous breathing during mechanical ventilation in patients with acute hypoxemic respiratory failure (AHRF) can prevent diaphragm disuse atrophy, reduce atelectasis, and improve oxygenation. However, excessive respiratory efforts can injure the lung and diaphragm. The feasibility of optimizing respiratory effort to protect both the lung and the diaphragm in AHRF is unknown. The objectives of this randomized cross-over trial were to determine 1) the feasibility of applying a lung- and diaphragm-protective ventilation (LDPV) strategy; and 2) whether the application of higher PEEP and/or VV ECMO increased the probability of achieving LDPV.

Methods. We included patients with AHRF, defined as $\mathrm{PaO} 2 /$ $\mathrm{FiO} 2<300$ and receiving invasive mechanical ventilation with or without venovenous extracorporeal membrane oxygenation. Patients were enrolled as early as possible after ICU admission and the study protocol was initiated once the clinical team decided that passive mechanical ventilation was no longer mandatory. Esophageal manometry was employed to measure respiratory effort (quantified as $\Delta \mathrm{Pes})$ and the dynamic transpulmonary pressure ( $\triangle \mathrm{PL}, \mathrm{dyn})$. The protocol proceeded in two phases: 1 ) after enrolment, sedatives and ventilator set rate were adjusted to initiate spontaneous breathing (sedation minimization phase); and 2) once the patient was spontaneously breathing, ventilation and sedation were systematically titrated using a pre-specified algorithm to achieve LDPV targets ( $\triangle$ Pes between -3 to $-8 \mathrm{~cm} \mathrm{H} 2 \mathrm{O}$ and a $\triangle \mathrm{PL}$, dyn $\leq 15 \mathrm{~cm} \mathrm{H} 2 \mathrm{O}$ ) (LDPV titration phase). This titration procedure was performed at both lower and higher PEEP (applied randomly), and in patients on VV ECMO, at both minimum tolerated and high sweep gas flow rates. Pendelluft from spontaneous 
breathing was quantified by regional ventilation delay measured by electrical impedance tomography. The primary outcome was the proportion of patients in whom the targets were achieved.

Results. Thirty patients were included in the primary analysis. Median [IQR] time from enrolment to LDPV titration phase was 1 [1-4] days. Median [IQR] duration of the LDPV titration phase was 1.5 (1-2.5) hours. At enrolment, LDPV targets were not met in any patients $(0 \%$, 95\% credible interval (Crl) 0-11). Once spontaneous breathing was initiated (phase 1) but prior to titration (phase 2), LDPV targets were met in $6 / 30(20 \%, 95 \% \mathrm{Crl} 10-40)$. Following titration, LDPV targets were achieved at either lower or higher PEEP in 20/30 (67\%,95\% Crl 49-81) patients. The effect of PEEP on $\triangle$ Pes varied among patients (median change in $\triangle \mathrm{Pes}$ from low to high PEEP was $-1 \mathrm{~cm} \mathrm{H} 2 \mathrm{O}$ and ranged between -9 and $7 \mathrm{~cm} \mathrm{H2O}$ ). Patients who had lung recruitment at higher PEEP, had reduced respiratory effort (mean change in $\triangle P e s$ $-3.2 \mathrm{~cm} \mathrm{H} 2 \mathrm{O}(95 \% \mathrm{Crl}-5-0)$ and reduced regional ventilation delay (-3\% (95\% Crl - 6-0), but not patients without lung recruitment (posterior probability for interaction $97 \%$ ). LDPV targets were more likely to be achieved in patients on VV ECMO compared to patients not on VV ECMO (OR 10, 95\% Crl, 2-81, Fig. 1 B). Increasing sweep gas flow from $5 \mathrm{~L} / \mathrm{min}$ (IQR 4-5) to $8 \mathrm{~L} / \mathrm{min}$ (IQR 7-10) reduced $\Delta$ Pes by $-3 \mathrm{~cm}$ $\mathrm{H} 2 \mathrm{O}(95 \% \mathrm{Crl}-4$ to -1.5$)$. Median (IQR) propofol dose at baseline was $40 \mu \mathrm{g} / \mathrm{kg} / \mathrm{min}(21-50)$. Propofol dose was increased in $17 / 30(57 \%$, $95 \% \mathrm{Cl} 40 \%-73 \%)$ patients. Median (IQR) change in propofol dose from baseline to protocol completion was $15 \mu \mathrm{g} / \mathrm{kg} / \mathrm{min}(0-37)$.

Conclusion. Achieving LDPV targets is feasible in patients with AHRF. In patients with lung recruitment at higher PEEP, increasing PEEP decreases inspiratory effort and ameliorates pendelluft. Extracorporeal Patients receiving VV ECMO had with a higher probability of achieving LDPV targets.

\section{7}

\section{Neurofilament light compared to neuron-specific enolase} as a predictor of poor outcome after cardiac arrest

L. Wihersaari'; M. Reinikainen, ; R. Furlan,,; A. Mandelli, ${ }^{2}$; J. Vaahersalo, ${ }^{3}$;

J. Kurola, ${ }^{1}$; M. Tiainen, ${ }^{3}$; V. Pettilä, ${ }^{3}$; S. Bendel, ${ }^{1}$; T. Varpula, ${ }^{3}$; R. Latini, ${ }^{4}$;

G. Ristagno, ${ }^{5} ;$ M. Skrifvars $^{6}$

${ }^{1}$ Department of anaesthesiology and intensive care, University of Eastern Finland and Kuopio University Hospital, Kuopio, Finland; ${ }^{2}$ Clinical neuroimmunology unit, institute of experimental neurology, division of neuroscience, Irccs Ospedale San Raffaele, Milan, Italy; ${ }^{3}$ University of helsinki, Helsinki University Hospital, Helsinki, Finland; ${ }^{4}$ Cardiovascular research, Mario Negri Institute for Pharmacological Research, Milano, Italy; ${ }^{5}$ University of milan, \{street_address\}, Milano, Italy; ${ }^{6}$ Deparment of emergency care and services, University of Helsinki and Helsinki University Hospital, Helsinki, Finland

Correspondence: $\mathrm{L}$. Wihersaari

Intensive Care Medicine Experimental 2020, 9(1): 001147.

Introduction. Blood biomarkers are practical tools in prognostication after cardiac arrest (CA) (1). The ERC-ESICM guidelines recommend measurement of neuron-specific enolase (NSE) after CA (2). NSE has some limitations (3-4), and the novel biomarker neurofilament light $(\mathrm{NfL})$ has demonstrated better prognostic ability in patients resuscitated from a shockable rhythm (5).

Objectives. Our target was to find out if high NfL concentrations in plasma predict poor outcome in post-CA patients regardless of initial rhythm. Moreover, we aimed to compare the prognostic ability of $\mathrm{NfL}$ to that of NSE.

Methods. In this post-hoc study of the FINNRESUSCI study (6), we analyzed blood samples of 262 adult patients treated in 21 Finnish ICUs after out-of-hospital cardiac arrest (OHCA) in 2010-2011. There were $183(69.8 \%)$ patients with a shockable initial rhythm and 79 (30.2\%) with a non-shockable rhythm. We measured NfL concentrations from plasma and NSE concentrations from serum at 24 and $48 \mathrm{~h}$ after cardiac arrest. We assessed the patients' outcomes at 12 months after CA as good (Cerebral Performance Category [CPC] 1-2) or poor (CPC 3-5). We evaluated the prognostic ability of the biomarkers by calculating the area under the receiver operating characteristic curve (AUROC) with 95\% confidence intervals (Cls) and compared them with the bootstrap method.

Results. The 12-month outcome was poor for 128/262 (48.9\%) patients. NfL concentrations were significantly higher for patients with poor outcome than for those with good outcome at $24 \mathrm{~h}$ (median, $621.3 \mathrm{pg} / \mathrm{ml}$ [inter-quartile range, $98.9-1480.8$ ] vs $29.5 \mathrm{pg} / \mathrm{ml}$ [16.668.5], $\mathrm{p}<0.001)$ and at $48 \mathrm{~h}(1193.6 \mathrm{pg} / \mathrm{ml}$ [143.0-4558.4] vs $38.1 \mathrm{pg} /$ $\mathrm{ml}$ [23.8-102.4], $\mathrm{p}<0.001)$. NSE concentrations for patients with poor outcome vs for those with good outcome were $12.9 \mu \mathrm{g} / \mathrm{l}(7.6-23.6) \mathrm{vs}$ $8.7 \mu \mathrm{g} / \mathrm{l}(5.9-13.4), \mathrm{p}<0.001$, at $24 \mathrm{~h}$ and $17.9 \mu \mathrm{g} / \mathrm{l}(8.1-56.4) \mathrm{vs} 8.2 \mu \mathrm{g} / \mathrm{l}$ $(5.9-12.1), p<0.001$, at $48 \mathrm{~h}$. The AUROCs for NfL as a predictor of poor outcome were $0.897(0.856-0.937)$ at $24 \mathrm{~h}$ and $0.882(0.830-0.933)$ at $48 \mathrm{~h}$. At the same time points the AUROCs for NSE were $0.643(0.570$ $0.717)$ and $0.711(0.629-0.792)$, respectively, being significantly lower than those for NfL, $p<0.001$ (Fig. 1).

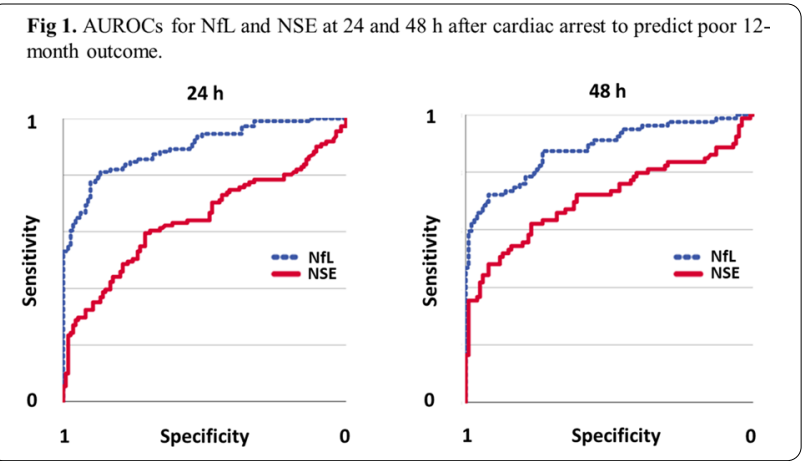

Conclusion. NfL demonstrated a better ability than NSE to predict poor 12-month outcome after OHCA.

Reference(s)

1. Sandroni C, Cariou A, Cavallaro F, Cronberg T, Friberg H, Hoedemaekers C, Horn J, Nolan JP, Rossetti AO, Soar J. Prognostication in comatose survivors of cardiac arrest: an advisorystatement from the European Resuscitation Council and the European Society of Intensive Care Medicine. Resuscitation. 2014 Dec;85(12):1779-89

2. Nolan JP, Sandroni C, Böttiger BW, Cariou A, Cronberg T, Friberg H, Genbrugge C, Haywood K, Lilja G, Moulaert VRM, Nikolaou N, Olasveengen TM, Skrifvars MB, Taccone F, Soar J. European Resuscitation Council and European Society of Intensive Care Medicine guidelines 2021: postresuscitation care. Intensive Care Med. 2021 Apr;47(4):369-421

3. Nielsen N, Cariou A, Hassager C (2019) Physiological interventions in cardiac arrest: passing the pilot phase. Intensive Care Med 45:287-289

4. Wihersaari L, Tiainen M, Skrifvars MB, Bendel S, Kaukonen KM, Vaahersalo J, Romppanen J, Pettila V, Reinikainen M, FINNRESUSCI study group (2019) Usefulness of neuron specifc enolase in prognostication after cardiac arrest: impact of age and time to ROSC. Resuscitation 139:214-221

5. Wihersaari L, Ashton NJ, Reinikainen M et al. (2021) Neurofilament light as an outcome predictor after cardiac arrest: a post hoc analysis of the COMACARE trial. Intensive Care Med 47(1):39-48

6. Vaahersalo J, Hiltunen P, Tiainen M, Oksanen T, Kaukonen KM, Kurola J, Ruokonen E, Tenhunen J, Ala-Kokko T, Lund V, Reinikainen M, Kiviniemi O, Silfvast T, Kuisma M, Varpula T, Pettilä V; FINNRESUSCI Study Group. Therapeutic hypothermia after out-of-hospital cardiac arrest in Finnish intensive care units: the FINNRESUSCI study. Intensive Care Med. 2013 May;39(5):826-37

7. Research grants from Sigrid Juselius Stiftelse and Finska Läkaresällskapet 


\section{Best Oral Presentations 5}

\section{7}

Association of deranged cerebrovascular autoregulation with brain injury following cardiac arrest: A post hoc analysis of the COMACARE trial

J. Laurikkala'; A. Aneman, ${ }^{2}$; A. Peng, ${ }^{3}$; M. Reinikainen ${ }^{4}$; P. Pham, ${ }^{5}$;

P. Jakkula, ${ }^{6}$; J. Hastbacka, ; E. Wilkman ${ }^{6}$; P. Loisa, ${ }^{7}$; J. Toppila, ${ }^{8}$;. Birkelund ${ }_{1}^{9}$;

K. Blennow, ${ }^{10} ;$ H. Zetterberg, ${ }^{11} ;$ M. Skrifvars ${ }^{12}$

${ }^{1}$ \{street_address\}, Helsinki, Finland; ${ }^{2}$ South western sydney local health district, Senior Staff Specialist (Intensive Care Unit), Sydney, Australia; ${ }^{3}$ Anaesthetics, Blacktown hospital, Blacktown, Australia; ${ }^{4}$ Department of intensive care, Kuopio University Hospital, Kuopio, Finland; ${ }^{5}$ South western sydney clinical school, University of New South Wales, Sydney, Australia; ${ }^{6}$ Department of anesthesiology and intensive care, HUS, Helsinki, Finland; ' Deparment of intensive care, Paijat-Hame Central Hospital, Lahti, Finland; ${ }^{8}$ Hus medical imaging centre, Helsinki University Hospital and University of Helsinki, Helsinki, Finland; ${ }^{9}$ Intensive care, Aarhus University Hospital, Aarhus, Denmark; ${ }^{10}$ Department of psychiatry and neurochemistry, institute of neuroscience and physiology, Gothenburg University, Mölndal, Sweden; ${ }^{11}$ Department of psychiatry and neurochemistry, institute of neuroscience and physiology, Gothenburg University, Gothenburg, Sweden; ${ }^{12}$ University of helsinki and helsinki university hospital, Perioperative, intensive care and pain medicine, Helsinki, Finland

Correspondence: J. Laurikkala

Intensive Care Medicine Experimental 2020, 9(1): 001197

Introduction. Out-of-hospital cardiac arrest (OHCA) carries a high overall mortality related to hypoxic-ischaemic brain injury (HIBI). After return of spontaneous circulation (ROSC), an initial phase of hyperae$\mathrm{mia}(0$ to $20 \mathrm{~min}$ ) is followed by vasospasm and hypoperfusion ( $20 \mathrm{~min}$ to $12 \mathrm{~h}$ ) which then subsides with normalization of cerebral blood flow (CBF) at about $72 \mathrm{~h}$ after cardiac arrest [1]. Cerebrovascular autoregulation (CVAR) refers to the ability of the brain vasculature to dilate and contract after changes in mean arterial blood pressure (MAP) aiming to maintain CBF relatively constant. Near-infrared spectroscopy (NIRS) may be used to monitor cerebral tissue oxygenation (cStO2) that reflect $\mathrm{CBF}$ and when correlated with simultaneous changes in MAP, allow for the tissue oxygenation index (TOx) to be derived as an index of dynamic CVAR [2]. Impaired CVAR monitored by TOx is associated with poor neurological outcome in acute neurocritical conditions [3] and also in OHCA as suggested by smaller cohort studies [4,5] Objectives. We assessed CVAR using NIRS and studied associations with markers of brain injury and outcome. We hypothesized that CVAR would be impaired in patients with poor outcome and that deviations of MAP outside the CVAR range would be associated with a more severe HIBI.

Methods. In the interventional multicentre COMACARE study, 120 comatose OHCA patients with an initial rhythm of ventricular fibrillation/tachycardia were randomized to low-normal or high-normal arterial oxygen ( $\mathrm{PaO} 2)$, carbon dioxide $(\mathrm{PaCO} 2)$ and MAP for $48 \mathrm{~h}$ with measurement of CStSO2 with NIRS [6]. In this post-hoc analysis, we measured TOx as the moving correlation coefficient between CStO2 and MAP as a dynamic measure of CVAR. Mean TOx values were derived for three time periods, $0-12 \mathrm{~h}, 12-24 \mathrm{~h}$ and $24-48 \mathrm{~h}$. A TOxfrom $>0$ to 1 indicated impaired autoregulation. We also measured the lower (LLA) and upper (ULA) limits and range (ULA-LLA) of CVAR. The primary outcome was the association between CVAR and 6-month functional outcome. We defined good outcome as Cerebral Performance Category (CPC) 1 or 2 and poor as CPC 3 to 5. Secondary outcomes included associations with biomarkers of brain injury (neurofilament light, NfL; neuron specific enolase, NSE; and S100B) at $48 \mathrm{~h}$. The protocol for this post-hoc analysis was published prior to commencing the study [7].

Results. We included 108 out of 120 patients with sufficient data to calculate TOx of whom 76 patients (70\%) had impaired CVAR. Chronic hypertension was more frequent in patients with impaired autoregulation $(58 \%$ vs $31 \%, p=0.002)$. Patients with poor outcome had lower ULA in all time intervals $(p=0.001)$ in the high-normal $\mathrm{PaO} 2$ $(p=0.002), \operatorname{PaCO} 2(p=0.012)$ and MAP $(p=0.001)$ groups. We did not find significant differences in the OptTOx values between different treatment arms and timepoints. The autoregulatory range was narrower in all time intervals $(p=<0.001)$ and in intervention groups in patients with poor outcome. The median NfL plasma concentration was 20.4 (interquartile range, $13.0-199.3$ ) $\mathrm{pg} / \mathrm{ml}$ in patients with intact CVAR compared to $43.0(15.2-650.3) \mathrm{pg} / \mathrm{ml}$ in patients with impaired CVAR $(p=0.042)$, whereas NSE and S100B levels were not different $48 \mathrm{~h}$ after $\mathrm{OHCA}$.

Conclusion. Impaired autoregulation is common especially in patients with chronic hypertension. A lower ULA and smaller range of CVAR associate with poor neurological outcome. The biomarker NfL was increased in patients with impaired autoregulation reflecting worse brain injury.

\section{Reference(s)}

1) van den Brule JMD, van der Hoeven JG, Hoedemaekers CWE: Cerebral Perfusion and Cerebral Autoregulation after Cardiac Arrest. Biomed Res Int 2018, 2018:4143636.

2) Steiner $L A$, Pfister $D$, Strebel SP, Radolovich D, Smielewski $P$, Czosnyka M: Near-infrared spectroscopy can monitor dynamic cerebral autoregulation in adults. Neurocrit Care 2009, 10(1):122-128

3) Rivera-Lara L, Geocadin R, Zorrilla-Vaca A, Healy R, Radzik BR, Palmisano C, White MA, Sha D, Ponce-Mejia L, Brown C et al.: Near-infrared Spectroscopy-derived Cerebral Autoregulation Indices Independently Predict Clinical Outcome in Acutely III Comatose Patients. J Neurosurg Anesthesiol 2020, 32(3):234-241.

4) Pham P, Bindra J, Chuan A, Jaeger M, Aneman A: Are changes in cerebrovascular autoregulation following cardiac arrest associated with neurological outcome? Results of a pilot study. Resuscitation 2015, 96:192-198.

5) Poot K, Genbrugge C, Meex I, Jans F, Boer W, Vander Laenen M, Ferdinande B, Mullens W, Dupont M, Dens J et al.: An observational nearinfrared spectroscopy study on cerebral autoregulation in post-cardiac arrest patients: time to drop 'one-size-fits-all' hemodynamic targets? Resuscitation 2015, 90:121-126

6) Jakkula P, Reinikainen M, Hastbacka J, Pettila V, Loisa P, Karlsson S, LaruSompa R, Bendel S, Oksanen T, Birkelund T et al.: Targeting low- or highnormal Carbon dioxide, Oxygen, and Mean arterial pressure After Cardiac Arrest and REsuscitation: study protocol for a randomized pilot trial. Trials 2017, 18(1):507

7) Aneman A, Laurikalla J, Pham P, Wilkman E, Jakkula P, Reinikainen M, Toppila J, Skrifvars MB: Cerebrovascular autoregulation following cardiac arrest: Protocol for a post hoc analysis of the randomised COMACARE pilot trial. Acta Anaesthesiol Scand 2019, 63(9):1272-1277.

8) This study was supported by a research grant from The Finnish Medical Foundation and Medicinska Understödsföreningen Liv och Hälsa to J.L.

\section{3}

Using proenkephalin as a novel biomarker to estimate glomerular filtration rate

R. Beunders ${ }^{1}$; L. Donato ${ }^{2}$; R. van Groenendael ${ }^{3}$; B. Arlt ${ }^{4}$; C. Carvalho ${ }^{4}$;

J. Schulte, ; A. Coolen ${ }^{5}$; J. Lieske6; J. Meeusen²; A. Jaffe ; P. Pickkers ${ }^{1}$

${ }^{1}$ Department of intensive care medicine, Radboud University Medical

Center, Nijmegen, Netherlands; ${ }^{2}$ Department of laboratory medicine and pathology, Mayo Clinic, Rochester, United States of America; ${ }^{3}$ Anesthesiology, pain and palliative medicine, Radboud University Medical Center, Nijmegen, Netherlands; ${ }^{4}$ Research and biostatistics, SphingoTec, $\mathrm{GmbH}$, Hennigsdorf, Germany; ${ }^{5}$ Donders institute for brain, cognition, and behaviour, Radboud University Medical Center, Nijmegen, Netherlands; ${ }^{6}$ Division of nephrology and hypertension, Mayo Clinic, Rochester, United States of America; ${ }^{7}$ Department of cardiology, Mayo Clinic, Rochester, United States of America

Correspondence: R. Beunders

Intensive Care Medicine Experimental 2020, 9(1): 001243

Introduction. Deterioration of kidney function is an independent predictor of clinical outcomes including mortality. Glomerular filtration rate (GFR) is the overall best indicator of kidney health. Accurate assessment of GFR is important for early diagnosis of 
acute kidney injury, monitoring of treatment regimens for affected patients, and proper dosing of renally excreted medications. Traditionally, GFR has been estimated (eGFR) using serum creatinine and demographic information including age, sex and race. However, currently available equations have limitations regarding accuracy and precision. In addition, changes in serum creatinine lag changes in GFR by up to $24 \mathrm{~h}-48 \mathrm{~h}$ when there is an acute injury. Although GFR can be measured using exogenous small molecules such as iohexol or iothalamate, these methods are labour-intensive and not practical in many circumstances. We have recently reported that serum Proenkephalin (PENK) is a promising biomarker of GFR. Here we report the performance of equations using PENK to predict mGFR as compared to available creatinine based equations.

Objectives. To develop an equation to estimate the GFR using PENK plasma concentrations.

Methods. In this international multicenter study, GFR was measured (mGFR) in 1354 adult patients using iohexol or iothalamate plasma clearance. GFR was calculated using the slope-intercept method and the Brøchner-Mortensen correction [1]. Plasma PENK concentrations were determined using the sphingotest ${ }^{\circledR}$ penKid ${ }^{\circledR}$ immunoassay [2]. Covariates for the equation-finding analysis were sex, age, race (African-American, American-Indian, Asian and Caucasian), patient type (cardiac surgery, sepsis, organ donor/recipient, chronic kidney disease), and plasma $\mathrm{Cr}$ and PENK concentration. A generalized linear model with a sigmoidal nonlinear transfer function was used for continuous output regression considering variable selection and equation building in a block randomized training sample cohort $(n=811)$. Bias, precision, and accuracy were assessed in a validation cohort $(n=543)$, and results were compared with the creatinine based Modification of Diet in Renal Disease (MDRD) and Chronic Kidney Disease Epidemiology Collaboration (CKD-EPI) eGFR equations.

Results.Mean \pm SD mGFR was $64 \pm 29 \mathrm{~mL} / \mathrm{min} / 1.73 \mathrm{~m} 2$. Data-driven covariate selection resulted in an optimal eGFR equation using PENK, creatinine, and age. Gender and race did not further improve the performance (see Fig. 1 for a scatter plot of the eGFR vs. mGFR). The R-squared of mGFR with eGFRpenk-cr was 0.73 ( $p<0.001$ ), compared to with eGFRmdrd: $0.66(p<0.001)$ and with eGFRckd-epi: 0.71 $(p<0.001)$. The mean bias \pm SD from mGFR was $-2.3 \pm 15.5$ for the eGFRpenk-cr, compared to eGFRmdrd: $-7.7 \pm 16.9$ and eGFRckdepi: $-5.0 \pm 15.7$. The accuracy of the eGFRpenk-cr was $82.7 \%$, compared to the eGFRmdrd: $77.3 \%$ and eGFRckd-epi: $75.0 \%$.

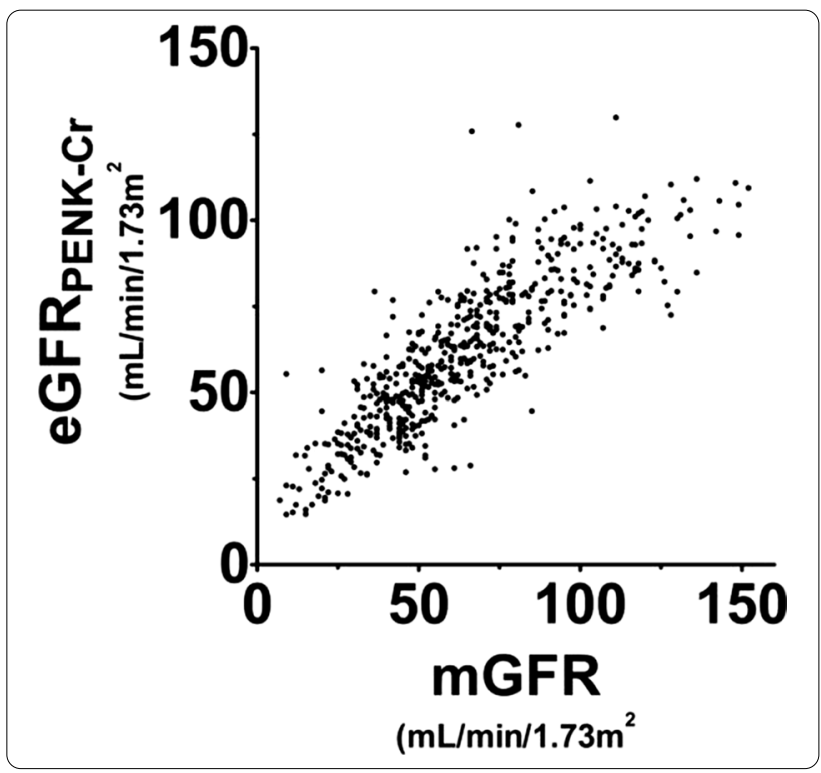

Conclusion. Our study demonstrates that a PENK-based equation including creatinine and age to estimate GFR performs better than existing Creatinine-based existing eGFR equations (MDRD and CKDEPI). Therefore, PENK represents a promising biomarker for accurate estimation of GFR. External validation in other patient cohorts is warranted.

\section{Reference(s)}

1) Donato, L.J., et al., Analytical performance of an immunoassay to measure proenkephalin. Clin Biochem, 2018. 58: p. 72-77.

2) Brochner-Mortensen, J., A simple method for the determination of glomerular filtration rate. Scand J Clin Lab Invest, 1972. 30(3): p. 271-4.

001416

Stroke in ECMO patients with COVID-19: An analysis of the COVID-19 Critical Care Consortium (CCCC) international, multicentre observational study

L. Premraj ${ }^{1}$; A. Zaagog ${ }^{2}$; A. Barnett ${ }^{3}$; J. Fanning, ${ }^{4}$; G. Peek ${ }^{5}$; S. Huth, ${ }^{4}$;

R. Arora ${ }^{6}$; J. Bellapart-Rubio ${ }^{4}$; D. Battaglini, ${ }^{7}$; G. Li Bassi ${ }^{8}$; J. Fraser ${ }^{9}$;

C. Robba ${ }^{10}$; M. Griffee ${ }^{11}$; SM. Cho ${ }^{12}$; JY. Suen ${ }^{9}$

${ }^{1}$ School of medicine, Griffith University, Gold Coast, Southport, Australia;

${ }^{2}$ Critical Care Medicine, MedStar Washington Hospital Center, Washington, United States of America; ${ }^{3}$ Faculty of health, school of public health \& social work, QUT Gardens Point Campus, Brisbane City, Australia; ${ }^{4}$ Faculty of Medicine, The University of Queensland, Saint Lucia, Australia; ${ }^{5}$ Congenital heart center, University of Florida, Gainesville, United States of America; ${ }^{6}$ Cardiac surgery, St. Boniface Hospital Research, Winnipeg, Canada; ${ }^{7}$ Anesthesia and intensive care, san martino policlinico hospital, irccs for oncology and neurosci, IRCCS AOU San Martino, Genova,

Italy; ${ }^{8}$ Applied research in respiratory diseases, Institut d'Investigacions Biomèdiques August Pi i Sunyer (IDIBAPS), Barcelona, Spain; ${ }^{9}$ Critical care research group, adult intensive care services, The Prince Charles Hospital, Chermside, Australia; ${ }^{10}$ Department of anesthesia and intensive care, University of Genoa, Genova, United Kingdom; ${ }^{11}$ Anesthesiologyassociate professor (clinical), University of Utah Hospital, Salt Lake City, United States of America; ${ }^{12}$ Neurocritical care, Johns Hopkins University, Baltimore, United States of America

Correspondence: L. Premraj

Intensive Care Medicine Experimental 2020, 9(1): 001416

Introduction. Neurological complications of COVID-19 are associated with increased morbidity and mortality, especially in the critically ill. Due to their complexity and coagulation status, cerebrovascular complications are of special concern in patients receiving ECMO therapy. As many COVID-19 patients admitted to the intensive care unit (ICU) require $E C M O$, it is important to understand prevalence, risk factors and outcome of ECMO-associated strokes in COVID-19.

Objectives. To determine prevalence, risk factors, and outcomes for stroke occurring as a complication of coronavirus disease 2019 (COVID-19) in critically ill patients requiring extracorporeal membrane oxygenation (ECMO).

Methods. The COVID-19 Critical Care Consortium (CCCC) is a prospective observational study enrolling patients over 18 requiring ICU admission for COVID-19. Adults with acute COVID-19 requiring ICU admission from January 1st through December 21st, 2020 were included in analysis. Survival models utilising parametric Weibull regression were used to investigate the impact of stroke on ICU death and discharge rates in ECMO and non-ECMO cohorts. These results were confirmed using semi-parametric Cox models. A multi-state survival model was used to analyse stroke risk in the ECMO cohort over time.

Results. Overall, 284 patients $(10.5 \%$ of 2,715$)$ required ECMO support, predominantly veno-venous (94\%). In the ECMO cohort, the overall stroke rate was $7.7 \%$ and was comprised of $15(5.3 \%)$ hemorrhagic, $3(1.1 \%)$ ischemic, and $4(1.4 \%)$ unspecified strokes. Among the 2,431 non-ECMO patients, only $37(1.5 \%)$ strokes occurred: $12(0.5 \%)$ hemorrhagic, $16(0.7 \%)$ ischemic, and nine $(0.4 \%)$ unspecified. ECMO 
patients were slightly younger (52yrs, STD 12.1) compared to nonECMO patients (59yrs, STD 14.4) and had lower SOFA scores. Median ECMO duration was 16 days (IQR 8-25). Neither cannula location, size, acute change in $\mathrm{PaCO} 2$ or $\mathrm{PaO} 2$ was associated with stroke. The multistate survival model found cumulative probabilities for hemorrhagic (Relative Risk $[R R]=10.5)$ and ischemic stroke $(R R=1.7)$ at 90 days was higher in ECMO patients compared to non-ECMO patients. ECMO use increased instantaneous (HR [Hazard Ratio] $=1.17$ ) and cumulative $(\mathrm{HR}=1.26)$ hazard of death.

Conclusion. In this study $7.7 \%$ of patients who received ECMO developed stroke. As patients who receive ECMO treatment had a significant higher risk of hemorrhagic stroke over 10 times that of nonECMO patients, a systematic protocolized neurological monitoring is warranted.

\section{5}

Is a simple bedside mobility score a useful predictor of long-term outcome for patients admitted to critical care with COVID-19 infection

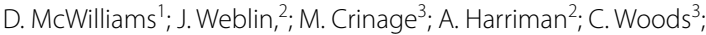
L. Cawtheray ${ }^{3}$

${ }^{1}$ Centre for care excellence, University Hospital Coventry \& Warwickshire, Coventry, United Kingdom; ${ }^{2}$ Therapy services, Queen Elizabeth Hospital Birmingham, Birmingham, United Kingdom; ${ }^{3}$ Physiotherapy, University Hospital Coventry \& Warwickshire, Coventry, United Kingdom Correspondence: D. McWilliams

Intensive Care Medicine Experimental 2020, 9(1): 001435

Introduction. Patients with severe COVID-19 infection often require prolonged periods of mechanical ventilation and associated prolonged stays in the ICU. This is associated with a high incidence of ICU acquired weakness, and significant rehabilitation needs both within hospital and following discharge home [1]. Due to the novel nature of the virus and already stretched clinical services, there is an urgent need to identify patients most at risk of ongoing morbidity who would benefit from targeted rehabilitation services. The Manchester Mobility Score (MMS) is a simple bedside tool used to assess mobility level and rehabilitation progress for patients within critical care [2].

Objectives. To assess the utility of the Manchester Mobility Score in predicting outcome for patients with COVID-19 discharged from critical care.

Methods. This was a prospective observational cohort study completed in 2 large university hospitals in the United Kingdom completed between 1st March 2020 and 31st March 2021. Inclusion criteria was patients admitted to critical care with a confirmed diagnosis of COVID 19 and surviving to critical care discharge. Mobility level was determined by the highest Manchester mobility score achieved before step down to the ward. Primary outcomes were post ICU length of stay and discharge location which was categorised as home, home with rehabilitation, or inpatient rehabilitation. Comparisons between scores and length of stay were performed using spearmans correlation coefficients.

Results. A total of 413 patients met eligibility criteria and were included in the analysis. The mean age of patients \pm SD was $55 \pm 12$ years and 269 $(65 \%)$ were male. The mean length of ICU stay was $21 \pm 16$ days and 336 (81\%) received mechanical ventilation. Mean time to first mobilise was 14 days, with a median MMS of 4 at ICU discharge. The MMS at ICU discharge was significantly negatively associated with length of stay (Spearman's rho of $-0.321, p<0.0001$ ). Figure 1 illustrates that the Manchester Mobility Score follows a near linear relationship with length of stay. An MMS of $\geq 4$ (standing with assistance) was associated with increased likelihood of being discharged home without any rehabilitation needs (56\% vs $42 \%, p<0.05)$ and reduced likelihood of needing inpatient rehabilitation $(10 \%$ vs $27 \%$, $\mathrm{p}<0.05)$.

Fig. 1 Relationship between MMS at ICU disharge and post ICU length of stay

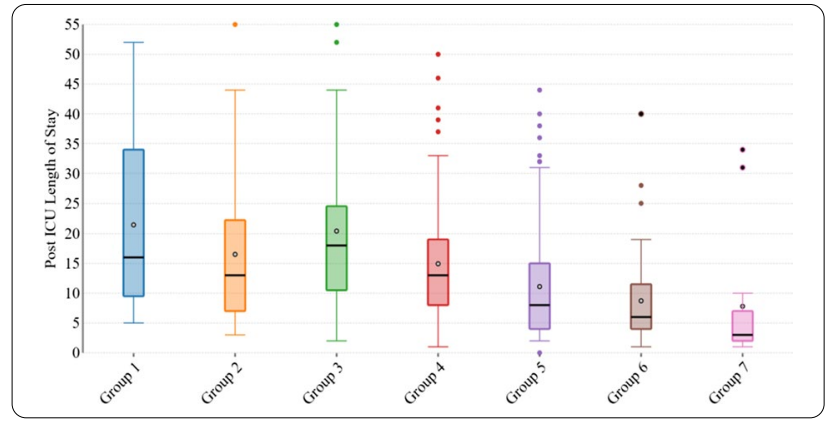

Table 1-Relationship between MMS at ICU discharge and discharge location

\begin{tabular}{llll}
\hline & MMS 1-3 (n=118) & MMS 4-7 (n=295) & p \\
\hline Home & $49(42 \%)$ & $164(56 \%)$ & $<0.05$ \\
Home with rehab & $36(31 \%)$ & $99(34 \%)$ & 0.5642 \\
Inpatient rehab & $32(27 \%)$ & $29(10 \%)$ & $<0.05$ \\
\hline
\end{tabular}

Conclusion. The Manchester Mobility Score showed excellent predictive value, with higher scores associated with shorter post ICU lengths of stay and the ability to stand before transfer to the ward associated with an increased likelihood of being discharged home. These results could help to inform decision making for supportive rehabilitation within hospital or facilitate earlier referral to inpatient rehabilitation facilities for those with lower MMS scores.

\section{Reference(s)}

1. McWilliams D, et al. (2020) Rehabilitation levels in COVID-19 patients admitted to intensive care requiring invasive ventilation: An observational study. Annals ATS. 18(1)

2. McWilliams D, et al. (2016) Feasibility and reliability of the Manchester Mobility Score as a measure of physical function within the Intensive Care Unit. ACPRC Journal. 48; 26-33

3. $\mathrm{n} / \mathrm{a}$

\section{1}

Long-term effects of the COVID-19 pandemic on burnout symptoms in ICU professionals: results from a 9-months follow-up longitudinal cohort study

N. Kok'; J. Van Gurp'; M. Fuchs'; H. Van Der Hoeven ${ }^{3}$;

CWE. Hoedemaekers ${ }^{3}$; M. Zegers ${ }^{3}$

${ }^{1}$ Iq healthcare, Radboud University Medical Center, Nijmegen, Netherlands; ${ }^{2}$ Intensive care, Canisius Wilhelmina Hospital, Nijmegen, Netherlands; ${ }^{3}$ Intensive care, Radboud University Medical Center, Nijmegen, Netherlands

Correspondence: N. Kok

Intensive Care Medicine Experimental 2020, 9(1): 001441

Introduction. In a previous study, we assessed burnout symptoms and moral distress in intensive care unit (ICU) professionals 3 months prior and 3-month after the outbreak of Coronavirus Disease 2019 (COVID19) pandemic.[1] We measured burnout symptoms and moral distress 9 months after the onset of COVID-19 to determine the long-term effects of the pandemic on professionals' wellbeing.

Objectives. To assess the prevalence and incidence of burnout symptoms in ICU professionals over time as well as explore which morally distressing issues affect burnout symptoms on the long term.

Methods. This is a longitudinal open cohort study. ICU professionals in two hospitals were sent surveys at three timepoints: a baseline survey prior to COVID-19 in October-December 2019 (252 respondents, response rate: $53 \%$ ), a survey 3-months after the start of COVID-19 in the 
Netherlands in May-June 2020 (233 respondents, response rate 50\%) and a 9-month follow-up in October-December 2020 (227 respondents, response rate: $51 \%$ ). Burnout symptoms and moral distress were measured with the Dutch Maslach Burnout Inventory (MBI) and the Moral Distress Scale. Burnout symptoms were defined with a MBI higher than -9.[1-2] Moral stressors were measured on a range from 0 (none) to 16 (very frequent and intense). We report prevalence and incidence rates of burnout symptoms and conduct Generalized Estimating Equations (GEE) on full cases at each time point to explore associations between moral distress and burnout.

Results. The prevalence of burnout symptoms was $23.0 \%$ at 3 months prior to the COVID-19 pandemic, 36.1\% at 3-month follow-up and $31.3 \%$ at 9 months. The incidence rate of new burnout cases was $22.9 \%$ at 3 months and $15.6 \%$ at 9 months. At 3 months, scarcity of resources, time and staff and working with non-ICU qualified colleagues were found to be significant moral stressors related to burnout.[1] From the 3-months to 9-months follow-up, the average scores on these stressors decreased from 3.6 to 3.4 and from 1.9 to 1.5 respectively. Through GEE, three morally distressing issues were found to be significantly associated with burnout at 9-month follow-up: scarcity of resources, time and staff (odds ratio $1.22 ; 95 \% \mathrm{Cl} 1.12$ to 1.32 ); continuing treatment of a patient on a ventilator without the prospect of recovery (odds ratio 1.18; $95 \% \mathrm{Cl} 1.09$ to 1.27); and witnessing that a patient is suffering due to a lack of continuity of caregivers (odds ratio $1.09 ; 95 \% \mathrm{Cl} 1.01$ to 1.19 ). We found no long-term effect of working with non-ICU qualified colleagues on burnout symptoms.

Conclusion. Prevalence of burnout symptoms increased at 3 months and decreased at 9 months but was still higher than prior to COVID-19. The 9-month follow-up data lays bare that different morally distressing issues are associated with burnout symptoms on the long-term. In order to minimize the impact of moral distress on burnout, ICU professionals and managers need to be constantly and keenly aware of where and when moral distress may develop.

Reference(s)

1. Azoulay E, De Waele J, Ferrer R, Staudinger T, Borkowska M, Povoa P, Iliopoulou K, Artigas A, Schaller SJ, Shankar Hari M, Pellegrini M, Darmon m, Kesecioglu J, Cecconi M, and ESICM (2020). Symptoms of burnout in intensive care unit specialists facing the COVID-19 outbreak. Annals of intensive care 10(110)

2. Kok N, Van Gurp J, Teerenstra S, Van der Hoeven H, Fuchs M, Hoedemaekers C, Zegers M (2020). Coronavirus Disease 2019 immediately increases Burnout Symptoms in ICU professionals: a Longitudinal Cohort Study. Critical Care Medicine 49(3): 419-427.

3. Supported by ZonMw, Project Number 516012513

\section{Oral Presentations}

\section{6}

"Perceptions of Anesthesiology Critical Care Medicine physicians on anxiety, depression, lack of diversity and bias in Critical Care Medicine-a follow-up survey of ASA, SOCCA and SCCM Members"

S. Siddiqui ${ }^{1}$

${ }^{1}$ Anesthesia and Intensive Care, Beth Israel Deaconess Medical

Center, Newton, Singapore

Correspondence: S. Siddiqui

Intensive Care Medicine Experimental 2020, 9(1): 000006.

Introduction. Background: A previous survey in 2018 of Anesthesiology Critical Care Medicine (ACCM) providers in the United States revealed a significant rate of burnout and stress. The COVID-19 pandemic has brought critical care to the forefront of the fight against the coronavirusand has created a particular burden for physicians of all specialties, bedside nurses and respiratory therapists working in the intensive care unit (ICU).The new public awareness of critical care has had a positive and negative impact on ACCM providers.

Objectives. We therefore designed a national survey of ACCM providers with the following goals: (1) to evaluate the impact of the pandemic on ACCM providers in the spheres of mental well-being, motivation and resilience; (2) to elicit suggestions from ACCM providers for resources they consider might mitigate stress during the pandemic; and (3) to garner their opinions on the presence of bias and diversity in the practice of critical care.

Methods. Approval and waiver of written informed consent by the Beth Israel Deaconess Medical Center Institutional Review Board. An anonymous validated online survey, the generalized anxiety disorder (GAD) screening tool, and the depression screening tool developed by the Anxiety and Depression Association of America were sent to all members of the American Society of Anesthesiologists (ASA) who selfidentified as being CCM trained or practicing critical care, members of the Society of Critical Care Anesthesiologists (SOCCA) as well as the Anesthesiology section of the Society of Critical Care Medicine (SCCM). Survey results were analyzed using a mixed-method approach.

Results. We received a total of 565 responses ( 300 complete and 265 incomplete) out of the 1255 members the survey was sent to, for an overall response rate of $45 \% .42 \%$ of the participants said they felt symptoms of anxiety since the start of the pandemic. There was an overall increase by $64 \%$ in contribution to CCM practice, a modest increase of $22.4 \%$ in the sense of achievement during the pandemic and an increase by $33 \%$ of a sense of Burnout. When symptoms of anxiety and depression were compared by gender, women were more likely than men to experience excessive symptoms ( $55.4 \%$ versus $36.7 \%$, respectively, $\mathrm{p}=0.003$ ).

Conclusion. This study reveals important challenges faced by anesthesiology critical care trained physicians. It is clear that bias and lack of diversity are more recognized by women and younger intensivists. The pandemic highlighted these disparities in much greater detail. Although overall there was satisfaction with the sense of respect and recognition, and low levels of stress and anxiety, there were differences between these perceptions by age and gender. Suggestions for reducing these disparities included diversity training of leadership, accountability for diversity and dealing with bias, and peer support.

\section{3}

The effect of rescue therapies on systemic and cerebral 02 in COVID

F. Iannuzzi ${ }^{1}$; L. Ball ${ }^{2}$; D. Battaglini, ${ }^{3}$; I. Brunetti, ${ }^{3}$; M. Bassetti, ${ }^{4}$, DR. Giacobbe, ${ }^{5}$; A. Vena, ${ }^{5}$; N. Patroniti, ${ }^{6}$; P. Rocco, ${ }^{7}$ BF. Matta, ${ }^{8}$; P. Pelosi ${ }^{9} ;$ C. Robba ${ }^{10}$

${ }^{1}$ Department of surgical sciences and integrated diagnostic (disc), university of genoa, genoa, it, IRCCS AOU San Martino, Genova, Italy; ${ }^{2}$ Anesthesia and intensive care, san martino policlinico hospital, irccs for oncology and neurosci, Department of surgical sciences and integrated diagnostic (disc), university of genoa, genoa, it, Genova, Italy; ${ }^{3}$ Anesthesia and intensive care, san martino policlinico hospital, irccs for oncology and neurosci, IRCCS AOU San Martino, Genova, Italy; ${ }^{4}$ Infectious disease unit, san martino policlinico hospital, irccs for oncology and neuroscience ", Department of Health Sciences, University of Genoa, Genoa, Italy., Genova, Italy; ${ }^{5}$ Infectious disease unit, san martino policlinico hospital, irccs for oncology and neuroscience, IRCCS AOU San Martino, Genova, Italy; ${ }^{6}$ Department of surgical sciences and integrated diagnostic (disc), university of genoa, genoa, it, Anesthesia and Intensive Care, San Martino Policlinico Hospital, IRCCS for Oncology and Neurosci, Genoa, Italy; ${ }^{7}$ Laboratory of pulmonary investigation, carlos chagas filho institute of biophysics, federal uni, Ministry of Science, Technology, and Innovation, Brasília, Brazil, Rio de Janeiro, Brazil: ${ }^{8}$ Neurosciences critical care unit, addenbrooke's hospital, cambridge university hospitals nhs fou, Department of Anesthesia,Addenbrooke's Hospital,Cambridge University Hospitals, Cambridge, United Kingdom; ${ }^{9}$ Departement of anesthesia and intensive care, University of Genoa, Genova, Italy; ${ }^{10}$ Department of anesthesia and intensive care, University of Genoa, Genova, United Kingdom

Correspondence: F. lannuzzi

Intensive Care Medicine Experimental 2020, 9(1): 000013

Introduction. In critically ill coronavirus disease (COVID-19) patients, the use of ventilatory rescue strategies remains uncertain, with controversial efficacy on systemic oxygenation. Moreover, no data are currently available regarding their effect on cerebral oxygenation and hemodynamics. 
Methods. Prospective observational study conducted at Policlinico San Martino Hospital Genova, Italy, including adult COVID-19 patients who underwent at least one type of rescue therapy. Arterial blood gases values (oxygen saturation, $\mathrm{SpO} 2$, partial pressure of oxygen, $\mathrm{PaO} 2$, and of carbon dioxide, $\mathrm{PaCO} 2$ ) and cerebral oxygenation ( $\mathrm{rSO} 3)$ were observed before (T0) and after the use of any rescue therapy (T1). Primary aim was to assess the effect of different ventilatory rescue therapies (such as recruitment maneuvers (RMs), prone positioning (PP), inhaled (iNO) and $\mathrm{CO} 2$ removal (ECCO2R)) on systemic blood gases values and cerebral oxygenation. Secondary aims were to assess the effect of rescue therapies on systemic hemodynamics and ICP and to evaluate the correlation between systemic and cerebral oxygenation in COVID-19 patients before and after rescue therapies.

Results. 45 rescue therapies in 22 patients were obtained. The median age of the population was 62 years [interquartile range, $\mathrm{IQR}=57-68,5]$, and $18 / 22(81,8 \%)$ were males. After RMs, no significant changes were observed in $\mathrm{PaO} 2$ and $\mathrm{PaCO} 2$ values, but cerebral oxygenation significantly decreased $(52 \%$ (3) vs $48,5 \%(39), p<0,001)$. After PP, systemic and cerebral oxygenation significantly increased $(\mathrm{PaO} 2=$ median $62, \mathrm{IQR}=15,8)$ vs $81,5(11,3) \mathrm{mmHg}, \mathrm{p}=0,005$; rSO3 $=53 \%(2,8)$ vs $59,5 \%(5,5), p=0,005$, respectively). After iNO, both systemic and cerebral oxygenation values increased (65 (7) vs 72 $(8,5) \mathrm{mmHg}, \mathrm{p}=0,015$ and $53 \%(6,5)$ vs $57 \%(7,5), \mathrm{p}=0,007$, respectively). The use of ECCO2R resulted in a decrease in $\mathrm{PaO} 2$ values (75 (6) vs $63,5(12,8) \mathrm{mmHg}, \mathrm{p}=0.009)$, with reduction of $\mathrm{rSO} 3$ values $(59 \%$ $(10,5)$ vs $56 \%(11,3), p=0.002)$. Finally, we found a statistically significantly correlation between $\mathrm{SpO} 2$ and $\mathrm{rSO} 3$ in the whole population both at T0 $(r=0.497, p=0.001)$, and T1 $(r=0.685, p<0.001)$.

Conclusion. Rescue therapies have specific pathophysiological mechanisms with different effect on systemic and cerebral oxygenation in critically ill COVID-19 patients. The choice of the strategy to use should take in account both lung and brain needs.

\section{Reference(s)}

1. Ball L, Serpa Neto A, Trifiletti V, Mandelli M, Firpo I, Robba C, Gama de Abreu M, Schultz MJ, Patroniti N, Rocco PRM, Pelosi P; PROVE Network: PROtective Ventilation Network. Effects of higher PEEP and recruitment manoeuvres on mortality in patients with ARDS: a systematic review, meta-analysis, meta-regression and trial sequential analysis of randomized controlled trials. Intensive Care Med Exp. 2020 Dec 18;8(Suppl 1):39. https://doi.org/10.1186/s40635-020-00322-2. PMID: 33,336,325; PMCID: PMC7746429.

2. Borsellino B, Schultz MJ, Gama de Abreu M, Robba C, Bilotta F. Mechanical ventilation in neurocritical care patients: a systematic literature review. Expert Rev Respir Med. 2016 Oct;10(10):1123-32. https://doi.org/10.1080/ 17476348.2017.1235976. Epub 2016 Sep 21. PMID: 27,635,737.

3. Della Torre V, Badenes R, Corradi F, Racca F, Lavinio A, Matta B, Bilotta F, Robba C. Acute respiratory distress syndrome in traumatic brain injury: how do we manage it? JThorac Dis. 2017 Dec;9(12):5368-5381. https:// doi.org/10.21037/jtd.2017.11.03. PMID: 29,312,748; PMCID: PMC5756968.

4. Young CC, Harris EM, Vacchiano C, Bodnar S, Bukowy B, Elliott RRD, Migliarese J, Ragains C, Trethewey B, Woodward A, Gama de Abreu M, Girard M, Futier E, Mulier JP, Pelosi P, Sprung J. Lung-protective ventilation for the surgical patient: international expert panel-based consensus recommendations. Br J Anaesth. 2019 Dec;123(6):898-913. https://doi.org/10.1016/j. bja.2019.08.017. Epub 2019 Oct 3. PMID: 31,587,835.

5. Robba C, Bonatti G, Battaglini D, Rocco PRM, Pelosi P. Mechanical ventilation in patients with acute ischaemic stroke: from pathophysiology to clinical practice. Crit Care. 2019 Dec 2;23(1):388. https://doi.org/10.1186/ S13054-019-2662-8. PMID: 31,791,375; PMCID: PMC6889568.

6. Writing Committee for the PROBESE Collaborative Group of the PROtective VEntilation Network (PROVEnet) for the Clinical Trial Network of the European Society of Anaesthesiology, Bluth T, Serpa Neto A, Schultz MJ, Pelosi P, Gama de Abreu M; PROBESE Collaborative Group, Bluth T, Bobek I, Canet JC, Cinnella G, de Baerdemaeker L, Gama de Abreu M, Gregoretti C, Hedenstierna G, Hemmes SNT, Hiesmayr M, Hollmann MW, Jaber S, Laffey J, Licker MJ, Markstaller K, Matot I, Mills GH, Mulier JP, Pelosi P, Putensen C, Rossaint R, Schmitt J, Schultz MJ, Senturk M, Serpa Neto A, Severgnini P, Sprung J, Vidal Melo MF, Wrigge H. Effect of Intraoperative High Positive End-Expiratory Pressure (PEEP) With
Recruitment Maneuvers vs Low PEEP on Postoperative Pulmonary Complications in Obese Patients: A Randomized Clinical Trial. JAMA. 2019 Jun 18;321(23):2292-2305. https://doi.org/10.1001/jama.2019.7505. Erratum in: JAMA. 2019 Nov 12;322(18):1829-1830. PMID: 31157366; PMCID: PMC6582260.

My biggest thanks goes to my mentors Chiara Robba and Lorenzo Ball for their great dedication in following my training path. Thanks to the Professors for their great contribution to training in both medical and research fields

\section{1}

Reduced hippocampal microglia in pigs mechanically ventilated plus diaphragm neurostimulation for $50 \mathrm{~h}$

T. Bassi'; R. Elizabeth²; F. Karl'²; N. Michelle ${ }^{3}$; M. Ornowska²; G. Matt ${ }^{4}$.

E. Doug ${ }^{4}$; S. Reynolds

${ }^{1}$ Physiology, Simon Fraser University, Burnaby, Canada; ${ }^{2}$ Critical care, Fraser Healthy Authority, New Westminster, Canada; ${ }^{3}$ Criti-

cal care, Fraser Health New Westminster, New Westminster, Canada:

${ }^{4}$ Research and development, Lungpacer Medical USA Inc., Exton, United States of America

Correspondence: T. Bassi

Intensive Care Medicine Experimental 2020, 9(1): 000111

Introduction. High percentages of hippocampal microglia in mechanically ventilated subjects have been demonstrated in both clinical and preclinical investigations. Microglia are key cells that promote and control neuroinflammation. To date, no studies have investigated strategies to prevent neuroinflammation during mechanical ventilation (MV).

Objectives. We evaluated whether diaphragm neurostimulation in synchrony with lung-protective MV for $50 \mathrm{~h}$ would result in lower percentages of hippocampal microglia in a normal-lung porcine model.

Methods. Twenty-eight healthy juvenile pigs (4-5 months, 48-66 kg) with non-injured lungs were divided into four groups: MV only (MV group, $\mathrm{n}=8), \mathrm{MV}$ in association with diaphragm neurostimulation delivered every other breath (TTDN50\% + MV group, $n=7$ ), MV in association with diaphragm neurostimulation delivered every breath (TTDN100\% + MV group, $\mathrm{n}=7$ ) and never ventilated (NV group, $n=6$ ). A central line catheter with embedded electrodes (Lungpacer LIVE Catheter) was inserted into the left subclavian vein for the TTDN50\% + MV andTTDN100\% + MV subjects, and the diaphragm was activated by temporary transvenous diaphragm neurostimulation (TTDN). Diaphragm neurostimulation in synchrony with MV targeted a reduction in pressure-time product between $15-20 \%$. MV in volume control was set to achieve and maintain a tidal volume of $8 \mathrm{ml} / \mathrm{kg}$ with a PEEP of $5 \mathrm{cmH} 2 \mathrm{O}$. The hippocampus was harvested from each pig and IBA-1 assay was used to mark microglia. Machine-learning software (ImageJ) was used to count and quantify IBA-1-positive cells as a percentage of total cells counted (Fig. 1A). Data are expressed as median and interquartile range. The Kruskal-Wallis test and Dunn's multiple comparison test were used for statistical analysis. P-values $£ 0.05$ were considered statistically significant.

Results. The microglia percentages found were 36.17 (30.71-48.27) for the MV group, 16.70 (10.82-22.42) for the TTDN50\% + MV group, 9.80 (7.86-11.19) for the TTDN100\% + MV group, and 10.12 (8.93-10.65) for the NV group, with statistically significant differences between groups, as determined by the Kruskal-Wallis test, $p=0.0002$ (Fig. 1A/B). Post hoc analysis using Dunn's multiple comparison test showed considerable statistical differences between the MV and NV groups (36.17 vs. $10.12, p=0.0016)$, and between the MV and TTDN100\% + MV groups (36.17 vs. $9.80, p=0.0005$ ).

Conclusion. In a porcine model, diaphragm neurostimulation in synchrony with MV for $50 \mathrm{~h}$ resulted in significantly less neuroinflammation in the hippocampus. Moreover, delivering diaphragm neurostimulation either every breath or every other breath resulted in microglia percentages statistically indistinguishable from the never ventilated group. 
Reference(s)

1. Bassi et al. Brain injury after $50 \mathrm{~h}$ of lung-protective mechanical ventilation in a preclinical model. Sci Rep. 2021

2. Kamuf J, Garcia-Bardon A, Ziebart A, et al. Lung injury does not aggravate mechanical ventilation-induced early cerebral inflammation or apoptosis in an animal model. PLoS One. 2018;13(8):1-11. 10.1371/journal.pone. 0202131

3. MITACS

4. TBVETS

5. Lungpacer Medical Inc.

\section{3}

Impact of Temporary Mechanical Circulatory Support on mortality in Cardiogenic Shock: an emulated target trial with a prospective, multicenter, French cohort study

A. Ughetto'; J. Eliet'; H. David'; N. Nagot'². F. Bazalgette'; G. Marin ${ }^{2}$.

M. Mourad'. S. Kollen 3. P. Gaudard'1. P. Colson

${ }^{1}$ Department of anesthesiology and intensive care, $\mathrm{CHU}$ Arnaud de

Villeneuve, Montpellier, France; ${ }^{2}$ Epidemiology and clinical research, University of Montpellier, Montpellier, France; ${ }^{3}$ Department of critical care medicine, PERPIGNAN HOSPITAL CENTRE, Perpignan, France

Correspondence: A. Ughetto

Intensive Care Medicine Experimental 2020, 9(1): 000133

Introduction. The field of temporary mechanical circulatory support (TMCS) has advanced in last decade justifying that TMCS is increasingly used for treatment of refractory cardiogenic shock (CS). Nevertheless, the efficacy of TMCS (extracorporeal life support (ECLS) and Impella) in CS remains controversial due to the lack of high-quality evidence.

Objectives. The aim of this prospective multicenter observational study simulating a randomized trial was to assess the impact of TMCS on the hospital mortality in patients with CS.

Methods. This study (ClinicalTrials.gov ID: NCT03528291) was conducted at 3 TMCS centers organized in a cardiac assistance network, one as a level 1 TMCS center (expert center), and 2 as level 2 centers (hub centers). The study was designed and led by the heart team of the expert center with input from the hub centers.All patients admitted to an intensive care unit between July 2017 and May 2020 either directly at the TMCS centers or after transfer from a non-specialized hospital, were screened for TMCS indication provided they were admitted for CS. CS was defined according to the European Society of Cardiology criteria. Were excluded patients younger than 18 years, CS after cardiac surgery, or after cardiac arrest if it was refractory or with a no flow $>3$ min and/or out-of-hospital cardiac arrest with non-shockable rhythm, or CS in the context of myocardial infarction complications, massive pulmonary embolism, and if TMCS was contraindicated TMCS indication was decided after a multidisciplinary discussion carried out by the "heart team". Implantation of TMCS resulted from an agreement of the heart team within the first $24 \mathrm{~h}$ after admission mainly based on the initial severity of the CS, or if CS was refractory to the medical treatment.The primary outcome was inhospital survival. A propensity score-weighted analysis was done for treatment-effect estimation. This method, which weights each patient according to their propensity score, includes all participants in the analysis.

Results. 246 patients with CS were included in the study: 121 in TMCS group (72\% ECLS, 14\% Impella, 14\% both ECLS and Impella) and 125 in control group. After adjustment by a propensity score, hospital mortality was comparable in the two groups (32\% TMCS group vs $27 \%$ control group; Odds ratio with TMCS, 1.28; $95 \%$ confidence interval, 0.87 to $1.88 ; \mathrm{p}=0.21$ ). Mortality at D180 was also similar in the two group (33\% vs $30 \%$ respectively; $p=0.51$ ). Mechanical ventilation duration was higher in the TMCS group (median (IQR) 9.0 days $(3.0 ; 16.0)$ vs 1.0 days $(0.0 ; 6.0) ; \mathrm{p}<0.01)$ ) as well as ICU and hospital length of stay (median (IQR) 29.0 days $(16.0 ; 49.0)$ vs 15.0 days $(10.0 ; 24.0) ; p<0.01)$ Concerning the side effects, thromboembolic events were significantly more frequent in the TCMS group $(14 \%$ vs $4 \% ; p<0.01)$ as well as the transfusion rate ((median (IOR); $4.0(0.0 ; 9.0)$ vs $0.0(0.0 ; 0.0)$; $\mathrm{p}<0.01$ ). In addition, hemolysis and blood infection were significantly higher in the TMCS group.

Quality of life as assessed by SF36 score was similar in both groups (TMCS group; $54.0(36.0 ; 80.0)$ vs control group 63.0 (36.0;79.0); $\mathrm{p}=0.73$ ).

Conclusion. In our study simulating a randomized trial, the use of TMCS does not seem to improve hospital survival in patients with cardiogenic shock. Thus, TMCS, which are iatrogenic side effects providers, should be reserved for the most severe patient and discussed by a multidisciplinary team.

\section{5}

COVID-19 emergencies management learning

through high-fidelity simulation for medical residents-Humanitas Simulation Centre Experience

A. Del Pozo'; E. Giustiniano, ${ }^{2}$; N. Fusilli, ${ }^{2}$; V. Simili, ${ }^{2}$; L. Calabro'ㄹ; L. Pugliese, ${ }^{2}$; N. Stomeo, ${ }^{2}$; C. Ebm, ${ }^{1}$; S. Brusa ${ }^{1}$

${ }^{1}$ Simulation centre, Humanitas University, Milan, Italy; ${ }^{2}$ Intensive care unit and anesthesiology, Humanitas Research Hospital, Cascina Perseghetto,

Italy

Correspondence: A. DEL POZO

Intensive Care Medicine Experimental 2020, 9(1): 000135

Introduction. The COVID-19 pandemic has caused an unprecedented global healthcare crisis. Multidisciplinary teams were stretched to care for exponentially rising case volumes. Contemporaneously, the pandemic has impacted the delivery of medical residents' education. Simulation-based medical education (SBME) is a well-established method for assessing and assisting in the process of readiness for practice in a variety of emergency situations [4,5] where the consequences of a flawed workflow algorithm can positively impact patients' and healthcare workers' safety. We propose the use of SBME to train medical residents of all specialties to manage the most common COVID 19-related emergencies.

Objectives. The aims of our study were to monitor multidisciplinary resident's knowledge using the latest guidelines for the management of critically ill adults with coronavirus through high-fidelity simulation; to stimulate the situational awareness of respiratory and cardiovascular complications and to apply non-technical skills such as shared mental models, team working and closed-loop communication.

Methods. At Humanitas Simulation Centre, SBME was organized for the COVID-19 elective training for Humanitas Hospital medical residents. Forty-five participants took part in high-fidelity clinical scenarios in respiratory and circulatory failure, bedside non-invasive and invasive procedures, and elements of COVID-19 specific care. To evaluate the baseline knowledge of the participants on the topic, a presimulation test consistent in ten questions was delivered electronically to all participants before the activity. The same test was reproposed immediately after the activity. Comparison of the outcomes of the preand post-activity tests was done to evaluate SBML effectiveness and clearness of the take home messages. Finally, a survey to get participants feedback was administered. It was validated by an expert, and the reliability calculated for each item. Responses were measured on 5-point Likert-scale items.

Results. Mean score obtained in the pre activity test was 4,5 while in the post activity test was 8,7 . Comparison of means of the two data sets using student t-test showed an important statistically significant difference $(P<0.0001)$. The survey consisted of 14 questions based on the satisfaction level regarding residents' goals achievements and technical and non-technical skills challenged. The activity overall satisfaction level was 4.3.

Conclusion. SBME is a value method to promote safe practice and enhance medical residents' ability to deal with COVID-19 emergency situations. The activities and debriefing had a significant positive impact on resident's specific knowledge. Suggestions to improve training during the specialization schools include increasing residents' access to high-fidelity simulation as themselves consider it a powerful method, adopting a more resident-centred approach and creating a safer learning environment. 
Reference(s)

1. Price JW, Price JR, Pratt DD, Collins JB, McDonald J. High-fidelity simulation in anesthesiology training: a survey of Canadian anesthesiology residents' simulator experience. Can J Anaesth. 2010 Feb;57(2):134-42. https://doi.org/10.1007/s12630-009-9224-5. PMID: 20,054,681.

2. Mileder LP, Schüttengruber G, Prattes J, Wegscheider T. Simulation-based training and assessment of mobile pre-hospital SARS-CoV-2 diagnostic teams in Styria, Austria. Medicine (Baltimore). 2020 Jul 17;99(29):e21081. https://doi.org/10.1097/MD.0000000000021081. PMID: 32,702,851; PMCID: PMC7373634

3. Benlolo S, Nensi A, Campbell DM, Assouad C, Taylor TS, Shore EM. The Use of In Situ Simulation to Enhance COVID-19 Pandemic Preparedness in Obstetrics. Cureus. 2021;13(1):e12906. Published 2021 Jan 25. 10.7759/ cureus.12906

4. Satin AJ. Simulation in Obstetrics. Obstet Gynecol. 2018 Jul;132(1):199209. https://doi.org/10.1097/AOG.0000000000002682. PMID: 29,889,745.

5. Malhotra A, Kumar A. Breaking the COVID-19 Barriers to Health Professional Team Training With Online Simulation. Simul Healthc. 2021 Feb 1;16(1):80-81. https://doi.org/10.1097/SIH.00000000000000518. PMID: 33,196,611: PMCID: PMC7853721.

6. Nair SS, Kaufman B. Simulation-Based Up-Training in Response to the COVID-19 Pandemic. Simul Healthc. 2020 Dec;15(6):447-448. https://doi. org/10.1097/SIH.0000000000000513. PMID: 33,003,128.

7. Fregene TE, Nadarajah P, Buckley JF, Bigham S, Nangalia V. Use of in situ simulation to evaluate the operational readiness of a high-consequence infectious disease intensive care unit. Anaesthesia. 2020 Jun;75(6):733738. https://doi.org/10.1111/anae.15048. Epub 2020 Mar 30. PMID: $32,221,964$.

8. Lim WY, Ong J, Vimal V, Lim H, Tan HC, Wong P, Ho VK, Ong SGK. Highfidelity simulation training with PPE may optimise resuscitation outcomes in the COVID-19 era. Resuscitation. 2021 Feb;159:42-44. https:// doi.org/10.1016/j.resuscitation.2020.12.015. Epub 2020 Dec 29. PMID: 33,385,472; PMCID: PMC7836637.

\section{5}

Epidemiology and Clinical Outcomes of Persistent Severe Acute Kidney Injury (AKI)

R. Mackey ${ }^{1}$; J. Textoris ${ }^{2}$; N. Rosenthal, ${ }^{1}$; LA. Carabuena, ${ }^{1}$; J. Kampf ${ }^{3}$;

A. Sanghani, ${ }^{3} ;$ J. Echeverri, ${ }^{4} ;$ MJ. Blackowicz, ${ }_{1}^{4}$ T. Rodriguez, ${ }^{3}$;

P. Mcpherson, ${ }^{3} ;$ J. Koyner ${ }^{5}$

'Premier Applied Sciences, Premier, Inc., Charlotte, United States

of America; ${ }^{2}$ Global medical affairs, bioMérieux-Campus de

l'étoile, Marcy-l'Étoile, France; ${ }^{3} G$ lobal medical affairs, bioMerieux Inc, Durham, United States of America; ${ }^{4}$ Global medical affairs, Baxter Healthcare, Deerfield, United States of America; ${ }^{5}$ Medicine-nephrology, University of Chicago, Chicago, United States of America

Correspondence: R. Mackey

Intensive Care Medicine Experimental 2020, 9(1): 000145

Introduction. Persistence of AKI is emerging as an important determinant of patient outcomes but is not well characterized.

Objectives. To describe and compare characteristics and outcomes for patients with KDIGO stage 2-3 AKI who developed persistent severe AKI (PS-AKI) vs. those who did not (NPS-AKI).

Methods. A retrospective observational study of hospitalized US adults was conducted using the Premier Healthcare Database from January 1, 2017 to December 31, 2019, with 30-day follow-up for outcomes and a 12-month look-back period (LBP) for baseline serum creatinine (SCr) level and comorbidities, including the Charlson Comorbidity Index (CCI). Index admission was the first admission during the study period that met inclusion criteria (age $\geq 18$ years, hospital length of stay (LOS) $\geq 3$ days, and $\geq 3 \mathrm{SCr}$ measures) and exclusion criteria ( $\geq 2$ dialysis visits during $L B P$, or ECMO, stage 5 CKD, eGFR $<15 \mathrm{~mL} / \mathrm{min} / 1.73 \mathrm{~m} 2$, baseline $\mathrm{SCr} \geq 4.0 \mathrm{mg} / \mathrm{dL}$, or renal transplant during index hospitalization or LBP). The study cohort was patients with
KDIGO stage 2 or 3 AKI (SCr criteria). PS-AKI was defined by AKI stage 3 lasting $\geq 3$ days, or with death within 3 days, without an intervening lower AKI stage, or stage 2 or 3 with dialysis within 3 days of the SCr criteria. An alternate definition, PS-AKISCrO, excluded PS-AKI defined by dialysis or death within 3 days of meeting $\mathrm{SCr}$ criteria. Outcomes were death during index hospitalization and during 30-day follow-up, and readmissions, death, dialysis and new dialysis (without index visit dialysis) during 30-day follow-up. Regression models adjusted for age, sex, race-ethnicity, CCl, hospital characteristics (number of beds, teaching status, region, urban/ rural), admission point of origin, admission type, (medical vs. surgical MS-DRG), primary payer, presence of CKD or sepsis, and ICU stay. Results.

Among 1,409,446 eligible patients, 9\% had stage 2-3 AKI $(n=126,528$, the study cohort) and $2.2 \%$ had PS-AKI. PS-AKI occurred in $24.4 \%$ of the study cohort, but in $32.5 \%$ of patients with an ICU stay and $17.6 \%$ of those without. Dialysis during index hospitalization occurred in $40.2 \%$ of PS-AKI and $16.9 \%$ of PS-AKISCrO. Compared to NPS-AKI, patients with PS-AKI had slightly lower mean age (64.9 vs. 66.9 years), higher mean $\mathrm{CCl}$ score (5.0 vs. 4.3$)$, and were more likely to be men ( $58.4 \%$ vs. $47.7 \%)$ ( $p<0.001$ for all). Patients with PS-AKI or PS-AKISCrO had worse outcomes than NPS-AKI ( $p \leq \mathbf{0 . 0 0 1}$ for all, Table). Adjusted for patient, clinical and hospital characteristics, PS-AKISCrO had 1.79 times higher odds of death than NPS-AKI during index hospitalization $(p<0.0001)$. During 30-day follow-up, adjusted odds for PS-AKISCrO vs. NPS-AKI were $7 \%$ higher for readmissions, $23 \%$ higher for death, 10.7 times higher for dialysis and 6.80 times higher for new dialysis ( $p \leq \mathbf{0 . 0 1}$ for all). Odds ratios were even higher for PS-AKI vs. NPS-AKI (not shown)

\begin{tabular}{|c|c|c|c|c|c|}
\hline & \multirow{2}{*}{$\begin{array}{l}\text { NPS-AKI } \\
95,612\end{array}$} & \multirow{2}{*}{$\begin{array}{l}\text { PS-AKI } \\
30,916\end{array}$} & \multirow{2}{*}{$\begin{array}{l}\text { (PS-AKIS- } \\
\text { CrO) } \\
17,285\end{array}$} & \multicolumn{2}{|c|}{$\begin{array}{l}\text { Odds Ratio(95\%Cl) PS- } \\
\text { AKIScrO vs. NPS-AKI }\end{array}$} \\
\hline & & & & Unadjusted & Adjusted \\
\hline Index ICU stay, \% & o 39,089 (40.9\%) & $18,858(61.0 \%)$ & $8322(48.1 \%)$ & $1.34(1.30,1.39)$ & \\
\hline Index Mortality & 8071 (8.4\%) & 9711 (31.4\%) & $2578(14.9 \%)$ & $1.90(1.81,1.99)$ & $1.79(1.70,1.89)$ \\
\hline $\begin{array}{l}\text { 30-day Readmis- } \\
\text { sions }\end{array}$ & 15,264 (17.4\%) & $4030(19.0 \%)$ & 2833 (19.3\%) & $1.13(1.08,1.18)$ & $1.07(1.02,1.12)$ \\
\hline 30-day Mortality & $1303(1.5 \%)$ & $419(2.0 \%)$ & $271(1.8 \%)$ & $1.24(1.09,1.42)$ & $1.23(1.07,1.40)$ \\
\hline 30-day Dialysis & $345(0.4 \%)$ & 1580 (7.5\%) & $799(5.4 \%)$ & $14.5(12.8,16.5)$ & $10.7(9.4,12.3)$ \\
\hline $\begin{array}{l}\text { 30-day New } \\
\text { Dialysis }\end{array}$ & $316(0.4 \%)$ & $402(3.3 \%)$ & $402(3.3 \%)$ & $9.26(7.98,10.75)$ & $6.80(5.84,7.93)$ \\
\hline
\end{tabular}

Conclusion. PS-AKI is prevalent among hospitalized US adults and is associated with significantly higher risk of death during index hospitalization, and readmissions, death and dialysis during 30-day follow-up.

Reference(s)

1. Perinel S, Vincent F, Lautrette A, et al. Transient and Persistent Acute Kidney Injury and the Risk of Hospital Mortality in Critically III Patients: Results of a Multicenter Cohort Study. Crit Care Med. 2015;43(8):e269-275.

2. Uhel F, Peters-Sengers $H$, Falahi F, et al. Mortality and host response aberrations associated with transient and persistent acute kidney injury in critically ill patients with sepsis: a prospective cohort study. Intensive Care Med. 2020 Aug;46(8):1576-1589.

3. Nagata K, Horino T, Hatakeyama Y, et al. Effects of transient acute kidney injury, persistent acute kidney injury and acute kidney disease on the long-term renal prognosis after an initial acute kidney injury event. Nephrology (Carlton). 2021 Apr;26(4):312-318

4. Chawla LS, Bellomo R, Bihorac A, et al. Acute Disease Quality Initiative Workgroup 16. Acute kidney disease and renal recovery: consensus report of the Acute Disease Quality Initiative (ADQI) 16 Workgroup. Nat Rev Nephrol. 2017 Apr;13(4):241-257.

5. N/A 
000162

Long-term health-related quality of life in patients undergoing ECMO for Acute Respiratory Distress Syndrome

AMHAM. Mostafa'; CJ.Tuttle ; MA. Mckie ${ }^{2}$; JA. Fowles ${ }^{3} ;$ A. Vuylsteke

${ }^{1}$ School of Clinical Medicine, University of Cambridge School of Clinical Medicine, Cambridge, United Kingdom; ${ }^{2}$ Medical research council biostatistics unit, University of Cambridge School of Clinical Medicine, Cambridge, United Kingdom; ${ }^{3}$ Department of anaesthesia and intensive care, Royal Papworth Hospital NHS Foundation Trust, Cambridge, United Kingdom

Correspondence: A.M.H.A.M. Mostafa

Intensive Care Medicine Experimental 2020, 9(1): 000162

Introduction. Veno-venous extracorporeal membrane oxygenation (VV-ECMO) is a form of life support used in cases of severe respiratory failure. While the short-term complications and mortality of VVECMO are established in the literature (1), few studies have reported the long-term health-related quality of life (HRQOL) and small sample sizes have made it difficult to establish predictors of HRQOL (2-6).

Objectives. This study aims to assess the long-term HRQOL of patients who underwent VV-ECMO for Acute Respiratory Distress Syndrome (ARDS) and explore determinants of poor HRQOL.

Methods. We performed a retrospective, observational study of a large cohort of adults who underwent VV-ECMO for ARDS at the Royal Papworth Hospital (June 2013-March 2019). Patients surviving critical care discharge were invited to a 6-month clinic, where they completed an EQ-5D-5L questionnaire to assess their HRQOL. Multivariate analysis was performed to assess prognostic factors for HRQOL.

Results. 245 patients were included in this study (median age of 45 years). 187 (76.3\%) survived until ECMO decannulation and 172 (70.2\%) were alive at discharge from their referring hospital. Of these patients, 98 (57.3\%) attended a follow-up clinic at a mean ( \pm SD) of 204 $( \pm 45)$ days post-discharge. $46 \%$ of patients reported problems with mobility, $21 \%$ with personal care, 53\% with usual daily activities, $56 \%$ reported pain/discomfort and $49 \%$ reported anxiety/depression. Multivariate analysis identified limb ischaemia $(-0.251 ; 95 \%$ C.I. -0.104 to $-0.398 ; p$-value 0.0008$)$, renal replacement therapy $(-0.151 ;-0.049$ to $-0.253 ; 0.0038)$, and receiving $>4$ platelet transfusions $(-0.208$; -0.081 to $-0.335 ; 0.0013$ ) as predictors of poor HRQOL.

Conclusion. We demonstrated that survivors of VV-ECMO have reduced $\mathrm{HRQOL}$ in multiple domains at a mean follow-up of over 6 months, with chronic pain being the most frequent complaint. Patients who had limb ischaemia, renal replacement therapy or $>4$ platelet transfusions are particularly at-risk of a poor HRQOL and may benefit from added support measures.

\section{Reference(s)}

1. ELSO. 2020. ECLS Registry Report [Online]. Available: https://www.elso. org/Registry/Statistics/InternationalSummary.aspx [Accessed April 2021].

2. WANG, Z. Y., LI, T., WANG, C. T., XU, L. \& GAO, X. J. 2017. Assessment of 1 -year Outcomes in Survivors of Severe Acute Respiratory Distress Syndrome Receiving Extracorporeal Membrane Oxygenation or Mechanical Ventilation: A Prospective Observational Study. Chin Med J (Engl), 130, $1161-1168$

3. PEEK, G. J, MUGFORD, M, TIRUVOIPATI, R, WILSON, A, ALLEN, E, THALANANY, M. M., HIBBERT, C. L., TRUESDALE, A., CLEMENS, F., COOPER, N. FIRMIN, R. K. \& ELBOURNE, D. 2009. Efficacy and economic assessment of conventional ventilatory support versus extracorporeal membrane oxygenation for severe adult respiratory failure (CESAR): a multicentre randomised controlled trial. Lancet, 374, 1351-63.

4. HODGSON, C. L., HAYES, K., EVERARD, T., NICHOL, A., DAVIES, A. R., BAILEY, M. J., TUXEN, D. V., COOPER, D. J. \& PELLEGRINO, V. 2012. Long-term quality of life in patients with acute respiratory distress syndrome requiring extracorporeal membrane oxygenation for refractory hypoxaemia. Crit Care, 16, R202.
5. GRASSELLI, G., SCARAVILLI, V., TUBIOLO, D., RUSSO, R., CRIMELLA, F., BICHI, F., CORINNA MORLACCHI, L., SCOTTI, E., PATRINI, L., GATTINONI, L., PESENTI, A. \& CHIUMELLO, D. 2019. Quality of Life and Lung Function in Survivors of Extracorporeal Membrane Oxygenation for Acute Respiratory Distress Syndrome. Anesthesiology, 130, 572-580.

6. GALAZZI, A., BRAMBILLA, A., GRASSELLI, G., PESENTI, A., FUMAGALLI, R. \& LUCCHINI, A. 2018. Quality of Life of Adult Survivors After Extra Corporeal Membrane Oxygenation (ECMO): A Quantitative Study. Dimens Crit Care Nurs, 37, 12-17.

7. No grants were received for this study

000163

Lack of improvement in sepsis care on the wards: results of four consecutive yearly point-prevalence studies in Wales

M. Kopczynska ${ }^{1}$; H. Unwin, ${ }^{1}$; R. Pugh²; B. Sharif, ; T. Chandy ${ }^{1}$; D. Davies ${ }^{1}$; M. Shield ${ }^{1}$; D. Purchase, ; S. Tilley ${ }^{1}$; A. Poacher ${ }^{1}$; L. Oliva ${ }^{1}$; S. Willis ${ }^{1}$; I. Ray'; J. Hui ${ }^{1}$; E. Wardle ${ }^{1}$; F. Andrew ${ }^{1}$; HMP. Chan, ; J. Barrington” ; L. Tan ${ }^{1}$.

T. Szakmany ${ }^{1}$

${ }^{1}$ Department of anaesthesia, intensive care and pain medicine, division of population medicine, Cardiff University, Cardiff, United Kingdom;

${ }^{2}$ Anaesthetic department, Glan Clwyd Hospital, Betsi Cadwaladr University Health Board, Bodelwyddan, United Kingdom

Correspondence: M. Kopczynska

Intensive Care Medicine Experimental 2020, 9(1): 000163

Introduction. Despite increased awareness, sepsis remains a major challenge and economic burden to healthcare globally. The 'Sepsis Six' bundle was promoted as a more deliverable tool outside of the critical care settings, but there is very little data available on the progress and change of sepsis care outside the critical care environment in the UK.

Objectives. Our aim was to compare the yearly prevalence, outcome and the 'Sepsis Six' bundle compliance in patients at risk of mortality from sepsis in non-intensive care environments.

Methods. Patients with a National Early Warning Score (NEWS) of 3 or above and suspected or proven infection were enrolled into four yearly 24-h point prevalence studies, carried out in fourteen hospitals across Wales from 2016-2019. We collected data on preadmission patient characteristics, co-morbidities, physiological and laboratory values, Dalhousie clinical frailty score, and management actions such as the completion of the 'Sepsis Six' bundle and involvement of critical care outreach.

Results. Out of the 26,947 patients screened 1,651 fulfilled inclusion criteria and were recruited. The full 'Sepsis Six' care bundle was completed on 223 (14.0\%) occasions, with no significant difference between the years. On $190(11.5 \%)$ occasions none of the bundle elements were completed (Fig. 1). There was no significant correlation between bundle element compliance, NEWS or year of study. One hundred and seventy $(10.7 \%)$ patients were seen by critical care outreach; the 'Sepsis Six' bundle was completed significantly more often in this group $(54 / 170,32.0 \%)$ than for patients who were not reviewed by critical care outreach $(168 / 1385,11.6 \% ; p<0.0001)$. Overall, 1349 (81.2\%) patients survived to 30 days with a mean survival time of 26.5 days $(95 \% \mathrm{Cl} 26.1-26.9)$ with no difference between each year of study. 90-day survival for years 2017-2019 was $74.7 \%$, with no difference between the years. In multivariate regression we identified older age, heart failure, recent chemotherapy, higher frailty score and do not attempt cardiopulmonary resuscitation orders as significantly associated with increased 30-day mortality. 


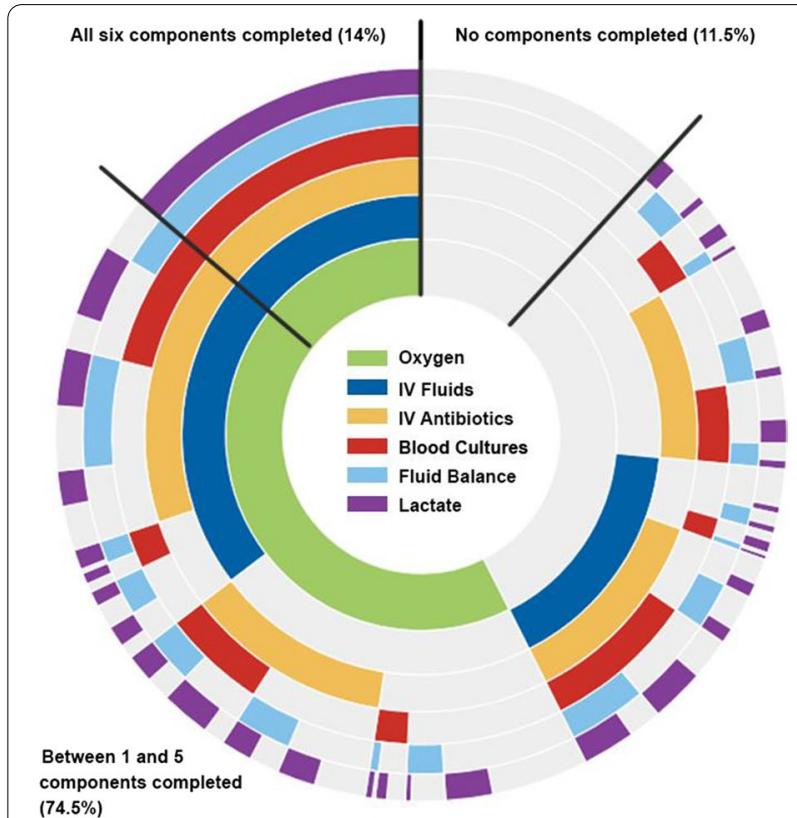

Fig. 1. 'Sepsis Six' bundle element completion rates

Conclusion. Our data suggests that despite efforts to increase sepsis awareness within the NHS, there is poor compliance with the sepsis care bundles and no change in the high mortality over the study period. Further research is needed to determine which time-sensitive ward-based interventions can reduce mortality in patients with sepsis and how can these results be embedded to routine clinical practice.

\section{6}

Reliability in proxies' assessment of preadmission health-related quality of life in elective surgery patients: a multicenter prospective observational study

K. Seidenspinner' ; J. Grunow,2; B. Ulm'; S. Weber-Carstens ${ }^{2}$; M. Blobner'; T. Wollersheim ${ }^{2}$; S. Schaller ${ }^{2}$

${ }^{1}$ Department of anaesthesiology and intensive care, Technical University of Munich, School of Medicine, Klinikum Rechts der Isar, München, Germany; ${ }^{2}$ Anaethesiology and operative intensive care medicine (ccm/cvk), CharitéUniversitätsmedizin Berlin, Charitéplatz 1, 10,117 Berlin, Germany Correspondence: K. Seidenspinner

Intensive Care Medicine Experimental 2020, 9(1): 000186

Introduction. In clinical studies it is common to obtain baseline data via proxies if patients are unconscious or not able to provide adequate information, e.g., in critically ill patients. The reliability of these proxy responses remains unclear.

Objectives. To evaluate the reliability of proxies' assessment of preadmission health-related quality of life (HRQOL) in patients undergoing elective surgery and investigate the difference between those admitted to a regular ward and an intensive care unit (ICU).

Methods. Elective surgery patients $\geq 18$ years of age with a proxy present during the postoperative period who were both able to independently answer the questionnaire were included. Preadmission HRQOL was evaluated via SF-36 by the patient prior to surgery and by their proxy postoperative on either regular ward or ICU. The patient as well as the proxy was interviewed in absence of each other. The Physical Health Component Summary Score (PCS) and Mental Health Component Summary Score (MCS) of the SF-36 were calculated. Reliability was evaluated via oneway-random, absolute agreement, single rater intraclass-correlation coefficients (ICC) as well as Bland-Altman-Plots. A univariate general linear model was used to assess the effect of factors on the difference between patient's and proxy's response.
Results. 368 patients participated in answering the SF-36 of which 204 patient-proxy pairs were included in the study (102 on the regular ward and ICU each). Baseline characteristics of patients are presented in Table 1. The median age of patients admitted to the ICU was significantly higher than in the regular ward. Majority (62\%) of patients admitted to ICU underwent heart surgery, whereas $56 \%$ of patients admitted to regular underwent visceral surgery.

ICC showed an overall moderate reliability of proxy responses for the PCS with no significant difference between the patients admitted to normal ward $(0.67(95 \% \mathrm{Cl} 0.54-0.77))$ or ICU $(0.62(95 \% \mathrm{Cl} 0.48-0.73))$. In contrast, there was only a poor reliability for the MCS but also without significant difference between the normal ward $(0.41(95 \% \mathrm{Cl} 0.22-$ $0.57)$ ) and ICU $(0.28(95 \% \mathrm{CI} 0.08-0.46))$.

The Bland-Altmann-Plots for the PCS and MCS showed an insufficient reliability of proxies' assessment in ICU and normal ward patients. Additionally, patients who were admitted to an ICU rated themselves systematically better than their proxies (Fig. 1).

In the univariate analysis the difference between proxy and patient assessment was significantly different for the PCS $(0.97(95 \% \mathrm{Cl}$ $-0.95-2.90)$ for the normal ward vs. $3.93(95 \% \mathrm{Cl} 2.04-5.82)$ for the ICU, $p=0.032)$ but not for MCS $(0.9595 \% \mathrm{Cl}-1.63-3.5$ vs. 3.68 $(95 \% \mathrm{Cl} 1.15-6.21), \mathrm{p}=0.14)$.

\begin{tabular}{|c|c|c|c|c|}
\hline Variable & All $(n=204)$ & Ward $(n=102)$ & $\operatorname{ICU}(n=102)$ & p-value \\
\hline Age (years) & $69[59-77]$ & $66.5[58-73]$ & $72[64-79]$ & 0.001 \\
\hline \multicolumn{5}{|l|}{ Gender } \\
\hline Male & $121(59)$ & 60 (59) & $61(60)$ & \multirow[t]{2}{*}{0.89} \\
\hline Female & $83(41)$ & $42(41)$ & $41(40)$ & \\
\hline Hospital length of stay (days) & $13[8-21]$ & $15[9-23]$ & $11[7-20]$ & 0.15 \\
\hline ICU length of stay (days) & n/a & n/a & $3[1-5]$ & $\mathrm{n} / \mathrm{a}$ \\
\hline Number of preexisting conditions & $3[2-6]$ & $2[1-4]$ & $6[3-9]$ & $<0.001$ \\
\hline Number of previous surgeries & $2[1-3]$ & $2[1-3]$ & $2[1-3]$ & 0.71 \\
\hline \multirow{2}{*}{\multicolumn{5}{|c|}{$\begin{array}{l}\text { Number of preexisting medication } \\
\text { Operation field }\end{array}$}} \\
\hline & & & & \\
\hline Orthopedic & $1(1)$ & $1(1)$ & $0(0)$ & \multirow[t]{10}{*}{$<0.001$} \\
\hline Trauma & $9(4)$ & $9(9)$ & $0(0)$ & \\
\hline Visceral & $89(44)$ & $57(56)$ & $32(31)$ & \\
\hline Heart & $63(31)$ & $0(0)$ & $63(62)$ & \\
\hline Thoracic & $11(5)$ & $10(10)$ & $1(1)$ & \\
\hline Vascular & $10(5)$ & $9(9)$ & $1(1)$ & \\
\hline Neuro & $11(5)$ & $10(10)$ & $1(1)$ & \\
\hline Gynecology & $7(3)$ & $4(4)$ & $3(3)$ & \\
\hline Plastics & $1(1)$ & $1(1)$ & $0(0)$ & \\
\hline Otorhinolaryngology & $2(1)$ & $1(1)$ & $1(1)$ & \\
\hline \multicolumn{5}{|l|}{$\begin{array}{l}\text { Location of postoperative } \\
\text { interview }\end{array}$} \\
\hline ICU & $38(19)$ & $0(0)$ & $64(63)$ & \multirow[t]{2}{*}{$<0.001$} \\
\hline Ward & $166(81)$ & $102(100)$ & $38(37)$ & \\
\hline
\end{tabular}

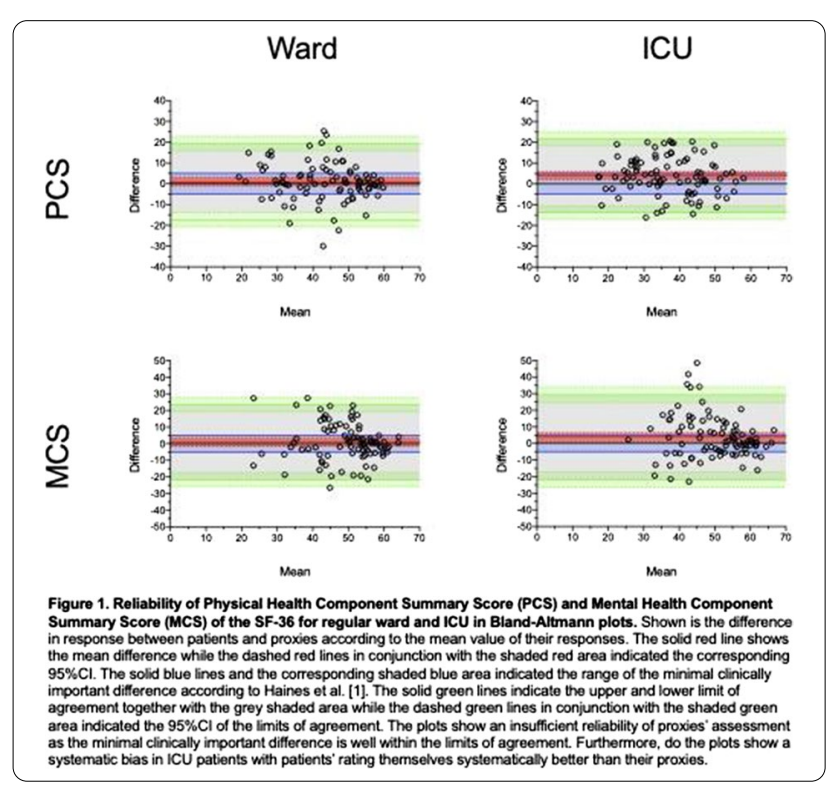


Conclusion. Preadmission HRQOL determination via a proxy seems not generally reliable. It should be tested whether factors such as the close relationship between patient and representative is a determining factor for an adequate assessment.

\section{Reference(s)}

1. Haines, K.J., et al. Long-term recovery following critical illness in an Australian cohort. JIC 6, 8 (2018). https://doi.org/10.1186/s40560-018-0276-X

2. Julius J. Grunow is participant in the BIH-Charité Junior Clinician Scientist Program funded by the Charité -Universitätsmedizin Berlin and the Berlin Institute of Health.

\section{7}

Under Pressure! A NHS Hospital ICU acquired Pressure Ulcers 3 year review: incidence and costs

L. Rodrigues Mendes

'Jack Steinberg Critical Care Unit, King's College Hospital, London, United Kingdom

Correspondence: $L$. Rodrigues Mendes

Intensive Care Medicine Experimental 2020, 9(1): 000187.

Introduction. Pressure Ulcers (PUs) have a profound impact on ICU patients health, rehabilitation, and quality of life. While their development has multifactorial causes, reporting its true incidence is still a challenge, as previous studies suggest wide variability in ICU acquired PUs incidence from 3.3 to $39.3 \%$ [1;2]. If the clinical burden of PUs is easy to recognize, their economical impact is still difficult to establish, often remaining unnoticed, with significant costs for healthcare organizations. Yearly estimations are between $£ 1.4-£ 2.1$ billion for the UK National Health Service (NHS), $€ 1.0-€ 2.3$ billion in Germany and \$9.1— $\$ 11.6$ billion in the USA, explaining why PUs costs are receiving increased attention [3;4]. In the UK, PUs can cost between $£ 1214$ to $£ 14,108$, per patient [5].

Objectives. The aim of our study was to assess incidence and estimated financial costs of adult ICU acquired PUs in King's College Hospital (KCH) NHS Trust.

Methods. Retrospective observational study, part of a Plan-Do-StudyAct service improvement approach, to analyse $\mathrm{KCH}$ ICU acquired PUs incidence and estimated costs for 2017/18, 2018/19 and 2019/20 financial years. Data was provided by KCH Tissue Viability Clinical Nurse Specialists, that assess the skin, validate and follow up all ICU PUs, reported via internal reporting systems. No formal research ethics approvals were required as patients were not directly involved. Cost estimation was done using the Department of Health and Social Care PU productivity calculator [6], created to help NHS organizations understand PUs associated cost elements.

Results. KCH NHS Trust, a London major teaching University hospital, with three general ICUs (ICU1; ICU2; ICU3), and one specialised in Liver and Hepatobiliary disorders (ICU4), in its main site. In these three years, 12,203 patients were admitted to ICU and 437 patients developed one or more PUs, with 503 reported PUs. In 2017/18 there were 137 patients with one or more PUs (149 reported PUs), and a 3.33\% PUs incidence, (2.99\% to $3.70 \%)$. In 2018/19 139 patients developed one or more PUs, (161 reported PUs), an 8.05\% increase from 2017/18, with 3.41\% PUs incidence (2.41\% to 4.39\%). In 2019/20, 161 patients developed PUs, (193 reported PUs), a $19.88 \%$ increase from $2018 / 19$, with a $4.01 \%$ incidence (2.98\% to $4.86 \%)$. We observed an incidence in medical devices related PUs, between $0.64 \%$ and $1.00 \%$, during this period. The three year ICU acquired PUs estimated costs were $£ 2,440$ million with: $£ 785,000$ costs for $2017 / 18$; $£ 812,000$ for $2018 / 19$ (3.4\% increase); $£ 843,000$ for $2019 / 20$ (3.8\% increase) (Fig. 1).

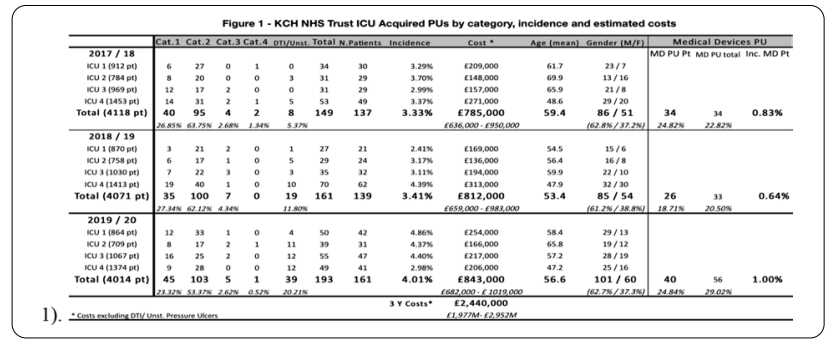

Conclusion. Our ICU acquired PUs incidences (from $2.41 \%$ to $4.86 \%$ ) are in line with other international studies, and the total estimated cost was $£ 2,440$ million. These results should influence investment in prevention strategies, therefore reducing impact to patient and organization.

\section{Reference(s)}

1. Lima-Serrano M, Méndez M, Cebollero F, Rodríguez J: Risk factors for pressure ulcer development in Intensive Care Units: A systematic review. Medicina Intensiva. 2017; Vol 41: p.339-346, Available in: https://www. medintensiva.org/en-risk-factors-for-pressure-ulcer-articulo-S217357271 7300942 [Accessed 12 Dec. 2020]

2. Chaboyer W, Thalib L, Harbeck E, Coyer F, Blot S, Bull C, Nogueira P, Lin F. Incidence and Prevalence of Pressure Injuries in Adult Intensive Care Patients: A Systematic Review and Meta-Analysis. Critical Care Medicine. 2018 Nov;46 (11): e1074-e1081. Available in https://journals.lww.com/ ccmjournal/Abstract/2018/11000/Incidence_and_Prevalence_of_Press ure_Injuries_in.39.aspx. [Accessed 17 Mar. 2021]

3. Saka, O; Pasmans R; Boodt K; Hoehne J; Radunz O: Do Healthcare Systems Promote the Prevention of Pressure Ulcers? Deloitte Consulting, 2014. Available in: https://www2.deloitte.com/content/dam/Deloitte/be/ Documents/life-sciences-health-care/2014\%20White\%20papers_Press ure\%20ulcers_A4-7.pdf [Accessed 02 Dec. 2020]

4. Padula W, Pronovost, P; Wald, H; Mishra M, Makic M, Moran, D; Meltzer $D$ : Value of hospital resources for effective pressure injury prevention: a cost-effectiveness analysis. BMJ Quality Safety 2019; Vol 28: p. 132-141, Available in: https://qualitysafety.bmj.com/content/28/2/132.abstract [Accessed 02 Dec. 2020]

5. Dealey C; Posnett, J; Walker, A: The cost of pressure ulcers in the United Kingdom. Journal of Wound Care 2012, Vol 21, N.6. p.261-266, Available in: https://www.magonlinelibrary.com/doi/abs/10.12968/jowc.2012.21.6. 261 [Accessed 24 Nov. 2020]

6. NHS Improvement (2016): Pressure Ulcer Productivity Calculator. Available in: https://improvement.nhs.uk/documents/2483/Pressure_ulcer_ productivity_calculator.xlsx [Accessed 02 Dec. 2020]

7. None

\section{8}

Long-term Effect of Antithrombin or Argatroban for the Treatment of Acute Lung Injury

M. Camprubí-Rimblas'; A. Areny-Balagueró ${ }^{2}$; E. Campaña-Duel, ${ }^{2}$; L. Morales-Quinteros ${ }^{3}$; L. Blanch'; A. Artigas ${ }^{4}$

${ }^{1}$ Institut d'investigació i innovació parc tauli (i3pt), ciberes, Universitat Autònoma de Barcelona, Cerdanyola del Vallès, Spain; ${ }^{2}$ Institut d'investigació i innovació parc tauli (i3pt), Universitat Autònoma de Barcelona, Cerdanyola del Vallès, Spain; ${ }^{3}$ Intensive care, Hospital Universitar Sagrat Cor-Grup Quirónsalut, Barcelona, Spain; ${ }^{4}$ Department of intensive care medicine, Corporacion Sanitaria Universitaria Parc Tauli, BarceIona, Spain

Correspondence: M. Camprubí-Rimblas

Intensive Care Medicine Experimental 2020, 9(1): 000208

Introduction. Coagulation and inflammation play a critical role in Acute Respiratory Distress Syndrome (ARDS). We previously demonstrated that nebulized Antithrombin (AT) decreased pulmonary 
coagulation and inflammation in a model of Acute Lung Injury (ALI) at $48 \mathrm{~h}$ without altering systemic coagulation and no bleeding (1). Herein we extent the AT treatment for as long as our ALI model lasts (72 h) and compare its effects with Argatroban (2).

Objectives. To evaluate the potential therapeutic effects of nebulized AT or Argatroban in an ALI model at $72 \mathrm{~h}$.

Methods. The ALI model was induced in Sprague-Dawley rats $(\sim 250 \mathrm{~g})$ by the intratracheal administration of $300 \mu \mathrm{l} \mathrm{HCl}(0.1 \mathrm{M})$ and $2 \mathrm{~h}$ later of $500 \mu \mathrm{l}$ Lipopolysaccharide (LPS $30 \mu \mathrm{g} / \mathrm{g}$ body weight). Two or 3 doses of AT ( $500 \mathrm{IU} / \mathrm{Kg}$ body weight) or 3 doses of Argatroban $(1 \mathrm{mg} / \mathrm{Kg}$ ) were nebulized through a mesh system called AeronebPro nebulizer system (Aerogen Limited). Control animals received saline $(0.9 \%)$ instead. Animals were sacrificed $72 \mathrm{~h}$ after injury. Procoagulant and fibrinolytic mediators and proinflammatory and chemoattractant molecules were evaluated in bronchoalveolar lavage (BAL) at protein level and in lung tissue at protein and mRNA levels. Statistics: One-Way-ANOVA and Newman Keuls posthoc test was used. When data failed the normality test in the oneway ANOVA, the Kruskal-Wallis was used (Statistical significance: $\mathrm{p} \leq$ 0.05).

Results. Increased levels of procoagulant and antifibrinolytic mediators were detected in BAL of injured animals treated with anticoagulants (thrombin antithrombin complex, TATC, and plasminogen activator inhibitor 1, PAI-1) at $72 \mathrm{~h}$ (data not shown), although decreased antifibrinolytic PAI-1 was found in lung tissue. Anticoagulant and profibrinolytic effects of 2 doses of AT in ALI at $48 \mathrm{~h}$ (data not shown) were partially maintained at $72 \mathrm{~h}$.

All anticoagulant treatments decreased significantly mRNA proinflammatory mediators (IL1 $\beta$ and TNFa) and chemoattractant molecules (Gro-kc $a$ and MCP1) in lung tissue at $72 \mathrm{~h}$, maintaining the effects found at $48 \mathrm{~h}$ (data not shown), although only 3 doses of AT decreased the protein levels of these mediators at $72 \mathrm{~h}$.

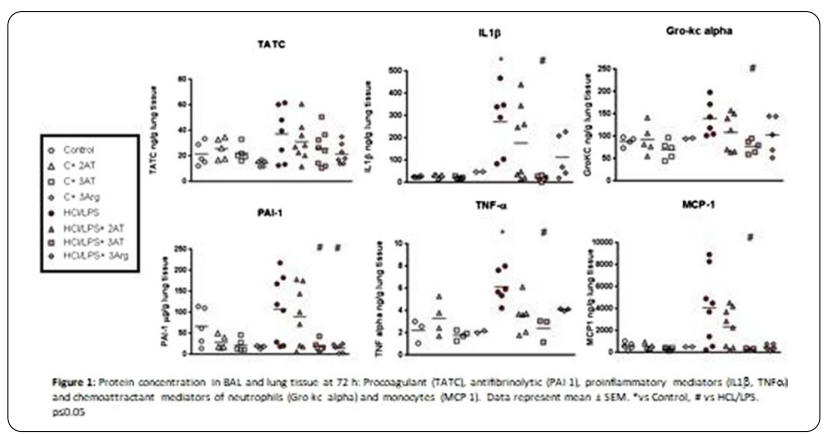

Conclusion. Nebulized anticoagulants are able to reduce inflammation, neutrophils and monocytes recruitment in an ALI model at $72 \mathrm{~h}$, but only 3 doses of AT or Argatroban promote the fibrinolytic activation.

\section{Reference(s)}

1. Camprubí-Rimblas M, Tantinyà N, Guillamat-Prats R, Bringué J, Puig F, Gómez MN, Blanch L, Artigas A. Effects of nebulized antithrombin and heparin on inflammatory and coagulation alterations in an acute lung injury model in rats. J Thromb Haemost. 2020 Mar;18(3):571-583. https:// doi.org/10.1111/jth. 14685

2. Puig F, Herrero R, Guillamat-Prats R, Gómez MN, Tijero J, Chimenti L, Stelmakh O, Blanch L, Serrano-Mollar A, Matthay MA, Artigas A. A new experimental model of acid- and endotoxin-induced acute lung injury in rats. Am J Physiol Lung Cell Mol Physiol. 2016 Aug 1;311(2):L229-37. https://doi.org/10.1152/ajplung.00390.2015.

3. This project has been financed by Grifols, S.A. Dr. Artigas received Grifols' Investor-Sponsored Research Program and was awarded with the Grifols Antithrombin Research Awards (GATRA).
000230

The impact of relative hypotension on acute kidney injury progression after cardiac surgery: a multicenter retrospective cohort study

Y. Kotani ${ }^{1}$; T. Yoshida ${ }^{2}$

${ }^{1}$ Department of intensive care medicine, Kameda Medical

Center, Kamogawa, Japan; ${ }^{2}$ Department of intensive care medicine, Tokyo

Women's Medical University Hospital, Tokyo, Japan

Correspondence: Y. Kotani

Intensive Care Medicine Experimental 2020, 9(1): 000230.

Introduction. The optimal blood pressure target to prevent acute kidney injury (AKI) after cardiac surgery remains unclear. In particular, no multicenter observational study has investigated the association between the deficit of postoperative blood pressure compared to the preoperative level and AKI among patients undergoing cardiac surgery.

Objectives. To explore the optimal blood pressure target among patients after cardiac surgery, and to determine whether the deficit between preoperative and postoperative mean perfusion pressure (MPP) is associated with AKI progression.

Methods. We conducted a multicenter retrospective cohort study in 14 ICUs in Japan. We enrolled adult patients undergoing elective coronary artery bypass grafting or valve surgery in 2018. We defined MPP as the difference between mean arterial pressure and central venous pressure. We defined MPP-deficit as the percentage difference between patient's preoperative and postoperative MPP. The exposure variables were time-weighted-average MPPdeficit and time spent with MPP-deficit $>20 \%$ during the first $24 \mathrm{~h}$ after ICU admission. The primary outcome was AKI progression, defined as one or more increase in the AKI stage based on the Kidney Disease: Improving Global Outcomes criteria between 24 and $72 \mathrm{~h}$ after ICU admission. We used multivariable logistic regression models to investigate the association between the exposure variables and the primary outcome. We also conducted an exploratory multivariable logistic regression analysis to examine the association of time-weighted-average postoperative MPP or time spent with MPP $<60 \mathrm{mmHg}$ during the first $24 \mathrm{~h}$ with subsequent AKI progression.

Results. Among the $\mathbf{7 4 6}$ patients enrolled, the median preoperative MPP was $80 \mathrm{mmHg}$ (interquartile range (IQR): $74-88 \mathrm{mmHg}$ ), and the median postoperative MPP was $66 \mathrm{mmHg}$ (IQR: $60-71 \mathrm{mmHg}$ ). The median time-weighted-average MPP-deficit was 20\% (IQR: $10-27 \%)$. The median duration with MPP-deficit $>20 \%$ was $12 \mathrm{~h}$ (IQR: 3-20 h). One hundred twenty patients (16.1\%) experienced AKI progression. The multivariable logistic regression analyses showed that every percent of increase in time-weighted-average MPP-deficit was not associated with AKI progression (odds ratio (OR): 1.01, $95 \%$ confidence interval $(95 \% \mathrm{Cl}): 0.99-1.03 ; p=0.46)$ and that every hour increase in time spent with MPP-deficit $>20 \%$ was not associated with AKI progression (OR: $1.01,95 \% \mathrm{Cl}: 0.99-1.04 ; \mathrm{p}=0.38$ ). The exploratory multivariable logistic regression analyses showed that every increase in time-weighted-average postoperative MPP and every hour increase in time spent with MPP $<60 \mathrm{mmHg}$ were associated with AKI progression (OR: 0.97, 95\% Cl: 0.94-0.99; $\mathrm{p}=0.0078$ and OR: $1.03,95 \% \mathrm{Cl}: 1.00-1.06 ; \mathrm{p}=0.024$, respectively).

Multivariable logistic regression analyses for AKI progression between 24 and $72 \mathrm{~h}$ after ICU admission

\begin{tabular}{lll}
\hline & $\begin{array}{l}\text { Adjusted OR } \\
\mathbf{( 9 5 \% ~ C l )}\end{array}$ & p value \\
\hline Time-weighted-average MPP, mmHg & $0.97(0.94-0.99)$ & 0.0078 \\
Time spent with MPP <60 mmHg, hour & $1.03(1.00-1.06)$ & 0.024 \\
Time-weighted-average MPP-deficit, \% & $1.01(0.99-1.03)$ & 0.46 \\
Time spent with MPP-deficit $>$ 20\%, hour & $1.01(0.99-1.04)$ & 0.38 \\
\hline
\end{tabular}


Conclusion. Although lower MPP and longer duration of $\mathrm{MPP}<60 \mathrm{mmHg}$ were associated with AKI progression, greater MPPdeficit or longer duration of MPP-deficit $>20 \%$ was not associated with AKI progression.

\section{Reference(s)}

1. KDIGO. KDIGO clinical practice guideline for acute kidney injury. Kidney Int Suppl. 2012;2:1-138.

2. Saito S, Uchino S, Takinami M, et al. Postoperative blood pressure deficit and acute kidney injury progression in vasopressor-dependent cardiovascular surgery patients. Critical care (London, England). 2016;20:74.

\section{4}

\section{Bayesian and heterogeneity of treatment effect analyses}

\section{of the HOT-ICU trial}

T. Klitgaard'; OL. Schjørring ; T. Lange, ; M. Hylander Møller³; A. Perner ${ }^{3}$; BS. Rasmussen, ; A. Granholm ${ }^{3}$

${ }^{1}$ Department of anesthesia and intensive care medicine, Aalborg University Hospital, Aalborg, Denmark; ${ }^{2}$ Department of public health, section of biostatistics, Copenhagen University, Copenhagen, Denmark; ${ }^{3}$ Department of intensive care, Copenhagen University Hospital, Rigshospitalet, Copenhagen, Denmark

Correspondence: T. Klitgaard

Intensive Care Medicine Experimental 2020, 9(1): 000234.

Introduction. Oxygen is the most commonly administrated medicine in the intensive care unit (ICU). However, its harms and benefits are still not fully elucidated. In the Handling Oxygenation Targets in the Intensive Care Unit (HOT-ICU) trial[1], 2928 adult ICU patients with acute hypoxaemic respiratory failure were randomised to a lower oxygenation target (arterial oxygen tension $\left(\mathrm{PaO}_{\mathrm{z}}\right): 8 \mathrm{kPa}$ ) or a higher oxygenation target $\left(\mathrm{PaO}_{2}: 12 \mathrm{kPa}\right)$ throughout their ICU admission for up to 90 days. The relative risk (RR) for the primary outcome (90-day all-cause mortality) in the primary, frequentist analysis was $1.02(95 \%$ confidence interval: 0.94-1.11, $p=0.64$ ). By using Bayesian analyses we assessed the probabilities of harmful and beneficial effects of a lower oxygenation target and the extent of such effects. Additionally, we explored whether heterogeneity of treatment effects (HTE) was present based on several baseline parameters.

Objectives. In the HOT-ICU intention-to-treat cohort we re-evaluated the effects of a lower versus a higher oxygenation target on mortality, assessed the probability of a range of effect sizes for mortality, and evaluated HTE regarding mortality according to the following baseline variables: Sequential Organ Failure Assessment (SOFA) score, $\mathrm{PaO}_{2} /$ fraction of inspired oxygen $\left(\mathrm{FiO}_{2}\right)$ ratio, noradrenaline dose, and plasma lactate concentration.

Methods. Prespecified[2] secondary Bayesian analysis of the primary outcome with assessment of HTE for prespecified baseline variables using adjusted Bayesian logistic regression models with weakly informative priors; models for assessing HTE included the baseline variable of interest and an interaction between this variable and the intervention (lower oxygenation target). Results are presented as posterior probability distributions, summarised with median values and 95\% credibility intervals (Crl). RRs $<1$ indicate benefit from the lower oxygenation target; RRs $>1$ indicate benefit of the higher oxygenation target. HTE is presented using conditional effect plots and as probabilities of negative/positive interactions between baseline variables and the intervention (relatively lower/higher risk of 90-day mortality, respectively, with the lower oxygenation target for increasing values of the baseline variable of interest).

Results. In total, 2888 of 2928 patients (98.6\%) were included adjusted $\mathrm{RR}=1.02$ (95\% Crl: 0.93-1.11). Probabilities of an $\mathrm{RR}<0.80$, $<0.90,<1.00,>1.00,>1.11$ and $>1.25$ were $0.0 \%, 0.3 \%, 36.5 \%, 63.5 \%$, $2.0 \%$ and $0.0 \%$, respectively. In the HTE analysis (Fig. 1) the probabilities of negative/positive interactions were $35 / 65 \%$ for increasing baseline SOFA scores; $14 / 86 \%$ for increasing baseline lactate concentrations, 76/24\% for increasing baseline $\mathrm{PaO}_{2} / \mathrm{FiO}_{2}$-ratios; and 5/95\% for increasing baseline noradrenaline doses.

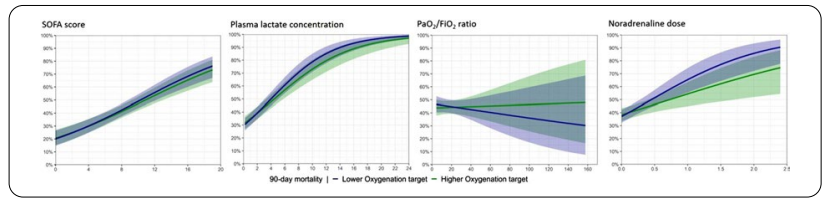

Fig. 1.Conditional effect plots illustrating the potential interaction between treatment allocation and 90-day all-cause mortality on the continuous scale. The values of the individual parameters of interest were plotted on the X-axes; the probabilities of mortality were plotted on the Y-axes.

Conclusion. In this secondary Bayesian analysis of the HOT-ICU trial, the adjusted RR for 90-day all-cause mortality, when comparing a lower and a higher oxygenation target, was with $95 \%$ probability between 0.93 and 1.11. Large effect sizes were unlikely. Our results suggested heterogeneity of treatment effects between baseline noradrenaline dose and oxygenation target allocation.

\section{Reference(s)}

1. Schjørring OL, Klitgaard TL, Perner A, Wetterslev J, Lange T, Siegemund M, Bäcklund M, Keus F, Laake JH, Morgan M, Thormar KM, Rosborg SA, Bisgaard J, Erntgaard AES, Lynnerup A-SH, Pedersen RL, Crescioli E, Gielstrup TC, Behzadi MT, Poulsen LM, Estrup S, Laigaard JP, Andersen C, Mortensen CB, Brand BA, White J, Jarnvig I-L, Møller MH, Quist L, Bestle MH, Schønemann-Lund M, Kamper MK, Hindborg M, Hollinger A, Gebhard CE, Zellweger N, Meyhoff CS, Hjort M, Bech LK, Grøfte T, Bundgaard H, Østergaard LHM, Thyø MA, Hildebrandt T, Uslu B, Sølling CG, MøllerNielsen N, Brøchner AC, Borup M, Okkonen M, Dieperink W, Pedersen UG, Andreasen AS, Buus L, Aslam TN, Winding RR, Schefold JC, Thorup SB, Iversen SA, Engstrøm J, Kjær M-BN, Rasmussen BS. Lower or Higher Oxygenation Targets for Acute Hypoxemic Respiratory Failure.N Engl J Med 2021; 384: 1301-11.

2. The Handling Oxygenation Targets in the Intensive Care Unit (HOT-ICU) trial is funded by Innovation Fund Denmark (4108- 00011A), the Novo Nordisk Foundation, the Obel Family Foundation, the Danish Regions' Medicine Foundation, the Danish Society of Anaesthesiology and Intensive Care Medicine, and the Danish Intensive Care Symposium Hindsgavl.

3. Klitgaard TL, Schjørring OL, Lange T, Møller MH, Perner A, Rasmussen BS, Granholm A. Bayesian and heterogeneity of treatment effect analyses of the HOT-ICU trial-a secondary analysis protocol.Acta Anaesthesiol Scand 2020; $1-6$

\section{5}

Correlation between nutrition, phosphate level and length of ventilation

L. Statlender'; M. Hellerman ${ }^{1}$; G. Fishman ${ }^{1}$; I. Kagan ${ }^{1}$; I. Bendavid';

M. Makalde ${ }^{1} ;$ R. Orit ${ }^{2} ;$ P. Singer ${ }^{1}$

${ }^{1}$ Intensive Care Unit, Rabin Medical Center, Petakh Tikwa, Israel: ${ }^{2}$ Industrial engineering and management, Ariel University, Ariel, Israel

Correspondence: $L$. Statlender

Intensive Care Medicine Experimental 2020, 9(1): 000235

Introduction. Hypophosphatemia has been related to prolonged ventilation and may be correlated with increased mortality. Starting or restarting nutrition to the critical care patient is associated with refeeding and hypophosphatemia. The aim of our study is to elucidate the relation between nutrition, hypophosphatemia and length of ventilation.

Methods. A retrospective cohort study included 1374 admissions in two consecutive years. Using the medical electronic chart, demographic and clinical data were obtained, including total administrated calories during admission. We correlated each patient minimal phosphate level with admission outcomes. ROC curve suggested a threshold level for defining hypophosphatemia. Based on this threshold, comparisons between baseline characteristics and outcomes of hypophosphatemic and non-hypophosphatemic patients were performed. Correlation between caloric intake, 
hypophosphatemia and length of ventilation were calculated, and finally multivariate analysis was performed.

The study was approved by the ethics committee.

Results. Cutoff level of phosphate level lesser than $2.5 \mathrm{mg} / \mathrm{dl}$ was found as best correlates with prolonged ventilation Based on this threshold, we separated the hypophosphatemic patients $(n=503)$ from the non-hypophosphatemic patients $(n=871)$. There were no significant differences between the groups, with regard to age, gender, BMI, APACHEE-II score at ICU admission, SOFA24 score, and deltaSOFA24-72. There were more trauma patients in the hypophosphatemic group, and less Ob-gyn and transplantation admissions in the hypophosphatemic group.

Mortality was significantly lower in the hypophosphatemic group $(10.74 \%$ vs $17.45 \%, p<0.002)$, but length of ventilation $(12.26 \pm 10.55$ vs $3.95 \pm 5.87$ days, $p<0.0001)$ and length of stay $(11.33 \pm 10.15$ vs $3.85 \pm 6.30$ days, $p<0.0001)$ were significantly longer. We found a negative correlation between \%calories administered and hypophosphatemia $(r=-0.3)$; between hypophosphatemia and length of ventilation $(r=-0.36)$. A positive correlation between \%calories administrated and length of ventilation $(r=0.55)$. Multivariate analysis revealed that these factors (along with male gender, SOFA score and delta SOFA) are all significant risk factors for prolonged ventilation.

Conclusion. Hypophosphatemia might be a marker of recovery in the critically ill patients. It is associated with increase length of ventilation and correlated with increased \%calories provided. Further research is needed to clarify this issue.

\section{Reference(s)}

1. Zusman O, Theilla M, Cohen J, Kagan I, Bendavid I, Singer P. Resting energy expenditure, calorie and protein consumption in critically ill patients: a retrospective cohort study. Crit Care Lond Engl. 2016;20(1):367. 10.1186/s13054-016-1538-4

2. Cohen J, Kogan A, Sahar G, Lev S, Vidne B, Singer P. Hypophosphatemia following open heart surgery: incidence and consequences. Eur J Cardiothorac Surg. 2004;26(2):306-310. 10.1016/j.ejcts.2004.03.004

3. Marik PE, Bedigian MK. Refeeding hypophosphatemia in critically ill patients in an intensive care unit. A prospective study. Arch Surg Chic III 1960. 1996;131(10):1043-1047. 10.1001/archsurg.1996.01430220037007

4. Shor R, Halabe A, Rishver S, et al. Severe hypophosphatemia in sepsis as a mortality predictor. Ann Clin Lab Sci. 2006;36(1):67-72.

5. Berger MM, Appelberg O, Reintam-Blaser A, et al. Prevalence of hypophosphatemia in the ICU—Results of an international one-day point prevalence survey. Clin Nutr Edinb Scotl. Published online December 29, 2020. 10.1016/j.clnu.2020.12.017

6. Sin JCK, King L, Ballard E, Llewellyn S, Laupland KB, Tabah A. Hypophosphatemia and Outcomes in ICU: A Systematic Review and Meta-Analysis. J Intensive Care Med. Published online August 12, 2020:885,066,620,940,274. 10.1177/0885066620940274

7. Suzuki S, Egi M, Schneider AG, Bellomo R, Hart GK, Hegarty C. Hypophosphatemia in critically ill patients. J Crit Care. 2013;28(4):536.e9-536.e19. 10. 1016/j.jcrc.2012.10.011

\section{6}

Lipopolysaccharide (LPS) treatment enhances the regenerative capacity of mesenchymal stromal cells-derived exosomes

A. Areny-Balagueró, '; M. Camprubí-Rimblas'; R. Guillamat-Prats'; A. Roig, ${ }_{1}^{3}$; D. Closa, ${ }^{4}$; A. Artigas ${ }^{5}$

${ }^{1}$ Institut d'investigació i innovació parc tauli (i3pt), Universitat Autònoma de Barcelona, Cerdanyola del Vallès, Spain; ${ }^{2}$ Ciberes, Centro de Investigación Biomédica en red de Enfermedades Respiratorias, Sabadell, Spain; ${ }^{3}$ Institut de ciència de materials de barcelona (icmab), Universitat Autònoma de Barcelona, Bellaterra, Spain; ${ }^{4}$ Institut d'investigacions biomèdiques de barcelona (iibb), Consell Superior d'Investigacions Científiques, Barcelona, Spain; ${ }^{5}$ Department of intensive care medicine, Corporacion Sanitaria Universitaria Parc Tauli, Barcelona, Spain Correspondence: A. Areny-Balagueró

Intensive Care Medicine Experimental 2020, 9(1): 000236
Introduction. Sepsis is a complex syndrome produced by a systemic infection that is accompanied by a dysregulated host immune response, affecting principally the lungs and causing, in many cases, an acute respiratory distress syndrome (ARDS) (1) associated with a mortality rate of $40 \%(2,3)$. Despite decades of research, there is still a lack of a specific treatment targeting the regeneration of lung tissue injury. It is well established that the administration of mesenchymal stromal cells (MSC) has a remarkable therapeutic effectiveness (4) and that its paracrine activity, mediated by the secretion of exosomes, has a crucial role on MSCs' action (5).

Objectives. In this study we aimed to determine the effect of the exosomes from MSC in vitro on cell proliferation, and to observe how pre-conditioning the MSCs to a septic environment, changes their exosomes' content and consequently, their regenerative capacity.

Methods. MSC were isolated from male Sprague-Dawley rats' femora and tibiae and cultured in non pre-stimulated and pre-stimulated (with lipopolysaccharide (LPS)) conditions. The secreted exosomes were obtained via standard ultracentrifugation protocol. Cell proliferation experiments were conducted by treating two different epithelial cell lines (BICR-18 and CAPAN-2) with exosomes from MSC and prestimulated MSC through an in vitro wound healing and a MTT assay. Exosomal protein profile was determined by a liquid chromatographymass spectrometry analysis.

Results. The treatment of epithelial cells with exosomes derived from pre-stimulated MSC (LPS exosomes) increases by an average of $10 \%$ its capacity to proliferate and to regenerate the wound healing in comparison with the epithelial cells treated with exosomes derived from non pre-stimulated MSC (Fig. 1a). The analysis of the protein content of both types of exosomes, which confirmed that pre-conditioning MSCs to a septic environment modifies the protein cargo of its exosomes (Fig. 1b),

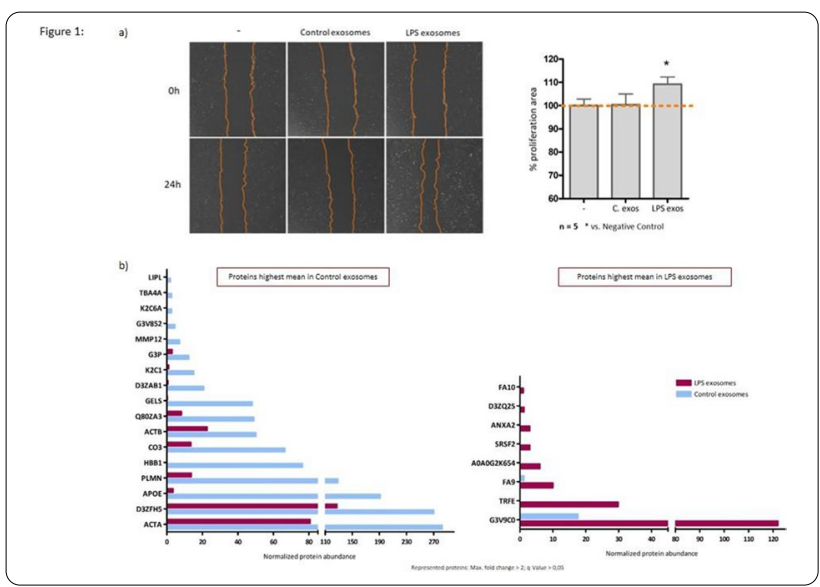

resulting in the appearance of ANXA2 a protein related to cell cycle regulation and cell proliferation (6).

Conclusion. In conclusion, pre-stimulating MSC with LPS (mimicking a septic environment) enhances their paracrine activity and improves the reparative capacity of their secreted exosomes by switching their protein expression profile.

\section{Reference(s)}

1. Niederman MS, Fein AM. Sepsis syndrome, the adult respiratory distress syndrome, and nosocomial pneumonia. A common clinical sequence. Clin Chest Med. 1990 Dec; 11(4):633-56. Review.

2. Villar J, Blanco J, Añón JM, Santos-Bouza A, Blanch L, Ambrós A, et al. The ALIEN study: incidence and outcome of acute respiratory distress syndrome in the era of lung protective ventilation. Intensive Care Medicine 2014; 37(12):1932-41.

3. Rudd KE, Johnson SC, Agesa KM, Shackelford KA, et al. Global, regional, and national sepsis incidence and mortality, 1990-2017: analysis for the Global Burden of Disease Study. Lancet. 2020; 395(10,219):200-211. 3. 
Rudd KE, Johnson SC, Agesa KM, Shackelford KA, et al. Global, regional, and national sepsis incidence and mortality, 1990-2017: analysis for the Global Burden of Disease Study. Lancet. 2020; 395(10,219):200-211.

4. Laffey J, Matthay M Cell-based Therapy for Acute Respiratory Distress Syndrome. American Journal of Respiratory and Critical Care Medicne 2017; 196 (3): 266-273.

5. 5.Wang X, Gu H, Qin D, et al. Exosomal miR-223 Contributes to Mesenchymal StemCell-Elicited Cardioprotection in Polymicrobial Sepsis. Sci Rep. 2015 Sep 8; 5: 13,721.

6. Wu M, Sun Y, Xu F, Liang Y, Liu H, Yi Y. Annexin A2 Silencing Inhibits Proliferation and Epithelial-to-mesenchymal Transition through p53-Dependent Pathway in NSCLCs. J Cancer. 2019; 10:1077-1085.

7. This project has been financed by Fundación Ramón Areces.

\section{8}

Perception and management of dysphagia in Spanish ICUs

L. Santana-Cabrera1; D. Simon-Bautista, 2. J. Rico-Rodriguez; ;

BN. Santana-Lopez³; JA. Alcaráz-Jimenez ; J. Martín-González ${ }^{1}$

${ }^{1}$ Intensive care unit, Complejo Hospitalario Universitario Insular Materno

Infantil de Gran Canaria, Las Palmas de Gran Canaria, Spain; ${ }^{2}$ Rehabilitation service, Complejo Hospitalario Universitario Insular Materno Infantil de Gran Canaria, Las Palmas de Gran Canaria, Spain; ${ }^{3}$ Intensive care unit, University Hospital of Gran Canaria Dr. Negrin, Las Palmas de Gran Canaria, Spain

Correspondence: B.N. Santana-Lopez

Intensive Care Medicine Experimental 2020, 9(1): 000238

Introduction. Oropharyngeal dysphagia is a problem in the ICU, although its incidence is not clearly known, nor is there enough information on how to evaluate it.

Objectives. To know the perception, diagnostic and therapeutic management of dysphagia in the ICUs of Spain.

Methods. Two-phase study: Phase 1, adaptation to Spanish of the survey used in the DICE study; Phase 2, analysis of the metric properties of the scales through prospective, observational and descriptive follow-up of a cohort of medical and nursing experts in the field of this research from the ICUs of Spain, from June to November 2020. The sampling was not probabilistic and consecutive, until obtaining the sample size. The present study has been funded by Fresenius Kabi Spain.

Results. 38 ICUs of which $86.8 \%$ were interdisciplinary $(n=33)$ and $81.5 \%$ treat less than 1500 patients annually $(n=31)$.

$89.5 \%$ consider dysphagia as a problem in ICU patients. $76.3 \%$ consider that dysphagia affects mortality, prolonging their stay in ICU (78.9\%) and increasing hospital costs (89.5\%). However, $52.6 \%$ do not have a standard operating procedure for its management and $36.8 \%$ do not routinely screen dysphagia in their patients.

In general, dysphagia screening is carried out in a greater proportion to those post-extubation or recently decannulated patients $(n=21$, $55.3 \%)$, to those who present clinical signs of dysphagia $(n=21$, $55.3 \%)$ and to those with baseline neurodeficits $(n=17,44.7 \%)$. The screening is normally carried out by nurses $(n=22,57.9 \%)$.

In a significant proportion, no specialized confirmatory exploratory tests are performed $(n=15,39.5 \%)$. The swallowing test $(n=20$, $52.6 \%)$ and clinical signs $(n=18,47.4 \%)$ are the most used techniques to diagnosis dysphagia.

Treatment of dysphagia is performed with modification of food consistency $(n=24,63.2 \%)$ or exclusive tube feeding $(n=15,39.5 \%)$.

Conclusion. Despite the fact that dysphagia is perceived as a very important problem in the prognosis of the ICU patient, a high percentage of units lack standardized work procedures on it. On the other hand, a significant proportion do not perform screening, do not use complementary tests and almost half reach the diagnosis through clinical signs.

\section{Reference(s)}

1. Zuercher P, Moret C, Schefold JC. Dysphagia in the intensive care unit in Switzerland (DICE) — results of a national survey on the current standard of care. Swiss Med Wkly. 2019 Jul 22;149:w20111. https://doi.org/10.4414/ smw.2019.20111

2. Perren A, Zürcher P, Schefold JC. Clinical Approaches to Assess Post-extubation Dysphagia (PED) in the Critically III. Dysphagia. 2019 Aug;34(4):475-486. https://doi.org/10.1007/s00455-019-09977-w

3. Zuercher P, Moret CS, Dziewas R, Schefold JC. Dysphagia in the intensive care unit: epidemiology, mechanisms, and clinical management. Crit Care. 2019;23(1):103. https://doi.org/10.1186/s13054-019-2400-2.

4. Skoretz SA, Flowers HL, Martino R. The incidence of dysphagia following endotracheal intubation: a systematic review. Chest. 2010;137(3):665-73. https://doi.org/10.1378/chest.09-1823.

000252

Dead space estimates may not be independently associated with 28-day mortality in COVID-19 ARDS

L. Morales-Quinteros ${ }^{1}$; A. Serpa Neto ${ }^{2}$; A. Artigas ${ }^{3}$; L. Blanch ${ }^{4}$; M. Botta ${ }^{5}$.

D. Kaufman, ; MJ. Schultz; ; A. Tsonas ${ }^{5}$; F. Paulus ${ }^{5} ;$ L. Bos $^{7}$

${ }^{1}$ Intensive care, Institut d'Investigació I Innovació Parc Taulí (I3PT), Barcelona, Spain; ${ }^{2}$ Department of critical care medicine, Hospital Israelita Albert Einstein, São Paulo, Brazil; ${ }^{3}$ Department of intensive care medicine, Corporacion Sanitaria Universitaria Parc Tauli, Barcelona, Spain; ${ }^{4}$ Institut d'investigació i innovació parc taulí i3pt, Parc Taulí Hospital Universitari, Sabadell, Spain; ${ }^{5}$ Intensive care, Amsterdam UMC, locatie AMC, Amsterdam, Netherlands; ${ }^{6}$ Icu, NYU School of Medicine, New York, United States of America; ${ }^{7}$ Intensive care, Academic Medical Cen-

tre, Amsterdam, Netherlands

Correspondence: L. Morales-Quinteros

Intensive Care Medicine Experimental 2020, 9(1): 000252

Introduction. Surrogates for impaired ventilation such as estimated dead-spacefractions and the ventilatory ratio have been shown to be independently associatedwith an increased risk of mortality in the acute respiratory distress syndrome and smallcase series of COVID-19 related ARDS.

Objectives. Quantify the dynamics and determine the prognostic value of surrogate markers of wastedventilation in patients with COVID-19 related ARDS.

Methods. Secondary analysis from the PRoVENT-COVID study. The PRoVENT-COVIDis a national, multicenter, retrospective observational study done at 22 intensive care units in the Netherlands. Consecutive patients aged at least 18 years were eligible forparticipation if they had received invasive ventilation for COVID-19 at a participating ICUduring the first month of the national outbreak in the Netherlands.

Results. 927 consecutive patients admitted with COVID-19 related ARDS were included in this study. Estimations of wasted ventilation such as the estimated dead space fraction(by Harris-Benedict and direct method) and ventilatory ratio were significantly higher innonsurvivors than survivors at baseline and during the following days of mechanicalventilation $(p<0.001)$. The end-tidal-to-arterial PCO2 ratio was lower in non-survivors thanin survivors $(p<0.001)$. As ARDS severity increased, mortality increased with successivetertiles of dead space fraction by Harris-Benedict and by direct estimation, and with anincrease in the VR. The same trend was observed with decreased levels in the tertiles forthe end-tidal-to-arterial PCO2 ratio. After adjustment for a base risk model that includedchronic comorbidities and ventilation- and oxygenation-parameters, none of the deadspace estimates measured at the start of ventilation or the following days weresignificantly associated with 28-day mortality.

Conclusion. There is significant impairment of ventilation in the early course of COVID-19 related ARDS but quantification of this impairment does not add prognostic information when added to a baseline risk-model.

\section{Reference(s)}

1. Kallet RH, Lipnick MS. End-tidal-to-arterial pco2 ratio as signifier for physiologic deadspace ratio and oxygenation dysfunction in acute respiratory distress syndrome. Respir Care. 2021;66:263-268 
2. Sinha P, Calfee CS, Beitler JR, et al. Physiologic Analysis and Clinical Performance of the Ventilatory Ratio in Acute Respiratory Distress Syndrome. Am J Respir Crit Care Med. 2019;199:333-341

3. Morales-Quinteros L, Schultz MJ, Bringué J, et al. Estimated dead space fraction and the ventilatory ratio are associated with mortality in early ARDS. Ann Intensive Care. 2019;9:128.

4. Instituto de Salud Carlos III

\section{8}

An useful alternative to diagnose brain death: portable gamma-camera for imaging with single-photon emission computed tomographic (SPECT) brain scintigraphy

C. Manso Murcia'; M. Mateos Llosa'; MD. Victoria Rodenas'; AM. GÓMEZ

DEL PULGAR VILLANUEVA; AB. Pérez Pérez'; N. López Hernández;

P. Rivera Sánchez ; M. Granados Madero ; J. Moya Sánchez ; R. Jara Rubio;

M. Royo-Villanova Reparaz

${ }^{1}$ Intensive care unit, Hospital clinico universitario Virgen de la Arrix-

aca, Murcia, Spain

Correspondence: C. Manso Murcia

Intensive Care Medicine Experimental 2020, 9(1): 000258.

Introduction. Brain death (BD) implies the permanent lack of brain functions and brain stem functions. Medical doctors have long used the most advanced technology and criteria to aid in the diagnosis of death, which requires the utmost professionalism and certainty. In search of the most reliable test, multiple options have been tried in order to find the one with the best attributes and that has the greatest number of advantages.

Objectives. The aim of this study is to correlate the results of transcranial doppler ecography (TCD) and gamma-camera (GC) using a portable system to establish a reliable diagnosis of brain death.

Methods. A prospective, observational study was carried out in Virgen de la Arrixaca Hospital, Murcia, Spain. All patients diagnosed with BD by clinical criteria underwent two supplementary tests: TCD and GC using the portable system Sentinella ${ }^{\circledR}$. Patients were recruited from January 2017 to December 2020. TCD was performed by experienced intensivists; GC, by specialists in Nuclear Medicine using technetium 99 by intravenous administration. The absence of perfusion in the cerebral hemispheres and brainstem was described as a pattern consistent with $\mathrm{BD}$, and typical patterns for TCD were diastolic reverberation and / or systolic peaks.

Results. 99 patients were studied. $64.7 \%$ were men aged 57.9 on average. Causes for BD were haemorrhagic stroke (40.4\%), traumatic brain injury $(24.2 \%)$, ischemic stroke $(16.1 \%)$, post-cardiac arrest anoxic encephalopathy (13.1\%), and subarachnoid haemorrhage (6\%). A clinical diagnosis of BD was made in all cases, and both tests were performed on patients. Portable gamma-camera confirmed BD in $100 \%$ of the patients. In 21 patients TCD patterns did not confirm the BD, the absence of an acoustic window being the most frequent cause of failure in this test. $74.5 \%$ were donors. No adverse effects were reported when using the portable GC.

Conclusion. Establishing a correct and early diagnosis of BD is important due to its legal and clinical implications, and to its relevant significance in organ donation. A portable GC could be a useful and feasible tool for the diagnosis.

\section{Reference(s)}

1. Thompson BB, Wendell LC, Potter NS, Fehnel C, Wilterdink J, Silver B, Furie $K$. The use of transcranial Doppler ultrasound in confirming brain death in the setting of skull defects and extraventricular drains. Neurocrit Care. 2014 Dec;21(3):534-8. https://doi.org/10.1007/s12028-014-9979-6. PMID: 24,718,963. Copy Download.nbib Format:

2. Powner DJ, Ackerman BM, Grenvik A. Medical diagnosis of death in adults: Historical contributions to current controversies [Internet]. Vol. 348, Lancet. Lancet Publishing Group; 1996 [cited 2021 Feb 21]. p. 1219-23. Available from: http://www.thelancet.com/article/S014067369 6040159/fulltext

3. Mclean S. The Definition of Death: Contemporary Controversies. Bmj [Internet]. 1999 [cited 2021 Feb 21]:319(7207):458. Available from: https:/
books.google.com/books?hl=es\&lr=\&id=eQ6OzvpZvp8C\&oi=fnd\&pg= PR12\&ots=u9gpRkGG_8\&sig=1AJEFaCZ_o2we0desR2nrCaKQul

4. Gardiner D, Shemie S, Manara A, Opdam $\bar{H}$. International perspective on the diagnosis of death. Br J Anaesth. 2011;108:114-28.

5. Yatim A, Mercatello A, Coronel B, Bret M, Colpart JJ, Moskovtchenko JF, et al. 99mTc-HMPAO cerebral scintigraphy in the diagnosis of brain death. In:Transplantation Proceedings [Internet]. 1991 [cited 2021 Apr 8]. p. 2491. Available from: https://pubmed.ncbi.nlm.nih.gov/1926447/

6. F.F. K, I.V. P. 99mTc-hexamethylpropyleneamine oxime (HMPAO)-its diagnostic potentials for assessing the cerebral blood supply (a review) [Internet]. Vol. 34, Meditsinskaia radiologiia. 1989 [cited 2021 Apr 8]. p. 29-35. Available from: https://pubmed.ncbi.nlm.nih.gov/2685504/

\section{0}

The antecedent characteristics of 124,234 subarachnoid haemorrhage patients and an analysis of risk in women vs. men aged 55-90

C. Harrison"; M. Taquet, 2. S. Luciano, 3. P. Harrison, 2; PJ. Watkinson";

MJ. Rowland

${ }^{1}$ Critical care research group, University of Oxford, Oxford, United Kingdom; ${ }^{2}$ Department of psychiatry, Department of Psychiatry, University of Oxford, Oxford, United Kingdom; ${ }^{3}$ Clinical sciences \& operations, TriNetX, Inc., Cambridge, United States of America

Correspondence: C. Harrison

Intensive Care Medicine Experimental 2020, 9(1): 000260

Introduction. Subarachnoid haemorrhage $(\mathrm{SAH})$ is associated with high levels of morbidity, mortality and economic burden. Previous research suggests that post-menopausal women are at an increased risk of SAH compared to men(1).

Objectives. This study aimed to; i) describe the antecedent demographics and characteristics of SAH patients, and ii) estimate the relative risk of SAH in women vs. men aged 55-90, exploring if the risk ratio changes with age.

Methods. A retrospective cohort study using the electronic health records of 80 million patients via the TriNetX Analytics Network was performed. The demographics, lifetime diagnoses, selected medications and lab values (e.g., blood pressure) recorded in the year prior to $\mathrm{SAH}$ are described for $124,234 \mathrm{SAH}$ patients. The risk ratios for $\mathrm{SAH}$ among women versus men aged 55-90 and in 5-year categories from 55 to 90 , including $95 \%$ confidence intervals, were calculated.

Results. The mean age of SAH was 56.8 years $( \pm 16.8)$, with a $51: 49$ female to male ratio. The average blood pressure was $133 / 76 \mathrm{mmHg}$ and at least $25 \%$ of patients had a prior diagnosis of hypertension. Unruptured intracranial aneurysms were recorded in $2 \%$ men and $6 \%$ women. There was no difference in risk of SAH between women and men aged 55-90 (RR 1.00, $\mathrm{Cl} 0.99-1.01)$. The risk ratio changed with increasing age, from a male preponderance aged 55-59 (RR $0.90, \mathrm{Cl} 0.88-0.93)$ to a female preponderance aged $85-90$ (RR $1.07, \mathrm{Cl}$ 1.01-1.13).

Conclusion. Differences in SAH risk between post-menopausal women and men are not seen in large real-world population data. Our findings support the emerging consensus that there is no difference in risk of SAH between men and women aged 55-90(2-4). The pattern of changing risk from male to female predominance with increasing age matches that seen in ischaemic stroke(5). Primary prevention of SAH is equally important in both sexes.

\section{Reference(s)}

1. Rothwell PM, Coull AJ, Silver LE, Fairhead JF, Giles MF, Lovelock CE, et al. Population-based study of event-rate, incidence, case fatality, and mortality for all acute vascular events in all arterial territories (Oxford Vascular Study). The Lancet. 2005;366(9499):1773-83.

2. Li X, Wang T, Feng D, Xu Z, Xu X, Gao H, et al. Sex-Specific Associations of Smoking with Spontaneous Subarachnoid Hemorrhage: Findings from Observational Studies. Journal of Stroke and Cerebrovascular Diseases. 2020:29(10):105,144.

3. Appelros P, Stegmayr B, Terént A. Sex Differences in Stroke Epidemiology. Stroke. 2009:40(4):1082-90. 
4. de Rooij NK, Linn FH, van der Plas JA, Algra A, Rinkel GJ. Incidence of subarachnoid haemorrhage: a systematic review with emphasis on region, age, gender and time trends. Journal of Neurology, Neurosurgery \& Psychiatry. 2007;78(12):1365-72

5. The authors would like to thank the University of Oxford and Oxford University Clinical Academic Graduate School.

6. Etminan N, Chang H-S, Hackenberg K, de Rooij NK, Vergouwen MDI, Rinkel GJE, et al. Worldwide Incidence of Aneurysmal Subarachnoid Hemorrhage According to Region, Time Period, Blood Pressure, and Smoking Prevalence in the Population: A Systematic Review and Meta-analysis. JAMA Neurology. 2019;76(5):588-97.

\section{5}

The use of different sepsis definitions on the ward uncovers different mortality risk: results of the three Welsh national multi-centre point-prevalence studies

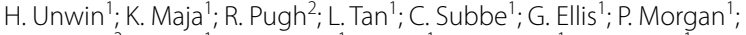

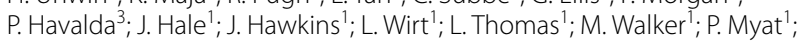

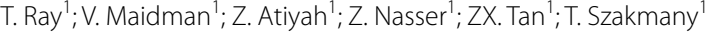

${ }^{1}$ Department of anaesthesia, intensive care and pain medicine, division of population medicine, Cardiff University, Cardiff, United Kingdom;

${ }^{2}$ Anaesthetic department, Glan Clywdd Hospital, Betsi Cadwaladar University Health Board, Bodelwyddan, United Kingdom; ${ }^{3}$ Anaesthetic department, Hywel Dda University Health Board, Haverfordwest, United Kingdom

Correspondence: $\mathrm{H}$. Unwin

Intensive Care Medicine Experimental 2020, 9(1): 000275

Introduction. In the last decade there has been significant attempts to enhance the recognition sepsis and its defining features. Resulting in emphasis on developing the most powerful method for detecting and risk stratification of sepsis outside of the intensive care units (ICU). This has led to the development of identification tools including: quick Sequential Organ Failure Assessment (quickSOFA) by the SEPSIS-3 definitions, Red Flag Sepsis by the UK Sepsis Trust and the sepsis risk stratification tool by the National Institute of Clinical Excellence (NICE). Despite these advancements a single most beneficial tool for identification of sepsis is yet to be found and adopted for use within the emergency department (ED) and on the general wards.

Objectives. Our aim was to compare the performance of Sequential Organ Failure Assessment (SOFA), systemic inflammatory response syndrome (SIRS), Red Flag Sepsis and NICE criteria's in the identification of patients at greatest risk of mortality from sepsis in non-ICU environments.

Methods. Secondary analysis of three annual 24-h point prevalence study periods. We screened all patients presenting to the ED and on the general wards of 14 acute hospitals across Wales. Those with a NEWS of 3 and proven or suspected infection were recruited.

Results. We recruited 1271 patients of which $724(56.9 \%)$ had SIRS $\geq 2,679$ (53.4\%) had SOFA $\geq 2,140$ (11.0\%) had $q S O F A \geq 2$ and $977(76.9 \%)$ had Red Flag Sepsis. When stratified according to NICE guidelines 450 (35.4\%) patients were in the "High risk" category in comparison to 665 (52.3\%) in "Moderate to High risk" and 156 $(12.3 \%)$ in "Low risk" category. None of the tools were able to reliably predict mortality at 90 -days. In a planned sensitivity analysis we have found that SOFA and NICE criteria had area under receiver operator curves (AUC) of 0.668 and 0.643 respectively at 7 days, this had reduced to 0.595 for SOFA and 0.591 for NICE by day 28 . Moreover, we could not find any significant correlation with any of the tools at any of the mortality timepoints.
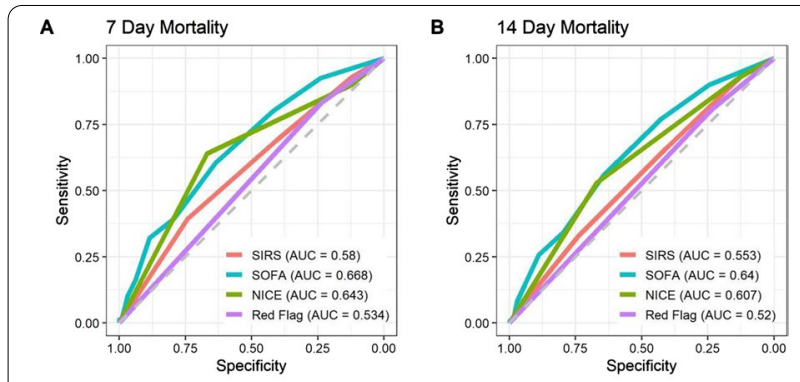

C
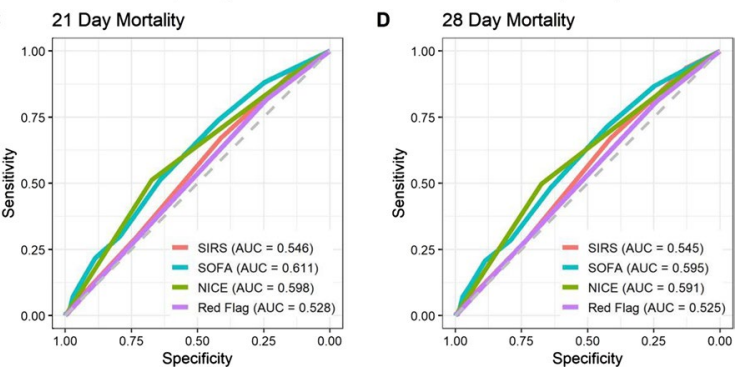

Receiver operating curves (ROC) and corresponding area under ROCs (AUC) for sepsis criteria for prediction of mortality at A: 7 days B: 14 days C: 21 days D: 28 days.

Conclusion. Our data suggests that the sepsis identification tools currently utilised do not predict mortality adequately. We found, SOFA and NICE criteria showed moderate discriminatory power for mortality at 7 and 14 days, however this disappeared with time and none of the tools accurately predicted mortality at 90 days. We believe this shows the two tools are at best moderately capable of identifying the acute changes in deterioration of sepsis patients. However for long term outcomes illness severity has less of an impact on outcome, which is more determined by comorbidity and frailty. These findings in combination with the poor correlation between the tools and mortality highlights their inadequacy for use on the general wards. We propose a largescale trial is needed in order to develop a new tool, to identify both the physiology of the initial deterioration and longer-term mortality risk of sepsis.

\section{Reference(s)}

1. Kopczynska M, Sharif B, Cleaver S, Spencer N, Kurani A, Lee C, et al. Sepsisrelated deaths in the at-risk population on the wards: attributable fraction of mortality in a large point-prevalence study. BMC research notes. 2018;11(1):720-

2. Keep JW, Messmer AS, Sladden R, Burrell N, Pinate R, Tunnicliff M, et al. National early warning score at Emergency Department triage may allow earlier identification of patients with severe sepsis and septic shock: a retrospective observational study. Emergency Medicine Journal. 2016;33(1):37.

3. Singer M, Deutschman CS, Seymour CW, Shankar-Hari M, Annane D, Bauer $M$, et al. The Third International Consensus Definitions for Sepsis and Septic Shock (Sepsis-3). JAMA. 2016;315(8):801-10.

4. Seymour CW, Liu VX, Iwashyna TJ, Brunkhorst FM, Rea TD, Scherag A, et al. Assessment of Clinical Criteria for Sepsis: For the Third International Consensus Definitions for Sepsis and Septic Shock (Sepsis-3). JAMA. 2016;315(8):762-74

5. Cecconi M, Evans L, Levy M, Rhodes A. Sepsis and septic shock. The Lancet. 2018;392(10,141):75-87. 


\section{3}

Incidence and outcomes in patients admitted to intensive care with acute kidney disease-a novel definition for prolonged acute kidney injury

M. Andonovic ${ }^{1}$; J. Traynor'; ${ }^{2}$ M. Shaw ${ }^{1}$; M. Sim ${ }^{1}$; P. Mark ${ }^{3}$; K. Puxty ${ }^{1}$

${ }^{1}$ Academic Department of Anaesthesia and Critical Care, University of Glasgow, Glasgow, United Kingdom; ${ }^{2}$ Department of nephrol-

ogy, Queen Elizabeth University Hospital, Glasgow, United Kingdom; 3 Institute of cardiovascular and medical sciences, University of Glasgow, Glasgow, United Kingdom

Correspondence: M. Andonovic

Intensive Care Medicine Experimental 2020, 9(1): 000293

Introduction. Development of acute kidney injury (AKI) in intensive care is common. Reported incidence of AKI varies significantly due to variation in classification systems used in the literature [1-3]. However, there is a well-documented link between AKI and increased mortality [4]. Recently, the term acute kidney disease (AKD) has been suggested to describe prolonged AKI lasting 7 days or more [5]. Currently, little data exists regarding differences between patients with AKD compared with rapid-reversal AKI.

Objectives. To determine the demographics of patients who develop AKD and compare these and short-term outcomes to patients with rapid-reversal AKI.

Methods. All patients aged 16 or older admitted to two large Scottish ICUs between July 2015 and June 2018 were identified. Baseline serum creatinine and subsequent values were used to identify patients with de-novo kidney injury according to KDIGO guidelines [6]. Renal recovery was defined as the point at which AKI criteria was no longer met.

Patients with recovery prior to day 7 were classified as AKI; patients with recovery at day 7 or beyond were classified as AKD. Patients who died prior to day 7 without renal recovery and patients on long-term renal replacement therapy (RRT) were excluded. Demographics were compared using relative proportions, median values and interquartile ranges, with multivariate analysis conducted to determine significance $(p<0.05)$.

Results. Out of 5,312 total patients, 1,620 developed de-novo kidney injury and survived to 7 days after first injury: 1,217 were classed as AKI and 403 were found to have AKD.

The population with AKD had higher proportions of male patients $(64.8 \%$ vs $59.4 \%)$, were more likely to be admitted from medical specialties $(49.1 \%$ vs $41.4 \%)$, had higher median APACHE II score $(24.0$ vs 19.0$)$, lower baseline eGFR (72.8 vs 84.9$)$ and higher rates of preexisting cardiovascular comorbidities ( $46.4 \%$ vs $42.2 \%)$ and diabetes ( $24.3 \%$ vs $17.1 \%)$. In addition, AKD patients had a greater requirement for RRT ( $58.6 \%$ vs $8.8 \%$ ) and multi-organ support ( $75.0 \%$ vs $52.1 \%)$.

Multivariate analysis revealed that male sex $(O R=1.26 ; p=0.043)$ and admission due to sepsis $(O R=1.36 ; p=0.003)$ were all statistically significant risk factors in development of AKD; increased baseline eGFR reduced risk $(O R=0.99 ; p<0.001)$. In-ICU $(16.3 \%$ vs $6.2 \% ; p<0.001)$ and in-hospital $(26.1 \%$ vs $11.6 \% ; p<0.001)$ mortality rates were higher in the AKD group.

Conclusion. Whilst AKI is recognised as a significant event with an associated increased risk of mortality, there is a paucity of data available on patients with AKD. The data produced from this study demonstrate that male sex, lower baseline eGFR and admission diagnosis of sepsis are all independent risk factors in the development of AKD. Short-term mortality of AKD patients is also significantly higher than patients with rapid reversal AKI. Further research is required to ascertain if the increased mortality risk of AKD is maintained over the long-term.

\section{Reference(s)}

1. Uchino S, Kellum JA, Bellomo R, Doig GS, Morimatsu H, Morgera S, et al. Acute renal failure in critically ill patients: a multinational, multicenter study. JAMA. 2005;294(7):813-8

2. Ostermann M, Chang RW. Acute kidney injury in the intensive care unit according to RIFLE. Crit Care Med. 2007;35(8):1837-43
3. Hoste EA, Bagshaw SM, Bellomo R, Cely CM, Colman R, Cruz DN, et al. Epidemiology of acute kidney injury in critically ill patients: the multinational AKI-EPI study. Intensive Care Med. 2015:41(8):1411-23.

4. Nisula S, Kaukonen KM, Vaara ST, Korhonen AM, Poukkanen M, Karlsson $\mathrm{S}$, et al. Incidence, risk factors and 90-day mortality of patients with acute kidney injury in Finnish intensive care units: the FINNAKI study. Intensive Care Med. 2013;39(3):420-8.

5. Chawla LS, Bellomo R, Bihorac A, Goldstein SL, Siew ED, Bagshaw SM, et al. Acute kidney disease and renal recovery: consensus report of the Acute Disease Quality Initiative (ADQI) 16 Workgroup. Nat Rev Nephrol. 2017;13(4):241-57.

6. Khwaja A. KDIGO clinical practice guidelines for acute kidney injury. Nephron Clin Pract. 2012;120(4):c179-84

7. No funding was received for this study

\section{6}

Time from hospital admission to ICU admission-does it really matter?

M. Gothen ${ }^{1}$; A. Marques²; I. Aragão ${ }^{3} ;$ R. Antunes ${ }^{3}$

${ }^{1}$ Medicine Integrated Master (MIM), Instituto de Ciências Biomédicas Abel Salazar (ICBAS), University of Porto (UP), Porto, Portugal; ' Emergency, Anaesthesia and Intensive Care Department (DACIE), Centro Hospitalar Universitário do Porto, Porto, Portugal; ${ }^{3}$ Intensive Care Unit (UCIP), Centro Hospitalar Universitário do Porto, Porto, Portugal

Correspondence: M. Gothen

Intensive Care Medicine Experimental 2020, 9(1): 000316.

Introduction. Several factors influence the outcome of critically ill patients. Ideally, these patients should be admitted to an intensive care unit (ICU) as soon as possible, but delays are frequent due to several factors.(1) The results from several studies regarding time from hospital admission to ICU and patient outcome are contradictory.(1-3) An emergency room (ER) staffed and run by the ICU in the emergency department (ED) can reduce the effect of time to ICU on mortality.

Objectives. To analyse the impact of time until intensive care admission on the clinical outcome of ICU patients and to determine whether patients admitted to the ICU from the ER have a better outcome.

Methods. We conducted a retrospective cohort study at a tertiary university hospital, including all adult patients admitted to the ICU from January 2016 to December 2019. Demographic data, comorbidity and disease severity scores, time from hospital admission to ER admission and to ICU admission, ICU and hospital length of stay (LOS), and outcome at ICU and hospital discharge were collected. The time from hospital admission to ICU was organized in quintiles to compare the sample characteristics of each quintile. Prolonged LOS was considered for hospital LOS over 25 days. All collected variables potentially associated with hospital mortality and LOS were studied through logistic regression models.

Results. We included 1705 admissions. The mean age was 61 years and $63 \%$ of the patients were male. $47 \%$ of the patients were admitted from the ER, $15 \%$ directly from the ED, $9 \%$ from other hospitals, $28 \%$ from hospital wards and 1\% from another ICU. The admission categories and sources for each quintile are shown in Figs. 1 and 2. The mean time from hospital admission to ICU was 81 h (h) 52 min (m) with a median of $5 \mathrm{~h} 34 \mathrm{~m}$. The mean time from hospital admission to ER admission was $2 \mathrm{~h} 52 \mathrm{~m}$ with a median of $9 \mathrm{~m}$. ICU mortality was $25 \%$ and hospital mortality was 32\%. A longer time to ICU was associated with a higher mortality $(\mathrm{OR}(95 \% \mathrm{Cl})=1,016(1,006-1,026), \mathrm{p}=0,001)$ and a prolonged LOS $($ OR $(95 \% C I)=1,168(1,136-1,200), p<0,001)$. The 5 th quintile had a higher mortality $(\mathrm{OR}(95 \% \mathrm{Cl})=1,921(1,394-2,646)$, $\mathrm{p}<0,001)$ and LOS (OR $(95 \% \mathrm{Cl})=5,976(4,272-8,360), \mathrm{p}<0,001)$ than the 1 st quintile (Fig. 3 ). In the multivariate analysis, time from hospital to ER was associated with hospital mortality $(\mathrm{OR}(95 \% \mathrm{Cl})=1,040$ $(1,013-1,068), p=0,004)$ and time to ICU was not. Patients admitted to the ICU from the ER had a shorter hospital LOS $(\mathrm{OR}(95 \% \mathrm{CI})=0,432$ $(0,349-0,534), p<0,001)$. 

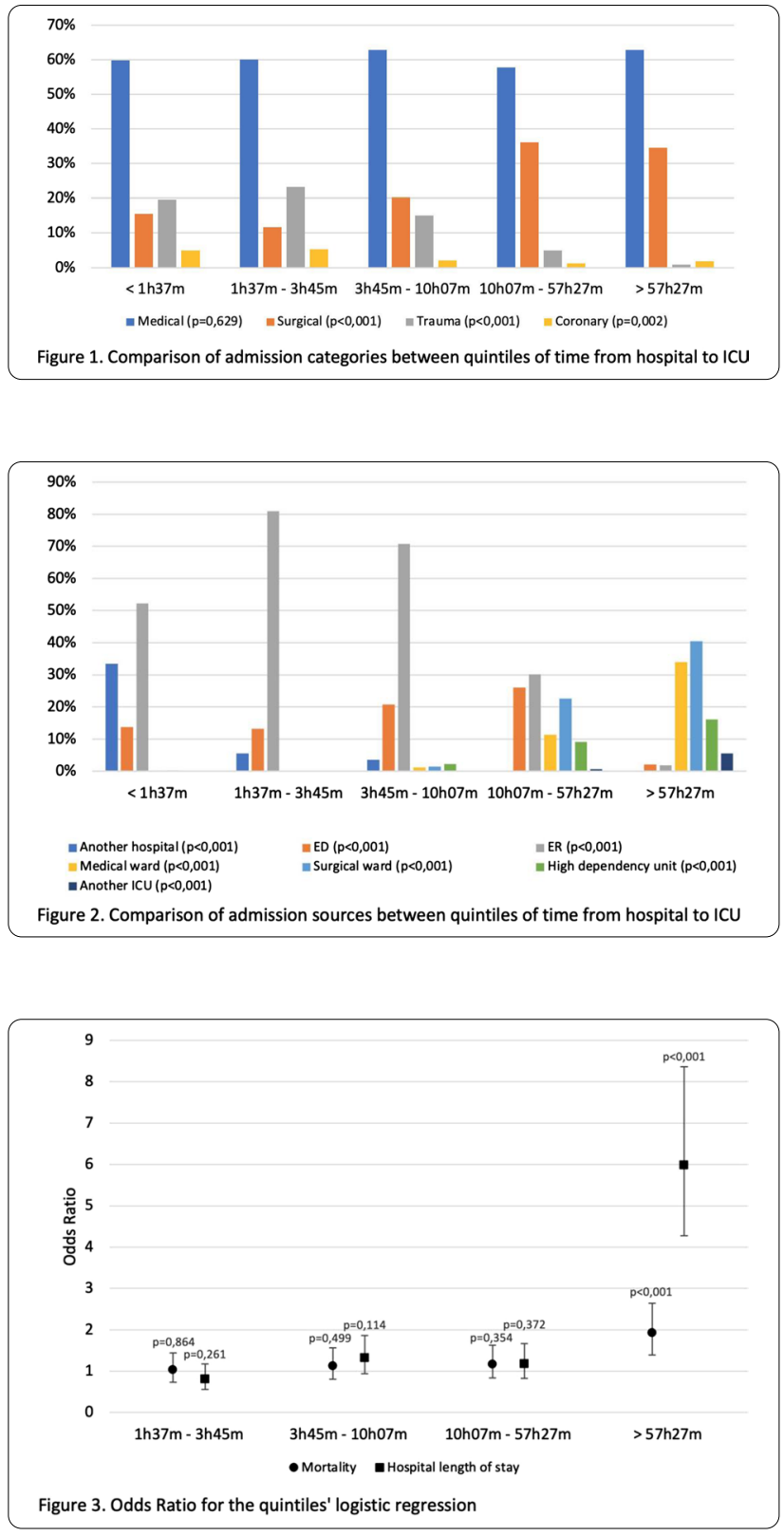

Conclusion. This study shows that a longer time from hospital admission to intensive care is associated with a higher mortality. With our model of organization, in which the ER has an ICU team, time from hospital to ER was more strongly associated with hospital mortality than time from hospital to ICU. It also shows that a longer time from hospital admission to ICU admission is associated with a longer hospital LOS.

\section{Reference(s)}

1. Groenland C., Termorshuizen F., Rietdijk W., et al. Emergency Department to ICU Time Is Associated With Hospital Mortality: A Registry Analysis of 14,788 Patients From Six University Hospitals in The Netherlands. Crit Care Med. 2019:47(11):1564-71.

2. Al-Qahtani S., Alsultan A., Haddad S., et al. The association of duration of boarding in the emergency room and the outcome of patients admitted to the intensive care unit. BMC Emerg Med. 2017;17:34.
3. O'Callaghan D., Jayia P., Vaughan-Huxley E., et al. An observational study to determine the effect of delayed admission to the intensive care unit on patient outcome. Crit Care. 2012;16(5):173.

\section{5}

Nociception Measurement In Ventilated Covid And Non-covid Patients In The Intensive Care Unit

J. van der Bos ${ }^{1}$; I. Van Der Wall'; E. de Jonge ; A. Dahan ${ }^{2}$

${ }^{1}$ intensive care, Leiden University Medical Center (LUMC), Leiden, Netherlands: ${ }^{2}$ Anesthesiology, Leiden University Medical Center (LUMC), Leiden, Netherlands

Correspondence: J. van der Bos

Intensive Care Medicine Experimental 2020, 9(1): 000325

Introduction. While during surgery nociception monitoring is gaining in popularity, it is still uncommon to measure the level of nociception in sedated and ventilated patients in the intensive care unit. Both in the operating room and intensive care unit, opioids are commonly administered to subdue nociception. In the current COVID-19 pandemic it became apparent that particularly COVID-19 patients ventilated in the intensive care unit needed a large amount of sedative and analgesic medication and sometimes muscle relaxants, to enable protective ventilation of the lungs. Several monitors are available to track nociception. One such monitor is the Nociception level (NOL) monitor (Medasense Biometrics Ltd, Ramat Gan, Israel). It is a reliable measure to monitor noxious stimulation during anesthesia and surgery. (1) The nociception level is a multiparameter monitor that combines information from the finger photoplethysmogram amplitude, skin conductance, skin conductance variation, heart rate, heart rate variability, and their time derivatives into one index ranging from 0 (absence of noxious stimulation) to 100 (severe noxious stimulation). NOL-index values $<10$ may indicate excessive analgesia (i.e. excessive suppression of nociception), $10<\mathrm{NOL}$-index values $<25$ suggests sufficient analgesia (or acceptable levels of nociception) and NOL-index values $>25$ suggests heightened nociception. Until now no study used nociception measurements in sedated and ventilated intensive care patients to systematically assess nociception levels using the NOL index. In this observational study, we monitored nociception in ventilated patients in the ICU (in COVID-19 patients and non-COVID-19 patients) for up to eight hours, to increase our knowledge on the behaviour of the NOL in sedated and ventilated patients in the ICU and correlate NOL values with use of sedatives and analgesics.

Objectives. Monitoring of nociception levels in ventilated COVID-19 and non-COVID-19 patients.

Methods. We conducted a observational and non-interventional study. The NOL was measured in adult patients during their stay in the intensive care unit for up to eight hours. To ensure that no interventions were based on the NOL, the screen of the device was covered. Ten COVID patients and nine non-COVID patients were included. All patients were sedated and received propofol, midazolam, clonidine or sufentanil or a combination of those drugs. We registered interventions, such as suction through the endotracheal tube and change of position and examined if those interventions influenced the instantaneous NOL values. We also calculated the average NOL index area above the threshold value of 25: TWA (time weighted average) $=$ (area of $\mathrm{NOL}>25$ )/(total measurement time). This number is indicative for the time that NOL values were above 25 , suggesting heightened nociception.

Results. Mean age in both groups was 69 year. $80 \%$ was male in the COVID cohort, against $78 \%$ in the non-COVID cohort. Average BMI in the COVID cohort was 28 , versus 27 in the non-COVID cohort.

No significant NOL reactions were observed during interventions (airway management and change of position) in the two patient cohorts (see Fig. 1).

TWA values were 0.2 (range 0.1-0.8) in COVID patients and 0.6 (range $0-1.5)$ in non-COVID patients $(p=0.400)$.

Conclusion. Sedated and ventilated patients with and without COVID19 disease ventilated in the intensive care were monitored for nociception level. All patients had low levels of nociception, suggesting 
excessive analgesic treatment. Based on NOL monitoring we conclude that nociceptive treatment may be lowered in both studied populations.

\section{2}

Dose-dependent impact of ventilation on the Renin-Angiotensin System: An experimental study of ventilator-induced lung injury in wild-type mice

X. Mao ; K. Krenn ${ }^{1}$; T. Tripp ${ }^{1}$; V. Tretter ${ }^{1}$; R. Reindl-Schweighofer ${ }^{2}$; F. Kraft'; B. Podesser ${ }^{3}$;. Zhu'; M. Poglitsch ${ }^{4}$; O. Domenig ${ }^{4}$; D. Abraham ${ }^{5}$; R. Ullrich ${ }^{1}$ Department of anaesthesia, general intensive care and pain medicine, Medical University of Vienna, Wien, Austria; ${ }^{2}$ Department of nephrology, Medical University of Vienna, Wien, Austria; ${ }^{3}$ Center for biomedical research, Medical University of Vienna, Wien, Austria; ${ }^{4}$ Attoquant diagnostics, Attoquant Diagnostics $\mathrm{GmbH}$, Wien, Austria; ${ }^{5}$ Center for anatomy and cell biology, Medical University of Vienna, Wien, Austria

Correspondence: X. Mao

Intensive Care Medicine Experimental 2020, 9(1): 000332

Introduction. Patients in need of mechanical ventilation may suffer additional damage to the lungs from ventilator-induced lung injury (VILI) that can increase risk of extrapulmonary organ failures (1) and affect outcome of intensive care treatment $(2,3)$. High tidal volumes (VT) and driving pressures have been associated with increased mortality in patients with and without acute respiratory distress syndrome (ARDS) (3-5). Although protective ventilatory regimens-with reduction of mechanical forces (volu-, baro-, atelectrauma) - reduce both, morbidity and mortality (6), the overall mortality of ARDS remains high. Additional non-mechanical factors are recently discussed that might play a promoting role in the development of VILI. We hypothesize that the renin angiotensin system (RAS) is implicated in the development of VILI as a component of biotrauma (7), and that pharmacological modification of the RAS activation pattern may be protective in this context.

Objectives. To better understand these pathomechanisms, and in order to develop alternative treatments, our study aimed to compare the impact of different VT on the development of VILI and RAS activity in plasma and lung tissue in mice and investigate the effects of treatment with angiotensin (Ang) 1-7 or angiotensin converting enzyme (ACE) inhibition as protective strategies.

Methods. Anesthetized mice were mechanically ventilated with low (LVT, $6 \mathrm{ml} / \mathrm{kg}$ ), high (HVT,15 ml/kg) or very high tidal volume (VHVT, $30 \mathrm{ml} / \mathrm{kg}$ ) for $4 \mathrm{~h}$, or sacrificed after $3 \mathrm{~min}$ of ventilation (sham). Additional mice ventilated with VHVT were treated with infusions of $60 \mu \mathrm{g} /$ $\mathrm{kg} / \mathrm{h}$ Ang $1-7$ or a single dose of $100 \mu \mathrm{g} / \mathrm{kg}$ captopril. Equilibrium angiotensin peptide levels were measured in plasma with liquid chromatography tandem mass spectrometry, lung tissue mRNA expression of ACE and ACE2 was measured by real time reverse transcription PCR and protein expression of ACE and ACE2 in lung tissue was evaluated by Western blotting.

Results. VILI in the VHVT group was characterized by increased lung wet/dry weight ratios $(p<0.05)$, and increased broncho-alveolar lavage fluid (BALF) total cell counts, interleukin (IL)-6, keratinocytederived cytokine (KC), and macrophage inflammatory protein (MIP) 2 levels in BALF (all $p<0.0001$ ), as compared to sham group. Equilibrium concentrations of Ang I, Ang II and Ang 1-7 showed highest plasma concentrations in the HVT group ( $p<0.01$ versus sham). While ACE mRNA expression in lung tissue was unchanged, its protein expression was VT-dependently increased $(p<0.05$ versus sham). ACE2 mRNA expression was decreased in all mechanically ventilated groups $(p<0.0001)$, while ACE2 protein levels were slightly increased in the LVT and HVT groups versus sham. Captopril increased Ang I and Ang $1-7$, and decreased Ang II in plasma $(p<0.01)$, resulting in decreased ACE-S (Ang II/Ang I ratio as marker of ACE activity) versus VHVT group $(p<0.0001)$. Ang 1-7 also decreased ACE-S $(p=0.002)$. Furthermore, captopril decreased ACE protein expression in lung tissue $(p=0.01$ versus VHVT group). Both treatments reduced levels of IL-6, KC and MIP2 in BALF ( $p<0.01$ versus VHVT group).

Conclusion. The RAS reacts to development of VILI. Classical ACEdependent and alternative ACE2-dependent RAS cascades were activated in the HVT group, whereas classical RAS activation prevailed with VHVT ventilation, as evidenced by circulating angiotensin metabolite levels and tissue expression of RAS enzymes. Ang 1-7 or captopril protected from VILI by modification of systemic more than local pulmonary RAS profile.

\section{Reference(s)}

1. The study was funded by the Dept. of Anesthesiology and General Intensive Care and a grant of Apeptico GmbH to KK

2. Madahar $\mathrm{P}$, Beitler JR. Emerging concepts in ventilation-induced lung injury. F1000Res 2020; 9. 2. Baumgardner JE, Markstaller K, Pfeiffer B, Doebrich M, Otto CM. Effects of respiratory rate, plateau pressure, and positive end-expiratory pressure on $\mathrm{PaO} 2$ oscillations after saline lavage. Am J Respir Crit Care Med 2002; 166: 1556- 1562. 3. Lanspa MJ, Peltan ID, Jacobs JR, Sorensen JS, Carpenter L, Ferraro JP, Brow n SM, Berry JG, Srivastava R, Grissom CK. Driving pressure is not associated with mortality in mechanically ventilated patients without ARDS. Crit Care 2019; 23: 424. 4. Amato MB, Meade MO, Slutsky AS, Brochard L, Costa EL, Schoenfeld DA, Stewart TE, Briel M, Talmor D, Mercat A, Richard JC, Carvalho CR, Brow er RG. Driving pressure and survival in the acute respiratory distress syndrome. N Engl J Med 2015; 372: 747-755. 5. Aoyama H, Pettenuzzo T, Aoyama K, Pinto R, Englesakis M, Fan E. Association of Driving Pressure with Mortality Among Ventilated Patients with Acute Respiratory Distress Syndrome: A Systematic Review and Meta-Analysis. Critical care medicine 2018; 46: 300-306. 6. Acute Respiratory Distress Syndrome Network, Brower RG, Matthay MA, Morris A, Schoenfeld D, Thompson BT, Wheeler A. Ventilation with lower tidal volumes as compared with traditional tidal volumes for acute lung injury and the acute respiratory distress syndrome. N Engl J Med 2000; 342: 1301-1308. 7. Hrenak J., Simko F. Renin-Angiotensin System: An Important Player in the Pathogenesis of Acute Respiratory Distress Syndrome. Int J Mol Sci. 2020;21:8038.

\section{6}

Preoperative iron deficiency with or without anemia and clinical outcome after elective cardiac surgery

F. Ampatzidou'; O. Drosos ${ }^{2}$; K. Diplaris ${ }^{3}$; N. Mihail'; G. Pistiolas ${ }^{4}$; F. Sifaki ${ }^{4}$;

A. Gkiouliava ${ }^{4}$; M. Nastou ${ }^{4}$; G. Drossos ${ }^{3}$

${ }^{1}$ Cardiothoracic icu, G.Papanikolaou Hospital, Thessaloniki, Greece;

${ }^{2}$ University of cambridge, University of Cambridge, Cambridge, United Kingdom; ${ }^{3}$ Cardiothoracic surgery, G.Papanikolaou Hospital, Thessaloniki, Greece; ${ }^{4}$ Cardiac anesthesia, G.Papanikolaou Hospital, Thessaloniki, Greece

Correspondence: F. Ampatzidou

Intensive Care Medicine Experimental 2020, 9(1): 000346

Introduction. Iron deficiency not only affects the production of haemoglobin but also suppresses the immune system by depleting its antioxidant capabilities.

Objectives. Aim of the study is to investigate whether iron deficiency defined as a level of ferritin $<100 \mathrm{mg} / \mathrm{L}$ with or without concomitant anaemia (WHO definition) has an impact on the clinical outcomes after cardiac surgery.

Methods. Patients who underwent elective cardiac surgical procedures during a 10 month period were prospectively investigated in our Cardiothoracic ICU. They were subsequently divided into two groups. Group A consisted of patients with Iron Deficiency (ID) (age 66.5 \pm 9.2 , Euroscore II 2.6 \pm 3.4 ) and Group B was the control Group (age 65.5 \pm 9.9 , Euroscorell $2.7 \pm 3.7)$. The following factors were investigated: Postoperative atrial fibrillation(AF), Acute Kidney Injury AKI (KDIGO criteria),need for Renal Replacement Therapy (RRT), need for postoperative non-invasive ventilation (NIV), stroke, prolonged ventilation ( $>24 \mathrm{~h}$ ) and Low Cardiac Output Syndrome (LCOS). We further subdivided Group A patient (ID) to those with and without anaemia. This allowed us to also investigated if anemia among Group A patients has any impact on outcome indices. Chi square test was used for statistical analysis.

Results. From a total of 361 patients, 167 consisted the ID Group. Results are shown in Tables 1 and 2. 
Table 1 Comparison of Group A (iron deficiency group) to Group B (control) shows a statistically significant variation in the use of NIV for iron deficient patients

\begin{tabular}{llll}
\hline & $\begin{array}{l}\text { ID group } \\
\text { (Group A) } \\
\mathbf{n = 1 6 7}\end{array}$ & $\begin{array}{l}\text { Control Group } \\
\text { (Group B) } \\
\mathbf{n = 1 9 4}\end{array}$ & p-value \\
\hline AF(n,\%) & $42(25)$ & $43(22)$ & 0.5 \\
AKI(n,\%) & $24(14)$ & $41(21.1)$ & 0.09 \\
RRT(n,\%) & $7(4.2)$ & $6(3.1)$ & 0.5 \\
NIV(n,\%) & $14(8.4)$ & $6(3.1)$ & $\mathbf{0 . 0 3}$ \\
Stroke(n,\%) & $1(0.6)$ & $3(1.5)$ & 0.4 \\
Prolonged ventilation(n,\%) & $8(4.8)$ & $3(1.5)$ & 0.07 \\
LCOS(n,\%) & $6(3.6)$ & $3(1.5)$ & 0.2 \\
\hline
\end{tabular}

Table 2 Comparison of the sub group of Group A (ID) patients with anaemia to Group B (control) shows a statistically significant different in the use of Renal Replacement Therapy in anaemic iron deficient patients

\begin{tabular}{llll}
\hline & $\begin{array}{l}\text { Anaemia group (sub- } \\
\text { group of Group A) } \\
\mathbf{n}=\mathbf{4 0}\end{array}$ & $\begin{array}{l}\text { Control Group } \\
\text { (Group B) } \\
\mathbf{n}=\mathbf{1 2 3}\end{array}$ & p-value \\
\hline $\operatorname{AF}(n, \%)$ & $6(15)$ & $35(28.5)$ & 0.08 \\
$\mathrm{AKI}(\mathrm{n}, \%)$ & $6(15)$ & $18(14.6)$ & 0.9 \\
$\mathrm{RRT}(\mathrm{n}, \%)$ & $4(10)$ & $3(2.4)$ & $\mathbf{0 . 0 4}$ \\
$\mathrm{NIV}(\mathrm{n}, \%)$ & $4(10)$ & $10(8.1)$ & 0.7 \\
Stroke(n,\%) & $1(02.5)$ & 0 & 0.4 \\
$\begin{array}{l}\text { Prolonged } \\
\text { ventilation(n,\%) }\end{array}$ & $1(02.5)$ & $7(5.7)$ & 0.4 \\
$\mathrm{LCOS}(\mathrm{n}, \%)$ & $1(02.5)$ & $5(4.1)$ & 0.64 \\
\hline
\end{tabular}

Conclusion. Patients with preoperative iron deficiency are more likely to require postoperative NIV due to respiratory complications. Furthermore, iron deficiency patients with concomitant anemia are more likely to require RRT due to Acute Kidney Injury.

\section{Reference}

1. Iron deficiency is associated with higher mortality in patients undergoing cardiac surgery: a prospective study British Journal of Anaesthesia, 124 (1): 25e34 (2020)

\section{2}

\section{Survival in very old patients admitted to the ICU for sepsis} is related to preexisting frailty and severity of organ dysfunction M. Ibarz'; L. Haas van Lelyveld ${ }^{2}$; A. Boumendil ${ }^{3}$; C. Jung ${ }^{4}$; B. Guidet ${ }^{5}$; H. Flaaten ${ }^{6}$; A. Artigas

${ }^{1}$ Intensive care, Hospital Universitari Sagrat Cor—Grup Quirónsalud, Barcelona, Spain; Intensive care, Diakonessenhuis Utrecht, Utrecht, Netherlands; ${ }^{3}$ Réanimation médicale, Hospital Saint-Antoine Ap-Hp, Paris, France; ${ }^{4}$ Dep. of cardiology, pulmonology and angiology, University Hospital of Düsseldorf, Düsseldorf, Germany; ${ }^{5}$ Réanimation Médicale, Hôpital
Saint-Antoine, Paris, France; ${ }^{6}$ Department of anaestesia and intensive care, University of Bergen, Bergen, Norway; ${ }^{7}$ Department of intensive care medicine, Corporacion Sanitaria Universitaria Parc Tauli, Barcelona, Spain Correspondence: M. Ibarz

Intensive Care Medicine Experimental 2020, 9(1): 000382

Introduction. Sepsis is a very frequent cause of admission of very old patients (VIPs) in Intensive Care Units (ICUs). Mortality rates remain high and although benefits of ICU admission are questioned, very few studies have investigated this age group.

Objectives. To analyze ICU, 30-day and 6-months mortality of VIPs admitted for sepsis. Secondly, to assess the incidence of sepsis diagnosis at admission and risk factors for mortality.

Methods. We evaluated the characteristics of 1025 VIPs admitted for sepsis from 2 large European prospective, observational, multicentric studies including patients aged $\geq 80$ years acutely admitted to the ICU; 307 ICUs in 21 European countries (VIP1 study; ID: NCTO3134807) and 241 ICUs in 22 European countries (VIP2 study; ID: NCT03370692). Sepsis was defined according to the sepsis 3.0 criteria. Frailty was determined according to Clinical Frailty Scale (CFS). We analyzed the severity of organ dysfunction (SOFA score), frailty, comorbidity-polypharmacy (CPS score), pre-existing physical (Katz score) and cognitive function (IQCODE score), therapeutic interventions, limitations on life support, risk factors for mortality and outcomes. Risk factors for mortality were determined by multivariate analysis and adjusted survival curves by Kaplan Meier test.

Results. Of 7465 acutely admitted VIPs, 1025 (14\%) had sepsis as admission diagnosis. $55 \%$ were male, median age was 83 years. Frailty (CFS $>4$ ) was present in $49 \%$ and median SOFA score at admission was 9 (IQR 6-12). Therapeutic interventions registered were vasoactive drugs (84\%), mechanical ventilation $(48 \%)$, noninvasive ventilation (19\%) and renal replacement therapies (19\%). Median ICU stay was 4 days (IQR 1.5-9). Life-support limitations were present as withholding in $29 \%$ and withdrawing in $15 \%$. ICU, 30 -day and 6 -month mortality were $31 \%, 45 \%$ and $54 \%$ respectively. Age, frailty, and SOFA score were the independent risk factors associated with 30-day and 6-month mortality.

Multivariate analysis (Cox). Predictors for 30-days mortality (VIP-1 study) and 6-months mortality (VIP-2 study)

\begin{tabular}{|c|c|c|c|c|}
\hline & $\begin{array}{l}\text { 30-day mortality } \\
\text { HR }(95 \% \mathrm{Cl})\end{array}$ & p-value & $\begin{array}{l}\text { 6-month mor- } \\
\text { tality } \\
\mathrm{HR}(95 \% \mathrm{Cl})\end{array}$ & p-value \\
\hline $\begin{array}{l}\text { Age } \\
\text { (per five-year } \\
\text { increase }\end{array}$ & $1.33(1.1-1.61)$ & 0.0029 & $1.16(1.09-1.25)$ & $<0.0001$ \\
\hline Frailty $(C F S>4)$ & $1.47(1.07-2.02)$ & 0.0182 & $1.34(1.18-1.51)$ & $<0.0001$ \\
\hline $\begin{array}{l}\text { SOFA score } \\
\text { (per one-point } \\
\text { increase }\end{array}$ & $1.13(1.1-1.17)$ & $<0.0001$ & $1.16(1.14-1.17)$ & $<0.0001$ \\
\hline
\end{tabular}

Kaplan-Meyer adjusted survival curves comparing septic and nonseptic acutely admitted VIPs (30-days in VIP-1; 6-months in VIP-2) 


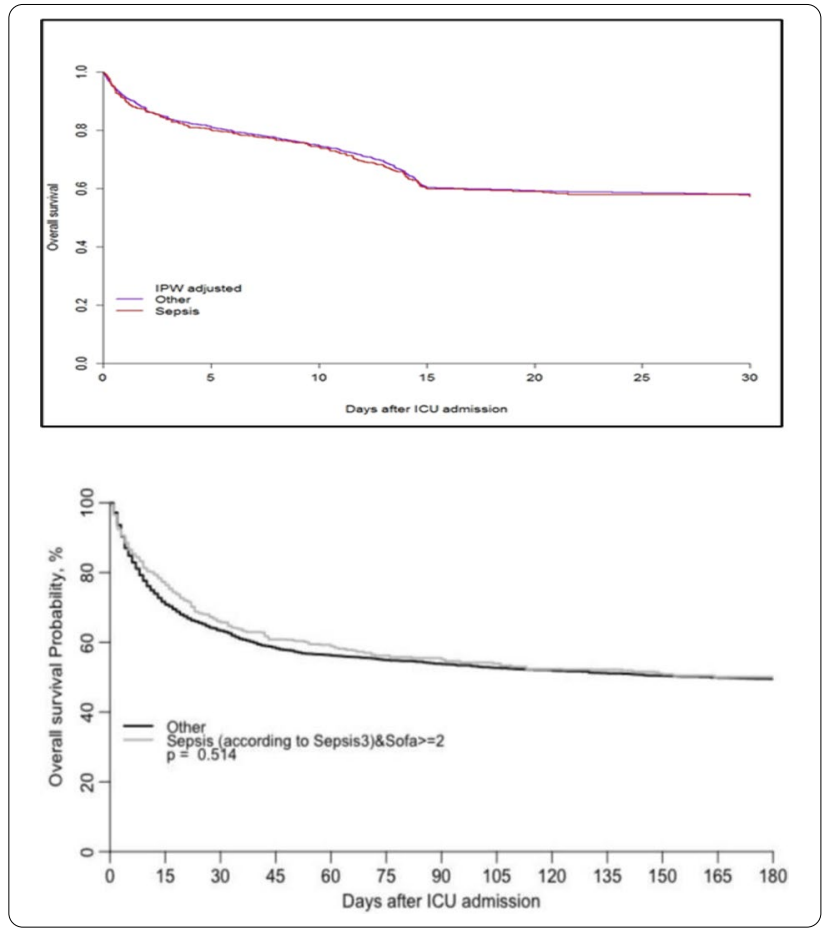

Conclusion. Our data indicate that sepsis at admission for elderly ICU patients is not associated with worse outcome compared to other admission categories. Further research is required to validate a predictive model and to define the optimal approach for VIPs who survive sepsis.

\section{Reference(s)}

1. 1.Ibarz M, Boumendil A, Haas LEM et al. (2020). Sepsis at ICU admission does not decrease 30-day survival in very old patients: a post-hoc analysis of the VIP1 multinational cohort study. Ann Intensive Care 2020; 10. 2. Haas LEM, Boumendil A, Flaatten H et al. (2021). Frailty is associated with long-term outcome in patients with sepsis who are over 80 years old: results from an observational study in 241 European ICUs. Age and ageing 1: 1-9. 3. Flaatten H, Morandi A, De Lange DW et al. (2017). The impact of frailty on $\mathrm{UCl}$ and 30-day mortality and the level of care in very elderly patients (> 80 years). Intensive Care Medicine. 43: 1820-1828. 4. Guidet B, de Lange DW, Boumendil A et al. (2020) The contribution of frailty, cognition, activity of daily life and comorbidities on outcome in acutely admitted patients over 80 years in European ICUs: the VIP2 study. Intensive Care Med; 46: 57-69.

\section{6}

Contracting the Diaphragm Every Breath Enhances Protection Against Ventilator-Induced Atrophy in Fast-Twich Diaphragm Myofibers

K. Fernandez ${ }^{1}$; E. Rohrs, ${ }^{1}$;T. Bassi, ${ }^{1}$; M. Ornowska, ${ }^{2}$; M. Nicholas, ${ }^{3}$;

S. Reynolds ${ }^{4}$

${ }^{1}$ Biomedical Physiology and Kinesiology, Simon Fraser University, Burnaby, Canada; ${ }^{2}$ Biomedical physiology and kinesiology, Simon Fraser University, Maple Ridge, Canada; ${ }^{3}$ Biomedical physiology and kinesiology, Simon Fraser University, New Westminster, Canada; ${ }^{4}$ Royal columbian hospital icu, Fraser Health Authority, North Vancouver, Canada

Correspondence: K. Fernandez

Intensive Care Medicine Experimental 2020, 9(1): 000396

Introduction. Mechanical ventilation (MV) provides non-physiological respirations to critically ill patients by unloading workload of the respiratory muscles, predominantly the diaphragm. This unloading quiesces the diaphragm muscle, thereby making it susceptible to atrophy associated with ventilator-induced diaphragmatic dysfunction[1-5]. This compounding injury can worsen morbidity and mortality in a critically ill patient, further complicating the hospital stay. A temporary transvenous diaphragm neurostimulation (TTDN) device (Lungpacer Medical Inc) that elicits diaphragm contractions synchronously with MV has previously been shown to mitigate MV-induced atrophy of all myofiber types in a preclinical model $[1,6]$. We have previously reported preliminary results on fiber-type-specific effects of TTDN after stimulating the diaphragm every alternate breath[6]; however, fiber-type-specific effects after stimulating every breath have not previously been reported.

Objectives. The goal of this study was to determine the extent of mitigation of MV-induced diaphragmatic atrophy in all myofiber types after contracting the diaphragm synchronously with every MV breath using TTDN (TTDN100\% + MV) and comparing it to the effects of contracting the diaphragm every alternate breath (TTDN50\%+MV).

Methods. This study design assigned 18 pigs $(48-70 \mathrm{~kg})$ to one of three ventilation conditions for $50 \mathrm{~h}$ : MV-Only, TTDN50\% + MV, and TTDN100\% + MV. Additionally, six pigs $(50-60 \mathrm{~kg})$ were assigned to a never-ventilated, never-paced (NV-NP) group. Diaphragm biopsies were fiber-typed by immunocytochemistry and myofiber cross-sectional areas (CSAs) were measured and normalized to each subject's weight.

Results. Animals in both treatment groups demonstrated significant mitigation of diaphragm myofiber atrophy compared to the MV-Only animals, for all myofiber types. The extent of atrophy mitigation in slow-twitch and fast-twitch diaphragm myofibers differed between the two treatment groups (Fig. 1A-D). The TTDN100\%+MV group showed a significantly larger median normalized CSA of Type IIA and IIX myofibers $(30.6 \mathrm{~mm} 2 / \mathrm{kg}$ and $47.3 \mathrm{~mm} 2 / \mathrm{kg}$, respectively)than the TTDN50\% + MV group $(28.0 \mathrm{~mm} 2 / \mathrm{kg}$ and $38.4 \mathrm{~mm} 2 / \mathrm{kg}$, respectively; $\mathrm{p}<0.0001$ for both types). The TTDN50\%+MV animals showed a significantly larger median normalized CSA of Type I myofibers $(26.6 \mathrm{~mm} 2 / \mathrm{kg})$ than the TTDN $100 \%+$ MV group $(23.2 \mathrm{~mm} 2 / \mathrm{kg}$; $\mathrm{p}<0.0001)$.

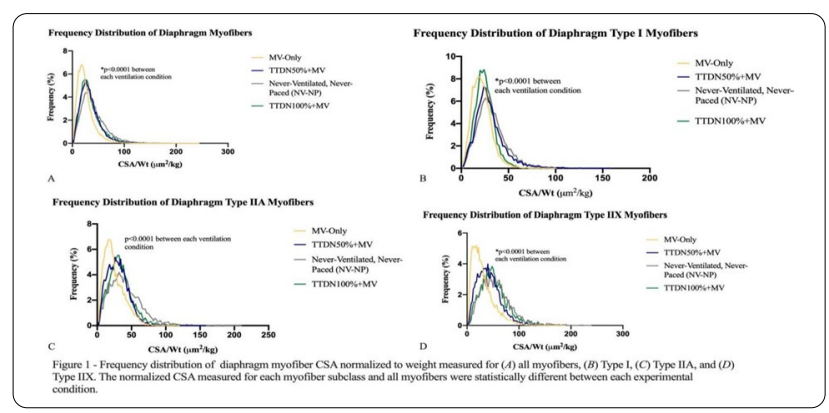

Conclusion. TTDN therapy to contract the diaphragm synchronously with MV for $50 \mathrm{~h}$ mitigates MV-induced atrophy in all myofibers; this protection was enhanced for Type II myofibers when TTDN contracted the diaphragm every breath compared to every alternate breath. These findings are clinically important as they demonstrate a dosedependent effect from synchronous stimulation with MV using TTDN. These results could translate into a targeted approach to diaphragm protection, by structuring a therapy specific to a myofiber type, to ensure effective and successful liberation from MV.

\section{Reference(s)}

1. 6.Fernandez K, Bassi T, Rohrs E, Ornowska M, Nicholas M, Reynolds S. Temporary Transvenous Diaphragm Neurostimulation Therapy With Mechanical Ventilation Mitigates Atrophy in All Diaphragm Myofiber Types. In: Chest. Vol 158. American College of Chest Physicians; 2020:A2287-A2288. 10.1016/j.chest.2020.08.1939

2. 5.Grosu HB, Lee YI, Lee J, Eden E, Eikermann M, Rose KM. Diaphragm muscle thinning in patients who are mechanically ventilated. Chest. 2012. 10. 1378/chest.11-1638 
3. 4.Supinski GS, Morris PE, Dhar S, Callahan LA. Diaphragm Dysfunction in Critical Illness. Chest. 2018;153(4). 10.1016/j.chest.2017.08.1157

4. 3.Grosu HB, Ost DE, Lee Yl, et al. Diaphragm muscle thinning in subjects receiving mechanical ventilation and its effect on extubation. Respir Care. 2017;62(7). 10.4187/respcare.05370

5. Levine S, Nguyen T, Taylor N, et al. Rapid Disuse Atrophy of Diaphragm Fibers in Mechanically Ventilated Humans. N Engl J Med. 2008;358(13):1327-1335.

6. Reynolds S, Meyyappan R, Thakkar V et al. Mitigation of VentilatorInduced Diaphragm Atrophy by Transvenous Phrenic Nerve Stimulation. Am J Respir Crit Care Med. 2017;195:339-348.

7. Student salary funding provided by Lungpacer Medical Inc. and partnership with Mitacs Accelerate Program

\section{2}

\section{Development of a dynamic prediction algorithm for early} detection of ICU-Acquired Infections

M. Varkila'; G. Lancia, ${ }^{2}$; C. Spitoni, ${ }^{2} ;$ OL. Cremer

${ }^{1}$ Intensive care, UMC Utrecht, Utrecht, Netherlands; ${ }^{2}$ Mathematics, Utre-

cht University, Utrecht, Netherlands

Correspondence: M. Varkila

Intensive Care Medicine Experimental 2020, 9(1): 000402

Introduction. Secondary infections are a major cause of morbidity and mortality in the ICU. Methods that enable earlier identification of patients who are potentially developing an ICU-acquired infection (ICU-AI) could help physicians in the management of these complications and improve outcomes for these patients.

Objectives. To investigate whether machine learning methods using analysis of high-resolution time series data from electronic health records could improve identification of high-risk patients and predict impending clinical deterioration ahead of clinically overt onset of infection.

Methods. We extracted longitudinal electronic health record data from patients admitted to a medical-surgical tertiary ICU in the Netherlands between January 2011 and December 2018. All patients older than 18 years with an ICU stay longer than 48 were included in the study. New-onset infectious episodes occurring $>48 \mathrm{~h}$ after ICU presentation were modelled using data from the MARS-cohort database. Reference time of infection diagnosis was defined by the start of empirical antibiotic therapy, or any blood culture that was followed by initiation of empirical antibiotic therapy, whichever occurs first. To be able to leverage longitudinal clinical data with different resolutions we employed a dual modelling approach. Firstly, to extract hidden patterns in the physiological state of a patient that might reflect the oncoming onset of infection, we trained a convolutional neural network (CNN) using high-resolution signals from six vital signs measurements (namely heart rate, mean arterial blood pressure, oxygen saturation, respiratory rate, end-tidal $\mathrm{CO} 2$ and minute ventilation signals). The CNN model was used to generate risk scores reflecting the probability of onset of infection in the next $24 \mathrm{~h}$. The risk scores derived from the CNN were subsequently employed as predictors in a landmarking competing risk Cox regression model together with other clinical time-fixed and time-varying candidate predictors. Equally spaced landmark time points were set every $8 \mathrm{~h}$ starting from day 2 of ICU admission to create a dynamic model that gives updated predictions across the course of the ICU admission.

Results. We studied 5388 ICU admissions amounting to 1344416 ICU observation hours. Over the course of these time series, we observed 1617 suspected ICU-acquired infections. ICU mortality was $17.6 \%$ (1037 patients). The CNN risk scores derived from the vital signs signals demonstrated a moderate ability to classify time windows as infected vs. non-infected with an averaged AUROC of 0.69 on the holdout test set (Fig. 1). When the CNN risk score was incorporated into the comprehensive landmarking Cox model, the CNN risk score emerged as one of the strongest predictors of ICU-AI (subdistribution hazard ratio $5.12,95 \% \mathrm{Cl} 3.14-8.34)$. Predictive performance of the comprehensive model, however, was only marginally improved by the addition of the CNN-score (Fig. 2). Other strong predictors of suspected infection were fever (SHR 1.83, 95\%Cl 1.61-2.09), a change in CRP (SHR 1.007,
95\% Cl 1.006-1.008), increased norepinephrine rate (SHR 1.30, 1.171.45), FiO2 (1.01, 95\% Cl 1.01-1.02) and lower platelet count (SHR 0.99, $95 \% \mathrm{Cl}$ 0.99-1.00).

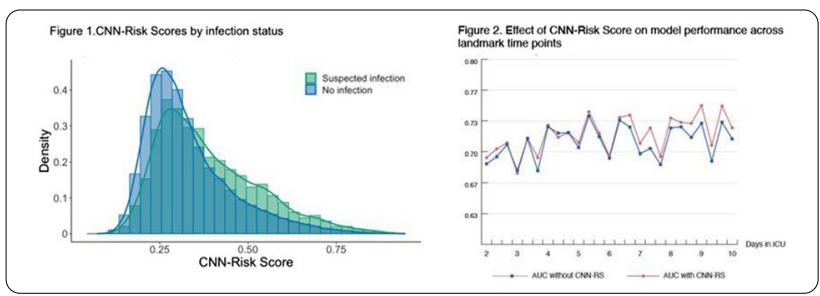

Conclusion. A CNN risk score based on high-resolution vital signs data achieved similar predictive performance to a conventional statistical model with a landmarking approach. While the CNN-score did not markedly improve predictive performance when added to a comprehensive clinical prediction model, a deep learning score may be useful in the context of a feature-rich data environment with continuous vital signs monitoring. The clinical relevance of such a real-time warning score, however, remains to be investigated.

\section{9}

Prone position is associated with recruitment and lower dead-space/shunt ratio in moderate and severe C-ARDS patients T. Fossalii; R. Colombo ${ }^{1}$; DF. Ottolina ${ }^{1}$; E. Catena ${ }^{1}$; MC. Basile ${ }^{2}$; A. Castelli ${ }^{1}$; R. Rech ${ }^{1}$; B. Borghi ; A. Ianniello ${ }^{3}$; N. Flor ${ }^{3}$; C. Fiorentini'; I. Marongiu ${ }^{2}$; B. Pavlovsky²; E. Spinelli²; T. Mauri ${ }^{2}$

${ }^{1}$ Anestesia e terapia intensiva, Sacco Hospital, Milano, Italy; ${ }^{2}$ Department of anesthesiology, Fondazione IRCCS Ca'Granda Ospedale Maggiore Policlinico, Milano, Italy; ${ }^{3}$ Unita operativa di radiologia, Sacco Hospital, Milano, Italy

Correspondence: B. Pavlovsky

Intensive Care Medicine Experimental 2020, 9(1): 000419

Introduction. Previous studies showed beneficial effects of prone position in intubated patients with COVID-19 Acute Respiratory Distress Syndrome (C-ARDS) in terms of improved oxygenation (1) and decreased mortality (2).

Objectives. We aimed to assess the physiological mechanisms enhancing lung protection and improving ventilation-perfusion (V/Q) matching during prone position in C-ARDS.

Methods. Twenty-one intubated, paralyzed patients with moderate and severe C-ARDS, admitted to the ICU of Sacco Hospital, Milan, Italy were enrolled between October 2020 and March 2021. Patients were on pressure-regulated volume control mode with Vt of $6-8 \mathrm{ml} / \mathrm{kg} \mathrm{PBW}$, PEEP $10 \mathrm{cmH} 2 \mathrm{O}$ and respiratory rate to correct $\mathrm{pH}$.

Patients were transported to the CT-scan facility in the prone position. Then, whole thorax scans were performed in the prone and supine position at end expiration, with pressure of $10 \mathrm{cmH} 2 \mathrm{O}$. Back to the ICU, electrical impedance tomography (EIT) monitoring was started and lung ventilation and perfusion (end-inspiratory $10 \mathrm{ml} 5 \%$ saline bolus method) (3) recordings were acquired in the supine and prone position. Gas exchange and respiratory mechanics were recorded together with EIT data.

Weight of different lung compartments and recruitment (i.e., the decrease of non-aerated tissue) were measured by quantitative CT scan analysis (4) Regional ventilation and a novel dynamic atelectrauma index computed as the impedance slope index (b-coefficient of $a y=a^{*}\left(x^{\wedge} b\right)+c$ power equation) were measured by EIT. From perfusion curve, the amount of only perfused (shunt) and only ventilated (dead space) units were measured.

Results. Median age was 67 [61-72] years. Patients were intubated from 2 [1-4] days.

$\mathrm{PaO} 2 / \mathrm{FiO} 2$ increased significantly in prone position $(108( \pm 41)$ vs 176 $( \pm 100), p=0.002)$.

We confirmed that C-ARDS patients, in the supine position, are characterized by relatively high normally aerated lung weight and low 
non-aerated compartment. Nonetheless, recruitment in the dorsal region of the lungs induced by prone position was large and significant, while ventral regions suffered de-recruitment, albeit by smaller extent (Table).

Respiratory system compliance was high in the supine position and didn't change when turned prone $(46( \pm 15)$ vs $45( \pm 18), p=0.957)$. This result, in combination with the observed reduction of the dynamic atelectrauma index (Table), may suggest that the same amount of alveoli were ventilated in both phases, but cyclically closing at end expiration only in the supine position.

Unmatched V/Q units didn't change significantly but oxygenation improved. An explanation may be the decrease of the dead space/ shunt ratio (Table), possibly causing larger increase in the fraction of cardiac output flowing through matched vs. shunted units.

\begin{tabular}{llll}
\hline & Supine $(\mathbf{n}=\mathbf{2 1})$ & Prone $(\mathbf{n = 2 1 )}$ & p-value \\
\hline Hyperinflated lung weight, g & $14( \pm 12)$ & $12( \pm 9)$ & $\mathbf{0 . 0 0 8}$ \\
Normally aerated lung weight, g & $356( \pm 132)$ & $400( \pm 164)$ & $\mathbf{0 . 0 0 4}$ \\
Poorly aerated lung weight, g & $525( \pm 192)$ & $505( \pm 173)$ & 0.335 \\
Non aerated lung weight, g & $571( \pm 294)$ & $477( \pm 249)$ & $\mathbf{0 . 0 0 1}$ \\
Recruitment, \% weight & - & $6.0( \pm 6.7)$ & $<\mathbf{0 . 0 0 1}$ \\
Ventral de-recruitment, \% weight & - & $-6.9( \pm 5.2)$ & $<\mathbf{0 . 0 0 1}$ \\
Dorsal recruitment, \% weight & - & $12.5( \pm 8.0)$ & $<\mathbf{0 . 0 0 1}$ \\
Dynamic atelectrauma index & $1.41( \pm 0.16)$ & $1.30(0.16)$ & $\mathbf{0 . 0 0 1}$ \\
Ventral Dynamic atelectrauma & $1.40( \pm 0.16)$ & $1.35( \pm 0.16)$ & 0.186 \\
$\quad$ index & & & $<0.001$ \\
Dorsal Dynamic atelectrauma & $1.45( \pm 0.20)$ & $1.25( \pm 0.19)$ & $<$ \\
$\quad$ index & & $8[4-19]$ & 0.105 \\
Only perfused units, \% & $5[1-12]$ & $22[15-31]$ & 0.301 \\
Only ventilated units, \% & $28[16-36]$ & $4.3[0.7-6.8]$ & $\mathbf{0 . 0 3 5}$ \\
Dead space /shunt ratio & $5.1[2.3-23.4]$ &
\end{tabular}

Conclusion. Prone position may enhance lung protection by recruiting the dorsal lung region, yielding decreased regional atelectrauma. Oxygenation improvement may be related to more physiologic V/Q matching.

\section{Reference(s)}

1. Langer T, Brioni M, Guzzardella A, et al. PRONA-COVID Group. Prone position in intubated, mechanically ventilated patients with COVID-19: a multi-centric study of more than 1000 patients. Crit Care. 2021 Apr 6;25(1):128.

2. Mathews KS, Soh H, Shaefi S, et al.; Study of the Treatment and Outcomes in Critically III Patients with Coronavirus Disease (STOP-COVID) Investigators. Prone Positioning and Survival in Mechanically Ventilated Patients With Coronavirus Disease 2019-Related Respiratory Failure. Crit Care Med. 2021 Feb 17.

3. Mauri T, Spinelli E, Scotti E, et al. Potential for Lung Recruitment and Ventilation-Perfusion Mismatch in Patients With the Acute Respiratory Distress Syndrome From Coronavirus Disease 2019. Crit Care Med. 2020 Aug;48(8):1129-1134.

4. Chiumello D, Busana M, Coppola S, et al. Physiological and quantitative CT-scan characterization of COVID-19 and typical ARDS: a matched cohort study. Intensive Care Med. 2020 Dec;46(12):2187-2196.

5. This work was supported only by academic funding.

\section{1}

Transient time-domain radars in clinical monitoring: a novel solution for continuous non-invasive intra-abdominal pressure monitoring

S. Tayebi'; A. Pourkazemi'; M. Mekeirele ${ }^{2}$; J. Poelaert ${ }^{3}$; M. Malbrain ${ }^{1}$. J. Stiens

${ }^{1}$ Department of electronics and informatics, Vrije Universiteit Brussel, Brussels, Belgium; ${ }^{2}$ Department of intensive care medicine, Universitair Ziekenhuis Brussel, Vrije Universiteit Brussel, Brussels, Belgium;

${ }^{3}$ Department of anesthesia and perioperative medicine, Universitair
Ziekenhuis Brussel, Vrije Universiteit Brussel, Brussels, Belgium Correspondence: $\mathrm{S}$. Tayebi

Intensive Care Medicine Experimental 2020, 9(1): 000421

Introduction. Reviewing recent literature reveals that the incidence of intra-abdominal hypertension (IAH) within the first week is around $50 \%$ in critically ill patients [1-4]. Since IAH is associated with higher morbidity and mortality, Intra-abdominal pressure (IAP) monitoring is highly recommended in ICU patients [1]. Finding a novel non-invasive, continuous IAP monitoring method that is also applicable to patients with pelvic hematomas or other masses could potentially improve patient care in the ICU. Using microwave (MW) reflectometry has been previously proposed as one of the most promising non-invasive alternatives for IAP measurement [5]. This abstract contains the preliminary results of an ongoing research project in developing a new radarbased sensor for non-invasive IAP measurements.

Methods. An artificial abdominal compartment was bought from "The Chamberlain Group" and used in this study (Fig. 1). The artificial abdominal wall (AW) comprises multiple layers representing skin, fat, muscle tissue, fascia, and peritoneum. A layer of water was also added to this structure to make it more realistic. Subsequently, the IAP was elevated from 10 to $24 \mathrm{mmHg}$ by increasing the intra-abdominal volume (IAV) via an air-filled balloon (instilling $30 \mathrm{ml}$ of air for every $2 \mathrm{mmHg}$ IAP elevation). The IAV increase results in changes in the geometric, mechanical, and electromagnetic properties of the AW. Therefore, MW reflection signals will be influenced with increasing IAP. Ultimately, IAP can be monitored through a novel radar-based monitoring technique known as Transient Radar Method (TRM) (Fig. 1).
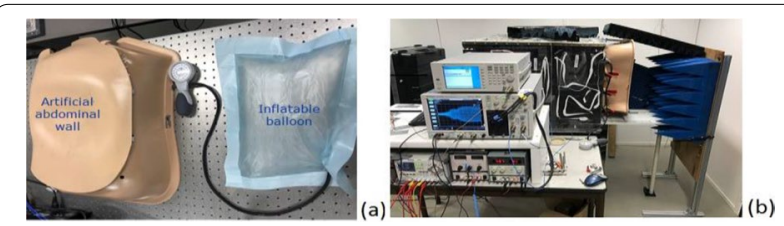

Fig. 1. The measurement set-up used in this investigation. (a) Using an inflatable balloon, IAP was elevated in the artificial abdominal model. (b) The TRM set-up used for IAP measurements

At each pressure value, the 10-GHz-transient-radar signal was directed towards the AW and its time-dependent reflection was recorded 3 times at each IAP value and further processed by applying advanced signal processing techniques [6].

Results. The results obtained from this investigation showed a promising future for non-invasive IAP measurements. On average, every $2 \mathrm{mmHg}$ IAP elevation decreased the reflection for $1.66 \pm 0.60 \mathrm{mV}$ with a $95 \%$ confidence interval (Fig. 2). The evolution of the reflection signal at different IAP values was in full agreement with the evolution of the IAV reported in previous studies [7]. A negative correlation of -0.97 with a $p$-value of 0.0001 was seen between the IAP and the reflection response of the AW as well (Fig. 2).

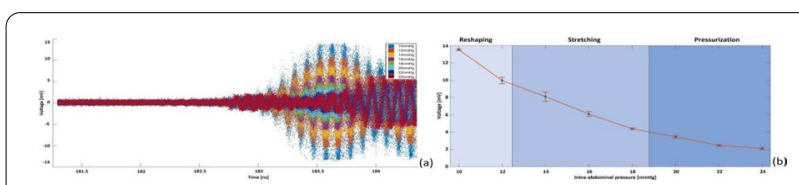

Fig. 2. Obtained results from this investigation (a) Reflection response of the abdominal model at different IAP values between 10 and $24 \mathrm{mmHg}$. (b) The reflection response of the abdominal model at a specific moment in time (183.5 ns)

Conclusion. TRM is a non-invasive, contact-free, continuous monitoring technique that can potentially be used in clinical monitoring. However, as TRM can only track relative IAP changes, the TRM calibration [8] in combination with IAP calibration before the start of a measurement cycle is an issue that needs to be solved in the future. Application of machine learning techniques is one of the options to solve this problem. 
Reference(s)

1. Pourkazemi, A., Tayebi, S., \& Stiens, J. H. (2020). Error Assessment and Mitigation Methods in Transient Radar Method. Sensors (Basel, Switzerland), 20(1), 263. https://doi.org/10.3390/s20010263

2. Malbrain, M. L., Peeters, Y., \&Wise, R. (2016). The neglected role of abdominal compliance in organ-organ interactions. Critical care (London, England), 20, 67. https://doi.org/10.1186/s13054-016-1220-x

3. Pourkazemi, A., Stiens, J., Becquaert, M., Vandewal, M. (2017). Transient Radar Method: Novel Illumination and Blind Electromagnetic/Geometrical Parameter Extraction Technique for Multilayer Structures. IEEE Transactions on Microwave Theory and Techniques, 65(6), 2171-2184. https:// doi.org/10.1109/TMTT.2017.2665633

4. Tayebi, S., Gutierrez, A., Mohout, I., Smets, E., Wise, R., Stiens, J., \& Malbrain, M. (2021). A concise overview of non-invasive intra-abdominal pressure measurement techniques: from bench to bedside. Journal of clinical monitoring and computing, 35(1), 51-70. https://doi.org/10.1007/ s10877-020-00561-4

5. Gyselaers, W., Lozada, M. J., Pacheco, L. D., Tayebi, S., \& Malbrain, M. (2020). Intra-abdominal pressure as an ignored parameter in the pathophysiology of preeclampsia. Acta obstetricia et gynecologica Scandinavica, 99(8), 963-965. https://doi.org/10.1111/aogs.13898

6. Kuteesa, J., Kituuka, O., Namuguzi, D., Ndikuno, C., Kirunda, S., Mukunya, D., \& Galukande, M. (2015). Intra-abdominal hypertension; prevalence, incidence and outcomes in a low resource setting; a prospective observational study. World journal of emergency surgery: WJES, 10, 57. https:// doi.org/10.1186/s13017-015-0051-4

7. Muturi, A., Ndaguatha, P., Ojuka, D., \& Kibet, A. (2017). Prevalence and predictors of intra-abdominal hypertension and compartment syndrome in surgical patients in critical care units at Kenyatta National Hospital. BMC emergency medicine, 17(1), 10. https://doi.org/10.1186/ s12873-017-0120-y

8. Malbrain, M. L., Chiumello, D., Pelosi, P., Wilmer, A., Brienza, N., Malcangi, V., Bihari, D., Innes, R., Cohen, J., Singer, P., Japiassu, A., Kurtop, E., De Keulenaer, B. L., Daelemans, R., Del Turco, M., Cosimini, P., Ranieri, M., Jacquet, L., Laterre, P. F., \& Gattinoni, L. (2004). Prevalence of intra-abdominal hypertension in critically ill patients: a multicentre epidemiological study. Intensive care medicine, 30(5), 822-829. https://doi.org/10.1007/ s00134-004-2169-9

The authors of the ETRO department of the Vrije Universiteit Brussel (VUB) acknowledge VUB for funding through the SRP-project M3D2, the IOF-GEAR project IOF3016, Tech4Health, Innoviris-Brussels through the Launch project BRGSOIB5, TRM4aSF, and VUB-FWO funding OZR3251: MZA Mwave Measurement Equipment

\section{7}

The association of fluid balance with Traumatic Brain Injury Outcomes: a systematic review

A. Kalakoutas ${ }^{1}$;T. Ashley²; P. Zolfaghari ${ }^{3}$

${ }^{1}$ Barts and The London School of Medicine and Dentistry, London, United Kingdom; ${ }^{2}$ Intensive care unit, St Bartholomew's Hospital, London, United Kingdom; ${ }^{3}$ Intensive care unit, Barts Health NHS Trust, London, United Kingdom

Correspondence: A. Kalakoutas

Intensive Care Medicine Experimental 2020, 9(1): 000427

Introduction. Traumatic brain injury (TBI) is a major cause of morbidity and mortality. It is associated with changes in cerebral autoregulation and disruption of the blood-brain barrier. As a result, patients are at increased risk of cerebral ischaemia, and cerebral oedema. There is debate about the association of excess fluid balance in TBI patients and worse outcomes (1-3). We carried out a systematic review to investigate the association of fluid balance and outcomes in TBI.

Methods. A systematic literature search on MEDLINE, Embase CINAHL, The Cochrane Database and bibliographies of included articles was performed. The citations were reviewed, selecting studies conducted in Critical Care Units (CCUs) assessing fluid volume management/balance and outcomes in moderate/severe TBI patients. Primary outcomes were mortality and neurological recovery. Secondary outcomes were intracranial pressure, osmotherapy requirement, development of Refractory Intracranial Hypertension, development of Acute Respiratory Distress Syndrome, length of mechanical ventilation, development of Acute Kidney Injury and ICU and hospital length of stay.

Results. Following review of the literature, fluid balance was categorised into restrictive, intermediate and liberal. Following screening, eight studies were included into the systematic review; four cohort studies, one case-control study and three RCTs. There was a lack of RCTs specific to fluid volume/balance management. Altogether, a total of $1611 \mathrm{TBI}$ patients were included. Meta-analysis on neurological outcomes was undertaken in two cohort studies only, due to the existing methodological heterogeneity amongst studies. Patients in intermediate fluid balance groups, compared to restrictive ones, were associated with decreased incidence of poor neurological outcomes $(\mathrm{OR}=0.39,95 \% \mathrm{Cl}=0.27-0.57, \mathrm{p}<0.00001)$. Similarly, patients in intermediate fluid balance groups compared to liberal ones, were also associated with a decreased incidence of poor neurological outcomes $(\mathrm{OR}=0.52,95 \% \mathrm{Cl}=0.36-0.75, \mathrm{p}=0.0004)$. Data on existing literature varies on the association of fluid balance with mortality. A RCT (4) and a cohort study (5) demonstrate increased mortality rate in higher fluid balance groups, whereas an RCT (6) and a case-control study (7) show no statistically significant difference in fluid administration volumes amongst survivors and non-survivors.

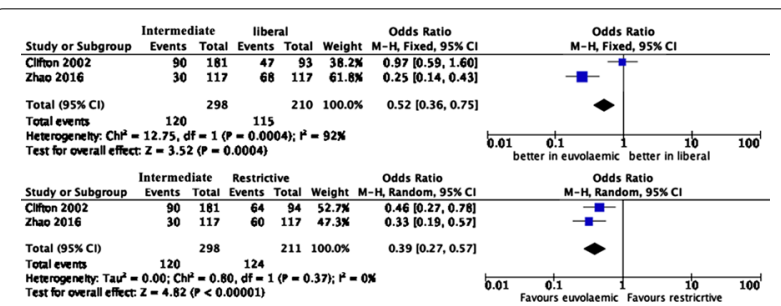

Figure. Forrest plot on Neurological Outcomes of Intermediate vs Liberal Fluid balance and Intermediate vs Restrictive fluid balance.

Conclusion. The review highlights the limited number of studies on this topic, which prevented formation of a meta-analysis in all outcomes. It allows early understanding of how euvolaemic fluid balance may improve neurological outcomes. Intervention plans based on euvolaemic fluid management, by means of fluid balance, can be assessed in RCTs. If results are significant, they could be recommended in clinical practice.

\section{Reference(s)}

1. Alvis-Miranda HR, Castellar-Leones SM, Moscote-Salazar LR. Intravenous fluid therapy in traumatic brain injury and decompressive craniectomy. Bulletin of Emergency \& Trauma. 2014 Jan;2(1):3.

2. Harris T, Thomas GOR, Brohi K. Early fluid resuscitation in severe trauma. BMJ 2012; 345:e5752

3. Bratton SL, Chestnut RM, Ghajar J, et al. Brain Trauma Foundation, American Association of Neurological Surgeons, Congress of Neurological Surgeons, Joint Section on Neurotrauma and Critical Care, AANS/CNSGuidelines for the management of severe traumatic brain injury. I. Blood pressure and oxygenation. J Neurotrauma 2007; 24 (Suppl. 1):S7-S13.

4. Ferrada P, Evans D, Wolfe L, et al. Findings of a randomized controlled trial using limited transthoracic echocardiogram (LTTE) as a hemodynamic monitoring tool in the trauma bay. Journal of Trauma and Acute Care Surgery. 2014 Jan 1;76(1):31-8.

5. Mauritz W, Janciak I, Wilbacher I, et al. Severe traumatic brain injury in Austria IV: intensive care management. Wiener Klinische Wochenschrift. 2007 Feb 1;119(1-2):46

6. Clifton GL, Valadka A, Zygun D, et al.: Very early hypothermia induction in patients with severe brain injury (the National Acute Brain Injury Study: Hypothermia II): a randomised trial. The Lancet Neurology. 2011 Feb $1 ; 10(2): 131-9$ 
7. Henzler D, Cooper DJ, Tremayne AB, et al. Early modifiable factors associated with fatal outcome in patients with severe traumatic brain injury: a case control study. Critical care medicine. 2007 Apr 1;35(4):1027-31

8. There was no grant involved in this study.

\section{2}

Efficacy of virtual reality-based early neurocognitive stimulation in critically ill patients: A pilot randomized clinical trial

G. Navarra-Ventura'; G. Gomà ; C. De Haro ${ }^{1}$; M. Jodar²; L. Sarlabous ${ }^{1}$; J. Aquino-Esperanza'; D. Hernando ${ }^{3}$; R. Bailón³; A. Ochagavía'; L. Blanch; J. López-Aguilar ${ }^{1}$; S. Fernández-Gonzalo ${ }^{4}$

'Critical care center, Parc Taulí Hospital Universitari, Institut d'Investigació i Innovació I3PT, UAB, CIBERES, Sabadell, Spain; Department of neurology, Parc Taulí Hospital Universitari, Institut d'Investigació i Innovació I3PT, UAB, CIBERSAM, Sabadell, Spain; ${ }^{3}$ Aragon institute of engineering research, Instituto de Investigación Sanitaria Aragón, UNIZAR, CIBERBBN, Zaragoza, Spain; ${ }^{4}$ Critical care center, Parc Taulí Hospital Universitari, Institut d'Investigació i Innovació I3PT, UAB, CIBERSAM, Sabadell, Spain

\section{Correspondence: G. Navarra-Ventura}

Intensive Care Medicine Experimental 2020, 9(1): 000442

Introduction. Survivors of critical illness often suffer from Post-Intensive Care Syndrome (PICS), an acquired debilitating condition that leads to significant functional and quality of life deficits.

Objectives. To evaluate the efficacy of a virtual reality (VR)-based early neurocognitive intervention to prevent cognitive and emotional deficits realted to PICS.

Methods. 72 critically ill adult patients undergoing or having undergone mechanical ventilation for $\geq 24 \mathrm{~h}$ were included in this pilot clinical trial. Patients were randomized into two groups: Treatment as usual (TAU, $n=38$ ) and Early neurocognitive stimulation (ENRIC, $\mathrm{n}=34$ ). All patients received standard ICU care. Patients in the ENRIC group received daily 20-min adjuvant neurocognitive stimulation when awake and alert during their ICU stay. The efficacy of this intervention in preventing post-ICU sequelae was assessed using a comprehensive neuropsychological battery and several mental health questionnaires at two follow-up visits.

Results. Forty-two patients (21 TAU, 21 ENRIC) completed the evaluation one month after ICU discharge and twenty-four of them (14 TAU, 10 ENRIC) one year later. One month after ICU discharge, ENRIC patients performed better on the working memory index than TAU patients (t-test: $p=0.009$; Fig. 1). Similarly, a general linear model of repeated measures reported a main effect of Group, but not Time or Group x Time interaction, on working memory index, with ENRIC patients outperforming TAU patients ( $p=0.008$; Fig. 2 ).

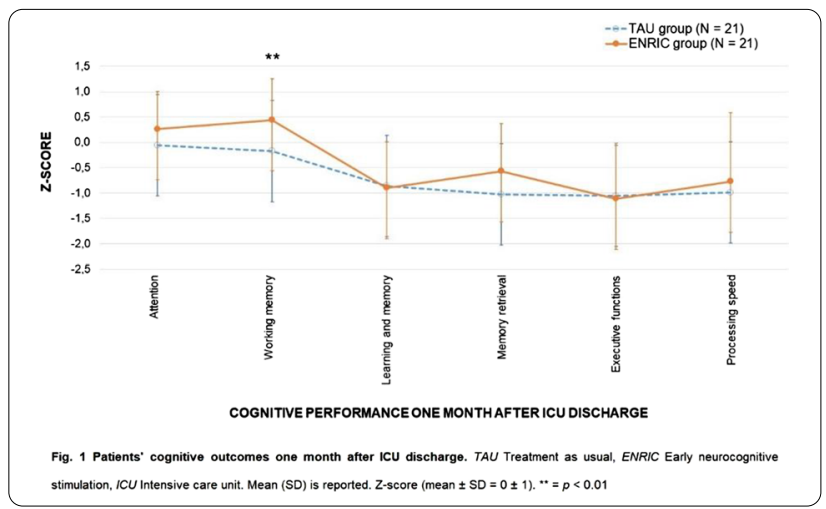

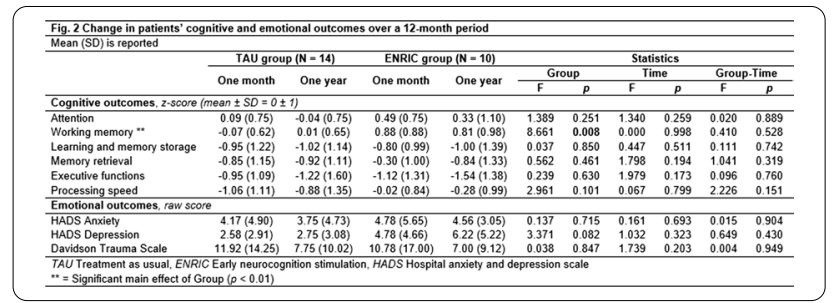

Conclusion. VR-based early neurocognitive interventions may be an effective solution to prevent short- and long-term working memory deficits in survivors of critical illness. Our results propose digital therapy as a safe and effective alternative to classical paper-and-pencil neurocognitive interventions.

\section{Reference(s)}

1. Inoue S, Hatakeyama J, Kondo Y et al. (2019) Post-intensive care syndrome: its pathophysiology, prevention, and future directions. Acute Med Surg ams2.415. https://doi.org/10.1002/ams2.415

2. Fernández-Gonzalo S, Navarra-Ventura G, Bacardit N et al. (2020) Cognitive phenotypes 1 month after ICU discharge in mechanically ventilated patients: a prospective observational cohort study. Crit Care 24:618. https://doi.org/10.1186/s13054-020-03334-2

3. Turon M, Fernandez-Gonzalo S, Jodar M et al. (2017) Feasibility and safety of virtual-reality-based early neurocognitive stimulation in critically ill patients. Ann Intensive Care 7:81. https://doi.org/10.1186/ s13613-017-0303-4

4. Brummel NE, Girard TD, Ely EW et al. (2014) Feasibility and safety of early combined cognitive and physical therapy for critically ill medical and surgical patients: the Activity and Cognitive Therapy in ICU (ACT-ICU) trial. Intensive Care Med 40:370-379. https://doi.org/10.1007/ s00134-013-3136-0

5. Navarra-Ventura G, López-Aguilar J, Blanch L et al. (2020) Characterization and management of cognitive and emotional alterations in COVID-19 critically ill patients after ICU discharge. Med Intensiva. https://doi.org/10. 1016/j.medin.2020.11.004

6. Guillem Navarra-Ventura, Mercè Jodar, Lluís Blanch, Josefina LópezAguilar, Sol Fernández-Gonzalo and Marc Turon are authors of the Early Neurocognitive Rehabilitation in Intensive Care (ENRIC) platform (source code protected under copyright and duly registered at SafeTheProof with registration number 960-951, 848, 947, 944-940).

7. The authors thank Marc Turon for his help in carrying out the study, Carles Forné for his contribution to the statistical analysis, and Michael Maudsley for his support in editing the abstract.

8. The development of the ENRIC platform has been funded by NeuroContent 2.0 (Ministerio de Ciencia e Innovación, Gobierno de España, IPT300000-2010-30) and Fundació La Marató TV3 (Corporació Catalana de Mitjans Audiovisuals, Generalitat de Catalunya, 181/U/2011). The project has been supported by the AGAUR-Producte 2019 grant (Departament d'Empresa i Coneixement, Generalitat de Catalunya, 2019PROD00108) and the European Regional Development Fund (European Commission).

\section{9}

Apoptotic, anti-apoptotic and innate immunity crosstalk in sepsis S. Ilia'; M. Miliaraki'; P. Briassoulis ${ }^{1}$; A. Polonifi'; M. Mantzourani ${ }^{2}$;

E. Briassouli ${ }^{2}$; K. Vardas ${ }^{3} ;$ A. Pistiki ${ }^{4}$; M. Theodorakopoulou ${ }^{5}$; S. Tsiodras ${ }^{4}$; A. Armaganidis ${ }^{5}$; S. Nanas ${ }^{3}$; G. Daikos ${ }^{2}$; G. Briassoulis ${ }^{1}$

${ }^{1}$ Pediatric intensive care unit, university hospital, medical school, University of Crete, Heraklion, Greece; ${ }^{2}$ First department of internal medicine laiko hospital, National and Kapodistrian University of Athens, Athens, Greece; ${ }^{3}$ First critical care department, National and Kapodistrian University of Athens, Athens, Greece; ${ }^{4} 4$ th department of internal medicine, attikon hospital, National and Kapodistrian University of Athens, Athens, Greece; ${ }^{5}$ 2nd department of critical care, Attikon General University Hospital, Haidari, Greece

Correspondence: M. Miliaraki 


\section{Intensive Care Medicine Experimental 2020, 9(1): 000449}

Introduction. The fragile balance among opposing forces of apoptotic/antiapoptotic and inflammatory or oxidative cascades in sepsis remains elusive. Recent studies have shown that apoptotic caspases $(-3,-9)$ are upregulated associated with anti-apoptotic molecules (survivin protein) (1). The extracellular heat shock proteins (HSPs), indicative of the innate immunity, are also upregulated in sepsis, but the intracellular HSPs are repressed $(2,3)$. We studied the combined apoptotic/antiapoptotic, inflammatory, oxidative stress response in septic patients.

Objectives. The present study seeks to explore the acute phase response and possible correlations among apoptotic (caspases) or anti-apoptotic biomolecules (survivin) and inflammatory or oxidative stress biomarkers, in adult ICU patients with sepsis and non-infectious (trauma-related) systemic inflammatory response syndrome (SIRS).

Methods. This prospective observational study was performed in ICU patients with sepsis or non-infectious (traumatic) SIRS, compared to healthy controls $(\mathrm{HC})$. The expression of survivin transcript variants (wild type/-WT, -2B, -3B,- $-\mathrm{E} \times 3$ ) was analyzed by real-time quantitative PCR in peripheral blood leukocytes, whereas the apoptotic or antiapoptotic equilibrium was specified by measuring caspases- 3 and -9 , along with survivin-WT serum protein concentrations and innate immunity dysregulation markers (IL-6, -8, -10, HSP90, HSP72), through enzyme-linked immunosorbent assay (ELISA). Total oxidative stress (TOS) and total antioxidant capacity (TAC) were measured by photometric testing.

Results. Out of 271 adult subjects enrolled in the study, 107 had sepsis, 75 SIRS and 89 were HC. Survivin protein (342 vs. 189 vs. 120 pg/ $\mathrm{ml}, \mathrm{p}<0.001)$, survivin-WT ( 0.21 vs. 0.02 vs. 0.01 copies $/ \mu \mathrm{l}, \mathrm{p}<0.001)$, caspase-3 ( 49 vs. 19 vs. $26 \mathrm{ng} / \mathrm{ml}, \mathrm{p}<0.02$ ), caspase-9 (146 vs. 118 vs. $97 \mathrm{ng} / \mathrm{ml}, \mathrm{p}<0.02)$, and TOS (1295 vs. 346 vs. $242 \mu \mathrm{mol} / \mathrm{l}, \mathrm{p}<0.001)$, were increased and TAC (130 vs. 230 vs. $248 \mu \mathrm{mol} / \mathrm{l}, \mathrm{p}<0.001)$ decreased in sepsis compared to SIRS and healthy controls. HSP90, IL-6, IL-10, IL-27 were also elevated in sepsis compared to controls (Fig. 1). Survivin-3B and $\triangle \mathrm{Ex} 3$ were positively correlated with IL-8 $(r=0.4, p=0.03)$. Compared to survivors, survivin protein (372 vs. $201 \mathrm{pg} / \mathrm{ml})$, caspase-3 (43 vs. $33 \mathrm{ng} / \mathrm{ml})$, TOS (1315 vs. $547 \mu \mathrm{mol} / \mathrm{l})$, HSP72 (3,3 vs. $0.97 \mathrm{ng} / \mathrm{ml}$ ), survivin-WT (0.19 vs. 0.06 copies $/ \mu \mathrm{l}),-3 B$ ( 0.76 vs. 0.29 copies $/ \mu$ l were increased and TAC decreased (138 vs. $210 \mu \mathrm{mol} / \mathrm{l})$ among non-survivors $(p<0.05)$.

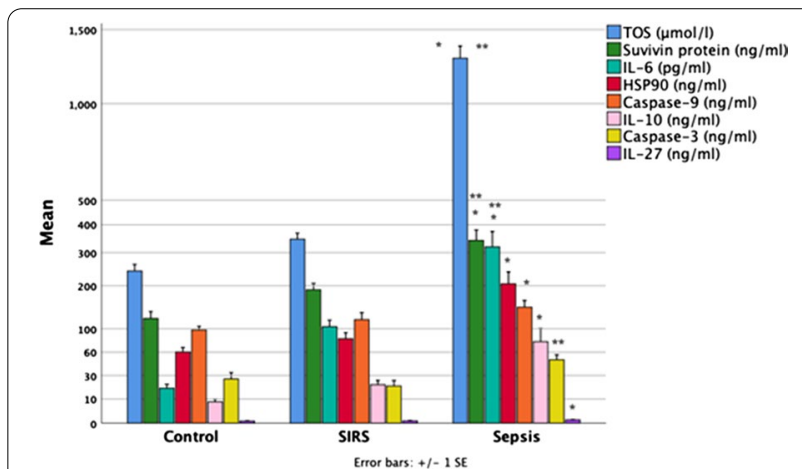

Fig. 1. Elevated levels of apoptotic (caspase-3, -9), anti-apoptotic (survivin), heat-shock response molecules (HSP90) or of inflammatory markers (IL-6, IL-10, IL-27) in sepsis, compared to non-infectious SIRS and to healthy controls

Bars represent means, error bars $1 \pm$ Error bars. Stars indicate significantly higher levels in sepsis $(p<0.05)$ compared to healthy controls* or SIRS**

Conclusion. The sepsis-induced increased apoptotic (caspases), antiapoptotic (survivin), inflammatory and oxidative stress response is related to outcome in adult ICU patients.
Reference(s)

1. Miliaraki, M. et al. Survivin and caspases serum protein levels and survivin variants mRNA expression in sepsis. Sci. Rep. 11, 1049 (2021).

2. Fitrolaki, M.-D. et al. Increased extracellular heat shock protein 90a in severe sepsis and SIRS associated with multiple organ failure and related to acute inflammatory-metabolic stress response in children. Medicine (Baltimore) 95, e4651 (2016).

3. Briassouli, E. et al. Glutamine suppresses Hsp72 not Hsp90a and is not inducing Th1, Th2, or Th17 cytokine responses in human septic PBMCs. Nutr. Burbank Los Angel. Cty. Calif 30, 1185-1194 (2014).

\section{2}

Arterial oxygen and cerebral oxygenation during clinical cardiopulmonary resuscitation

A. Nelskylä ; M. Skrifvars'; S. ÅNgerman ${ }^{1}$; J. Nurmi ${ }^{1}$

${ }^{1}$ Department of emergency care and services, University of Helsinki

and Helsinki University Hospital, Helsinki, Finland

Correspondence: A. Nelskylä

Intensive Care Medicine Experimental 2020, 9(1): 000452

Introduction. Resuscitation guidelines recommend $100 \%$ oxygen during cardiopulmonary resuscitation (CPR). Improving brain oxygenation assessed with near infrared spectroscopy (NIRS) values have been associated with a higher likelihood of return of spontaneous circulation.

Objectives. We assessed factors associated with NIRS values with a focus on arterial hyperoxia, during clinical CPR and immediately after return of spontaneous circulation (ROSC).

Methods. A prospective observational cohort study of out-of-hospital cardiac arrest patients treated by a physician-staffed helicopter unit. Intra-arrest brain regional oxygen saturation ( $\mathrm{rSO} 2$ ) with NIRS, invasive blood pressure and end-tidal CO2 (etCO2) were monitored during CPR. Arterial blood samples were drawn during CPR and immediately after ROSC. Moderate hyperoxia was defined as arterial oxygen partial pressure (paO2) 20.0-39.9 $\mathrm{kPa}$ and severe as values $\geq 40 \mathrm{kPa}$. Intraarrest factors correlated with rSO2 were assessed with the Spearman's correlation.

Results. Of 80 recruited patients, 73 (91\%) had rSO2 recorded during CPR and 28 patients achieved ROSC. Intra-arrest paO2 was analysed in 46 patients and after ROSC in 20 patients. The rSO2 during CPR correlated with intra-arrest systolic $(r=0.28, p<0.001)$ and diastolic blood pressure $(p=0.32, p<0.001)$ but not with paO2 $(r=0.13, p=0.41)$, paCO2 $(r=0.18, p=0.22)$ or etCO2 $(r=0.008, p=0.9)$. Moderate intraarrest hyperoxia was seen in one patient and in four patients $(20 \%$, 95\% Cl 7-42\%) after ROSC. No patient had severe hyperoxia during CPR, and one patient (5\%, Cl 95\% 0-25\%) immediately after ROSC.

Conclusion. Cerebral oxygenation during CPR appears more dependent on hemodynamic conditions than arterial oxygen concentration. Severe hyperoxia during CPR or immediately after ROSC is rare in clinical CPR performed by physician-staffed helicopter units.

\section{Reference(s)}

1. Genbrugge C, Meex I, Boer W, et al. Increase in cerebral oxygenation during advanced life support in out-of-hospital patients is associated with return of spontaneous circulation. Crit Care 2015;19(1):112.

2. Sanfilippo F, Murabito P, Messina A, et al. Cerebral regional oxygen saturation during cardiopulmonary resuscitation and return of spontaneous circulation: A systematic review and meta-analysis. Resuscitation 2020;159:19-27.

3. Soar J, Nolan JP, Böttiger BW, et al. European Resuscitation Council Guidelines for Resuscitation 2015: Sect. 3. Adult advanced life support. Resuscitation 2015:95:100-47.

4. This study was funded by grants from Sigrid Jusélius stiftelse, Finska Läkaresällskapet, Medicinska Understödsföreningen Liv och Hälsa and Stiftelsen Dorothea Olivia, Karl Walter och Jarl Walter Perklens minne. 


\section{5}

\section{The Norwegian ICU TRIAGE study}

A. Robertsen ${ }^{1}$; TA. Aasmundstad ${ }^{2}$; R. Sexe, ${ }^{3}$; C. Nyvold, ${ }^{4}$; PH. Christensen, ${ }^{5}$; J. Dahlberg, ${ }^{6}$; M. Magelssen, ; TM. Olasveengen, ${ }^{8}$; H. Ersdal, ${ }^{9}$; P. Klepstad, ${ }^{10}$; K. Sunde,

${ }^{1}$ Department of research and development, division of emergencies and critical care, Oslo University hospital, Oslo, Norway; ${ }^{2}$ Department of anesthesiology, division of emergencies and critical care, Oslo University hospital Rikshospitalet, Oslo, Norway; ${ }^{3}$ Department of anesthesiology, division of emergencies and critical care, Oslo University hospital, Oslo, Norway: ${ }^{4}$ Depeartment of medicine, Oslo University hospital, Oslo, Norway; ${ }^{5}$ Department of anesthesiology, Hamar hospital, Hamar, Norway; ${ }^{6}$ Department of anesthesiology, Akerhus University hospital, Oslo, Norway; ${ }^{7}$ Clinical ethics, Center of medical ethics, Oslo, Norway; ${ }^{8}$ Department of anesthesiology, Oslo University hospital, Oslo, Norway; ${ }^{9}$ Department of anesthesiology, Stavanger University hospital, Stavanger, Norway; ${ }^{10}$ Department of anesthesiology, St.Olavs university hopsital, Trondheim, Norway; ${ }^{11}$ Department of anesthesiology, Oslo University hospital Ullevål, Oslo, Norway

Correspondence: A. Robertsen

Intensive Care Medicine Experimental 2020, 9(1): 000475

Introduction. How to allocate intensive care resources during the ongoing Covid-19 pandemic is a major concern. We wanted to explore how ICU triage decisions actually were handled by ICU clinicians in Norway.

Objectives. Identify number of ICU rejections among potential ICU patients with and without Covid-19, and to explore decision categories used for ICU rejections.

Methods. A prospective observational study was conducted between March and September 2020 in 16 Norwegian ICUs from all health regions. Only rejections with an intensivist involved in decision-making were included. Patients' variables, hospitals' emergency level at time of decision, predefined decision categories, and outcome were collected, linked to national Covid-19 data.

Results. A total of 25 rejections were identified all in ICUs located in southeast of Norway, which was the most charged region during the first wave of the pandemic. Rejected patients were mean 73 years (range $37-95$ ), 80\% were men, $76 \%$ had serious co- morbidities, $44 \%$ lacked decision-making capacity, and one had an advance directive. Covid-19 status at time of decision was $48 \%$ positive, $44 \%$ negative and $8 \%$ unknown. Reasons behind rejections were "too sick to benefit" (56\%), "judgement based on several considerations" (36\%) and "must wait" (8\%). Main considerations were high age (16\%), co-morbidity $(32 \%)$, frailty $(20 \%)$ and signs of irreversible organ failure $(32 \%)$. Frequently there were multiple considerations. No rejections were related to patient's wishes. Only $8 \%$ of rejections were categorized as related to capacity strain (no available beds or health care personnell), not lack of ventilators or personal protection equipment. In total, $72 \%$ of rejections occurred.

during times of "yellow" emergency levels, meaning no elective surgical activity, intensified collaboration between hospitals and increased ICU capacity by expanding number of ICU beds in specialized Covid-19 ICU cohorts. Rejected patients received treatment either in the ward or at an intermediate level, and 32\% survived to discharge. We did not capture decisions about treatment limitations made in the Emergency Department or at the ward, if the intensivist was not involved.

Conclusion. A low number of ICU rejections and specifically nearly no rejections related to capacity strain was identified in a country with a relatively low total Covid-19 ICU pressure compared to other European countries. These baseline-rejections were even lower than expected.
000486

A follow-up and accompaniment programme to assess the Post-Intensive Care syndrome seqüelae in COVID-19 ICU survivors: the PICS-COVID19 project

S. Fernandez-Gonzalo1; G. Navarra-Ventura, ${ }^{2}$; G. Gomà²; C. De Haro²; C. Espinal Sacristan ${ }^{3}$; C. Fortià Palahi ${ }^{3}$; N. Ridao Sais ${ }^{4}$; J. Lopez-Aguilarr'; M. Godoy Gonzalez ${ }^{3}$; L. Oliveras Furriols, ; N. Miguel Rebanal ${ }^{3}$; C. Subirà, ; M. Jodar ; $;$ L. Sarlabous 2 ; R. Fernandez ${ }^{5} ;$ A. Ochagavia²; L. Blanch ${ }^{2}$

${ }^{1}$ Critical care center, Hospital Universitari Parc Taulí, Institut d'Investigació i Innovació (I3PT), UAB, CIBERSAM, Sabadell, Spain; ${ }^{2}$ Critical care center, Hospital Universitari Parc Taulí, Institut d'Investigació i Innovació (I3PT), UAB, CIBERES, Sabadell, Spain; ${ }^{3}$ Critical care center, Hospital Universitari Parc Taulí, Institut d'Investigació i Innovació (I3PT), UAB, Sabadell,

Spain; ${ }^{4}$ Rehabilitation medicine, Hospital Universitari Parc Taulí, Institut d'Investigació i Innovació (I3PT), UAB, Sabadell, Spain; ${ }^{5}$ Critical care center, Fundació Althaia, Xarxa Assistencial Universitària de Manresa, CIBERES, Manresa, Spain; ${ }^{6}$ Neurology department, Parc Taulí Hospital Universitari, Institut d'Investigació i Innovació (I3PT), UAB, CIBER-

SAM, Sabadell, Spain

Correspondence: S. Fernandez-Gonzalo

Intensive Care Medicine Experimental 2020, 9(1): 000486

Introduction. Post-Intensive Care Syndrome(PICS) affects 30-50\% of the ICU survivors (1-5), and may last years after ICU discharge, impacting on their quality of life (6-8). Acute Respiratory Distress Syndrome (ARDS) patients undergoing invasive mechanical ventilation (IMV) are highly vulnerable to develop PICS-related sequelae, i.e. ICU-acquired muscle weakness, cognitive difficulties and anxietydepressive symptoms (9). The diagnosis of ARDS and the need for IMV in severe COVID19 patients, along with the conditions of the hospital emergency situation (eg. isolation during admission, impossibility for applying conventional protocols for sedation/mobilization,etc.), and the difficulty of following-up discharged patients during the pandemic, may rise up PICS incidence in ICU COVID19 survivors.

Objectives. (1) To describe a telematic follow-up and accompaniment programme developed to assess and manage PICS in critical ill patients with COVID19 for a year after ICU discharge. 2) To show the preliminary data about the characterization of PICS in ICU survivors affected by COVID19 from 1 to 9 months after ICU discharge.

Methods. The telematic follow-up and accompaniment programme includes two sections: an assessment monitoring system (AMS), based on auto-administered questionnaires and expert-made questions for evaluating PICS domains;and an action protocol, based on the screening PICS profile generated by the answers in the AMS. Participants answered the questionnaires 1, 3, 6, 9 and 12 months after ICU stay. We evaluated: functionality (Barthel Index), fatigue/ dyspnea and pain (0-10 visual analogue scale), anxiety-depressive symptoms (Hospital of Anxiety and Depression Scale HADS), selfperception of cognition (Perceived Deficit Questionnaire). Beta mixed-effects models have been used.

Results. 117 COVID19 ICU survivors (37.6\% female; Age $59.6 \pm 10.72$ years old) were analyzed. $63.2 \%$ received IMV. Median length of ICU stay was 10 days (Min/Max=1-73).Progression of symptoms from 1 to 9 months are shown in Fig. 1. Nine months after ICU discharge patients manifest fatigue and/or dyspnea during moderate efforts (26.3\%), pain (39.5\%), anxiety $(38.5 \%)$, depression (30.8\%), and post-traumatic stress disorder (PTSD) symptoms $(35.9 \%)$, and cognitive difficulties (27.8\%). Female gender was related to functionality $(p=0.002)$, anxiety $(p=0.01)$, depression $(p=0.004)$ and PTSD $(p=0.05)$. IMV was associated with depressive symptoms $(p<0.001)$.

\section{Reference}




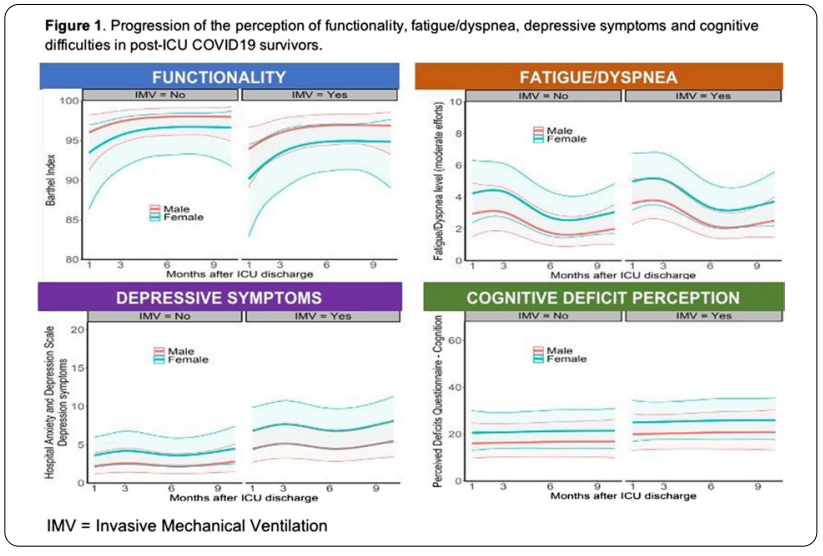

Conclusion. The telematic follow-up programme allows a remote screening assessment and management of PICS in the COVID19 ICU survivors. Preliminary results suggest that,although functionality improves, some physical symptoms and cognitive/mental health difficulties persist 9 months after ICU discharge. The role of female gender and IMV in both, the emotional estate and the functionality level of the COVID19 ICU survivors, deserves further exploration.

\section{Reference(s)}

1. This project (COV20/00595) is funded by the Extraordinary call for the SARS-COV-2 and COVID-19 disease by the Instituto de Salud Carlos III, Ministry of Health of the Spanish government (Spain).

2. Herridge MS, Moss M, Hough CL, Hopkins RO, Rice TW, Bienvenu OJ, Azoulay E. Recovery and outcomes after the acute respiratory distress syndrome (ARDS) in patients and their family caregivers. Intensive Care Med. 2016 May:42(5):725-738

3. Hopkins, R.O., Weaver, L.K., Collingridge, D. et al. (2005). Two years cognitive, emotional, and quality of life outcomes in acute respiratory distress syndrome. Am J RespirCrit Care Med, 171, 340-47.

4. Hopkins RO, Herridge MS. Quality of life, emotional abnormalities,and cognitive dysfunction in survivors of acute lung injury/acute respiratory distress syndrome. Clin Chest Med. 2006:27:679-89.

5. Mikkelsen ME, Shull WH, Biester RC, Taichman DB, Lynch S, Demissie E, Hansen-Flaschen J, Christie JD. Cognitive, mood and quality of life impairments in a select population of ARDS survivors. Respirology. 2009 Jan;14(1):76-82

6. Fernández-Gonzalo S, Navarra-Ventura G, Bacardit N, Gomà Fernández G, de Haro C, Subirà C, López-Aguilar J, Magrans R, Sarlabous L, Aquino Esperanza J, Jodar M, Rué M, Ochagavía A, Palao DJ, Fernández R, Blanch L. Cognitive phenotypes 1 month after ICU discharge in mechanically ventilated patients: a prospective observational cohort study. Crit Care. 2020 Oct 21;24(1):618

7. Jackson JC, Hart RP, Gordon SM, Shintani A, Truman B, May L, et al. Six-month neuropsychological outcome of medical intensive care unit patients. Crit Care Med. 2003.

8. Hodgson C, Bellomo R, Berney S et al. Early mobilization and recovery in mechanically ventilated patients in the ICU: a bi-national, multi-centre, prospective cohort study. Crit Care. 2015;19-81.

9. Pandharipande PP et al. Long-Term Cognitive impairment after critical illness. New Eng J Med. 2013; 369(14):1306-1316.

10. Needham DM, Davidson J, Cohen H, Hopkins RO, Weinert C, Wunsch $\mathrm{H}$, et al. Improving long-term outcomes after discharge from intensive care unit: report from a stakeholders' conference. Crit Care Med. 2012 Feb;40(2):502-9.
000517

Implementation of a Critical Care Follow-Up Clinic in COVID-19 Pandemic: A prospective evaluation of functional status and Post-Intensive Care Syndrome beyond ICU walls and hospital discharge

I. Canas-Pérez ; C. Díaz-Rodríguez'; R. Herrán-Monge ; C. Cuenca-Rubio'; MM. García-García'; AM. Prieto-Lamo'; D. Pérez-Torres'; JÁ. Berezo-García';

T. Ruiz-Albi'; PM. Enríquez-Giraudo

'Servicio de medicina intensiva, Hospital Universitario Río Hortega, Valladolid, Spain; ${ }^{2}$ Servicio de neumología, Hospital Universitario Río Hortega, Valladolid, Spain

Correspondence: D. Pérez-Torres

Intensive Care Medicine Experimental 2020, 9(1): 000517

Introduction. The Post-Intensive Care Syndrome (PICS) encompasses the physical (especially respiratory and neuromuscular), psychic/ emotional (anxiety, depression...) and cognitive sequelae of patients hospitalized in Intensive Care Units (ICU). Prolonged mechanical ventilation and lengthy hospital stays make this syndrome a common complication of COVID-19 patients.

Objectives. To evaluate the functional status and prevalence of PICS in critical COVID-19 patients admitted to the ICU, in an outpatient critical care follow-up clinic, 3 months after hospital discharge.

Methods. We created a multidisciplinary critical care follow-up clinic leaded by intensivists and also teamed by pulmonologists and nurses. We prospectively screened all the PCR-confirmed COVID19 patients admitted to the ICU of a single University Hospital in Spain during the study period (March to May 2020, corresponding to the first epidemic wave of COVID-19 in the country) and referred those with potential impaired functional status or risk factors for PICS to the outpatient clinic. We retrieved epidemiological, clinical and outcome data from the electronic medical record. We assessed functional status and PICS three months after hospital discharge by performing a protocolized scale-based evaluation of the physical, psychological and cognitive dimensions (Barthel, MoCA, HAD, PTSD and SF12), estimating muscle strength by handheld dynamometry, and measuring respiratory function with spirometry and diffusion. The results are expressed as frequency (\%), mean \pm standard deviation or median (interquartile range), as appropriate.

Results. We included 37 patients, from 50 eligible survivors to hospital discharge, $70 \%$ male, aged $53 \pm 12$, body mass index $27(25-32)$ $\mathrm{Kg} / \mathrm{m}^{2}$, Barthel index 100 points $100 \%$. Past medical history: $24 \%$ dyslipidaemia, $19 \%$ hypertension, $8 \%$ diabetes, $13 \%$ asthma or chronic obstructive pulmonary disease. $100 \%$ of the patients fulfilled Berlin criteria of ARDS, with an APACHE-II 14 (11-16) points, a median ICU stay 20 (12-36) days and a hospital stay 31 (21-57) days. Respiratory management: $95 \%$ of the patients were managed with invasive mechanical ventilatory support, during a median of 18 (10-29) days, $78 \%$ of which required prone position ventilation, with a median of $2(1-4)$ sessions; the remainder $5 \%$ was managed with high-flow oxygen therapy. $11 \%$ of the patients underwent extracorporeal life support (vv-ECMO). $24 \%$ of the patients required tracheostomy during the weaning process.

Sedation management: $92 \%$ of the patients underwent neuromuscular blockade, with a median of $8 \pm 5$ days. All the patients requiring mechanical ventilation received sedative medications, $92 \%$ of which midazolam, with a median duration of therapy of $18 \pm 10$ days.

Complications during ICU stay: 65\% ICU-acquired weakness, 38\% delirium, $31 \%$ pressure injuries, $22 \%$ shock, $19 \%$ ventilator-associated pneumonia, $16 \%$ deep vein thrombosis.

Preventive strategies to reduce the burden of functional status impairment or PICS: $59 \%$ physiotherapy, starting after $24 \pm 12$ days, $35 \%$ alternative media, $5 \%$ family visitation, $30 \%$ referral to functional rehabilitation centre for further recovery after discharge.

Three-month after-discharge visit in the outpatient critical care follow-up clinic: body mass index $25(23-31) \mathrm{Kg} / \mathrm{m}^{2}, 100 \%$ Barthel index 100 points, 39\% alopecia, 30\% insomnia, 3\% ICU-acquired swallowing disorders. $76 \%$ had visited their General Practitioner, whereas $16 \%$ had visited the Emergency Department. 51\% were 
under physiotherapy follow-up. $19 \%$ were still on sick leave. $40 \%$ had not recovered their hobbies. $35 \%$ still had impaired functional respiratory tests. $62 \%, 22 \%$ and $10 \%$ experienced alterations of physical, psychological and cognitive dimensions, respectively (68\% experienced alterations of at least one dimension). $32 \%$ were referred to other specialists for further follow-up.

Conclusion. Critical COVID-19 patients with ARDS required prolonged mechanical ventilation and lengthy ICU stays, and were commonly complicated with delirium or ICU-acquired weakness. Our critical care follow-up clinic revealed about two out of three critical COVID-19 patients developed alterations consistent with PICS three months after discharge. This might be influenced by the fact that strategies to prevent PIC were suboptimally implemented due to an overwhelming workload.

\section{2}

\section{End-of-life care within the intensive care unit}

E. Page ${ }^{1}$; G. Erez ${ }^{2}$

${ }^{1}$ Intensive care medicine, Northwick Park Hospital, London, United

Kingdom; ${ }^{2}$ Palliative medicine, Northwick Park Hospital, London, United Kingdom

Correspondence: E. Page

Intensive Care Medicine Experimental 2020, 9(1): 000532

Introduction. Advances in life-sustaining treatments have translated into improved intensive care survival rates over recent years, however, the mortality rate remains significant 1 .

Therefore, alongside life-saving interventions, intensive care must also provide good end-of-life care 2 .

Palliative care within critical care presents different challenges, but the core domains recognised for intensive care 3 align with the principles in UK guidelines4.

The COVID-19 pandemic brought new difficulties; rapid expansion of critical care capacity, visitor restrictions and the increased mortality rate of COVID-19 patients in intensive care5.

Objectives. The objective of this audit was to review end-of-life care within our intensive.

care unit (ICU) and how the pandemic may have impacted on this care. Methods. This was an initial retrospective audit, based on the English and Welsh National Audit of Care at the End of Life and the five Priorities of Care of the dying person; recognising the possibility of death and dying, communication, patient and family involvement in decisions, exploring their needs and creating an individual care plan for the dying person 6,7 .

The audit included all patients who died in our ICU in July 2020 and those admitted to ICU in July 2020 who died later in their admission. Electronic and paper notes were reviewed.

Results. There were 14 deaths in total-one patient's paper notes were unavailable so was excluded.

11 of 13 were expected deaths. While 11 of 13 patients had discussions with next of kin about the possibility of dying during admission, only 7 out of the 13 next of kin were informed the patient was dying. This may have been due in part to the use of unclear language.

In no cases was the possibility of death discussed with the patient, for 6 of the 13 this would have been possible, based on their Glasgow Coma Score and Confusion Assessment Method for ICU. For 2 of 13 patients, their wishes were explored with the next of kin.

11 of 13 patients had an individual care plan; 4 of these involved the next of kin. For 6 of 13, there was consideration of end-of-life symptoms. In 4 of 13, there was consideration of spiritual needs, but this may not account for undocumented discussions between nursing staff and families.

Due to visitor restrictions, most of the communication with families was by telephone. For 9 of 13 patients, visitation was offered to the next of kin.

The findings were presented at a local departmental meeting and work to improve care continues.

Conclusion. Talking about death and dying is often required in ICU, which was reflected in family conversations, but communication with the patient themselves needs improving. Where possible, best prac tice would be to speak to the patient about their prognosis, even if it's uncertain and explore their wishes around dying and what matters most to them. We also recommend that end-of-life care in ICU be included as a quality indicator for critical care by the Faculty of Intensive Care Medicine.

\section{Reference(s)}

1. NHS Benchmarking Network-National Audit of Care at the End of Life. Accessed 23/06/20 https://www.nhsbenchmarking.nhs.uk/nacel

2. 6. Leadership Alliance for the Care of Dying People-One Chance to Get it Right: Improving people's experience of care in the last few days and hours of life. June 2014. Accessed 23/06/20 https://assets.publishing.servi ce.gov.uk/government/uploads/system/uploads/attachment_data/file/ 323188/One chance to get it right.pdf

3. Mahase E. Covid-19: Intensive care mortality has fallen by a third since pandemic began, researchers find. BMJ 2020;370:m2882. Accessed 11/02/202.

4. NICE guideline [NG31]: Care of dying adults in the last days of life. December 2015. Accessed 23/06/20. https://www.nice.org.uk/guidance/ ng31

5. Clarke EB, Curtis JR, Luce JM, Levy M, Danis M, Nelson J, Solomon MZ; Robert Wood Johnson Foundation Critical Care End-Of-Life Peer Workgroup Members. Quality indicators for end-of-life care in the intensive care unit. Crit Care Med. 2003 Sep;31 (9):2255-62. https://doi.org/10.1097/ 01.CCM.0000084849.96385.85. PMID: 14,501,954. Accessed 23/06/20.

6. NHS National Services Scotland - Scottish Intensive Care Society Audit Group: Minimum Standards and Quality Indicators for Critical Care in Scotland. December 2015. Accessed 30/03/21. https://www.sicsag.scot. nhs.uk/Quality/20151215-Quality-Indicators-Booklet-V3-0.pdf

7. The Faculty of Intensive Care Medicine: Care at the End of Life-A guide to best practice, discussion and decision-making in and around critical care. September 2019. Accessed 23/06/20

8. No grant or funding received

\section{8}

Uneven distribution of ventilation in a porcine model of unilateral ligation of the pulmonary artery may explain bilateral lung injury I. Marongiu ${ }^{1}$; E. Spinellii ${ }^{2}$ E. Scotti ${ }^{2}$; A. Caccioppola ${ }^{1}$; G. Dal Santo ${ }^{1}$;

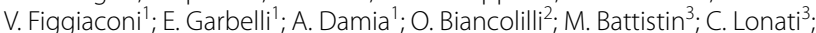

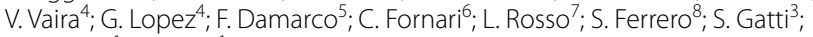
A. Zanella ${ }^{1}$ T. Mauri $^{1}$

${ }^{1}$ Department of pathophysiology and transplantation, University of Milan, Milano, Italy; ${ }^{2}$ Department of anesthesiology, Fondazione IRCCS Ca'Granda Ospedale Maggiore Policlinico, Milano, Italy; ${ }^{3}$ Center for preclinical research, Fondazione IRCCS Ca'Granda Ospedale Maggiore Policlinico, Milano, Italy; ${ }^{4}$ Department of biomedical surgical and dental sciences, University of Milan, Milano, Italy; ${ }^{5}$ Department of thoracic surgery and lung transplants, Fondazione IRCCS Ca'Granda Ospedale Maggiore Policlinico, Milano, Italy; ${ }^{6}$ School of medicine and surgery, University of Milano-Bicocca, Monza, Italy; ${ }^{7}$ Department of health science, University of Milan, Milano, Italy; ${ }^{8}$ Pathology unit, Fondazione IRCCS Ca'Granda Ospedale Maggiore Policlinico, Milano, Italy

Correspondence: I. Marongiu

Intensive Care Medicine Experimental 2020, 9(1): 000568

Introduction. The authors previously reported that ligation of the left pulmonary artery in healthy pigs is associated with bilateral lung injury, but the mechanisms involved remain elusive [1]. Alveolar hypocapnia in the ligated lung might play a key role, leading to local apoptosis, reduction of surfactant secretion and pneumo-constriction, with hyper-ventilation of contralateral perfused lung [2, 3]. Supplementation of inhaled $\mathrm{CO} 2$ might prevent lung injury, by limiting the mechanical consequences of alveolar hypocapnia.

Objectives. To study regional ventilation distribution in a model of unilateral ligation of the left pulmonary artery with and without administration of inhaled $\mathrm{CO} 2$.

Methods. Eleven mechanically ventilated healthy pigs with surgical ligation of the left pulmonary artery were allocated to two groups: 4 animals were ventilated with $\mathrm{Vt} 10 \mathrm{ml} / \mathrm{Kg}$, PEEP $5 \mathrm{cmH} 2 \mathrm{O}$, rate $25 \mathrm{bpm}$ 
and $\mathrm{FiO} 250 \%$ for $48 \mathrm{~h}$ (Ligation group) and 7 received the same ventilation plus $5 \% \mathrm{FiCO} 2$ (Ligation + $\mathrm{FiCO} 25 \%$ group). Distribution of ventilation measured by electrical impedance tomography and regional respiratory mechanics were assessed in both groups at 2, 12, 24, 36 and $48 \mathrm{~h}$ from surgical artery ligation in both study groups. The histological score, plateau pressure and gas exchanges were compared at T48.

Results. At T48, animals in the Ligation group developed bilateral lung injury while inhaled $\mathrm{CO} 2$ prevented it. Specifically, in the ligation group oxygenation was poorer, respiratory mechanics were impaired and the histological score was higher (Table 1), although it did not differ between the right and left lung $(8.5[7.5 ; 10.5]$ vs $8.3[7.3 ; 8.9]$, $p=0.686$ ). While lung injury of the ligated side was already described in previous experiments [4], we focused on the distribution of ventilation as possible mechanism for contralateral lung injury. The Ligation group showed higher fraction of tidal volume $(\mathrm{Vt})$ reaching the right perfused lung (Fig. 1A) from T2 to T36 compared to the Ligation + FiCO2 5\% group. At T48, right and left lungs were subject to a more equal distribution of $\mathrm{Vt}$, probably due to a fall in right lung compliance (Fig. 1B), consistent with development of lung injury in the perfused lung. Right lung compliance, instead, remained stable in the Ligation + FiCO2 group (Fig. 1B), as well as left side compliance in both groups (Fig. 1B).

\section{Table 1}

\begin{tabular}{llll}
\hline & Lig + CO2 5\% (n=7) & Lig $(\mathbf{n}=\mathbf{4})$ & P value \\
\hline $\begin{array}{l}\text { Compliance rs }(\mathrm{mL} / \\
\text { (mH2O) }\end{array}$ & $40[31 ; 44]$ & $24[18 ; 26]$ & 0.006 \\
$\begin{array}{l}\text { Plateau Pressure } \\
(\mathrm{cmH} 2 \mathrm{O})\end{array}$ & $14[13 ; 16]$ & $23[21 ; 29]$ & 0.006 \\
$\begin{array}{l}\text { PaO2/FiO2 } \\
\text { Histological score }\end{array}$ & $520[384 ; 542]$ & $298[141 ; 391]$ & 0.039 \\
\hline
\end{tabular}

Data are expressed as median [quartiles]. Comparisons are obtained with Mann-Whitney test. rs = respiratory system.

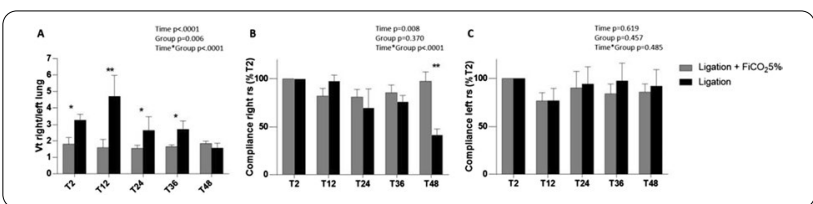

Fig. 1. Data were analyzed with generalized estimating equation models. $V t=$ Tidal Volume; $r s=$ respiratory system

Conclusion. Redistribution of $\mathrm{Vt}$ to the perfused lung may be implied in the development of regional lung injury following ligation of contralateral pulmonary artery. Mechanisms inducing injury in the nonperfused lung remain to be elucidated.

\section{Reference(s)}

1. American Thoracic Society 2020 International Conference, May 15-20, 2020_Philadelphia, PA. Mauri T., Spinelli E., Scotti E., et al., Occlusion of the Left Pulmonary Artery Induces Bilateral Lung Injury in Healthy Swines.

2. Langer T, Castagna V, Brusatori S, et al. Short-term Physiologic Consequences of Regional Pulmonary Vascular Occlusion in Pigs. Anesthesiology. Aug 2019;131(2):336-343.

3. Kiefmann M, Tank S, Tritt MO, et al. Dead space ventilation promotes alveolar hypocapnia reducing surfactant secretion by altering mitochondrial function. Thorax. 2019 Mar;74(3):219-228. https://doi.org/10.1136/ thoraxjnl-2018-211864.

4. Edmunds LH, Jr., Holm JC. Effect of inhaled CO2 on hemorrhagic consolidation due to unilateral pulmonary arterial ligation. J Appl Physiol. Jun 1969:26(6):710-5. 10.1152/jappl.1969.26.6.710

\section{2}

Alternative Substrates in the Critically III Subject (ASICS):

Preliminary Safety, Feasibility and Tolerability Data

A. Langan 1 ; A. McNelly ; D. Bear ${ }^{3}$; F. Santos ${ }^{4}$; K. Rooney ; S. Eaton ${ }^{6}$;

S. Heales ; J. Prowle ; R. Pearse ${ }^{4}$; H. Montgomery ${ }^{8}$; ZA. Puthucheary ${ }^{4}$;

T. Martin ${ }^{4} ;$ F. Seidu ${ }^{4}$

${ }^{1}$ Dietetics, Adult critical care unit, The Royal London Hospital, London,

United Kingdom; ${ }^{2}$ William harvey research institute, Queen Mary Univer-

sity of London, London, United Kingdom; ${ }^{3}$ Dietetics, critical care unit, St

Thomas' Hospital, London, United Kingdom; ${ }^{4}$ Adult critical care unit, Royal London Hospital, London, United Kingdom; ${ }^{5}$ Critical care, Bristol Royal

Infirmary, Bristol, United Kingdom; ${ }^{6}$ Developmental biology \& cancer, UCL Great Ormond Street Institute of Child Health, London, United Kingdom;

${ }^{7}$ Genetics and genomic medicine, UCL Great Ormond Street Institute of Child Health, London, United Kingdom; ${ }^{8}$ Institute of sport, excercise \& health, University College London, London, United Kingdom

Correspondence: A. McNelly

Intensive Care Medicine Experimental 2020, 9(1): 000582

Introduction. Increasing ketone body production by altering nutrition composition may mitigate muscle loss in early critical illness, improving related outcomes and reducing healthcare costs in survivors of an Intensive Care Unit (ICU) stay.

Objectives. To perform a randomised controlled feasibility study to determine whether:

i) ICU patients in early critical illness can be recruited to and retained in a study of ketogenic enteral feeding; ii) The modular enteral feed can be prepared, safely administered and is well-tolerated; iii) The 10-day enteral feeding regimen significantly raises plasma and urine ketone body levels.

Methods. Patients were recruited $\leq 48 \mathrm{~h}$ after ICU admission from two UK ICUs; randomised to ketogenic or standard enteral feed for 10 days. Inclusion criteria: (i) $>18$ years old (ii) due to receive enteral nutrition (iii)likely to be mechanically ventilated $>48 \mathrm{~h}$, on ICU $\geq 5$ days and to survive $\geq 10$ days (iv)multi-organ failure (SOFA score $>2$ in $>2$ categories). ClinicalTrials.gov NCT04101071[1].

Dietitian-prescribed modular ketogenic feed was reconstituted on ICU [80\% fat (40-80\% medium chain triglycerides (Betaquik ${ }^{\circledR}$, Vitaflo)); $20 \%$ protein (Renapro ${ }^{\circledR}$, Stanningley Pharma); 5\% carbohydrate (Maxijul ${ }^{\circledR}$, Nutricia)].

Data were collected for feasibility of recruiting and retaining study participants (retention rate: patients receiving 10 days randomised feed and those on ICU $\geq 5$ days if $<10$ days feed); Adverse Events (AEs): diarrhoea (Bristol Stool Score $>5$ ), high gastric residual volumes $(G R V) \geq 350 \mathrm{mls}$, vomiting ( $\geq 10 \mathrm{mls}$ ) (percent days of events/total possible days), number of hypoglycaemic events; and point-of-care urine and plasma ketone body levels.

Results. Of 286 patients screened (29/09/19-07/03/21), 29 were recruited and 24 retained (12/14: control group;12/15 intervention group; retention rate: $82.6 \%$ ). It proved possible but labour-intensive for dietitians/research nurses to calculate feed constituents to meet patients' daily nutritional targets and prepare the feed, and for bedside nurses to administer it. Follow-up to 12 months has been completed in 4 of 29 patients; data collection and analysis is ongoing.

No related or unexpected serious adverse events were reported. For the first 17 patients recruited, AEs were similar between control and intervention groups, respectively, for rates of high GRV $22.4 \%$ vs $19.0 \%$; vomiting $5.7 \%$ vs $10.8 \%$; and diarrhoea $49.8 \%$ vs $62.0 \%$ ( $p>0.05$ for all). Two episodes of hypoglycaemia and one case of Acute Kidney Injury occurred in the control arm. One patient transferred to total parenteral nutrition from ketogenic feed.

Ketogenic enteral feeding was associated with mild plasma ketosis (up to $2.4 \mathrm{mmol} / \mathrm{L}$ ) and a greater urinary ketosis (up to $8 \mathrm{mmol} / \mathrm{L}$ ) (see Figure: Mean blood (left panel) and urine (right panel) ketone concentrations. 


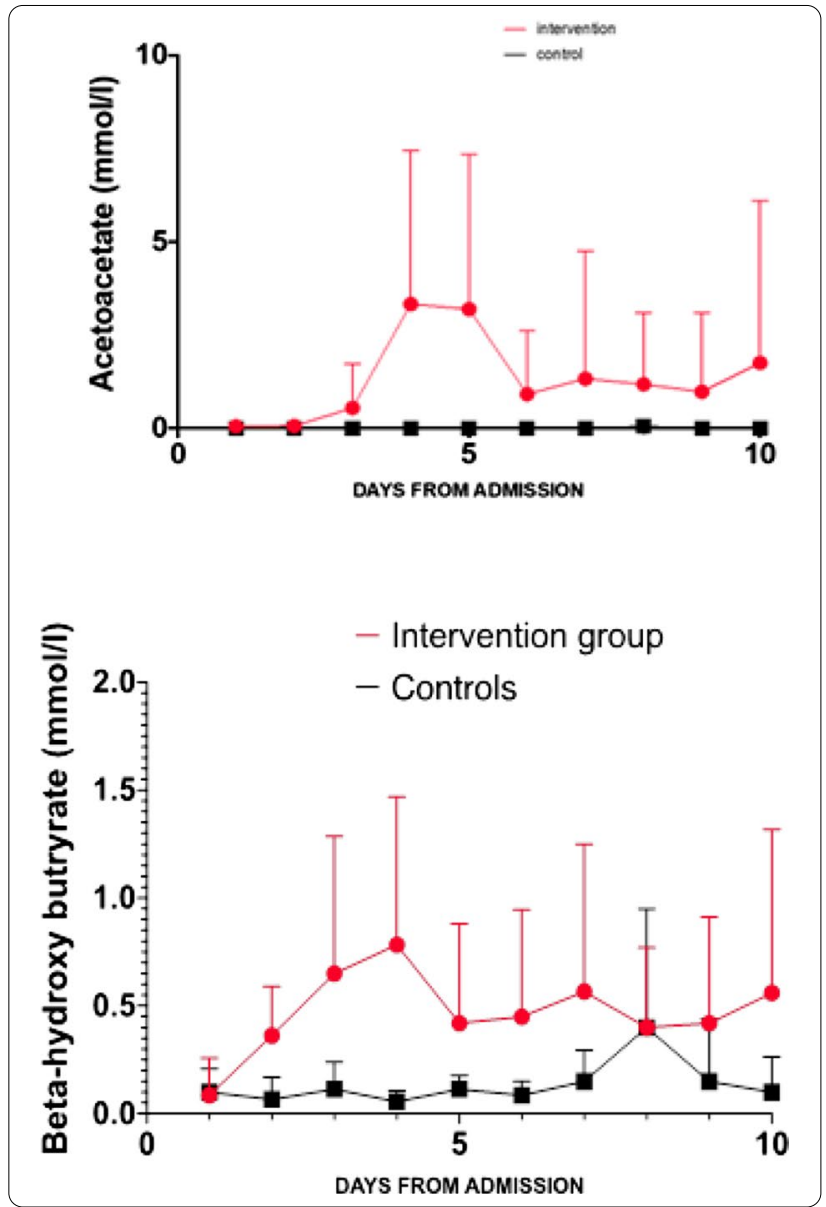

Conclusion. Administration of a ketogenic enteral feed to ICU patients appears safe, well-tolerated and effectively induces ketone body production. Ready-made feed would improve feasibility of delivering the intervention.

\section{Reference(s)}

1. Langan, A., Alternative substrates in the critically ill subject (ASICS): protocol for a feasibility study. Intensive care medicine experimental, 2020; 8(SUPPL 2):43.

2. National Institute for Health Research: Research for Patient Benefit Grant PB-PG-0317-20,006

\section{8}

Quantitative capillary refill time as a tissue perfusion indicator in postcardiac surgery patients: Single-center prospective cohort study

Y. Miyuki'; H. Naoki'; H. Takahiro'; U. Yoshihiro'; D. Kent

'Department of acute medicine, The University of Tokyo, Tokyo, Japan Correspondence: Y. Miyuki

Intensive Care Medicine Experimental 2020, 9(1): 000598

Introduction. Capillary refill time (CRT) is clinically used as a simple and non-invasive index for evaluating peripheral circulation in disaster medicine, however many environmental factors are known to reduce its accuracy. Quantitative CRT (Q-CRT) and delta $A b(\triangle A b)$ measured by applying the principle of pulse oximeter has been developed to evaluate peripheral perfusion quantitively. Delta $A b$ reflects the following three factors; oxygen saturation, hemoglobin, and circulating blood volume. These two new indexes have been shown to be associated with lactate in sepsis and perioperative outcomes after liver transplantation.

Objectives. The purpose of this study was to investigate the relationship between the clinically used circulatory index and two CRT measurements of Q-CRT and $\triangle \mathrm{Ab}$ in postcardiac surgery patients.

Methods. Adult patients who underwent cardiac surgery from August 2018 to July 2019 and admitted to the ICU of the University of Tokyo Hospital were enrolled prospectively. Patients who did not consent to participate, patients after heart transplantation, and patients who did not use cardiopulmonary bypass (CPB) were excluded. Quantitative CRT and $\triangle \mathrm{Ab}$ were measured at ICU admission, and investigated the relationship with intraoperative factors, hemodynamic parameters, and laboratory data. We also evaluated the change in lactate $6 \mathrm{~h}$ after the surgery.

Results. We included sixty-eight patients (39 males and 29 females) who underwent valve surgery, coronary graft surgery, artificial vessel replacement, and ventricular assist device implantation. The median operation time was $6.5 \mathrm{~h}$ (5.1-7.7), CPB time $198 \mathrm{~min}$ (147-243). The median values of Q-CRT and $\triangle \mathrm{Ab}$ at ICU admission were $3.39 \mathrm{~s}$ (2.55$4.88)$ and $0.024(0.003-0.044)$, respectively. Significant correlations were observed between Q-CRT and SvO2 $(p=0.0447, r=-0.2693)$ and $\triangle \mathrm{Ab}$ and the $6 \mathrm{~h}$ lactate change $(\mathrm{p}=0.0391, r=-0.2508)$.

Conclusion. It was suggested that Q-CRT and $\triangle A b$ may be useful as peripheral circulatory evaluation indexes after cardiac surgery.

\section{Reference(s)}

1. Nicole T, Sameena L, Beniot V, et al. Outcomes of post-cardiac surgery patients with persistent hyperlactatemia in the intensive care unit: a matched cohort study. J Cardiothorac Surg. 2016; Feb 24; 11:33.

2. Yamamoto M, Doi K, Hayase N, et al. Pulse oximetry-based capillary refilling evaluation predicts postoperative outcomes in liver transplantation: a prospective observational cohort study. BMC Anesthesiol. 2020 Sep 29; 20 (1): 251.

3. Oi Y, Sato K, Nogaki A, et al. Association between venous blood lactate levels and differences in quantitative capillary refill time. Acute Med Surg. 2018;5(4):321-8.

4. Morimura N, Takahashi K, Doi T, et al. A pilot study of quantitative capillary refill time to identify high blood lactate levels in critically ill patients. Emerg Med J. 2015; 32(6):444-8.

5. Schriger $\mathrm{DL}$, Baraff $\mathrm{L}$. Defining normal capillary refill: variation with age, sex, and temperature. Ann Emerg Med. 1988;17(9):932-5.

6. The authors wish to thank Hideaki Hirahara and Naoki Kobayashi (Nihon Kohden Corporation, Tokyo, Japan) for their support with data analysis.

000601

Tidal volume challenge to predict preload responsiveness in patients with acute respiratory distress syndrome under prone position

R. Shi ${ }^{1}$; S. Ayed, ${ }^{1}$; F. Moretto ${ }^{1}$; N. De Vita ${ }^{1}$; F. Gavelli ${ }^{1}$; S. Carelli ${ }^{1}$; A. Pavot ${ }^{1}$; C. Lai ${ }^{1}$; X. Monnet ${ }^{1}$; JL. Teboul ${ }^{1}$

${ }^{1}$ Médecine intensive_réanimation, inserm umr s_999, fhu sepsis, groupe de recherche carmas, Bicêtre Hospital, Université Paris Saclay, AP-HP, Le Kremlin-Bicêtre, France

Correspondence: R. Shi

Intensive Care Medicine Experimental 2020, 9(1): 000601

Introduction. Prone position is frequently used in patients with acute respiratory distress syndrome (ARDS), especially during the COVID19 pandemic. Hemodynamic instability might happen during prone position sessions. In this setting, testing preload responsiveness without requiring cardiac index $(\mathrm{Cl})$ measurements has been poorly investigated.

Objectives. Our study aimed to investigate the ability of pulse pressure variation (PPV) and its changes during a 1-min tidal volume (TV) challenge (TVC) to assess preload responsiveness in ARDS patients under prone position. (ClinicalTrials.gov registration: NCT04457739). Methods. Patients with ARDS ventilated with a $6 \mathrm{~mL} / \mathrm{kg}$ TV under prone position were prospectively included from 2019 to 2021. By using a pulse contour analysis monitor, we measured PPV and 
changes in $\mathrm{Cl}$ during a Trendelenburg maneuver $(\triangle \mathrm{Cl}$ TREND). After transiently increasing VT to $8 \mathrm{~mL} / \mathrm{kg}$, we measured first absolute changes in PPV during TVC ( $\triangle$ PPV TVC6-8), and then changes in Cl during end-expiratory occlusion (EEO) $(\triangle \mathrm{Cl}$ EEO8). Preload responsiveness was defined by both $\triangle \mathrm{CI}$ TREND $\geq 8 \%$ (according to Yonis et al. [1])and $\triangle \mathrm{Cl} \mathrm{EEO} 8>5 \%$ (according to Gavelli et al. [2]). Preload unresponsiveness was defined by both $\triangle \mathrm{CI}$ TREND $<8 \%$ and $\triangle \mathrm{Cl}$ EEO $8<5 \%$.

Results. Eight-four sets of measurements were analyzed in 58 patients, whose 44 had COVID-19 (76\%). The mean age was $65 \pm 11$ years old The inclusions were conducted after $11(2-14)$ hours of prone position. The mean arterial pressure was $82(75-90) \mathrm{mmHg}$ (under norepinephrine in $83 \%$ cases at a dose of $0.25(0.15-0.42) \mu \mathrm{g} / \mathrm{kg} / \mathrm{min})$. The driving pressure was $12(10-18) \mathrm{cmH} 2 \mathrm{O}$, the respiratory system compliance was $32(21-40) \mathrm{mL} / \mathrm{cmH} 2 \mathrm{O}$ and the positive end-expiratory pressure was $14(11-16) \mathrm{cmH} 2 \mathrm{O}$. The ratio of partial pressure arterial oxygen and fraction of inspired oxygen was $104 \pm 27 \mathrm{mmHg}$. In 42 cases, patients were classified as preload responders. The baseline PPV predicted preload responsiveness with an area under the receiver operating characteristic curve (AUROC) of $0.85 \pm 0.04$ (threshold 5\%; sensitivity: $74 \%$, specificity: $79 \%$ ). The $\triangle$ PPV TVC6-8 predicted preload responsiveness with an AUROC of $0.94 \pm 0.03$ (threshold $2 \%$; sensitivity: $98 \%$, specificity: $88 \%)$. ( $p=0.03$ vs. PPV). The grey zone of baseline PPV to predict preload responsiveness ranged from 4 to $6 \%$ and included 36 measurements. Analysis of these 36 cases shows that $\triangle$ PPV TVC6-8 predicted preload responsiveness with an AUROC of $0.90 \pm 0.07$ (threshold $2 \%$, sensitivity: $93 \%$, specificity: $90 \%)(p=0.02$ $v$ s. baseline PPV: $0.60 \pm 0.09$ ). We excluded 13 cases in 11 patients from the analysis where only $\Delta \mathrm{CI}$ TREND $\geq 8 \%$ or only $\Delta \mathrm{CI}$ EEO $8 \geq 5 \%$

Conclusion. In patients with ARDS under protective ventilation during prone position, the changes in PPV during a 1-min TVC could reliably assess preload responsiveness without the need of $\mathrm{Cl}$ measurements.

\author{
Reference(s) \\ 1. Yonis et al. Crit Care 2017;21:295 \\ 2. Gavelli et al. Ann Intensive Care 2020;10:65 \\ 3. None.
}

\section{5}

Biomarkers of oxidative injury after cardiac arrest in patients treated with low or high arterial oxygen target

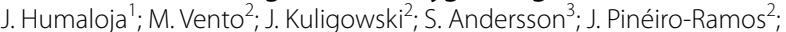

Á. Sanchez-Illana'; E. Litonius'; P. Jakkula ${ }^{4}$; M. Reinikainen ${ }^{5}$; M. Skrifvars'

${ }^{1}$ Department of emergency care and services, University of Helsinki and Helsinki University Hospital, Helsinki, Finland; ${ }^{2}$ Neonatal research group, Health Research Institute \& University and Polytechnic Hospital La Fe, Valencia, Spain; ${ }^{3}$ Children's hospital, pediatric research center, Helsinki University \& Helsinki University Hospital, Helsinki, Finland; ${ }^{4}$ Department of anaesthesiology, intensive care and pain medicine, Helsinki University \& Helsinki University Hospital, Helsinki, Finland; ${ }^{5}$ Department of intensive care, Kuopio University Hospital, Kuopio, Finland

Correspondence: J. Humaloja

Intensive Care Medicine Experimental 2020, 9(1): 000605

Introduction. After resuscitation from cardiac arrest, reperfusion injury of varying degree is inevitable.1,2 Reperfusion triggers formation of harmful reactive oxygen species (ROS). Isoprostanes, isofurans, neuroprostanes, and neurofurans are chemical byproducts formed through the direct interaction of cell lipid structures and ROS, and are currently considered the most reliable biomarkers for determining oxidative injury in vivo.3 Levels of these biomarkers and their association with arterial oxygen have not been studied in adults with cardiac arrest.

Objectives. We investigated the extent of oxidative injury related to ischemia-reperfusion by determining the levels of isoprostanes, isofurans, neuroprostanes, and neurofurans after out of hospital cardiac arrest (OHCA). Additionally, we tested if biomarker levels differed between low or high arterial oxygen tension $(\mathrm{PaO} 2)$ targets during intensive care unit (ICU) care.

Methods. We performed a post-hoc analysis of plasma samples collected during the COMACARE trial (NCT02698917)0.4 After resuscitation from OHCA, 123 patients were treated with a lower ( $\mathrm{PaO} 210-15 \mathrm{kPa})$ or higher ( $\mathrm{PaO} 220-25 \mathrm{kPa}$ ) arterial oxygen target during the first $36 \mathrm{~h}$ in the ICU. We measured plasma levels of isoprostane, isofuran, neuroprostane, and neurofuran at ICU admission, and at 24,48 , and $72 \mathrm{~h}$ thereafter. $5 \mathrm{We}$ compared biomarker levels over time between oxygen groups with linear mixed model analysis.

Results. Plasma samples were available for 112 patients who were included in this study. All four biomarker levels followed a similar time pattern, highest level peaking at $24 \mathrm{~h}$. In linear mixed model analysis there were no significant differences in any of the biomarker levels between low and high arterial oxygen target groups, $\mathrm{p}$-values are shown in Fig. 1.

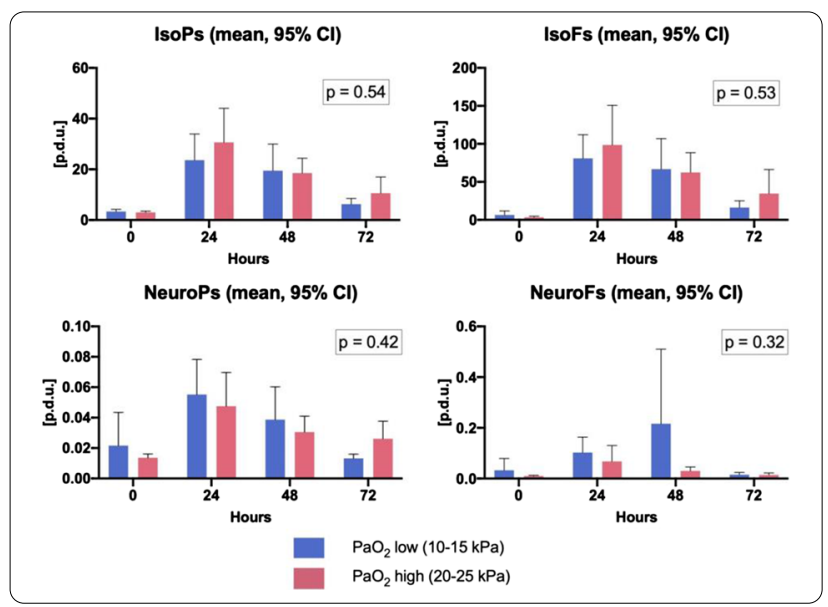

Conclusion. We did not find any association between lipid peroxidation byproducts and arterial oxygen tensions. As a post hoc analysis it is possible that the clinical setting is not optimal to detect the biochemical difference. All four lipid peroxidation biomarker levels followed a certain time pattern, with levels peaking around $24 \mathrm{~h}$ after CA, revealing the timing of maximum oxidative stress intensity.

\section{Reference(s)}

1. Kalogeris T, Baines CP, Krenz M, Korthuis RJ. Ischemia/reperfusion. Compr Physiol 2017;7(1):113-70.

2. Granger DN, Kvietys PR. Reperfusion injury and reactive oxygen species: The evolution of a concept. Redox Biol 2015;6:524-51.

3. Marrocco I, Altieri F, Peluso I. Measurement and Clinical Significance of Biomarkers of Oxidative Stress in Humans. Oxid. Med. Cell. Longev. 2017;2017

4. Jakkula P, Reinikainen M, Hästbacka J, et al. Targeting low- or high-normal Carbon dioxide, Oxygen, and Mean arterial pressure After Cardiac Arrest and REsuscitation: Study protocol for a randomized pilot trial. Trials 2017;18(1).

5. Sánchez-Illana Á, Thayyil S, Montaldo P, et al. Novel free-radical mediated lipid peroxidation biomarkers in newborn plasma. Anal Chim Acta 2017:996:88-97

6. This study has been supported by Finska Läkaresälskapet, Sigrid Jusélius Stiftelse, University of Helsinki and Helsinki University Hospital Funding. (State funding, Finland), and Instituto de Salud Carlos III (CP16/00034; PI17/00127).

\section{1}

\section{Potential for lung recruitment in patients} with SARS-CoV2-associated acute respiratory distress syndrome: a multicenter study

T. Pham ${ }^{1}$; C. Brault, ${ }^{2}$; F. Beloncle 3 ; M. Combet'; AF. Haudebourg ${ }^{4}$; M. Rodriguez ${ }^{5}$; M. Dres ${ }^{6}$; L. Piquilloud ${ }^{7}$;. Zerbib ${ }^{2} ;$ B. Pavlovsky ${ }^{3} ;{ }^{\prime}$. Bony $y^{6}$; R. Coudroy ${ }^{5}$; G. Carteaux ${ }^{4}$

${ }^{1}$ Médecine intensive-réanimation, Bicetre Hospital AP-HP, Le Kremlin-Bicêtre, France: ${ }^{2}$ Médecine intensive-réanimation, Amiens University Hospital, Amiens, France; ${ }^{3}$ Médecine intensive-réanimation, Angers University Hospital Center, Angers, France; ${ }^{4}$ Médecine intensive-réanima- 
tion, Hôpital Henri-Mondor Ap-Hp, Créteil, France; ${ }^{5}$ Médecine intensive-réanimation, Poitiers University Hospital, Poitiers, France; ${ }^{6}$ Médecine intensive-réanimation, University Hospitals Pitié Salpêtrière—Charles Foix, Paris, France; ${ }^{7}$ Service de médecine intensive adulte, Lausanne University Hospital, Lausanne, Switzerland

Correspondence: $\mathrm{T}$. Pham

Intensive Care Medicine Experimental 2020, 9(1): 000611

Introduction. Respiratory mechanics and potential for lung recruitability of COVID-19 related acute respiratory distress syndrome (C-ARDS) have been reported only in small series with heterogeneous results. Precise measurement of these variables may provide valuable information to personalize ventilation.

Objectives. Main objectives were the description of respiratory system characteristics in a large multicenter cohort of intubated patients with C-ARDS and identification of factors associated with recruitability.

Methods. This is a multicenter observational study performed in 6 Intensive Care Units (ICUs) in France. We included intubated C-ARDS patients. Within $72 \mathrm{~h}$ following intubation we measured respiratory mechanics, lung recruitability and airway opening pressure (AOP). Low respiratory system compliance was define as $<40 \mathrm{ml} / \mathrm{cmH} 2 \mathrm{O}$. We assessed lung recruitability using recruitment-to-inflation ratio $(\mathrm{R} / \mathrm{I})$, obtained with a drop in PEEP over a single breath maneuver, as previously described [1]. Patients were deemed high recruiters if $\mathrm{R} / \mathrm{l}>0.5$. AOP was assessed with a low-flow inflation.

Results. We included 190 patients, $78 \%$ were men, with a mean (SD) age of $61(13)$, BMI of $29.7(6.2) \mathrm{kg} / \mathrm{m}^{2}$, SAPS2 of 41 (15), and SOFA of 6 (3). Their median [IQR] duration of invasive ventilation was 14 [9;27] days and $57 \%$ were discharged alive from the ICU. A total of 103 patients (55\%) exhibited a low respiratory system compliance and an AOP above $5 \mathrm{cmH} 2 \mathrm{O}$ was retrieved in 40 (23\%) patients. A total of 102 patients (54\%) were high recruiters with a R/I above 0.5 . Comparisons between high recruiters and low recruiters are shown in the table. The mean (SD) expired volume in a single breath maneuver was 1061 (289) $\mathrm{mL}$ and did not differbetween the two groups $(p=0.103)$. There was no statistical difference except absolute tidal volume being lower and PEEP set higher in the recruiters. When tidal volumes were standardized to predicted body weight, the difference was no longer significant.

Table: characteristics of the patients according to recruitability. Data are shown as mean $\pm S D$, meadian $[I Q R]$ or $n(\%)$.

\begin{tabular}{|c|c|c|c|c|}
\hline & $\begin{array}{l}\text { All patients } \\
\mathrm{N}=190\end{array}$ & $\begin{array}{l}\text { Low recruit- } \\
\text { ers } N=88\end{array}$ & $\begin{array}{l}\text { High recruit- } \\
\text { ers } N=102\end{array}$ & p-value \\
\hline $\mathrm{pH}$ & $7.35 \pm 0.08$ & $7.36 \pm 0.08$ & $7.34 \pm 0.08$ & 0.184 \\
\hline $\mathrm{PaO} 2 / \mathrm{FlO} 2, \mathrm{mmHg}$ & $147 \pm 78$ & $141 \pm 57$ & $152 \pm 92$ & 0.309 \\
\hline Tidal volume, mL & $403 \pm 50$ & $412 \pm 49$ & $396 \pm 51$ & 0.035 \\
\hline $\begin{array}{l}\text { Tidal volume } \\
\text { per kg } \\
\text { of predicted body } \\
\text { weight, } \mathrm{mL} / \mathrm{kg}\end{array}$ & $6.1 \pm 0.5$ & $6.1 \pm 0.5$ & $6.1 \pm 0.5$ & 0.885 \\
\hline PEEP, cmH2O & $14.5[11 ; 16]$ & $13[10 ; 15]$ & $15[13 ; 16]$ & 0.002 \\
\hline $\begin{array}{l}\text { Plateau pressure, } \\
\mathrm{cmH} 2 \mathrm{O}\end{array}$ & $25[22 ; 27]$ & $24[21 ; 26]$ & $25[23 ; 28]$ & 0.007 \\
\hline $\begin{array}{l}\text { Driving pressure, } \\
\mathrm{cmH} 2 \mathrm{O}\end{array}$ & $11[90 ; 13]$ & $10[90 ; 13]$ & $11[90 ; 13]$ & 0.918 \\
\hline $\begin{array}{l}\text { Respiratory system } \\
\text { Compliance, } \\
\mathrm{mL} / \mathrm{cmH} 2 \mathrm{O}\end{array}$ & $36.5[30 ; 47]$ & $38[31 ; 47]$ & $36[29 ; 47]$ & 0.536 \\
\hline $\begin{array}{l}\text { Respiratory } \\
\text { system Compli- } \\
\text { ance }<40 \mathrm{~mL} / \\
\mathrm{cmH} 2 \mathrm{O}\end{array}$ & $103(55)$ & $46(52)$ & $57(56)$ & 0.732 \\
\hline$R / I$ & $0.52[0.33 ; 0.75]$ & $0.32[0.23 ; 0.41]$ & $0.72[0.59 ; 0.88]$ & $<0.001$ \\
\hline $\mathrm{AOP}>5 \mathrm{cmH} 2 \mathrm{O}$ & $40(23)$ & $20(24.7)$ & $20(21.5)$ & 0.751 \\
\hline
\end{tabular}

Conclusion. In a cohort of 190 C-ARDS patients receiving invasive mechanical ventilation, approximatelyhalf were deemed highly recruitable and $23 \%$ presented an $A O P>5 \mathrm{cmH} 2 \mathrm{O}$ within $72 \mathrm{~h}$ after intubation. We could not identify specific parameters associated with recruitability. This emphasizes the importance of measuring respiratory mechanics and assessing recruitability to personalize mechanical ventilation in this population.

\section{Reference}

1. Chen L, Del Sorbo L, Grieco DL, Junhasavasdikul D, Rittayamai N, Soliman I, et al. Potential for Lung Recruitment Estimated by the Recruitment-toInflation Ratio in Acute Respiratory Distress Syndrome. A Clinical Trial. Am J Respir Crit Care Med. 2020;201

\section{2}

Serum Phosphate levels and its impact on outcome in critically ill surgical patients: A prospective observational study

V. Sundarsingh $\mathrm{S}^{1} ; \mathrm{T}$. Joseph, ${ }^{2} ; \mathrm{S}$. Joshi ${ }^{2}$

${ }^{1}$ Critical care medicine, DR. DY Patil Medical College \& Research

Centre,Pimpri, Pune, India; ${ }^{2}$ Anaesthesiology \& critical care, DR. DY Patil

Medical College,Pune, Pune, India

Correspondence: $V$. Sundarsingh $S$

Intensive Care Medicine Experimental 2020, 9(1): 000622

Introduction. Phosphorus plays a vital role in many biological processes, including energy metabolism, cellular signalling, nucleic acid metabolism, membrane integrity, and bone mineralization..(1)Maintaining normal serum phosphate levels is extremely important as phosphate supply disturbance can result in multiple organ system dysfunction, which is not limited to systems, such as the respiratory, cardiac, immunologic, hematologic,or neuromuscular.(2)The aim of this study was to assess the association of phosphate concentration with key clinical outcomes in a surgical cohort of critically ill patients. Objectives. To find the impact of hypophosphatemia in critically ill surgical patients and correlating the day 1 serum phosphate levels with the following outcomes (after following up the patient for a duration of 28 days).

\section{- PRIMARY OUTCOME:}

1. 28 day mortality

- SECONDARY OUTCOME:

1. Length of stay in ICU

2. Length of stay in hospital

3. Duration of Mechanical ventilation

4. Vasopressor days

5. Renal replacement therapy

Methods. The study was conducted from 1 January 2020 to 31 August 2020 after approval by the Institutional Ethics Committee.This prospective observational study was conducted in a 15 bedded surgical ICU in a tertiary care teaching institute in western part of India. A total number of 125 patients were included at ICU admission. The following inclusion criteria were used: 1 ) age $\geq 18$ years; 2 ) patients with serum phosphate level measured at admission at ICU; 3 ) survival status for a follow-up period of 28 days after ICU admission. The criteria for exclusion were: 1 ) age below 18 years; 2 ) pregnancy; 3 ) patients who could not be followed up; 4) Those without serum phosphate level measured at admission to ICU.Statistical analysis was conducted by SPSS 20.0 software.

Results. The median phosphate measurement was $3.17 \mathrm{mg} / \mathrm{dL}(2.2-$ 3.77). Hyperphosphatemia( $>4.5 \mathrm{mg} / \mathrm{dL})$ occurred in $0.02 \%(3 / 125)$ and hypophosphatemia $(\leq 2.5 \mathrm{mg} / \mathrm{dL})$ in $30.4 \%(38 / 125)$. Patients who had hypophosphatemia at admission had a higher ICU mortality than those without hypophosphatemia $(P=0.034)$. In addition, ICU nonsurvivors had lower minimum phosphate concentrations than did survivors $(P=0.005)$. Similar results were seen for hospital mortality. 
Even on multivariable logistic regression analysis, hypophosphatemia was independently associated with ICU mortality (adjusted odds ratio, 2.93 [95\% confidence interval, 1.34-6.43]; $\mathrm{P}=0.007$ ). None of the other outcomes had any statisical significance when compared between patients with and without hypophosphatemia.

Conclusion. Hypophosphatemia at admission is an independent risk factor for 28-day mortality in critically ill surgical patients. The development of worsening hypophosphatemia should be diagnosed early to prevent its harmful effects in critically ill surgical patients.

\section{Reference(s)}

1. Wang, L., Xiao, C., Chen, L. et al. Impact of hypophosphatemia on outcome of patients in intensive care unit: a retrospective cohort study. BMC Anesthesiol 19, 86 (2019).

2. RL Wadsworth, MB ChB FRCA, S Siddiqui, MB ChB FRCA, Phosphate homeostasis in critical care, BJA Education, Volume 16, Issue 9, September 2016, Pages 305-309

\section{3}

Simulation based Objective Structured Clinical Evaluations (OSCE) to determine the competency level of ICU trainees. Cobalidation trial phase I

E. Sancho Ferrando ${ }^{1}$; A. Castellanos Ortega ${ }^{1}$; MJ. Broch Porcarr ${ }^{1}$; C. Vicent Perales'; ;. Madrid López'; N. Martinez Sanz'; D. Palacios Castañeda²; M. Valdivia De La Fuente ${ }^{2} ;$ V. Gómez Tello ${ }^{3}$; MJ. Párraga ${ }^{4}$; MC. Fuentes-Dura $;$; MD. Sancerni-Beitia ${ }^{5} ;$ R. Garcia-Ros

${ }^{1}$ Intensive care unit, Hospital Universitario y Politécnico La Fe, Valencia, Spain; ${ }^{2}$ Intensive care unit, Hospital Puerta de Hierro-Majadahonda, Majadahonda, Spain; ${ }^{3}$ Intensive care unit, University Hospital Moncloa, Madrid, Spain; ${ }^{4}$ Intensive care unit, Hospital Universitario Morales Meseguer, Murcia, Spain; ${ }^{5}$ Department of methodology of the behavioral sciences, Faculty of Psychology, University of Valencia, Valencia, Spain; ${ }^{6}$ Department of developmental and educational psychology, Faculty of Psychology, University of Valencia, Valencia, Spain

Correspondence: E. Sancho Ferrando

Intensive Care Medicine Experimental 2020, 9(1): 000633

Introduction. CoBaTriCE collaboration program was created in 2003 to assure a high-quality education and training for intensive care residents in Europe. It offers resources for mentors and residents to consolidate the basic knowledge considered essential to define an intensivist and it is based in practical evaluations, simulation and feedback. Even though, in Spain and many other countries, the classical time-based model of training based on exposure to clinical practice is still used.

Objectives. The main objective of this study was to evaluate the level of competency (level I: full supervision required, level II: supervision in most of situations, level III: supervision in complex situations, level IV: indirect supervision, level V: independent practitioner) acquired by Spanish residents after the third of five years of training in intensive care medicine. Secondary objectives were to identify gaps in their current education and to investigate the reliability and feasibility of conducting simulationbased assessment in multiple sites at the same time.

Methods. This is a national multicentric observational study. An independent panel of 10 experts designed an objective structured clinical evaluation (OSCE) with 5 high fidelity simulation scenarios (septic shock with ARDS, neurocritical care, myocardial infarction with cardiac arrest, polytrauma and postoperative haemorrhagic shock). They also defined critical essential performance elements (CEPE) and critical non-essential performance elements (CNEPE) for each scenario and assessed the performance of the residents rating 15-min video records of each scenario. Thirty-six ICU residents in their third year of training from 13 Spanish teaching hospitals participated in the OSCE. Intraclass correlation coefficients were used to measure the inter-rater reliability, and several differential analyses were carried out to measure the discriminant validity of each scenario.

Results. A total of 176 performances were analysed as the video records of 4 performances were lost. The mean score achieved for all residents was 69.6 out of 100 points. The percentage of accomplished CEPE and CNEPE was $74.7 \%$ and $61.6 \%$ respectively. The distribution of the residents among the different competency levels was: $18.8 \%$ achieved level I; 35\% level II; $43 \%$ level III; 3.4\% level IV or V. Inter-rater reliability was satisfactory, significant differences between scenarios scores were obtained. and there were not differences in the scores achieved for the residents among the different simulation centers.

Conclusion. Most of the residents reached level III or less. There was a great heterogeneity in the performance of the residents in their third year of ICM training program regarding scenarios, hospitals and level of competency acquired. Reliance on the traditional experience-based training model alone is insufficient for ensuring quality and safety in patient care. Multiple center simulation-based assessment showed feasibility, validity and reliability as an evaluation method of competency.

\section{Reference(s)}

1. van Bockel EAP, Walstock PA, van Mook WNKA, Arbous MS, Tepaske R, van Hemel TJD, et al. Entrustable professional activities (EPAs) for postgraduate competency based intensive care medicine training in the Netherlands: The next step towards excellence in intensive care medicine training. J Crit Care 2019;54:261-7. https://doi.org/10.1016/j.jcrc.2019.09. 012.

2. CoBaTrlCE Collaboration. The educational environment for training in intensive care medicine: structures, processes, outcomes and challenges in the European region. Intensive Care Med 2009;35:1575-83. https://doi. org/10.1007/s00134-009-1514-4.

3. CoBaTrlCE Collaboration. International standards for programmes of training in intensive care medicine in Europe. Intensive Care Med 2011;37:385-93. https://doi.org/10.1007/s00134-010-2096-x.

4. Castellanos-Ortega A, Broch MJ, Barrios M, Fuentes-Dura MC, SancerniBeitia MD, Vicent C, et al. Acceptance and validity of the methods used to implement a competency based medical education programme in an Intensive Care Department of a teaching referral center. Med Intensiva 2020. https://doi.org/10.1016/j.medin.2019.12.007.

5. ESICM Awards 2018

6. Valencian Community public grant (DOCV núm. 8164)

000642

Muscle mass loss as assessed by urinary creatinine and potassium excretion in COVID-19 patients and the impact of neuro-muscular blocking and prone positioning

AV. Burgel, '; L. Hulshof, ${ }^{1}$; M. Volbeda'; M. Renes, ${ }^{1}$; M. Nijsten ${ }^{1}$

${ }^{1}$ Department of critical care, University Medical Center Groningen, Groningen, Netherlands

Correspondence: M. Nijsten

Intensive Care Medicine Experimental 2020, 9(1): 000642

Introduction. Urinary creatinine excretion (UCE) reflects muscle mass and changes in muscle mass in ICU patients $[1,2]$. A negative potassium balance, as partially reflected by urinary potassium excretion may also indicate muscle mass loss in ICU patients [3]. Follow-up studies of muscle mass loss during ICU stay with serial UCE measurements or imaging techniques indicate that this loss is $\geq 1 \%$ / day in non-COVID-19 patients [2].

Objectives. Treatment of COVID-19 patients in the ICU frequently involves neuromuscular blockage (NMB) and prone positioning. As these interventions may affect loss of muscle mass, we assessed their relation with UCE and UPE in COVID-19, in addition to the known effect of sex and day of ICU stay.

Methods. In the 90 consecutive ICU patients admitted for COVID-19, we analyzed daily UCE and UPE in $24 \mathrm{~h}$ urine collections during the first 30 ICU days starting on the ICU day 2. UCE and UPE values were excluded when the measured creatinine clearance ( $\mathrm{mCC}$ defined as $694^{*}$ UPE/plasma creatinine) was $<35 \mathrm{ml} / \mathrm{min}$ or when the patient received renal replacement therapy (RRT). NMB was induced by continuous rocuronium infusion titrated to the patients' response. Supine or prone position was recorded each day. Regression analysis was performed with UCE and UPE as dependent variables and ICU-day, sex, NMB and prone positioning as independent variables. Since females have a considerably lower muscle mass than males $[1,2]$, sex was included as an independent variable. 
Results. We included 73 patients of whom 5 underwent RRT on some days. A total of 787 UCE and UPE measurements were available. Mean \pm SD age was $61 \pm 10$ years and hospital mortality was $16 \%$. Prone positioning was performed in $33(45 \%)$ of the patients on 122 ICU days, NMB was induced in $50(68 \%)$ of the patients on 256 ICU days. Mean daily decrease of UCE as a reflection of muscle mass loss was $1.2 \%(95 \% \mathrm{Cl} 1.0$ to $1.5 \% ; \mathrm{P}<0.001)$. Multivariate linear regression analysis identified ICU-day $(P<0.001)$ and sex $(P<0.001)$ as independently associated with UCE. Males had a $36 \%$ higher UCE than females $(P<0.001)$. Neither prone positioning nor NMB were independently associated with UCE. With respect to potassium excretion, we found that ICU-day $(P<0.001)$, sex $(P<0.001)$ and NMB $(P=0.007)$ were independently associated with UPE. Prone positioning was not independently associated with UPE.

Conclusion. Using UCE as a measure of muscle mass we found that COVID-19 patients display similar loss of muscle mass as nonCOVID-19 patients [2], i.e. $\geq 1 \%$ per day. Although we did not find an association between UCE and NMB use, the increased potassium excretion during days when NMB was applied may indicate that this treatment may result in accelerated muscle loss. Future research to address this hypothesis should include potassium balances or repeated imaging studies to assess muscle mass.

\section{Reference(s)}

1. [1] Hessels et al. Urinary creatinine excretion is related to short-term and long-term mortality in critically ill patients. Intensive Care Med 2018

2. [2] Volbeda $\mathrm{M}$ et al. Time courses of urinary creatinine excretion, measured creatinine clearance and estimated glomerular filtration rate over 30 days of ICU admission. J Crit Care 2020.

3. [3] Van Bakelen M et al. Impact of sex and ICU treatment duration on creatinine and potassium excretion in critically ill patients. Abstract ESICM 2020

4. None

\section{5}

Antiapoptotic antioxidant and innate immunity responses to the oxidative inflammatory stress in pediatric sepsis

S. Ilia'; M. Miliaraki'; P. Briassoulis'; ; A. Polonifi'; M. Mantzourani';

E. Briassouli ${ }^{2}$;. Tavladaki'; A. Spanaki ${ }^{1}$; H. Dimitriou ${ }^{3}$; M. Venihaki ${ }^{4}$;

M. Katrinaki ${ }^{4}$; G. Daikos ${ }^{2}$; G. Briassoulis ${ }^{1}$

${ }^{1}$ Pediatric intensive care unit, university hospital, medical school, University of Crete, Heraklion, Greece; ${ }^{2}$ First department of internal medicine, laiko hospital, National and Kapodistrian University of Athens, Athens, Greece; ${ }^{3}$ Division of mother and child health, school of medicine, University of Crete, Heraklion, Greece: ${ }^{4}$ Department of clinical chemistry, school of medicine, University of Crete, Heraklion, Greece

Correspondence: M. Miliaraki

Intensive Care Medicine Experimental 2020, 9(1): 000665

Introduction. Programmed cell death is executed through apoptotic processes and regulated by anti-apoptotic responses, probably having central roles during profound immunosuppression or hyperactivation of innate immunity and determining the risk of mortality in sepsis1,2. Based on these findings apoptotic/antiapoptotic, inflammatory or oxidative stress biomolecules could have key roles in pediatric sepsis.

Objectives. The present study seeks to explore possible correlations between antiapoptotic (survivin), antioxidant and innate immunity biomolecules compared to biomarkers of inflammatory-oxidative stress in pediatric intensive care unit (PICU) patients with sepsis.

Methods. This prospective observational study was performed in critically ill pediatric patients with sepsis compared to trauma (noninfectious critical illness) and healthy controls ( $\mathrm{HC}$ ). The expression of survivin transcript variants (wild type-WT, $-2 \mathrm{~B},-3 \mathrm{~B},-\Delta \mathrm{E} \times 3$ ) was analyzed by real-time quantitative PCR in peripheral blood leukocytes, whereas the survivin-WT serum protein concentrations and innate immunity heat shock proteins (HSP)-72, -90 and IL-6, $-8,-10$ dysregulation markers through enzyme-linked immunosorbent assay (ELISA). Total oxidative stress (TOS) and total antioxidant capacity (TAC) were quantified by photometric testing.
Results. Among the 163 children enrolled in the study, 63 had sepsis, 49 trauma and 51 were HC. Elevated serum levels of survivin ( 272 vs $111 \mathrm{vs} 85 \mathrm{pg} / \mathrm{ml})$ and TOS (1186 vs 329 vs $198 \mu \mathrm{mol} / \mathrm{l})$ and repressed of TAC ( 186 vs 217 vs $308 \mu \mathrm{mol} / \mathrm{l}$ ) were found in septic children compared to trauma and $\mathrm{HC}$, respectively, (all, $p=0.001$ ). Significantly increased HSP90 (571 vs 163 vs $134 \mathrm{ng} / \mathrm{ml}, \mathrm{p}=0.001)$, HSP72 (1.1 vs $0.37 \mathrm{vs} 0.3 \mathrm{ng} / \mathrm{ml}, \mathrm{p}=0.01)$, IL-6 (143 vs 132 vs $22 \mathrm{pg} / \mathrm{ml}, \mathrm{p}=0.007$ ) and IL-10 (77 vs 32 vs $17.4 \mathrm{ng} / \mathrm{ml}, \mathrm{p}=0.001$ ) were also recorded among septic children (Fig. 1). Survivin protein was positively related to HSP90 $(r=0.41, p=0.02)$ and TOS $(r=0.85, p=0.001)$ and inversely to TAC $(r=-0.47, p=0.04)$. Among non-survivors TOS (909 vs $401 \mu \mathrm{mol} / \mathrm{l})$, HSP90 (761 vs $215 \mathrm{ng} / \mathrm{ml}$ ), HSP72 (1.65 vs $0.47 \mathrm{ng} / \mathrm{ml}$ ), and IL-8 (248 vs $99 \mathrm{ng} / \mathrm{ml}$ ) were increased compared to survivors (all, $\mathrm{p}<0.05$ ).

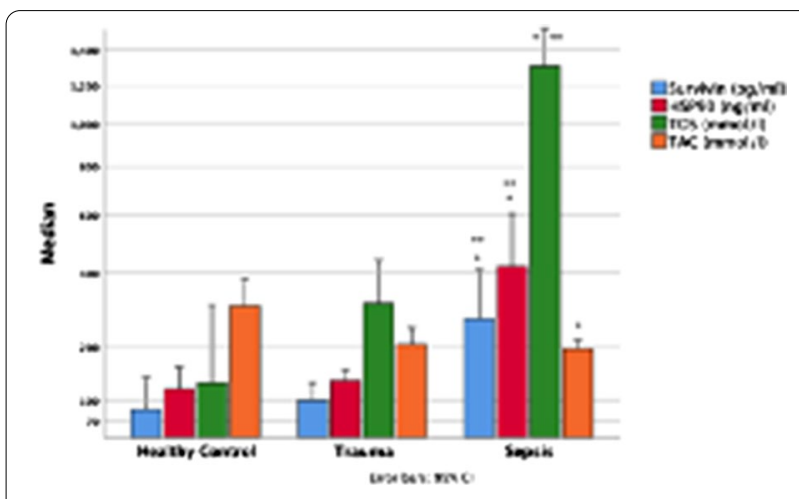

Fig. 1 Stars indicate significantly higher levels $(p<0.05)$ in pediatric sepsis compared to healthy controls* or trauma**

Conclusion. Antiapoptotic (survivin), inflammatory, innate immunity (HSP) and oxidative stress biomolecules are induced, and antioxidant capacity repressed, in pediatric sepsis. Early onset exaggeration of these responses is related to outcome.

\section{Reference(s)}

1. Cao, C., Yu, M. \& Chai, Y. Pathological alteration and therapeutic implications of sepsis-induced immune cell apoptosis. Cell Death Dis. 10, 782 (2019).

2. Altznauer, F. et al. Inflammation-associated cell cycle-independent block of apoptosis by survivin in terminally differentiated neutrophils. J. Exp. Med. 199, 1343-1354 (2004).

\section{6}

Non-invasive monitoring of pulmonary blood flow in mechanically ventilated adults

N. Fleming ${ }^{1}$; B. Cholley, ${ }^{2}$ N N. Ayoubi ${ }^{3}$; H. Bath, ${ }^{3}$; S. Bustin ${ }^{3}$

${ }^{1}$ Department of anesthesiology and pain medicine, UC Davis School of Medicine, Sacramento, United States of America; ${ }^{2}$ Department of anesthesia and intensive care medicine, Hôpital Européen Georges-Pompidou, AP-HP, Paris, France; ${ }^{3}$ Department of clinical research, Rostrum Medical Innovations Inc., Vancouver, Canada

Correspondence: N. Ayoubi

Intensive Care Medicine Experimental 2020, 9(1): 000666

Introduction. Parameters that can provide insight into ventilationperfusion matching can help clinicians understand underlying pathophysiological processes and guide individual patient care. Pulmonary Blood Flow (PBF) provides a measurement of blood flow participating in gas exchange. Monitoring of PBF, (cardiac output minus shunt fraction), can help optimize therapy in mechanically ventilated patients. The current Reference method to measure cardiac output, are based on thermodilution via a pulmonary artery catheter. We introduce a novel device-VQm Pulmonary Health Monitor $(\mathrm{PHM})^{\mathrm{TM}}-$ to measure PBF non-invasively and semi-continuously in mechanically-ventilated adults. 
Objectives. To evaluate measurements of PBF by means of a new, non-invasive cardio-pulmonary health monitor $\left(\mathrm{VQm} \mathrm{PHM}^{\mathrm{TM}}\right.$, Rostrum Medical Innovations Inc., Canada) compared to clinical gold standards. Methods. We conducted a non-interventional pilot study on mechanically ventilated cardiac patients undergoing cardiopulmonary bypass $(\mathrm{CPB})$ to measure PBF from the VQm PHM ${ }^{\mathrm{TM}}$ and the gold standard. The VQm PHM ${ }^{\mathrm{TM}}$ estimates PBF through a modified differential Fick equation and sequential gas delivery (SGD) technology by administrating 3 -breath inspiratory boluses of $\mathrm{CO}_{2}$ at an $\mathrm{FiCO}_{2}$ of $10 \%$. These measurements were compared to PBF obtained from intermittent bolus thermodilution cardiac output (TDCO) method and shunt fraction estimated from central venous and arterial blood gases with the use of the Berggren equation. The Berggren shunt was subtracted from the thermodilution derived cardiac output to calculate the Reference PBF (Berggren, 1942). Agreement between the VQm $\mathrm{PHM}^{\mathrm{TM}}$ and Reference PBF was assessed by Bland-Altman representation while the trending ability of the VQm PHM ${ }^{\mathrm{TM}}$ was assessed by a polar plot and a fourquadrant plot analysis.

Results. 13 patients, all male, age 69 (49-85) years, were included in this preliminary analysis. The mean difference between paired PBF values was $0.1 \mathrm{~L} / \mathrm{min}$ and the $95 \%$ limits of agreement were 1.3 and $-1.0 \mathrm{~L} / \mathrm{min}$ (Fig. 1A). The angular limits of agreement were $29^{\circ}$ and $-36^{\circ}$, with a bias of $-4^{\circ}$ (Fig. 1B) and a concordance rate of $90.3 \%$ was obtained (Fig. 1C), indicating comparable trending ability between VQm PHM ${ }^{\mathrm{TM}}$ and our Reference PBF. These preliminary results represent the first in-human data collected using a new, non-invasive cardiopulmonary health monitor.

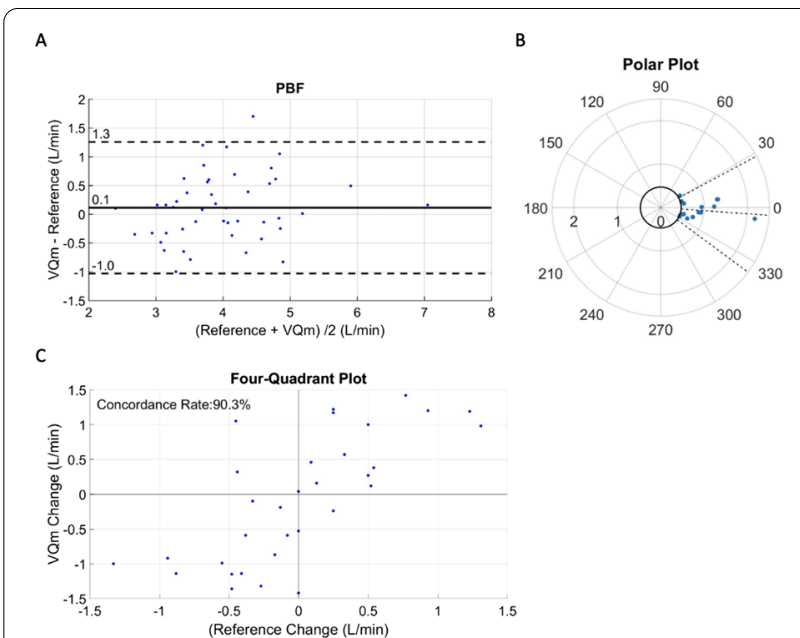

Fig. 1 Agreement between our Reference PBF (TDCO-Shunt) and values obtained using $\mathrm{VQm} \mathrm{PHM}^{\mathrm{TM}}$ (panel $\mathrm{A}$ ), changes between sequential PBF measurements obtained using VQm PHM ${ }^{\mathrm{TM}}$ PBF and our Reference PBF (panel B) and direction of change between VQm $\mathrm{PHM}^{\mathrm{TM}}$ PBF measurements and our Reference PBF (panel C) for 46 total measurements

Conclusion. Our preliminary results indicate both good trending and agreement between PBF measured using VQm and our Reference value calculated as TDCO-Shunt.

\section{Reference(s)}

1. This study was funded by Rostrum Medical Innovations Inc

2. Berggren SM. The oxygen deficit of arterial blood caused by non-ventilating parts of the lung. Acta Physiol Scand Suppl. 1942:4:1-92
000675

Positive reappraisal coping strategies among intensive care professionals in France in response to the COVID-19 pandemic A. Fournier ${ }^{1}$; V. Deltour ${ }^{2}$; F. Lheureux ${ }^{3}$; AL. Poujol ${ }^{4}$; F. Ecarnot ${ }^{5}$; N. Meunier-Beillard ${ }^{6}$; M. Loiseau ${ }^{7}$; C. Binquet ${ }^{8}$; JP. Quenot ${ }^{9}$; A. Laurent ${ }^{2}$

${ }^{1}$ Psychology, University of Burgundy, Dijon, France; ${ }^{2}$ Psy-drepi, University of Burgundy, Dijon, France; ${ }^{3}$ Psychologie, University of Franche-Comté, Besançon, France; ${ }^{4}$ Psychopathologies, Ecole de Psychologues Praticiens, Paris, France; ${ }^{5}$ Département de cardiologie, Centre hospitalier régional universitaire de Besançon, Besançon, France; ${ }^{6} \mathrm{Cic}$ 1432, clinical epidemiology, University of Burgundy, Dijon, France; ${ }^{7}$ Service de médecine légale, Hospital Center University François Mitterand, Dijon, France; ${ }^{8}$ Inserm, cic 1432, clinical epidemiology, Hospital Center University François Mitterand, Dijon, France; ${ }^{9}$ Medical intensive care unit, Chu Dijon, Dijon, France

Correspondence: A. Fournier

Intensive Care Medicine Experimental 2020, 9(1): 000675

Introduction. Before the epidemic, the working conditions in intensive care units (ICU) were already identified as stressful for professionals $(1,2)$. The COVID crisis added an additional degree of stress. Uncertainty, risk of being contaminated or contaminating others, a lack of personal protective equipment for personnel, a shortage of healthcare workers trained in intensive care, a lack of specific treatment for the infection, and the wide variability in the course of the disease contributed to increase stress perception. Finally, numerous ethical and moral dilemmas within the caregiving teams were raised.

Objectives. The objective of the PsyCOVID-ICU research team was to evaluate, in a French multicenter and cross-sectional survey, the effectiveness of coping strategies used by ICU professionals to deal with the first pandemic wave of COVID-19 in France.

Methods. From 22 April to 13 May 2020, online questionnaires were offered in intensive care units of 77 French hospitals. These questionnaires assessed perceived stress [adapted versions of the PS-ICU (3) and the Khalid scales (4)], use of coping strategies [Brief-COPE $(5,6)]$ and psychological distress [GHQ-12 (7)]. The intensity of the epidemic for each region was defined according to the criteria of the French public health agency.

Results. A total of 2,643 ICU professionals (physicians, residents, nurses, nurses' aides, medical students and nursing managers) participated in the study. More than half of the caregivers $(63.5 \%)$ suffered from psychological distress. The intensity of the epidemic had an indirect effect on psychological distress, via the perceived stress score $(b=0.23,95 \%$-confidence interval $[\mathrm{Cl}]=0.05,0.41$ ), and the use of positive reappraisal coping strategies decreased the relationship between perceived stress and symptom severity, $\mathrm{b}=-0.32,95 \%-\mathrm{Cl}=-0.54,-0.11$. None of the other coping strategies was found to have a moderating effect. Among the six specific stress dimensions measured by the stress scale, the effect of the epidemic intensity on mental health was mainly driven by dimension "Patient- and family-related emotional load" $(b=0.13,95 \%-C l=0.03$, 0.22 ) and dimension "Care provided in sub-optimal or conflictual condition", $\mathrm{b}=0.09,95 \%-\mathrm{Cl}=0.02,0.18$.

Conclusion. Professionals working in areas where the epidemic was of high intensity were more affected by stress and psychological distress, driven mainly by the emotional and ethical burden. Our study highlights the importance to target strategies focusing on positive thinking, notably in terms of positive leadership approaches by department chiefs and nursing managers, could help to mitigate the stress perceived by frontline healthcare workers in the epidemic context (8).

Reference(s)

1. Donchin Y, Seagull FJ. The hostile environment of the intensive care unit. Curr Opin Crit Care. 2002 Aug;8(4):316-20.

2. Embriaco N, Papazian L, Kentish-Barnes N, Pochard F, Azoulay E. Burnout syndrome among critical care healthcare workers. Curr Opin Crit Care. 2007 Oct;13(5):482-8

3. Laurent A, Fournier A, Lheureux F, Martin Delgado MC, Bocci MG, Prestifilippo A, et al. An international tool to measure perceived stressors in intensive care units: the PS-ICU scale. Annals of intensive care. Accepted 
4. Khalid I, Khalid TJ, Qabajah MR, Barnard AG, Qushmaq IA. Healthcare Workers Emotions, Perceived Stressors and Coping Strategies During a MERS-CoV Outbreak. Clin Med Res [Internet]. 2016 Mar 1;14(1):7-14.

5. Carver CS. You want to measure coping but your protocol's too long: Consider the Brief COPE. Int J Behav Med. 1997;4(1):91-100.

6. Baumstarck K, Alessandrini M, Hamidou Z, Auquier P, Leroy T, Boyer L. Assessment of coping: a new french four-factor structure of the brief COPE inventory. Health Qual Life Outcomes [Internet]. 2017 Jan 11;15(1):8.

7. Salama-Younes M, Montazeri A, Ismail A, Roncin C. Factor structure and internal consistency of the 12-item General Health Questionnaire (GHQ12) and the Subjective Vitality Scale (VS), and the relationship between them: a study from France. Health Qual Life Outcomes [Internet]. 2009; 7(1):22.

8. Rad A, Yarmohammadian M. A study of relationship between managers'leadership style and employees'job satisfaction. Leadersh Heal Serv [Internet]. 2006 Apr;19(2):11-28.

9. This study was funded by a grant (PHRC-COVID 2020) from the Programme Hospitalier de Recherche Clinique National, funded by the French Ministry of Health.

\section{7}

\section{Response to prone positioning in SARS-CoV2}

mechanically-ventilated patients

M. Combet'; A. Pavot, ${ }^{1}$; C. Lai' ${ }^{1}$; Q. Fossé ${ }^{1}$; S. Ayed, ${ }^{1}$; L. Jelinski ${ }^{1}$; F. Lardet' ${ }^{1}$;

N. Fage ${ }^{1}$; L. Guerin, ; N. Anguel, ${ }^{1}$; D. Osman, ; JL. Teboul ${ }^{1}$; X. Monnet';

T. Pham ${ }^{1}$

${ }^{1}$ Médecine intensive réanimation, Bicetre Hospital AP-HP, Le Kremlin-Bicê-

tre, France

Correspondence: M. Combet

Intensive Care Medicine Experimental 2020, 9(1): 000677

Introduction. Intensive care units worldwide are dealing with an overwhelming inflow of patients with SARS-CoV2-associated ARDS. It has been hypothesized [1] that, in SARS-CoV2 ARDS, two phenotypes exist: type L for low elastance, low shunt and low lung weight and type $\mathrm{H}$ for high elastance, high shunt and high lung weight. It has been suggested $[1,2]$ that these two phenotypes should be distinguished from one another because they could benefit from different management in ICU in terms of tidal volume, PEEP and prone positioning.

Objectives. We aimed to assess response to prone positioning in SARS-CoV2-patients and according to ARDS phenotype.

Methods. This is a single-center study in a French medical ICU.

We included patients receiving mechanical ventilation for SARS-CoV2ARDS and undergoing at least one proning session. Patients were categorized in the $\mathrm{L}$ or $\mathrm{H}$ groups according to their respiratory system compliance before the 1st proning session: patients with a respiratory compliance $\geq 40 \mathrm{~mL} / \mathrm{cmH} 2 \mathrm{O}$ were classified in the $\mathrm{L}$ group.

We considered patients to be prone responders if they verified at least one of the following criteria: an increase in $\mathrm{PaO} 2$ to $\mathrm{FiO} 2$ (PF) ratio $\geq 20 \mathrm{mmHg}$, a PF ratio increase $\geq 20 \%$ or a decrease in $\mathrm{PaCO} 2$ of at least $5 \mathrm{mmHg}$.

Results. Seventy patients were included in our study. Median age was $61 \pm 12$ years and median SOFA on admission was $6 \pm 4$. Overall mortality was $65 \%$.

Most patients (85\%) had low compliance $<40 \mathrm{~mL} / \mathrm{cmH} 2 \mathrm{O}$ (H group) before prone positioning. Respiratory characteristics before prone positioning and response to proning are shown in Table 1. Type $L$ patients were taller, and consequently received higher tidal volume. Tidal volume standardized with predicted body weight were similar in both groups.

Most patients (74\%) responded to proning according to at least one criterion. Patients with high compliance had higher initial $\mathrm{PaCO} 2$ and a non-statistically significant rate of patients having a $\mathrm{PaCO} 2$ decrease of $5 \mathrm{mmHg}$ or more.
Table 1 Respiratory parameters before and during prone positioning session

\begin{tabular}{|c|c|c|c|c|}
\hline & $\begin{array}{l}\text { All patients } \\
\mathrm{N}=70\end{array}$ & $\begin{array}{l}\text { L phenotype } \\
\mathrm{N}=10\end{array}$ & $\begin{array}{l}\mathrm{H} \text { phenotype } \\
\mathrm{N}=60\end{array}$ & p-value \\
\hline Height, $\mathrm{cm}$ & $172 \pm 8.98$ & $176 \pm 3.75$ & $171 \pm 9.39$ & 0.004 \\
\hline \multicolumn{5}{|l|}{ Before proning } \\
\hline $\mathrm{Vt}, \mathrm{mL}$ & $397 \pm 42$ & $425 \pm 34$ & $393 \pm 42$ & 0.019 \\
\hline Vt per $\mathrm{kg}$ of IBW, $\mathrm{mL} / \mathrm{kg}$ & $6.0 \pm 0.6$ & $5.9 \pm 0.4$ & $6.0 \pm 0.7$ & 0.544 \\
\hline Plateau pressure, $\mathrm{cmH} 2 \mathrm{O}$ & $27[26 ; 30]$ & $25[24 ; 26]$ & $28[26 ; 30]$ & 0.001 \\
\hline Driving pressure, $\mathrm{cmH} 2 \mathrm{O}$ & $13[11 ; 16]$ & $10[10 ; 10]$ & $14[12 ; 16]$ & $<0.001$ \\
\hline Compliance, $\mathrm{mL} / \mathrm{cmH} 2 \mathrm{O}$ & $31[25 ; 35]$ & $43[42 ; 45]$ & $30[24 ; 33]$ & $<0.001$ \\
\hline Arterial pH & $7.35 \pm 0.10$ & $7.23 \pm 0.14$ & $7.37 \pm 0.07$ & 0.016 \\
\hline $\mathrm{PCO} 2, \mathrm{mmHg}$ & $43 \pm 7$ & $47 \pm 8$ & $42 \pm 8$ & 0.089 \\
\hline $\mathrm{PF}, \mathrm{mmHg}$ & $108 \pm 30$ & $112 \pm 35$ & $108 \pm 29$ & 0.691 \\
\hline \multicolumn{5}{|l|}{ Response to proning } \\
\hline $\begin{array}{l}\Delta \text { Plateau pressure, } \\
\mathrm{cmH2O}\end{array}$ & $0[-2 ; 1]$ & $0[-2 ; 0]$ & $0[-2 ; 1]$ & 0.833 \\
\hline $\begin{array}{l}\Delta \text { Driving pressure, } \\
\mathrm{cmH} 2 \mathrm{O}\end{array}$ & $-2[-3 ; 0]$ & $0[-1 ; 0]$ & $-2[-3 ; 0]$ & 0.080 \\
\hline $\begin{array}{l}\Delta \text { Compliance, } \mathrm{mL} / \\
\mathrm{cmH} 2 \mathrm{O}\end{array}$ & $3.47[0 ; 8]$ & $5[-2 ; 11]$ & $3[0 ; 7]$ & 0.835 \\
\hline$\triangle \mathrm{PF}$ & $65 \pm 77$ & $56 \pm 79$ & $66 \pm 77$ & 0.707 \\
\hline $\begin{array}{l}\text { PPF }>20 \mathrm{mmHg} \text { or } 20 \%, \\
\mathrm{~N}(\%)\end{array}$ & $48(70)$ & $6(60)$ & $42(71)$ & 0.479 \\
\hline$\searrow \mathrm{PCO} 2>5 \mathrm{mmHg}, \mathrm{n}(\%)$ & $13(19)$ & $4(40)$ & $9(15)$ & 0.085 \\
\hline Prone responder, $\mathrm{n}(\%)$ & $51(74)$ & $8(80)$ & $43(73)$ & 1.000 \\
\hline
\end{tabular}

Conclusion. Most patients with SARS-COV2-associated ARDS presented altered compliance and responded to proning, regardless of their initial respiratory system compliance. Further research is needed to better understand the determinants of $\mathrm{L}$ and $\mathrm{H}$ phenotypes and their response to prone positioning in terms of $\mathrm{PCO} 2$ reduction.

\section{Reference(s)}

1. Gattinoni L, Chiumello D, Rossi S (2020) Covid 19 pneumonia: ARDS or not? Critical Car

2. Fan E., Beitler J, Brochard L et al. (2020) COVID-19-associated acute respiratory distress syndrome: is a different approach to management warranted? Lancet Respir Med

\section{2}

Burn out syndrome in ICU workers amidst covid-19 pandemic B. Gimenez Beltrán ${ }^{1}$; B. Diez Del Corral'; M. Perez Ruiz'; A. Breval Flores'; J. Flores Azofra²; A. Estella García

${ }^{1}$ University hospital of jerez., Intensive care unit, Jerez, Spain; ${ }^{2} \mathrm{Gp}$, University Hospital of Puerto Real, Puerto Real, Spain

Correspondence: B. Gimenez Beltrán

Intensive Care Medicine Experimental 2020, 9(1): 000692

Introduction. The covid-19 pandemic, which has been affecting for mores than a year, has taken its toll in the mental health of multiple health workers, specially those in the ICU.

Objectives. The aim of the present study is to describe the incidence of burn-out syndrome and anxiety/depression within the ICU workers during the covid-19 pandemic in a hospital which ICU material beds have doubled.

Methods. This is an observational, cross-sectional study. All of the health workers, currently working in an adult Intensive Care Unit in a 
Regional Hospital, were invited to participate through an anonymous online questionnaire, after the first 10 month of the pandemic. We've used two validated tests: Maslach Burnout Inventory-MBI for the burn-out syndrome diagnostic and the Hospital Anxiety and Depression Scale-HADS for the screening of anxiety and depression, as well as asked for demographic data. Statistical analysis: Data was analyzed by SPSS v22.

Results. A total of 166 workers were invited to participate obtaining a feed-back response of the $52.4 \%$ (87 after two weeks in December 2020. Of the participants, the $55.2 \%$ (48) were Nurses, $19.5 \%$ (17) Care Assistants, 10.3\% (9) Hospital Porters, 10.3\% (9) Attending physicians and $4.6 \%$ (4) of Resident physicians. Of all of them the $72.4 \%$ (63) were women with a mean age of $43.2 \pm 10.9$ years and the $27.6 \%$ (24) were men with mean age of $46.1 \pm 8.7$ years.

Of the 87 participants, $24.1 \%$ (21) present all the criteria that defines the Burn-out syndrome and the $52.9 \%$ (46) present only some of them-being classified as "potential case"-. Out of the professionals with all the criteria defining the syndrome, the $71.4 \%(15)$ were nurses, followed by the Attending physicians 19\% (4) and the Care assistants $9.5 \%$ (2). Health workers that had been working within the ICU for more than 10 years were the sub-group with more prevalence of burn-out syndrome, $47.6 \%$ (10), and the sub-group of workers that started working within the pandemic i.e., less than a year working in ICU, 33.33\% (7).

Furthermore, a total of $53 \%$ (46) participants were considered "potential cases" because the presented some of the characteristic of the syndrome described in the $\mathrm{MBl}$, especially frequent the "emotional exhaustion" subscale, being in high levels in $50 \%$ (43) of the participants.

Regarding the HADS, 48.3\% (42) of the asked workers present clinically diagnosed Anxiety, with higher prevalence within the Nurses and Care assistants-54.7\% (23) and 24\% (1) respectively -. It's worth mentioning that the total of respondent Resident doctors had Anxiety. In the depression scale, we have detected 9 cases (10.3\%)-4 within the Nurses, 3 within Care Assistants and 2 within Attending physicians Conclusion.

- The incidence of the Burn-out syndrome was higher than previously considered, being affected 1 in 4 ICU workers.

- The "emotional exhaustion" subscale in the MBI was in high rank in most of the respondents.

- Half of the questioned professionals had anxiety criteria and 1 in 10 depression criteria, both of them being more prevalent within the Nurses.

\section{6}

\section{Differences in resting energy expenditure among critically ill intubated COVID-19 patients according to age group}

D. Karayiannis ${ }^{1}$; A. Maragkouti ${ }^{2}$;. Mikropoulos ${ }^{2}$; A. Sarri' ${ }^{2}$; A. Kanavou ${ }^{3}$;

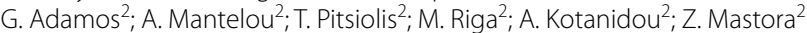

${ }^{1}$ Clinical nutrition, Evaggelismos, Athina, Greece; ${ }^{2} 1$ st department of critical care medicine \& pulmonary services, Evangelismos Hospital, Medical School, National and Kapodistrian University of Athens, Athens, Greece; ${ }^{3}$ First department of critical care medicine and pulmonary services, Evaggelismos, Athina, Greece

Correspondence: D. Karayiannis

Intensive Care Medicine Experimental 2020, 9(1): 000716

Introduction. Although there are some data published regarding resting energy expenditure (REE) of critically ill intubated patients with Covid-19 $(1,2)$ the effects of various factors that exert influence on REE such as age have not been clarified yet.
Objectives. This study described REE of intubated patients with COVID-19 during their first 14 days of hospitalization and explores whether patients' age group ( $>65$ vs $<65$ years) is an independent factor in determining energy needs.

Methods. Resting energy expenditure (REE) values were evaluated among critically ill intubated COVID 19 patients until 14th day of intensive care unit stay (ICU-S) by indirect calorimetry, using a medical device to determine energy needs (Cosmed Q NRG +). All measurements were performed according to guidelines from a task force of medical experts from the European Society of Intensive Care Medicine in the international calorimetry study initiative (ICALIC) project $(3,4)$. Data normality was assessed using the Shapiro Wilk test. REE values were expressed as median values (interquartile range, IQR). Mann Whitney $\mathrm{U}$ test was used to for comparison of REE values between according to age group, at certain time points (days), while generalized linear models were used to test associations between REE and patients' age group.

Results. 21 adult COVID-19 patients $(54.2 \%$ male, $31.4 \%$ obese, $51.7 \%>65$ years) requiring mechanical ventilation were assessed prospectively. REE was negatively and significantly associated with age (Spearman $\mathrm{rho}=-0,28)$, indicating a gradual decrease of energy needs according to age group. Individuals in the age group $>65$ years had lower median REE values compared to those in the age group $<65$ years, during their 7 th ( $1731 \mathrm{vs} 1950 \mathrm{kcal} /$ day, $\mathrm{p}=0.077)$ and 14th day of hospitalization, respectively ( $1910 \mathrm{vs} 2350 \mathrm{kcal} /$ day, $\mathrm{p}=0.011$ ). After adjusting for comorbidities, gender, type of nutrition support (enteral vs parenteral nutrition) and neuromuscular blockade administartion (yes or no), people $<65$ years of age were significantly more likely to experience increased energy expenditure ( $>30 \mathrm{kcal} / \mathrm{kgr}$ body weight) compared to those $>65$ years of age $(R R=1.52,1.22-1.83, p<0.001)$.

Conclusion. In this project, we identified the energy needs of critical ill COVID-19 intubate patients until 14 th day of intensive care unit stay and highlighted that age seems to be an important determinant of resting energy expenditure values.

\section{Reference(s)}

1. Singer P, Pichard C, De Waele E. Practical guidance for the use of indirect calorimetry during COVID 19 pandemic. Clin Nutr Exp. 2020;33:18-23

2. Oshima T, Delsoglio M, Dupertuis YM, Singer P, De Waele E, Veraar C, et al. The clinical evaluation of the new indirect calorimeter developed by the ICALIC project. Clin Nutr. 2020

3. Yu PJ, Cassiere H, DeRosa S, Bocchieri K, Yar S, Hartman A. Hypermetabolism and Coronavirus Disease 2019. JPEN J Parenter Enteral Nutr. 2020;44:1234-

4. Whittle J, Molinger J, MacLeod D, Haines K, Wischmeyer PE. Persistent hypermetabolism and longitudinal energy expenditure in critically ill patients with COVID-19. Crit Care. 2020;24:581.

000738

The effect of endothelial dysfunction on liberation from mechanical ventilation-a cohort study

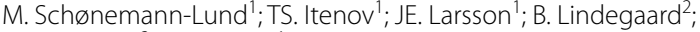

P. Johansson ${ }^{3}$; MH. Bestle

${ }^{1}$ Department of anaesthesiology and intensive care, Copenhagen University Hospital-North Zealand, Copenhagen, Denmark; ${ }^{2}$ Department of respiratory medicine and infectious diseases, Copenhagen University Hospital—North Zealand, Copenhagen, Denmark; ${ }^{3}$ Transfusion medicine, Copenhagen University Hospital, Rigshospitalet, København,

Denmark

Correspondence: M. Schønemann-Lund

Intensive Care Medicine Experimental 2020, 9(1): 000738 
Introduction. Endothelial damage is associated with worse prognosis and organ failure in sepsis, trauma and acute myocardial infarction (1, 2). How endothelial damage relates to patient-important outcomes in acute respiratory failure is undetermined.

Objectives. To determine if higher levels of Syndecan-1 $(3,4)$, soluble Thrombomodulin (STM) $(5,6)$ and Platelet Endothelial Cell Adhesion Molecule-1 $(\operatorname{PECAM}-1)(7,8)$ are associated with lower rates of liberation from mechanical ventilation (LFMV) or all-cause mortality.

Methods. From a prospective, single-center, cohort study, we included patients receiving mechanical ventilation on the 1st day in the intensive care unit and followed them for 30 days. We grouped patients in to three groups according to quartiles of Syndecan-1, STM and PECAM- 1 levels respectively (Group $1=1$ st quartile, Group $2=2$ nd $\& 3$ rd quartile, Group $3=4$ th quartile). We analyzed the rate of LFMV along with the rate of dying prior to LFMV using Cox-regression and report the results as adjusted cause-specific hazard rates (HR) with $95 \%$ confidence intervals $(95 \% \mathrm{Cl})(9)$. We adjusted for gender, age, a history of chronic obstructive pulmonary disease, septic shock, a history of heart failure, $\mathrm{PaO} 2 / \mathrm{FiO} 2$-ratio, respiratory infection, acute kidney injury and bilirubin. The rate of death from any cause was analyzed with Cox-regression and controlled for the same variables as the primary analysis.

Results. Of 459 patients, 309 were liberated from mechanical ventilation and 98 died before LFMV. 163 patients died within 30 days. sTM (HR 0.53 for 4 th vs. 1st quartile [95\% confidence interval (Cl) $0.35-0.78, p<0.01])$ was associated with a lower rate of LFMV within 30 days (Fig. 1). Syndecan-1 (HR 1.10 for 4 th vs. 1st quartile $[95 \% \mathrm{Cl}$ $0.77-1.57, p=0.60]$ ) and PECAM-1 (HR 0.86 for 4th vs. 1st quartile [95\% Cl 0.60-1.22, $\mathrm{p}=0.39]$ ) were not (Fig. 1). Nevertheless, patients with a PECAM-1 in the 4th quartile had a lower rate of LFMV within the first 5 days of ICU admission (HR 0.52 for 4 th vs. 1st quartile [95\% $\mathrm{Cl} 0.32-0.84, \mathrm{p}=0.01]$ ). For sTM (HR 0.37 for 4 th vs. 1st quartile $[95 \% \mathrm{Cl} 0.22-0.63, \mathrm{p}<0.01])$ the effect was also largest during the first 5 days, whereas for Syndecan-1 (HR 0.94 for 4th vs. 1st quartile $[95 \% \mathrm{Cl} 0.60-1.48, \mathrm{p}=0.8]$ ) there was still no effect. Syndecan-1 and PECAM-1 were associated with the rate of dying prior to LFMV (Fig. 1). All three biomarkers were associated with 30-day all-cause mortality (Fig. 1).

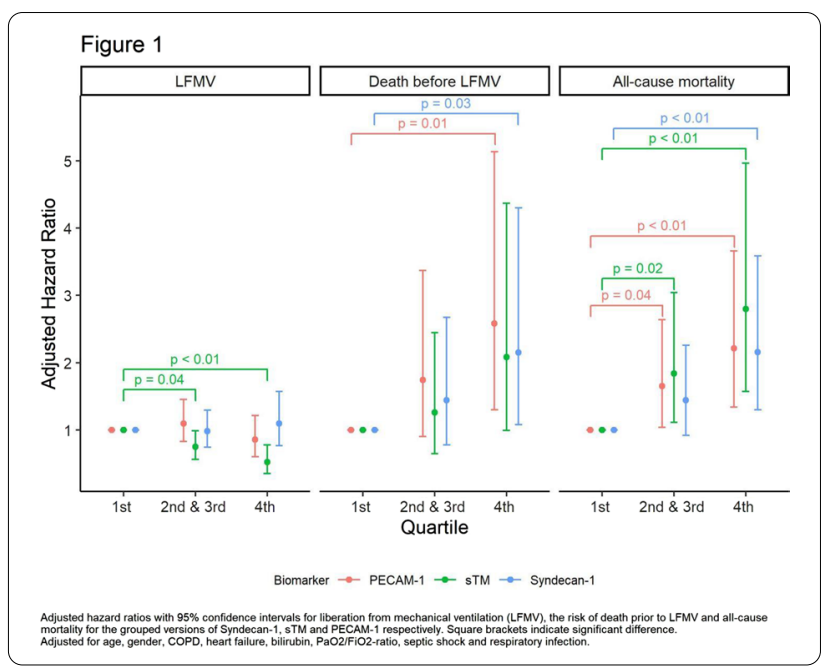

Conclusion. In acute respiratory failure, endothelial damage is associated with both a lower rate of liberation from mechanical ventilation and higher all-cause mortality. The results suggest that endothelial damage is an important factor behind the development of acute respiratory failure.

\section{Reference(s)}

1. Johansson PI, Stensballe J, Ostrowski SR. Shock induced endotheliopathy (SHINE) in acute critical illness - a unifying pathophysiologic mechanism. Crit Care 2017;21:1-7.

2. Johansen ME, Johansson PI, Ostrowski SR, Bestle MH, Hein L, Jensen ALG, Søe-Jensen P, Andersen MH, Steensen M, Mohr T, Thormar K, Lundgren B, Cozzi-Lepri A, Lundgren JD, Jensen J-U. Profound Endothelial Damage Predicts Impending Organ Failure and Death in Sepsis. Semin Thromb Hemost 2015:41:16-25.

3. Hahn RG, Patel V, Dull RO. Human glycocalyx shedding: Systematic review and critical appraisal. Acta Anaesthesiol Scand 2021;aas.13797.10.1111/ aas.13797

4. Jedlicka J, Becker BF, Chappell D. Endothelial Glycocalyx. Crit Care Clin 2020;36:217-232.

5. Thrombomodulin (IPR016316)—InterPro entry —InterPro. at < http:// www.ebi.ac.uk/interpro/entry/InterPro/IPR016316/>

6. Ishii H, Majerus PW. Thrombomodulin is present in human plasma and urine. J Clin Invest 1985:76:2178-2181.

7. Villar J, Zhang H, Slutsky AS. Lung Repair and Regeneration in ARDS Chest 2019:155:587-594.

8. Woodfin A, Voisin M-B, Nourshargh S. PECAM-1: A Multi-Functional Molecule in Inflammation and Vascular Biology. Arterioscler Thromb Vasc Biol 2007:27:2514-2523.

9. Andersen PK, Geskus RB, de Witte T, Putter H. Competing risks in epidemiology: possibilities and pitfalls. Int J Epidemiol 2012;41:861-870.

10. Innovation Fund Denmark

11. Hindsgavl Symposium

12. Research Foundation at North Zealands Hospital

13. AP Møller Foundation

\section{9}

Standardised nursing handover in the centre for intensive care of the North Estonia Medical Centre Foundation

R. Kaljurand ${ }^{1}$; K. Zirel ${ }^{2}$

${ }^{1}$ Intensive care unit, North Estonia Medical Centre Foundation, Tallinn, Estonia; ${ }^{2}$ Director of nursing, North Estonia Medical Centre Foundation, Tallinn, Estonia

Correspondence: $\mathrm{R}$. Kaljurand

Intensive Care Medicine Experimental 2020, 9(1): 000749

Introduction. The North Estonia Medical Centre Foundation is one of the leading health care institutions in Estonia and a driver of innovation in the health sector. The aim of the organisation is to raise patient safety to an important place in all activities (4). International studies indicate a lack of a systemic structure for nursing handover (1). The data of the Joint Commission International $(\mathrm{JCl})$, indicates that deficient nursing handovers account for $80 \%$ of all adverse events (2) and only $8 \%$ of health care higher education institutions teach nurses, official nursing handover (3). The $\mathrm{JCl}$ has created quality requirements for ensuring the safety of the patient (2), based on which different quality standards (1).

Objectives. Development and application of a standardised nursing handover for the nurses of the Centre of Intensive Care of the North Estonia Medical Centre Foundation.

Methods. The aim was achieved by using a qualitative research method. The theoretical framework for the development project was created by describing evidence based scientific articles relevant to the field, written in English, which provided an overview of standardised nursing handover, as well as the factors affecting the nursing handover. The research was based on the model of change of Burke and Litwin, which described the activities of the planning and implementation process of changing an organisation. An observational study was used as the data collection method, during the period 09.07.2020-23.07.2020, for mapping the bottlenecks in the department III of the Centre of Intensive Care of the North Estonia Medical Centre Foundation. 
Results. The analysis of the data of the observational study revealed the bottlenecks of the nursing handover. No information was forwarded concerning the need for isolation and occurrence of allergies in $97 \%$ of the cases, there was a complete lack of identifying patients, according to the requirements. $17 \%$ of nurses forwarded information concerning the close ones of the patient. A checklist in Estonian was compiled called STOP-Situation (Situatsioon), Background Information (Taustinformatsioon), Situation Evaluation (Olukorra hindamine), Day Plan (Päevaplaan), suitable to the intensive care department profile, relying on the the SBAR standard (Situation, Background, Assessment, Recommendations).

Conclusion. The standardised checklist STOP, reflects the condition of the patient as a whole, by organ systems. It has a systematic structure, providing informative insights and reducing the probability of error occurrence. Universities and hospitals must invest in the training activities of health care employees, in order to ensure a quality nursing handover. The confirming of the teaching of nursing handover into the curriculum of health care of the university, allows students to develop and practice required communication skills, to be better prepared for their future roles. Increasing awareness gives nurses a greater professional satisfaction and feeling of security, through which are improved team work and patient centred nursing care.

The standardisation of nursing handover, together with the application of a checklist STOP in an electronic environment, gives a better possibility for verbally forwarding more complex information, reduces handover mistakes connected with unreadable handwriting and increases the quality of nursing assistance.

\section{Reference(s)}

1. The North Estonia Medical Centre consolidated annual report, 2019

2. Methangkool, E., Tollinche, L., Sparling, J., \& Agarwala, A. V. (2019). Communication: Is There a Standard Handover Technique to Transfer Patient Care. International Anesthesiology Clinics, 57(3), 35-47.

3. JCl. (2018). Communicating Clearly and Effectively to Patients How to Overcome Common Communication Challenges in Health Care. 11. Retrieved from http://www.jointcommissioninternational.org

4. Eggins, S., \& Slade, D. (2015). Communication in clinical handover: improving the safety and quality of the patient experience. Journal of Public Health Research, 4(3), 197-199.

\section{6}

\section{Sphingosine 1-phosphate (S1P) mediates endothelial} permeability and organ failure in trauma. A translational study DP. van den Brink'; RB. Van Amstel' ; J. Vos ${ }^{1}$; NC. Weber ${ }^{2}$; DJ. Kleinveld'; NP. Juffermans ${ }^{1}$

${ }^{1}$ Department of intensive care medicine, Amsterdam UMC, locatie AMC, Amsterdam, Netherlands; ${ }^{2}$ Laboratory of experimental intensive care and anesthesiology, Amsterdam UMC, locatie AMC, Amsterdam, Netherlands

Correspondence: D.P. van den Brink

Intensive Care Medicine Experimental 2020, 9(1): 000776

Introduction. Trauma-induced shock is characterized by endothelial activation, leading to increased endothelial permeability, resulting in edema formation and contributing to multiple organ failure (MOF). The underlying mechanisms are not entirely understood. Sphingosine-1-phosphate (S1P) is a signaling lipid involved in maintaining endothelial barrier function. We hypothesized that S1P levels are reduced in severely injured patients with organ failure and that augmenting S1P reduces endothelial permeability.
Objectives. To investigate the role of S1P in trauma-induced organ failure by measuring S1P levels in trauma patients and to investigate the effects of S1P on thrombin induced endothelial permeability in vitro.

Methods. From 23 severely injured trauma patients who developed MOF within $48 \mathrm{~h}$ (defined as a SOFA score $\geq 6$ ), blood was sampled at emergency department admission, 1 day, and 3 days after trauma. Plasma S1P concentrations were measured by ELISA. For cell studies, human microvascular lung endothelial cells were seeded on transwells and stimulated with thrombin $(15 \mathrm{IU} / \mathrm{mL})$ or vehicle. Cells were incubated with S1P $(1 \mu \mathrm{M})$, fingolimod (FTY720 $=$ S1P agonist) $(1 \mu \mathrm{M})$ or W146 (S1P antagonist) (1 $\mu \mathrm{M})$. In the upper compartment, FITC-dex$\operatorname{tran}(70 \mathrm{kDa}, 5 \mathrm{mg} / \mathrm{mL}$ ) was added. To assess endothelial permeability, FITC-dextran leakage to the lower compartment was measured 3 and $6 \mathrm{~h}$ later.

Results. The median age of the patients was 62 (IQR 54-67) of which $78.3 \%$ were male. Median injury severity score was 29 (25-36), ICU length of stay was 10 days (4-18) and 28-day mortality was $17.4 \%$. S1P levels decreased significantly in the first day after trauma $(1.77 \mu \mathrm{M}$ $(0.50)$ vs $1.57(0.45) \mu \mathrm{M}, \mathrm{p}=0.043)$ and showed a trend towards recovery 3 days after trauma (1.57 (0.45) $\mu \mathrm{M}$ vs $1.71(0.49), p=0.056)$ In vitro, supplementation with S1P in thrombin stimulated endothelial cells reduced FITC-dextran leakage compared to thrombin only (165.52 $\mu \mathrm{g} / \mathrm{mL}$ (14.88) vs $123.42 \mu \mathrm{g} / \mathrm{mL}$ (32.33) $\mathrm{p}=0.014)$. Similarly, fingolimod $(237.25 \mu \mathrm{g} / \mathrm{mL}$ (57.85) vs $155.12 \mu \mathrm{g} / \mathrm{mL}$ (27.66), $\mathrm{p}<0.001)$ reduced endothelial permeability while incubation with W146 showed no difference $3 \mathrm{~h}$ after thrombin stimulus $(187.04 \mu \mathrm{g} / \mathrm{mL}$ (28.76) vs $195.91 \mu \mathrm{g} / \mathrm{mL}$ (49.23), $\mathrm{p}=1.000$ ).

Conclusion. S1P levels in trauma patients developing MOF decreases in the first $24 \mathrm{~h}$ after injury. Augmenting S1P availability improved endothelial barrier function in vitro. Therefore, S1P may play a role, at least partly, in mitigating endothelial permeability and subsequent progression of MOF. Further research should delineate whether S1P is a novel therapeutic target to reduce trauma induced organ failure.

Fig. 1. Effects of S1P (A) and FTY720 (B) on thrombin induced permeability in an in vitro transwell model
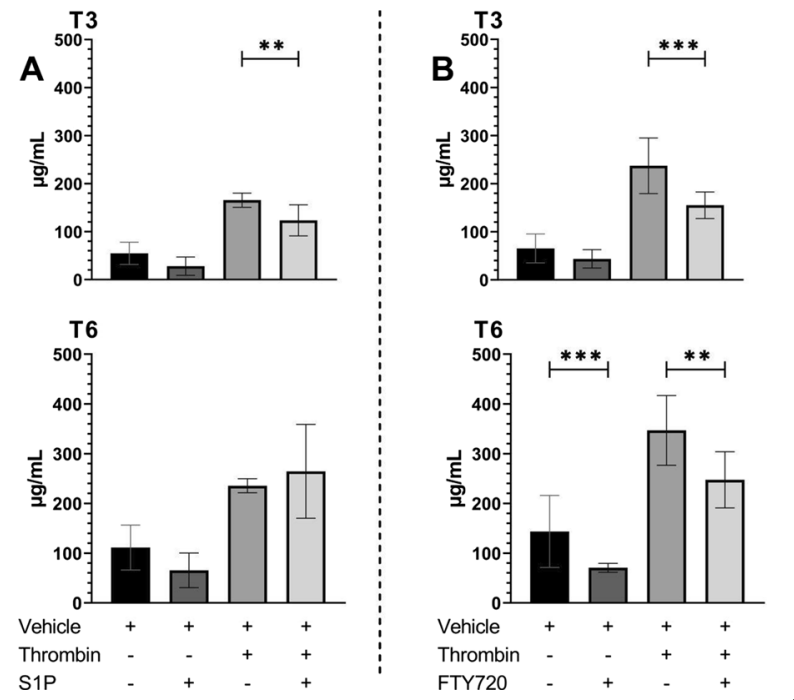
000787

Evaluation of guideline adherence during preoperative evaluation for elective surgery using an electronic decision support system

F. Biegert'; D. Andonov' ; B. Ulm¹'; M. Blobner ${ }^{1}$; B. Jungwirth²;

S. Kagerbauer ${ }^{1}$

${ }^{1}$ Department of anaesthesiology and intensive care, Klinikum rechts der Isar der Technischen Universität München, München, Germany; ${ }^{2}$ Department of anaesthesiology and intensive care, University Hospital Of Ulm, Ulm, Germany

Correspondence: F. Biegert

Intensive Care Medicine Experimental 2020, 9(1): 000787

Introduction. Different international and national guidelines exist for evaluating patients undergoing elective surgery. There is a wide consensus that proper implementation of clinical guidelines can significantly improve patient outcome. However, few clinical studies exist to assess how well these recommendations are followed in day-to-day practice. Past surveys have indicated a generally poor adherence to current guidelines $[1,2]$. Here, we present the results of a retrospective study to evaluate the preoperative management of 2000 patients that underwent elective surgery as part of an ongoing project to implement an electronic decision support system (DSS).

Methods. After ethical approval (ethics committee number 450/20 S-EB, ClinicalTrials.gov ID NCT04843202), a total of 2000 patients who underwent elective non-cardiac surgery between June 2018 and June 2020 were randomly selected and their data extracted from the electronic premedication protocol for retrospective evaluation. Eligibility criteria included an age of 18 or older, as well as a planned surgery classified as elective. The patient population consisted of $57.1 \%(n=1142)$ male and $42.9 \%(n=858)$ female patients. Mean age was $55.4(S D=17.8)$, mean $B M I$ was $26.3(S D=4.9)$, proportions of patients' ASA-scores I through IV were $21.8 \%(n=435)$, $44.8 \%(n=895), 32.5 \%(n=651)$ and $0.9 \%(n=19)$ respectively. The attained data from each premedication questionnaire was entered into a newly developed software which then presented the appropriate guideline recommendations applicable for the particular case. Included in the tool at the time of the study were the current guidelines of the European Society of Anaesthesiology (ESA) $[3,4]$ and of the German Society for Anaesthesiology and Intensive Care Medicine (DGAI) [5]. By comparing the original premedication documentation with the outputs of the software, guideline compliance was assessed. For evaluation, guideline recommendations were grouped together according to the aim of the specific recommendation, for example comments that advised for an ECG to be performed, resulting in 17 subgroups.

Results. In total, 9774 individual recommendations occurred of which around $81 \%(n=7955)$ originated from ESA guidelines. $46.5 \%$ of all recommendations were correctly followed with the overall rate of adherence being $44.9 \%$ in ESA and $53.5 \%$ in DGAI recommendations. Among ESA comments, grade 1 recommendations had a better rate of adherence than grade 2 comments ( $64.1 \%$ vs. $38.5 \%)$. When grouping together recommendations that target the same procedures, the largest ones were those for renal values (17.8\%), ECG (16.0\%) and haemoglobin (11.6\%). Groups with the best rate of adherence were imaging stress testing $(86.1 \%, 62 / 72)$, ECG $(78.2 \%, 1221 / 1562)$ and haemoglobin $(71.3 \%, 811 / 1138)$. Worst adherence was observed in coronary angiography $(0 \%, 0 / 15)$, spirometry $(4.2 \%, 15 / 353)$ and oximetry $(11.4 \%, 49 / 428)$ groups. In the patient population, better adherence rates were associated with a higher ASA score $(24 \%$ vs. $41 \%$ vs. $52 \%$ vs. $66 \%$ ), a lower $\mathrm{BMI}(54 \%$ in $\mathrm{BMI}<30$ vs. $36 \%$ in $\mathrm{BMI}>30)$, a higher risk surgery (46\% in low and medium risk surgery vs. $61 \%$ in high-risk surgery) and a higher age ( $35 \%$ in patients $<65 \mathrm{y} / \mathrm{o}$ vs. $56 \%$ in patients $>65 \mathrm{y} / \mathrm{o}$ ). No significant differences were observed between male and female patients, smokers and non-smokers and alcoholics vs. non-alcoholics.

Conclusion. With around every second recommendation being followed, overall guideline adherence is consistent with self-assessment of anesthesiologists in former studies. However one possible reason for weak guideline adherence in our study could be due to the retrospective study design. A personal discussion with the patient, the content of which was not fully documented on the premedication sheet, may have rendered one or another guideline recommendation obsolete. In general, guidelines published by the DGAI had a better adherence than ESA guidelines. Probable contributing factors are national guidelines tending to be more commonly known due to the familiar language, as well as the fact that the German publication compared with latest European releases relies more on graphs and tables rather than text, which improves readability. Wether implementation of a DSS improves adherence or not, as well as how high dependency might be, is subject to evaluation through an ongoing prospective study and remains to be shown.

\section{Reference(s)}

1. The project was funded by the Central Innovation Program for small and medium-sized enterprises of the German Federal Ministry for Economic Affairs and Energy (ZF4544902TS8) as a joint project between TUM and HIM (Health Information Management GmbH, Bad Homburg, Germany) acting as cooperation partners.

2. Schweizer R, Godet G, Petit PY, et al. Adherence of French cardiologists to guidelines for non-cardiac surgery. Anaesth Crit Care Pain Med. 2016;35(4):249-253. 10.1016/j.accpm.2015.12.009

3. Koers L, Schlack WS, Hollmann MW, de Hert S, Preckel B. European implementation of the "2014 ESC/ESA guideline on non-cardiac surgery: cardiovascular assessment and management". Minerva Anestesiol. 2017:83(5):457-464. 10.23736/S0375-9393.16.11504-4

4. De Hert S, Staender S, Fritsch G, et al. Pre-operative evaluation of adults undergoing elective noncardiac surgery: Updated guideline from the European Society of Anaesthesiology. Eur J Anaesthesiol. 2018;35(6):407465. 10.1097/EJA.0000000000000817

5. Kristensen SD, Knuuti J, Saraste A, et al. 2014 ESC/ESA Guidelines on noncardiac surgery: cardiovascular assessment and management: The Joint Task Force on non-cardiac surgery: cardiovascular assessment and management of the European Society of Cardiology (ESC) and the European Society of Anaesthesiology (ESA). Eur Heart J. 2014;35(35):2383-2431. 10. 1093/eurheartj/ehu282

6. Präoperative Evaluation erwachsener Patienten vor elektiven, nicht herz-thoraxchirurgischen Eingriffen. Gemeinsame Empfehlung der DGAI, DGCH und DGIM. Anästh Intensivmed 2017;58:349-364. https://doi.org/ 10.19224/ai2017.349

\section{2}

Lactate is associated with mortality in very old intensive care patients suffering from COVID-19: results from a observational study of 2860 patients

RR. Bruno'; B. Wernly; H. Flaaten ${ }^{3}$; J. Fjølner ${ }^{4}$; A. Artigas ${ }^{5}$; B. Bollen Pinto ${ }^{6}$; S. Joerg ${ }^{7}$; S. Binneboessel ${ }^{1}$; PH. Baldia ${ }^{1} ;$ M. Kelm ${ }^{1}$; M. Beil ${ }^{8}$; S. Sigal ${ }^{9}$;

P. Vernon Van Heerden ${ }^{10}$; W. Szczeklik ${ }^{11}$; J. Wollborn ${ }^{12}$; MJA. Banzo ${ }^{13 .}$ S. Leaver ${ }^{14}$; D. De Lange ${ }^{15} ;$ B. Guidet ${ }^{16}$

${ }^{1}$ Cardiology, Pulmonary Diseases, Vascular Medicine, Universität Düsseldorf, Düsseldorf, Germany; ${ }^{2}$ Department of department of anaesthesiology, perioperative medicine and intensive care medicie, Para- 
celsus Medical University, Salzburg, Austria; ${ }^{3}$ Department of anaestesia and intensive care, University of Bergen, Bergen, Norway; ${ }^{4}$ Department of intensive care, Aarhus University Hospital, Aarhus, Denmark; ${ }^{5}$ Department of intensive care medicine, Corporacion Sanitaria Universitaria Parc Tauli, Barcelona, Spain; ${ }^{6}$ Anesthesiology, Hôpitaux Universitaires de Genève (HUG), Genève, Switzerland; ${ }^{7}$ Medical surgical intensive care unit, University Hospital of Bern, Bern, Switzerland; ${ }^{8}$ Medical intensive care unit, Hadassah Medical Center, London, United Kingdom; ${ }^{9}$ Medical intensive care unit, Hadassah Medical Center, Jerusalem, Israel, Israel; ${ }^{10}$ Department of anesthesiology, critical care and pain medicine, Hadassah Medical Center, The Hebrew University of Jerusalem, Jerusalem, Israel; ${ }^{11}$ Intensive care and perioperative medicine, Jagiellonian University Medical College, Kraków, Poland; ${ }^{12}$ Critical care, Harvard Medical School, Boston, United States of America; ${ }^{13} \mathrm{Icu}$, Hospital Clinico Universitario Lozano Blesa, Zaragoza, Spain; ${ }^{14}$ Research lead critical care directorate, St George's Hospital, London, United Kingdom; ${ }^{15}$ Department of intensive care medicine, Utrecht University, Utrecht, Netherlands; ${ }^{16}$ Réanimation Médicale, Hôpital Saint-Antoine, Paris, France

Correspondence: R.R. Bruno

Intensive Care Medicine Experimental 2020, 9(1): 000792

Introduction. Lactate is an established prognosticator in critical care. However, there still is insufficient knowledge about its role in predicting outcome in COVID-19. This especially concerns older patients who have been affected most by that condition during the initial surge in 2020.

Methods. This prospective international observation study (The COVIP study) recruited ICU 2860 patients aged 70 years or older (ClinicalTRials.gov ID: NCT04321265) 70 years and older admitted with COVID-19 disease from March 2020 to February 2021. In addition to serial lactate values (arterial blood gas analysis), we have recorded an electronic database with several parameters including SOFA score, ICU procedures, limitation of care, length of stay in the ICU, and ICU mortality. A lactate concentration $\geq 2.0 \mathrm{mmol} / \mathrm{L}$ at baseline as abnormal.

Results. In total 2860 patients were analysed. In most patients (68\%), serum lactate was less than $2 \mathrm{mmol} / \mathrm{L}$. Elevated baseline lactate resulted in significantly higher ICU mortality $(71 \%$ vs. $57 \%$, p < 0.001$)$. In the multivariable analysis, the maximum lactate concentration on day one was independently associated with ICU mortality (aOR $1.0695 \% \mathrm{Cl} 1.02-1.11 ; p=0.007)$, 30-day-mortality (aOR $1.0795 \% \mathrm{Cl}$ $1.02-1.13 ; p=0.005)$ and 3-month-mortality (aOR $1.1595 \% \mathrm{Cl} 1.08$ $1.24 ; \mathrm{p}<0.001$ ) after adjustment for age, sex, SOFA, and frailty. In 859 patients with baseline lactate $\geq 2 \mathrm{mmol} / \mathrm{l}$ sufficient data to calculate the difference between maximal levels on day 1 and 2 ( $\Delta$ serum lactate) was available. Falling lactate was inversely associated with ICU mortality (OR $0.6395 \% \mathrm{Cl} 0.46-0.87 ; \mathrm{p}=0.004$ ) and remained so after multivariate adjustment for SOFA, age, CFS and sex (aOR $0.6095 \% \mathrm{Cl}$ $0.42-0.85 ; p=0.004$ ).

Conclusion. In critically ill old intensive care patients suffering from COVID-19, lactate and its kinetics are valuable tools for outcome prediction. However, most critically ill old COVID-19 patients had normal serum lactate on admission.

\section{Reference(s)}

(1) Masyuk M et al. Intensive Care Med 2019, 45:55-61

(2) Bruno RR et al. Diagnostics (Basel). 2020 Dec 18;10(12):1105

(3) This study was endorsed by the ESICM. Free support for running the electronic database and was granted from the dep. of Epidemiology, University of Aarhus, Denmark. The support of the study in France by a grant from Fondation Assistance Publique-Hôpitaux de Paris pour la recherche is greatly appreciated. In Norway, the study was supported by a grant from the Health Region West. In addition, the study was supported by a grant from the European Open Science Cloud (EOSC). EOSCsecretariat. eu has received funding from the European Union's Horizon Programme call H2020-INFRAEOSC-05-2018-2019, grant agreement number 831644.
This work was supported by the Forschungskommission of the Medical Faculty of the Heinrich-Heine-University Düsseldorf, No. 2018-32 to GW and No. 2020-21 to RRB for a Clinician Scientist Track.

000793

Study of the dyspnea and the electromyographic activity of the diaphragm and extradiaphragmatic inspiratory muscles during weaning from mechanical ventilation

C. Bureau' ; M. Dres ; ' L. Dangers ${ }^{1}$; J. Mayaux'; E. Morawiec ${ }^{1}$; T. Similowski'; A. Demoule ${ }^{1}$

${ }^{1}$ Service de médecine intensive-réanimation-r3s, University Hospitals Pitié Salpêtrière-Charles Foix, Paris, France

Correspondence: $\mathrm{C}$. Bureau

Intensive Care Medicine Experimental 2020, 9(1): 000793

Introduction. Success or failure of the spontaneous breathing trial (SBT) is defined by objective and subjective criteria of acute respiratory failure (ARF).(1) Although dyspnea is a key symptom of ARF, dyspnea is not considered as common criteria of SBT success or failure. Here, we assessed dyspnea during SBT. Our first objective was to determine whether dyspnea is a reliable criterion of weaning success or failure. Our second objective was to quantify during SBT the relationship between dyspnea and the respective electromyographic activity (EMG) of the diaphragm (EMGdi) and of the Alae nasi (EMGan), an extradiaphragmatic inspiratory muscle.

Methods. Patients hospitalized in an intensive care unit and in whom a SBT was initiated by the physician in charge were included in the study. Were continuously recorded during the $30-\mathrm{min}$ SBT or until SBT failure: airway flow and pressure, EMGdi with nasogastric probe and EMGan with surface electrodes. Dyspnea intensity was assessed by the Dyspnea-Visual Analogic Scale (Dyspnea-VAS) at initiation and end of the SBT. At the end of the SBT, patients were classified as success or failure.(1).

Results. Thirty-one patients were included, $71 \%$ male, aged (median [interquartile range]) 65 [61-71] years, SAPS2 53 [37-74]. They were invasively mechanically ventilated for 6 [3-10] days. Seventeen patients succeeded SVT. Baseline Dyspnea-VAS was similar in patients who succeeded $(0[0-2])$ and in those who failed $(2[2-4] \mathrm{cm}, \mathrm{p}=0.18)$ SBT. Dyspnea-VAS at the end of SBT was lower in patients who succeeded $(0[0-4]$ vs $10[8-10] \mathrm{cm}, p=0.03)$. The increase in Dyspnea-VAS was lower in those who succeeded $(0[0-1]$ vs $6[4-8] \mathrm{cm}$, $p=0.01$ ). The area under the curve of Dyspnea-VAS was 0.909 . During SBT, the EMGan increased was lower in patients who succeeded (0.93 [0.6-1.1] vs 1.43 [0.91-2.4], $\mathrm{p}=0.01)$. The EMGdi increased was lower in patients who succeeded $(0.87$ [0.62-1.64] vs 1.87 [1.20-2.92], $p=0.02$ ). The increase of Dyspnea-VAS was significantly correlated to the increase in EMGan (Rho $=0.43[0.05-0.71], \mathrm{p}<0.05)$, but was not correlated to EMGdi (Rho $=-0.26[-0.68-0.28], p=0.33$ ).

Conclusion. During an SBT, dyspnea is a reliable marker of weaning success and failure, suggesting that Dyspnea-VAS could be used as a monitoring tool of SBT. In addition, Dyspnea seems to be more closely related to the EMG activity of extradiaphragmatic inspiratory muscles than the diaphragm.

000797

Outcomes for patients admitted to dedicated neurosciences and other critical care units following Traumatic Brain Injury in the largest collocated critical care unit in Europe

D. Nice ${ }^{1}$

${ }^{1}$ Critical Care, Queen Elizabeth Hospital Birmingham, Birmingham, United Kingdom

Correspondence: $\mathrm{D}$. Nice

Intensive Care Medicine Experimental 2020, 9(1): 000797 
Introduction. Traumatic brain injury (TBI) is a worldwide health issue that is the leading cause of death and disability in people under forty $(1,2)$. TBI research has improved our understanding of the pathophysiological processes underlying $\mathrm{TBI}$, giving rise to potential new therapies. However, no specific treatments have been found to have positive effects on outcomes for patients (3). The development of Neurocritical Care Unit (NCC) and parameter guided therapy to meliorate the potentially devastating effects of secondary brain injury have demonstrated positive improvements to patient mortality rates and neurological recovery post TBI $(4,5)$. Length of stay, which is often used as a surrogate marker for the quality of care, varies between studies that have examined TBI patients cared for in a dedicated NCC compared to those, cared for in a General Critical Care unit (GCC) $(6,7)$. The extended Glasgow Outcome Score (GOS-E) (8).is a validated tool for stratifying neurological recovery post TBI. It is the most widely used disability tool in TBI research and has been demonstrated to have good correlations with other psycho-cognitive assessments (9).

Objectives. The aim of this study is to determine whether there is a difference in outcome for patients who have sustained a TBI and cared for in a dedicated NCC or a GCC unit. The hypothesis is that there will be a difference in patient outcomes when comparing the four different units and that the TBI patients cared for on the NCC will have better outcomes than those patients cared for on the GCC units. Therefore the null hypothesis is that there will be no difference in patient outcome when comparing NCC to GCC.

The primary outcome of this study will be the GOS-E score at 28 days and three months.

The secondary outcomes will be hospital and Critical care length of stay and mean days ventilated.

Methods. Single centre retrospective longitudinal cohort study that used data collated from the Intensive Care National Audit and Research Centre (ICNARC) database. The GOS-E was scored for each patient at twenty-eight days, three months. The score was calculated by the researcher using the electronic patient record. Statistical analyses were conducted using Statview. All data are expressed and displayed as mean \pm standard deviation (SD) unless otherwise stated. Results were compared using analysis of variance (ANOVA). All P-values are quoted after Bonferroni corrections (where appropriate), and corrected $\mathrm{P}$-values $<0.05$ were considered significant.

Results. In total 396 patients were admitted to the four co-located Critical Care units following TBI, situated at a tertiary neurosciences and major trauma centre in the West Midlands over two years. The primary outcomes of GOS-E, no statistical difference was detected comparing the NCC to the three GCCs at 28 days $(p=0.4539)$ or three months $(p=0.2431)$. Multi-variate analysis of the secondary outcomes detected no statistical difference for critical care length of stay $(p=0.2233)$,hospital length of stay $(p=0.2272)$, and number of days ventilated $(p=0.427)$. Conclusion. The research study conducted demonstrated no statistical difference in the primary and secondary outcomes comparing the NCC to the 3 GCCs in Europe's largest co-located Critical Care unit. This is attributed to the implementation of a protocol-driven therapy guideline in all 4 Critical Care Units. This has resulted in standardised practice in the management of ICP and CPP post TBI and reduced variability in patient outcomes. The results support the use of protocol-driven therapy guidelines in the management of patients with TBI.

\section{Reference(s)}

1. Maas, A.I.R., Stocchetti, N., Bullock, R. (2008) 'Moderate and severe traumatic brain injury in adults', The Lancet.Neurology, 7(8) pp. 728-741. 10. 1016/S1474-4422(08)70164-9

2. National Institute for Clinical Excellence (2017) Head injury: assessment and early management. Clinical guideline [CG176] Available online: https://www.nice.org.uk/guidance/cg176 (Accessed: 10/05/2019)

3. Roberts, I., Yates, D., Sandercock, P., Farrell, B., Wasserberg, J., Lomas, G., Cottingham, R., Svoboda, P., Brayley, N., Mazairac, G., Laloë, V.,
Muñoz-Sánchez, A., Arango, M., Hartzenberg, B., Khamis, H., Yutthakasemsunt, S., Komolafe, E., Olldashi, F., Yadav, Y., Murillo-Cabezas, F., Shakur, H., Edwards, P. \& CRASH, t.c. (2004) 'Effect of intravenous corticosteroids on death within 14 days in 10,008 adults with clinically significant head injury (MRC CRASH trial): randomised placebo-controlled trial', Lancet, 364(9442), pp. 1321-1328. 10.1016/S0140-6736(04)17188-2

4. Patel, H.C., Menon, D.K., Tebbs, S., Hawker, R., Hutchinson, P.J., Kirkpatrick, P.J. (2002) 'Specialist neurocritical care and outcome from head injury', Intensive Care Medicine, 28, pp. 547-553. https://doi.org/10.1007/ s00134-002-1235-4

5. Sekhon, M.S., Gooderham, P., Toyota, B., Kherzi, N., Hu, V., Dhingra, V., Hameed, M., Chittock, D., Griesdale, D. (2017) 'Implementation of Neurocritical Care Is Associated With Improved Outcomes in Traumatic Brain Injury', The Canadian Journal of Neurological Sciences, 44, pp. 350 -357. http:/doi.org/https://doi.org/10.1017/cjn.2015.25

6. Egawa, S., Hifumi, T., Kawakita, K., Okauchi, M., Shindo, A., Kawanishi, M., Tamiya, T. Kuroda, Y. (2016) 'Impact of neurointensivist-managed intensive care unit implementation on patient outcomes after aneurysmal subarachnoid hemorrhage', Journal of critical care, 32 pp. 52-55 10.1016/j.jcrc. 2015.11.008

7. Kurtz, P., Fitts, V., Sumer, Z., Jalon, H., Cooke, J., Kvetan, V., Mayer, S.A.(2011) 'How Does Care Differ for Neurological Patients Admitted to a Neurocritical Care Unit Versus a General ICU?' Neurocritical Care, 15(477) https://doi. org/10.1007/s12028-011-9539-2

8. Jennett, B., Snoek, J., Bond, M. R., \& Brooks, N. (1981) 'Disability after severe head injury: observations on the use of the Glasgow Outcome Scale', Journal of Neurology, Neurosurgery, and Psychiatry, 44(4) pp. 285-293. 10.1136/jnnp.44.4.285

9. Weir, J., Steyerberg, E.W., Butcher, I., Lu, J., Lingsma, H.F., Mchugh, G.S., Roozenbeek, B., Maas, A.I.R. And Murray, G.D (2012)'Does the extended Glasgow Outcome Scale add value to the conventional Glasgow Outcome Scale?' Journal of Neurotrauma, 29(1), pp. 53-58. 10.1089/neu.2011. 2137

\section{1}

Distinct waveforms of peripheral arterial blood pressure tracings reveal preload-, cardiac contractility- and afterload- deficient hemodynamic instability: an in-silico simulation study

M. Mulder ; M. Broomé2 ; D. Donker ${ }^{3}$; B. Westerhof

${ }^{1}$ Cardiovascular and respiratory physiology, University

of Twente, Enschede, Netherlands; ${ }^{2}$ Anesthesia and intensive care, dept. of physiology and pharmacology, Karolinska Institutet, Stockholm, Sweden; ${ }^{3}$ Department of intensive care medicine, UMC Utrecht, Utrecht, Netherlands

Correspondence: M. Mulder

Intensive Care Medicine Experimental 2020, 9(1): 000811

Introduction. Critically ill patients frequently suffer from hemodynamic (HD) instability, basically driven by decreased preload, cardiac contractility or afterload, or a combination of those. Since peripheral arterial blood pressure waveforms hold information on both cardiac and vascular function, we hypothesized that these signals allow to distinguish underlying causes of HD instability, which is relevant for guidance of HD treatment.

Objectives. To investigate whether peripheral arterial waveform morphology differs between distinct causes of HD instability.

Methods. In this in-silico experimental study, we used 'Aplysia CardioVascular Lab', a closed-loop lumped parameter model to study patient-specific hemodynamics [1]. We defined a 60-year-old individual with normal physiology as control. We simulated HD instability based on well-defined preload, contractility and afterload reduction by stepwise decrease of these three input variables separately: blood volume ( $2 \%$-steps), left ventricular contractility and systemic vascular resistance (both 5\%-steps). The peripheral arterial waveforms were analysed using MATLAB ${ }^{\circledR}$ software. We defined 
45 features describing the waveform morphology, such as absolute and relative pressure, and slopes of different parts of the waveform. The calculated features were used as input for a principle component analysis (PCA), to quantitatively analyse the variance in waveform morphology between the underlying causes.

Results. A total of 50 waveforms were simulated and analysed. The arterial waveforms of the control and of three hemodynamic instability cases all with a MAP of $60 \mathrm{mmHg}$, are shown in Fig. 1. During hemodynamic instability the waveform changed characteristically and it was possible to differentiate between the three causes based on the morphology. The waveform features describing this morphology were further investigated with PCA: the first principle component explained $94 \%$ of the variance in the features and a second explained 5\%. Shown in more detail in Fig. 2, the three underlying HD instability causes can be distinguished from each other using only the first two principle components. Features with the highest correlation with these principle components are describing the systolic upstroke and downstroke, and anacrotic and dicrotic notches of the waveforms.

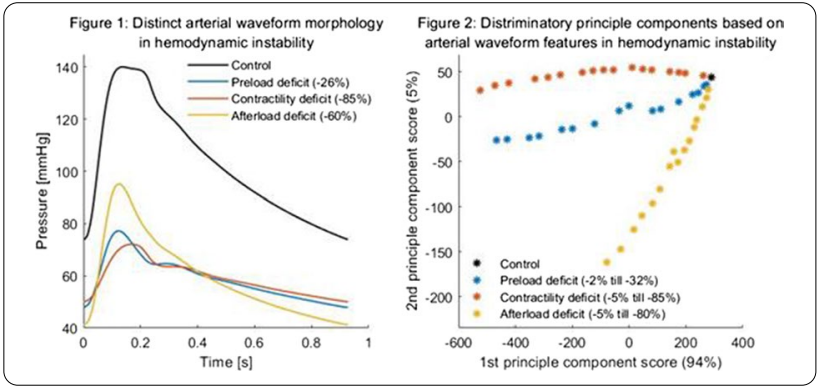

Conclusion. In this simulation study we identified distinct peripheral arterial waveform morphologies that distinguish between deficits in preload, contractility and afterload during HD instability. This sets the stage for personalised clinical decision support based on arterial line tracings to optimise HD treatment in critically ill patients.

\section{Reference(s)}

1. M. Broomé, E. Maksuti, A. Bjällmark, B. Frenckner, and B. Janerot-Sjöberg, "Closed-loop real-time simulation model of hemodynamics and oxygen transport in the cardiovascular system," Biomed. Eng. Online, vol. 12, no. 1, pp. 1-20, 2013, https://doi.org/10.1186/1475-925X-12-69.

\section{5}

\section{The profile of organ failure at ICU admission and outcomes} in elderly COVID-19 patients

H. Flaatten ${ }^{1}$; C. Jung ${ }^{2}$; M. Beil ${ }^{3}$; J. Fjølner ${ }^{4}$; B. Guidet ${ }^{5}$; P. Vernon Van Heerden $^{3}$; S. Leaver ${ }^{6}$; S. Sigal ${ }^{7}$; W. Szczeklik ${ }^{8}$; RP. Moreno ${ }^{9}$

${ }^{1}$ Department of anaesthesia and intensive care, Haukeland University Hospital, Bergen, Norway; ${ }^{2}$ Dep. of cardiology, pulmonology and angiology, University Hospital of Düsseldorf, Düsseldorf, Germany; ${ }^{3}$ Medical intensive care unit, Hadassah Medical Center, Jerusalem, Israel; ${ }^{4}$ Department of intensive care, Aarhus University Hospital, Aarhus, Denmark; ${ }^{5}$ Réanimation Médicale, Hôpital Saint-Antoine, Paris, France; ${ }^{6}$ Research lead critical care directorate, St George's Hospital, London, United Kingdom; ${ }^{7}$ Medical intensive care unit, Hadassah Medical Center, Jerusalem, Israel, Israel; ${ }^{8}$ Intensive care and perioperative medicine, Jagiellonian University Medical College, Kraków, Poland; ${ }^{9}$ Unidade de cuidados intensivos neurocríticos, Hospital de São José, Centro Hospitalar de Lisboa Central, Nova Médical School, Lisbon, Portugal

Correspondence: $\mathrm{H}$. Flaatten

Intensive Care Medicine Experimental 2020, 9(1): 000825

Introduction. The occurrence of organ failure in elderly ICU patients during the pandemic are seldomly reported in papers from this pandemic, although have been suggested as a triage criterium.
Objectives. This study is a part of the COVIP, a prospective clinical study conducted by the VIP network within the HSRO section of ESICM, with the aim to study the elderly ICU patients during the pandemic. Organ failure (OF) assessment was a part of the study. Our aim is to describe the occurrence of OF at admission and the short-term outcomes of our patients.

Methods. Study period was from mid-March 2020 until January 2021, using an online Case Record Form (1). Participating countries were responsible for obtaining National Ethical Clearance. At ICU admission all patients were scored using the Sequential Organ Dysfunction Assessment (SOFA) score (2), within the first $24 \mathrm{~h}$. Organ failure (OF) was described as any individual SOFA score $>2$. Frailty was obtained using the clinical frailty scale (CFS). Common ICU procedures (mechanical/ non-invasive ventilation, use of vasoactive drugs and renal replacement therapy) were registered as well as their start and duration. Outcome was measured as 30 days mortality.

Results. In total 3383 patients were admitted to 207 ICUs in 35 countries. Mean age was 76.9 years and $69.6 \%$ were male. Table 1 reveals the individual SOFA scores in the patients. Acute respiratory (55\%) and circulatory failure (31\%) were most prevalent. Very few had hepatic or coagulation failure $(<1 \%)$. The mean SOFA score was 5.7 and Fig. 1 reveals the distribution of sum SOFA score. Overall, 30 -day survival was $50.8 \%$.

\begin{tabular}{|c|c|c|c|c|c|c|}
\hline \multicolumn{7}{|c|}{ The occurrence of organ dysfunction and failure (SOFA score) } \\
\hline $\begin{array}{l}\text { SOFA } \\
\text { score }\end{array}$ & $\begin{array}{l}\text { Respira- } \\
\text { tory }\end{array}$ & $\begin{array}{l}\text { Circula- } \\
\text { tory }\end{array}$ & Renal & CNS & Hepatic & $\begin{array}{l}\text { Coagula- } \\
\text { tion }\end{array}$ \\
\hline 1 & 167 & 397 & 723 & 533 & 303 & 480 \\
\hline 2 & 1123 & 82 & 363 & 194 & 97 & 182 \\
\hline 3 & 1041 & 489 & 114 & 130 & 9 & 24 \\
\hline 4 & 824 & 561 & 119 & 181 & 3 & 5 \\
\hline $\begin{array}{l}\text { Sum } \\
\text { failure }\end{array}$ & $\begin{array}{l}1823 \\
(55.1 \%)\end{array}$ & $\begin{array}{l}1050 \\
(31 \%)\end{array}$ & $\begin{array}{l}233 \\
(6.9 \%)\end{array}$ & $\begin{array}{l}311 \\
\quad(9.2 \%)\end{array}$ & $12(0.4 \%)$ & $29(0.9 \%)$ \\
\hline
\end{tabular}

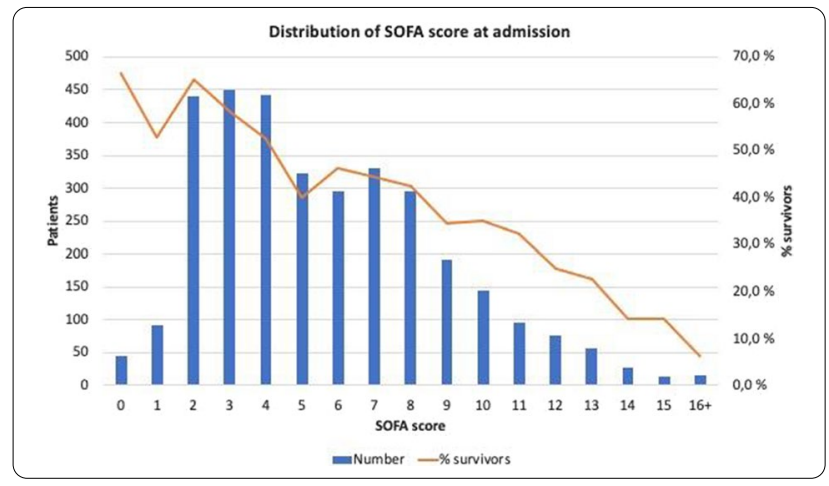

Conclusion. Most patients had severe respiratory or circulatory failure but comparatively few with other OF. Deterioration beyond the first day in hepatic and coagulation functions cannot be ruled out (3). SOFA score was inversely correlated with 30 -day survival and a score of $>10$ was associated with survival $<20 \%$. Our study confirms that severe acute $O D$ is important to understand short-term mortality in elderly ICU COVID-19 patients.

\section{Reference(s)}

1. Moreno R., Vincent J.-L., Matos R., et al. The use of maximum SOFA score to quantify organ dysfunction/failure in intensive care. Results of a prospective, multicentre study. Intensive Care Medicine 1999:25:686-96

2. Vincent, J. et al. The SOFA (Sepsis-related Organ Failure Assessment) score to describe organ dysfunction/failure. On behalf of the Working Group on Sepsis-Related Problems of the European Society of Intensive Care Medicine. 22, Intensive Care Med (1996) 22:707-710 
3. Jung C, Flaatten H, Fjølner J, et al. The impact of frailty on survival in elderly intensive care patients with COVID-19: the COVIP study. Critical care (London, England) 2021;25:149. 10.1186/s13054-021-03551-3 4. The VIP study group and all its collaborators.

5. Open Access funding enabled and organized by Projekt DEAL. The support of the study in France by a grant from Fondation Assistance Publique-Hôpitaux de Paris pour la recherche is greatly appreciated. In Norway, the study was supported by a grant from the Health Region West. In addition, the study was funded by a grant from the European Open Science Cloud (EOSC) by the European Commission. EOSCsecretariat.eu has received funding from the European Union's Horizon Programme call H2020-INFRAEOSC-05-2018-2019, grant agreement number 831644. No further specific funding was received.

\section{2}

Loop diuretics in adult intensive care patients with fluid overload. A systematic review of randomised clinical trials with meta-analysis and Trial Sequential Analysis

S. Wichmann ${ }^{1}$; M. Barbateskovic, ${ }^{2}$; N. Liang, ${ }^{3}$; TS. Itenov ${ }^{1}$; RE. Berthelsen, ;

J. Lindschou, ${ }^{2}$; A. Perner ${ }^{4}$; C. Gluud, ${ }^{2}$; MH. Bestle ${ }^{1}$

${ }^{1}$ Department of anaesthesiology and intensive care, Copenhagen University Hospital-North Zealand, Copenhagen, Denmark; ${ }^{2}$ Copenhagen trial unit, centre for clinical intervention research, Copenhagen University Hospital, Rigshospitalet, Copenhagen, Denmark; ${ }^{3}$ Institute of basic research in clinical medicine, China Academy of Chinese Medical Sciences, Beijing, China; ${ }^{4}$ Department of intensive care, Copenhagen University hospital, Rigshospitalet, Copenhagen, Denmark Correspondence: S. Wichmann

Intensive Care Medicine Experimental 2020, 9(1): 000832

Introduction. Fluid overload is a common condition in intensive care unit (ICU) patients and a risk factor for organ dysfunction and death (1-4). Loop diuretics are the predominant way to treat fluid overload (5), but evidence of benefits, timing, dosing, and harms appear sparse. Objectives. In this systematic review we assessed benefits and harms of loop diuretics in ICU patients with fluid overload. Primary outcomes were all-cause mortality, health-related quality of life, and number of patients experiencing one or more serious adverse events (SAEs).

Methods. Systematic review with meta-analysis of randomised clinical trials assessing the effect of loop diuretics versus placebo/no intervention/other diuretics/different doses of loop diuretics in adult ICU patients with fluid overload. We adhered to our published protocol (6), the Cochrane Handbook, and used an eight step procedure to assess if the threshold for clinical significance were crossed (7), conducted Trial Sequential Analysis (TSA) to control for random errors, assessed the certainty of evidence with GRADE, and reported the review according to PRISMA.

Results. We included 10 trials (804 participants), all at high risk of bias. The trials assessed three comparisons: 1) loop diuretics vs. placebo/ no intervention/standard of care; 2 ) loop diuretics vs. loop diuretics in other doses; and 3) loop diuretics vs. other diuretics. Analyses were only possible for comparison 1. Meta-analysis showed no evidence of a difference of loop diuretics vs. control on all-cause mortality (RR 0.72; $95 \% \mathrm{Cl} 0.49$ to $1.06 ; \mathrm{I} 2=0 \% ; 4$ trials, 359 patients; TSA-adjusted $\mathrm{Cl} 0.15$ to 3.48 with only $11.5 \%$ of diversity-adjusted required information size achieved; very low certainty of evidence). No data were reported on health-related quality of life. Meta-analysis showed fewer SAEs in the loop diuretic group compared with the control but the TSA adjusted result was not significant (RR $0.81 ; 95 \% \mathrm{Cl} 0.66$ to $0.99 ; 12=0 \% ; 6$ trials, 476 patients; TSA adjusted $\mathrm{Cl} 0.5$ to 1.20 with only $34.7 \%$ of the diversity-adjusted required information size achieved; very low certainty of evidence).

Conclusion. The evidence for treating fluid overload in ICU patients with loop diuretics is sparse and the certainty of evidence is very low and inconclusive.

\section{Reference(s)}

1. Messmer AS, Zingg C, Müller M, Gerber JL, Schefold JC, Pfortmueller CA. Fluid Overload and Mortality in Adult Critical Care Patients-A Systematic
Review and Meta-Analysis of Observational Studies*. Critical Care Medicine. 2020;48(12):1862-70.

2. Wang $N$, Jiang L, Zhu B, Wen Y, Xi X-M. Fluid balance and mortality in critically ill patients with acute kidney injury: a multicenter prospective epidemiological study. Crit Care. 2015;19:371.

3. Acheampong A, Vincent J-L. A positive fluid balance is an independent prognostic factor in patients with sepsis. Critical Care. 2015;19:251.

4. Kelm DJ, Perrin JT, Cartin-Ceba R, Gajic O, Schenck L, Kennedy CC. Fluid Overload in Patients With Severe Sepsis and Septic Shock Treated With Early Goal-Directed Therapy Is Associated With Increased Acute Need for Fluid-Related Medical Interventions and Hospital Death: Shock. 2015;43(1):68-73.

5. McCoy IE, Chertow GM, Chang TI-H. Patterns of diuretic use in the intensive care unit. PLoS ONE. 2019;14(5):e0217911. https://doi.org/10.1371/ hiyrbak.prone.0217911

6. Wichmann S, Barbateskovic M, Lindschou J, Gluud C, Perner A, Bestle $\mathrm{MH}$. Loop diuretics in adult intensive care patients with fluid overload: A protocol for a systematic review of randomised clinical trials with meta-analysis and Trial Sequential Analysis. Acta Anaesthesiol Scand. 2020;64(9):1327-34.

7. Jakobsen JC, Wetterslev J, Winkel P, Lange T, Gluud C. Thresholds for statistical and clinical significance in systematic reviews with meta-analytic methods. BMC Med Res Metodol. 2014;14:120. http://www.biomedcent ral.com/1471-2288/14/120

8. None

\section{7}

Aerobic exercise capacity in long-term survivors of critical illness: secondary analysis of the post-EPaNIC follow-up study

N. Van Aerde ${ }^{1}$; P. Meersseman, ${ }^{2}$; Y. Debaveye, ${ }^{1}$; A. Wilmer, ${ }^{1}$; MP. Casaer, ${ }^{1}$;

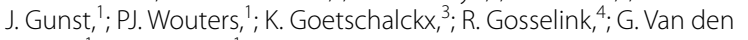
Berghe $^{1} ;$ G. Hermans ${ }^{1}$

${ }^{1}$ Department of cellular and molecular medicine, Katholieke Universiteit Leuven, Leuven, Belgium; ${ }^{2}$ Medical intensive care unit, department of general internal medicine, University Hospitals Leuven, Leuven, Belgium; ' 3 epartment of cardiovascular sciences, Katholieke Universiteit Leuven, Leuven, Belgium; ${ }^{4}$ Department of rehabilitation sciences, Katholieke Universiteit Leuven, Leuven, Belgium

Correspondence: N. Van Aerde

Intensive Care Medicine Experimental 2020, 9(1): 000847

Introduction. Physical impairments after intensive care (ICU) are well-documented by statical evaluations of strength and by sixminute-walk-distance. However, data on aerobic exercise capacity as assessed with cardiopulmonary exercise testing (CPET) in long-term ICU-survivors are scarce.

Objectives. To describe aerobic exercise capacity in 5-year ICU-survivors as compared to healthy controls and to assess the association between severity of organ failure in ICU and exercise capacity up to 5-years follow-up.

Methods. Secondary analysis of the EPaNIC follow-up cohort [1-3] (NCT00512122) including 433 patients screened with CPET between 1 to 5-years following ICU admission. Aerobic exercise capacity in 5-year ICU survivors $(\mathrm{N}=361)$ was compared to demographically matched controls $(\mathrm{N}=49)$. We described the prevalence of abnormal exercise capacity, defined as peak oxygen consumption (VO2peak) $<85 \%$ of predicted maximal oxygen consumption (\%predVO2max) in maximal CPETs (defined as respiratory exchange ratio $>1.05$ ), and identified exercise limiting factors. To study the association between severity of organ failure, quantified as the maximal Sequential Organ Failure Assessment score during ICUstay (SOFA-max), and aerobic exercise capacity as assessed with VO2peak, a linear mixed model was built, adjusting for predefined confounders and including all follow-up CPET studies.

Results. Aerobic exercise capacity was lower in 5-year follow-up patients than in controls (VO2peak: $24.0 \pm 9.7 \mathrm{ml} / \mathrm{min} / \mathrm{kg}$ versus $31.7 \pm 8.4 \mathrm{ml} / \mathrm{min} / \mathrm{kg}, \mathrm{p}<0.001$; \%predVO2max: $94 \% \pm 31 \%$ versus $123 \% \pm 25 \%, p<0.001 ;$ VO2AT: $15.3 \pm 8.7 \mathrm{ml} / \mathrm{min} / \mathrm{kg}$ versus $18.5 \pm 6.0 \mathrm{ml} / \mathrm{min} / \mathrm{kg}, \mathrm{p}=0.015$; \%predVO2max: $55 \% \pm 16 \%$ versus $71 \% \pm 21 \%, p<0.001)$. Abnormal exercise capacity was present in 
$118 / 313(37.7 \%)$ patients versus $1 / 48(2.1 \%)$ controls, $p<0.001$. Muscular limitation frequently contributed to impaired exercise capacity at 5-years [71/118 (60.2\%)]. SOFA-max independently associated with VO2peak throughout follow-up.

Conclusion. Critical illness survivors often display abnormal aerobic exercise capacity, frequently involving muscular limitation. Severity of organ failure throughout the ICU stay independently associates with these impairments.

\section{Reference(s)}

1. Casaer MP, Mesotten D, Hermans G, Wouters PJ, Schetz M, Meyfroidt G, Van Cromphaut S, Ingels C, Meersseman P, Muller J, Vlasselaers D, Debaveye Y, Desmet L, Dubois J, Van Assche A, Vanderheyden S, Wilmer A, Van den Berghe G, (2011) Early versus late parenteral nutrition in critically ill adults. N Engl J Med 365: 506-517

2. Hermans G, Van Aerde N, Meersseman P, Van Mechelen H, Debaveye $Y$, Wilmer A, Gunst J, Casaer MP, Dubois J, Wouters P, (2019) Five-year mortality and morbidity impact of prolonged versus brief ICU stay: a propensity score matched cohort study. Thorax 74: 1037-1045

3. Van Aerde N, Meersseman P, Debaveye Y, Wilmer A, Gunst J, Casaer MP, Bruyninckx F, Wouters PJ, Gosselink R, Van den Berghe G, (2020) Five-year impact of ICU-acquired neuromuscular complications: a prospective, observational study. Intensive Care Med 46: 1184-1193

4. Research Foundation Flanders, Belgium [G.0399.12 (GH), 1805116 N (GH): 1700111 N (MPC), 1131618 N (NVA)]; KOOR, University Hospitals Leuven (JG); Methusalem, Flemish Government, METH/14/06 (GVdB); ERC AdvG2012-321,670 \& AdvG-2017-785,809 (GVdB).

\section{1}

\section{Effect of female sex on endothelial activation and fibrinolysis} in trauma patients

R. Dujardin'; DJ. Kleinveld²; C. Van Den Brom³ ; L. Geeraedts Jr'; E. Beijer C. Gaarder ; K. Brohi'; S. Stanworth ${ }^{8}$; P. Johansson ${ }^{9}$; J. Stensballe ${ }^{10}$; M. Maegele ${ }^{11}$; NP. Juffermans ${ }^{2}$

${ }^{1}$ Department of intensive care medicine, Amsterdam UMC, locatie AMC, Amsterdam, Netherlands; ${ }^{2}$ Laboratory of experimental intensive care and anesthesiology, Amsterdam UMC, locatie AMC, Amsterdam, Netherlands; ${ }^{3}$ Department of anesthesiology, Amsterdam UMC, locatie VUmc, Amsterdam, Netherlands; ${ }^{4}$ Department of surgery, Amsterdam UMC, locatie VUmc, Amsterdam, Netherlands; ${ }^{5}$ Anesthesiology, Amsterdam UMC, locatie VUmc, Amsterdam, Netherlands; ${ }^{6}$ Department of traumatology, University Hospital Oslo, Oslo, Norway; ${ }^{7}$ Center for trauma sciences, Queen Mary University of London, London, United Kingdom; ${ }^{8} \mathrm{Nhs}$ blood and transplant, John Radcliffe Hospital, Oxford, United Kingdom; ${ }^{9} T$ ransfusion medicine, Copenhagen University Hospital, Rigshospitalet, København, Denmark; ${ }^{10}$ Department of anesthesiology, Copenhagen University Hospital, Rigshospitalet, København, Denmark; ${ }^{11}$ Department of traumatology and orthopedic surgery, Krankenhaus Merheim, Köln, Germany

Correspondence: R. Dujardin

Intensive Care Medicine Experimental 2020, 9(1): 000851

Introduction. Premenopausal female sex is suggested to provide a survival advantage in response to traumatic injury that is attributed to protective effects of estrogen on endothelial and coagulation disturbances[1]. However, previous studies report contradictory results.

Objectives. To compare markers of endothelial activation and fibrinolysis between male and female trauma patients of different age groups.

Methods. This study is performed as part of the prospective, multicenter, Activation of Coagulation and Inflammation in Trauma (ACIT) trial. All trauma patients $\geq 18$ years with full trauma team activation were included between January 2008 and April 2015. Exclusion criteria were penetrating trauma, traumatic brain injury (TBI) defined as an abbreviated injury score (AIS) of head and neck $\geq 3$ and total other AIS $<3$, administration of $>2 \mathrm{~L}$ i.v. fluids before hospital admission, hospital arrival $>2 \mathrm{~h}$ after injury or burns covering $>5 \%$ of the body.
Trauma patients were divided in the following three categories: male patients $<45$, female patients $<45$, and female patients $\geq 45$ years of age. At hospital admission, blood was drawn for analysis of von Willebrand factor antigen (vWF:ag) and thrombomodulin (TM) as markers for endothelial activation and D-dimer, plasmin antiplasmin complexes (PAP) and tissue plasminogen activator (tPA) as markers for fibrinolysis. Groups were compared using a Kruskal Wallis test, followed by a Dunn's post hoc test.

Results. In total, 743 patients were enrolled. Groups did not differ in injury severity or shock indices (Table 1). Compared to males, early mortality was significantly higher in the female $\geq 45$ group, but not in female $<45$ patients.

Levels of VWF:ag and TM were lower in both the female $<45$ and female $\geq 45$ group when compared to men, but not different between the female age groups.

Female $\geq 45$ patients showed increased PAP and tPA levels compared to female $<45$ patients, and increased PAP levels compared to male $<45$ patients. Although D-dimer levels were also higher in female $\geq 45$ patients, this increase was not statistically significant.

Table 1. Baseline characteristics, endothelial and fibrinolysis markers. Data reported as median (IQR) unless otherwise specified.

\begin{tabular}{|c|c|c|c|c|}
\hline Variable & $\begin{array}{l}\text { Male }<45 \\
\mathrm{~N}=485\end{array}$ & $\begin{array}{l}\text { Female }<45 \\
N=119\end{array}$ & $\begin{array}{l}\text { Female } \geq 45 \\
N=139\end{array}$ & p value \\
\hline Age & $29(22-36)$ & $29(22-35)$ & $64(54-74)$ & $<0.01$ \\
\hline ISS & $17(9-29)$ & $14(7-29)$ & $17(9-29)$ & 0.091 \\
\hline $\begin{array}{l}\text { Lactate } \\
\qquad(\mathrm{mmol} / \mathrm{L})\end{array}$ & $2.1(1.3-3.4)$ & $2.0(1.3-3.1)$ & $2.1(1.3-3.3)$ & 0.73 \\
\hline $\begin{array}{c}\text { Base Excess } \\
(\mathrm{mEq} / \mathrm{L})\end{array}$ & $-1.5(-4.1-0.4)$ & $\begin{array}{l}-2.1(-5.3-- \\
0.2)\end{array}$ & $-1.6(-5.4-0.7)$ & 0.16 \\
\hline $\begin{array}{l}\text { Mortality, } \\
12 \mathrm{~h}, \%\end{array}$ & $2.3 \%$ & $1.7 \%$ & $6.5 \%$ & 0.024 \\
\hline VWF:ag (IU) & $\begin{array}{l}228.2 \\
(160.2-293.5)\end{array}$ & $\begin{array}{l}186.0 \\
(140.0-252.1)\end{array}$ & $\begin{array}{l}208.0 \\
(161.2-274.1)\end{array}$ & $<0.01$ \\
\hline TM (ng/mL) & $5.7(4.1-10.4)$ & $4.4(2.9-13.7)$ & $4.1(3.2-7.2)$ & $<0.01$ \\
\hline $\begin{array}{l}\text { D-dimer (ng/ } \\
\mathrm{mL} \text { ) }\end{array}$ & $\begin{array}{c}13,724.0 \\
(3324.5- \\
42,305.8)\end{array}$ & $\begin{array}{c}13,480.0 \\
(2780.0- \\
56,089.0)\end{array}$ & $\begin{array}{l}20,365.0 \\
(5121.8- \\
68,073.8)\end{array}$ & 0.062 \\
\hline PAP $(\mu \mathrm{g} / \mathrm{L})$ & $\begin{array}{l}3284.3(1546.1- \\
7034.8)\end{array}$ & $\begin{array}{l}2797.9 \\
(1548.8- \\
6606.4)\end{array}$ & $\begin{array}{l}4779.8 \\
\quad(2178.8- \\
12,376.2)\end{array}$ & $<0.01$ \\
\hline tPA (ng/mL) & $12.4(7.0-19.1)$ & $8.3(4.4-13.7)$ & $\begin{array}{l}13.9 \\
(9.1-21.4)\end{array}$ & $<0.01$ \\
\hline
\end{tabular}

Conclusion. This study shows that female patients $\geq 45$ years of age have an increased risk of mortality compared to female and male patients $<45$ years of age, associated with an increase in markers of fibrinolysis. Reduced endothelial activation as observed in women did not provide a survival benefit. Whether suppletion of estrogen in women $>45$ confers protection by decreasing fibrinolysis warrants further research.

\section{Reference(s)}

1. [1] Deitch EA, Livingston DH, Lavery RF, Monaghan SF, Bongu A, Machiedo GW. Hormonally active women tolerate shock-trauma better than do men: a prospective study of over 4000 trauma patients. Ann Surg. 2007;246(3):447-53

\section{6}

Management of acute exacerbations of COPD in the intensive care unit: Trends and outcomes in the OUTCOMEREA database, 1997-2018

LM. GALERNEAU1'; S. Bailly²; S. Ruckly, ; M. Garrouste-Orgeas ${ }^{4}$; S. Siami ${ }^{5}$; C. DUPUIS ${ }^{6}$; M. Darmon ${ }^{7}$; G. Rigault ; C. Adrie ${ }^{8}$;. Laurent ${ }^{9}$; 
E. de Montmollin ${ }^{10} ;$ L. Argaud ${ }^{11} ;$ N. Terzi, ${ }^{1} ;$ JL. Pepin²; JF. Timsit ${ }^{10} ; .$. The Outcomerea Network ${ }^{10}$

${ }^{1}$ Médecine intensive—réanimation, C.H.U de Grenoble, La Tronche, France; ' ${ }^{2}$ Laboratoire hp2, C.H.U de Grenoble, La Tronche, France; ${ }^{3}$ Umr 1137, INSERM, Paris, France; ${ }^{4}$ Medical unit, Hôpital Franco-Britannique, Levallois-Perret, France; ${ }^{5}$ Réanimation, Hospital Center Sud Essonne -, Étampes, France; ${ }^{6}$ Médecine intensive-réanimation, $\mathrm{CHU}$ Gabriel-Montpied, Clermont-Ferrand, France; ${ }^{7}$ Medical icu, Hôpital Saint-Louis, Paris, France; ${ }^{8}$ Service d'explorations fonctionnelles, Cochin Hospital, Paris, France; ${ }^{9}$ Réanimation, C.H. de Versailles, Le Chesnay, France; ${ }^{10}$ Intensive care medicine department, Assistance Publique Hopitaux De Paris, Paris, France; ${ }^{11}$ Service de médecine intensive-réanimation, Hospital Édouard Herriot, Lyon, France

Correspondence: L.M. GALERNEAU

Intensive Care Medicine Experimental 2020, 9(1): 000866

Introduction. The natural history of COPD is punctuated of acute exacerbations of COPD (AECOPDs) leading to hospitalization in intensive care units (ICU) for the most severe cases. The daily practice of corticosteroids/antibiotics prescriptions and the respective proportion of invasive and non-invasive ventilation (NIV) have evolved over time in the ICU. These contextual changes might have implications regarding AECOPDs management and ICU outcomes such as length of stay, intubation rates and mortality.

Objectives. The aim of the current study was to assess over 22 years the evolution of AECOPDs changes in the management practices and the impact on main outcomes including length of stay and mortality in ICU and in post-ICU stay.

Methods. Included patients were participants in the Outcomerea prospective national database, in 32 ICUs centers between 1997 and 2018 with a main diagnosis of AECOPD. Patient characteristics or status at the end of stay in ICU was taken from the database, as well as data related to corticosteroids therapy, antibiotic therapy, NIV or Invasive Mechanical Ventilation (IMV). Evaluation of time trends were performed with analysis of the evolution of these variables over time out using a mixed model. Time series analyses were performed to assess the relationship between mortality in ICU and use of corticosteroids, antibiotics and invasive mechanical ventilation, using a dynamic regression model.

Results. We found limited changes in the profile of the 1848 patients with AECOPD admitted to ICUs except a slight increase in body mass index $(+0.29 \% /$ year, $p=0.01)$ and severity was greater at ICU admission $(+0.55 \%$ of SAPS Score/year, $p=0.01)$. There was overtime a significant reduction in the prescription of corticosteroids $(-4.7 \%$ / year, $p=<0.01)$ and antibiotics $(-5.6 \% /$ year, $p=<0.01)$, without these changes being correlated with the evolution of mortality in ICU in a time series analysis. The proportion of patients treated with Invasive Mechanical Ventilation (IMV) also gradually declined (-3.37\%/year, $p=0.01)$ with this time a significant correlation between variations of use of IMV and variations of deaths in ICU $(p=<0.01)$. Rate of NIV failures decreased over time $(-6.3 \%$ year, $p<0.01)$ with a diffusion of NIV for facilitating IMV weaning $(+8.1 \% /$ year, $p=<0.01)$. There was a significant shortening of length of stay in ICU $(-3.2 \% /$ year, $p=<0.01)$. We observed an improvement of the prognosis of patients $(-4.13 \% / y$ year of deaths in ICU, $p=0.03$ and $-4.29 \% /$ year of deaths in hospital post-ICU stay, $p=0.01$ ).

Conclusion. Lengths of stay decreased with a better prognosis of AECOPD in ICU. The management of ventilatory support has improved. And if prescriptions for corticosteroids and antibiotics have been reduced, prescription strategies for these therapeutics in ICU have to be precise.

\section{8}

Renal mitochondrial dysfunction in ovine experimental Sepsis Associated Acute Kidney ilnjury

T. Luther' ; S. Bülow-Anderberg'; P. Persson²; S. Franzén '; P. Skorup33; K. Hultenby ; F. Palm²; T. Schiffer'; R. Frithiof

${ }^{1}$ Dept of Surgical Sciences, Anesthesia and Intensive Care, Uppsala University, Uppsala, Sweden; ${ }^{2}$ Department of medical cell biology, integrative physiology, Uppsala University, Uppsala, Sweden; ${ }^{3}$ Section of infectious diseases, department of medical sciences, Uppsala University, Uppsala, Sweden; ${ }^{4} 6$. department of laboratory medicine, division of biomolecular and cellular medicine, Karolinska Institute, Stockholm, Sweden; ${ }^{5}$ Department of physiology and pharmacology, Karolinska Institute, Stockholm, Sweden

Correspondence: T. Luther

Intensive Care Medicine Experimental 2020, 9(1): 000868

Introduction. Sheep develop Sepsis Associated Acute Kidney Injury (SA-AKI) during experimental sepsis despite normal to increased renal oxygen delivery (DO2). Renal mitochondrial dysfunction has been demonstrated in hypodynamic models of sepsis in rodents. Changes in renal lactate/pyruvate have been observed in ovine models while renal adenosine triphosphate (ATP)-levels are initially preserved. A disturbed relation between oxygen consumption (VO2) and renal sodium transport, which is a major source of ATP-demand, has been demonstrated in clinical studies of AKI. This phenomena could be explained by mitochondrial dysfunction.

Objectives. To investigate the function of isolated renal mitochondria in comparison to renal oxygen handling in an ovine hyperdynamic model of SA-AKI.

Methods. Mixed breed ewes were anesthetized and either subjected to an infusion of live Escherichia Coli with resuscitative measures for $28 \mathrm{~h}$ or severe deterioration (Sepsis group, $\mathrm{n}=13$ ), or served as controls $(n=8)$. Renal VO2 and sodium resorption (transport) were repeatedly measured. Live cortical mitochondria were isolated at baseline and end of experiment and assessed in vitro with high resolution respirometry. Mitochondrial Complex I (Cl) mediated Respiratory Control Ratio (RCR) was determined as a division of respiration during adenosine diphosphate (ADP) depletion (State 2) and during ADP excess (State 3). Complex II (CII) mediated State 3 respiration was assessed by adding $\mathrm{Cll}$ substrate succinate and $\mathrm{Cl}$-inhibitor rotenone. Mitochondrial efficiency defined as produced ATP per $\mathrm{O} 2$ consumed (P/O-ratio) was calculated using respiration during steady state infusion of ADP. Mitochondrial uncoupling was measured as respiration in presence of ATP-syntase inhibitor oligomycin.

Results. Five ewes in Sepsis group were terminated prematurely after a mean of $22 \pm 2.5 \mathrm{~h}$. In Sepsis group compared to controls there were a markedly reduced creatinine clearance ( $6 \pm 5 \mathrm{~mL} / \mathrm{min}$ vs. $70 \pm 36 \mathrm{~mL} /$ $\min ; \mathrm{p}<0.001)$ and reduced sodium resorption $(0.8 \pm 0.7 \mathrm{mmol} / \mathrm{min}$ vs. $9 \pm 5 \mathrm{mmol} / \mathrm{min} ; \mathrm{p}<0.001)$ at end of experiment, while left renal VO2 was unaffected $(3.6 \pm 1.6 \mathrm{~mL} / \mathrm{min}$ vs. $3.5 \pm 1.5 \mathrm{~mL} / \mathrm{min} ; \mathrm{n} . \mathrm{s}$.). The relation between sodium transport and $\mathrm{VO} 2$ was markedly disturbed by sepsis $(0.1 \pm 0.1 \mathrm{mmolNa} / \mathrm{mLO} 2$ v.s. $1.4 \pm 0.8 \mathrm{mmolNa} / \mathrm{mLO} 2$; $p<0.001)$. Septic sheep developed altered cortical mitochondrial function at end of experiment, evident as reduced RCR (6.0 \pm 1.5 vs. $8.2 \pm 1.6 ; \mathrm{p}=0.006)$ and increased $\mathrm{Cl} / \mathrm{Cl}$-ratio during State $3(1.6 \pm 0.2$ vs. $1.3 \pm 0.1 ; p=0.0014)$ mainly due to decreased $\mathrm{Cl}$-dependent State 3 respiration $(p=0.016)$. There were however no significant differences in renal mitochondrial efficiency or mitochondrial uncoupling.

Conclusion. Renal mitochondrial dysfunction comprised of a reduction of the RCR and an increased CII/Cl-relation in State 3, can be demonstrated in an ovine model of SA-AKI. The disturbed relation between renal oxygen consumption and renal sodium transport cannot be explained by a change in renal cortical mitochondrial efficiency or uncoupling.

\section{Reference(s)}

1. Schiffer TA, Gustafsson H, Palm F. Kidney outer medulla mitochondria are more efficient compared with cortex mitochondria as a strategy to sustain ATP production in a suboptimal environment. Am J Physiol Renal Physiol 2018; 315: F677-F81

2. Redfors B, Bragadottir G, Sellgren J, Swärd K, Ricksten S-E. Acute renal failure is NOT an "acute renal success" - a clinical study on the renal oxygen supply/demand relationship in acute kidney injury. Crit Care Med 2010; 38: 1695-701

3. Skytte Larsson J, Krumbholz V, Enskog A, Bragadottir G, Redfors B, Ricksten SE. Renal Blood Flow, Glomerular Filtration Rate, and Renal Oxygenation in Early Clinical Septic Shock. Crit Care Med 2018; 46: e560-e66 
4. May CN, Ishikawa K, Wan L, Williams J, Wellard RM, Pell GS, Jackson GD, Bellomo R. Renal bioenergetics during early gram-negative mammalian sepsis and angiotensin II infusion. Intensive Care Med 2012; 38: 886-93

5. Fenhammar J, Rundgren M, Hultenby K, Forestier J, Taavo M, Kenne E, Weitzberg E, Eriksson S, Ozenci V, Wernerson A, Frithiof R. Renal effects of treatment with a TLR4 inhibitor in conscious septic sheep. Crit Care 2014; 18: 488

6. This study was in part funded by the Swedish Research Council (grant no 2014-02,569 and 2014-07,606)

\section{3}

Serial bedside ultrasonography for estimating urinary bladder wall thickness as a predictor for catheter associated urinary tract infection in critically ill patients: A reliable "cut-off" finding study BP. Das ${ }^{1}$; M. Gupta ${ }^{2}$

${ }^{1}$ sstreet_address\}, Varanasi, India; ${ }^{2}$ Microbiology, IMS BHU, Varanasi, India Correspondence: B.P. Das

Intensive Care Medicine Experimental 2020, 9(1): 000883

Introduction. Indwelling catheters are used routinely in the Intensive Care Units (ICUs), one of the integral problem of which is urinary tract infection (CAUTI), and is the most common nosocomial infection worldwide. Diagnosing CAUTI in critically ill patients is a challenge for intensivists. Hence, there was an unmet need of developing a quick, reproducible, accurate objective method of screening and/or diagnosing CAUTI.

Objectives. Now, we all have a portable USG machine in our ICU, which is a real-time bedside modality; though has been used extensively in OPD, but was never explored in critically ill patients for screening or diagnosing CAUTI in ICU. With this background, we have proceeded with an aim to evaluate bladder wall thickness (BWT) with serial bedside ultrasonography in catheterized critically ill patients as a tool to predict urinary tract infection (CAUTI).

Methods. After ethics committee approval \& written informed consent, a single-centric, prospective, observational cohort study was conducted from March2019 to February2020 in ICU,Dept of Anesthesia,IMS-BHU, where consecutive adult patients aged 20-50 years, admitted with any critical illness, were enrolled into the study and assessed for eligibility (without prior catheterization/ UTI, requiring ICU stay for $\geq 5$ days, requiring catheterisation within 2 days of admission and for a period of at least 3 days). All eligible patients were evaluated for bladder wall thickness(BWT), both in empty bladder(BWTe) and full bladder after $250 \mathrm{ml}$ normal saline instillation(BWTf) as per protocol, with serial bedside USG using a curvilinear probe $(3.5 \mathrm{MHz})$ over a stretch of 21 days or till the patient was in ICU [on day1 of ICU admission, then day3, day5, day7, day10, day14, day17, day21, and whenever there was an episode of fever with suspicion of CAUTI (fluffy sediments in high colored urine)]. Also urine \& blood cultures sent on those particular days from day 3 onwards as per protocol or whenever there was fever. Then those evaluated "BWT values", were allocated into one of the three cohorts based on urinary culture reports [CAUTI group; significant colony count (>105) but without CAUTI (CCS group); insignificant colony count (CCI group)]. Demographics,SOFA/APACHE scoring,ventilator days,length of ICU stay,survival rate noted.

Primary outcomes noted were performance of BWT to predict CAUTI using receiver operating characteristics curve (AUC-ROC) and to find out reliable "cut-off" with highest sensitivity \& specificity. Also correlation of BWT measurements with urine culture reports (CAUTI/ colonization/insignificant colony counts) and 30-day mortality were noted. Secondary outcomes noted were incidence of nosocomial UTI (bacterial \& fungal) and antibiogram. Chi-square/student-t test were used for qualitative/quantitative data with statistical significance at $p$-value $\leq 0.05$.

Results. Demographic parameters were comparable in the 3 cohorts. There were total of 1242 evaluated "BWT values" obtained on 180 enrolled patients, [476 values in CAUTI group, 362 values in significant colony count but without CAUTI (CCS group), 404 in insignificant colony count (CCI group)]. Mean BWTe in CAUTI group was $5.54 \mathrm{~mm} \pm 1.95 \mathrm{~mm}$ vs $4.12 \pm 1.62 \mathrm{~mm}$ in colonization group vs $3.42 \pm 1.54 \mathrm{~mm}$ in $\mathrm{CCl}$ group), $\mathrm{p}=0.002$. BWT $>5.54 \mathrm{~mm}$ had a good positive correlation with CAUTI with Spearman's coefficient of +0.834 with better correlation in empty bladder (BWTe) than in $250 \mathrm{ml}$ filled bladder (BWTf). The reliable cut-off value of BWTe for diagnosing CAUTI was found to be $5.6 \mathrm{~mm}$ with highest AUC-ROC (mean 0.887 with $95 \% \mathrm{Cl}$ of $0.863-0.942$ ) with $91.2 \%$ sensitivity and $78 \%$ specificity, with PPV $88.8 \%$ and NPV $85.2 \%$. At the optimum cut-off defined at $3.8 \mathrm{~mm}$, NPV was $100 \%$, leaving no probability of significant colony count with BWTe $<3.8 \mathrm{~mm}$; PPV was also very high $(95.2 \%$ ) with AUC-ROC (mean 0.872 with $95 \% \mathrm{Cl}$ of $0.822-0.980$ ) with $89.4 \%$ sensitivity and $76.2 \%$ specificity. Incidence of CAUTI overall was 38\%.

Conclusion. Bladder wall thickness with serial bedside USG is not only simple, non-invasive \& reproducible but quite a reliable objective method to predict CAUTI in ICU, which may increase diagnostic accuracy for screening out CAUTI from colonization, with attributes of performance being best in empty bladder as compared to full bladder, with cut-off value of $5.6 \mathrm{~mm}$.

\section{Reference(s)}

1. Nil

2. We acknowledge the relatives of the patient for their permission and the staffs and doctors in the ICU team for their good work and constant support.

\section{3}

Effects of spontaneous respiratory efforts during MV

on the distribution of pulmonary ventilation and regional

inflammation in an experimental model of ARDS

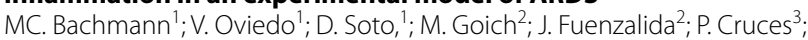
F. Diaz ${ }^{4}$; G. Bugedo ${ }^{1}$; A. Bruhn ${ }^{1}$; J. Retamal ${ }^{1}$

${ }^{1}$ Departamento de medicina intensiva, Pontificia Universidad Católica de Chile, Santiago, Chile; ${ }^{2}$ Medicina veterinaria, UNAB, Santiago, Chile;

${ }^{3}$ Unidad de pacientes críticos, Hospital El Carmen de Maipú, Santiago, Chile; ${ }^{4}$ Unidad de cuidados intensivos pediátricos, Clinica Alemana, Vitacura, Chile

Correspondence: M.C. Bachmann

Intensive Care Medicine Experimental 2020, 9(1): 000893

Introduction. Spontaneous breathing (SB) in patients with acute respiratory distress syndrome (ARDS) on mechanical ventilation (MV) has been associated with strong diaphragmatic activity and high pleural pressures, generating excessive stress in the dependent regions of the lung [1]. However, these mechanisms may be present before intubation [2]. Our hypothesis is that spontaneous respiratory efforts during the acute phase of lung injury increase regional lung stress, and therefore regional inflammation, protective MV can reverse these phenomena.

Objectives. The aim of this study is to determine the effects of spontaneous ventilation, during acute respiratory failure, on the regional distribution of ventilation and inflammation.

Methods. We studied 18 pigs subjected to a lung injury model and randomly distributed to one of 3 groups $(n=6): 1)$ Controlled MV $(\mathrm{CMV}), 8 \mathrm{~h}$ in volume-controlled ventilation, with VT of $6 \mathrm{ml} / \mathrm{kg}$, RR of $30 \mathrm{bpm}, \mathrm{PEEP}$ of $5 \mathrm{cmH} 2 \mathrm{O}$ and $\mathrm{FIO} 2$ of 1. 2) Spontaneous Ventilation (SB), $8 \mathrm{~h}$ in pressure support ventilation (PSV), with a pressure adjusted for an initial VT of 6 to $8 \mathrm{ml} / \mathrm{kg}$, PEEP between 0 and $5 \mathrm{cmH} 2 \mathrm{O}$ and FIO2 of 1. 3) Mixed, sequentially ventilated with PSV for $4 \mathrm{~h}$ and then CMV for the next $4 \mathrm{~h}$. For the three groups, gas exchange, pulmonary mechanics, electrical impedance tomography (EIT) and hemodynamics were evaluated at baseline, after lung injury $(\mathrm{H} 0)$ and at hours 4 and 8 ( $\mathrm{H} 4$ and $\mathrm{H} 8$ ). Bronchoalveolar lavages (BAL) were performed in $\mathrm{H} 4$ and $\mathrm{H} 8$ for cytokine analysis and tissue samples from 3 lung regions (ventral-intermediate and dorsal) were obtained at the end of the study.

Results. RR was higher in the SB group ( $37 \pm 4.6)$ compared to CMV group $(30 \pm 0.8, p=0.002)$. We observed significant differences (throughout the experiment) in esophageal pressure (Pes) between 
the CMV and SB groups $(p=0.0001)$. In the mixed group, esophageal oscillation pressure increased from -8.2 (-16-3.6) during PSV to 2.8 (1.9-3.3) during CMV $(p=0.002)$. The SB and mixed groups showed deterioration of oxygenation over time and $\mathrm{CMV}$ improved $\mathrm{PaO} 2 /$ $\mathrm{FiO} 2$ at the end of the experiment. When analyzing the EIT data, we found a tendency to decrease in the Impedance Ratio at $\mathrm{H} 8(\mathrm{p}=0.06)$ compared with CMV group. Furthermore, in the mixed group a greater heterogeneity was found in the distribution of ventilation in $\mathrm{H} 4$, a phenomenon that was reversed after $4 \mathrm{~h}$ in controlled ventilation. No differences were found in the concentration of cytokines in BAL fluid nor plasma. The histological score was lower in the CMV group compared to the SB and mixed group $(p=0.04)$. Finally, we found a linear correlation between Pes swing and histological damage in CMV and SB groups.

Conclusion. Ventilatory efforts were associated with changes in the regional pattern of ventilation in mixed and SB group compared with CMV. This change in the ventilatory pattern was accompanied by greater lung damage as observed in the histological score.

\section{Reference(s)}

1. Mascheroni D, Kolobow T, Fumagalli R, Moretti MP, Chen V, Buckhold D. Acute respiratory failure following pharmacologically induced hyperventilation: an experimental animal study. Intensive Care Med. 1988;15:8-14.

2. Retamal J, Hurtado D, Villarroel N, Bruhn A, Bugedo G, Amato MBP, et al. Does Regional Lung Strain Correlate With Regional Inflammation in Acute Respiratory Distress Syndrome During Nonprotective Ventilation? An Experimental Porcine Study. Crit. Care Med. 2018;591-9.

3. FONDECYT $1,171,810$

\section{5}

Transpulmonary end expiratory pressure during express peep setting in ARDS COVID-19 patients

F. Oller Sanchez ${ }^{1}$; FJ. Parrilla'2; A. Castellví-Font, ${ }^{2}$; C. Vilà-Vilardell, 2 ;

V. Boutonnet ${ }^{3}$; O. Festa ${ }^{4}$; M. Blanes ${ }^{5}$; C. Mestre ${ }^{5}$; P. Pratsopraroca ${ }^{5}$. JR. Masclans-Enviz'; J. Marin-Corral ${ }^{2}$

${ }^{1}$ Critical care department, Hospital Universitari Parc Tauli, Barcelona, Spain; ${ }^{2}$ Critical care department, Hospital del Mar de Barcelona, GREPAC (Grup de Recerca en Patologia Crítica)—IMIM, Barcelona, Spain; ${ }^{3}$ Grepac (grup de recerca en patologia crítica), IMIM, Barcelona, Spain; ${ }^{4}$ Anesthesia department, Hospital de Sant Boi, Barcelona, Spain; ${ }^{5}$ Critical care department, Hospital del Mar de Barcelona, Barcelona, Spain

Correspondence: F. Oller Sanchez

Intensive Care Medicine Experimental 2020, 9(1): 000895

Introduction. Selecting an optimal positive end expiratory pressure (PEEP) in acute respiratory distress syndrome (ARDS) remains controversial. Selecting PEEP to achieve but not exceed a plateau pressure target (EXPRESS PEEP) is widely used in ICU as protective mechanical ventilation management(1). Maintaining positive transpulmonary end expiratory pressure (PLee) improves oxygenation and ameliorate lung mechanics(2). In addition to EXPRESS PEEP management, maintaining positive transpulmonary end expiratory pressure (PLee) could be relevant to improve outcomes.

Objectives. 1) To evaluate the association between EXPRESS PEEP and PLee in ventilated COVID-19 patients with ARDS and its repercussion in lung mechanics (transpulmonary driving pressure or DPL and lung compliance or $\mathrm{CL}$ ) and oxygenation (PaO2/FiO2). 2) To evaluate the response of those variables when prone positioning is needed.

Methods. Prospective, physiological and observational single-center study where patients were treated according to our unit ARDS protocol. COVID-19 patients with ARDS were included at intubation (ETI) when optimal PEEP by EXPRESS PEEP was selected. Esophageal pressure monitoring (nasogastric tube with esophageal balloon-catheter) was performed to calculate PLee. PLee and lung mechanics were measured after $1 \mathrm{~h}$ of intubation (T1) and oxygenation at $\mathrm{T} 1$ and after $5 \mathrm{~h}$ of ETI (T2). PLee was also measured in those patients who needed prone positioning after $1 \mathrm{~h}$ of the maneuver (T3). Oxygenation was evaluated at $24 \mathrm{~h}$ after prone positioning (T4). In all patients, lung mechanics were measured after 3 days of inclusion (T5). Patients were stratified regarding PLee at $\mathrm{T} 1$ (PLee $>0 \mathrm{cmH} 2 \mathrm{O}$ or PLee + vs PLee $\leq \mathbf{c m H} 2 \mathrm{O}$ or PLee-). Demographics, comorbidities and clinical data was also recorded. Statistical analysis was done using SPSS and $p<0.05$ was considered statistically significant. The study was approved by our ethics committee.

Results. Fifteen patients were included. At T1, 10 (67\%) patients were included in PL+ group vs 5 (33\%) patients in PL- group (Fig. 1A). No differences were shown in anthropometric, comorbidities and clinical parameters at T1. PL- group showed higher DPL and lower $\mathrm{CL}$ and $\mathrm{PaO} 2 / \mathrm{FiO} 2$ at $\mathrm{T} 1$ (Fig. 1B-D). Oxygenation improvement (DPaO2/ $\mathrm{FiO} 2$ ) between $\mathrm{T} 1$ and $\mathrm{T} 2$ was also lower in PL- group (Fig. 1E). While no patient in $\mathrm{PL}+$ group needed prone positioning at $\mathrm{T} 2$, all patients in PL- needed it. The maneuver resulted in a positivization of PLee in all these patients at T3 (Fig. 2A). This finding was accompanied by an improvement in oxygenation at T4 (Fig. 2B) and in lung mechanics at T5 (Fig. 2C).

Conclusion. When using EXPRESS PEEP to select optimal PEEP, taking PLee into account could help to improve patient management and outcomes. Protective mechanical ventilation should include higher PEEP with both targets: positive PLee and prone positioning if needed.

Figure 1: Transpulmonary end expiratory pressure, lung mechanics and oxigenation after PEEP EXPRESS setting in invasive mechanical ventilation of patients with COVID-19 ARDS.
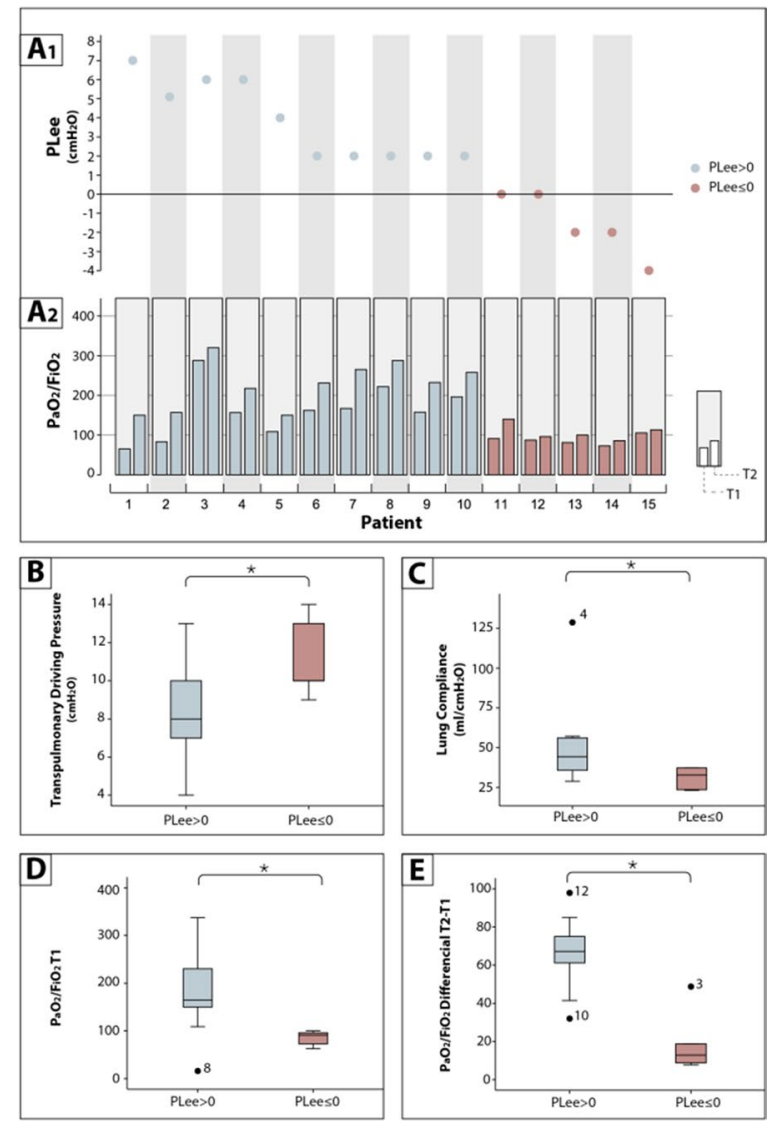

Dend expiratory pressure (PLee) at $\mathrm{T} 1$ is shown for each patient.

A2: $\mathrm{P}_{3} \mathrm{O}_{2} / \mathrm{F}_{1} \mathrm{O}_{2}$ at $\mathrm{T} 1$ and $\mathrm{T} 2$ is shown for each patient.

B: Boxplots of driving transpulmonary pressure for Plee $>0$ group and PLee s0 group at $\mathrm{T1}$. " $p<0.05$ between group by Mann-Whitney test

Compliance for PLee $>0$ group and PLee $\leq 0$ group at T1. " $p<0.05$ between groups by Mann-Whitn: Boxploso

test. 0 group and Phee s0 group at th of PEEP EXPRESS setting. "P< OS between groups by Mann-Whitney $\mathrm{U}$ test. 


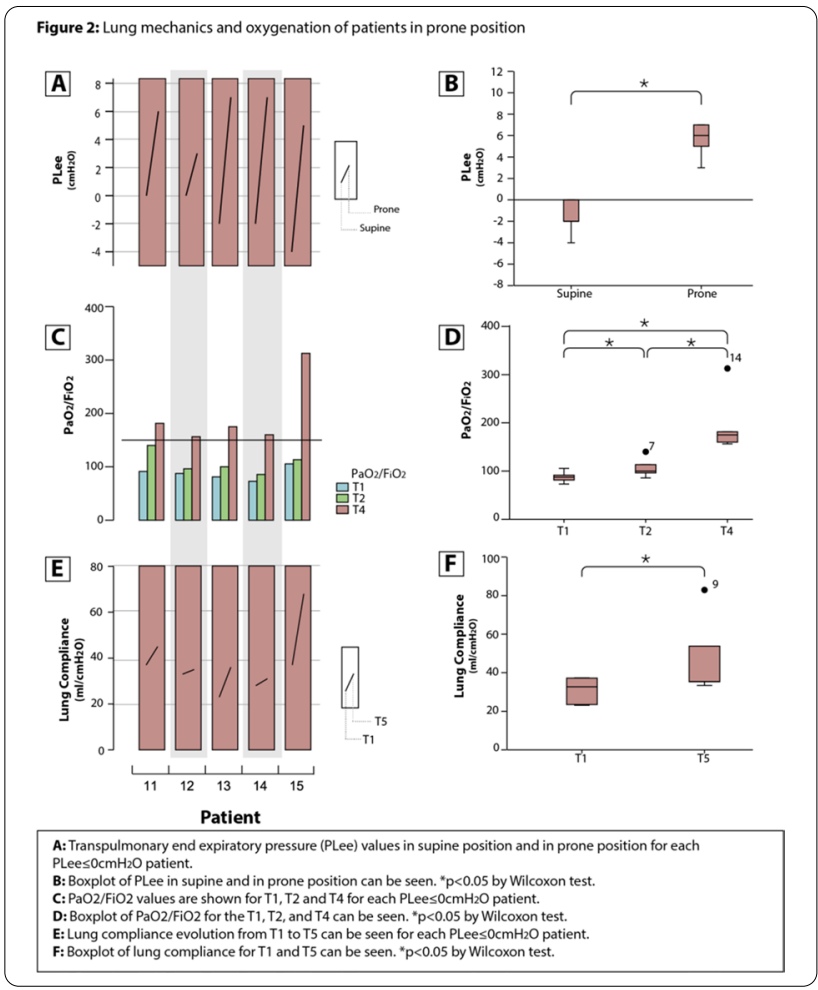

\section{Reference(s)}

1. Talmor D, Sarge T, Malhotra A, O'Donnell CR, Ritz R, Lisbon A, et al. Mechanical ventilation guided by esophageal pressure in acute lung injury. N Engl J Med [Internet]. 2008 Nov 13 [cited 2014 Aug 30];359(20):2095-104. Available from: http://www.pubmedcentral.nih. gov/articlerender.fcgi?artid=3969885\&tool=pmcentrez\&rendertype $=$ abstract

2. Mercat A, Richard JM, Vielle B, Jaber S, Osman D, Diehl J-L, et al. Positive end-expiratory pressure setting in adults with acute lung injury and acute respiratory distress syndrome: a randomized controlled trial. JAMA [Internet]. 2008 Feb 13;299(6):646-55. Available from: http://www.ncbi. nlm.nih.gov/pubmed/18270353

\section{9}

Contrast-enhanced ultrasound and Doppler ultrasound for evaluation of microcirculation in ischemia-reperfusion injury

C. Behem ${ }^{1}$; W. Schierling ${ }^{2}$; T. Friedheim ${ }^{1}$; H. Holthusen ${ }^{1}$; A. Rapp ${ }^{3}$;

T. Suntrop ; M. Gräßler '; HO. Pinnschmidt ${ }^{4}$; SH. Wipper ${ }^{5}$; K. Pfister ${ }^{2}$; CJC. Trepte

${ }^{1}$ Anesthesiology, University Medical Center Hamburg-Eppendorf, Hamburg, Germany; ${ }^{2}$ Vascular surgery, University Medical Center Regensburg, Regensburg, Germany; ${ }^{3}$ Cardiology, Rostock University Medical Center, Rostock, Germany; ${ }^{4}$ Medical biometry \& epidemiology, University Medical Center Hamburg-Eppendorf, Hamburg, Germany; ${ }^{5}$ Vascular surgery, Medical University of Innsbruck, Innsbruck, Austria

Correspondence: C. Behem

Intensive Care Medicine Experimental 2020, 9(1): 000899

Introduction. Microcirculatory perfusion is of utmost importance for tissue oxygenation and organ function with paramount implications for patient outcome in several diseases and clinical settings including ischemia/reperfusion. So far, there has been limited use of direct microcirculatory evaluation of vital organs due to limitations of existing methods. Recently, contrast enhanced ultrasound as well as
Doppler ultrasound have been proposed for microcirculatory evaluation of both liver and kidneys. $(1,2,3)$.

Objectives. Aim of this study was to evaluate the behaviour of hepatic and renal contrast enhanced ultrasound as well as Doppler ultrasound in experimental ischemia/reperfusion induced by aortic cross clamping.

Methods. In 16 pigs, ischemia/reperfusion was induced by supracoeliac aortic cross clamping. Contrast enhanced ultrasound was used to quantify time to peak while Doppler ultrasound was used to assess resistive index of both liver and kidney. In addition, laser speckle contrast imaging was used to assess hepatic and renal microcirculatory Flux. Macrohaemodynamic parameters were measured simultaneously. Measurements were performed at baseline, after reperfusion as well 4,5 h after ischemia/reperfusion. In addition, measurements were performed after haemodynamic optimization prior and after aortic cross clamping.

Results. Ischemia/reperfusion led to decreases of both hepatic as well as renal time to peak (18.42 (15.91-21.33) vs. $5.59(4.83-6.47)$ sec., $\mathrm{p}<0.001 ; 5.72(4.94-6.62)$ vs. $4.67(4.03-5.41)$ sec., $p<0.001$, respectively). Renal resistive index increased after ischemia/reperfusion $(0.66$ $(0.63-0.69)$ vs. $0.79(0.77-0.82), p<0.001)$. While hepatic Flux increased after ischemia/reperfusion (398.57 (336.80-460.33) vs. 465.92 (404.16527.68) p.u., $p=0.035)$, renal Flux decreased (807.41 (745.65-869.17) vs. 617.04 (555.28-678.80) p.u., $\mathrm{p}<0.001)$. Comparison of micro- and macrocirculatory parameters revealed loss of haemodynamic coherence. Renal as well as hepatic resistive index decreased following therapeutic interventions both prior and after ischemia/reperfusion.

Conclusion. Ischemia/reperfusion induced by aortic cross clamping led to marked decreases of both hepatic and renal contrast enhanced ultrasound derived time to peak as well an increase in Doppler ultrasound derived renal resistive index. Haemodynamic optimization influenced resistive index of liver and kidneys. Contrast enhanced ultrasound as well as Doppler ultrasound may be useful for assessment of microcirculatory behaviour in ischemia/reperfusion although further studies are needed to assess these effects.

\section{Reference(s)}

1. Schierling W, Wipper S, Behem CR, et al. Sonographic real-time imaging of tissue perfusion in a porcine haemorrhagic shock model. Ultrasound Med Biol 2019

2. Lima A, van Rooij T, Ergin B, et al. Dynamic Contrast-Enhanced Ultrasound Identifies Microcirculatory Alterations in Sepsis-Induced Acute Kidney Injury. Crit care med 2018;46:1284-92.

3. Harrois A, Grillot N, Figueiredo S, Duranteau J. Acute kidney injury is associated with a decrease in cortical renal perfusion during septic shock. Critical care 2018;22:161.

4. This study was supported by the Baxter-ESICM Fluid Management Award 2016.

\section{1}

\section{Oliguria in Critically III Patients: Impact on AKI Classification} and Outcomes Prediction

N. Bianchi' ${ }^{1}$ LL. Stavart ${ }^{2}$; M. Altarelli ${ }^{1}$; T. Kelevina ${ }^{1}$; M. Faouzi ${ }^{3}$; A. Schneider ${ }^{1}$ ${ }^{1}$ Adult intensive care unit, Lausanne University Hospital, Lausanne, Switzerland; ${ }^{2}$ Faculty of biology and medicine, University of Lausanne, Lausanne, Switzerland; ${ }^{3}$ Division of biostatistics, Center for Primary Care and Public Health (Unisanté), University of Lausanne, Switzerland, Lausanne, Switzerland

Correspondence: N. Bianchi

Intensive Care Medicine Experimental 2020, 9(1): 000921

Introduction. Current definition and staging of acute kidney injury (AKI) considers both serum creatinine ( $\mathrm{s} C \mathrm{Cr}$ ) and urinary output (UO) alterations. However, the relevance of oliguria-based criteria is disputed.

Objectives. To determine the contribution of oliguria, as defined by KDIGO criteria, to AKI diagnosis, severity assessment and mortality prediction. 
Methods. We conducted a cohort study including all adult patients admitted within our multi-disciplinary intensive care unit between January 1st 2010 and June 15th 2020. Daily sCr and hourly UO measurements along with socio-demographic characteristics and severity scores were extracted from our electronic medical charts. Long-term mortality was assessed by cross-referencing our database with the Swiss national death registry. We determined the onset and severity of AKI according to KDIGO classification using $\mathrm{UO}$ and $\mathrm{s} C \mathrm{Cr}$ criteria separately and assessed their agreement. Using a multivariable model accounting for baseline characteristics, severity scores and $\mathrm{s} C \mathrm{C}$ stages, we evaluated the relative influence of UO criteria on 90-day mortality. Sensitivity analyses were conducted to assess the impact of missing $\mathrm{sCr}$, body weight and $\mathrm{UO}$ values.

Results. Among the $15^{\prime} 620$ patients included in the study $\left[10^{\prime} 330\right.$ (66.1\%) males, median age 65.0 years (IQR, 53.0-75.0), median SAPS score 40.0 (IQR, 30.0-53.0), median follow-up 67.0 months (IQR, $34.0-100.0)], 12^{\prime} 143(77.7 \%)$ fulfilled AKI criteria. SCr and UO criteria had poor agreement on AKI diagnosis and staging (Cohen's weighted kappa $=0.36,95 \% \mathrm{Cl} 0.34-0.37, \mathrm{p}<0.001)$. Compared to the isolated use of $\mathrm{s} C \mathrm{Cr}$ criteria, consideration of $\mathrm{UO}$ criteria enabled to identify AKI in $5^{\prime} 630(36.0 \%)$ patients. Those patients had a higher 90 -day mortality than no-AKI patients (respectively $12.9 \%$ and $8.3 \%, p<0.001$ ). On multivariable analysis accounting for $\mathrm{s} C \mathrm{~S}$ stage, comorbidities and illness severity, UO stage 2 and 3 were associated with a higher 90-day mortality [OR 2.4 (1.6-3.8), $\mathrm{p}<0.001$, and $6.2(3.7-10.5), \mathrm{p}<0.001$, respectively]. These results remained significant in all sensitivity analyses.

Conclusion. Oliguria lasting more than $12 \mathrm{~h}$ (KDIGO stage 2 or 3) has major diagnostic and prognostic implications, irrespective of $\mathrm{sCr}$ elevations.

\section{Reference(s)}

1. The study was funded by the intensive care unit research fund.

\section{4}

Effectiveness of sleep promotion strategies in improving patients' sleep quality in intensive care-a systematic review

A. Carra'; J. Mellinghoff ${ }^{2}$

${ }^{1}$ Critical Care, St George's, University of London, London, United Kingdom; ${ }^{2}$ Faculty of health, social care and education, Kingston University London, London, United Kingdom

Correspondence: A. Carra

Intensive Care Medicine Experimental 2020, 9(1): 000964

Introduction. Sleep deprivation (SD) is the sustained lack of sleep resulting in physical and cognitive sequalae. It affects patients in intensive care units with possible negative effects on their immune system, impaired memory consolidation, altered cognitive capacity and attention, and emotions regulation. It may further lead to agitation, delirium, post-traumatic stress disorder and depression. While SD is mentioned in clinical practice guidelines, newer evidence in support of non-pharmacological interventions to improve it has recently been published.

Objectives. To investigate if the application of non-pharmacological sleep promotion interventions in comparison to usual care would be effective in improving subjective sleep quality in adults admitted to intensive care units (ICU).

Methods. A systematic literature review was undertaken to answer the research question with the aim to identify which strategies have been tested in improving sleep quality in adult critical care patients. The PICOS framework [1] was used to retrieve primary studies from medical databases including Embase, Medline, CINAHL and Cochrane. Inclusion criteria comprised worldwide studies performed between 2009 and 2020 and published in English language. Quality assessment of the articles was performed using the CASP-tool [2].

Results. Eleven studies $[3,4,5,6,7,8,9,10,11,12,13]$ were included for the review, four randomised controlled trials and seven quasiexperimental studies. The interventions identified included light and noise reduction methods using eye-mask (EM) and earplugs (EP) and a quiet-time protocol (QT), relaxation techniques such as musicotherapy and aromatherapy or combined strategies. Statistically significant results showing improved quality of sleep was reported by all the interventions apart from the QT protocol. The greatest effect was found by EM and EP. This was the most investigated intervention by seven studies, four of which were RCTs, presenting the highest level of evidence. The second most effective interventions were musicotherapy, investigated by one RCT, and aromatherapy by two quasi-experimental designs. The least effective were the combined strategies studies in particular one RCT investigating EM+EP with musicotherapy and one quasi-experimental design investigating musicotherapy with aromatherapy. Sleep quality was measured by validated tools such as the Richards-Campbell Questionnaire Score, Verran and Snyder-Halpern Scale and Medical Outcomes Study Scale.

Conclusion. All of the investigated interventions reported a significant improvement in sleep quality compared to standard care or no intervention except for the QT study. Although the interventions were reported as effective in improving sleep quality, the quality of evidence supporting the interventions is not strong. Further studies are needed to support the implementation of these interventions in clinical practice, in particular larger multicentre RCT.

\section{Reference(s)}

1. The author(s) received no financial support for the research, authorship, and/or publication of this study.

2. [1] Schardt, C. et al. Utilization of the PICO framework to improve searching Pubmed for clinical questions. BMC Medical Informatics and Decision Making, 2007, 7, 16-20. [2] Critical Appraisal Skills Programme. CASP Checklist, 2018 [online] Available at: https://casp-uk.net/casp-toolschecklists/ Date last accessed: 15/05.2021 [3] Su, C. et al. A randomized controlled trial of the effects of listening to non-commercial music on quality of nocturnal sleep and relaxation indices in patients in medical intensive care unit. Wiley, 2012, 69(6):1377-89 [4] Dave, K., Qureshi, A. and Gopichandran, L. Effects of Earplugs and Eye Masks on Perceived Quality of Sleep during Night among Patients in Intensive Care Units, Asian Journal of Nursing Education and Research,2015, 5(3), pp. 319-322. [5] Le Guen, M. et al. Earplugs and eye masks vs routine care prevent sleep impairment in post-anaesthesia care unit: a randomized study, British Journal of Anaesthesia: BJA, 2014, 112(1), pp. 89-95. [6] Hu, R. et al. Effects of earplugs and eye masks combined with relaxing music on sleep, melatonin and cortisol levels in ICU patients: a randomized controlled trial, Critical Care, 2015, 19(1), pp. 115. [7] Ebrahimi, T. et al. (2019) 'Effect of Eye Masks, Earplugs, and Quiet Time Protocol on Sleep Quality of Patients Admitted to the Cardiac Care Unit: A Clinical Trial Study', MedicalSurgical Nursing Journal, 2019, 8(3). [8] Younis, B., Mohammad, Ferial, H. A. and Hamza, A. Effectiveness of using eye mask and earplugs on sleep length and quality among intensive care patients: A quasi-experimental study, International Journal of Nursing Practice, 2019, 25(3), 127-140 [9] Pagnucci, N. et al. Promoting nighttime sleep in the intensive care unit: Alternative strategies in nursing, Intensive \& Critical Care Nursing, 2019, 51, 73-81. [10] Özlü, K. Z. and Bilican, P. Effects of aromatherapy massage on the sleep quality and physiological parameters of patients in a surgical intensive care unit, 2017, African Journal of Traditional, Complementary and Alternative Medicines 83-88. [11] Cho, E. H., Lee, M. and Hur, M. The Effects of Aromatherapy on Intensive Care Unit Patients'Stress and Sleep Quality: A Nonrandomised Controlled Trial, Evidence-Based Complementary and Alternative Medicine, 2017, pp. 2892-2910. [12] Scotto, C. J. et al. Earplugs improve patients' subjective experience of sleep in critical care, Nursing in Critical Care, 2009, 14(4), pp. 180-184. [13] Yazdannik, A. R. et al. The effect of earplugs and eye mask on patients' perceived sleep quality in intensive care unit, Iranian Journal of Nursing and Midwifery Research, 2014, 19(6), pp. 673-678.

\section{7}

Venovenous Extracorporeal Membrane Oxygenation in Patients with Acute Respiratory Failure from COVID-19

M. Urner'; AG. Barnett ${ }^{2}$; G. Li Bassi ${ }^{3}$; D. Brodie, ; HJ. Dalton, ; ND. Ferguson, ${ }^{1}$; S. Heinsarr ; C. Hodgson; ; G. Peek ${ }^{8}$; K. Shekar, ; JY. Suen, J. Fraser ${ }^{6}$; E. Fan ${ }^{1}$

${ }^{1}$ Interdepartmental division of critical care medicine, University of Toronto, Toronto, Canada; ${ }^{2}$ School of public health \& social work, QUT Gardens Point Campus, Brisbane City, Australia; ${ }^{3}$ Applied research 
in respiratory diseases, Institut d'Investigacions Biomèdiques August Pi i Sunyer (IDIBAPS), Barcelona, Spain; ${ }^{4}$ ntensive care, Columbia University College of Physicians and Surgeons/New York-Presbyterian Hospital, New York, United States of America; ${ }^{5} \mathrm{Heart}$ and vascular institute and department of pediatrics, Inova Fairfax Hospital, Falls Church, United States of America; ${ }^{6} \mathrm{Critical}$ care research group, adult intensive care services, The Prince Charles Hospital, Chermside, Australia; ${ }^{7}$ Department of epidemiology and preventive medicine, Monash University, Melbourne, Australia; ${ }^{8}$ Congenital heart center, University of Florida, Gainesville, United States of America

Correspondence: M. Urner

Intensive Care Medicine Experimental 2020, 9(1): 000977

Introduction. The optimal indications for extracorporeal membrane oxygenation (ECMO) and modifiers of treatment effectiveness in patients with acute respiratory failure from COVID-19 are currently unknown. Established protocols are largely based on a randomized controlled trial in patients without COVID-19 [1, 2].

Objectives. We emulated a pragmatic clinical trial using observational data from the COVID-19 Critical Care Consortium to determine the effectiveness of ECMO therapy compared to conventional mechanical ventilation and to analyze different clinical criteria for the decision to initiate ECMO in patients with acute respiratory failure from COVID-19.

Methods. Patients were included in the analysis if they were admitted to participating Intensive Care Units with clinically suspected or laboratory-confirmed SARS-CoV-2 infection between January 3, 2020, and January 26,2021 . We compared the receipt of usual care, which included treatment with ECMO if deemed clinically indicated, to treatment with conventional mechanical ventilation without the use of ECMO. The primary outcome was hospital mortality up to 60 days after intensive care unit admission. Adherence-adjusted estimates were calculated using marginal structural models with inverse probability weighting, accounting for baseline and time-varying confounding, as well as for competing events (death versus hospital discharge) $[3,4]$.

Results. A total of 3,248 patients from 25 countries were eligible for analysis. Hospital mortality was 38.2\% (95\% confidence interval [Cl]: $36.1 \%$ to $40.3 \%$ ) under usual care compared to $40.7 \%$ ( $95 \%$ $\mathrm{Cl}: 35.7 \%$ to $45.8 \%$ ) had patients received conventional mechanical ventilation without ECMO (risk ratio $0.94 ; 95 \% \mathrm{Cl} 0.84$ to 1.05 ). ECMO therapy would have been most effective if provided to patients with age $\leq 65$ years and ratio of arterial partial pressure of oxygen-to-fraction of inspired oxygen $(\mathrm{PaO} 2 / \mathrm{FiO} 2) \leq 80 \mathrm{mmHg}$ (risk ratio $0.58,95 \%$ $\mathrm{Cl}: 0.45$ to 0.76 ) or with driving pressures $>15 \mathrm{cmH} 2 \mathrm{O}$ (risk ratio 0.73 , $95 \% \mathrm{Cl}: 0.60$ to 0.89 ) during the first 10 days of mechanical ventilation. Conclusion. Age and severity of hypoxemia, as well as the duration and intensity of mechanical ventilation should be considered when deciding to initiate ECMO in patients with COVID-19.

\section{Reference(s)}

1. [1] Combes, A., Hajage, D., Capellier, G., Demoule, A., Lavoué, S., Guervilly, C., ... \& Mercat, A. (2018). Extracorporeal membrane oxygenation for severe acute respiratory distress syndrome. New England Journal of Medicine, 378(21), 1965-1975.

2. [2] Bartlett, R. H., Ogino, M. T., Brodie, D., McMullan, D. M., Lorusso, R., MacLaren, G., .. \& Paden, M. L. (2020). Initial ELSO guidance document: ECMO for COVID-19 patients with severe cardiopulmonary failure. Asaio Journal, 66(5), 472.

3. [3] Hernán, M. A. (2018). How to estimate the effect of treatment duration on survival outcomes using observational data. bmj, 360:k182

4. [4] Young, J. G., Stensrud, M. J., Tchetgen Tchetgen, E. J., \& Hernán, M. A. (2020). A causal framework for classical statistical estimands in failuretime settings with competing events. Statistics in medicine, 39(8), 1199-1236

5. We recognize the crucial importance of the ISARIC and SPRINT-SARI networks for the development and expansion of the COVID-19 Critical Care Consortium. We acknowledge all members of the COVID-19 Critical Care Consortium and various collaborators. Dr. Urner is supported by a Vanier Canada Graduate Scholarship from the Canadian Institutes of Health Research. Dr Barnett is supported by a National Health and
Medical Research Council fellowship. Dr. Heinsar is supported by a PhD Scholarship from the Prince Charles Hospital Foundation. Dr. Fan reports personal fees from Abbott, ALung Technologies, Fresenius Medical Care, Getinge, MC3 Cardiopulmonary, and Vasomune outside the submitted work. Dr. Ferguson reports personal fees from Baxter, Getinge, and Xenios, outside the submitted work. Dr. Brodie receives research support from ALung Technologies. He reports personal fees from Baxter, Abiomed, and Xenios; and has been on the medical advisory board of Hemovent. Dr. Fraser reports grant support from FP, Xenios, Getinge, Cynata, CSL and Queensland Health outside the submitted work. Dr Hodgson is supported by a National Health and Medical Research Council Investigator Grant from Australia. Dr Shekar acknowledges research support from Metro North Hospital and Health Service. Dr. Suen is supported by the Advance Queensland Fellowship from the Queensland Government. The COVID-19 Critical Care Consortium received funding from The University of Queensland, Wesley Medical Research, The Prince Charles Hospital Foundation, Queensland Department of Health, The Health Research Board of Ireland; Biomedicine international training research programme for excellent clinician-scientists; European Union's research and innovation programme (Horizon 2020); la Caixa Foundation and Fisher and Paykel Healthcare. The funders had no role in study design, data collection, data analysis, data interpretation, or writing of the report.

\section{8}

Anti-inflammatory treatment in ovine hypoand and hyperinflammatory ARDS subphenotypes: An experimental study

K. Wildi'; C. Ainola ${ }^{2}$; S. Livingstone ${ }^{2}$; SM. Colombo ${ }^{3}$; N. Sato ${ }^{2} ;$ K. Sato $^{2}$. S. Heinsar'; G. Abbate'; M. Bouquet'; K. Hyslop²; E. Wood²; M. Passmore²; G. Libassi²; J. Suen'; J. Fraser'

${ }^{1}$ CCRG, Clinical Sciences, The Prince Charles Hospital, Chermside, Australia; ${ }^{2}$ Critical care research group, The Prince Charles Hospital, Chermside, Australia; ${ }^{3}$ Department of pathophysiology and transplantation, University of Milan, Milan, Metropolitan City of Milan, Italy, Italy; ${ }^{4}$ Critical care research group, adult intensive care services, The Prince Charles Hospital, Chermside, Australia

Correspondence: K. Wildi

Intensive Care Medicine Experimental 2020, 9(1): 000978

Introduction. Acute Respiratory Distress Syndrome (ARDS) is a lifethreatening condition without treatment available to reduce the associated high mortality. Retrospective analyses of $>4000$ patients provided evidence for the presence of two distinct subphenotypes, based on clinical and biological variables (1-5): namely, a hypoinflammatory (P1) and a hyperinflammatory (P2) subphenotype. In our laboratory, we developed ovine models of $\mathrm{P} 1$ and $\mathrm{P} 2 \mathrm{ARDS}$ subphenotypes as reported previously (6).

Objectives. In a highly controlled pre-clinical trial, we aimed to evaluate in these models the effectiveness of anti-inflammatory treatment with corticosteroids and erythromycin on oxygenation and haemodynamic parameters.

Methods. We studied 30 anesthetized sheep on mechanical ventilation up to $48 \mathrm{~h}$, and randomized into two distinct subphenotypes: 1) P1 received an intravenous infusion (IV) of oleic acid (OA) $(n=15)$; 2) P2 received IV OA and subsequent IV infusion of lipopolysaccharide $(n=15)$ to achieve a ratio between partial pressure of oxygen and inspiratory fraction of oxygen $(\mathrm{PaO} 2 / \mathrm{FiO} 2)$ of $<150 \mathrm{mmHg}$. After development of ARDS, animals were further randomly allocated to receive either a bolus of $100 \mathrm{mg}$ of methylprednisolone IV followed by a $48 \mathrm{~h}$ intravenous infusion of $2 \mathrm{mg} / \mathrm{kg} / 24 \mathrm{~h}$ (P2-pred and P1-pred), erythromycin $100 \mathrm{mg}$ IV (P2-ery and P1-ery) or no treatment (control: $\mathrm{P} 2-\mathrm{ctr}$ and P1-ctr). Clinicians conducting the experiment were blinded to treatment groups. Pulmonary mechanics and hemodynamic parameters were assessed hourly, blood sampling and bronchoscopy with bronchoalveolar lavage (BAL) was performed in regular intervals. Results. Severe impairment in $\mathrm{PaO} 2 / \mathrm{FiO}_{2}$ was found at the time of ARDS diagnosis in all animals. As shown in Fig. 1, treatment with corticosteroids provided the highest therapeutic benefit in the P2-pred group, leading to a significant increase in $\mathrm{PaO} 2 / \mathrm{FiO} 2$ by reducing 
pulmonary edema (extravascular lung water index: EVLWI), dead space ventilation and shunt fraction. In addition, a significant increase in mean arterial pressure and decrease in heart rate was evident in P2-pred and P1-pred with a trend towards better values in P2-pred. Fitting a linear mixed-effect model, these differences persisted with a significant effect of time and group:time interaction. In P2-ery and P1-ery, there was no obvious treatment effect while in some parameters, erythromycin even appeared to be deleterious. Upon autopsy, wet-dry lung tissue ratio was comparable among groups.

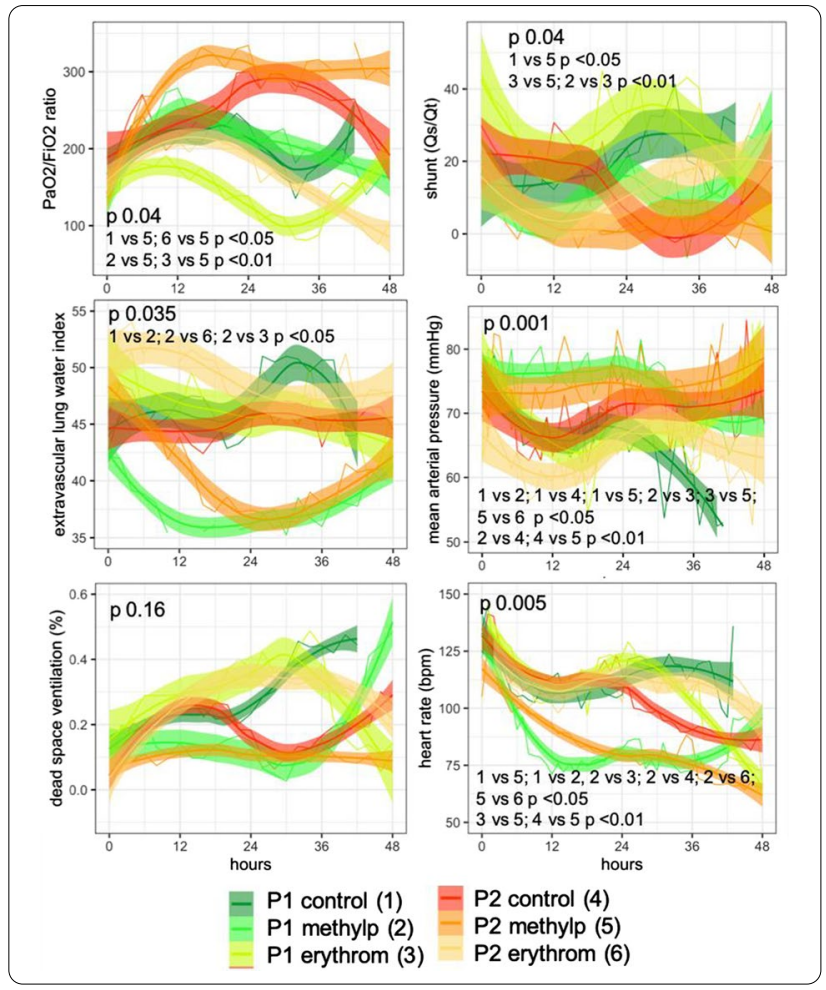

Conclusion. Treatment with methylprednisolone improves oxygenation and hemodynamic parameters, especially in the ovine hyperinflammatory ARDS subphenotype, proposing personalized early treatment for ARDS patients.

\section{Reference(s)}

1. Famous KR, Delucchi K, Ware LB, Kangelaris KN, Liu KD, Thompson BT, et al. Acute respiratory distress syndrome subphenotypes respond differently to randomized fluid management strategy. Am J Respir Crit Care Med. 2017;195(3):331-8.

2. Sinha P, Delucchi KL, Thompson BT, McAuley DF, Matthay MA, Calfee CS. Latent class analysis of ARDS subphenotypes: a secondary analysis of the statins for acutely injured lungs from sepsis (SAILS) study. Intensive Care Med [Internet]. 2018;44(11):1859-69.

3. Calfee CS, Delucchi K, Parsons PE, Taylor B, Ware LB, Matthay MA. Latent Class Analysis of ARDS Subphenotypes: Analysis of Data From Two Randomized Controlled Trials. Lancet Respir Med. 2014;2(8):611-20.

4. Calfee CS, Delucchi KL, Sinha P, Matthay MA, Hackett J, Shankar-Hari M, et al. Acute respiratory distress syndrome subphenotypes and differential response to simvastatin: secondary analysis of a randomised controlled trial. Lancet Resp Med [Internet]. 2018;0(0):1-8.

5. Bos LD, Schouten LR, van Vught LA, Wiewel MA, Ong DSY, Cremer O, et al. Identification and validation of distinct biological phenotypes in patients with acute respiratory distress syndrome by cluster analysis. Thorax [Internet]. 2017;72(10):876-83.

6. Jonathan E Millar, Wildi K, Bartnikowski N, Bouquet M, Hyslop K, Passmore $M R$, et al. Characterising pre-clinical sub-phenotypic models of Acute
Respiratory Distress Syndrome: an experimental ovine study. BioRxiv. 2020;

7. The study was supported by the Prince Charles Hospital Foundation and funding from CRE Action (NHMRC).

\section{2}

Current knowledge of attitudes towards basic and advanced pediatric and adult life support among emergency department, emergency medical service, and public health unit personnel in Crete

G. Briassoulis'; A. Papoutsakis²; P. Briassoulis ${ }^{3}$; M. Miliaraki ${ }^{3}$ E. Briassouli4; P. Aggouridakis²; S. llia

${ }^{1}$ Postgraduate program "emergency and intensive care of children, adolescents and young adults", University of Crete, School of Medicine, PICU, Heraklion, Greece; ${ }^{2}$ Postgraduate program "emergency and intensive care of children, adolescents and young adults", University of Crete, School of Medicine, Heraklion, Greece; ${ }^{3}$ Pediatric intensive care unit, university hospital, University of Crete, School of Medicine, Heraklion, Greece; ${ }^{4}$ Pediatric department children hospital "aghia sophia", National and Kapodistrian University of Athens, Athens, Greece

Correspondence: S. Ilia

Intensive Care Medicine Experimental 2020, 9(1): 000982

Introduction. It is currently well appreciated that a medical team's level of knowledge regarding emergency situations defines the quality of health care services. Studies have also highlighted the importance of a lifelong and ongoing training of medical and nursing staff, based on clinical scenarios for children and adults, and with a structured approach.

Objectives. The present study evaluated a tertiary Hospital Emergency Department (ED), the National Ambulance Service (NAS), the Health Centers (HC) and the Local Public Health Services (LPHS) in the prefecture of Heraklion, with regard to their medical staff's knowledge on the necessary structured approach for severe disease, trauma or cardiac arrest emergencies in children and adults. In addition, medical practitioners' self-assessments and perspectives on the emergency medicine related research were also recorded.

Methods. This 6-month cross sectional study was based on a targeted survey questionnaire, in order to collect extensive feedback from the clinical staff in ambulatory settings (ED, NAS, HC, and LPHS) regarding their knowledge on the latest ALS, APS, BLS, PLS and APLS protocols. Results. Among the survey participants ( $48.3 \%$ doctors, $39.6 \%$ nurses and $12.1 \%$ paramedics), a superiority of medical staff in the ED or NAS was recorded, with a $65.1 \%$ education level in specialized adult life support, compared to HC or LPHS $(p=0.019)$, while the corresponding education rates in pediatric life support $(P L S)$ and in advanced pediatric life support (APLS) were only $12.1 \%$ and $6 \%$ respectively. Last training in BLS was recorded $11.5 \pm 7.9$ years ago, specialized support $4.7 \pm 6$ years ago, while $8.1 \%$ have not attended any emergency seminar (LPHS 25\%, HC 10\%, $\mathrm{p}=0.046$ ). More frequent repetitive seminars were recorded among rescuers $(77.8 \%)$ compared to doctors $(38.1 \%)$ or nurses $(39.7 \%, p=0.003)$. In everyday practice, there were significant differentiations of the cases in which workers had to apply CPR $(p<0.002)$, administer oxygen $(p<0.001)$, resuscitation drugs $(p<0.005)$, or attach the defibrillator $(p<0.001)$ among the different centers. More than half of the participants were unaware of the initial approach to a victim with loss of consciousness or sepsis, convulsions $(56.4 \%)$, burns $(42.3 \%)$, drowning $(25.5 \%)$, or supraventricular tachycardia (35.6\%). Survey participants characterized their basic knowledge in BLS and PLS as moderate or below average, while a high demand for continuing lifelong education was recorded, particularly among physicians (98.6\%) compared to nurses (96.6\%) and rescuers $(83.3 \%, p=0.012)$.

Conclusion. The results of this study highlight the inadequate level of training of medical, nursing staff, and rescuers working in any emergency reception structure, with little education on pediatric emergency protocols. Medical practitioners characterize their knowledge as moderate and express their desire for continuous education on adult and pediatric emergencies. 
001011

Predicting preload responsiveness by decreasing positive end-expiratory pressure in mechanically ventilated patients in intensive care unit: the PEEP-test study

C. Lai ${ }^{1}$; R. Shi'; JL. Teboul'; F. Moretto ${ }^{1}$; S. Ayed, ${ }^{1}$; A. Pavot ${ }^{1}$; A. Beurton²; N. Anguel, ${ }^{1} ;$ T. Pham $^{3} ;$ X. Monnet $^{1}$

${ }^{1}$ Médecine intensive —réanimation, inserm umr s_999, fhu sepsis, groupe de recherche carmas, Bicêtre Hospital AP-HP Université Paris-Saclay DMU CORREVE, Le Kremlin-Bicêtre, France; ${ }^{2}$ Service de pneumologie et réanimation médicale, University Hospitals Pitié Salpêtrière-Charles Foix, Paris, France; ${ }^{3}$ Médecine intensive-réanimation, inserm umr u1018, fhu sepsis, groupe de recherche carmas, Bicêtre Hospital AP-HP Université Paris-Saclay DMU CORREVE, Le Kremlin-Bicêtre, France

Correspondence: C. Lai

Intensive Care Medicine Experimental 2020, 9(1): 001,011

Introduction. In mechanical ventilated patients, positive end-expiratory pressure (PEEP) decreases cardiac preload by increasing the right atrial pressure and increases right ventricle afterload.

Objectives. We hypothesized that decreasing the level of PEEP (PEEP-test) may be used as a preload challenge to detect preload responsiveness.

Methods. In patients under mechanical ventilation with no spontaneous breathing and PEEP $\geq 10 \mathrm{cmH} 2 \mathrm{O}$ ("high level"), preload responsiveness was assessed by a passive leg raising (PLR) maneuver. Preload responsiveness was defined as an increase in cardiac index $(\mathrm{Cl})$ during $P L R \geq 10 \%$. The PEEP-test consisted in reducing PEEP from high level to $5 \mathrm{cmH} 2 \mathrm{O}$. Pulse-contour-derived $\mathrm{Cl}$ (PiCCO2) were monitored during PLR and PEEP-test.

Results. We enrolled 59 patients among whom 27 were preload responsive (median increase in Cl during PLR: 14\% (interquartile range (IQR): 11-18\%). The median PEEP level at baseline was 12 (IQR: 11-15) $\mathrm{CmH} 2 \mathrm{O}$ and the PEEP-test resulted in a median decrease of PEEP by 7 (IQR: 6-10) $\mathrm{cmH} 2 \mathrm{O}$, with no difference between preload responsive and unresponsive patients. Among preload responsive patients, PEEP-test induced an median increase in $\mathrm{Cl}$ of 14 (IQR: 9-18)\% (from $2.4 \pm 0.7$ to $2.8 \pm 0.8 \mathrm{~L} / \mathrm{min} / \mathrm{m} 2, \mathrm{p}<0.0001)$. In preload unresponsive patients, PLR and PEEP-test increased $\mathrm{Cl}$ by respectively $3(1-6) \%$ and $6(2-8) \%$, respectively. These changes were significantly lower than in preload responsive patients. Preload responsiveness was predicted by an increase in $\mathrm{Cl} \geq 8 \%$ during PEEP-test with a sensitivity of $96 \%$ (95\% confidence interval: $81-99 \%)$ and a specificity of 78 (95\%Cl: $60-91) \%$. The mean area under the curve of PEEP-test for detecting preload responsiveness was 0.91 (standard deviation (SD): 0.05) $(p<0.0001$ vs. 0.5). The Spearman's correlation coefficient between the PLR- and the PEEP-test-induced changes in $\mathrm{Cl}$ was 0.81 (95\% Cl: $0.70-0.89$, $\mathrm{p}<0.0001)$.

Conclusion. The $\mathrm{Cl}$ increases during a PEEP-test, which consists in reducing PEEP to $5 \mathrm{cmH} 2 \mathrm{O}$, reliably detect preload responsiveness in mechanically ventilated patients with a PEEP level $\geq 10 \mathrm{cmH} 2 \mathrm{O}$.

\section{4}

Role of the Activities of Daily Living in outcome prediction of very old intensive care patients suffering from COVID-19

RR. Bruno'; B. Wernly²; H. Flaaten ${ }^{3}$; J. Fjølner'; A. Artigas ${ }^{5}$; B. Bollen Pinto ${ }^{6}$; S. Joerg ${ }^{7}$; PH. Baldia'; S. Binneboessel' ${ }^{1}$; M. Kelm ${ }^{1}$; M. Beil ${ }^{8}$; S. Sigal ${ }^{\text {9 }}$; P. Vernon Van Heerden ${ }^{10}$; J. Wollborn ${ }^{11}$; D. De Lange ${ }^{12}$; W. Szczeklik ${ }^{13}$; B. Guidet ${ }^{14} ;$ S. Leaver ${ }^{15} ;$ C. Jung

${ }^{1}$ Cardiology, Pulmonary Diseases, Vascular Medicine, Universität Düsseldorf, Düsseldorf, Germany; ${ }^{2}$ Department of department of anaesthesiology, perioperative medicine and intensive care medicie, Paracelsus Medical University, Salzburg, Austria; ${ }^{3}$ Department of anaestesia and intensive care, University of Bergen, Bergen, Norway; ${ }^{4}$ Department of intensive care, Aarhus University Hospital, Aarhus, Denmark; ${ }^{5}$ Department of intensive care medicine, Corporacion Sanitaria Universitaria Parc Tauli, Barcelona, Spain; ${ }^{6}$ Anesthesiology, Hôpitaux Universitaires de Genève (HUG), Genève, Switzerland; ${ }^{7}$ Medical surgical intensive care unit, University Hospital of Bern, Bern, Switzerland; ${ }^{8}$ Medical intensive care unit, Hadassah Medical Center, London, United Kingdom; ${ }^{9}$ Medical intensive care unit, Hadassah Medical Center, Jerusalem, Israel, Israel; ${ }^{10}$ Department of anesthesiology, critical care and pain medicine, Hadassah Medical Center, The Hebrew University of Jerusalem, Jerusalem, Israel; ${ }^{11}$ Critical care, Harvard Medical School, Boston, United States of America;

${ }^{12}$ Department of intensive care medicine, Utrecht University, Utrecht, Netherlands; ${ }^{13}$ Intensive care and perioperative medicine, Jagiellonian University Medical College, Kraków, Poland; ${ }^{14}$ Réanimation Médicale, Hôpital Saint-Antoine, Paris, France; ${ }^{15}$ Research lead critical care directorate, St George's Hospital, London, United Kingdom

Correspondence: R.R. Bruno

Intensive Care Medicine Experimental 2020, 9(1): 001,014

Introduction. Critically ill old ICU patients suffering from COVID-19 are at increased risk for adverse Outcome. Guidelines recommend using the assessment of Activities of Daily Living (ADL) for risk stratification, but the evidence is low.

Objectives. This study investigates the value of ADL for outcome prediction in this particular vulnerable subgroup.

Methods. The COVIP study is a prospective international observational study that recruited ICU patients 70 years and older admitted with COVID-19 disease. Several parameters, including the Activities of Daily Living (ADL), Clinical Frailty Scale (CFS), SOFA score, usual ICU procedures, limitation of care, ICU- and 3 months survival were recorded. The study was registered at ClinicalTrials.gov (ID: NCT04321265).

Results. This prespecified subgroup analysis included 2379 patients with a documented ADL. Most patients did not evidence any dependence in their daily living before hospital admission. With rising independence, patients were older, more frail, and suffered significantly more from comorbidities and higher SOFA. Patients with an ADL $>4$ evidenced significantly lower ICU mortality and 3-month-mortality. In univariate Cox regression analysis, the $\mathrm{ADL}$-Score was inversely associated with ICU mortality (OR 0.91 ( $95 \% \mathrm{Cl} 0.88-0.94, \mathrm{p}<0.001)$. After adjustment for SOFA, age, gender, and treatment limitations, the ADL-score was statistically significantly associated with ICU mortality (aOR $0.93(95 \% \mathrm{Cl} 0.90-0.97, \mathrm{p}<0.001)$. After additional adjustment for CFS, no significant association with ICU-mortality remained (aOR 0.98 $(95 \% \mathrm{Cl} 0.93-1.03, \mathrm{p}=0.384)$. The same observation was true for threemonth mortality and $A D L$ as a binary variable.

Conclusion. In critically ill old intensive care patients suffering from COVID-19, ADL provides limited additional information for outcome prediction in addition to CFS.

\section{Reference(s)}

1. Intensive Care Med. 2020 Jan:46(1):57-69. https://doi.org/10.1007/ s00134-019-05853-1.

2. Crit Care. 2021 Apr 19;25(1):149. https://doi.org/10.1186/ s13054-021-03551-3.

\section{6}

Moving from bytes to bedside: artificial intelligence in the ICU, where do we stand?

D. Van De Sande'; M. van Genderen '; J. Huiskens²; D. Gommers ${ }^{1}$; J. Van Bommel $^{1}$

'Department of intensive care, Erasmus MC, Rotterdam, Netherlands; ${ }^{2}$ Health care analytics, SAS Institute, Huizen, Netherlands

Correspondence: D. Van De Sande

Intensive Care Medicine Experimental 2020, 9(1): 001,056

Introduction. Due to the increasing demand for intensive care unit (ICU) treatment, and to improve quality and efficiency of care, there is a need for adequate and efficient clinical decision-making $(1,2)$. The advancement of artificial intelligence (Al) technologies has resulted in the development of prediction models, which might aid clinical decision-making. As a result, the number of publications increased rapidly in the recent years (3). However, at this moment it remains undetermined whether ICU patients already clinically benefit from Al. In addition, the research methods behind these studies have not been described in detail and the risk of bias is unknown. 
Objectives. To give a contemporary overview of the current maturity of $\mathrm{Al}$ in the ICU, the research methods behind these studies, and the risk of bias in these studies.

Methods. A systematic search was conducted in Embase, Medline, Web of Science Core Collection and Cochrane Central Register of Controlled Trials databases to identify eligible studies. Studies using Al to analyze ICU data were considered eligible. Specifically, the study design, study aim, dataset size, level of validation, level of clinical readiness, and the outcomes of clinical trials were extracted. The level of clinical readiness was assessed over time, by applying the general concept of technology readiness levels introduced by National Aeronautics and Space Administration (NASA), which previously has been translated to the ICU environment $(4,5)$. Risk of bias in retrospective studies was evaluated by the Prediction model Risk Of Bias ASsessment Tool (PROBAST) (6).

Results. Out of 6,455 studies identified through literature search, 494 were included. The most common study design was retrospective (476 studies [ $96.4 \%$ of all studies]) followed by prospective (8 $[1.6 \%])$ and observational $(10[2.0 \%])$ trials. Most studies aimed to predict complications (110 studies [22.2\%]) and mortality (102 studies [20.6\%]). In total 441 studies $(89.3 \%)$ scored level 4 or below (that is, the development \& prototyping environment) on the 'level of readiness' scale. No studies were identified that reported on the outcome evaluation of an Al model integrated in routine clinical practice. $378(80.9 \%)$ retrospective studies were classified as high risk of bias.

Conclusion. The vast majority of developed ICU-AI models remain within the testing and prototyping environment; only a handful were actually evaluated in clinical practice. Over time, the direction of the maturation of ICU-AI is mainly horizontal rather than diagonal, i.e. expansion of retrospective models instead of moving towards the clinical implementation. A uniform and structured approach can support the development, safe delivery, and implementation of $\mathrm{Al}$ to determine clinical benefit in the ICU.

\section{Reference(s)}

1. Fleuren LM, Thoral P, Shillan D, Ercole A, Elbers PWG, Collaborators RDRN. Machine learning in intensive care medicine: ready for take-off? Intens Care Med. 2020;46(7):1486-8.

2. JC M. Technology readiness levels 1995. Available from: http://www.artem isinnovation.com/images/TRL_White_Paper_2004-Edited.pdf.

3. Shillan D, Sterne JAC, Champneys A, Gibbison B. Use of machine learning to analyse routinely collected intensive care unit data: a systematic review. Crit Care. 2019;23(1):284

4. Adhikari NK, Rubenfeld GD. Worldwide demand for critical care. Curr Opin Crit Care. 2011:17(6):620-5.

5. Adhikari NK, Fowler RA, Bhagwanjee S, Rubenfeld GD. Critical care and the global burden of critical illness in adults. Lancet. 2010;376(9749):1339-46.

6. Moons KGM, Wolff RF, Riley RD, Whiting PF, Westwood M, Collins GS, et al. PROBAST: A Tool to Assess Risk of Bias and Applicability of Prediction Model Studies: Explanation and Elaboration. Ann Intern Med. 2019:170(1):W1-W33.

7. There were no funding sources.

\section{1}

Pain measurement variation in critically ill patients throughout time of day in different admission groups in a general

ICU

E. Giannelou'; E. Papathanassoglou²; M. Karanikola'; M. Giannakopoulou ${ }^{3}$; E. Bozas ${ }^{3}$; N. Middleton ${ }^{1}$; M. Mpouzika'

${ }^{1}$ Nursing department, Cyprus University of Technology, Limassol, Cyprus; ${ }^{2}$ Faculty of nursing, University of Alberta, Edmonton, Canada; ${ }^{3}$ Nursing department, National and Kapodistrian University of Athens, Athens, Greece

Correspondence: E. Giannelou

Intensive Care Medicine Experimental 2020, 9(1): 001061

Introduction. Critically ill patients experience pain during their hospitalization in Intensive Care Unit (ICU) as many painful procedures are performed, mainly in the morning [1-3]. Pain assessment is challenging because numerous patients cannot express themselves verbally $[4,5]$.

Objectives. The Critical Care Pain Observation Tool (CPOT) was used to measure pain in uncommunicative critically ill patients [1-4] before, during and $20 \mathrm{~min}$ after the turning procedure both at morning and afternoon and, among groups with different reason for admission.

Methods. A total of 1164 observations were carried out in this prospective, observational study with repeated measures. 121 adult surgical, medical, trauma and burn critically-ill patients were followed up for 5 days. Pain was assessed by CPOT at rest just before, during and 20 min after turning procedure, twice daily, morning and afternoon.

Results. In the morning measurement, total mean CPOT score (Mean + SD) of 121 patients during turning procedure was $1.84(0.84)$ on the 1st day, 1.91(0.89), 1.83(0.83), 1.68(0.85) and 1.54(1.02) for the next 4 days respectively $(p=0.001)$. In the afternoon, total mean CPOT score during turning was $1.82(0.86), 1.82(0.86), 1.61(0.89), 1.46(0.91)$, $1.43(0.97)$ on each day respectively $(p<0.001)$. At the 1 st and 2 nd morning, burn patients had the highest mean CPOT score $[3.00(0.58)$, $2.63(0.92)]$ when compared to surgical $[1.60(0.74), 1.57(0.77)]$, medical $[1.86(0.85), 2.06(0.86)]$ and trauma $[1.89(0.80), 2.00(0.95)]$ respectively $(p=0.001)$. Mean CPOT scores during turning among groups was also significant in the 1st and 5th afternoon measurements. More specifically, at the 1st and 5th afternoon, burn patients had the highest mean CPOT score [3.14(0.59), 2.00(0.58)] when compared to surgical $[1.49(0.64), 1.11(1.08)]$, medical $[1.94(0.87), 1.43(0.95)]$ and trauma $[1.83(0.85), 1.69(0.79)]$ respectively $(p=0.004)$

Conclusion. During the first two days of ICU admission, mean pain scores did not differ between morning and afternoon measurement, whereas, in the fifth afternoon mean CPOT score was higher for the burn patients. Time of day and reason for admission could be further examined in ICU patients' pain assessment and management. Pain treatment protocols initiated upon ICU admission could be considered for further investigation as patients seem to experience pain from the 1 st day.

\section{Reference(s)}

1. Gelinas C, Arbour C, Michaud C, Vaillant F, Desjardins S, Implementation of the critical-care pain observation tool on pain assessment/ management nursing practices in an intensive care unit with nonverbal critically ill adults: a before and after study. Int J Nurs Stud. 2011; 48(12): 1495-504. https://doi.org/10.1111/j.1478-5153.2008.00294.x

2. Gelinas C, Melody R, Boitor M et al., Nurses' evaluation of the CPOT use at 12-month post implementation in the intensive care unit. Brit Assoc Crit Care Nurs. 2014; 19(6): 272-280. https://doi.org/10.1111/nicc.12084

3. Payen JF, Gelinas C, Measuring pain in non-verbal critically ill patients: which pain instrument? Crit Care. 2014; 18(5): 554. https://doi.org/10. 1186/s13054-014-0554-5412

4. Georgiou E, Paikousis L, Lambrinou E, Merkouris A, Papathanassoglou $E D E$, The effectiveness of systematic pain assessment on critically ill patient outcomes: A randomised controlled trial. Aust Crit Care. 2020; 33(5): 412-419. https://doi.org/10.1016/j.aucc.2019.09.004

5. Puntillo KA, Max A, Timsit JF et al., Determinants of procedural pain intensity in the intensive care unit. The Europain study. Am J Respir Crit Care Med. 2014; 189(1): 39-47. https://doi.org/10.1164/rccm.201306-11740C

\section{9}

Beneficial Effect of Prone Positioning During Venovenous Extracorporeal Membrane Oxygenation for COVID-19

A. Zaaqoq ${ }^{1}$; AG. Barnett ${ }^{2}$; M. Griffee, ${ }^{3}$; G. Maclaren, ; J. Jacobs, ; S. Heinsar, ; JY. Suen, ${ }^{7} ;$ G. Li Bassi ${ }^{2}$; J. Fraser ${ }^{8}$; HJ. Dalton, ${ }^{9} ;$ G. Peek ${ }^{10}$

${ }^{1}$ Critical Care Medicine, MedStar Washington Hospital Center, Washington, United States of America; ${ }^{2}$ School of public health \& social work, QUT Gardens Point Campus, Brisbane City, Australia; ${ }^{3}$ Anesthesia, University of Utah School of Medicine, SALT LAKE, United States of America; ${ }^{4}$ Cardiothoracic intensive care unit, National University of Singapore, singapore, Singapore; ${ }^{5}$ Department of surgery, University of Florida, Gainesville, United States of America; ${ }^{6}$ Critical care research group, University of Queensland and The Prince Charles Hospital, Brisbane, Australia; ${ }^{7}$ Critical care research group, adult intensive care services, The Prince Charles 
Hospital, Chermside, Australia; ${ }^{8} \mathrm{Ccrg}$, clinical sciences, The Prince Charles Hospital, Chermside, Australia; ${ }^{9}$ Heart and vascular institute and department of pediatrics, Inova Fairfax Hospital, Falls Church, United States of America; ${ }^{10}$ Congenital heart center, University of Florida, Gainesville, United States of America

Correspondence: A. Zaaqoq

Intensive Care Medicine Experimental 2020, 9(1): 001079

Introduction. Prone positioning has been shown to improve outcomes in patients with severe acute respiratory distress syndrome (ARDS). Venovenous extracorporeal membrane oxygenation (VV ECMO) has recently been shown to have acceptable outcomes for refractory COVID-19-related ARDS. Whether prone-positioning during VV ECMO for COVID-19 improves outcomes is unknown.

Objectives. The study investigated the impact of prone positioning during venovenous extracorporeal membrane oxygenation (VV ECMO) support for COVID-19 acute respiratory failure on the patient outcome.

Methods. An observational study of VV ECMO patients. We used a multi-state survival model to compare the outcomes of patients treated with or without prone positioning during ECMO which incorporates the dynamic nature of prone positioning and adjusts for potential confounders. 72 international institutions participating in the COVID-19 Critical Care Consortium (COVID Critical) international registry. COVID-19 patients who were supported by VV ECMO during the study period.

Results. There were 232 COVID-19 patients at 72 participating institutions who were supported with VV ECMO during the study period from February 16, 2020, through October 31, 2020. Proning was used in 176 patients $(76 \%)$ before initiation of ECMO and in 67 patients (29\%) during ECMO. Survival to hospital discharge was 33\% in the ECMO prone group vs. $22 \%$ in the ECMO supine group. Prone positioning during ECMO support was associated with reduced mortality (hazard ratio $0.31,95 \% \mathrm{Cl}, 0.14$ to 0.68 ).

Conclusion. Our study highlights that prone-positioning during VV ECMO support for refractory COVID-19-related ARDS is associated with reduced mortality. Given the observational nature of the study, a randomized controlled trial of prone positioning on VV ECMO is needed to confirm these findings.

\section{0}

Persistent acute kidney injury and not transient azotaemia was associated with lower transplant free survival in patients with acute liver failure: a multicenter cohort study

F. S. Cardoso ${ }^{\text {1 }}$ P. Fidalgo, ${ }^{2}$; M. Gottfried, ${ }^{3}$; S. Tujios, ${ }^{4}$; J. Olson, ; W. Lee, ${ }_{1}^{4}$ C. Karvellas ${ }^{6}$

${ }^{1}$ Intensive care unit, Hospital Curry Cabral, CHULC, Lisboa, Portugal; ${ }^{2}$ Intensive care, Hospital de São Francisco Xavier, Lisboa, Portugal; ${ }^{3}$ Public health sciences, Medical University of South Carolina, Charleston, United States of America; ${ }^{4}$ Digestive and liver diseases, UT Southwestern Medical Center, Dallas, United States of America; ${ }^{5}$ nternal medicine, University of Kansas School of Nursing, Kansas City, United States of America; ${ }^{6}$ Critical care, University of Alberta, Edmonton, Canada

Correspondence: F. S. Cardoso

Intensive Care Medicine Experimental 2020, 9(1): 001080

Introduction. Acute kidney injury (AKI) has been associated with worse outcomes in patients with acute liver failure (ALF) [1]. The differential impact of transient azotaemia (TA) versus persistent AKI has been studied in general critically-ill patients, but data on patients with ALF is lacking [2].

Objectives. We aimed to assess the differential impact of TA versus persistent AKI on short-term survival in patients with ALF.

Methods. We retrospectively included all patients $(\geq 15$ years of age) with ALF from the multicenter United States Acute Liver Failure Study Group prospective registry between 1998 and 2016. Patients with $<3$ days of follow-up or without kidney function evaluation on day 3 post inclusion were excluded. AKI was defined according to the Kidney Disease Improving Global Outcomes guidelines (2012) on day one post inclusion. Kidney recovery was assessed on day 3 post inclusion and defined as TA, by a return to the no-AKI range within $48 \mathrm{~h}$ of the initial AKI diagnosis, or persistent AKI if no such recovery or the need for renal replacement therapy (RRT) was observed. Primary outcome was transplant-free survival at 21 days post inclusion (TFS). Associations were studied using logistic regression analysis.

Results. Among 1071 patients with ALF included, 339 (31.7\%) were males and median age (interquartile range) was 39 (29-51) years. Paracetamol-related ALF was present in 497 (46.4\%) patients. On day one post inclusion, $485(45.3 \%)$ patients had grade $3-4$ hepatic encephalopathy (HE), $500(46.7 \%)$ required invasive mechanical ventilation (IMV), 197 (18.4\%) were on vasopressors, and 219 (20.4\%) required RRT. On day one post inclusion, 673 patients (62.8\%) patients had AKI. On day 3 post inclusion, 601 (56.1\%) patients had persistent AKI, 72 (6.7\%) had TA, 71 (6.6\%) had de-novo AKI, and 327 (30.5\%) remained without AKI. Following adjustment for significant confounders (age, sex, race, etiology, HE grade, use of IMV and vasopressors, and INR), while persistent AKI (adjusted odds ratio (aOR) (95\% confidence interval $(95 \% \mathrm{Cl})) 0.64(0.46-0.90))$ or de-novo AKI (aOR $(95 \% \mathrm{Cl}) 0.51(0.28$ $0.94)$ ) on day 3 post inclusion were associated with lower TFS, TA was not $(\mathrm{aOR}(95 \% \mathrm{Cl}) 1.88(0.98-3.61))$

Conclusion. In a large multicenter cohort of patients with ALF, persistent AKI and not TA was independently associated with lower shortterm TFS.

\section{Reference(s)}

1. Uchino S, Bellomo R, Bagshaw SM, Goldsmith D. Transient azotaemia is associated with a high risk of death in hospitalized patients. Nephrol Dial Transplant. 2010 Jun;25(6):1833-9. https://doi.org/10.1093/ndt/gfp624. Epub 2010 Jan 6. PMID: 20,054,022.

2. Tujios SR, Hynan LS, Vazquez MA, Larson AM, Seremba E, Sanders CM, Lee WM; Acute Liver Failure Study Group. Risk factors and outcomes of acute kidney injury in patients with acute liver failure. Clin Gastroenterol Hepatol. 2015 Feb;13(2):352-9. https://doi.org/10.1016/j.cgh.2014.07.011. Epub 2014 Jul 11. PMID: 25,019,700; PMCID: PMC4937794.

3. NIH. Grant Number: U-01 58,369

\section{9}

Structural muscle alterations due to severe COVID-19 infection

J. Marin-Corral'; PG. S²; A. Salazar Degracia'; J. Bigas, ; R. Conesa,

J. Lloreta, 3. J. Gea, 2. JR. Masclans ${ }^{1}$

${ }^{1}$ Critical care department, Hospital del Mar-IMIM, Barcelona, Spain

${ }^{2}$ Respiratory department, Hospital del Mar-IMIM, Barcelona, Spain;

${ }^{3}$ Pathology department, Hospital del Mar-IMIM, Barcelona, Spain

Correspondence: J. Marin-Corral

Intensive Care Medicine Experimental 2020, 9(1): 001099

Introduction. Muscle pain and weakness are present from the early stages and persist as late manifestation of COVID-19 in a high percentage of patients, particularly in those with the most severe forms of the disease.

Objectives. To identify muscle structural and ultrastructural alterations due to COVID-19 infection in critically ill patients.

Methods. ICU patients admitted for COVID-19 infection without a history of muscle diseases were included. Biopsies of the vastus lateralis of the quadriceps muscle and clinical and demographic data were collected from all subjects. A fraction of each sample was processed in formalin and embedded in paraffin for histological analysis: 1) immunohistochemistry for percentages of type I / II fibers and morphometries and 2) hematoxylin-eosin staining for muscle abnormalities. Another fraction was processed in glutaraldehyde for the evaluation of the ultrastructure by means of electron microscopy. A historical cohort of healthy subjects was used as a control group. For statistical analysis, the Mann Whitney test by SPSS was used. Protocol was approved by the IRB and informed consent was obtained.

Results. 25 patients admitted to the ICU with COVID-19 and 5 healthy subjects were included. There were no differences in age or gender between both groups [64 (58-75) vs 61 (59-68) years, $p>0.05$ and $80 \%$ vs $80 \%$ men, $p>0.05$ ]. COVID- 19 patients had a higher BMI than control subjects [28 (27-31) vs $25(23-29) \mathrm{Kg} / \mathrm{m} 2, \mathrm{p}<0.05]$ 
COVID-19 patients presented a decrease in the size of type II fibers [1736 (1096-3137) vs $3318(2171-5612) \mu \mathrm{m} 2, p=0.037]$. No changes were observed in the fibrillar percentages, in the size of type I fibers, or in the abnormalities. Alterations in muscle capillaries with thickening of the basement membranes were repeatedly observed in the COVID19 group. Viral particles were also isolated in some cases.

Conclusion. Changes in terms of atrophy, vascular alterations and presence of viral particles were observed in the peripheral muscles of patients with severe COVID19.

\section{5}

External Validation of Urinary C-C motif Chemokine Ligand 14 (CCL14) for Prediction of Persistent Acute Kidney Injury

S. Bagshaw ${ }^{1}$; A. Al-Khafaji, ${ }^{2}$; A. Artigas ${ }^{3}$; D. Davison, ${ }^{4}$; M. Haase, ${ }^{5}$

M. Lissauer ${ }^{6}$; K. Zacharowski ; M. Chawla ${ }^{8}$;. Kwan' $;$;. Kampf, ${ }^{10}$;

P. Mcpherson, ${ }^{10} ;$ J. Kellum ${ }^{2}$

${ }^{1}$ \{street_address\}, Edmonton, Canada; ${ }^{2}$ Department of critical care medicine, University of Pittsburgh, Pittsburgh, United States of America; ${ }^{3}$ Critical care department, Corporacion Sanitaria Universitaria Parc

Tauli, Barcelona, Spain; ${ }^{4}$ Anesthesiology and critical care medicine, School of Medicine \& Health Sciences, George Washington University, Barcelona, United States of America; ${ }^{5}$ Otto von-guericke-university magdeburg, Diaverum Renal Care Center, Magdeburg, Germany; ${ }^{6}$ Department of surgery, division of acute care surgery, Rutgers-Robert Wood Johnson Medical School, New Brunswick, United States of America; ${ }^{7}$ Anesthesiology, intensive care medicine \& pain therapy, University Hospital Frankfurt, Goethe University, Frankfurt, Germany; ${ }^{8}$ Critical care medicine, Veterans Affairs Medical Center, San Diego, United States of America; ${ }^{9}$ Astute medical inc., bioMerieux, San Diego, United States of America; ${ }^{10} \mathrm{Global}$ medical affairs, bioMerieux Inc, Durham, United States of America

Correspondence: S. Bagshaw

Intensive Care Medicine Experimental 2020, 9(1): 001105

Introduction. Persistent acute kidney injury (AKI) is associated with suboptimal clinical outcomes and represents a therapeutic dilemma for clinicians. Recent, the biomarker urinary $\mathrm{C}-\mathrm{C}$ motif chemokine ligand 14 (CCL14) was found to predict the development of persistent AKI.

Objectives. To externally validate urinary CCL14 for the prediction of persistent AKI in heterogenous cohort of critically ill patients.

Methods. This was a secondary analysis of the multi-national prospective SAPPHIRE study. Critically ill patients with cardiac and/or respiratory dysfunction who developed Kidney Disease: Improving Global Outcomes (KDIGO) stage 2-3 AKI within one-week of enrollment were included. The primary exposure of interest was the urine concentration of CCL14 measured at the onset of AKI stage 2-3. The primary outcome was the development of persistent severe $\mathrm{AKI}$, defined as $\geq 72 \mathrm{~h}$ of $\mathrm{KDIGO}$ stage $3 \mathrm{AKI}$ or death or renal-replacement therapy (RRT) prior to $72 \mathrm{~h}$. The secondary outcome was a composite of RRT or death by 90 -days. Receiver operating characteristic (ROC) curve analyses were used to assess the discrimination urinary CCL14 for the development of persistent severe $\mathrm{AKI}$ and multivariate analyses to compare tertiles of urinary CCL14 and outcomes.

Results. 195 patients who developed KDIGO stage 2-3 AKI were included. In total, 28 (14\%) developed persistent severe AKI. Of these, 15 had $A K I \geq 72$ h, 12 received RRT and 1 died prior to $\geq 72 \mathrm{~h}$. Persistent severe AKI was associated with pre-morbid chronic kidney disease, diabetes mellitus, greater non-renal APACHE III score, greater positive fluid balance, use of vasopressor agents, and larger relative change in baseline serum creatinine. The AUC for urinary CCL14 to predict persistent severe $\mathrm{AKI}$ was 0.81 ( $95 \% \mathrm{Cl}, 0.72$ to 0.89 ). The risk of persistent severe AKI increased with greater values of urinary CCL14. RRT and/or death at 90-days increased within tertiles of greater urinary CCL14 concentration.

Conclusion. This secondary analysis externally validates urinary CCL14 to predict persistent severe AKI in critically ill patients.
001117

Prevalence and risk factors for the use of physical restraints in ICU patients: a multinational cohort study

M. Collet ${ }^{1}$; J. Caballero López ${ }^{2}$; R. Sonneville ${ }^{3}$; P. Nydahl4 ; A. Schandl ${ }^{5}$;

H. Woien ${ }^{6}$; MVD. Boogaard, ; J. Hästbacka ${ }^{8}$; M. Haenggi ${ }^{9}$; K. Colpaert ${ }^{10}$;

L. Rose ${ }^{11} ;$ T. Lange, ${ }^{12} ;$ M. Krog ${ }^{13} ;$ I. Egerod $^{14} ;$ A. Perner $^{15}$

${ }^{1}$ \{street_address\}, Copenhagen, Denmark; ${ }^{2}$ Intensive care depart-

ment, University Hospital Arnau de Vilanova, Lleida, Spain; ${ }^{3}$ Medical and infectious intensive care unit, Bichat-Claude Bernard Hospi-

tal, Paris, France; ${ }^{4}$ Department of anaesthesiology and intensive care medicine, Universitätsklinikum Schleswig-Holstein, Campus Kiel, Kiel, Germany; ${ }^{5}$ Department of molecular medicine and surgery, Karolinska Institutet, Stockholm, Sweden; ${ }^{6}$ Division of emergencies and intensive care, Oslo University Hospital, Oslo, Norway; ${ }^{7}$ Intensive care medicine, Radboud University Medical Center, Nijmegen, Netherlands; ${ }^{8}$ Intensive care units, department of anaesthesiology, intensive care and pain medicine, University of Helsinki and Helsinki University Hospital, Helsinki, Finland; ${ }^{9}$ Department of intensive care, Inselspital, Bern University Hospital and University of Bern, Bern, Switzerland; ${ }^{10}$ Intensive care, Ghent University Hospital, Gent, Belgium; ${ }^{11}$ King's college, Florence Nightingale Faculty of Nursing, Midwifery and Palliative Care, London, United Kingdom; ${ }^{12}$ Center for statistical science, Peking University, Beijing, China; ${ }^{13}$ University hospital aarhus, Department of Intensive Care, Aarhus, Denmark; ${ }^{14}$ Department of neuroanaesthesiolgoy, Rigshospitalet, København, Denmark; ${ }^{15}$ Department of intensive care, Copenhagen University Hospital, Rigshospitalet, Copenhagen, Denmark

Correspondence: $M$. Collet

Intensive Care Medicine Experimental 2020, 9(1): 001117

Introduction. Physical restraints potentially promote safety for patients in the ICU. However, the use of physical restraints is highly variable (range $0 \%$ to $100 \%$ ) and may be associated with adverse outcomes, e.g. delirium, unplanned extubation and increased posttraumatic stress. We aimed to assess the prevalence and variables associated with the use of physical restraints, as reported in patients in the AID-ICU multinational prospective observational cohort study with a 2 week-inception period1. The aim of the AID-ICU cohort study was to describe the prevalence and risk factors for intervening with haloperidol in ICU patients.

Methods. This planned post-hoc analysis of the AID-ICU study included adult acutely admitted ICU patients not meeting an exclusion criterion (e.g. mental illness/retardation, dementia). Follow-up was until 90 days after ICU admission. Data were registered in a secure web-based case report form where physical restraints was assessed daily as yes/no. Patient consent was obtained according requirements in each country. A statistical analysis plan was published (http:// www.cric.nu/aid-icu-sap-restrain/) before the analyses. Hazard ratios (HR) were computed using cox model censored for death and ICU discharge.

Results. Preliminary results.

Between March 7th and June 30th, 2016, 99 ICUs in 13 countries in Europe, and North and South America screened 1922 patients where 1260 patients had complete data and were included in the analysis. Physical restraints were used at least once in 217 of 1260 patients $(17 \%(95 \% \mathrm{Cl} 15-19)$ with a variation between country of a minimum of $1 \%$ to a maximum of $48 \%$ of included patients per country and for a median of 2 days (IQR 1-6). Those using physical restraints in ICU more frequently had coma and delirium and were mechanically ventilated within the first $24 \mathrm{~h}$ of ICU admission. Among those restrained $33 \%$ (95\% Cl 27-39) were treated with anti-psychotics (haloperidol, olanzapine, or quetiapine), $41 \%$ (34-48) with benzodiazepines, and $85 \%(80-90)$ with sedatives at least once during ICU admission. While physically restrained, $61 \%(95 \% \mathrm{Cl} 55-68)$ of the patients were comatose, $83 \%$ (77-88) were mechanically ventilated and $42 \%(36-49)$ were delirious. Scandinavian countries had fewer restrained patients, but similar numbers of sedated patients as compared with the total cohort.

Time from ICU admission to the use of physical restraints was shorter in comatose patients (hazard ration $[\mathrm{HR}]=1.96,95 \% \mathrm{Cl} 1.3-3.97)$ and in those with hyperactive delirium $(H R=2.09,1.48-2.93)$. Time from 
ICU admission to the use of physical restraints was longer in patients with hypoactive delirium ( $\mathrm{HR}=0.60,0.40-0.91)$ ).

Conclusion. Physical restraints were commonly used in ICU patients with high variation between countries. Coma and hyperactive delirium subtypes were associated with the use of physical restraints.

\section{Reference(s)}

1. Collet MO, Caballero J, Sonneville R, et al. Prevalence and risk factors related to haloperidol use for delirium in adult intensive care patients: the multinational AID-ICU inception cohort study. Intensive Care Med. May 2018. 10.1007/s00134-018-5204-y

\section{4}

Long-term survival of mechanically ventilated patients with severe COVID-19: an observational cohort study O. Peñuelas'; L. Del Campo Albendea; A. Lesmes González De Aledo ${ }^{3}$ JM. Añón ${ }^{4}$; C. Rodriguez Solis ${ }^{5}$; J. Jimenez, ${ }_{1}^{6}$;. Vera, ${ }^{7}$; D. Ballesteros, ${ }_{1}^{8}$; N. Franco, ${ }^{9}$ Á. Algaba, 10; JP. Avilés, ${ }^{11}$; B. Abad, ${ }^{12}$; A. Abella, ${ }^{13}$; J. Cedeño ${ }^{14}$ J. García Suarez ${ }^{8}$; Ó. Martinez, ${ }^{15}$; B. Tommaso ${ }^{16}$; J. Mancebo; A. Muriel2 ; F. Frutos-Vivar ${ }^{17}$

'Intensive care medicine, Getafe University Hospital. CIBER de Enfermedades Respiratorias, CIBERES, Spain., Getafe, Spain; ${ }^{2}$ Department of clinical biostatistics, Hospital Ramón y Cajal, Madrid, Spain; ${ }^{3}$ Hospital universitario doce de octubre, Intensive Care Unit, Madrid, Spain; ${ }^{4}$ Department of intensive care medicine, Hospital La Paz-madrid, Madrid, Spain; ${ }^{5}$ Intensive care unit, Hospital Universitario de Getafe, Getafe, Spain; ${ }^{6}$ Intensive care medicine, Getafe University Hospital., Getafe, Spain: ${ }^{7}$ Intensive care unit, Hospital de la Santa Creu i Sant Pau, Barcelona, Spain; ${ }^{8}$ Hospital universitario puerta de hierro majadahonda, Intensive Care Unit, Madrid, Spain: ${ }^{9}$ Intensive care unit, Hospital Universitario de Móstoles, Madrid, Spain; ${ }^{10}$ Intensive care unit, Hospital Universitario de Torrejón, Torrejón, Spain; ${ }^{11}$ Intensive care unit, Hospital Universitario Severo Ochoa, Leganés, Spain; ${ }^{12}$ Intensive care unit, Hospital Universitario La Princesa, Madrid, Spain; ${ }^{13}$ Intensive care unit, Hospital del Henares, Coslada, Madrid, Spain; ${ }^{14}$ Intensive care unit, Hospital Universitario Gregorio Marañón, Madrid, Spain: ${ }^{15}$ Intensive care unit, Hospital Universitario del Tajo, Aranjuez, Madrid, Spain; ${ }^{16}$ Department of anesthesiology, Hospital Ramón y Cajal, Madrid, Spain; ${ }^{17}$ Intensive care unit, Getafe University Hospital., Getafe, Spain

Correspondence: O. Peñuelas

Intensive Care Medicine Experimental 2020, 9(1): 001124

Introduction. Information is lacking regarding long-term survival and predictive factors for mortality in patients with acute hypoxemic respiratory failure due to coronavirus disease 2019 (COVID-19) and undergoing invasive mechanical ventilation. We aimed to estimate 90 -day and 180-day survival of patients with COVID-19 requiring invasive ventilation and to develop a predictive model for intensive care unit mortality.

Objectives. To analyze the long-term survival of patients who required invasive mechanical ventilation for severe COVID-19 pneumonia.

To assess a predictive model of ICU mortality.

Methods. A multicentre, national cohort study between March 8 and April 30, 2020 in 16 intensive care units (ICU) in Spain (clinicaltrials.gov \#NCT04379258). Participants were consecutive adults who received invasive mechanical ventilation for COVID-19. Severe acute respiratory syndrome coronavirus 2 (SARS-CoV-2) infection detected in positive testing of a nasopharyngeal sample and confirmed by real time reverse-transcriptase polymerase chain reaction (rt-PCR). The primary outcomes were 90 -day and 180-day survival after hospital admission. Secondary outcomes were length of ICU and hospital stay, and ICU and in-hospital mortality. A predictive model and a nomogram were developed to estimate the probability of ICU mortality. The Ethics Committee of Hospital Universitario de Getafe approved the study in a waiver consent manner.

Results. 868 patients were included (median age, 64 years [interquartile range [IQR], 56-71 years]; $72 \%$ male). Severity at ICU admission, estimated by SAPS3, was 56 points [IQR 50-63]. Prior to intubation, $26 \%$ received some type of noninvasive respiratory support. The 90-day and 180-day survival rates were $69 \%$ (95\% confidence interval
[Cl] 66\%-72\%) and 59\% (95\% Cl 56\%-62\%) respectively. The predictive factors associated with ICU mortality were: age (odds ratio [OR] 1.049 [95\% Cl 1.032-1.066] per 1-year increase), SAPS3 (OR $1.025[95 \% \mathrm{Cl}$ 1.008-1.041] per 1-point increase), neutrophil to lymphocyte ratio (OR 1.009 [95\% Cl 1.002-1.016]), a failed attempt of noninvasive positive pressure ventilation previous to orotracheal intubation(OR $2.131[95 \%$ $\mathrm{Cl} 1.279-3.550])$, and use of selective digestive decontamination (OR 0.587 [95\% $\mathrm{Cl} 0.358-0.963]$ ).

Conclusion. The long-term survival of mechanically ventilated patients with severe COVID-19 reaches more than $50 \%$ and may help to provide individualized risk stratification and potential treatments.

\section{Reference(s)}

1. Foundation for Biomedical Research of the University Hospital of Getafe, Spain (COVID-19 No.ISCIII:COV20/00977, 2020. Madrid $(0,010,604)$

001137

PRospective multicenter Observational study on Transfusion practice in vv-ECMO patients: The PROTECMO Study. Preliminary data on hemoglobin values

G. Martucci': M. Schmidtt ${ }^{2}$ G. Grasselli ${ }^{3}$; A. Tabatabai ${ }^{4}$ : M. Giani ${ }^{5}$;

C. Agerstrand ${ }^{6} ;$ S. Buabbas $;$; P. Schellongowski ${ }^{8} ;$ G. Vojka $^{9} ;$ T. Duburcq $^{10}$ AA. Hssain ${ }^{11}$; V. Fanelli' ${ }^{12}$. F. Tuzzolino ${ }^{13}$; M. Rizzo ${ }^{14}$ : L. Broman ${ }^{15}$; K. Tanaka ${ }^{16 .}$ M. Ranieri ${ }^{17}$; A. Pesenti ${ }^{18}$; D. Brodie, ${ }^{19}$; A. Arcadipane ${ }^{20}$

${ }^{1}$ Anesthesia and intensive care unit, ISMETT, Palermo, Italy; ${ }^{2}$ Service de reanimation medicale, Paris University, Paris, France; ${ }^{3}$ Intensive care unit, Policlinico of Milan, Milano, Italy; ${ }^{4}$ Lung rescue unit, University of Maryland, Baltimora, United States of America; ${ }^{5}$ Dipartimento di emergenza e urgenza, ASST Monza, University Of Milano-Bicocca, Monza, Italy; ${ }^{6}$ Medical ecmo program, Columbia university, New York, United States of America; ${ }^{7}$ Icu, Ministry of Health, Kuwait, Kuwait; ${ }^{8}$ Department of medicine i, Medical University of Vienna, Wien, Austria; ${ }^{9}$ Micu, University Medical Center, Ljubljana, Zaloška cesta, Ljubljana, Slovenia, Ljubljana, Slovenia; ${ }^{10}$ Intensive care unit, Chu De Lille, Lille, France: ${ }^{11} \mathrm{I}$ Icu, Hamad Medical Corporation, Doha, Qatar; ${ }^{12}$ Surgical sciences department, Città della Salute e della Scienza, University of Torino, Turin, Italy; ${ }^{13}$ Research office, IRCCS-ISMETT, Palermo, Italy; ${ }^{14}$ Research office, ISMETT, Palermo, Italy; ${ }^{15}$ Icu, Karolinska, Stockholm, Sweden; ${ }^{16}$ Department of anesthesiology, University of Oklahoma, Oklahoma City, United States of America; ${ }^{17}$ Department of medical and surgical sciences, Alma Mater StudiorumUniversità di Bologna, Bologna, Italy; ${ }^{18}$ Department of Pathophysiology and Transplantation, University of Milan, Milano, Italy; ${ }^{19}$ Intensive care, Columbia University College of Physicians and Surgeons/New York-Presbyterian Hospital, New York, United States of America; ${ }^{20}$ Anesthesia and intensive care unit, IRCCS-ISMETT, Palermo, Italy

Correspondence: G. Martucci

Intensive Care Medicine Experimental 2020, 9(1): 001137

Introduction. The optimal hemoglobin $(\mathrm{Hb})$ during $\mathrm{V}-\mathrm{V}$ ECMO is still debated. A previous survey showed that high volume centers $(>20$ run/year) tolerate lower $\mathrm{Hb}$ values.

Objectives. To describe the level of $\mathrm{Hb}$ and the trigger for transfusion adopted worldwide in a large cohort of V-V ECMO in a defined timeframe and explore its relations with clinical outcomes.

Methods. Prospective multicenter observational international cohort study on consecutive V-V ECMO patients (during one year or up to 20 patients, having complete data) enrolled by 40 centers in 19 countries from Jan 2019 to Dec 2020. Patient were described for preECMO characteristics and followed daily for 28 days or less if the time on ECMO was shorter. Generalized Estimating Equations (GEE) models were applied in order to consider within subject repeated measurements over time. $\mathrm{Hb}$ was registered daily and before the first daily transfusion episode. Centers were considered at low $(<12)$, medium (12-20), high $(>20)$ volume, according to the number of cases per year.

Results. 635 patients were enrolled with the following baseline pre-ECMO characteristics [median, $95 \% \mathrm{Cl}$ or $\mathrm{N}(\%)$ ]: age 52 years (40-60), male gender $444(70 \%)$, BMl $28.5 \mathrm{~kg} / \mathrm{m} 2$ (24.9-34), hospital stay 5.4 days (1.7-11), ICU stay 3 days $(0.9-7)$, mechanical ventilation 2.1 days (0.6-5.5), PaO2/FiO2 ratio 70 (59-94), SAPS-2 40 (30-55), 
SOFAscore 10 (7-12), PRESERVE score 4 (2-5), RESPscore 2 (0-4), Hb 10.8 (9.4-12.30), and hematocrit 33.5\% (29.5-38). Main causes of ARF were COVID-19 (N 204, 32.2\%), other viral pneumonia (N 119, 18.8\%) and bacterial pneumonia ( $\mathrm{N} \mathrm{102,16.1 \% ).} \mathrm{Main} \mathrm{ECMO} \mathrm{configurations}$ were fem-jug (64.7\%) and fem-fem $25.2 \%$. At baseline $\mathrm{Hb}$ was not significantly higher in patients discharged alive from the ICU compared to non-survivors: $\mathrm{Hb} 11.1 \pm 2.4 \mathrm{~g} / \mathrm{dl}$ vs $10.8 \pm 2.3 \mathrm{~g} / \mathrm{dl}, \mathrm{p}=0.15$. A lower baseline value was tolerated at higher volume center: 11.3 (low and medium volume) vs 10.7 high volume; $p=0.02$. (Fig. 1a) But in a Kaplan-Meier survival analysis the baseline quartiles of Hb didn't show a statistically significant difference in ICU survival, log-rank $P=0.56$. $\mathrm{N} 430(67.6 \%)$ patients were successfully weaned from ECMO (average ECMO run length 10.9 days [5.9-20)] and 383 (60.22\%) were discharged alive from the ICU and 374 (59.4\%) were discharged from the hospital. Longitudinal data include 8182 days on ECMO. Median $\mathrm{Hb}$ considering all the ECMO days was $9 \mathrm{~g} / \mathrm{dl}(8-9.8)$ and considering the average per patient (weighting for ECMO duration) was $9.2 \mathrm{~g} / \mathrm{dl}$ (8.3-9.9). Despite the differences at baseline all patients tent to reach a similar $\mathrm{Hb}$ value in longer ECMO stays.

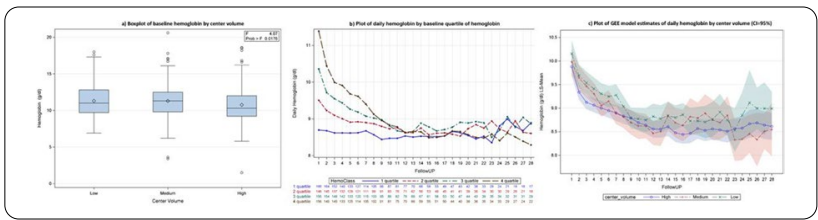

Patients in the lower quartile maintained a stable value over time, while the higher quartiles demonstrated a statistically significant decrease towards the mean and the progression is higher for the higher baseline values (Fig. 1b). In high volume centers $\mathrm{Hb}$ is usually lower than in the others, $\mathrm{p}<0.01$ (Fig. 1c). In the whole cohort median pretransfusion $\mathrm{Hb}$ was $7.9 \mathrm{~g} / \mathrm{dl}(7.1-9)$. No statistical difference in the $\mathrm{Hb}$ during ECMO was found between survivors and non-survivors. Conclusion. In a large multicenter cohort of V-V ECMO patients, $\mathrm{Hb}$ was usually kept at a lower level than normal range, between 8 and $10 \mathrm{~g} / \mathrm{dl}$ as proposed by the EOLIA trial. Considering these range values, variations in $\mathrm{Hb}$ was not associated with clinical outcome. A tendency over lower $\mathrm{Hb}$ in higher volume centers was confirmed by these large observational data.

\section{Reference(s)}

1. PROTECMO study was endorsed by ESICM and funded by 2019 ELSO grant. We acknowledge all the participants of the PROTECMO study group

\section{0}

Virtual post-intensive-care rehabilitation for survivors of COVID-19: a feasibility study

F. Howroyd'; N. Earle, ; J. Weblin,

${ }^{1}$ Therapy services, Queen Elizabeth Hospital Birmingham, Birmingham, United Kingdom

Correspondence: F. Howroyd

Intensive Care Medicine Experimental 2020, 9(1): 001200

Introduction. The COVID-19 pandemic has led to a rapid increase in intensive care admissions; the survivors of which are anticipated to have extensive physical and psychocognitive morbidity [1-5]. An innovative, virtual post-intensive-care rehabilitation service has been developed for survivors of COVID-19, however its feasibility and impact have not yet been evaluated.

Methods. A non-randomised, feasibility study was performed. Adults admitted to the intensive care of a large UK hospital with a confirmed diagnosis of COVID-19 and surviving to hospital discharge were included. Patients were invited to participate in a virtual rehabilitation programme consisting of a weekly exercise class and support group, led by critical care physiotherapists and follow-up nurses. Primary outcomes were recruitment, retention and adherence. Secondary outcomes included physical and non-physical clinical outcomes; including the One Minute Sit-to-Stand Test (1MSTS), Medical Research Council Dyspnoea Scale (MRC), Quick Dash Shoulder Disability Questionnaire (QD), Hospital Anxiety and Depression Scale for Anxiety (HADS-A) and Depression (HADS-D), Intensive Care Psychological Assessment Tool (IPAT) and the EQ-5D Perceived Health Rating Scale (EQ5D).

Results. 38 out of 76 eligible patients $(50 \%)$ agreed to participate in virtual rehabilitation, of which 28 (74\%) completed the 8-week programme. The baseline characteristics of patients invited to the postintensive-care rehabilitation programme are in Table 1. Patients who attended virtual rehabilitation were predominantly male $(79 \%)$, of BAME ethnic groups (79\%) with a mean age of 54 years (SD 11).

Table 1 : Demographic and clinical characteristics of patients who completed virtual rehabilitation and those who declined $(n=66)$

\begin{tabular}{|c|c|c|c|}
\hline & $\begin{array}{l}\text { Completed } \\
(n=28)\end{array}$ & Declined $(n=38)$ & $p$ \\
\hline Mean Age (SD) & $54(11)$ & $54(12)$ & 0.816 \\
\hline $\begin{array}{l}\text { Gender; Male: } \\
\text { Female }\end{array}$ & 22 (79\%): $6(21 \%)$ & 28 (74\%): $10(26 \%)$ & 0.647 \\
\hline $\begin{array}{l}\text { BAME Ethnicity; } \\
\text { Yes: No }\end{array}$ & 22 (79\%): 6 (21\%) & 24 (63\%): $14(37 \%)$ & 0.178 \\
\hline $\begin{array}{l}\text { Median ICU } \\
\text { Length of Stay }\end{array}$ & $\begin{array}{l}21 \text { days }(18.75- \\
27.75)\end{array}$ & $\begin{array}{l}21.5 \text { days } \\
(13.5-28)\end{array}$ & 0.421 \\
\hline $\begin{array}{l}\text { Median Hospital } \\
\text { Length of Stay }\end{array}$ & 33 days (30-46) & 38 days (19-38) & 0.765 \\
\hline $\begin{array}{l}\text { Median Ventilator } \\
\text { Days }\end{array}$ & 20 days (16-24) & $\begin{array}{l}17.5 \text { days } \\
(10-26.5)\end{array}$ & 0.381 \\
\hline
\end{tabular}

Following completion of the virtual rehabilitation programme, there was a statistically significant improvement in all physical and non-physical outcomes (Table 2). After rehabilitation, there was an observed increase in exercise capacity (1MSTS 20 vs 25, p<0.001) and reduction in perceived breathlessness (MRC 3 vs $2 p<0.001$ ) and shoulder disability (QD 43.18 vs 19.31 p 0.001). Scores for anxiety (HADS-A), depression (HADS-D) and psychological distress (IPAT) were low prior to commencing rehabilitation, yet showed further reduction on completion of the programme (HADS-A: 4 vs $3 p=0.021$; HADS-D: 4 vs $2.5 p=0.010$; IPAT: 3 vs $2 p=0.002$ ). Perceived health ratings also showed significant improvement after rehabilitation (EQ5D 60 vs $76 p=0.001)$.

Table 2: Results of physical and non-physical outcomes pre and post rehabilitation $(n=28)$

\begin{tabular}{lllr}
\hline & $\begin{array}{l}\text { Pre-rehabilitation } \\
\text { (median) }\end{array}$ & $\begin{array}{l}\text { Post-rehabilita- } \\
\text { tion (median) }\end{array}$ & $\boldsymbol{p}$ \\
\hline 1MSTS & $\begin{array}{c}20 \text { stands (IQR } \\
15-27)\end{array}$ & $\begin{array}{c}25 \text { stands }(\mathrm{IQR} \\
19-31)\end{array}$ & $<0.001$ \\
QD & $43.18(\mathrm{IQR}$ & $19.31(\mathrm{IQR}$ & 0.001 \\
& $7.86-53.95)$ & $6.80-37.49)$ & \\
MRC & $3(2-3)$ & $2(1-2)$ & $<0.001$ \\
HADS-A & $4(\mathrm{IQR} 2-8)$ & $3(\mathrm{IQR} 1-6.75)$ & 0.021 \\
HADS-D & $4(\mathrm{IQR} 2-7)$ & $2.5(\mathrm{IQR} 1-5.5)$ & 0.010 \\
IPAT & $3(\mathrm{IQR} 1.25-6.75)$ & $2(\mathrm{IQR} 1-4)$ & 0.002 \\
EQ5D & $60(\mathrm{IQR} 50-80)$ & $76(\mathrm{IQR} 65-90)$ & 0.001 \\
\hline
\end{tabular}

Conclusion. It is feasible to recruit and retain survivors of severe COVID-19 to virtual post-intensive-care rehabilitation. Those that attended showed significant improvements in physical and non-physical outcomes. Further research, including an appropriately powered multi-centre clinical trial, is required to further validate these findings. 
Reference(s)

1. [1] Wang, D., Hu, B., Hu, C., et al. Clinical characteristics of 138 hospitalized patients with 2019 novel coronavirus-infected pneumonia in Wuhan, China. JAMA 2020; 323 (11): 1061-1069. 10.1001/jama.2020.1585.

2. [2] Remuzzi, A., Remuzzi, G. COVID-19 and Italy: what next? Lancet 2020 395: 1225-1228. https://doi.org/10.1016/S0140-6736(20)30627-9.

3. [3] Grasselli, G., Zangrillo, A., Zanella, A., et al. Baseline characteristics and outcomes of 1591 patients infected with SARS-CoV-2 admitted to ICUs of the Lombardy region, Italy. JAMA 2020; 323 (16): 1574. 10.1001/jama. 2020.5394.

4. [4] McWilliams, D., Weblin, J., Hodson, J., Veenith, T., Whitehouse, T., Snelson, C., and the QEHB COVID-19 Research Team. Rehabilitation Levels in COVID-19 Patients Admitted to Intensive Care Requiring Invasive Ventilation: An Observational Study. Annals of the American Thoracic Society 2020; https://doi.org/10.1513/AnnalsATS.202005-5600C

5. [5] Philips, M., Turner-Stokes, L., Wade, D., Walton, K. British Society of Rehabilitation Medicine. Rehabilitation in the wake of COVID-19-A phoenix from the ashes. https://www.bsrm.org.uk/downloads/covid-19bsr missue1-published-27-4-2020.pdf; 2020 [accessed 16 December 2020] 6. N/A

\section{0}

Sex-specific treatment characteristics and clinical outcomes of elderly critically-ill COVID-19 patients-results from the COVIP study

G. Wolff'; J. Fjølner²; B. Wernly ${ }^{3}$; R. Bruno, ${ }^{1} ;$ M. Beill ${ }^{4}$; B. Guidet ${ }^{5} ;$ P. Vernon Van Heerden ${ }^{6}$; D. De Lange7; B. Bollen Pinto ${ }^{8}$; J. Schefold ${ }^{9}$; M. Kelm'; S. Leaver ${ }^{10}$; S. Sigal ${ }^{11}$; W. Szczeklik ${ }^{12}$; RP. Moreno ${ }^{13}$; A. Artigas ${ }^{14}$; H. Flaatten ${ }^{15}$; C. Jung

${ }^{1}$ Cardiology, pulmonology and vascular medicine, Uniklinik Düsseldorf, Düsseldorf, Germany; ${ }^{2}$ Department of intensive care, Aarhus University Hospital, Aarhus, Denmark; ${ }^{3}$ Cardiology, Paracelsus Medical University, Salzburg, Austria; ${ }^{4}$ Medical intensive care unit, Hadassah Medical Center, London, United Kingdom; ${ }^{5}$ Réanimation Médicale, Hôpital Saint-Antoine, Paris, France; ${ }^{6}$ Intensive care unit, Hadassah University Hospital_Ein Kerem, Jerusalem, Israel; ${ }^{7}$ Department of intensive care medicine, Utrecht University, Utrecht, Netherlands; ${ }^{8}$ Anesthesiology, Hôpitaux Universitaires de Genève (HUG), Genève, Switzerland; ${ }^{9}$ Department of intensive care medicine, University Hospital Bern, Bern, Switzerland; ${ }^{10}$ Research lead critical care directorate, St George's Hospital, London, United Kingdom; ${ }^{11}$ Medical intensive care unit, Hadassah Medical Center, Jerusalem, Israel, Israel; ${ }^{12}$ Intensive care and perioperative medicine, Jagiellonian University Medical College, Kraków, Poland; ${ }^{13}$ Unidade de cuidados intensivos neurocríticos, Hospital de São José, Centro Hospitalar de Lisboa Central, Nova Médical School, Lisbon, Portugal; ${ }^{14}$ Critical care department, Corporacion Sanitaria Universitaria Parc Tauli, Barcelona, Spain; ${ }^{15}$ Department of anaesthesia and intensive care, Haukeland University Hospital, Bergen, Norway

Correspondence: G. Wolff

Intensive Care Medicine Experimental 2020, 9(1): 001210

Introduction. Elderly critically-ill patients with COVID-19 are most vulnerable during the ongoing pandemic, men are known to more likely develop severe disease than women. However, it remains unknown whether there are sex-specific differences after admission to an intensive care unit (ICU) regarding treatment characteristics and clinical outcomes.

Objectives. We aimed to perform a sex-specific analysis in elderly critically-ill patients with COVID-19 $\geq 70$ years of age from the prospective multicentric international COVIP study (organized by VIP group of ESICM)

Methods. All patients included in the COVIP study were analyzed for sex-specific ICU admission and treatment characteristics including frailty (according to CFS), as well as ICU and 30-day mortality. A multilevel adjusted analysis was performed; counts and percentages, medians with interquartile range (IQR) and odds ratios (OR) with 95\% confidence intervals $(\mathrm{Cl})$ are reported.

Results. A total of 3,159 patients $(69.8 \%$ male, $30.2 \%$ female 75 years median age) were included, cardiovascular comorbidities were frequent (arterial hypertension 67\%, diabetes mellitus $37 \%$, cardiovascular disease 23\%). Median SOFA score at admission was 5 (IQR 3-8). Female patients were significantly more frail than male patients $(p<0.001)$. Male patients more often underwent tracheostomy $(20 \%$ vs. $14 \%$; OR $1.57 ; p<0.001)$, vasopressor therapy $(69 \%$ vs. $62 \%$; OR $1.25 ; \mathrm{p}=0.02)$ and renal replacement therapy (17\% vs. $11 \%$; OR 1.96; $p<0.001)$. There was no difference in life-sustaining treatment limitations. 30 -day mortality (50\% male vs. $49 \%$ female; OR $1.11 ; p=0.19$ ) showed no difference, ICU mortality (48\% male vs. $47 \%$ female; OR 1.18; $p=0.04$ ) very slightly favored women. After multilevel adjustment for disease severity, frailty, age and treatment limitations, this small effect remained statistically significant (OR 1.32, 95\% Cl 1.05-1.67; $p=0.02$ )

Conclusion. In this analysis of sex-specific treatment characteristics and clinical outcomes of critically-ill elderly patients with COVID-19, we found more intensive treatment in male vs. female patients. After multilevel adjustment, there was a tiny but statistically significant association of male sex with higher ICU mortality.

001211

Critical Care Ultrasound Goal-directed Versus Early Goal-directed Therapy in Septic Shock:A Randomized Controlled Study

W. Zhang

${ }^{1}$ Critical Care Medicine, Affiliated Hospital of Zunyi Medical Univer-

sity, Zunyi, China

Correspondence: $W$. Zhang

Intensive Care Medicine Experimental 2020, 9(1): 001211

Introduction. In the early stage of septic shock, critical care ultrasound (CCUS) has the potential to guide multi-targeted treatment through the evaluation of cardiac function, fluid responsiveness, lung status and efficacy response to vasopressor infusions. Several trials have shown that CCUS-guided fluid management improved outcomes of patients. Nevertheless, a randomized controlled trial showed that echocardiogram-guided sepsis resuscitation did not significantly improve the outcomes of patients with septic shock.

Objectives. We aimed to compare the differences between critical care ultrasound goal-directed therapy (CCUGDT) and early goaldirected therapy (EGDT) in patients with septic shock.

Methods. We conducted a prospective, single center, randomized controlled study in the 35-bed general intensive care unit of an academic tertiary care hospital. Critically ill patients who were first diagnosed as septic shock in our department from December 2018 to December 2019, were recruited as the subjects of the study. The enrolled patients were randomly divided into the control group (EGDT group) and study group (CCUGDT group). The relevant conventional monitoring indicators and ultrasound parameters were recorded respectively at the time of enrollment $(0 \mathrm{~h}), 6$ th $\mathrm{h}, 12 \mathrm{th} \mathrm{h}$, and 24 th $\mathrm{h}$ in both groups. Resuscitation targeting rate and lactate clearance rate $(\mathrm{LRC})$ at 6 th $\mathrm{h}$ were primary clinical outcomes. Secondary endpoints consisted of ICU mortality and 28-day mortality.

Results. Eight-six of whom were enrolled in this study, then randomly divided into the EGDT group ( $n=44)$ and the CCUGDT group $(n=42)$ The 6-h LRC in the CCUGDT group was significantly higher than thatin EGDT group ( $23.8 \%$ vs. $9.7 \%, P=0.010)$. The cumulative fluid infusion volume and fluid balance at $12 \mathrm{hand} 24 \mathrm{~h}$ in the CCUGDT group were significantly lower than those in the EGDT group $(P<0.05)$.

Conclusion. CCUGDT increased $6 \mathrm{~h}$ LRC, improved the 24 th $\mathrm{h}$ resuscitation targeting rate and reduced the cumulative fluid infusion compared with EGDT at 12th $\mathrm{h}$ and 24th $\mathrm{h}$ in patients with septic shock.

\section{Reference(s)}

1. Kanji HD, McCallum J, Sirounis D, MacRedmond R, Moss R, Boyd JH. Limited echocardiography-guided therapy in subacute shock is associated with change in management and improved outcomes. J Crit Care 2014:29:700-5. 2. Wang XT, Liu DW, Zhang HM, et al. [Impact of extended focus assessed transthoracic echocardiography protocol in septic shock patients]. Zhonghua Yi Xue Za Zhi 2011;91:1879-83. 3. Lanspa MJ, Burk RE, Wilson EL, Hirshberg EL, Grissom CK, Brown SM. Echocardiogramguided resuscitation versus early goal-directed therapy in the treatment of septic shock: a randomized, controlled, feasibility trial. J Intensive Care 
2018;6:50. 4. Singer M, Deutschman CS, Seymour CW, et al. The Third International Consensus Definitions for Sepsis and Septic Shock (Sepsis-3). JAMA 2016;315:801-10. 5. Rivers E, Nguyen B, Havstad S, et al. Early goal-directed therapy in the treatment of severe sepsis and septic shock. N Engl J Med 2001;345:1368-77.

2. This study was supported by the Growth Project of Young Scientific and Technological Talents of Guizhou Provincial Department of Education (CJ981), the Science and Technology Support Plan of Guizhou Province in 2019 (Qian Ke He Support [2019] 2834), and the Science and Technology Plan of Guizhou Province in 2020 (Foundation of Guizhou Science and Technology Cooperation [2020]1Z061).

\section{4}

Association between timing of tracheostomy and 3-month mortality among patients aged $\geq \mathbf{7 0}$ years with COVID-19 K. Polok ; J. Fronczek ; C. Jung ${ }^{2}$; H. Flaaten ${ }^{3}$; J. Fjølner ${ }^{4}$; B. Wernly ${ }^{5}$; D. De Lange $^{6}$; S. Leaver ${ }^{7}$; B. Guidet ${ }^{8}$; P. Vernon Van Heerden ${ }^{9}$; M. Czuczwar ${ }^{10}$; W. Szczeklik ${ }^{1}$

${ }^{1}$ Intensive care and perioperative medicine, Jagiellonian University Medical College, Kraków, Poland; '2Dep. of cardiology, pulmonology and angiology, University Hospital of Düsseldorf, Düsseldorf, Germany; ${ }^{3}$ Department of anaestesia and intensive care, University of Bergen, Bergen, Norway; ${ }^{4}$ Department of intensive care, Aarhus University Hospital, Aarhus, Denmark; ${ }^{5}$ Cardiology, Paracelsus Medical University, Salzburg, Austria; ${ }^{6}$ Department of intensive care medicine, Utrecht University, Utrecht, Netherlands; ${ }^{7}$ Research lead critical care directorate, St George's Hospital, London, United Kingdom; ${ }^{8}$ Réanimation Médicale, Hôpital Saint-Antoine, Paris, France; ${ }^{9}$ Department of anesthesiology, critical care and pain medicine, Hadassah Medical Center, The Hebrew University of Jerusalem, Jerusalem, Israel; ${ }^{10}$ 2nd department of anaesthesiology and intensive therapy, Medical University of Lublin, Lublin, Poland Correspondence: K. Polok

Intensive Care Medicine Experimental 2020, 9(1): 001214

Introduction. It is believed that tracheostomy offers several benefits for patients with prolonged mechanical ventilation. Some studies suggest that early tracheostomy in critically ill patients may improve outcomes including long-term mortality. However, the available evidence concerning the population of patients with COVID-19 is scarce.

Objectives. To evaluate whether there is an association between tracheostomy timing and 3-month mortality in critically-ill patients aged $\geq 70$ years with confirmed COVID-19.

Methods. This is a prospective multicentre study enrolling consecutive patients $\geq 70$ years old admitted to the ICU with confirmed COVID-19 (COVIP, NCT04321265). Recruitment took place in 164 centres in 15 European countries, Turkey, Israel and Libya from 19 March to 31 December 2020. Baseline demographic and clinical data as well as information about timing and duration of mechanical ventilation and tracheostomy were gathered. The primary endpoint was mortality within 3 months after admission to the ICU. Multivariable analyses were performed using proportional hazard Cox regression to evaluate association between 3-month mortality and early tracheostomy defined using three different thresholds i.e., $<7$ days, $<10$ days and $<14$ days from endotracheal intubation. To account for survivor bias patients were included into survival analysis in the moment of tracheostomy.

Results. The final study sample comprised 2892 patients at the mean age of 75.8 (SD 4.7) years among whom 2050 (70.9\%) were male. Tracheostomy was performed in $19.8 \%$ of entire cohort $(574 / 2892)$ and $27.4 \%$ of mechanically ventilated patients (574/2096). The 3-month mortality among patients with tracheostomy and available 3-month survival status was $50.2 \%$ (229/456). A multivariable analysis adjusted for age, gender, comorbidities, baseline Clinical Frailty Scale and Sequential Organ Failure Assessment scores revealed no association between 3-month mortality and early tracheostomy defined as performed $<7$ days (HR $0.83,95 \% \mathrm{Cl} 0.53$ to 1.31 ), $<10$ days (HR 0.82, $95 \% \mathrm{Cl} 0.57$ to 1.18$)$ and $<14$ days $(1.02,95 \% \mathrm{Cl} 0.75$ to 1.38$)$ from endotracheal intubation.
Conclusion. In this prospective observational study we showed that early tracheostomy was not associated with improved 3-month mortality in elderly patients with COVID-19 admitted to the ICU.

\section{Reference(s)}

1. Hosokawa, K., Nishimura, M., Egi, M. et al. Timing of tracheotomy in ICU patients: a systematic review of randomized controlled trials. Crit Care 19, 424 (2015). https://doi.org/10.1186/s13054-015-1138-8

\section{6}

MicroRNAs as biomarkers of disease severity in patients with COVID-19

A. Ferruelo, ; Y. Douhal ${ }^{2}$; R. Herrero, ; R. Murillo, ${ }^{3}$; A. Silva ${ }^{4}$; D. Carriedo, ; C. Jaramillo, ${ }^{5}$ O. Peñuelas ${ }^{6} ;$ JÁ. Lorente,

${ }^{1}$ Getafe university hospital, CIBERES, Madrid, Spain; ${ }^{2}$ Biochemistry department, Getafe University Hospital, Getafe, Spain; ${ }^{3}$ Getafe university hospital. ciberesucicovid project (cov20/00110, isciii), CIBERES, Madrid, Spain; ${ }^{4}$ Getafe university hospital, Universidad Europea de Madrid (Campus Villaviciosa), Villaviciosa de Odón, Spain; ${ }^{5}$ ntensive care unit, Getafe University Hospital, Getafe, Spain; ${ }^{6}$ Getafe university hospital. on behaf of ciberesucicovid project (cov20/00110, isciii), CIBERES, Madrid, Spain Correspondence: $O$. Peñuelas

Intensive Care Medicine Experimental 2020, 9(1): 001216

Introduction. The time course of patients with SARS-CoV-2 infection (coronavirus disease [COVID]-19) is not predictable based on clinical grounds, and biomarkers of disease severity are lacking. MiRNAs play important roles in the regulation of gene expression and are considered essential modulators during viral infections.

Objectives. To identify miRNAs differentially expressed in patients with COVID-19 of different severity.

Methods. We conducted a case control study of next generation sequencing in a discovery cohort of 30 patients. We used serum samples from patients coming to the Emergency Department (ED) and not admitted to the hospital (mild group), patients admitted to the hospital ward (moderate group), and patients admitted to the intensive care unit (ICU) requiring mechanical ventilation (severe group) $(n=10$ each group). Patients were matched for age, sex and previous corticosteroid treatment. None had received other immunosuppressive therapy. Serum samples obtained on the day of ED visit, hospital admission, or ICU admission, respectively, were analyzed for miRNA expression (miRNAseq) and bioinformatic analysis using CLC Genomics (Qiagen). Target prediction and enrichment analysis was conducted using webbased tools (mirNet 2.0). RT-qPCR was used to validate the differential expression of miRNAs identified in the discovery cohort. In addition, RT-qPCR was done in serum samples from a validation cohort of 60 patients with the same inclusion criteria as the discovery cohort ( $n=20$ each group).

Results. We identified 4 miRNAs differentially expressed in the severe group as compared to the mild and moderate groups. Differential expression was validated by RT-qPCR both in the discovery and the validation cohorts. The top 10 pathways and networks (Reactome) involved were cellular responses to stress, activated TLR4 signaling, cellular senescence, MAPK targets/ Nuclear events mediated by MAP kinases, Toll Like Receptor 4 (TLR4) Cascade, Toll Like Receptor 10 (TLR10) Cascade, Toll Like Receptor 5 (TLR5) Cascade, MyD88 cascade initiated on plasma membrane, TRAF6 mediated induction of NFkB and MAP kinases upon TLR7/8 or 9 activation, and Toll Like Receptor 7/8 (TLR7/8) Cascade (adjusted $p$ value $<10$ e- 6 for all cases).

Conclusion. We have identified by miRNAseq miRNAs characteristic of severe disease in COVID-19. These miRNAs are involved in a large number cell pathways, mostly related to TLR-mediated cell signaling, which plays key roles in innate immunity. Our findings may have implications for biomarker and innovative therapeutic target discovery in COVID-19.

\section{Reference(s)}

1. CIBERESUCICOVID Project (COV20/00110, ISCIII) 
001241

In a multi-centre, multi-country observational study of major trauma admissions to European intensive care units, frailty was not predictive of outcome. High SOFA and head injury were associated with higher mortality

C. Duncan, ; ; DO. Lonsdale, ; H. Farah'; S. Farnell-Ward'; C. Ryan'; X. Watson ${ }^{1}$; M. Cecconi ${ }^{3}$; H. Flaaten ${ }^{4}$; J. Fjølner ${ }^{5}$; C. Jung ${ }^{6}$; B. Guidet';

W. Szczeklik ${ }^{8}$; D. De Lange ${ }^{9} ;$ S. Leaver ${ }^{1}$

'Department of critical care, St George's University Hospitals NHS Foundation Trust, London, United Kingdom; ${ }^{2}$ Departments of clinical pharmacology and intensive care, St George's, Univeristy of London and St George's University Hospitals NHS Foundation Trust, London, United Kingdom; ${ }^{3}$ Departments of biomedical sciences and anesthesia and intensive care, Humanitas University and IRCCS Humanitas Research Hospital, Milan, Italy; ${ }^{4}$ Department of anaestesia and intensive care, University Hospital Bergen, Bergen, Norway; ${ }^{5}$ Department of intensive care, Aarhus University Hospital, Aarhus, Denmark; ${ }^{6}$ Division of cardiology, pulmonology and vascular medicine, University Hospital Düsseldorf, Heinrich-Heine- University, Düsseldorf, Germany; ${ }^{7}$ Institut pierre louis d'epidémiologie et de santé publique and service de réanimation, Sorbonne Université and Hôpital Saint-Antoine, Paris, France; ${ }^{8} \mathrm{Center}$ for intensive care and perioperative medicine, Jagiellonian University Medical College, Kraków, Poland; ${ }^{9}$ Department of intensive care medicine and dutch poisons information center (dpic), University Medical Center, Utrecht University, Utrecht, Netherlands

\section{Correspondence: D. Lonsdale}

Intensive Care Medicine Experimental 2020, 9(1): 001241

Introduction. Cases of major trauma in the very old (over 80 years) is increasingly common in the intensive care. Patients and their relatives/carers expect clinicians to provide information on the potential benefits and risks of invasive therapies associated with an admission to critical care before making choices about the care they wish to receive. Predicting outcome is challenging in this group of patients as age is a poor marker of health and poor predictor of outcome. Increasingly, decisions are guided with the use of sickness severity scores of both the acute condition (e.g. SOFA) and chronic health issues (e.g. clinical frailty scale, CFS). Recent work in a single UK trauma centre (1) and two-Australian ICUs (2) suggests that increased CFS is associated with worse outcome in elderly major trauma patients (defined as $>65$ and $>50$ years respectively). We aimed to test whether this association held true in the very old (over 80 ) or whether more traditional critical care related sickness severity scores had stronger associations with outcome (SOFA). Data for this study came from two large prospective observational studies of very old intensive care patients (VIP-1 and -2). These studies recruited participants from over 300 critical care units in 21 countries across Europe.

Methods. Data from the VIP-1 and VIP-2 studies for patients with major trauma admission were obtained. Ethical approval for these studies was obtained in the host countries/organisations. Baseline characteristics, level of care provided and outcome (ICU and 30 day mortality) were summarised. Uni- and multi- variable regression analysis were undertaken to determine associations between CFS and SOFA scores, type of major trauma and outcome (30-day mortality).

Results. Of the 8062 patients recruited to the VIP studies, 498 patients were admitted to intensive care because of major trauma. Participants in this analysis were recruited from 20 countries across Europe. Baseline characteristics and oucome are presented in Table 1. Median age was 84 years old. The youngest participant was 80 and the oldest participant 100. Median SOFA score was 6 (inter quartile range, IQR, 3-9) and CFS was 3 (IQR 2-5). Survival to 30-days was just $52 \%$. Median and inter-quartile range of CFS was the same in survivors and non-survivors. In the logistic regression analysis, CFS was not associated with increased mortality. SOFA score $(p<0.001)$ and multi-trauma with head injury $(p<0.05)$ were significantly associated with increased mortality.
Table 1. Patient characteristics, life-support interventions and outcome

\begin{tabular}{ll}
\hline & $\mathrm{N}=498$ \\
\hline $\begin{array}{l}\text { Baseline characteristics } \\
\text { Gender }\end{array}$ & \\
$\quad$ Male & $282(57 \%)$ \\
$\quad$ Female & $216(43 \%)$ \\
Age & $84(82,87)$ \\
Clinical frailty scale & $3(2,5)$ \\
$\quad$ SOFA (Total) & $6.0(3.0,9.0)$ \\
Reason for admission & \\
$\quad$ Multitrauma without head injury & $154(31 \%)$ \\
Multitrauma with head injury & $147(30 \%)$ \\
$\quad$ Isolated head injury & $197(40 \%)$ \\
Life support intervention & \\
$\quad$ Non-invasive ventilation & $61(12 \%)$ \\
Invasive ventilation & $308(62 \%)$ \\
$\quad$ Vasopressors & $259(52 \%)$ \\
$\quad$ Renal replacement therapy & $28(5.6 \%)$ \\
Limitations in care & \\
Life-sustaining care witheld & $133(27 \%)$ \\
Life-sustaining care withdrawn & $90(18 \%)$ \\
Outcome & \\
ICU length of stay (days) & $4(1,9)$ \\
Survival to ICU discharge & $339(68 \%)$ \\
30-day survival & $247(52 \%)$ \\
\hline 19tatistics presented: n (\%); Median $(\mathrm{IQR})$ \\
$\quad$
\end{tabular}

Conclusion. Major trauma admissions in the very old is not uncommon and 30-day mortality is high. We found that CFS was not a helpful predictor of mortality. SOFA and trauma with head injury are associated with worse outcomes in this patient group. To our knowledge this is the largest study of the associations between frailty and outcome in major trauma cases in the very old.

\section{Reference(s)}

1. Rickard F, Ibitoye S, Deakin H et al. The Clinical Frailty Scale predicts adverse outcome in older people admitted to a UK major trauma centre. Age and Ageing 2020; 50: 891-897.

2. Tipping CJ, Bilish E, Harrold M, Holland AE, Chan T, Hodgson CL. The impact of frailty in critically ill patients after trauma: A prospective observational study. Australian Critical Care 2020; 33: 228-235.

3. The ESICM supported VIP1 with a research award. Free support for running the electronic database and was granted from the dep. of Epidemiology, University of Aarhus, Denmark. Financial support for creation of the e-CRF and maintenance of the database was possible from a grant (open project support) by Western Health region in Norway) 2018 who also funded the participating Norwegian ICUs. DRC Ile de France and URC Est helped conducting VIP2 in France.

001289

The effect of shock duration on trauma-induced coagulopathy in a murine model

P. Sloos ${ }^{1}$; M. Adrie'; V. Jan²; N. Juffermans'3 ; DJ. Kleinveld ${ }^{4}$

${ }^{1}$ LEICA, AMC, Amsterdam, Netherlands; ${ }^{2}$ Department of plasma proteins, Sanquin, Amsterdam, Netherlands; ${ }^{3}$ Intensive care, Academic Medical Centre, Amsterdam, Netherlands; ${ }^{4}$ Intensive care medicine, Amsterdam UMC, locatie AMC, Amsterdam, Netherland

Correspondence: P. Sloos

Intensive Care Medicine Experimental 2020, 9(1): 001289

Introduction. Trauma-induced coagulopathy $(\mathrm{TIC})$ is associated with high morbidity and mortality. TIC originates endogenously from damaged and hypoxic endothelial cells during traumatic shock. TIC is initially characterized by platelet dysfunction, consumption of coagulation factors and hyperfibrinolysis. Specific TIC phenotypes have been identified. The mechanisms underlying these phenotypes are unknown, but shock duration may play a key role. We hypothesize that TIC phenotypes can shift as the duration of shock persists and that a shorter shock duration is associated with more fibrinolysis. 
Objectives. We aimed to elucidate the effect of shock duration on TIC and to examine which shock duration is best suited to study hyperfibrinolysis after traumatic injury in a mouse model.

Methods. Male $\mathrm{BI} / 6 \mathrm{~J}$ mice were anesthetized and subjected to one of the following groups: 1) 90 min of mechanical ventilation (MV) $(n=5)$ 2) $90 \mathrm{~min} \mathrm{MV}$ with trauma (bilateral tibia/fibula fractures + laparotomy with crush injury) $(n=5), 3) 30$ min MV with trauma and 30 min shock $(n=10)$ 4) 90 min MV with trauma and 90 min shock $(n=10)$. Shock was achieved by controlled blood draw until a MAP of $25-30 \mathrm{mmHg}$ was achieved. Outcomes included arterial blood gas analysis, hemodynamic parameters, rotational thromboelastometry (ROTEM) and D-dimer.

Results. Shock was induced by equal amount of blood withdrawal in group 3: 330ul ( \pm 40ul) and group 4: 350ul ( $\pm 60 \mathrm{ul})$. Only mice in shock died: $10 \%$ in the 30 min shock group and $30 \%$ in the 90 min shock group. ROTEM results are shown in Fig. 1. Trauma +30 min shock is associated with a decreased clotting amplitude, compared to $90 \mathrm{~min}$ shock, but does not differ significantly from control groups. Median level of maximum lysis did not differ between groups. However, half the mice in the 30 min shock group had a maximum lysis of more than $15 \%$, compared to none in the 90 min shock group. D-dimer levels did not differ between groups.

Conclusion. During shock duration, TIC characteristics change. A trauma model with 30 min shock seems more suitable to study TIC with fibrinolysis, compared to a model with 90 min shock duration. After 90 min of shock, a transition towards less fibrinolysis may occur.

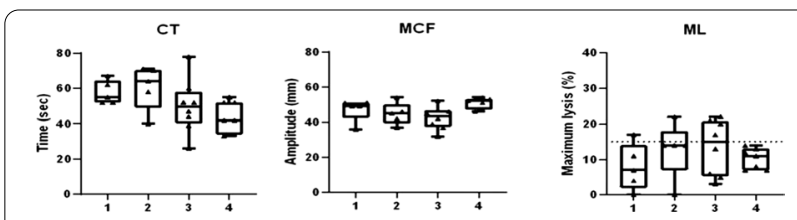

Fig. 1. Rotational thromboelastometry (ROTEM), EXTEM results. Data are represented as median with IQR. 1) Control. 2) Trauma only. 3) Trauma + 30 min shock. 4) Trauma + 90 min shock. A) EXTEM clotting time. B) EXTEM alpha angle. C) EXTEM maximum clot firmness. D) EXTEM Maximum lysis

\section{6}

Pathogenic Role of Vascular Endothelial Growth Factor-A (VEGF-A) in Sepsis-Induced Acute Kidney Injury

A. Lopez-Ruiz ; LA. Juncos ${ }^{2}$

${ }^{1}$ Department of Critical Care, AdventHealth, Orlando, United States of America; ${ }^{2}$ Department of internal medicine-division of nephrology, Central Arkansas Veterans Health Care System, Little Rock, United States of America

Correspondence: A. Lopez-Ruiz

Intensive Care Medicine Experimental 2020, 9(1): 001316

Introduction. Septic shock is the most common reason for admission to ICU and is frequently associated with distant organ complications such as acute kidney injury (AKI). The development of sepsis-induced AKI (S-AKI) contributes to higher mortality than sepsis itself and is associated with longer ICU stay. The mechanisms responsible for S-AKI are multifactorial which greatly reflex the difficulty in finding specific therapies for this condition. The use of biomarkers than can aid to predict or recognize S-AKI is a topic of major interest in the field of intensive care. We previously described that circulating level of vascular endothelial growth factor (VEGF-A) is significantly elevated in rodents with sepsis and is strongly associated with S-AKI. In addition, we further demonstrated that exogenous administration of soluble fms-like tyrosin kinase-1, a soluble inhibitor of VEGF-A (sFlt-1) attenuated S-AKI by reducing intrarenal inflammation [1]. We now expand our research to explore the role of these two endothelial biomarkers in the development of S-AKI in patients with septic shock.

Objectives. Our primary outcome was to evaluate whether circulating level of VEGF-A and sFlt-1 are associated with S-AKI in patients with septic shock. We also examined if these biomarkers are associated with duration of vasopressor support and with the need for renal replacement therapy (RRT).

Methods. We measured circulating blood level of VEGF-A and sFIt-1 in 93 patients admitted to an academic ICU in the United States of America. These biomarkers were measured within $24 \mathrm{hs}$ of admission after appropriate consent. We then retrospectively categorized these patients in 2 groups; septic shock (SS $n=42$ ) and no septic shock (NSS $n=39$ ) and we added an additional healthy control group ( $\mathrm{HC}$ $n=12$ ). Septic shock was determined based on Sepsis-3 definition [2] and these patients received therapy following the Surviving Sepsis Campaign Guidelines [3]. We followed these patients for 30 days. During this period we determined whether the initial level of VEGF-A and sFlt-1 are associated with the diagnosis and severity of AKI based on KDIGO criteria, the duration of vasopressor support and the need for RRT. Data was analyzed using 2-way ANOVA and Pearson correlation.

Results. Of the 93 patients, 50 (54\%) were male. The SS group had a median age $61 \pm 6$ years and SOFA score $8 \pm 3$. The median age for NSS group was $59 \pm 3$ years and SOFA score of $2 \pm 2$. The patients with SS had an admission VEGF-A level higher than NSS $(1780 \pm 108$ vs $615 \pm 78 \mathrm{pg} / \mathrm{ml}, \mathrm{p}<0.05)$. The level of sFlt-1 was also higher in SS compared to NSS $(670 \pm 64$ vs $296 \pm 35 \mathrm{pg} / \mathrm{ml}, \mathrm{p}<0.05)$. In the HC the level of VEGF $(285 \pm 66 \mathrm{pg} / \mathrm{ml})$ and sFlt-1 $(171 \pm 37 \mathrm{pg} / \mathrm{ml})$ was significantly lower than SS and NSS $(p<0.01)$. In the group of patients with SS, (32/42-76\%) developed AKI and most of these patients (24/32-75\%) developed AKI stage 2 or 3 . Need for RRT occurred in $33 \%$ of the patients with SS. The level of VEGF was greater than $1000 \mathrm{pg} /$ $\mathrm{ml}$ in $100 \%$ patients who developed AKI stage 2-3 and in those who required RRT. In patients with SS, a level of VEGF greater than 600 pg/ $\mathrm{ml}$ on admission to ICU strongly correlated with development of AKI $(r=0.76, p<0.05)$, need for RRT $(r=0.73, p<0.05)$ and more than $72 \mathrm{hs}$ of vasopressor support $(r=0.71, p<0.05)$. However, the strongest correlation with $A K I$ and $A K I$ requiring RRT was found in patients with with VEGF-A/sFlt-1 ratio greater than $2.0([r=0.91$ for $A K I, p<0.01]$, $[r=0.86, A K I$ on RRT $p<0.01])$. The ratio also strongly correlated with greater than 72 hs of vasopressor support $(r=0.83, p<0.01)$. Data is presented as Median \pm SEM.

Conclusion. Our data demonstrate that VEGF-A is consistently elevated in patients with SS and is a strong predictor for AKI, duration of vasopressor support and need for RRT. Given the significant and more robust increase in circulating VEGF-A than sFlt-1 during SS, we believe that VEGF-A/sFlt-1 ratio is a stronger and more reliable predictor for AKI during septic shock than measuring VEGF-A. Further studies are needed to determine if the VEGF-A/sFlt-1 ratio can be implemented to predict the progression from sepsis to septic shock as well as other distant organ complications related to septic shock.

\section{Reference(s)}

[1] Vascular Endothelial Growth Factor (VEGF) Contributes to Sepsis-induced Acute Kidney Injury. Lopez-Ruiz A et al. J. Am. Soc. Nephrol 26 (11), 2015

[2] The Third Consensus Definition of Sepsis and Septic Shock. Singer M. JAMA 315, 2016.

[3] The Surviving Sepsis Campaign Bundle; 2018 Update. Levy M.M et al. Intensive Care Med 44, 2018

\section{9}

\section{Measuring and Enhancing Physiotherapy Staff Well-being} during the SARS-CoV-2 3rd Wave

\section{E. Liston'; J. Dowds, ${ }^{2}$}

${ }^{1}$ Physiotherapy, St James's Hospital, Dublin, Ireland; ' 2 Department of physiotherapy, St James"s Hospital, Dublin, Ireland

Correspondence: E. Liston

Intensive Care Medicine Experimental 2020, 9(1): 001329

Introduction. The impact of SARS-CoV-2 pandemic on healthcare worker mental health and well-being has been recognised by The World Health Organisation (WHO)0.1 Anticipating possible effects of the 3rd wave on the Cardiorespiratory Physiotherapy team well-being we aimed to perform a weekly measure of staff well-being. In an effort to enhance well-being, we implemented team-based interventions and strategies, with a secondary aim to provide peer support. From 
the beginning of the pandemic the Cardiorespiratory Physiotherapy team led on treating the Covid-19 patient cohort across critical care and medical wards, including teaching and support to the broader physiotherapy team. In response to demands faced by critical care colleagues the team also worked extended hours to assist in Proning rounds, including evenings and weekends.

Methods. The Warwick and Edinburgh Mental Wellbeing Scale (WEMWBS) was anonymously completed every week by Cardiorespiratory Physiotherapy team members, from January to April 2021. Scores were collated and an average weekly score calculated. Weekly average scores were fed back to the team with opportunities to discuss possible causes and potential actions. A final team de-brief was completed at the end of April 2021.

Results. In total 9 data collection points were completed with a median number of participants of 11 (Range 8-12). The average team score ranged from 43.1 to 52 , where scores of $41-44$ correspond with possible depression and scores of 45-59 with average mental wellbeing. 2 As admissions and deaths attributable to Covid continued to increase we found a persistent low score in our team, with our lowest score corresponding with peak admissions in mid-February. Consistently low scores were found in "I've been feeling relaxed" and "I've had energy to spare". In contrast, there was a steady increase from this point associated with reduced case numbers, implementation of wellbeing interventions, improved weather, and vaccination program role out. Consistently high scores in "I've been feeling loved" and "I've been feeling useful" were recorded.

Conclusion. Our results demonstrated the negative impact of dealing with the Sars-CoV-2 pandemic on the mental health and well-being of our physiotherapy team. Due to implementing team interventions, in response to low mental health and well-being, as a team were able to maintain average mental health and well-being. Measuring and monitoring our collective well-being provided time for self-reflection and facilitated our de-brief discussions. Our results demonstrate the ongoing effects of the Sars-CoV-2 pandemic and highlight the need for additional support for healthcare workers at this time.

\section{Reference(s)}

1. Tennant, R., Hiller, L., Fishwick, R., Platt, S., Joseph, S., Weich, S., \& StewartBrown, S. (2007). The Warwick-Edinburgh mental well-being scale (WEMWBS): development and UK validation. Health and Quality of Life Outcomes, 2007; 5(1), 63.

2. Mental health and psychosocial considerations during the COVID-19 outbreak. World Health Organization (WHO), 2020

\section{4}

DNA Methylation signatures as potential prognostic biomarker in critical illness

D. Pestana ${ }^{1}$; C. J. Walsh²; B. Costa ${ }^{3}$; P. C. Liaw ${ }^{4}$; D. Dwivedi'; J. L. Y. Tsang ${ }^{5}$; P. Castelo-Branco ${ }^{3} ;$ C. C. Dos Santos ${ }^{6}$; A. Binnie ${ }^{3}$

${ }^{1}$ Epigenetics and human disease laboratory, Algarve Biomedical

Center, Faro, Portugal; ${ }^{2}$ Department of respirology, Michael Garron Hospital, Toronto, Canada; ${ }^{3}$ Faculty of medicine and biomedical sciences, University of Algarve, Faro, Portugal; ${ }^{4}$ Thrombosis and atherosclerosis research institute, department of medicine, McMaster University, Hamilton, Ontario, Canada; ${ }^{5}$ Department of medicine, McMaster University, Hamilton, Ontario, Canada; ${ }^{6}$ Institute of medical sciences and department of medicine, University of Toronto, Toronto, Canada

Correspondence: D. Pestana

Intensive Care Medicine Experimental 2020, 9(1): 001334

Introduction. Multiple clinical scoring systems have been developed to predict outcomes after critical illness including the Multiple Organ Dysfunction Score (MODS), the Sequential Organ Failure Assessment (SOFA) score, and the APACHE-II score, however their prognostic performance is only moderate. In this respect, biological markers might be helpful in improving prognostication. DNA methylation is an epigenetic mark that is relatively stable and can be easily measured in whole blood. In this study we examined the performance of DNA methylation as a marker of prognosis amongst critically-ill patients.
Methods. DNA methylation profiling was performed on whole blood samples from 134 critically-ill patients (66 septic and 68 non-septic), drawn on the first day of ICU admission. Methylation levels were analyzed using the Illumina Infinium HumanMethylation450 array. To identify $\mathrm{CpG}$ sites at which methylation levels were associated with severity of illness, patients were divided into "high MODS" (MODS score $\geq 9$ ) and "low MODS" (MODS score $\leq 8$ ) groups and Differentially Methylated Regions (DMRs) were identified that distinguished the two groups. This approach yielded 63 DMRs, including 241 individual CpG sites.

Patients were then subdivided into three groups according to ICU outcome: (1) survival, (2) early mortality ( $\leq 5$ day) and (3) late mortality ( $>6$ days). For each of the $241 \mathrm{CpG}$ sites, we performed an analysis of variance (ANOVA) between the three groups and, subsequently, a post-hoc analysis using Tukey's test.

Results. Our analysis revealed five CpG sites that showed significant variance across the three groups: two located in the body of TRPV 3 gene, two in the intergenic regions of chromosomes 2 and 7, and one upstream of the PPT2 transcription start site. Linear Discriminant Analysis was performed using the five $\mathrm{CpG}$ sites as predictor variables and the dataset was split into a training set (60\% of the patients) and a testing set ( $40 \%$ of the patients) to test the model. The resultant model correctly predicted the outcome for $71.14 \%$ of the observations in the test set. The accuracy of the model, although not perfect, reveals that an epigenetic signature might be useful in predicting outcomes of critical illness.

Conclusion. Our study suggests that DNA methylation may be an early prognostic biomarker in critically-ill patients. Further investigation is required to identify the optimal methylation signature and to validate these findings in other patient cohorts.

\section{Reference(s)}

1. Marshall JC, Cook DJ, Christou NV, Bernard GR, Sprung CL, Sibbald WJ. Multiple organ dysfunction score: a reliable descriptor of a complex clinical outcome. Crit Care Med. 1995;23(10):1638-1652. 10.1097/00003 246-199510000-00007

2. Rossaint J, Zarbock A. Pathogenesis of Multiple Organ Failure in Sepsis. Crit Rev Immunol. 2015;35(4):277-291. 10.1615/critrevimmunol.20150 15461

3. Ramírez M. Multiple organ dysfunction syndrome. Curr Probl Pediatr Adolesc Health Care. 2013;43(10):273-277. 10.1016/j.cppeds.2013.10.003

\section{3}

Long-term psychological burden of intensive care survivors

S. Rai ${ }^{1}$;. Neeman'; R. Brown ${ }^{3}$; F. van Haren ${ }^{4}$; A. Rajamani ${ }^{5}$;

K. Sundararajan ${ }^{6}$; I. Mitchell?; D. Needham ${ }^{8}$

${ }^{1}$ Intensive Care Unit, Canberra Health Services, Canberra, Australia;

${ }^{2}$ Biological data science institute, anu college of science, The Australian National University, Canberra, Australia; ${ }^{3}$ Research school of psychology, The Australian National University, Canberra, Australia; ${ }^{4}$ College of health and medicine, The Australian National University, Canberra, Australia; ${ }^{5}$ Intensive care unit, Nepean Hospital, Sydney, Australia; Intensive care unit, Royal Adelaide Hospital, Adelaide, Australia; ${ }^{7}$ Intensive care unit, Canberra Health Services, Canberra, Australia, Canberra, Australia;

${ }^{8}$ Pulmonary \& critical care medicine, Johns Hopkins University, Baltimore, United States of America

Correspondence: S. Rai

Intensive Care Medicine Experimental 2020, 9(1): 001403

Introduction. Over the last few decades, there has been emerging literature on the long-term outcomes of intensive care unit (ICU) survivors with respiratory failure requiring invasive ventilation. However, there are little published data on the long-term psychological outcomes of general ICU survivors and those not requiring invasive ventilation.

Objectives. The primary objective was to describe the severity of affective psychological symptoms [Post Traumatic Stress Disorder (PTSD), Depression, Anxiety and Stress] and impact on health related quality of life (HRQOL) in a general population of ICU survivors at 
12-months. The secondary aim was to compare outcomes between the intubated and non-intubated groups of ICU survivors.

Methods. Prospective, multicentre observational cohort study from 4 Australian teaching hospitals, with a 12-month follow up of ICU survivors. Study participants included intubated ICU survivors with a length of ICU stay $>72 \mathrm{~h}$ and non-intubated ICU survivors who received organ support in the ICU. Validated tools for screening for affective symptoms included the Post Traumatic Stress Scale 14 (PTSS14), Depression Anxiety Stress Scale-21 items (DASS21) and EQ5D-5L tool for HRQOL. Established scales for the assessment tools were used to classify severity of clinical symptoms.

Results. Of the 172 patients recruited, $9(5 \%)$ died at hospital discharge, 12 (7\%) withdrew consent before any follow-up and 19 (11\%) were lost to all follow up. Overall, 133 patients were included in the final data analysis and over $80 \%$ completed follow-up assessments. At 12-months, clinically significant PTSD symptoms were evident in $10 \%$ of the overall ICU survivors, with a reasonable proportion depicting moderate or worse symptoms of depression $(20 \%)$, anxiety (15\%) and stress (9\%). There was no difference in the severity of affective symptoms between the intubated and nonintubated groups at follow-up. On a visual analogue scale (0-100), the survivors perceived their overall health status at a median of 70 [IQR 21], with overall mean EQ5D index value at $0.65(0.03)$ at 12 months. HRQOL problems were noted in every domain assessed mobility $(42 \%)$, selfcare $(26 \%)$, usual activities $(46 \%)$, pain or discomfort (48\%) and anxiety or depression (38\%).

Conclusion. At 12-months, a significant proportion of ICU survivors had symptoms suggestive of PTSD, depression, anxiety, stress and problems with their HRQOL. The non-intubated ICU survivors had a similar psychological burden as the invasively ventilated and should be included in future research and strategies to improve outcomes.

\section{Reference(s)}

1. Grants by Canberra Hospital Private Practice Fund and Royal Adelaide Hospital Maurice Sando Foundation Sponsorship Scheme

\section{7}

\section{Microbiological Point of Care analysis of endotracheal aspirate} from intubated patients admitted to the Intensive Care Unit

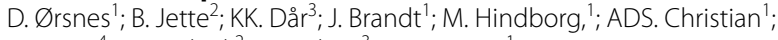
K. Mette ${ }^{4} ;$ H. Frederik'; AJJ. Claus ${ }^{3} ;$ HB. Morten ${ }^{1}$

${ }^{1}$ Dept. of anaesthesiology and intensive care, Nordsjaellands Hospital, Hilleroed, University of Copenhagen, Copenhagen, Denmark; ${ }^{2}$ Dept. of clinical microbiology, Herlev Hospital, Hilleroed, University of Copenhagen, Copenhagen, Denmark; ${ }^{3}$ Dept. of clinical biochemistry, Nordsjaellands Hospital, Hilleroed, University of Copenhagen, Copenhagen, Denmark; ${ }^{4}$ Dept. of clinical microbiology, Rigshospitalet, University of Copenhagen, Denmark, Copenhagen, Denmark

Correspondence: D. Ørsnes

Intensive Care Medicine Experimental 2020, 9(1): 001417

Introduction. The paraclinical diagnosis of lower respiratory tract infection(LRTI) still relies heavily on culturing of respiratory tract samples which are quite insensitive, hence resulting in false negative response. There is a growing market for commercially available multiplex polymerase chain reaction( $\mathrm{PCR}$ ) assays promising fast turnaround time(TAT) and high sensitivity towards a variety of common pathogens, which may improve antibiotic stewardship.

Objectives. The purpose of this study was to assess the applicability of the FILMARRAY ${ }^{\circledR}$ Pneumonia Panel Plus(PP) in the clinical setting of an Intensive Care Unit(ICU).

Methods. Tests were performed on undiluted specimens from mechanically ventilated patients at the ICU on the first day of intubation. The FILMARRAY ${ }^{\circledR}$ Pneumonia Panel Plus was used to test 76 endotracheal aspirate samples frozen in a biobank. Comparison with pathogen detection using conventional microbiological methods(CMM) was performed, and concordance rates between methods was assessed taking into account incomplete investigation by conventional methods. Evaluation on the usage of antibiotics was done retrospectively by a clinical microbiologist and an Intensive Care physician assessing a potential change in treatment based on FILMARRAY $^{\circledR}$ results and patient status.

Results. In 42(55,3\%) samples PP and CMM are in full concordance. Additional findings by CMM were seen in $2(2,6 \%)$ samples. Additional findings by PP were seen in $31(40,7 \%)$ samples. Complete discrepancy between methods were seen in 1(1,3\%) sample. In 2 samples, PP detected methicillin resistant staphylococcus Aureus, even though CMM showed methicillin sensitive staphylococcus Aureus. By CMM, extended virus panel TAT was a median of $111,28 \mathrm{~h}$. TAT for culture and susceptibility a median 52,82 h. PCR for atypical $40,04 \mathrm{~h}$. PP TAT was estimated $2 \mathrm{~h}$, making de-escalation possible in $35(46,1 \%)$ patients, among these discontinuation of macrolide would have been possible in $24(31,6 \%)$.

Conclusion. The FILMARRAY ${ }^{\circledR}$ Pneumonia Panel Plus may offer valuable information, however it detected several additional pathogens compared to conventional microbiological methods and the interpretation of results appears difficult in terms of disease causality and antibiotic resistance. The biggest impact may be on fast-delivered negative results, which resonated in the possible discontinuation of macrolide. Even though our study showed potential for theFILMAR$R A Y^{\circledR}$ Pneumonia Panel Plus, further investigations on clinical effect should be performed prospectively.

\section{Reference(s)}

Conflict of interest:

FILMARRAY ${ }^{\circledR}$ Pneumonia Plus Panels and QC-material was sponsored by BioMérieux SA along with cost of shipment. All agreements have been revised and approved by the Unit for Research an Innovation, The Capital Region of Denmark.

\section{9}

Determination of Serum Zinc and Copper Levels and Their Relation with Mortality in Critically III Patients

M. Yildirim, ; B. Kesikli, ; L. Talan, ; MA. Tekes, ; N. Yazıhan, ${ }^{3}$; ND. Altintas ${ }^{3}$

${ }^{1}$ Interdisciplinary food, metabolism and clinical nutrition department, Ankara University, Institute of Health Sciences, Ankara, Turkey;

${ }^{2}$ Department of internal medicine, division of physiopathology, Ankara University, Faculty of Medicine, Ankara, Turkey; ${ }^{3}$ Department of internal medicine, division of intensive care medicine, Ankara University, Faculty of Medicine, Ankara, Turkey; ${ }^{4}$ Department of internal medicine, Ankara University, Faculty of Medicine, Ankara, Turkey

Correspondence: N.D. Altintas

Intensive Care Medicine Experimental 2020, 9(1): 001419

Introduction. It is known that nutrition and trace elements are important in critically ill patients and trace element levels change during inflammation[1]. Since zinc and copper are included in the structure of superoxide dismutase enzyme, which is involved in antioxidant defense systems, it may be crucial to consider serum zinc and copper levels during treatment in intensive care units (ICU) [2].

Objectives. To determine the relationship between serum zinc and copper levels, serum biomarkers of inflammation and mortality during critical illness.

Methods. During the study period 55 critically ill patients admitted to ICU who met study criteria and age and sex matched 20 control patients from outpatient clinics were included in our study. Blood samples for copper, zinc, IL-6, IL-10, and ceruloplasmin levels were collected from ICU patients on the 1st, 3rd and 7th days and once from the control group.

Results. Of the 55 patients included in the study $25(45,5 \%)$ was male. The mean age was $72.9 \pm 12.9$ years. Mean body mass index of patients was $25.4 \pm 5.6$. Main admission diagnoses were sepsis, pneumonia and respiratory failure. Mean APACHEII score was $23.6 \pm 6.5$, mean SOFA score was $6.1 \pm 3.4$, mean NUTRIC score was $5.8 \pm 1.6$. Of the patients $36(65 \%)$ received mechanical ventilation, $27(\% 49)$ received vasopressors. Length of ICU stay was $13.8 \pm 9.01$ days. 28th day mortality was $36 \%$. Serum zinc levels were significantly lower $(53.0 \mathrm{ug} / \mathrm{dl}$ vs 95.0 
$\mathrm{ug} / \mathrm{dl}, \mathrm{p}<0,001)$ and serum copper levels were significantly higher (119.2 vs $93.8, p=0.003)$ in critically ill patients. Serum zinc levels showed an increase through day 1 to day 7 as CRP and procalcitonin levels decreased, statistically significant in survivors. The increase in serum zinc levels was inversely correlated with the change in positive acute phase reactant levels (CRP: $R=-0,353 p=0,017 ; P C T: R=-0,547$ $p \leq 0,000)$. Serum copper and ceruloplasmin levels were significantly higher in survivors than in non-survivors on the 1 st and 3rd days (1st day copper levels: $128.8 \mathrm{ug} / \mathrm{dl}$ vs $102.5 \mathrm{ug} / \mathrm{dl}, \mathrm{p}=0.004 ; 1$ st day ceruloplasmin levels: $0.41 \mathrm{~g} / \mathrm{l}$ vs $0.32 \mathrm{~g} / \mathrm{l}, \mathrm{p}=0.005$; 3 rd day copper levels: $125.08 \mathrm{ug} / \mathrm{dl}$ vs $123.7 \mathrm{ug} / \mathrm{dl}, \mathrm{p}=0.004 ; 3 \mathrm{rd}$ day ceruloplasmin levels $0.38 \mathrm{~g} / \mathrm{l}$ vs $0.30 \mathrm{~g} / \mathrm{l}, \mathrm{p}=0.004$ ). For all days, serum copper/ceruloplasmin ratio did not differ significantly between groups. Between the $3 \mathrm{rd}$ and the 7th days of the study IL-10 levels decreased in survivors, while it increased in non-survivors. Rate of change in IL-10 level on 7th day was significantly different between survivors and non-survivors $(p=0.005)$. When logistic regression analysis was performed, copper and zinc were not found to be independent factors affecting mortality. Conclusion. The decrease in zinc levels and the increase in serum copper and ceruloplasmin levels during acute illness may be associated with the systemic inflammatory response of the body. In this study, serum zinc and copper levels were not found to be independently associated with mortality.

\section{Reference(s)}

1. Besecker B. Dietary and Nutritional Aspects of Zinc in Critically III Adult Patients. In: Rajendram R, Preedy VR, Patel VB (eds) Diet and Nutrition in Critical Care. Springer, New York, NY, 2015.

2. Singer P, Blaser AR, Berger MM, Alhazzani W, Calder PC, Casaer MP, et al. ESPEN guideline on clinical nutrition in the intensive care unit. Clin Nutr. 2019:38(1):48-79.

3. Grant was received from Ankara University Scientific Research Projects Coordination Unit (Project no: 20L0230006)

\section{1}

Early Versus Late Sustained Low Efficiency Dialysis in Critically III Cirrhotics With Septic Shock and Acute Kidney Injury- An Open Label Randomized Controlled Trial-NCT02937961

R. Maiwall ${ }^{1}$; A. Hidam ${ }^{1}$; V. Nayak'; RP. Mathur ${ }^{1}$; S. Sinha ${ }^{1}$; A. Kumar ${ }^{1}$; S. Sarin ${ }^{1}$ ${ }^{1}$ Hepatology, Institute of Liver and Biliary Sciences, New Delhi, India

Correspondence: R. Maiwall

Intensive Care Medicine Experimental 2020, 9(1): 001431

Introduction. Renal dialysis is a key to the management of acute kidney injury (AKI) in the intensive care unit (ICU). Critically ill cirrhotics (CIC) pose a management challenge due to severe and progressive metabolic derangements and the ideal timing of initiation of dialysis support in the absence of life-threatening complications in $\mathrm{CIC}$ is not known.

Objectives. We aimed to compare the safety and efficacy of early (EG) versus late (LG) initiation of sustained-low-efficiency dialysis (SLED) in $\mathrm{CIC}$ with septic shock and AKI. The primary end-point was deaths due to renal failure at day 28 while secondary outcomes included the impact of initiation of dialysis strategy on hemodynamics, renal recovery, reversal of shock, and overall 28-day mortality. We also aimed to assess the role of urine neutrophil gelatinase-associated lipocalin( $(\mathrm{NNGAL})$ and cystatin c (CysC) in predicting clinical outcomes.

Methods. CIC with septic shock and AKI after initial fluid resuscitation were randomized to EG wherein SLED was initiated within 6-12 h of presentation while in the LG was initiated once the patient met absolute criteria (i.e.refractory hyperkalemia, metabolic acidosis, fluid overload, uremia, or anuria $>24 \mathrm{~h}$ ). Renal biomarkers were done for all patients at enrolment CysC was evaluated using nephelometry and UNGAL by spectrophotometry. SLED sessions were targeted to $8-10 \mathrm{~h}$ in duration with blood and dialysate flows of $150-200 \mathrm{~mL} / \mathrm{min}$ and $300 \mathrm{ml} / \mathrm{min}$, respectively. SLED was delivered using the dialysis machines (Fresenius, 4008S, Dialyser F6 HPS). In patients who developed hemodynamic instability, continuous renal replacement therapy was administered as the rescue strategy as continuous venovenous hemodiafiltration (CVVHDF) using Prisma and Prismaflex (Gambro) devices, with blood flows ranging from $150-180 \mathrm{~mL} / \mathrm{hr}$ and target effluent rates of $20-25 \mathrm{~mL} / \mathrm{kg} / \mathrm{hr}$. Anticoagulation was not used during dialysis.

Results. Fifty CIC (aged $46 \pm 9$ years, 90\% males, $87 \%$ alcohol-related) were randomized. Pneumonia (72\%), and spontaneous bacterial peritonitis (4\%) were common. Baseline demographic and renal parameters were comparable including baseline lactate $(\mathrm{mg} / \mathrm{dl})[2.7 \pm 1.8 \mathrm{vs}$. $3.2 \pm 2.1]$, MELD[ $34.8 \pm 6.1$ vs. $34.7 \pm 6.9]$ and SOFA scores[12.9 \pm 2.1 vs. $13.6 \pm 3.9]$. The levels of CysC $[2.7 \pm 0.6$ vs. $3.0 \pm 0.7](\mathrm{mg} / \mathrm{L})$ and UNGAL [ $2091.3 \pm 1928.1$ vs. $2359.7 \pm 1738.4] \mathrm{ng} / \mathrm{ml}$ respectively in EG vs.LG. Median time to dialysis (in hours)was 7(IQR 4-8) in EG vs 24(17-48) in LG. Patients in the EG at $48 \mathrm{~h}$ had significantly higher serum bicarbonate $[23.2 \pm 4.9$ vs. $21.3 \pm 4.6 ; p=0.02]$, lower serum potassium [3.9 \pm 0.5 vs. $4.2 \pm 0.7 ; p=0.04]$, CVP $(12.7 \pm 2.3 v s .14 \pm 2.04 ;]$ and serum phosphate levels $[3.4 \pm 0.9$ vs.4.0 $\pm 1.3 ; p=0.04]$ than $L G$ respectively. Mortality at 28 -days $[60 \%$ vs. $76 \% ; p=0.23]$ was similar, however, a much lower proportion of deaths were noted in the EG due to renal failure [4\%vs.24\%; $p=0.04]$.A lower incidence of intradialytic hypotension(IDH)[12\% vs. $48 \% ; p=0.005]$, higher urea reduction ratio[75\% vs. $41 \% ; p=0.019]$, lactate clearance $[54 \%$ vs. $9 \% ; p=0.001]$, improvement in SOFA scores(by 2 points) at $48 \mathrm{~h}[40 \%$ vs. $17 \% ; p=0.07]$ and reversal of shock [60\% vs.16\%; $p=0.001]$ was noted in the EG than LG. The hemodynamic stability (any increase in vasopressors) was similar in both groups. A greater frequency of recovery of renal functions [68\% vs. $12 \% ; p<0.001]$ was noted in $E G$ which also translated into improved 28-day survival [HR 0.39, 0.18$0.84]$ after adjusting for the baseline SOFA score. Renal biomarkers did not predict 28-day mortality, however, $\mathrm{Cys} C$ predicted renal recovery [OR 0.30, 0.11-0.83) while levels of UNGAL predicted reversal of shock $(0.61,0.39-0.95)$.

Conclusion. Critically ill cirrhotics with septic shock and severe AKI have high short-term mortality. Early initiation of dialysis could timely avert the metabolic complications, decrease the incidence of IDH and deaths due to renal failure. A higher frequency of recovery of renal functions and reduced AKI-related mortality could be achieved by early initiation of renal dialysis in $\mathrm{CICs}$.

\section{Reference(s)}

1. Zarbock A, Kellum JA, Schmidt C, et al.Effect of Early vs Delayed Initiation of Renal Replacement Therapy on Mortality in Critically III Patients With Acute Kidney Injury: The ELAIN Randomized Clinical Trial. JAMA. 2016 May 24-31;315(20):2190-9.

2. Gaudry S, Hajage D, Schortgen F et.al AKIKI Study Group. Initiation Strategies for Renal-Replacement Therapy in the Intensive Care Unit. N Engl J Med. 2016 Jul 14;375(2):122-33.

3. STARRT-AKI Investigators; Canadian Critical Care Trials Group; Australian and New Zealand Intensive Care Society Clinical Trials Group; United Kingdom Critical Care Research Group; Canadian Nephrology Trials Network; Irish Critical Care Trials Group, Bagshaw SM, Wald R, Adhikari NKJ, et al,. Timing of Initiation of Renal-Replacement Therapy in Acute Kidney Injury. N Engl J Med. 2020 Jul 16;383(3):240-251

4. ICMR project ID-2019-0084

\section{7}

Moral problems among ICU professionals in the COVID-19 pandemic: a longitudinal qualitative exploration of moral injury and moral distress

N. Kok'; 'CWE. Hoedemaekers'²; H. Van Der Hoeven²; M. Zegers, ${ }^{2}$; J. Van Gurp

${ }^{1}$ Iq healthcare, Radboud University Medical Center, Nijmegen, Netherlands; ${ }^{2}$ Intensive care, Radboud University Medical Center, Nijmegen, Netherlands

Correspondence: N. KoK

Intensive Care Medicine Experimental 2020, 9(1): 001447

Introduction. The Coronavirus Disease 2019 (COVID-19) pandemic has mainstreamed the use of moral injury to describe the most impactful/complex/emotionally charged experiences of intensive care unit (ICU) professionals. Symptoms of moral injury are persisting feelings 
of guilt and/or shame, loss of trust in goodness, moral detachment and/or disorientation.[1] Moral injury stems from military psychology. It is yet understudied in medicine and it is unclear how it relates to the more predominant notion of moral distress, which is thought to be of a more ephemeral nature.[2].

Objectives. To uncover relevant conceptual distinctions between moral injury and moral distress of/with ICU professionals by studying moral problems related experienced while working/facing the COVID19 pandemic.

Methods. This is a prospective longitudinal qualitative study in which ICU physicians and nurses are serially interviewed over a period of 14 months during 2020-2021. The semi-structured interviews were guided by a topic list based on sensitizing concepts stemming from the current literature on moral injury and distress. Interviews were transcribed and then analyzed using CAQDAS ATLAS.TI 8.4.20. Transcripts were coded using a thematic approach, using open, axial and constant comparison coding strategies.

Results. The current dataset comprises 45 interviews with 25 unique ICU physicians and nurses. Prevailing situations that caused moral problems where downscaling quality of care, impersonal care, looming triage, prioritizing work activities due to lack of time/personnel, the relative absence of family members of patients and intrusive thoughts about patients. Feelings of catastrophe were mainly experienced in the first COVID-19 wave (March-April 2020). Nurses indicated to experience personal failure, guilt and powerlessness about care provision mostly during this time. In subsequent interviews, however, they described a realization that there was nothing they could do to avoid failure. This stimulated acceptance and self-forgiveness. Most did not experience persistent negative feelings. Physicians had anticipatory anxiety about becoming triagists. Some worried that they were becoming increasingly indifferent towards patients. They reported anxiousness and/or a sense of shame at the normalization of feeling morally detached from patients. Most professionals were initially angry at the public's relaxing commitment to prevent the spread of COVID-19 and blamed policymakers for not seeing ICU professionals plight. Some even felt betrayed and lost trust, and later grew progressively resigned or defeated about this.

Conclusion. No clear-cut distinction between moral injury and moral distress was found. As of yet, most of the ICU professionals' experiences of failure and guilt are consistent with conceptualizations of moral distress and less so with those of moral injury, as the distressing episodes seemingly do not persist. Growing moral detachment and normalization of deviance may, however, be signs of a developing moral injury.

\section{Reference(s)}

1. Dean W, Talbot SG, Caplan A (2020). Clarifying the Language of Clinician Distress. JAMA 323(10): 923-924.

2. Jinkerkson J (2016). Defining and Assessing Moral Injury: a Syndrome Perspective. Traumatology 22(2): 122-130.

\section{1}

\section{Critical Care-Associated Dysglycaemia in Covid-19:}

Steroid-Induced or Occult Diabetes Mellitus?

M. Sisley"; N. Daines ; A. Trimmings,

${ }^{1}$ Intensive care, East Sussex Healthcare NHS Trust, Eastbourne, United

Kingdom

Correspondence: M. Sisley

Intensive Care Medicine Experimental 2020, 9(1): 001461

Introduction. Covid-19 can be a life-threatening disease with significant implications for critical care resources. Data suggests that the presence and severity of obesity, type two diabetes and metabolic syndrome are independent prognostic indicators (Popkin et al., 2020). In addition, diabetes has been identified as the most modifiable independent risk factor for morbidity and mortality in Covid-19 (NHS England, 2020).
Recent UK guidelines (COVID: Diabetes) have recommended $\mathrm{HbA1C}$ testing for all patients with suspected or confirmed Covid-19 infection at the time of initial assessment (Joint British Diabetes Societies for Inpatient Care, 2021). HbA1c is a measure of glycosylated haemoglobin, obtained from a relatively cheap blood biochemistry test. The level reflects average blood glucose over a period of around 120 days. It can therefore be used to rapidly detect chronic hyperglycaemia (i.e., diabetes mellitus).

Current routine practice across our trust does not involve testing $\mathrm{HbA1c}$ on admission to intensive care in either known diabetic or non-diabetic patients. The implication of this is that new diagnosis of diabetes mellitus may be missed, information about ongoing diabetic control may be overlooked, and hyperglycaemia may be falsely attributed to the administration of steroids in the treatment of Covid-19.

Objectives. Our primary objective was to assess critical care compliance with national guidance for inpatient care of patients with COVID-19 across our trust. Looking forward we hope to aid the early recognition of a disease that has a long-term financial burden if left untreated, improve prognostication of critically ill patients, and avoid misdiagnoses, such as when hyperglycaemia is attributed to the administration of steroids or critical illness itself.

Methods. Data from the 120 patients admitted to intensive care with Covid-19 pneumonitis over a three-month surge period were retrospectively reviewed and analysed, including admission biometrics of weight, height, $\mathrm{BMI}, \mathrm{HbA} 1 \mathrm{c}$ result and documented diagnoses of diabetes mellitus.

Results. Only $16.7 \%$ of patients with Covid-19 pneumonitis requiring organ support for critical illness had their $\mathrm{HbA1c}$ measured during admission, with just 3.3\% checked at the point of admission. Moreover, $90.0 \%$ of the HbA1c values measured were in the diabetic or pre-diabetic range. The average $\mathrm{HbA1c}$ of those measured was $70.5 \mathrm{mmol} / \mathrm{mol}$ which reflects an average blood glucose measurement of $11.1 \mathrm{mmol} / \mathrm{L}$ over the preceding three months. A pre-existing diagnosis of diabetes mellitus was documented for $25.0 \%$ of the patients admitted over this time. Of the thirteen patients who had an $\mathrm{HbA} 1 \mathrm{c}$ measurement with no prior history of diabetes, $53.8 \%$ were in the diabetic range (i.e., greater than $48 \mathrm{mmol} / \mathrm{mol}$ ). This represented seven new diagnoses of diabetes mellitus. Furthermore, out of the 108 admissions where height and weight were recorded, $90.0 \%$ were overweight or obese. Despite overweight and obesity being significant risk factors for type two diabetes, only $13.4 \%$ of this group had their $\mathrm{HbA1c}$ measured at any point. Only $42.9 \%$ of patients with obesity had a recorded diagnosis of obesity or "high BMI" in their past medical history.

Conclusion. Taking broader lessons from the Covid-19 pandemic, ITU admission is currently a missed opportunity to identify new diagnoses of diabetes in a patient population extremely likely to have comorbid metabolic disease. As an easily modifiable risk factor for morbidity and mortality, not just in concurrent Covid-19 infection, this represents overlooked potential within our trust to improve health outcomes and provide future cost savings through the prevention of the burdensome complications of the disease.

\section{Reference(s)}

1. Popkin, B., Du, S., Green, W., Beck, M., Algaith, T., Herbst, C. Alsukait, R. Alluhidan, M., Alazemi, N. and Shekar, M., 2020. Individuals with obesity and COVID-19: A global perspective on the epidemiology and biological relationships. Obesity Reviews, 21(11).

2. NHS England. (2020). Delivering Diabetes Care during the COVID-19 Pandemic-the'new normal' [Version 2]. https://www.diabetes.org.uk/ resources-s3/public/2020-06/Delivering\%20Diabetes\%20Care\%20dur ing\%20the\%20COVID-19\%20Pandemic\%20220620.pdf

3. Joint British Diabetes Societies for Inpatient Care. (2021). Concise advice on inpatient diabetes (COVID:Diabetes): hyperglycaemia/diabetes guidance for people with Covid-19 infections [Version 1.2]. https://diabetesresources-production.s3.eu-west-1.amazonaws.com/resources-s3/public/ 2021-03/COvID_Virtual_Ward_v1.2.pdf 
000259

A qualitative exploration of respiratory physiotherapists experiences of lung ultrasound training and its adoption in critical care within the United Kingdom

S. Hayward'; S. Innes'; M. Smith ${ }^{3}$

'Physiotherapy department, Blackpool Teaching Hospitals NHS Trust, Blackpool, United Kingdom; ${ }^{2}$ School of sport, rehabilitation and exercise sciences, University of Essex, Colchester, United Kingdom; ${ }^{3}$ School of healthcare sciences, Cardiff University, Cardiff, United Kingdom Correspondence: S. Hayward

Intensive Care Medicine Experimental 2020, 9(1): 000259

Introduction. Lung ultrasound (LUS) is gaining popularity amongst respiratory physiotherapists as an imaging modality to aid pulmonary assessments, guide intervention selection and monitor the efficacy of interventions. The ability of respiratory physiotherapists to incorporate LUS into their practice is influenced by multiple factors.

Objectives. The aim of this study was to explore the experiences of respiratory physiotherapists who have initiated the adoption and implementation of LUS into their practice in critical care.

Methods. This study used qualitative research methodology through semi-structured interviews to explore the varied experiences of eight senior respiratory physiotherapists of adopting and implementing LUS into their practice in critical care within the United Kingdom. Our previously published national survey was used to inform the semistructured interview questions via the identification of concepts for exploration and elaboration. The interviews took place by telephone, were digitally recorded and transcribed verbatim. The transcribed data were thematically analysed.

Results. Thematic analysis resulted in the identification of five overarching themes that participants highlighted in relation to the research topic. The themes were: (i) support for physiotherapists using LUS; support was generally high across the critical care multi-disciplinary team but with some hesitation coming from physiotherapy managers. (ii) Knowledge and understanding of LUS evidence; as LUS is an emerging field within physiotherapy it therefore requires some evidence translations from other professions. (iii) Governance; participants believe LUS enhances their clinical effectiveness, the educational pathways were perceived as adequate and the risks of LUS are low but require consideration. (iv) Physiotherapists' motivation to use LUS; participants reported a range of personal, professional, and patient focused factors as rationale for engaging with LUS. (v) Resources; need to be in place to ensure mentorship and ultrasound equipment are available along with protected time to practice. Once accreditation has been achieved, LUS was considered a time effective skill to attain by enabling targeted resource allocation.

Conclusion. Participants reported a range of factors that influenced their ability to adopt and implement LUS into practice: several were enabling while others were barriers to progress. Enabling factors from within the themes included support from senior clinicians, peers, colleagues and mentors, motivation for personal and professional development, optimising patient care and efficient allocation of resources. Barriers included difficulty accessing mentorship, lack of machine availability, limited time to train, lack of governance clarity and reluctance from some physiotherapy managers to support LUS adoption. The adoption of LUS by physiotherapists remains in its infancy but participants believed it showed potential for the profession.

\section{Reference(s)}

1. Hayward S, Smith M, Innes S. Diagnostic thoracic ultrasound imaging An exploration of respiratory physiotherapists' interest and use in clinical practice: A national survey. Ultrasound. 2020 Feb;28(1):14-22. https://doi. org/10.1177/1742271X19861131

2. Hayward SA, Janssen J. Use of thoracic ultrasound by physiotherapists: a scoping review of the literature. Physiotherapy. 2018 Dec;104(4):367-375. https://doi.org/10.1016/j.physio.2018.01.001

3. Le Neindre A, Mongodi S, Philippart F, Bouhemad B. Thoracic ultrasound: Potential new tool for physiotherapists in respiratory management. A narrative review. J Crit Care. 2016 Feb;31 (1):101-9. https://doi.org/10.1016/j. jerc.2015.10.014
e-Posters: Acute Kidney Injury and haemofiltration

\section{9}

Adding antibiotics in dialysate fluid during renal replacement therapy: proof of concept

I. Bartuševičiené ${ }^{1}$; V. Vicka'; D. Ringaitiene ${ }^{3}$; J. Sipylaite ${ }^{4}$

${ }^{1}$ Faculty of Medicine, Vilnius University, Vilnius, Lithuania; ${ }^{2}$ Anesthesiology and reanimatology clinic, Vilnius university Hospital Santaros Clinic, Vil-

nius, Lithuania; ${ }^{3} \mathrm{Clinic}$ of anesthesiology and intensive care, Vilnius University Hospital, Santaros Clinic, Vilnius, Lithuania; Institute of clinical medicine, clinic of anaesthesiology and intensive care, Faculty of Medicine of Vilnius University, Vilnius, Lithuania

Correspondence: I. Bartuševičienè

Intensive Care Medicine Experimental 2020, 9(1): 000019

Introduction. Studies have shown significant variability in antibiotic trough concentrations in critically ill patients receiving RRT. Unintended under-dosing leads to increased resistance of the microflora and sub-optimal effect, prolonged hospitalization and worse clinical outcome.

Objectives. The purpose of this study was to assess whether adding beta-lactam antibiotics to dialysate solution can maintain stable antibiotic concentrations during CRRT in experimental conditions.

Methods. A single compartment model reflecting the patient was constructed and connected to the RRT machine. CVVHD modality of RRT was chosen. Dialysate fluid was prepared in three different concentrations of meropenem $(0 \mathrm{mg} / \mathrm{L} ; 16 \mathrm{mg} / \mathrm{L} ; 64 \mathrm{mg} / \mathrm{L})$. For each dialysate concentration various combinations of dialysate and blood flow rates were tested by taking different samples. Meropenem concentration in all samples was calculated using spectrophotometry method.

Results. Constructed experimental model results suggest that decrease in blood meropenem concentration can be up to $35.6 \%$. Moreover, experimental data showed that antibiotic loss during CVVHD can be minimized and stable plasma antibiotic concentration can be achieved with the use of a $16 \mathrm{mg} / \mathrm{L}$ Meropenem dialysate solution. Furthermore, increasing meropenem concentration up to $64 \mathrm{mg} / \mathrm{L}$ is associated with an increase antibiotic concentration up to $18.7-78.8 \%$.

Conclusion. Our experimental study suggests that the administration of antibiotics to dialysate solutions may be an effective method of ensuring a constant concentration of antibiotics in the blood of critically ill patients receiving RRT. Further research confirming the safety and efficiency of the method application in clinical practice is required.

\section{4}

Prevalence and Outcomes of Acute Kidney Injury in COVID-19 Critically III Patients

C. Roriz'; D. Castro'; O. Gomes ' F. Henriques ${ }^{1}$; C. Santos ${ }^{1}$; F. Sequeira1;

N. Ferreira'; A. Araujo ${ }^{1}$; P. Viana ${ }^{1}$; L. Pereira ${ }^{1}$

'Intensive Care Unit, Centro Hospitalar de Leiria, Leiria, Portugal

Correspondence: C. Roriz

Intensive Care Medicine Experimental 2020, 9(1): 000314

Introduction. The outbreak of coronavirus disease 2019 (COVID-19) has rapidly evolved into a global pandemic. Although most patients with COVID-19 have mild symptoms, some develop severe symptoms, which can include acute respiratory distress syndrome, septic shock and multiple organ failure. Acute kidney injury is common among critically ill patients with COVID-19, affecting approximately $20-40 \%$ of patients admitted to intensive care units (ICU) according to reports from Europe and USA, and it is considered a marker of disease severity and a negative prognostic factor for survival.The pathogenesis of acute kidney injury (AKI) in patients with COVID-19 is likely multifactorial, involving the direct effects of the SARS-CoV-2 virus on the kidney and the indirect mechanisms resulting from systemic consequences of viral infection on distant organs including the lung, in addition to mechanisms relating to the management of COVID-191. 
Objectives. The purpose of this study is to evaluate the prevalence of AKI and outcomes of all the COVID-19 critically ill patients admitted to our intensive care unit between 1st March 2020 to 1st March 2021.

Methods. A retrospective cohort study of all COVID-19 patients admitted in a ten-bedded combined medical-surgical ICU of a Portuguese hospital between 1st March 2020 to 1st March 2021. Acute kidney injury was defined using the Kidney Disease Improving Global Outcome (KDIGO) serum creatinine criteria. We considered the baseline serum creatinine as the serum creatinine level that was measured on the day of admission. AKI recovery was defined as a decrease of serum creatinine value to a value less than 1.20 times the baseline creatinine level. Comparisons were performed using the Mann-Whitney U-test for quantitative variables and Pearson-Chi-square for qualitative variables.

Results. A total of 103 patients were admitted during this period. The median patient's age was 67 years (minimum 29 years; maximum 85 years) being $78.6 \%$ of them men (81). The median ICU length of stay was 12 days [7.75-19], 85 patients needed mechanical ventilation $(82 \%)$ and the median time with mechanical ventilation was 11 days [5-19], being that all patients had acute hypoxic respiratory failure and needed either noninvasive or invasive mechanical ventilatory support. Regarding the prognostic indices the median SAPS 2 was 43 [15-26] and the median APACHE II was 19 [15-26]. Forty-three patients $(41,7 \%)$ had evidence of AKI (Stage I-12,6\%, Stage II-13,6\%, Stage III-15,5\%). Eight patients needed renal replacement therapy $(7,8 \%)$. Regarding prognostic scores patients with AKI had higher APACHE II scores ( 26 vs $18 p<0.01)$ and higher SAPS 2 levels (51 vs $38 p<0.01$ ). We also observe that patients who developed AKI were statistically older (72 vs $64 \mathrm{p}<0.01)$ and had higher rates of previous arterial hypertension ( 39 vs $37 p=0.01$ ). Among the twenty-six patients who recovered from AKI 18 patients survive while 8 patients die in the ICU $(p<0.01)$. Compared with non-AKI group, development of AKI was associated with a greater ICU mortality (24 vs $11 \mathrm{p}<0.001)$.

Conclusion. Acute kidney injury is common in critically ill patients presenting with COVID-19. It is associated with higher rates of previous arterial hypertension, increased severity of illness on admission to ICU and increased mortality. We also observe that patients who recover from AKI had a higher probability of survive.

\section{Reference(s)}

1. Lowe R., Ferrari M., Clinical characteristics and outcome of critically ill COVID-19 patients with acute kidney injury: a single centre cohort study. BMC Nephrology. 2021

2. Acute kidney injury in COVID-19 patients. ESICMtv Webinar. Posted April 17, 2020. https://mediatheque.cyim.com/mediatheque/media.aspx? mediald $=81938 \&$ channel $=71460$ (Accessed on April 11, 2021)

3. Nadim M., Forni L., Mehta R., et al. COVID-19-associated acute kidney injury: consensus report of the 25th Acute Disease Quality Initiative (ADQI) Workgroup. Nature Reviews Nephrology. 2020

\section{5}

Incidence of de-novo renal injury in patients admitted to intensive care with sepsis and associated in-ICU and in-hospital mortality

R. Shemilt' ${ }^{1}$ M. Andonovic ${ }^{2}$; J. Traynor ${ }^{3}$; M. Shaw ${ }^{2}$; M. Sim² ${ }^{2}$ P. Mark' K. Puxty ${ }^{2}$

${ }^{1}$ University of Glasgow, Glasgow, United Kingdom; ${ }^{2}$ Academic Department of Anaesthesia and Critical Care, University of Glasgow, Glasgow, United Kingdom; ${ }^{3}$ Department of nephrology, Queen Elizabeth University Hospital, Glasgow, United Kingdom; ${ }^{4}$ Institute of cardiovascular and medical sciences, University of Glasgow, Glasgow, United Kingdom

Correspondence: R. Shemilt

Intensive Care Medicine Experimental 2020, 9(1): 000345

Introduction. Acute kidney injury (AKI) is common in patients admitted to the intensive care unit (ICU) and has a widely variable reported incidence due to the use of various classifications $[1,2]$. When defined by Kidney Disease Improving Global Outcomes (KDIGO) [3] criteria, incidence of $\mathrm{AKI}$ in ICU patients has been reported to be $57.3 \%$, with sepsis the most common aetiology [1]. Renal injury associated with sepsis has a higher mortality than AKI in the overall ICU population [3]. Objectives. To determine the incidence of de-novo renal injury in patients with a primary diagnosis of sepsis and compare baseline characteristics, co-morbidity, organ support requirements and mortality between those with and without renal injury.

Methods. A retrospective cohort study of all patients aged over 16 admitted to the ICU with a primary diagnosis of sepsis in two large teaching hospitals in Glasgow, United Kingdom, between 2015 and 2018. Patients with a history of diabetes mellitus were included patients with pre-existing established renal failure were excluded. De-novo kidney injury was identified using KDIGO criteria and was defined as at least a Stage 1 AKI by this classification.

Results. Of the 5,312 patients admitted during the study period, 1,102 (20.7\%) were admitted with sepsis: 636 of these patients had de-novo renal injury during their admission; 466 patients did not. Patients with and without AKI had similar baseline characteristics, though those with new kidney injury tended to have higher APACHE II scores and lower baseline estimated glomerular filtration rate.

In patients with sepsis and AKI compared to those with sepsis and no renal injury, there was higher prevalence of cardiovascular disease ( $45.6 \%$ vs $32.4 \%)$, diabetes $(20.1 \%$ vs $12.9 \%)$ and increased incidence of invasive mechanical ventilation (76.1\% vs $51.3 \%$ ) and cardiovascular support $(78.6 \%$ vs $48.7 \%)$. Patients with sepsis and AKI required renal replacement therapy during their ICU stay in $28.3 \%$ of cases and multiorgan support in $68.4 \%$ of cases. This compared to $34.8 \%$ requiring multi-organ support in the sepsis without renal injury group.

All-cause mortality was significantly greater in the group with de-novo renal injury, both in-ICU $(31.3 \%$ vs $11.4 \% ; p<0.001)$ and in-hospital $(36.2 \%$ vs $15.2 \% ; p<0.001)$.

Conclusion. These data highlight the high incidence of de-novo renal injury in septic patients admitted to ICU. De-novo renal injury in patients with sepsis was associated with increased in-ICU and in-hospital mortality; there was also a higher proportion of pre-existing cardiovascular co-morbidity or diabetes mellitus in this group. Patients with kidney injury were more likely to require invasive ventilation, cardiovascular support and multi-organ support. This demonstrates the high prevalence of de-novo kidney injury within the septic population and the significant impact this has on all cause in-hospital mortality in this patient cohort.

\section{Reference(s)}

1. Hoste, E, Bagshaw, SM, Bellomo, R, et al. Epidemiology of acute kidney injury in critically ill patients: the multinational AKI-EPI study. Intensive Care Med 2015; 41: 1411-1423

2. Bagshaw SM, Uchino S, Bellomo R, et al. Septic acute kidney injury in critically ill patients: clinical characteristics and outcomes. CJASN. 2007:2(3):431-439

3. Khwaja A. KIDGO clinical practice guidelines for acute kidney injury. Nephron Clin Pract 2012; 120: 179-184.

4. No grants to acknowledge

\section{5}

Acute kidney injury in patients with pre-existing diabetes admitted to intensive care

K. Ross' ; M. Andonovic ${ }^{2}$; J. Traynor ${ }^{3}$; M. Shaw²; M. Sim²; P. Mark' ${ }^{4}$ K. Puxty 2 ${ }^{1}$ Intensive care, University Hospital Crosshouse, Kilmarnock, United Kingdom; ${ }^{2}$ Academic Department of Anaesthesia and Critical Care, University of Glasgow, Glasgow, United Kingdom; ${ }^{3}$ Department of nephrology, Queen Elizabeth University Hospital, Glasgow, United Kingdom; ${ }^{4}$ Institute of cardiovascular and medical sciences, University of Glasgow, Glasgow, United Kingdom

Correspondence: K. Ross

Intensive Care Medicine Experimental 2020, 9(1): 000355

Introduction. The rate of diabetes amongst the general population has been increasing for several decades [1]; an estimated 3.8 million adults in England have diabetes [2]. Diabetes is recognised as an independent risk factor for acute kidney injury (AKI), which is associated 
with a longer hospital stay and increased risk of requiring long term renal replacement therapy (RRT) $[1,3]$.

Objectives. This study aimed to identify the prevalence of AKI within the diabetic critical care population and its effect on requirement for organ support and short-term outcomes.

Methods. Data was collected retrospectively for all diabetic adults (aged 16 years or older) admitted to two tertiary level intensive care units in Scotland between 01/07/2015 and 30/06/2018. The presence or absence of AKI was defined by Kidney Disease Improving Global Outcomes (KDIGO) guidelines [4]. Patients with pre-existing established renal failure were excluded.

Results. 746 patients were identified for inclusion: 402 of these patients had an AKI. The median age of patients was 65 years, with a slight male predominance (55\%). Patients with AKI were more likely to present from a medical speciality and the most common admission diagnosis was sepsis (compared to malignancy in those without kidney injury). There was a greater prevalence of respiratory $(20.7 \% \mathrm{v}$ $18.3 \%$ ) and hepatic (12.4\% v $7.2 \%$ ) comorbidities in the AKI group. $30.6 \%$ of AKI patients required RRT; they were also more likely to require mechanical ventilation $(72.9 \%$ v $30.4 \%)$ and cardiovascular support $(64.4 \%$ v $37.5 \%)$. Patients with diabetes who developed AKI had higher in-ICU mortality $(29.9 \%$ v $11.3 \% ; \mathrm{p}<0.001)$ and in-hospital mortality $(36.6 \%$ v $16.0 \% ; p<0.001)$ compared to those without AKI.

Conclusion. The prevalence of AKI in this population is similar to previous studies in the general ICU population [5]. However, the hospital mortality of AKI patients in the diabetic population is greater [6]. This is likely multifactorial: this population had a high degree of comorbidity, patients required greater levels of organ support and $30.6 \%$ of patients required RRT which is associated with higher mortality [6]. Differences between mortality in the subgroups may be related to admission diagnosis and speciality. Most non-AKI patients were admitted from surgical specialities due to malignancy, perhaps suggesting that these were post-operative admissions following a curative procedure. Conversely, sepsis was identified as the leading cause of ICU admission in the AKI group.

This study found that patients with diabetes who develop AKI have a higher in-hospital mortality. A significant proportion of these patients require RRT. Further follow up of these patients is required to evaluate the long-term impact of AKI following ICU admission as this will have significant associated morbidity and healthcare costs.

\section{Reference(s)}

1. Nisula, S., Kaukonen, KM., Vaara, S.T. et al. Incidence, risk factors and 90-day mortality of patients with acute kidney injury in Finnish intensive care units: the FINNAKI study. Intensive Care Med. 2013 Jan 5; 39: $420-428$.

2. Hoste EAG, Bagshaw SM, Bellomo R. et al. Epidemiology of acute kidney injury in critically ill patients: the multinational AKI-EPI study. Intensive Care Med. 2015 Jul 11:41:1411-1423.

3. Kidney Disease: Improving Global Outcomes (KDIGO). KDIGO clinical practice guideline for acute kidney injury. Kidney inter. 2012. 2(1): 1-138

4. Mehta RH, Grab JD, O'Brien SM et al. Bedside tool for predicting the risk of postoperative dialysis in patients undergoing cardiac surgery. Circulation. 2006 Nov 6; 14(21): 2208-2216.

5. Public Health England, Diabetes Prevalence Model [internet]0.2016 [cited 2021 Apr 4]. Available from: https://assets.publishing.service.gov.uk/gover nment/uploads/system/uploads/attachment_data/file/612306/Diabe tesprevalencemodelbriefing.pdf

6. Patschan D, Müller GA. Acute Kidney Injury in Diabetes Mellitus. Int J Neph 2016 Nov 15; 2016 (6,232,909): 1-7.

7. No funding was received for this study

\section{9}

Risk factors of acute kidney injury in severe critically ill patients with Covid-19 under mechanical ventilation

I. Chavez'; P. Lopez z' VH. Villalobos Alvarez ${ }^{3}$; A. Sanchez-Calzada ${ }^{3}$; R. Chaires Gutierrez ${ }^{4}$; J. Aguirre Sánchez ${ }^{5}$

${ }^{1}$ Intensive Care Unit, The American British Cowdray Medical Center, Mexio City, Mexico; ${ }^{2}$ Critical care, ABC Medical Center, Ciudad de México,
Mexico; ${ }^{3}$ Intensive care unit, ABC Medical Center, Ciudad de México, Mexico; ${ }^{4}$ Intensive care unit, The American British Cowdray Medical Center, Mexico City, Mexico; ${ }^{5}$ Intensive care unit, The American British Cowdray Medical Center, Ciudad de México, Mexico

Correspondence: I. Chavez

Intensive Care Medicine Experimental 2020, 9(1): 000399

Introduction. Since December 2019, when respiratory coronavirus type 2 and the resulting disease, coronavirus acute respiratory syndrome type 2 (Coronavirus Disease 2019 [COVID-19]), developed in Wuhan, China, it has become a global pandemic, with 105,333,798 cases reported on February 4, 2021. On February 27, 2020, Mexico City reported the first case of COVID-19, followed by a massive growth of infections throughout the country. The total number of cases up today is $1,886,245$ with 81,223 estimated active cases. $18.77 \%$ of patients have required hospitalization. The total number of deaths is 164,290 with an estimated 184,125. Acute kidney injury (AKI)was found in $28 \%$ of hospitalized patientes and in $46 \%$ of critically ill patients contributing to a significant higher mortality. Identification of risk factors is important to guide early decissions early triage of patients for more intensive monitoring and prevent increased mortality.

Objectives. Determine whether high PEEP has a greater impact than increased cytokines in AKI in patients with COVID-19.

Methods. It was a cohort observational restrospective transversal study at Mexico City, the clinical data was extracted from $A B C$ medical center's electronic records by other intensivists and another intensivist subsequently checked for data accuracy.

We enrolled 100 severly or critically ill patients, over 18 years old who were positive in reverse transcription polymerase chain reaction (RTPCR) for diagnosis of Covid-19, who were admitted to the intensive care unit and need mechanical ventilation. For patients who had multiple hospital admissions we included only the first hospitalization. Patients were excluded if there was not possible to collect data, had a history of chronic kidney disease, had a previous kidney transplant,a glomerular filtration rate $<30 \mathrm{ml} / \mathrm{min} / 1.73 \mathrm{~m} 2$ or serum creatinine levels $>2 \mathrm{mg} / \mathrm{dl}$ during admission. Renal acute failure was defined by using KDIGO criterias (Kidney Disease:Improving Global Outcomes) Laboratory and respiratory variables, including Dimer- D, IL-6, ferritin, PCR, and PEEP at the beginning of mechanical ventilation, were recorded to perfom a Koogorov Smirnoff for normal distribution and then ANOVA test to compare groups. The significance thereshold was set at a $p$ value of less than 0.05 .

Results. We initially included 100 critically ill patients which 10 patients were excluded because missing data. 45 patients developed AKI during hospitalization in which 11 patients need kidney replacement therapy and 45 had normal renal function. We performed a correlation between cytokines and acute renal failure with ROC curve finding significant differences in IL6 and Dimer D with an área under ROC curve of $0.868(\mathrm{Cl}: 0.78-0.94)$ and $0.752(\mathrm{Cl}: 0.64-0.85)$, respectively. When we compared groups using ANOVA the one's that end in kidney replacement therapy and the one's with no renal failure we found no significal difference in PEEP, IL-6, PCR $(p=0.314, p=0.834$, $p=0.596)$. However, we found significant differences in $D$-dimer for developing renal failure and need of kidney replacement therapy $(\mathrm{p}=0.023)$

We sought to find the association between AKI and risk factors in severe and clinical patients with COVID-19, in order to treat them earlier and prevent worst clinical outcome. The incidente of AKI in this study was $50 \%$, in which $57 \%$ present AKI stage $1,22 \%$ AKI stage 2, 20\% AKI stage 3. SARS-COV-2 binds to the angiotesin-convertng enzyme 2 receptor, highly expressed in the kidney providing a route for direct cytopathic infection, severe inflammation and cytokine storm. The development of coagulopathy its a well studied mechanism in COVID-19 patients, therefore, an imbalanace between vasoconstrictors, vasodilators and oxidative stress at endothelium can lead to compromised microvascular perfusión at kidneys. In our study, we found that increased IL6 and Dimer D are risk factors for developing $\mathrm{AKI}$ and specifically the higher the Dimer D is at admission worst prognosis for requiring kidney replacement therapy. 
Conclusion. In this study, we found that incidence of renal acute failure in patients under mechanical ventilation and Covid19 was high so its important to find risk factors associated to renal acute failure and treat them on time to get a better clinical outcome and reduce mortality at ICU.

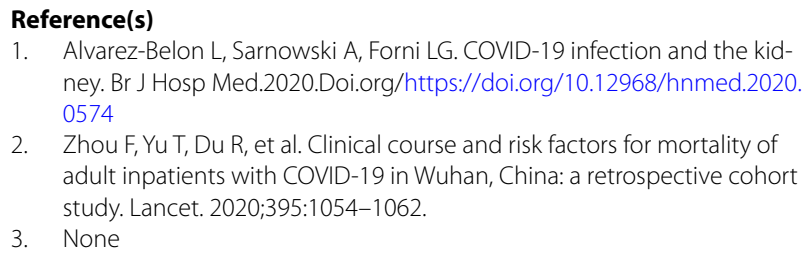

1. Alvarez-Belon L, Sarnowski A, Forni LG. COVID-19 infection and the kidney. Br J Hosp Med.2020.Doi.org/https://doi.org/10.12968/hnmed.2020. 0574

2. Zhou F, Yu T, Du R, et al. Clinical course and risk factors for mortality of adult inpatients with COVID-19 in Wuhan, China: a retrospective cohort study. Lancet. 2020;395:1054-1062.

3. None

\section{2}

Incidence and outcomes of acute kidney injury in patients admitted to intensive care with underlying cardiovascular disease T. Watson ; M. Andonovic ${ }^{2}$; J. Traynor ${ }^{3}$; M. Shaw ${ }^{4}$; M. Sim, ; ;. Mark, ; K. Puxty ${ }^{4}$

${ }^{1}$ Intensive Care Unit, University Hospital Crosshouse, Crosshouse, United Kingdom; ${ }^{2}$ Academic Department of Anaesthesia and Critical Care, University of Glasgow, Glasgow, United Kingdom; ${ }^{3}$ Nephrology, Queen Elizabeth University Hospital, Glasgow, United Kingdom; ${ }^{4}$ Intensive care unit, Glasgow Royal Infirmary, Glasgow, United Kingdom; ${ }^{5}$ Institute of cardiovascular and medical sciences, University of Glasgow, Glasgow, United Kingdom

Correspondence: T. Watson

Intensive Care Medicine Experimental 2020, 9(1): 000412

Introduction. Acute kidney injury (AKI) is widely recognised as a significant contributor to morbidity and mortality in hospitalised patients $(1,2)$. It is a common occurrence in critical illness (3) and is associated with poorer outcomes and an increased mortality rate $(4,5)$. However, it is unclear how much of an effect underlying cardiovascular disease has on incidence of $\mathrm{AKI}$, and how this relates to patient outcomes.

Objectives. The goal of this study was to research the incidence of AKI in patients with underlying cardiovascular disease, associated risk factors and short-term outcomes including mortality.

Methods. This retrospective cohort study identified all adult patients aged 16 or over admitted to two large general ICUs over a 3-year period. Cardiovascular disease was defined as patients with prior documented history of ischaemic heart disease, hypertension, cerebrovascular disease or peripheral vascular disease. Patients with pre-existing established renal failure were excluded. AKI was identified according to KDIGO guidelines (6). Baseline demographics, additional co-morbidities, interventions and outcomes were then compared between the AKI and non-AKI groups.

Results. From a total of 5,312 patients 2,002 (37.7\%) had underlying cardiovascular disease. AKI occurred in $48.0 \%$ of patients with underlying cardiovascular disease admitted to ICU. Renal replacement therapy was used in $21.4 \%$ of these patients. There was a higher proportion of male patients with AKI compared to without (60.4\% vs $49.4 \%)$; there was also a higher proportion of diabetes in the AKI group (26.2\% vs $20.8 \%$ ). Patients with AKI had a higher APACHE II score (23.0 to 14.0$)$ and predicted mortality $(45.8 \%$ to $15.9 \%)$, with more patients with AKI requiring multi organ support (59.0\% to $22.3 \%$ ). The mortality during ICU admission was $31.7 \%$ in the AKI group compared to $9.8 \%$ in non-AKI group $(p<0.001)$, with a similar trend in hospital mortality $(39.2 \%$ to $13.7 \% ; p<0.001)$.

Conclusion. Approximately half of patients with underlying cardiovascular disease developed an AKI whilst critically unwell. AKI is recognised as a common occurrence in critically unwell patients $(3,4)$ but the exact incidence varies significantly (7); this is likely due to variation in the classification system used $(8,9)$. However, this data is in keeping with current literature on the prevalence of AKI in ICU patients (3). The group of patients who developed an AKI required higher levels of organ support and were found to have higher median APACHE II scores and predicted mortality. However, the inICU and in-hospital mortality rates in this population with KDIGO defined AKI are higher than in the undifferentiated ICU population (4). This data contributes to the prior literature describing the association between AKI and increased morbidity and mortality. However, further research into the pathophysiological mechanisms and long-term outcomes of AKI on the background of cardiovascular comorbidities is warranted.

\section{Reference(s)}

1. Zeng X, McMahon GM, Brunelli SM, Bates DW, Waikar SS. Incidence, outcomes, and comparisons across definitions of AKI in hospitalized individuals. Clinical Journal of the American Society of Nephrology. 2014 Jan 7;9(1):12-20.

2. Sutherland SM, Byrnes JJ, Kothari M, Longhurst CA, Dutta S, Garcia P, Goldstein SL. AKI in hospitalized children: comparing the pRIFLE, AKIN, and KDIGO definitions. Clinical Journal of the American Society of Nephrology. 2015 Apr 7;10(4):554-61.

3. Case J, Khan S, Khalid R, Khan A. Epidemiology of acute kidney injury in the intensive care unit. Critical care research and practice. $2013 \mathrm{Jan}$ $1 ; 2013$

4. Kidney Disease: Improving Global Outcomes (KDIGO). KDIGO clinical practice guideline for acute kidney injury. Kidney inter. 2012. 2(1): 1-138.

5. Hoste EA, Schurgers M. Epidemiology of acute kidney injury: how big is the problem?. Critical care medicine. 2008 Apr 1;36(4):S146-51.

6. Srisawat N, Sileanu FE, Murugan R, Bellomo R, Calzavacca P, Cartin-Ceba R, Cruz D, Finn J, Hoste EA, Kashani K, Ronco C. Variation in risk and mortality of acute kidney injury in critically ill patients: a multicenter study. American journal of nephrology. 2015;41(1):81-8.

7. Hoste EA, Bagshaw SM, Bellomo R, Cely CM, Colman R, Cruz DN, Edipidis K, Forni LG, Gomersall CD, Govil D, Honoré PM. Epidemiology of acute kidney injury in critically ill patients: the multinational AKI-EPI study. Intensive care medicine. 2015 Aug:41 (8):1411-23.

8. Truche AS, Ragey SP, Souweine B, Bailly S, Zafrani L, Bouadma L, Clec'h C, Garrouste-Orgeas M, Lacave G, Schwebel C, Guebre-Egziabher F. ICU survival and need of renal replacement therapy with respect to AKI duration in critically ill patients. Annals of intensive care. 2018 Dec;8(1):1-1.

9. Susantitaphong P, Cruz DN, Cerda J, Abulfaraj M, Alqahtani F, Koulouridis I, Jaber BL. World incidence of AKI: a meta-analysis. Clinical Journal of the American Society of Nephrology. 2013 Sep 6;8(9):1482-93.

10. No funding was received for this study.

\section{8}

Severe Acute Kidney Injury (AKI) in Critically III Patients with COVID-19 treated by Extracorporeal Membrane Oxygenation (ECMO): Incidence, Risk Factors, and Outcomes

A. Kogann ; E. Karni²; D. Volvovitch²; J. Tamer²; Y. Kasiff²; E. Ram²; E. Raanani²; L. Sternik ${ }^{2}$

${ }^{1}$ Cardiac Surgery ICU, Sheba Medical Center, Ramat Gan, Israel; ${ }^{2}$ Department of cardiac surgery, Sheba Medical Center, Ramat Gan, Israel

Correspondence: A. Kogan

Intensive Care Medicine Experimental 2020, 9(1): 000478

Introduction. Acute kidney injury (AKI) commonly occurs in critically ill patients with severe COVID-19 illness, required treatment by ECMO for acute respiratory failure. However, data are limited in these patients.

Objectives. We aimed to report the incidence, risk factors, and prog nostic impact of severe AKI, defined as KDIGO (Kidney Disease Improving Global Outcomes) stages 2 or 3 as well as the efficacy of CRRT (Continuous Renal Replacement Therapy) for survival in these patients. Methods. We performed observational study in two Israeli hospitals between 3.2020 and 2.2021. Data from all Covid-19 patients treated with ECMO regarding kidney function and treatment as well as survival rates were analyzed.

Results. During study period 58 patients were treated by ECMO and from them we identified 33 patients (59\%) with severe AKI (staged $2-3)$. Twenty eight patients with AKI were treated with by different kind of CRRT. From whole cohort 32 patients (55\%) survived. Among patients suffering from severe AKI there were only 10 patients 
survived (30\%). In multivariate logistic regression analysis, severe AKI was independently associated with age $(\mathrm{OR}=1.08(95 \% \mathrm{Cl} 1.03-$ 1.14), $p=0.003$ and creatinine level on admission $(\mathrm{OR}=1.04(95 \%$ $\mathrm{Cl}$ : 1.008-1.065), $p=0.012$ ) Inflammatory (interleukin-6, C-reactive protein, and ferritin) or thrombotic (D-dimer and fibrinogen) markers were not associated with severe AKI.

Conclusion. Severe AKI was common in critically ill patients with COVID-19. AKI is a major risk factor and predictor for mortality in COVID-19 patients required ECMO treatment.

\section{0}

\section{Comparison of renal replacement therapy and renal recovery} before and during the COVID-19 pandemic- A single centre

\section{experience}

R. Ryan, ${ }^{1}$;. Taylor ${ }^{1}$; C. Laing ${ }^{2}$; M. Singer ${ }^{1}$; N. Maccallum, ${ }^{1}$; N. Arulkumaran, 'Intensive care department, University College London Hospital, London, United Kingdom; ${ }^{2}$ Department of renal medicine, University College Hospital, London, United Kingdom

Correspondence: I. Taylor

Intensive Care Medicine Experimental 2020, 9(1): 000520.

Introduction. At the peak of the COVID-19 pandemic, we were required to modify our hemofiltration protocols to facilitate the increased demand for renal replacement therapy (RRT) capacity. Patients with COVID-19 are at increased risk of thrombotic complications, creating additional challenges around optimizing the anticoagulation for RRT. This was further compounded by the supply issues of citrate regional anticoagulation.

Methods. We conducted a single centre retrospective case-control study of patients with COVID-19 requiring RRT, compared with consecutive RRT-requiring non-COVID-19 ICU patients. Our objectives were to ascertain if there were any differences in the initiation criteria for RRT during the pandemic, incidence of filter clotting, duration of RRT prior to renal recovery, hospital survival, and renal recovery.

Results. Of 154 patients admitted to ICU with COVID-19, 47 (30.5\%) received continuous venovenous haemofiltration (CVVHF). Compared to patients without COVID-19 $(n=37)$, patients with COVID-19 were younger, had a lower serum creatinine on hospital admission, and incidence of CKD was lower. Despite reduced CVVHF exchange rates in COVID-19 patients, metabolic acidosis was corrected. Incidence of haemofilter circuit clotting is higher and duration of RRT requirement is longer compared to non-COVID-19 patients. Requirement for ongoing mechanical ventilation precludes intermittent RRT in a nephrology acute dialysis unit. Hospital mortality is $60 \%$ among COVID-19 patients requiring RRT on ICU, although renal recovery among survivors is favourable.

Conclusion. Despite lower CVVHF exchange rates, metabolic abnormalities were corrected and there were no immediate complications. Among survivors, renal function recovers despite prolonged periods on CVVHF.

\section{Reference(s)}

1. Goshua G, Pine AB, Meizlish ML, et al. Endotheliopathy in COVID-19-associated coagulopathy: evidence from a single-centre, cross-sectional study. Lancet Haematol 2020; 7(8): e575-e82.

2. Liu C, Mao Z, Kang H, Hu J, Zhou F. Regional citrate versus heparin anticoagulation for continuous renal replacement therapy in critically ill patients: a meta-analysis with trial sequential analysis of randomized controlled trials. Crit Care 2016; 20(1): 144.

3. Munshi L, Del Sorbo L, Adhikari NKJ, et al. Prone Position for Acute Respiratory Distress Syndrome. A Systematic Review and Meta-Analysis. Ann Am Thorac Soc 2017; 14(Supplement_4): S280-S8.

4. Shankaranarayanan D, Neupane SP, Varma E, et al. Peritoneal Dialysis for Acute Kidney Injury During the COVID-19 Pandemic in New York City. Kidney Int Rep 2020

5. Grissom CK, Hirshberg EL, Dickerson JB, et al. Fluid management with a simplified conservative protocol for the acute respiratory distress syndrome*. Crit Care Med 2015; 43(2): 288-95.
6. National Heart L, Blood Institute Acute Respiratory Distress Syndrome Clinical Trials N, Wiedemann HP, et al. Comparison of two fluid-management strategies in acute lung injury. N Engl J Med 2006; 354(24): 2564-75.

7. Docherty A, Harrison E, Green C, et al. Features of 16,749 hospitalised UK patients with COVID-19 using the ISARIC WHO Clinical Characterisation Protocol. medRxiv and bioRxiv, 2020

8. Helms J, Tacquard C, Severac F, et al. High risk of thrombosis in patients with severe SARS-CoV-2 infection: a multicenter prospective cohort study. Intensive Care Med 2020; 46(6): 1089-98

9. Robbins-Juarez SY, Qian L, King KL, et al. Outcomes for Patients With COVID-19 and Acute Kidney Injury: A Systematic Review and Meta-Analysis. Kidney Int Rep 2020; 5(8): 1149-60.

10. Roberts LN, Bramham K, Sharpe CC, Arya R. Hypercoagulability and Anticoagulation in Patients With COVID-19 Requiring Renal Replacement Therapy. Kidney Int Rep 2020

\section{1}

"Fluid Balance and CRRT-impact on Mortality"

C. Sousa'; I. Jesus Pereira²; G. Cabral Campello

${ }^{1}$ Nephrology, Centro Hospitalar De Trás-Os-Montes E Alto Douro,

E.P.E., Vila Real, Portugal; ${ }^{2}$ Serviço de medicina intensiva polivalente, Centro Hospitalar de Vila Nova de Gaia/Espinho, Vila Nova de Gaia, Portugal; ${ }^{3}$ Unidade de cuidados intensivos, Centro Hospitalar do Tâmega e Sousa, Penafiel, Portugal

Correspondence: $C$. Sousa

Intensive Care Medicine Experimental 2020, 9(1): 000531

Introduction. Positive fluid balance (FB) has been associated with an increased length of stay in the intensive care unit (ICU) and with a higher mortality in critically ill patients1,2,3. Nevertheless, optimal fluid balance management in critically ill patients, especially those requiring continuous renal replacement therapy (CRRT), still requires further investigation4,5,6.

Objectives. We aimed to investigate the association between cumulative fluid balance and ICU mortality, and 30-day and 90-day mortality in a cohort of critically ill ICU patients that required CRRT during ICU stay.

Methods. We conducted an observational retrospective study of patients admitted in a mixed medical-surgical ICU in a tertiary referra hospital between the 1st of January and 31 of December of 2019.

We collected demographic data, SOFA and SAPSII score, data on CRRT, ICU length of stay, daily and cumulative fluid balances (CFB), and mortality in the ICU, at 30 and at 90-days.

The data was analyzed using the SPSS statistical program v. 26.0 The significance level used was 0,05. Descriptive results are given as mean \pm standard deviation (SF) for normally distributed parameters and as median and interquartile range for non-normally distributed. According to the variables characteristics and the test results, we performed the Student T test and the Mann Whitney test for independent sample, chi-squared test and Fisher's exact test whenever the chisquare test assumptions were violated. Logistic regression modeling was used to understand factors associated with mortality.

Results. During the referral period, 330 patients were admitted in the ICU, with a mean SOFA of 5.56 and a mean SAPSII of 44 . A total of 69 (21\%) patients were submitted to CRRT.

In our cohort, $55,1 \%$ were male and the mean age was $64.7 \pm 15.2$ years, the mean SOFA at admission was $9,55 \pm 3,0$ and SAPSII was $54,43 \pm 18,3$, and the median length of stay was $5,0[5,0-9,0]$ days.

Septic shock was responsible for $42 \%$ of the admissions, followed by metformin-associated lactic acidosis (17,4\%).

Regional anticoagulation with citrate was used in $53 \%$ of the patients, with a median of $37,0[20,5-119,8]$ hours of total effective treatment The need to change the set at $48 \mathrm{~h}$ was significantly associated with the type of coagulation used $(p=, 018)$. The use of regional anticoagulation with citrate was associated with a lower need to change the set, and had no relationship with mortality.

There was no relationship between the lactate value (initial lactate level was $6,1 \pm 4,9 \mathrm{mmol} / \mathrm{s})$, citrate technique and mortality $(p=, 133)$. 
Lactate clearance at $24 \mathrm{~h}$ was consistently associated with the 30-days mortality $(p<, 10)$, for each unit increase in the lactate clearance, the chance of death decreases approximately $36 \%(O R=0.64, p=0.042)$. Higher CFB was significantly associated with an increase in ICU and 30 - and 90 -days mortality $(p=0,002 ; p=0,017 ; p=0,015$ respectively). The mean daily fluid balance was also significantly associated with an increase in 30 and 90 -days mortality $(p=0,005 ; p=0,011)$.

We found other variables associated with ICU mortality: absence of renal function recovery $(p<0.001)$; sepsis shock or systemic inflammatory response syndrome diagnosis $(p=, 024)$; and higher SOFA and SAPSII scores $(p=, 019 ; p<, 001$ respectively). In the multivariate analysis, significant predictors of mortality were older age $(O R=1,11$; $p=, 030)$ and absence of renal function recovery $(O R=0,001 ; p=, 032)$. Conclusion. A higher cumulative fluid balance is independently associated with ICU, 30-day and 90-day mortality in patients on CRRT. This underlines the importance of implementing restrictive fluid therapy in critically ill patients, and support physicians towards the prescription of fluid removal during CRRT as fluids need to be considered a drug with important implications in mortality5,7.

Mortality was associated with older age and absence of renal function recovery, to reinforce the importance of avoiding nephrotoxicity(aminoglycosides, AINEs, contrast...) in these patients.

\section{Reference(s)}

1. 6- Jhee et al. The interactive effects of input and output on managing fluid balance in patients with acute kidney injury requiring continuous renal replacement therapy. Critical Care(2019) 23:329.

2. 5- Naorungroj T, Neto AS, Zwakman-Hessels L, Yanase F, Eastwood G, Bellomo R. (2019) Hourly Fluid Balance in Patients Receiving Continuous Renal Replacement Therapy. Blood Purif 2020;49:93-101.https://doi.org/ 10.1159/000503771.

3. 4-Wald R, MCArthur E, Adhikari NK, Bagshaw SM, Burns KE, Garg AX, Harel Z, Kitchlu A, Mazer CD, Nash DM, et al. Changing incidence and outcomes following dialysis-requiring acute kidney injury among critically ill adults: a population-based cohort study. Am J Kidney Dis. 2015;65(6):870-7.

4. 3- Kim IY, Kim JH, Lee DW, Lee SB, Rhee H, Seong EY, Kwak IS, Song SH. Fluid overload and survival in critically ill patients with acute kidney injury receiving continuous renal replacement therapy. PLoS One. 2017;12(2): e0172137

5. 2-van Mourik N, Metske HA, Hofstra JJ, Binnekade JM, Geerts BF, Schultz MJ, et al. (2019) Cumulative fluid balance predicts mortality and increases time on mechanical ventilation in ARDS patients: An observational cohort study. PLoS ONE 14(10): e0224563. https://doi.org/10.1371/journ al. pone. 0224563

6. Lee J, de Louw E, Niemi M, Nelson R, Mark RG, Celi LA, et al. Association between fluid balance and survival in critically ill patients. J Intern Med. 2015; 277(4):468-77. Epub 2014/06/17. https://doi.org/10. 1111/ joim.12274 PMID: 24,931,482; PubMed Central PMCID: PMC4265574.

7. 7-van Mourik N, Metske HA, Hofstra JJ, Binnekade JM, Geerts BF, Schultz MJ, et al. (2019). Cumulative fluid balance predicts mortality and increases time on mechanical ventilation in ARDS patients: An observational cohort study. PLoS ONE 14(10): e0224563. https://doi.org/10.1371/ journal. pone. 0224563

8. Intensive care service of the Tamega e Sousa Hospital Center

\section{5}

\section{Creatinine-based formula versus cistatin c-based formula} for estimation of GFR in critically ill COVID patients

R. Marinho'; DA. Ferreira ${ }^{2}$; RP. Medeiros ${ }^{3}$; R. Rego ${ }^{4}$; C. Costa ${ }^{4}$; R. Ribeiro ${ }^{4}$; R. Sousa ${ }^{5}$; A. Marinho ${ }^{3}$

${ }^{1}$ Serviço Cuidados Intensivos, Hospital Geral de Santo António, Porto, Portugal; ${ }^{2}$ Internal medicine, Hospital Dr. Nélio Mendonça, Funchal, Portugal; ${ }^{3}$ Serviço cuidados intensivos, Hospital Geral de Santo António, Porto, Portugal; ${ }^{4}$ Internal medicine, Hospital Geral de Santo António, Porto, Portugal; ${ }^{5}$ Mgf, USF Terras de Souza, Paredes, Portugal

Correspondence: R. Marinho

Intensive Care Medicine Experimental 2020, 9(1): 000535
Introduction. Plasma or serum creatinine is the most commonly used diagnostic marker for the estimation of glomerular filtration rate (GFR) in clinical routine. Equations to estimate GFR based on serum creatinine have been introduced and the most validated and applied are the MDRD equation. Lately, the low molecular weight protein cystatin $C$ was introduced as a GFR estimate (eGFR) superior to creatinine.

Objectives. We sought to compare the predictive equations for calculating the glomerular filtration rate (GFR) using creatinine and cystatin C in COVID-19 patients admitted to the Intensive Care Unit (ICU), on admission and in the first 4 weeks of hospitalization.

Methods. Retrospective study of COVID patients admitted to the Intensive Care Service between April 2020 and April of 2021, using demographic variables, urea, creatinine and cystatin $C$ values, as well as outcome. Statistical analysis performed in SPSS 25 , with a correlation coefficient between the formulas and $\mathrm{ROC}$ curve regarding outcome.

Results. 282 patients were evaluated, with a mean age of $67 \pm 12.4$ years (22-92), 187 of whom were male (66.3\%), with an average body mass index of $28.1 \pm 5.6$, with length of stay in intensive care of $13.4 \pm 12.8$ days (1-94) and mortality rate in intensive care of $44 \%$ $(n=124)$.

There was a great variability in the calculation of GFR when comparing formulas based on creatinine and Cystatin C. The average GFR calculated by the Cockcroft-Gault equation was 77.9 at admission and then $76.5 ; 80.5 ; 97.2$ and 97.9 between the 1st and 4th week. The MDRD, it was $92.2 \mathrm{ml} / \mathrm{min}$ at admission and later $91.6 ; 91.2 ; 114.6$ and 131.6. The CKD-EPI was 79.1 on admission and then 79; 77.5; 82 and 87.2. Compared to the Larsson Equation based on Cystatin C was 55.3 at admission and then 48.6; 41.1; 43.4 and 40.1. The CKD-EPI with Cystatin C was 52.7 at admission and then $46.3 ; 38.6 ; 42.2$ and 37.5 . Finally the CKD-EPI using Cystatin C and creatinine was 64 at admission and then 59.2;53.1; 58.2 and 61.3 .

The difference was confirmed whith the correlation coefficient, in which there was a very strong correlation between the 3 creatininebased formulas with Pearson's coefficient $>0.9$; however, the Pearson's coefficient between 0.7 and 0.81 when comparing creatinine to cystacin C formulas.

The formula that correlated better with outcome was the CKD-EPI using Cystatin C with an AUC of 0.61 at admission and 0.72 at the 1 st week.

Conclusion. The correlation analysis showed the eGFRs from every formula could all to some extent reflect the glomerular function or GFR accurately. The formula that correlated better with outcome was the CKD-EPI suing Cystatin C.

\section{2}

"Intermittent veno-venous hemodia-dialysis": a new approach for renal replacement therapy in the ICU setting

N. Zucman ${ }^{1}$; C. Verney ${ }^{1}$; F. Uhel' ${ }^{1}$; JD. Ricard'; D. Dreyfuss ${ }^{1}$; D. Roux ${ }^{1}$

${ }^{1}$ Intensive Care Unit, Louis-Mourier Hospital, Colombes, France

Correspondence: N. Zucman

Intensive Care Medicine Experimental 2020, 9(1): 000572

Introduction. The optimal renal replacement therapy (RRT) modality during acute kidney injury (AKI) remains debated. Continuous and intermittent techniques can be used equally, based on availability and medical experience (1). Intermittent hemodialysis requires a water treatment system that limits its use and increases the cost. Here, we describe an intermittent approach we called "intermittent venovenous hemodia-dialysis" (IVVHDD) which circumvents these limitations. No funding nor honoraria were provided by the manufacturer of the device.

Methods. The manufacturer of the device (Fresenius Medical Care) proposes a special setting for its hemodiafiltration machine MultiFiltrate Pro ${ }^{\circledR}$ with "Post-dilution CVVHDF" which allows for delivery of intermittent hemodialysis. To do this, the manufacturer provides a T-union system that allows using both dialysis and fluid replacement pumps for dialysis. This allows for a doubling of the dialysate fluid flow and increased diffusive transfers. The dialysate presents as bags containing $5 \mathrm{~L}$ of the appropriate solution supplied by the manufacturer. 
Schematic representations of extracorporeal circuits of IVVHDD (compared with conventional Post-dilution (VVHDF) are shown in the Figure.

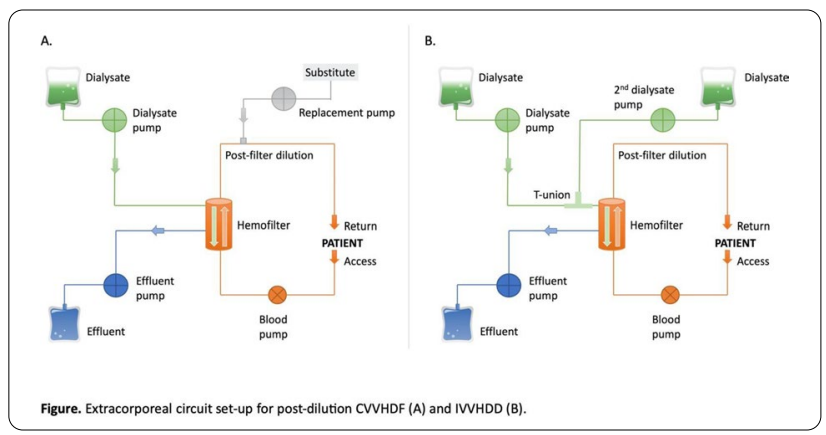

Standard initial settings were a dialysate flow of $9000 \mathrm{ml} / \mathrm{h}(4500 \mathrm{ml} / \mathrm{h}$ per pump), a blood flow of $250 \mathrm{ml} / \mathrm{min}$, and ultrafiltration rate as required, up to $1000 \mathrm{ml} / \mathrm{h}$.

Results. This retrospective study reports results of this technique in 18 patients who underwent a total of 88 IVVHDD sessions in our ICU. Median age was 63 years, four patients had end-stage kidney disease requiring chronic dialysis. Thirteen patients presented with AKI on ICU admission and one with profound metabolic acidosis. Median duration of IVVHDD sessions was $5 \mathrm{~h}$ (IQR $[4 ; 6])$. At time of initiation, median plasma urea and creatinine levels were $32 \mathrm{mmol} / \mathrm{L}$ (IQR [23.5; 38.6]) and $454 \mu \mathrm{mol} / \mathrm{L}$ (IQR [352; 619]), respectively. Median plasma urea reduction ratio was $38.2 \%$ (IQR [29.2; 48.6]). Hemodynamic impairment requiring fluid infusion or catecholamine increase occurred in $21.6 \%$ of sessions. The technique allowed for adequate control of hyperkalemia (maximum level $7.8 \mathrm{mmol} / \mathrm{L}$ which was lowered to $4.9 \mathrm{mmol} / \mathrm{L}$ after a 6-h session) when present. Hypokalemia or hypophosphatemia were observed in less than $5 \%$ of sessions. Other adverse events were scarce but emphasize the need for staff training and standardized procedures.

Conclusion. In conclusion, this technique is a proof-of-concept of a form of intermittent RRT using the Multifiltrate Pro ${ }^{\circledR}$ machine. Although its performance is inferior to conventional hemodialysis (because of dialysate flow rate that is one-third that with conventional hemodialysis) it is however acceptable and the absence of need for water treatment system makes it an interesting alternative to traditional RRT modalities, especially when logistic and financial considerations are at stake.

\section{Reference(s)}

1. Vinsonneau C, Allain-Launay E, Blayau C, Darmon M, du Cheyron D, Gaillot T, et al. Renal replacement therapy in adult and pediatric intensive care: Recommendations by an expert panel from the French Intensive Care Society (SRLF) with the French Society of Anesthesia Intensive Care (SFAR) French Group for Pediatric Intensive Care Emergencies (GFRUP) the French Dialysis Society (SFD). Ann Intensive Care. 2015 Dec;5(1):58.

\section{6}

Acute Kidney Injury Incidence, Progression, and Long-term Outcomes in Critically III Patients with COVID-19

E. Cooney ${ }^{1}$; W. Kok ${ }^{1}$; U. Blanco ${ }^{1}$; L. Pirondini' ${ }^{2}$, J. Gregson² ${ }^{2}$ T. Soares ${ }^{1}$; M. Ostermann ${ }^{3}$; N. Lumlertgul

${ }^{1}$ Intensive care, Guy's and St Thomas' NHS Foundation Trust, London, United Kingdom; ${ }^{2}$ Lshtm, London School of Hygiene \& Tropical Medicine, London, United Kingdom; ${ }^{3}$ Intensive care, Guys \& St Thomas Nhs Foundation Trust, London, United Kingdom

Correspondence: N. Lumlertgul

Intensive Care Medicine Experimental 2020, 9(1): 000596

Introduction. Acute kidney injury (AKI) occurs commonly in critically ill patients with coronavirus disease-19 (COVID-19). However, data is limited on the clinical course, progression, and long-term renal outcomes in these patients.

Methods. This retrospective observational study includes all adult patients admitted to a tertiary-care intensive care unit (ICU) in London, United Kingdom, between 1st March 2020 and 24th July 2020. We examined the incidence, clinical course, risk factors for AKI development and progression, short-term and long-term survival, dialysis dependence, and renal recovery.

Results. Of 313 patients ( $70 \%$ male, mean age $54.5 \pm 13.9$ years), AKI developed in 240 (76.7\%) patients: 63 (20.1\%) stage 1, $41(13.1 \%)$ stage $2,136(43.5 \%)$ stage 3 . The median onset from ICU admission was 1 (interquartile range $0-2$ ) days. Progression to AKI stage $2 / 3$ occurred in $36 \%$. Risk factors of AKI progression were mechanical ventilation [HR (hazard ratio) $4.11 ; 95 \%$ confidence interval $(\mathrm{Cl}) 1.61-10.49$ ] and positive fluid balance [HR $1.21(95 \% \mathrm{Cl} 1.11-1.31)]$, while steroid therapy was a protective factor ( $\mathrm{HR} 0.73[95 \% \mathrm{Cl} 0.55-0.97])$. Kidney replacement therapy (KRT) was initiated in $31.9 \%$. AKI patients had a higher ICU mortality than non-AKI patients (33\% vs.12\%). Dialysis dependence was $4 \%$ at hospital discharge and $5 \%$ at 90 days. Renal recovery was identified in $81.6 \%$ at discharge and $90.9 \%$ at 90 days in survivors. Conclusion. During the first COVID wave, AKI was highly prevalent among severely ill COVID-19 patients. About one-third of patients progressed to severe AKI and required KRT. Recognition of risk factors and subsequent outcomes might help inform future practice, generate hypotheses for potential management, and develop follow-up strategies.

\section{2}

Polymyxin B hemoperfusion and endotoxic shock in COVID-19

S. De Rosa'; M. Decal ${ }^{2}$;. Cattin ${ }^{3}$; M. Proglio ${ }^{2}$ : A. Lorenzin ${ }^{4}$ : E. Boni, ${ }^{5}$

G. Golino ${ }^{6}$; V. Rizzello ${ }^{6}$; J. Santi ${ }^{1}$; C. Ronco, ${ }^{7}$; V. Danzi, ${ }^{6}$

${ }^{1}$ Department of anesthesia and intensive care unit, St. Bortolo Hospital, Vicenza, Italy; ${ }^{2}$ International renal research institute of vicenza, San Bortolo Hospital Of Vicenza, Vicenza, Italy: ${ }^{3}$ Anestesia e Rianimazione, Ospedale San Bortolo di Vicenza, Vicenza, Italy; ${ }^{4}$ International renal research institute of vicenza, St. Bortolo Hospital, Vicenza, Italy; ${ }^{5}$ Department of anesthesia and intensive care unit, Ospedale San Bortolo di Vicenza, Vicenza, Italy; ${ }^{6}$ Department of anesthesia and intensive care unit, San Bortolo Hospital Of Vicenza, Vicenza, Italy; ${ }^{7}$ Department of nephrology, dialysis, and transplantation, San Bortolo Hospital Of Vicenza, Vicenza,

Italy

\section{Correspondence: S. De Rosa}

Intensive Care Medicine Experimental 2020, 9(1): 000602

Introduction. Many evidences showed that enterocytes dysfunction allows direct translocation of the Gl content into the blood or lymphatic system. Specifically, due to the characteristics of the microbiota, Gram negative bacteria or endotoxin may translocate into the systemic circulation leading to inflammatory activation, immune dysfunction and sepsis. In COVID 19 population, recent evidences showed that the interaction in the Gl system between SARS-CoV-2,angiotensin converting enzyme-2 (ACE-2) receptor and transmembrane serine protease 2 (TMPRSS2) leads to virus penetration into cell and ends up in enterocytes dysfunction and malabsorptive syndrome (e.g. diarrhea).

Objectives. The aim of this study was to evaluate the role of extracorporeal endotoxin removal by Polymyxin B hemoperfusion (PMX-HP), in the treatment of patients with COVID-19.

Methods. Single-center prospective, observational study of 10 patients with confirmed COVID-19 who were affected by septic shock requiring treatment with PMX-HP and admitted in a large, independent tertiary care medical center in Northern, Italy from February 2020 to February 2021. Each treatment is registered in a web-based database (EUPHAS2 registry).

Results. Ten patients with severe acute respiratory syndrome coronavirus 2 infection confirmed by real-time reverse transcriptase-polymerase chain reaction from nasal/oral swab, were included in the analysis. The median age was 62.5 years (34.0-68.0) and BMI was 28.2 (20.835.8). The median APACHE II score was 27 (15-35) and SAPS II score 
was 73 (52-85). Sequential Organ Failure Assessment (SOFA) score progressively improved over the next $120 \mathrm{~h}$ following PMX-HP simultaneously to Vasoactive inotropic score (VIS). It was associated with median endotoxin activity assay (EAA) decrease from 0.77 [0.55-0.84] at T0 to 0.57 [0.24-0.87] at T120 associated to a simultaneous decrease in procalcitonin from $7.45[0.3-70.55]$ at T0 to 1.18 [0.41-10.83] at T120. No PMX-HP-related complications were observed.

Conclusion. In a COVID-19 case series of patients with endotoxic shock, PMX-HP was associated with organ function recovery, hemodynamic improvement, and contemporary EAA level reduction.

\section{Reference(s)}

1. Cutuli SL, Artigas A, Fumagalli R, Monti G, Ranieri VM, Ronco C, Antonelli M; EUPHAS 2 Collaborative Group. Polymyxin-B hemoperfusion in septic patients: analysis of a multicenter registry. Ann Intensive Care. 2016 Dec;6(1):77. https://doi.org/10.1186/s13613-016-0178-9.

2. Cutuli SL, Artigas A, Fumagalli R, Monti G, Ranieri VM, Ronco C, Antonelli M; EUPHAS 2 Collaborative Group. Polymyxin-B hemoperfusion in septic patients: analysis of a multicenter registry. Ann Intensive Care. 2016 Dec;6(1):77. https://doi.org/10.1186/s13613-016-0178-9.

3. Cutuli SL, Artigas A, Fumagalli R, Monti G, Ranieri VM, Ronco C, Antonelli M; EUPHAS 2 Collaborative Group. Polymyxin-B hemoperfusion in septic patients: analysis of a multicenter registry. Ann Intensive Care. 2016 Dec:6(1):77. https://doi.org/10.1186/s13613-016-0178-9.

4. Cutuli SL, Artigas A, Fumagalli R, Monti G, Ranieri VM, Ronco C, Antonelli M; EUPHAS 2 Collaborative Group. Polymyxin-B hemoperfusion in septic patients: analysis of a multicenter registry. Ann Intensive Care. 2016 Dec;6(1):77. https://doi.org/10.1186/s13613-016-0178-9.

5. Cutuli SL, Artigas A, Fumagalli R, Monti G, Ranieri VM, Ronco C, Antonelli M; EUPHAS 2 Collaborative Group. Polymyxin-B hemoperfusion in septic patients: analysis of a multicenter registry. Ann Intensive Care. 2016 Dec;6(1):77. https://doi.org/10.1186/s13613-016-0178-9.

6. Cutuli SL, Artigas A, Fumagalli R, Monti G, Ranieri VM, Ronco C, Antonelli M; EUPHAS 2 Collaborative Group. Polymyxin-B hemoperfusion in septic patients: analysis of a multicenter registry. Ann Intensive Care. 2016 Dec;6(1):77. https://doi.org/10.1186/s13613-016-0178-9.

7. Cutuli SL, Artigas A, Fumagalli R, Monti G, Ranieri VM, Ronco C, Antonelli M; EUPHAS 2 Collaborative Group. Polymyxin-B hemoperfusion in septic patients: analysis of a multicenter registry. Ann Intensive Care. 2016 Dec;6(1):77. https://doi.org/10.1186/s13613-016-0178-9.

8. Cutuli SL, Artigas A, Fumagalli R, Monti G, Ranieri VM, Ronco C, Antonelli M; EUPHAS 2 Collaborative Group. Polymyxin-B hemoperfusion in septic patients: analysis of a multicenter registry. Ann Intensive Care. 2016 Dec;6(1):77. https://doi.org/10.1186/s13613-016-0178-9.

9. Cutuli SL, Artigas A, Fumagalli R, Monti G, Ranieri VM, Ronco C, Antonelli M; EUPHAS 2 Collaborative Group. Polymyxin-B hemoperfusion in septic patients: analysis of a multicenter registry. Ann Intensive Care. 2016 Dec;6(1):77. https://doi.org/10.1186/s13613-016-0178-9.

10. Cutuli SL, Artigas A, Fumagalli R, Monti G, Ranieri VM, Ronco C, Antonelli M; EUPHAS 2 Collaborative Group. Polymyxin-B hemoperfusion in septic patients: analysis of a multicenter registry. Ann Intensive Care. 2016 Dec;6(1):77. https://doi.org/10.1186/s13613-016-0178-9.

11. Cutuli SL, Artigas A, Fumagalli R, Monti G, Ranieri VM, Ronco C, Antonelli M; EUPHAS 2 Collaborative Group. Polymyxin-B hemoperfusion in septic patients: analysis of a multicenter registry. Ann Intensive Care. 2016 Dec;6(1):77. https://doi.org/10.1186/s13613-016-0178-9.

12. Cutuli SL, Artigas A, Fumagalli R, Monti G, Ranieri VM, Ronco C, Antonelli M; EUPHAS 2 Collaborative Group. Polymyxin-B hemoperfusion in septic patients: analysis of a multicenter registry. Ann Intensive Care. 2016 Dec;6(1):77. https://doi.org/10.1186/s13613-016-0178-9.

13. Cutuli SL, Artigas A, Fumagalli R, Monti G, Ranieri VM, Ronco C, Antonelli M; EUPHAS 2 Collaborative Group. Polymyxin-B hemoperfusion in septic patients: analysis of a multicenter registry. Ann Intensive Care. 2016 Dec:6(1):77. https://doi.org/10.1186/s13613-016-0178-9.

14. Cutuli SL, Artigas A, Fumagalli R, Monti G, Ranieri VM, Ronco C, Antonelli M; EUPHAS 2 Collaborative Group. Polymyxin-B hemoperfusion in septic patients: analysis of a multicenter registry. Ann Intensive Care. 2016 Dec;6(1):77. https://doi.org/10.1186/s13613-016-0178-9.

15. Cutuli SL, Artigas A, Fumagalli R, Monti G, Ranieri VM, Ronco C, Antonelli M; EUPHAS 2 Collaborative Group. Polymyxin-B hemoperfusion in septic patients: analysis of a multicenter registry. Ann Intensive Care. 2016 Dec;6(1):77. https://doi.org/10.1186/s13613-016-0178-9.

16. Cutuli SL, Artigas A, Fumagalli R, Monti G, Ranieri VM, Ronco C, Antonelli M; EUPHAS 2 Collaborative Group. Polymyxin-B hemoperfusion in septic patients: analysis of a multicenter registry. Ann Intensive Care. 2016 Dec;6(1):77. https://doi.org/10.1186/s13613-016-0178-9.

17. Cutuli SL, Artigas A, Fumagalli R, Monti G, Ranieri VM, Ronco C, Antonelli M; EUPHAS 2 Collaborative Group. Polymyxin-B hemoperfusion in septic patients: analysis of a multicenter registry. Ann Intensive Care. 2016 Dec;6(1):77. https://doi.org/10.1186/s13613-016-0178-9.

18. Cutuli SL, Artigas A, Fumagalli R, Monti G, Ranieri VM, Ronco C, Antonelli M; EUPHAS 2 Collaborative Group. Polymyxin-B hemoperfusion in septic patients: analysis of a multicenter registry. Ann Intensive Care. 2016 Dec;6(1):77. https://doi.org/10.1186/s13613-016-0178-9.

19. Cutuli SL, Artigas A, Fumagalli R, Monti G, Ranieri VM, Ronco C, Antonelli M; EUPHAS 2 Collaborative Group. Polymyxin-B hemoperfusion in septic patients: analysis of a multicenter registry. Ann Intensive Care. 2016 Dec;6(1):77. https://doi.org/10.1186/s13613-016-0178-9.

20. Cutuli SL, Artigas A, Fumagalli R, Monti G, Ranieri VM, Ronco C, Antonelli M; EUPHAS 2 Collaborative Group. Polymyxin-B hemoperfusion in septic patients: analysis of a multicenter registry. Ann Intensive Care. 2016 Dec;6(1):77. https://doi.org/10.1186/s13613-016-0178-9.

21. Cutuli SL, Artigas A, Fumagalli R, Monti G, Ranieri VM, Ronco C, Antonelli M; EUPHAS 2 Collaborative Group. Polymyxin-B hemoperfusion in septic patients: analysis of a multicenter registry. Ann Intensive Care. 2016 Dec;6(1):77. https://doi.org/10.1186/s13613-016-0178-9.

22. Cutuli SL, Artigas A, Fumagalli R, Monti G, Ranieri VM, Ronco C, Antonelli M; EUPHAS 2 Collaborative Group. Polymyxin-B hemoperfusion in septic patients: analysis of a multicenter registry. Ann Intensive Care. 2016 Dec;6(1):77. https://doi.org/10.1186/s13613-016-0178-9.

23. Cutuli SL, Artigas A, Fumagalli R, Monti G, Ranieri VM, Ronco C, Antonelli M; EUPHAS 2 Collaborative Group. Polymyxin-B hemoperfusion in septic patients: analysis of a multicenter registry. Ann Intensive Care. 2016 Dec;6(1):77. https://doi.org/10.1186/s13613-016-0178-9.

24. Cutuli SL, Artigas A, Fumagalli R, Monti G, Ranieri VM, Ronco C, Antonelli M; EUPHAS 2 Collaborative Group. Polymyxin-B hemoperfusion in septic patients: analysis of a multicenter registry. Ann Intensive Care. 2016 Dec;6(1):77. https://doi.org/10.1186/s13613-016-0178-9.

25. Cutuli SL, Artigas A, Fumagalli R, Monti G, Ranieri VM, Ronco C, Antonelli M; EUPHAS 2 Collaborative Group. Polymyxin-B hemoperfusion in septic patients: analysis of a multicenter registry. Ann Intensive Care. 2016 Dec;6(1):77. https://doi.org/10.1186/s13613-016-0178-9.

26. Cutuli SL, Artigas A, Fumagalli R, Monti G, Ranieri VM, Ronco C, Antonelli M; EUPHAS 2 Collaborative Group. Polymyxin-B hemoperfusion in septic patients: analysis of a multicenter registry. Ann Intensive Care. 2016 Dec;6(1):77. https://doi.org/10.1186/s13613-016-0178-9.

27. Cutuli SL, Artigas A, Fumagalli R, Monti G, Ranieri VM, Ronco C, Antonelli M; EUPHAS 2 Collaborative Group. Polymyxin-B hemoperfusion in septic patients: analysis of a multicenter registry. Ann Intensive Care. 2016 Dec;6(1):77. https://doi.org/10.1186/s13613-016-0178-9.

28. Cutuli SL, Artigas A, Fumagalli R, Monti G, Ranieri VM, Ronco C, Antonelli M; EUPHAS 2 Collaborative Group. Polymyxin-B hemoperfusion in septic patients: analysis of a multicenter registry. Ann Intensive Care. 2016 Dec;6(1):77. https://doi.org/10.1186/s13613-016-0178-9.

29. Cutuli SL, Artigas A, Fumagalli R, Monti G, Ranieri VM, Ronco C, Antonelli M; EUPHAS 2 Collaborative Group. Polymyxin-B hemoperfusion in septic patients: analysis of a multicenter registry. Ann Intensive Care. 2016 Dec;6(1):77. https://doi.org/10.1186/s13613-016-0178-9.

30. Cutuli SL, Artigas A, Fumagalli R, Monti G, Ranieri VM, Ronco C, Antonelli M; EUPHAS 2 Collaborative Group. Polymyxin-B hemoperfusion in septic patients: analysis of a multicenter registry. Ann Intensive Care. 2016 Dec;6(1):77. https://doi.org/10.1186/s13613-016-0178-9.

31. Cutuli SL, Artigas A, Fumagalli R, Monti G, Ranieri VM, Ronco C, Antonelli M; EUPHAS 2 Collaborative Group. Polymyxin-B hemoperfusion in septic patients: analysis of a multicenter registry. Ann Intensive Care. 2016 Dec;6(1):77. https://doi.org/10.1186/s13613-016-0178-9.

32. Cutuli SL, Artigas A, Fumagalli R, Monti G, Ranieri VM, Ronco C, Antonelli M; EUPHAS 2 Collaborative Group. Polymyxin-B hemoperfusion in septic patients: analysis of a multicenter registry. Ann Intensive Care. 2016 Dec;6(1):77. https://doi.org/10.1186/s13613-016-0178-9. 
33. De Rosa S, Cutuli SL, Ferrer R, Antonelli M, Ronco C; COVID-19 EUPHAS2 Collaborative Group. Polymyxin B hemoperfusion in coronavirus disease 2019 patients with endotoxic shock: Case series from EUPHAS2 registry. Artif Organs. 2020 Dec 30.

34. None

\section{6}

Comparison of de-novo acute kidney injury in intensive care patients requiring and not requiring renal replacement therapy S. Singh ${ }^{1}$; M. Andonovic ; J. Traynor ${ }^{2}$; M. Shaw ${ }^{1}$; M. Sim ${ }^{1}$; P. Mark ${ }^{3}$; K. Puxty ${ }^{1}$ ${ }^{1}$ Academic department of anaesthesia and critical care, University of Glasgow, Glasgow, United Kingdom; ²Department of nephrology, Queen Elizabeth University Hospital, Glasgow, United Kingdom; ${ }^{3}$ Institute of cardiovascular and medical sciences, University of Glasgow, Glasgow, United Kingdom

Correspondence: S. Singh

Intensive Care Medicine Experimental 2020, 9(1): 000626.

Introduction. Acute kidney injury (AKI) is a common occurrence in intensive care unit (ICU) patients [1]. Some AKIs in ICU patients require renal replacement therapy (RRT). Mortality in this population has been reported to be up to $80 \%$ [2]. There is limited work comparing ICU patients with de novo $A K I$ requiring and not requiring RRT.

Objectives. We aimed to compare characteristics and outcomes of ICU patients with de novo AKI requiring RRT and not requiring RRT.

Methods. The Scottish Intensive Care Society Audit Group WardWatcher database was interrogated for patients admitted to 2 Scottish teaching hospital ICUs between July 2015 and June 2018. All patients older than 16 were added to the Strathclyde Electronic Renal Patients Records database.

Data were extracted from both databases. Patients' demographics, pre-existing co-morbidities and admission characteristics were extracted for all patients. Mortality and $95 \%$ confidence intervals $(95 \% \mathrm{Cl})$ were calculated to ICU discharge and hospital discharge. Analyses were stratified into patients requiring and not requiring RRT.

Results. 2,147 of $5,312(40.4 \%)$ patients developed de novo renal injury and 498 (23.2\%) of these patients required RRT. Comparing RRT and non-RRT cohorts we observed similar ages (61.0 [IQR $=48.0-73.0]$ vs $61.0[\mathrm{IQR}=49.0-70.0])$ and genders $(60.4 \%$ vs $59.6 \%$ male, $\mathrm{p}=0.76)$. No differences were observed in pre-existing cardiovascular, respiratory or liver disease between groups; higher rates of pre-existing diabetes mellitus were observed in the RRT group compared to the non-RRT group $(24.7 \%$ vs $16.9 \%$, p < 0.001$)$. Median baseline eGFR was lower in the patients requiring RRT (70.0 [IQR $=42.3-95.9]$ vs 84.5 [IQR=63.8-100.7]).

Most patients requiring RRT were admitted from medical specialties $(54.6 \%)$ compared to those not requiring RRT who were mostly admitted from surgical specialties (53.4\%). Sepsis/infection was the most common reason for admission in both non-RRT $(27.7 \%)$ and RRT (36.1\%) groups.

112 patients (5.2\%) lacked full APACHE-II and predicted mortality data and thus were excluded from associated analyses. The non-RRT group had lower APACHE-Il scores (21 vs 27 ) and predicted mortality (37.8\% vs $62.9 \%)$ compared to the RRT group.

Observed in-ICU mortality was significantly higher in the RRT group (42.4\% $[95 \% \mathrm{Cl}=38.1 \%-46.7 \%]$ vs $26.6 \%[95 \% \mathrm{Cl}=24.2 \%-28.5 \%]$ ). Significantly higher mortality was observed at hospital discharge in the RRT group $(47.2 \%$ [95\% $\mathrm{Cl}=43.0 \%-51.8 \%]$ vs $32.4 \%[95 \% \mathrm{Cl}=30.3 \%-34.9 \%]$ ).

Conclusion. In ICU patients with de novo AKI, nearly one in four required RRT. These patients were more likely to have pre-existing diabetes mellitus, lower pre-admission eGFRs and were mostly admitted from medical specialties. Predicted mortality and APACHE-II score were higher in the RRT group. This was also observed clinically with significantly higher mortality rates to ICU and hospital discharge in the RRT group. Further research is required looking at longer-term outcomes in RRT vs non-RRT cohorts.

\section{Reference(s)}

1. Melo F de AF, Macedo E, Fonseca Bezerra AC, et al. A systematic review and meta-analysis of acute kidney injury in the intensive care units of developed and developing countries. PLoS One. 2020;15(1).

2. Palevsky PM. Renal replacement therapy in acute kidney injury. Adv Chronic Kidney Dis. 2013;20(1):76-84.

3. No funding was received for this study.

\section{6}

ACUTE KIDNEY INJURY AS A PROGNOSTIC FACTOR IN CRITICALLY ILL PATIENTS WITH SEVERE SARS-COV-2 PNEUMONIA

C. Ferré ${ }^{1}$; A. Pobo ${ }^{1}$; X. Daniel'; M. Roure ; C. Villavicencio Lujan";

M. Cartanyà'; J. Leache Irigoyen ${ }^{1}$; M. Bodí ; I. Oliva ${ }^{1}$

${ }^{1}$ Intensive care, Hospital Universitari de Tarragona Joan XXIII, Tarragona,

Spain

Correspondence: C. Ferré

Intensive Care Medicine Experimental 2020, 9(1): 000636.

Introduction. Nowadays, the world is facing a pandemic due to the disease appeared in December 2019 in the city of Wuhan (Hubei province) caused by a new virus named "Severe acute respiratory syndrome coronavirus 2" (SARS-CoV-2) (1). SARS-CoV-2 not only induces acute respiratory failure, but it is also associated with the involvement of different organs and systems, such as kidney, liver, heart, gastrointestinal, hematological and nervous system with a high rate of mortality and multiorgan failure (2).

The aim of this study was to measure the incidence of AKI and its impact on intensive care unit (ICU) mortality of patients with severe SARS-Cov-2 pneumonia admitted to ICU.

Methods. Observational cohort study carried out in the ICU at Joan XXIII University Hospital (Tarragona, Spain), during 11 months. The data were collected during the first $72 \mathrm{~h}$ of admission.

AKI was defined as a rise in creatinine of 1.5 times baseline value or decrease in urine output below $0.5 \mathrm{~mL} / \mathrm{kg} / \mathrm{h}$ for $6 \mathrm{~h}$ (3).

Results. 181 patients were admitted to ICU with severe SARS-CoV-2 pneumonia. The median age was 65 years old and $71.3 \%$ were male. The most coexisting conditions were overweight (50.3\%), obesity (33.1\%), hypertension (47\%), diabetes (28.3\%) and cardiovascular disease (13.2\%). $25.41 \%$ developed AKI, $17.4 \%$ of whom needed continuous renal replacement therapy (CRRT). Patients with AKI were significantly older [69.5 (58.5-74.25) years vs 64. (54-71) years; $p=0.03]$, had a significantly higher prevalence of hypertension $(67.4 \%$ vs $40 \% ; p=0.002)$, chronic ischemic heart disease ( $13 \%$ vs $4.4 \% ; p=0.002)$, significantly higher SAPS-III [55 (5258 ) vs $52(48-55) ; p=0.000]$ and SOFA at $24 \mathrm{~h}$ [5 (4-7) vs $4(3-6) ; p=0.000]$.

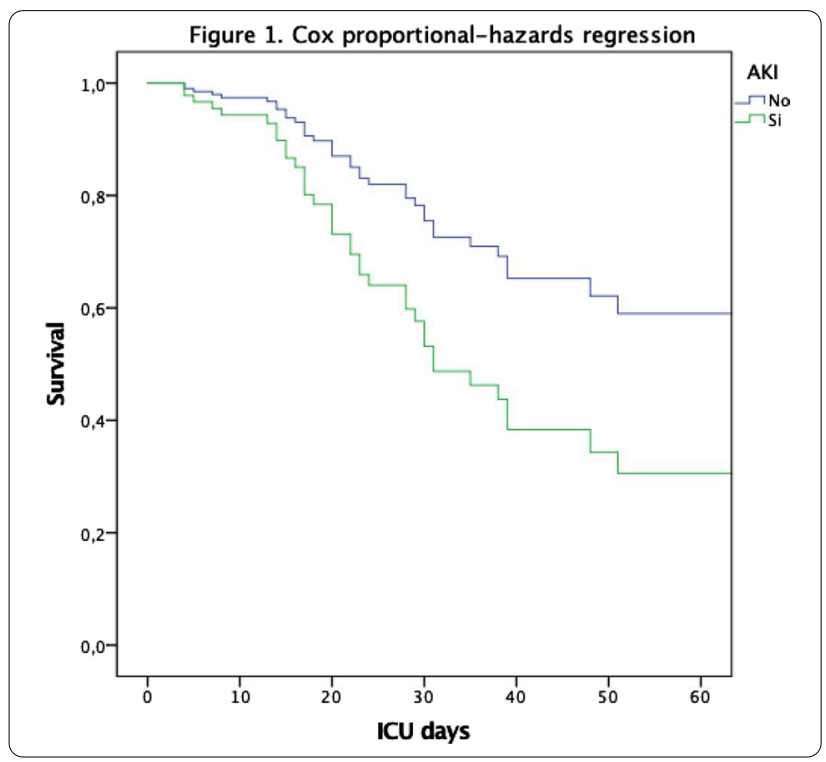


AKI was significantly associated to higher use of norepinephrine ( $87 \%$ vs $63 \% ; p=0.003)$, dobutamine $(10.9 \%$ vs $0.7 \% ; p=0.004)$ and furosemide ( $63 \%$ vs $33.3 \% ; p=0.001$ ). These patients also had higher incidence of shock ( $21 \%$ vs $16 \% ; p=0.000)$, left ventricular disfunction $(15.2 \%$ vs $3 \% ; p=0.03)$ and need for mechanical ventilation ( $100 \%$ vs $80.7 \% ; p=0.000$ ).

AKI was an independent predictor of ICU-mortality in adjusted survival analysis with HR 2.24 (95\% Cl 1.06-4.74). Fig. 1

Conclusion. AKI is a frequent complication in patients with SARS CoV-2 pneumonia and was associated with greater development of shock and left ventricular dysfunction, as well as greater need for mechanical ventilation. Furthermore, AKI is an independent predictor of ICU mortality. Therefore, we suggest early diagnosis and strict monitoring of AKI in these patients.

\section{Reference(s)}

1. Khwaja A. KDIGO clinical practice guidelines for acute kidney injury. Nephron Clin Pract. 2012;120(4):c179-84. https://doi.org/10.1159/00033 9789. Epub 2012 Aug7. PMID: 22890468.

2. Mokhtari T, Hassani F, Ghaffari N, Ebrahimi B, Yarahmadi A, Hssanzadeh G. COVID-19 and multiorgan failure: a narrative review on potential mechanisms. J Mol Histol, 2020 Oct 4: 1-16. https://doi.org/10.1007/s10735-02009915-3. PMCID: PMC7533045. https://www.ncbi.n/m.nih.gov/pmc/artic les/PMC7533045/

3. Arentz M, Yim E, Klaff L, Lokhandwala S, Riedo FX, Chong M, Lee M. Characteristics and Outcomes of 21 Critically III Patients With COVID-19 in Washington State. JAMA. 2020 Apr 28;323(16):1612-1614. https://doi.org/ 10.1001/jama.2020.4326. PMID: 32191259; PMCID: PMC7082763. https:// www.ncbi.nlm.nih.gov/pmc/articles/PMC7082763/.

\section{7}

Evaluation of an on-line registry for the assessment of renal depuration techniques: DialyReg

M. Gonzalez-Fernandez ; N. Quílez-Trasobares²; JA. Barea-Mendoza ${ }^{3}$; MD. Arias Verdú4; J JE. Barrueco Francioni ${ }^{5}$; G. Seller Pérez ${ }^{5}$; ME. Herrera Gutiérrez ${ }^{5}$ J JA. Sanchez-lzquierdo

${ }^{1}$ Critical care, University Hospital 12 de Octubre, Madrid, Spain; ${ }^{2}$ Intensive care, University Hospital 12 de Octubre, Madrid, Spain; Intensive care medicine, Hospital 12 De Octubre, Madrid, Spain; ${ }^{4}$ Intensive care, Hospital Carlos Haya, Málaga, Spain; ${ }^{5} \mathrm{CU}$, Hospital Regional Universitario de Málaga (Carlos de Haya), Málaga, Spain

Correspondence: M. Gonzalez-Fernandez

Intensive Care Medicine Experimental 2020, 9(1): 000647

Introduction. On-line databases have developed as an alternative method for storing and analysing scientific data. They respect confidentiality, provide security and are easy to use. Based on international databases, we present the evaluation of an on-line registry designed for the tracing of renal depuration techniques.

Objectives. To analize the results from a multicenter registry which allows the monitoring of patients with Continuous Renal Replacement Therapy (CRRT) in order to detect and solve problems that appear during its implementation.

Methods. Prospective multicenter observational and descriptive study from the registry DialyReg. This is an on-line database located on a REDCap web-based platform. It provides legal guarantees regarding security and confidentiality and it allows data analysis in real time. Statistical analysis was performed with SPSS IBM ${ }^{\circledR}$. It had Bioethics Comittee's approval from both Hospitals.

Results. From january 2019 to december 2020, 322 patients have been included with the main admission diagnosis of medical illness $(51 \%)$, sepsis $(24 \%)$, postsurgical $(23 \%)$ or trauma $(2 \%)$. The principal indication for the therapy was internal homeostasis $(41.4 \%)$, oliguria (26.3\%), hydric disbalance (14.7\%) and hemodynamics (13\%).

The median of catheters per patient was 1 (IQR1-1) with a median duration of 5 days (IQR 3-9). The $82 \%$ of the catheters were coaxial dual lumen and the preferential vascular access was right femoral vein $(57 \%)$. The main associated complications were infection (1.7\%), hematoma (1.4\%) and thrombosis (1.2\%). The median of haemofilters per patient was 4 (IQR 2-6) with a mean duration of therapy set of $31 \mathrm{~h}$ (SD: 24).

Initial therapy dose was set in $32 \mathrm{ml}$ per kilogram (SD: 12) and dynamic adjustment was perform in $61 \%$ of the sample. The main reason for discontinuation of the theraphy was recovery $(52 \%)$, death $(33 \%)$ and transition to intermittent renal replacement (11\%). Interruption was due to end of the treatment (52\%), planned $(33 \%)$ or set coagulation (11\%).

The main complications from the therapy were hypotermia (16\%), hypophosphataemia (13\%), metabolic acidosis $(10 \%)$, hypocalcemia $(6 \%)$ and hypokalemia (5\%). The weaning from the therapy was nocturnal renal replacement therapy $(25.6 \%)$, progressive SLED (18\%), fast without diuretics (43\%) and fast with diuretics (13.5\%). 52\% of the patients were discharged from the hospital while $43 \%$ died in the ICU and $5.6 \%$ died during hospitalization.

Conclusion. The DialyReg register is a feasible and affordable tool for real time control of the results of renal replacement theraphy in our units. It could be useful for the maintenance of security programs in these thecniques.

\section{Reference(s)}

1. Harris P, Taylor R, Minor B, Elliott V et al. The REDCap Consortium: Building an International Community of Software Platform Partners. J Biomed Inform. 2019

2. Rachoin JS, Weisberg L. Renal replacement therapy in the ICU. Crit Care Med 2019; 47(5): 715-21

000650

Clinical usefulness of urinary NGAL and L-FABP for prediction of AKI that require renal replacement therapy

M. Akatsuka'; H. Tatsumi'; S. Kazuma'; H. Kuroda'; W. Aisaka' ;. Goto ${ }^{1}$; S. Tanahashi'; Y. Masuda ${ }^{1}$

${ }^{1}$ Department of intensive care medicine, Sapporo Medical University School of Medicine, Sapporo, Japan

Correspondence: M. Akatsuka

Intensive Care Medicine Experimental 2020, 9(1): 000650

Introduction. Acute kidney injury (AKI) often occurs in the ICU and its incidence in the ICU has been reported to be more than $50 \% 1$ ). The use of continuous renal replacement therapy (CRRT) is often required in the patients with developed AKI. Therefore, early diagnosis and prompt intervention are important for preventing the progression of renal damage and improving the prognosis. In recent studies, the usefulness of kidney biomarkers including neutrophil gelatinaseassociated lipocalin (NGAL) and liver-type fatty-acid binding protein (L-FABP) for predicting of AKI has been investigated2), 3).However, the usefulness of those biomarkers in clinical practice is not clear.

Objectives. The aim of this study was to determine the clinical usefulness of the urinary biomarkers for early diagnosis of AKI, prediction of the need for CRRT, and prediction of prognosis.

Methods. The study subjects were patients who were admitted to the ICU during the period from June 2016 to August 2017 and in whom urinary NGAL and L-FABP were determined on ICU admission. We divided the patients into an AKI group and a non-AKI group. The outcomes were the relationships of AKI biomarkers with indication of CRRT and 28-day mortality. We also investigated the usefulness of the AKI biomarkers for early detection of the development of AKI in patients in whom AKI occurred after ICU admission. AKI was diagnosed by the KDIGO criteria.

Results. A total of 74 patients were included in this study. There were 48 patients in the AKI group and 26 patients in the non-AKI group. CRRT was performed in 37 patients in the AKI group. Urinary NGAL and urinary L-FABP levels were significantly higher in patients who received CRRT than in patients who did not receive CRRT $(p=0.0037$ and 0.049 , respectively). As for 28-day mortality, 58 patients survived and 16 patients died. Urinary L-FABP level on ICU admission was associated with 28-day mortality $(p=0.038)$. On the other hand, there was no significant difference in urinary NGAL level between patients who survived and patients who died $(p=0.188)$. Among the 26 
patients in the non-AKI group on ICU admission, AKI later occurred in seven patients. Urinary NGAL and L-FABP levels in patients in whom AKI occurred after ICU admission were not significantly higher than the levels in patients in whom AKI did not occur after ICU admission $(\mathrm{p}=0.919$ and $\mathrm{p}=0.991$, respectively).

Conclusion. Urinary AKI biomarkers have the potential to predict indication of CRRT. Also, urinary L-FABP might be useful for predicting 28-day prognosis. On the other hand, this study showed the limitation of using those biomarkers for predicting early diagnosis of AKI. Further study will be needed to determine whether the use of urinary biomarkers leads to improved prognosis of AKI through early diagnosis and appropriate therapeutic intervention.

\section{Reference(s)}

1) Hoste EA, Bagshaw SM, Bellomo R, et al. Epidemiology of acute kidney injury in critically ill patients: the multinational AKI-EPI study. Intensive Care Med 2015; 41: 1411-23.

2) Huelin P, Solà E, Elina Chiara, et al. Neutrophil Gelatinase-Associated Lipocalin for Assessment of Acute Kidney Injury in Cirrhosis: A Prospective Study. Hepatology 2019; 70: 319-333.

3) Naruse $\mathrm{H}$, Ishii J, Takahashi $\mathrm{H}$, et al. Predicting acute kidney injury using urinary liver-type fatty-acid binding protein and serum $\mathrm{N}$-terminal proB-type natriuretic peptide levels in patients treated at medical cardiac intensive care units. Crit Care 2018; 22: 197.

\section{1}

Continuous Renal Replacement Therapy with hemoadsorption $\left(O x i r i{ }^{\circledR}\right)$ in patients with severe acute respiratory failure due to COVID-19 and associated renal failure

M. Gonzalez-Fernandez' ; N. Quílez-Trasobares²; JA. Barea-Mendoza3; MD. Arias Verdú4; JE. Barrueco Francioni ${ }^{5}$; G. Seller Pérez ${ }^{5}$; ME. Herrera Gutiérrez5; JA. Sanchez-Izquierdo

${ }^{1}$ Critical care, University Hospital 12 de Octubre, Madrid, Spain; ${ }^{2}$ Intensive care, University Hospital 12 de Octubre, Madrid, Spain; ${ }^{3}$ Intensive care medicine, Hospital 12 De Octubre, Madrid, Spain; ${ }^{4}$ Intensive care, Hospital Carlos Haya, Málaga, Spain; ${ }^{5} \mathrm{CU}$, Hospital Regional Universitario de Málaga (Carlos de Haya), Málaga, Spain

Correspondence: M. Gonzalez-Fernandez

Intensive Care Medicine Experimental 2020, 9(1): 000651

Introduction. The utilization of continuous renal replacement therapy with hemoadsorption (Oxiris ${ }^{\circledR}$ ) in patients with severe acute respiratory failure due to COVID-19 and associated renal failure may modify the systemic inflammatory response associated with this disease based on cytokine clearance.

Objectives. To describe the characteristics of our series as well as the therapy applied and to evaluate the impact of hemoadsorption on the evolution of our patients.

Methods. Prospective unicenter observational study in patients admitted to the ICU from march to december 2020 due to SARS-CoV-2 infection with respiratory failure and acute kidney injury (AKI) in need for Continuous Renal Replacement Therapy (CRRT) in which hemoadsorption with Oxiris ${ }^{\circledR}$ membrane is set up. We analyze epidemiology, severity scores, respiratory status and inflammatory biomarkers before and after therapy as well as dose and complications of CRRT, ICU lenght of stay and mortality. Statistical analysis is performed with SPSS IBM ${ }^{\circledR}$.

Results. 246 patients were analized: $25 \%$ of them presented acute kidney injury, $80 \%$ of which required CRRT and in $72 \%$ of them hemoadsorption was associated. The characteristics of hemoadsorption group were: median age of 55 years with male predominance $(72 \%), 57 \%$ of them were hypertensive, $43 \%$ obese and $21 \%$ chronic pneumopaths. The worst SOFA at $24 \mathrm{~h}$ of admission was 7.9 (IQR: 3-11), the worst PaFi was 95 (IQR: 66-110) and the worst compliance was $45 \mathrm{ml} / \mathrm{cmH} 2 \mathrm{O}$ (IQR: 40-52). Neuromuscular blockade and prone position were used in $93 \%$ of them, and veno-venous ECMO was applied in 12 cases.

The main indication of CRRT was homeostasis (81\%) and fluid balance $(19 \%)$ with median of initial dose employed of $35 \mathrm{ml} / \mathrm{kg}$ (IQR: $35-40$ ), final of $25 \mathrm{ml} / \mathrm{kg}$ (IQR 30-45) and median duration of 5 days. The anticoagulation system used was: $93 \%$ citrate, $4 \%$ nothing and
$3 \%$ heparin, without any case of citrate accumulation. We detected several complications, all of them in mild degree: hypothermia 35\%, persistence of metabolic acidosis $27 \%$, metabolic alkalosis $11 \%$ and hypophosphataemia, hypomagnesemia and hypokalemia $2 \%$ respectively. No statistically significant differences were found between inflammatory biomarkers $(P C R, P C T$, ferritin, lymphocytes, fibrinogen), SOFA or PaFi pre and post-CRRT maybe due to the small sample size, althought they were found between pre and post-CRRT D-dimer $(p<0.05)$. ICU lenght of stay was 12 days (IQR $6-18)$ and ICU mortality rate was $30 \%$.

Conclusion. In our series, CRRT with hemoadsorption has been an useful and safe technique in the management of acute renal failure in COVID-19 patients. This therapy could lower D-dimer levels.

\section{Reference(s)}

1. Ronco C, Reis T. Kidney involvement in Covid-19 patients and the role of extracorporeal therapies. Nat Rev Nephrol. 2020.

2. Ronco $\mathrm{C}$ et al. Extracorporeal Blood Purification and Organ Support in the Critically III patient during COVID-19 pandemic: expert review and recommendation. Blood Purif 2020.

\section{3}

Analysis of the economic impact of the different anticoagulation strategies used for renal replacement therapy (RRT)

MD. Arias Verdúl; G. Seller-Pérez ; J. Barrueco-Francioni ${ }^{1}$;

M. Gonzalez-Fernandez²; N. Quílez-Trasobares²; J. Barea-Mendoza, ${ }^{2}$;

JA. Sanchez-Izquierdo²; M. Herrera-Gutiérrez

${ }^{1}$ Intensive care unit, Hospital Regional Universitario de Málaga (Carlos de Haya), Málaga, Spain; ${ }^{2}$ Intensive care, University Hospital 12 de Octu-

bre, Madrid, Spain

Correspondence: M.D. Arias Verdú

Intensive Care Medicine Experimental 2020, 9(1): 000673

Introduction. cc.

Objectives. To assess the economic impact of the different anticoagulation strategies used for renal replacement therapy (RRT).

Methods. To calculate the theoretical cost according to the anticoagulation regimen and duration of the filter. We analyzed the cost of filters, anticoagulant and fluids.

Filter: for RRT or citrate (Ci-Ca) ST-150 Baxter ${ }^{\circledR}$, and for sled technique, used in the night, F10HPS Fresenius ${ }^{\circledR}$.

Fluids: Hemosol Baxter ${ }^{\circledR}$ (CRRT without $\mathrm{Ci}-\mathrm{Ca}$ or nocturnal sled) and Foxsilium Baxter ${ }^{\circledR}$ (Ci-Ca).

Citrate: Regiocit Baxter ${ }^{\circledR}$ for a dose of $3.2 \mathrm{~mm} / \mathrm{l}$ and Qb of $120 \mathrm{ml} / \mathrm{min}$. Data are shown as mean $\pm S D$, median $\pm I Q R$, or $\%$.

Non parametric analyses are performed for $\mathrm{p}<0.05$.

Results. Data from 159 patients were collected from the RegTDE. (data record-TDE) The mean duration of the treatments was $9.3 \pm 46.6$ days, with a total consumption of 690 filters $(4.26 \pm 4.4$ / patient) and a mean duration of $31.2 \pm 23.5 \mathrm{~h} /$ filter. $35.8 \%$ were anticoagulated with heparin, $15 \%$ with citrate, and no anticoagulation in $43 \%$, with an average duration of $55 \mathrm{~h}$ (24-72) for citrate, $33 \mathrm{~h}(13-51)$ for prostacyclins $+\mathrm{HnF}, 23 \mathrm{~h}(12-48)$ with heparin, and $12 \mathrm{~h}(12-31)$ without anticoagulation $(p<0.05)$. The reason for removing the hemofilter was programmed (37\%): clot (36\%) and end of treatment (18\%). The average dose was $34.7 \mathrm{ml} / \mathrm{Kg} / \mathrm{h}$, which means an effluent of 2,300 $\mathrm{ml} / \mathrm{h}$ for normal patient.

According to the results in our patients, we assume that the duration of the citrate filters is $>48 \mathrm{~h}$.

The average cost / day assuming a filter duration of $72 \mathrm{~h}$ would be $€ 80$ for conventional anticoagulation, $€ 102$ for nocturnal sled and $€ 180$ for citrate. However, if the duration of the filter with $\mathrm{HnF}$ or without anticoagulation were $24-48 \mathrm{~h}$, the daily cost would be $€ 150$, and if it lasts $24 \mathrm{~h}$ it will rise to $€ 230$ /day.

Conclusion. if the filters, with $\mathrm{HnF}$ or without anticoagulation, extend their duration beyond $48 \mathrm{~h}$, this could be the most profitable regimen and, if the average duration does not exceed $48 \mathrm{~h}$, both nocturnal sled and citrate are more efficient options in terms of cost. The existence of a defined anticoagulant management protocol and the monitoring of 
its results may show advantages over the use of a single anticoagulation regimen in terms of cost.

\section{4}

\section{AKI in COVID-19 ARDS patients: Incidence, clinical presentation} and outcome

A. Lavrentieva ${ }^{1}$; C. Giannaki ${ }^{1}$; A. Dimaki ${ }^{1}$; M. Tzimou' ; B. Voutsas ${ }^{1}$; M. Bitzani

'Icu, General Hospital of Thessaloniki "George Papanikolaou", Thessaloniki, Greece

Correspondence: A. Lavrentieva

Intensive Care Medicine Experimental 2020, 9(1): 000674

Introduction. There are limited data on COVID-19-associated acute kidney injury (AKI) in critically ill patients.

Objectives. The objective of the study was to investigate the incidence, severity, clinical features, and outcome of COVID-19-associated AKI in an ICU setting.

Methods. Single-centre cohort study performed in a COVID-19 ICU from March 2020-March 2021. AKI incidence, clinical presentation, outcome, the association with admission severity of illness and organ function, as well as the association with admission biomarkers of inflammation [C-reactive protein (CRP), Procalcitonin (PCT) and ferritin] were evaluated. AKI was defined by Kidney Disease Improving Global Outcomes (KDIGO) criteria.

Wilcoxon-MWU test was used for comparison of parameters between patients with and without AKI.

Results. A total of 197 patients were included in the analysis. The incidence of AKI was $24.4 \%$ (48 patients). The mean LOS among critically ill COVID-19 patients with and without AKI was $16.5 \pm 10$ (SD) vs $16.8 \pm 9.2(\mathrm{SD})$ days, $p>0.05$. The median time from the onset of COVID19 symptoms to $A K I$ was 12 days (IQR, 3-15) and from ICU admission to $A K I 3$ days (IQR, 1-9.5). AKI patients were older (69.9 \pm 12.5 vs. $63.8 \pm 12.3$ years, $p=0.009)$, had higher APACHE II score (17.1 \pm 5 vs. $15.3 \pm 6.7, p=0.006)$, and SOFA score ( $7.8 \pm 1.9$ vs. $6.9 \pm 2, p=0.008)$. There was no difference on admission values for CRP, PCT and ferritin between the 2 groups.

AKI diagnosis was based on serum creatinine value in $83.3 \%$ of patients; in $44.4 \%$ urine output criterion was used for AKI diagnosis. Stage I was diagnosed in $8(17.8 \%)$ patients, stage II in $23(51.1 \%)$ patients and stage III in $14(31.1 \%)$ patients. AKI was considered sepsisrelated in $72 \%$ of the patients, while nephrotoxic drugs and contrast media were incriminated in $57.8 \%$ and $6.8 \%$ of cases.

Renal replacement therapy was required in $24.4 \%$ of the patients due to fluid overload $(84.6 \%)$, acid-base balance disorders $(69.2 \%)$, and electrolyte abnormalities (72\%). On discharge, improvement of renal function was observed in $41.5 \%$ of patients. Survival rate was lower in the AKI group compared to the no AKI group (24.5\% vs $70 \%)$. Stage III AKI was associated with the lower survival rate in comparison to stages I and II (7.1\% vs. $34.4 \%)$.

Conclusion. High incidence of AKI was observed in COVID-19 ICU patients. AKI diagnosis was related to increased mortality; survival rate was lower in the more severe cases of AKI. Sepsis was the most common cause of acute kidney injury. Age, higher APACHE II and SOFA scores were associated with development of AKI in Covid-19 ARDS patients.

\section{8}

Could nocturnal dialysis (nocturnal SLED) be an appropriate option for patients admitted to the ICU ? Analysis of the Reg-TDE multicentre registry

MD. Arias Verdú'; G. Seller-Pérez'; JA. Sanchez-Izquierdo²;

J. Barea-Mendoza, ${ }^{2}$; J. Barrueco-Francioni'; ; N. Quílez-Trasobares²;

M. Gonzalez-Fernandez²; M. Herrera-Gutiérrez'

${ }^{1}$ Intensive care, Hospital Regional Universitario de Málaga (Carlos de Haya), Málaga, Spain; ${ }^{2}$ Intensive care, University Hospital 12 de Octubre, Madrid, Spain

Correspondence: M.D. Arias Verdú

Intensive Care Medicine Experimental 2020, 9(1): 000678
Introduction. Hybrid CRRT have presented promising results in some series but they still have little application in our country, mainly for economic reasons, as there are no monitors capable of treating fluids on-line.

Objectives. To analyze the behaviour of nocturnal dialysis (nocturnal SLED) compared to conventional techniques in terms of operability and safety.

Methods. We analyze the prospective multicentre Reg-TDE registry during 2019. The nocturnal dialysis technique (Nocturnal SLED) is performed preferably using a low-flow polysulfone filter, without anticoagulation, and with a programmed duration of $12 \mathrm{~h} /$ day. It is compared with continuous techniques (CRRT): types of anticoagulation, filter losses due to clots and the most common complications. Data are shown as mean + standard deviation, median + interquartile range, or $\%$. Non-parametric analyses are performed for a $p<0.05$.

Results. We analysed 159 treatments, in which 689 filters were used: $522(75.8 \%)$ in CRRT and $167(24.2 \%)$ in nocturnal SLED. 100\% phosphorous-free fluids were used to perform nocturnal SLED and a lowflux polysulfone membrane dialysis filter was used in $77.8 \%$.

There were no differences in the ability to perform dynamic dose adjustment (73.1 vs $68 \%$, p ns), although the dose applied in TDEN was $20(11-29)$ vs $30(20-40) \mathrm{ml} / \mathrm{Kg}$ in CRRT (p ns).

This modality was performed without anticoagulation in $75.4 \%$ of the cases vs $34.5 \%$ in CRRT $(p<0.001)$, despite the fact that the losses due to clot were 7.8 vs $44.8 \%(p<0.001)$.

The number of complications was significantly lower $(0.48+-0.72$ vs $0.63+-0.8, p 0.005)$, specifically fewer hypothermic episodes (0.6 vs $13.4 \%, p<0.001$ ), lower frequency of hypokalemia ( 3 vs $6.9 \%, p 0.09$ ), and lower transfusion requirement $(0.08+-0.34$ vs $0.26-0.7$ units, $p$ $0.003)$. Hypophosphataemia did not show differences between groups (18 vs $16.1 \%$, p ns), although for CRRT performed with P fluids the percentage was only $2.9 \%$.

Conclusion. Nocturnal dialysis or nocturnal SLED has clear advantages over continuous dialysis for stable patients: no anticoagulation is required, the entire prescribed dose is ensured and administered, metabolic complications are fewer, and the workload is reduced. The foreseeable introduction into the market of new monitors with the capacity to prepare fluids on-line would justify the generalisation of this modality for the management of stable patients.

\section{9}

Impact of therapy characteristics in terms of efficacy, safety, and complications due to citrate use versus other anticoagulation modalities in Continuous Renal Replacement Therapy

N. Quílez-Trasobares'; M. Gonzalez-Fernandez²; JA. Barea-Mendoza ${ }^{3}$; MD. Arias Verdú4; JE. Barrueco Francioni ${ }^{5}$; G. Seller Pérez ; ME. Herrera Gutiérrez; ; JA. Sanchez-Izquierdo ${ }^{2}$

${ }^{1}$ Intensive care, University Hospital 12 de Octubre, Madrid, Spain; ${ }^{2}$ Critical care, University Hospital 12 de Octubre, Madrid, Spain; ${ }^{3}$ Intensive care medicine, Hospital 12 De Octubre, Madrid, Spain; ${ }^{4}$ Intensive care, Hospital Carlos Haya, Málaga, Spain; ${ }^{5} \mathrm{ICU}$, Hospital Regional Universitario de Málaga (Carlos de Haya), Málaga, Spain

Correspondence: M. Gonzalez-Fernandez

Intensive Care Medicine Experimental 2020, 9(1): 000689

Introduction. Citrate therapy is a well-studied anticoagulation system for Continuous Renal Replacement Therapy (CRRT) in critically ill patients with great efficacy and scarce adverse effects. It seems to prolong filter lifetime, reduce bleeding complications and provide excellent control of acid-base status. Due to its liver metabolization it must be used with caution in patients with hepatopathy or severe multiorgan failure and persistent hyperlactatemia.

Objectives. To compare citrate therapy to other anticoagulation modalities in terms of efficacy and safety in our cohort and to analyze the potential complications of citrate utilization in patiens with hepatopathy. 
Methods. Prospective multicenter observational cohort study based on the DialyReg registry with the Clinical Research Ethics Committee's approval from both hospitals. We compare therapy dose, clot filter loss and common complications in patients undergoing CRRT in ICU since 2019 to 2020. Statistical analysis is performed with SPSS system 20.

Results. We analyze data from 311 patients, corresponding to 1216 haemofilters. The distribution in anticoagulation systems was: heparin $(40 \%)$, citrate (38\%), and without anticoagulation (22\%).

The initial and final median therapy dose was similar in the different groups respectively, citrate $(32 \mathrm{ml} / \mathrm{kg}$ and $28 \mathrm{ml} / \mathrm{kg})$, heparin $(33 \mathrm{ml} /$ $\mathrm{kg}$ and $30 \mathrm{ml} / \mathrm{kg}$ ) and without anticoagulation $(29 \mathrm{ml} / \mathrm{kg}$ and $28 \mathrm{ml} /$ $\mathrm{kg})$. Dynamic adjustment was performed more frequently in the citrate group $(76 \%)$ than in heparin $(58 \%)$ and without anticoagulation groups $(54 \%)(p<0.05)$.

Median duration of the set was higher in citrate group ( $55 \mathrm{~h}$, IQR 24-72) versus $23 \mathrm{~h}$ in heparine group (IQR 12-48) and $12 \mathrm{~h}$ in no anticoagulation group (IQR 12-31) $(p<0.05)$. Coagulation of the system was present in $35 \%$ of the total (16\% with heparin, $12 \%$ witouth coagulation and $7 \%$ with citrate) with a higher incidence with heparin $(46.7 \%)$ than with citrate $(26 \%)(p<0.05)$.

Complications associated with the technique were: hypothermia in the $16 \%$ of the total (4\% citrate vs $5 \%$ heparin and $6 \%$ nothing), hypokalemia ( $3 \%$ citrate vs $1 \%$ rest) and hypomagnesemia $(6.6 \%$ citrate vs $2 \%$ rest) $(p<0.05)$; hypophosphatemia was present in the $12 \%$ of the total and it was higher in the groups no anticoagulation (6\%) or heparin $(5 \%)$ versus citrate $(1 \%)$; metabolic acidosis was similar in the different types of anticoagulation (3\% respectively). Following proper use of the technique with citrate and the use of phosphate dialysis fluids, complications are similar to the other types of therapy.

Citrate anticoagulation was also analyzed in the subgroup of patients with hepatopathy (37 patients corresponding to 145 haemofilters): $50 \%$ cirrhosis, 37\% liver transplantation, $7 \%$ acute liver failure, and $4 \%$ acute-on-chronic failure). Heparin was used in 39\%, citrate in $36 \%$, and no anticoagulation was used in $25 \%$ of the sample. Therapy had to be discontinued due to clotting in $17 \%$ of the hemofilters without anticoagulation, $9 \%$ with heparin and $3 \%$ with citrate. No statistically significant differences were found in terms of complications due to therapy. There were also no changes in the calcium dose adjustment in the $77 \%$ of the cases, two changes in $16 \%$ of the cases and one in the $2 \%$. Citrate accumulation did not occur in any of the following cases and there were no transfusions related to the technique.

Conclusion. Citrate anticoagulation increases the duration of the haemofilters and allows better adjustment of the therapy dose in our series, possibly being more cost-effective. It is a safe anticoagulation system with no major adverse effects and this safety is also applied to patients with hepatopathy.

\section{Reference(s)}

1. Zhang W, Bai M, Yu Y, Li L, Zhao L, Sun S, Chen X. Safety and efficacy of regional citrate anticoagulation for continuous renal replacement therapy in liver failure patients: a systematic review and meta-analysis. Crit Care 2019: 23(1)

2. Wu MY, Hsu YH, Bai CH, et al. Regional citrate versus heparin anticoagulation for continuous renal replacement therapy: a meta-analysis of randomized controlled trials. Am J Kidney Dis 2012;59(6):810-818

3. Oudemans-van Straaten HM, Ostermann M. Bench-to-bedside review: Citrate for continuous renal replacement therapy, from science to practice. Crit Care 2012;16:249

4. Liu C, Mau Z, Kang H, et al. Regional citrate versus heparin anticoagulation for continuous renal replacement therapy in critically ill patients: a meta-analysis with trial sequiential analysis of randomized controlled trials. Crit Care 2016;20:144

\section{8}

Asthma as a risk factor of contrast-associated acute kidney injury in patients with coronary artery disease

O. Mironova ${ }^{1}$; O. Sivakova ${ }^{2}$; V. Fomin ${ }^{3}$

${ }^{1}$ Sechenov University, Moskva, Russia; ${ }^{2}$ Department of arterial hypertension, National Medical Research Center of Cardiology, Moscow, Russia;

${ }^{3}$ Chair of internal diseases \#1, Sechenov University, Moskva, Russi

Correspondence: O. Mironova

Intensive Care Medicine Experimental 2020, 9(1): 000748

Introduction. The role of underlying inflammation in the setting of acute kidney injury is well established. Asthma is a chronic inflammatory disease of respiratory system, so the inflammation is seen in both of the conditions, as well as in the atherosclerotic plaques of the patients with stable coronary artery disease (CAD). The number of patients with asthma and stable coronary artery disease is increasing each year.

Objectives. The aim of the study was to assess the role of asthma as a potential risk factor predisposing to the development of contrastassociated acute kidney injury (CA-AKI) in patients with stable coronary artery disease.

Methods. 1023 patients with stable CAD were enrolled in a prospective, open, cohort study. CA-AKI was defined as an increase of $25 \%$ or more, or an absolute increase of $0,5 \mathrm{mg} / \mathrm{dl}$ or more in serum creatinine from baseline value, assessed at $48-72 \mathrm{~h}$ following the administration of the contrast media. The primary endpoint of the study was the development of contrast-induced acute kidney injury according to KDIGO criteria. Most of the patients, included in the study, were males aged $61,7 \pm 10,1$ years with arterial hypertension and overweight. 26 patients $(2,5 \%)$ suffered from asthma. In order to compare groups of patients with and without asthma the Mann-Whitney test was used, the exact two-tailed $p$-value was computed.

Results. The rate of $\mathrm{Cl}-\mathrm{AKI}$ was $12,9 \%$ (132 patients). The rate of $\mathrm{Cl}$ AKI using the absolute creatinine rise definition was $1,8 \%$ cases (18 cases). Patients with asthma had almost twice lower rate of CA-AKI than patients without asthma (7,7\% versus $13 \%)$. The number of male patients was significantly lower in the asthma group (58\% vs. $73 \%$ ). Patients suffering from asthma were mostly obese (body mass index (BMI) $32,4 \pm 18,4 \mathrm{~kg} / \mathrm{m} 2$ ), unlike patients without asthma, who were overweight $(29,1 \pm 4,7 \mathrm{~kg} / \mathrm{m} 2)$. Although our results were not statistically significant $(p=0,56)$, the difference found might seem promising for further research, including the potential influence of the drugs used for the treatment of asthma and thus reducing the inflammation. The difference in gender and weight and $\mathrm{BMI}$, as well as the relatively low number of patients with asthma might have influenced the results of the analysis. The larger studies with more strict inclusion criteria should be conducted to better assess the importance of asthma as a risk factor of CA-AKI in patients with normal weight, overweight ones and with obesity.

Conclusion. The prevalence of contrast-associated acute kidney injury was lower in patients with asthma (7,7\% vs. $13 \%)$, but didn't meet statistical significance and needs further evaluation in larger studies, as well as a separate analysis in patients in different weight groups. The potential influence of the drugs used to reduce inflammation and treat asthma remains to be studied in patients with stable coronary artery disease undergoing percutaneous coronary interventions with contrast media administration.

000820

Urinary L-FABP variation using the semi-quantitative kit in ICU patients

G. Suzuki ${ }^{1}$; R. Ichibayashi ${ }^{1}$; M. Honda

${ }^{1}$ Critical care center, Toho University Medical Center Omori Hospital, Ota

City, Japan

Correspondence: G. Suzuki

Intensive Care Medicine Experimental 2020, 9(1): 000820

Introduction. Predicting the prognosis of intensive care unit (ICU) patients is crucial. This study aimed to investigate the use of the 
urinary liver-type fatty acid-binding protein (L-FABP) semi-quantitative kit in rapidly predicting the prognosis of patients admitted in the ICU.

Methods. We conducted a single-center, prospective, observational study wherein 100 consecutive patients admitted to the ICU with an indwelling bladder catheter were enrolled between April and October 2020. Urine specimens were collected at the time of admission (T1) and after $6 \mathrm{~h}(\mathrm{~T} 2)$, and urinary L-FABP levels were semi-quantitatively measured. Based on the L-FABP results, we divided patients into three groups (L-FABP decreased group/unchanged group/increased group), following which we compared their 14-day mortality.

Results. Finally, a total of 79 patients were included in the analysis. In multivariate analysis, urinary L-FABP variation (Odds ratio $(\mathrm{OR})=14.327,95 \%$ confidence interval $(\mathrm{Cl})=1.819-112.868, \mathrm{p}=0.01)$ and lactate $(\mathrm{OR}=1.234,95 \% \mathrm{Cl}=1.060-1.437, \mathrm{p}=0.01)$ were significantly associated with 14-day mortality.

Conclusion. Urinary L-FABP variation at $6 \mathrm{~h}$ after admission was significantly associated with 14-day mortality. Moreover, this study depicts how a semi-quantitative assay kit may help in easily and rapidly evaluating urinary L-FABP levels.

\section{Reference(s)}

1. McMahon BA, Galligan M, Redahan L, Martin T, Meaney E, Cotter EJ, et al. Biomarker Predictors of Adverse Acute Kidney Injury Outcomes in Critically III Patients: The Dublin Acute Biomarker Group Evaluation Study. Am J Nephrol. 2019;50:19-28.

2. Suzuki G, Ichibayashi R, Yamamoto S, Nakamichi Y, Watanabe M, Honda M. Clinical significance of urinary L-FABP in the emergency department. Int J Emerg Med. 2019;12:24

3. Xu Y, Xie Y, Shao X, Ni Z, Mou S. L-FABP: A novel biomarker of kidney disease. Clin Chim Acta. 2015;445:85-90.

\section{7}

Renal replacement therapy and outcomes in elderly COVID-19 patients

H. Flaatten ${ }^{1}$; C. Jung ${ }^{2}$; M. Beil ${ }^{3}$; J. Fjølner ${ }^{4}$; B. Guidet ${ }^{5}$; P. Vernon Van Heerden ${ }^{3}$; D. De Lange ${ }^{6}$; S. Leaver ${ }^{7}$;. Sigal ${ }^{8}$; W. Szczeklik' ; M. Joannidis ${ }^{10}$ ${ }^{1}$ Department of anaesthesia and intensive care, Haukeland University Hospital, Bergen, Norway; ${ }^{2}$ Dep. of cardiology, pulmonology and angiology, University Hospital of Düsseldorf, Düsseldorf, Germany; ${ }^{3}$ Medical intensive care unit, Hadassah Medical Center, Jerusalem, Israel; ${ }^{4}$ Department of intensive care, Aarhus University Hospital, Aarhus, Denmark ${ }^{5}$ Réanimation Médicale, Hôpital Saint-Antoine, Paris, France; ${ }^{6}$ Department of intensive care medicine, Utrecht University, Utrecht, Netherlands; ${ }^{7}$ Research lead critical care directorate, St George's Hospital, London, United Kingdom; ${ }^{8}$ Medical intensive care unit, Hadassah Medical Center, Jerusalem, Israel, Israel; ${ }^{9}$ Intensive care and perioperative medicine, Jagiellonian University Medical College, Kraków, Poland; ${ }^{10}$ Division of intensive care and emergency medicine, Medizinische Universität Innsbruck, Innsbruck, Austria

Correspondence: $\mathrm{H}$. Flaatten

Intensive Care Medicine Experimental 2020, 9(1): 000827

Introduction. The Covid-19 epidemic has provided many challenges for ICUs worldwide. One particular problem is related to acute kidney injury which effects many COVID-19 patients admitted to the ICU (1).

Objectives. This study is a part of the prospective COVIP study conducted by the VIP network within the HSRO section in ESICM, which focused on elderly ICU patients during the pandemic. Assessment and treatment of acute organ dysfunction were an important part of the study. Our aim is to describe the occurrence of renal replacement therapy (RRT) during ICU admission in elderly COVID-19 patients and the short-term outcomes.

Methods. The period studied was from mid-March 2020 until January 2021, using an online Case Record Form set up at the University of Aarhus as in previous VIP studies (2). Participating countries were responsible for obtaining National Ethical Clearance. At ICU admission all patients were scored using the Sequential Organ Dysfunction Assessment (SOFA) score (2), and common ICU procedures including RRT were recorded with time commenced and duration of treatment. Short term outcomes are described as LOS in the ICU and short-term survival in the RRT treated group.

Results. In total 3383 patients were admitted to 207 ICUs in 35 countries. Mean age was 76.9 years and $69.6 \%$ were male. 497 patients received RRT (14.7\% of all patients). Table 1 reveals further data regarding these patients. RRT was initiated at day 1 in 171 patients, day 2 in 92 and day $3+$ in 245 patients. The mean duration of RRT was 7.8 days, and RRT was commenced after initiation of MV in $84.5 \%$ of the patients, (mean start after 5.0 days).

\begin{tabular}{|c|c|c|c|c|c|c|c|}
\hline \multicolumn{8}{|c|}{ Comparison of RRT treated and not treated patients } \\
\hline & $n=$ & $\begin{array}{l}\text { Age } \\
\text { (mean } \\
\text { years) }\end{array}$ & $\begin{array}{l}\text { SOFA } \\
\text { renal }\end{array}$ & $\begin{array}{l}\text { SOFA } \\
\text { sum }\end{array}$ & MV & $\begin{array}{l}\text { ICU } \\
\text { LOS } \\
\text { days }\end{array}$ & $\begin{array}{l}\text { 30-day } \\
\text { survival }\end{array}$ \\
\hline RRT+ & $\begin{array}{l}497 \\
(14.7 \%)\end{array}$ & 75 & 1.6 & 7.7 & $\begin{array}{l}449 \\
(90.3 \%)\end{array}$ & 23.1 & $34.6 \%$ \\
\hline RRT - & 2802 & 76.1 & 0.5 & 4.7 & $\begin{array}{l}1831 \\
(65.3 \%)\end{array}$ & 13.9 & $50.4 \%$ \\
\hline
\end{tabular}

$\mathrm{MV}=$ mechanical ventilation.

Conclusion. $15 \%$ of elderly COVID-19 patients were treated with RRT during their ICU stay. These patients utilised more resources than nonRRT patients, requiring RRT on average for 8 days, and had an increase in ICU LOS of 10 days. RRT patients were treated more frequently with MV prior to initiation of RRT, and survival at 30 days was considerably lower. In a comparably sized, prospective study (3) in adult COVID-19 ICU patients the rate of AKI-RRT was found to be $20.6 \%$. $45 \%$ of these patients survived to 28 days. Their median age was considerably lower which may explain the differences in mortality compared with this study. Hospital survival of non-COVID patients $>73$ years old requiring RRT has been demonstrated to be less than $30 \%$ (4).

Our study demonstrates that development of AKI necessitating RRT in elderly COVID-19 patients is associated with an increased utilisation of resources and a lower survival rate.

\section{Reference(s)}

1. 4. Commereuc M, Guérot E, Charles-Nelson A, et al. ICU Patients Requiring Renal Replacement Therapy Initiation: Fewer Survivors and More Dialysis Dependents From 80 Years Old. Crit Care Med. 2017 Aug;45(8):e772-e781. https://doi.org/10.1097/CCM.0000000000002407

2. 3. Gupta S, Coca SG, Chan L, et al. AKI Treated with Renal Replacement Therapy in Critically III Patients with COVID-19. Journal of the American Society of Nephrology. 2021;32(1):161-176. 10.1681/ASN.2020060897.

3. 2. Flaatten $H$, de Lange DW, Morandi $A$, et al. The impact of frailty on ICU and 30-day mortality and the level of care in very elderly patients ( $\geq 80$ years). Intensive Care Med. 2017:43(12):1820-1828. 10.1007/ s00134-017-4940-8

4. Yang X, Tian S, Guo H. Acute kidney injury and renal replacement therapy in COVID-19 patients: A systematic review and meta-analysis. Int Immunopharmacol. 2021;90:107,159. 10.1016/j.intimp.2020.107159

5. The VIP study group and all its collaborators.

6. Open Access funding enabled and organized by Projekt DEAL. The support of the study in France by a grant from Fondation Assistance Publique-Hôpitaux de Paris pour la recherche is greatly appreciated. In Norway, the study was supported by a grant from the Health Region West. In addition, the study was funded by a grant from the European Open Science Cloud (EOSC) by the European Commission. EOSCsecretariat.eu has received funding from the European Union's Horizon Programme call H2020-INFRAEOSC-05-2018-2019, grant agreement number 831644. No further specific funding was received. 
000857

Effect of acute renal failure and renal replacement therapy of Procalcitonin and C-reactive Protein serum levels in COVID19 patients

JP. Cidade ${ }^{1}$; V. Costa, ; P. Fidalgo, ; R. Morais, ${ }^{1}$; B. Valerio, ${ }^{1}$; C. Paulino, ; S. Brito' ; A. Tralhão'; L. Morais, ; ;. Moniz, ; L. Coelho, ; P. Povoa'; D. Nora'; C. Tapadinhas,

${ }^{1}$ Polyvalent Intensive Care Unit, Hospital São Francisco De Xavier, Lisboa, Portugal

Correspondence: J.P. Cidade

Intensive Care Medicine Experimental 2020, 9(1): 000857

Introduction. Procalcitonin (PCT) is a surrogate biomarker of infection in critically ill patients namely with COVID19. COVID19 patients frequently develop acute renal failure, requiring ICU admission and continuous renal replacement therapy (CRRT). A decrease in glomerular filtration rate and CRRT could have a profound impact on PCT levels, considering its low molecular weight, limiting its use as biomarker of infection and its clinical application in this population.

Objectives. This study aimed to describe the kinetics of PCT and C-reactive protein (CRP) levels in ICU COVID19 patients and determine the impact of acute renal failure and CRRT on its levels.

Methods. We performed a single-center retrospective cohort study. COVID19 was diagnosed using clinical and radiologic criteria with a SARS-CoV-2 positive RT-PCR test. All adult patients admitted to ICU with COVID19 were eligible and were further divided in 2 groups according to the presence of acute renal failure needing of CRRT: "CRRT-group" and "control group". Patients' pair matching between the two groups was made, for statistical comparison of PCT and CRP levels at correspondent days, using two-step clustering, considering age, sex, SOFA at admission and ICU length of stay as pairing variables. Qui-square test was used for categorical variables and Kruskal-Wallis and Mann-Whitney-U were used on continuous variables for statistical assessment of outcomes between groups.

Results. 110 patients (mean age 63 years, $73.6 \%$ males) were included in the analysis ( $\mathrm{N}=29$ CRRT-group; $\mathrm{N}=81$ control group). SOFA score at ICU admission, ICU length of stay and mortality rate were different between groups $(p<0.001)$. Antibacterial therapy was not different between CRRT and control groups. Using paired patients between groups, no statistical difference was observed, in CRP levels, before, after and in its change with CRRT, in comparison the control group $(p=0.296, p=0.227$ and $p=0.681$, respectively). PCT levels were statistically different before and after CRRT, between groups ( $p=0.001$ and $p<0.001$, respectively), but the changes with CRRT were not statistically different $(p=0.497)$ (Fig. 1). The comparison of relative PCT changes with CRRT, with the relative changes of CRP, were also not statistically different $(p=0.522)$.

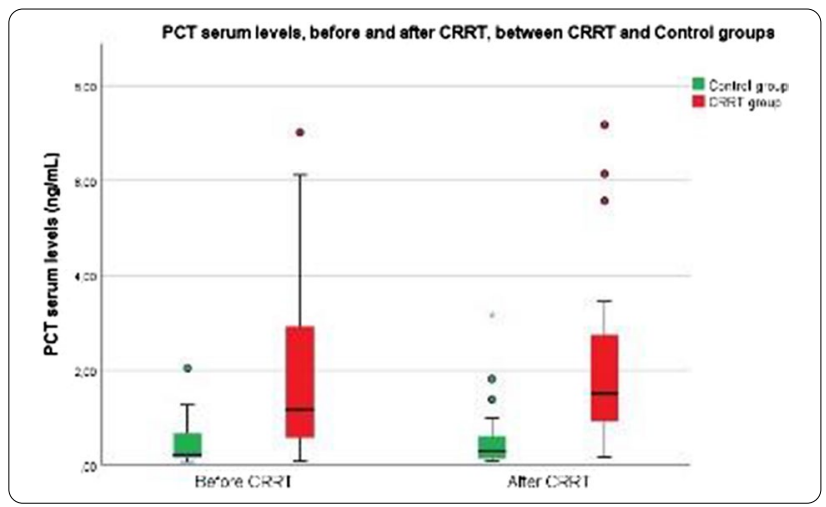

Conclusion.: In severe COVID19 patients the presence of acute renal failure had a marked impact on PCT levels but not on CRP, but CRRT had no effect on PCT and CRP levels. Consequently, the clinical use of PCT in COVID19 patients with acute renal failure is compromised.
Reference(s)

1. 4-Dahaba AA, Elawady GA, Rehak PH, List WF. Procalcitonin and proinflammatory cytokine clearance during continuous venovenous haemofiltration in septic patients. Anaesth Intensive Care. 2002 Jun;30(3):269-74. https://doi.org/10.1177/0310057X0203000302. PMID: 12075632.

2. 3 -Rombolà G, Brunini F. COVID-19 and dialysis: why we should be worried. J Nephrol. 2020 Jun;33(3):401-403. https://doi.org/10.1007/s40620020-00737-w. PMID: 32323202; PMCID: PMC7175820

3. 2-Heidari-Beni F, Vahedian-Azimi A, Shojaei S, Rahimi-Bashar F, Shahriary A, Johnston TP, Sahebkar A. The Level of Procalcitonin in Severe COVID-19 Patients: A Systematic Review and Meta-Analysis. Adv Exp Med Biol. 2021;1321:277-286. https://doi.org/10.1007/978-3-030-59261-5_25. PMID: 33656733.

4. 1-Zare ME, Wang Y, Nasir Kansestani A, Almasi A, Zhang J. Procalcitonin Has Good Accuracy for Prognosis of Critical Condition and Mortality in COVID-19: A Diagnostic Test Accuracy Systematic Review and Meta-analysis. Iran J Allergy Asthma Immunol. 2020 Dec 19;19(6):557-569. https:// doi.org/10.18502/ijaai.v19i6.4926. PMID: 33,463,126.

\section{8}

COVID-19: Impact on Circuit Lifetime and Performance during Continuous Renal Replacement Therapy

L. Whiting ${ }^{1}$; N. Bianchi ${ }^{1}$; S. Abed-Maillard ${ }^{1}$; A. Schneider ${ }^{1}$

${ }^{1}$ Service de médecine intensive adulte, Lausanne University Hospital, Lausanne, Switzerland

Correspondence: $L$. Whiting

Intensive Care Medicine Experimental 2020, 9(1): 001008

Introduction. SARS-CoV-2 infection is associated with a coagulopathy characterized by increased fibrinogen and D-dimers levels. The impact of this coagulopathy on continuous renal replacement therapy (CRRT) circuit lifespan and performance remains unknown.

Methods. In this prospective observational study, we enrolled all consecutive patients who received CRRT in the intensive care unit of a tertiary hospital between September and December 2020. All therapies were administered in continuous veno-venous hemodialysis mode with regional citrate anticoagulation. We collected patients' baseline characteristics, laboratory results, CRRT circuit lifespan as well as plasma and effluent samples at 12 (T1), 24 (T2), 48 (T3) and $72 \mathrm{~h}$ (T4) of CRRT circuit initiation. At each study time point, we computed urea, creatinine and $\beta 2$-microglobulin clearance. Results obtained in patients with COVID-19 (C19 group) were compared to those without COVID-19 (control group). Circuits' lifespan was assessed using Kaplan-Meier estimates and compared using log-rank test. Filter clearances at each study time point were compared using Student's T-test. Mixed models analyses were conducted to assess determinants of circuit lifetime and filter clearance.

Results. We included 35 patients, $26(74 \%)$ males with a median age of 68 [IQR 57- 71] years. Of those $16(45 \%)$ were COVID-19 positive. We analyzed 150 CRRT circuits: 77 (51.3\%) in the C19 group and 73 (48.7\%) in the control group. Compared to patients in the control group, those in the C19 group had a significantly shorter median circuit lifespan (57 [49.0-66.0] versus 68 [66-71] hours, $p=0.016$ ). They had a lower median urea (T1 27.5 vs $31.4 ;$ T2 26.8 vs $32.6 ;$ T3 27.3 vs 30.4 and T4 26.4 vs $30.7 \mathrm{ml} / \mathrm{kg} / \mathrm{h}$, all $\mathrm{p}<0.05)$ and creatinine (T1 22.7 vs $24.7 ; \mathrm{T} 2$ 23.0 vs $24.5 ;$ T3 20.4 vs 22.9 and T4 21.3 vs $22.8 \mathrm{ml} / \mathrm{kg} / \mathrm{h}$, respective $p$ values: $0.03,0.02,0.06$ and 0.08 ) clearance at all study time points. However, there was no difference in $\beta 2$-microglobulin's clearance between the two groups (respective $p$ values: $0.6,1.0,0.8$ and 0.7 ).

Conclusion. Patients with COVID-19 disease had a shorter CRRT circuit lifetime and a lower urea and creatinine clearance. The magnitude of this difference was, however, limited and further studies are required to explore clinical implications of such differences. 
001032

Elimination of 3 doses of gentamicin over 3 consecutive days using the same ST150 polyacrylonitrile-derived filter. An in vitro assessment

F. Baud ${ }^{1}$; L. Lamhaut ${ }^{1}$

${ }^{1}$ Département Anesthésie-Réanimation-SAMU de Paris, Assistance Publique-Hôpitaux de Paris hôpital Necker, Paris, France

Correspondence: F. Baud

Intensive Care Medicine Experimental 2020, 9(1): 001032

Introduction. Adsorption of gentamicin in polyacrylonitrile filters is well documented and in previous study we evidenced a mean adsorption rate of $67.7 \pm 2.1 \%$ in sessions lasting $6 \mathrm{~h}$ using the ST150, BaxterGambro filter (1).

Objectives. We extended the study over three consecutive days using the same filter to mimic adsorption of a daily dose of gentamicin over the 72-h life span of a filter.

Methods. Prismaflex ${ }^{\circledR}$ monitor and $\mathrm{ST} 150^{\circledR}$ filter were used in the continuous diafiltration (CDF) mode at a $2.5 \mathrm{~L} / \mathrm{h}$ flowrate (D/F ratio: $1.5 / 1 \mathrm{~L} / \mathrm{h}$ ). The daily session started with a $6-\mathrm{h}$ session of CDF. Thereafter, the 5 -Liter central compartment was changed using a bag free of gentamicin to assess gentamicin release over the following $18 \mathrm{~h}$. Experiments were repeated on Day 2 and stopped at the end of the 6-h session of CDF on Day 3. The experiment was performed in duplicate, one session used pre-dilution and the other post-dilution. Results were analyzed using the NeckEpur model $(1,2,3)$.

Results. The initial concentration of gentamicin in the CC was $40.0 \mathrm{mg} / \mathrm{L}$. Ninety-five percent of the initial dose was eliminated within the 6 -h session. At a $2.5 \mathrm{~L} / \mathrm{h}$ diafiltration flowrate, the mean daily clearances of gentamicin were $5.5,4.0$, and $3.3 \mathrm{~L} / \mathrm{h}$, respectively. The mean diafiltration and adsorption ratios in the daily elimination of gentamicin were $32 / 68,58 / 42$, and $88 / 12 \%$, respectively. During day 1 and 2 , the mean amount of gentamicin released from the ST150 ${ }^{\circledR}$ filter were 14 and $34 \mathrm{mg}$, respectively.

Conclusion. The pharmacokinetics of gentamicin over three days is strongly altered by adsorption in the same filter with a progressive decrease of elimination by adsorption, suggesting saturation of the filter. One limitation of our study results from the mode of administration using a bolus dose instead of an infusion over $30 \mathrm{~min}$. Adsorption adds a clearance to those of diafiltration. The time-dependency of gentamicin clearance precludes using a constant dosage regimen over the filter's life span.

\section{Reference(s)}

1. Baud F, Houzé P, Carli P, Lamhaut L: Elimination of fluconazole by polysulfone- and AN69-derived filters during continuous renal replacement therapy. An in vitro assessment. Internat J Artif Organ 2020. 10.1177/ 0391398820976144

2. Baud FJ, Houze P, Raphalen JH, Winchenne A, Philippe P, Carli P, Lamhaut $L$ : Diafiltration flowrate is a determinant of the extent of adsorption of amikacin in renal replacement therapy using the ST150((R))-AN69 filter: An in vitro study. Int J Artif Organs 2020, 43(12):758-766.

3. Baud FJ, Houze P, Carli P, Lamhaut L: Alteration of the pharmacokinetics of aminoglycosides by adsorption in a filter during continuous renal replacement therapy. An in vitro assessment. Therapie 2020. https://doi. org/10.1016/j.therap.2020.10.005
001034

Time spent at elevated intra-abdominal pressures within the first 24 postoperative hours after surgery is associated with the development of acute kidney injury in cardiac surgery patients

V. Moll'; AK. Khanna ${ }^{2}$; S. Minear ${ }^{3}$; K. Stanton ${ }^{4}$; A. Prabhakar' ; L. Essakalli; ; A. Kurz

${ }^{1}$ Department of anesthesiology, division of critical care medicine, Emory University School of Medicine, Atlanta, United States of America;

${ }^{2}$ Department of anesthesiology, section of critical care medicine, Wake Forest School of Medicine, Winston-Salem, United States of America; ${ }^{3}$ Department of anesthesiology, Cleveland Clinic Florida, Weston, United States of America; ${ }^{4}$ Analytics, Potrero Medical, Hayward, United States of America; ${ }^{5}$ Departments of general anesthesiology and outcomes research, anesthesiology institute, Cleveland Clinic, Cleveland, United States of America

Correspondence: $\vee$. Moll

Intensive Care Medicine Experimental 2020, 9(1): 001034

Introduction. Increased intra-abdominal pressure (IAP) frequently occurs in critically ill patients and is independently associated with acute kidney injury (AKI) and mortality $(1,2)$. Up to a third of all postoperative cardiac surgery have acute kidney injury and intra-abdominal hypertension (IAP $\geq 12 \mathrm{mmHg}$ ) (IAH) occurs in approximately 33-46\% $(3,4)$. Most IAP is measured intermittently and only when clinical suspicion dictates it. The relationship of high fidelity IAP measurements with postoperative $\mathrm{AKI}$ in this cohort is largely unknown.

Objectives. We hypothesize that time spent with IAH above certain thresholds is associated with increased incidence of acute kidney injury in cardiac surgery patients.

Methods. 40 cardiac surgery patients from a single academic center part of an ongoing, large multicenter observational registry study evaluating the associations between IAP and kidney injury in cardiac surgery patients were studied. Patients with a history of chronic kidney disease (ICD-10 codes), or with a foley dwell time of fewer than $24 \mathrm{~h}$ were excluded (22 patients analyzed). AKI was assessed per KDIGO criteria. All patients received the Accuryn monitoring system (Potrero Medical, Hayward, CA) which automatically tracks urine output (UO), IAP, and core temperature. The system samples UO approximately $10 \mathrm{~s}$ after each active drain line clearance event to ensure that all urine is accounted for the rate is then linearly interpolated between clearance events. IAP is recorded at $100 \mathrm{~Hz}$ and downsampled to $1 \mathrm{~Hz}$. A $30 \mathrm{~s}$ running minimum was applied to calculated end-expiratory pressure. To do this the $1 \mathrm{~Hz}$ IAP signal was grouped into $30 \mathrm{~s}$ windows with an overlap of $1 \mathrm{~s}$. The minimum value in this $30 \mathrm{~s}$ window is then called the $30 \mathrm{~s}$ running minimum. This minimum value corresponds to end-expiratory phase. Sources of noise such as patient coughing and position changes creating outliers were eliminated applying a $10 \mathrm{~min}$ running median. Data were then resampled every 15 min. Summary statistics are reported using python with the pandas and numpy packages. Fisher exact test was used for descriptive analytics.

Results. 14/22 patients developed AKI stage 2 post-cardiac surgery. Demographics are shown in Table 1. Sustained time spent in intraabdominal hypertension at various grades of IAH is associated with the development of $\mathrm{AKI}$ stage 2 (Table 2). Figure 1 shows the percentage of patients versus time spent at various IAP thresholds in the first $24 \mathrm{~h}$ post-cardiac surgery stratified by AKI grade 2 . 


\begin{tabular}{|c|c|c|c|c|}
\hline$(\mathrm{N}=22)$ & Value & $\begin{array}{l}\text { IAP>20 muHg } \\
\text { for at least } \\
\text { hours } 6\end{array}$ & $\begin{array}{l}\text { IAP NOT >20 } \\
\text { mingl for at } \\
\text { least } 6 \text { hours }\end{array}$ & p-value \\
\hline \multicolumn{5}{|l|}{ Age } \\
\hline & $(30,40]$ & 0 & 1 & 0.875 \\
\hline & $(50,60]$ & 2 & 1 & 0.875 \\
\hline & $(60,70]$ & 5 & 5 & 0.875 \\
\hline & $(70,80]$ & 2 & 4 & 0.875 \\
\hline вMI & & & & \\
\hline & (20,30] & 6 & 8 & 0.814 \\
\hline & $\begin{array}{ll}(30,40] \\
\mathrm{NA}\end{array}$ & $3_{0}^{4}$ & $\begin{array}{l}3 \\
1\end{array}$ & $\begin{array}{l}0.814 \\
0.814\end{array}$ \\
\hline \multicolumn{5}{|l|}{ Race } \\
\hline & Black & 3 & 4 & 0.900 \\
\hline & Asian & 1 & 0 & 0.900 \\
\hline & Caucasian or White & 5 & 7 & 0.900 \\
\hline & Unknown & 1 & 1 & 0.900 \\
\hline \multicolumn{5}{|l|}{ Gender } \\
\hline & Female & 5 & 4 & 0.666 \\
\hline & Male & 5 & 8 & 0.666 \\
\hline AKI grade 2 status & No & 3 & 5 & 0.675 \\
\hline & Yes & 7 & 7 & 0.675 \\
\hline \multicolumn{5}{|c|}{ Hospital length of stay (days) } \\
\hline & $(0,10]$ & 8 & 7 & 0.646 \\
\hline & $(10,20]$ & 2 & 4 & 0.646 \\
\hline & $\mathrm{NA}$ & 0 & 1 & 0.646 \\
\hline \multicolumn{5}{|c|}{ Surgery duration (hours) } \\
\hline & $\begin{array}{l}\left(\begin{array}{l}(0.2,2.3] \\
(2.55,50]\end{array}\right. \\
-\end{array}$ & $5_{5}^{2}$ & $\frac{1}{5}$ & 0.782 \\
\hline & $(5.0,7.5]$ & 3 & 5 & 0.782 \\
\hline & $\mathrm{NA}$ & 0 & 1 & 0.782 \\
\hline \multicolumn{5}{|c|}{ Bypass duration (hours) } \\
\hline & $(0,1]$ & 1 & 2 & 0.813 \\
\hline & $(1,2]$ & 2 & 4 & 0.813 \\
\hline & $(2,3]$ & 5 & 5 & 0.813 \\
\hline & $\mathrm{NA}$ & 2 & 1 & 0.813 \\
\hline \multicolumn{5}{|l|}{ Surgery } \\
\hline & $\begin{array}{l}\text { CABG and alve } \\
\text { CABG only }\end{array}$ & $\begin{array}{l}0 \\
3\end{array}$ & $\begin{array}{l}3 \\
5\end{array}$ & 0.323 \\
\hline & No CABG no Valve & 2 & 1 & 0.323 \\
\hline & Valve only & 5 & 3 & 0.323 \\
\hline
\end{tabular}

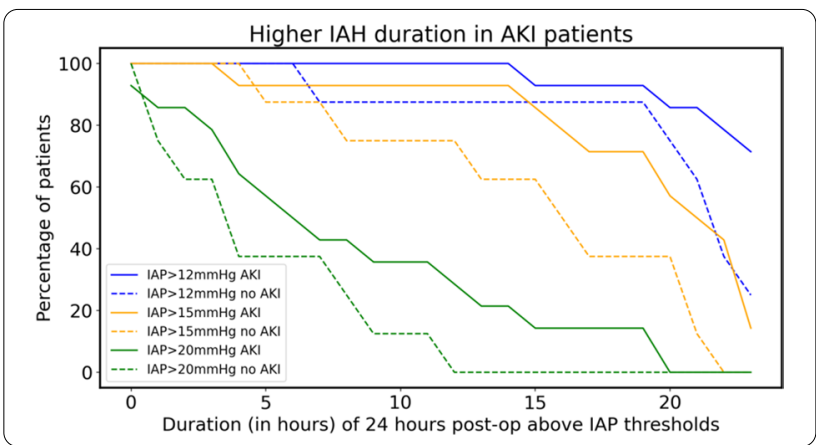

Table 2: AKI stage 2 vs Hours IAP above 20 controlling for confounders

Table 2: AKI stage 2 vs Hours IAP above 20 controlling for confounders
\begin{tabular}{|l|l|l|}
\hline & Adjusted odds ratio $(95 \% \mathrm{CI})$ & $\mathrm{p}$-value \\
\hline Hours spend in IAP>20 mmHg & $1.31(0.97,1.77)$ & 0.078 \\
\hline Age & $0.9(0.78,1.04)$ & 0.152 \\
\hline BMI & $1.09(0.88,1.34)$ & 0.425 \\
\hline Race* & $0.88(0.18,4.33)$ & 0.880 \\
\hline Gender & $0.26(0.02,3.04)$ & 0.282 \\
\hline Hospital length of stay & $1.39(0.9,2.13)$ & 0.138 \\
\hline Surgery type* & $0.77(0.14,4.32)$ & 0.769 \\
\hline Surgery duration & $1.42(0.34,5.97)$ & 0.634 \\
\hline Cardio-pulmonary bypass duration & $1.41(0.1,20.6)$ & 0.803 \\
\hline
\end{tabular}

* converted to 1-hot format and compressed to a vector using PCA
Conclusion. In critically ill post-cardiac surgery patients the time spent at higher IAP levels is associated with the development of AKI. Interventions directed towards decreasing sustained IAP or improving abdominal perfusion pressure (mean arterial pressure-intra-abdominal pressure) in these patients might thus reduce the risk of AKI and subsequently improve outcomes using a novel therapeutic target.

\section{Reference(s)}

1. Iyer, D et al. Crit. Care Resusc. 2014

2. Dalfino, L. et al. Interact. Cardiovasc. Thorac. Surg. 2013

3. Malbrain, M. L. et al. Minerva Anestesiol. 2014

4. Malbrain, M. et al.. Critical Care Med 2005

5. This study is supported by Potrero Medical.

\section{0}

The incidence and management of acute kidney injury in COVID-19 during the unexpected first wave: Italian SIN-SIAARTI Survey and Recommendations for Practice

S. De Rosa'; M. Marengo, ${ }^{2}$ S. Romagnoli ${ }^{3} ;$ M. Fiorentino ${ }^{4}$;V. Fanelli ${ }^{5}$;

E. Fiaccadori ${ }^{6}$; N. Brienza ${ }^{7}$;. Morabito ${ }^{8}$;V. Pota ${ }^{9}$; F. Valente ${ }^{10}$; G. Grasselli ${ }^{11}$; V. Cantaluppi ${ }^{12}$

${ }^{1}$ Department of anesthesia and intensive care unit, St. Bortolo Hospital, Vicenza, Italy: ${ }^{2}$ Nephrology and dialysis unit, ASL CN1, Cuneo, Italy; ${ }^{3}$ Health sciences department, section of anesthesiology, intensive care and pain medicine, Careggi, Florence, Italy; ${ }^{4}$ Department of emergency and organ transplantation, nephrology dialysis and transplantation unit, "Aldo Moro" University, Bari, Italy; ${ }^{5}$ Surgical sciences department, Città della Salute e della Scienza, University of Torino, Turin, Italy; ${ }^{6}$ Dipartimento di medicina e chirurgia, Azienda Ospedaliera-Universitaria di Parma, Parma, Italy; ${ }^{7}$ Unit of anesthesia and resuscitation, department of emergencies and organ transplantations, Aldo Moro University, Bari, Italy; ${ }^{8}$ Department of nephrology and urology, hemodialysis unit, Umberto I, Policlinico di Roma, "Sapienza" University, Roma, Italy; ${ }^{9}$ Department of woman, child and general and specialized surgery, University of Campania Luigi Vanvitelli, Napoli, Italy; ${ }^{10}$ Usc nefrologia e dialisi, Ospedale S. Chiara di Trento, Trento, Italy; ${ }^{11}$ Intensive care unit, Policlinico of Milan, Milano, Italy; ${ }^{12}$ Nephrology and kidney transplantation unit, department of translational medicine, University of Piemonte Orientale, "Maggiore della Carità"University Hospital, Novara, Italy

Correspondence: S. De Rosa

Intensive Care Medicine Experimental 2020, 9(1): 001140

Introduction. The pandemic of Novel Coronavirus Disease 2019 (COVID-19) is challenging, given the large number of hospitalized patients. Cardiovascular co-morbidities are linked to a higher mortality risk. Thus, patients with acute kidney injury might represent a high-risk population. Hence, we conducted a nationwide survey to assess incidence and management of AKI in patients by COVID19 and invited all Italian Society of Nephrology (SIN) and (SIAARTI) members undertaking survey to complete over three weeks commencing 8th April 2020.

Objectives. We conducted a nationwide survey to assess the incidence and management of AKI in COVID-19 patients and invited all members of the Italian Society of Nephrology (SIN) and (SIAARTI) to complete within one week starting April 8, 2020.

Methods. This is a multi-centre, observational, nationwide online survey, involving Italian Nephrology and Critical care centres in Italy, developed in partnership between SIAARTI- SIN Society. Invitations to participate distributed through emails, social Network (a link for email registration was disseminated via social media (i.e. twitter facebook and Linkedin) Data Collected April 8, 2020, through April 15,2020 . The survey was divided into 3 main parts: A) Acute Kidney Injury (AKI); B) Renal Replacement Therapies (RRT); C) extracorporeal methods for the removal of inflammatory mediators (EBPT).

Results. A total of 141 responses were collected in the SIN-SIAARTI survey: 77 (54.6\%) intensivists, 63(44.6\%) nephrologists and only one $(0.8 \%)$ from another specialist. About 19,000 cases of COVID19 infection have been recorded in hospitalized patients; among these, 1393 AKI cases were found, of which 1146 were observed in intensive care units. For the determination of AKI, only $43 \%$ of clinicians 
routinely used the international criteria (KDIGO; AKIN, RIFLE) based on serum creatinine and urinary output, with a slight prevalence among nephrologists. Both groups agree on the underestimation of AKI ( $82.7 \%$ among nephrologists and $67 \%$ among intensivists). About $50 \%$ of recognized AKI was not directly related to COVID19 interstitial pneumonia. Overall, $72.4 \%$ of clinicians used urine tests with sediment, a disproportionate figure among nephrologists $(76.7 \%)$ and intensivists $(47.3 \%)$. On the other hand, only 2 nephrological centers used biomarkers (reported use of cystatin-C) compared to 37 of the intensivists (reported the use of cystatin-C, NGAL and Nephrocheck). The renal replacement treatment was started in 628 patients with a prevalence of continuous techniques in both groups. Continuous techniques was the preferred choice in both groups and in most cases RRT was started because of oliguria $(74.05 \%)$ or the classic indications of urgency $(67.94 \%)$ or serum creatinine/urea $(60.31 \%)$ or fluid overload $(52.67 \%)$. Early initiation was preferred and RRT was excluded in case of therapeutic withdrawal, severe comorbidities, severe hemodynamic instability. Regional anticoagulation with citrate was the most common choice among both resuscitators and nephrologists. The $41.04 \%$ of the physician interviewed never used EBPT. A percentage of 4.33 used these techniques only in AKI settings while $24.63 \%$ resorted to these techniques even in the absence of AKI. Nephrologists have made greater use of EBPT, especially in the presence of AKI. High-volume haemofiltration was never used in by $58.54 \%$ of respondents; $\mathrm{HCO}$ membranes (35.14\%) and adsorbents (21.14\%) were used in more than $50 \%$ of cases.

Conclusion. This survey shows that during the first wave there was an underestimation of AKI in Italy based on based on the non-use of the diagnostic criteria of aki, especially by intensivists. Likewise, the management of blood purification therapies is very heterogeneous. Future national clinical collaborative studies are advocated in order to investigate the role of a collaborative approach between nephrologists and intensivists on the patient outcome and other controversial topics about aki and RRT in COVID19 patients.

\section{Reference(s)}

1. Hirsch JS, Ng JH, Ross DW, Sharma P, Shah HH, Barnett RL, et al. Acute kidney injury in patients hospitalized with COVID-19. Kidney Int. 2020;98(1): 209-18.

2. Batlle D, Soler MJ, Sparks MA, Hiremath S, South AM, Welling PA, et al. Acute kidney injury in COVID-19: emerging evidence of a distinct pathophysiology. J Am Soc Nephrol. 2020;31(7):1380-3.

3. This study was supported in part by the Italian Society of Nephrology and the Italian Society of Anaesthesia Analgesia Resuscitation and Intensive care

\section{7}

\section{Thromboprophylaxis and acute kidney injury in patients} with ARDS due to severe COVID 19 during the first pandemic surge

VD. Gumucio Sanguino'; A. Betbesé-Roig, 2, J. Puentes Yáñez; ;

MA. Gordillo-Benitez ' F. Di-Paolo, '; SM. Pons'; AL. Corral' ${ }^{1}$; K. Maisterra Santos ${ }^{1}$; J. Sabater Riera ${ }^{1}$; JL. Pérez Fernández

${ }^{1}$ Intensive care medicine, Bellvitge Hospital, Hospitalet de Llobregat, Spain; ${ }^{2}$ Intensive care, Hospital de la Santa Creu i Sant Pau, Barcelona, Spain

Correspondence: V.D. Gumucio Sanguino

Intensive Care Medicine Experimental 2020, 9(1): 001267

Introduction. Severe forms of coronavirus disease-2019 (Covid-19) are associated with high D-dimer values and high incidence of deep vein thrombosis (DVT) or pulmonary embolism (PE), Despite reports, the incidence of Acute Kidney Injury (AKI) is high and clearly related with mortality although pathophysiology pathways remain unclear. Thromboprophylaxis protocols were increased during the Covid-19 strain, and clinical trials are providing evidence that anticoagulation regimens are beneficial for survival.

Objectives. To assess the effect of different thromboprophylaxis regimens in the incidence of AKI (stage 1 or more of KDIGO classification) in patients with Covid-19 presenting ARDS and treated with invasive MV.

Methods. We performed a retrospective observational study in $2 \mathrm{Uni}-$ versity hospitals of Barcelona metropolitan area (Spain) from March 01 to March 31,2020, including adults with ARDS receiving invasive MV within the first 3 days from ICU admission and positive SARS-Cov-2 testing by RT-PCR in respiratory fluids. Patients with end-stage renal disease (ESRD) were not included. We collected all clinical data during the first 7 days from ICU admission including the maximum thromboprophylaxis regimen received. Renal replacement therapy (RRT) requirement and the survival outcome after 28 days from ICU admission were also registered. AKI was defined within the first 7 days from ICU admission as the new appearance of AKI or the progression of AKI (at least one KDIGO stage) after ICU admission. Standard thromboprophylaxis regimen (STR) was subcutaneous enoxaparin $40 \mathrm{mg} /$ day. Intermediate thromboprophylaxis regimen (ITR) was prescribed in those patients with $D$-dimer $\geq 1000 \mathrm{ng} / \mathrm{mL}$ using subcutaneous enoxaparin $0.5 \mathrm{mg} / \mathrm{kg} / 12 \mathrm{~h}$. Systemic anticoagulation was prescribed when DVT or PE were present.

Results. A total of 269 patients with SARS-Cov2 infection were admitted at ICU during the study period. Ninety did not receive invasive MV and 5 already had ESRD before infection. Among the remaining 174 patients, 36 patients (21\%) already presented AKI at ICU admission. New AKI or progression of AKI stage within the first week appeared in 78 patients (45\%). High D-Dimer values over $1000 \mathrm{ng} / \mathrm{mL}$.

were present at ICU admission in 93 (55\%) patients and in 122 (77\%) patients during the first week from ICU admission. Maximum thromboprophylaxis during the first week was STR in $53(31 \%)$ patients, ITR in $76(45 \%)$ patients and systemic anticoagulation in 41 (24\%). Mortality at 28 days after ICU admission was $56 \%$ and $39 \%$ in those patients with or without AKI respectively. Multivariable analysis showed that the use of an ITR compared to a STR was associated with a reduc tion of AKI within the first week from ICU admission (OR $0.37,95 \% \mathrm{Cl}$ $0.16-0.83$ )

Conclusion. This bi-center study suggests that an increased regimen of thromboprophylaxis in Covid19 critically ill patients may decrease the incidence of AKI within the first week from ICU admission, and subsequently could influence mortality.

\section{6}

Quality metrics and outcome of slow low efficiency dialysis(SLED) in critically ill patients with acute kidney injury(AKI)

L. o'connor'; C. Mitchell, ; H. Blackman,

${ }^{1}$ Intensive Care, South Tyneside and Sunderland NHS Foundation trust, Sunderland, United Kingdo

Correspondence: $L$. o'connor

Intensive Care Medicine Experimental 2020, 9(1): 001286

Introduction. SLED has been the primary renal replacement therapy(RRT) on ICU at SRH since 2014. Since introducing SLED, a number of quality metrics were used to appraise the service. Markers of the efficiency of continuous RRT in ICU have been extensively studied, however there is comparatively scant literature about quality metrics for intermittent RRT in ICU. Those such as Kt/V are extrapolated from the chronic RRT programme \& rely on estimating the volume of distribution of urea, which is variable \& difficult to estimate in the ICU population. Urea reduction ratio(URR) is a simpler method of measuring adequacy of intermittent haemodialysis(IHD) in patients with AKI, but it also has limitations failing to take into account ultrafiltration. A URR of 0.67 correlated with a Kt/V of $>1.2$ and $\mathrm{KIDIGO}$ recommend a weekly $\mathrm{Kt} / \mathrm{V}$ of 3.9. [i] [ii] A retrospective observational study found improved survival in those with an intermediate illness severity score when a URR $>0.58$ /treatment was achieved.[iii].

Objectives. Annual appraisal of the SLED sessions delivered in 2019 using the following; target URR $>0.58$ per session, $100 \%$ achieved prescribed SLED targets, mortality \& need for RRT at 3 months post admission.

Methods. 485 treatment records for 115 patients receiving SLED from Jan-Dec 2019 were reviewed. 79\% of sessions were the standard 
prescription; dialysate flow $300 \mathrm{ml} / \mathrm{min}$, blood flow $150 \mathrm{ml} / \mathrm{min}$ for $6 \mathrm{~h}$ using Citrusate or unfractionated heparin anticoagulation.

Results. Complete data was available for $78.5 \%$ of sessions.

- Median duration of delivered RRT $=6 \mathrm{~h}$ (Range $0.3-24 \mathrm{~h}$ )

- Median URR of all sessions=0.49 (Range 0.1-0.85) irrespective of intentional or accidental premature termination of sessios.

- Median URR of uninterrupted sessions $=0.62=\mathrm{Kt} / \mathrm{V} 1.12$

- $28 \%$ failed to meet RRT prescribed targets, $72 \%$ met targets.

- $50 \%$ mortality \& $5 \%$ Dialysis dependant at 3 months
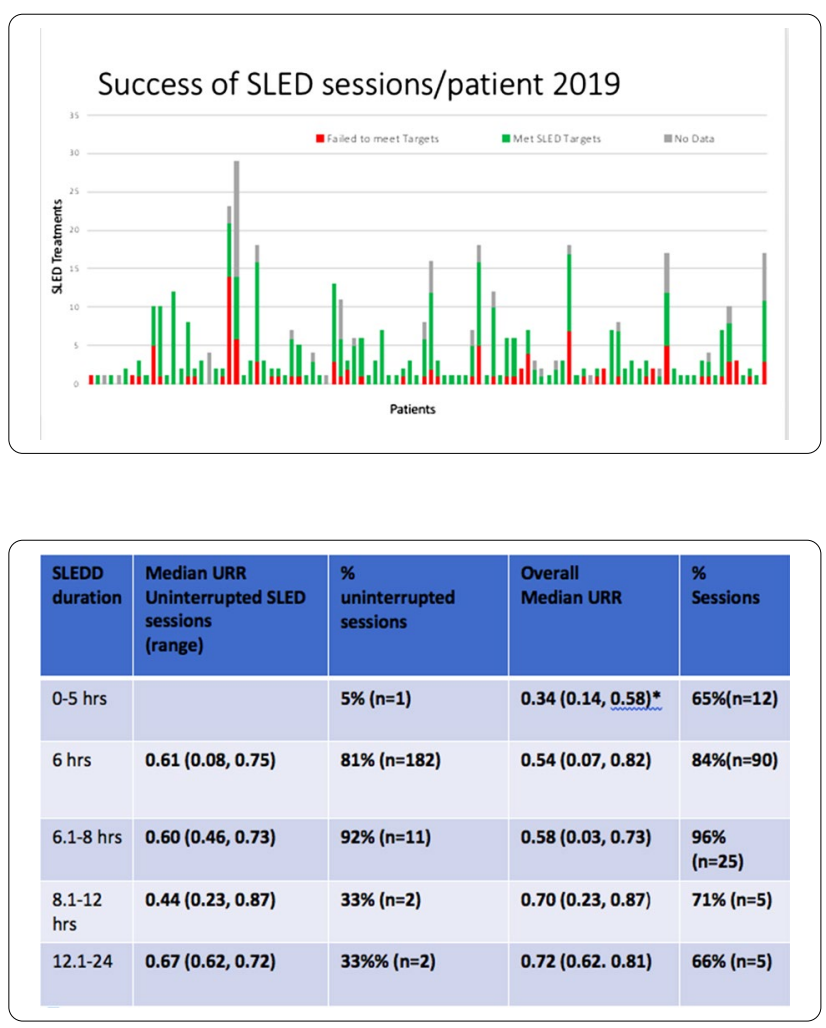

Conclusion. The overall median URR falls below the recommended 0.67 \& only $72 \%$ of sessions met the prescribed SLED targets. However in view of the adequacy of URR in uninterrupted sessions, underestimation due to ultrafiltration \& delivery of daily sessions this data suggests our unit meets the recommended weekly dialysis dose in those needing multiple sessions.

\section{Reference(s)}

1. [i] Liang, K.V., Zhang, J.H. \& Palevsky, P.M. Urea reduction ratio may be a simpler approach for measurement of adequacy of intermittent hemodialysis in acute kidney injury. BMC Nephrol. 2019; (20) 82:1272-1277 [ii] Kidney Disease. Improving global outcomes (KDIGO) Acute kidney injury work group. KDIGO clinical practice guideline for Acute kidney injury. Kidney Int Suppl. 2012;2:1-138 [iii] Paganini E, Tapolyai M, Goormastic M, Halstenberg W, Kozlowski L, Leblanc M, Lee JC, Moreno L, Sakai K. Establishing a dialysis therapy/patient outcome link in intensive care unit acute dialysis for patients with acute renal failure. Am J Kidney Dis. 1996; 28:S81-S89
001381

Descriptive Study of Hemoperfusion in patients with severe COVID 19

S. Cruz Montesinos ${ }^{1}$; G. Del Pozo²; M. Llano ${ }^{1}$; W. Montaluisa';

D. Velastegui ${ }^{1}$; M. Lascano ${ }^{1}$; A. Paez ${ }^{1}$; W. Osorio ${ }^{3}$; B. Villamagua ${ }^{1}$; P. Freire ${ }^{1}$; M. Argotti' ; M. Araujo ${ }^{3}$; V. Narvaez'; P. Romero'; J. Giron' ${ }^{1}$; A. Gross ${ }^{1}$ ${ }^{1}$ Intensive Care, Hospital de Especialidades de la FF.AA N1., Quito, Ecuador; ${ }^{2}$ Intensive care, Hospital Militar de Quito, Quito, Ecuador; ${ }^{3} \mathrm{Neph}-$ rology department, Hospital de Especialidades de la FF.AA N1., Quito,

Ecuador

Correspondence: S. Cruz Montesinos

Intensive Care Medicine Experimental 2020, 9(1): 001381

Introduction. There are many therapeutic interventions that have been proposed as part of the treatment of Covid 19, many aimed at stopping the development of the cytokine storm and thus reducing mortality, for which we have decided to evaluate the impact of hemoperfusion on the mortality of patients with severe covid 19.

Objectives. Certain studies have suggested that renal replacement therapy may have beneficial effects on pathological processes such as sepsis, acute respiratory disease and acute respiratory distress syndrome (ARDS) (1,2), whereas other studies have questioned (3) arguing that continuous renal replacement therapy (CRRT) was correlated with higher mortality during the Middle East respiratory coronavirus (MERS-CoV) outbreak. In this context, we have set out to describe our experience with early intervention with hemoperfusion therapy in patients with severe covid-19 and high levels of interleukin- 6 in a tertiary care center in Ecuador, in particular the association of haemoperfusion with mortality.

Methods. It is a study with a Descriptive Longitudinal design, which is carried out in patients with severe Covid 19 who underwent at least one hemoperfusion session in a tertiary hospital in the city of Quito in the intensive care area. All patients included had a positive RT PCR for SARS COV 2, between the months of June 2020 to February 2021.

Results. Data was collected from 11 patients undergoing the hemoperfusion procedure, in the context of treatment for uncontrolled immune response and IL- 6 clearance. Three patients were excluded due to incomplete data, leaving a total sample of eight patients. The average age was 54 years ( \pm 4 years). No patient had renal, pulmonary (COPD) or hypertension as comorbidities; three patients presented other types of comorbidities (37.5\%).

The severity markers at admission showed APACHE-II values of 6.0 (IQR: 5.0, 14.0 points), admission SOFA 7.0 (IQR: 6.0, 8.0 points). Average Neutrophil-Lymphocyte Index (INL) 13.6 (IQR: 4.8, 15.4). LDH (prior) median 462.0 (IQR: 311.0, 509.5 U / L) Ferritin (prior) 1929.0 (IQR: 1719.8, 2000.0 ug / L) Procalcitonin (prior) 0.33 (IQR: 0.19, $0.75 \mathrm{ng} / \mathrm{ml}$ ) D-Dimer (prior) 2415.5 (IQR: 697.5,4668.0 ug / L) IL-6 (prior) 134.5 (IQR: $114.3,399.7 \mathrm{pg} / \mathrm{ml}$ ). The mortality rate in the present series was $37.5 \%$ $(\mathrm{n}=3)$.

Conclusion. In our series of cases, it is observed that after hemoperfusion, there is no significant decrease in mortality and rather it is similar to that reported worldwide.

\section{Reference(s)}

1. Schefold JC, von Haehling S, Pschowksi R, Bender TO, Berkmann C, Briegel $S$, Hasper D, Jorres A. The effect of continuous versus intermittent renal replacement therapy on the outcome of critically ill aptients with acute renal failure (CONVINT): a prospective randomized controlled trial. Crit Care. 2014;18(1):R11.52.Huang C, Wang Y, Li X, et al. Clinical features of patients infected with 2019 novel coronavirus in Wuhan China. Lancet. 2020;395:497-506.

2. Miao H, Shi J, Wang C, Lu G, Zhu X, Wang Y, Cui Y, Zhang Y. Continuous Renal Replacement Therapy in Pediatric Severe Sepsis: A Propensity Score-Matched Prospective Multicenter Cohort Study in the PICU. Crit Care Med 2019; 47(10):e806-e13.

3. Han F, Sun R, Ni Y, Hu X, Chen X, Jiang L, Wu A, Ma L, Chen M, Xv Y, Tu Y. Early initiation of continuous renal replacement therapy improves clinical outcomes in patients with acute respiratory distress syndrome. Am J Med Sci 2015; 349(3): 199-205

4. Dra. Mayra Gutierrez 
5. Dra. Villaba Cinthia

6. Dr. Alexis Paredes

7. Dra. Maria Jose Auz

8. Dr. Daniel Dominguez

9. Dra. Wachi Adriana

\section{3}

Clinical features and coagulation profile of COVID 19 patients with Acute Kidney Injury

M. Sileli'; E. Siomos '; I. Karageorgiou'; A. Kosmas'; D. Kampranis';

E. Kerezidou'; N. Kapravelos'; C. lasonidou'

'B icu, GENERAL HOSPITAL "GEORGE PAPANIKOLAOU", Thessaloniki,

Greece, Greece

Correspondence: M. Sileli

Intensive Care Medicine Experimental 2020, 9(1): 001433

Introduction. Acute Kidney Injury (AKI) is a common complication in COVID-19 patients. Existing literature suggests various possible causative factors, including hypercoagulability, as a possible culprit.

Objectives. To explore for factors involved in new-onset AKI in COVID19 patients and secondarily to investigate whether the presence of hypercoagulability upon admission to the ICU, as determined by ROTEM, is implicated.

Methods. A single-center observational cohort study on COVID-19 patients under mechanical ventilation (December 2020-May 2021). Patients on the medical history of hypercoagulable state, chronic anticoagulant treatment, chronic dialysis, thromboembolic events and exposure to nephrotoxic contrast media or nephrotoxic drugs before or during ICU stay were excluded. All patients received a local protocol of thromboprophylaxis (LMWH) at the ward. ROTEM assays were performed in each patient upon ICU admission. We recorded for demographics, BMI, comorbid conditions quantified by Charlson's comorbidity index (CCI), SOFA score, APACHE II and serum creatinine. The eGFR was also calculated just after admission to the ICU.

Moreover, we recorded the following ROTEM parameters: CFT EXTEM, MCF EXTEM and MCF FIBTEM. Patients were allocated into two groups (AKI /non-AKI). AKI was defined according to KDIGO criteria. Chisquare, Fisher's exact, Student's t, and Mann-Witney $U$ test were used as appropriate. The $p$-value was set at 0.05 . ROC curves were constructed and the best cut-off points were determined.

Results. Fifty-two patients were included with a median age of 62.5 (IQR: 56 to 69.25$)$. The majority were males $(61,5 \%)$. The median BMI was 31 (IQR: 28 to 37.25) and the median CCI 2 (IQR:1 to 3). All EXTEM CFT values were within normal limits. As reflected by supranormal EXTEM MCF and FIBTEM MCF values in most patients $157.7 \%$ and $84.6 \%$, respectively), a hypercoagulable state was noticed. A total of $29(55,8 \%)$ patients developed AKI in our cohort. $6(20,7 \%)$ of them were categorized as AKI stage I, 10 (34,5\%) as stage II and $13(44,8 \%)$ as stage III. Among them, $11(37,9 \%)$ required renal replacement therapy (RRT). Age, BMI and APACHE II were similar among groups $(p>0.05)$. Patients who developed AKI were in a higher percentage of women (60 vs 53.1), had more comorbidities as quantified by $\mathrm{CCl}$ ( 3 vs 1 ), higher SOFA ( 7 vs 6 ) and lower eGFR ( 81 vs 105) $p<0.05$. The best cut-off points were for $\mathrm{CCl}>1$, for SOFA $>7$ and eGFR $\leq 90 \mathrm{~mL} /$ $\mathrm{min} / 1.73 \mathrm{~m}^{2}$. Interestingly enough, there was no evidence of an association between AKI and the tested ROTEM parameters in our cohort $(p>0.05)$. The same results were shown when the ROTEM parameters were tested in patients who underwent RRT.

Conclusion. Of the factors studied, female gender, eGFR $\leq 90$ upon ICU admission, SOFA score $>7$ and $\mathrm{CCl}>1$ related to AKI. The present study does not support hypercoagulability as a culprit. Our findings extend the current observations. A further universal assessment of risk factors involved in AKI after SARS-CoV-2 infection is mandatory.

\section{Reference(s)}

1. R Lowe, M Ferrari, M Nasim-Mohi, A Jackson, R Beecham, K Veighey, R Cusack, D Richardson, Mpw Grocott, Dzh Levett. A Dushianthan. Clinical characteristics and outcome of critically ill COVID-19 patients with acute kidney injury: a single center cohort study. BMC Nephrol. 2021;22(1):92.
2. Luca Spiezia, Annalisa Boscolo, Francesco Poletto, Lorenzo Cerruti, Ivo Tiberio, Elena Campello, Paolo Navalesi, and Paolo Simioni. COVID19-Related Severe Hypercoagulability in Patients Admitted to Intensive Care Unit for Acute Respiratory Failure. Thromb Haemost. 2020; 120(6): 998-1000.

3. None

001455

Evaluation of urinary creatinine and urea excretion in critically ill patients with various acute kidney injury stages

HW. Zijlstra ${ }^{1}$; M. Nijsten ${ }^{1}$; M. Volbeda

${ }^{1}$ Department of critical care, University Medical Center Groningen, Groningen, Netherlands

Correspondence: H.W. Zijlstra

Intensive Care Medicine Experimental 2020, 9(1): 001455

Introduction. Acute kidney injury (AKI) is a common complication of critical illness, with various underlying causes. In the intensive care unit (ICU) plasma creatinine, plasma urea (or blood urea nitrogen), or measured creatinine clearance are used as a parameters of renal function. Plasma creatinine in the ICU is a notoriously unreliable measure of renal function, partially as a result of loss of muscle mass in the critically ill. 24-h creatinine clearance is probably most reliable, yet not routinely done. To the best of our knowledge the course of plasma urea and urinary urea excretion and their relation with $\mathrm{AKI}$ are unknown.

Objectives. To evaluate the relationship between urinary excretion of creatinine and urea in different AKI stages.

Methods. We collected blood samples and 24-h urine samples in ICU patients with AKI stage 0,1 and 2 as assessed for the first 7 days. We excluded patients with renal replacement therapy (AKI 3). We looked at the overall ICU population, without considering the underlying disease.

We evaluated daily plasma creatinine and urea, and urinary creatinine (UCE) and urea (UUE) excretion in 24-hour urine samples in the first 10 days of ICU stay. We calculated the urea to creatinine ratio (UCR) plasma and the fractional urea excretion (FUE) as UUE/UCE.

Results. We evaluated 7312,1570 , and 402 patients with AKI stage 0,1 and 2 respectively. The mean ( \pm SD) UCR increased in the first 10 days of ICU stay, from $0.09( \pm 0.05)$ on day 1 to $0.11( \pm 0.06)$ on day 10 , $p<0.001$. There was no difference in the time course of UCR for the different AKI stages (Fig. 1).

We found an increase in the FUE in the first 10 days of ICU stay, from 29.4 ( \pm 18.7 ) on day 1 to $54.5( \pm 29.3)$ on day 10 in the overall group, $\mathrm{p}<0.001$. There was no marked difference in in the time courses of FUE for the different AKI stages.

Figure 1 shows the UCR and FUE in the different AKI stages in the first 10 days of ICU stay.

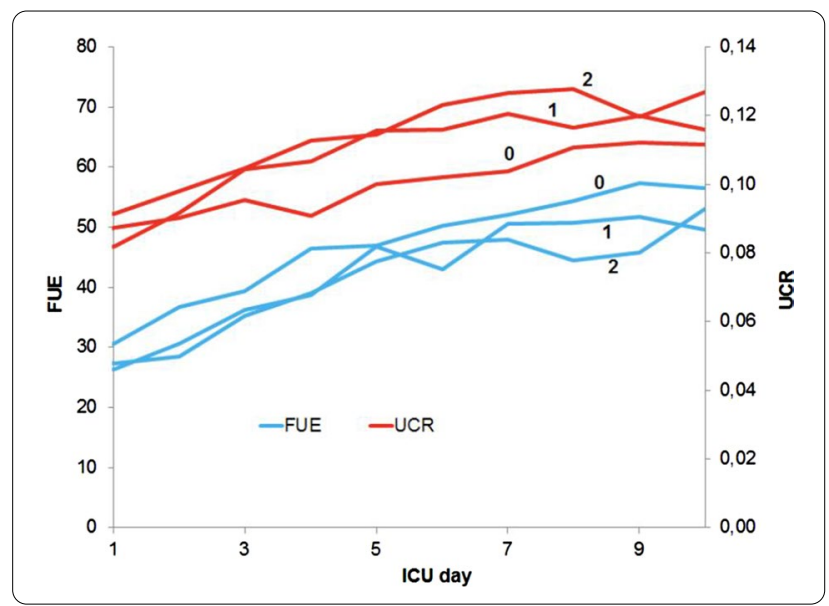


Conclusion. The UCR and UUE increase in the overall group in the first 10 days of ICU stay, but with similar time courses for AKI stage 0,1 and 2 . The catabolic state of critically ill patients apparently has a larger impact on the UCR and UUE, than renal function.

e-Posters: Acute respiratory failure and ventilation

\section{6}

Association of fever with High Flow Nasal Cannula Oxygen Therapy failure in critically ill COVID-19 patients

C. Billiou'; A. Gros, ${ }^{2}$; N. Maziers'; P. Labedade'; F. Bruneel ${ }^{2}$; L. Ensenyat ${ }^{1}$. S. Legriel'; G. Chevrel'; A. Ferré2; S. Marque

${ }^{1}$ Icu, Hospital Center Sud Francilien, Corbeil-Essonnes, France; ${ }^{2}$ Icu, C.H. de Versailles, Le Chesnay, France

Correspondence: S. Marque

Intensive Care Medicine Experimental 2020, 9(1): 000016

Introduction. High Flow Nasal Cannula oxygen therapy (HFNC) has been commonly used in COVID-19 patients. According to recent publications, from 19 to $39 \%$ of ICU patients may benefit from $\operatorname{HFNC}(1,2)$. Yet few data are available on factors associated with HFNC failure in severe COVID-19 patients $(3,4)$.

While fever is a frequent presenting symptom of COVID-19 and has been identified as a predictor of mortality in mechanically ventilated patients, its impact on HFNC patients' outcome has seldom been studied (5).

Objectives. The aim of our study was to report the clinical course of severe COVID-19 patients treated with HFNC, describe the clinical characteristics of patients for whom HFNC was a failure and determine features associated with HFNC failure, hypothesizing that hyperthermia would be one of them.

Methods. We conducted a retrospective bicentric study of COVID-19 patients admitted to two non-university tertiary hospitals. All adult patients requiring ICU admission and HFNC between 1st March 2020 and 31st December 2020 were included. Patients were classified in the HFNC success group if they were discharged alive from ICU without requirement for invasive mechanical ventilation. Hyperthermia was defined as a temperature strictly superior to $38.3^{\circ} \mathrm{C}$ within the first $120 \mathrm{~h}$ of HFNC therapy. As a bivariate exploration, non-parametric tests were performed, among which Kruskal-Wallis, Mann-WhitneyWilcoxon and Fisher's exact tests. Additionally and in order to describe the patients' profiles according to their response to HFNC, multivariate data mining using techniques from the factorial analysis family is ongoing.

Results. A total of 195 patients were included in the study. This population had a median age of 63 years, a median BMI of $29 \mathrm{~kg} / \mathrm{m}^{2}$, while $32 \%(n=62)$ were women. The median length of stay (LOS) in ICU was 14 days, with an overall ICU mortality of $25 \%(n=48)$ and $26 \%(n=50)$ in hospital.

HFNC failure was observed in $58 \%(n=114)$ of patients. In bivariate analysis, besides the classical variables linked to an unfavourable outcome in ICU, patients who failed HFNC had a lower $\mathrm{PaO} 2 / \mathrm{FiO} 2$ on the first $24 \mathrm{~h}$ of HFNC (median of 80 vs 107; $p<0.001$ ), experienced more frequently hyperthermia $(O R=2.84 ; p<0.001)$, had a more frequent history of hypertension ( 86 patients with; $\mathrm{OR}=2.34 ; \mathrm{p}=0.005$ ), were older (median of 64 vs 59 years; $p=0.016$ ) and presented a lower ROX score on HFNC initiation (median of 4.35 vs $5.25 ; p=0.021$ ).

Interestingly, the median LOS in ICU significantly differed according to HFNC duration $(p<0.001)$. In the 68 patients who were treated for less than $24 \mathrm{~h}$ with HFNC, median LOS in ICU was 26 days, while it turned out to be shorter with $9(n=64)$ and 10 days $(n=63)$ for the patients who were treated from 2 to 3 days with HFNC $(n=64)$ or a longer duration $(n=63)$ respectively. Moreover, this categorisation in 3 subgroups tended to significantly split patients in terms of hospital mortality and history of hypertension, with a higher mortality $(p=0.082)$ and a more frequent history of hypertension $(p=0.011)$ in the sub-group of patients with a shorter duration of HFNC.
Conclusion. Though HFNC has been commonly used in critically ill COVID-19 patients, few factors have been identified as predictors of its failure.

In our study, patients for whom HFNC failed were older, had a lower $\mathrm{PaO} 2 / \mathrm{FiO}_{2}$ and a lower ROX on HFNC initiation, but they had also a more frequent history of hypertension and experienced hyperthermia more often than the ones who survived ICU without requiring mechanical ventilation.

HFNC duration subgroups seemed to be of interest to address the multivariate exploration of these preliminary data.

\section{Reference(s)}

1. 5-Monitoring of high flow nasal cannula for SARS-CoV-2 severe pneumonia: less is more, better look at the respiratory rate. Damien Blez and al, Intensive Care Med 2020

2. 4- Application of High-Flow nasal cannula in hypoxemic patients with COVID-19: a retrospective cohort study. Ming Hu and al, BMC Pulm Med 2020

3. 3- Predictors of failure with high-flow nasal oxygen therapy in COVID-19 patients with acute respiratory failure: a multicenter observational study. Ricard Mellado-Artigas and al, J Intensive Care 2021

4. 2-Clinical characteristics and day-90 outcomes of 4244 critically ill adults. COVID-ICU group, Intensive Care Med 2021

5. High-Flow Nasal Cannula in Critically ill Patients with severe COVID-19. Alexandre Demoule and al, AJRCCM 2020

\section{8}

Prediction of successful weaning from mechanical ventilation by continuous ultrasound monitoring of diaphragm excursion A. Demoule ${ }^{1}$; S. Virolle, ; A. Mercat ${ }^{2}$; D. Bergum, ${ }^{3}$; C. Bureau,

${ }^{1}$ Medical intensive care unit, APHP-La Pitié-Salpêtrière Hospital and Sorbonne University, Paris, France; ${ }^{2}$ Medical intensive care unit, University Hospital of Angers, Angers, France; ${ }^{3}$ Department of anaesthesia and intensive care medicine, St Olav University Hospital, Trondheim,

Norway

Correspondence: A. Demoule

Intensive Care Medicine Experimental 2020, 9(1): 000018

Introduction. There is compelling evidence that diaphragm weakness is frequent in critically ill patients, and is associated with increased risk of weaning failure. It is well established that diaphragm ultrasound may help diagnose diaphragm dysfunction and predict weaning failure. However, standard ultrasound methods require skilled operators and continuous monitoring is not feasible. The aim of the present study was to evaluate a novel ultrasound-based medical device for continuous monitoring of the diaphragm, RESPINOR DXT.

Objectives. The specific objectives were: 1) to compare the measure of diaphragm excursion (DE) with DXT and standard ultrasound, 2) to establish a relationship between DXT measure of DE and simultaneous measure of transdiaphragmatic pressure (Pdi), 3) to determine the performance of DE measured with the DXT to predict weaning success or weaning failure.

Methods. Two prospective observational studies were performed in three intensive care units in Europe. Data were pooled for the purpose of this analysis. Mechanically ventilated patients who met the criteria for a spontaneous breathing trial (SBT) were enrolled. Continuous measurements of diaphragm excursion with DXT were compared to 1) standard ultrasound measurements using Spearman correlation and Bland \& Altman analysis, and 2) simultaneously measured Pdi using Spearman correlation. To evaluate DXTs ability to predict weaning success, median values for DE during specific timepoints in the SBT grouped according to failed and successful weaning was analyzed using Mann-Whitney $U$ test and receiver operating characteristic (ROC) curve using Youden's index.

Results. 49 patients were included in the analysis. Among them, 25 patients (51\%) were successfully weaned from mechanical ventilation. There was a strong positive correlation between DE measured with DXT and standard ultrasound $(r=0.88, p=<0.001, n=122$ breaths from different time points). Values of DE measured with DXT and Pdi 
were highly correlated for patients that succeeded weaning $(r=0.85)$. No correlation was observed in the weaning failure group $(r=-0.07)$. Median DE was lower among patients who failed weaning compared to patients who successfully weaned during the second minute of the SBT $(0.77 \mathrm{~cm}$ vs. $1.35 \mathrm{~cm}, \mathrm{p}<0.01)$. A ROC analysis revealed an area under the curve of 0.76 for the second minute of the SBT and a cut off value of $1.1 \mathrm{~cm}$, with a sensitivity of $72 \%$ and specificity of $82 \%$.

Conclusion. The RESPINOR DXT device quantifies DE similar to standard ultrasound and the changes in DE align with simultaneously measured Pdi. In addition, the DXT can reliably predict weaning failure.

\section{Reference(s)}

1. This study was initiated by the investigators and was funded by Respinor, Oslo, Norway

\section{4}

Evaluation of early and late complications in our Percutaneous tracheomies opened with the Combined Forceps and Dilatation Method (CFDY)

C. Balci ${ }^{1}$; T. Murat Emre ${ }^{1}$; E. Yildiz ; ${ }^{1}$ L. Demir ${ }^{1}$; M. Dinler ${ }^{1}$; M. Turgut ${ }^{1}$ ${ }^{1}$ Anaesthesiology and Reanimation, Healty Science Un., Kütahya, Turkey Correspondence: C. Balci

Intensive Care Medicine Experimental 2020, 9(1): 000044

Introduction. Percutaneous Tracheostomy (PT) is a procedure commonly used in intensive care units. Indications for PT include longterm mechanical ventilation, airway preservation, and pulmonary care. Today, in all intensive care units, Ciaglia (sequential dilatation) (CSDY) or Forceps Dilatation (FD-Griggs) method is frequently used using two methods. In 2018-2019, we developed a method in which we expanded with forceps before sequential dilatation, which is a combination of sequential dilatation and forceps method, and completed the procedure with sequential dilation again. We named this method as "Combined Forceps and Dilatation Method" (CFDY).

Objectives. In this study, we aimed to determine early and late complications by retrospectively screening patients who underwent tracheostomy with CFDY between 2019-2021 in our ICU.

Methods. This study was planned as a single center retrospective. All patients hospitalized in our intensive care unit between 2019-2021 and underwent treceostomy with PT were retrospectively screened. Early complications such as bleeding, hypoxia, subcutaneous emphysema, PNX, and late-term complications such as treceaesophageal fistula, stenosis, and infection were recorded in all patients.

Results. It was found that 100 patients out of a total of 1300 patients hospitalized in our intensive care unit between 2019-2021 had PT. Among the early and late complications, no complications occurred in two patients, except minimal bleeding. Data collection continues. Conclusion. PT can be performed with different methods in intensive care units. We also think that the sequential dilatational and percutaneous treceostomy method we developed based on our own experience can be safely applied.

\section{Reference(s)}

1. 1.Early versus late tracheostomy for critically ill patients Brenda NG Andriolo, Regis B Andriolo, Humberto Saconato, Álvaro N Atallah, Orsine Valente, Cochrane Emergency and Critical Care Group Cochrane Database Syst Rev. 2015 Jan; 2015(1): CD007271

\section{6}

Evaluation of early and late complications of Percutaneous Treceostomy performed with Combined Forceps and Dilatation Method in COVID 19 Intensive Care Unit

C. Balci'; T. Murat Emre'; E. Yildiz'; H. Engin ${ }^{2} ;$ O. Kayıkcl ${ }^{1}$

${ }^{1}$ Anaesthesiology and Reanimation, Healty Science Un., Kütahya, Turkey;

${ }^{2} \mathrm{ICU}$, Kocaeli Derince Traning Hospital, KOCAELI, Turkey

Correspondence: C. Balci

Intensive Care Medicine Experimental 2020, 9(1): 000046

Introduction. Percutaneous Tracheostime is a method used frequently in intensive care and increases patient comfort. The most common indications are long-term mechanical ventilation, airway preservation and pulmonary care. In the Combined Forceps and Dilatation Method (CFDY) developed by ourselves, we first expand the tissue and trachea with forceps and complete the procedure with sequential dilation again. We think this method causes less bleeding.

Objectives. The use of anticoagulants in patients with COVID 19 treatment increases the tendency to bleed.In this study, we aimed to determine early and late complications by retrospectively screening patients who underwent tracheostomy with CFDY between 20192021 in our covid 19 intensive care clinic.

Methods. This study was planned as a single center retrospective. Bleeding, hypoxia, subcutaneous emphysema, early complications such as PNX, and late complications such as treceaesophageal fistula, stenosis, and infection were recorded in all patients hospitalized in our COVID 19 intensive care unit between 2019-2021 and underwent treceostomy with the CFYD method.

Results. It was determined that 15 patients out of 299 patients hospitalized in our COVID 19 (pandemic 1) intensive care unit between 2019-2021 had PT. Among the early and late complications, no complications occurred in two patients, except minimal bleeding. Data collection continues.

Conclusion. We think that the Sequential Dilastational and Percutaneous Trachesotomy (CFYD) method, which we developed with our own clinical experience, is safer in COVID 19 Intensive Care Units.

\section{Reference(s)}

1. Early versus late tracheostomy for critically ill patients. Brenda NG Andriolo, Regis B Andriolo, Humberto Saconato, Álvaro N Atallah, Orsine Valente, Cochrane Emergency and Critical Care Group. Cochrane Database Syst Rev. 2015 Jan; 2015(1): CD007271

\section{5}

Incidence of Submassive Pulmonary Embolism in Intensive Care Unit (NONCOVID19) and Our Results After Thrombolytic Therapy C. Balci ${ }^{1}$; E. Yildiz'; B. Ozel'; O. Ece'; A. Gunes ${ }^{1}$

${ }^{1}$ Anaesthesiology and Reanimation, Healty Science Un., Kütahya, Turkey Correspondence: C. Balci

Intensive Care Medicine Experimental 2020, 9(1): 000065

Introduction. Covid 19 often affects vascular structures and causes thrombus and pulmonary embolism. Because of this feature, it is important to investigate the cause of pulmonary embolism in patients without PCR (-) and COVID 19 clinic. Otherwise, patients diagnosed with PE may be mistakenly admitted to COVID19 intensive care units.

Objectives. In the last two years, we opened to present the incidence and prognosis of submassive PE cases admitted to our intensive care unit.

Methods. It was aimed to determine the incidence and treatment of patients who were hospitalized in intensive care units under the name of Anaesthesiology and Reanimation between 2019-2021 and who were diagnosed with $\mathrm{PE}$.

Results. Number of inpatients in four care units (37 beds in total) under KSBU Department of Anesthesiology and Reanimation between 2019-2021, 1300.Four of these patients were accepted to be PE with the diagnosis of embolism in the main pulmonary artery on CT Angio. Thrombolytic agent was given for treatment and patients were followed up in the intensive care unit for $48 \mathrm{~h}$. Manageable hemoptysis developed in one of our patients. All of them were discharged.

Conclusion. PE developing due to COVID 19 is common in the last year. It is vital that the diagnosis and treatment of NonCOVID PE occurring in the main pulmonary artery is not delayed. PE developing in the main pulmonary artery is fatal. Rapid administration of thrombolytic therapy is life-saving. The most common complication is hemoptysis and close follow-up of the patients is important.

\section{Reference(s)}

1. Meyer G, Vieillard-Baron A, Planquette B (2016) Recent advances in the management of pulmonary embolism: focus on the critically ill patients. Ann Intensive Care 6:19 


\section{7}

Prone position in COVID-19 and non-COVID-19 acute respiratory distress syndrome: an international multicentre observational comparative study

L. Camporota1; B. Sanderson 1; D. Chiumello²; N. Terzi, ; L. Argaud ${ }^{4}$; T. Rimmelé ${ }^{5}$; R. Metuor, ; A. Verstraete, ; M. Cour ${ }^{4}$; J. Bohé ${ }^{6}$; V. Piriou ${ }^{6}$; P. Beuret ${ }^{7}$; C. Guérin ${ }^{8}$

${ }^{1}$ Criticalcare, St Thomas' Hospital, London, United Kingdom; ${ }^{2}$ Anesthesia and intensiva care, San Paolo, Milano, Italy; ${ }^{3}$ Médecine intensive réanimation, C.H.U de Grenoble, La Tronche, France; ${ }^{4}$ Service de médecine intensive-réanimation, Hospital Édouard Herriot, Lyon, France; ${ }^{5}$ Anesthesia \& critical care, Hospices Civils de Lyon, Lyon, France; ${ }^{6}$ Service d'anesthésie-réanimation médecine intensive, Lyon Sud Hospital Center, Pierre-Bénite, France, ${ }^{7}$ Service de réanimation polyvalente, Centre Hospitalier Général, Roanne, France, Roanne, France; ${ }^{8}$ Service de réanimation médicale, hôpital de la croix rousse, Grande Rue de la Croix Rousse, Lyon, France

Correspondence: L. Camporota

Intensive Care Medicine Experimental 2020, 9(1): 000067.

Introduction. Prone position (PP) is used in acute respiratory distress syndrome (ARDS) and in COVID-19 (C-ARDS). However, it is unclear how responders may be identified and whether an oxygenation response translates into improved outcomes.

Objectives. The objective of this study was to quantify response to PP in C-ARDS, describe the differences between C-ARDS and ARDS, and explore variables associated to survival.

Methods. This was an retrospective, observational, multicentre, international cohort study in seven ICUs in Italy, UK and France. We enrolled 376 adults (220 C-ARDS and 156 ARDS) who underwent at least one episode of PP.

Results. Pre-proning, a greater proportion of C-ARDS had severe disease (53\% vs 40\%)- PaO2/FiO2 (13.0 [IQR:10.5-15.5] vs 14.1 [IQR:10.518.6] $\mathrm{kPa} ; \mathrm{p}=0.017$ ) but greater compliance (38 [IQR:27-53] vs 31 [IQR:21-37] $\mathrm{ml} / \mathrm{cmH} 2 \mathrm{O} ; \mathrm{p}<0.001)$. Patients with C-ARDS had a longer median time from intubation to PP (2.0 [IQR: 0.7-5.0] vs 1.0 [IQR: 0.52.9] days; $p=0.03$ ).

The proportion of responders (increase in $\mathrm{PaO} 2 / \mathrm{FiO} 2 \geq 2.67 \mathrm{kPa}$ (20 $\mathrm{mmHg}$ ) or $\geq 20 \%$ ) was similar (ARDS $79 \%$ vs C-ARDS $76 \% ; \mathrm{p}=0.5)$. Responders had earlier PP [1.4 [IQR:0.7-4.2] vs 2.5 [IQR:0.8-6.2] days; $=0.06)] . \mathrm{PP}<24 \mathrm{~h}$ achieved greater improvement in oxygenation (11 kPa [IQR: 4-21] vs $7 \mathrm{kPa}$ [IQR: 2-13]; $\mathrm{p}=0.002$ ).

The variables independently associated with 'responder' category were: $\mathrm{PaO} 2 / \mathrm{FiO} 2$ pre-proning (OR 0.89 [95\%Cl 0.85-0.93]; $\mathrm{p}<0.001)$; earlier proning (OR 0.94[0.89-0.99]; $\mathrm{p}=0.019$, per day).

The overall mortality was $45 \%$. Responders with C-ARDS showed a trend for lower mortality (39\% vs $54 \% ; p=0.07$ ). Variables independently associated with mortality were: age (OR 1.04 [95\% Cl 1.02-1.06]; $p<0.001$ ); disease severity (OR 1.01 [95\% Cl 1.00-1.02]; $p=0.007)$, later proning (OR 1.06 [95\%Cl $1.0-1.13] ; p=0.04$, per day); delta-PaO2/FiO2prone-supine (OR 0.97 [95\% Cl 0.95-0.99]; $p=0.002$ ).

Conclusion. Prone position, particularly when delivered early, achieved a significant oxygenation response in $\sim 80 \%$ of C-ARDS, similar to ARDS. Response to prone position was independently associated to improved survival.

\section{8}

\section{VV-ECMO retrieval teams. Project feasibility in COVID-19 times}

S. Alcántara Carmona ; N. Martínez Sanz ; M. Pérez Redondo;

H. Villanueva Fernández ; J. Duerto ÁLvarez; I. Fernández Simón1;

A. Ortega López ${ }^{1}$; P. Matía Almudévar'; S. Bravo Tejado ${ }^{1}$; D. Ballesteros

Sanz ${ }^{1}$; JJ. Rubio Muñoz

${ }^{1}$ Intensive care department, Hospital Universitario Puerta de Hierro Majadahonda, Madrid, Spain

Correspondence: S. Alcántara Carmona

Intensive Care Medicine Experimental 2020, 9(1): 000068

Introduction. In the midst of the COVID-19 pandemic, the region of Madrid created its first veno-venous ECMO (VV-ECMO) retrieval team (two hospitals, on a one week-on/one week-off roster), in order to provide extracorporeal respiratory support to patients with severe acute respiratory distress syndrome (ARDS).

Objectives. To describe the feasibility of the project and the experience of our hospital as part of the VV-ECMO retrieval team.

Methods. Retrospective study (Sept 2020-Feb 2021). We included all patients evaluated as VV-ECMO candidates by our center during our weeks-on. The inclusion/exclusion criteria for VV-ECMO together with the retrieval protocol were developed by a panel of intensivists of different hospitals across Madrid together with the emergency medical services, and both of them were afterwards evaluated and agreed upon by all the Intensive Care Departments of the region. There were no absolute exclusion criteria for VV-ECMO (Fig. 1). The retrieval team was composed of an intensive care physician, a cardiac surgeon and a perfusionist.

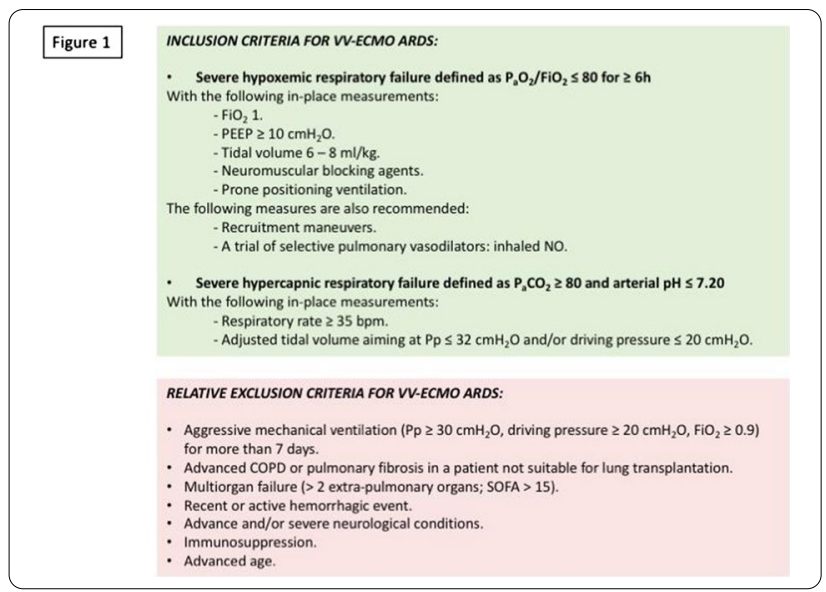

Results. During the period studied, a total of 30 patients were referred for VV-ECMO, with 13 being considered as non-suitable due to different reasons and 17 considered suitable for VV-ECMO treatment. In seven out of the 17 cases, retrieval was performed under VV-ECMO due to poor gas exchange, while the other 10 patients were retrieved without VV-ECMO. There were no complications during patient retrieval, either with or without VV-ECMO. Out of the 10 patients retrieved without VV-ECMO, five ended up with extracorporeal respiratory support after arrival to our center. Overall, 12 of the 17 patients (70.6\%) deemed as candidates for VV-ECMO ended up needing the therapy (Fig. 2).

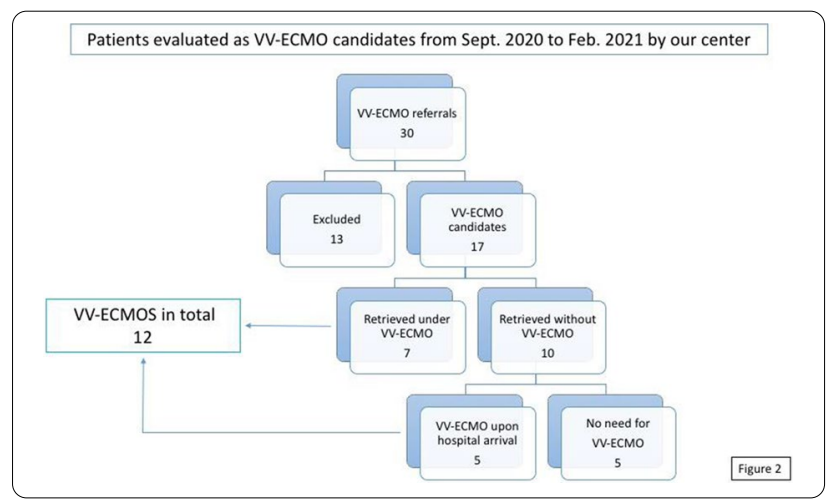

Of the 12 actual VV-ECMO patients, nine were males (75\%) and mean age was $53 \pm 7$ years. The mean duration of the VV-ECMO run was 19 [14-35] days, with shorter runs for patients that survived: 13 [11.5-14] vs. 35 [30.5-49.5]. Five patients died during VV-ECMO (41.6\%) due to futility of treatment, three are still in ICU (one weaned and two still on VV-ECMO) and four (33.3\%) have already been discharged from ICU. 
Conclusion. The creation of a VV-ECMO retrieval team was feasible during convulsive times thanks to the combined effort of the Intensive Care Departments of Madrid making it possible for patients across the region to access a highly complex treatment.

\section{1}

The use of methylprednisolone in patients with Coronavirus Disease (COVID-19) requiring Intensive Care Hospitalization: a longitudinal observational study

S. Hoflack'; L. Doucet Equal Author'; L. Van Slambrouck'; P. Lormans 'Intensive care, AZ Delta campus Rumbeke, Roeselare, Belgium

Correspondence: S. hoflack

Intensive Care Medicine Experimental 2020, 9(1): 000071

Introduction. For a long time, the use of corticosteroids on severely ill patients with coronavirus disease (COVID-19) was a controversial treatment. However, given the conflicting evidence on this topic, we studied the effects of methylprednisolone on the critically ill patients and hereby share our experience on laboratory findings and $\mathrm{PaO2} /$ FiO2 ratio (ratio of partial oxygen concentration on arterial blood gas sample and fraction of inspired oxygen).

Methods. Out of a population of 68 patients who were hospitalized at the intensive care unit due to a COVID-19 infection, 28 patients with severe respiratory failure received methylprednisolone in a fixed scheme of 12 days ( $125 \mathrm{mg}$ IV for 2 days, followed by $2 \times 0,5 \mathrm{mg} / \mathrm{kg}$ IV twice a day for 5 days followed by a declining schedule for 4 days until stop). After day 5 and day 10 we analyzed the CRP (C-reactive protein) levels, lymphocytosis, D-dimers, LDH (lactate dehydrogenase) and $\mathrm{Pa} 02 / \mathrm{FiO} 2$ ratio of our patients.

Results. We observed a significant decrease in median CRP levels between day 0 (start of methylprednisolone treatment) and day 5 $(p=0,001)$, and between day 0 and day $10(p=0,005)$. Between day 5 and $10(p=0,352)$ no decrease was found. The same increase in Pa02/ $\mathrm{FiO} 2$ was recorded between day 0 and day $5(p=0,009)$, and between 0 and day $10(p=0,019)$. For $D$-dimers only a significant difference was found between day 0 and day $10(p=0,018)$. No significant difference could be observed for lymphocytosis and LDH levels between start of treatment and day 5 or day 10 .

Conclusion. There is a strong and lasting significant decrease in CRP levels and incline in $\mathrm{PaO} 2 / \mathrm{FiO} 2$ ratio after starting methylprednisolone. A slower, but also significant decrease was found for D-dimers. Further research and control group analysis are required to confirm this effect is due to the treatment with corticosteroids. However, it does indicate that methylprednisolone could play a very important role in the treatment of the severely ill COVID-19 patients requiring ICU admission.

\section{5}

Complications Associated with the Use of Invasive Mechanical Ventilation in the Treatment of Acute Respiratory Distress

\section{Syndrome: A Systematic Review}

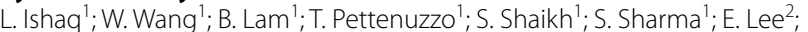

M. Azam ; ; K. Gorsky ; M. Englesakis'; S. Munawer'; E. Fan

${ }^{1}$ Interdepartmental division of critical care medicine, University

of Toronto, Toronto, Canada; ${ }^{2}$ Department of medicine, University

of Toronto, Toronto, Canada; ${ }^{3}$ Department of anesthesiology, University

of Toronto, Toronto, Canada; ${ }^{4}$ Library and information services, University

Health Network, Toronto, Canada

Correspondence: L. Ishaq

Intensive Care Medicine Experimental 2020, 9(1): 000075

Introduction. The cornerstone of supportive therapy in Acute Respiratory Distress Syndrome (ARDS) is the use of invasive mechanical ventilation (IMV) to maintain gas exchange. Though lifesaving in patients with ARDS, the use of IMV is associated with a number of potential harms. We conducted a systematic review to characterize the range and frequency of adverse effects associated with the use of IMV in ARDS.
Methods. We used a sensitive search strategy developed by an information specialist to query the following databases from inception to September 2019: 1) Medline 2) Embase 3) CCTR 5) CDSR 6) Clinicaltrials. gov. We included studies in which adult patients (Age $>18$ ) with ARDS received IMV, with the exception of case reports, editorials, and conference abstracts. We excluded studies with a small sample size $(n<50)$. Outcomes of interest included barotrauma, ventilator-associated pneumonia (VAP), and all other adverse effects potentially related to IMV. Quality assessments were done using the Cochrane Risk of Bias Tool for RCTs and the Newcastle-Ottawa Quality Assessment Scale for cohort and case-control studies. The review was registered with PROSPERO (CRD42020161960).

Results. 7816 unique articles were identified by our search strategy, of which, 136 met the eligibility criteria (38,198 patients). There were 51 RCTs, 81 observational studies, and 4 quasi-experimental studies published between 1993 and 2019. The risk of bias varied across articles. The most frequently described complications were barotrauma and VAP The median (IQR) reported rate of barotrauma was $8.1 \%(4.0 \%-12.1 \%$, $\mathrm{n}=23,196)$. It declined from a median (IQR) of $11.5 \%(8.0 \%-16.0 \%)$ in articles published from 1990-1999 (35 articles, $\mathrm{n}=7380$ ), to $6.0 \%(4.0 \%$ $10.8 \%$ ) in $2000-2010$ (33 articles, $\mathrm{n}=9701$ ), to $5.6 \%$ (2.9\%-10.2\%) in 2010-2019 (24 articles, $\mathrm{n}=6115$ ). The median rate of barotrauma did not correlate with the severity of ARDS. The median rate (IQR) of VAP was $28.0 \%(16.4 \%-44.5 \%, n=17,582)$. The rate was stable over time, but in 10 studies $(n=1489)$ whose populations had more severe ARDS, the median (IQR) rate was higher compared to the 30 studies $(n=14,692)$ with moderate ARDS and 1 study $(\mathrm{n}=238)$ with mild ARDS $(40.7 \%$ [23.5\%-48.7\%] vs $27.5 \%$ [ $15.7 \%-43.2 \%$ ] vs $21.0 \%$, respectively). Complications involving other organ systems were less frequently described and not clearly attributed to IMV (Table 1).

Conclusion. Barotrauma and VAP in patients with ARDS undergoing IMV are relatively common, and differ according to severity of underlying lung injury, and may be changing over time. The reduction in barotrauma over time may reflect increasing use of lung protective ventilation in patients with ARDS. In contrary, the rates of potential complications from IMV involving other organ systems are not as well described.

Table 1: Summary of data collected from included articles

\begin{tabular}{llll}
\hline Variable & Median & IQR & \# articles \\
\hline Age (yrs) & 54.3 & $49.2-60.0$ & 128 \\
APACHE II & 21.5 & $19.0-24.1$ & 63 \\
\% Female & $37.0 \%$ & $30.0-42.0 \%$ & 118 \\
Causes of ARDS (\%) & & & \\
Sepsis & $20.8 \%$ & $13.5-33.2 \%$ & 81 \\
Pneumonia & $46.7 \%$ & $35.6-59.6 \%$ & 87 \\
Aspiration & $14.0 \%$ & $9.1-18.8 \%$ & 54 \\
Trauma & $9.9 \%$ & $5.0-17.4 \%$ & 58 \\
Complications (\%) & & & \\
Barotrauma & $8.1 \%$ & $4.0-12.1 \%$ & 92 \\
VAP & $28.0 \%$ & $16.4-44.5 \%$ & 48 \\
Gastrointestinal & $16.0 \%$ & $5.5-31.8 \%$ & 10 \\
Cardiac & $20.2 \%$ & $10.0-53.0 \%$ & 21 \\
Renal & $24.9 \%$ & $16.6-34.8 \%$ & 43 \\
Hematological & $25.0 \%$ & $19.1-32.0 \%$ & 13 \\
Neurological & $18.6 \%$ & $6.4-35.7 \%$ & 16 \\
\hline
\end{tabular}




\section{1}

Effects of prone position in COVID and non-COVID ARDS

B. Küçükerdem ${ }^{1}$; S. Ugur, ${ }^{1}$; E. Senturk'; G. Kirr ; F. Yurdakul, ; E. Canan²; N. Cakar ${ }^{1}$

${ }^{1}$ Anesthesiology and reanimation, Koc University, Istanbul, Turkey; ${ }^{2}$ Metavision, Qlinicu, Istanbul, Turkey

Correspondence: B. Küçükerdem

Intensive Care Medicine Experimental 2020, 9(1): 000101

Introduction. Acute respiratory distress syndrome (ARDS) is a life threatening condition. Although COVID and non-COVID ARDS may be different in characteristic ( $\mathrm{L}$ and $\mathrm{H}$ type) ${ }^{*}$ we wanted to compare the effectiveness of the prone position in these two groups.

Methods. Retrospectively, single-center study was conducted between January 2017 and May 2020 in Intensive Care Unit (ICU) of American Hospital. Between these dates, 44 of 2204 patients hospitalized in the intensive care unit were diagnosed with ARDS who were invasively mechanically ventilated. While 16 of these patients had ARDS due to COVID, 28 of them had ARDS due to other reasons (Diagram 1). During hospitalization patients APACHE II and SOFA scores, arterial blood gases, Horowitz index, mechanical ventilation parameters and duration, the number of prone positioning and mortality rates were evaluated. The difference of the arterial blood gas values between prone and supine position with the best and the worst Horowitz score were calculated, respectively.

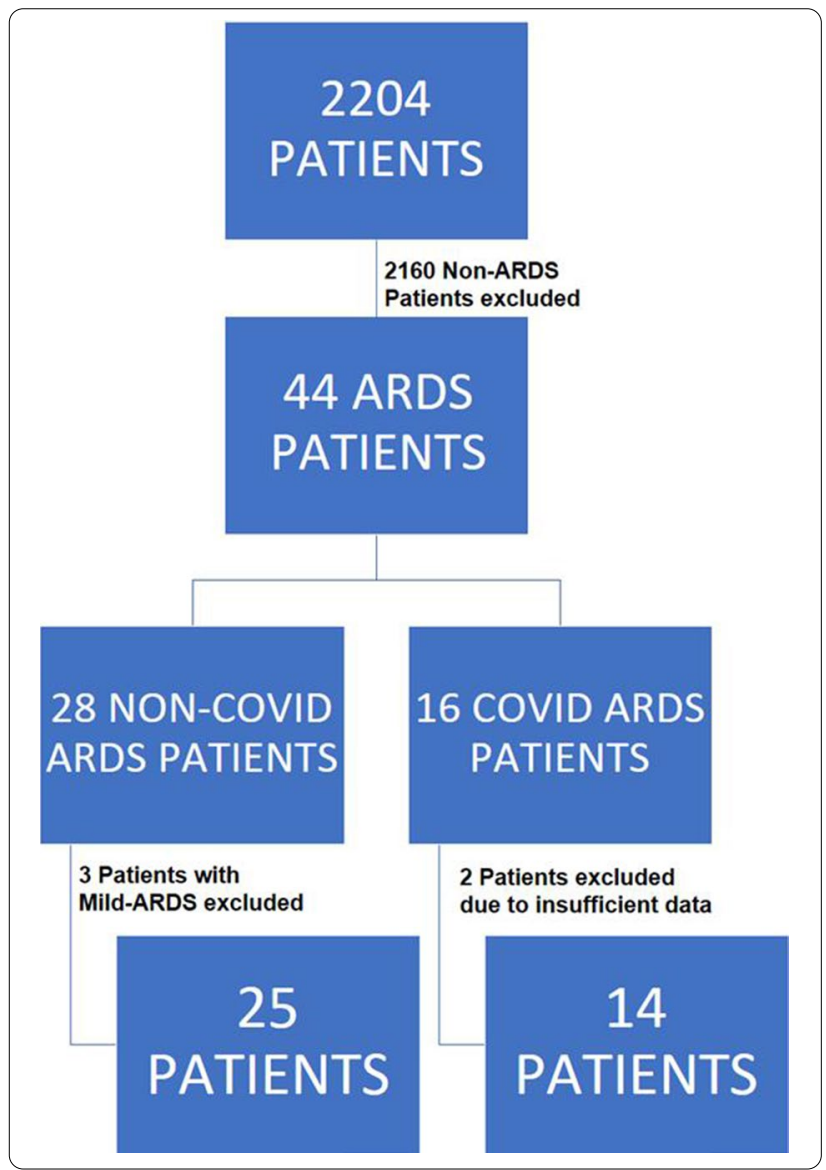

Results. The responses of the patients in the two groups with similar demographic data to prone positions were compared; there was an increase in oxygenation during prone positioning, but no significant difference was observed between the groups. Mortality rates $35 \%$ vs $36 \%, p=0.986$ ), mechanical ventilation durations (398 vs $375 \mathrm{~h}$ $p=0.828)$ and other parameters were similar between COVID and
Non-COVID ARDS (Table 1). However, during the hospitalization period of the patients, the number of prone positioning in the COVID group was approximately 5 times higher than the number of prone positioning in the non-COVID group. (6.2 vs 1.3 times during hospitalization).

\begin{tabular}{|c|c|c|c|c|}
\hline $\begin{array}{l}\text { Mechanical } \\
\text { ventilation and } \\
\text { demographic } \\
\text { data }\end{array}$ & $\begin{array}{l}\text { Overall } \\
(n=39)\end{array}$ & $\begin{array}{l}\text { Covid-ARDS } \\
(\mathrm{n}=14, \\
35.9 \%)\end{array}$ & $\begin{array}{l}\text { NonCovid- } \\
\text { ARDS } \\
(25,64.1 \%)\end{array}$ & $p$ value \\
\hline SOFA Score & 6.15 & 5.35 & 6.6 & 0.119 \\
\hline APACHE Score & 20.1 & 19.28 & 20.56 & 0.393 \\
\hline ICU Mortality & $35.8 \%$ & $35.71 \%$ & $36 \%$ & 0.972 \\
\hline $\begin{array}{l}\text { Mechanical Venti- } \\
\text { lation (hours) }\end{array}$ & 364 & 398 & 375 & 0.453 \\
\hline $\begin{array}{l}\mathrm{PaO} 2 \\
\text { change }(\mathrm{mmHg})\end{array}$ & +72.9 & +71.4 & +73.8 & 0.347 \\
\hline
\end{tabular}

$\triangle \mathrm{PaO} 2(\mathrm{mmHg})$ Partial oxygenation difference between supine and prone position.

Conclusion. There was no difference between oxygenation response, mechanical ventilation duration, and mortality in the COVID and nonCOVID ARDS groups with prone positioning. However, it was observed that COVID patients need more frequent prone positioning.

Reference(s)

1. Gattinoni L, Chiumello D, Caironi P, Busana M, Romitti F, Brazzi L, Camporota L. COVID-19 pneumonia: different respiratory treatments for different phenotypes? Intensive Care Med. 2020 Jun;46(6):1099-1102. https://doi.org/10.1007/s00134-020-06033-2. Epub 2020 Apr 14. PMID: 32,291,463; PMCID: PMC7154064.

\section{7}

\section{PRONE POSITION IN PATIENTS WITH ARDS COVID 19 IN ICU HIGH} ALTITUDE > 2600 mts

D. Molano ${ }^{1}$; A. Estupiñan²; L. Merino, ; ${ }^{3}$ A. Tinoco ${ }^{4}$; A. Viruez, ${ }^{5}$; M. Ayala ${ }^{6}$

${ }^{1}$ Medicina crítica y cuidado intensivo, Los Cobos Medical Center, Bogotá, Colombia; ${ }^{2}$ Intensive c, Sociedad de Cirugía de Bogotá-Hospital de San José, Bogotá, Colombia; ${ }^{3}$ Intensive c, San Pablo Clinic_-Huaraz, Huaraz, Peru; ${ }^{4}$ Intensive c, Hospital Ramiro Priale Priale, Huancayo, Peru; ${ }^{5}$ Intensive c, Hospital Agramont M.R./CENE S.A., El Alto, Bolivia; ${ }^{\circ}$ ntensive care, Los

Cobos Medical Center, Bogotá, Colombia

Correspondence: D. Molano

Intensive Care Medicine Experimental 2020, 9(1): 000107

Introduction. Among the management strategies in patients with pneumonia and ARDS, the use of low tidal volumes, positive pressure at the end of expiration (PEEP) and ventilation in the prone position have been implemented, as the cornerstone in the management of the patients with refractory hypoxemia. Regarding the prone position, several benefits have been suggested, including improvement in perfusion ventilation, recruitment of dependent alveolar areas, optimization of chest wall mechanics, and adequate drainage of pulmonary secretions.

Despite these benefits described, there is still no consensus on its generalized use in patients with severe hypoxemia, on the duration of the cycles and on monitoring regarding the adequate response or not to this strategy.

Methods. Multicenter observational study carried out in Reference Intensive Care Units for the care of COVID-19 cases at three altitude levels in Latin America:

- San José Hospital and Los Cobos Clinic in Bogotá (Colombia) at $2,650 \mathrm{~m}$.

- San Pablo Clinic Huaraz Headquarters (Peru) at 3,100 m.

- Hospital del Norte, El Alto (Bolivia) at 4,150 m.

Each center collected information on adult patients (over 18 years of age) admitted between June 1 and August 31, 2020, the moment corresponding to the start of the peak of the pandemic in Latin America. 
Continuous variables are expressed using the median (p50) and confidence interval (IC) to rule out extreme values. Analysis of the KruskalWallis test were used to analyze the difference between quantitative numerical data between more than two groups. The chi square test (for data with normal distribution) were used to compare categorical variables. The level of statistical significance considered was $p<0.05$. The study has the approval of the Institutional Research Bioethics Committee of each healthcare center according to local regulations.

Results. We analyzed 100 patients, of which $93.4 \%$ of the patients received mechanical ventilation in the first $24 \mathrm{~h}$ of admission to the ICU, with $70.8 \%$ of the patients managed in controlled ventilatory modes upon admission. Prone ventilation was performed in $100 \%$ of the patients, at least for one cycle, during the first 24 years of admission to the ICU. It was divided into 3 groups, according to the duration of the cycles, with $<16 \mathrm{~h}$, between 16 and $30 \mathrm{~h}$, and $>30 \mathrm{~h}$. In our sample, the majority of patients were pronated for more than $16 \mathrm{~h}$, data that persist in the subgroup analyzes of three categories (Table 1 and 2). There were important differences in mortality, which was lower in pronation cycles between 16 and $30 \mathrm{~h}$ and in cycles greater than $30 \mathrm{~h}$. In the group $<16 \mathrm{~h}, 48 \%$ patients are 4 cycles of pronation, in the $16-30 \mathrm{~h}$, the $41 \%$ are 3 cycles and in the $>30 \mathrm{~h}$, the $86 \%$ are 2 cycles of pronation. (Tab 3) Overall mortality was $43.3 \%$ with important variations in the city of Huaraz (n: 7, 17.5\%) vs Bogotá and El Alto (55 and $57.5 \%$ ) respectively. Survival in the subgroups, according to pronation time, was $5.3 \%$ in the group $<16 \mathrm{~h}, 73 \% \%$ in the group between 16 and $30 \mathrm{~h}$, and $75 \%$ in the group $>30 \mathrm{~h} .(\mathrm{p}<0.005)$.

Conclusion. With these initial clinical data, we observed that ventilation in the prone position is an early and generalized strategy used in mechanically ventilated patients with COVID 19 pneumonia and ARDS managed in high-altitude cities. Increase duration pronation cycles for more than $16 \mathrm{~h}$ was a safe practice, which did not show an increase in mortality. The prolongation of pronation hours is associated with a lower number of necessary cycles. It is not yet clear the number of cycles and the duration of these, which combined would have the greatest benefit in these patients. Additional studies are needed to confirm these data.

\section{Reference(s)}

1. Hallifax RJ, Porter BM, Elder PJ, Evans SB, Turnbull CD, Hynes G, Lardner R, Archer K, Bettinson HV, Nickol AH, Flight WG, Chapman SJ, Hardinge M, Hoyles RK, Saunders P, Sykes A, Wrightson JM, Moore A, Ho LP, Fraser E, Pavord ID, Talbot NP, Bafadhel M, Petousi N, Rahman NM; Oxford Respiratory Group. Successful awake proning is associated with improved clinical outcomes in patients with COVID-19: single-centre high-dependency unit experience. BMJ Open Respir Res. 2020 Sep;7(1):e000678 https:// doi.org/10.1136/bmjresp-2020-000678. PMID: 32,928,787; PMCID: PMC7490910

2. Sryma PB, Mittal S, Madan K, Mohan A, Hadda V, Tiwari P, Guleria R Reinventing the Wheel in ARDS: Awake Proning in COVID-19. Arch Bronconeumol. 2020 Nov;56(11):747-749. English, Spanish. https://doi.org/10. 1016/j.arbres.2020.06.002. Epub 2020 Jul 3. PMID: 32,736,877.

\section{0}

Diaphragm neurostimulation mitigates hippocampal apoptosis in pigs mechanically ventilated for $50 \mathrm{~h}$

T. Bassi'; R. Elizabeth2'; F. Karl'; N. Michelle ${ }^{3}$; M. Ornowska ${ }^{2}$; G. Matt ${ }^{4}$.

E. Doug ${ }^{4}$; S. Reynolds

${ }^{1}$ Physiology, Simon Fraser University, Burnaby, Canada; ${ }^{2}$ Critical care, Fraser Healthy Authority, New Westminster, Canada; ${ }^{3} \mathrm{Criti}-$ cal care, Fraser Health New Westminster, New Westminster, Canada; ${ }^{4}$ Research and development, Lungpacer Medical USA Inc., Exton, United States of America

Correspondence: T. Bassi

Intensive Care Medicine Experimental 2020, 9(1): 000110

Introduction. Recent preclinical studies have demonstrated ventilation-associated brain injury; 1 despite this, there have been no studies to date which have investigated strategies to mitigate brain insult during mechanical ventilation (MV).
Objectives. We evaluated whether diaphragm neurostimulation in synchrony with lung-protective MV for $50 \mathrm{~h}$ prevents hippocampal apoptosis in a normal-lung porcine model.

Methods. Twenty-eight healthy juvenile pigs (4-5 months, 48-66 kg) with non-injured lungs were divided into four groups: MV only (MV group, $\mathrm{n}=8), \mathrm{MV}$ in association with diaphragm neurostimulation delivered every other breath (TTDN50\%+MV group, $n=7$ ), MV in association with diaphragm neurostimulation delivered every breath (TTDN100\% + MV group, $\mathrm{n}=7$ ) and never ventilated (NV group, $n=6$ ). A central line catheter with embedded electrodes (Lungpacer LIVE Catheter) was inserted into the left subclavian vein for the TTDN50\% + MV andTTDN100\% + MV subjects, and the diaphragm was activated by temporary transvenous diaphragm neurostimulation (TTDN). Diaphragm neurostimulation in synchrony with MV targeted a reduction in pressure-time product between $15-20 \%$. MV in volume control was set to achieve and maintain a tidal volume of $8 \mathrm{ml} / \mathrm{kg}$ with a PEEP of $5 \mathrm{cmH} 2 \mathrm{O}$. The hippocampus was harvested from each pig and TUNEL assay was used to mark cellular apoptosis. Machine-learning software (ImageJ) was used to count and quantify TUNEL-positive cells as a percentage of total cells counted ( Fig. 1A-inset). Data are expressed as median and interquartile range. The Kruskal-Wallis test and Dunn's multiple comparison test were used for statistical analysis. P-values $£ 0.05$ were considered statistically significant.

Results. The apoptotic index results were $31.70(29.79-43.76)$ for the MV group, 20.53 (10.85-26.46) for the TTDN50\%+MV group, 6.57 (4.94-11.26) for the TTDN100\% + MV group, and $0.96(0.50-1.61)$ for the NV group, with statistically significant differences between groups, as determined by the Kruskal-Wallis test, $p<0.0001$ ( Fig. 1A/B). Post hoc analysis using Dunn's multiple comparison test showed a considerable statistical difference between the MV and NV groups ( 31.70 vs. $0.96, p<0.0001$ ), between the TTDN50\%+MV and NV groups ( 20.53 vs. $0.96, p=0.0172$ ), and between the MV and TTDN $100 \%+M V$ groups ( 31.70 vs. $6.57, p=0.0107$ ).

Conclusion. In a porcine model, diaphragm neurostimulation in synchrony with $\mathrm{MV}$ for $50 \mathrm{~h}$ resulted in significantly less cellular apoptosis in the hippocampus than mechanical ventilation alone. Moreover, diaphragm neurostimulation demonstrated a neuroprotective effect; this neuroprotective effect demonstrated a dose-response where TTDN on $100 \%$ of breaths showed a greater effect than TTDN on $50 \%$ of breaths. Finally, diaphragm neurostimulation on every breath resulted in an apoptotic index statistically indistinguishable from the never ventilated group.

\section{Reference(s)}

1. Bassi et al. Brain injury after $50 \mathrm{~h}$ of lung-protective mechanical ventilation in a preclinical model. Sci Rep. 2021.

2. TBVETS

3. MITACS

4. Lungpacer Medical Inc.

\section{2}

Diaphragm neurostimulation reduces neuroinflammation in pigs mechanically ventilated for $50 \mathrm{~h}$

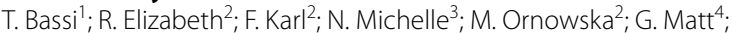

E. Doug ${ }^{4}$; S. Reynolds

${ }^{1}$ Physiology, Simon Fraser University, Burnaby, Canada; ${ }^{2}$ Critical

care, Fraser Healthy Authority, New Westminster, Canada; ${ }^{3}$ Criti-

cal care, Fraser Health New Westminster, New Westminster, Canada;

${ }^{4}$ Research and development, Lungpacer Medical USA Inc., Exton, United States of America

Correspondence: T. Bassi

Intensive Care Medicine Experimental 2020, 9(1): 000112

Introduction. While neuroinflammation due to mechanical ventilation (MV) has been recently demonstrated in preclinical studies, no studies have investigated strategies to prevent neuroinflammation during MV.1,2 The percentage of reactive astrocytes is a well-accepted measure of neuroinflammation. 3

Objectives. We evaluated whether diaphragm neurostimulation in synchrony with lung-protective MV for $50 \mathrm{~h}$ would result in a lower 
percentage of hippocampal reactive astrocytes in a normal-lung porcine model.

Methods. Twenty-eight healthy juvenile pigs (4-5 months, 48-66 kg) with non-injured lungs were divided into four groups: MV only (MV group, $\mathrm{n}=8$ ), $\mathrm{MV}$ in association with diaphragm neurostimulation delivered every other breath (TTDN50\%+MV group, $n=7$ ), MV in association with diaphragm neurostimulation delivered every breath (TTDN100\% + MV group, $\mathrm{n}=7$ ) and never ventilated (NV group, $\mathrm{n}=6$ ). A central line catheter with embedded electrodes (Lungpacer LIVE Catheter) was inserted into the left subclavian vein for the TTDN50\% + MV andTTDN100\% + MV subjects, and the diaphragm was activated by temporary transvenous diaphragm neurostimulation (TTDN). Diaphragm neurostimulation in synchrony with MV targeted a reduction in pressure-time product between $15-20 \%$. MV in volume control was set to achieve and maintain a tidal volume of $8 \mathrm{ml} / \mathrm{kg}$ with a PEEP of $5 \mathrm{cmH} 2 \mathrm{O}$. The hippocampus was harvested from each pig and GFAP assay was used to mark reactive astrocytes. Machinelearning software (ImageJ) was used to count and quantify GFAPpositive cells as a percentage of total cells counted (Fig. 1A). Data are expressed as median and interquartile range. The Kruskal-Wallis test and Dunn's multiple comparison test were used for statistical analysis. P-values of $£ 0.05$ were considered statistically significant.

Results. The percentages of reactive astrocytes found for each group were: $25.63 \%(21.21-28.66)$ for the MV group, $11.93 \%(5.81-$ 15.78) for the TTDN50\%+MV group, $10.41 \%$ (7.10-11.56) for the TTDN100\%+ MV group, and $10.69 \%(9.31-12.85)$ for the NV group, with statistically significant differences between groups, as determined by the Kruskal-Wallis test, $p=0.0009$ (Fig. 1A/B). Post hoc analysis using Dunn's multiple comparison test showed considerable statistical differences between the MV and NV groups $(25.63 \%$ vs.10.69\%, $p=0.0108$ ), between the MV and TTDN50\%+MV groups $(25.63 \%$ vs. $11.93 \%, p=0.0478)$, and between the MV and TTDN100\% + MV groups (25.63\% vs. $10.41 \%, \mathrm{p}=0.0016$ ).

Conclusion. In a porcine model, diaphragm neurostimulation in synchrony with MV for $50 \mathrm{~h}$ resulted in less neuroinflammation in the hippocampus. Moreover, delivering diaphragm neurostimulation either every breath or every other breath resulted in reactive astrocyte percentages statistically indistinguishable from the never ventilated group.

\section{Reference(s)}

1. Chen T, Chen C, Zhang Z, Zou Y, Peng M, Wang Y. Toll-like receptor 4 knockout ameliorates neuroinflammation due to lung-brain interaction in mechanically ventilated mice. Brain Behav Immun. 2016:56:42-55. 10.1016/j.bbi.2016.04.004 2. Bassi, T. et al. Brain injury after $50 \mathrm{~h}$ of lung-protective mechanical ventilation in a preclinical model. Sci Rep. 2021;11(5105). https://doi.org/10.1038/s41598-021-84440-1 3. Schimmel S, Acosta S, Lozano D. Neuroinflammation in traumatic brain injury: A chronic response to an acute injury. Brain Circ. 2017;3(3):135. 10.4103/bc. bc 18 18

2. Bassi, T. et al. Brain injury after $50 \mathrm{~h}$ of lung-protective mechanical ventilation in a preclinical model. Sci Rep. 2021;11(5105). https://doi.org/10. 1038/s41598-021-84440-1

3. Schimmel S, Acosta S, Lozano D. Neuroinflammation in traumatic brain injury: A chronic response to an acute injury. Brain Circ. 2017;3(3):135. 10. 4103/bc.bc_18_17

4. TBVETS

5. MITACS

6. Lungpacer Medical Inc.

\section{0}

Bedside Echocardiography in COVID-19 pandemic, don't miss the curable diseases

W. Alhabashy'; T. Aisa²; J. Thomas²; F. Colreavy ${ }^{3}$

${ }^{1}$ Anesthesia and intensive care, The Mater Misericordiae University Hospital, Dublin, Ireland; ${ }^{2}$ Anesthesia and intensive care, Our Lady of Lourdes hospital, Drogheda, Ireland; ${ }^{3}$ Intensive care, The Mater Misericordiae

University Hospital, Dublin, Ireland

Correspondence: T. Aisa

Intensive Care Medicine Experimental 2020, 9(1): 000140
Introduction. Many studies recommended to use the bed side echocardiography during the management of COVID-19 patient with the focus on the hemodynamic management and fluid responsiveness assessment. Although there are recommendations to use the echocardiography in complex shock states, but still there is no guidance for patients with hypoxemic respiratory failure in the context of stable hemodynamics in the diagnosis of bilateral lung infiltrates/ pulmonary oedema during the COVID-19 pandemic. It is not uncommon that patients were admitted during the pandemic with hypoxemic respiratory failure in a picture which could be misinterpreted as the COVID-19 pneumonitis.

Objectives. To highlight the role of the bedside echocardiography in the management of hypoxic respiratory failure during the COVID-19 pandemic.

Methods. We report case series of four patients who had been admitted with pneumonia versus pulmonary oedema with hypoxic respiratory failure suspected due to COVID-19. Critical care echocardiography (CCE) was performed by the intensivist led to a change of the diagnosis and management plan subsequently, which all were proven negative COVID-19 after $48 \mathrm{~h}$ from admission.

Results. Case presentations: All patients were admitted to the intensive care unit (ICU) with hypoxic respiratory failure (RF). Initial diagnosis was COVID pneumonitis, which required noninvasive \pm invasive ventilation.

Case \# 1: 42- year old male patient who required invasive ventilation, CCE revealed a big left atrial myxoma obstructing the mitral valve and causing severe pulmonary edema. Thus, he was referred to cardiac surgery where the tumor has been excised and the patient was successfully extubated and discharged home.

Case \# 2: 54-year-old male patient who required invasive ventilation, when transesophageal echocardiography (TOE) showed a flail anterior mitral leaflet with severe regurgitation and bicuspid aortic valve. Thus; He was referred to open cardiac surgery where aortic and mitral valves replacement was done. He was tracheostomized and discharged out of ICU.

Case \# 3: 18-year-old woman, who required non invasive ventilation. CCE revealed severe mitral stenosis, giant left atrium, and left ventricular function (LV) was preserved. The initial diagnosis was confirmed as cardiogenic pulmonary edema which was treated accordingly with diuretics and Esmolol infusion. The patient improved and discharged from the ICU.

Case \# 4: 18-year-old female who required invasive ventilation. CCE showed severely dilated and dysfunctional LV with ejection fraction $10-15 \%$ with thrombus versus sarcoma. The patient had orthotropic heart transplant and was extubated after few days and discharged from the ICU.

Conclusion. The intensivist should be vigilant to diagnose the treatable underlying pathologies which mimic COVID19 hypoxic respiratory failure. Echocardiography is a valuable tool for the intensivist that helps change of the diagnosis and plan of management dramatically.

\section{2}

Increases of inflammatory markers occurring after stopping steroids in critical COVID-19 patients is associated with higher mortality

F. Martínez-Sagasti, ; A. Nuñez-Reiz, ${ }^{1}$; P. González-Arenas, ${ }^{1}$;

S. Domingo-Marín, ; M. Rodríguez-Gómez, ; C. Cardenal-Sánchez, ;

J. Duerto-Alvarez,'; A. Pino-Ramírez, ; P. Matía-Almudevar, ;

C. Galban-Malagón, ; M. Calle-Romero, ; A. Palazón-Blanco, ;

M. Sánchez-García,

${ }^{1}$ Critical care, Hospital Clínico San Carlos, Madrid, Spain

Correspondence: F. Martínez-Sagasti,

Intensive Care Medicine Experimental 2020, 9(1): 000142.

Introduction. Two randomized conrolled trials showed reduced mortality in patients receiving a pre-stablished dosage and duration scheme of steroids for Covid-19-associated Acute Respiratory Distress Syndrome (ARDS)(1),(2). After universal implementation in our ICU, we reviewed systemic inflammatory markers over 5 days after stopping 
steroid administration, to study the association of inflammatory status of the individual patient in this particular scenario and outcome.

Objectives. Comparison of the time course of plasma inflammatory marker concentrations and lung function over the first 5 days following removal of steroids in survivors and non-survivors of COVID-19 ARDS receiving invasive mechanical ventilation (MV).

Methods. We performed a retrospective analysis of all consecutive ARDS COVID-19 patients admitted between March 4, 2020 and February 28, 2021 who received a standard protocol of corticosteroids. Daily $\mathrm{PaO} 2 / \mathrm{FiO} 2, \mathrm{D}$-Dimer, $\mathrm{C}$ reactive protein (CRP), procalcitonin (PCT), and LDH plasma levels 2 days before to 5 days after end of steroid therapy were collected and compares with the Mann-Whitney U test. Variables are presented as median [interquartile range].

Results. 405 ARDS COVID-19 patients were admitted during the study period, of whom 321 (79\%) received corticosteroids. A subgroup of $131(40.8 \%)$ improved early and were discharged while still on steroids. In the study group of 148 patients who completed the entire course of steroids and were mechanically ventilated, $82(59 \%)$ survived, $57(41 \%)$ died in the ICU and 9 remain in critical care at the time of study. $105(70.9 \%)$ were male and median values were, age 63 (57.4-70) years, BMI (Kg/m2): 27.7 (26.0-31.1), APACHE II-score 16 (10.5-19.5), symptoms to ICU 8 (6-13) days, ICU stay 25 (16-43.25) days. Survivors were younger than non-survivors, 60 (52-68) vs 66 $(58-71)$ years $(p<0.05)$. Day 0,2 and 5 plasma levels of biomarkers are listed in the table.

Table. Biomarkers and oxygenation index after end of steroid therapy according to outcome

\begin{tabular}{|c|c|c|c|c|c|c|}
\hline \multirow[b]{2}{*}{ Value } & \multicolumn{2}{|c|}{0 day (withdrawal) } & \multicolumn{2}{|l|}{2 days } & \multicolumn{2}{|l|}{5 days } \\
\hline & $\begin{array}{l}\text { Alive } \\
(N=82)\end{array}$ & $\begin{array}{l}\text { Dead } \\
(N=57)\end{array}$ & $\begin{array}{l}\text { Alive } \\
(\mathrm{N}=82)\end{array}$ & $\begin{array}{l}\text { Dead } \\
(N=57)\end{array}$ & $\begin{array}{l}\text { Alive } \\
(\mathrm{N}=82)\end{array}$ & $\begin{array}{l}\text { Dead } \\
(N=57)\end{array}$ \\
\hline $\begin{array}{l}\text { LDH } \\
(\mathrm{UI} / \mathrm{L})\end{array}$ & $\begin{array}{l}742 \\
(635- \\
896)\end{array}$ & $\begin{array}{l}896 \\
(693- \\
1170)^{\wedge}\end{array}$ & $\begin{array}{l}725 \\
(586- \\
915)\end{array}$ & $\begin{array}{l}903 \\
(760- \\
1256)+\end{array}$ & $\begin{array}{l}664 \\
(546- \\
962)\end{array}$ & $\begin{array}{l}870 \\
(678- \\
1192)^{\wedge}\end{array}$ \\
\hline $\begin{array}{l}\text { CRP } \\
(\mathrm{mg} / \mathrm{L})\end{array}$ & $\begin{array}{l}4.4 \\
(1.5-9.4)\end{array}$ & $\begin{array}{l}7 \\
(2.8-12.1)\end{array}$ & $\begin{array}{l}5.1 \\
(2.3-11.5)\end{array}$ & $\begin{array}{l}12.2 \\
(7.6- \\
16.1)^{\wedge}\end{array}$ & $\begin{array}{l}6.1 \\
(2-12.1)\end{array}$ & $\begin{array}{l}13.4 \\
(8.9- \\
19.6)+\end{array}$ \\
\hline $\begin{array}{l}\text { РCT } \\
(\mathrm{ng} / \mathrm{mL})\end{array}$ & $\begin{array}{l}0.1 \\
(0.1-0.2)\end{array}$ & $\begin{array}{l}0.2 \\
(0.1- \\
1.1)+\end{array}$ & $\begin{array}{l}0.1 \\
(0.1-0.2)\end{array}$ & $\begin{array}{l}0.3 \\
(0.1-1)+\end{array}$ & $\begin{array}{l}0.1 \\
(0.2-0.2)\end{array}$ & $\begin{array}{l}0.4 \\
(0.2- \\
0.8)+\end{array}$ \\
\hline $\begin{array}{l}\text { D-dimer } \\
\text { (mcg/L) }\end{array}$ & $\begin{array}{l}1721 \\
(1275- \\
2567)\end{array}$ & $\begin{array}{l}1955 \\
(1557- \\
3213)\end{array}$ & $\begin{array}{l}1853 \\
(1290- \\
2299)\end{array}$ & $\begin{array}{l}2700 \\
(2330- \\
3630)^{\wedge}\end{array}$ & $\begin{array}{l}1766 \\
(1146- \\
2239)\end{array}$ & $\begin{array}{l}2793 \\
(1663- \\
3840)^{\wedge}\end{array}$ \\
\hline $\begin{array}{r}\mathrm{PaO} 2 / \mathrm{FiO} 2 \\
(\mathrm{mmHg})\end{array}$ & $\begin{array}{l}168 \\
(136- \\
204)\end{array}$ & $\begin{array}{l}132 \\
(106- \\
153)+\end{array}$ & $\begin{array}{l}167 \\
(135- \\
228)\end{array}$ & $\begin{array}{l}125 \\
(93- \\
154)+\end{array}$ & $\begin{array}{l}163 \\
(130- \\
228)\end{array}$ & $\begin{array}{l}109 \\
(83- \\
132)+\end{array}$ \\
\hline
\end{tabular}

Comparison median values for each day between survivors and non-survivors. ${ }^{*} \mathrm{p}<0.05, \wedge p<0.01_{,}+\mathrm{p}<0.001$, NS: Not significant.

Conclusion. The significantly increased plasma levels of $\mathrm{LDH}_{\text {, }}$ D-Dimers, CRP and PCT within 5 days of end of standard protocol steroid therapy in mechanically ventilated non-survivors of COVID-19-associated ARDS, suggests reactivation of inflammation after removal of steroid treatment. The impact of customizing the duration of therapy to the degree of inflammation on mortality in this patient population merits further research.

\section{Reference(s)}

1. Tomazini BM, Maia IS, Cavalcanti AB, Berwanger O, Rosa RG, Veiga VC, et al. Effect of Dexamethasone on Days Alive and Ventilator-Free in Patients With Moderate or Severe Acute Respiratory Distress Syndrome and COVID-19: The CoDEX Randomized Clinical Trial. JAMA. 2020:324(13):1307-16

2. Horby P, Lim WS, Emberson JR, Mafham M, Bell JL, Linsell L, et al. Dexamethasone in Hospitalized Patients with Covid-19. N Engl J Med. 2021:384(8):693-704.
000185

Lung mechanics and clinical implications in patients with acute respiratory distress syndrome (ARDS) caused by the novel coronavirus disease (COVID-19)

L. Lopez Garcia

1 University Hospital 12 de Octubre, Madrid, Spain

Correspondence: L. Lopez Garcia

Intensive Care Medicine Experimental 2020, 9(1): 000185

Introduction. As a result of the great number of novel coronavirus disease cases treated at Intensive Care Units (ICU) around the world hypothesis have been set out about the existence of two different clinical phenotypes according to pulmonary compliance, with potential implications related to the response to therapeutic measures and the prognosis.

Objectives. To determine the role of lung mechanics in the evolution, the response to therapeutic measures and the prognosis of patients with ARDS caused by COVID-19.

Methods. We carried out a descriptive, observational, prospective study of 125 patients with ARDS caused by novel coronavirus disease who required invasive mechanical ventilation and were admitted to the ICU of a tertiary hospital in Madrid, from March to May 2020. Demographic variables were collected, as well as mechanical ventilation parameters, therapies applied and clinical course. Quantitative variables are reported as median and interquartile range, and qualitative variables as frequency. Chi-square test was applied, and a $p$ value $<0.05$ was considered statistically significant.

Results. We obtained data about mechanical ventilation parameters of 97 patients. Of these, $50.5 \%$ had a compliance above $40 \mathrm{ml} / \mathrm{cm}$ $\mathrm{H} 2 \mathrm{O}$ (L phenotype) and $49.5 \%$ below $40 \mathrm{ml} / \mathrm{cm} \mathrm{H} 2 \mathrm{O}$ (H phenotype). We observed that patients with $\mathrm{H}$ phenotype required more prone position periods $(2,23$ versus $1,40, p=0,005)$, received treatment with corticoids in a greater proportion $(68.7 \%$ versus $36.7 \%, p=0,002)$, and had worse oxygenation measured as $\mathrm{PaO} 2 / \mathrm{FiO} 2$ index $(92,75$ versus $111,84, p=0,008)$. There were no statistically significant differences in demographic variables, maximum PEEP applied, days of mechanical ventilation or admission in ICU, tracheostomy, need of veno-venous extra corporeal membrane oxygenation and mortality.

Conclusion. Although there are still doubts about the existence of two different phenotypes, we have observed that patients with lower pulmonary compliance had worse oxygenation that led to more aggressive treatments, but this had no correlation with a worse outcome. More studies are needed to determine if these phenotypes are separate entities or if they just reflect different moments of the same clinical course.

\section{Reference(s)}

1. Gattinoni L, Chiumello D, Caironi P, Busana M, Romitti F, Brazzi L, et al. COVID-19 pneumonia: different respiratory treatments for different phenotypes? Intensive Care Med. 2020;46(6):1099-102.

\section{6}

Targeted Maximal Likelihood Estimation to Determine the Effect of Diuretics on Covid-19 Mortality

K. Chavez ; B. Lee ; C. Schorr ${ }^{1}$; N. Puri ${ }^{1}$; H. Bach²; A. Banerjee ${ }^{2}$; K. Quevada ${ }^{2}$. S. Patel

${ }^{1}$ Critical care medicine, Cooper University Hospital, Camden, United

States of America; ${ }^{2}$ Critical care medicine, Cooper Medical School

of Rowan University, Camden, United States of America

Correspondence: K. Chavez

Intensive Care Medicine Experimental 2020, 9(1): 000196

Introduction. Covid-19 infection contributes to a wide range of organ dysfunction, but the most common pathology is pulmonary. A significant proportion of patients infected with COVID will progress to ARDS The management of Covid related ARDS was unclear at the start of the pandemic, but there is now evidence of some efficacious therapies. A conservative fluid balance using diuretics remains a mainstay for traditional ARDS management; it is unclear if the benefit remains within the pathophysiologic milieu of COVID ARDS. Given the physiologic 
plausibility, we aim to assess the mortality benefit of diuretics in the management of COVID through a retrospective observational analysis. Observational studies are wrought with confounding, making causal relations challenging to ascertain. We propose using Causal Inference to determine the Average Treatment Effect of diuretics for Covid mortality. Specifically, we will use a Targeted Maximal Likelihood Estimation (TMLE) as our estimator, which expects both the conditional outcome and treatment exposure, which optimizes the bias-variance tradeoff.

Methods. A retrospective review of adult patients with COVID-19 admitted to an ICU between March 2020 and June 2020 was performed. ICU mortality was the primary outcome for our study. We used TMLE with an ensemble of machine learning algorithms as the primary model via Zepid and Supylearner Python libraries. The machine learning ensemble included Logistic Regression, a Neural Network, Naive Bayes, and XGboost, with the best models chosen for prediction. We included Acute Kidney Injury requiring dialysis, gender, age, ethnicity, ECMO, oxygen support level on admission, Corticosteroid, and Tociluzumab treatment as our covariates. Covariate Absolute Standardized Differences pre and post weighting demonstrated in Fig. 1. The primary metric was the Average Treatment Effect (ATE). Secondary metrics included Odds Ratio and Risk Ratio.

Results. Our initial dataset included 215 patients with 16 missing values, resulting in a final count of 199. Using TMLE, mortality analysis on the diuretics group $(n=199)$ demonstrated an ATE (RD) of $-0.125,95 \%$ $\mathrm{Cl}[-0.239,-0.011]$, risk ratio (RR) $0.715,95 \% \mathrm{Cl}[0.519,0.986]$ and odds ratio $(\mathrm{OR})$ of $0.585[0.3,0.986]$.

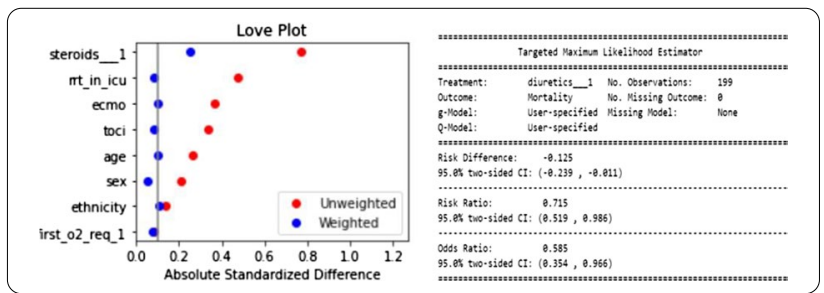

Conclusion. Observational studies are predisposed to confounding, limiting causal inference, but TMLE attenuates many of these limitations. Using TMLE, in this cohort, we demonstrate that diuretics reduce mortality in patients with Covid-19 related hypoxic respiratory failure.

\section{Reference(s)}

1. Schuler MS, Rose S. Targeted Maximum Likelihood Estimation for Causal Inference in Observational Studies. American Journal of Epidemiology. 2017;185(1):65-73. 10.1093/aje/kww165 American Journal of Epidemiology

2. Grams ME, Estrella MM, Coresh J, Brower RG, Liu KD. Fluid balance, diuretic use, and mortality in acute kidney injury. Clin J Am Soc Nephrol. May 2011;6(5):966-73. 10.2215/cjn.08781010

3. Horby P, Lim WS, Emberson JR, et al. Dexamethasone in Hospitalized Patients with Covid-19_Preliminary Report. N Engl J Med. Jul 17 2020;10. 1056/NEJMoa2021436

4. Chen W, Janz DR, Shaver CM, Bernard GR, Bastarache JA, Ware LB. Clinical Characteristics and Outcomes Are Similar in ARDS Diagnosed by Oxygen Saturation/Fio2 Ratio Compared With Pao2/Fio2 Ratio. Chest. Dec 2015;148(6):1477-1483. 10.1378/chest.15-0169

5. Zaim S, Chong JH, Sankaranarayanan V, Harky A. COVID-19 and Multiorgan Response. Curr Probl Cardiol. Aug 2020;45(8):100,618. 10.1016/j.cpcar diol.2020.100618
000197

Retrospective Evaluation of Cytokine Storm Parameters In Severe Covid-19 Pneumonia

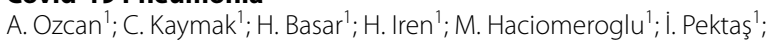

MT. Goc

${ }^{1}$ Department of anesthesiology and reanimation, University of Health Sciences, Ankara Health Application and Research Center, Ankara, Turkey

Correspondence: C. Kaymak

Intensive Care Medicine Experimental 2020, 9(1): 000197

Introduction. Corona virus disease 2019 (COVID-19) is primarily a respiratory system infection caused by severe acute respiratory syndrome corona virus 2 (SARS-CoV-2). Studies have revealed that the hyperinflammatory response induced by the infection is the major cause of disease severity and death.

Objectives. The purpose of the present study was to evaluate the levels of interleukin-6 (IL-6) and other inflammatory parameters in patients with severe COVID-19 pneumonia and ARDS.

Methods. After the approval of ethics committee, this retrospective study was conducted in COVID-19 patients admitted to intensive care unit with severe pneumonia and acute respiratory distress syndrome (ARDS) during a 10-month period. Demographic characteristics, APACHE II score at admission and laboratory data including IL-6, CRP, ferritin, LDH, lymphocyte count, neutrophil / lymphocyte ratio, D-Dimer and fibrinogen on days $0,7,14$ and 28 of admission to ICU were recorded.

Results. A total of 399 patients with severe COVID-19 pneumonia and ARDS were included in the study. The $59,4 \%$ of the patients was male. The meanage of the patients was $68,8 \pm 14,8$. The mean APACHE II score at admission was $13,8 \pm 8,7$. IL-6, ferritin and D-Dimer were remarkably high in the screened patients and started to decrease on the fourth week of ICU stay in the survivors (Table 1).

Table 1. Inflammatorybiomarkers of patients

\begin{tabular}{lcccc}
\hline & Day 0 & Day 7 & Day 14 & Day 28 \\
\hline LL-6 & $105,9 \pm 212,4$ & $112,4 \pm 200$ & $111,9 \pm 161,5$ & $67,5 \pm 124$ \\
CRP (mg/L) & $116,7 \pm 92,5$ & $90,8 \pm 98,7$ & $105,5 \pm 92$ & $104,5 \pm 94,5$ \\
Ferritin ( $\mathrm{\mu g} / \mathrm{L})$ & $1293 \pm 4619,3$ & $1743,8 \pm 7677,2$ & $1046,4 \pm 2727,4$ & $630,3 \pm 545,2$ \\
Fibrinogen & $545,7 \pm 191$ & $476,2 \pm 200,6$ & $574,3 \pm 245$ & $533,3 \pm 148,8$ \\
$\quad$ (mg/dl) & & & & \\
D-Dimer ( $\mathrm{Mg} / \mathrm{L})$ & $4070,4 \pm 10,675,8$ & $5624,9 \pm 7715,1$ & $5976,8 \pm 18,110,4$ & $3033,5 \pm 2510,3$ \\
Lymphocyte & $936 \pm 1232,7$ & $1072,4 \pm 1172,6$ & $1018,5 \pm 668,9$ & $1373,8 \pm 866,3$ \\
$\quad$ (mmm3) & $17,8 \pm 18,1$ & $19,5 \pm 25,2$ & $15,2 \pm 15,9$ & $11,4 \pm 16,2$ \\
NLR & $516,9 \pm 402,1$ & $587,3 \pm 541$ & $380,1 \pm 172,2$ & $435,9 \pm 336$ \\
LDH (U/L) & & & & \\
\hline
\end{tabular}

*Data arepresented as mean \pm SD, IL-6:Interleukin-6, CRP:C-Reactive Protein,

NLR: Neutrophillymphocyteratio, LDH: Lactatedehydrogenase.

Conclusion. Elevation of circulating cytokines was significantly associated with presence of pneumonia in COVID-19 and the severity of lung injury. IL-6 together with other inflammatory biomarkers are associated with severe disease and poor outcome. Anticytokine therapies may help in inhibiting inflammatory response and could be considered in patients with high serum IL-6 levels.

\section{Reference(s) \\ 1. None.}

\section{6}

Optimal Titration Of PEEP In COVID ARDS-Are We Undershooting?

A. Sasidharan Nair ${ }^{1}$; A. Katamreddy'; JY. You'; ${ }^{1}$ P. Gulani ${ }^{2}$

${ }^{1}$ Internal medicine, NYC Health + Hospitals/Jacobi, New York, United

States of America; ${ }^{2}$ Internal medicine, critical care, NYC Health + Hospitals/Jacobi, New York, United States of America

Correspondence: A. Sasidharan Nair 
Intensive Care Medicine Experimental 2020, 9(1): 000206.

Introduction. Acute respiratory distress syndrome (ARDS) from coronavirus disease 2019 (COVID 19) is associated with a high mortality rate (1) and worse hypoxemia (2). The optimal titration of positive end expiratory pressure (PEEP) is challenging and usually executed by following the ARDSnet PEEP/inspired oxygen fraction (FiO2) table (3). Titration of PEEP by measuring end expiratory transpulmonary pressure (TPPexp) has been studied (4) (5), however, not specifically in patients at risk for decreased chest wall compliance-like morbidly obese, on neuromuscular blocking agents or in prone position. COVID ARDS is associated with increased frequency of the latter two (6) (2), and predilection for unfavorable outcomes in morbidly obese patients (7). It stands to reason that such patients would benefit from routine monitoring of TPPexp and subsequent titration of PEEP to maintain TPPexp $\geq$ zero, to ensure maximum alveolar recruitment. Objectives. To determine if mean PEEP differed among patients with ARDS due to COVID 19 and comorbidities known to decrease chest wall compliance.

Methods. This was a retrospective single-center observational study in which all consecutive patients admitted to the medical intensive care unit requiring invasive mechanical ventilation (IMV) from hypoxemic respiratory failure secondary to polymerase chain reaction confirmed COVID-19 were included. A total of 113 patients were included. Data on demographics, prior medical history, BMI, vital signs, average ventilator settings (PEEP, $\mathrm{FiO} 2$ and tidal volume), parameters needed to calculate Acute Physiology and Chronic Health Evaluation (APACHE) II and therapeutic interventions like anticoagulation, prone positioning, neuromuscular blockade, and systemic corticosteroids were collected.

Results. In-hospital mortality was seen in 74 of 113 patients (65.5\%). Table 1 shows the number of patients who were morbidly obese $(\mathrm{BMI} \geq 40 \mathrm{~kg} / \mathrm{m} 2)$, placed in prone position or received neuromuscular blockade, along with their respective mean PEEP.

\begin{tabular}{lll}
\hline & $\begin{array}{l}\text { PEEP } \\
\text { Mean (SD) }\end{array}$ & P value \\
\hline Total $(n=113)$ & $13.3(3.87)$ & \\
Morbidly obese $(n=18)$ & $14.5(3.33)$ & 0.13 \\
Prone position $(n=51)$ & $14.98(3.23)$ & 1.49 \\
Neuromuscular blockade $(n=70)$ & $14.4(3.58)$ & 7.49 \\
\hline
\end{tabular}

Conclusion. Here we see PEEP was not higher in patients with poor chest wall compliance, as would be expected. Of the two forces acting on the alveoli [airway pressure and pleural pressure (Ppl)] only the former is measured in routine practice (8). TPPexp is the difference between PEEP and Ppl and is responsible for keeping the alveoli open (9). Patients with poor chest wall compliance like with morbid obesity, on neuromuscular blockading agents or in prone position, have a higher Ppl, and therefore PEEP needs to be titrated to a higher level to maintain TPPexp $\geq 0$, to ensure maximum alveolar recruitment and improvement in oxygenation.

\section{Reference(s)}

a. Hasan SS, Capstick T, Ahmed R, Kow CS, Mazhar F, Merchant HA, et al. Mortality in COVID-19 patients with acute respiratory distress syndrome and corticosteroids use: a systematic review and meta-analysis. Expert Rev Respir Med. 2020;14(11):1149-63.

b. Gibson PG, Qin L, Puah SH. COVID-19 acute respiratory distress syndrome (ARDS): clinical features and differences from typical pre-COVID-19 ARDS. Med J Aust. 2020;213(2):54-6 e1.

c. NHLBI ARDS Network | Tools [Internet]. Ardsnet.org. 2021 [cited 4 April 2021]. Available from: http://www.ardsnet.org/tools.shtml

d. Talmor D, Sarge T, Malhotra A, O'Donnell CR, Ritz R, Lisbon A, et al. Mechanical Ventilation Guided by Esophageal Pressure in Acute Lung Injury. New England Journal of Medicine. 2008;359(20):2095-104. e. Beitler JR, Sarge T, Banner-Goodspeed VM, Gong MN, Cook D, NovackV, et al. Effect of Titrating Positive End-Expiratory Pressure (PEEP) With an Esophageal Pressure-Guided Strategy vs an Empirical High PEEP-Fio2 Strategy on Death and Days Free From Mechanical Ventilation Among Patients With Acute Respiratory Distress Syndrome: A Randomized Clinical Trial. JAMA. 2019;321(9):846-57.

f. Courcelle R, Gaudry S, Serck N, Blonz G, Lascarrou JB, Grimaldi D, et al. Neuromuscular blocking agents (NMBA) for COVID-19 acute respiratory distress syndrome: a multicenter observational study. Crit Care. 2020;24(1):446.

g. Kompaniyets L GA, Belay B, et al.. Body Mass Index and Risk for COVID19-Related Hospitalization, Intensive Care Unit Admission, Invasive Mechanical Ventilation, and Death — United States, March-December 2020. MMWR Morb Mortal 2021;Wkly Rep (70):355-61.

h. Suarez-Sipmann F, Ferrando C, Villar J. PEEP titration guided by transpulmonary pressure: lessons from a negative trial. J Thorac Dis. 2019;11(Suppl 15):S1957-S62.

i. Turbil E, Galerneau LM, Terzi N, Schwebel C, Argaud L, Guerin C. Positiveend expiratory pressure titration and transpulmonary pressure: the EPVENT 2 trial. J Thorac Dis. 2019:11(Suppl 15):S2012-S7.

2. None

\section{1}

A machine learning-based algorithm to predict endotracheal intubation in covid-19 patients with acute respiratory failure: a potential clinical decision support tool

J. Montomoli' ${ }^{1}$; M. Bitondo'; L. Romeo ${ }^{2}$; LS. Menga ${ }^{3}$; M. Cesarano ${ }^{3}$;T. Rosà ${ }^{3}$. S. Spadaro ${ }^{4}$; E. Frontoni ${ }^{2}$; S. Maggiore ${ }^{5}$; DL. Grieco ${ }^{3}$; M. Antonelli ${ }^{3}$

${ }^{1}$ Anaesthesia and intensive care, Infermi Hospital, AUSL della Romagna, Rimini, Italy; ${ }^{2}$ Department of information engineering, Università Politecnica delle Marche, Ancona, Italy; ${ }^{3}$ Anesthesia and intensive care, Agostino Gemelli University Polyclinic, Roma, Italy; ${ }^{4}$ Ferrara, Ospedale Sant'Anna, Ferrara, Italy; ${ }^{5}$ Anesthesiology, D'Annunzio University of Chieti-Pescara, Chieti, Italy

Correspondence: J. Montomoli

Intensive Care Medicine Experimental 2020, 9(1): 000211

Introduction. Non-invasive respiratory support (NIRS) has been proposed as a first line therapy for the treatment of acute hypoxemic respiratory failure in COVID-19 patients. These approaches may reduce the need for invasive mechanical ventilation but may also delay endotracheal intubation leading to worse outcome. In a context of scarce resources and high pressure on the healthcare system, a predictive model based on a machine learning algorithm could help physicians to identify patients at high risk for deterioration and enable prompt escalation of care.

Objectives. To assess whether a machine learning algorithm can correctly predict the need of endotracheal intubation in a cohort of COVID-19 patients admitted to ICU with moderate to severe hypoxemic respiratory failure.

Methods. We used the population of the HENIVOT Randomized Clinical Trial consisting of 109 patients with COVID-19 with a ratio of partial pressure of arterial oxygen to fraction of inspired oxygen (P/F ratio) $\leq 200$. The XGBoost method was applied as a prediction model in consideration of its characteristics of high generalization performance, the low risk of overfitting, and high interpretability. We included in the model information available at the inception of NIRS (either helmet noninvasive ventilation or high-flow oxygen).

Results. At the inception of the NIRS, among the 109 patients (median age 65 years, 88 males), the median P/F ratio, SOFA, and SAPS score were 102 (IQR:82-125), 2 (IQR:2-3), and 30 (IQR:24-35), respectively. The model correctly predicted the need of endotracheal intubation among 27 (sensitivity 61\%) of the 44 COVID-19 patients that were intubated during the follow-up while it correctly identified 41 (specificity $63 \%$ ) of the 65 patients with a successful trial with a NIVS (Table). 


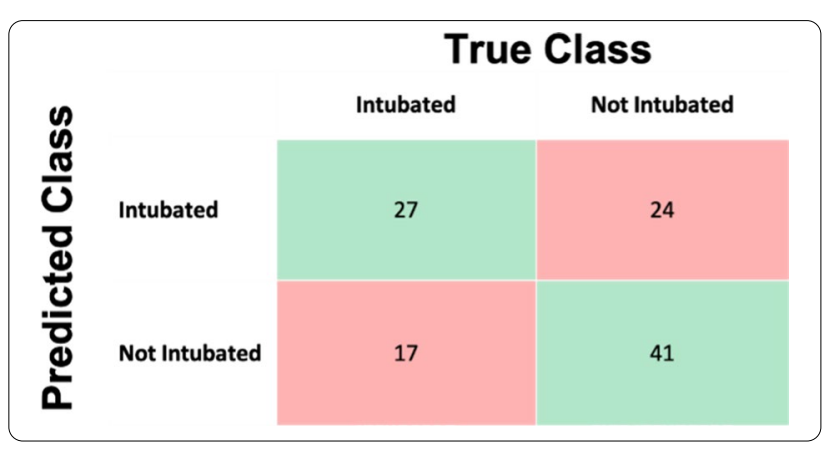

The positive and negative predictive values were $53 \%$ and $71 \%$, respectively, with an overall model accuracy of $62 \%$. The most relevant feature to predict endotracheal intubation was the oxygen saturation followed by the duration of the respiratory symptoms, the mean arterial pressure, and the $\mathrm{P} / \mathrm{F}$ ratio (Figure).

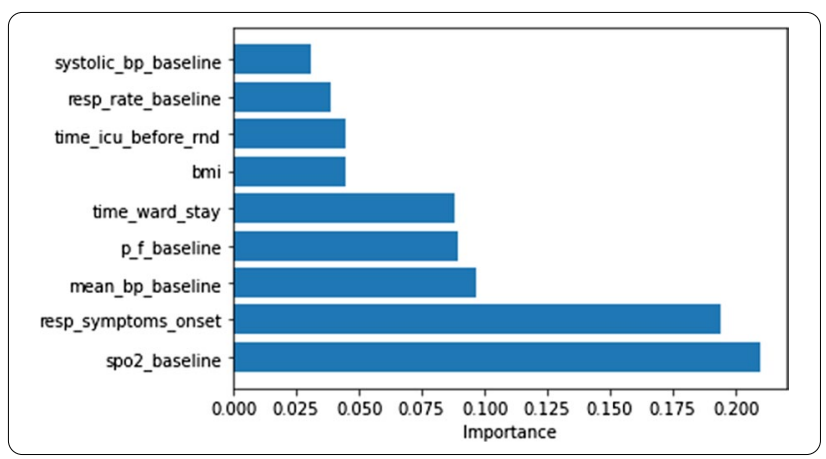

Conclusion. Our results suggest that machine learning-based approaches may support physicians in the triage of COVID-19 patients in terms of the ventilatory management. In particular, the model showed the best performance in the identification of patients that are more likely to benefit from a trial with a NIRS. The actual model should be further validated in other populations of COVID-19 patients.

\section{Reference(s)}

1. Chen T, Guestrin C. XGBoost: A Scalable Tree Boosting System. In: Proceedings of the 22nd ACM SIGKDD International Conference on Knowledge Discovery and Data Mining. San Francisco California USA: ACM 2016. 785-94. 10.1145/2939672.2939785

2. Effect of Helmet Noninvasive Ventilation vs High-Flow Nasal Oxygen on Days Free of Respiratory Support in Patients With COVID-19 and Moderate to Severe Hypoxemic Respiratory Failure: The HENIVOT Randomized Clinical Trial. Grieco DL, Menga LS, Cesarano M, et al. JAMA. 2021 Mar 25. https://doi.org/10.1001/jama.2021.4682. Online ahead of print. PMID: $33,764,378$

\section{7}

\section{Covid19 pneumonia:Spontaneous pneumothorax}

\section{and pneumomediastinum}

N. Shaikh ${ }^{1}$; E. Jamal ${ }^{2}$; AD. Hani ${ }^{3}$; Mustafa ${ }^{4}$; M. Arif ${ }^{4}$; K. Muhmmad ${ }^{5}$

${ }^{1}$ Hamad Medical Corporation, Doha, Qatar; ${ }^{2}$ Surgical intensive care unit, Hamad Medical Corporation, Doha, Qatar; ${ }^{3}$ Hmgh intensive care, Hamad Medical Corporation, Doha, Qatar; ${ }^{4} \mathrm{Hmgh}$ icu, Hamad Medical Corporation, Doha, Qatar; ${ }^{5}$ Dirctor hmgh intensive care units, Hamad Medical Corporation, Doha, Qatar

Correspondence: N. Shaikh

Intensive Care Medicine Experimental 2020, 9(1): 000217
Introduction. Covid-19 pneumonia can occasionally complicate into spontaneous pneumothorax (SP) and/or spontaneous pneumomediastinum (SM). The literature about occurrence of SP and SM is available in the form of individual case reports or smaller case series.

Objectives. Our study was to know the occurrence of SP and or SM, risk factors and outcome in Covid-

Methods. All patients with Covid -19 pneumonia which complicated into SP and/or SM in a dedicated health facility from march to September 2020 were retrospectively enrolled in the study. SP and SM diagnosis were confirmed by chest $\mathrm{x}$-ray and CT (computer tomography). Between-group comparisons were performed by using the chi-square test and the t-test. Differences were considered statistically significant at $\mathrm{p} \leq 0.05$.

Results. A total of 1100 patients were admitted with Covid-19 pneumonia and 43 patients developed SP, SP + SM or SM. Majority of patients were male (42/97.9\%) and a commonly occurring comorbidity was diabetes mellitus (DM) (13/30.2\%). All patients had a diagnosis of ARDS (acute respiratory distress syndrome) and a majority of patients had low lung compliance at the time of developing SP or SM (Less than $25 \mathrm{ml} / \mathrm{cm} \mathrm{H} 2 \mathrm{O}) .22$ of the patients developed SP (51.2\%), 11 patients developed both SP and SM (25.6\%) and 10 patients developed SM only (23.3\%). There was no significant difference in development of SP or SM and patients' gender or blood group $(P>0.05)$. There was no significant difference in occurrence of SP or SM whether patients were on invasive or noninvasive ventilation $(P=0.4 / 0.9$ respectively). Lung compliance was significantly $(P<0.05)$ lower in patients complicated with SP and or SM. Patients with SP required significantly higher $(P<0.001)$ chest drain insertion. There was no significant difference $(P=0.61)$ in mortality whether patient had $S P, S P+S M$ or $S M$. Conclusion: Patients with severe COVID-19 pneumonia can complicate into Spontaneous pneumothorax and pneumomediastinum. These complications are relatively more common in male diabetic patients. Patients with diagnosis of ARDS and having a low lung compliance are at a higher risk of developing SP, SP + SM or SM.

Conclusion. Spontaneous pneumothorax and spontaneous pneumomediastinum more common in male diabetic patients. Patients with diagnosis of ARDS and having a low lung compliance are at a higher risk of developing SP, SP + SM or SM.

\section{5}

Airways management in SARS-COV2 related respiratory failure: a prospective observational multi-center study

L. Cattin ${ }^{1}$; S. Mongodi ${ }^{2}$; F. Ferrari'i ; G. Bettini ${ }^{2}$; E. Pariani ${ }^{2}$; F. Daverio ${ }^{2}$;

E. Polati ${ }^{3}$; F. Mojoli'i ;. Danzi, ; S. De Rosa

'Department of anesthesia and intensive care unit, San Bortolo Hospital

Of Vicenza, Vicenza, Italy; ${ }^{2}$ Department of anesthesia and intensive

care, Fondazione I.R.C.C.S. Policlinico San Matteo, Pavia, Province of Pavia,

Italy, Italy; ${ }^{3}$ Anesthesia and intensive care b unit, University of Verona,

AOUI-University Hospital Integrated Trust of Verona, Verona, Italy

Correspondence: L. Cattin

Intensive Care Medicine Experimental 2020, 9(1): 000245

Introduction. The Emergency Endotracheal intubation (ETT) of a patient who is COVID-19 positive is a high-risk procedure and an additional challenge to an intensivist due to barrier enclosures that have been developed to reduce the risk of COVID-19 transmission to healthcare providers during intubation. Although the incidence of difficult airways is commonly higher in critically ill patients, the evidence of severe hypoxemia without sign of respiratory distress could complicate the scenario.This silent hypoxia often leads to a delayed recognition of the severity of respiratory failure and to a late intubation which is often characterized by a high risk of complications related to the actual airways' management, hemodynamic and cardiac. It has been shown that non-survivors had worse blood gas analyzes than survivors, both before and after intubation. Few studies have reported the implications and adverse events of performing endotracheal intubation for critically ill COVID-19 patients admitted to intensive care units (ICUs).

Objectives. The primary objective was to determine adverse events related to tracheal intubation in COVID-19 patients. Secondary 
objectives were to determine the intubation related-mortality, 7-days mortality, intra-hospital mortality and mortality after discharge from the ICU.

Methods. We have performed a prospective, observational, dualcenter study of consecutive COVID-19 patients undergoing advanced airway management for respiratory failure and admitted two large, independent tertiary care medical centers in Northern, Italy from November 2020 to April 2021.We reported data about patients'dem ographics,comorbidities and parameters related to the intubation procedure and the expertise.Within $30 \mathrm{~min}$ from the intubation,we recorded the occurrence of severe hypoxia $(\mathrm{SpO} 2<80 \%)$,cardiac arrest,hemodynamic instability (one SBP value $<65 \mathrm{mmHg}$, or $\mathrm{SBP}<90 \mathrm{mmHg}$ for at least $30 \mathrm{~min}$ while on fluid challenge or the need of vasopressors).We collected data about difficult airways, as defined as more than 3 attempts of intubation, the need of front of neck airways position, death within $30 \mathrm{~min}$ from the intubation,arrhythmia, oesophageal intubation,pneumomediastinum and pneumothorax recognized within $6 \mathrm{~h}$ from the intubation,7-days mortality,intra-hospital mortality,mortality after discharge from the ICU.

Results. We enrolled 103 patients (age $62.13 \pm 10.43$ years; $74.76 \%$ men).Primary outcome data were available for all patients.Among the study patients, $61.63 \%$ experienced at least 1 major adverse peri-intubation event.The predominant event was cardiovascular instability, observed in $58.14 \%$ of all patients undergoing emergency intubation,followed by cardiac arrest (3.5\%).The worst SpO2 during intubation was $80.34 \pm 14.86 \%$ and the average time of the procedure was $55.87 \pm 44.59 \mathrm{~s}$.

Conclusion. In this observational study of intubation practices in critically ill patients with COVID-19, major adverse peri-intubation events were observed frequently.

\section{Reference(s)}

1. Zhang L, Li J, Zhou M, Chen Z. Summary of 20 tracheal intubation by anesthesiologists for patients with severe COVID-19 pneumonia: retrospective case series. J Anesth. 2020;34(4):599-606.

2. Yao W, Wang T, Jiang B, Gao F, Wang L, et al.; collaborators. Emergency tracheal intubation in 202 patients with COVID-19 in Wuhan, China: lessons learnt and international expert recommendations. $\mathrm{Br} J$ Anaesth. 2020 Jul;125(1):e28-e37.

\section{3}

\section{COVID-19-associated acute respiratory distress syndrome: two} different methods of oxygenating patients in first and second wave of the pandemic

JL. Martinez Melgar'; E. Moreno Lopez²; I. Gallego Barbachano'; A. Ortega Montes ; E. Sanmartin Mantiñan'; A. De La Campa Gestido'; J. Casal Lorenzo ; I. Cenoz Osinaga ${ }^{1}$; T. Sanchez De Dios ${ }^{1}$; P. Posada Gonzalez ${ }^{1}$ Intensive care unit, Complexo Hospitalario Universitario de Pontevedra, Pontevedra, Spain; ${ }^{2}$ Anesthesia and perioperative care, Complexo Hospitalario Universitario de Ferrol, Ferrol, Spain

Correspondence: E. Moreno Lopez

Intensive Care Medicine Experimental 2020, 9(1): 000253

Introduction. In December 2019, an acute respiratory distress syndrome (ARDS) caused by a novel coronavirus (SARS-CoV-2) emerged from China becoming a pandemic.

Objectives. To describe the oxygen therapy for patients with ARDS admitted to our Intensive Care Unit (ICU) in the first (March-April 2020) and second (September- December 2020) pandemic wave.

Methods. Retrospective and descriptive study of patients with COVID19-associated ARDS admitted in the first (1WV) and second (2WV) wave of the pandemic. The following parameters were analyzed: gender, age range, APACHE II, SOFA, comorbidities and respiratory parameters: invasive mechanical ventilation (IMV): pressure (P) / volume (V) mode, FiO2, PEEP, prone positioning (PP) and neuromuscular blockade needed (NMB); non-invasive ventilation (nIV): mode (BIPAP/ CPAP), high nasal flow cannula (HNFC) or both, FiO2 (patients needing less than $24 \mathrm{~h}$ of nIV or those extubated requiring rescue therapy with
nIV were excluded). Complications: reintubation, endotracheal tube exchange and tracheostomy (Tr): surgical (STr) or percutaneous (PTr).

Results. 62 patients were analyzed ( 22 from the $1 \mathrm{WV}$ and 40 from $2 \mathrm{WV})$. From the first wave: 19 male $(\mathrm{M}) / 3$ female $(\mathrm{F}),<60$ years old: 4 between $60-70: 13$ and $>70$ years old:7. APACHE II: $26 \pm 7$, SOFA: $7.5 \pm 1.9$, asthma/COPD: 4 (3/1), BMl>30 kg/m2:5, immunocompromised: 4, hypertension: 13, diabetes:4. Mechanical ventilation was needed in 22 patients in the 1WV (100\%), Pmode 14, Vmode 8 , FiO2 < 0.6: 5, between 0.6 and 0.8: 12 and >0.8: 5. PEEP< 9 mmHg: 3 , between $9-12: 13,>12: 6$. Prone positioning: 18 patients ( 8 within the first $24 \mathrm{~h}$ ) and NMB: 14. Of 40 patients from the second wave, 13 required IMV and 27 nIV. Gender: $32 \mathrm{M} / 8 \mathrm{~F}$ (IMV: $10 \mathrm{M} / 3 \mathrm{~F}$, nIV: $22 \mathrm{M} / 5 \mathrm{M}$ ), age range: $<60$ years old: 10 (3IMV/7nIV), 60-70: 23 (7 IMV/ $16 \mathrm{nIV})$ ), and $>70$ years old: 6 (2IMV/4 nIV), APACHE II: $26.5 \pm 8(\mathrm{IMV})$ and $25.8 \pm 8.4$ (nIV), SOFA $7.3 \pm 2.7$ (IMV) and 7.8 $\pm 1.8(\mathrm{nIV})$, COPD/ asthma: 5 (3IMV/2 nIV), BMI>30 kg/m2: 17 (6IMV/11 nIV), immunocompromised: 7 (4 IMV/3 nIV), hypertension: 17 (7 IMV/10 nIV), diabetes: $12(3 \mathrm{IMV} / 9 \mathrm{nIV})$. In the second wave, 13 patients required mechanical ventilation, Pmode in 8 and Vmode in $5, \mathrm{FiO} 2<0.6: 1$, between 0.6 and 0.8: 6 and >0.8:6. PEEP < 9: 4, between 9-12: 7 and > 12: 2: Prone positioning: 6 ( 4 patients within the first 24 h), NMB:6. In the second wave, nIV was required in 27 patients: BIPAP mode 6/ CPAP 2, HNFC: 14 and mixed in 5 patients. $\mathrm{FiO} 2<0.6: 4$, between 0.6and 0.8: 16 and >0.8: 7 . Respiratory complications: reintubation: 4 (1WV) $/ 2$ (2WV), endotracheal tube exchanged: 7 (1WV) /3(2WV), tracheostomy: 5 (1WV): 4 $\mathrm{STr} / 1 \mathrm{PTr}, 4$ in the 2WV: $2 \mathrm{STr} / 2 \mathrm{PTr}$.

Conclusion. In the first wave $100 \%$ patients required mechanical ventilation, Pmode: $64 \%$, $\mathrm{FiO} 20.6-0.8: 55 \%$, PEEP 9-12: 60\%, prone positioning: $81 \%$ and NMB $64 \%$. In the second wave mechanical ventilation was required in $32.5 \%$ of the patients (Pmode $61.5 \%$, $\mathrm{FiO} 2$ 0.6-0.8: 46\%, PEEP 9-12: 54\%, prone positioning: $46 \%$ and NMB: $46 \%$ ) compared with $67.5 \%$ who needed non invasive ventilation (HNFC: 52\%, BIPAP/CPAP: 30\%) and FiO2 0.6-0.8: 59\%

\section{5}

Effects of an interleukin- 6 receptor antagonist on critically ill COVID-19 patients admitted to an ICU unit in a middle-income country

PP. Arias-Sanchez'; PD. Wendel Garcia²; H. Tirape-Castro ${ }^{1}$; J. Apolo, ;

A. Martinez, ${ }_{1}^{1}$ H. Aguirre-Bermeo

${ }^{1}$ Intensive care unit, Hospital Vicente Corral Moscoso, Cuenca, Ecuador;

${ }^{2}$ Institute of intensive care medicine, University Hospital Zürich, Zürich, Switzerland

Correspondence: $\mathrm{H}$. Aguirre-Bermeo

Intensive Care Medicine Experimental 2020, 9(1): 000265

Introduction. The COVID-19 disease is associated with a dysregulated inflammatory host response, which can lead to high morbidity and mortality in critically ill patients requiring intensive care unit admission. The interleukin-6 (IL-6) receptor antagonist (Tocilizimab) might modulate the inflammatory effects of IL-6. Its early administration on critically ill patients may improve survival and clinical outcomes. [1].

Objectives. To determine if early administration of Tocilizumab added to usual care improves clinical outcomes in COVID-19 patients with a hyperinflammatory state.

Methods. Retrospective analysis of all critically ill COVID-19 patients admitted to an ICU in a middle-income country (Ecuador) between June and December 2020. Tocilizumab was given when the following criteria were met: positive SARS-CoV-2 PCR test, 18 years old or older, $\mathrm{PaO} 2 / \mathrm{FiO} 2$ ratio $<200 \mathrm{mmHg}$, and hyperinflammatory state (defined as serum ferritin levels $>1000 \mathrm{ng} / \mathrm{mL}$ and IL-6 levels $>40 \mathrm{pg} / \mathrm{mL}$ ). The exclusion criteria for Tocilizumab administration were: active infection, high risk of Gl perforation, thrombocytopenia, and neutropenia. Two doses of Tocilizumab ( $8 \mathrm{mg} / \mathrm{kg}$ maximum $800 \mathrm{mg}$ ) were infused within a temporal span of $12 \mathrm{~h}$. Tocilizumab had to be administered within the first $36 \mathrm{~h}$ of intensive care unit admission. All patients were administered steroids (Dexamethasone $6 \mathrm{mg}$, once a day, ten days).

To assess the causal effect of Tocilizumab, a matching of patients having received Tocilizumab to patients having received standard of care, was undergone. Matching was performed considering: age, sex, BMI, 
C-reactive protein, procalcitonin, interleukin-6, leukocyte count, ferritin, D-dimer, SAPS II, Pao2/FiO2 ratio, and respiratory support at intensive care unit admission, targeting a standardized mean deviation of $<0.1$ between groups.

Results. The main results are presented in the table.

\begin{tabular}{|c|c|c|c|}
\hline Variables & $\begin{array}{l}\text { Usual care } \\
\text { group } \\
(n=46)\end{array}$ & $\begin{array}{l}\text { Tocilizumab } \\
\text { group } \\
(n=23)\end{array}$ & $p=$ \\
\hline Age, years & $54 \pm 16$ & $54 \pm 12$ & 0.76 \\
\hline $\mathrm{BMl}, \mathrm{kg} / \mathrm{m} \wedge 2$ & $27 \pm 3.2$ & $28 \pm 4.2$ & 0.51 \\
\hline $\begin{array}{l}\text { Time from symptom onset to ICU } \\
\text { admission, days }\end{array}$ & $9 \pm 6$ & $7 \pm 4$ & 0.25 \\
\hline C-reactive protein, mg/L & $21 \pm 13$ & $21 \pm 12$ & 0.92 \\
\hline Procalcitonin, ng/mL & $1 \pm 1.3$ & $1.3 \pm 3.4$ & 0.53 \\
\hline $\mathrm{IL}-6, \mathrm{pg} / \mathrm{mL}$ & $195 \pm 449$ & $186 \pm 235$ & 0.93 \\
\hline 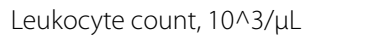 & $11.3 \pm 5.7$ & $11.6 \pm 5.3$ & 0.83 \\
\hline Ferritin, $\mathrm{ng} / \mathrm{mL}$ & $1206 \pm 1136$ & $1430 \pm 865$ & 0.41 \\
\hline D-dimer, $\mu \mathrm{g} / \mathrm{mL}$ & $1.9 \pm 2.6$ & $1.4 \pm 2.0$ & 0.41 \\
\hline SAPS $\|$ & $45 \pm 18$ & $44 \pm 14$ & 0.89 \\
\hline $\mathrm{PaO}$ /FiO2 Ratio, mmHG & $153 \pm 97$ & $143 \pm 134$ & 0.71 \\
\hline Non-high flow Oxygen therapy* & $22(47.9)$ & $12(52.2)$ & 0.31 \\
\hline $\begin{array}{l}\text { Non-Invasive Ventilation and high } \\
\text { flow Oxygen therapy* }\end{array}$ & $9(19.6)$ & $7(30.4)$ & 0.48 \\
\hline Invasive Mechanical Ventilation* & $15(32.6)$ & $6(26.1)$ & 0.78 \\
\hline Length of ICU stay, days & $12 \pm 6$ & $11 \pm 7$ & 0.42 \\
\hline Bacteremia & $6(13)$ & $6(26)$ & 0.17 \\
\hline Respiratory tract infection & $15(33)$ & $7(30)$ & 0.85 \\
\hline In-ICU mortality & $14(30)$ & $8(35)$ & 0.80 \\
\hline
\end{tabular}

Data are presented as mean \pm standard deviation or number (\%) as correspond. *Respiratory support at admission.

Conclusion. In this cohort of hyperinflammated critically ill COVID-19 patients, we did not find a statistical difference in clinical outcomes between patients having received usual care and patients having received early therapy with Tocilizumab.

\section{Reference(s)}

1. REMAP-CAP Investigators, Gordon AC, Mouncey PR, Al-Beidh F, Rowan KM, Nichol AD, Arabi YM, et al. Interleukin-6 receptor antagonists in critically ill Patients with Covid-19. N Engl J Med. 2021. https://doi.org/10. 1056/NEJMoa2100433.

\section{9}

\section{Pneumomediastinum in patients with severe SARS COV-2}

A. Castro ${ }^{1}$; Cl. Olvera-Guzman ${ }^{1}$; J. Franco-Granillo'; JS. Aguirre-Sanchez ${ }^{1}$ ${ }^{1}$ Critical care unit, ABC Medical Center, Ciudad de México, Mexico Correspondence: A. Castro

Intensive Care Medicine Experimental 2020, 9(1): 000289

Introduction. Pneumomediastinum is the presence of air in the mediastinum after alveoli are broken and air dissects sheaths of the pulmonary blood vessels and travels to the mediastinum and cervical fascia. (1) In $1-2 \%$ of patients under mechanical ventilation, pneumomediastinum results from barotrauma after excessive intra-alveolar pressure, high tidal volumes and/or intrinsic PEEP and dynamic hyperinflation.(2) Incidence of pneumomediastinum in mechanical ventilation without COVID 19 is $0.3 \%$ and $0.7-0.91 \%$ in COVID 19 (2-3) It is not known whether pneumodeiastinum in COVID 19 is associated with mortality. (4).
Objectives. Describe mechanical ventilation parameters and respiratory mechanics of patients with pneumomediastinum in COVID 19 to determine risk factors and outcomes.

Methods. All patients files with severe COVID19 and pneumomediastinum admitted to the ICU from April 2020 to March 2021 were included. Demographics, respiratory mechanics and ventilatory parameters were obtained as well as treatments and outcomes.

Results. Of 434 included, 17 (3.9\%) developed pnemomediastium. Mean age of 17 patients was $59 \pm 14$ (41-81) years, $3(17 \%)$ female. Risk factors: 11 (64\%) smoked, Body Mass Index (BMI) 27.8 4.8 (2144) and 16 (94\%) were under invasive mechanical ventilation. Days under mechanical ventilation (MV) were $23 \pm 13(6-47)$ and days in the ICU: $27 \pm 16(8-57) .70 \%(n=12)$ received non invasive MV before invasive $\mathrm{VM}$. Mean $\mathrm{PaO} 2 / \mathrm{FiO} 2$ at the time of intubation was $144 \pm 72$ (58-300). Tracheostomy was performed in 7 patients (41\%) and 6 (35\%) died. When comparing patients that survived vs. the ones that died, only tracheostomy was statistically significant. Table 1 shows the differences between survivors and non survivors.

Conclusion. Demographics, respiratory mechanics and ventilatory parameters were not different between survivors and non survivors, but the presence of tracheostomy was more frequent in the patients that died. Further research is needed to compare whether all results analyzed and reported above are different from patients with COVD 19 and mechanical ventilation without pneummedistinum.

\begin{tabular}{llll}
\hline & Survivors & Non-survivors & $\boldsymbol{p}$ \\
\hline Age (years) & $55 \pm 13(41-78)$ & $66 \pm 14(42-81)$ & 0.12 \\
Gender female & $1(33 \%)$ & $2(67 \%)$ & 0.51 \\
Smoking & $9(81 \%)$ & $2(33 \%)$ & 0.10 \\
Days under MV & $27 \pm 19(6-46)$ & $28 \pm 12(12-47)$ & 0.9 \\
Days in ICU & $26 \pm 18(6-46)$ & $28 \pm 12(12-48)$ & 0.85 \\
Tracheostomy & $2(28 \%)$ & $5(90 \%)$ & 0.034 \\
Pleural drainage & $3(66 \%)$ & $2(34 \%)$ & 1 \\
PaO2/FiO2 (mmHg) & $139 \pm 67(69-45)$ & $152 \pm 87(58-300)$ & 0.73 \\
Static compliance (ml/ & $26 \pm 5(16-39)$ & $28 \pm 8(20-38)$ & 0.46 \\
$\quad$ cmH2O) & & & \\
Plateau pressure (cmH2O) & $27 \pm 3(23-30)$ & $29 \pm 2(25-30)$ & 0.35 \\
Driving pressure & $17 \pm 3(13-22)$ & $18 \pm 4(12-18)$ & 0.35 \\
\hline
\end{tabular}

Reference(s)

1. Macklin CC. Transport of air along sheaths of pulmonic blood vessels from alveoli to mediastinum: clinical implications. Arch Intern Med 1939; 64: 913-926. 2. Zhang XY et al. Diagnosis and prognosis of spontaneous pneumomediastinum in eighteen children. Chinese Journal of Pediatrics 2013: 51: 849-851 3. Chu CM, Leung YY, Hui JY, et al. Spontaneous pneumomediastinum in patients with severe acute respiratory syndrome. Eur Respir J 2004; 23: 802-804. 4. Sethi et al. Subcutaneous emphysema and pneumomediastinum in patients with COVID-19 disease; case series from a tertiary care hospital in Pakistan. Epidemiology and Infection 2021; 149: $1-8$.

2. None

\section{1}

Efficacy and feasibility of awake proning in patients with COVID-19 related acute respiratory failure

T. Aisa'; MA. Malik'; K. Algrni ${ }^{2}$

${ }^{1}$ Anesthesia and intensive care, Our Lady of Lourdes Hospital,

Drogheda, Drogheda, Ireland; ${ }^{2}$ Health promotion and education, Umm Al Qura University, Makkah, Saudi Arabia

Correspondence: T. Aisa 
Intensive Care Medicine Experimental 2020, 9(1): 000291

Introduction. Acute respiratory distress syndrome (ARDS) is a major complication of COVID-19 that occurs in $20-41 \%$ of patients with severe disease $(1,2)$. Proning is one of the management options proven to to improve oxygenation and reduce mortality in nonCOVID-19- related ARDS (3) The application of prone positioning in conscious, non intubated patients (awake proning) during COVID19 related ARDS has shown to improve the respiratory parameters and improve oxygenation (4). However, the reported duration of proning was short in some studies and the sample size was small in the others. Moreover, the studies reported its application and feasibility outside ICU settings are still few. As a response to COVID19 pandemic surge, we established, together with the respiratory team, a dedicated COVID 19 respiratory ward for the management of mild to moderate ARDS patients who require oxygen therapy, non invasive ventilation (NIV) or high flow nasal cannula (HFNC). We adopted a policy of early awake proning in such patients and conducted a prospective observational study to report the physiological changes, improvement of oxygenation, the need for intubation, alongside the duration and tolerance of awake proning.

Methods. In this single-centre, prospective observational cohort study, we included all awake, non intubated, spontaneously breathing patients with COVID-19 and hypoxemic acute respiratory failure requiring oxygen supplementation, NIV or HFNC. The main exclusion criteria were acute respiratory failure requiring intubation, haemodynamic unstability or decreased conscious level. Arterial blood gases were measured just before proning, $30 \mathrm{~min}$ and 1 -h post proning. Wherever possible, oxygen delivery interface, and PEEP were not changed between before or after proning. The subsequent prone positioning sessions were allowed according to the clinicians' discretion. Patients were followed-up until intensive care unit (ICU) discharge (if being admitted to ICU) for occurrence of intubation, time to intubation, and its duration. If patients tolerated the prone position $>3 \mathrm{~h}$ without adverse effects, discomfort or asking for supination, the prone position was considered feasible. Comfort was assessed by asking the patient how they would evaluate their comfort on a scale of "well tolerated", "somewhat tolerated", or "untolerable". The main outcome was the proportion of responders' improvement in oxygenation (the increase in the PFR $\geq 20 \%$ between before and during proning). Secondary outcomes included improvement in the respiratory distress parameters (RR, HR, BP, SpO2, Pao2), the need for intubation, its feasibility, comfort and adverse effects.

Results. In the period between January 9 and April 1, 2021 we enrolled 50 patients, of whom $23(46 \%)$ male, 27 (54\%) female with mean age 56.20 (11.91) years and mean body mass index 29.46 (3.77). There was a significant improvement in oxygenation from supine to prone position alongside with NIV and HFNC (PFR 85.06 (13.76) in supine position while it was 124.06 (34.08) in prone position with substantial increase in PFR 1-h post proning 138.34 (28.01 and $P$ value 0.0001 , these findings were reported in $43(86 \%)$ patients of our sample. There was a significant decrease in the HR (99.92 (7.05) versus 93.14 (10.20); P value 0.0001 , RR (38.32 (4.37) versus 29.53(4.60); $P$ value 0.0001 between supine and prone position respectively. Furthermore, 7 (14\%) Patients required intubation with mean duration of ventilation 16.5 days. Prone positioning was feasible in $41(82 \%)$ of the patients (mean duration 8.54 (SD 3.13) hours) and 38 (76\%) reported that it was well tolerated. Only $5(10 \%)$ patients had complications in the form of back pain, anxiety and agitation.

Conclusion. Awake proning was feasible, tolerable and effective in improving the oxygenation in patients with COVID-19 related pneumonia and hypoxic respiratory failure. Further studies are required to determine the outcome of awake proning and whether it could be a preventive measure rather than recue therapy in such patients.

\section{Reference(s)}

1. Guérin C, Reignier J, Richard JC, et al. Prone positioning in severe acute respiratory distress syndrome. N Engl J Med 2013; 368: 2159-68
2. Coppo A, Bellani G, Winterton D, et al. Feasibility and physiological effects of prone positioning in non-intubated patients with acute respiratory failure due to COVID-19 (PRON-COVID): a prospective cohort study. Lancet Respir Med 2020; 8:765-74

3. We acknowledge the whole team in anesthesia and ICU department at Our Lady of Lourdes hospital, Drogheda.

4. Wang D, Hu B, Hu C, et al. Clinical characteristics of 138 hospitalized patients with 2019 novel coronavirus-infected pneumonia in Wuhan, China. JAMA 2020; 323: 1061-69

5. Wu C, Chen X, Cai Y, et al. Risk factors associated with acute respiratory distress syndrome and death in patients with coronavirus disease 2019 pneumonia in Wuhan, China. JAMA Intern Med 2020; published online March 13. DOl:10.1001/ jamainternmed.2020.0994

\section{1}

Early vs Late Intubation in a single Center Cohort of COVID-19 patients require Intensive Care

D. Castro ${ }^{1}$; C. Roriz, ; N. Ferreira ${ }^{1}$; H. Fernando ${ }^{1}$; F. Sequeira ${ }^{1}$; C. Santos ${ }^{1}$;

O. Gomes ${ }^{1}$; A. Araujo ${ }^{1}$; P. Viana1; L. Pereira

${ }^{1}$ Serviço de Medicina Intensiva, Centro Hospitalar de Leiria, Leiria, Portugal

Correspondence: D. Castro

Intensive Care Medicine Experimental 2020, 9(1): 000311

Introduction. Coronavirus disease 2019 (COVID-19) is associated with a spectrum of presentations, from milder symptoms to severe hypoxic respiratory failure. During the beginning of pandemic most recommendations suggested an early intubation but now clinicians are more comfortable with noninvasive modes of oxygenation such as high flow nasal canula (HFNC) or non-invasive positive pressure ventilation (NIV).

Objectives. The aim of this study was to compare ventilatory parameters and lung mechanics of early and late intubation in COVID-19 critical ill patients and assess the impact of noninvasive modes in these patients.

Methods. An observational retrospective study of all COVID-19 patients admitted in our ICU from 1st March 2020 to 1st March 2021. Data was collected regarding the mode of ventilation (noninvasive and invasive ventilation), mechanical invasive ventilation parameters in the first $24 \mathrm{~h}$ post intubation and clinical outcomes. Comparisons were performed using the Mann-Whitney U-test for quantitative variables and Pearson Chi-square test for qualitative variables.

Results. One hundred and three patients with confirmed COVID-19 were admitted in our ICU in one year. Seventy-nine percent patients were male and the median age was 67 years (minimum 29; maximum 85). Of all COVID-19 ICU-patients, 51 (49,5\%) were under NIV; 17 $(16,5 \%)$ under HFNC and $15(14,6 \%)$ under NIV and HFNC. 85 patients $(82,5 \%)$ needed invasive mechanical ventilation (IMV). Median of days in VMI were $11(5,0-19,0)$; ICU length of stay was $12(7,8-19,0)$ and ICU mortality rate was $34,0 \%$.

Median time to intubation was 3 days from presentation. Patients were divided into an early intubation ( $\leq 3$ days) or late intubation (>3 days) group for analysis. Late intubation group had a lower static compliance in first $24 \mathrm{~h}(39$ vs $32: p=0,012)$ and more hours in NIV (15 vs $72 ; p<0,001)$ than early intubation group suggesting that NIV can delay intubation. No differences were found in days of $\mathrm{IMV}, \mathrm{PO} 2 / \mathrm{FiO} 2$ ratio at intubation time, plateau pressure in first $24 \mathrm{~h}$, hours of HFCN, ICU length of stay or mortality between groups.

Few patients were submetted to HFNC, so we separated patients into NIV and no-NIV group. Patients who had been under NIV before IMV had a lower static compliance (39 vs $36, p=0,004)$, lower PO2/ FiO2 ratio (91 vs $80, p=0,007$ ) and higher ICU mortality ( 23 vs 27 , $p=0,006)$. In relation to respiratory mechanics, there was a significantly lower static compliance in first $24 \mathrm{~h}$ in ICU non-survivors (36 vs $36, p=0,007)$.

Conclusion. In our cohort, ventilated COVID-19 patients who were later intubated and all non-survivors appear to have had worst compliances. Both groups had similar $\mathrm{PO} 2 / \mathrm{FiO} 2$ ratios suggesting that 
the extent lung injury was similar. In conclusion, we suggest that the intubation time does not seem to be significantly associated with poor outcomes and may be related with severity of disease. On the other hand, NIV seems to delay intubation and having a negative impact on lung mechanics and outcome.

\section{Reference(s)}

1. Rola P, Farkas J, Spiegel R, Kyle-Sidell C, Weingart S, Duggan L, Garrone M, Thomas A. Rethinking the early intubation paradigm of COVID-19: time to change gears? Clin Exp Emerg Med. 2020 Jun;7(2):78-80. https://doi. org/10.15441/ceem.20.043. Epub 2020 Jun 10. PMID: 32,521,584; PMCID: PMC7348679

2. Zuo MZ, Huang YG, Ma WH, Xue ZG, Zhang JQ, Gong YH, Che L; Chinese Society of Anesthesiology Task Force on Airway Management, Airway Management Chinese Society of Anesthesiology Task Force on. Expert Recommendations for Tracheal Intubation in Critically ill Patients with Noval Coronavirus Disease 2019. Chin Med Sci J. 2020 Feb 27;35(2):105-9. https://doi.org/10.24920/003724. Epub ahead of print. PMID: 32,102,726; PMCID: PMC7367670

3. Bhatraju PK, Ghassemieh BJ, Nichols M, Kim R, Jerome KR, Nalla AK, Greninger AL, Pipavath S, Wurfel MM, Evans L, Kritek PA, West TE, Luks A, Gerbino A, Dale CR, Goldman JD, O'Mahony S, Mikacenic C. Covid-19 in Critically III Patients in the Seattle Region-Case Series. N Engl J Med. 2020 May 21;382(21):2012-2022. https://doi.org/10.1056/NEJMoa2004 500. Epub 2020 Mar 30. PMID: 32,227,758; PMCID: PMC7143164.

4. Pandya A, Kaur NA, Sacher D, O'Corragain O, Salerno D, Desai P, Sehgal S, Gordon M, Gupta R, Marchetti N, Zhao H, Patlakh N, Criner GJ, University T; COVID-19 Research Group. Ventilatory Mechanics in Early vs Late Intubation in a Cohort of Coronavirus Disease 2019 Patients With ARDS: A Single Center's Experience. Chest. 2021 Feb;159(2):653-656. https://doi. org/10.1016/j.chest.2020.08.2084. Epub 2020 Aug 31. PMID: 32,882,246; PMCID: PMC7456835.

5. Matta A, Chaudhary S, Bryan Lo K, DeJoy R 3rd, Gul F, Torres R, Chaisson $\mathrm{N}$, Patarroyo-Aponte G. Timing of Intubation and Its Implications on Outcomes in Critically III Patients With Coronavirus Disease 2019 Infection. Crit Care Explor. 2020 Oct 23;2(10):e0262. https://doi.org/10.1097/CCE. 0000000000000262 . PMID: 33,134,950; PMCID: PMC7587415.

\section{0}

The effect of high flow oxygen via tracheostomy on diaphragm function in patients with prolonged weaning from mechanical ventilation: a randomized crossover study

E. Lytra'; I. Poularas²; D. Cokkinos'; Y. Papachatzakis²; V. Vlachakos²;

P. Ampatzis'; D. Exarchos'; ; D. Filippiadis ${ }^{3}$; S. Zakynthinos ${ }^{2}$; C. Routsi ${ }^{2}$ ${ }^{1}$ Department of radiology, Evangelismos Hospital, Athens, Greece; 2Department of intensive care, Evangelismos Hospital, Medical School, National and Kapodistrian University of Athens, Athens, Greece; ${ }^{3}$ Department of radiology, Attikon Hospital, Medical School, National and Kapodistrian University of A thens, Athens, Greece

Correspondence: C. Routsi

Intensive Care Medicine Experimental 2020, 9(1): 000320

Introduction. High flow oxygen therapy (HFOT) delivered through nasal cannulas has demonstrated clinical benefits, as compared to low-flow oxygen devices, and it is increasingly being utilized in every day clinical practice. Limited data exist on whether such effects are also present in HFOT through tracheostomy, thus it remains controversial.

Objectives. We undertook a physiological study to examine the shortterm effects of HFOT on diaphragmatic function, and hemodynamic and respiratory parameters in tracheostomized ICU patients with prolonged weaning from mechanical ventilation.

Methods. After disconnection from the ventilator, patients underwent a 30-min spontaneous breathing trial (SBT) receiving oxygen either conventionally via T-piece, or by HFOT delivered via tracheostomy, followed by a washout period of 15 min breathing through T-piece and 30 min receiving oxygen with the other modality in a randomized crossover manner. At the start and end of each study period, patients underwent an assessment, which consisted of blood gases, hemodynamic and respiratory measurements, i.e., respiratory rate (RR) and tidal volume (TV) measured with a calibrated handheld Wright's spirometer through the endotracheal tube, along with right hemidiaphragm ultrasonography which included excursion of diaphragmatic dome and thickness of diaphragmatic zone of apposition at end-inspiration and end-expiration. Then, diaphragmatic thickening fraction was calculated as the difference between end-inspiratory and end-expiratory thickness divided by end-expiratory thickness, and expressed inspiratory muscle effort.

Results. Eleven patients were enrolled in whom 19 sessions were completed uneventfully; eight patients were studied twice in 2 different days with alternate sessions and the remaining 3 patients were studied only once. The patients were randomly assigned to start the SBT with T-piece $(n=10)$, or with HFOT $(n=9)$. No significant carryover effect on the measured variables was detected. With HFOT compared to T-piece, TV and VE increased [\{median difference (interquartile range)\} 83.0 (38.0-105.0) vs. $-24.5(-87.3-29.3) \mathrm{mL}, \mathrm{p}=0.001$ and $0.01(-0.04-1.98)$ vs. $-0.01(-2.00-0.03) \mathrm{L}, \mathrm{p}=0.044$, respectively], RR/VT decreased [-7.4 $(-31.8--4.1)$ vs. $3.0(-8.6-17.1)$ breaths $/ \mathrm{min} / \mathrm{L}, \mathrm{p}=0.01], \mathrm{SaO} 2$ increased [1.1 (-0.5-3.3) vs. $-1.1(-2.7--0.1) \%, p=0.002$, respectively], and diaphragmatic excursion increased $[0.24(0.19-0.36)$ vs. $0.04(-0.12-0.11)$ $\mathrm{cm}, \mathrm{p}<0.001]$, whereas diaphragmatic thickening fraction did not change [-1.9 (-8.7- -0.2$)$ vs. $-0.5(-2.8-4.8) \%, p=0.235]$.

Conclusion. In ICU patients with prolonged weaning from mechanical ventilation, HFOT delivered through tracheostomy compared with low-flow oxygen delivery through T-piece, improves ventilation, pattern of breathing and blood oxygenation, without increasing inspiratory muscle effort.

\section{6}

Measuring peak cough flow to predict extubation failure in intensive care unit

G. RAGER ${ }^{1}$; D. Tran-Van ${ }^{1}$; B. Fontaine ${ }^{1}$

${ }^{1}$ Service anesthésie-réanimation, HIA Robert Picqué, villenave d'ornon,

France

Correspondence: G. RAGER

Intensive Care Medicine Experimental 2020, 9(1): 000336

Introduction. During weaning from mechanical ventilation, after recovery from respiratory failure and successful completion of a spontaneous breathing trial (SBT), removing the endotracheal tube represents a crucial step for every patients. Reintubation is associated with higher mortality and poorer outcomes in intensive care unit. Predictive parameters of unsuccessful extubation are controversial with divergent results in studies. Peak cough flow (PCF) is a measurement of cough strength which is suggested to predict extubation outcome. Methods. We conducted a prospective observational study at a single military hospital in France. Critically ill patients were consecutively screened for eligibility after $48 \mathrm{~h}$ under mechanical ventilation and considered by their physicians ready for weaning. The purpose of this study was to describe the predictive capacity of PCF with a cut off value of $35 \mathrm{~L} / \mathrm{min}$ (1) for extubation outcome in patients admitted to mixed medical-surgical intensive care unit. The PCF was measured by the respiratory therapist with a portable device connected to the tracheal tube before extubation.

Results. Out of a total of 154 patients who were extubated, 17 were failed extubation (11\%).

In the general population, the sensitivity and specificity of PCF with a cut off value of $35 \mathrm{~L} / \mathrm{min}$ was $59.1 \%(50.4 ; 67.4)$ and $52.9 \%(27.8 ; 77.0)$ respectively. The positive predictive value was $91.0 \%$ and the negative predictive value was $13.8 \%$. The area under the curve was 0.57 [0.44; $0.71]$.

In the multivariate analysis of our predictive model, four parameters were associated with extubation outcome when PCF was measured such as age $(\mathrm{OR}=0,96[0,93 ; 0,99] \mathrm{p}=0.01)$, Medical Research Council $(\mathrm{MRC})$ score $<48(\mathrm{OR}=0.24[0.09 ; 0.60] \mathrm{p}<0.001)$, duration of mechanical ventilation $(\mathrm{OR}=1.08[0.96 ; 1.25] \mathrm{p}=0.26)$ and endotracheal secretions with a frequency endotracheal suctioning $\leq 6 / 24 \mathrm{~h}(\mathrm{OR}=0.74$ $[0.27 ; 1.97] p=0.55)$. 
The sensitivity (80.0\%) and the positive predictive value (94.1\%) were improved when PCF was measured, in this specific population, to determine post-extubation outcome.

Conclusion. As a conclusion, PCF could be a predictive tool of successful extubation after passing a spontaneous breathing trial in selected group of critically patients.

\section{Reference(s)}

(1) Beuret P, Roux C, Auclair A, Nourdine K, Kaaki M, Carton M-J. Interest of an objective evaluation of cough during weaning from mechanical ventilation. Intensive Care Med. juin 2009;35(6):1090-3.

\section{6}

Ab ingestis in Acute Motor Axonal Neuropathy: an uncommon cause of severe ARDS requiring extra-corporeal support G. Salve'; M. Cavagnino ${ }^{1}$; L. Civardi²; A. Stella ${ }^{2}$; S. Bonaiti²; M. Pozzi ${ }^{2}$;

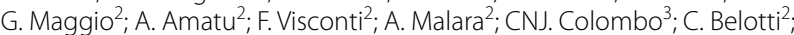
DM. Piovera ${ }^{2}$; C. Pellegrini' ${ }^{2}$; A. Degani ${ }^{2}$; F. Sciutti ${ }^{2}$; S. Mongodi' ${ }^{2}$ F. Mojoli ${ }^{1}$ ${ }^{1}$ Department of clinical-surgical, diagnostic and pediatric sciences, unit of anaesthesia and inte, The University of Pavia, Pavia, Italy; ${ }^{2}$ Intensive care unit, IRCCS Policlinico San Matteo Pavia, Pavia, Italy; ${ }^{3}$ Intensive Care Department, Fondazione Policlinico San Matteo IRCCS, Pavia, Italy Correspondence: G. Salve

Intensive Care Medicine Experimental 2020, 9(1): 000356

Introduction. A 44-year-old obese patient presented on March 13th to the Emergency Department (ED) with stomach pain and vomiting; he was discharged with symptomatic therapy after a negative headCT. $24 \mathrm{~h}$ later, he returned to ED with progressive cranial nerves palsy (dysphonia, dysarthria, dysphagia, bilateral mydriasis, ptosis) with normal conscious state and peripheral motricity. A second contrastenhanced head-CT ruled out acute haemorrhagic/ischemic events. While hospitalized for in-depth analyses, bilious vomit due to paralytic ileus caused massive aspiration, requiring emergency intubation. Botulism/Guillain-Barré syndrome were suspected, and antitoxins and immunoglobulin administered; however, all exams resulted negative (blood, urine, cerebral spine fluid, bronchoalveolar lavage cultures, C. Botulinum toxin in serum/faeces, head-MRI, electroencephalogram) and no improvement was observed in neurological status during daily sedation interruptions. On the other hand, the respiratory picture rapidly evolved to severe ARDS with refractory hypoxemia despite PEEP titration to $18 \mathrm{cmH} 2 \mathrm{O}$ and pronation, leading to non-protective ventilation ( $\mathrm{PaO} 2 / \mathrm{FiO} 260, \mathrm{Cl} 35 \mathrm{ml} / \mathrm{cmH} 2 \mathrm{O}$, driving pressure $17 \mathrm{cmH} 2 \mathrm{O}$ ). Methods. On March 21st a veno-venous femoro-femoral $23 \mathrm{Fr}-24 \mathrm{Fr}$ ECMO was placed by our ECMO-team and the patient transferred to our ICU. Second level diagnostic tests were performed in the hypothesis of an uncommon variant of Guillain-Barré syndrome: GQ1B antibodies and Immunohistochemestry were negative, but electromyography showed a severe motor axonal damage. A diagnosis of Acute Motor Axonal Neuropathy (AMAN) was made; having already received immunoglobulins, no specific therapy was indicated. His neurological status slowly recovered and now the patient is conscious, with no cranial nerves deficit and normal peripheral motricity. The lungs recovered after daily bronchoscopies and aspiration of bilious material and 18 days of ultra-protective ventilation (PEEP $18 \mathrm{cmH} 2 \mathrm{O}$ set with oesophageal pressure, tidal volume $4 \mathrm{ml} / \mathrm{kg}$ ) with extra-corporeal support (Fig. 1: lung-CT pre- and post-ECMO).

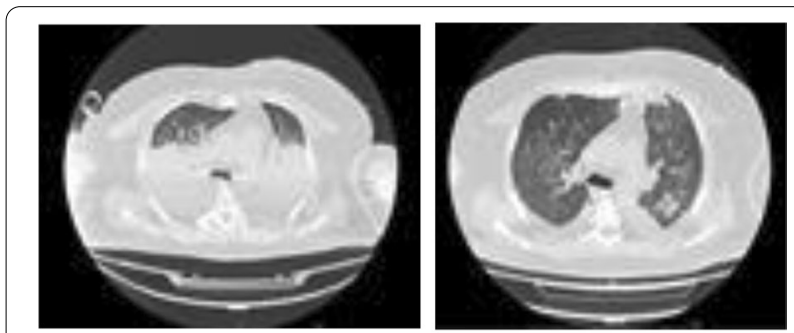

Figure 1

Results. Patients with AMAN may develop severe acute respiratory failure because of cranial nerves palsy and severe ab ingestis, despite normal conscious status; this has to be taken into account to adequate monitor deglutition capacity and establish correct timing for intubation.

Conclusion. It could be difficult to diagnose AMAN and to identify the trigger cause; AMAN is generally related to Campylobacter Jejun enteritis; our patient presented gastrointestinal symptoms, however cultures remained negative.

\section{Reference(s)}

2. Axonal Guillain-Barré syndrome: concepts and controversies Prof Satoshi Kuwabara, MD Prof Nobuhiro Yuki, MD. Lancet Neurol. 2013 Dec;12(12):1180-8 doi.org/https://doi.org/10.1016/S1474-4422(13) $70,215-1$

\section{1}

Dexamethasone and endotracheal obstruction in COVID-19 pneumonia. A preliminary study

G. Pérez-Acosta'; L. Santana-Cabrera²; J. Blanco-Lopez';

JM. González-Martín²; T. Carrillo-Garcia'; P. Padrón Espinosa';

J. Martín-González

${ }^{1}$ ICU, C.H.U. Insular-Materno Infantil, Las Palmas de Gran Canaria, Spain;

${ }^{2}$ Intensive care unit, Maternal and child Hospital, Las Palmas de Gran

Canaria, Spain

Correspondence: G. Pérez-Acosta

Intensive Care Medicine Experimental 2020, 9(1): 000361

Introduction. During the current COVID-19 pandemic, high percentage of patients require critical management with mechanical ventilation, due to of acute hypoxemic respiratory failure.

The pathogenesis causes diffuse alveolar damage with inflammatory infiltrates,immune response constitutes a fundamental factor in the organic damage.

Endotracheal-obstruction (EO) is one of the possible respiratory complications presented by the COVID-19 patients admitted to Intensive Care Units (ICU), is frequently associated with necrohemorrhagic tracheobronchitis

At the beginning of the pandemic, there has been controversy regarding the use of corticosteroids. However the use of corticosteroids appears to modulate lung inflammation and thereby reduce respiratory progression and mortality in most critical patients.

Objectives. Study the presentation of EO in COVID-19 patients admitted to the ICU. Analyze the relationship between the use of dexamethasone and the possible appearance of this complication.

Methods. During the first two waves (between February and December 2020) 96 patients were admitted to ICU with COVID-19 pneumonia Mean time to onset EO was 13.05 days ( \pm 13.34$)$. We performed a Kaplan-Meier curve to analyze EO and dexamethasone treatment. 


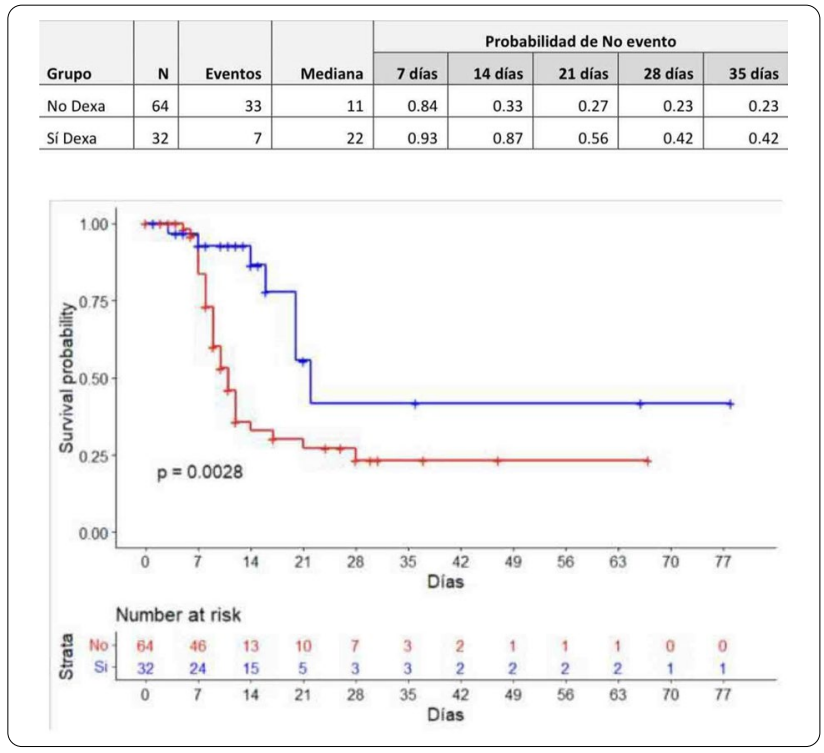

Multivariate regression analysis was performed to predict obstruction based on the demographic variables involved.

\begin{tabular}{lcllll}
\hline & Beta & EE & HR & IC 95 & p value \\
\hline Female Gender & 0.869 & 0.417 & 2.385 & $1.05-5.4$ & 0.037 \\
Age & 0.035 & 0.02 & 1.035 & $1-1.08$ & 0.081 \\
Height & 0.007 & 0.03 & 1.007 & $1-1.01$ & 0.017 \\
Dexamethasone & -1.802 & 0.881 & 0.165 & $0.03-0.93$ & 0.041 \\
\hline
\end{tabular}

Results. A total of 72 patients $(75 \%)$ required invasive mechanical ventilation. EO occurred in 40 patients (55\%).Patients treated with dexamethasone have a significantly lower risk of developing EO $(p=0.0028)$ compared to those patients who did not receive it. We find as risk factors for EO: female sex (Hazard ratio $H R=2.38$, $p=0.037)$, age $(H R=1.03, p=0.08)$ and height $(H R=1.007$, $\mathrm{p}=0.017$ ).

Conclusion. Patients receiving dexamethasone have a lower risk of endotracheal obstruction.

The possible protective relationship has not been described in the medical literature, it seems interesting to develop a complementary multicenter study to corroborate the results obtained.

\section{Reference(s)}

1. RECOVERY Collaborative Group, Horby P, Lim WS, et al. Dexamethasone in Hospitalized Patients with Covid-19-Preliminary Report. N Engl J Med. 2020

2. Wang D, Hu B, Hu C, et al. Clinical characteristics of 138 hospitalized patients with 2019 novel coronavirus-infected pneumonia in Wuhan, China. JAMA 2020;323:1061-9.

3. Dagens A, Sigfrid L, Cai E, et al. Scope, quality, and inclusivity of clinical guidelines produced early in the covid-19 pandemic: rapid review. BMJ 2020;369: m1936.

4. Pérez Acosta G, Santana-Cabrera L. Necrotizing tracheobronchitis with endotracheal tube obstruction in COVID-19 patients. Rev Clin Esp. 2020;220:531-533. https://doi.org/10.1016/j.rce.2020.05.002.
5. Moore JB, June CH. Cytokine release syndrome in severe COVID-19. Science 2020;368:473-4.

7. Zhu N, Zhang D, Wang W, et al. A novel coronavirus from patients with pneumonia in China, 2019. N Engl J Med. 2020;382:727-733.

8. COVID-19, Artificial intelligence-based prevention. Illustrious College of Physicians Las palmas de Gran Canaria

\section{5}

Modifications in lung compliance in COVID-19 patients during ICU admission

G. Pérez-Acosta ${ }^{1}$; J. Blanco-Lopez' ; L. Santana-Cabrera²; P. Padrón Espinosa'; ;. Carrillo-Garcia'; F. Suárez-Diaz ${ }^{3}$; J. Navarro-Mesa';

C. Suárez-Araujo ${ }^{5}$ j. Martín-González ${ }^{1}$

${ }^{1}$ Intensive care unit, C.H.U. Insular-Materno Infantil, Las Palmas de Gran

Canaria, Spain; ${ }^{2}$ Intensive care unit, Maternal and child Hospital, Las

Palmas de Gran Canaria, Spain; ${ }^{3}$ Idetic, University of Las Palmas de Gran Canaria, Las Palmas de Gran Canaria, Spain: ${ }^{4}$ Idetic, Universidad de las

Palmas de Gran Canaria, Las Palmas de Gran Canaria, Spain; ${ }^{5}$ luctc, Universidad de las Palmas de Gran Canaria, Las Palmas de Gran Canaria, Spain

Correspondence: $\mathrm{G}$. Pérez-Acosta

Intensive Care Medicine Experimental 2020, 9(1): 000375

Introduction. Coronavirus infection can have different clinical manifestations, pulmonary failure is the most frequent and serious problem in patients requiring admission to intensive care units (ICU). The patients with the highest risk profile are men older than 55 years and associated comorbidities such as Hypertension, Diabetes and Obesity, among others.

During admission to the ICU, pulmonary involvement by COVID-19 has been described, that the pattern of pulmonary complications changes, thus separating two clinical phenotypes based on lung mechanics. An early phase with high lung compliance, while other patients present a decreased compliance initially and even with the passage of time, possibly as a consequence of the lung disease.

Objectives. Study the pulmonary characteristics in ICU admitted patients with COVID-19 pneumonia. Describe the demographic factors and their possible relationship with the different COVID-19 lung phenotypes based on lung compliance. Analyze the relationship between changes in lung compliance with days of mechanical ventilation, ICU admission time, and mortality.

Methods. 96 patients were admitted to the ICU with COVID-19 pneumonia, demographic variables were collected. Compliance respiratory data were studied during ICU admission. We analyzed the clinical characteristics according two groups of lung compliance. Comparing the group of patients with high compliance with the group with decreased compliance.

Results. The mean lung compliance was $42.1( \pm 13.8) \mathrm{mL} / \mathrm{cmH} 20$ with quasimodal distribution. Compliance changes in day 19th of admission. Most patients with higher lung compliance were men, no other differences in clinical factors were found. Ventilation days and ICU length were the same in the two compliance groups.
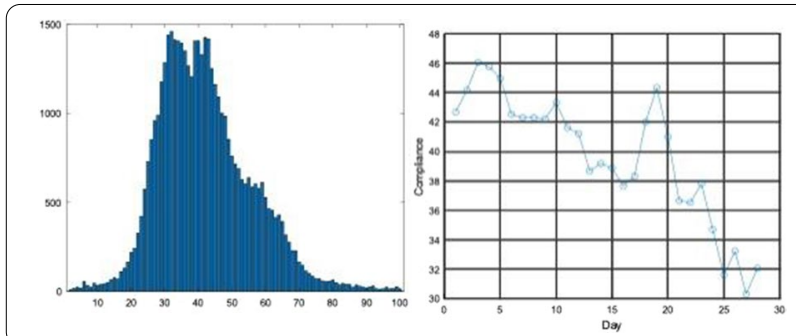


\begin{tabular}{|c|c|c|c|}
\hline $\mathrm{N}=72$ & $\begin{array}{c}\text { Crs }<42.08 \\
\mathrm{~N}=37\end{array}$ & $\begin{array}{c}\text { Crs }>42.08 \\
\mathrm{~N}=35\end{array}$ & p value \\
\hline Age, mean \pm SD & $64,3 \pm 11$ & $58.97 \pm 15.89$ & 0.182 \\
\hline Gender, men, $\mathbf{n}(\%)$ & $17(45.95)$ & $31(88.57)$ & 0.0003 \\
\hline Hypertension, n(\%) & $24(64.86)$ & $23(65.71)$ & 0.863 \\
\hline Heart disease, $n(\%)$ & $7(18.92)$ & $6(17.14)$ & 0.911 \\
\hline Diabetes, $\mathbf{n}(\%)$ & $19(51.35)$ & $13(37.14)$ & 0.329 \\
\hline Obesity, n(\%) & $18(48.65)$ & $14(40)$ & 0.616 \\
\hline \begin{tabular}{|l}
$\begin{array}{l}\text { Pulmonar Chronic Disease, } \\
\mathrm{n}(\%)\end{array}$ \\
\end{tabular} & $6(16.22)$ & $3(8.57)$ & 0.532 \\
\hline Cancer, n(\%) & $4(10.81)$ & $3(8.57)$ & 0.938 \\
\hline Prone Positioning, $\mathbf{n}(\%)$ & $21(56.76)$ & 19 (54.29) & 0.979 \\
\hline \begin{tabular}{|l|}
$\begin{array}{l}\text { Neuromuscular blocking } \\
\text { agents, } \mathrm{n}(\%)\end{array}$ \\
\end{tabular} & $33(89.19)$ & $34(97.14)$ & 0.388 \\
\hline Corticoesteroids, n(\%) & $28(75.68)$ & $32(91.43)$ & 0.139 \\
\hline \begin{tabular}{|l} 
Endotracheal obstruction, \\
$n(\%)$
\end{tabular} & $21(56.76)$ & $18(51.43)$ & 0.828 \\
\hline \begin{tabular}{|l} 
Time from symptoms onset, \\
days, median (laR)
\end{tabular} & $6(4-7)$ & $5(3-7)$ & 0.277 \\
\hline Intubation days, median (IQR) & $11(7-15)$ & $14(8-17)$ & 0.252 \\
\hline $\begin{array}{l}\text { Mechanical ventilation days, } \\
\text { median (IQR) }\end{array}$ & $16(7.5-28.5)$ & $21.5(8-31)$ & 0.464 \\
\hline ICU days, median (IQR) & $20.5(9-27.5)$ & $26(12-39.5$ & 0.009 \\
\hline ICU deaths, $n(\%)$ & 0 & $1(2.86)$ & 0.977 \\
\hline Hospital deaths, $n(\%)$ & $10(27.03)$ & $3(8.57)$ & 0.083 \\
\hline
\end{tabular}

Conclusion. In patients with severe respiratory failure due to COVID19 pneumonia, we observed a quasi unimodal pulmonary compliance distribution with a mean of $42.1( \pm 13.8) \mathrm{mL} / \mathrm{cmH} 20$. Men has higher compliance. No differences in risk factors were found. During ICU admission compliance changes in day 19th. Lower compliance values were not associated with higher ICU stay. Complementary studies are needed to corroborate these results.

\section{Reference(s)}

1. Gattinoni L, Coppola S, Cressoni M, Busana M, Rossi S, Chiumello D. COVID-19 does not lead to a "typical" acute respiratory distress syndrome. Am J Respir Crit Care Med. 2020;201(10):1299-300.

2. Guan W, Ni Z, Hu Y, Liang W, Ou C, He J, et al. Clinical characteristics of coronavirus disease 2019 in China. N Engl J Med. 2020;382(18):1708-20.

3. Zhou F, Yu T, Du R, Fan G, Liu Y, Liu Z, et al. Clinical course and risk factors for mortality of adult inpatients with COVID-19 in Wuhan, China: a retrospective cohort study. Lancet. 2020;395(10,229):1054-62.

4. None

5. COVID-19, Artificial intelligence-based prevention. Illustrious College of Physicians Las palmas de Gran Canaria

\section{6}

\section{Clinical-histopathologic correlation of severe pulmonary}

\section{SARS-CoV-2 infection}

M. Wu'; G. Lopez²; D. Ottolina ${ }^{3}$; J. Montomoli4; T. Fossali ; $;$ L. Carsana ${ }^{3}$; R. Rech ${ }^{3}$; A. Castelli ${ }^{3}$; C. Cogliati ${ }^{3}$; E. Catena ${ }^{3}$; M. Nebuloni ${ }^{5}$; R. Colombo 6 ${ }^{1}$ Division of internal medicine, ASST Fatebenefratelli Sacco-Ospedale Luigi Sacco, Milan, Italy; ${ }^{2}$ Pathology unit, ASST Fatebenefratelli SaccoOspedale Luigi Sacco, Milano, Italy; ${ }^{3}$ Division of anesthesiology and intensive care, ASST Fatebenefratelli Sacco-Ospedale Luigi Sacco, Milan, Italy; 4Division of anesthesiology and intensive care, Ospedale degli Infermi, Rimini, Italy; ${ }^{5}$ Pathology unit, ASST Fatebenefratelli SaccoOspedale Luigi Sacco, Milan, Italy; ${ }^{6}$ Division of anesthesiology and intensive care, ASST Fatebenefratelli Sacco—Ospedale Luigi Sacco, Milano, Italy

Correspondence: R. Colombo

Intensive Care Medicine Experimental 2020, 9(1): 000376

Introduction. Although autoptic pulmonary findings have been described in patients who died from severe COVID-19 evidence regarding the correlation between clinical data and histopathologic pulmonary damage is lacking.
Objectives. To examine clinical-histopathologic correlation in patients deceased because of COVID-19 pneumonia.

Methods. We analyzed lung tissue samples from consecutive patients who died from COVID-19 between February 29 and June 30, 2020, at Luigi Sacco Hospital in Milan. Lung histopathologic patterns were scored in a semiquantitative manner according to the percentage of the tissue involved: $0(0 \%), 1(1-33 \%), 2(34-66 \%)$, and $3(67-100 \%)$ of each pulmonary lobe [1]. Therefore, the sum of scores ranged from 0 to 15 for each pattern. The pre-specified histopathologic patterns were [1-3]: i) Exudative diffuse alveolar damage (DAD), characterized by edema with hyaline membrane formation; ii) Proliferative DAD, with prominent type-II pneumocyte hyperplasia and atypia; iii) Organizing pneumonia, with prominent fibroblast proliferation; iv) Acute fibrinous organizing pneumonia (AFOP), with intra-alveolar fibrin aggregates with inflammatory cells; $v$ ) Interstitial pneumonia, with lymphocytic infiltrates within alveolar septa and interstitium; vi) Bronchopneumonia, characterized by foci of granulocytic infiltrates with or without necrosis; vii) Arteriolar thrombi, within arteriolar blood vessels; viii) Intracapillary megakaryocytes; ix) Areas of normal lung (without pathological features).

Since different patterns coexist, we performed a hierarchical clustering from histopathologic findings of consecutive autopsies and then used them in the clinical data analysis.

Results. Full pulmonary examination was available in 75 of 92 autopsies. 48 patients (64\%), 44 from ICU and 4 from medical ward, had full clinical data. Histopathologic patterns and main differences between clusters are summarized in Fig. 1. Cluster 3 had prevalence of Proliferative DAD and organizing pneumonia, while cluster 4 had prevalence of areas of normal lung. Length-of-stay (LOS) in ICU ( $p<0.0001)$, LOS in hospital $(p<0.0001)$, days on mechanical ventilation $(p<0.0001)$, days on positive airway pressure $(p<0.0001)$, mean positive end-expiratory pressure (PEEP) $(p=0.007)$, PEEP $x$ days on mechanical ventilation $(p=0.003)$, PEEP $x$ days on positive pressure airway $(p=0.003)$ worst serum albumin $(p=0.017), \mathrm{IL}-6(p=0.047)$, and kidney SOFA $(p=0.001)$ differed among histopathological clusters.

Conclusion. Our study draws a comprehensive link between the clinical and histopathologic findings in a large cohort of COVID-19 patients. These results, far from being merely descriptive, provide valuable insights into the pathophysiological mechanisms underlying the disease course and highlight that the impact of therapeutic strategies (e.g. mechanical ventilation) on lung tissues should be reminded to accurately tailor patients' management.

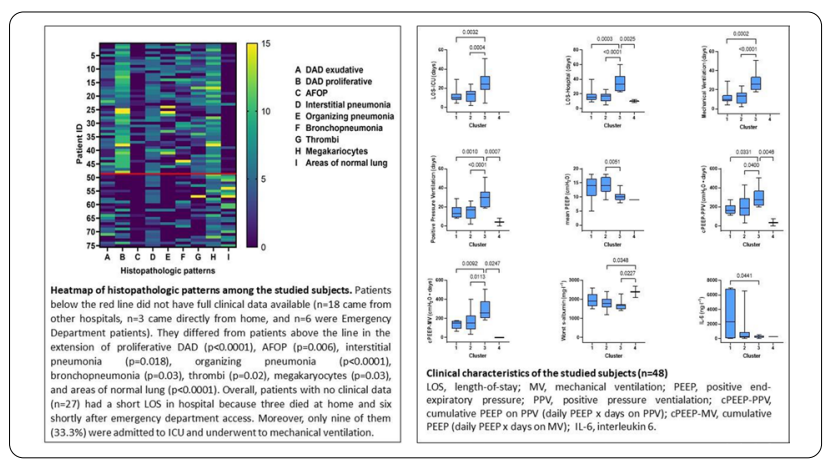

\section{Reference(s)}

1. Carsana, L., et al., Pulmonary post-mortem findings in a series of COVID19 cases from northern Italy: a two-centre descriptive study. Lancet Infect Dis, 2020. 20(10): p. 1135-1140.

2. Menter, T., et al., Postmortem examination of COVID-19 patients reveals diffuse alveolar damage with severe capillary congestion and variegated findings in lungs and other organs suggesting vascular dysfunction. Histopathology, 2020. 77(2): p. 198-209.

3. Schaller, T., et al., Postmortem Examination of Patients With COVID-19. JAMA, 2020. 323(24): p. 2518-2520. 
000390

Long-term symptom burden of COVID-19 according to grade of severity

A. Liao' ${ }^{1}$; R. Sarkarr ${ }^{1}$; R. O'reilly' ${ }^{1}$;. Matthews ${ }^{1}$; S. Enyioma ${ }^{1}$; K. Brignall ${ }^{1}$

${ }^{1}$ Respiratory medicine and critical care, Medway Maritime Hospital, Gill-

ingham, United Kingdom

Correspondence: A. Liao

Intensive Care Medicine Experimental 2020, 9(1): 000390

Introduction. It is important that every hospital, serving its unique population, develops an understanding of the long-term sequelae of COVID-19 which is still being investigated. This study was set up to examine the multi-system symptomatology of patients admitted with various degrees of severity in a single centre in the UK.

Methods. We performed a retrospective analysis of symptoms and markers of function in surviving patients admitted to this district general hospital with COVID-19 during the "first wave". Data on persistent and initial symptoms were collected at first follow-up. Severity was defined according to where patients were treated in the following way: "Mild"- general medical ward $(n=49)$. "Moderate"- respiratory ward $(n=50)$. "Severe"- critical care $(n=17)$. Availability of respiratory support varied between areas. Continuous positive airway pressure (CPAP), non-invasive ventilation (NIV) and high flow nasal oxygen (HFNO) were available on the respiratory ward and the high dependency unit (HDU), with scope for invasive monitoring on HDU. Intubated patients were treated in ICU. Chi-squared testing was used to determine crude associations between severity and presence of symptoms at follow-up.

Results. Median age in the cohort $(\mathrm{N}=116)$ was 58.5 years (IQR 52-69), majority male (60\%). Median length of admission and time to follow-up were 9 days (IQR 4-13.5) and 11 weeks post-discharge (IQR 9-13) respectively. Dyspnoea was the most reported symptom initially (97\%) and at follow-up (62\%). Severity of dyspnoea was graded for 81 patients using the Medical Research Council Dyspnoea Scale. Twenty-three (28\%) were graded 4 or 5 at follow-up. Psychological symptoms were common. $38 \%$ scored $\geq 8$ in the Hospital Anxiety and Depression Scale for anxiety and 34\% for depression. Of 81 previously employed patients, 34 (42\%) had not returned to work. New onset memory problems were associated most strongly to the severe group $(P<0.001)$, as was persistent fatigue $(P=0.004)$. Association with severity was also seen in patient reported anxiety $(P=0.02)$ and depression $(P=0.03)$. Severity was not associated with the presence of other physical symptoms.

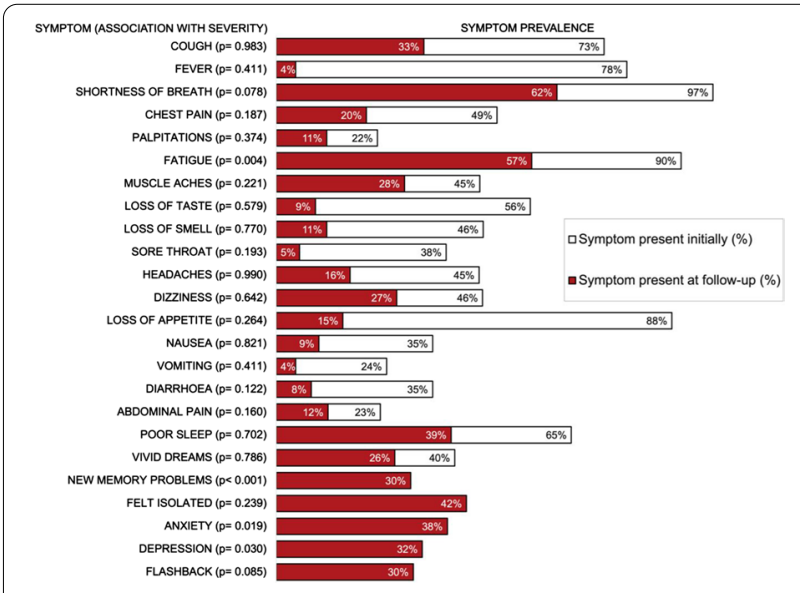

Conclusion. The significant amount of multi-system residual symptoms, especially in patients discharged from critical care warrant a coherent and systematic follow-up. This service should include comprehensive psychological and physical, mainly pulmonary assessment capabilities.
000394

Accuracy of pulse oximetry (SpO2) with different oximeters.

Oxygap study

MA. Blanchet'; G. Mercier'; PA. Bouchard ${ }^{1}$; E. Rousseau'; F. Lellouche

${ }^{1}$ Centre de recherche, Institut Universitaire de Cardiologie et de Pneu-

mologie de Québec-Université Laval, Québec, Canada

Correspondence: M.A. Blanchet

Intensive Care Medicine Experimental 2020, 9(1): 000394

Introduction. An accurate $\mathrm{SpO} 2$ value is critical in order to optimally titrate the $\mathrm{O} 2$ flow or FiO2 delivered to patients and to follow oxygenation guidelines. It has been shown with closed-loop oxygen titration that small variations in $\mathrm{SpO} 2$ target greatly affect the oxygen flow required, which may have a relevant impact on clinical decisions. However, the oximeters' accuracy appears to vary widely from model to model, leading to an underestimation or an overestimation of actual $\mathrm{SaO} 2$ values. This variability may represent an obstacle to an optimal delivery of oxygen therapy, including the implementation of guidelines. Objectives. The objective of this study is to assess the accuracy and bias of the $\mathrm{SpO} 2$ value measured by several oximeters compared to the Reference value, arterial oxygen saturation ( $\mathrm{SaO} 2)$ measured by arterial gases in intubated and spontaneously breathing patients in the intensive care unit.

Methods. (ClinicalTrials.gov ID: NCT04772183).

The study was approved by the local ethics board with a waiver of consent. We include stable patients hospitalized in the intensive care unit with an arterial catheter in place. Main exclusion criteria are: instability requiring frequent ventilator parameters modifications, poor $\mathrm{SpO} 2$ signal, infectious isolation, $\mathrm{SpO} 2>96 \%$. We evaluate six oximeters: Nonin (Plymouth, MN), Massimo (Irvine, CA), Philips (Eindhoven, Netherlands) and Nellcor (Pleasanton, CA), as well as two low-cost oximeters (Contec CMS50DL and Beijing Choice C20). The oximeter sensors are placed on the finger in a randomized order. The $\mathrm{SpO} 2$ values are collected simultaneously at least two minutes after all oximeters were put in place and are compared to simultaneous arterial blood gas (gold standard). The $\mathrm{SpO} 2$ values from each oximeter will be compared to the $\mathrm{SaO} 2$ value with the Bland \& Altman method. We plan to include 210 patients in the study.

Results. We present here the preliminary results of 40 measurements in 36 patients ( 29 men and 7 women, mean age $68.7 \pm 8.1$ years). The patients skin colour was graded with the Fitzpatrick scale (type 1: 14, type 2: 20, type 3: 2). 7 patients were under invasive ventilation with a mean $\mathrm{FiO} 2$ of $38 \pm 11 \%, 17$ under conventional oxygen therapy with a mean $\mathrm{O} 2$ flow of $2.5 \pm 2.3 \mathrm{~L} / \mathrm{min}$ and 12 did not have respiratory support. Three oximeters underestimated the $\mathrm{SaO} 2$ while two oximeters overestimated $\mathrm{SaO} 2$ (Philips). The mean gap between $\mathrm{SpO} 2$ value and the $\mathrm{SaO} 2$ for each oximeter varies from $-2.9 \%$ (Nonin) to $0.7 \%$ (Philips) (Table).

\begin{tabular}{|c|c|c|c|c|c|c|c|}
\hline & \multirow{2}{*}{$\begin{array}{l}\text { SaO2 } \\
\text { (Refer- } \\
\text { ence) }\end{array}$} & \multicolumn{6}{|l|}{$\mathrm{SpO} 2$} \\
\hline & & Nonin & $\begin{array}{l}\text { Nell- } \\
\text { cor }\end{array}$ & $\begin{array}{l}\text { Mas- } \\
\text { simo }\end{array}$ & Philips & $\begin{array}{l}\text { Con- } \\
\text { tec }\end{array}$ & Beijing \\
\hline $\begin{array}{l}\text { Mean } \pm S D \\
(\%)\end{array}$ & $\begin{array}{l}93.7 \\
\pm 1.8\end{array}$ & $\begin{array}{l}90.9 \\
\pm 2.4\end{array}$ & $\begin{array}{l}93.2 \\
\pm 2.3\end{array}$ & $\begin{array}{l}93.7 \\
\pm 2.2\end{array}$ & $\begin{array}{l}94.4 \\
\pm 2.5\end{array}$ & $\begin{array}{l}90.8 \\
\pm 2.5\end{array}$ & $\begin{array}{l}93.4 \\
\pm 2.3\end{array}$ \\
\hline $\begin{array}{r}\text { Gap SaO2- } \\
\text { SpO2 (\%) }\end{array}$ & - & -2.9 & -0.5 & 0.0 & 0.7 & -2.8 & -0.3 \\
\hline $\begin{array}{l}\text { Underesti- } \\
\text { mation } \\
\text { (\% measure- } \\
\text { ments) }\end{array}$ & - & 100 & 41 & 43 & 23 & 89 & 49 \\
\hline $\begin{array}{l}\text { Overestima- } \\
\text { tion } \\
\text { (\% measure- } \\
\text { ments) }\end{array}$ & - & 0 & 18 & 25 & 50 & 5 & 36 \\
\hline
\end{tabular}

Conclusion. We found significant gaps between the tested oximeters. These informations will be helpful to specify the gap between main oximeters, to adapt the guidelines for oxygen therapy to the oximeters used and to choose the target with closed-loop oxygen titration. 
000428

Respiratory Intra-Cycle Worsening of Pulmonary Hemodynamics during ARDS

Correspondence: A. Santos

Intensive Care Medicine Experimental 2020, 9(1): 000428

Introduction. Pulmonary hemodynamics worsening during ARDS is frequent and clinically relevant. However, little information exists about how pulmonary hemodynamics changes during the respiratory cycle.

Objectives. To evaluate how pulmonary hemodynamics changes during the respiratory cycle in a porcine model of ARDS.

Methods. 6 pigs were subjected to lung saline lavages followed by $2 \mathrm{~h}$ of injurious mechanical ventilation to create a model of ARDS. Pulmonary hemodynamics was evaluated by means of a transonic flow sensor and a high fidelity microtip pressure catheter placed in the pulmonary artery trunk. Signals from these sensors were acquired during a 2 min priod before (Baseline) and $2 \mathrm{~h}$ after the ARDS model was established. Mechanical ventilation was set in control volume with PEEP $8 \mathrm{cmH} 2 \mathrm{O}$, tidal volume $6 \mathrm{ml} / \mathrm{kg}$, FiO2 1 and respiratory rate to keep an EtCO2 around $45 \mathrm{mmHg}$. Pulmonary hemodynamic variables were calculated beat by beat and then subjected to a custom algorithm that allows their evaluation at each moment of the respiratory cycle. This analysis allows expressing how the variables behaves during the whole respiratory cycle, during expiration and how it changes during inspiration.

Results. No differences were found in tidal volume and PEEP between the experimental conditions but driving pressure significantly increased during ARDS when compared with Baseline (18 \pm 3 vs $6 \pm 1 \mathrm{cmH} 2 \mathrm{O}, \mathrm{p}<0.001)$. Main results of hemodynamic evaluation are described in Table 1.

Table 1. Expiratory and inspiratory Pulmonary artery hemodynamics

\begin{tabular}{|c|c|c|c|}
\hline Variable & Baseline & ARDS & $p^{a}$ \\
\hline \multicolumn{4}{|c|}{ Stroke Volume (mI) } \\
\hline Expiratory & $38(3)$ & $33(6)$ & 0.046 \\
\hline Inspiratory & $-3(1)^{b}$ & $-3(1)^{b}$ & 0.99 \\
\hline Total & $35(3)$ & $30(6)$ & 0.046 \\
\hline \multicolumn{4}{|c|}{ Mean pressure $(\mathrm{mmHg})$} \\
\hline Expiratory & $19.5(2.0)$ & $27.3(4.3)$ & 0.004 \\
\hline Inspiratory & $-0.5(0.3)^{b}$ & $-0.7(0.3)^{b}$ & 0.004 \\
\hline Total & $19.1(2.1)$ & $26.7(4.2)$ & 0.004 \\
\hline \multicolumn{4}{|c|}{ Pulse Pressure (mmHg) } \\
\hline Expiratory & $11.9(4.1)$ & $19.4(3.6)$ & 0.007 \\
\hline Inspiratory & $-0.8(0.4)^{b}$ & $-1.5(0.7)^{b}$ & 0.028 \\
\hline Total & $11.1(3.8)$ & $17.9(3.0)$ & 0.008 \\
\hline \multicolumn{4}{|c|}{ Compliance $\left(\mathrm{ml} / \mathrm{cmH}_{2} \mathrm{O}\right)$} \\
\hline Expiratory & $3.59(1.34)$ & $1.77(0.49)$ & 0.01 \\
\hline Inspiratory & $-0.07(0.04)^{b}$ & $-0.04(0.00)^{b}$ & 0.169 \\
\hline Total & $3.52(1.32)$ & $1.72(0.49)$ & 0.01 \\
\hline \multicolumn{4}{|c|}{ Resistance (dyn. $\mathrm{c}^{\mathrm{cm}} \mathrm{cm}^{-5}$ ) } \\
\hline Expiratory & $483(120)$ & $682(198)$ & 0.069 \\
\hline Inspiratory & $34(13)^{b}$ & $50(12)^{b}$ & 0.306 \\
\hline Total & $517(130)$ & $732(222)$ & 0.074 \\
\hline \multicolumn{4}{|c|}{ Effective arterial elastance $(\mathrm{mmHg} / \mathrm{ml})$} \\
\hline Expiratory & $0.51(0.07)$ & $0.84(0.19)$ & 0.003 \\
\hline Inspiratory & $0.04(0.01)^{\mathbf{b}}$ & $0.08(0.04)^{b}$ & 0.041 \\
\hline Total & $0.55(0.08)$ & $0.92(0.22)$ & 0.003 \\
\hline
\end{tabular}

${ }^{a} \mathrm{p}$ for the comparison between baseline an ARDS ate the corresponding respiratory stage.

${ }^{b} \mathrm{p}<0.01$ testing if the change during inspiration was significant.

Comparing with Baseline, ARDS decreased stroke volume and pulmonary artery compliance and increased mean pulmonary artery pressure and pulmonary effective arterial elastance (lumped parameter of arterial load) during the whole respiratory cycle and during expiration. Comparing with expiration, inspiration caused a decrease in the pulmonary artery mean and pulse pressure and an increase in pulmonary artery resistance and effective arterial elastance both in Baseline and in ARDS; these inspiratory effects were significantly larger during ARDS than in Baseline for all variables except for resistance.

Conclusion. In this experimental ARDS model, changes in pulmonary hemodynamics caused by breathing during mechanical ventilation were described. Especially, a larger tidal increase in pulmonary arterial load and in consequence a respiratory intra-cycle worsening on pulmonary hemodynamics caused by ARDS was demonstrated. This might be related with mechanics and functional change in the respiratory system cause by ARDS as well as mechanical ventilation.

\section{Reference(s) \\ 1. 2020 Professor Burkhard Lachmann Award for Experimental Research \\ 2. 2012 ESICM ECCRN Basic Science Award}

\section{9}

In silico testing of a non-invasive, individualised PEEP titration method for optimal lung compliance during pressure support ventilation

R. Warnaar'; E. Oppersma ${ }^{1}$; A. Cornet ${ }^{2}$; D. Donker ${ }^{3}$

${ }^{1}$ Cardiovascular \& Respiratory Physiology, University of Twente, Enschede, Netherlands; ${ }^{2}$ Department of intensive care medicine, MST, Enschede, Netherlands; ${ }^{3}$ Department of intensive care medicine, UMC Utrecht, Utrecht, Netherlands

Correspondence: R. Warnaar

Intensive Care Medicine Experimental 2020, 9(1): 000429

Introduction. Comprehensive quantification of respiratory system mechanics during pressure support ventilation (PSV) classically requires invasive measurements or interruptions of spontaneous breathing. This limits broad clinical applicability of these methods for lung compliance optimisation during PSV. Therefore, we present a non-invasive approach for individualised PEEP titration based on dynamic lung compliance measurements at the bedside.

Objectives. This study focuses on the sensitivity of a non-invasive method for bedside quantification of dynamic lung compliance in response to simulated PEEP interventions in patients receiving PSV.

Methods. An individual patient's respiratory system was modelled in Simulink R2020b (The MathWorks, Inc., MA, USA) as an electrical analogue with a pressure dependent lung compliance (Fig. 1a). This in silico patient was ventilated in PSV mode applying 7 different PEEP levels: 0,2 , $4,6,8,10,12$, and $14 \mathrm{cmH} 2 \mathrm{O}$. Simulations were run during spontaneous breathing at each PEEP level. The expiratory time constant (RC-time = airway resistance * respiratory system compliance $=$ RAW * CRS) was estimated [1]. The airway resistance was determined as the time derivative of the airway pressure divided by the time derivative of the flow, both around zero flow at end-inspiration. CRS was calculated as the RC-time over the median airway resistance over all breaths. The correlation between true, i.e. modelled, and measured compliance was quantified with the Pearson correlation coefficient.

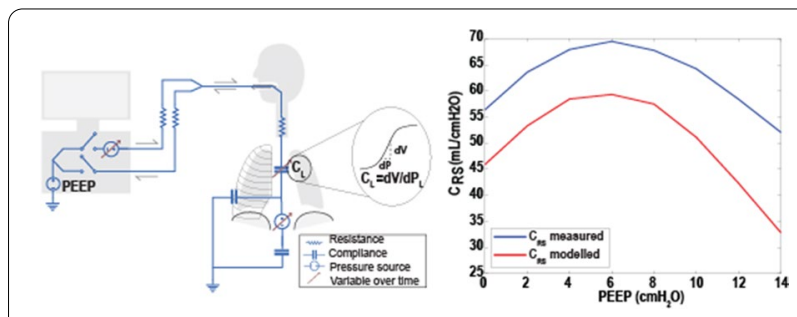

Fig. 1. a) Individual ventilated patient model represented as an electrical analogue. The respiratory system compliance (CRS) is estimated at various PEEP levels, affecting the working point on the lung compliance (CL) curve. b) Respiratory system compliance (CRS): measured (blue), and modelled (red) 
Results. Both measured and modelled CRS at each PEEP level are shown in Fig. 1b. The measured compliances correlate well with the modelled compliances $(r=0.85, p<0.01)$, although they show a systematic offset. Importantly, individualised titration of PEEP was possible using the proposed method, as the PEEP level for maximal lung compliance was identical in both curves.

Conclusion. We demonstrate that this method allows for non-invasive, individualised PEEP titration during PSV ventilation in a modelled clinical case. This approach has great potential for future bedside use, and therefore deserves further clinical testing, aiming to optimise pressure support ventilation, and promote patient-ventilator synchrony and weaning.

\section{Reference(s)}

1. N. Al-Rawas et al., "Expiratory time constant for determinations of plateau pressure, respiratory system compliance, and total resistance," Crit. Care, vol. 17, no. 1, p. R23, 2013, https://doi.org/10.1186/cc12500.

\section{4}

\section{A Novel CPAP Weaning Protocol for COVID-19 used} across the Critical Care and Respiratory Medicine Interface L. Skelly' ; D. Devis ${ }^{1}$; J. Thomas '; P. Smith, ; E. Scholes-Pearson ${ }^{1}$; T. Furniss, ${ }^{1}$ ${ }^{1}$ Critical care, Warrington Hospital, Warrington, United Kingdom Correspondence: L. Skelly

Intensive Care Medicine Experimental 2020, 9(1): 000434

Introduction. COVID-19 was declared a pandemic on the 12th March 2020. Due to the novel nature of the disease and the pressure it forced upon healthcare systems the management of the disease varied. Initially early intubation and ventilation was advised but this rapidly changed due to the volume of patients that required respiratory support. The effectiveness of Continuous Positive Airway Pressure (CPAP) in acute respiratory failure had previously been investigated, prior to the pandemic. This suggested that management of respiratory failure with CPAP contributed to a reduction in several outcomes including length of stay in ICU and the number of patients that required intubation (1,2). By April 2020, the National Institution for Health and Care Excellence (NICE) revised guidance recommending CPAP in COVID-19 patients whilst recognising a lack of evidence of its efficacy (3). International evidence proposed that hypoxaemic patients with COVID-19 pneumonitis responded well to CPAP which indicated a vital role for its use. In addition to this, using CPAP could reduce the need for intubation and ventilation and therefore reduce the need for advanced respiratory support (4). A CPAP weaning protocol was used to guide patient care using intermittent CPAP and high flow nasal oxygen (HFNO) in our District General Hospital (DGH).

Methods. This is a retrospective, observational study conducted at Warrington general hospital in the United Kingdom. In normal circumstances Warrington DGH has a 20 bed ICU. Due to the constraints of COVID-19 the bed base was expanded into theatre recovery. The CPAP weaning protocol was initiated for each patient that was admitted to Intensive Care for CPAP due to COVID-19 pneumonitis. The weaning plan provided guidance for the use of intermittent CPAP and HFNO during breaks. Patient demographics and outcomes were recorded as part of ICNARC data and compared with national statistics. After the demand of the second wave reduced, data was collected through a questionnaire that was completed by the multidisciplinary team (MDT) working on Intensive Care to gain feedback regarding the use of the weaning plan.

Results. The CPAP weaning regimen was used primarily in Intensive care and provided continuity of care when patients were subsequently stepped down to ward level care under the medical team. Local statistics show a mortality rate was $16.6 \%$ that was similar to national Figs. $17.8 \%$ (5). The median ICU length of stay was 5.5 days (IQR: 3.2, 8.3). Data was collected from the MDT that used the regimen. $94 \%$ of staff found the weaning protocol easy to use, and $92.5 \%$ felt that it assisted with patient handover. This was particularly important during the pandemic due to the demand on Intensive care it was important to provide consistency and aid communication within the MDT. In times where staffing was stretched, temporary staffing increased and nursing ratios were placed under strain this tool provided guidance for patient care. $97 \%$ of users found that it assisted the assessment of patient progress which in turn emphasised flow from Intensive care to ward level care.

Conclusion. This novel CPAP weaning pathway aided continuity of care and assessment of patient progress during their stay in ICU. In our hospital it added clarity to the handover, particularly from intensive care to ward level care. The CPAP weaning plan did involve the use of CPAP in combination with HFNO which may have limited availability in other centres. However, if available could be a useful tool to consider when treating patients with COVID-19 pneumonitis in the future.

\section{Reference(s)}

1. Faria DAS, da Silva EMK, Atallah Álvaro N, et al. Noninvasive positive pressure ventilation for acute respiratory failure following upper abdominal surgery. Cochrane Database Syst Rev 2015.

2. 4.Wu Z, McGoogan JM. Characteristics of and Important Lessons From the Coronavirus Disease 2019 (COVID-19) Outbreak in China: Summary of a Report of 72314 Cases From the Chinese Center for Disease Control and Prevention. JAMA 2020:323:1239-42.

3. No Conflicts of Interest.

4. Williams TA, Finn J, Perkins GD, et al. Prehospital continuous positive airway pressure for acute respiratory failure: a systematic review and meta-analysis. Prehosp Emerg Care 2013;17:261-73.

5. NHS England. Guidance for the role and use of non-invasive respiratory support in adult patients with COVID19 (confirmed or suspected), 2020

6. Intensive Care National Audit \& Resource Centre. ICNARC report on COVID19 in critical care: England, Wales and Northern Ireland 26th March 2021. ICNARC 2021

\section{7}

High rate of endotracheal tube occlusions reported in COVID-19 patients: analyse of humidification devices use

F. Lellouche'; CA. Lavoie-Bérard, ${ }^{2}$; E. Rousseau, ${ }^{3}$; PA. Bouchard, ${ }^{3}$.

M. Simon, ; JC. Lefebvre, ${ }^{4}$

${ }^{1}$ Critical care and research center, University Institute of Cardiology and Respirology of Quebec, Québec, Canada; ${ }^{2}$ Medicine, Laval University, Québec, Canada; ${ }^{3}$ Research center, University Institute of Cardiology and Respirology of Quebec, Québec, Canada; ${ }^{4}$ Département d'anesthésiologie et de soins intensifs, division de soins intensifs, Laval University, Québec, Canada

Correspondence: F. Lellouche

Intensive Care Medicine Experimental 2020, 9(1): 000437

Introduction. High rates of endotracheal tube occlusions have been reported in COVID-19 patients (1-7). Underhumidification of inspiratory gases may explain this complication during prolonged mechanical ventilation(8).

Objectives. To evaluate humidification performances of heat and moisture exchangers and heated humidifiers used in these reports and compare to humidity output according to manufacturers.

Methods. We conducted a bench study with the psychrometric method to evaluate several HME with a previously described method(8): The Hygrobac S (Medtronic, Minneapolis, MN, USA) used at our centre, the Sunmed FH603008 and the Medline DYNJAAHME1B used in Wiles et al. study(4), Intertherm, Intersurgical used in Sugimoto study(6) and Aero-Pro ${ }^{\mathrm{TM}}$ HEPA Light Machine and AirLife ${ }^{\circledR}$ Edith 1000 used in Panchamian study(1). We compared to the data of humidity delivered provided by the manufacturers. We also evaluated heated wire heated humidifiers used with ambient temperature at $22-24^{\circ} \mathrm{C}, 28-30^{\circ} \mathrm{C}$ and turned off $(7)$.

Results. Preliminary results are presented, with 2 to 5 measurements done at steady state for each device). Other humidity measurements are ongoing and still NA. 


\begin{tabular}{|c|c|c|c|c|c|}
\hline $\begin{array}{l}\text { Authors, } \\
\text { (Reference) }\end{array}$ & $\begin{array}{l}\mathrm{N} \text { patients } \\
\text { with } \\
\text { COVID-19 }\end{array}$ & $\begin{array}{l}\text { Rate of } \\
\text { ETO }\end{array}$ & $\begin{array}{l}\text { Humidification } \\
\text { device used }\end{array}$ & $\begin{array}{l}\mathrm{AH}(\mathrm{mgH} 2 \mathrm{O} / \mathrm{L}) \\
\text { according to } \\
\text { the manu- } \\
\text { facturer (ISO } \\
9360 \text { ) }\end{array}$ & $\begin{array}{l}\mathrm{AH}(\mathrm{mgH} 2 \mathrm{O} / \mathrm{L}) \\
\text { measured } \\
\text { with psy- } \\
\text { chrometric } \\
\text { method }\end{array}$ \\
\hline $\begin{array}{l}\text { Lavoie- } \\
\text { Bérard } \\
\text { et al., }\end{array}$ & $\begin{array}{l}6 \mathrm{HME} \\
14 \mathrm{HH}\end{array}$ & $\begin{array}{l}\text { HME: } 0 \\
(0 \%) \\
\text { HH: } 3 \\
(21 \%)\end{array}$ & $\begin{array}{l}\text { HME (1) } \\
\text { HH (MR 850) }\end{array}$ & $\begin{array}{l}\text { HygrobacS: } \\
33.6\end{array}$ & $\begin{array}{c}\text { HygrobacS: } \\
29.1 \pm 1.2 \\
\text { HH: NA }\end{array}$ \\
\hline Wiles et al & 187 & $12(6.4 \%)$ & $\begin{array}{l}\text { HME (7 ETO) (2) } \\
\text { HH (4 ETO) }\end{array}$ & $\begin{array}{l}\text { Medline: } 31 \\
\text { Sunmed: } 33.4\end{array}$ & $\begin{array}{l}\text { Medline: } \\
24.9 \pm 1.3 \\
\text { Sunmed: } \\
25.7 \pm 0.4\end{array}$ \\
\hline $\begin{array}{l}\text { Rubano } \\
\text { et al }\end{array}$ & 110 & $28(25 \%)$ & NA & NA & NA \\
\hline $\begin{array}{l}\text { Perez } \\
\text { Acosta } \\
\text { et al }\end{array}$ & 22 & $16(72 \%)$ & NA & NA & NA \\
\hline $\begin{array}{l}\text { Panchamian } \\
\text { et al }\end{array}$ & 48 & $14(29 \%)$ & $\begin{array}{l}\text { HME (3) } \\
\text { (anesthesia } \\
\text { machines) }\end{array}$ & $\begin{array}{l}\text { Aero-Pro (Not } \\
\text { reported) } \\
\text { AirLife Edith } \\
\text { 1000: } 30\end{array}$ & $\begin{array}{c}\text { Aero-Pro: NA } \\
\text { AirLife Edith } \\
\text { 1000: NA }\end{array}$ \\
\hline $\begin{array}{l}\text { Zaidi \& } \\
\quad \text { Narasim- } \\
\text { han }\end{array}$ & NA & $\begin{array}{l}\text { Frequent } \\
\text { ETO }\end{array}$ & $\mathrm{HH}$ turned off & NA & $8 \mathrm{mgH} 2 \mathrm{O} / \mathrm{L}$ \\
\hline $\begin{array}{l}\text { Bottirolli } \\
\text { et al }\end{array}$ & 17 & $\begin{array}{c}3(18 \%) 1 \\
\text { death }\end{array}$ & $\begin{array}{l}\text { HME (anesthe- } \\
\text { sia machines) }\end{array}$ & NA & NA \\
\hline $\begin{array}{l}\text { Sugimoto } \\
\text { et al }\end{array}$ & Case report & 1 & HME (4) & Inter-Therm: 34 & $\begin{array}{l}\text { Inter-Therm: } \\
27.4 \pm 1.8\end{array}$ \\
\hline $\begin{array}{l}\text { Van Boven } \\
\text { et al }\end{array}$ & $\begin{array}{l}\text { Case } \\
\text { reports }\end{array}$ & 2 & HME (5) & $\begin{array}{l}\text { Hydro-Guard: } \\
23\end{array}$ & NA \\
\hline
\end{tabular}

Table: Studies reporting endotracheal tube occlusions in COVID-19 patients. Rate of endotracheal tube occlusions, type of humidification device, absolute humidity according to the manufacturer and measured on bench with the psychrometric method.

(1) DARTM Adult-Pediatric Electrostatic Filter HME (Small) (Previously HygrobacS),

(2) Sunmed FH603008 and the Medline DYNJAAHME1B,

(3) Aero-Pro ${ }^{\mathrm{TM}}$ HEPA Light Machine and AirLife ${ }^{\circledR}$ Edith 1000

(4) Inter-Therm, Intersurgical,

(5) Hydro-Guard Mini, Intersurgical.

Conclusion. High rates of ETO have been reported in COVID-19 patients. Prolonged duration of intubation, reduction of suctioning possibilities may explain in part this complication. The main explanation is the low humidity delivered by humidification devices involved in ETO (below $28 \mathrm{mgH} 2 \mathrm{O} / \mathrm{L}$ ). The difference between the manufacturer's claims and measured humidity is worrying. The ISO method does not allow to predict HME's clinical performances.

\section{Reference(s)}

1. Panchamia RK, Piracha MM, Samuels JD. Desperate times: Repurposing anesthesia machines for mechanical ventilation in COVID-19. J Clin Anesth 2020; 66: 109,967. 2. Perez Acosta G, Santana-Cabrera L. [Necrotizing tracheobronchitis with endotracheal tube obstruction in COVID-19 patients]. Rev Clin Esp 2020; 220: 531-533. 3. Rubano JA, Jasinski PT, Rutigliano DN, Tassiopoulos AK, Davis JE, Beg T, Poovathoor S, Bergese SD, Ahmad S, Jawa RS, Vosswinkel JA, Talamini MA. Tracheobronchial Slough, a Potential Pathology in Endotracheal Tube Obstruction in Patients With Coronavirus Disease 2019 (COVID-19) in the Intensive Care Setting. Annals of surgery 2020; 272: e63-e65. 4. Wiles S, Mireles-Cabodevila E, Neuhofs S, Mukhopadhyay S, Reynolds JP, Hatipoglu U. Endotracheal Tube Obstruction Among Patients Mechanically Ventilated for ARDS Due to COVID-19: A Case Series. Journal of intensive care medicine 2020: $885,066,620,981,891$. 5. Bottiroli M, Calini A, Pinciroli R, Mueller A, Siragusa A, Anelli C, Urman R, Nozari A, Berra L, Mondino M, Fumagalli R. The repurposed use of anesthesia machines to ventilate critically ill patients with Coronavirus Disease 2019 (COVID-19). Research Square 2021: 1-20. 6. Sugimoto R, Kenzaka T, Fujikawa M, Kawasaki S, Nishisaki H. Humidifier Use and Prone Positioning in a Patient with Severe COVID-19 Pneumonia and Endotracheal Tube Impaction Due to Highly Viscous Sputum. Cureus 2020; 12: e8626. 7. Zaidi G, Narasimhan M. Lessons Learned in Critical
Care at a 23 Hospital Health System in New York During the Coronavirus Disease 2019 Pandemic. Chest 2020; 158: 1831-1832. 8. Lellouche F, Taille S, Lefrancois F, Deye N, Maggiore SM, Jouvet P, Ricard JD, Fumagalli B, Brochard L. Humidification performance of 48 passive airway humidifiers: comparison with manufacturer data. Chest 2009; 135: 276-286.

\section{3}

High Emergency Lung Transplant for late refractory ARDS: primary experience in Foch hospital

E. CUQUEMELLE'; G. Tachon²; B. Zuber' ; J. Devaquet, ${ }^{2}$; M. Glorion, ${ }^{3}$.

WJ. De ${ }^{3}$; C. Pricopi ${ }^{3}$; F. Parquin' $;$ A. Roux ${ }^{4}$; C. Picard ${ }^{4}$; M. Le Guen ${ }^{5}$; E. Sage ${ }^{3}$;

C. Cerf ${ }^{2}$

${ }^{1}$ Respiratory intensive care unit, Hospital Foch, Suresnes, France;

${ }^{2}$ Intensive care unit, Hospital Foch, Suresnes, France; ${ }^{3}$ Thoracic surgery, Hospital Foch, Suresnes, France; ${ }^{4}$ Pneumology department, Hospital Foch, Suresnes, France; ${ }^{5}$ Anesthesiology department, Hospital Foch, Suresnes, France

Correspondence: E. CUQUEMELLE

Intensive Care Medicine Experimental 2020, 9(1): 000453

Introduction. In some patients with late refractory ARDS on maximal support by ECMO, considering switch from "ECMO to recovery" to bridge to lung transplantation (LTX) could be an option. We report our preliminary experience in such strategy.

Methods. Between 2016 and 2020 patients with late refractory ARDS on ECMO to recovery strategy failure referred to our center for LTX evaluation were reviewed. Patients with pre-existing lung disease or severe comorbidities before ARDS onset were not considered for evaluation. In all cases, lung damage irreversibility was established by multidisciplinary staff. Results are expressed in median [min;max].

Results. 13 patients $(4 \mathrm{~F} ; 9 \mathrm{M})$ aged of $56 \mathrm{y}[20 ; 63]$ were included. The etiologies were Sars-cov-2 ARDS (9), toxic lung damage (2), Anti-mda5 AIP (1), and unknown (1). After multidisciplinary staff, only 6 patients were transferred in our center. Only those data were analyzed. Among 6 patients, 4 were transplanted and 2 died before LT. ECMO duration, MV duration and ICU stay before LT were respectively 35 days [20;88], 38 [20;89], 34 [3;94]. All patients had sarcopenia and severe ICU acquired paresis with $\mathrm{MRC}<12$. All patients were tracheotomized and none had bedsores. Contra-indications for LTx were severe extrarespiratory failure (1/6) and non-controlled infection (1/6). All transplant patients are alive follow-up 445d [95;1790]. Post-operative MV duration was 24 days [9;126]; LOD in ICU was 59 days [29;202]. Every induction protocol included basiliximab and post-operative immunosuppression was usual. In 2 cases patients were informed of and accepted LTx surgery and 2 cases were not. Though, patients graft acceptance and treatment adhesion were good. In all cases relatives were extensively informed of risks and benefits, and accepted the treatment. All patients were listed in high emergency program after expert panel acceptation.

Conclusion. LTx seems to be a reasonable option in irreversible late refractory ARDS under maximal support therapy in very selected patients in an expert center with a specific task force (transplantationARDS@hopital-foch.com). Incidence of such young potentially eligible patient is increased due to the current COVID-19 pandemic. As further evaluations are needed, we propose to create a national registry to evaluate this new strategy.

\section{6}

Study of the variables associated to ECMO free days at 28 days in patients with ARDS treated with ECMO

P. Blanco-Schweizer'; MM. García-García²; D. Pérez-Torres³;

J. Sánchez-Ballesteros²; R. Herrán-Monge ${ }^{2}$; JA. Ayala-Fernández;

C. Díaz-Rodríguez'; GJ. Posadas-Pita' ${ }^{1}$ I. Canas-Pérez²; P. Enríquez

${ }^{1}$ Department of intensive care medicine, Hospital Universitario Río Hortega, Valladolid, Spain; ${ }^{2}$ Servicio de medicina intensiva, Hospital Universitario Río Hortega, Valladolid, Spain; ${ }^{3}$ Intensive care department, Hospital Universitario Río Hortega, Valladolid, Spain

Correspondence: P. Blanco-Schweizer

Intensive Care Medicine Experimental 2020, 9(1): 000456 
Introduction. Extracorporeal membrane oxygenation (ECMO) is a highly sophisticated technique associated to improved survival in patients with severe acute respiratory distress syndrome (ARDS) and refractory hypoxemia and/or hypercapnia $(1,2)$. However, variables associated to the length of the ECMO run are yet to be described.

Methods. Observational, retrospective, unicentric study in patients with ARDS managed with ECMO in an ICU that is Reference for 1.8 million inhabitants with a mobile-ECMO service. All patients treated between 01/01/2019 and 01/12/2020 were included. Data were collected until 15/12/2020. Data about comorbidity, ventilatory treatment, lung function, complications and respiratory mechanics pre and during ECMO were collected. Data are expressed as mean $\pm \mathrm{sd}$, median (iqr) and proportions. Association between variables were studied with t-test, Wilcoxon rank test, chi-square, fisher exact test and spearman correlation coefficient as appropriate A multivariable forward analysis to investigate variables related to ECMO free days at 28 days was performed. It included all variables associated with a $\mathrm{p}<0.2$ in the univariant analysis and those associated in literature. Patients who died on ECMO were considered to have 0 ECMO free days.

Results. $\mathrm{N}=37$, all patients were treated with veno-venous ECMO, ECMO free days at 28 days was 9 (0-19).

Associtation of variables to outcome in the univariate analysis.

\begin{tabular}{lll}
\hline Variable & $\begin{array}{l}\text { Spearman correlation } \\
\text { coef, mean difference } \\
(\mathbf{9 5} \% \mathbf{C l})\end{array}$ & $\mathbf{P}$ \\
& $9(4-15)$ & 0.05 \\
SOFA preECMO $^{3} 7$ & 0.3 & 0.07 \\
RESPscore & $10(-0.8-22)$ & 0.07 \\
COPD & 0.01 & 0.9 \\
PaCO2 preECMO & $4(-2-10)$ & 0.19 \\
Retrieved on ECMO & -0.25 & 0.15 \\
Tidal volume per PBW preECMO & -0.28 & 0.09 \\
Plateau pressure preECMO & -0.27 & 0.13 \\
Mechanical power preECMO & -0.28 & 0.11 \\
Driving power preECMO & 0.33 & 0.06 \\
Tidal volume per PBW day 3 & & 0.015 \\
ECMO & 0.42 & 0.008 \\
Mechanical power day 3 ECMO & 0.45 & 0.09 \\
Driving power day 3 ECMO & -0.29 & 0.007 \\
Sweep gas flow day 3 ECMO & $9(3-15)$ & 0.04 \\
Hospital acquired pneumonia & $7(0.18-13)$ & \\
Continuous renal replacement & & \\
therapy & & \\
\hline
\end{tabular}

Variables associated to outcome in the multivariate analysis.

\begin{tabular}{lll}
\hline Variable & Coeficient & $\mathbf{P}$ \\
\hline Hospital acquired pneumonia & -13.5 & 0.002 \\
Continuous renal replacement therapy & -10 & 0.016 \\
PaCO2 preECMO & -0.2 & 0.038 \\
\hline
\end{tabular}

Conclusion. 1. The only variables associated to ECMO free days at 28 days were hospital acquired pneumonia, the need for renal replacement therapy and preECMO PaCO2 in the multivariate analysis. 2 . In spite of the inverse relationship between preECMO mechanical power and driving power and ECMO free days at 28 days, this relationship became direct with these variables on day 3 of ECMO.

\section{Reference(s)}

1. Combes A, Hajage D, Capellier G, Demoule A, Lavoue S, Guervilly C, Da Silva D, Zafrani L, Tirot P, Veber B, Maury E, Levy B, Cohen Y, Richard C, Kalfon P, Bouadma L, Mehdaoui H, Beduneau G, Lebreton G, Brochard L, Ferguson ND, Fan E, Slutsky AS, Brodie D, Mercat A. Extracorporeal membrane oxygenation for severe acute respiratory distress syndrome. N Engl J Med. 2018:378:1965-1975. https://doi.org/10.1056/NEJMoa1800385.

2. Peek GJ, Mugford M, Tiruvoipati R, Wilson A, Allen E, Thalanany MM, Hibbert CL, Truesdale A, Clemens F, Cooper N, Firmin RK, Elbourne D. Efficacy and economic assessment of conventional ventilatory support versus extracorporeal membrane oxygenation for severe adult respiratory failure (CESAR): a multicentre randomised controlled trial. Lancet. 2009;374:1351-1363. https://doi.org/10.1016/S0140-6736(09)61069-2.

\section{0}

Aerosol deposition in the respiratory tract under mechanical ventilation: impact of the nebulizer type and ventilation mode

J. bonsignore ${ }^{1}$ Y. Montigaud ${ }^{2}$; L. Leclerc ${ }^{3}$; E. Odouard ${ }^{4}$; N. Prevot ${ }^{5}$; J. Pourchez ${ }^{6} ;$ S. Perinel Ragey

${ }^{1}$ Réanimation g, University Hospital of Saint-Étienne, Saint-Priest-en-Jarez, France; ${ }^{2} \mathrm{Cis}$, Ecole des Mines de Saint-Étienne, Saint-Étienne, France;

${ }^{3}$ Cis, EMSE, Saint-Étienne, France; ${ }^{4}$ Médecine nucléaire, University Hospital of Saint-Étienne, Saint-Priest-en-Jarez, France; ${ }^{5}$ Nuclear medicine, hopital nord, St-Priest-en-Jarez, France; ${ }^{6}$ Cis, EMSE, St-Priest-en-Jarez, France;

${ }^{7}$ Réanimation G, University Hospital of Saint-Etienne, Saint-Priest-en-Jare z, France

Correspondence: $\mathrm{J}$. bonsignore

Intensive Care Medicine Experimental 2020, 9(1): 000470

Introduction. Nebulization of various drugs is a common route of administration, particularly for intensive care patients under mechanical ventilation (MV)(1). Nevertheless, despite frequent use, nebulization practices in ICU are numerous, frequently led only by data from in vitro studies(2). To investigate how we can improve our practices, we developed an ex vivo preclinical model of porcine respiratory tract (RT) to assess the regional aerosol deposition in MV conditions. The AIM of this study is to evaluate the impact of the type of ventilation mode and of nebulizer on the aerosol deposition in the RT.

Methods. The ex vivo respiratory model, used in this study, was previously validated (3) and, was composed of a porcine RT, placed in a sealed enclosure and mechanically ventilated through an endo-tracheal tube connected to the trachea, with or without spontaneous ventilation according to the condition. The model was set in $30^{\circ}$ proclive. It was ventilated with two modes: pressure support volume (PSV) or volume controlled (VC) and we compared a jet nebulizer (JN) and a vibrating mesh nebulizer (VMN) in each mode filed with 99mtechnetium-labeled diethylene-triamine-penta-acetic acid. The nebulizer was set $15 \mathrm{~cm}$ upstream the Y-piece in the inspiratory limb. For each condition we used au heated humidifier. We used a gamma camera acquisition to quantify the deposited fractions in each part of the setting.

Results. The model was setup 3 times for each condition. The deposited fractions in each component were determined thanks to scintigraphy images and lead to the Fig. 1. For PSV (PART A), deposited fractions reached respectively (results express in \% of nominal dose, JN then VMN: $11 \%( \pm 3 \%)$ and $39 \%( \pm 8 \%)$ in inspiratory limb (IL), $2 \%( \pm 1 \%)$ and $2 \%( \pm 0 \%)$ in the endo tracheal tube (ETT), $9 \%( \pm 2 \%)$ and $47 \%$ $( \pm 7 \%)$ in RT, $10 \%( \pm 3 \%)$ and $8 \%( \pm 2 \%)$ in expiratory limb (EL). For VC (PART B), deposited fractions reached: $11 \%( \pm 4 \%)$ and $22 \%( \pm 16 \%)$ in $\mathrm{IL}, 2 \%( \pm 0 \%)$ and $2 \%( \pm 0 \%)$ in ETT, $13 \%( \pm 5 \%)$ and $55 \%( \pm 7 \%)$ in RT, $5 \%( \pm 0 \%)$ and $18 \%( \pm 8 \%)$ in EL. 


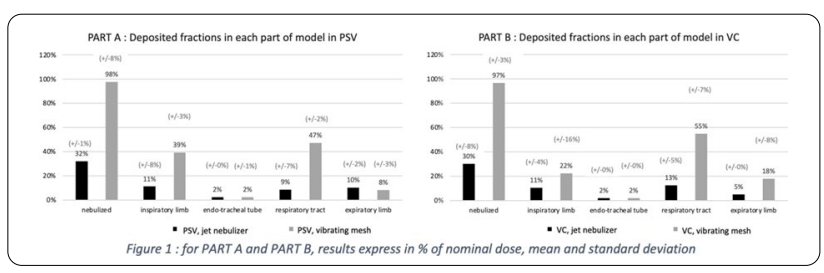

Conclusion. This study shows that, other conditions being similar, the type of nebulizer can modify the deposited fraction in the RT from $9 \%$ for JN to $47 \%$ for VMN in PSV and from 13 to $55 \%$ respectively in VC. Moreover, the data are in accordance with literature for the impact of ventilation mode which finds a better deposition of the aerosol for VC than in PSV (4). However, in VC literature showed that the patient must be more sedated which increase the risk of ventilated acquire pneumonia or prolonged ventilation time. These data are preclinical and it is their principal limitation, needing clinical trials to be confirmed. The ventilation mode impacts the aerosol deposition, as previously described in literature, other conditions being reproductive. But the major difference due to the choice of a VMN observed in this study must be taken in consideration by clinicians to optimize the nebulization practices.

\section{Reference(s)}

1. Ehrmann S, Roche-Campo F, Bodet-Contentin L, Razazi K, Dugernier J, Trenado-Alvarez J, et al. Aerosol therapy in intensive and intermediate care units: prospective observation of 2808 critically ill patients. Intensive Care Med. 1 févr 2016;42(2):192-201.

2. Fink JB, Dhand R, Grychowski J, Fahey PJ, Tobin MJ. Reconciling In Vitro and In Vivo Measurements of Aerosol Delivery from a Metered-Dose Inhaler during Mechanical Ventilation and Defining Efficiency-enhancing Factors. Am J Respir Crit Care Med. 1 janv 1999;159(1):63-8.

3. Montigaud Y, Georges Q, Pourchez J, Leclerc L, Goy C, Clotagatide A, et al. Aerosol delivery during invasive mechanical ventilation: development of a preclinical ex vivo respiratory model for aerosol regional deposition. Sci Rep. 29 nov 2019;9(1):17,930.

4. Dugernier J, Reychler G, Wittebole X, Roeseler J, Depoortere V, Sottiaux $T$, et al. Aerosol delivery with two ventilation modes during mechanical ventilation: a randomized study. Ann Intensive Care. 22 juill 2016;6(1):73.

\section{7}

\section{ECMO therapy in ARDS patients: comparison of SARS-CoV-2} cohort with other etiologies cohort

P. Blanco-Schweizer ; MM. García-García²; J. Sánchez-Ballesteros ${ }^{2}$

R. Herrán-Monge²; D. Pérez-Torres'; ;. Díaz-Rodríguez';

JA. Ayala-Fernández ${ }^{3}$; GJ. Posadas-Pita'i I. Canas-Pérez ${ }^{2}$; P. Enríquez

${ }^{1}$ Department of intensive care medicine, Hospital Universitario Río Hortega, Valladolid, Spain; ${ }^{2}$ Servicio de medicina intensiva, Hospital Universitario Río Hortega, Valladolid, Spain; ${ }^{3}$ Intensive care department, Hospital Universitario Río Hortega, Valladolid, Spain

Correspondence: P. Blanco-Schweizer

Intensive Care Medicine Experimental 2020, 9(1): 000487

Introduction. Extracorporeal membrane oxygenation (ECMO) is a highly sophisticated technique associated to improved survival in patients with severe acute respiratory distress syndrome (ARDS) and refractory hypoxemia and/or hypercapnia $(1,2)$. Data about patients with severe SARS-CoV-2 infection managed with ECMO have been published $(3,4)$, however, comparison of their characteristics and outcomes with patients treated with ECMO due to other causes is yet to be published.

Methods. Observational, retrospective, unicentric study in patients with ARDS managed with ECMO in an ICU that is Reference for 1.8 million inhabitants with a mobile-ECMO service. SARS-CoV-2 cohort: patients with ARDS treated with ECMO between 15/03/2020 and 01/12/2020. Non-SARS-CoV-2 cohort: patients with ARDS due to other causes treated with ECMO in the previous 12 months. Epidemiological and clinical variables before and during ECMO therapy were collected. Data are expressed as mean $\pm \mathrm{sd}$, median(IQR) and proportions. Comparison were performed using T-test, Wilcoxon rank test, chi-square and Fisher exact test.

\section{Results.}

\begin{tabular}{|c|c|c|c|c|}
\hline Variable & $\begin{array}{l}\text { SARS-CoV-2, } \\
\mathrm{n}=21 \\
\text { Mean } \pm \mathrm{sd}, \\
\text { median (iqr), } \\
\mathrm{n}(\%)\end{array}$ & $\begin{array}{l}\text { Non-SARS- } \\
\text { CoV-2, } \mathrm{n}=16 \\
\text { Mean } \pm \mathrm{sd}, \\
\text { median (iqr), } \\
\mathrm{n}(\%)\end{array}$ & $\begin{array}{l}\text { Mean differ- } \\
\text { ence }(95 \% \\
\mathrm{Cl}), \%\end{array}$ & $\mathbf{P}$ \\
\hline Age & $54 \pm 10$ & $48 \pm 15$ & $6(-14-2)$ & Ns \\
\hline Male & $18(85)$ & $9(56)$ & 29 & 0.046 \\
\hline $\begin{array}{l}\text { Risk factors } \\
\text { - Hypertension } \\
\text { - Diabetes } \\
\text { - COPD }\end{array}$ & $\begin{array}{l}7(33) \\
6(29) \\
2(10)\end{array}$ & $\begin{array}{l}2(13) \\
0(0) \\
1(6)\end{array}$ & $\begin{array}{l}20 \\
29 \\
4\end{array}$ & 0.019 \\
\hline $\begin{array}{l}\text { Hospital admis- } \\
\text { sion to ICU } \\
\text { days }\end{array}$ & $3(1-5)$ & $2(0-6)$ & $1(-2-5)$ & Ns \\
\hline NIMV pre-MV & $6(29)$ & $0(0)$ & 29 & 0.019 \\
\hline $\begin{array}{l}\text { NIMV days pre- } \\
\text { ECMO }\end{array}$ & $5(1-7)$ & - & - & Ns \\
\hline $\begin{array}{l}\text { MV days pre- } \\
\text { ECMO }\end{array}$ & $8(5-12)$ & $2(1-5)$ & $4(1-7)$ & 0.001 \\
\hline $\begin{array}{l}\text { Tidal volume } \\
\text { pre-ECMO (ml/ } \\
\text { PBW) }\end{array}$ & $6.8 \pm 1$ & $6.4 \pm 1.3$ & $0.4(-1.2-0.4)$ & Ns \\
\hline $\begin{array}{l}\text { Dring pressure } \\
\text { pre-ECMO }\end{array}$ & $15(15-18)$ & $16(15-18)$ & $1(-2-4)$ & Ns \\
\hline PEEP pre-ECMO & $14(8-14)$ & $10(7-14)$ & $2(-5-0.4)$ & Ns \\
\hline $\begin{array}{l}\text { Compliance pre- } \\
\text { ECMO }\end{array}$ & $30(24-32)$ & $24(17-30)$ & $5(-11-0.3)$ & Ns \\
\hline Prone pre-ECMO & $21(100)$ & $14(88)$ & 12 & Ns \\
\hline $\begin{array}{l}\text { Paralysis pre- } \\
\text { ECMO }\end{array}$ & $21(100)$ & $16(100)$ & 0 & Ns \\
\hline $\begin{array}{l}\text { P/F ratio pre- } \\
\text { ECMO }\end{array}$ & $84(74-89)$ & 78 (65-99) & $4(-21-30)$ & Ns \\
\hline $\begin{array}{l}\mathrm{PaCO} 2 \text { pre- } \\
\text { ECMO mmHg }\end{array}$ & $63(54-84)$ & $62(50-75)$ & $5(-19-9)$ & Ns \\
\hline SOFA pre-ECMO & $5(4-7)$ & $7(6-11)$ & $3(0.4-4)$ & Ns \\
\hline $\begin{array}{l}\text { Veno-venous } \\
\text { strategy }\end{array}$ & $21(100)$ & $16(100)$ & 0 & Ns \\
\hline AKI & $6(29)$ & $9(56)$ & 27 & Ns \\
\hline CRRT & $6(29)$ & $8(50)$ & 21 & Ns \\
\hline On ECMO VAP & $8(38)$ & $5(31)$ & 7 & Ns \\
\hline Awake ECMO & $12(57)$ & $8(50)$ & 7 & Ns \\
\hline Tracheostomy & $9(-42)$ & $5(31)$ & 11 & Ns \\
\hline MV days & $34(18-40)$ & $20(14-40)$ & $6(-16-4)$ & Ns \\
\hline ECMO days & $18(8-30)$ & $10(7-16)$ & 6 & Ns \\
\hline ICU days & $34 \pm 18$ & $30 \pm 17$ & 4 & Ns \\
\hline ECMO survival & $17(81)$ & $12(75)$ & 2 & Ns \\
\hline ICU survival & $15(71)$ & $10(62)$ & 2 & Ns \\
\hline
\end{tabular}

Conclusion. 1. Higher proportion of male, diabetes, use of NIMV and pre-ECMO MV days was found in SARS-CoV-2 chort. No difference in clinical charactertistics or outcome could be found.

\section{Reference(s)}

1. Schmidt M, Hajage D, Lebreton G, Monsel A, Voiriot G, Levy D, Baron E, Beurton A, Chommeloux J, Meng P, Nemlaghi S Pierre B, Leprince P, Demoule A, Guidet B, Constantin JM Fartoukh M, Dres M, Combes A, Groupe de recherche clinique en reanimation et soins intensifs du patiente en insuffisance Respiratorire aigue (GRC-RESPIRE). Extracorporeal 
membrane oxygenation for severe acute respiratory distress syndrome associated with COVID-19: a retrospective cohort study. Lancet Respir Med. 2020 Nov;8(11):1121-1131. https://doi.org/10.1016/S2213-2600(20) 30328-3

2. Barbaro RP, MacLaren G, Boonstra PS, Iwashyna TJ, Slutsdy AS, Fan E, Bartlett RH, Tonna JE, Hyslop R, Fanning JJ, Rycus PT, Huer SJ, Anders MM, Agerstrand CL, Hryniewicz K, Diaz R, Lorusse R, Combes A, Brodie D, for the Extracorporeal Life Support Organization. Extracorporeal membrane oxygenation support in COVID-19: an international cohort study of the Extracorporeal Life Support Organization registry.

3. Peek GJ, Mugford M, Tiruvoipati R, Wilson A, Allen E, Thalanany MM, Hibbert CL, Truesdale A, Clemens F, Cooper N, Firmin RK, Elbourne D. Efficacy and economic assessment of conventional ventilatory support versus extracorporeal membrane oxygenation for severe adult respiratory failure (CESAR): a multicentre randomised controlled trial. Lancet. 2009;374:1351-1363. https://doi.org/10.1016/S0140-6736(09)61069-2.

4. Combes A, Hajage D, Capellier G, Demoule A, Lavoue S, Guervilly C, Da Silva D, Zafrani L, Tirot P, Veber B, Maury E, Levy B, Cohen Y, Richard C, Kalfon P, Bouadma L, Mehdaoui H, Beduneau G, Lebreton G, Brochard L, Ferguson ND, Fan E, Slutsky AS, Brodie D, Mercat A. Extracorporeal membrane oxygenation for severe acute respiratory distress syndrome. N Engl J Med. 2018;378:1965-1975. https://doi.org/10.1056/NEJMoa1800385

\section{9}

Impact of the airway humidification strategy on ventilation efficiency in COVID-19 ARDS patient

CA. Lavoie-Bérard, ; J J. Lefebvre, ; PA. Bouchard, 3, E. Rousseau, ${ }_{1}^{3}$; M. Simon, ; F. Lellouche ${ }^{5}$

${ }^{1}$ Medicine, Laval University, Québec, Canada; ${ }^{2}$ Département d'anesthésiologie et de soins intensifs, division de soins intensifs, Laval University, Québec, Canada; ${ }^{3}$ Research center, University Institute of Cardiology and Respirology of Quebec, Québec, Canada; ${ }^{4}$ Critical care and research center, University Institute of Cardiology and Respirology of Quebec, Québec, Canada; ${ }^{5}$ Reaserch center, University Institute of Cardiology and Respirology of Quebec, Québec, Canada

Correspondence: $F$. Lellouche

Intensive Care Medicine Experimental 2020, 9(1): 000489

Introduction. Patients with COVID-19 disease requiring invasive mechanical ventilation frequently meet criteria of ARDS and benefit from protective ventilation $(1,2)$. The inspiratory gases humidification system recommended when low tidal volumes and high respiratory rate are set is the heated humidifiers $(\mathrm{HH})$ to minimize dead space impact and improve $\mathrm{CO} 2$ clearance (3). We report here the experience of two centres in Quebec City using different humidification strategies and discuss the impact on ventilation efficiency.

Methods. We conducted a retrospective observational study including all intubated COVID-19 patients in the two designated COVID-19 centers for adults in Quebec City between March 23rd and June 6th 2020. In the Québec Heart and Lung Institute, HH (MR 850, Fisher\&Paykel, Auckland, New Zealand) were used to reduce instrumental dead space. In the CHU Québec-Laval University, HME (Hygrobac S, Medtronic, Minneapolis, MN, USA) were preferred for their filtering characteristics to prevent air contamination in case of accidental disconnection of the ventilator. In both centres, an electrostatic filter was placed at the expiratory port of the ventilator. We collected informations about demographics and ventilator data, total dead space, blood gases and outcome. Mechanical power was calculated with the following formula $M P=0.098 \times R R$ $x$ Vt $x($ Ppeak-(0.5 $\times$ DP))(4). Total dead space was calculated by the addition of endotracheal tube volume, HME internal volume (45 ml, when present), CO2 sensor (5 to $9 \mathrm{ml})$, closed suction system $(9 \mathrm{ml})$, and physiologic dead space ( 1.1 ml/kg PBW)(5).

Results. Twenty-six patients with confirmed COVID-19 were admitted in ICU during the study period, among which 20 patients were intubated and included in the analysis. In total $\mathrm{HH}$ was used in 14 patients and HME in 6. Fourteen over 20 patients were male, mean age was $60 \pm 14$ years, mean $\mathrm{PaO} 2 / \mathrm{FiO} 2$ ratio at day one was $156 \pm 45 \mathrm{mmHg}$, all patients had $\mathrm{PaO} 2 / \mathrm{FiO} 2$ ratio below 300 .
The total calculated dead space in the HME group was $147 \pm 11 \mathrm{ml}$ and $\mathrm{Vd} / \mathrm{Vt}$ was $33.7 \pm 3 \%$. By comparison, in the $\mathrm{HH}$ group, the total estimated dead space was $102 \pm 14 \mathrm{ml}$ leading to a Vd/ Vt of $26.7 \pm 3 \%$. The ventilator ratio was higher in the HME group (2.19 \pm 0.6 vs. $1.61 \pm 0.5)$. In the HME group, $\mathrm{PaCO} 2$ was higher (48.0 vs. $42.3 \mathrm{mmHg}$ ), despite higher minute ventilation (171 vs. $145 \mathrm{ml} /$ $\mathrm{min} / \mathrm{kg}$ PBW). Driving pressure $(10.8 \pm 3.7$ vs. $9.9 \pm 2.9 \mathrm{cmH} 2 \mathrm{O})$ and mechanical power $(30.0 \pm 9.6$ vs. $23.0 \pm 6.5 \mathrm{~J} / \mathrm{min}$ ) were higher in the HME group. During the course of the hospitalization, HME was replaced by $\mathrm{HH}$ in 3 patients (50\%) to increase alveolar ventilation because of acidosis associated with high plateau pressure.

Conclusion. The humidification strategy in COVID-19 patients may have significant impact for ventilatory efficiency. When instrumental dead space was minimized (with $\mathrm{HH}$ ), alveolar ventilation was higher (lower PaCO2) despite lower minute ventilation.

\section{Reference(s)}

1. Alhazzani W, Moller MH, Arabi YM, Loeb M, Gong MN, Fan E, Oczkowski S, Levy MM, Derde L, Dzierba A, Du B, Aboodi M, Wunsch H, Cecconi M, Koh Y, Chertow DS, Maitland K, Alshamsi F, Belley-Cote E, Greco M, Laundy M, Morgan JS, Kesecioglu J, McGeer A, Mermel L, Mammen MJ, Alexander PE, Arrington A, Centofanti JE, Citerio G, Baw B, Memish ZA, Hammond N, Hayden FG, Evans L, Rhodes A. Surviving Sepsis Campaign: guidelines on the management of critically ill adults with Coronavirus Disease 2019 (COVID-19). Intensive Care Med 2020. 2. Marini JJ, Gattinoni L. Management of COVID-19 Respiratory Distress. JAMA 2020. 3. Papazian L, Aubron C, Brochard L, Chiche JD, Combes A, Dreyfuss D, Forel JM, Guerin C, Jaber S, Mekontso-Dessap A, Mercat A, Richard JC, Roux D, Vieillard-Baron A, Faure $\mathrm{H}$. Formal guidelines: management of acute respiratory distress syndrome. Annals of intensive care 2019; 9: 69. 4. Urner M, Juni P, Hansen B, Wettstein MS, Ferguson ND, Fan E. Time-varying intensity of mechanical ventilation and mortality in patients with acute respiratory failure: a registry-based, prospective cohort study. Lancet Respir Med 2020; 8: 905-913. 5. Lellouche F, Delorme M, Brochard L. Impact of respiratory rate and dead space in the current era of lung protective mechanical ventilation Chest 2020; 158(1):45-47.

\section{3}

Non-protective tidal volumes can result in safe driving pressure and trans-pleural pressure values

M. Varela'; R. Sá Lopes²; A. Silva ${ }^{1}$; C. Oliveira ${ }^{1}$; A. Krystopchuk'; D. Nuñez ${ }^{1}$; J. Moreno ${ }^{1}$

${ }^{1}$ Intensive Care Medicine 1, University Hospital Center of Algarve, Faro, Faro, Portugal; ${ }^{2}$ Internal medicine 1, University Hospital Center of Algarve, Faro, Faro, Portugal

Correspondence: M. Varela

Intensive Care Medicine Experimental 2020, 9(1): 000493

Introduction. Driving pressure (DP) has been proposed as a means to better titrate tidal volume and PEEP. It has gained utility together with tidal volume divided by predicted body weight (TV/PBW).

However, literature is scarce on whether TV/PBW is correlated with DP in passively breathing patients and, especially, in those in spontaneous breathing through a ventilator.

Objectives. We aimed to compare patients'TV/PBW values with their DP values, in patients ventilated passively and those spontaneously breathing.

Methods. During one month, for each patient admitted to our nonCovid19 polyvalent Intensive Care Unit, we measured their leg length and calculated their height using the Chumlea equation, to obtain their PBW.

Every weekday, we collected data from the ventilator to then calculate TV/PBW and DP. If the patient was spontaneously breathing, we calculated delta Pooc to obtain transpulmonary driving pressure (tpDP).

Data was analyzed using Microsoft Office 365 Excel v16.0.13901.20148. Results. Of the 84 records, only $32.1 \%$ (27) of measurements showed patients being ventilated below $8 \mathrm{~mL} / \mathrm{kg}$.

Although having this low incidence of protective TV/PBW, 83.3\% of all records showed patients having a DP below 15 . 
Of those being passively ventilated over $8 \mathrm{~mL} / \mathrm{Kg}, 76 \%$ had a $\triangle \mathrm{P}$ below 15 , while $95 \%$ of those with TV/PBW between 6 and $8 \mathrm{~mL} / \mathrm{Kg}$ had a DP below 15 .

In spontaneously breathing patients, of the 8 records of patients with TV/PBW below $8 \mathrm{~mL} / \mathrm{Kg}, 62.5 \%$ were being safely ventilated with a DP below 15 and tpDP below 16. Yet, on those 12 with a TV/PBW over $8 \mathrm{~mL} / \mathrm{Kg}, 58.3 \%$ had both DP and tpDP within normal values.

Conclusion. Our results indicate that a safe DP can thus be attained not only with lung protective tidal volumes, but also with TV/PBW over the threshold.

\section{Reference(s)}

1. Umbrello M, Marino A, Chiumello D. Tidal volume in acute respiratory distress syndrome: how best to select it. Annals of Translational Medicine. 2017;5(14):287-287

2. Camporota L. Respiratory failure: choose your own adventure. Presentation presented at ESICM LIVES 2020

3. Berger M, Cayeux M, Schaller M, Soguel L, Piazza G, Chioléro R. Stature estimation using the knee height determination in critically ill patients. e-SPEN, the European e-Journal of Clinical Nutrition and Metabolism. 2008;3(2):e84-e88

4. Bertoni M, Telias I, Urner M, Long M, Del Sorbo L, Fan E et al. A novel non-invasive method to detect excessively high respiratory effort and dynamic transpulmonary driving pressure during mechanical ventilation. Critical Care. 2019;23(1)

5. Poole J, McDowell C, Lall R, Perkins G, McAuley D, Gao F et al. Individual patient data analysis of tidal volumes used in three large randomized control trials involving patients with acute respiratory distress syndrome. British Journal of Anaesthesia. 2017;118(4):570-575

6. Sahetya S, Mallow C, Sevransky J, Martin G, Girard T, Brower Ret al. Association between hospital mortality and inspiratory airway pressures in mechanically ventilated patients without acute respiratory distress syndrome: a prospective cohort study. Critical Care. 2019;23(1)

\section{5}

Injurious driving pressure values are better predictors than TV/ PBW of higher mechanical power and normalized mechanical power

M. Varela ${ }^{1}$; R. Sá Lopes ${ }^{2}$; A. Silva ${ }^{1}$; C. Oliveira ${ }^{1}$; A. Krystopchuk'; D. Nuñez'; J. Moreno

${ }^{1}$ Intensive Care Medicine 1, University Hospital Center of Algarve, Faro, Faro, Portugal; ${ }^{2}$ Internal medicine 1, University Hospital Center of Algarve, Faro, Faro, Portugal

Correspondence: M. Varela

Intensive Care Medicine Experimental 2020, 9(1): 000495

Introduction. Mechanical power values over $17 \mathrm{~J} / \mathrm{min}$ have recently been shown to be associated with higher mortality in critically ill patients. Using predicted body weight (PBW) to normalize MP (norMP) has also been suggested as a measure to estimate injurious ventilation.

Currently, driving pressure (DP) and tidal volume (TV) normalized to predicted body weight are one of the main parameters to assess for injurious ventilation. However, the relationship between these currently used parameters and mechanical power and norMP has not been fully elucidated.

Objectives. Our goal was to estimate whether DP correlates with MP and norMP and whether it is superior to ventilation based on TV/PBW in predicting harm in patient ventilated under Pressure Control (PC).

Methods. During one month, for each patient admitted to our nonCovid19 polyvalent Intensive Care Unit, we measured their leg length and calculated their height using the Chumlea equation, to obtain their PBW.

Every weekday, we collected data from the ventilator to then calculate TV/PBW and DP. If the patient was ventilated in PC, we calculated MP using the equation proposed by Van der Meijden et al. and Becher et al. We also calculated norMP, as described by Zhang et al.

Data was analyzed using RStudio v1.4.1103.

Results. 65 records of patients while on PC were obtained.
DP was positively associated with MP and norMP values (Spearman correlation test rho 0,863 and 0,859 respectively, $p$-value $<0,05$ ).

TV/PBW was also positively correlated, albeit lower, with MP and norMP values (Spearman correlation test rho 0,643 and 0,696, respectively, p-value $<0,05$ ).

Of the 12 records showing injurious DP values, $11(91,6 \%)$ showed a MP over $17 \mathrm{~J} / \mathrm{min}$. Of the 45 records showing TV/PBW over $8 \mathrm{~mL} / \mathrm{Kg}$, only $10(22,2 \%)$ had MP values over $17 \mathrm{~J} / \mathrm{min}$.

Conclusion. This small study shows that DP is superior to TV/PBW to estimate MP and norMP. As MP continues gaining evidence as a predictor of mechanical ventilation-associated mortality, mechanical ventilation guided by DP seems superior to TV/PBW in estimating harm during mechanical ventilation.

\section{Reference(s)}

1. Serpa Neto A, Deliberato R, Johnson A, Bos L, Amorim P, Pereira S et al. Mechanical power of ventilation is associated with mortality in critically ill patients: an analysis of patients in two observational cohorts. Intensive Care Medicine. 2018;44(11):1914-1922

2. Zhang Z, Zheng B, Liu N, Ge H, Hong Y. Mechanical power normalized to predicted body weight as a predictor of mortality in patients with acute respiratory distress syndrome. Intensive Care Medicine. 2019;45(6):856-864

3. Umbrello M, Marino A, Chiumello D. Tidal volume in acute respiratory distress syndrome: how best to select it. Annals of Translational Medicine. 2017:5(14):287-287

4. Berger M, Cayeux M, Schaller M, Soguel L, Piazza G, Chioléro R. Stature estimation using the knee height determination in critically ill patients. e-SPEN, the European e-Journal of Clinical Nutrition and Metabolism. 2008;3(2):e84-e88

5. Becher T, van der Staay M, Schädler D, Frerichs I, Weiler N. Calculation of mechanical power for pressure-controlled ventilation. Intensive Care Medicine. 2019:45(9):1321-1323

6. none

000501

The impact of Non-Invasive Ventilation delivered by oro-nasal mask, full-face-mask or helmet on time to intubation in COVID-19 patients

L. Cattin ${ }^{1}$; F. Ferrari ${ }^{2}$; N. Rossi ${ }^{3}$; J. Santi ${ }^{1}$;V. Rizzello ${ }^{1}$; N. Brumana ${ }^{4}$;

A. Marinello ${ }^{3}$; E. Polati ${ }^{5} ;$ V. Danzi, ${ }^{1}$ : S. De Rosa ${ }^{6}$

${ }^{1}$ Department of anesthesia and intensive care unit, San Bortolo Hospital Of Vicenza, Vicenza, Italy; ${ }^{2}$ Intensive care unit, San Matteo University Hospital, Pavia, Italy; ${ }^{3}$ Anesthesia and intensive care unit, University of Verona, Verona, Italy: ${ }^{4}$ Anestesia e rianimazione, Ospedale San Bortolo di Vicenza, Vicenza, Italy; ${ }^{5}$ Anesthesia and intensive care b unit, University of Verona, AOUI-University Hospital Integrated Trust of Verona, Verona, Italy; ${ }^{6}$ Department of anesthesia and intensive care unit, St. Bortolo Hospital, Vicenza, Italy

Correspondence: $L$. Cattin

Intensive Care Medicine Experimental 2020, 9(1): 000501

Introduction. Non-invasive ventilation (NIV) is currently a strategy obviate the need for endotracheal intubation in patients with acute respiratory failure. NIV through a mask device is that higher levels of positive end-expiratory pressure (PEEP) may lead to mask intolerance and air leaks around the sides of the mask. Helmet NIV may have several advantages over face masks including better tolerability and less air leaks. However, also NIV can cause lung injury from excessive negative pressure forces. Invasive mechanical ventilation (IMV) is a lifesaving strategy for critically ill patients with coronavirus disease 2019 (COVID-19). Optimal timing of initiation of invasive mechanical ventilation in patients with acute hypoxemic respiratory failure due to COVID-19 is unknown. In addition,the effect of intubation timing on the prognosis of critically ill patients with coronavirus 2019 (COVID-19) is not yet well understood.

Objectives. We examined the impact of time to intubation and use of NIV delivered by oro-nasal mask, full face-mask or helmet in COVID-19 patients. Secondary outcomes included 28-day invasive ventilator-free 
days (ie, days alive without mechanical ventilation), duration of ICU and hospital length of stay, and hospital and 28-day mortality.

Methods. Single-center prospective, observational study of 90 patients with confirmed COVID-19 who require treatment for respiratory failure with NIV a) via oro-nasal masks b) via full face-mask and c) NIV via Helmet and admitted in a large, independent tertiary care medical center in Northern, Italy from November 2020 to April 2021.

Results. Among 88 patients admitted to the ICU, 87 received NIV: $17(19.32 \%)$ were treated with NIV via oro-nasal masks, 29 (32.95\%) NIV via full face-mask and 41 (46.59\%) NIV via Helmet. All patients received invasive mechanical ventilation (IMV). Among 88 COVID-19 patients with IMV, 56 (63.664\%) died, and 32 (36.36\%) survived and was discharged. The median age was 63.0 years $(56.0-$ 69.5). The median period from the noninvasive mechanic ventilation (NIV) to intubation was 96 (24-192) hours in IMV survivors and 84 (36$156)$ hours in IMV non-survivors $(p=0.72)$. The mortality odds ratio for each full increment of $1 \mathrm{~h}$ of NIV was $0.9996(95 \% \mathrm{Cl} 0.99-1.005)$ $\mathrm{P}=0.87$.

Conclusion. In our analysis, all patients failed NIV trial and received IMV. The duration of NIV did not affect the mortality in our COVID-19 population..

\section{Reference(s)}

1. Zhang Q, Shen J, Chen L, Li S, Zhang W, Jiang C, Ma H, Lin L, Zheng X, Zhao Y. Timing of invasive mechanic ventilation in critically ill patients with coronavirus disease 2019. J Trauma Acute Care Surg. 2020 Dec;89(6):1092-1098. https://doi.org/10.1097/TA.000000 0,000002,939. PMID: 32,925,581; PMCID: PMC7687875.

2. Patel BK, Wolfe KS, Pohlman AS, Hall JB, Kress JP. Effect of Noninvasive Ventilation Delivered by Helmet vs Face Mask on the Rate of Endotracheal Intubation in Patients With Acute Respiratory Distress Syndrome: A Randomized Clinical Trial. JAMA. 2016 Jun 14;315(22):2435-41. https:// doi.org/10.1001/jama.2016.6338.

\section{4}

\section{Usefulness of transpulmonary pressure monitoring} in the ventilatory management of morbidly obese C-ARDS

\section{patients}

M. Sánchez Galindo '; JA. Sanchez Giralt²; BM. San³; MA. Pérez Lucendo²;

B. Abad Santamaría ${ }^{2}$; LG. J.'; B. Muñoz Molina ${ }^{2} ;$ M. Trigueros Genao;

S. Portillo Sánchez²; I. Magaña Bru²; I. Temprano García; A. Canabal Berlanga '; F. Suarez Sipmann²

${ }^{1}$ ITU, Hospital de La Princesa, Madrid, Spain; ${ }^{2} \mathrm{CCU}$, Hospital de La

Princesa, Madrid, Spain; ${ }^{3}$ Intensive care medicine, Hospital de La Princ-

esa, Madrid, Spain

Correspondence: M. Sánchez Galindo

Intensive Care Medicine Experimental 2020, 9(1): 000504

Introduction. Obesity is a well described risk factor for developing covid related acute respiratory distress syndrome (C-ARDS). Morbidly obese C-ARDS patients are particularly difficult to ventilate as, even though initial respiratory system compliance is moderately impaired applied airway pressures are often in the high non-protective range. The use of esophageal manometry can be particularly useful in this population with a decreased chest wall compliance, as it allows to separate lung from chest wall mechanics and thus target mechanical ventilation to transpulmonary instead of airway pressures.

Objectives. To study the usefulness of transpulmonary pressure monitoring to individualize PEEP settings and ventilatory support in morbidly obese C-ARDS patients.

Methods. We studied morbidly obese patients $(B M I \geq 40)$ with the diagnosis of severe C-ARDS monitored with esophageal manometry by means of a modified nasogastric catheter with a distal balloon(Nutrivent ${ }^{\circledR}$ ).After radiologic confirmation of correct catheter positioning and adequate balloon air inflation, measurements were confirmed by a passive positive pressure occlusion test observed in the airway and esophageal pressure tracings of the ventilator(Servo$\mathrm{U}$, Maquet Critical Care).Patients were ventilated with lung protective settings and submitted to an automated sequential recruitment maneuver combining a short recruitment maneuver(RM)in a PC mode followed by a decremental PEEP titration in a VC mode.Optimum PEEP was established according to compliance $(2 \mathrm{cmH} 2 \mathrm{O}$ above the PEEP level resulting in maximal compliance and according to the change in polarity of end-expiratory transpulmonary pressure(PLee)selecting the PEEP resulting in a PLee of $+2 \mathrm{cmH} 2 \mathrm{O}$ ).

Results. We included 7 morbidly obese patients (BMI $48 \pm 8$, mean $\pm S D$ ), man/woman 5/2;aged $50(43-60)$ years, in this preliminary analysis.All were ventilated in a PC mode and lung mechanics and ventilator parameters at baseline, during recruitment and at PEEP according to compliance and to PLee are presented in Table 1. A total of 21 sequential RM were analyzed.Mean inspiratory RM airway pressures were $51 \pm 5 \mathrm{cmH} 2 \mathrm{O}$ applied during $2 \mathrm{~min}$. $56 \%$ of the applied $\mathrm{RM}$ airway pressure was dissipated in expanding the chest wall resulting in an end-inspiratory transpulmonary pressure of $32 \pm 7 \mathrm{cmH} 2 \mathrm{O}$ (Table 1). Optimum PEEP according to compliance was $15.4 \pm 4.6$ and to PLee $16.3 \pm 3.7 \mathrm{cmH} 2 \mathrm{O}(\mathrm{p}=0.12)$. Compliance, and airway plateau and driving pressures significantly decreased at both optimum PEEP levels compared to baseline conditions.On averagetranspulmonary end-inspiratory pressures were a $50 \%$ lower than the airway plateau pressures.Transpulmonary driving pressure was significantly lower than airway driving pressure at all studied conditions (Table 1).

\begin{tabular}{|c|c|c|c|c|c|c|}
\hline Mechanics & $\begin{array}{l}\text { Base- } \\
\text { line }\end{array}$ & $\mathrm{RM}$ & $\begin{array}{l}\text { Post-RM } \\
\text { (C.dyn PEEP) }\end{array}$ & $\begin{array}{l}\text { Post-RM } \\
\text { (PLee PEEP) }\end{array}$ & $\begin{array}{l}\text { Baseline vs } \\
\text { C.dyn PEEP }\end{array}$ & $\begin{array}{l}\text { Baseline vs } \\
\text { PLee PEEP }\end{array}$ \\
\hline $\begin{array}{l}\text { Plateau } \\
\text { pressure }(\mathrm{cmH} 2 \mathrm{O})\end{array}$ & $29 \pm 3$ & $51 \pm 5$ & $24 \pm 4$ & $26 \pm 4$ & $P=0.0001$ & $P=0.004$ \\
\hline PEEP(cmH2O) & $17 \pm 4$ & $36 \pm 5$ & $15 \pm 5$ & $16 \pm 4$ & $P=0.06$ & $P=0.26$ \\
\hline $\begin{array}{l}\text { Driving } \\
\text { pressure }(\mathrm{cmH} 2 \mathrm{O})\end{array}$ & $12 \pm 2$ & 15 & $9 \pm 3$ & $10 \pm 3$ & $P<0.0001$ & $P<0.0001$ \\
\hline T.Volume(ml/kg) & $6.8 \pm 1.4$ & - & $6.1 \pm 1.3$ & $6.1 \pm 1.3$ & - & - \\
\hline C. dyn $(\mathrm{ml} / \mathrm{cmH} 2 \mathrm{O})$ & $42 \pm 15$ & - & $51 \pm 16$ & $50 \pm 17$ & $P<0.0001$ & $P<0.0001$ \\
\hline PLei (cmH2O) & $13 \pm 3$ & $32 \pm 7$ & $8 \pm 3$ & $10 \pm 3$ & - & - \\
\hline PLee $(\mathrm{cmH} 2 \mathrm{O})$ & $3 \pm 2$ & $17 \pm 5$ & $2 \pm 3$ & $3 \pm 1$ & - & - \\
\hline Driving PL(cmH2O) & $10 \pm 3$ & 15 & $6 \pm 4$ & $7 \pm 3$ & 0,0005 & 0,0004 \\
\hline
\end{tabular}

Conclusion. Transpulmonary pressure monitoring was useful in the ventilatory management of morbidly obese C-ARDS patients. It helped to individualized PEEP, although non-significant there was a small difference between both compliance and PLee determined PEEP values indicating that there may are individual differences of clinical relevance. The large proportion of pressure needed to expand the chest-wall of these patients helps to identify the real pulmonary stress during recruitment and lung protective ventilation in these patients.

\section{6}

Non-targeted proteomics in critical patients with COVID-19 (ProteoCrític)

M. Martín Cerezuela'; MJ. Broch Porcar ${ }^{1}$; I. Guillen Bernat ${ }^{1}$; C. Vicent Perales'; A. Martínez Yañez'; C. Maya Gallegos'; A. Congost Mestre ${ }^{2}$; O. Antunez Temporal2 ${ }^{2}$; A. Castellanos Ortega'; M. Sanchez Del Pino ${ }^{3}$ ${ }^{1}$ Intensive care unit, Hospital Universitario y Politécnico La Fe, Valencia, Spain, Spain; ${ }^{2}$ Proteomic, University of Valencia, València, Spain; ${ }^{3}$ Proteomic unit, University of Valencia, València, Spain

Correspondence: M. Martín Cerezuela

Intensive Care Medicine Experimental 2020, 9(1): 000506

Introduction. Despite rapid and extensive efforts made to study the emerging COVID-19 disease, pathophysiological conditions are still poorly understood. Non-targeted proteomics examines many proteins and could help to identify proteomic profiles that would allow us to know the biological mechanism in which they are involved and to delve into the pathophysiology of this disease, which would help to discover potential therapeutic targets.

Objectives. To characterize and quantify plasma proteins in COVID-19 patients with moderate-severe ARDS at different evolutionary times. Methods. Prospective, observational study of a cohort of patients with moderate-severe ARDS ( $\mathrm{PaO} 2 / \mathrm{FiO} 2<200 \mathrm{mmHg}$ ) by SARS-CoV- 2 . 
Demographic, clinical, evolutionary data (severity and mortality score) and plasma samples (MP) under study at admission (TO) and at 7 days (T1) are collected. Approved by the hospital's CEIB. The MPs are analyzed in the Proteomics Service (UV) after being prepared in LC-MS/ MS in SWATH mode for data independent acquisition. By bioinformatics (mixOmics) a multilevel multivariate approach is performed analyzing the internal variance considering significance an $\mathrm{RDF}<1 \%$. Quantitative variables are described as median (interquartile range) and qualitative variables as absolute values and percentages. The continuums are compared with the Student $t$ and the categorical ones with the $\mathrm{X} 2$ test or Fisher test $(\mathrm{p}<0.05)$ with the STATA 14 statistical package.

Results. 40 samples from 17 patients and 6 healthy volunteers were studied: severe ARDS $35.3 \%$, moderate $64.7 \%$, age 52 (46-64), males $58.82 \%$, BMI 30.86 (27.25-33.98), SAPS-3 45 (42-47), SOFA admission 3 (2-3), Invasive Mechanical Ventilation (IMV) 53\%, High-flow Nasal Oxygen (HFNO) 47\%, ECMO 11\%, Acute Kidney Injury 17\%, mortality $23.5 \%$. The IMV group had significantly more SAPS 3, basal SOFA, more superinfections, greater use of vasopressors and longer stays than the HFNO group $(p<0,05)$. We identified $>385$ proteins with $R D F<1 \%$, with 40 being those that best discriminated T0 and T1 (Fig. 1), mainly participating in biological processes (Gene Ontology): neutrophil aggregation, zinc sequestration, triglyceride transport and lipoprotein and lipase regulation. The interactions between them are shown in Fig. 2.

Fig. 1. https://we.tl/t-0GjiydHx7g

Fig. 2. https://we.tl/t-YIMEhOX1f2

Conclusion. The analysis allowed the identification of differential proteins between the evolutionary times under investigation and their biological function and interactions were studied. In a second phase, their relationship with the evolution of the disease and their potencial use as biomarkers will be studied.

\section{7}

Ketamine association for the difficulty in weaning of COVID-19 pneumonia ventilated patients in our Intensive Care Unit

O. Moreno Romero'; M. Muñoz Garach²

${ }^{1}$ Intensive care unit, San Cecilio University Clinical Hospital, Granada,

Spain; ${ }^{2}$ Intensive care unit, Hospital Universitario Clínico San Cecilio, Granada, Spain

Correspondence: O. Moreno Romero

Intensive Care Medicine Experimental 2020, 9(1): 000507

Introduction. High respiratory drive leads to weaning failure in severe ARDS in ventilated COVID 19 patients and thus, probably increases ICU length of stay and worsens prognosis.

Objectives. To analyse the asociation of ketamine to standard sedation in patients with high ventilatory impulse helped weaning during the first days of mechanical ventilation of COVID-19 pneumonia patients in our intensive care unit.

Methods. We performed a 3-month prospective study (September 2020-November 2020) of patients with severe COVID-19 pneumoniae under invasive mechanical ventilation (IMV) in our intensive care unit. From all IMV patients, we selected a small sample with early weaning maneuvers in the first $48 \mathrm{~h}$ and with a high respiratory drive/impulse $(P 0.1>6)$ after decreasing standard sedation and who needed to start of reintroduce neuromuscular blockers (NMBAs) in a maximum of $24 \mathrm{~h}$ to readapt the ventilation. In these patients, we added an infusion of $2 \mathrm{mg} / \mathrm{kg} / \mathrm{h}$ of ketamine for a minimum of $24 \mathrm{~h}$ and a maximum of $72 \mathrm{~h}$. Weaning was reinitiated after 24-72 h maximum. Variables registered: sex, age, use of prone position in the first $24 \mathrm{~h}$, use of NMBAs, mean time to extubation in these patients, death rate of all COVID-19 patients and these sample.

Results. In 3 months, from 110 covid-19 patients under IMV in our ICU, we selected 25 patients for our study. Variables: men 18 (72\%); mean age 57y (37-69); $13(52 \%)$ patients needed prone position in the first $24 \mathrm{~h}$ and 10 (40\%) NMBAs; mean time to extubation 6.5 days (4-11), death rate $4(16 \%), 2$ with septic shock and 2 with cardiogenic shock. Death rate of global IMV covid 19 patients in these period was 38\%.
Conclusion. In our sample, mean time to extubation was 6.5 days. Compared to the global IMV COVID 19 patients death rate in our ICU, our study patients had less mortality (16 vs $38 \%$ ). Adding ketamine to standard sedation in patients with high ventilatory impulse during early weaning might be an efective tool to help in weaning process in these particularly patients, although our sample is small and there is no group control to compare results.

\section{Reference(s)}

1. High respiratory drive and excessive respiratory efforts predict relapse of respiratory failure in critically ill patients with COVID-19. Am J Respir Crit Care Med 2020;202:1173-1178.

2. The respiratory drive: an overlooked tile of COVID-19 pathophysiology. Gattinoni et al. Am J Respir Crit Care Med 2020.Oct 15;202(8):1079-1080.

\section{8}

Results of videolaryngoscopy in the intubation of COVID-19 patients in our intensive care unit

O. Moreno Romero'; M. Muñoz Garach'; P. Fernandez Morales';

MT. Cruces Moreno'

${ }^{1}$ Intensive care unit, Hospital Universitario Clínico San Cecilio, Granada, Spain

Correspondence: $\mathrm{O}$. Moreno Romero

Intensive Care Medicine Experimental 2020, 9(1): 000508

Introduction. Intubation procedure in severe ARDS COVID-19 patients is essential to prevent severe complications of critical hypoxia and thus, morbi-mortality.

Objectives. To analyse the use of videolaringoscopy for the intubation of patients with COVID-19 severe pneumoniae in our intensive care unit (ICU).

Methods. We performed a 8-month retrospective study (Agost 2020March 2021) of patients with severe COVID 19 pneumoniae under invasive mechanical ventilation (IMV) in our intensive care unit who where intubated with videolaryngoscopy Kalrstorz ${ }^{\mathrm{TM}}$. Variables registered: sex, age, APACHE II at admision, Cormack, duration of intubation, use of Frova guide, no possibility for intubation, death rate during intubation if present.

Results. In 8 months, from 295 covid19 patients under IMV in our ICU, we registered 185 patients intubated with videolaryngoscopy. Variables: men 55 (64.7\%); mean age 55y (28-76); mean APACHE II of $19 \pm 6.5$; Cormack I and II 181 (61\%), Cormack III 70 (24\%), Cormack IV 44 (15\%); mean time of intubation procedure 15 s (5-72); Use of Frova guide in $17(9 \%) ; 5$ patients (3\%) were impossible to intubate and needed emergent tracheostomy: 3 with high Cormack and severe cervical artrosis and 2 with unknown laryngotracheal stenosis; no death were registered during technique.

Conclusion. The use of videolaryngoscopy for the intubation of severe hypoxemic covid 19 patients minimizes the time and thus the complications in these particularly patients. In our sample, only in $3 \%$ the intubation was impossible and needed emergent tracheostomy. No death were reported during technique.

\section{Reference(s)}

1. Emergency tracheal intubation in 202 patients with COVID-19 in Wuhan, China: lessons learnt and international expert recommendations. British Journal of Anaesteshia (2020), 125(1):e28-e37

2. Summary of 20 tracheal intubation by anesthesiologists for patients with severe COVID-19 pneumonia: retrospective case series. Li Zhang et al. Journal of Anesthesia (2020) 34:599-606

\section{4}

$\mathrm{PaO2} / \mathrm{FiO} 2$ and $\mathrm{PaCO} 2$ dynamics benefit from Time-Controlled Adaptive Ventilation (TCAV)-report of an early experience in an APRV-naïve ICU

M. Varela'; S. Duarte ${ }^{1}$; L. Melão ${ }^{1}$; A. Krystopchuk'; D. Nuñez ${ }^{1}$; J. Moreno

${ }^{1}$ Intensive Care Medicine 1, University Hospital Center of Algarve,

Faro, Faro, Portugal

Correspondence: M. Varela

Intensive Care Medicine Experimental 2020, 9(1): 000514 
Introduction. We recently started using Airway Pressure Release Ventilation (APRV) in our polyvalent ICU, according to Time Controlled Adaptive Ventilation (TCAV) protocol proposed by Habashi and later by Zhou.

APRV, which resembles a CPAP mode with expiratory releases, was first used with these expiratory releases in a fixed time. TCAV is APRV with expiratory cycles adapted to the patient's respiratory system, with potential benefits, namely in ARDS, atelectasis, sedation and vasopressor dose requirements.

Medical and nurse staff had no previous experience with APRV or TCAV, hence formal review of our early experience was needed.

Objectives. Our aim was to study our polyvalent ICU's early experience with TCAV and whether benefit was obtained from it.

Methods. We retrospectively analyzed the electronic clinical records of all patients admitted to our polyvalent ICU who were ventilated on TCAV for more than $2 \mathrm{~h}$, between the 1st of January and the 30th of April of 2021. We excluded Sars-Cov2 positive patients.

TCAV was used according to the Habashi protocol, with an initial PHigh of 28-32 and Plow of $0 \mathrm{mmHg}$, TLow between 0,4 and $0,8 \mathrm{~s}$ and adjusted so that the end of expiration has a flow between 50 and $75 \%$ of peak expiratory flow, Thigh between 2 and $4 \mathrm{~s}$ and increased as soon as possible. Automatic tube compensation was not used. Maquet Servo $U$ ventilators were used.

We searched for patients' demographics, number of days while on invasive mechanical ventilation, date and reasons for starting and ending TCAV, ventilation modes before and after TCAV, FiO2, PO2, PCO2 before, $2 \mathrm{~h}$ and $6 \mathrm{~h}$ after initiating TCAV.

Data was analyzed using Microsoft Office 365 Excel v16.0.13901.20148. Results. 8 patients were put on TCAV, of which only 5 had information on their clinical records that could be used to correctly determine $\mathrm{PaO} 2 / \mathrm{FiO} 2$ and $\mathrm{PCO} 2$ before and hours after TCAV was initiated.

Reasons for switching to TCAV included ARDS (1 patient), recurrent atelectasis while on PC (3), $\mathrm{FiO} 2>60 \%$ of difficult weaning (3) and to gain experience with TCAV (1).

Reasons for switching from TCAV to another mode of ventilation were: patient-ventilator asynchrony (1 patient), weaning to CPAP (1), weaning to Pressure Support (4) and lack of experience with TCAV (2).

The average number of days while on TCAV was 3 days.

Average $\mathrm{PaO} 2 / \mathrm{FiO} 2$ before initiating TCAV was 156 , with a decrease to 146 after $2 \mathrm{~h}$, however there was an increase to 219 after $6 \mathrm{~h}$. Average PCO2 before TCAV was $52,8 \mathrm{mmHg}$ and of $45,4 \mathrm{mmHg}$ after $2 \mathrm{~h}$ of TCAV.

No complications occurred during the period that the patients were on TCAV.

Conclusion. TCAV implementation in an APRV-naïve ICU is safe and can have a positive impact in $\mathrm{PaO} 2 / \mathrm{FiO} 2$ and $\mathrm{PCO} 2$ values. ICU staff should consider waiting at least $6 \mathrm{~h}$ after TCAV initiation to assess if benefit exists in $\mathrm{PaO} 2 / \mathrm{FiO} 2$ values.

Lack of experience was responsible for $25 \%$ of TCAV terminations, which shows that more formal training might be advised before implementing TCAV.

\section{Reference(s)}

1. Fredericks A, Bunker M, Gliga L, Ebeling C, Ringqvist J, Heravi H et al. Airway Pressure Release Ventilation: A Review of the Evidence, Theoretical Benefits, and Alternative Titration Strategies. Clinical Medicine Insights: Circulatory, Respiratory and Pulmonary Medicine. 2020;14:1-9

2. Nieman G, Gatto L, Andrews P, Satalin J, Camporota L, Daxon B et al. Prevention and treatment of acute lung injury with time-controlled adaptive ventilation: physiologically informed modification of airway pressure release ventilation. Annals of Intensive Care. 2020;10(1).

3. Zhou Y, Jin X, Lv Y, Wang P, Yang Y, Liang G et al. Early application of airway pressure release ventilation may reduce the duration of mechanical ventilation in acute respiratory distress syndrome. Intensive Care Medicine. 2017:43(11):1648-1659.

4. Kollisch-Singule M, Andrews P, Satalin J, Gatto L, Nieman G, Habashi N. The time-controlled adaptive ventilation protocol: mechanistic approach to reducing ventilator-induced lung injury. European Respiratory Review. 2019;28(152):180,126.
000514

Evaluation of a disposable flexible bronchoscope: a randomized, controlled, cross-over trial

R. Lenhardt'; O. Akca²; R. Latif ${ }^{2}$; F. Arnold ${ }^{3}$

${ }^{1}$ Anesthesiology and Critical Care, University of Louisville, Louisville,

France; ${ }^{2}$ Anesthesiology critical care, University Hospital, Louisville, United States of America; ${ }^{3}$ Infectious diseases, University of Louisville, Louisville,

France

Correspondence: R. Lenhardt

Intensive Care Medicine Experimental 2020, 9(1): 000514

Introduction. Flexible bronchoscopy is a frequently performed procedure in the critical care unit. Flexible bronchoscopes are typically reusable and therefore need high-level disinfection to prevent inadvertent spread of microbial pathogens from patient to patient. To overcome the process of disinfection, disposable bronchoscopes were developed.

Objectives. The aim of the study was to compare image clarity and handling performance of a reusable flexible bronchoscope (Storz8402 $2 x$, El Segundo, CA) to a disposable flexible bronchoscope (aScope 3 Ambu, Glen Burnie, MD).

Methods. With IRB approval and after obtaining informed consent, a total of 12 critically ill patients, who needed to undergo bronchoscopy, were enrolled in this prospective, randomized, controlled, crossover study. The view-clarity, image, and light -brightness of each bronchoscope was assessed by one unblinded and four blinded observers using offline video tapes. Flexibility and general ease of use was assessed by the operating observer. A visual analogue scale (VAS) from 0-100 was used for each measurement. Values above a VAS of 50 were considered good, above 70 very good and above 90 excellent.

Results. Results from the operating observer: View-clarity with the disposable bronchoscope was $75 \pm 12$ and $80 \pm 7$ with the reusable bronchoscope $(p=0.06)$, Image was $73 \pm 12$ and $80 \pm 8(p=0.01)$, respectively, light-brightness was $79 \pm 13$ and $80 \pm 13 \quad(p=0.75)$ respectively. Flexibility was rated $87 \pm 10$ with the disposable bronchoscope versus $74 \pm 13(p=0.04)$ with the reusable bronchoscope. Lastly, general ease of use was rated $79 \pm 15$ versus $79 \pm 11(p=1)$. Results from blinded observers rating video quality of the bronchoscopes: View-clarity with the disposable bronchoscope was $53 \pm 17$ and $71 \pm 13$ with the reusable bronchoscope $(p<0.01)$, Image was $57 \pm 15$ and $73 \pm 12 \quad(p<0.01)$, respectively, light-brightness was $57 \pm 18$ and $75 \pm 13(p<0.01)$ respectively.

Conclusion. The reusable bronchoscope was superior in terms of view-clarity, image quality and light-brightness compared to the disposable bronchoscope. However, there was a significant difference in flexibility favoring the disposable bronchoscope. General use was rated equivalent between the compared bronchoscopes. The disposable bronchoscope performed good in all categories and very good in flexibility and general use, rendering it a reasonable alternative to reusable bronchoscopes in the ICU environment.

\section{Reference(s)}

1. Bellomo, R., Tai, E., \& Parkin, G. (1992). Fibreoptic bronchoscopy in the critically ill: a prospective study of its diagnostic and therapeutic value. Anaesth Intensive Care, 20(4), 464-469. 2. Kristensen, M. S., \& Fredensborg, B. B. (2013). The disposable Ambu aScope vs. a conventional flexible videoscope for awake intubation-a randomised study. Acta Anesth Scand, 57(7), 888-895. 3. Evaluation of a single-use intubating videoscope (Ambu aScope ${ }^{\mathrm{TM}}$ ) in three airway training manikins for oral intubation, nasal intubation and intubation via three supraglottic airway devices. Anaesthesia, 66(4), 293-299.

\section{4}

Differences between first and second wave among critically ill COVID-19 patients admitted to an ICU

G. Pérez-Acosta' ; L. Santana-Cabrera'; J. Blanco-Lopez ; P. Padrón Espinosa'; T. Carrillo-Garcia'; JM. González-Martín; J. Martín-González ${ }^{1}$ Intensive care unit, C.H.U. Insular-Materno Infantil, Las Palmas de Gran Canaria, Spain; ${ }^{2}$ Intensive care unit, Maternal and child Hospital, Las 
Palmas de Gran Canaria, Spain

Correspondence: G. Pérez-Acosta

Intensive Care Medicine Experimental 2020, 9(1): 000544

Introduction. Spain, like the rest of the world, has faced various waves of COVID during 2020. Different treatments were applied since the beginning of the pandemic. The continuous analysis of the infection course, has changed during admission to intensive care units (ICU). In our unit, different protocols were used between the first and second waves, taking into account the therapeutic recommendations and the expert committee. Specifically, the first wave included the use of Lopinavir / Ritonavir and Chloroquine, being replaced in the second wave by Emtricitabine / Tenofovir and Remdesivir. In both waves, the administration of Tocilizumab was available, its use being greater during the first wave, although without reaching statistical significance ( $p$ 0.053).

Objectives. Compare the demographic characteristics and prognosis of patients admitted to our ICU for COVID-19 pneumonia during the first wave (March-July) with those admitted during the second wave (August-December).

Methods. A prospective, observational, descriptive, cohort study with patients admitted to the ICU for COVID-19 pneumonia, during the first wave admitted from March-July 2020 and, the second, from AugustDecember. The demographic characteristics, comorbidities, treatment, complications and mortality between the two pandemic waves were studied.

Results. Seventy-two patients were included, 26 during the first wave and 46 in the second. Males predominated, $61.5 \%$ vs $73.9 \%$. The most associated comorbidities, in both periods, was arterial hypertension (65.4\% vs $65.2 \%)$, diabetes mellitus ( $46.2 \%$ vs $34.8 \%)$ and overweight, according to $\mathrm{BMI},(29.13 \pm 3.38$ versus $28.98 \pm 4.25)$. There is a greater use of non-invasive mechanical ventilation during the second wave $(p<0.01)$ with a lower incidence of atelectasis $(p<0.01)$ and bronchial obstruction du ring the second wave $(p=0.055)$, without observing significant differences. in the appearance of other complications.

\begin{tabular}{|c|c|c|c|c|c|c|c|}
\hline & $\sum_{n \rightarrow \infty}$ & $\begin{array}{l}m a \\
\text { mate }\end{array}$ & Promen & & 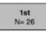 & 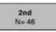 & svous \\
\hline Amomomin & 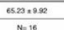 & $82 x+163$ & 0.560 & nuvv & $\begin{array}{ll}\mathrm{N}=1 \\
3 \mathrm{ss}\end{array}$ & 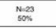 & $\infty$ \\
\hline anomernation & tist & 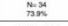 & $020 \mathrm{~A}$ & 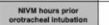 & 5.0060 & $4 * x+40$ & sen \\
\hline Pan & $22,13.3 .38$ & $2890+\angle 28$ & $0.05 s$ & 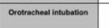 & $\begin{array}{l}n=2 \\
\text { aceis }\end{array}$ & 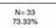 & 028 \\
\hline nomenom & 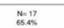 & 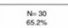 & 1 & neamberaston & 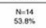 & $\prod_{n \rightarrow \infty}$ & 0.003 \\
\hline 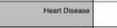 & 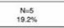 & 1BR & , & 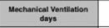 & $1000 \times 1404$ & 1300.1300 & 0.073 \\
\hline Dososent & 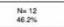 & $\begin{array}{l}\text { vall } \\
\text { sine }\end{array}$ & 0.451 & nonateon & 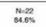 & 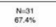 & 0.1es \\
\hline ontencis & 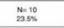 & $\begin{array}{ccc}\cos \theta \\
\cos \end{array}$ & (oon & 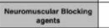 & sing & 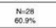 & $0.0 \times 1$ \\
\hline 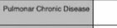 & istis & iss & ozem & 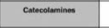 & 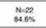 & 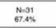 & 0.65 \\
\hline and & 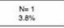 & 162 & 024 & 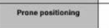 & 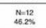 & $\begin{array}{lll}1423 \\
503\end{array}$ & 0000 \\
\hline 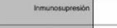 & 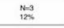 & 1.05 & 1 & 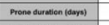 & $184 \times 255$ & $26+343$ & oust \\
\hline fromenry & 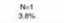 & 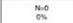 & 0.351 & 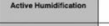 & $\underbrace{n=1}_{10 x}$ & 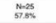 & 0.06 \\
\hline APACHE & $142 \pm 4.1$ & $17 \pm 8$ & 0.184 & 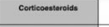 & 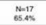 & 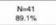 & 0.077 \\
\hline SoFA & 8004.5 & $7 \neq 4$ & 0.162 & 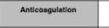 & 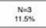 & 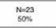 & 0.00 \\
\hline Chantsow & $286+168$ & 3.2 & 0.658 & Treschestomy & 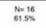 & stid & e.097 \\
\hline 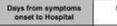 & 0.10 .460 & 580.203 & oort & rescumanes & 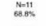 & 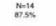 & o9s \\
\hline 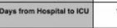 & $1052+62$ & $204+322$ & osese & surveleat & $x_{3, \infty}^{n-s}$ & $\mathrm{~N}_{125 \mathrm{~s}}$ & \\
\hline icuoms & $20.83+1326$ & $1740 \mathrm{~A}=1302$ & 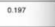 & uncomplest & 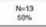 & Nost & 0268 \\
\hline 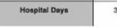 & $3188 \times 199$ & $3218 x+194$ & 085 & Hescuin Probsecel & wa & 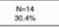 & 0.008 \\
\hline & & 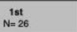 & 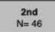 & P valuo & & & \\
\hline 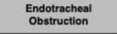 & & $\begin{array}{l}\text { N=17 } \\
7.27 \%\end{array}$ & $\begin{array}{l}\substack{N=23 \\
50 \%} \\
5\end{array}$ & 0.055 & & & \\
\hline Cardlac arthythmias & & $\begin{array}{c}\text { No8 } \\
30.8 \% \\
\text { 3. }\end{array}$ & $\begin{array}{l}=15 \\
32.6 \% \\
32.6\end{array}$ & 1 & & & \\
\hline Myocarditis & & $\begin{array}{l}N=0 \\
0 \%\end{array}$ & $\begin{array}{l}\mathrm{N}=2 \\
4.3 \%\end{array}$ & 0.532 & & & \\
\hline Pneumothorax & & $\begin{array}{l}\mathrm{N}=1 \\
3.8 \% \\
-10 \%\end{array}$ & $\begin{array}{l}N=3 \\
6.5 \%\end{array}$ & 1 & & & \\
\hline Atolectasis & & 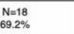 & $\begin{array}{l}N=11 \\
239 \%\end{array}$ & 80.01 & & & \\
\hline Delitium & & $\begin{array}{l}N=13 \\
50 \%\end{array}$ & $\begin{array}{l}\begin{array}{l}N=18 \\
39.1 \%\end{array} \\
39 \%\end{array}$ & 0.459 & & & \\
\hline Prone ulcers & & 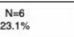 & $\begin{array}{l}N=15 \\
32.6 \%\end{array}$ & 0.433 & & & \\
\hline Acute Renal Falluro & & $\begin{array}{l}N=7 \\
N=9 \%\end{array}$ & $\begin{array}{l}N=11 \\
23.9 \%\end{array}$ & 0.784 & & & \\
\hline Masive hemorrhage & & 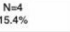 & $\begin{array}{l}\mathrm{N}=3 \\
6.5 \%\end{array}$ & 0.244 & & & \\
\hline $\begin{array}{l}\text { Tromboembollic } \\
\text { complications }\end{array}$ & & $\begin{array}{l}N=0 \\
0 \%\end{array}$ & $\begin{array}{l}N=1 \\
2.2 \%\end{array}$ & 1 & & & \\
\hline Deatn & & $\begin{array}{l}\text { Nes } \\
192 \%\end{array}$ & $\begin{array}{l}\text { No6 } \\
13 \%\end{array}$ & 0.511 & & & \\
\hline
\end{tabular}

Conclusion. No significant differences were observed in the demographic characteristics, or in the comorbidities of the hospitalized patients, between the two waves. High blood pressure, diabetes and overweight were the most common risk factors. Finally, knowing the complications that these patients develop is fundamental in the tendency to reduce the appearance of some such as endotracheal tube obstruction or atelectasis and the greater use of non-invasive ventilation therapies.

\section{Reference(s)}

1. Pérez Acosta G, Santana-Cabrera L. Necrotizing tracheobronchitis with endotracheal tube obstruction in COVID-19 patients. Rev Clin Esp. 2020;220:531-533. https://doi.org/10.1016/j.rce.2020.05.002.

2. J.M. Casas-Rojo, J.M. Antón-Santos, J. Millán-Núñez-Cortés, C. LumbrerasBermejo, J.M. Ramos-Rincón, E. Roy-Vallejo, et al.Clinical characteristics of patients hospitalized with COVID-19 in Spain: results from the SEMICOVID-19 Registry, Revista Clínica Española. 2020;220:480-494.

3. Contou D, Fraissé M, Pajot O, Tirolien JA, Mentec H, Plantefève G. Comparison between first and second wave among critically ill COVID-19 patients admitted to a French ICU: no prognostic improvement during the second wave? Crit Care. 2021;25(1):3. https://doi.org/10.1186/ s13054-020-03449-6.

4. Gao X, Ma C, Ma Y, Wang X, Wei J, Feng T, et al. Clinical efficacy and safety of different antiviral regimens in patients with coronavirus disease 2019 Zhonghua Wei Zhong Bing Ji Jiu Yi Xue. 2020;1423-1427. https://doi.org/ 10.3760/cma.j.cn121430-20201019-00679.

5. Dauner DG, Dauner KN. Summary of adverse drug events for hydroxychloroquine, azithromycin, and chloroquine during the COVID-19 pandemic. J Am Pharm Assoc. 2021; S1544-3191. doi: 10.1016 j.japh.2021.01.007. Epub ahead of print.

6. COVID-19, Artificial intelligence-based prevention. Illustrious College of Physicians Las palmas de Gran Canaria

\section{1}

Early P/F ratio evolution and Low-PEEP invasive mechanical ventilation in COVID-19 patients

S. Ceruti ${ }^{1}$; A. Saporito, ${ }^{2}$; M. Biggiogero ${ }^{3}$; A. Glotta ${ }^{4}$; P. Maida, ${ }^{5}$; C. Garzoni, ${ }^{5}$ ${ }^{1}$ Department of critical care, Clinica Luganese Moncucco, Via Moncucco, Lugano, Switzerland, Lugano, Switzerland; ${ }^{2}$ Anesthesiology division, EOC Ente Ospedaliero Cantonale, Bellinzona, Switzerland, Bellinzona, Switzerland; ${ }^{3}$ Clinical research unit, Clinica Luganese Moncucco, Lugano, Switzerland; ${ }^{4}$ Department of critical care, Clinica Luganese Moncucco, Lugano, Switzerland; Internal medicine department, Clinica Luganese Moncucco, Lugano, Switzerland

Correspondence: $\mathrm{S}$. Ceruti

Intensive Care Medicine Experimental 2020, 9(1): 000551

Introduction. Invasive MV is the gold standard treatment in patients with severe respiratory distress, including critically ill COVID-19 patients.Local approach to improve critically ill COVID-19 patients' management was performed using strict patient criteria for Intensive Care Unit (ICU) admission, standard treatment for thromboembolism and a low-positive end-expiratory pressure (PEEP) strategy.

Objectives. The main objective was to report the effects of low-PEEP ventilatory strategy on P/F-ratio evolution during first three days of MV, also reporting the ICU length of stay (LOS), MV length and mortality rate.

Methods. Patients on invasive MV were treated with a PEEP adapted to ARDSnet table according to BMI (PEEP $10 \mathrm{cmH} 2 \mathrm{O}$ if BMl $<30 \mathrm{~kg} / \mathrm{m} 2$, PEEP $12 \mathrm{cmH} 2 \mathrm{O}$ if BMI $30-50 \mathrm{~kg} / \mathrm{m} 2$, PEEP $15 \mathrm{cmH} 2 \mathrm{O}$ if BMl $>50 \mathrm{~kg} /$ $\mathrm{m} 2$ ). $\mathrm{FiO} 2$ was adapted to maintain a $\mathrm{SpO} 2$ greater than $92 \%$ and a $\mathrm{PaO} 2>60 \mathrm{mmHg}$. A protective ventilation strategy (TV 6-8 $\mathrm{ml} \mathrm{Kg-1}$, Pplat $<30 \mathrm{cmH} 2 \mathrm{O})$ with permissive hypercapnia $(\mathrm{pH}>7.20)$ was adopted, with pronation cycles of 16-h.

Results. Seventy-nine critically ill COVID-19 patients were on invasive MV. Average applied PEEP resulted $11 \pm 2.9 \mathrm{cmH} 2 \mathrm{O}$ for $\mathrm{BMI}<30 \mathrm{~kg} /$ $\mathrm{m} 2,16 \pm 3.18 \mathrm{cmH} 2 \mathrm{O}$ for $\mathrm{BMI}>30 \mathrm{~kg} / \mathrm{m} 2$. No patient presented a BMI greater than $50 \mathrm{~kg} / \mathrm{m} 2$. After low-PEEP strategy application, patients' $\mathrm{P} / \mathrm{F}$ ratio presented daily improvement from admission during next $72 \mathrm{~h}(\mathrm{p}<0.001 ; \mathrm{Cl} 99 \%)$ that resulted statistically significant for each 
single day after oro-tracheal intubation [Fig. 1]. Median ICU length of stay (LOS) was 15 days (10-28); median MV length was 12 days (8-26). The ICU mortality rate was $31.6 \%$.

Conclusion. A low-PEEP management in COVID-19 patients on invasive MV showed a rapid and progressive improvement in $\mathrm{P} / \mathrm{F}$ ratio during the first $72 \mathrm{~h}$. This approach appeared also to be associated with a shorter MV length and ICU LOS, as well as lower mortality rate compare to other trials, but studies were too heterogeneous to obtain significantly strong comparative results. A low-PEEP strategy could be beneficial at multiple level, but further studies are still necessary to confirm this hypothesis.

\section{Reference(s)}

1. Marini JJ, Gattinoni L (2020) Management of COVID-19 Respiratory Distress. JAMA—J Am Med Assoc 323:2329-2330

2. Gattinoni L, Coppola S, Cressoni M, Busana M, Rossi S, Chiumello D (2020) COVID-19 does not lead to a "typical" acute respiratory distress syndrome. Am J Respir Crit Care Med 201:1299-1300

3. Bouadma L, Lescure FX, Lucet JC, Yazdanpanah Y, Timsit JF (2020) Severe SARS-CoV-2 infections: practical considerations and management strategy for intensivists. Intensive Care Med 46:579-582

4. Brower RG, Hopkins Uni-versity J, Lanken PN, et al. (2004) Higher versus Lower Positive End-Expiratory Pressures in Patients with the Acute Respiratory Distress Syndrome. N Engl J Med 351:327-336

\section{2}

\section{ARDS in SARS-COV2 pneumonia: Adjuvant measures} of mechanical ventilation

N. Rodrigo Castroviejo'; G. Ferrigno ${ }^{2}$; V. Madrid Romero ${ }^{3}$; R. Beltrán Bernáldez'; C. Sanmartino Gonzalez'; M. Berenguer Rodriguez';

LL. Beltran Martínez'; D. Monge Donaire'; A. Tejero Pedregosa'; S. Cortés Díaz ${ }^{1}$

${ }^{1}$ Intensive care, Hospital Virgen De La Concha, Zamora, Spain; ${ }^{2}$ Intensive Care Unit, Hospital Virgen De La Concha, Zamora, Spain; Internal medicine, Hospital Virgen De La Concha, Zamora, Spain

Correspondence: G. Ferrigno

Intensive Care Medicine Experimental 2020, 9(1): 000562

Introduction. Acute respiratory distress syndrome (ARDS) is a frequent cause of respiratory failure in the critically ill patient, viral etiology stands out for its high mortality, close to $50 \%$ of cases. This syndrome is characterized by pulmonary edema of non-cardiogenic origin causing hypoxemia, which in most cases will require invasive ventilatory support. Adjuvant measures to invasive mechanical ventilation ( IMV) have been widely used as a protective mechanical ventilation strategy. Prone positioning with $16 \mathrm{~h}$ sessions decreases mortality in patients with moderate and/or severe ARDS (1). Despite this, its use in routine clinical practice prior to the pandemic is less than recommended and less than $50 \%$ of patients with an indication receive this technique (2). However, its use during the COVID-19 pandemic has reached rates of over $80 \%$.

Objectives. To describe the characteristics of our patients admitted for COVID-19 pneumonia and the use of adjuvant invasive mechanical ventilation measures. Also, to analyze intra-ICU mortality.

Methods. Retrospective study including patients admitted to the ICU with a diagnosis of COVID-19 pneumonia during the pandemic period. Demographic data, severity scales, need for ventilatory support, VMI days, need for prone and repeat therapy, need for muscle blockers, and use of recruitment maneuvers were recorded. Results are expressed as mean $(95 \% \mathrm{Cl})$.

Results. 101 patients were studied, $81 \%$ male, 19\% female, age 64.5 (61.7-67.3) years, APACHE 14.24 (12.1-16.2), SAPSII 38.55 (35.1-43.9), SOFA 6 (5.28-6.7). 85\% of our patients required IMV for 13.71 (10.317.07 ) days. Of these, $28.6 \%$ required tracheostomy. A total of $52.4 \%$ of patients were treated with prone therapy, with a mean of 1.23 days to prone therapy from the day of admission. Almost $50 \%$ of the patients were prone on the first day of admission, the rest between the second and third day. Of the patients with prone positioning, 38\% required 1 or 2 sessions, the rest required 3 or more, with a maximum of 5 sessions in $2.3 \%$ of patients. As for the use of neuroblocking agents, $96 \%$ of the patients used them; it should be noted that this measure was used intermittently. Finally, the use of recruitment maneuvers was performed in almost $100 \%$ of the patients; these maneuvers were performed in an early and individualized manner, aiming for the maximum possible pulmonary re-expansion and its maintenance over time.

ICU stay was 16.5 (11.7-21.21) days. Mortality of all patients was $25 \%$ intra-ICU and $85 \%$ of those who were intubated and survived received prone therapy.

Conclusion. Covid 19 pneumonia causes severe ARDS in patients admitted to the ICU and in most patients hypoxemia refractory to the use of IMV (2). The use of adjuvant measures in ARDS in the specific case of COVID- 19 pneumonia helps us to achieve our objectives for a protective mechanical ventilation strategy for our patients: homogenize ventilation, minimize alveolar collapse and cyclic changes in transpulmonary pressure and provide the lowest Fio2. The use of muscle blockers and recruitment maneuvers were used in almost $100 \%$ of patients requiring IMV. Prone therapy was performed in a high percentage of survivors without being able to establish its impact on mortality. It is worth highlighting the use of nitric oxide therapy (iNo), which is not available in our hospital and could improve mortality in our patients with combined strategies of the previous therapies (3).

\section{Reference(s)}

1. Johannigman, Jay A. MD; Davis, Kenneth Jr., MD; Miller, Sandra L. MD; Campbell, Robert S. RRT; Luchette, Fred A. MD; Frame, Scott B. MD, and Branson, Richard D. BA, RRT Prone Positioning and Inhaled Nitric Oxide: Synergistic Therapies for Acute Respiratory Distress Syndrome, The Journal of Trauma: Injury, Infection, and Critical Care: April 2001_Volume 50-Issue 4-p 589-596

2. Grasselli, G., Cattaneo, E., Florio, G. et al. Mechanical ventilation parameters in critically ill COVID-19 patients: a scoping review. Crit Care 25, 115 (2021).

3. Guérin C, Reignier J, Richard J-C, Beuret P, Gacouin A, Boulain T, et al, Prone positioning in severe acute respiratory distress syndrome. N Engl J Med. 2013;368:2159-68.

\section{3}

Venovenous ECMO support in patients with SARS-COV-2 infection complicated with severe acute respiratory failure-a single centre cohort study

AB. Rodrigues ${ }^{1}$; I. Neves ${ }^{1}$; D. Gomes, ${ }^{1}$; SM. Fernandes ${ }^{1}$; JM. Ribeiro,

${ }^{1}$ Serviço de Medicina Intensiva, Centro Hospitalar Universitário Lisboa Norte-Hospital de Santa Maria, Lisbon, Portugal

Correspondence: A.B. Rodrigues

Intensive Care Medicine Experimental 2020, 9(1): 000583

Introduction. Implementation of extracorporeal membrane oxygenation (ECMO) in patients with severe hypoxemic respiratory failure due to SARS-CoV-2 infection has been challenged due to potentially scarse resources and doubt about its effectiveness. Demonstration of field results from ECMO centres is thus essential to mount sufficient evidence for recommendation design.

Objectives. We report the characteristics and outcome of patients with severe SARS-CoV-2 pneumonia treated with venovenous ECMO support from one single referral centre in a large urban university hospital.

Methods. Retrospective review of prospectively collected data from a protocol driven ECMO program. Patients with a diagnosis of COVID19 and severe hypoxemic respiratory treated with ECMO support were included. From March 2020 to March 2021, we collected patient's baseline characteristics, including clinical, respiratory and ventilatory parameters. Triage and ECMO run were started according to EURO ELSO criteria. Data is reported for survivors and non-survivors. Statistical analysis was performed to compare discharged and deceased patients.

Results. A total of 51 patients were treated with ECMO support. We included forty-seven patients, excluding 4 patients from our final 
report ( 3 still on ECMO run and 1 venoarterial ECMO due to cardiogenic shock without respiratory failure). Mean age was $52 \pm 11.9$ years, $72 \%$ were male. Most prevalent comorbidities were dyslipidemia (32\%), obesity (19\%), hypertension (17\%), and type 2 diabetes (9\%). Average SAPS II score was $36 \pm 15$, admission SOFA score $6 \pm 3$, and mean SOFA score on day 1 of cannulation was $7 \pm 4$. Of note, $17 \%$ were outreached from other hospitals. COVID-19 symptoms to ECMO cannulation was $15 \pm 7$ days. Oxygen therapy prior to ECMO included high flow nasal cannula (31\% of patients) for an average of 5 days, noninvasive ventilation (33\%) for 2 days and invasive mechanical ventilation (100\%) for 6 days. Pre-ECMO PaO2:FiO2 ratio was $87 \pm 4 \mathrm{mmHg}$, static compliance was $31 \pm 4 \mathrm{~mL} / \mathrm{cmH} 2 \mathrm{O}$, plateau pressure $25 \pm 4 \mathrm{mmHg}$ and driving pressure $14 \pm 4 \mathrm{mmHg}$. Patients had an average ECMO run duration of $26 \pm 19$ days and ICU length of stay of $38 \pm 20$ days. ICU mortality was $27 \%$. All other patients were discharged from the ICU and were alive after a mean follow-up of 126 days, apart from 5 with undefined hospital outcome (2 still in the ICU, 3 in the ward). Patients who survived were younger (49 vs 59 years, $p=0.01$ ), had lower SAPS II score (33 vs $44, p=0.03$ ), had shorter disease course (14 vs 19 days, $p=0.02)$, shorter time of mechanical ventilation before ECMO ( 5 vs 11 days, $p=0.006$ ) and higher lung compliance (33 vs $22 \mathrm{~mL} / \mathrm{cmH} 2 \mathrm{O}, p=0.01$ )

Conclusion. Our single centre experience revealed that venovenous ECMO support is safe and is associated with a high probability of survival in patients with the most severe forms of COVID-19 pneumonia. Early cannulation showed a survival benefit and should be strongly considered in the relatively young with severe hypoxemia.

\section{Reference(s)}

1. Zhang J, Merrick B, Correa GL, Camporota L, Retter A, Doyle A, et al. Venovenous extracorporeal membrane oxygenation in coronavirus disease 2019: a case series. ERJ Open Research [Internet]. 2020;6(4):00,463-2020. Available from: http://dx.doi.org/10.1183/23120541.00463-2020

2. Combes A, Hajage D, Capellier G, Demoule A, Lavoué S, Guervilly C, et al. Extracorporeal Membrane Oxygenation for Severe Acute Respiratory Distress Syndrome. New England Journal of Medicine [Internet]. 2018 May 24 [cited 2021 Apr 9];378(21):1965-75. Available from: http://www. nejm.org/doi/10.1056/NEJMoa1800385

\section{0}

\section{Point-of-Care-Ultrasound of pleural alterations in patients} after prolonged weaning caused by COVID-19-pneumonia F. Andres ; M. Spacek'; G. Maisuradze

${ }^{1}$ ICU, KLINIK BAVARIA Kreischa, Kreischa, Germany Correspondence: F. Andres

Intensive Care Medicine Experimental 2020, 9(1): 000600

Introduction. The COVID-19 (Corona virus disease 2019) pandemic poses an enormous challenge for the health care system around the world. There is an admission to ICU (intensive care unit) for invasive ventilation required in up to $16 \%$ of positive tested patients (Grasselli et al., 2020; Kluge et al., 2020). For accurate diagnostic of COVIDpneumonia POCUS (point-of-care-ultrasound) is an essential tool (Xirouchaki et al., 2011). The typic sonography findings in COVIDpneumonia are the following (Peng et al., 2020):

Thickening of pleural line with irregularities

Increased occurrence of b-lines

Consolidations

The POCUS of pleural alterations has been well researched with regard to early diagnostic and acute risk stratification of COVID-pneumonia (Brahier et al., 2020; Mohamed et al., 2020). Alterations in patients with successful prolonged weaning from mechanical ventilations have not been studied so far, especially regarding longterm COVID syndrome (Mohamed et al., 2020; Mendelson et al., 2021).

Objectives. Aim of this study was the description of pleural alterations using POCUS in patients after prolonged weaning caused by COVIDpneumonia. We scanned different POCUS patterns and compared them with the duration of ventilation and hospital stay, age, gender and pre-existing pulmonary conditions.
Methods. Between May 2020 and February 2021 we retrospectively investigated 30 patients with successful weaning from prolonged ventilation due to COVID-pneumonia who were required to the ICU. POCUS examination according to protocol was performed between the 5th and 15th day after weaning by three experienced ICU physicians. The examination followed a modified BLUE and Rouby's protocol consisting of twelve pleural zones (Lichtenstein and Mezière, 2008; Monteiro Pereira Jr. et al., 2020). For pattern distribution we developed the score system LUSS (lung ultrasound score) reaching 0 to 72 points as an amount of the B-sector (B-lines) and C-sector (consolidations, pleural thickening). For the statistical analysis regression analysis was applied.

Results. During the study period POCUS was performed on 30 patients (22 male, 8 female) with a mean age of $71 \mathrm{y}$. Median length of ventilation constituted 37,5 days. Pre-existing lung disease was present in $36 \%$ of patients. The median LUSS was 17 (2-18) and was worse in the anterior pulmonal zones. Consolidations and pleural thickening (median LUSS 11) were more present than B-lines (median LUSS 5). Performing the analysis no correlation between the duration of ventilation and the LUSS could be shown $(p=0.68)$. Splitted LUSS in $\mathrm{C}$-sector und $\mathrm{B}$-sector revealed no correlation to the ventilation duration either $(\mathrm{pC}=0.71, \mathrm{pB}=0,65)$. There was no interaction of duration of ventilation and pre-existing pulmonary illness to LUSS $(p=0.64)$.

Conclusion. The main finding of the study was, that there had been no significant correlation between the duration of ventilation followed COVID-pneumonia and the pleural alternations detected by ultrasound in patients after prolonged weaning. But COVID-typical changes could be detected in all patients who were successfully weaned. In conclusion there should be further studies regarding a follow-up POCUS at discharge. More patient characteristics and preexisting conditions could be considered.

\section{Reference(s)}

1. Peng QY, Wang XT, Zhang LN. 2020. Findings of lung ultrasonography of novel corona virus pneumonia during the 2019-2020 epidemic. Intensive Care Med, 46(5):849-850 https://doi.org/10.1007/s00134-020-05996-6.

2. Monteiro Pereira Jr. EF, de Carvalo Bethlem M, Silveira Vieira M, Muniz Pazeli Jr. J. 2020. Lung Ultrasound in Covid 19 Pandemic. Winfocus [accessed: 02/14/2021] URL: https://www.winfocus.org/lung-ultrasoundin-covid-19-pandemic/.

3. Mohamed MFH, Al-Shokri S, Yousaf Z, Danjuma M, Parambil J, Mohamed S, Mubasher M, Dauleh MM, Hasanain B, AlKahlout MA, Abubeker IY. 2020. Frequency of abnormalities detected by point-of-care lung ultrasound in symptomatic COVID-19 patients: Systematic review and meta-analysis. Am J Trop Med Hyg, 103(2):815-821 https://doi.org/10.4269/ajtmh. 20-0371.

4. Mendelson M, Nel J, Blumberg L, Madhi SA, Dryden M, Stevens W, Venter FWD. 2021. Long-COVID: An evolving problem with an extensive impact. South African Med J, 111(1):10-13 https://doi.org/10.7196/SAMJ.2021. v111i1.15433.

5. Lichtenstein DA, Mezière GA. 2008. Relevance of lung ultrasound in the diagnosis of acute respiratory failure the BLUE protocol. Chest, 134(1):117-125 https://doi.org/10.1378/chest.07-2800.

6. Kluge S, Janssens U, Welte T, Weber-Carstens S, Marx G, Karagiannidis C. 2020. S2k-Leitlinie-Empfehlungen zur intensivmedizinischen Therapie von Patienten mit COVID-19. AWMF Regist 113/001:Stand 23.11.2020 https://doi.org/10.5414/ATX02525.

7. Grasselli G, Pesenti A, Cecconi M. 2020. Critical Care Utilization for the COVID-19 Outbreak in Lombardy, Italy. JAMA, 323(16):1545-1546 https:// doi.org/10.1056/nejmoa2002032.

8. Brahier T, Meuwly J-Y, Pantet O, Brochu Vez M-J, Gerhard Donnet H, Hartley M-A, Hugli O, Boillat-Blanco N. 2020. Lung ultrasonography for risk stratification in patients with COVID-19: a prospective observational cohort study. Clin Infect Dis https://doi.org/10.1093/cid/ciaa1408.

9. Xirouchaki N, Magkanas E, Vaporidi K, Kondili E, Plataki M, Patrianakos A, Akoumianaki E, Georgopoulos D. 2011. Lung ultrasound in critically ill patients: Comparison with bedside chest radiography. Intensive Care Med, 37(9):1488-1493 https://doi.org/10.1007/s00134-011-2317-y.

10. The authors received no financial support for the research, authorship, and/or publication of this article. 


\section{9}

Dexamethasone vs Methylprednisolone in the treatment of patients with COVID-19 pneumonia admitted to our ICU J. Martin Rasero'; LM. Vega'; N. Cruza Leganés'; F. Arbol Linde'; E. Humanes ${ }^{2}$; MA. Taberna Izquierdo'; A. Simón Martin

'Intensive care unit, General Hospital Nuestra Señora del Prado, Talavera de la Reina, Spain; ${ }^{2}$ Emergencies, General Hospital Nuestra Señora del

Prado, Talavera de la Reina, Spain

Correspondence: J. Martin Rasero

Intensive Care Medicine Experimental 2020, 9(1): 000609

Introduction. COVID-19 pandemic has been a major challenge for ICUs worldwide, not only because of the large number of patients, but also because of the lack of knowledge in the initial management and treatment of the first critical patients admitted. The introduction of corticosteroids brought about a change in patients management. Now the difficult task of discerning which is the best therapeutic option and the optimal doses of the same is presented.

Objectives. To compare treatment with dexamethasone $20 \mathrm{mg}$ every $24 \mathrm{~h}$ for 5 days and methylprednisolone 500 mg every $24 \mathrm{~h}$ for 5 days in terms of days of ICU stay and days of mechanical ventilation (MV), in patients with severe COVID-19 pneumonia who required admission to the ICU and required MV.

Methods. A retrospective observational cohort study of patients admitted with severe COVID-19 pneumonia in our ICU between 03/14/2020 and 12/21/2020.

During the study period, 122 patients were admitted, excluding those who took a different corticosteroid regimen and those who did not require $M V$ during their admission.

The analysis was performed with ANOVA test for a single value, in which we wanted to calculate whether there was a significant difference between dexamethasone $20 \mathrm{mg} / 24$ for 5 days and methylprednisolone $500 \mathrm{mg} / 24 \mathrm{~h}$ for 5 days. The reason for using one treatment or the other is based on literature at that time. During the first and the beginning of the second wave, patients were treated with methylprednisolone and later, based on data from new publications, this was replaced by dexamethasone.

Results. During the study period, 122 patients were admitted, including those who required $\mathrm{MV}$ and received the study treatment, 76 patients. Thirty nine patients received dexamethasone, with a mean age of $60,74(95 \% \mathrm{Cl}, 56,8-64,68)$ years old, $\mathrm{PaO} 2 / \mathrm{FiO} 2$ at admission $99,62(95 \% \mathrm{Cl}, 86,9-112,33)$ and APACHE II score 15,46 $(95 \% \mathrm{Cl}, 14,08-16,85) ; 37$ patients received methylprednisolone, with a mean age of $58,95(95 \% \mathrm{Cl}, 55,71-62,19)$ years old, $\mathrm{PaO} 2 / \mathrm{FiO} 2$ at admission 109,97 $(95 \% \mathrm{Cl}, 90,62-129,32)$ and APACHE II score $18,38(95 \% \mathrm{Cl}, 16,32-20,44)$. ICU stay in the group treated with dexamethasone was 22,64 days $(95 \% \mathrm{Cl}, 16,56-28,72, p=0,2488)$ and in the group treated with methylprednisolone 27,65 days $(95 \% \mathrm{Cl}$, $21,8-33,49, p=0,2488)$. Regarding days of MV, those treated with dexamethasone had $17,56(95 \% \mathrm{Cl}, 12,13-23, \mathrm{p}=0,1663)$ days of $\mathrm{MV}$ and those treated with methylprednisolone had $23,02(95 \% \mathrm{Cl}$, $17,63-28,42, p=0,1663$ ) days of MV.

Conclusion. We cannot conclude the superiority of one treatment over the other in the reduction of days of stay in the ICU, nor in days of $\mathrm{MV}$, since there are no statistically significant differences, at least at the doses used in this cohort.

\section{8}

The relationship between respiratory system compliance, ventilatory ratio, and d-dimer in COVID-19 ARDS

A. Agarossi'; J. Montomoli, ; A. Carsetti, ; S. Spadaro, ${ }^{4}$; R. Colombo, ${ }^{1}$;

R. Ciraolo, ${ }^{2}$; A. Donati, ${ }^{3}$ S. Guarino, ${ }^{4}$; D. Ottolina, ; A. Potalivo, ${ }^{2}$;

P. Giaccaglia, ${ }^{3} ;$ A. Fogagnolo, ${ }^{4}$; E. Clemenza, ; T. Fossali, ; E. Catena, ${ }^{1}$

${ }^{1}$ Anesthesiology and Intensive Care, ASST Fatebenefratelli Sacco, Milano, Italy: ${ }^{2}$ Anesthesiology and intensive care, Ospedale "Infermi" di

Rimini, Rimini, Italy; ${ }^{3}$ Anesthesiology and intensive care, Ospedali Riuniti Ancona, Ancona, Italy; ${ }^{4}$ Anesthesiology and intensive care, Arcispedale Sant'Anna, Ferrara, Italy

Correspondence: A. Agarossi
Intensive Care Medicine Experimental 2020, 9(1): 000618

Introduction. The Acute Respiratory Distress Syndrome secondary to COVID-19 (C-ARDS) has shown peculiar features, such as a higher respiratory system compliance (CRS) and a higher ventilatory ratio (VR) when compared to ARDS due to other aetiologies. In addition, C-ARDS-induced hypoxaemia might depend, at least in part, to alterations in pulmonary perfusion secondary to thrombotic phaenomena. Consistently, $\mathrm{d}$-dimer plasma levels are usually elevated in these patients.

Objectives. We aim to explore the correlation between CRS, VR and plasma d-dimer in C-ARDS patients.

Methods. All adult C-ARDS patients admitted to intensive care unit in four Italian hospitals (Sacco Hospital, Milan; Infermi Hospital, Rimini; Ospedali Riuniti, Ancona; Sant'Anna Hospital, Ferrara) were screened. Invasively mechanically ventilated patients who met the Berlin ARDS diagnostic criteria were included in the database. Exclusion criteria were: absence of blood gas analysis or respiratory mechanics data within 24 hours from admission, spontaneous breathing at the time of inclusion, age $<18$ years. Data were retrieved from digital patients records and included in a dedicated database. The need for informed consent was waived by local ethics committees. Data were checked for normality of distribution with Kolmogorov-Smirnov test. Given the non-normal distribution of all the variables but age, tidal volume and CRS Spearman's Rho was used to assess between-variable correlations. Mann-Whitney $U$ test or Student's t test were for comparisons. CRS was measured under static conditions. VR was calculated as: [minute ventilation - paCO2] / (ideal body weight . $100 \cdot 37.5$ ). Data are presented as either mean \pm standard deviation, frequency (percentage) or median [1st-3rd quartile] according to their nature and distribution.

Results. Of the 130 patients included in the database, 46 met one or more of the above-mentioned exclusion criteria, leaving 84 for the analysis. The only significant correlations were those between $\mathrm{CRS}$ and $\mathrm{paO} 2 / \mathrm{FiO} 2$ ratio (Rho 0.417, $\mathrm{p}$-value $<0.001$ ), and between VR and plasma d-dimer (Rho 0.481, p-value $<0.001$ ). The study population was divided according to the population mean CRS into a higher and a lower CRS group (H-CRS and L-CRS, respectively). When analysing the two subgroups separately, only the correlation between VR and plasma d-dimer retained significance (L-CRS: Rho 0.362 , p-value $0.012 ; \mathrm{H}$-CRS: Rho 0.611 , p-value $<0.001$ ). Clinical and demographic features of the study population and between-group comparisons are reported in Table 1.

\begin{tabular}{|c|c|c|c|c|}
\hline & All patients & Lower CRS & Higher CRS & $p$-value \\
\hline Age (years) & $61.93 \pm 12.0$ & $62.7 \pm 13.1$ & $60.8 \pm 10.4$ & $>0.05$ \\
\hline Male sex [n (\%)] & $68(81.0 \%)$ & $34(70.8 \%)$ & 34 (94.4\%) & $>0.05$ \\
\hline$B M I(\mathrm{~kg} / \mathrm{m} 2)$ & $\begin{array}{c}28.3[26.2- \\
31.1]\end{array}$ & $\begin{array}{l}29.01 \\
\quad[26.2-31.3]\end{array}$ & $27.7[26.2-31.1]$ & $>0.05$ \\
\hline SOFA score & $8.0[7.0-11.0]$ & $8.5[8.0-11.0]$ & $8.0[6.2-10.5]$ & 0.042 \\
\hline$p H$ & $7.33[7.2-7.39]$ & $7.32[7.26-7.38]$ & 7.34 [7.29-7.39] & $>0.05$ \\
\hline $\mathrm{PaCO} 2(\mathrm{mmHg})$ & $47.0[42.2-53.7]$ & $47.0[43.0-54.0]$ & $47.0[42.0-52.7]$ & $>0.05$ \\
\hline $\mathrm{PaO} 2 / \mathrm{FiO} 2$ ratio & $114[92-165]$ & $106[82-148]$ & 130 [109-181] & 0.01 \\
\hline$V T(m / / k g \mid B W)$ & $7.4 \pm 1$ & $7.5 \pm 1$ & $7.2 \pm 0.8$ & $>0.05$ \\
\hline $\begin{array}{l}\text { Respiratory rate } \\
\quad(b p m)\end{array}$ & $18[16-20]$ & 18 [16-20] & 18 [16-20] & $>0.05$ \\
\hline PEEP (cmH2O) & $10.0[8.0-12.0]$ & $10.0[8.0-12.0]$ & $12.0[10.0-12.0]$ & $>0.05$ \\
\hline CRS $(\mathrm{ml} / \mathrm{cmH} 2 \mathrm{O})$ & $45.1 \pm 13.0$ & $36.0 \pm 6.4$ & $57.2 \pm 9.1$ & $<0.001$ \\
\hline Haematocrit (\%) & $\begin{array}{c}37.9[34.0- \\
40.2]\end{array}$ & 36.0 [32.0 39.1] & $\begin{array}{c}38.1[34.7- \\
41.0]\end{array}$ & 0.046 \\
\hline $\begin{array}{c}\text { C-Reactive Pro- } \\
\text { tein }(\mathrm{mg} / \mathrm{L})\end{array}$ & $\begin{array}{c}110.4[37.9 \\
-232.8]\end{array}$ & $\begin{array}{c}176.9[45.4- \\
244.5]\end{array}$ & $\begin{array}{c}83.0[22.3- \\
182.8]\end{array}$ & $>0.05$ \\
\hline
\end{tabular}

Conclusion. Our data show that, in C-ARDS patients, a relationship between VR and plasma d-dimer exists, albeit weak to moderate. However, the correlation between these two variables becomes 
stronger when analysing separately H-CRS patients. This could point out the role of impaired efficiency of ventilation in the pathophysiology of COVID-19-related respiratory failure, especially in those patients with a higher CRS. In addition, d-dimer might prove helpful to identify patients at higher risk of decompensation due to high VR.

\section{Reference(s)}

1. Huang C, Wang Y, Li X, et al. Clinical features of patients infected with 2019 novel coronavirus in Wuhan, China. Lancet 2020; 395: 497-506.

2. Grieco DL, Bongiovanni F, Chen L, Menga LS, Cutuli SL, Pintaudi G, Carelli S, Michi T, Torrini F, Lombardi G, Anzellotti GM, De Pascale G, Urbani A, Bocci MG, Tanzarella ES, Bello G, Dell'Anna AM, Maggiore SM, Brochard L, Antonelli M. Respiratory physiology of COVID-19-induced respiratory failure compared to ARDS of other etiologies. Crit Care. 2020 Aug 28;24(1):529. https://doi.org/10.1186/s13054-020-03253-2.

3. Carsana L, Sonzogni A, Nasr A, Rossi RS, Pellegrinelli A, Zerbi P, Rech R, Colombo R, Antinori S, Corbellino M, Galli M, Catena E, Tosoni A, Gianatti A, Nebuloni M. Pulmonary post-mortem findings in a series of COVID-19 cases from northern Italy: a two-centre descriptive study. Lancet Infect Dis. 2020 Oct;20(10):1135-1140. https://doi.org/10.1016/S1473-3099(20) 30434-5.

4. Liu X, Liu X, Xu Y, Xu Z, Huang Y, Chen S, Li S, Liu D, Lin Z, Li Y Ventilatory ratio in hypercapnic mechanically ventilated patients with COVID-19-associated acute respiratory distress syndrome. Am J Resp Crit Care Med 2020; 201:1297-99. https://doi.org/10.1164/rccm.202002-0373LE.

5. ARDS Definition Task Force, Ranieri VM, Rubenfeld GD, Thompson BT, Ferguson ND, Caldwell E, et al. Acute respiratory distress syndrome: the Berlin Definition. JAMA 2012; 307:2526-33. https://doi.org/10.1001/jama. 2012.5669

\section{5}

High Flow Nasal Cannula in patients admitted in Intensive Care Unit with SARS-COV-2 pneumonia and Acute Respiratory Distress Syndrome. Is the failure of this therapy related to obesity?

B. Lence'; C. Rivero'; JR. Fernández Villanueva'; I. Granja Gomez';

R. Hernández Vaquero'; A. Virgos Pedreira'; Al. Suarez Freire'; I. Acosta

Vidal'; A. Santiago Langarica'; ' C. Galbán

${ }^{1}$ Intensive care medicine department, Complejo Hospitalario Universi-

tario de Santiago de Compostela, Santiago de Compostela, Spain

Correspondence: $\mathrm{B}$. Lence

Intensive Care Medicine Experimental 2020, 9(1): 000635

Introduction. In 2020, COVID 19 disease spread and became a global health problem. Intensive Care Units (ICU) have hughely increased the number of patients admitted due to pneumonia and acute respiratory distress syndrome (ARDS). In this context, High Flow Nasal Cannula (HFNC) has been considered an option to support respiratory failure to provide not only higher concentration but also higher flow of oxygen to treat hypoxemia. Before the COVID-19 pandemic, studies demonstrated that HFNC could avoid orotraqueal intubation (OTI) and reduce mortality. The present study examines whether HFCN can be consider an option in treatment for hypoxemia in COVID-19 patients admitted in ICU and if its failure could be related to obesity, based on a Body Mass Index (BMI) over 30 .

Objectives. Firstly, knowing if HFNC can avoid OTI in patients with ARDS due to COVID-19 pneumonia. Secondly, to see if HFCN failure is related to $\mathrm{BMI}$.

Methods. This single center descriptive restrospective study is been realized in Hospital Clínico de Santiago de Compostela (Spain), and has analyzed at this moment 159 patients admitted in ICU. Data collection included the respiratory support at the admission and the BMI. Data analysis was made through SPSS statistics with Pearson's Chisquare test.
Results. 159 patients were admitted in ICU with ARDS requiring oxygen support due to respiratory failure. At the time of admission, $85(53 \%)$ were treated with HFNC, 74 (47\%) required OTI.

In the HFNC subgroup, $75(88 \%)$ of those patients needed OTI and mechanical ventilation: 18 (21\%) patients in less than $1 \mathrm{~h} ; 61$ (72\%) in less than 1 day. Criteria for OTI and mechanical ventilation were regular: refractory hypoxemia and tachypnea despite of non-invasive respiratory support.

89 patients, over de 159 included in this study, had overweight considering it a $\mathrm{BMI}>30$. In only the subgroup of patients treated with HFNC, 50 patients ( $60 \%$ of the subgroup) had a BMI $>30$, in 43 of those patients, the $86 \%$ of the patients with a BMI $>30$ supported with HFNC, presented hypoxemia and tachypnea, requiring OTI, almost all of them in the first hour (38 patients over 43).

To summarize, 149 (94\%) patients received mechanical ventilation and 89 were obese.

Conclusion. ARDS caused by COVID-19 requires OTI and mechanical ventilation in most of the patients admitted in ICU. While, more data is required to confirm a relation between BMI and HFNC failure. Besides, HFNC support is likely to fail the very first hour in patients with obesity.

\section{Reference(s)}

1. Li J, Fink JB, Ehrmann S. High-flow nasal cannula for COVID-19 patients: low risk of bio-aerosol dispersion. Eur Respir J 2020; 55: 2,000892 [https:// doi.org/10.1183/13993003.00892-2020].

2. Rochwerg B, Granton D, Wang DX, et al. High flow nasal cannula compared with conventional oxygen therapy for acute hypoxemic respiratory failure: a systematic review and meta-analysis. Intensive Care Med 2019; 45: $563-572$

3. Clinical consensus recommendations regarding non-invasive respiratory support in the adult patient with acute respiratory failure secondary to SARS-CoV-2 infection. Cinesi et al. Medicina Intensiva https://doi.org/ https://doi.org/10.1016/j.medin.2020.03.005

\section{1}

AirWayShield ${ }^{\circledR}$, a novel protection barrier to protect clinicians while intubating patients with COVID-19 or other respiratory diseases

JM. Alonso Babarro'; DCG. Jose Fidel2; J. Medrano ${ }^{3}$

${ }^{1}$ Intensive care unit, The Prince Charles Hospital, Chermside, Australia;

${ }^{2}$ Anesthesiology department, Araba University Hospital, Gasteiz, Spain;

${ }^{3}$ Bioaraba, Basque Country University, UPV/EHU, Vitoria-Gasteiz, Spain

Correspondence: J. Medrano

Intensive Care Medicine Experimental 2020, 9(1): 000641.

Introduction. The COVID-19 pandemic has raised an urgent need for adequate protective measures during high-risk aerosol-generating procedures such as endotracheal intubation (ETI). Current clinical guidelines for airway management encourage clinicians to focus on using appropriate contact and airborne precautions. The Airway Shield ${ }^{\mathrm{TM}}$ is a new and cost-effective barrier device that protects medical staff during intubation while facilitating the procedure itself, in contrast to other available alternatives such as barrier enclosures ('aerosol boxes').

Objectives. To conduct a randomized cross-over trial to determine whether intubation with the Airway Shield ${ }^{\mathrm{TM}}$ is non-inferior to standard intubation. We hypothesized that endotracheal intubation with Airway Shield $^{\mathrm{TM}}$ in a mannequin model results in similar first-pass success rates and intubation times when compared to endotracheal intubation without the device.

Methods. 


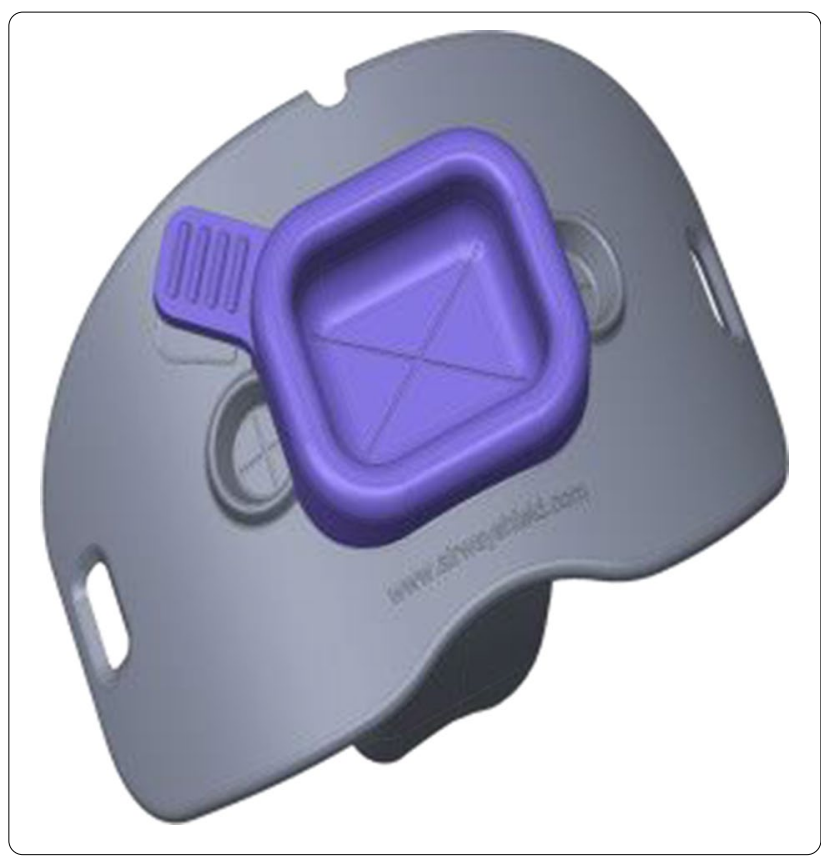

We conducted a randomized cross-over trial to test feasibility of endotracheal intubation (ETI) with a Maclntosh laryngoscope and Airway Shield ${ }^{\mathrm{TM}}$ compared to standard of care (Maclntosh laryngoscope) in a manikin model (AirSim, TruCorp ${ }^{\circledR}$ ). Primary endpoints were intubation time and success rate. Participants included 15 anaesthesiologists (10 females, 5 males) from the Hospital Universitario Araba (Vitoria, Spain) with no previous experience with the device. Participants were randomized to start with (9) and without (6) the Airway Shield ${ }^{\mathrm{TM}} .3$ attempts with and 3 without the device were made by each participant (total intubations $=90$ ).

Results. 45 intubations were carried out with each method. The mean time for ETI with the Airway Shield ${ }^{\mathrm{TM}}$ and Maclntosh Laryngoscope was 4,9 sg [2-9] compared to 9,6 sg [3-43] for ETI without the device $(p<0,001)$. The ETI success rate was $100 \%$ with both methods. Finally, participants scored the feasibility of intubation with Airway Shield. On a scale of $0-10$, six gave Airway Shield ${ }^{\mathrm{TM}} 10$ points (very feasible), three gave it 9 points, and six 8 points. Regarding ease of intubation, participants gave Airway Shield ${ }^{\mathrm{TM}}$ a mean of $9 / 10$ points compared to $8 / 10$ without Airway Shield ${ }^{\mathrm{TM}}$.

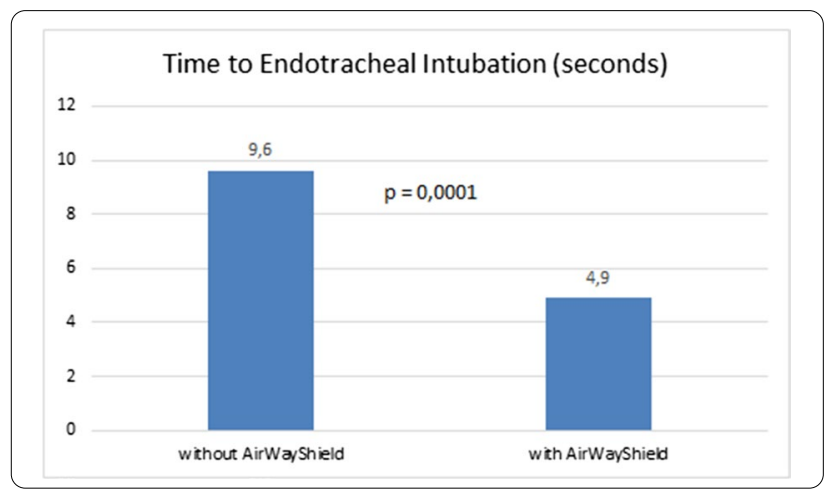

Conclusion. The Airway Shield ${ }^{\mathrm{TM}}$ is a cost-effective, safe, and innovative solution for avoiding clinician exposure to aerosols and droplets during airway management. This study demonstrates that it facilitates laryngoscopy by reducing intubation times in a mannequin model.

\author{
Reference(s) \\ 1. TruCorp ${ }^{\circledR}$, mannequin provider \\ 2. https://airwayshield.com
}

\section{4}

\section{STUDY OF THE MAIN RISK FACTORS OF POST EXTUBATION}

\section{STRIDOR IN CRITICAL ILL PATIENTS AFFECTED BY SARS-CoV2}

A. Ruiz García'; M. Trascasa Muñozz; FC. Serrano'; DR. Beltran²; R. Arraoz Illanes ${ }^{1}$; AB. Oñoro Morales²; L. Monge Sola²; MC. Pintado ${ }^{3}$; A. Acha²; E. Nevado ${ }^{2}$

${ }^{1}$ Medicina intensiva, Hospital Universitario Príncipe de Asturias, Meco, Spain; ${ }^{2}$ Intensive care unit, Hospital Príncipe de Asturias, Alcalá de Henares, Spain; ${ }^{3}$ Unidad de Cuidados Intensivos, Hospital Universitario Príncipe de Asturias, Alcala de Henares, Spain

Correspondence: A. Ruiz García

Intensive Care Medicine Experimental 2020, 9(1): 000644

Introduction. Post-extubation stridor is an uncommon entity in critical ill patients, with a global incidence of $<10 \%$ in the Intensive Care Units. It however implies more re-intubations; more days of mechanical ventilation and longer hospital stay.

Objectives. To establish the incidence and main risk factors of postextubation stridor in the critical ill patients affected by Sars-CoV2.

Methods. An observational retrospective study in an Intensive Care Unit of a Spanish University hospital, between December 2020 and March of 2021.

Patients included were diagnosed with an acute respiratory failure due to COVID 19 pneumonia and developed stridor during ICU stay.

We collected demographic data (age, sex), comorbidities (arterial hypertension, previous pulmonary pathology, dyslipidaemia, diabetes mellitus and obesity), days of ICU stay and days of endotracheal intubation, neuromuscular blockers and pronation manoeuvres, treatment with corticosteroids, need of reintubation and mortality.

Results. From the 112 COVID-19 patients accepted in our ICU during the chosen period, we analysed 8 cases of stridor (7.14\%). The mean age of our sample was $61.63 \pm 8.23$ years. The demographic data is shown in the table below.

\begin{tabular}{|c|c|c|c|c|}
\hline & & YES & NO & $\%$ \\
\hline Sex $q$ & & 7 & 1 & $87.5 \%$ \\
\hline \multirow{3}{*}{$\begin{array}{l}\text { CV medi- } \\
\text { cal } \\
\text { history }\end{array}$} & Hypertension & 3 & 5 & $37.5 \%$ \\
\hline & Obesity & 5 & 3 & $62.5 \%$ \\
\hline & Dyslipidaemia & 3 & 5 & $37.5 \%$ \\
\hline \multicolumn{2}{|c|}{ Mellitus diabetes } & 0 & 8 & $0 \%$ \\
\hline \multirow{2}{*}{$\begin{array}{l}\text { Pulmonary medical } \\
\text { history }\end{array}$} & Asthma & 2 & 6 & $25 \%$ \\
\hline & COPD & 0 & 8 & $0 \%$ \\
\hline \multicolumn{2}{|l|}{ Tobacco } & 1 & 7 & $12.5 \%$ \\
\hline
\end{tabular}

$100 \%$ of the patients required intubation with no complications associated and on the first try.

8 -gauge tubes with subglottic aspiration were used in the $87.5 \%$ of the patients (8/7). 4. patients required pronation manoeuvres; neuromuscular blockers were used in the $75 \%$ of the patients with a median of $2.5 \pm 0.82$ days.

The mean number of days of endotracheal intubation was $8.88 \pm 2.8$. $50 \%$ of the patients had completed treatment with corticosteroids (Dexamethasone 6-10 $\mathrm{mg} /$ day during 10 days).

Due to stridor, 4 of 8 patients (50\%) were reintubated, during 5.25 days on average. $100 \%$ of the patients received high dose of corticosteroid therapy ( $>1 \mathrm{mg} / \mathrm{Kg} /$ day). Only 1 patient was tracheotomised. The average stay in ICU was $13.87 \pm 2.67$ days, with no relationship between the need of reintubation or the conservative treatment ( $p$ 0.272) All of our patients were discharged of ICU.

Conclusion. In our sample, the number of cases of stridor was less than the literature cases, probably due to performing tracheostomy early, specifically at the 10th-12th days of intubation. 
In our study, we found a higher frequency of stridor in women between 55-65 years, with hypertension and/or obesity. We found no relationship between stridor and complications in the intubation technique, in pronation manoeuvres or neuromuscular blockers. There was no relationship between active corticosteroid therapy and need of reintubation. Stridor extended ICU stay in all the patients. We found no differences in mortality.

000655

Impact in oxygen indexes with PEEP titration before supination in pronated patients with moderate to severe ARDS due to SARS-COV2 infection

C. Rayzha, ${ }^{1}$; DC. Ortiz, ; AG. Lopez, ; N. Garzon, ${ }^{3}$; J. Carrizosa ${ }^{4}$

${ }^{1}$ Critical care medicine, Universidad del Rosario, Bogotá, Colombia; ${ }^{2}$ Department of critical and intensive care, Fundacion Santa Fe de Bogota University Hospital, Bogotá, Colombia; ${ }^{3}$ Department of critical and intensive care medicine, Fundacion Santa Fe de Bogota University Hospital, Bogotá, Colombia; ${ }^{4}$ Department of critical and intensive care medicine, University Hospital Fundación Santa Fe de Bogotá, Bogota, Colombia

Correspondence: J. Carrizosa

Intensive Care Medicine Experimental 2020, 9(1): 000655

Introduction. Acute respiratory distress syndrome (ARDS) in patients with SARS-COV2 infection has been related to a remarkable mortality rate affecting thousands of people worldwide. The prone position is one of the most relevant non-ventilatory strategies intended to improve oxygenation. However, clinical results are still on the path to achieve ways to optimize and standardize those strategies with the hope to decrease mortality and poor functional outcomes.

Objectives. This study aims to identify if PEEP titration right before the change of position from prone to supine is related to better oxygen levels measured by the $\mathrm{PaO} 2 / \mathrm{FiO} 2$ relationship $4 \mathrm{~h}$ after supination.

Methods. A prospective observational study comparing patients who did not receive PEEP titration vs patients who received PEEP titration at a University Hospital in Bogota, Colombia. At least two $\mathrm{mmHg}$ of PEEP right before supination after a complete cycle of prone position guided by the institutional protocol of 16 to $24 \mathrm{~h}$ was considered in the "PEEP titration group". A bivariate analysis was done to identify if the pre-supine PEEP titration is related to better oxygen indexes, shorter stay, and mortality.

Results. 29 patients in the "PEEP titration group" (PTG) and 22 patients in the "control group" (CG) were included for analysis. Mean age was 70 years old (SD 10 years), $60 \%$ male, median SOFA score was 4 points in both groups (IQR 2-8). Radiographic patterns for PTG: mixed 43\%, ground-glass opacification $20 \%$, alveolar pattern $17 \%$, consolidation 10\%; for CG: mixed $50 \%$, alveolar pattern $37 \%$ and ground-glass opacification $12.5 \% .100 \%$ of the patients of both groups received neuromuscular blockade at the time of evaluation. Arterial blood gas analysis at pre-supine and control $4 \mathrm{~h}$ later in the supine position was as follows: PTG PaO2/FiO2 195 (SD 40), $\mathrm{PaCO} 246$ (SD 11), $\mathrm{PaO} 2 / \mathrm{FiO} 2$ control 200; CG: PaO2/FiO2 197 (SD 42), $\mathrm{PaCO} 242$ (SD 8), PaO2/FiO2 control 177. In the bivariate analysis, there were no significant differences in terms of mortality. However, an absolute variation change in $\mathrm{PaO} 2 / \mathrm{FiO} 2$ favours PTG and less time of invasive mechanical ventilation (14 vs 18.5 days), and lesser length of ICU stay (16 vs 20 days).

Conclusion. Most favourable parameters of mechanical ventilation support are mandatory in every ICU setting as COVID-19 units. PEEP titration to an optimal level before the change of position from prone to supine seems to be related to less time requiring invasive mechanical ventilation, fewer prone cycles, and shorter length of ICU stay. These results need to be evaluated in greater trials.

\section{Reference(s)}

1. Koulouras, V., Papathanakos, G., Papathanasiou, A., \& Nakos, G. (2016). Efficacy of prone position in acute respiratory distress syndrome patients: A pathophysiology-based review. World journal of critical care medicine, 5(2), 121-136. https://doi.org/10.5492/wjccm.v5.i2.121
2. Weiss, T. T., Cerda, F., Scott, J. B., Kaur, R., Sungurlu, S., Mirza, S. H., Alolaiwat, A. A., Kaur, R., Augustynovich, A. E., \& Li, J. (2021). Prone positioning for patients intubated for severe acute respiratory distress syndrome (ARDS) secondary to COVID-19: a retrospective observational cohort study. British journal of anaesthesia, 126(1), 48-55. https://doi.org/10.1016/j.bja. 2020.09.042

3. Peck, T. J., \& Hibbert, K. A. (2019). Recent advances in the understanding and management of ARDS. F1000Research, 8, F1000 Faculty Rev-1959. https://doi.org/10.12688/f1000research.20411.1

000656

Respiratory function in patients recovered from COVID-19 according to the received ventilatory support

S. Terenzi'; M. Bitondo ; AF. Ferravante ${ }^{2}$; G. Sanson ${ }^{3}$; L. Tassinari ${ }^{1}$;

F. Facondini i ; R. Ciraolo, ; E. Gamberini'; A. Potalivo, ; LA. Lazzari Agli';

J. Montomoli ${ }^{1}$

${ }^{1}$ Anesthesiology and intensive care, Ospedale "Infermi" di Rimini, Rimini, Italy; ${ }^{2}$ Department of pneumology, Infermi Hospital, AUSL della Romagna, Rimini, Italy; ${ }^{3}$ Dept. of medicine, surgery and health sciences, University of Trieste, Trieste, Italy

Correspondence: J. Montomoli

Intensive Care Medicine Experimental 2020, 9(1): 000656

Introduction. There is limited evidence about chronic impairment in the respiratory function in patients that recovered from COVID-19. In particular, it has not been examined if the need of different intensity of care correlates with the degree of chronic dysfunction.

Objectives. To assess differences in the respiratory test performance at 6 months from COVID-19 diagnosis among patients never hospitalized and patients hospitalized receiving different respiratory support.

Methods. We identified all patients recovered from COVID-19 with no previous chronic respiratory disease that received 6 months follow-up at the pneumology department of Infermi Hospital, in Rimini (Northern Italy). We classified patients as hospitalized and not hospitalized with mild pneumonia. Furthermore, hospitalized patients were classified according to the respiratory support: oxygen mask, CPAP or highflow oxygen, mechanical ventilation (MV). All patients performed the walking test and spirometry. Results among groups were compared using the ANOVA test and a generalized model was used to examine the walking test results among groups.

Results. We enrolled 122 patients, $68(55.7 \%)$ male, with a median age of 65 (IQR: 51-72) (table). Diffusion capacity of the lungs for carbon monoxide (DLCO) showed a clinical significant impairment in all groups of patients with a statistically significant difference among the groups $(p=0.002)$. Patients receiving MV showed the lowest DLCO (Figure). On the contrary, the forced ventilatory capacity (FVC) was above the normal expected value in all patients. During the walking test, the change in the peripheral oxygen saturation but not the Borg score was statistically significant in all the groups $(p<0.001)$.

\section{Descriptive variables}

\begin{tabular}{lllll}
\hline & $\begin{array}{l}\text { not hospital- } \\
\text { ized } \\
(\mathbf{n}=\mathbf{4 8})\end{array}$ & $\begin{array}{l}\text { oxygen } \\
(\mathbf{n}=\mathbf{2 5})\end{array}$ & $\begin{array}{l}\text { CPAP/HFNC } \\
(\mathbf{n}=\mathbf{3 2})\end{array}$ & $\begin{array}{l}\text { mechanical } \\
\text { ventilation } \\
(\mathbf{n}=17)\end{array}$ \\
\hline $\begin{array}{l}\text { age, median } \\
\text { (IQR) }\end{array}$ & $54(37-68)$ & $73(67-80)$ & $65(54-70)$ & $65(55-71)$ \\
smokers & $5(10 \%)$ & $2(8 \%)$ & $1(3 \%)$ & $0(0 \%)$ \\
ex-smokers & $15(31 \%)$ & $9(36 \%)$ & $11(34 \%)$ & $6(35 \%)$ \\
no smokers & $28(58 \%)$ & $14(56 \%)$ & $21(65 \%)$ & $11(65 \%)$ \\
\hline
\end{tabular}




\begin{tabular}{|c|c|c|c|c|c|}
\hline \multicolumn{6}{|c|}{ Respiratory test results } \\
\hline & $\begin{array}{l}\text { not hospi- } \\
\text { talized } \\
(n=48)\end{array}$ & $\begin{array}{l}\text { oxygen } \\
(n=25)\end{array}$ & $\begin{array}{l}\text { CPAP/HFNC } \\
(n=32)\end{array}$ & $\begin{array}{l}\text { mechanical } \\
\text { ventilation } \\
(n=17)\end{array}$ & $\begin{array}{l}\text { ANOVA } \\
\text { p-value }\end{array}$ \\
\hline FVC \% (SD) & $109(17)$ & $103(21)$ & $102(17)$ & $101(18)$ & 0.231 \\
\hline $\begin{array}{l}\text { DLCO \% } \\
\text { (SD) }\end{array}$ & $73(16)$ & $62(20)$ & $66(18)$ & $55(13)$ & 0.002 \\
\hline $\begin{array}{l}\text { Distance } \\
\text { WT, m } \\
\text { (SD) }\end{array}$ & $454(111)$ & 384 (128) & $454(98)$ & $401(149)$ & 0.066 \\
\hline $\begin{array}{l}\text { SpO2 } \\
\text { baseline, } \\
\%(\mathrm{SD})\end{array}$ & $97.6(1.1)$ & $96.2(1.5)$ & $96.6(1.3)$ & $96.8(1.2)$ & $<0.001$ \\
\hline $\begin{array}{l}\text { SpO2 } \\
\text { minimal, } \\
\%(S D)\end{array}$ & $95.3(1.1)$ & $93.6(2.4)$ & $93.8(2.9)$ & $93.2(2.7)$ & 0.005 \\
\hline $\begin{array}{l}\text { Borg } \\
\text { baseline, } \\
\text { n (SD) }\end{array}$ & $0.36(0.86)$ & $0.33(0.97)$ & $0.07(0.25)$ & $0.18(0.43)$ & 0.324 \\
\hline $\begin{array}{l}\text { Borg end, } n \\
\text { (SD) }\end{array}$ & $2.63(1.45)$ & $2.57(1.32)$ & $2.38(1.25)$ & $2.49(1.18)$ & 0.887 \\
\hline
\end{tabular}

Conclusion. Among patients recovered from COVID-19, our findings suggest impairment in lung oxygenation but not restrictive lung disease regardless of the need of hospitalization. However, the severity of the DLCO impairment correlated with the intensity of care received.

\section{Reference(s)}

1. Torres-Castro R, Vasconcello-Castillo L, Alsina-Restoy X, Solis-Navarro L, Burgos F, Puppo H, Vilaró J. Respiratory function in patients post-infection by COVID-19: a systematic review and meta-analysis. Pulmonology. 2020 Nov 25:S2531-0437(20)30,245-2. https://doi.org/10.1016/.pulmoe.2020. 10.013. Epub ahead of print. PMID: 33,262,076; PMCID: PMC7687368.

\section{8}

Waveform-based algorithm to improve patient ventilator asynchrony in PSV: a pilot study

I. Bianchi ${ }^{1}$; A. Orlando, ${ }^{2}$; M. Pozzi, ; R. Puce, ${ }^{2}$; E. Arisi, ; F. Mojoli ${ }^{2}$

${ }^{1}$ Department of critical care and anesthesia, ASST Papa Giovanni

XXIII, Bergamo, Italy; ${ }^{2}$ Anaesthesia and intensive care, Fondazione I.R.C.C.S.

Policlinico San Matteo, Pavia, Italy; ${ }^{3}$ Department of clinical-surgical,

diagnostic and pediatric sciences, Unit of anaesthesia and intensive care,

University of Pavia, Pavia, Italy

Correspondence: I. Bianchi

Intensive Care Medicine Experimental 2020, 9(1): 000658

Introduction. Waveforms-based algorithms have been developed to automatically detect patient respiratory activity and to guide ventilator's triggering and cycling.

Objectives. To assess the performance of an automated waveformbased software (IntelliSync + ) available on G5 and C6 ventilators (Hamilton Medical, $\mathrm{CH}$ ).

Methods. In 13 patients under PSV, IntelliSync + (IS+) was compared to standard triggering and cycling-off (ST) with default setting (2L/min and $25 \%$ peak inspiratory flow, respectively). Assisted breaths (AB) were classified as well, acceptably or poorly assisted (WA, AA, PA) as follows. WA: trigger delay (TD) shorter than $100 \mathrm{~ms}$ and either early cycling (EC) or cycling delay (CD) shorter than $100 \mathrm{~ms}$. PA: TD longer than $300 \mathrm{~ms}$ and/or either EC longer than $100 \mathrm{~ms}$ or LC longer than $300 \mathrm{~ms}$. AA: all the other breaths. The asynchrony index (Al) was calculated as the sum of the major asynchronies (auto triggering (AT), double triggering (DT), ineffective efforts (IE), reverse triggering (RT)) plus the poorly assisted breaths $(\mathrm{PA})$, on the total breath number (patient and machine triggered). $A B$ were classified as optimally triggered, triggered with acceptable or severe delay according to TD $(<100,100-300,>300 \mathrm{~ms})$. AB were classified as optimally cycled, cycled with acceptable, severe delay or early cycled according to either $C D(<100,100-300,>300 \mathrm{~ms})$ or $\mathrm{EC}>100 \mathrm{~ms}$.
Results. Al was significantly lower with IS + ( Table 1, Fig. 1). This was mainly explained by a better inspiratory synchronization of assisted breaths. TD was significantly shorter (137 [116-199] vs 180 [162-227] $\mathrm{ms} ; \mathrm{p}=0.02$ ) and optimally triggered breaths were more than doubled with IS+compared with ST. The percentage of IE and AT was similar and extremely low with both ST and IS +. CD was not different (IS + vs. ST: 160 [131-231] vs. 129 [70-316]), but an acceptable CD was more frequent with IS+.

\begin{tabular}{llll}
\hline & Standard & Intellisync + & p value \\
\hline Asynchrony Index (\%) & $36.3[9-69.5]$ & $16.9[4.3-39]$ & 0.0303 \\
Ineffective Efforts (\%) & $2.0[1.0-5.5]$ & $1.1[0.0-3.5]$ & 0.7537 \\
Auto Triggering (\%) & $0.3[0.0-3.4]$ & $0.0[0.0-3.5]$ & 0.6744 \\
Well assisted breaths (\%) & $2.7[0.6-15.3]$ & $7.1[1.3-28.7]$ & 0.1401 \\
Acceptably assisted & $68.4[37.7-80.6]$ & $69.6[60.0-81.0]$ & 0.3967 \\
$\quad$ breaths (\%) & & & \\
Poorly assisted breaths (\%) & $21.2[3.6-62.3]$ & $9.0[2.6-29.7]$ & 0.1240 \\
Optimal triggering (\%)* & $13.5[6.2-17.1]$ & $28.9[11.0-44.5]$ & 0.0043 \\
Acceptable trigger & $76.3[73.0-79.5]$ & $63.0[45.7-71.7]$ & 0.0052 \\
$\quad$ delay (\%)* & & & \\
Severe trigger delay (\%)* & $9.4[0.6-20.2]$ & $3.4[0.4-14.4]$ & 0.1823 \\
Optimal cycling (\%)* & $38.4[9.9-87.2]$ & $21.5[9.0-56.3]$ & 0.4326 \\
Acceptable cycling & $18.6[9.7-38.4]$ & $58.8[15.2-78]$ & 0.0107 \\
$\quad$ delay (\%)* & & & \\
Severe cycling delay (\%)* & $1.9[0.0-29.4]$ & $4.1[0.5-19.2]$ & 0.8139 \\
Early cycling (\%)* & $0.0[0.0-1.7]$ & $0.0[0.0-3.3]$ & 1.000 \\
\hline
\end{tabular}

*expressed as percentage of the number of assisted breaths. Assisting Perfomance
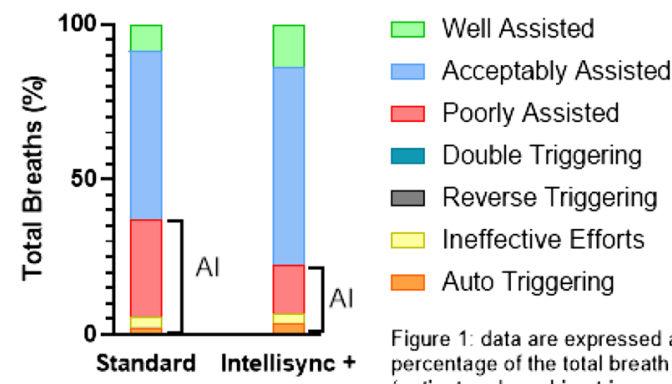

Figure 1: data are expressed as mean percentage of the total breath number (patient and machine triggered)

Conclusion. In a small population of ICU patients undergoing invasive assisted mechanical ventilation, waveform-based algorithms improved patient-ventilator interaction compared to default settings.

\section{8}

Prone positioning in SRDA due to COVID19 experience in a Spanish ICU

O. Martinez Moreno ${ }^{1}$; M. Nuñez Talavera ${ }^{1}$; CM. Rodríguez Mejías;

P. Fernandez Florido'; A. Sanchez Gonzalez ${ }^{1}$

${ }^{1}$ Intensive care unit, Hospital Universitario Virgen de las Nieves, Granada, Spain

Correspondence: $\mathrm{O}$. Martinez Moreno

Intensive Care Medicine Experimental 2020, 9(1): 000668

Introduction. COVID-19 causes acute respiratory distress syndrome an important percentage of the patients require ICU admission and most of them precise invasive mechanical ventilation to treat hypoxemia, being the mainstay of supportive therapy. Several strategies 
for ARDS have been evaluated including prone positioning which is one of the most effective interventions and associated with survival improvements. Prone positioning frequently leads to an improvement of gas exchange mainly due to a better overall ventilation/perfusion matching also related to a decrease in ventilator-induced lung injury due to a more uniform distribution of volume and distending forces.

Objectives. Describe the epidemiological features and premorbid clinical characteristics of patients admitted to our ICU for SARS-Cov2 pneumonia and who have required at least one prone cycle during their admission. Also describe the ventilatory parameters that have been established and the changes after the prone position, as well as the changes in the oxygenation parameters of the patients.

Methods. Descriptive study carried out in the ICU of the Virgen de las Nieves hospital in Granada. Forty-four patients out of the total of 200 admitted to the ICU with diagnosis of SARS-Cov2 pneumonia between 1 of January 2021 and 31 March 2021 and who required intubation and a prone position during their admission were included. Epidemiological characteristics on admission have been collected. Subsequently, the parameters of oxygenation of the patients were noted prior to prone positioning, after it and at $24 \mathrm{~h}$. It has also been specified if any complications derived from the prone are observed, and the mortality of the patients included in the study. We considered that a prone responder is one in which there is an increase in $\mathrm{PaO} 2 / \mathrm{FiO} 2$ at $24 \mathrm{~h}$ greater than $20 \%$.

Results. Of the 44 patients studied, $54.5 \%$ are men with a mean age of 60 years with a mean score on the APACHE scale of 11.59 being the most prevalent risk factors overweight (88.63\%), diabetes mellitus (36.4\%), arterial hypertension (47.7\%) and previous respiratory diseases (20.5\%).

All patients have required at least one prone cycle during admission, of which $81.8 \%$ meet the criteria for responders. Of those requiring a second prone cycle, 24 patients, $79.2 \%$ are responders. 16 patients required a third prone cycle with a total of $62.5 \%$ responders, 7 required a fourth cycle with a response rate of $57.1 \%$, and finally only 3 patients required a fifth prone cycle with $33.3 \%$ responders.

The only secondary effect reported was facial edema. Of the total of those studied, $34.1 \%$ die during their stay in the hospital.

Conclusion. The most prevalent factors among the patients included are overweight, diabetes and hypertension. Prone positioning is a secure strategy of treatment which achieves an improvement in oxygen exchange in a high percentage of patients. This achievement decreases with further cycles of prone.

\section{Reference(s)}

1. Menk M, Estenssoro E, Sahetya SK, Neto AS, Sinha P, Slutsky AS, et al. Current and evolving standards of care for patients with ARDS. Intensive Care Med. 1 de diciembre de 2020;46(12):2157-67.

2. Weiss TT, Cerda F, Scott JB, Kaur R, Sungurlu S, Mirza SH, et al. Prone positioning for patients intubated for severe acute respiratory distress syndrome (ARDS) secondary to COVID-19: a retrospective observational cohort study. British Journal of Anaesthesia. 1 de enero de 2021;126(1):48-55.

3. Carsetti A, Damia Paciarini A, Marini B, Pantanetti S, Adrario E, Donati A. Prolonged prone position ventilation for SARS-CoV-2 patients is feasible and effective. Critical Care. 15 de mayo de 2020;24(1):225.

4. Prone Positioning in Moderate to Severe Acute Respiratory Distress Syndrome due to COVID-19: A Cohort Study and Analysis of Physiology [Internet]. 2020 [citado 12 de abril de 2021].

5. Guérin C, Albert RK, Beitler J, Gattinoni L, Jaber S, Marini JJ, et al. Prone position in ARDS patients: why, when, how and for whom. Intensive Care Med. 1 de diciembre de 2020;46(12):2385-96.

\section{9}

Non-invasive respiratory support in COVID-19 is associated with a high risk of failure but no increase in mortality or complications of ventilation

O. Mahroof, ; M. Jeffrey ${ }^{1}$; J. Martin, ; A. Conway-Morris, ${ }^{2}$

${ }^{1} \mathrm{Jvf}$ intensive care unit, Addenbrooke's Hospital, Cambridge, United Kingdom; ${ }^{2}$ Department of anaesthetics \& intensive care medicine, Cambridge
University Hospitals NHS Foundation Trust, Cambridge, United Kingdom Correspondence: M. Jeffrey

Intensive Care Medicine Experimental 2020, 9(1): 000669

Introduction. Severe COVID-19 is associated with a significant risk of severe respiratory failure, need for ICU support and death. There remains uncertainty as to whether non-invasive support such as high flow nasal oxygenation (HFNO) or non-invasive ventilation (NIV) is preferable to immediate mechanical ventilation (MV)1,2.

Methods. We analysed all severe COVID-19 patients admitted to our large tertiary university ICU during the UK's '2nd wave' over a 14-week period from Oct 2020. All patient data was contemporaneously captured by an eMR system and were subsequently anonymised and analysed. Any nosocomial COVID-19 was excluded.

Results. Of 80 patients treated for COVID-19, 72 met inclusion criteria. Demographic and clinical factors are shown in the table. Initial mode of support was HFNO for 32 patients, NIV for 13 patients and MV for 27 patients. Of those starting on HFNO, 11 (34\%) ultimately received MV (8 as next escalation, 3 following NIV). Of those starting NIV, 9 (69\%) ultimately received MV ( 8 as next escalation, 1 having been stepped to HFNO first). The median duration of MV for those initially managed with non-invasive modes was longer $15 \mathrm{~d}$ (IQR 10) vs initial MV cohort $10 \mathrm{~d}$ (IQR 17), but with a lower crude mortality of 50 vs $66 \%$. Suspected VAP, pneumothorax and tracheostomy occurred more frequently in those ventilated immediately on admission.

Conclusion. Although non-invasive support was associated with frequent progression to invasive mechanical ventilation, especially amongst patients in receipt of NIV, we did not find a signal to increased mortality or complications of ventilation amongst this deteriorating group.

\section{Reference(s)}

1. NHS England. Guidance for the role and use of non-invasive respiratory support in adult patients with COVID19 (confirmed or suspected), 2020. 2. Ashish A, et al. BMJ Open Resp Res 2020;7:e00692. 10.1136/bmjre sp-2020-000692

\section{9}

Neuromuscular blocking agents in COVID 19 patients

L. Caley ${ }^{1}$; AC. Dinis ${ }^{1}$; T. Oliveira' ${ }^{1}$; AM. Neto Real' ${ }^{1}$;. Narciso ${ }^{1}$; T. Pereira'; C. Nuno ${ }^{1}$

${ }^{1}$ Intensive medicine, Centro Hospitalar Médio Tejo, Abrantes, Portugal

Correspondence: L. Caley

Intensive Care Medicine Experimental 2020, 9(1): 000679

Introduction. Neuromuscular blocking agents (NMBA) has been suggested as an adjunctive therapy for in ICU mechanically ventilated patients due to three potential benefits: eliminate ventilatorpatient dyssynchrony, facilitate lung protective ventilation and improve oxygenation. Because hypoxemia is the hallmark characteristic of the coronavirus disease 2019 (COVID 19), based in acute respiratory distress syndrome (ARDS) RCTs there was an increased use of NMBA in ICU worldwide in severe COVID 19 patients, beyond the $24 \pm 48 \mathrm{~h}$ recommended by most guidelines.

Objectives. To describe the clinical features of severe COVID 19 patients subject to more $24 \mathrm{~h}$ NMBA infusions.

Methods. Retrospective analysis of severe COVID 19 patients, admitted to the ICU of a public hospital in Portugal, between March 2020 to March 2021. We selected all mechanically ventilated patients subjected to NMBA infusions for more than $24 \mathrm{~h}$. Punctual or less of $24 \mathrm{~h}$ NMBA infusions were not considered.

Results. We identified 72 patients subject to NMBA more than $24 \mathrm{~h}$. Thirty-seven (51.4\%) patients died. The mean age was 61.1 years. Male sex was observed in $70.8 \%(n=21)$. The mean comorbidities were 1.75: hypertension and diabetes mellitus were the most frequents $65.3 \%(n=47)$ and $43.1 \%(n=31)$, respectively. The mean of APACHE II and SAPS II score were 18.09 and 41.7. Hypoxemia measured by the $\mathrm{PaO} 2 / \mathrm{FiO} 2$ ratio at admission was less than $100 \mathrm{mmHg}$ in $59.7 \%(n=43)$ of the patients. The two subsequent evaluations 
showed $\mathrm{PaO} 2 / \mathrm{FiO} 2$ ratio less than $100 \mathrm{mmHg}$ in $50 \%(n=36)$ and $41.7 \%(n=30)$. After $24 \mathrm{~h}$ of ICU admission the $\mathrm{PaO} / \mathrm{FiO} 2$ ratio observed was $26.4 \%(n=19)$ between $150-200 \mathrm{mmHg}$ and $20.8 \%$ between $200-250 \mathrm{mmHg}$. Nevertheless, $25 \%(n=18)$ of the patients were in persisting severe hypoxemia $(\mathrm{PaO} 2 / \mathrm{FiO} 2<100 \mathrm{mmHg}$ ). Dexamethasone at the dose of $6 \mathrm{mg}$ was used in $68.1 \%(n=49)$. The prone position was applied to $76.4 \%(n=55)$, with the time interval between 18 to $113 \mathrm{~h}$. The NMBA used to be rocuronium and Atracurium, $81.9 \%(n=59)$ and $30.6 \%(n=22)$, respectively. The mean curarization time was $109.6 \pm 77.8$ h. Only $20.8 \%(n=15)$ patients were on NMBA infusion within 24 to $48 \mathrm{~h}$.

Conclusion. The use of NMBA in severe COVID 19 patients was broad and prolong. We still need to learn the benefits and consequences we derive from such strategy.

\section{Reference(s)}

1. Courcelle R et al. Neuromuscular blocking agents (NMBA) for COVID19 acute respiratory distress syndrome: a multicenter observational study. Crit Care. 2020 Jul 19;24(1):446. https://doi.org/10.1186/ s13054-020-03164-2.

2. Navas-Blanco JR, Dudaryk R. Management of Respiratory Distress Syndrome due to COVID-19 infection. BMC Anesthesiol 20, 177 (2020). doi. org/https://doi.org/10.1186/s12871-020-01,095-7.

3. Bourenne J et al. Sedation and neuromuscular agents blocking in acute respiratory syndrome. Ann Transl Med. 2017 Jul;5(14):291. https://doi.org/ 10.21037/atm.2017.07.19

4. Awareness of NMBA formal indications and its rational use in severe COVID 19 patients.

\section{0}

First versus Second Wave of the COVID-19 Pandemics Among the Critically III Requiring Invasive Mechanical Ventilation in a Portuguese ICU-What Changed?

M. Mourisco'; F. Brochado'; F. Machado ${ }^{2}$; D. Dias ${ }^{2}$; M. Fernandes

${ }^{1}$ Intensive Care Medicine, Centro Hospitalar de Entre Douro e Vouga, Santa Maria da Feira, Portugal; ${ }^{2}$ Internal medicine, Centro Hospitalar de Entre Douro e Vouga, Santa Maria da Feira, Portugal

Correspondence: M. Mourisco

Intensive Care Medicine Experimental 2020, 9(1): 000680

Introduction. Similarly to other European countries [1], Portugal experienced a second wave of SARS-CoV-2 infections since September 2020, increasing the number of patients requiring Intensive Care Unit (ICU) admission and invasive mechanical ventilation (IMV).

Objectives. To describe differences regarding demographics, clinical and gas exchange parameters, as well as 90-day mortality among patients admitted to the ICU during the first and second waves of the COVID-19 pandemics.

Methods. Single-center retrospective cohort study including adults admitted in a portuguese ICU with critical COVID-19 disease, featuring severe hypoxemia requiring over $48 \mathrm{~h}$ of invasive mechanical ventilation-from March 18th to May 23rd, 2020 (first wave) and from September 14th to 31st December, 2020 (second wave). Unlike the first wave, all the patients admitted during the second wave received early glucocorticoids and non-invasive ventilation trials using Helmet CPAP were performed when appropriate. Data regarding demographics, known comorbidities, SAPS II and SOFA score (for the first $24 \mathrm{~h}$ of ICU admission) and the last arterial oxygen partial pressure to fractional inspired oxygen ( $\mathrm{PaO} 2 / \mathrm{FiO} 2)$ ratio before endotracheal intubation were collected. The lowest daily $\mathrm{PaO} 2 / \mathrm{FiO}_{2}$ ratio was retrieved from arterial blood samples collected every $4 \mathrm{~h}$ throughout the first 5 days of IMV. Data were analysed using IBM ${ }^{\circledR}$ SPSS $^{\circledR}$ Statistics 26.0. Measures were presented as [mean \pm standard deviation (SD)] and [median; interquartile range (IQR)], as appropriate.

Results. 65 patients were included, 33 corresponding to first wave admissions and 32 to admissions during the second wave. $62.5 \%$ $(n=20)$ of patients had a Helmet CPAP trial upon ICU admission in the second wave, with a length of non-invasive mechanical ventilation of $1.1 ; 3.3$ days. No relevant differences were found regarding age ( $62 \pm 12$ vs. $63 \pm 8$ years, $p=0.66)$, pre-existing comorbidities, SAPS II or SOFA score. $40.6 \%$ of the second wave ICU admissions came directly from the Resuscitation Room vs. 12.1.\% during the first wave $(p=0.02)$. Length of stay was significantly higher in the second wave cohort $(11.3 ; 6.9$ vs. $17.7 ; 24.4$ days, $p<0.01)$. Such tendency was verified regarding length of IMV (9.5; 8.1 vs. 12.0; 21.8 days, $\mathrm{p}=0.09)$. $\mathrm{PaO} 2 / \mathrm{FiO} 2$ ratio before endotracheal intubation was lower in the second wave cohort $(165 \pm 10$ vs. $134 \pm 12 \mathrm{mmHg}$, $\mathrm{p}=0.05$ ), as well as the worst daily $\mathrm{PaO} 2 / \mathrm{FiO} 2$ ratios during the first 5 days of IMV, although only relevant on day 5 of IMV (171 \pm 11 vs. $145 \pm 7 \mathrm{mmHg}, \mathrm{p}=0.05)$. 90-day mortality was significantly higher on the second wave $(15.2 \%$ vs. $46.9 \%, p<0.01)$.

Conclusion. We report a significantly higher 90 -day mortality rate on second wave ICU admissions requiring IMV, a group of patients who was preferentially ventilated non-invasively upon admission. Therefore, second wave patients requiring IMV may represent a selected cohort in terms of disease severity throughout ICU stay.

\section{Reference(s)}

1. Contou D, Fraissé M, Pajot O, Tirolien JA, Mentec H, Plantefève G. Comparison between first and second wave among critically ill COVID-19 patients admitted to a French ICU: no prognostic improvement during the second wave?. Crit Care. 2021 Jan 4;25(1):3. https://doi.org/10.1186/ s13054-020-03449-6. PubMed PMID: 33,397,421; PubMed Central PMCID: PMC7780592.

000681

Complications of mechanical ventilation amongst patients with severe COVID-19

O. Mahroof, ; M. Jeffrey ; J. Martin, ; A. Conway-Morris, ${ }^{2}$

${ }^{1} \mathrm{Jvf}$ intensive care unit, Addenbrooke's Hospital, Cambridge, United Kingdom; ${ }^{2}$ Department of anaesthetics \& intensive care medicine, Cambridge University Hospitals NHS Foundation Trust, Cambridge, United Kingdom Correspondence: M. Jeffrey

Intensive Care Medicine Experimental 2020, 9(1): 000681

Introduction. Severe COVID-19 pneumonitis causes widespread bilateral lung injury. Mechanical ventilation (MV) has been widely used in managing this form of severe respiratory failure. Despite lung protective ventilation, complications from MV is commonplace1, 2. We reviewed our local data for the 2nd COVID wave in the UK.

Methods. For 3 months from Oct'20, all patients who were MV following community acquired COVID-19 were included. Their characteristics, therapeutics, ventilatory management and arterial blood gas values were captured in real time, anonymised and analysed.

Results. 47 patients were mechanically ventilated within this period with 27 requiring MV as their initial mode of respiratory support. Of this group $45(96 \%)$ were muscle relaxed by infusion, $36(77 \%)$ were turned prone and $44(94 \%)$ received epoprostenol (PGI2) by nebulisation.

Tracheostomy was required in $22(47 \%), 35$ (74\%) were suspected of developing a VAP (rate of 51/1000 ventilator days) and 40 (85\%) developed a secondary infection of any type. CTPA proven PE was present or developed in $13(18 \%)$ and pneumothorax developed in $5(7 \%)$. ICU median length of stay was $17 \mathrm{~d}$ (IQR 13). The overall mortality of MV patients was $60 \%$. Subgroup mortality for those who required MV as their initial mode was $66 \%$ and those requiring $\mathrm{MV}$ as a secondary mode was $50 \%$.

Independent factors associated with higher mortality as assessed by regression analysis in this cohort, MV was one of 4 variables, with others being male, lower PF ratio and a higher APACHE II.

Conclusion. Mechanical ventilation remains an important mode of managing severe respiratory failure from COVID pneumonitis. However, it is associated with significant risks including need for ventilatory adjunctive therapies such as relaxation, prone position and nebulised epoprostenol. Also, with significant high rates of need for tracheostomy, suspected VAP, secondary infection and pneumothoraces. Severity of illness and MV in COVID-19 are associated with death. 
Reference(s)

1. Zhou F et al. Clinical course and risk factors for mortality of adult inpatients with COVID-19 in Wuhan, China: a retrospective cohort study. Lancet 2020;395:1054-62.

2. Zhu $\mathrm{N}$ et al. A novel coronavirus from patients with pneumonia in China, 2019. N Engl J Med 2020;382:727-33

\section{8}

Comparison of the management and outcomes of ICU patients between UK Wave 1 and Wave 2 of COVID

O. Mahroof, ; M. Jeffrey ${ }^{1}$; J. Martin, ${ }^{1}$; A. Conway-Morris, ${ }^{2}$

${ }^{1} \mathrm{Jvf}$ intensive care unit, Addenbrooke's Hospital, Cambridge, United Kingdom; ${ }^{2}$ Department of anaesthetics \& intensive care medicine, Cambridge University Hospitals NHS Foundation Trust, Cambridge, United Kingdom Correspondence: M. Jeffrey

Intensive Care Medicine Experimental 2020, 9(1): 000688

Introduction. The UK has now experienced 2 serious COVID-19 waves affecting the whole nation. The second wave predominantly incorporated the newer recognised SARS CoV-2 variant B.1.1.7 (Kent variant)1. Large trials have shown the use of therapeutics such as dexamethasone 2 and remdesivir (and later tocilizumab) to be helpful in treating severe COVID-19. The characteristics and findings from our UK 2nd Wave ICU patients (also in comparison to Wave 1) are documented below.

Methods. We analysed all community acquired severe COVID-19 patients admitted to our large tertiary university ICU during Wave 1 (Mar-Jun 2020) and Wave 2 (Oct-Dec 2020). Anonymised contemporaneous data from an eMR system was collated and analysed.

Results. We managed 93 patients with COVID-19 in Wave 1 and 72 in Wave 2 to December. Clinical and demographic factors are shown in the table. In Wave 1, corticosteroids were given to $17 \%$ of patients, no other immunomodulatory therapies were given.

In Wave 2, 70 (97\%) received dexamethasone, 31 (43\%) remdesivir, 1 tocilizumab and $8(11 \%)$ convalescent plasma. In Wave 1, $81(87 \%)$ were mechanically ventilated (MV), 4 (4\%) with non-invasive support (HFNO/NIV alone) and 8 (9\%) with simple oxygen therapy. Those ventilated in Wave 1 had a median duration of ventilation of 15 days. In Wave 2, 25 (34\%) were managed with non-invasive support alone (HFNO/NIV) and 47 (64\%) required MV overall for a median of 10 days. Crude mortality in Wave 1 was 32\%, in Wave 2 it was 43\%.

Conclusion. Management of patients in Wave 2 was altered by the accumulated experience and evidence from Wave 1, with increased use of immunomodulatory and anti-viral drugs, a lower proportion of mechanical ventilation and greater use of prone ventilation for those who were mechanically ventilated. Mortality was, however, higher in Wave 2. Whether this reflects novel variants, increased use of pre-ICU immunomodulation with increased proportion of 'non-responders' being admitted to ICU or other factors remains to be determined.

\section{Reference(s)}

1. The RECOVERY Collaborative Group. Dexamethasone in Hospitalised Patients with Covid-19. N Engl J Med 2021;384:693-704. https://doi.org/ 10.1056/NEJMoa2021436.

2. Challen R. Risk of mortality in patients infected with SARS-CoV-2 variant of concern 202,012/1: matched cohort study. BMJ 2021;372:n579.

\section{7}

Ventilatory management of COVID-19 related ARDS in a Tunisian MICU

R. Toumi ${ }^{1}$; K. Meddeb ${ }^{1}$; E. Bedhiafi²; MY. Kallala²; M. zghidi²; J. Mahmoud ${ }^{2}$ I. Ben Saida'; M. Boussarsar

${ }^{1}$ Medical intensive care unit, farhat hached hospital, université de sousse, Faculté de Médecine de Sousse, LR Nº LR12SP09. Heart Failure, Sousse, Tunisia; ${ }^{2}$ Medical intensive care unit, Farhat Hached University Hospital, Sousse, Tunisia

Correspondence: R. Toumi

Intensive Care Medicine Experimental 2020, 9(1): 000697
Introduction. The management of COVID-19-related ARDS has been challenging. The introduction of new concepts such as "happy hypoxemia" has changed the approach of intensivists regarding ventilatory management of ARDS.

Objectives. To describe ventilatory support management of critically ill adults admitted in for COVID-19 related ARDS.

Methods. This is a retrospective study carried out in a 12-bed medical intensive care unit (MICU) of Farhat Hached University Hospital in Sousse, Tunisia, analyzing patient records from July 17th 2020 to February 18th 2021, who were admitted for a critical COVID-19 related ARDS. Patients' characteristics at ICU admission, severity, ventilatory management and outcomes were studied.

Results. During the study period, 145 patients were admitted for COVID-19 pneumonia. They were $66.2 \pm 10.8$ mean years aged, hypertension, 72(49.7\%) and diabetes 77(53.1\%). Mean SAPS II was at $30.5 \pm 10.6,64(44.1 \%)$ patients presented severe ARF with mean $\mathrm{P} / \mathrm{F}$ ratio at $146.6 \pm 76.5$. Chest CT-scan was performed in $98(67.6 \%)$ patients, $61(62.2 \%)$ had more than $50 \%$ of pulmonary parenchyma lesions. Ventilatory support was required in 138(95.2\%) patients. On admission, only $11(8 \%)$ patients received initial IMV, 66(47.8\%), HFNC; $61(44.2 \%)$, NIV. Seventy-five(59.1\%) patients failed non-invasive procedures with a mean delay to intubation at $3.3 \pm 1.9$ dys. Overall, 86(62.3\%) patients required IMV. Mean HFNC duration was $4.5 \pm 3$ dys and mean NIV duration was $2.9 \pm 2.4$ dys. Overall IMV duration was $8.9 \pm 5.5$ dys, respectively, $10.5 \pm 7.5$ dys for initial IMV and $8.7 \pm 5.2$ dys for delayed IMV. Overall mortality was at $80(55.2 \%)$, $76(89.4 \%)$ in patients who received IMV.

Conclusion. The present study demonstrated the poor prognosis of COVID-19 related ARDS. HFNC, when started early, achieved the better prognosis. NIV was used as an attempt to screen responsive patients.

\section{9}

A Year of the COVID-19 Pandemics in the ICU: Predicting 90 day-Mortality

M. Mourisco'; F. Brochado '; F. Machado ${ }^{2}$; D. Dias ${ }^{2}$; M. Fernandes'

${ }^{1}$ Intensive Care Medicine, Centro Hospitalar de Entre Douro e Vouga, Santa Maria da Feira, Portugal; 2 Internal medicine, Centro Hospitalar de Entre Douro e Vouga, Santa Maria da Feira, Portugal

Correspondence: M. Mourisco

Intensive Care Medicine Experimental 2020, 9(1): 000699

Introduction. The COVID-19 pandemic put an unparalleled strain upon Intensive Care Units (ICU) worldwide, as a growing number of patients were admitted due to severe hypoxic respiratory failure. Evidence abounds regarding in-hospital mortality [1, 2, 3], but little is known regarding how the clinical course during ICU impacts mediumterm mortality.

Objectives. This study aims to propose variables relating to demographics, clinical and gas exchange parameters as potential 90-day mortality predictors among the critically ill requiring invasive mechanical ventilation due to SARS-CoV-2 pneumonia.

Methods. Single-center retrospective study including adults admitted in a portuguese ICU featuring severe hypoxemia requiring over $48 \mathrm{~h}$ of invasive mechanical ventilation due to critical COVID-19 disease, from March 18th to 31st December, 2020. Data regarding demographics, known comorbidities, Age-Adjusted Charlson Comorbidity Index (aaCCI) [4], SAPS II score and the last arterial oxygen partial pressure to fractional inspired oxygen $(\mathrm{PaO} 2 / \mathrm{FiO} 2)$ ratio before endotracheal intubation were collected. The lowest daily $\mathrm{PaO} 2 / \mathrm{FiO} 2$ ratio was retrieved from arterial blood samples collected during the first 5 days of invasive mechanical ventilation. Data were analysed using IBM ${ }^{\circledR}$ SPSS $^{\circledR}$ Statistics 26.0. The outcome of interest was defined as "alive 90 days after ICU admission" and data were collected by means of electronic process revision. Univariate logistic regression models were computed for each variable individually and data were interpreted according to clinical context. 
Results. 65 patients were included. 90-day mortality in this series was $30.8 \%(n=20)$. Table 1 shows the variables that proved statistically significant impact on 90-day mortality in this subset of patients.

\begin{tabular}{|c|c|c|c|c|c|c|c|}
\hline Variables & $B$ & SE & Wald & df & Sig & $\operatorname{Exp}(B)$ & $\begin{array}{l}95 \% \text { C.I. for } \\
\operatorname{Exp}(B)\end{array}$ \\
\hline $\begin{array}{l}\text { Worst } \mathrm{PaO} 2 / \\
\text { FiO2 ratio } \\
\text { on Day 2, } \\
\text { mmHg }\end{array}$ & 0.034 & 0.010 & 10.668 & 1 & 0.001 & 1.034 & $1.014 ; 1.056$ \\
\hline $\begin{array}{l}\text { Worst } \mathrm{PaO} 2 / \\
\mathrm{FiO} 2 \text { ratio } \\
\text { on Day 3, } \\
\text { mmHg }\end{array}$ & 0.022 & 0.008 & 7.336 & 1 & 0.007 & 1.023 & $1.006 ; 1.039$ \\
\hline $\begin{array}{c}\text { Worst } \mathrm{PaO} 2 / \\
\text { FiO2 ratio } \\
\text { on Day 4, } \\
\text { mmHg }\end{array}$ & 0.018 & 0.007 & 6.726 & 1 & 0.010 & 1.018 & $1.004 ; 1.032$ \\
\hline $\begin{array}{l}\text { Worst } \mathrm{PaO} 2 / \\
\text { FiO2 ratio } \\
\text { on Day } 5 \text {, } \\
\text { mmHg }\end{array}$ & 0.028 & 0.009 & 9.170 & 1 & 0.002 & 1.028 & $1.013 ; 1.048$ \\
\hline SAPS II score & -0.086 & 0.030 & 8.408 & 1 & 0.005 & 0.918 & $0.865 ; 0.974$ \\
\hline $\mathrm{aaCCl}{ }^{*}$ & -0.754 & 0.245 & 9.499 & 1 & 0.002 & 0.471 & $0.291 ; 0.760$ \\
\hline
\end{tabular}

Conclusion. This study brings perspective on how clinical evolution during ICU stay of the patients who underwent mechanical ventilation due to severe COVID-19 predicts 90 -day mortality. In fact, a higher SAPS II score and aaCCI significantly decrease the odds of being alive 90 days after ICU admission. Higher $\mathrm{PaO} 2 / \mathrm{FiO} 2$ ratios thoughout days 2 to 5 of IMV significantly increase the odds of a favourable outcome at day 90 after ICU admission. These findings stress how patient management in the ICU impacts on clinical outcomes even after hospital discharge.

\section{Reference(s)}

1. [1] Wang K, Zhang Z, Yu M, Tao Y, Xie M. 15-day mortality and associated risk factors for hospitalized patients with COVID-19 in Wuhan, China: an ambispective observational cohort study. Intensive Care Med. 2020 Jul;46(7):1472-1474. https://doi.org/10.1007/s00134-020-06047-w. Epub 2020 Apr 23. PubMed PMID: 32,328,724; PubMed Central PMCID: PMC7176814. [2] Domecq JP, Lal A, Sheldrick CR, Kumar VK, Boman K, Bolesta S, Bansal V, Harhay MO, Garcia MA, Kaufman M, Danesh V, Cheruku S, Banner-Goodspeed VM, Anderson HL 3rd, Milligan PS, Denson JL, St Hill CA, Dodd KW, Martin GS, Gajic O, Walkey AJ, Kashyap R. Outcomes of Patients With Coronavirus Disease 2019 Receiving Organ Support Therapies: The International Viral Infection and Respiratory IIIness Universal Study Registry. Crit Care Med. 2021 Mar 1;49(3):437-448. https:// doi.org/10.1097/CCM.0000000000004879. PubMed PMID: 33,555,777. [3] Yang X, Yu Y, Xu J, Shu H, Xia J, Liu H, Wu Y, Zhang L, Yu Z, Fang M, Yu T, Wang Y, Pan S, Zou X, Yuan S, Shang Y. Clinical course and outcomes of critically ill patients with SARS-CoV-2 pneumonia in Wuhan, China: a single-centered, retrospective, observational study. Lancet Respir Med. 2020 May;8(5):475-481. https://doi.org/10.1016/S2213-2600(20)30079-5. Epub 2020 Feb 24. PubMed PMID: 32,105,632; PubMed Central PMCID: PMC7102538. [4] Setter NW, Peres ML, de Almeida BMM, Petterle RR, Raboni SM. Charlson comorbidity index scores and in-hospital prognosis of patients with severe acute respiratory infections. Intern Med J. 2020 Jun;50(6):691-697. https://doi.org/10.1111/imj.14398. PubMed PMID: $31,180,163$.

\section{2}

\section{Pneumomediastinum rising on the waves of Covid-19}

B. Hajdu ${ }^{1}$; C. Rookes, ; R. Whittaker, ; P. Szawarski,

${ }^{1}$ Critcal care and theatres, Frimley Health NHS Foundation Trust-Wex-

ham Park Hospital, Slough, United Kingdom

Correspondence: B. Hajdu

Intensive Care Medicine Experimental 2020, 9(1): 000702
Introduction. Pneumomediastinum has previously been a rare condition occurring at an estimated rate of 1:45,000 emergency department admissions. Recent covid-19 pandemic has changed this as demonstrated by the growing number of cases reported in the literature $(1,2,3,4,5,67,8,9)$. We performed retrospective analysis of Covid-19 cases admitted to a DGH intensive care unit in the months September 2020 to March 2021 (second wave) with a specific focus on pneumomediastinum and possible predisposing factors including drugs, noninvasive ventilation and covid severity. Mortality in patients with pneumomediastinum appeared to match overall crude mortality of our Covid-19 patient population. The opinions varied on the effect of this complication on outcome.

Objectives. A retrospective case analysis to identify the common factors in cases admitted with pneumomediastinum in light of the new clinical practices. Highlight the potential causes for this complication, and the effect on outcome.

Methods. Based on our ICNARC reported cases we performed a retrospective case analysis, of our COVID-19 admissions during the second wave in our ICU between the 30th of September 2020 until the 28th of February 2021. All admissions with COVID-19 pneumonitis to ICU have been identified and their admission imaging reports reviewed. We identified the patients with pneumomediastinum at admission to ICU evidenced by CT pulmonary angiogram, which was already done at the admission decision or performed en-route to ICU. Subsequently the admission notes were consulted, and the pre-admission care analysed, with particular regard to CPAP, Dexamethasone and anticoagulant use, as well as study medications. Lastly the outcome of the pneumomediastinum group is compared to the mortality of the rest of the admissions.

Results. We have screened 151 admissions with COVID-19 in the above period and found that 20 patients were admitted with pneumomediastinum to ICU. All patients were admitted from our respiratory ward where they have spent between 3-16 days (mean: 8.9 days) and received CPAP for $1-14$ days (mean: 4.2 ).

Dexamethasone and VTE prophylaxis was a standard of care from admission to hospital, which all 151 patients received.

There were three instances of study medication use in this cohort (Tocilizumab, Colchicine, Aspirin-one patient each).

Admissions of these patients were triggered by the clinical decline in $17 / 20$ cases and the diagnosis was identified on the CT scan en-route to ICU and 3/20 was known to have this diagnosis before the decision of admission. We have also observed that the diagnosis of pneumomediastinum was a trigger for ICU referral.

Pneumomediastinum was further complicated by pneumothorax in 10 of the cases, and by surgical emphysema in another 2 .

Conclusion. In comparison to the previous wave in our hospital the changes are remarkable. We had no cases with pneumomediastinum in the period from March to June 2020 during our first wave, but had 20 cases in the period from October 2020 to March 2021 during the second.

The main difference between the two periods were the universal use of dexamethasone from hospital admission onwards, and the slightly longer period of non-invasive ventilation pre-admission to ICU.

There was a $13 / 20(65 \%)$ mortality among the pneumomediastinum patients, which is generally very similar to our ICNARC based overall mortality for this period of $61 \%$.

Therefore the team has the two impressions, firstly that the pneumomediastinum is potentially a complication from dexamethasone use alone or in combination with the use of CPAP. Secondly, as we have seen half as many cases with pneumothorax, than that with pneumomediastinum, we are hypothesizing, that the development of the pneumomediastinum may precede that of the pneumothorax, and hence would put the source of gas leak into the mediastinum rather than the chest cavity, but further studies are required to confirm these views.

\section{Reference(s)}

1. References: Wang W, Gao R, Zheng Y, Jiang L. COVID-19 with spontaneous pneumothorax, pneumomediastinum and subcutaneous emphysema. J Travel Med. 2020 Aug 20;27(5):taaa062. https://doi.org/10.1093/jtm/taaa0 
62. PMID: 32,330,274; PMCID: PMC7188125. Quincho-Lopez A, QuinchoLopez DL, Hurtado-Medina FD. Case Report: Pneumothorax and Pneumomediastinum as Uncommon Complications of COVID-19 PneumoniaLiterature Review. Am J Trop Med Hyg. 2020 Sep;103(3):1170-1176. https://doi.org/10.4269/ajtmh.20-0815. PMID: 32,705,978; PMCID: PMC7470555. Shan S, Guangming L, Wei L, Xuedong Y. Spontaneous pneumomediastinum, pneumothorax and subcutaneous emphysema in COVID-19: case report and literature review. Rev Inst Med Trop Sao Paulo. 2020 Oct 9;62:e76. https://doi.org/10.1590/S1678-9946202062 076. PMID: 33,053,145; PMCID: PMC7552989. López Vega JM, Parra Gordo ML, Diez Tascón A, Ossaba Vélez S. Pneumomediastinum and spontaneous pneumothorax as an extrapulmonary complication of COVID-19 disease. Emerg Radiol. 2020 Dec;27(6):727-730. https://doi.org/10. 1007/s10140-020-01806-0. Epub 2020 Jun 11. PMID: 32,524,296; PMCID: PMC7286744. Elhakim TS, Abdul HS, Pelaez Romero C, Rodriguez-Fuentes Y. Spontaneous pneumomediastinum, pneumothorax and subcutaneous emphysema in COVID-19 pneumonia: a rare case and literature review. BMJ Case Rep. 2020 Dec 12;13(12):e239489. https://doi.org/10.1136/bcr2020-239489. PMID: 33,310,838; PMCID: PMC7735137. Lemmers DHL, Abu Hilal M, Bnà C, Prezioso C, Cavallo E, Nencini N, Crisci S, Fusina F, Natalini G. Pneumomediastinum and subcutaneous emphysema in COVID-19: barotrauma or lung frailty? ERJ Open Res. 2020 Nov 16:6(4):00,385-2020 https://doi.org/10.1183/23120541.00385-2020. PMID: 33,257,914; PMCID: PMC7537408. Martinelli AW, Ingle T, Newman J, Nadeem I, Jackson K, Lane ND, Melhorn J, Davies HE, Rostron AJ, Adeni A, Conroy K, Woznitza N, Matson M, Brill SE, Murray J, Shah A, Naran R, Hare SS, Collas O, Bigham S, Spiro M, Huang MM, labal B, Trenfield S, Ledot S, Desai S, Standing L, Babar J, Mahroof R, Smith I, Lee K, Tchrakian N, Uys S, Ricketts W, Patel ARC, Aujayeb A, Kokosi M, Wilkinson AJK, Marciniak SJ. COVID-19 and pneumothorax: a multicentre retrospective case series. Eur Respir J. 2020 Nov 19;56(5):2,002,697. https://doi.org/10.1183/13993003.02697-2020. PMID: 32,907,891; PMCID: PMC7487269. Manna S, Maron SZ, Cedillo MA, Voutsinas N, Toussie D, Finkelstein M, Steinberger S, Chung M, Bernheim A, Eber C, Gupta YS, Concepcion J, Libes R, Jacobi A. Spontaneous subcutaneous emphysema and pneumomediastinum in non-intubated patients with COVID-19. Clin Imaging. 2020 Nov;67:207-213. https://doi. org/10.1016/j.clinimag.2020.08.013. Epub 2020 Aug 26. PMID: 32,871,424; PMCID: PMC7448957. Al-Azzawi M, Douedi S, Alshami A, Al-Saoudi G, Mikhail J. Spontaneous Subcutaneous Emphysema and Pneumomediastinum in COVID-19 Patients: An Indicator of Poor Prognosis? Am J Case Rep. 2020 Jul 24;21:e925557. https://doi.org/10.12659/AJCR.925557. PMID: 32,703,927; PMCID: PMC7405921.

\section{5}

\section{Outcomes and clinical characteristics comparison} amongst intubated, non-intubated COVID-19 positive, and intubated COVID-19 negative patients

N. Wadud ${ }^{1}$; N. Ahmed, ${ }^{1}$; M. Shergill, ${ }^{1}$; M. Khan, ${ }^{1}$; M. Krishna, ${ }^{2}$; A. Gilani, ${ }^{2}$; S. El Zariff'; J. Galaydick'; K. Linga, ${ }^{2}$; S. Koor ${ }^{2}$; J. Galea ${ }^{3}$; L. Stuczynski ${ }^{3}$; M. Osundele ${ }^{3}$

${ }^{1}$ Internal Medicine, Garnet Health Medical Center, Middletown, United States of America; ${ }^{2}$ Critical care medicine, Garnet Health Medical Center, Middletown, United States of America; ${ }^{3}$ Pharmacy, Garnet Health Medical Center, Middletown, United States of America

Correspondence: N. Wadud

Intensive Care Medicine Experimental 2020, 9(1): 000715

Introduction. Over one hundred and fifty million confirmed cases and with almost three million deaths worldwide, COVID-19 is still a global pandemic. Many counties still facing third wave of infection, certain chronic medical conditions are associated with increased risk of mortality and hence an urgent need to find effective therapeutic modalities.

Objectives. To compare outcomes and clinical characteristics amongst intubated, non-intubated COVID-19 positive, and intubated COVID-19 patients.
Methods. Retrospective study, included 245 patients, adults over 18 years admitted to Garnet Health Medical Center with confirmed COVID-19 results from March 2020 to May 2020. Further classified into 3 groups; COVID-19 positive and intubated, COVID-19 negative and intubated and COVID-19 positive and non-intubated. Information collected included demographics: age, sex, race, comorbidities; treatment modalities. Primary outcome for mortality, ventilator duration, ICU and length of hospital stays were compared. Statistical analysis was done by 1 way ANOVA and statistical significance defined as $P>00.05$.

Results. 245 patients were analyzed; Grouped into intubated COVID19 positive (105), intubated COVID-19 negative (115) and non-intubated COVID-19 positive (25). Mean age was $60.45,63.22$ and 65.96 with male predominance $72.38 \%, 53.04 \%$ and $60 \%$ respectively. Caucasians seemed more affected $53.33 \%, 73.91 \%$ and $40 \%$ followed by African Americans. Chronic medical conditions included hypertension $(63.80 \%, 68.69 \%$ and $84 \%)$, diabetes mellitus $(44.76 \%, 34.78 \%$ and $48 \%)$ and asthma $(13.33 \%, 10.43 \%$ and $8 \%)$ respectively. Mortality was $45.71 \%, 34.23 \%$ and $16 \%$. COVID-19 positive patients were intubated for a mean duration of 22.14 days compared to 8.69 days in COVID-19 negative patients. Mean ICU and hospital length of stay were 16.01 and 23.7 days in intubated COVID-19 positive patients, 8.83 and 11.25 days in intubated COVID-19 negative patients, while mean ICU and hospital durations were 2.84 and 12.12 days in non-intubated COVID-19 positive patients; $\mathrm{P}=0.0001$. Acute Respiratory Distress Syndrome (ARDS), in intubated COVID-19 positive patients (82.85\%), acute kidney injury (AKI) (80\%) with $32.38 \%$ needing continuous renal replacement therapy (CRRT). While in intubated COVID-19 negative patients $66.95 \%$ developed AKI with $17.3 \%$ needing CRRT and only $5.21 \%$ patient developed ARDS. Non -intubated COVID-19 positive patients AKI was predominant in $64 \%$ with no CRRT and $28 \%$ developed ARDS. Inflammatory markers were also significantly elevated in COVID-19 positive intubated patients comparatively. In addition, 53 $(50.47 \%)$ patients received Tocilizumab, $10(9.52 \%)$ received Remdesivir in COVID-19 positive intubated group. While 6 (24\%) and 1 (4\%) received Tocilizumab and Remdesivir respectively, in non-intubated COVID-19 positive group.

Conclusion. Intubated COVID-19 positive patients had greatest mortality, ventilator duration, length of ICU and hospital stays. This study identified risk factors and complications that may be associated in COVID-19.

\section{Reference(s)}

1. Webb Hooper M, Nápoles AM, Pérez-Stable EJ. COVID-19 and Racial/Ethnic Disparities. JAMA. 2020;323(24):2466-2467

2. Armstrong RA, Kane AD, CookTM. Outcomes from intensive care in patients with COVID-19: a systematic review and meta-analysis of observational studies. Anaesthesia. 2020 Oct;75(10):1340-1349

3. Gupta S, Hayek SS, Wang W, et al. Factors Associated With Death in Critically III Patients With Coronavirus Disease 2019 in the US. JAMA Intern Med. 2020;180(11):1436-1446.

4. Armstrong RA, Kane AD, Cook TM. Outcomes from intensive care in patients with COVID-19: a systematic review and meta-analysis of observational studies. Anaesthesia. 2020 Oct;75(10):1340-1349.

5. Karlsen APH, Wiberg S, Laigaard J, Pedersen C, Rokamp KZ, Mathiesen O. A systematic review of trial registry entries for randomized clinical trials investigating COVID-19 medical prevention and treatment. PLoS One. 2020 Aug 20

6. Salama C, Han J, Yau L, Reiss WG, Kramer B, Neidhart JD, Criner GJ, Kaplan-Lewis E, Baden R, Pandit L, Cameron ML, Garcia-Diaz J, Chávez V, Mekebeb-Reuter M, Lima de Menezes F, Shah R, González-Lara MF, Assman B, Freedman J, Mohan SV. Tocilizumab in Patients Hospitalized with Covid-19 Pneumonia. (EMPACTA trial) N Engl J Med. 2021 Jan 7;384(1):2030. https://doi.org/10.1056/NEJMoa2030340.

7. None. 
000734

Characterization of endothelial dysfunction in COVID-19 patients admitted to intermediate respiratory and intensive care units. Preliminary results of the HEMOCOVID-19 multicenter study

C. Vilà-Vilardell, ; J. Mesquida²; A. Caballer ${ }^{2}$; L. Cortese ${ }^{3}$; A. Perez Pacheco ${ }^{4}$; P. Castro, ${ }^{5} ;$ M. García de Acilu ${ }^{6} ;$ RC. Mesquita ${ }^{7}$;. Durduran ${ }^{3}$

${ }^{1}$ Intensive care medicine, Hospital del Mar de Barcelona, GREPAC (Grup

d'Investigació en Patologia Crítica)_IMIM, Barcelona, Spain, Spain;

${ }^{2}$ Àrea de crítics, Parc Tauli Hospital Universitari, Sabadell, Spain; ${ }^{3}$ Medi-

cal optics group, ICFO-The Institute of Photonic Sciences, Castelldefels,

Spain; ${ }^{4}$ Intensive care department, Hospital General de Mexico, Mexico

City, Mexico; ${ }^{5}$ Medical intensive care unit, Hospital Clínic de Barce-

Iona, Barcelona, Spain; ${ }^{6}$ Critical care department, Hospital Universitari

Vall d'Hebron, Barcelona, Spain; ${ }^{7}$ Institute of physics, University of Campi-

nas, Campinas, Brazil

Correspondence: J. Mesquida

Intensive Care Medicine Experimental 2020, 9(1): 000734

Introduction. Although COVID-19 mainly causes respiratory forms of disease, post-mortem pathological examinations have revealed that it might affect the microvasculature of several organs, causing endothelial damage, and behaving as a systemic vascular disease [1,2].

Objectives. To characterize the microcirculatory endothelial function, on peripheral skeletal muscle, of severe COVID-19 patients admitted to respiratory (IRCU) and intensive care units (ICU).

Methods. Prospective observational study carried out in 6 intensive care units of Spain, Mexico and Brazil (ClinicalTrials.gov NCT04689477). Severe COVID-19 patients admitted to the IRCU/ICU due to hypoxemia were included, and compared to a group of healthy individuals. Respiratory, hemodynamic, and microcirculatory parameters were simultaneously evaluated within the first week of admission. Local tissue oxygen saturation (StO2), and local hemoglobin content (THC) were measured on the forearm (brachioradialis muscle) by means of near-infrared spectroscopy (PortaMon, Artinis). A transient vascular occlusion test (VOT), was performed in order to obtain dynamic StO2derived parameters: deoxygenation rate (DeO2), reoxgenation rate (ReO2), and hyperemic response (AUCH). The VOT consisted in a threeminute ischemic challenge by means of a proximal tourniquet system. A sample size of 33 controls and 66 patients was anticipated in order to detect a difference in $\mathrm{ReO} 2$ greater than $30 \% / \mathrm{min}$, assuming a SD of $\mathrm{ReO} 2$ of $50 \% / \mathrm{min}$.

Results. Thirty-two healthy controls and 73 COVID-19 patients were studied. Patients were older and with higher body mass index (BMI).Baseline StO2 and THC did not differ between the two groups. Dynamic VOT-derived parameters were significantly impaired in the COVID-19 patients group, showing lower metabolic rate (DeO2), and diminished endothelial reactivity as compared to the healthy population (Table). Regression models showed no association between StO2derived parameters and age or BMI.

Table. Main characteristics of the studied healthy volunteers and COVID-19 critically ill patients at inclusion. ${ }^{*} p<0.05$

\begin{tabular}{lll}
\hline & $\begin{array}{l}\text { Healthy volunteers } \\
(\mathbf{n}=\mathbf{3 2})\end{array}$ & $\begin{array}{l}\text { COVID-19 patients } \\
(\mathbf{n}=\mathbf{7 3})\end{array}$ \\
\hline Age (years) & $34 \pm 10$ & $59 \pm 13^{*}$ \\
Gender (male), n(\%) & $16(50)$ & $51(70)^{*}$ \\
BMI & $23 \pm 3$ & $30 \pm 5^{*}$ \\
Pre-existing comor- & - & $33(45)$ \\
$\quad$ bidities $(\mathrm{n}, \%)$ & - & $17(23)$ \\
$\cdot$ Hypertension & - & $18(25)$ \\
- Diabetes Mellitus & & $7(10)$ \\
- Smoker & & \\
- COPD & & $36.0 \pm 0.7$ \\
Temperature $\left({ }^{\circ} \mathrm{C}\right)$ & $36.3 \pm 0.3$ &
\end{tabular}

\begin{tabular}{lll}
\hline & $\begin{array}{l}\text { Healthy volunteers } \\
(\mathbf{n}=\mathbf{3 2})\end{array}$ & $\begin{array}{l}\text { COVID-19 patients } \\
(\mathbf{n}=\mathbf{7 3})\end{array}$ \\
\hline HR $($ beats/min) & $75 \pm 13$ & $77 \pm 16$ \\
MAP $(\mathrm{mmHg})$ & $83 \pm 9$ & $86 \pm 12$ \\
RR (resp/min) & $18 \pm 3$ & $22 \pm 5^{*}$ \\
SpO2 $(\%)$ & $97 \pm 2$ & $95 \pm 4$ \\
FiO2 $(\%)$ & $21 \pm 0$ & $54 \pm 21^{*}$ \\
StO2 $(\%)$ & $65 \pm 3$ & $67 \pm 6$ \\
THC $(U)$ & $45 \pm 11$ & $43 \pm 16$ \\
DeO2 $(\% / m i n)$ & $-9.1 \pm 2.4$ & $-5.2 \pm 2.0^{*}$ \\
ReO2 $(\% /$ min) & $120 \pm 55$ & $77 \pm 37^{*}$ \\
Hyperemia AUC $(U)$ & $14.8 \pm 6.8$ & $8.5 \pm 5.0^{*}$
\end{tabular}

Conclusion. Severe COVID-19 patients admitted to the intermediate respiratory and intensive care units due to hypoxemic respiratory failure showed significant alterations in the systemic microcirculation, both in the local metabolic rate and in the endothelial function. Whether these alterations have prognostic and/or therapeutic implications needs to be further evaluated.

\section{Reference(s)}

1. On behalf of the HEMOCOVID-19 Consortium

2. [2] Varga Z, et al. Endothelial cell infection and endotheliitis in COVID-19. Lancet. 2020; 395(10,234):1417-1418

3. [1] Jonigk D, et al. COVID-19: what the clinician should know about postmortem findings. Intensive Care Med 2021; 47(1):86-89

\section{5}

Peripheral microcirculatory alterations are associated with the severity of ARDS in COVID-19 patients admitted to intermediate respiratory and intensive care units. Preliminary results of the HEMOCOVID-19 multicenter study

A. Caballer ${ }^{1}$; J. Mesquida ${ }^{1}$; C. Vilà-Vilardell, ${ }^{2}$; L. Cortese ${ }^{3}$; A. Perez Pacheco ${ }^{4}$; P. Castro, ${ }^{5} ;$ M. García de Acilu ${ }^{6} ;$ RC. Mesquita7 ; T. Durduran ${ }^{3}$

${ }^{1}$ Àrea de crítics, Parc Tauli Hospital Universitari, Sabadell, Spain; ${ }^{2}$ Intensive care medicine, Hospital del Mar de Barcelona, GREPAC (Grup d'Investigació en Patologia Crítica) —IMIM, Barcelona, Spain, Spain; ${ }^{3}$ Medical optics group, ICFO-The Institute of Photonic Sciences, Castelldefels, Spain; ${ }^{4}$ Intensive care department, Hospital General de Mexico, Mexico City, Mexico; ${ }^{5}$ Medical intensive care unit, Hospital Clínic de Barcelona, Barcelona, Spain; ${ }^{6} \mathrm{Critical}$ care department, Hospital Universitari Vall d'Hebron, Barcelona, Spain; ${ }^{7}$ Institute of physics, University of Campinas, Campinas, Brazil

Correspondence: J. Mesquida

Introduction. Although COVID-19 mainly causes respiratory forms of disease, it might affect the microvasculature of several organs, behaving as a systemic vascular disease. We previously demonstrated that COVID-19 patients show altered peripheral microcirculation, measured by means of non-invasive near-infrared spectroscopy (NIRS) on skeletal muscle.

Objectives. To evaluate the relationship between pulmonary disease severity and systemic microcirculatory alterations of severe COVID-19 patients admitted to respiratory (IRCU) and intensive care units (ICU).

Methods. Prospective observational study carried out in 6 intensive care units of Spain, Mexico and Brazil (ClinicalTrials.gov NCT04689477). Severe COVID-19 patients admitted to the IRCU/ICU due to hypoxemia were included. Respiratory, hemodynamic, and microcirculatory parameters were simultaneously evaluated within the first week of admission. Local tissue oxygen saturation (StO2), and local hemoglobin content (THC) were measured on the forearm (brachioradialis 
muscle) by means of near-infrared spectroscopy (PortaMon, Artinis). A vascular occlusion test (VOT), consisting in a three-minute induced ischemia, was performed in order to obtain dynamic StO2 parameters: deoxygenation rate (DeO2), reoxgenation rate $(\mathrm{ReO} 2)$, and hyperemic response (AUCH).

The severity of ARDS was evaluated by the ratio between pulse-oximetric oxygen saturation ( $\mathrm{SpO} 2$ ), and the fraction of inspired oxygen (FiO2) (SF ratio). Cut-off values of SF ratio of 144, 235, and 315 were used in order to classify patients into severe, moderate, and mild ARDS, respectively $[1,2]$.

Results. Seventy-three COVID-19 patients were studied. Mean age was $59 \pm 13,70 \%$ were males, and days from ICU admission were $3 \pm 3$. Respiratory support at inclusion: $53 \%$ invasive mechanical ventilation (MV), 32\% high-flow nasal cannula (HFNC), 4\% non-invasive mechanical ventilation (NIV), and $11 \%$ Venturi mask (Vmk). None of the patients were hemodynamically unstable. All MV patients were receiving sedative agents, and $15(38 \%)$ of them needed low doses of norepinephrine. Neither sedatives nor vasopressors were used in other respiratory support conditions.

Baseline StO2, and DeO2 negatively correlated with SF ratio, while $\mathrm{ReO} 2$ showed a positive correlation with SF ratio. ANOVA analysis showed significant differences in baseline $\mathrm{StO} 2$ and $\mathrm{ReO} 2$ among the different group of patients according to SF ratio (Fig. 1). No significant differences were observed according to the respiratory support therapy used

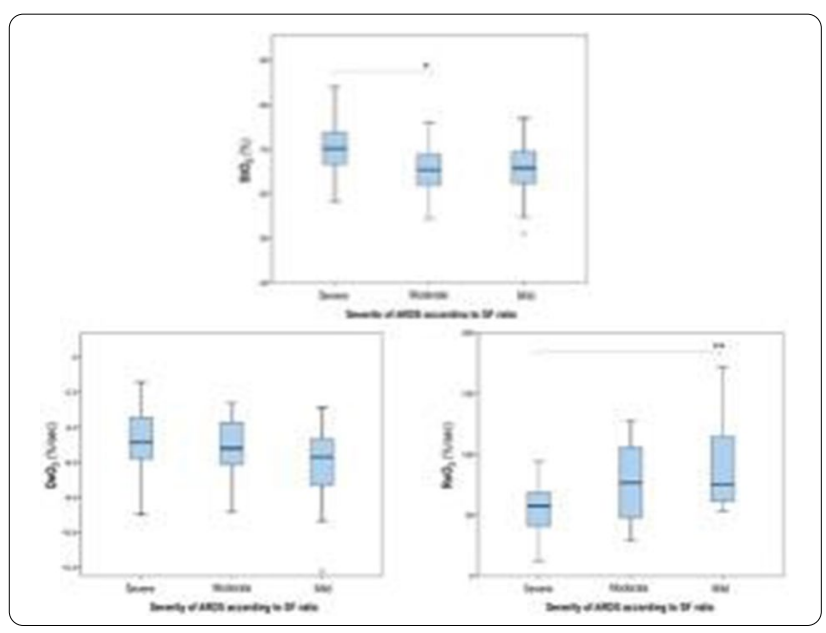

Conclusion. Microcirculatory alterations, and mainly endothelial dysfunction, measured by NIRS, are associated with the severity of ARDS in COVID-19 patients admitted to the IRCU/ICU. These alterations are not explained by the use of sedative agents or vasopressors. Whether these alterations have prognostic and/or therapeutic implications deserves to be further evaluated.

\section{Reference(s)}

1. Rice TW, et al. Comparison of the $\mathrm{SpO} 2 / \mathrm{FlO} 2$ ratio and the $\mathrm{PaO} 2 / \mathrm{FlO} 2$ ratio in patients with acute lung injury or ARDS. Chest 2007; 132(2):410-7 [2] Chen W, et al. Clinical Characteristics and Outcomes Are Similar in ARDS Diagnosed by Oxygen Saturation/Fio2 Ratio Compared With Pao2/ Fio2 Ratio. Chest 2015; 148(6):1477-1483

2. On behalf of the HEMOCOVID-19 Consortium

\section{3}

The role of respiratory support in the formation of insomnia in patients with COVID-19

O. Boduliev'; N. Adamchuk

${ }^{1}$ Anaesthesiology and intensive care, Ukraine Medical Stomatological Academy, Poltava, Ukraine

Correspondence: $\mathrm{O}$. Boduliev
Intensive Care Medicine Experimental 2020, 9(1): 000753

Introduction. The frequency of insomnia in patients with COVID-19 is much higher compared with the general population. The available literature does not provide data on the association of insomnia with the severity of hypoxia and the type of respiratory support.

Methods. The cohort study included 198 patients hospitalized with PCR-confirmed SARS-CoV-2 infection. Non-invasive ventilation (NIV) was performed in 64 patients, 78 patients received oxygen therapy with a face mask, and 56 patients were included in the group without respiratory support. There were no significant differences between the groups in terms of gender, age composition and comorbidity. To evaluate the severity of hypoxia expected value $\mathrm{SpO} 2 / \mathrm{FiO}^{2}$. The presence of insomnia was assessed by the Athens Insomnia Scale (AIS).

Results. SpO2/FiO2 for the NIV group was $156.4 \pm 32.0$; for the oxygen therapy group $206.4 \pm 28.2$ and without respiratory support $369.9 \pm 56.8$. Insomnia was detected in $96.9 \%$ for the NIV group, in $82.1 \%$ for the oxygen therapy group and in $71.4 \%$ of patients in the group without respiratory support. The correlation between the AIS score and $\mathrm{SpO} 2$ / FiO2 was 0.58; between the AIS and the type of respiratory support 0.35 .

Conclusion. Thus, a higher degree of dependence of insomnia on $\mathrm{SpO} 2 / \mathrm{FiO} 2$ than on the type of respiratory support may indicate the prevalence of hypoxia in the insomnia formation in COVID-19 patients.

\section{Reference(s)}

1. Silva ESME, Ono BHVS, Souza JC. Sleep and immunity in times of COVID19. Rev Assoc Med Bras (1992). 2020 Sep 21;66Suppl 2(Suppl 2):143-147. https://doi.org/10.1590/1806-9282.66.S2.143. PMID: 32,965,373.

2. Vitale JA, Perazzo P, Silingardi M, Biffi M, Banfi G, Negrini F. Is disruption of sleep quality a consequence of severe Covid-19 infection? A case-series examination. Chronobiol Int. 2020 Jul;37(7):1110-1114. https://doi.org/ 10.1080/07420528.2020.1775241. Epub 2020 Jun 23. PMID: 32,573,293.

3. De Felice FG, Tovar-Moll F, Moll J, Munoz DP, Ferreira ST. Severe Acute Respiratory Syndrome Coronavirus 2 (SARS-CoV-2) and the Central Nervous System. Trends Neurosci. 2020 Jun;43(6):355-357. https://doi.org/ 10.1016/j.tins.2020.04.004. Epub 2020 Apr 21. PMID: 32,359,765; PMCID: PMC7172664.

000782

Azithromycin in Covid-19 patients: really no benefits?

A. Trifi'; A. Mehdi' ${ }^{2}$; O. Benjima ${ }^{3}$; E. Sghir ${ }^{2}$; B. Jeribi ${ }^{3}$; H. Cherif ${ }^{3}$; Y. Touil, ${ }^{1}$;

Y. Masseoudi ${ }^{3}$; S. Abdellatifi,; A. Ammous ${ }^{3}$; S. Ben Lakhal ${ }^{1}$

${ }^{1}$ Intensive care unit, Hospital la Rabta, Tunis, Tunisia; ${ }^{2}$ Medical icu, Hospital Rabta, Tunis, Tunisia; ${ }^{3}$ Surgical icu, Hospital Rabta, Tunis, Tunisia

Correspondence: A. Trif

Intensive Care Medicine Experimental 2020, 9(1): 000782

Introduction. Azithromycin, because of its immunomodulatory properties and some activity against SARS-CoV2, rose to fame in the Covid19 crisis. Yet, its wide use began to decline from December 2020 (date of disclosure of the RECOVERY results concluding on no survival benefit). Otherwise, this antimicrobial increased the susceptibility of Covid19 patients to invasive pulmonary aspergillosis (IPA).

Objectives. to assess our experience with azithromycin in critically ill Covid-19 patients.

Methods. a retrospective double cohort study including all successive Covid-19 patients, hospitalized in two ICUs, between September 2020 and February 2021. Azithromycin was used until December 2020 then it was no longer prescribed until today. Thus, we compared 2 groups: Azithromycine group with usual care versus usual care alone. Outcomes were: 28 -day mortality, acute respiratory distress syndrome (ARDS), septic shock, vasopressors use, requirement of mechanical ventilation (MV), duration of MV, length of stay (LOS) in ICU and side effects (SE).

Results. of 144 patients, 80 received azithromycine (either before or during hospitalization) versus 64 who not received. Age and comorbidities didn't differ, female gender tended to be more present in azithromycin group (sex-ratio: $52 / 28$ vs $51 / 13, p=0,062$ ). Also, 
obesity and anosmia were more likely observed in this same group with respectively $(80 \%$ vs $53 \%, p=0,001$ and $10 \%$ vs $1,5 \%, p=0,044)$. Severity scores and laboratory findings were comparable. Outcomes: No significant difference was showed between Covid-19 patients receiving azithromycine in addition to usual care versus those who received only usual care regarding mortality $(56,2 \%$ vs $53,2 \%, p=0,8)$, septic shock ( $43 \%$ vs $31 \%, p=0,16)$, requirement of $\operatorname{MV}(45,6 \%$ vs $46,7 \%, p=1)$, vasopressors use $(50,6 \%$ vs $54 \%, p=0,73)$, duration of MV $(6,5$ [3-10] vs 4 [2-9], $p=0,24)$ and ICU-LOS (8 [5-12] vs $9[5-14], p=0,64)$. Whereas, the occurrence of ARDS tended to be lower in azithomycin group ( $72 \%$ vs $86,2 \%, p=0,06)$. Two SE were noted in azithomycin group: one case of IPA and Atrioventricu$\operatorname{lar}(\mathrm{AV})$ conduction disorder.

Conclusion. our results further consolidated the inefficiency of azithromycin, in critical Covid-19 patients, to improve survival. Yet, its potential to reduce lung inflammation seemed to be not only theoretical; as it reduced the evolution to ARDS with a difference near to significance.

\section{Reference(s)}

1. Delliere S, Dudoignon E, Fodil F, et al. Risk factors associated with COVID19-associated pulmonary aspergillosis in ICU patients: a French multicentric retrospective cohort. Clinical Microbiology and Infection 27(5) http:// dx.doi.org/10.1016/j.cmi.2020.12.005

2. RECOVERY Collaborative Group. Azithromycin in patients admitted to hospital with COVID-19 (RECOVERY): a randomised, controlled, openlabel, platform trial. Lancet. 2021 Feb 13;397(10,274):605-612. https://doi. org/10.1016/S0140-6736(21)00149-5. Epub 2021 Feb 2. PMID: 33,545,096; PMCID: PMC7884931.

\section{8}

Comparison of patients admitted for COVID19 pneumonia in a polyvalent Intensive Care Unit (ICU): first wave versus the second wave pandemic

L. López de la Oliva Calvo ${ }^{1}$; A. Abella Alvarez ; B. Lobo Valbuena'; S. Garcia Manzanedo'; M. Mohamed Mohamed'; S. Pajares Martínez'; F. Gordo

Vidal' $^{1}$

${ }^{1}$ Intensive care unit, Henares University Hospital, Madrid, Spain

Correspondence: L. López de la Oliva Calvo

Intensive Care Medicine Experimental 2020, 9(1): 000788

Introduction. The COVID19 pandemic has challenged ICU teams worldwide. Different strategies were developed in response to a demand that exceeded the available means. First wave caught us unaware, with little response time and trying to solve problems in the field. For the second wave we had all the necessary equipment, but ICU teams were physically and mentally exhausted.

Objectives. To analyze the characteristics of the patients and their evolution during the first wave compared to the data from the second wave in a polyvalent Spanish ICU with 8 available beds in a non-COVID situation. Methods. Descriptive cross-sectional study during the months of February-May 2020 (first wave) and September-December 2020 (second wave). Patients transferred to another center were excluded. Demographic data, organ failure, mechanical ventilation, ICU and hospital stay and mortality, among others, were analyzed. In the descriptive analysis, quantitative variables are shown as median and 25th and 75th percentile, and qualitative variables are expressed as percentages. The data were analyzed with the Mann-Whitney $\mathrm{U}$ or Chi-square test.

Results. 58 patients were included in the 1st wave vs 81 in the 2 nd wave. No differences were found in age or sex, with a median age of 65 years and $30 \%$ of women. Table 1 shows significant differences between 1st wave and 2 nd wave. The need for invasive mechanical ventilation was higher in the 1st wave, with a higher percentage of high flow oxygen therapy and/or non-invasive mechanical ventilation in the 2nd wave. No differences were found in origin (emergency department, ward, etc.), frailty index, need for tracheostomy, renal replacement techniques, reintubation and days on invasive mechanical ventilation. Regarding overall mortality, we observed a decrease of $5 \%$ during the second wave (regarding deceased patients), although it was not statistically significant.

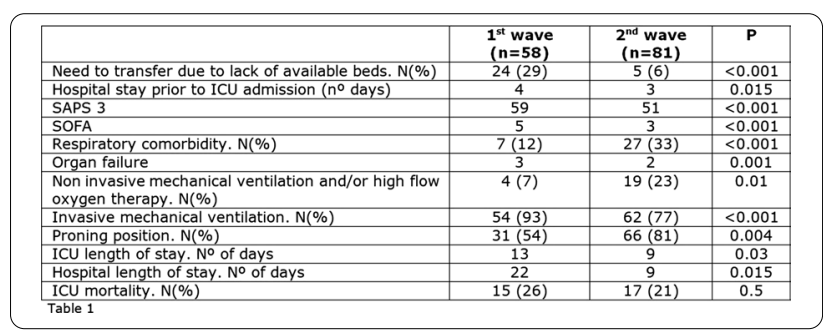

Conclusion. There are important differences regarding available resources between the 1 st and 2 nd waves, highlighting the need for more transfers during the first wave. Patients in the second wave showed a lower level of severity with greater respiratory comorbidity, fewer days of stay prior to ICU admission, and greater success on high-flow oxygen therapy and non-invasive mechanical ventilation, and shorter ICU and hospital length of stays.

\section{Reference(s)}

1. Giacomo Grasselli, MD, Alberto Zangrillo, MD, Alberto Zanella, MD, et al. Baseline Characteristics and Outcomes of 1591 Patients Infected With SARS-CoV-2 Admitted to ICUs of the Lombardy Region, Italy. JAMA. 2020;323(16):1574-1581.

2. Sho Saito, Yusuke Asai, Nobuaki Matsunaga, Kayoko Hayakawa, Mari Terada, Hiroshi Ohtsu, Shinya Tsuzuki, Norio Ohmagari. First and second COVID-19 waves in Japan: A comparison of disease severity and characteristics. J Infect. 2021 Apr;82(4):84-123.

3. Domenico Luca Grieco, MD, Luca S. Menga, MD, Melania Cesarano, MD, et al. Effect of Helmet Noninvasive Ventilation vs High-Flow Nasal Oxygen on Days Free of Respiratory Support in Patients With COVID-19 and Moderate to Severe Hypoxemic Respiratory Failure. The HENIVOT Randomized Clinical Trial. JAMA. 2021; 325(17):1731-1743.

000802

The role of increased steroid dosing in Intensive Care patients with COVID-19: a single centre review of practice and outcomes C. Kaye ${ }^{1}$; A. Clarkin ${ }^{1}$; K. Sim ${ }^{1}$

${ }^{1}$ Intensive Care Unit, NHS Grampian, Aberdeen, United Kingdom

Correspondence: C. Kaye

Intensive Care Medicine Experimental 2020, 9(1): 000802

Intoduction. Patients with severe COVID-19 routinely receive dexamethasone to improve mortality, but many patients remain critically ill after this 10-day course. There are varying beliefs amongst clinicians about the benefit of increasing steroid dose in these patients. Reports suggest the use of higher doses of steroids in these patients, especially in those deteriorating. Despite many proponents of higher dose or longer duration steroid therapy, there remains concern about the potential adverse effects of such a strategy.

Objectives. In this review of our practice, we aimed to describe the population \& outcome of patients receiving steroids in excess of the dosing regimen in the RECOVERY trial, compared to patients who followed that regimen.

Methods.Patients admitted to Aberdeen Royal Infirmary ICU with COVID-19 over 6 months were identified from the electronic patient record. Patients were classified based on the course of steroids received: standard (RECOVERY trial), additional (any dose between standard dose \& a $1 \mathrm{~g}$ pulse of methylprednisolone) or pulsed (1 g methylprednisolone). Routine data from the patient record, labs \& radiology were combined. Before extraction, the outcomes of interest were defined as:

- ICU mortality

- P/F ratio 
- additional infections

- thrombosis

- GI bleeds

- hyperglycaemia

Results. Of the 74 patients, 57 received standard steroids, 16 received additional steroids and 1 received pulsed steroids. Patients receiving additional steroid were more likely to be older (71.9 vs 62.9 years, P0.005), heavier ( 99.7 vs $88.2 \mathrm{~kg}, \mathrm{P} 0.078$ ) \& with a greater number of comorbidities ( 2.76 vs $1.98 \mathrm{P} 0.079$ ). On presentation to the ICU, the additional steroid group had an increased oxygenation deficit (lowest $\mathrm{P} / \mathrm{F} 11.07$ vs $14.91 \mathrm{P} 0.01$ ) however at the point of commencing additional steroids, this difference was not as evident (13.42 additional vs 14.91 standard, P0.356).

Patients receiving additional steroids had a $40.7 \%$ (P0.007) increase in mortality, compared to standard steroid use. Surviving patients on standard steroids showed an improved mean P/F (19.9 kPa vs $14.79 \mathrm{kPa}$ P0.003), which improved daily. There was no increase in the rates of DVT/PE or Gl bleed. Patients receiving additional steroids showed a trend towards increased bloodstream infections ( 0.53 vs 0.23 P0.12). Glucose control was similar between both groups with mean maximum daily glucose of 13.1 and $12.8 \mathrm{mmol} / \mathrm{l}$ (P0.54) in the standard $\&$ additional groups respectively.

Conclusion. This single centre evaluation observed no benefit from the use of additional steroids beyond the RECOVERY dexamethasone guidance \& suggests an increased incidence of bloodstream infections. We believe this suggests patients who fail to improve with initial dexamethasone have steroid-resistant disease \& will have little benefit from additional steroids. Established interventional trials investigating management of COVID-19 should consider further investigation of this group, to provide a definitive answer \& give reassurance to clinicians.

\section{Reference(s)}

1. Berton AM, Prencipe N, Giordano R, Ghigo E, Grottoli S. Systemic steroids in patients with COVID-19: pros and contras, an endocrinological point of view. J Endocrinol Invest. 2021;44(4):873-875. 10.1007/ s40618-020-01325-2

2. Camporota L, Meadows C, Ledot S, et al. Consensus on the referral and admission of patients with severe respiratory failure to the NHS ECMO service. Lancet Respir Med. 2021;9(2):e16-e17. 10.1016/\$2213-2600(20) 30581-6

3. Richards-Belle A, Orzechowska I, Gould DW, et al. COVID-19 in critical care: epidemiology of the first epidemic wave across England, Wales and Northern Ireland. Intensive Care Med. 2020;46(11):2035-2047. 10.1007/ s00134-020-06267-0

4. The RECOVERY Collaborative Group. Dexamethasone in Hospitalized Patients with Covid-19. N Engl J Med. 2021;384(8):693-704. 10.1056/ NEJMoa2021436

\section{0}

Elderly COVID-19 patients with acute hypoxemic respiratory failure given NIV during the ICU stay

H. Flaatten ${ }^{1}$; C. Jung ${ }^{2}$; M. Beil ${ }^{3}$; J. Fjølner ${ }^{4}$; B. Guidet ${ }^{5}$; P.Vernon Van Heerden $^{3}$; D. De Lange ${ }^{6}$; S. Leaver ; S. Sigal ${ }^{8}$; W. Szczeklik ${ }^{9}$; A. Artigas ${ }^{10}$ 1 Department of anaesthesia and intensive care, Haukeland University Hospital, Bergen, Norway; ${ }^{2}$ Dep. of cardiology, pulmonology and angiology, University Hospital of Düsseldorf, Düsseldorf, Germany; ${ }^{3}$ Medical intensive care unit, Hadassah Medical Center, Jerusalem, Israel; ${ }^{4}$ Department of intensive care, Aarhus University Hospital, Aarhus, Denmark; ${ }^{5}$ Réanimation Médicale, Hôpital Saint-Antoine, Paris, France; ${ }^{6}$ Department of intensive care medicine, Utrecht University, Utrecht, Netherlands; ${ }^{7}$ Research lead critical care directorate, St George's Hospital, London, United Kingdom; ${ }^{8}$ Medical intensive care unit, Hadassah Medical Center, Jerusalem, Israel, Israel; ${ }^{9}$ Intensive care and perioperative medicine, Jagiellonian University Medical College, Kraków, Poland; ${ }^{10}$ Department of intensive care medicine, Corporacion Sanitaria Universitaria Parc Tauli, Barcelona, Spain

Correspondence: $\mathrm{H}$. Flaatten

Intensive Care Medicine Experimental 2020, 9(1): 000830
Introduction. The Covid-19 pandemic has provided many challenges for ICUs worldwide. One particular problem is related to the use of noninvasive ventilation (NIV), and its use has been discussed especially at the start of the pandemic (1).

Objectives. This study is a part of COVIP, a prospective clinical study conducted by the VIP network within the HSRO section in ESICM, with the aim of studying elderly ICU patients (age $\geq 70$ years) during the pandemic. Assessment and treatment of acute organ dysfunction were an important part of the study. Our aim is to describe the use of NIV during ICU admission in elderly COVID-19 patients and the short-term outcomes.

Methods. Study period was from mid-March 2020 until January 2021. Participating countries were responsible for obtaining National Ethical Clearance. At ICU admission all patients were scored using the Sequential Organ Dysfunction Assessment (SOFA) score (2), and common ICU procedures including NIV and invasive mechanical ventilation (MV) were recorded with start and duration of such treatment. Short term outcomes are described as LOS in the ICU and 30-day survival in the group given NIV. This group is subdivided according to use of NIV alone (no MV given), NIV use prior to MV or use after MV. For comparison a group given only $\mathrm{MV}$ is provided.

Results. In total 3383 patients were admitted to 207 ICUs in 35 countries. Mean age was 76.9 years and $69.6 \%$ were male. In total 860 patients (25.4\%) received NIV during their ICU stay. Most received NIV before or after MV but a large group received NIV only (Table 1). Patients given NIV prior to start of MV had the shortest time on NIV and the worst outcome with fewer than 1 in 3 patients surviving to 30 days. The best outcome was found in the group where NIV was given immediately after MV probably supporting weaning from ventilator dependence, where 3 out of 4 survived to 30 days. Patients given NIV only had outcomes between these two groups.

\begin{tabular}{|c|c|c|c|c|c|c|c|}
\hline \multicolumn{8}{|c|}{ NIV in different settings in COVID-19 patients (data as mean) } \\
\hline & $\mathrm{n}=$ & $\begin{array}{l}\text { Days } \\
\text { ICU }\end{array}$ & $\begin{array}{l}\text { Hours } \\
\text { NIV }\end{array}$ & SOFA & SOFAresp & Age & $\begin{array}{l}30-d \\
\text { survival }\end{array}$ \\
\hline NIV only & 359 & 7.2 & 85 & 4.3 & 2.2 & 77.3 & $51.3 \%$ \\
\hline $\begin{array}{l}\text { NIV to } \\
\text { MV }\end{array}$ & 331 & 17 & 57 & 5.6 & 2.5 & 75.6 & $32.6 \%$ \\
\hline $\begin{array}{l}\text { MV to } \\
\text { NIV }\end{array}$ & 170 & 21 & 77 & 5.0 & 3.0 & 76.6 & $76.5 \%$ \\
\hline MV only & 1777 & 18.7 & 0 & 6.8 & 3.0 & 75.4 & $42.3 \%$ \\
\hline
\end{tabular}

Conclusion. These results clearly demonstrate that NIV is used in different clinical settings especially during this pandemic. Knowledge of how NIV is used during the ICU trajectory is important for outcome. There are important differences between these three groups. The group receiving only NIV were older, had less organ dysfunction measured as SOFA score on admission (in particular the respiratory component). They had the shortest ICU stay and the longest time on average on NIV, nearly 4 days. The group with the worst outcome, those starting with NIV and subsequently converting to MV, are most probably patients who failed NIV (3). This group also has more OD than the two other groups, a marker of worse outcome in our patients. The best outcome was unsurprisingly found when NIV was given as a weaning tool after MV, here 3 out of 4 patients survived to 30 days.

\section{Reference(s)}

1. Winck JC, Ambrosino N. COVID-19 pandemic and non invasive respiratory management: Every Goliath needs a David. An evidence based evaluation of problems. Pulmonology. April 2020. 10.1016/j.pulmoe.2020.04. 013.

2. Vincent, J. et al. The SOFA (Sepsis-related Organ Failure Assessment) score to describe organ dysfunction/failure. On behalf of the Working Group on Sepsis-Related Problems of the European Society of Intensive Care Medicine. 22, Intensive Care Med (1996) 22:707-710

3. Bertaina M, Nuñez-Gil IJ, Franchin L, et al. HOPE COVID-19 investigators. Non-invasive ventilation for SARS-CoV-2 acute respiratory failure: a subanalysis from the HOPE COVID-19 registry. Emerg Med J. 2021 Mar 
16:emermed-2020-210,411. https://doi.org/10.1136/emermed-2020210411. Epub ahead of print. PMID: 33,727,235; PMCID: PMC7970657.

4. Grant Open Access funding enabled and organized by Projekt DEAL. The support of the study in France by a grant from Fondation Assistance Publique-Hôpitaux de Paris pour la recherche is greatly appreciated. In Norway, the study was supported by a grant from the Health Region West. In addition, the study was funded by a grant from the European Open Science Cloud (EOSC) by the European Commission. EOSCsecretariat.eu has received funding from the European Union's Horizon Programme call H2020-INFRAEOSC-05-2018-2019, grant agreement number 831644. No further specific funding was received. Acknowledgement: The VIP study group and all its collaborators.

\section{7}

Management of severe ARDS due to SARS-Cov2 pneumonia using low-flow extracorporeal $\mathrm{CO} 2$ extraction and changes in ventilatory support

R. Molina'; B. Lobo-Valbuena'; A. Abella, ; L. López de la Oliva Calvo'; MM. Mohamed ${ }^{1}$; F. Gordo ${ }^{1}$

${ }^{1}$ Intensive care unit, H. U del Henares, Madrid, Spain

Correspondence: R. Molina

Intensive Care Medicine Experimental 2020, 9(1): 000837.

Introduction. Extracorporeal $\mathrm{CO} 2$ extraction (ECCO2R) is a technique that uses an extracorporeal device to extract excess $\mathrm{CO} 2$ from the blood by passing it through an air exchange membrane using low blood flows and requiring systemic anticoagulation. Its main use is to reduce the hypercapnic acidosis seen in patients with COPD or to apply a lung protective strategy with low tidal volume in patients with respiratory failure (RF) due to ARDS.

Objectives. To describe our current experience with ECCO2R during COVID-19 pandemic in patients who developed severe ARDS due to COVID-19 (C-ARDS).

Methods. We present eight cases of patients with severe global RF of more than ten days' evolution with insufficient response to protective $\mathrm{MV}$ and proning and in whom the possibility of transfer for ECMO was ruled out due to poor prognostic factors. ECCO2R support offered the possibility of trying to increase the degree of oxygenation by increasing the level of PEEP and lowering Pplat and tidal volume to achieve protective lung ventilation. We used ProLUNG ${ }^{\circledR}$ system, a device with a $1.8 \mathrm{~m} 2$ biocompatible non-porous poly-4-methyl-1-pentene membrane. It allows a sweep airflow of up to $15 \mathrm{Lpm}$ and blood flow from 100 to $450 \mathrm{ml} / \mathrm{min}$. The therapeutic objectives of ProLUNG ${ }^{\circledR}$ were achieved within the first $12 \mathrm{~h}$ of treatment.

Results. Table 1 shows patients' characteristics, Table 2 results obtained after initiating ECCO2R. Regarding complications, non-heparin-induced thrombopenia was observed in one patient. Regarding outcome, the mean duration of therapy was 5.25 days, with a mean blood flow of $400 \mathrm{ml} / \mathrm{min}$ and air flow of $12 \mathrm{Lpm}$. Mortality at ICU discharge was $37.5 \%$, one of the patients still weaning. Two died.

\begin{tabular}{lllllllllll}
\hline $\mathbf{N}$ & $\begin{array}{l}\text { Sex } \\
(\mathbf{F} / \mathrm{M})\end{array}$ & $\begin{array}{l}\text { Age } \\
(\mathbf{y r})\end{array}$ & $\begin{array}{l}\text { BMI } \\
\mathbf{( k g} / \\
\mathbf{m} 2)\end{array}$ & $\begin{array}{l}\text { Days } \\
\text { Symp/ } \\
\text { ECCO2R }\end{array}$ & $\begin{array}{l}\text { Days EIT/ } \\
\text { ECCO2R }\end{array}$ & $\begin{array}{l}\text { Compli- } \\
\text { ance (ml/ } \\
\mathbf{c m H 2 O})\end{array}$ & $\begin{array}{l}\text { PO2/ } \\
\text { FiO2 }\end{array}$ & $\begin{array}{l}\text { Days on } \\
\text { ECCO2R }\end{array}$ & $\begin{array}{l}\text { ICU } \\
\text { LOS } \\
\text { (days) }\end{array}$ & Out \\
\hline 1 & F & 53 & 33 & 26 & 15 & 17 & 64 & 5 & 47 & $\mathrm{a}$ \\
2 & $\mathrm{~F}$ & 55 & 37 & 25 & 11 & 13 & 70 & 7 & 33 & $\mathrm{a}$ \\
3 & $\mathrm{M}$ & 74 & 28 & 53 & 13 & 34 & 96 & 1 & 24 & $\mathrm{~d}$ \\
4 & M & 67 & 33 & 29 & 19 & 8 & 71 & 6 & 23 & $\mathrm{~d}$ \\
5 & $\mathrm{~F}$ & 61 & 32 & 16 & 21 & 28 & 61 & 4 & 12 & $\mathrm{a}$ \\
6 & $\mathrm{M}$ & 69 & 36 & 20 & 9 & 42 & 78 & 3 & $(\mathrm{x})$ & $\mathrm{a}$ \\
7 & $\mathrm{M}$ & 69 & 36 & 23 & 14 & 35 & 81 & 5 & 8 & $\mathrm{a}$ \\
8 & $\mathrm{M}$ & 64 & 25 & 23 & 14 & 14 & 80 & 8 & 1 & $\mathrm{~d}$ \\
\hline
\end{tabular}

${ }^{*} \mathrm{~N}=\mathrm{n}^{\circ}$ case. $\mathrm{F}=$ female, $\mathrm{M}=$ male. Days Symp/ECCO2R=days from symptom onset to ECCO2R. Days EIT/ECCO2R = days from intubation to $E C C O 2 R$. LOS = length of stay after ECCO2R. $(x)=$ patient awaiting discharge from ICU. Out= Outcome, $a=$ alive, $d=$ death.

Table 2 Data after initiating ECCO2R

\begin{tabular}{lllllllll}
\hline $\mathbf{N}$ & $\begin{array}{l}\text { Target TV } \\
(\mathbf{m l} / \mathbf{k g})\end{array}$ & $\begin{array}{l}\text { Pplat } \\
(\mathbf{c m H 2 O})\end{array}$ & $\begin{array}{l}\text { Driving } \\
\text { Pressure } \\
(\mathbf{c m H 2 O})\end{array}$ & $\begin{array}{l}\text { PEEP } \\
(\mathbf{c m H 2 O})\end{array}$ & $\mathbf{p H}$ & FiO2 & $\begin{array}{l}\text { SOFA } \\
\text { score }\end{array}$ & $\begin{array}{l}\text { Prone } \\
\text { posi- } \\
\text { tion }\end{array}$ \\
\hline 1 & $6->4.5$ & $32->30$ & $24->16$ & $8->14$ & $7.29->7.32$ & $0.8->0.6$ & 5 & $\mathrm{~N}$ \\
2 & $6.4->4.5$ & $27->23$ & $16->10$ & $6->10$ & $7.26->7.32$ & $1->0.75$ & 7 & $\mathrm{~N}$ \\
3 & $7.2->5$ & $28->28$ & $20->18$ & $8->10$ & $7.28->7.35$ & $1->0.85$ & 5 & $\mathrm{Y}$ \\
4 & $7.8->4.6$ & $31->28$ & $18->13$ & $12->14$ & $7.26->7.33$ & $1->0.9$ & 5 & $\mathrm{Y}$ \\
5 & $5.6->5$ & $28->28$ & $16->10$ & $12->18$ & $7.21->7.48$ & $1->0.7$ & 6 & $\mathrm{Y}$ \\
6 & $6.7->6.5$ & $28->25$ & $11->9$ & $14->16$ & $7.26->7.33$ & $0.9->0.7$ & 7 & $\mathrm{Y}$ \\
7 & $5.1->4$ & $29->28$ & $17->12$ & $12->16$ & $7.19->7.33$ & $1->0.65$ & 9 & $\mathrm{Y}$ \\
8 & $6->4.2$ & $30->26$ & $18->14$ & $8->12$ & $7.26->7.35$ & $80->75$ & 9 & $\mathrm{Y}$ \\
\hline
\end{tabular}

* TV = tidal volume. $\mathrm{N}=$ no. $\mathrm{Y}=$ yes.

Conclusion. These cases, as in previous studies, demonstrate the usefulness of the application of ECCO2R as a tool to optimize the degree of lung protection, essential in patients with severe ARDS. Furthermore, by lowering Pplat, which in turn allows PEEP to be increased safely, pulmonary oxygenation increased, giving time for the resolution of the underlying condition with little complications.

\section{Reference(s)}

1. Burki NK, Mani RK, Herth FJ, Schmidt W, Teschler H, Bonin F, et al. A novel extracorporeal $\mathrm{CO}(2)$ removal system: Results of a pilot study of hypercapnic respiratory failure in patients with COPD. Chest. 2013;143:678-86

2. Morelli A, Del Sorbo L, Pesenti A, Ranieri VM, Fan E. Extracorporeal carbondioxide removal (ECCO2R) in patients with acute respiratory failure. Intensive Care Med. 2017;43(4):519-30

3. Fitzgerald, M., Millar, J., Blackwood, B. et al. Extracorporeal carbon dioxide removal for patients with acute respiratory failure secondary to the acute respiratory distress syndrome: a systematic review. Crit Care 18, 222 (2014).

4. B. Lobo-Valbuena, Ma . García-Arias, R.B. Pérez, D.V. Delgado, F. Gordo, Characteristics of critical patients with COVID-19 in a Spanish secondlevel hospital, Medicina Intensiva, Volume 45, Issue 1, 2021, Pages 56-58, ISSN 0210-5691, https://doi.org/10.1016/j.medin.2020.06.020.

\section{3}

Risk factors associated with mortality during the first prone cycle in patients with ARDS secondary to SARS- CoV-2 pneumonia C. González'; G. Musso'; JM. Dominguez²; J. Rodriguez Louzan';

L. Berloni' ; G. Appendino ; M. Manago ${ }^{1}$; C. Lovesio, ; ${ }^{1}$ E. Estenssoro ${ }^{3}$

${ }^{1}$ Intensive Care, Sanatorio Parque, Rosario, Argentina; ${ }^{2}$ Intensive care, Cardiovascular Intitute of Rosario, Rosario, Argentina; ${ }^{3}$ Unidad de terapia intensiva, Hospital Interzonal de Agudos San Martín de La Plata, La Plata, Argentina

Correspondence: C. González

Intensive Care Medicine Experimental 2020, 9(1): 000843

Introduction. SARS-CoV2 pandemic has led to a greater need to rescue treatments such as prone position.

Objectives. Our objective was to identify factors associated with mortality after the first $24 \mathrm{~h}$ of prone position in patients on mechanical ventilation due to moderate and severe ARDS caused by SARS- CoV- 2 .

Methods. This was a retrospective cohort study. We included all patients with ARDS caused by SARS- CoV-2 pneumonia and required prone. We registered sex, age, APACHE II, SOFA, number of prone sessions-which lasted $24 \mathrm{~h}$-, hospital-acquired infections and hospital mortality. $\mathrm{PaO} 2 / \mathrm{FiO} 2$, plateau pressure and driving pressure were recorded and compared before and after the first prone session.

Results. Table 1. Respiratory variables before and after prone and other variables 


\begin{tabular}{|c|c|c|c|c|}
\hline & $\begin{array}{l}\text { ALL } \\
(n=126)\end{array}$ & $\begin{array}{l}\text { Survivors } \\
(n=44)\end{array}$ & $\begin{array}{l}\text { Nonsurvivors } \\
(n=82)\end{array}$ & "p" \\
\hline Age (years) & $60 \pm 15$ & $60 \pm 16$ & $61 \pm 15$ & 0.42 \\
\hline APACHE ॥ & $12 \pm 5$ & $10 \pm 4$ & $13 \pm 6$ & $<0.001$ \\
\hline SOFA & $4 \pm 2$ & $4 \pm 3$ & $4 \pm 2$ & 0.47 \\
\hline $\begin{array}{l}\text { Baseline } \mathrm{PaO} 2 / \mathrm{FiO} \\
\text { (mmHg) }\end{array}$ & $112 \pm 26$ & $111 \pm 24$ & $113 \pm 28$ & 0.92 \\
\hline $\begin{array}{l}\text { Prone } \mathrm{PaO} 2 / \mathrm{FiO} 2 \\
\quad(\mathrm{mmHg})\end{array}$ & $186 \pm 59$ & $220 \pm 48$ & $169 \pm 57$ & $<0.001$ \\
\hline $\begin{array}{l}\% \text { of increase in } \mathrm{PaO} 2 / \\
\mathrm{FiO} 2 \text { after prone }(\%)\end{array}$ & $71 \pm 68$ & $100 \pm 65$ & $54 \pm 57$ & $<0.001$ \\
\hline $\begin{array}{l}\text { Baseline plateau pressure } \\
\left(\mathrm{cmH}_{2} \mathrm{O}\right)\end{array}$ & $25.7 \pm 3.6$ & $25.8 \pm 2.7$ & $25.8 \pm 4$ & 0.89 \\
\hline $\begin{array}{l}\text { Prone Plateau pressure } \\
\left(\mathrm{cmH}_{2} \mathrm{O}\right)\end{array}$ & $25.4 \pm 3.7$ & $26 \pm 4$ & $26 \pm 4.2$ & 0.07 \\
\hline $\begin{array}{l}\text { Baseline driving pressure } \\
\left(\mathrm{CmH}_{2} \mathrm{O}\right)\end{array}$ & $14 \pm 4$ & $14.2 \pm 3.8$ & $13.8 \pm 3.8$ & 0.49 \\
\hline $\begin{array}{l}\text { Prone driving pressure } \\
\left(\mathrm{cmH}_{2} \mathrm{OH}_{2} \mathrm{O}\right)\end{array}$ & $13.5 \pm 4$ & $13.4 \pm 3.4$ & $13.6 \pm 4.2$ & 0.67 \\
\hline $\begin{array}{l}\text { Baseline Tidal volume } \\
(\mathrm{ml})\end{array}$ & $401 \pm 62$ & $395 \pm 60$ & $403 \pm 64$ & 0.52 \\
\hline $\begin{array}{l}\text { Tidal volume after prone } \\
(\mathrm{ml})\end{array}$ & $399 \pm 67$ & $390 \pm 53$ & $402 \pm 59$ & 0.24 \\
\hline$N^{\circ}$ of prone cycles $(n, \%)$ & $1.8 \pm 1.2$ & $1.4 \pm 0.7$ & $2.1 \pm 1.3$ & $<0.001$ \\
\hline $\begin{array}{l}\text { Hospital-acquired infec- } \\
\text { tion }(n, \%)\end{array}$ & $52(40)$ & $10(10)$ & $32(30)$ & $<0.001$ \\
\hline
\end{tabular}

Table 2 Independent risk factors fo hospital mortality. Logistic regression analysis

\begin{tabular}{llll}
\hline & Odds Ratio & Cl (95\%) & $\mathbf{p}$ \\
\hline \% of increase in PaO2/FiO2 after prone & 0.99 & $0.98-0.99$ & 0.01 \\
APACHE II & 1.2 & $1.08-1.34$ & 0.001 \\
Hospital-acquired infection & 5.8 & $1.78-2$ & 0.001 \\
N $^{\circ}$ of prone cycles & 1.84 & $1.08-3.12$ & 0.02 \\
Age & 1.06 & $1.01-1.10$ & 0.001 \\
\hline
\end{tabular}

Conclusion. In this cohort of patients the increase in $\mathrm{PaO} 2 / \mathrm{FiO} 2$ ratio after $24 \mathrm{~h}$ of prone position was independently associated with decreased mortality; while age, APACHE II, number of prone cycles, and presence of hospital-acquired infection were associated with increased mortality.

\section{9}

Value of modified calculation methods and potential usage areas of ROX index in ICU patients

E. Eris ${ }^{1}$; A. Mammadova²; ZS. Solmaz'; A. Atasoy ${ }^{1}$; AT. Kara²; G. Gursel ${ }^{2}$

${ }^{1}$ Critical care fellowship programme, Gazi University School of Medicine, Ankara, Turkey; ${ }^{2}$ Department of pulmonary critical care medicine, Gazi University School of Medicine, Ankara, Turkey

Correspondence: E. Eriş

Intensive Care Medicine Experimental 2020, 9(1): 000869

Introduction. Respiratory failure is a very widespread life threatening clinical problem worldwide. Recent studies suggest that ROX index is promising parameter to predict HFNC treatment success in hypoxemic respiratory failure. On the other hand there is few data related using arterial blood gas values to calculate ROX and to evaluate if ROX can predict mortality, intubation and NIV, weaning, extubation failure in patients with respiratory failure.

Objectives. Aim of this study is to compare the ROX index calculated from $\mathrm{PaO} 2, \mathrm{SaO} \%$ and $\mathrm{SpO} 2 \%$ and to investigate the role of $\mathrm{ROX}$ index in predicting intubation indication, mortality, NIV, weaning and extubation failure in patients with respiratory failure.

Methods. In this retrospective cohort study ROX index was calculated with classical formula of $[(\mathrm{SpO} 2 \% / \mathrm{FiO} 2) /$ respiratory rate(RR)] and also with arterial blood gas $\mathrm{PaO} 2$ and $\mathrm{SaO} \%$ values. $\mathrm{ROX}$ calculated from baseline, and first 3rd day of non invasive ventilation(NIV) and mechanical ventilation(MV) treatments' $\mathrm{SpO} 2, \mathrm{FiO} 2$ and $\mathrm{RR}$ values. Pressure support, PEEP, peak inspiratory pressure values were also recorded during these measurements. These ROX values were used to analyse if they predict intubation, mortality, NIV, weaning and extubation failure. Student t, Chi square, logistic regression, ROC curve tests were used for statistical analysis.

Results. 290 patients were included in the study. Mean age of the patients was $71+14$ and $46 \% \%$ of them were female. Mean APACHE II and SOFA scores were $20+8$ and $5+3$ respectively. Mortality, intubation rate, NIV failure, weaning failure and extubation failure rates were $19 \%, 37 \%, 12 \%, 7 \%, 6 \%$ respectively. ROX values and theresholds for predicted parameters that have highest sensitivity and specificity values were given in table. NIVROXD3 calculated for 3rd day of NIV with median PIP: $20 \mathrm{cmH} 2 \mathrm{O}, \mathrm{FiO} 2: 32 \%$ and RR:20/min. MVROXD3 values were calculated with median PIP:25 cmH2O, FiO2:35\% and RR:18/min. NIVROXD3SpO2<9 ((OR:15(Cl95\%2-114)p:0.008) and MVROXD3PaO2<11 ((OR:10)( Cl95\%: 3.2-33), p:0.005)were found to be an independent risk factor for mortality together with APACHE II score((OR:0.8)( Cl95\%: 0.7-1) p:0.006), and sepsis((OR:8)( Cl95\%: 2-24),p:0.001) in logistic regression analysis.

Conclusion. Results of this study suggest that calculation of ROX index not only with $\mathrm{SpO} 2 \%$ but also arterial blood gas $\mathrm{PaO} 2$ and $\mathrm{SaO} 2 \%$ values may also be useful to predict outcome of NIV and MV treatment and mortality. Further studies with more patients are necessary to verify and standardise of these results.

\begin{tabular}{|c|c|c|c|c|c|c|c|}
\hline ROX & $\begin{array}{l}\text { Cut- } \\
\text { off }\end{array}$ & $\begin{array}{l}\text { Pre- } \\
\text { dicted }\end{array}$ & $\begin{array}{l}\text { Sensi- } \\
\text { tivity }\end{array}$ & $\begin{array}{l}\text { Speci- } \\
\text { ficity }\end{array}$ & $\mathbf{p}$ & $\begin{array}{l}\text { PS } \\
\text { median }\end{array}$ & $\begin{array}{l}\text { PEEP } \\
\text { median }\end{array}$ \\
\hline $\begin{array}{l}\text { Baseline ROX } \\
\text { SpO2\% }\end{array}$ & $<7$ & $\begin{array}{l}\text { intuba- } \\
\text { tion }\end{array}$ & 80 & 60 & 0.005 & & \\
\hline $\begin{array}{l}\text { NIVROXD- } \\
1 \mathrm{SaO} \%\end{array}$ & $<10$ & mortality & 76 & 73 & 0.001 & 12 & 6 \\
\hline $\begin{array}{l}\text { NIVROXD- } \\
\text { 3PaO2 }\end{array}$ & $<10$ & $\begin{array}{l}\text { NIV } \\
\text { failure }\end{array}$ & 82 & 70 & 0.005 & 12 & 7 \\
\hline $\begin{array}{l}\text { NIVROXD- } \\
\text { 3SpO2\% }\end{array}$ & $<9$ & Mortality & 92 & 75 & 0.005 & 12 & 7 \\
\hline $\begin{array}{l}\text { MVROXD- } \\
3 \mathrm{PaO} 2\end{array}$ & $<11$ & $\begin{array}{l}\text { Extuba- } \\
\text { tion } \\
\text { failure }\end{array}$ & 81 & 70 & 0.001 & 30 & 5 \\
\hline $\begin{array}{l}\text { MVROXD- } \\
\text { 3PaO2 }\end{array}$ & $<11$ & $\begin{array}{c}\text { Weaning } \\
\text { failure } \\
\text { Mortality }\end{array}$ & 74 & 67 & 0.005 & 30 & 5 \\
\hline $\begin{array}{l}\text { Weanin- } \\
\text { gROXP- } \\
\text { SPaO2 }\end{array}$ & $<10$ & $\begin{array}{l}\text { Weaning } \\
\text { failure }\end{array}$ & 77 & 74 & 0.004 & 13 & 5 \\
\hline $\begin{array}{l}\text { Weanin- } \\
\text { gROXSBT- } \\
\text { SpO2\% }\end{array}$ & $<11$ & $\begin{array}{l}\text { Weaning } \\
\text { failure }\end{array}$ & 76 & 60 & 0.017 & & \\
\hline
\end{tabular}

ROX values calculated with: Baseline ROX SpO2\%: Admission SpO2, NIVROXD1SaO2\%: SaO2 recorded first day of NIV, NIVROXD3PaO2: $\mathrm{PaO} 2$ recorded first day of NIV, NIVROXD3SpO2\%:SpO2 recorded third day of NIV,MVROXD3PaO2: $\mathrm{PaO} 2$ recorded third day of MV, WeaningROXP$\mathrm{SPaO} 2 \mathrm{PaO} 2$ recorded during weaning day pressure support ventilation. WeaningROXSBTSPO2\%: SpO2\% recorded during weaning day spontan breathing trial.

\section{Reference(s)}

1. 2-Roca, O., Messika, J., Caralt, B., Garcia-de- Acilu, M., Sztrymf, B., Ricard, J., \& Masclans, J. R. (2016). Predicting success of high-flow Nasal cannula in pneumonia patients with hypoxemic respiratory failure: The utility of the 
ROX index. Journal of Critical Care, 35, 200-205. https://doi.org/10.1016/j. jcrc.2016.05.022

2. 1-Roca O, Caralt B, Messika J, Samper M, Sztrymf B, Hernández G, et al. An index combining respiratory rate and oxygenation to predict outcome of nasal high-flow therapy. Am J Respir Crit Care Med 2019;199:1368-76.

\section{7}

Assessment of Admission COVID-19 Associated

Hyper-inflammatory Syndrome (cHIS) Score in Critically-III COVID-19 Patients

M. Yildirim ${ }^{1}$; B. Halacli ${ }^{1}$; Y. Gunegul ${ }^{1}$; E. Ulusoydan ${ }^{1}$; G. Guven ${ }^{1}$; E. Ortac Ersoy'; A. Topeli'

${ }^{1}$ Division of intensive care, department of internal medicine, Hacettepe University Faculty of Medicine, Ankara, Turkey

Correspondence: M. Yildirim

Intensive Care Medicine Experimental 2020, 9(1): 000887

Introduction. COVID-19 related cytokine storm has got interest regarding individualised approach to especially severe cases. COVID-19 associated hyperinflammatory syndrome (cHIS) is one of the proposed phenotypes which has been defined as 1 . fever, 2. macrophage activation (ferritin $\geq 700 \mu \mathrm{g} / \mathrm{L}$ ), 3. haemotological dysfunction (NLR $\geq 10$ or haemoglobin $\leq 9.2 \mathrm{~g} / \mathrm{dL}$ and platelet $\leq 110,000$ cells $/ \mathrm{L}), 4$. coagulopathy (D-dimer $\geq 1.5 \mu \mathrm{g} / \mathrm{mL}$ ), 5 . hepatic injury ( $\mathrm{LDH} \geq 400 \mathrm{U} / \mathrm{L}$ or $\mathrm{AST} \geq 100 \mathrm{U} / \mathrm{L})$ and 6 . cytokinemia (IL-6 $\geq 15 \mathrm{pg} / \mathrm{mL}$ or triglyceride $\geq 150 \mathrm{mg} / \mathrm{dL}$ or $\mathrm{CRP} \geq 15 \mathrm{mg} / \mathrm{dL}$ ). Each 6 criteria increase cHIS score one point and a previous study showed that a maximum cHIS score of $\geq 3$ during follow-up was associated with hyperinflammation and poor prognosis among hospitalized COVID-19 patients (1). There has been no study so far to evaluate admission CHIS score to predict ICU outcomes.

Objectives. We aimed to investigate effects of ICU admission cHIS score on prognosis and outcomes of critically-ill COVID-19 patients. Methods. Laboratory confirmed COVID-19 patients who were admitted to ICU between 21st March 2020 and 21st March 2021 were included. A cHIS score $\geq 3$ was accepted as hyperinflammatory phenotype.

Results. Among 328 patients, 17 patients who received tocilizumab and anakinra during ICU stay were excluded and remaining 311 patients were studied. At admission, 167 (53.7\%) patients had a cHIS score of $\geq 3$ and 144 patients (46.3\%) had less than 3 . Patients with $\geq 3$ cHIS score had higher SOFA score $(p<0.01)$. In patients with $\mathrm{cHIS}$ score $\geq 3$, invasive mechanical ventilation were more frequently performed on admission $(p=0.04)$. Systemic steroid use was seen more in $\geq 3$ cHIS group $(p=0.03)$. Length of ICU and hospital stay in patients with $\geq 3 \mathrm{cHIS}$ score was longer than other patients $(p<0.01)$. ICU mortality in patients with $\geq 3$ CHIS score was higher than in those with less than 3 cHIS score $(p=0.03)$ and there was a trend towards hospital mortality $(p=0.06)$ (Table).

Table: General characteristics and outcomes of critically-ill COVID19 patient.

\begin{tabular}{|c|c|c|c|c|}
\hline & $\begin{array}{l}\text { Total } \\
(n=311)\end{array}$ & $\begin{array}{l}\text { cHIS score }<3 \\
(n=144)\end{array}$ & $\begin{array}{l}\text { cHIS score } \geq 3 \\
(n=167)\end{array}$ & $p$ value \\
\hline $\mathrm{Age}^{*}$ & $66[56-76]$ & $68[60-78]$ & 63 [54-74] & $<0.01$ \\
\hline Male sex, n (\%) & $199(64.0)$ & $91(63.2)$ & $108(64.7)$ & 0.78 \\
\hline $\begin{array}{l}\text { Hypertension, } \\
\text { n (\%) } \\
\text { Diabetes, n (\%) } \\
\text { Cardiac disease, } \\
\text { n (\%) } \\
\text { Chronic lung } \\
\text { disease, n (\%) }\end{array}$ & $\begin{array}{l}167(53.7) \\
112(36.0) \\
109(35.0) \\
54(17.4)\end{array}$ & $\begin{array}{l}88(61.1) \\
53(36.8) \\
56(38.9) \\
30(20.8)\end{array}$ & $\begin{array}{l}79(47.6) \\
59(35.5) \\
53(32.1) \\
24(14.5)\end{array}$ & $\begin{array}{l}\mathbf{0 . 0 2} \\
0.54 \\
0.21 \\
0.14\end{array}$ \\
\hline $\begin{array}{l}\text { Clinical Fraility } \\
\text { Scale* }\end{array}$ & $3[2-6]$ & $3[2-6]$ & $4[2-6]$ & 0.97 \\
\hline APACHE $\|^{*}$ & 15 [12-19] & 15 [11-19] & $16[12-21]$ & 0.08 \\
\hline SOFA* & $4[3-6]$ & $3[2-5]$ & $4[3-6]$ & $<0.01$ \\
\hline $\mathrm{PaO} 2 / \mathrm{FiO}_{2} \mathrm{~T}^{*}$ & 145 [113-217] & 149 [119-235] & 142 [109-203] & 0.10 \\
\hline
\end{tabular}

\begin{tabular}{|c|c|c|c|c|}
\hline & $\begin{array}{l}\text { Total } \\
(n=311)\end{array}$ & $\begin{array}{l}\text { cHIS score }<3 \\
(n=144)\end{array}$ & $\begin{array}{l}\text { cHIS score } \geq 3 \\
(n=167)\end{array}$ & $\mathrm{p}$ value \\
\hline $\begin{array}{l}\text { Systemic steroid } \\
\text { use, } n(\%)\end{array}$ & $256(82.3)$ & $112(77.8)$ & $144(86.7)$ & 0.03 \\
\hline IMVT, n (\%) & $125(40.2)$ & $49(34.0)$ & $76(45.5)$ & 0.04 \\
\hline $\begin{array}{l}\text { Acute kidney } \\
\text { injuryT, n (\%) }\end{array}$ & $66(21.2)$ & $24(16.6)$ & $42(25.1)$ & 0.15 \\
\hline $\begin{array}{l}\text { Septic shockT, } \\
\text { n (\%) }\end{array}$ & $47(15.1)$ & $16(11.1)$ & $31(18.6)$ & 0.09 \\
\hline ICU LOS*, days & 11 [5-18] & $9[4-17]$ & 12 [7-22] & $<0.01$ \\
\hline $\begin{array}{l}\text { Hospital LOS*, } \\
\text { days }\end{array}$ & 19 [12-31] & 17 [11-27] & 23 [13-35] & $<0.01$ \\
\hline $\begin{array}{l}\text { ICU mortality, } \\
\text { n (\%) }\end{array}$ & $103(33.1)$ & $39(27.1)$ & $64(38.3)$ & 0.03 \\
\hline $\begin{array}{l}\text { Hospital mortal- } \\
\text { ity, } \mathrm{n}(\%)\end{array}$ & $108(34.8)$ & $42(29.6)$ & $66(39.5)$ & 0.06 \\
\hline
\end{tabular}

*median [IQR],Ton admission,text in bold in $\mathrm{p}$ value indicated statistical significance. APACHE:acute physiology and chronic health evaluation, SOFA:sequential organ failure assessment, IMV:invasive mechanical ventilation, ICU:intensive care unit,LOS:length of stay.

Conclusion. Our study showed patients with hyperinflammatory phenotype on admission had worse ICU outcomes. Further investigations in more patients are needed to validate these findings and whether cHIS score could be used in the context of immunomodulatory treatments decision apart from steroids.

\section{Reference(s)}

1. Webb BJ et al. Clinical criteria for COVID-19-associated hyperinflammatory syndrome: a cohort study. Lancet Rheumatol. (2020) 2(12):e754-e763.

\section{2}

\section{Treating moderate-severe interstitial pneumonia due to CoViD} 2019 by c-PAP helmet

A. CORONA ${ }^{1}$; G. Richini'; M. Pasqua ${ }^{1}$; C. Santorsola ${ }^{1}$; I. Gatti ${ }^{1}$; A. Capone ${ }^{1}$; E. Zendra'; M. Mendeni ${ }^{2}$; A. Augenti ${ }^{2}$

'DEPARTMENT OF EMERGENCY AND CASUALTY AND ICU, ASST Vallecamonica, Esine, Province of Brescia, Italy, Esine, Italy; ${ }^{2}$ Department of internal medicine, ASST Vallecamonica, Esine, Province of Brescia, Italy, Esine, Italy

Correspondence: A. CORONA

Intensive Care Medicine Experimental 2020, 9(1): 000912

Introduction. COVID-19 was first reported in late 2019 and declared a pandemic on 12 March 2020. At the beginning of the pandemic, international guidelines did not address the use of CPAP in COVID-19 patients, focusing instead on high-flow nasal oxygen (HFNO), noninvasive ventilation (NIV) and invasive mechanical ventilation once patients are intubated. However, eventually few studies showed that Early use of CPAP may provides quantified evidence of benefit in treating interstitial pneumonia due to CoViD 2019, leading to a significant reduction in mortality.

Objectives. To assess efficacy of Helmet c-PAP to treat moderate severe interstitial pneumonia due to CoViD 2019.

Methods. Prospective observational study conducted at ASST Valcamonica Hospital during the CoViD 2019 third wave, between February-April 2021. We collected demographic, anamnestic, respiratory, laboratory and outcome data on all patients admitted to medical and pneumological ward treated with c-PAP for confirmed COVID-19 disease and severe acute respiratory syndrome.

Results. We recruited a total of 72 whose main characteristics [expressed in median (IQR) value or percentage] are: (i) age: 66 (60-74) with $81 \%$ with at least one comorbidity; (ii) admission SOFA 2 (2-3), SAPS II 37 (34-39); (iii) CT scan alveolar involvement 50\% (30-55); (iv) $\mathrm{paO2} / \mathrm{FiO} 2242$ (224-281), lactates $1.6(1.2-1.9)$ and with dyspnea in $81 \%$ of cases. The median (IQR) c-PAP (i) profile was PEEP $=10$ (1012.5 ) associated with a $\mathrm{FiO} 2$ of 0.5 ; (ii) duration $9(6-13)$ days. Most of 
patients improved, despite about of $25 \%$ of $\mathrm{CT}$ imaging impairing. The median (IQR) 262 (212-395) paO2/FiO2 was recorded in spontaneous breathing at the discarging time. Penumothorax/Pneumomediastinum were recorded in only 3 patients. Only $6(8.3 \%)$ patients deterioreted and needed admission to ICU. Death occurred only in $7(12.7 \%)$ patients including the 6 patients admitted to ICU (4/6). Therefore only $3 / 66(4.5 \%)$ among remaining in the general ward.

Conclusion. In conclusion, our results suggest that a number of patients with moderate-to-severe AHRF due to Covid-19 pneumonia may be amenable to high-flow CPAP, even in the presence of gas exchange and CT findings usually considered as indications for IMV.

\section{Reference(s)}

1. Brusasco C, Corradi F, Di Domenico A, Raggi F, Timossi G, Santori G, Brusasco V; Galliera CPAP-Covid-19 study group; collaborators of the Galliera CPAP-COVID-19 study group are. Continuous positive airway pressure in COVID-19 patients with moderate-to-severe respiratory failure. Eur Respir J. 2021 Feb 17;57(2):2,002,524

2. None

\section{3}

\section{Outcome of high flow nasal cannula in COVID-19 pneumonia}

A. Pavalascu'; E. Abril Palomares'; N. Moliner Arrebola ${ }^{1}$; I. Rodado Muñoz ${ }^{1}$; L. Riesco De Vega'; MÁ. Estévez Hidalgo'; ME. González González'; N. Camino Redondo'; MC. Martín Delgado

'Icu, Torrejón University Hospital, Madrid, Spain

Correspondence: A. Pavalascu

Intensive Care Medicine Experimental 2020, 9(1): 000913

Introduction. COVID-19 pneumonia can result in acute hypoxemic respiratory failure (hARF), requiring supportive respiratory therapy (1). High-flow nasal cannula (HFNC) is considered a key issue in proper patients (pts.) and early application (2).

Objectives. To determine if HFNC can reduce the requiring of endotracheal intubation (ET) in pts. with hARF secondary to COVID-19, and if it can decrease the length of intensive care unit (ICU) stay.

Methods. A retrospective, descriptive study for 14 months (Mar. 2020-Apr. 2021) of pts. admitted to a public hospital in Madrid. The pts. included were those treated with HFNC, tested positive for SARS CoV-2, and failure of non-rebreathing masks with oxygen reservoir bag to maintain arterial oxygen saturation $(\mathrm{SaO} 2) \geq 92 \%$. The pts. who required immediate endotracheal intubation (ET) were excluded. The group of pts. who had a successful outcome as defined by reversal of hypoxemia $(\mathrm{SaO} 2 \geq 92 \%)$, and no need for HFNC (Gp-N), was compared with the group of pts. who needed ET during the ICU stay (Gp-T). SaO2, ROX index, as well as age, sex, underlying diseases and complications, were entered into logistic regression analysis. Data are presented as percentage and mean, and standard deviation, $p<0.01$ being considered significant. All analyses were done with IBM SPSS Statistics for macOS, version 25 .

Results. A total of 74 pts. with COVID-19 pneumonia treated with HFNC, which accounts for $28 \%$ of the pts. with COVID-19 pneumonia admitted to the unit during this period, were studied. Overall characteristics: $69 \%$ males, mean age: $59 \pm 11$ years $(95 \% \mathrm{Cl}), \mathrm{Gp}-\mathrm{T}$ : $40 \%$ of total pts., mean admission APACHE II: $8 \pm 3(95 \% \mathrm{Cl}$; Gp-C $7 \pm 3$ vs. Gp-T $10 \pm 4$ ), mean ICU stay in discharged pts.: Gp-C $5 \pm 3$ days vs. Gp-T $27 \pm 20$ days $(95 \% \mathrm{Cl}, \mathrm{p}<0.001)$, mean hospital stay in discharged pts.: Gp-C $13 \pm 5$ days vs. Gp-T $36 \pm 18$ days $(95 \% \mathrm{Cl}, \mathrm{p}<0.001)$. The pts. in the $\mathrm{Gp}-\mathrm{C}$ were changed to a venturi mask in $4 \pm 2$ days $(95 \% \mathrm{Cl})$. Mean invasive mechanical ventilation (IMV) period in discharged pts.: $24 \pm 19$ days $(95 \% \mathrm{Cl})$, and mean HFNC period until ET was performed: $3 \pm 2$ days. $10 \%$ of pts. were exitus during ICU stay. The most common complication in $\mathrm{Gp}$-T was acute kidney failure, followed by ventilatorassociated pneumonia (53\%, $40 \%$ pts.). In Gp-C $98 \%$ of pts. presented respiratory failure, without other complications. Minimal ROX index on the 1st day of admission resulted in an independent factor associated with the need for ET, Gp-T $5.4 \pm 1.6$ vs. Gp-C $7.0 \pm 2.7$ (OR 2.1, p=0.02). Conclusion. High-flow therapy is a useful treatment in pts. with COVID19 moderate-severe acute respiratory distress syndrome to avoid ET, or as a bridge therapy. Using Rox index to identify the risk for ET, the pts. improved progressively on Gp-C, and ET was avoided in up to $60 \%$ of cases. Positive response to HFNC therapy in selected pts., avoiding inappropriately delayed ET, led to a favourable outcome in the length of ICU stay and hospital stay, and avoided complications related to IMV.

\section{Reference(s)}

1. Demoule A, Vieillard Baron A, Darmon M, Beurton A, Géri G, Voiriot G, et al. High flow nasal cannula in critically ill severe COVID-19 patients. Am J Respir Crit Care Med. 2020

2. Yang $X, Y u, Y, X u$ J, et al. Clinical course and outcomes of critically ill patients with SARS-CoV-2 pneumonia in Wuhan, China: a singlecentered, retrospective, observational study. Lancet Respir Med 2020;8:475-81.

\section{3}

Awake ECMO strategy in COVID-19 patients. A subanalysis of the ECMOVIBER study

M. Martinez Martinez ${ }^{1}$; M. Santafe, ${ }_{1}^{1}$; A. Cortina ${ }^{1}$; A. Pacheco, ; AE. Mera ${ }^{1}$; A. Vaz $z^{2}$ MP. Fuset ${ }^{3}$; P. Millán ${ }^{4}$; MV. Boado ${ }^{5}$; J. Rubio ${ }^{6}$; A. González-Pérez; E. Gallart ${ }^{1}$; R. Roncon-Albuquerque ${ }^{2}$; J. Riera ${ }^{1}$

${ }^{1}$ Critical care department, Vall d'Hebron University Hospital, Vall d'Hebron Research Institute, Barcelona, Spain; ${ }^{2}$ Intensive care department \& cirurgia e fisiologia department, Centro Hospitalar São João \& Faculdade de Medicina da Universidade do Porto, Porto, Portugal; ${ }^{3} \mathrm{Critical}$ care department, Bellvitge University Hospital, L'Hospitalet de Llobregat, Spain; ${ }^{4}$ Critical care department, La Paz University Hospital, Madrid, Spain; ${ }^{5}$ Critical care department, Hospital Universitario Cruces, Barakaldo, Spain; ${ }^{6} \mathrm{Critical}$ care department, University Hospital October 12, Madrid, Spain; ${ }^{7}$ Critical care department, Central University Hospital of Asturias, Oviedo, Spain Correspondence: J. Riera

Intensive Care Medicine Experimental 2020, 9(1): 000933

Introduction. ECMO runs of COVID-19 patients are longer than habitual. An awake ECMO strategy may optimize outcomes.

Objectives. Describe the population of COVID-19 patients with an awake ECMO strategy. Compare outcomes with the rest of the population.

Methods. The ECMOVIBER investigation included 334 consecutive patients with confirmed SARS-CoV-2 infection that needed extracorporeal respiratory support among 25 centers (23 in Spain, 2 in Portugal) between March 1 and December 1, 2020. End of follow up and recruitment was December 1 . This is a subanalysis focusing on those patients with an awake ECMO strategy defined by as the patient being awake, cooperative, performing rehabilitation and physiotherapy.

Results. Of the 325 patients with available information, 71 (21.8\%) received the awake ECMO strategy at some point of their ECMO run. The description of the cohort and the comparison with the rest of the population are detailed in Table 1. Of note, those patients with an awake ECMO strategy were younger and the clinical condition was slightly better than the rest of the population. They were more frequently supported in high volume ECMO centers. Larger drainage cannula was used in this cohort. At day 3 of ECMO support less patients received neuromuscular blockade and tidal volume was higher at this time point, with no difference in the driving pressure. Time on ECMO, in the ICU and in hospital were longer but survival was higher in patients with the awake ECMO strategy.

\begin{tabular}{lllll}
\hline Variable & \multicolumn{2}{l}{ Awake $\mathbf{n}=\mathbf{7 1}$ Non awake $\mathbf{n}=\mathbf{2 5 4}$} & p.value & $\mathbf{n}$ \\
\hline Age & $50[44.5,58]$ & $56[48,61]$ & 0.007 & 325 \\
Sex (male) & $57(80.3 \%)$ & $203(79.9 \%)$ & 1 & 325 \\
Hypertension & $24(33.8 \%)$ & $97(38.2 \%)$ & 0.591 & 325 \\
Immunocompromised & $5(7 \%)$ & $16(6.3 \%)$ & 0.788 & 325 \\
COPD & $3(4.2 \%)$ & $16(6.3 \%)$ & 0.775 & 325 \\
PaO2/FIO2 preECMO & $79[70,94]$ & $76[63,89.8]$ & 0.048 & 325 \\
pH preECMO & $7.3[7.2,7.4]$ & $7.3[7.2,7.4]$ & 0.289 & 321 \\
PaCO2 preECMO & $59.5[52,69]$ & $62[51,74]$ & 0.431 & 321 \\
\hline
\end{tabular}




\begin{tabular}{|c|c|c|c|c|}
\hline Variable & Awake $n=71$ & Non awake $n=254$ & p.value & $\mathrm{n}$ \\
\hline Driving P preECMO & $16[13.8,20]$ & $18[15,21]$ & 0.014 & 317 \\
\hline Tidal volume preECMO & $\begin{array}{l}450[380, \\
480]\end{array}$ & $422.5[380,480]$ & 0.516 & 317 \\
\hline Resp rate preECMO & $25[20.5,28]$ & $25[22,30]$ & 0.551 & 320 \\
\hline PEEP preECMO & $12[8,14]$ & $12[10,14]$ & 0.897 & 320 \\
\hline $\mathrm{PAM} \geq 70 \mathrm{mmHg}$ preECMO & $66(26.1 \%)$ & $33(46.5 \%)$ & 0.009 & 324 \\
\hline Prone position preECMO & $67(94.4 \%)$ & $245(96.5 \%)$ & 0.491 & 325 \\
\hline MV days preECMO & $5[3,9]$ & $5.5[3,9]$ & 0.947 & 325 \\
\hline $\begin{array}{l}\text { Center Volume }<15 \text { cases } \\
\text { /year }\end{array}$ & $2(2.8 \%)$ & $94(37 \%)$ & $<0.001$ & 325 \\
\hline Fr drainage cannula & $25.3(2)$ & $24(1.9)$ & $<0.001$ & 315 \\
\hline Driving pressure day 3 & $12[10,14]$ & $12[10,14]$ & 0.682 & 308 \\
\hline Tidal volume day 3 & $\begin{array}{l}260[200 \\
370]\end{array}$ & $247.5[150,326.5]$ & 0.024 & 309 \\
\hline Respiratory rate day 3 & $12[10,15]$ & $12[10,15]$ & 0.66 & 311 \\
\hline PEEP day 3 & $10[10,12]$ & $10[10,12]$ & 0.904 & 309 \\
\hline NMB day 3 & $27(38 \%)$ & $161(66.3 \%)$ & $<0.001$ & 314 \\
\hline ECMO days & $\begin{array}{l}19.5[11.8, \\
33]\end{array}$ & $14.5[8,27]$ & 0.004 & 300 \\
\hline ICU days & $45[31,68.5]$ & $34[21.2,50]$ & 0.004 & 265 \\
\hline Hospital days & $53[41,76]$ & $41[25,59.5]$ & 0.002 & 256 \\
\hline 1st DEC: Home no oxygen & $37(52.1 \%)$ & $74(29.1 \%)$ & $<0.001$ & 325 \\
\hline $\begin{array}{l}\text { 1st DEC: Home with } \\
\text { oxygen }\end{array}$ & $0(0 \%)$ & $7(2.8 \%)$ & & \\
\hline 1st DEC: Dead & $11(15.5 \%)$ & $117(46.1 \%)$ & & \\
\hline 1st DEC: Still on ECMO & $15(21.1 \%)$ & $31(12.2 \%)$ & & \\
\hline $\begin{array}{l}\text { 1st DEC: Hospital without } \\
\text { ECMO }\end{array}$ & $8(11.2 \%)$ & $25(9.8 \%)$ & & \\
\hline
\end{tabular}

Conclusion. An awake ECMO strategy in COVID-19 patients is feasible and might be associated with better outcomes.

\section{Reference(s)}

1. Lebreton et al. Extracorporeal membrane oxygenation network organisation and clinical outcomes during the COVID-19 pandemic in Greater Paris, France: a multicentre cohort study. Lancet Respir Dis 2021 S2213-2600(21)00,096-5.

2. Hoeper et al. Extracorporeal membrane oxygenation instead of invasive mechanical ventilation in patients with acute respiratory distress syndrome. ICM 2013. 39:2056-2057

3. Riera et al. Extracorporeal membrane oxygenation retrieval in coronavirus disease 2019: a case-series of 19 patients supported at a high-volume extracorporeal membrane oxygenation center. Crit Care Expl 2020; 2:e0228.

\section{7}

Assessing the Predictive Accuracy of the ROX index with ITU patients admitted for High-Flow Nasal Oxygen with confirmed COVID-19

K. Butler'; J. Patel, ${ }^{2}$; J. Hodson, ${ }^{3}$; J. Weblin ${ }^{4}$

${ }^{1}$ Therapy Services, Queen Elizabeth Hospital Birmingham, Birmingham, UK, United Kingdom; ${ }^{2}$ Critical care, Queen Elizabeth Hospital Birmingham, Birmingham, United Kingdom; ${ }^{3}$ Department of critical care, Queen Elizabeth Hospital Birmingham, Birmingham, United Kingdom; ${ }^{4}$ Specialist surgery critical care, Queen Elizabeth Hospital Birmingham, Birmingham, United Kingdom

Correspondence: K. Butler

Intensive Care Medicine Experimental 2020, 9(1): 000957

Introduction. High Flow Nasal Oxygen (HFNO) is an adjunct used for the treatment of hypoxaemia [1]. Increasingly utilised during the SARS-CoV-2 pandemic [2], recent evidence has suggested that HFNO could decrease the need for invasive mechanical ventilation (IMV) in this cohort [3]. However, delaying IMV may be associated with worse outcomes [4]. The ROX index [5], calculated as the ratio of partial pressure of arterial oxygen to inspired oxygen divided by respiratory rate, has been shown to be a validated predictor of HFNO success in patients with hypoxemia secondary to pneumonia, with a score of $\leq 4.88$ after $12 \mathrm{~h}$ predicting the need for IMV [5]. Its utility in predicting HFNO success within the COVID-19 cohort is uncertain, with various ROX threshold scores reported in the literature $[6,7,8,9,10]$.

Objectives. To assess the predictive accuracy of the ROX score for patients admitted to a tertiary centre ITU for HFNO with a confirmed diagnosis of SARS-CoV-2.

Methods. Retrospective data was collected using electronic patients records for all patients with confirmed SARS-CoV-2 admitted to ITU for HFNO between September 2020-January 2021. ROX scores were calculated immediately prior to HFNO commencement, and 2, 4, 6 , 12,18 , and $24 \mathrm{~h}$ post HFNO commencement. Patients were excluded from analysis if they had received IMV prior to HFNO, had HFNO at other sites, had prolonged periods of CPAP/NIV or HFNO was ceiling of care. The predictive accuracy of the ROX score, with respect to HFNO treatment success, was assessed using receiver operating characteristic (ROC) curves, and quantified using the area under the ROC curve (AUROC).

Results. 78 patients were included in analysis. HFNO treatment successfully prevented intubation in 38 cases (49\%), with the remainder requiring IMV a median of $23 \mathrm{~h}$ (IQR: 8-48) after commencing HFNO. Patients with failed HFNO treatment were significantly older (mean: 59 vs. 52 years, $p=0.027$ ), had a higher APACHE II score (mean: 15 vs. 12, $p=0.001$ ), and a longer ITU stay (median: 14 vs. 4 days, $p<0.001$ ). No significant difference in clinical frailty score (median: 2 vs. $2, p=0.573$ ), BMI (mean: 28.2 vs. $29.1 \mathrm{~kg} / \mathrm{m} 2, p=0.366$ ) or gender (\% male: $65 \%$ vs. $66 \%, p=1.000$ ) was observed between groups. The ROX score was found to be significantly predictive of treatment success at all of the time points considered, with AUROCs ranging from $0.67-0.81$ (Table 1). The predictive accuracy was greatest where ROX was measured $2 \mathrm{~h}$ post-HFNO commencement $(A U R O C=0.81, p<0.001)$, with median scores of 7.43 vs. 4.44 for those with treatment success vs. failure.

Table 1 AUROC for ROX index at measured time points for HFNO success and failure

\begin{tabular}{|c|c|c|c|c|c|c|}
\hline \multirow{3}{*}{$\begin{array}{l}\text { Time from } \\
\text { HFNOCom- } \\
\text { mencement } \\
\text { to ROX Score }\end{array}$} & \multicolumn{4}{|c|}{ Outcome } & \multirow{3}{*}{$\begin{array}{l}\text { AUROC } \\
(95 \% \mathrm{CI})\end{array}$} & \multirow{3}{*}{$\begin{array}{l}\text { p- } \\
\text { Value }\end{array}$} \\
\hline & \multicolumn{2}{|c|}{ Failure } & \multicolumn{2}{|c|}{ Success } & & \\
\hline & $N$ & Median (IQR) & $N$ & Median (IQR) & & \\
\hline Pre-HFNO & 40 & $\begin{array}{c}3.38(2.73- \\
4.30)\end{array}$ & 38 & $\begin{array}{c}4.43(3.37- \\
5.87)\end{array}$ & $\begin{array}{l}0.72(0.61- \\
0.83)\end{array}$ & 0.001 \\
\hline 2 Hours & 39 & $\begin{array}{l}4.44(3.76- \\
5.64)\end{array}$ & 38 & $\begin{array}{c}7.43(5.71- \\
9.39)\end{array}$ & $\begin{array}{l}0.81(0.71- \\
0.91)\end{array}$ & $<0.001$ \\
\hline 4 Hours & 33 & $\begin{array}{c}5.45(4.48- \\
6.57)\end{array}$ & 37 & $\begin{array}{l}6.78(5.31- \\
9.40)\end{array}$ & $\begin{array}{c}0.69(0.56- \\
0.81)\end{array}$ & 0.007 \\
\hline 6 Hours & 32 & $\begin{array}{c}5.48(4.51- \\
6.80)\end{array}$ & 37 & $\begin{array}{l}6.77(5.48- \\
8.80)\end{array}$ & $\begin{array}{l}0.67(0.54- \\
0.79)\end{array}$ & 0.018 \\
\hline 12 Hours & 26 & $\begin{array}{c}6.19(4.85- \\
7.67)\end{array}$ & 36 & $\begin{array}{c}7.13(5.74- \\
11.40)\end{array}$ & $\begin{array}{l}0.67(0.54- \\
0.81)\end{array}$ & 0.021 \\
\hline 18 Hours & 22 & $\begin{array}{l}5.74(5.11- \\
7.75)\end{array}$ & 35 & $\begin{array}{c}7.83(5.66- \\
10.44)\end{array}$ & $\begin{array}{l}0.74(0.61- \\
0.87)\end{array}$ & 0.003 \\
\hline 24 Hours & 17 & $\begin{array}{l}5.14(4.23- \\
7.38)\end{array}$ & 32 & $\begin{array}{c}7.81(6.31- \\
10.11)\end{array}$ & $\begin{array}{l}0.75(0.59- \\
0.90)\end{array}$ & 0.005 \\
\hline
\end{tabular}

Conclusion. This retrospective cohort study indicates a high predictive accuracy of the ROX index when used in the SARS-CoV-2 cohort of patients admitted to a tertiary centre ITU. The highest accuracy was demonstrated at $2 \mathrm{~h}$ post commencement of HFNO when a ROX index of $\leq 4.44$ predicts failure of HFNO and the consideration for IMV. 
This study adds to the body of evidence in the use of HFNO and the ROX index, however further research, including a meta-analysis and systematic review, is required to identify a consensus of the ROX index score in the SARS-CoV-2 cohort.

\section{Reference(s)}

1. J.-P. Frat, A. W. Thille, A. Mercat, C. Cirault, S. Ragot, S. Perbet, G. Prat, T. Boulain, E. Morawiec, A. Cottereau, J. Devaquet, S. Nseir, K. Razazi, J.-P. Mira, L. Argaud, J.-C. Chakarian, J.-D. Ricard, X. Wittebole, S. Chevalier, A. Herbland, M. Fartoukh, J.-M. Constantin, J-M. Tonnelier, M. Pierrot, A. Mathonnet, G. Béduneau, C. Delétage-Métreau, J.-C. M. Richard, L. Brochard and R. Robert, "High-Flow Oxygen through Nasal Cannula in Acute Hypoxemic Respiratory Failure,"The New England Journal of Medicine, vol. 372, no. 23, pp. 2185-2196, 2015.

2. W. Alhazzani, L. Evans, F. Alshamsi, M. H. Møller, M. Ostermann, H. C. Prescott, Y. M. Arabi, M. Loeb, M. Ng Gong, E. Fan, S. Oczkowski, M. M. Levy, L. Derde, A. Dzierba, B. Du, F. Machado, H. Wunsch, M. Crowther, M. Cecconi, Y. Koh, L. Burry, D. S. Chertow, W. Szczeklik, E. Belley-Cote, M. Greco, M. Bala, R. Zarychanski, J. Kesecioglu, A. McGeer, L. Mermel, M. J. Mammen, S. Nainan Myatra, A. Arrington, R. Kleinpell, G. Citerio, K. Lewis, E. Bridges, Z. A. Memish, N. Hammond, F. G. Hayden, M. Alshahrani, Z. Al Duhailib, G. S. Martin, L. J. Kaplan, C. M. Coopersmith, M. Antonelli and A. Rhodes, "Surviving Sepsis Campaign Guidelines on the Management of Adults With Coronavirus Disease 2019 (COVID-19) in the ICU: First Update" Critical Care Medicine, vol. 49, no. 3, pp. pe219-e234, March 2021.

3. A. Demoule, A. V. Baron, M. Darmon, A. Beurton, G. Géri, G. Voiriot, T. Dupont, L. Zafrani, L. Girodias, V. Labbé, M. Dres, M. Fartoukh and E. Azoulay, "High-Flow Nasal Cannula in Critically III Patients with Severe COVID-19," American Journal of Respiratory and Critical Care Medicine, vol. 202, no. 7, pp. 1039-1042, 2020.

4. E. Spinelli, T. Mauri, J. R. Beitler, A. Pesenti and D. Brodie, "Respiratory drive in the acute respiratory distress syndrome: pathophysiology, monitoring, and therapeutic interventions," Intensive Care Medicine, vol. 46, no. 4, pp. 606-618, 2020.

5. O. Roca, B. Caralt, J. Messika, M. Samper, B. Sztrymf, G. Hernández, M. García-de-Acilu, J.-P. Frat, J. R. Masclans and J.-D. Ricard, "An Index Combining Respiratory Rate and Oxygenation to Predict Outcome of Nasal High-Flow Therapy," American Journal of Respiratory and Critical Care Medicine, vol. 199, no. 11, pp. 1368-1376, 2019.

6. G. L. Calligaro, U. Lalla, G. Audley, P. Gina, M. G. Miller, M. Mendelson, S. Dlamini, S. Wasserman, G. Meintjes, J. Peter, D. Levin, J. A. Dave, N. Ntusi, S. Meier, F. Little, D. L. Moodley, E. H. Louw, A. Nortje, A. Parker, J. J. Taljaard, B. W. Allwood, K. Dheda and C. F. N. Koegelenberg," The utility of high-flow nasal oxygen for severe COVID-19 pneumonia in a resource-constrained setting: A multi-centre prospective observational study,"The Lancet, vol. 28, 2020.

7. [7] M. Hu, Q. Zhou, R. Zheng, X. Li, J. Ling, Y. Chen, J. Jia and C. Xie, "Application of high-flow nasal cannula in hypoxemic patients with COVID-19: a retrospective cohort study," BMC Pulmonary Medicine, vol. 20, p. 324, 2020.

8. J. Xu, X. Yang, C. Huang, X. Zou, T. Zhou, S. Pan, L. Yang, Y. Wu, Y. Ouyang, Y. Wang, D. Xu, X. Zhao, H. Shu, Y. Jiang, W. Xiong, L. Ren, H. Liu, Y. Yuan, H. Qi S. Fu, D. Chen, D. Zhang, S. Yuan and Y. Shang, "A Novel Risk-Stratification Models of the High-Flow Nasal Cannula Therapy in COVID-19 Patients With Hypoxemic Respiratory Failure," Frontiers in Medicine, vol. 7, no. 607821,2020

9. A. Chandel, S. Patolia, W. A. Brown, C. A. Collins, D. Sahjwani, V. Khangoora, P. C. Cameron, M. Desai, A. Kasarabada, J. K. Kilcullen, S. D. Nathan and C. S. King, "High-flow nasal cannula in COVID-19: Outcomes of application and examination of the ROX index to predict success," Respiratory Care, 2020.

10. M. Patel, J. Chowdhury, N. Mills, R. Marron, A. Gangemi, Z. Dorey-Stein, I. Yousef, M. Zheng, L. Tragesser, J. Giurintano, R. Gupta, P. Rali, G. D'Alonzo, H. Zhao, N. Patlakh, N. Marchetti, G. J. Criner and M. Gordon, "ROX Index Predicts Intubation in Patients with COVID-19 Pneumonia and Moderate to Severe Hypoxemic Respiratory Failure Receiving High Flow Nasal Therapy," medRxiv, no. 20143867, 2020
000973

Incidence of intracranial hemorrhage in Covid-19

and non-Covid-19 associated ARDS patients on ECMO

K. Stahl1 ${ }^{1}$; B. Seeliger, ${ }^{2} ;$ DA. Hofmaenner, $;$; M. Doebler ${ }^{4}$; PD. Wedel Garcia ${ }^{3}$. HJ. Gillmann"; MM. Hoeper'; C. Bode'; S. David, ${ }^{3}$

${ }^{1}$ Department of gastroenterology, hepatology and endocrinology, Hannover Medical School, Hannover, Germany; ${ }^{2}$ Department of pneumology, Hannover Medical School, Hannover, Germany; ${ }^{3}$ Institute of intensive care medicine, University Hospital of Zürich, Zürich, Switzerland; ${ }^{4}$ Department of anesthesia and intensive care medicine, Universitätsklinik, Bonn, Brunei Darussalam; ${ }^{5}$ Department of anesthesia and intensive care medicine, Medizinische Hochschule Hannover, Hannover, Germany; ${ }^{6}$ Department of respiratory medicine, Hannover Medical School, Hannover, Germany; ${ }^{7}$ Department of anesthesia and intensive care medicine, Universitätsklinik, Bonn, Germany

Correspondence: K. Stahl

Intensive Care Medicine Experimental 2020, 9(1): 000973

Introduction. Coronavirus disease (Covid)-19 shows unique pathophysiological features compared to other respiratory viruses such as a generalized endothelio-/ and coagulopathy that is clinically associated with an extraordinary high rate of thromboembolic events. It has therefore been recommended to use an intensified prophylactic anticoagulation regimen in critical ill Covid-19 patients. It is however unclear if such a strategy might simultaneously leads to increased bleeding complications. We systematically investigated both the rate and the factors associated with intracranial hemorrhage $(\mathrm{ICH})$ during ECMO support in Covid-19 and non-Covid-19 ARDS patients.

Methods. All patients with Covid-19 associated ARDS receiving ECMO support from March 2020 to March 2021 in three tertiary care ECMO referral centers were investigated in this study. All ICH events confirmed on cerebral imaging were recorded. Patients were compared to a historical matched cohort of patients on ECMO support for viral (non-Covid) associated ARDS.

Results. A total of 142 patients receiving ECMO support for Covid-19 and 44 matched patients for viral (non-Covid) associated ARDS were included. Of those, 29/142 (20.4\%) of Covid-19 patients had an ICH event with $9 / 142$ (6.3\%) being fatal. In the matched historic cohort of ECMO patients with viral non-Covid associated ARDS 2/44 (4.5\%) had an ICH events with only $1 / 44(2.3 \%)$ being fatal $(p=0.03)$. Relevant differences were found between the three different centers concerning both frequencies of $\mathrm{ICH}$ as well as anticoagulation strategies.

Conclusion. ICH is an often fatal complication of ECMO support in ARDS patients that we observed $3 \times$ fold more often in severe Covid19 . We hypothesize that this bleeding susceptibility might be a direct consequence of cerebrovascular endothelialitis. Both the associated mortality and the long-term morbidity for thrombosis vs. bleeding highlight the importance of a tailored individualized approach to choose the right degree of anticoagulation in critically ill Covid-19 patients.

\section{5}

Assessment of Three Different Computing Methods of Inspiratory Transpulmonary Pressure in Patients With Multiple Mechanical Problems

K. Inci'; N. Boyacl, ${ }^{1}$; I. Kara ${ }^{1}$; G. Gursel ${ }^{2}$

${ }^{1}$ Critical care fellowship program, Gazi University School of Medi-

cine, Ankara, Turkey; ${ }^{2}$ Department of pulmonary critical care medicine, critical care fellowship program, Gazi University School of Medi-

cine, Ankara, Turkey

Correspondence: G. Gursel

Intensive Care Medicine Experimental 2020, 9(1): 000985

Introduction. While plateau airway pressure alone is an unreliable estimate of lung distension in same pathologies inspiratory transpulmonary pressure(TPP) is an important parameter to reflect lung overdistension in patients with ARDS and there is no concensus about 
which computation method should be used to calculate it. Studies suggest that different formulas may lead different ventilator set ups.

Objectives. The aim of this study is to compare 3 different TPP measurement method in patients with multiple mechanical abnormalities.

Methods. 34 patients were included in this prospective obsevational study. Eosephageal pressure was measured in all patients. Measurements were obtained during volume controlled mechanical ventilation in sedated and paralyzed patients. Patients' diagnosis, comorbidities, respiratory mechanics were recorded. During the study day airway and eosephageal pressures, flow, tidal volume were measured and elastance, elastance derived, directly measured and release derived inspiratory transpulmonary pressures were calculated.

Results. Mean plateau pressure of the patients was $22 \pm 5 \mathrm{cmH} 2 \mathrm{O}$ and mean respiratory system elastance was $36.7 \pm 13 \mathrm{cmH} 2 \mathrm{O} / \mathrm{L}$. We found mean elastance derived TPP $(18 \pm 9 \mathrm{H} 2 \mathrm{O})$ was significantly higher than directly measured $(13 \pm 9 \mathrm{cmH} 2 \mathrm{O})$ and release derived methods $(11 \pm 9 \mathrm{cmH} 2 \mathrm{O})$ in our mixed study population. But there was a good agreement, correlation and there was no bias between the different formulas.

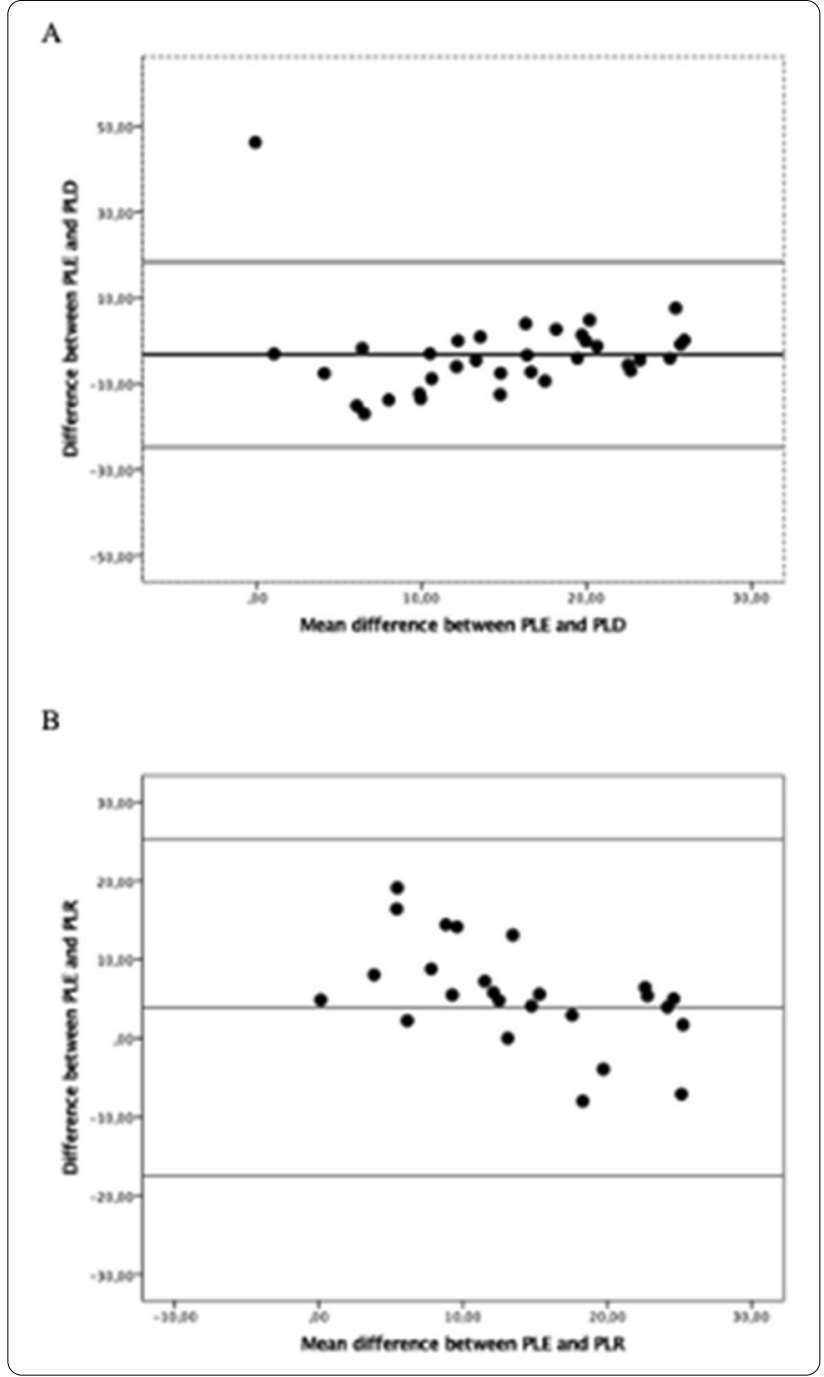

Figure Comparison of different formulas with Blant Altman graphic. Conclusion. Our results suggest that standardization of calculation method of inspiratory TPP is necessary before using it to estimate alveolar overdistension.
000987

Prone position in COVID-19 patients with extracorporeal respiratory support: a subanalysis of the ECMOVIBER registry M. Martinez Martinez'; C. Bonilla'; C. Díaz'; L. Chiscano'; M. Díez, ; C. Maldonado Toral' ; P. Blanco-Schweizer ${ }^{2}$; P. Fortuna, ${ }^{3}$; P. Ricart ${ }^{4}$; P. Ruiz de Gopegui Miguelena ${ }^{5}$; E. Sandoval ${ }^{6}$; J. Duerto-Alvarez, ; V. Gómez ; M. Alfonso-García9' E. Gallart'; J. Riera'

${ }^{1}$ Intensive care, Vall d'Hebron University Hospital, Barcelona, Spain; ${ }^{2}$ Department of intensive care medicine, Hospital Universitario Río Hortega, Valladolid, Spain; ${ }^{3}$ Unidade de urgência médica, Hospital S. José, Centro Hospitalar Universitário Lisboa Central, Lisboa, Portugal; ${ }^{4}$ Intensive care, Hospital Universitari Germans Trias i Pujol, Badalona, Spain; ${ }^{5}$ Intensive care, Hospital Universitario Miguel Servet, Zaragoza, Spain; ${ }^{6} \mathrm{Cardio-}$ vascular surgery, Hospital Clínic de Barcelona, Barcelona, Spain; ${ }^{7}$ Critical care, Hospital Clínico San Carlos, Madrid, Spain; ${ }^{8}$ Intensive care, Hospital Álvaro Cunqueiro, Vigo, Spain; ${ }^{9}$ Intensive care, Hospital Universitario del Vinalopó, Elx, Spain

Correspondence: M. Martinez Martinez

Intensive Care Medicine Experimental 2020, 9(1): 000987

Introduction. Extracorporeal membrane oxygenation (ECMO) is a rescue technique for severe hypoxemia refractory to conventional therapies. The use of prone positioning while on ECMO support is a controversial intervention for improving oxygenation and pulmonary mechanics in this population.

Objectives. To describe the characteristics and outcomes of patients who were placed in the prone position while on ECMO support.

Methods. Observational retrospective analysis of the ECMOVIBER registry, including 25 ECMO centers in Spain (23) and Portugal (2). All adult ( $>18$ years old) COVID-19 patients requiring extracorporeal respiratory support between 1st March and 1st December 2020 were included. End of follow up and recruitment was December 1. Demographic data, comorbidities, and complications during ECMO were recorded. Results are described using mean (standard deviation), median (interquartile range) or frequency (percentage). Comparisons are performed using Chi2 test, Fisher's exact test, T-test or Mann-Whitney $\mathrm{U}$ test as appropriate.

Results. A total of 334 patients [age 53 (10.3) years, $268(80.2 \%)$ male] were included. Information about prone positioning was obtained in 328. Prone positioning had been used in most patients (315 [96\%]) prior to cannulation. Only 43 patients (13.1\%) were placed on prone position while on ECMO support. There were no differences in patient characeristics between this group and the rest of the cohort (Table 1).

Patients placed in prone position during ECMO had a lower PaO2/ $\mathrm{FIO} 2$ at cannulation. Complications during ECMO support were slightly more frequent in this group, with a higher incidence of thrombosis on both the patient and the circuit and a higher incidence of ventilator-associated pneumonia (VAP). Prone positioning while on ECMO was significantly associated with a longer ECMO run duration with no differences on the duration of mechanical ventilation or short and long term survival.

Table 1 Characteristics and outcomeSs

\begin{tabular}{lllll}
\hline & Prone $=43$ & No prone=285 & p-value & N \\
\hline -Age (years) & $58(53.6-60)$ & $54(46-61)$ & 0.103 & 328 \\
-Sex (male) & $39(90.7 \%)$ & $224(78.6 \%)$ & 0.099 & 328 \\
$\begin{array}{l}\text {-Chronic respiratory } \\
\text { disease }\end{array}$ & $5(11.6 \%)$ & $15(5.3 \%)$ & 0.16 & 328 \\
-Ischemic cardiomyo- & $2(4.7 \%)$ & $12(4.2 \%)$ & 1 & 328 \\
$\quad \begin{array}{l}\text { pathy } \\
\text {-Chronic kidney disease }\end{array}$ & $1(2.3 \%)$ & $11(3.9 \%)$ & 0.752 & 327 \\
-BMI (kg/m2) & $30.7(7.4)$ & $30.4(5.9)$ & 0.985 & 327 \\
-P/F ratio pre ECMO & $70(60-79.2)$ & $78(65-91)$ & 0.003 & 328 \\
-Driving pressure pre & $17(4.6)$ & $18.2(4.9)$ & 0.166 & 320 \\
ECMO & & & & \\
\hline
\end{tabular}




\begin{tabular}{lllll}
\hline & Prone=43 & No prone=285 & p-value & N \\
\hline $\begin{array}{l}\text {-Noradrena- } \\
\text { line }>0.1 \mathrm{mcg} / \mathrm{kg} / \mathrm{min}\end{array}$ & $4(9.3 \%)$ & $61(21.5 \%)$ & 0.015 & 327 \\
$\begin{array}{l}\text {-Coinfection at ECMO } \\
\text { initiation }\end{array}$ & $11(25.6 \%)$ & $86(30.2 \%)$ & 0.663 & 328 \\
$\begin{array}{l}\text {-Prone position pre } \\
\text { ECMO }\end{array}$ & $39(90.7 \%)$ & $276(96.8 \%)$ & 0.076 & 328 \\
-MV pre ECMO (days) & $5(3-8)$ & $5(3-9)$ & 0.159 & 328 \\
-Hemorrhagic shock & $7(16.3 \%)$ & $34(12 \%)$ & 0.59 & 326 \\
-Acute kidney injury & $13(31.7 \%)$ & $70(24.8 \%)$ & 0.425 & 323 \\
-VAP & $32(74.4 \%)$ & $127(44.9 \%)$ & 0.001 & 326 \\
-Thrombosis (patient) & $15(34.9 \%)$ & $37(13 \%)$ & 0.039 & 326 \\
-Thrombosis (circuit) & $25(58.1 \%)$ & $91(31.9 \%)$ & 0.002 & 326 \\
-Duration of ECMO & $25.5(16.7)$ & $18.3(17.2)$ & 0.006 & 300 \\
-Duration of MV & $44.9(26.8)$ & $37(31.5)$ & 0.056 & 268 \\
-Status on 1st December & & & 0.214 & 328 \\
Weaned & $17(39.5 \%)$ & $134(47 \%)$ & & \\
Ongoing & $10(23.3 \%)$ & $39(13.7 \%)$ & & \\
Deceased & $16(37.2 \%)$ & $112(39.3 \%)$ & & \\
Six month survival & $10(38.5 \%)$ & $89(44.1 \%)$ & 0.74 & 228 \\
\hline
\end{tabular}

Conclusion. Prone position while on extracorporeal support is not associated with improved outcomes.

\section{Reference(s)}

1. Giani et al. Prone Positioning during Venovenous Extracorporeal Membrane Oxygenation in Acute Respiratory Distress Syndrome. A Multicenter Cohort Study and Propensity-matched Analysis. Ann Am Thorac Soc 2021; 18(3):495-501.

\section{8}

\section{COVID-19 patients on anaesthesia ventilators: a retrospective}

\section{outcome evaluation}

M. Abdelrazik ${ }^{1}$; M. Elhassan ${ }^{1}$; SY. Ong ${ }^{1}$; R. Balasubramaniam ${ }^{1}$; C. Jeronimo ${ }^{1}$ 'Intensive care unit, North Middlesex University Hospital, London, United Kingdom

Correspondence: M. Elhassan

Intensive Care Medicine Experimental 2020, 9(1): 000988

Introduction. The COVID-19 pandemic hit the United Kingdom in late January 2020. By May 1st 2021, there have been 4.41 million confirmed cases and 127,524 deaths among those who tested COVID positive. This is ranked the world's fifth highest death rate and the top country in Europe in respect of death rate related to COVID-19 pneumonia (1). The NHS Hospitals across the UK were overwhelmed by the mounting numbers of COVID- 19 patients which was unprecedentedly consuming the medical resources.

Intensive care units were suffering from shortage of medical equipment including mechanical ventilators which was the main pillar in the treatment of the critically ill COVID-19 patients. This was a challenging situation which forced medical experts to repurpose the anaesthesia ventilators for supporting these patients. Using these ventilators was burdensome for the medical staff in the ICU down to the lack of experience in dealing with these machines for ventilating ICU patients. Anaesthesia machines have comparatively distinctive technical features from the standard ICU ventilators.

Intensive care Unit at North Middlesex University Hospital was one of the earliest centres to be affected by the COVID-19 pandemic. The numbers of COVID-19 patients admitted to the ICU surmounted the capacity of ICU supplies. The ICU ran out of the standard ICU ventilators making it imperative using anaesthesia machines for significant number of our patients. Unfortunately, the literature concerning the use of anaesthesia machines in ICU was scarce aside from some guidelines released by regulatory bodies and the ventilator manufacturers that aimed to safely use these types of ventilators during the pandemic (2). Higher rate of complications and mortality related to anaesthesia ventilators was questionable.

The main purpose of this retrospective study is to evaluate the outcome of patients on anaesthesia ventilators in patients with COVID-19 pneumonia.

Objectives. To determine whether patients with COVID-19 disease fare worse on anaesthesia ventilators compared with COVID-19 patients on standard ICU ventilators.

Methods. ICNARC data after the first UK COVID-19 wave was merged with data from the mechanical ventilators. Only patients with complete data were included. The data of Sixty-seven patients mechanically ventilated -thirty-six on Anaesthesia-Ventilators (AVEN) and thirty-one on standard ICU-Ventilators (IVEN)- were collected and analysed. Descriptive analysis was used to define mean and 95\% Confidence Intervals using bootstrapping. Modelling using ICU outcome as response variable was performed with the lasso regression for variable selection. Variables were identified along defined times in ICU. Those selected variables were included in a logistic regression model.

Results. Patients on AVEN or IVEN neither differ in regard to demographic data (age, sex, ethnicity, BMI, help on daily activities, ICNARC score, APACHE score, of first $24 \mathrm{~h} \mathrm{PaO2} / \mathrm{FiO} 2$ [P/F] ratio), nor on critical care variables (lung compliance, $\mathrm{FiO} 2, \mathrm{PaO} 2$, total PF ratio, $\mathrm{SaO} 2, \mathrm{PaCO} 2$, respiratory rate, tidal volume, peak inspiratory pressure, PEEP, fluid balance and organ support days).There was no difference in mortality between both groups (chi-squared 3.5, $\mathrm{p}=1$ ). $\mathrm{PaCO} 2$ (OR 0.22 [95\% Cl 0.06-0.80]), basic respiratory $(0.52$ [0.28-0.96]) and cardiovascular support (0.88 [0.79-0.99]) were inversely related with mortality. This model predicted mortality regardless of type of mechanical ventilator with an area under the curve (AUC) of 0.94 (0.85-0.97).

Conclusion. Patients with COVID-19 disease mechanically ventilated with anaesthesia ventilators during the first UK wave did not fare worse than patients on standard ICU ventilators. Variables related with ventilation ( $\mathrm{PaCO} 2)$, basic respiratory and cardiovascular support predicted ICU mortality.

\section{Reference(s)}

1. World Health Organization. Available at: https://covid19.who.int/table. Accessed May 1, 2021

2. APSF/ASA guidance on purposing anesthesia machines as ICU ventilators. Available at: https://www.asahq.org/in-the-spotlight/coronaviruscovid-19-information/purposing-anesthesia-machines-for-ventilators. Accessed May 1, 2021

\section{9}

Can we use Adaptive Support Ventilation (ASV) in COVID-19?

R. Knafelj'; M. Noc

${ }^{1}$ Univesity medical center ljubljana, MICU, Ljubljana, Slovenia

Correspondence: R. Knafelj

Intensive Care Medicine Experimental 2020, 9(1): 000989

Introduction. When used appropriately automated ventilation modes reduce workload and enhance patient safety, fasten weaning and increase ventilator free days. Inflow of COVID-19 patients imposed new challenges to technology and human resources. Adaptive Support Ventilation (ASV) is automated mode enabling spontaneous, assisted and spontaneous (supported) breaths under same set of settings (PEEP, $\mathrm{FiO} 2$ and \% of minute ventilation).

Objectives. To assess feasibility of ASV in Covid-19 patients ventilated for acute respiratory insufficiency.

Methods. 25 consecutive patients with COVID-19 referred to tertiary center for advanced ventilatory support and/or rescue therapies were ventilated in ASV mode (C6 Hamilton, Switzerland). All received propofol and fentanyl in continuous infusion targeting RAAS -4. \%MV, PEEP and FiO2 were adjusted by physician targeting physiological acceptable limits $(\mathrm{pH} \geq 7.2, \mathrm{pO} 2 \geq 10 \mathrm{kPa}$ $\mathrm{pCO} 2 \leqslant 8 \mathrm{kPa}$ ). Recruitability was assessed and recruitment 
manoeuvre performed in recruiters (PV tool, C6, Hamilton, Switzerland). In all patients oesophageal manometry via dedicated gastric catheter (Nutrivent, Sidam, Italy) was performed.

Results. In cohort go 25 patients $69 \%$ were male with BMI $29 \pm 5$ and APACHE $17 \pm 5$. Ventilator parameters are presented in Table 1. * IBW $\times 100 \mathrm{~mL} / \mathrm{kg}$, PLPEEP transpulmonary pressure @PEEP, PLplato transpulmonary pressure @plato; $\mathrm{MP}=0.098 \times \mathrm{RR} \times \mathrm{Vt} \times \mathrm{DP}$, $\mathrm{VR}=(\mathrm{MV} \times \mathrm{PaCO} 2) / \mathrm{IBM} \times 100 \times 5 \mathrm{kPa})$.

\begin{tabular}{llll}
\hline & Day 1 & Day 3 & P \\
\hline $\begin{array}{l}\text { \%predictive minute ventilation (L/ } \\
\text { min) }\end{array}$ & $167 \pm 37$ & $143 \pm 61$ & NS \\
PEEP (cmH2O) & $10 \pm 3$ & $12 \pm 3$ & NS \\
FiO2 & $0.8 \pm 0.24$ & $0.55 \pm 0.1$ & $<0.005$ \\
Respiratory Rate (/min) & $25 \pm 6$ & $18 \pm 4$ & 0,024 \\
Vt (mL) & $450 \pm 0.59$ & $448 \pm 0.58$ & NS \\
Vt (mL/kg IBM) & $6.7 \pm 0.8$ & $6.2 \pm 0.5$ & NS \\
Driving Pressure (DP, cmH2O) & $14 \pm 6$ & $11 \pm 4$ & NS \\
Mechanical Power (MP, J/min) & $31 \pm 11$ & $17 \pm 9$ & NS \\
Compliance (mL/cmH2O) & $34 \pm 12$ & $30 \pm 9$ & NS \\
PL PEEP (cmH2O) & $1,5 \pm 0.5$ & $1,7 \pm 0.5$ & NS \\
PL plato (cmH2O) & $9.8 \pm 1.8$ & $11.8 \pm 1.8$ & NS \\
Ventilatory Ratio(VR) & $2.9 \pm 0.7$ & $2.2 \pm 0.3$ & NS \\
pO2 (kPa) & $10.3 \pm 2.8$ & $11.8 \pm 1.2$ & NS \\
pCO2 (kPa) & $6.33 \pm 1.2$ & $6.1 \pm 0.5$ & NS \\
\hline
\end{tabular}

Conclusion. In group of selected COVID19 patients use of ASV proved to be save, effective and feasible. Although relatively high minute ventilation was used, ASV delivered Vt in protective range and no airtrapping was documented.

\section{Reference(s)}

1. Arnal JM et al. Airway and transpulmonary driving pressures and mechanical powers selected by INTELLiVENT-ASV in passive, mechanically ventilated ICU patients. Heart \& Lung (2019).

2. none

\section{7}

\section{Increasing angiopoietin-2 predicts AKI, ARDS and mortality}

\section{in COVID-19 patients}

C. Volleman'; R. Ibelings ${ }^{2}$; APJ. Vlaar'; C. Van Den Brom²;. Amsterdam Umc Covid Biobank ${ }^{3}$

'Department of intensive care medicine, leica laboratory, Amsterdam UMC, location AMC, Amsterdam, Netherlands: ${ }^{2}$ Department of anesthesiology, experimental laboratory for vital signs, Amsterdam UMC, locatie VUmc, Amsterdam, Netherlands: ${ }^{3}$ Amsterdam umc, location AMC, Amsterdam, Netherlands

Correspondence: C. Volleman

Intensive Care Medicine Experimental 2020, 9(1): 000997

Introduction. Endotheliopathy is suggested to be part of the development of AKI and ARDS in COVID-19, probably due to vascular leakage and edema formation $(1,2,3)$. The endothelial angiopoietin/Tie2 system tightly regulates endothelial barrier function, and disturbances in this system can cause vascular leakage $(4,5)$. However, the angiopoietin/Tie2 system has not yet been linked to endothelial barrier function in this patient group.

Objectives. To examine the effect of plasma from COVID-19 patients with mild and severe symptomatology on endothelial permeability and its relation to the angiopoietin/Tie2 system.

Methods. Plasma was received from the Amsterdam UMC COVID Biobank and obtained from COVID-19 patients admitted to the ward ( $n=14$, mild symptoms) or intensive care unit ( $n=20$, severe symptoms) and during week 1 and week 2 of hospital admission. Human kidney and pulmonary endothelial cells were exposed to patient plasma and in vitro endothelial barrier function was assessed using electric cellsubstrate impedance sensing. Circulating levels of angiopoietin-2 were measured by sandwich enzyme-linked immunosorbent assay.

Results. Of 34 patients ( $64.7 \%$ male; age $63 \pm 11$ years old), $32.4 \%$ developed AKI, 58.8\% developed ARDS, and 90-day mortality occurred in $32.4 \%$. Plasma from COVID-19 patients reduced in vitro renal and pulmonary endothelial resistance, whereas no significant differences were found at admission between patients at the ward or at the ICU (renal $0.45[0.44-0.50]$ vs 0.46 [0.43-0.52] $\mathrm{p}=0.944$; pulmonary 0.37 [0.34-0.38] vs $0.38[0.37-0.42] p=0.064)$. Nonetheless, endothelial resistance significantly increased following exposure with plasma from ward patients obtained at week 1 (renal 0.45 [0.44-0.50] vs 0.50 $[0.48-0.69] p=0.039$; pulmonary $0.37[0.34-0.38]$ vs 0.40 [0.37-0.46] $\mathrm{p}=0.001$ ) whereas this increase was only significant after two weeks in patients at the ICU (renal $0.46[0.43-0.52]$ vs $0.77[0.56-0.86]$ $\mathrm{p}<0.001$; pulmonary 0.38 [0.37-0.42] vs 0.72 [0.46-0.93] $\mathrm{p}<0.001$ ). Patients admitted to the ICU showed an increase in circulating angiopoietin-2 levels after one week (3.04 [2.08-4.42] vs 5.58 [3.62-8.23] $\mathrm{ng} / \mathrm{ml}, \mathrm{p}=0.002$ ) whereas levels remained stable in ward patients. The increase in angiopoietin-2 showed good discrimination for the development of AKI (AUC $=0.788,95 \% \mathrm{Cl}=0.584-0.992, \mathrm{p}=0.013)$ and ARDS (AUC $=0.838,95 \% \mathrm{Cl}=0.687-0.989, \mathrm{p}<0.001$ ) and 90-day mortality (AUC $=0.914,95 \% \mathrm{Cl}=0.807-1.000 \mathrm{p}<0.001$ ).

Conclusion. Plasma from COVID-19 patients induced in vitro renal and pulmonary endothelial hyperpermeability. Interestingly, an increase in circulating angiopoietin-2 levels over time is able to predict AKI, ARDS and 90-day mortality with high prognostic accuracy.

\section{Reference(s)}

1. Brindle NPJ, Saharinen P, Alitalo K. Signaling and functions of angiopoietin-1 in vascular protection. Circ Res. 2006;98(8):1014-23.

2. Sack KD, Kellum JA, Parikh SM. The Angiopoietin-Tie2 Pathway in Critical Illness. Crit Care Clin. 2020;36(2):201-16.

3. Libby P, Lüscher T. COVID-19 is, in the end, an endothelial disease. Eur Heart J. 2020;41(32):3038-44.

4. Varga Z, Flammer AJ, Steiger P, Haberecker M, Andermatt R, Zinkernagel AS, et al. Endothelial cell infection and endotheliitis in COVID-19. Vol. 395, Lancet (London, England). 2020. p. 1417-8.

5. Gupta A, Madhavan M V, Sehgal K, Nair N, Mahajan S, Sehrawat TS, et al. Extrapulmonary manifestations of COVID-19. Nat Med. 2020:26(7):1017-32

6. CEvdB is supported by the European Society of Intensive Care Medicine (Levi-Montalcini Award 2017), the Dutch Society of Anesthesiology (Young Investigator Grant 2017) and the Dutch Research Council (Veni 2019).

\section{9}

Evaluation of inspiratory efforts in awake prone positioning in COVID-19 patients during NIV trials

V. Punzi ${ }^{1}$; L. Zacchetti ${ }^{2}$;. Bianchi ${ }^{2}$; F. Raimondi'; S. Cazzaniga ${ }^{3}$; L. Novelli ${ }^{4}$; M. Brivio ${ }^{2}$; L. Grazioli², F. Mojolí, F. Di Marco ${ }^{4}$; FL. Lorini ${ }^{2}$

${ }^{1}$ School of medicine and surgery, University of Milan, Milano, Italy;

${ }^{2}$ Department of critical care and anesthesia, ASST Papa Giovanni

XXIII, Bergamo, Italy; ${ }^{3}$ School of medicine and surgery, Università degli Studi di Milano—Bicocca, Milan, Italy; ${ }^{4}$ Pulmonary medicine unit, ASST Papa Giovanni XXIII, Bergamo, Italy; ${ }^{5}$ Department of clinical-surgical, diagnostic and pediatric sciences, Unit of anaesthesia and intensive care, University of Pavia, Pavia, Italy

Correspondence: $\mathrm{V}$. Punzi

Intensive Care Medicine Experimental 2020, 9(1): 001019

Introduction. Non-invasive ventilation (NIV) preserves respiratory muscle functions, reduces sedation requirements and might prevent endotracheal intubation. However, excessive inspiratory efforts exerted during spontaneous breathing may worsen lung injury (1) due to inhomogeneous lung stress and increased lung oedema, and may lead to delayed intubation and increased mortality. Prone positioning (PP) has been recently proposed in spontaneously breathing 
patients to improve oxygenation (2). Our study explores the hypothesis that prone positioning (PP) may act in synergy with NIV to reduce respiratory efforts and improve gas exchange in patients with Covid19 hypoxemic respiratory failure.

Objectives. To assess the effect of PP on the magnitude of respiratory efforts, measured by esophageal pressure swings ( $\triangle$ Pes) during inspiration, in patients affected by COVID-related pneumonia and undergoing NIV trial.

Methods. In this single-center prospective observational study, we enrolled consecutive patients aged $>18$ years old, affected by Covid19 acute respiratory failure (defined by respiratory rate $(\mathrm{RR}) \geq 25$ breaths/min and oxyhemoglobin saturation $\left(\mathrm{SpO}_{2}\right) \leq 92 \%$ or $\mathrm{PaO}_{2} /$ $\mathrm{FiO}_{2}$ ratio (P/F) $<200$ despite supplemental oxygen) and candidates for receiving NIV trial and prone positioning. At admission to ICU, a multifunctional nasogastric tube (NutriVent) was inserted, and the first NIV trial started. Ventilatory parameters were left unchanged during the period of study. Gas exchange and $\triangle P$ Pes were collected in supine position ( $\mathrm{S} 1$ ), after $2 \mathrm{~h}$ of prone positioning (P) and after $30 \mathrm{~min}$ from resupination (S2). NIV/PP trial was repeated the day after the ICU admission. Sedation was permitted to maintain Richmond AgitationSedation Scale (RASS) equal to zero. PP was considered feasible if tolerated for at least $2 \mathrm{~h}$. Data are presented as median and interquartile range (IQR). One way analysis of variance for non-parametric repeated measurements (Kruskal Wallis) with a post hoc Tukey's correction was used to compare data.

Results. Patients' characteristics and mechanical support parameters during NIV are summarized in Tables 1 and 2. Ten out of the eleven patients enrolled were able to perform both NIV/PP trials; one patient was intubated before the second trial. Only in one case the NIV/PP trial was interrupted before two-hours, due to intolerance to PP. $\triangle \mathrm{Pes}$ decreased significantly from $\mathrm{S} 1$ to $\mathrm{P}(5.2[3-6.8]$ vs $2.7[2-4.1] \mathrm{cmH} 2 \mathrm{O}$, $\mathrm{p}<0.05$ ]; similarly, $\mathrm{P} / \mathrm{F}$ increased from $\mathrm{S} 1$ to $\mathrm{P}(177$ [133-233] vs 280 [215-361], $\mathrm{p}<0.05)]$. Both values returned closer to the first NIV measurement after resupination ( $\triangle$ Pes 3.9 [2.8-4.7] cmH2O, P/F 217 [146244]) (Fig. 1). No significant differences were found in neither RR (19 [17-22], 17 [14-21], 20 [17-22] breaths per minute) nor tidal volume (7.7 [6.9-8.2], 7.8 [7-8.5], 7.3 [6.8-8.1] mL per kilogram of predicted body weight) in S1, P, S2, respectively. No major complications were recorded.

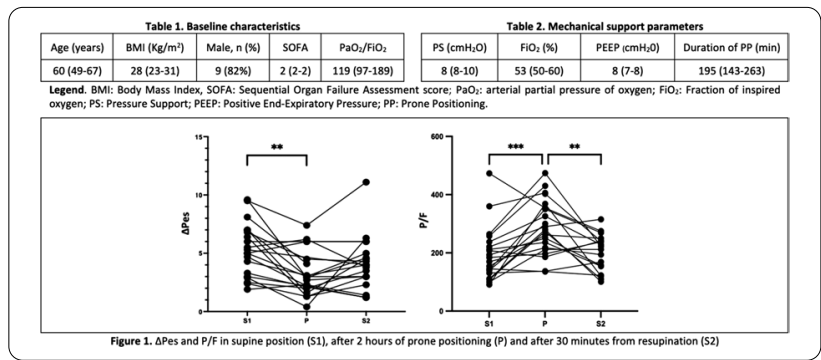

Conclusion. Prone position during spontaneous breathing is feasible and associated with a significant reduction inspiratory efforts and improvement in oxygenation, which cannot be explained only by the application of NIV.

\section{Reference(s)}

1. Coppo A et al. Feasibility and physiological effects of prone positioning in non-intubated patients with acute respiratory failure due to COVID-19 (PRON-COVID): a prospective cohort study. Lancet Respir Med 2020;8: 765-74

2. Brochard L, Slutsky A, Pesenti A. Mechanical Ventilation to Minimize Progression of Lung Injury in Acute Respiratory Failure. Am J Respir Crit Care Med. 2017;195(4):438-442
001020

Differences in the emotional state in COVID-19 and non-COVID-19 ICU survivors at early phases of the post-ICU recovery

N. Miguel Rebanal'; G. Gemma ${ }^{2}$; C. De Haro ${ }^{2}$; C. Espinal Sacristan ${ }^{3}$;

C. Fortià Palahi ${ }^{3}$; N. Ridao Sais ${ }^{4}$ j. López-Aguilar²; M. Godoy Gonzalez;

L. Oliveras Furriols, ${ }^{4}$; C. Subirà, ; M. Jodar, ; R. Fernandez, ; A. Ochagavía²; L. Blanch²; S. Fernández-Gonzalo

${ }^{1}$ Critical care center, Hospital Universitari Parc Taulí, Institut d'Investigació i Innovació (I3PT), UB, Sabadell, Spain; ${ }^{2}$ Critical care center, Institut d'Investigació i Innovació (I3PT), Hospital Parc Taulí de Sabadell, UAB, CIBERES, Sabadell, Spain; ${ }^{3}$ Critical care center, Hospital Universitari Parc Taulí, Institut d'Investigació i Innovació (I3PT), UAB, Sabadell, Spain; ${ }^{4}$ Rehabilitation medicine, Parc Taulí Hospital Universitari, Institut d'Investigació i Innovació Parc Taulí I3PT, UAB, Sabadell, Spain; ${ }^{5}$ Critical care center, Fundació Althaia, Xarxa Assistencial Universitària de Manresa, CIBERES, Manresa, Spain, Manresa, Spain; ${ }^{6}$ Neurology department, Parc Taulí Hospital Universitari, Institut d'Investigació i Innovació I3PT, UAB, CIBERES, Sabadell, Spain; ${ }^{7}$ Critical care center, Parc Taulí Hospital Universitari, Institut d'Investigació i Innovació I3PT, UAB, CIBERSAM, Sabadell, Spain

Correspondence: N. Miguel Rebanal

Intensive Care Medicine Experimental 2020, 9(1): 001020

Introduction. Post-Intensive Care Syndrome (PICS) refers to a set of physical, cognitive and emotional impairments that persists after hospital discharge (1) and has a high impact on the survivors' quality of life (QoL) (2-4). The emotional state is one of the most affected domains after ICU stay. The prevalence for anxiety and depression is estimated at $16-62 \%$ (5). Moreover, depressive symptomatology has been related to higher mortality in the next 2 years after ICU discharge (6). ICU survivors with Acute Respiratory Distress Syndrome (ARDS) and those undergoing invasive mechanical ventilation (IMV) are especially vulnerable to develop PICS (6). Most of the critically ill COVID19 patients present both conditions, that added to the adverse conditions of the COVID19 hospital emergency situation (isolation during admission, impossibility for applying conventional protocols for sedation/mobilization during ICU, etc.) may increase the level of emotional distress in the COVID-19 ICU survivors.

Objectives. 1) To compare the emotional state (anxious, depressive and post-traumatic stress symptomatology) between two cohorts of ICU survivors, with and without COVID-19, 1 month after ICU discharge. 2) To explore the relationship between the emotional state and the QoL (physical and mental health) in the initial phase of the post-ICU recovery.

Methods. A pre-pandemic cohort of ICU survivors undergoing IMV was compared with a cohort of COVID-19 ICU survivors. The emotional state was assessed 1 month after ICU discharge using the following scales: Hospital Anxiety and Depression Scale (HADS) and Davidson Trauma Scale (DTS). QoL was assessed using the Short Form Health Survey SF-12 (Physical health and Mental Health indexes). Mann Whitney $U$ tests and Spearman correlations were used.

Results. Non-COVID-19 ICU IMV survivors cohort: 40 patients, of which $50 \%$ female, mean age $63.16 \pm 15.16$ years old and mean duration of IMV of $13.3 \pm 17.9$ days. COVID-19 ICU survivors cohort: 73 patients of whom $38.4 \%$ female, mean age $58.29 \pm 11.22$ years old and with a mean duration of IMV of $17.85 \pm 12.24$ days. $47 \%$ of ICU survivors in the COVID-19 cohort did not receive IMV.

Differences in the emotional state are shown in Fig. 1. COVID-19 ICU survivors with and without IMV showed significantly higher levels of anxiety $(p=0.025$ and $p=0.04$, respectively) and PTSD symptoms $(p<0.001 ; p=0.04)$ than non-COVID-19 ICU survivors. No differences were observed regarding depression. 


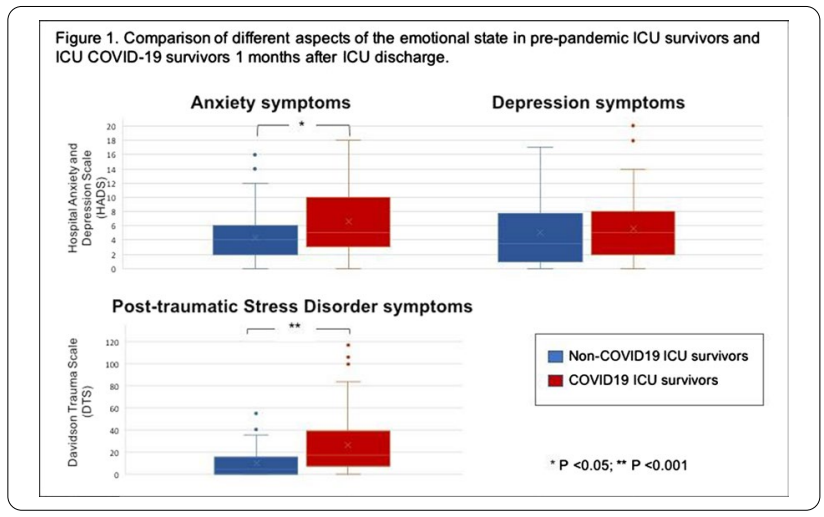

QoL-Physical health index was statistically related to the depressive symptoms in non-COVID19 survivors $(r s=-0.354 ; p=0.025)$, while it was related to PTSD symptoms ( $r s=-0.296 ; p=0.013$ ) in COVID19 survivors. QoL-Mental health index was statistically related to the anxiety, depression and PTSD symptomatology of both non-COVID19 ( $r s \leq-0.513$; all $p \leq 0.001$ ) and COVID19 ICU survivors ( $r s \leq-0.47$; all $\mathrm{p}<0.001)$.

Conclusion. COVID19 ICU survivors manifested higher anxiety and PTSD symptoms than non-COVID19 ICU survivors. No differences were found in depressive symptomatology. Emotional state was not related to IMV. The emotional state in the early post-ICU recovery phase was associated with a worst physical and mental health QoL.

\section{Reference(s)}

1. Herridge MS, Moss M, Hough CL, Hopkins RO, Rice TW, Bienvenu OJ, Azoulay E. Recovery and outcomes after the acute respiratory distress syndrome (ARDS) in patients and their family caregivers. Intensive Care Med. 2016 May;42(5):725-738. https://doi.org/10.1007/s00134-016-43218. Epub 2016 Mar 30. PMID: 27,025,938

2. Hatch, R, Young, D, Barber, V, Griffiths, J, Harrison, DA, \& Watkinson, P. (2018, 23 noviembre). Anxiety, Depression and Post Traumatic Stress Disorder after critical illness: a UK-wide prospective cohort study. Critical Care, 22(310). https://doi.org/10.1186/s13054-018-2223-6.

3. LaBuzzeta, JN, Rosand, J, \& Vranceanu, AM. (2020). Review: Post-Intensive Care Syndrome: Unique Challenges in the Neurointensive Care Unit. Neurocrit Care, 31(3). https://doi.org/10.1007/s12028-019-00826-0

4. Hopkins, RO, Weaver, LK, Collingridge, D. et al. (2005). Two years cognitive, emotional, and quality of life outcomes in acute respiratory distress syndrome. Am J RespirCrit Care Med, 171, 340-47.

5. Hopkins RO, Herridge MS. Quality of life, emotional abnormalities, and cognitive dysfunction in survivors of acute lung injury/acute respiratory distress syndrome. Clin Chest Med. 2006;27:679-89.

6. Mikkelsen ME, Shull WH, Biester RC, Taichman DB, Lynch S, Demissie E, Hansen-Flaschen J, Christie JD. Cognitive, mood and quality of life impairments in a select population of ARDS survivors. Respirology. 2009 Jan;14(1):76-82. https://doi.org/10.1111/j.1440-1843.2008.01419.x. PMID: $19,144,052$.

7. Needham DM, Davidson J, Cohen H, Hopkins RO, Weinert C, Wunsch $\mathrm{H}$, et al. Improving long-term outcomes after discharge from intensive care unit: report from a stakeholders' conference. Crit Care Med. 2012 Feb;40(2):502-9. https://doi.org/10.1097/CCM.0b013e318232da75. PMID: $21,946,660$

8. These results are linked to the PICS-COVID19 project (COV20/00595), funded by the Extraordinary call for the SARS-COV-2 and COVID-19 disease by the Instituto de Salud Carlos III, Ministry of Health of the Spanish government.
001044

Ventilator Associated Pneumonia and Complementary

Diagnosing Tools Among Patients With and Without Covid-19: 2020 Study at a Portuguese ICU

S. Duarte'; M. Varela'; A. Krystopchuk'2; J. Moreno, ${ }^{3}$; D. Nuñez, ${ }^{4}$

${ }^{1}$ Intensive care medicine 1, University Hospital Center of Algarve, Faro, Faro, Portugal; ${ }^{2}$ Emergency and intensive care medicine department, Algarve University Hospital Centre, Faro, Portugal; ${ }^{3}$ Intensive care departement, Centro Hospitalar e Universitário do Algarve, Faro, Portugal, Portugal; ${ }^{4}$ Intensive care unit, Centro Hospitalar Universitário do Algarve-Faro, Faro, Portugal

Correspondence: S. Duarte

Intensive Care Medicine Experimental 2020, 9(1): 001044

Introduction. Ventilator-associated pneumonia (VAP) is the most common infection acquired in the intensive care unit (ICU). To date, there is no diagnostic gold standard for VAP. Some guidelines suggest the use of semiquantitative cultures. Also, community acquired pneumonia are still a diagnosis of admission in ICU patients, together with complicated nosocomial pneumonia.

Methods. We performed a retrospective observational study, where we compared the incidence of VAP among covid and non-covid patients in our unit, the methods of diagnosis and the incidence of positive semiquantitative cultures. We also compared the patients admitted due to community acquired pneumonia or hopital associated. The study involved 81 COVID-19 and 32 non-COVID-19 patients receiving invasive ventilation in a single University teaching hospital between January 1st 2020 and December 31th 2020.

Results. From the total of 113 ventilated patients (covid-19 and noncovid-19) diagnosed with pneumonia, 10 of them had more tha one diagnosis of pneumonia during the stay in the ICU. 88 were nosocomial pneumonias, in which, 68 VAP (40 early onset and 28 late onset) and 20 HAP. The remaining, 22 were due to aspiration and 13 community acquired. From all the VAP diagnosis, early onset VAP, 53.6\% were diagnosed on the 2 nd day of admission, $35.7 \%$ on the $3 \mathrm{rd}$, and $10.7 \%$ on the 4th day. Regarding the late onset VAP, $53.84 \%$ diagnosed between the 5 th and 7 th days, and $46.16 \%$ between the 8 th and 15th days of admission.From 32 covid-19 patients only $37,5 \%$ had positive bronchial secretions, compared to $39,5 \%$ non-covid- 19 patients.Regarding the thorax CT-scan, non-covid-19 patients showed higher incidence of imaging characteristics of pneumonia than covid19 patients. And $41 \%$ didn't perform thorax CT-scan.

Conclusion. We observed that Covid-19 patients had less VAP and less positive cultures, probably associated with the beginning of antimicrobials before ICU admission. There is a high rate of CT-scan that wasn't performed, and the assumption of pneumonia was only through thorax X-Ray imaging, this is due to the difficulty in moving ventilated patients to and from the CT-scan room, which brings us the need for a more quicker, easier, and bedside way to help in the diagnosis of pneumonia in this setting, for example, pulmonary echography.

\section{Reference(s)}

1. Torres A, Niederman MS, Chastre J, et al. International ERS/ESICM/ ESCMID/ALAT guidelines for the management of hospital-acquired pneumonia and ventilator-associated pneumonia: Guidelines for the management of hospital-acquired pneumonia (HAP)/ventilatorassociated pneumonia (VAP) of the European Respiratory Society (ERS), European Society of Intensive Care Medicine (ESICM), European Society of Clinical Microbiology and Infectious Diseases (ESCMID) and Asociación Latinoamericana del Tórax (ALAT). Eur Respir J. 2017;50(3):1,700,582

2. Garcia-Vidal C, Sanjuan G, Moreno-García E, et al. Incidence of coinfections and superinfections in hospitalized patients with COVID-19: a retrospective cohort study. Clin Microbiol Infect. 2020;27:83-8

3. Vincent J-L, Sakr Y, Singer M, et al. Prevalence and outcomes of infection among patients in intensive care units in 2017. JAMA. 2020;323:1478-510

4. None 


\section{9}

Efficiency of non-invasive ventilation in acute severe asthma

M. kallel'; A. Jamoussi'; S. AYED ${ }^{1}$; E. Rachdi' ${ }^{1}$; F. JARRAYA ${ }^{1}$; H. Maamouri';

T. Merhebene ${ }^{1}$; M. Besbes ${ }^{1}$; J. Ben Khelil ${ }^{1}$

'Medical icu, Hôpital Abderrahmen Mami de pneumo-phtisiologie, Ari-

ana, Tunisia

Correspondence: A. Jamoussi

Intensive Care Medicine Experimental 2020, 9(1): 001049

Introduction. Noninvasive ventilation (NIV) has been shown to be effective in severe exacerbations of chronic obstructive pulmonary disease and acute pulmonary edema. Its role in severe acute asthma nevertheless remains uncertain.

Objectives. Our study aimed to evaluate the efficiency of NIV in severe acute asthma particularly concerning the need of endotracheal intubation (ETI).

Methods. We conducted a retrospective cohort study in intensive care unit (ICU) between September 1989 and December 2017 including all asthmatic patients with severe acute asthma. Two groups of patients were identified depending on the use of NIV on admission: a NIV + group and a NIV- group. These two groups were matched according to six criteria: i) age, gender, severity of asthma, $\mathrm{PaCO} 2$ on admission, ii) a clinical score and the Salmeron score on admission. The data and clinical outcomes for these patients were retrieved and compared with a multivariate analysis.

Results. The study included 354 patients with 177 events in each group. Demographic characteristics, vital signs, $\mathrm{PaO} 2$ and $\mathrm{PaCO} 2$ at baseline were similar for both groups. The comparison of clinical and blood gas parameters recorded during the first $24 \mathrm{~h}$ had showed an improvement in both groups without significant difference except for the $\mathrm{PaO} 2$ / FiO2 ratio (The mean of $\mathrm{PaO} 2$ / $\mathrm{FiO} 2$ ratio at $\mathrm{H} 24$ was $280.7 \pm 110.5 \mathrm{~mm} \mathrm{Hg}$ in the NIV + group against $238 \pm 107.7 \mathrm{~mm} \mathrm{Hg}$ in the NIV-group).

The application of NIV combined to medical treatment had significantly decreased the need of ETI $(O R=0.188[0.064-0.55](p=0.005)$. In fact, only 17 patients in the NIV + group (9.6\%) against 38 patients in the NIV- group (21.5\%) had been intubated. Analysis of independent predictors of ETI for NIV failure was a value of $\mathrm{PaCO} 2 \geq 47.9 \mathrm{mmHg}$ at the first hour and an IGSII score $\geq 26$.

Complications were observed in 75 patients (21.2\%). Although the incidence of these complications was similar in both groups, hemodynamic complications were significantly less frequent in NIV + group [5 cases $(2.8 \%)$ versus $18(10.2 \%)]$.

Finally, the use of NIV in severe acute asthma had no impact on mortality or length of stay.

Conclusion. Cautious and monitored use of NIV during severe acute asthma decreases the need of ETI and can probably accelerate the improvement of asthmatic patients in the ICU.

\section{7}

\section{Sensitivity or specificity for a predictor? Diaphragmatic} ultrasound predictors of failure with high-flow nasal cannula as ventilatory therapy in patients with respiratory failure secondary to SARS-CoV-2 pneumonia

M. Bruna'; G. Hidalgo, ${ }_{1}$; S. Castañeda, ; M. Galvez, ; D. Bravo, ${ }_{1}^{1}$; R. Benitez, ${ }^{1}$; R. Tobar, ${ }^{1}$ J. Quevedo, ${ }_{1}^{1}$ JM. Rodriguez, ${ }^{1}$; C. Murua ${ }^{1}$; R. Madariaga ${ }_{1}^{1}$;

C. Benavides, ; M. Huilcaman, ${ }^{1}$; F. Martinez, ${ }^{2}$

${ }^{1}$ Critical care unit, Hospital Gustavo Fricke, Viña del Mar, Chile; ${ }^{2}$ Critical care unit, Hospital Naval Almirante Nef, Viña del Mar, Chile Correspondence: M. Bruna

Intensive Care Medicine Experimental 2020, 9(1): 001057

Introduction. High Flow Nasal Cannula (HFNC) is a therapy frequently used in critically ill patients with respiratory failure. However, prior to the onset of the SARS-Cov2 pandemic, there was limited literature available regarding predictors that would allow to establish subgroups of patients at risk of therapeutic failure.
Objectives. The primary objective was to assess diaphragmatic ultrasound criteria as predictors of failure to this therapy. The secondary objective was to evaluate clinical variables as predictors of failure. Methods. Prospective cohort study including patients over 18 years old who were consecutively admitted to the Critical Care Unit of Gustavo Fricke Hospital ( Viña del mar, Chile), from July 24 to October 20 2020 with respiratory failure secondary to SARS-CoV-2 pneumonia confirmed by RT-PCR or suspected; and in need of ventilatory support with HFNC.

Baseline measurements of arterial blood gas, modified BORG dyspnea scales, respiratory rate and use of accessory muscles, were made prior to the start of therapy.

Subsequently, measurements of the following variables were performed after $12 \mathrm{~h}$ and daily: ROX index, excursion and diaphragmatic contraction speed (diaphragmatic excursion / inspiratory time) by ultrasound both in supine and prone position.

The end of follow-up was determined by any of the following situations: weaning from HFNC, intubation, or death.

Demographic data such as age, sex, comorbidities, APACHE and SOFA on admission, time of disease evolution, and laboratory tests were also analyzed.

Results. A total of 61 patients were admitted, 21 of them were excluded due to various criteria. Of the remaining 40 , an analysis of the first 29 patients was performed.

$68.9 \%$ were men, the mean age was $60.1 \pm 15.1$ years, and $73.9 \%$ had comorbidities, with cardiovascular disease being the most prevalent.

The supine diaphragmatic contraction speed evaluated after $12 \mathrm{~h}$, obtained good performance as a predictor of failure, (AUC $=0.83 ; 95 \%$ $\mathrm{Cl} 0.63-1.0)$. A value above $1.49 \mathrm{~cm} / \mathrm{sec}$, had a $100 \%$ sensitivity and $58.3 \%$ specificity to detect patients who required intubation $(p=0.03)$. The supine ROX index measured after $12 \mathrm{~h}$ presented adequate performance (AUC $=0.95 ; 95 \% \mathrm{Cl} 0.86-1.0$ ). A value lower than 7.9 showed a sensitivity of $90.9 \%$ and a specificity of $100 \%$ to predict failure with HFNC $(p=0.02)$.

Conclusion. As in other etiologies of respiratory failure, in patients with SARS-CoV2 infection, the ROX index was an effective tool for predicting HFNC failure. Measurement of diaphragmatic contraction speed by ultrasound emerges as a novel diagnostic complement.

\section{Reference(s)}

1. Mauri T, Turrini C, Eronia N, Grasselli G, Volta CA, Bellani G, et al. Physiologic effects of high-flow nasal cannula in acute hypoxemic respiratory failure. Am J Respir Crit Care Med 2017;195:1207-1215

2. Frat J-P, Thille AW, Mercat A, Girault C, Ragot S, Perbet S, et al.; FLORALI Study Group; REVA Network. High-flow oxygen through nasal cannula in acute hypoxemic respiratory failure. N Engl J Med 2015;372:2185-2196

3. Alain Boussuges,Sarah Rives,Julie Finance, Fabienne Brégeon. Assessment of diaphragmatic function by ultrasonography: Current approach and perspectives. World J Clin Cases 2020. June 26; 8(12): 2408-2424

4. Alain Boussuges, MD, PhD; Yoann Gole, MSc; and Philippe Blanc, MD. Diaphragmatic Motion Studied byM-Mode Ultrasonography.Methods, Reproducibility, and Normal Values. CHEST 2009; 135:391-400

\section{8}

An in vitro appraisal of a novel catheter for esophageal pressure monitoring in comparison with commercially available catheters SM. Colombo ; ; G. Abbate ; L. Keibun, ${ }^{1}$; C. Semenzin, ; N. Sato, ${ }^{1}$; C. Ainola, ${ }_{1}^{1}$ N. Obonyo ; ; J. Suen, ; J. Pauls, ; J. Fraser'; ; G. Li Bassi ${ }^{1}$

${ }^{1}$ Critical care research group, The Prince Charles Hospital, Chermside, Australia

Correspondence: S.M. Colombo

Intensive Care Medicine Experimental 2020, 9(1): 001068

Introduction. Naso-gastric catheters (NG-C), comprising esophageal balloons for estimation of pleural pressure and transpulmonary pressure are becoming essential tools in the management of mechanically ventilated patients.\# A novel NG-C has been recently developed to monitor esophageal and gastric pressure and avoid gastro-esophageal 
reflux. Herein, we comprehensively test its esophageal balloon vs. commercially available alternatives.

Methods. We tested two novel NG-Cs (Aspisafe NG and NG+, NY, $\mathrm{NY}$ ), in comparison with 5 commercially-available alternatives. Balloon elastance was computed in triplicate, through inflation of air up to $40 \mathrm{cmH} 2 \mathrm{O}$. We developed a novel in-vitro model comprising pig's esophagus, mounted into a pressurized, temperature-controlled plastic box. The transesophageal pressure was computed in triplicate, as the difference between the NG-C esophageal balloon internal pressure and the box pressure, upon various inflation volumes (up to 30 $\mathrm{cmH} 2 \mathrm{O})$ and 9 box pressures $(-20-+20 \mathrm{~cm} \mathrm{H} 2 \mathrm{O})$. We defined range of accuracy as transesophageal pressure of $0 \pm 1 \mathrm{~cm} \mathrm{H} 2 \mathrm{O}$. Continuous variables were described as median (IQR). Categorical variables were described as percentages. Kruskal-Wallis test was applied for comparisons among catheters.

Results. Esophageal balloon elastance differed among catheters $(\mathrm{N}: 21, \mathrm{p}<0.0001)$. In particular, the Cooper ${ }^{\circledR}$ presented the highest median elastance, $15.7 \mathrm{cmH} 2 \mathrm{O} / \mathrm{mL}$ (IQR 8.3-24.8), while the Aspisafe NG had the lowest $1.9 \mathrm{cmH} 2 \mathrm{O} / \mathrm{mL}$ (IQR $1.1-2.6$ ). Figure 1 shows the transesophageal pressure, which differs among NG-C types $(\mathrm{N}=2032$, $\mathrm{p}<0.001$ ). In addition, when recorded transesophageal pressure measurements were in the range of accuracy, the inflation volume varied among NG-C types $(\mathrm{N}=422, \mathrm{p}<0.001)$. Upon negative box internal pressure, measurements were accurate in only $17.2 \%$ of the cases; conversely, when the box internal pressure was positive, accurate measurements occurred in $24.6 \%$ of the cases.

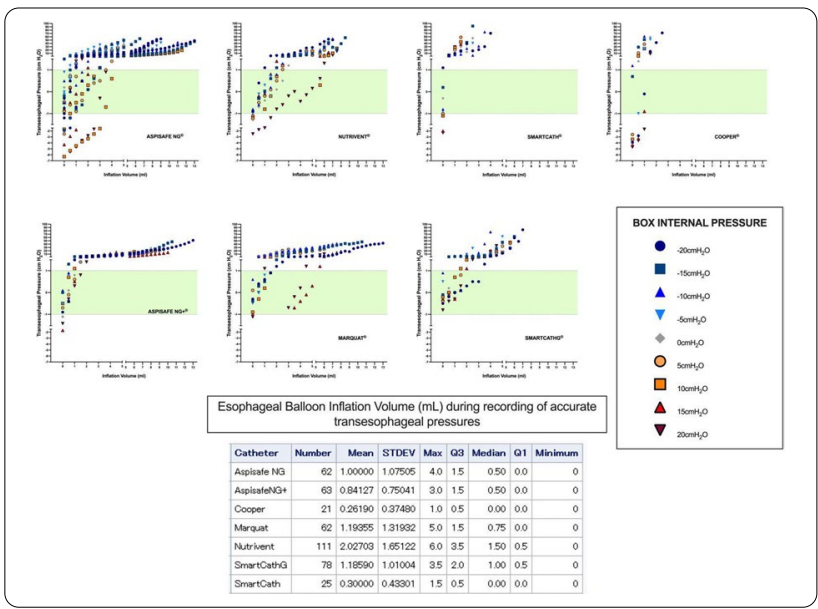

Conclusion. We characterized a novel NG-C comprising esophageal pressure monitoring. In comparison with commercially available $\mathrm{NG}-\mathrm{Cs}$, the novel esophageal balloon presents relatively low elastance and comparable accuracy in transesophageal pressure measurement. Of note, when box pressure was set to negative values, the accuracy of all tested esophageal balloons was poor.

\section{Reference(s)}

1. \# Mauri T., et al. Esophageal and transpulmonary pressure in the clinical setting: meaning, usefulness and perspectives. Intensive Care Med. 2016 Sep;42(9):1360-73. https://doi.org/10.1007/s00134-016-4400-x.

\section{1}

Study protocol for the Oral Hygiene in Critically ill Patients (OHCP) trial: a randomized controlled trial of alternatives to oral chlorhexidine in ventilator-associated pneumonia prevention bundles

PC. Vieira ${ }^{1}$; M. Bernardino Neto ${ }^{1}$; RB. De Oliveira ${ }^{2}$; MF. Mendonça ${ }^{2}$ ${ }^{1}$ Programa de pós-graduação em ciências da saúde, Faculdade de Medicina da Universidade Federal de Uberlândia, Uberlândia, Brazil; ${ }^{2}$ Unidade de terapia intensiva, Hospital das Clínicas da Universidade Federal de Uberlândia, Uberlândia, Brazil

Correspondence: P.C. Vieira

Intensive Care Medicine Experimental 2020, 9(1): 001071

Introduction. Oral hygiene of critically ill patients on mechanical ventilation is an important strategy for the prevention of ventilator-associated pneumonia (VAP) and many intensive care societies and institutions still recommend that it should be performed with chlorhexidine digluconate, given its possible benefits [1]. However, meta-analyzes of double-blind randomized trials reported increased mortality with the use of oral chlorhexidine $[2,3]$ and no reduction in VAP rates $[4,5]$. Therefore, once there are safety concerns, the routine application of this substance, in all patients of a general Intensive Care Unit (ICU), requires further evaluation [6-8]. The mechanism that could explain this increase in mortality is speculated to be a possible direct pulmonary toxicity after chlorhexidine aspiration, causing lung injury and acute respiratory distress syndrome (ARDS) $[5,8]$. Recently, the Ministry of Health's Advisory Council on the Critical Patient Safety Project, in collaboration with the Spanish Society of Intensive and Critical Medicine and Coronary Units, made an addendum to the Pneumonia Zero Project, modifying the recommendation of oral care with chlorhexidine, in ventilated patients, from mandatory to not mandatory [9]. Additionally, the European Respiratory Society, European Society of Intensive Care Medicine, European Society of Clinical Microbiology and Infectious Diseases and Asociación Latinoamericana del Tórax, have decided not to issue a recommendation on the use of oral chlorhexidine, in their last VAP prevention international guidelines, until more safety data becomes available [10].

Methods. The OHCP trial (ICTRP: RBR-7p6568) is an ongoing doubleblind, randomized trial, that evaluates the use of oral chlorhexidine in terms of efficacy and safety and possible alternatives. 150 adult patients admitted in the ICU will be recruited. Eligible and consenting patients will be randomly allocated to three different groups: (1) oral hygiene with chlorhexidine; (2) cetylpyridinium; (3) sterile water. Included patients must be tested for COVID-19 and positive cases excluded. To make the measures as unbiased as possible, the observer, trial statistician, participants and executers of the procedure are blinded to the intervention. The experimental protocols have been approved by local ethics committee and conform to the CONSORT guidelines. Recruitment is intended to be complete up to 12 months. Multiple regression statistical analysis, structural equation modeling and the statistical package Bioestat 5.3 or similar will be used to analyze the collected data.

Results. The primary endpoint is prevention of VAP. Secondary outcome measures are occurrence of adverse effects, ARDS and mortality. Principal analysis is which of the substances is more effective in preventing VAP and safer.

Conclusion. This trial assesses the association between oral care, in critically ill ventilated patients, with different antiseptics and rates of VAP, ARDS and mortality. Perhaps its results fill gaps in knowledge.

\section{Reference(s)}

1. Klompas M, Li L, Kleinman K, Szumita PM, Massaro AF. Associations between ventilator bundle components and outcomes. JAMA Intern Med. 2016;176:1277-83, http://dx.doi.org/10.1001/jamainternmed.2016. 2427

2. Bouadma L, Karpanen T, Elliott T. Chlorhexidine use in adult patients on ICU. Intensive Care Med. 2018;44:2232-4, http://dx.doi.org/10.1007/ s00134-018-5137-5.20

3. Cantón-Bulnes ML, Garnacho-Montero J. Antisepsia orofaríngea en el paciente crítico y en el paciente sometido a ventilación mecánica. Med Intensiva. 2019;43(S1):23-30, http://dx.doi.org/10.1016/j.medin.2018.06. 011.52

4. Gobierno de España. Seguridad del Paciente. Proyecto Neumonía Zero. En colaboración con la Sociedad Española de Medicina Intensiva, Crítica y Unidades Coronarias (SEMICYUC) y la Sociedad Española de Enfermería Intensiva y Unidades Coronarias (SEEIUC). Declaración del Consejo Asesor del Ministerio de Sanidad de los Proyecto de Seguridad en Pacientes Críticos sobre el lavado con clorhexidina em pacientes ventilados; 2018. https://www.seguridaddelpaciente.es/resources/documentos/2018/07/ 
declaracion-del-consejo-sobre-el-lavado-oral-con-clorhexidina-en-pacie ntes-ventilados.pdf [accessed 10 August 2020]

5. Torres A, Niederman MS, Chastre J, Ewig S, Fernandez-Vandellos P, Hanberger $\mathrm{H}$, et al. International ERS/ESICM/ESCMID/ALAT guidelines for the management of hospital-acquired pneumonia and ventilator-associated pneumonia: Guidelines for the management of hospital-acquired pneumonia (HAP)/ventilator-associated pneumonia (VAP) of the European Respiratory Society (ERS), European Society of Intensive Care Medicine (ESICM). European Society of Clinical Microbiology and Infectious Diseases (ESCMID) and Asociación Latinoamericana del Tórax (ALAT). Eur Respir J. 2017;50, http://dx.doi.org/10.1183/13993003.00582-2017

6. We acknowledge the important contributions from the Uberlandia Clinical Hospital and the Health Science pHD Program of Uberlandia Federal University. In addition, we thank the participating ICU staff, patients and families.

7. Vieira PC, de Oliveira RB, da Silva Mendonça TM. Should oral chlorhexidine remain in ventilator-associated pneumonia prevention bundles? Med Intensiva. 2020. https://doi.org/10.1016/j.medin.2020.09.009

8. Klompas M, Speck K, Howell MD, Greene LR, Berenholtz SM. Reappraisal of routine oral care with chlorhexidine gluconate for patients receiving mechanical ventilation: systematic review and meta-analysis. JAMA Intern Med. 2014;174:751-61, http://dx.doi.org/10.1001/jamainternmed.2014. 359

9. Price R, MacLennan G, Glen J. Selective digestive or oropharyngeal decontamination and topical oropharyngeal chlorhexidine for prevention of death in general intensive care: systematic review and network meta-analysis. BMJ. 2014;348:g2197, http://dx.doi.org/10.1136/bmj. g2197.17

10. Deschepper M, Waegeman W, Eeckloo K, Vogelaers D,Blot S. Effects of chlorhexidine gluconate oral care on hospital mortality: a hospital-wide, observational cohort study. Intensive Care Med. 2018;44:1017-26, http:// dx.doi.org/10.1007/s00134-018-5171-3

11. Ricard JD, Lisboa T. Caution for chlorhexidine gluconate use for oral care: insufficient data. Intensive Care Med. 2018;44:1162-4, http://dx.doi.org/ 10.1007/s00134-018-5217-6

\section{3}

Epidemiology and pronostic factors of elderly Covid-19 patients in intensive care unit: analysis of 71 patients

G. MORATELLI ${ }^{1}$; K. Regaieg ${ }^{1}$; A. Al Harach ${ }^{1}$; O. Remets ${ }^{1}$;

D. Goldgran-Toledano ${ }^{1}$

${ }^{1}$ Médecine intensive et réanimation, Intercommunal Hospital Group Le

Raincy Montfermeil, Montfermeil, France

Correspondence: G. MORATELLI

Intensive Care Medicine Experimental 2020, 9(1): 001083

Introduction. The decision of admission of elderly patients to intensive care unit (ICU) is often difficult1. It becomes delicate in a pandemic context.

Objectives. The objective of our study is to describe the epidemiological and clinical characteristics of covid-19 patients over 70 years old hospitalized in our ICU, and to identify predictive factors of poor prognosis in this patients group.

Methods. It is an observational retrospective single-center study. We have included all covid 19 patients older than 70 years hospitalized into our ICU from March 2020 to April 2021.

Categorical variables were expressed as frequencies and percentage, whereas quantitative variables were expressed as median.

Two groups were compared in univariate analysis: survivors and deceased. Multivariate analysis was performed to identify the independent predictors of poor prognosis.

Results. During the study, 71 patients were included and the mortality rate was $54 \%(n=38)$. The epidemiological analysis has demonstrated that the median age was $75(70-86)$ years, median BMI was $29(15-51) \mathrm{Kg} / \mathrm{m} 2$, median SAPS was 39 (18-89) and the sex ratio was $68 \%(n=48) .73 \%$ of patients $(n=52)$ have a history of cardiovascular disease, $49 \%$ of them $(n=35)$ have diabetes and $24 \%(n=17)$ have a chronic renal disease. $30 \%(n=21)$ of the patients have presented an acute kidney injury at the admission and $17 \%(n=12)$ of them have needed renal replacement therapy. During the hospitalisation $30 \%$ $(n=21)$ of patients have received vasopressor. $83 \%(n=59)$ of the patients have received non-invasive ventilation and $44 \%(n=31)$ of them have needed oral intubation following non-invasive ventilation failure. The mean of duration of mechanical ventilation was 14,6 days (2-30). The mortality in mechanical ventilated group was $81 \%(n=25)$. In univariate analysis: SAPS, AKI, diabetes, use of mechanical ventilation, ventilation associated pneumonia (VAP) and shock were associated with high risk of mortality.In multivariate analysis, only mechanical ventilation was associated with a poor of prognosis (oddsratio $10.0[2.31 ; 56.6], \mathrm{p}<0.01)$.

Conclusion. The mortality of elderly covid 19 patients in ICU is high. Mechanical ventilation was associated with a poor of prognosis. The use of mechanical ventilation must be indicated on a case-by-case basis. More studies are needed on this subject.

\section{Reference(s)}

1. Grasselli G, Greco M, Zanella A, et al. Risk Factors Associated with Mortality among Patients with COVID-19 in Intensive Care Units in Lombardy, Italy. JAMA Intern Med. 2020;180(10):1345-1355. 10.1001/jamainternmed. 2020.3539

\section{4}

Digoxin improves diaphragmatic strength and outcome in patients with chronic obstructive pulmonary disease. A prospective randomized controlled study

M. Benlabed ; ;. Benlabed²; R. Gaudy'; S. Aissaoui³; S. Nedjari4;

A. Ladjouze 5

${ }^{1}$ Anesthesiology and intensivecare, University of Lille, Lille, France;

${ }^{2}$ Saint pierre hospital, Université Libre de Bruxelles, Bruxelles, Belgium;

${ }^{3}$ Anesthesiology, Algiers university, Algiers, Algeria, Algeria; ${ }^{4}$ Anesthesiology, Algiers university, Alger Centre, Algeria, Algeria; ${ }^{5}$ Anesthesiology, Algiers University, Algiers, Algeria

Correspondence: M. Benlabed

Intensive Care Medicine Experimental 2020, 9(1): 001084

Introduction. Patients with acute exacerbation of chronic obstructive pulmonary disease (AECOPD), present a diaphragmatic fatigue which contributes to the difficult weaning of mechanical ventilation. In experimental and clinical studies, Theophyllin and digoxin [1]have been associated with improvement of diaphragmatic strength.

Objectives. So the aim of our study was to investigate in patients with chronic obstructive pulmonary disease(COPD) under face mask non invasive ventilation for acute exacerbation, the effect of IV digoxin on the diaphragmatic strength and consequently on weaning process outcome.

Methods. After ethical committee consentment,we perfomed a prospective randomized controlled study and enrolled 30 patients with AECOPD from september 2019 to september 2020. They were $75+-10$ years old, under non invasive pressure support ventilation, with the same SOFA score Day 1. The patients were randomized in two groups:

A first group receiving intravenous digoxin $0.02 \mathrm{mg} / \mathrm{kg}$ infused during $10 \mathrm{mn}$.

A second group receiving placebo (saline solution) infused also during the same time.

Pressure support was the same in the 2 groups i.e. $7 \mathrm{cmh} 20$ plus Peep 0 $\mathrm{cmh} 20$ set for the weaning trial.

We assessed in the two groups using diaphragmatic ultrasonography[2] the amplitude of right diaphragmatic excursion(EXdi) and thickening fraction of the right diaphragm (TFdi) atT90mn.TFdi is defined as thickness at end-inspiration-thickness at end-expiration/ thickness at end-expiration.

The right hemi-diaphragm was visualized in the zone of apposition using a $10-\mathrm{MHz}$ linear ultrasound probe. Diaphragmatic ultrasonography was performed before and after the administration of either digoxin or placebo.We monitored at T90mn digoxin blood level and blood gases. We recorded also ICU stay,Total non invasive ventilation time(TNIVT) and ICU mortality.

Results. Statistical analysis used Mann Whitney test and results expressed as mean+-standard deviation.We observed that arterial 
blood gases and $\mathrm{pH}$ were not significantly different between the 2 groups.Digoxin blood level was in the therapeutic range with a mean value, of $2.80 \pm 0.10 \mathrm{nmol} / \mathrm{L}$ at T $90 \mathrm{~min}$ after digoxin administration. Diaphragmatic excursion(EXdi) and Diaphragm thickening fraction(TFdi) were not different between the 2 groups before the administration of either digoxin or placebo respectively for EXdi $1.3+-0.5 \mathrm{cmvs} 1.25+-0.4 \mathrm{~cm}$. P $<0.4$ and for TFdi $33 \%+-2 \mathrm{vs} 36 \%+-4$ $p<0.3$. First we observed essentially that maximal excursion atT90mn was higher in the digoxin group than in placebo group respectively $4.2 \mathrm{~cm}+-0.2 \mathrm{vs} 1.2 \mathrm{~cm}+-0.15 \mathrm{p}<0.002$.

Secondly, diaphragm thickening fraction atT90mn was more elevated in digoxin group than in placebo group respectively $55 \%+-5 \mathrm{vs}$ $35 \%+-3 . P<0.003$. ICU length of stay(ICULOS) was shorter in digoxin group compared to placebo group respectively 4.8 days $+-2 \mathrm{vs}$ 9.5 days $+-2 \mathrm{P}<0.004$.

Total non invasive ventilation time(TNIVT) (hours) was significantly shorter in digoxin group than in placebo group respectively group 120 hours +-2 vs $237+-3 p<0.0001$.ICU mortality was more elevated in placebo group than in digoxin group respectively $20 \%$ vs $6 \%$

\begin{tabular}{llll}
\hline & Digoxin Group & Placebo Group & $\mathbf{P}$ \\
\hline EXdi cm T90mn & $4.2+-0.2$ & $1.2+-0.15$ & $<0.002$ \\
TFdi \% T90mn & $55+-5$ & $35+-3$ & $<0.003$ \\
ICULOS (days) & $4.8+-2$ & $9.5+-2$ & $<0.004$ \\
TNIVT (hours) & $120+-2$ & $237+-3$ & $<0.0001$
\end{tabular}

Conclusion. We observed that Diaphragmatic strength improves significantly after digoxin administration in AECOPD patients and consequently shortens the weaning process with beneficial effects in terms of outcome.

\section{Reference(s)}

1. Ferrari G.et al. Diaphragm ultrasound as a new index of discontinuation from mechanical ventilation. Critical Ultrasound Journal. 6,1, 2014

2. Aubier et al. Effects of Digoxin on Diaphragmatic Strength Generation in Patients with Chronic Obstructive Pulmonary Disease During Acute Respiratory Failure.Am Rev Resp Dis. 135, 3, 1987

3. Thanks to my colleagues of Algiers for their collaboration.

\section{3}

Comparing high-flow nasal cannula to bi-level positive pressure ventilation for hypercapnic respiratory failure in adults: systematic review and meta-analysis of randomized controlled trials

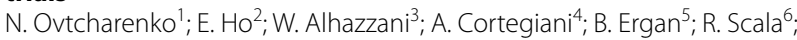
G. Sotigu' $;$ S. Oczkowski ${ }^{3} ;$ K. Lewis $^{1}$

${ }^{1}$ Department of medicine, McMaster University, Hamilton, Canada; ${ }^{2}$ Faculty of health sciences, McMaster University, Hamilton, Canada; ${ }^{3}$ Department of medicine and department of health research methods, evidence and impact, McMaster University, Hamilton, Canada; ${ }^{4}$ Section of anesthesia, intensive care and emergency, University of Palermo, Palermo, Italy; ${ }^{5}$ Department of pulmonary and critical care, Dokuz Eylul University School of Medicine, Izmir, Turkey; ${ }^{6}$ Pulmonology and respiratory intensive care unit, S Donato Hospital, Arezzo, Italy; ${ }^{7}$ Clinical epidemiology and medical statistics unit, department of medical, surgical, experimental, University of Sassari, Sassari, Italy

Correspondence: N. Ovtcharenko

Intensive Care Medicine Experimental 2020, 9(1): 001093

Introduction. Bi-level positive pressure ventilation (BiPAP) is a firstline intervention for hypercapnic respiratory failure in adults, with demonstrated mortality benefit and reduction in intubation as compared to invasive mechanical ventilation (Osadnik et al.; Rochwerg et al.). However, it is often not tolerated due to patient discomfort, claustrophobia, and skin breakdown (Carron et al.). High-flow nasal cannula (HFNC) is an alternative form of non-invasive ventilation currently indicated in multiple settings for hypoxemic respiratory failure. However, given its effects of elevation in mean airway pressure and washout of dead space, it may also have a role in the hypercapnic patient population (Ischaki et al.; Mündel et al.; Parke et al.;Bräunlich et al.).

Objectives. To assess current evidence from randomized controlled trials (RCTs) for HFNC as a treatment for hypercapnic respiratory failure. Methods. Literature search was performed from inception to December 2020 in EMBASE, Medline, and the Cochrane library. Titles and abstracts were screened in pairs by four independent reviewers using Covidence systematic review software. 4932 studies were imported for screening, 261 underwent full text review and 7 studies were included in the final analysis. Pooled risk ratios (RR) were used for dichotomous outcomes and mean differences (MD) for continuous outcomes, utilizing a random-effects model.

Results. Seven RCTs were included in the final analysis. For mortality, there was no significant difference between interventions (RR 0.86 , $95 \% \mathrm{Cl} 0.48-1.56$, low certainty). For intubation, there was no significant difference between interventions (RR $0.80,95 \% \mathrm{Cl} 0.46-1.39$, low certainty). There was no difference in ICU length of stay (MD 0.08, 95\% $\mathrm{Cl}-1.16-1.32$, low certainty) but a trend towards lower hospital length of stay favouring HFNC (MD $-0.82,95 \% \mathrm{Cl}-1.83-0.20$, high certainty). There was no significant difference in partial pressure of $\mathrm{CO} 2$ between groups (MD -1.87, 95\% Cl-5.34-1.60, moderate certainty).

Conclusion. Current evidence suggests that HFNC may be an effective alternative to BiPAP for patients with hypercapnic respiratory failure. However, given low certainty of evidence, further studies are needed to better understand the role of HFNC in this setting.

\section{Reference(s)}

1. No funding to disclose.

2. Rochwerg, Bram, et al. "Official ERS/ATS Clinical Practice Guidelines: Noninvasive Ventilation for Acute Respiratory Failure." European Respiratory Journal, vol. 50, no. 4, 2017, 10.1183/13993003.02426-2016.

3. Parke, R., et al. "Nasal High-Flow Therapy Delivers Low Level Positive Airway Pressure." British Journal of Anaesthesia, vol. 103, no. 6, 2009, pp. 886-90, 10.1093/bja/aep280.

4. Osadnik, Christian R., et al. "Non-Invasive Ventilation for the Management of Acute Hypercapnic Respiratory Failure Due to Exacerbation of Chronic Obstructive Pulmonary Disease." Cochrane Database of Systematic Reviews, vol. 2017, no. 7, 2017, 10.1002/14651858.CD004104.pub4.

5. Mündel, Toby, et al. "Mechanisms of Nasal High Flow on Ventilation during Wakefulness and Sleep." Journal of Applied Physiology, vol. 114, no. 8, 2013, pp. 1058-65, 10.1152/japplphysiol.01308.2012.

6. Ischaki, Eleni, et al. "Nasal High Flow Therapy: A Novel Treatment Rather than a More Expensive Oxygen Device." European Respiratory Review, vol. 26, no. 145, 2017, 10.1183/16000617.0028-2017.

7. Carron, M., et al. "Complications of Non-Invasive Ventilation Techniques: A Comprehensive Qualitative Review of Randomized Trials." British Journal of Anaesthesia, vol. 110, no. 6, 2013, pp. 896-914, 10.1093/bja/aet070,

8. Bräunlich, Jens, et al. "Nasal Highflow Improves Ventilation in Patients with COPD." International Journal of Chronic Obstructive Pulmonary Disease, vol. Volume 13, May 2016, p. 1077, 10.2147/COPD.S104616.

\section{6}

Incidence and outcome of barotrauma in patients with coronavirus disease 2019 (COVID-19) on invasive mechanical ventilation

E. Abril Palomares ${ }^{1}$; A. Pavalascu'; ÁL. Córdoba Sánchez ; Á. Algaba Calderón'; C. Muñoz De Cabo'; DO. Stanescu'; EM. Gómez Peñalver'; G. Navarro Velasco ${ }^{1}$; MC. Martín Delgado

${ }^{1}$ Icu, Torrejón University Hospital, Madrid, Spain

Correspondence: A. Pavalascu

Intensive Care Medicine Experimental 2020, 9(1): 001096

Introduction. Barotrauma (BT), a potentially lethal complication of invasive mechanical ventilation (IMV), includes different types of lung injury including pneumothorax (PTX), subcutaneous emphysema (SE), and pneumomediastinum (PM) (1). Studies have shown higher 
incidence of BT related to IMV in acute respiratory distress syndrome (ARDS) due to COVID-19 (2).

Objectives. To determine risk factors, management and evolution of the patients (pts.) with ARDS secondary to COVID-19 receiving IMV in whom BT was diagnosed.

Methods. A retrospective, descriptive study for 14 months (Mar 2020-Apr. 2021) of pts. admitted to a 16 beds intensive care unit (ICU) in Madrid. The pts. included were those who tested positive for SARS CoV-2, requiring IMV, and experiencing BT. Pts. who experienced PTX caused by procedures other than IMV were excluded. Lung protective ventilation strategies were used in all cases. Data were based on clinical and imaging features of cases. Maximal values of airway pressures, peak inspiratory pressure (PIP), positive end-expiratory pressure $(\mathrm{PEEP})$, tidal volume (TV), respiratory rate (RR), compliance (C), partial pressure of carbon dioxide (PCO2), as well as age, sex, underlying diseases, need for vasoactive medications (VAM), prone position, management and complications, were analyzed. We also have studied the relationship between BT and the pathological features of ARDS. Values are presented as percentage and mean, and standard deviation, $\mathrm{p}<0.01$ being considered significant. All analyses were done with IBM SPSS Statistics for macOS, version 25.

Results. A total of 18 pts., out of a total of 266 pts. with COVID-19 admitted to the unit during this period, were analyzed. Overall characteristics: $78 \%$ males, mean age: $68 \pm 6$ years $(95 \% \mathrm{Cl})$, mean admission APACHE II: $17 \pm 7(95 \% \mathrm{Cl})$, mean VMI duration in discharged pts.: $51 \pm 27$ days $(95 \% \mathrm{Cl} ; \mathrm{p}<0.001)$, mean ICU stay in discharged pts.: $61 \pm 33$ days $(95 \% \mathrm{Cl}, \mathrm{p}<0.001) .44 \%$ of pts. were exitus during ICU stay. PTX occurred in 13 pts. (72\%), PM in 12 pts. (61\%), and SE in 8 pts. (44\%). Diagnosis of PTX and PM was made with imaging (X-Ray, CT Scan) in $90 \%$ of pts., vs. clinical diagnosis in $10 \%$ of cases. The most common PTX location was unilateral, in $85 \%$ of cases. Chest tubes were inserted in $61 \%$ of PTX cases. Ventilator-associated pneumonia (VAP) before BT event was diagnosed in 12 pts., $67 \%\left(95 \% \mathrm{Cl}_{\text {; }}\right.$ $p<0.001)$.The incidence of BT was higher in the proliferative, and fibrotic ARDS phase (40\%, 47\%). Maximal values of PIP, PEEP, TV, RR, C and $\mathrm{PCO} 2$ before $\mathrm{BT}$ diagnosis were higher, without statistical significance. Analyzing prone position and need for VAM before BT diagnosis, no correlation was found.

Conclusion. Barotrauma mainly occurs in the late stages of ARDS, and VAP during ICU stay was identified as a risk factor for BT events. In most PTX and PM cases diagnosis was a radiological finding without clinical manifestations. BT events were not associated with higher respiratory pressures or volumes, prone position, or hemodynamic instability.

\section{Reference(s)}

1. McGuinness G, Zhan C, Rosenberg N, et al. Increased Incidence of Barotrauma in Patients with COVID-19 on Invasive Mechanical Ventilation. Radiology. 2020;297(2): E252-E262

2. Theodore W Marcy, M.D., F.C.C.P.t. Barotrauma: Detection, Recognition and Management. Chest 1993; 104:578-84

\section{3}

\section{Extracorporeal membrane oxygenation retrieval} during the COVID-19 pandemic: a subanalysis of the ECMOVIBER registry

M. Martinez Martinez' ; E. Argudo'; C. Palmada'; M. García-De-Acilu'; S. García'; RS. Contreras'; S. Alcántara Carmona²; C. Albacete³; A. Blandino Ortiz'; P. Santa-Teresa ; H. Pérez Chomón ${ }^{6}$; R. Gimeno ; G. Renedo Sánchez-Girón ${ }^{8}$; E. Rodriquez-Ruiz ${ }^{9}$; E. Gallart ${ }^{1}$; J. Riera ${ }^{1}$

'Intensive care, Vall d'Hebron University Hospital, Barcelona, Spain; ${ }^{2}$ Intensive care, \{street_address\}, Madrid, Spain; ${ }^{3}$ Intensive care, Hospital Universitario Virgen de la Arrixaca, Murcia, Spain; ${ }^{4}$ Intensive care, Hospital Universitario Ramón y Cajal, Madrid, Spain; ${ }^{5}$ Intensive care unit, H.G.U Gregorio Marañón, Madrid, Spain; ${ }^{6}$ Servicio medicina intensiva, hospital universitario virgen macarena, Sevilla, Spain; Intensive care, Hospital Universitari i Politècnic La Fe, Valencia, Spain; ${ }^{8}$ Intensive care, Hospital Clínico Universitario, Valladolid, Spain; ${ }^{9}$ Medicina intensiva, Complejo Hospitalario Santiago de Compostela, Santiago de Compostela, Spain

Correspondence: M. Martinez Martinez
Intensive Care Medicine Experimental 2020, 9(1): 001103

Introduction. Extracorporeal membrane oxygenation (ECMO) is a resource intensive and highly complex technique for severe hypoxemia refractory to conventional therapies. The management of these patients in high volume centres (at least 30 cases/year) is associated with improved survival. To ensure equal access to the therapy, mobile ECMO teams are deployed.

Objectives. To describe the population and outcomes of patients who required ECMO retrieval during the COVID-19 pandemic in the Iberian Peninsula.

Methods. Observational retrospective analysis of the ECMOVIBER registry, including 25 ECMO centers in Spain (23) and Portugal (2). All adult COVID-19 patients requiring extracorporeal respiratory support between 1st March and 1st December 2020 were included. End of follow up and recruitment was 1st December. Demographic data, comorbidities, and complications during ECMO were recorded. ECMO retrieval was defined as a primary ECMO transport, in which a patient is cannulated by a mobile ECMO team. Results are described using mean (standard deviation), median (interquartile range) or frequency (percentage). Comparisons are performed using Chi2 test, T-test or Mann-Whitney $\mathrm{U}$ test as appropriate.

Results. A total of 334 patients [age 53 (10.3) years, 268 (80.2\%) male] were included. 130 (38.9\%) required ECMO retrieval. The description of this cohort and a comparison with the rest of the population are detailed in Table 1. No significant differences in comorbidities, respiratory condition prior to ECMO and complications during the support were identified.

Even though high volume ECMO centres only treated $28.7 \%$ of the total population, $48.4 \%$ of the retrievals were performed by these centres. The preferred cannulation strategy was femoro-jugular (88 [67.6\%]), although femoro-femoral was more frequently used than in the rest of the population (37 [28.7\%] vs 23 [11.3\%], $p<0.001)$. Drainage cannulae were also larger ( $25 \mathrm{~F}$ vs. $23.9 \mathrm{~F}, \mathrm{p}<0.001$ ).

At the end of follow up, the overall mortality of retrieved patients was $30.8 \%$, compared to $46 \%$ in the rest of the population, $p=0.01$.

Table 1 Retrieved patients vs. the rest of the population

\begin{tabular}{|c|c|c|c|c|}
\hline & $\begin{array}{l}\text { Retrieved } \\
N=130\end{array}$ & $\begin{array}{l}\text { Rest } \\
N=204\end{array}$ & p-value & $\mathrm{n}$ \\
\hline -Age (years) & $52.7(10.6)$ & $53(10.1)$ & 0.79 & 334 \\
\hline -Sex (male) & $105(80.8 \%)$ & $163(79.9 \%)$ & 0.958 & 334 \\
\hline -Chronic respiratory disease & $7(5.4 \%)$ & $14(6.9 \%)$ & 0.769 & 333 \\
\hline -Chronic kidney disease & $4(3.1 \%)$ & $8(3.9 \%)$ & 0.078 & 333 \\
\hline -Body mass index (kg/m2) & $130(6)$ & $30.1(6.2)$ & 0.684 & 333 \\
\hline - $\mathrm{PaO} 2 / \mathrm{FiO} 2$ ratio pre ECMO & $79(22.6)$ & $79.7(23.2)$ & 0.684 & 334 \\
\hline -Driving pressure pre ECMO & $17.3(5)$ & $18.6(4.8)$ & 0.008 & 325 \\
\hline -Noradrenaline $>0.1 \mathrm{mcg} / \mathrm{kg} / \mathrm{min}$ & $27(20.9 \%)$ & $40(19.7 \%)$ & 0.358 & 332 \\
\hline -Coinfection at ECMO initiation & $38(29.5 \%)$ & $61(29.9 \%)$ & 1 & 333 \\
\hline -Ventilation pre ECMO (days) & $6.3(4.7)$ & $7.3(6.9)$ & 0.773 & 333 \\
\hline -Death at cannulation & $5(13.9 \%)$ & $6(6.4 \%)$ & 0.43 & 130 \\
\hline -Hemorrhagic shock & $20(15.6 \%)$ & $23(11.4 \%)$ & 0.353 & 329 \\
\hline -Acute kidney injury & $34(27 \%)$ & $50(25.1 \%)$ & 0.808 & 325 \\
\hline -Ventilator-associated pneumonia & $70(55.1 \%)$ & $89(44.3 \%)$ & 0.072 & 328 \\
\hline -Thrombosis (patient) & $28(22 \%)$ & $24(12.2 \%)$ & 0.039 & 324 \\
\hline -Thrombosis (circuit) & $46(35.4 \%)$ & $70(34.3 \%)$ & 0.995 & 328 \\
\hline -Duration of ECMO & $20.8(20.7)$ & $17.6(14.9)$ & 0.43 & 284 \\
\hline -Status on 1st December 2020 & & & 0.01 & 334 \\
\hline Weaned & $67(51.5 \%)$ & $84(41.2 \%)$ & & \\
\hline Ongoing & $23(17.7 \%)$ & $26(12.7 \%)$ & & \\
\hline Deceased & $40(30.8 \%)$ & $94(46.1 \%)$ & & \\
\hline
\end{tabular}


Conclusion. ECMO retrieval is safe, feasible and allows to concentrate the patients in high volume centres, which may play a role in the outcomes.

\section{Reference(s)}

1. Broman LM, Holzgraefe B, Palmer K, et al. The Stockholm experience: interhospital transports on extracorporeal membrane oxygenation. Crit Care 2015;19:278.

2. Riera et al. Extracorporeal membrane oxygenation retrieval in coronavirus disease 2019: a case-series of 19 patients supported at a high-volume extracorporeal membrane oxygenation center. Crit Care Expl 2020; 2:e0228.

3. Lebreton et al. Extracorporeal membrane oxygenation network organisation and clinical outcomes during the COVID-19 pandemic in Greater Paris, France: a multicentre cohort study. Lancet Respir Dis 2021; S2213-2600(21)00,096-5.

\section{4}

Low lung compliance does not impact on mortality in severe SARS-CoV-2 pneumonia

R. Costa-Reis ; M. Adao-Serrano ; ; M. Sousa, ; D. Regateiro, 2; J. Gouveia'; C. Candeias, ${ }^{1} ;$ JM. Ribeiro, ${ }^{1}$; SM. Fernandes,

'Serviço de medicina intensiva, Centro Hospitalar Universitário Lisboa Norte, Lisbon, Portugal; ${ }^{2}$ Clínica universitária de medicina intensiva, Faculdade de Medicina da Universidade de Lisboa, Lisbon, Portugal

Correspondence: R. Costa-Reis

Intensive Care Medicine Experimental 2020, 9(1): 001104

Introduction. Since the first cases of severe COVID-19 were reported, there has been an interest in respiratory physiology. In patients requiring invasive mechanical ventilation (IMV), a dispute emerged on whether differences in lung phenotype affects prognosis. In order to evaluate the clinical impact of lung mechanics, we studied lung compliance during the first 7 days of IMV in patients with severe pneumonia associated with SARS-CoV-2 infection.

Methods. A single-centre, ethical commission approved, retrospective study was conducted in a large urban university referral hospital. Protocolled prospective collected data was collected from consecutive patients admitted with severe COVID-19 pneumonia. Patients submitted to ECMO support were excluded. Demographic, clinical and laboratory data were analysed and compared with variables related to lung mechanics. Patients were divided in two arbitrary groups, based on the value of static compliance $(40 \mathrm{~mL} / \mathrm{cmH} 2 \mathrm{O}$ as a cut-off value), that we believe identify two different phenotypes. Statistical analysis was performed using STATA SE 15.1, and comparisons were made using $t$-test and $X 2$, as well as multivariate logistic regression.

Results. One hundred patients were included in our study. Male patients were predominant (79\%). Mean SAPS II was $39.5 \pm 10$, with a global mortality rate of $44 \%$. At admission, mean static compliance value was $40.9 \pm 12.2 \mathrm{~mL} / \mathrm{cmH} 2 \mathrm{O}$. Fifty-one patients were grouped in the low compliance cohort (LC: below $40 \mathrm{~mL} / \mathrm{cmH} 2 \mathrm{O}$ ) and 49 patients were grouped in the high compliance (HC: above $40 \mathrm{~mL} / \mathrm{cmH} 2 \mathrm{O}$ ). LC group had more females. Age, SAPS II, time from symptom-to-hospital and time from hospital-to-IMV were similar between the two groups. LC patients tended to be more hypoxemic, as revealed by a lower PO2:FiO2 ratio on day one of IMV (133.8 \pm 7.6 vs $164.8 \pm 10.9, p=0,02)$; this difference was lost at day six of IMV. As expected, tidal volume was higher in the HC group $(6.5 \pm 0.1$ vs $6.8 \pm 0.1 \mathrm{ml} / \mathrm{kg}$ of ideal body weight, $p=0.02$ ), without compromising safe plateau and driving pressures, although driving pressure was higher in the LC group $(12.6 \pm 0.6$ vs $9.3 \pm 0.5 \mathrm{cmH} 2 \mathrm{O}, p=0.0001)$. Use and duration of proning were similar (3.3 vs 2.9 days, $p=0.38$ ). Laboratory data showed no difference on C-reactive protein levels nor lymphocyte count between the two groups. Static compliance on day one of IMV did not impact on ventilator-free days and did not affect ICU mortality. Mortality remained unchanged when adjusted for SAPS II, age or tidal volume per ideal body weight used.

Conclusion. Lung mechanics differ between patients with severe SARS-CoV-2 pneumonia, requiring personalised ventilatory strategies.
However, these two different phenotypes do not seem to affect duration of ventilatory support nor prognosis.

001109 Time to Intubation and Initiation of Extracorporeal Membrane Oxygenation (ECMO) in Critically III Patients with Coronavirus Disease 2019 (COVID-19) Pneumonia

C. Merley, ${ }_{1}^{1}$; LA. Galloway, ${ }_{1}$; A. Zaaqoq ${ }^{2}$

${ }^{1}$ School of medicine, Georgetown University, Washington, United States

of America; ${ }^{2}$ Critical Care Medicine, MedStar Washington Hospital

Center, Washington, United States of America

Correspondence: A. Zaaqoq

Intensive Care Medicine Experimental 2020, 9(1): 001109

Introduction. Beginning in December 2019, the highly contagious coronavirus pneumonia (COVID-19) rapidly spread through China and around the world. As of April 2021, there are estimated over 132,000000 confirmed cases with over 2,700,00 fatalities. The World Health Organization (WHO) has estimated that approximately $14 \%$ of infections develop into severe disease, with $5 \%$ of patients critically ill. Venovenous (VV) Extracorporeal Membrane Oxygenation (ECMO) is an effective rescue intervention when the conventional management of severe ARDS deemed ineffective. However, it is unclear if the duration of symptoms before ECMO has impact on the patient outcome.

Methods. A cross-sectional cohort study of 27 patients with COVID-19 pneumonia who required VV ECMO for respiratory support. The study period extended from April 2020 to December 2020. Data captured include baseline patient characteristics, comorbidities, pre-ECMO respiratory support, duration of symptoms before ECMO support, laboratory results, and therapeutic interventions for COVID-19. Survival to hospital discharge was the primary end-point.

Results. from 27 patient supported with ECMO for acute respiratory failure, $10(37 \%)$ patients were discharged from the hospital. The median age of the survivors is 40.5 years old ( $27-52$ years old). Half the survived patients are females with median BMI 37.7 (22.8-65.7). there are no differences in co-morbid conditions between both the survivors and the non-survivors. Also both groups received the same adjunctive therapies for hypoxia and therapeutics for COVID-19 pneumonia. The median duration from symptoms onset to ECMO cannulation is $19( \pm 3.9)$ days in ECMO survivors and $10.5( \pm 6.6)$ days in the non-survivors $(p=0.009)$.

Conclusion. in our limited experience, the duration of symptoms before initiation of ECMO seems to have an impact on the patient outcome. Survivors have longer duration of symptoms in comparison to non-survivors. Our results need to be confirmed in a larger cohort of COVID-19 pneumonia supported by ECMO.

\section{0}

Efficacy of noninvasive ventilation according to ventilation modes in adult patients with acute hypoxemic respiratory failure: A systematic review and network meta-analysis

M. Sakuraya ${ }^{1}$; H. Okano ${ }^{2}$; M. Tomoyuki ${ }^{3}$; S. Kimata ${ }^{4}$; H. Satoshi ${ }^{5}$

${ }^{1}$ Department of emergency and intensive care medicine, JA Hiroshima General Hospital, Hatsukaichi, Japan; ${ }^{2}$ Department of critical and emergency medicine, National hospital organization yokohama medical, Yokohama, Japan; ${ }^{3}$ Department of emergency and critical care medi-

cine, Misato Kenwa Hospital, Saitama, Japan; ${ }^{4}$ Department of preventive services, school of public health, Kyoto University, Kyoto, Japan; ${ }^{5}$ Department of respiratory medicine and infectious diseases, Niigata University Graduate School of Medical and Dental Sciences, Niigata, Japan Correspondence: M. Sakuraya

Intensive Care Medicine Experimental 2020, 9(1): 001110

Introduction. Excessive tidal volume was reported to be associated with treatment failure in patients undergone noninvasive ventilaton. Noninvasive ventilation failure increased hospital mortality. Continuous positive airway pressure (CPAP) may more useful avoiding alveolar overdistention, rather than pressure support ventilation (PSV).

We conducted a network meta-analysis to compare the efficacy of noninvasive ventilation according to ventilation modes with high-flow 
nasal oxygen (HFNO), standard oxygen therapy (SOT) and invasive mechanical ventilation (IMV) in adult patients with acute hypoxemic respiratory failure.

Methods. The Cochrane Central Register of Controlled Trials, MEDLINE, EMBASE, and Ichushi databases were searched. Studies including adults with acute hypoxemic respiratory failure and randomized-controlled trials that compared two different respiratory management (CPAP, PSV, HFNO, SOT or IMV) were included. A network metaanalysis was performed via a frequentist approach with multivariate random-effects meta-analysis. The certainty of evidence was assessed based on GRADE Working Group approach. The outcome measures included a primary outcome of short-term mortality at the end of the follow-up period ( $<100$ days), ICU discharge, and hospital discharge. The secondary outcome was the incidence of intubation during ICU stay.

Results. The search strategy identified 12,618 records, including 21 RCTs (2,995 participants; range, 30-776 participants) that were eligible for inclusion. Nine trials compared PSV with SOT, five trials compared CPAP with SOT, three trials compared HFNO with SOT, two trials compared PSV with IMV and a trial compared PSV vs HFNO. In addition, a 3-group study directly compared PSV with HFNO and also with SOT. No studies compared CPAP or HFNO with IMV. There were total of 23 comparisons for 21 RCTs.

\section{Risk of Short-term Mortality}

Using SOT as the Reference, CPAP (risk ratio [RR], 0.54; 95\% Cl, 0.300.95 ; moderate certainty) was significantly associated with a lower risk of mortality. PSV (RR, $0.79 ; 95 \% \mathrm{Cl}, 0.59-1.05$; very low certainty) and HFNO (RR, $0.81 ; 95 \% \mathrm{Cl}, 0.52-1.26$; low certainty) was not associated with a statistically significant lower risk of mortality compared with SOT.

Compared with IMV, all noninvasive oxygenation strategies were not associated with a statistically significant lower risk of mortality, but all certainties of evidence were very low.

The probability of being best in reducing short-term mortality among all possible interventions was higher for CPAP, followed by PSV, HFNO, IMV, and SOT.

\section{Risk of Endotracheal Intubation}

Using SOT as the Reference, CPAP (RR, 0.49; $95 \% \mathrm{Cl}, 0.30-0.78$; low certainty) and PSV (RR, 0.69; $95 \% \mathrm{Cl}, 0.52-0.91$; moderate certainty) were associated with a lower risk of endotracheal intubation. HFNO (RR, 0.77; 95\% Cl, 0.52-1.13; low certainty) was not associated with a statistically significant lower risk of endotracheal intubation compared with SOT.

The probability of being best in reducing endotracheal intubation among all possible interventions was higher for CPAP, followed by PSV, HFNO, and SOT.

Conclusion. CPAP was associated with lower risk of death compared with SOT among the patients with acute hypoxemic respiratory failure, meanwhile PSV and HFNO did not decrease mortality. Noninvasive oxygenation strategies did not decrease short-term mortality compared with IMV.

\section{2}

Utilization of Diaphragm Pacing During the COVID-19 Pandemic: Decreasing Respiratory Insufficiency and Mechanical Ventilation R. Onders ${ }^{1}$; M. Elmo ${ }^{1}$; R. Schilz'; M. Pelletier

${ }^{1}$ Surgery, University Hospitals Cleveland Medical Center, Cleveland, United States of America

Correspondence: R. Onders

Intensive Care Medicine Experimental 2020, 9(1): 001112

Introduction. Diaphragm pacing(DP) was developed and approved to liberate spinal cord injured(SCl) patients from mechanical ventilation(MV). DP improves ventilator induced diaphragm dysfunction and recovery from injured phrenic nerves while also decreasing wean times by $64 \%$. The FDA recognized the need to decrease MV burden during the COVID-19 pandemic and authorized a temporary DP system. In 2020 we expanded our DP indications using the new and chronic systems. This report is the largest combined experience of DP data.

Methods. This is a retrospective analysis of an IRB approved prospective, non-randomized interventional experience at a single institution. A chronic DP system was laparoscopically implanted in those who would require DP for greater than 30 days. A temporary DP system was implanted in patients at risk for prolonged $\mathrm{MV}$ at the time of surgery via median sternotomy, thoracotomy, laparotomy or independently laparoscopically. DP stimulation was utilized to aid in weaning or diaphragm dysfunction recovery.

Results. DP electrodes were placed in 125 patients in 2020. Age range was 2 months to 83 years. Chronic system were implanted in 64: idiopathic(25), SCI(21), injured phrenic nerve(9), bilateral diaphragm dysfunction on MV(4), amyotrophic lateral sclerosis(2), intractable hiccups(2), myasthenia gravis(1). Temporary DP system were implanted in 61: cardiac procedures via median sternotomy(46), open vascular procedures(6), lung transplants(4), heart transplants(3), COVID-19(2). There were no adverse events related to electrode implantation. Al electrodes recorded diaphragm electromyography assessing diaphragm function and identifying phrenic nerve injuries. All stimulated patients had improved diaphragm function. In the temporary DP group, $40 \%(25)$ did not require stimulation because of rapid MV weaning. The other $60 \%$ were stimulated with improved ventilation. All temporary electrodes were removed completely with no device related adverse events. Five deaths occurred within 30 days in the temporary DP group, all unrelated to pacing. In a case match analysis of the high risk cardiac patients DP increased the likelihood of extubation by $48 \mathrm{~h}(83 \%$ versus $25 \%)$.

Conclusion. Prolonged MV causes significant morbidity, mortality and costs. The pandemic ICU burden highlighted the need to improve ventilator weaning. Chronic DP therapy showed continued safety and efficacy. New temporary DP was safe and improved liberation from MV, potentially reducing ICU resources. Further trials are suggested in these expanded indications.

\section{Reference(s)}

1. Onders R, Elmo MJ, Kaplan C, Katirji B, Schilz R. Extended Use of Diaphragm Pacing in Patients with Unilateral of Bilateral Diaphragm Dysfunction: A New Therapeutic Option. Surgery 2014;156:772-86.

2. Onders RP, Elmo MJ, Kaplan C, Schilz R, Katirji B, Tinkoff G. Long-term experience with diaphragm pacing for traumatic spinal cord injury: Early implantation should be considered. Surgery 2018. 164(4):705-711.

3. Onders RP, Markowitz A, Ho VP, Hardacre J, Novitsky Y, Towe C, Elmo M, Kaplan C, Schilz R. Completed FDA feasibility trial of surgically placed temporary diaphragm pacing electrodes: A promising option to prevent and treat respiratory failure.Am J Surg. 2018 Mar;215(3):518-521

001116

Treatment of Tocilizumab in Addition to Corticosteroid Improves Outcome in Hospitalized Patients with COVID-19: A Meta-analysis K. Rivera ${ }^{1}$; RA. Cordovez ${ }^{2}$; M. Patricio ${ }^{3}$; C. Permejo, ${ }^{2}$

${ }^{1}$ Department of Adult Cardiology, Philippine Health Center, Quezon City, Philippines; ${ }^{2}$ Department of Adult Cardiology, Philippine Heart Center, Quezon City, Philippines; ${ }^{3}$ Department of Ambulatory, Emergency and Critical Care, Philippine Heart Center, Quezon City, Philippines

Correspondence: K. Rivera

Intensive Care Medicine Experimental 2020, 9(1): 001116

Introduction. Coronavirus Disease (COVID-19) has been declared a global pandemic. Worse COVID-19 disease severity and outcomes are directly related with higher levels of inflammatory markers. 1,2,3,4. Interleukin-6 (IL-6) is one of the inflammatory markers monitored in COVID- 19. Tocilizumab, an anti-human IL-6 receptor monoclonal antibody currently used as treatment for rheumatoid arthritis, has been recently studied whether its addition to standard care affects COVID19 outcomes. 5 
Objectives. This study focuses on the effect of Tocilizumab in combination with standard care which includes the use of corticosteroid to therapy in patients hospitalized with covid- 19 .

Methods. Systematic review and meta-analysis conducted revealed 765 potentially relevant papers. Randomized-controlled trials on the effect of Tocilizumab for treatment of patients with severe to critical COVID-19 were included in the study with a population of at least 100 patients. Articles involving Tocilizumab without the use of any steroid as part of the therapy was excluded in the study. Electronic search was made using PubMed, Cochrane Library, Cochrane Covid-19 Registry, ScienceDirect and Medrxiv of all relevant articles. The last search was conducted on April 23, 2021. Cochrane risk tool bias was used to mitigate bias. Results are plotted down and analyzed using RevMan 5.4 using the hazard ratio, frequency and time to recovery respectively by 4 researchers individually.

Results. A total of seven randomized-controlled trial were included in the final analysis. A total of 6,116 patients were included, of which 3,134 patients received tocilizumab and 2,982 received standard care. There is a significant reduction in mortality outcome with initiation of Tocilizumab in addition to a corticosteroid therapy than the standard care alone (RR $0.89,95 \% \mathrm{Cl} 0.82-0.97$, and $\mathrm{p}=0.005$ ). Progression of disease defined as need for use of mechanical ventilation, non-invasive ventilation, high-flow nasal cannula oxygen therapy and/or ICU admission was decreased (RR of 0.79 and $95 \% \mathrm{Cl} 0.70-0.89$ and $p=<0.0001$. Median time to clinical improvement with Tocilizumab therapy in addition to a corticosteroid was decreased (HR 1.25, 95\% Cl 1.12-1.38, $\mathrm{p}=<0.0001$ ).
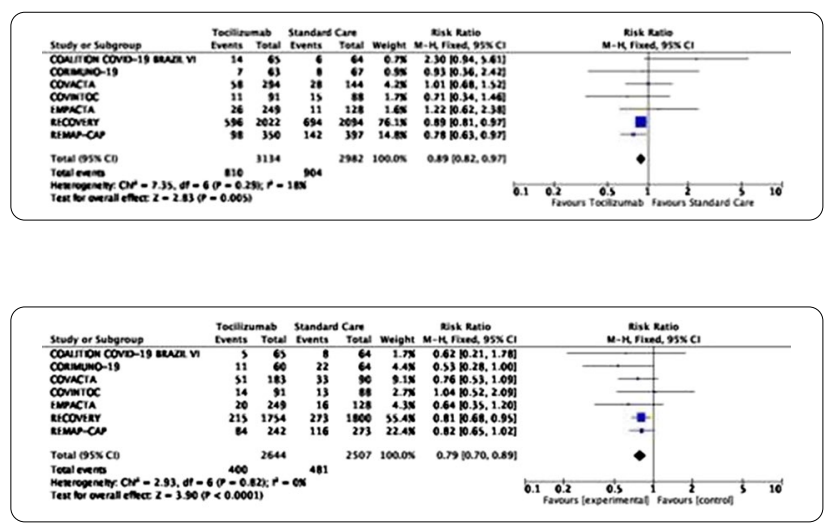

Conclusion. Tocilizumab in combination with a corticosteroid treatment improves clinical outcomes in hospitalized patients suffering from COVID-19. What makes Tocilizumab still relevant as part of the armamentarium against the COVID-19 is its judicious use given at the right time and to the right set of patients.

\section{Reference(s)}

1. Page M J, McKenzie J E, Bossuyt P M, Boutron I, Hoffmann T C, Mulrow C D et al. The PRISMA 2020 statement: an updated guideline for reporting systematic reviewsBMJ 2021; 372:n71 10.1136/bmj.n71

2. Kaneko A. Tocilizumab in rheumatoid arthritis: efficacy, safety and its place in therapy. Ther Adv Chronic Dis. 2013;4(1):15-21. 10.1177/20406 22312466908

3. Liu Z, Li J, Chen D, Gao R, Zeng W, Chen S, et al. Dynamic interleukin-6 level changes as a prognostic indicator in patients with COVID-19. Front Pharmacol. (2020) 11:1093. https://doi.org/10.3389/fphar.2020.01093

4. Liu T, Zhang J, Yang Y, Ma H, Li Z, Zhang J, et al. The role of interleukin-6 in monitoring severe case of coronavirus disease 2019. EMBO Mol Med. (2020) 12:1-12. https://doi.org/10.15252/emmm.202012421

5. Han H, Ma Q, Li C, Liu R, Zhao L, Wang W, et al. Profiling serum cytokines in COVID-19 patients reveals IL-6 and IL-10 are disease severity predictors. Emerg Microbes Infect. (2020) 9:1123-30. https://doi.org/10.1080/22221 751.2020 .1770129
6. Iannaccone G, Scacciavillani R, Del Buono MG, Camilli M, Ronco C, Lavie $\mathrm{CJ}$, et al. Weathering the cytokine storm in COVID-19: therapeutic implications. CardioRenal Med. (2020) 10:277-87. https://doi.org/10.1159/00050 9483

7. None

001119

Use of neuromuscular blocking agents in mechanically ventilated patients with COVID-19: A propensity score analysis

G. Li Bassi' ; K. Gibbons, ${ }^{2}$; JY. Suen, ${ }^{3}$; HJ. Dalton, ${ }^{4}$; N. White, ; A. Corley, ${ }_{1}$;

S. Forsyth, ${ }^{1}$; J. Laffey ${ }^{6}$; E. Fann ; J. Fanning, ${ }^{8}$; M. Panigada ${ }^{9}$; R. Bartlett ${ }^{10}$;

D. Brodie, ${ }^{11}$; A. Burrell ${ }^{12}$; D. Chiumello ${ }^{13}$; A. Elhazmi ${ }^{14}$; M. Esperatti ${ }^{15}$,

G. Grassellii ${ }^{16} ;$ C. Hodgson $^{17} ;$ J. Fraser ${ }^{18}$

1 University of queensland, Critical Care Research Group, Brisbane, Spain; ${ }^{2}$ Child health research centre, University of Queensland, Brisbane,

Australia; ${ }^{3}$ Critical care research group, adult intensive care services, The Prince Charles Hospital, Chermside, Australia; ${ }^{4}$ Heart and vascular institute and department of pediatrics, Inova Fairfax Hospital, Falls Church, United States of America; ${ }^{5}$ Statistics, Queensland University of Technology, Brisbane, Australia; ${ }^{6}$ Anaesthesia and intensive care medicine, National University of Ireland Galway, Galway, Ireland; ${ }^{7}$ Interdepartmental division of critical care medicine, University of Toronto, Toronto, Canada;

${ }^{8}$ Faculty of Medicine, The University of Queensland, Saint Lucia, Australia; ${ }^{9}$ Department of anesthesiology, Fondazione IRCCS Ca'Granda Ospedale Maggiore Policlinico, Milano, Italy; ${ }^{10}$ Medical center, University of Michigan, Ann Arbor, United States of America; ${ }^{11}$ Intensive care, Columbia University College of Physicians and Surgeons/New York-Presbyterian Hospital, New York, United States of America; ${ }^{12}$ Australian and new zealand intensive care research centre, University of Melbourne, Melbourne, Australia; ${ }^{13}$ Anesthesia and intensiva care, San Paolo, Milano, Italy; ${ }^{14}$ Intensive care medicine, King Faisal Specialist Hospital and Research Centre, Riyadh, Saudi Arabia; ${ }^{15}$ Intensive care medicine, Hospital Privado de Comunidad, Mar del Plata, Argentina; ${ }^{16}$ Intensive care unit, Policlinico of Milan, Milano, Italy; ${ }^{17}$ Department of epidemiology and preventive medicine, Monash University, Melbourne, Australia; ${ }^{18} \mathrm{Ccrg}$, clinical sciences, The Prince Charles Hospital, Chermside, Australia

Correspondence: G. Li Bassi

Intensive Care Medicine Experimental 2020, 9(1): 001119

Introduction. Controversy surrounding neuromuscular blocking agents (NMBA) use in patients with early acute respiratory distress syndrome (ARDS) has persisted throughout the years. However, whether NMBAs are useful in Coronavrus Disease 2019 (COVID-19) patients requiring invasive mechanical ventilation (IMV) is unknown.

Objectives. To appraise the effect of NMBAs in COVID-19 mechanically ventilated patients on 28-day intensive care unit (ICU) mortality.

Methods. We investigated NMBA use in COVID-19 patients on IMV from February 1 to November 24, 2020, in 147 hospitals across six continents, all part of the COVID-19 Critical Care Consortium. We performed univariate analyses to compare patients with or without NMBA treatment. A propensity score matched Cox proportional hazards analysis, incorporating time-varying NMBA treatment, was carried out to appraise the effect of NMBA on 28-day intensive care unit (ICU) mortality.

Results. 1019 out of 1885 (54\%) patients received NMBA therapy, with a median (IQR) time from ICU admission to commencement of NMBA therapy of $0(0-2)$ days. The median (IQR) duration of NMBA therapy was 3 (2-6) days. Upon commencement of IMV, patients who received NMBA therapy had a lower mean $( \pm \mathrm{SD}) \mathrm{PaO} 2 / \mathrm{FiO} 2(143 \pm 76$ vs $160 \pm 93 ; P=0.002$ ). After propensity score matching, Cox proportional hazard modelling demonstrated that NMBA therapy was associated with significantly higher 28-day ICU mortality (adjusted $\mathrm{HR}=2.20,95 \% \mathrm{Cl} 1.67,2.89, \mathrm{P}<0.001)$. Sensitivity analyses testing various NMBA therapeutic regimens confirmed similar associations with mortality.

Conclusion. Early use of NMBA is common in COVID-19 patients on IMV and associated with a 2.2-fold increase 28-day mortality risk. In the absence of definitive data from clinical trials, our data suggest that NMBA should be applied cautiously in this setting. 
Reference(s)

1. University of Queensland, Wesley Medical Research, The Prince Charles Hospital Foundation, The Health Research Board of Ireland; Biomedicine international training research programme for excellent clinician-scientists; European Union's research and innovation programme (Horizon 2020); la Caixa Foundation.

001131

Acute Eosinophilic Pneumonia as a late presentation of refractory respiratory failure in COVID patients

R. Caetano Garcês ${ }^{1}$; J. Santos-Limaa ; M. Cascão ${ }^{1}$;. T. Marta ${ }^{1}$; R. Cavaco'; L. Bento $^{1}$

'Unidade de Urgência Médica (UUM), Centro Hospitalar Universitário de Lisboa Central—Hospital de São José, Lisboa, Portugal

Correspondence: R. Caetano Garcês

Intensive Care Medicine Experimental 2020, 9(1): 001131

Introduction. Acute eosinophilic pneumonia (AEP), although uncommon, has been previously associated with viral infections [1]. Recent data suggests that SARS-CoV2 could be a possible etiological agent for the development of this entity [2,3].

AEP is characterized by an acute respiratory illness with an eosinophil infiltration of the pulmonary parenchyma, ranging from an asymptomatic presentation till a fulminant pneumonia. Its timely diagnosis and prompt treatment is associated with a good outcome. Objectives. We present a case series that hypothesizes an association between severe cases of SARS-CoV2 pneumonia and AEP.

Methods. A retrospective study and data analysis of our intensive care unit (ICU) database was performed. All patients admitted in our unit due to SARS-CoV2 infection were included. A presumptive diagnosis of AEP was assumed in patients with eosinophilia $\left(>0,5 \times 10^{\wedge} 9 / \mathrm{L}\right)$ and evidence of diffusion or oxygenation deficit with typical radiological findings of AEP, presenting during the recovery phase of SARS-CoV2 infection. Patients with another identifiable etiology for this clinical presentation were excluded.

Although crucial for the definitive diagnosis, bronchoalveolar lavage for cell count was not performed due to technical difficulties in sample processing.

Response to treatment was defined as an improvement in $\mathrm{PaO} 2 /$ $\mathrm{FiO} 2$ ratio in the invasive mechanically ventilated (IMV) and a reduction in veno-venous extracorporeal membrane oxigenation (VVECMO) blood flow $(\mathrm{Qb})$ requirement, after the administration of glucocorticoids therapy.

Results. Since the beginning of the COVID outbreak, 352 SARS-Cov2 patients were treated in our ICU: $66 \%$ were male, with a mean age of $62,1 \pm 14,0$ years; mean length of stay was $11,1 \pm 3,2$ days and allcause mortality was $37,5 \% .76 \%$ required invasive mechanical ventilation for a mean of 10,2 $\pm 9,7$ days and $12,5 \%$ underwent VV-ECMO. A presumptive diagnosis of AEP was made in 5 patients $(3$ males; mean age: $43,2 \pm 11,0$ years) within a mean of $38,0 \pm 5,2$ days from the initial SARS-Cov2 infection symptom presentation; previous underlying conditions were found in only one patient with past medical history of rheumathoid arthritis, all other patients were otherwise healthy. At the day of the presumptive AEP diagnosis 4 patients presented with patchy bilateral opacities, only one had unilateral patchy opacities; 2 were on IMV and 3 were on VV-ECMO.

Glucocorticoids were started on the 5 patients after the diagnosis. Four patients had a significant reduction in respiratory support after the 3rd day of steroid administration; the latter, mechanically ventilated, had a discrete increase in $\mathrm{PaO} 2 / \mathrm{FiO} 2$ ratio.

Conclusion. This case series points out a possible association between AEP and severe SARS-CoV2 infection. Although rare, AEP should be considered as an important comorbidity in the recovery phase SARS CoV2 patient presenting with a refractory respiratory failure.
Reference(s)

1) Murao K, Saito Atsishi, et al. 2020. Acute eosinophilic pneumonia as a complication of Influenza A (H1N1) pulmonary infection. Sarcoidosis Vasc Diffuse Lung disease. 35:95-97.

2) Murao K, Saito A, Kuronuma K, et al. 2020. Acute eosinohpilic pneumonia accompanied with COVID-19: a case report. Respirol Case Rep.

3) Descalzo $V$, et al. 2021. Acute eosinophilic pneumonia associated with SARS-CoV2 Infection; Arch Bronconeumol. 57:50-52.

001142

Transpulmonary pressures with an air-filled esophageal catheter J. Berg ; P. Massion²; B. Lambermont ${ }^{3}$; G. Parzibut ${ }^{4}$; N. Samalea $;$

D. Ledoux, ${ }^{2}$

${ }^{1} \mathrm{ICU}, \mathrm{CHU}$ liege, liege, Belgium; ${ }^{2}$ Department of intensive care, Chu De Liège, Liège, Belgium; ${ }^{3}$ Intensive care, University Hospital of Liège, Liège, Belgium; ${ }^{4}$ pneumology, CHU de Liège, Avenue de L'Hòpital, Liège, Belgium, Liège, Belgium; ${ }^{5}$ Anesthoology, CHU liege, liege, Belgium

Correspondence: J. Berg

Intensive Care Medicine Experimental 2020, 9(1): 001142

Introduction. Transpulmonary pressures measurements could be useful to better characterize respiratory mechanics and to guide lung protective ventilation in ARDS1. An easy, bedside, disposable and reliable tool for esophageal pressure measurements is lacking.

Objectives. to explore the feasibility of our new transpulmonary pressure measurement method by an air-filled esophageal catheter ${ }^{2}$ and its clinical benefit for detecting ventilator induced lung injury and for individualizing protective ventilation.

Methods. We tested our novel method with an air-filled esophageal catheter without balloon, positioned in the lower third of the esophagus and connected to an air-filled pressurized blood pressure transducer bounded to the monitor. We collected data from 21 ventilated patients (11 covid-19 ARDS, 4 neurological injuries, 2 cardiopathies, 3 pulmonary sepsis, 1 thoracic trauma), measured end-inspiratory and end-expiratory pressures of both airway and esophageal pressures. We calculated transpulmonary pressures via our new available online calculator (www.esophageal-pressure-calculator.be).

Results. We obtained esophageal and transpulmonary pressure measurements with our new method in all our 21 patients (male $n=17$ ). Signals were stable and reliable as reported ${ }^{2}$. According to airway pressures, 19/21 patients were under protective ventilation in volumecontrolled mode (see Table 1a). Esophageal pressure measurements allowed i) determination of chest wall/lung elastances, elastance ratio and esophageal delta pressure; ii) detection of risk for barotrauma (PLei,ER $>20 \mathrm{cmH} 2 \mathrm{O})$, for atelectrauma (PLee $<0 \mathrm{cmH} 2 \mathrm{O})$ and for lung stress with the transpulmonary driving pressure (delta $\mathrm{PL}>12 \mathrm{cmH} 2 \mathrm{O}$ ) (see Table 1b). According to the transpulmonary approach, 16/21 patients presented either barotrauma $(n=5)$ and/or atelectrauma $(n=12)$ and/or lung stress $(n=4)$. Respiratory settings were adapted in all 16 patients accordingly (modification of Peep $n=11$ or of $V t$ $\mathrm{n}=6$ ). Of note, Covid-19 patients compared with non-covid patients had a higher plateau pressure, a higher PEEP, a higher ventilatory ratio $^{3}$ and a higher risk of barotrauma.

\begin{tabular}{llll}
\hline & Total $(\mathbf{n}=\mathbf{2 1})$ & $\begin{array}{l}\text { Covid 19 } \\
(\mathbf{n}=\mathbf{1 1})\end{array}$ & $\begin{array}{l}\text { Non-covid } \\
(\mathbf{n}=\mathbf{1 0})\end{array}$ \\
\hline $\begin{array}{l}\text { 1a. Airway- } \\
\text { derived } \\
\text { parameters: }\end{array}$ & & & \\
$\begin{array}{c}\text { Tidal volume } \\
\text { (ml/kg PBW) }\end{array}$ & $6.3 \pm 1.4$ & $6.5 \pm 1.1$ & $6.6 \pm 0.5$ \\
$\begin{array}{l}\text { PEEP (cmH2O) } \\
\text { Plateau pressure } \\
(\mathrm{cmH} 2 \mathrm{O})\end{array}$ & $10 \pm 4$ & $12 \pm 2.8^{*}$ & $8 \pm 3.6$ \\
$\begin{array}{c}\text { Driving pressure } \\
(\mathrm{cmH} 2 \mathrm{O})\end{array}$ & $10 \pm 4$ & $23.6 \pm 4.7^{* *}$ & $16.8 \pm 4$ \\
\hline
\end{tabular}




\begin{tabular}{|c|c|c|c|}
\hline & Total $(n=21)$ & $\begin{array}{l}\text { Covid } 19 \\
(n=11)\end{array}$ & $\begin{array}{l}\text { Non-covid } \\
(n=10)\end{array}$ \\
\hline $\begin{array}{l}\text { Elastance } \\
\text { of respira- } \\
\text { tory system } \\
(\mathrm{Ers}, \mathrm{cmH} 2 \mathrm{O} / \mathrm{L})\end{array}$ & $24.4 \pm 11.9$ & $29.1 \pm 14.3$ & $19.4 \pm 5.4$ \\
\hline Ventilatory ratio & $2.5 \pm 1.3$ & $2.9 \pm 1.1^{*}$ & $2.1 \pm 1.4$ \\
\hline \multicolumn{4}{|l|}{$\begin{array}{l}\text { 1.B Esophageal- } \\
\text { derived } \\
\text { parameters: }\end{array}$} \\
\hline $\begin{array}{l}\text { Esophageal pla- } \\
\text { teau pressure } \\
(\mathrm{cmH2O})\end{array}$ & $15 \pm 4$ & $15 \pm 1$ & $15 \pm 2$ \\
\hline $\begin{array}{l}\text { Eso. End-expira- } \\
\text { tory pressure } \\
(\mathrm{cmH} 2 \mathrm{O})\end{array}$ & $12 \pm 4$ & $12 \pm 1$ & $12 \pm 2$ \\
\hline $\begin{array}{l}\text { Esophageal } \\
\text { Delta Pressure } \\
(\mathrm{cmH2O})\end{array}$ & $3 \pm 2$ & $3 \pm 2$ & $4 \pm 2$ \\
\hline $\begin{array}{l}\text { Chest wall } \\
\text { elastance } \\
(\mathrm{cmH} 2 \mathrm{O} / \mathrm{L})\end{array}$ & $7.3 \pm 3.6$ & $6.8 \pm 3.5$ & $8.3 \pm 3.4$ \\
\hline $\begin{array}{l}\text { Lung elastance } \\
(\mathrm{El}, \mathrm{cmH} 2 \mathrm{O} / \mathrm{L})\end{array}$ & $17.1 \pm 13.1$ & $22.3 \pm 15.9$ & $11.1 \pm 5.7$ \\
\hline $\begin{array}{c}\text { Elastance ratio } \\
(E R=E l / E r s)\end{array}$ & $0.64 \pm 0.22$ & $0.70 \pm 0.07$ & $0.55 \pm 0.05$ \\
\hline $\begin{array}{l}\text { Barotrauma: } \\
\text { PLei,ER } \\
\text { (cmH2O) }\end{array}$ & $13 \pm 7$ & $17 \pm 2^{* *}$ & $9 \pm 1$ \\
\hline $\begin{array}{l}\text { Atelectrauma: } \\
\text { PLee (cmH2O) }\end{array}$ & $-1.5 \pm 4.4$ & $0 \pm 1$ & $-3 \pm 2$ \\
\hline $\begin{array}{l}\text { Lung stress: } \\
\text { delta } \mathrm{PL} \\
(\mathrm{cmH2O})\end{array}$ & $7 \pm 5$ & $9 \pm 5$ & $5 \pm 3$ \\
\hline
\end{tabular}

${ }^{*} \mathrm{p}<0.05 ;{ }^{* *} \mathrm{p}<0.01 ; \mathrm{PL}$, transpulmonary pressure; ei, end-inspiratory; ee, end-expiratory.

Conclusion. Transpulmonary pressures can easily be obtained at the bedside by our air-filled esophageal catheter's method and allow individualized protective ventilation in ICU patients.

\section{Reference(s)}

2. Sinha $P$ et al. (2019) AJRCCM 333-341.

3. Massion PB et al. (2021) ICM experimental, submitted.

4. Yoshida T et al. (2019) ICM 535-538.

\section{3}

Implementation of the veno-venous ExtraCorporeal Membrane Oxygenation(ECMO) Retrieval program in the region of Madrid (Spain). Experience of Hospital Universitario 12 de Octubre ECMO referral centre

A. Delgado Tellez de Cepeda'; E. Renes Carreño ${ }^{1}$; JL. PerezVela'; JA. Rubio Mateo-Sidron ; A. Lesmes ${ }^{1}$; T. Grau Carmon ${ }^{1}$; JC. Montejo-Gonzalez ${ }^{1}$

${ }^{1}$ Intensive Care, Hospital 12 de Octubre, Madrid, Spain

Correspondence: A. Delgado Tellez de Cepeda

Intensive Care Medicine Experimental 2020, 9(1): 001143

Introduction. ECMO retrieval programs are important in order to achieve equal accessibility to the extracorporeal membrane oxygenation in every region of the country.

Objectives. The objetives of this study are: first to describe the cases trasferred to an ECMO referral centre( Hospital Universitario 12 de Octubre), second to investigate charactheristics before ECMO and while the patient was on ECMO, third to analyse the presence or not of complications secondary to transfer and cannulation and finally to analyse the ICU outcome.

Methods. This is a prospective descriptive study of ECMO retrieval program done from November 1st, 2020 to May 15 th, 2021. The cases were acepted either for emergency ECMO cannulation in the hospital of origin and retrieval or for conventional transfer. We analysed basic descriptive variables such as male proportion, age, IMC and etiology of ARDS. We also analysed variables before ECMO such as duration of non-invasive ventilation, invasive ventilation and ICU leght of stay before ECMO. We recorded severity scores such as ELSO, SOFA and APACHE Scores. We also analysed several variables while the patient was on ECMO, whether prone position on ECMO was done, median days of ECMO and succesful weaning from ECMO. We also recorded whether there were complications during cannulation or transfer. Finally ICU survival was examined.

Results. 17 cases were accepted on the ECMO Retrieval program from November 1st, 2020 to May 15th, 2021. 13 (76\%) of a total of 17 cases were male. 15 cases were accepted for emergency ECMO cannulation in the hospital of origin and transfer and 2 were accepted for conventional transfer and cannulation was done on the ECMO referral centre. Basic characteristics of the cases were: median age of 48 ( IQ 18) and IMC 31.2 (IQR 9.8). The etiology of SDRA was predominantly secondary to Sars-Cov-2( COVID 19) infection, 16 cases of a total of 17 cases (94\%). Lenght of non invasive ventilation and invasive ventilation before ECMO were 7.5 days( $10.3 \mathrm{IQR})$ and 2 days( $2.5 \mathrm{IQR}$ ) respectively and lenght of ICU admission before ECMO was 3 days ( IQR 5). Severity scores were APACHE 8 (IQR 4), SOFA 4 (IQR 2.5), ELSO 3 (IQR 3). While the cases where on ECMO Prone position was done on 9 cases(53\%). Median days on ECMO were 13 days(IRQ 10). Succesfull weaning from ECMO were achieved on 9 cases(53\%). No complications were seen on transfer or cannulation. ICU Survivors were 6(35\%). Conclusion. This is the first pilot study for ECMO retrieval in the region of Madrid, Spain. Despites high mortality in the context of COVID 19 pandemia, the program assures ECMO availability and it allows to rescue severe ARDS cases in the whole region.

001145

Safety and outcome of prolonged prone positioning in severe acute respiratory distress syndrome in COVID-19 patients

M. Tzimou'; C. Giannaki; SC. Kotoulas'; M. Endiaroglou'; A. Lavrentieva; V. Soulountsi ${ }^{1}$; G. Vasileiadou'; M. Bitzani ${ }^{1}$

${ }^{1}$ A-icu, General Hospital of Thessaloniki "George Papanikolaou", Thessaloniki, Greece

Correspondence: A. Lavrentieva

Intensive Care Medicine Experimental 2020, 9(1): 001145

Introduction. Prone position ventilation (PPV) is a potentially lifesaving intervention; however, limited data are available on the use of prolonged prone position in invasively ventilated patients with coronavirus disease-19 (COVID-19).

Objectives. The aims of this study were to determine the outcome and the safety of prolonged PPV in ARDS patients with COVID-19.

Methods. Retrospective, single-center study conducted between March 13, 2020 and April 30, 2021, on adult patients who need invasive mechanical ventilation for respiratory failure caused by COVID19. The respiratory effects of the first prone position were studied in a subset of 42 patients. Patient demographics, clinical, and mechanical ventilation (MV) variables, presence of complications and outcome were evaluated. Physiologic variables, including severity of illness, oxygenation, and measures of lung mechanics before, during and after prone positioning were compared in survivors and non survivors; $p<0.05$ was considered statistically significant.

Results. Data of 41 patients placed in prone position were evaluated (age $62.7 \pm 11$, mean $\pm S D$, Charlson comorbidity score-2.9 \pm 1.8 , APACHE I| score-15.4 \pm 5.3 , SAPS II score-7.1 \pm 1.4 , admission SOFA score- $6.8 \pm 1.2$, (mean $\pm S D$ for all variables). Median (interquartile range, IQR) time from intubation to prone position ventilation was $59 \mathrm{~h}(1-130 \mathrm{~h})$. Total prone position ventilation duration was $29 \mathrm{~h}$ (24-52 h). Twenty-seven (65\%) developed complications [ventral pressure wounds (11 patients), hypotension (6 patients), tachycardia (6 
patients), pneumothorax (1 patient) accidental catheter removal (1 patients), atrial fibrillation (2 patients)]. Twenty patients survived (48.7\%). Non survivors were elder ( $68.7 \pm 10$ vs. $56.6 \pm 9, p=0.001)$, had higher admission SOFA score (7.3 \pm 1.4 vs. $6.8 \pm 1.2, \mathrm{p}=0.04)$ and APACHE II score ( $17.1 \pm 5.8$ vs. $13.5 \pm 3.9, \mathrm{p}=0.01)$, higher Charlson comorbidity index ( $3.3 \pm 1.6$ vs. $2.4 \pm 1.9, p=0.05)$; as well as had significantly lower static compliance, higher driving pressure and lower oxygenation before prone position, during prone position, and after turning into supine position $(p<0.05)$. Sixty five percent of survived patients ( $n: 13)$ were extubated successfully on day 11 , median(10-16, IQR) from admission. Non- survivors had longer median time from admission to prone positioning [median $79 \mathrm{~h},(7-96 \mathrm{~h}, \mathrm{IQR})$, vs. median $38 \mathrm{~h},(1-68 \mathrm{~h}, \mathrm{IQR})], \mathrm{p}=0.001$. In multivariable models, age (OR, 1.2/yr; $95 \% \mathrm{Cl}, 1.05-1.2, \mathrm{p}=0.001)$, admission APACHE II score (OR, 1.25 per unit; $95 \% \mathrm{Cl}, 1.18-1.3 ; \mathrm{p}<0.001)$, were independently associated with mortality.

Conclusion. A high mortality rate was observed in COVID-19 patients with ARDS placed in the prone position. The most common complications were ventral pressure wounds, hypotension, and tachycardia. Late onset of prone position, age, severity of illness on admission, as well as the levels of physiologic variables (static compliance, driving pressure and oxygenation index) were associated with poor outcome.

\section{7}

Ventilatory efficiency is improved during flow controlled ventilation in ARDS

E. Van Dessel'; GR. De Meyer'; S. Morrisonn²; PG. Jorens' ${ }^{1}$;. Schepens, ${ }^{1}$

'Department of critical care medicine, Antwerp University Hospital

(UZA), Edegem, Belgium; '2Department of anesthesiology, Antwerp Uni-

versity Hospital (UZA), Edegem, Belgium

Correspondence: G.R. De Meyer

Intensive Care Medicine Experimental 2020, 9(1): 001167

Introduction. Flow controlled ventilation (FCV) is a recently developed mode of mechanical ventilation, consisting of a constant inspiratory and expiratory flow. Whereas the inspiration is comparable to volume controlled ventilation (VCV), the actively controlled, constant flow during expiration is novel. FCV is known to minimize dissipated energy to the lung[1] and thus has the potential to promote lung protective ventilation in the acute respiratory distress syndrome (ARDS). Furthermore, some pre-clinical studies have demonstrated that the controlled expiratory flow results in more efficient alveolar gas exchange[2].

Objectives. To compare the ventilatory efficiency between FCV and VCV in patients with ARDS.

Methods. The study was designed as a prospective crossover trial in adults with moderate ARDS admitted in a large tertiary referral hospital. After approval of the ethics board, a total of eleven patients requiring invasive mechanical ventilation for moderate $A R D S$ ( $P / F$ ratio 100-200) due to COVID-19 were recruited.

The intervention consisted of a sequence of respiratory modes: after initial baseline measurements in pressure controlled ventilation (PCV), patients were ventilated in FCV mode for a total of $30 \mathrm{~min}$ with the Evone ventilator (Ventinova Medical B.V., Eindhoven, The Netherlands) followed by $30 \mathrm{~min}$ of VCV. Respiratory rate (RR), positive end-expiratory pressure (PEEP) and inspiratory fraction of oxygen ( $\mathrm{FiO} 2$ ) were held constant. The inspiratory/expiratory (I:E) ratio of 1:1 was pursued during FCV, I:E of 1:1.5 was set during PCV and VCV. During FCV, the ventilator was set with the same peak inspiratory pressure (PIP) as during baseline PCV. For VCV, the same tidal volume as during baseline PCV was set. Respiratory mechanics and vital signs were recorded every $5 \mathrm{~min}$. Arterial blood gases were sampled every $15 \mathrm{~min}$. Statistical comparison of FCV and VCV was performed using the Wilcoxon rank sum test.

Results. A total of eleven patients successfully completed the study protocol. Results from one patient were excluded from the analysis because of a protocol violation that was detected during data analysis. With the applied settings, minute ventilation (MV) was lower in the FCV interval compared to the VCV interval of the study (VCV $7.9 \mathrm{~L} / \mathrm{min}$ versus FCV $6.6 \mathrm{~L} / \mathrm{min}, \mathrm{P}<0.001)$. However, the arterial levels of $\mathrm{CO} 2$
(PaCO2) were similar at the end of the 30-min ventilation periods for each setting $(P=0.428)$. This indicates that the ventilatory efficiency, defined as $\mathrm{MV} / \mathrm{PaCO} 2$, was significantly improved during FCV (VCV 0.16 versus $F C V 0.14, P=0.002$ ). Respiratory mechanics and ventilation parameters are summarized in Table 1.

Table 1. Comparison of flow controlled and volume controlled ventilation

\begin{tabular}{llll}
\hline & FCV & VCV & p value \\
\hline PaCO2 $(\mathrm{mmHg})$ & $49(13)$ & $50(12)$ & 0.428 \\
Minute volume $(\mathrm{L} / \mathrm{min})$ & $6.60(2)$ & $7.90(2)$ & $<0.001$ \\
Peak inspiratory pressure $(\mathrm{cmH} 20)$ & $26(6)$ & $28(9)$ & 0.009 \\
Respiratory rate $($ breath/min $)$ & $20(4)$ & $20(4)$ & 0.948 \\
PaO2 / FiO2 & $168(64)$ & $168(65)$ & 0.635 \\
Tidal volume $(\mathrm{ml})$ & $327(81)$ & $389(80)$ & $<0.001$ \\
Ventilatory efficiency $\left(\mathrm{L}^{*} \mathrm{mmHg} / \mathrm{min}\right)$ & $0.14(0.04)$ & $0.16(0.04)$ & 0.002 \\
Dynamic compliance $(\mathrm{ml} / \mathrm{cmH} 2 \mathrm{O})$ & $21(17)$ & $29(24)$ & $<0.001$ \\
\hline
\end{tabular}

Table 1 Respiratory mechanics measured during each ventilation mode. Data is represented as median (IQR). Statistics were performed with the Wilcoxon rank sum test

Conclusion. Ventilation with a controlled expiratory flow resulted in a significantly improved ventilatory efficiency compared to conventional volume controlled ventilation in patients with moderate ARDS. Further research is needed to study the physiological effects of expiratory flow limitation on alveolar ventilation.

Reference(s)

1. Dr. Schepens is supported by a grant from Research Foundation-Flanders (FWO -TBM T004620N)

2. Barnes T, Enk D. Ventilation for low dissipated energy achieved using flow control during both inspiration and expiration. Trends in Anaesthesia and Critical Care. 2019:24:5-12

3. Schmidt J, Wenzel C, Mahn M, et al. Improved lung recruitment and oxygenation during mandatory ventilation with a new expiratory ventilation assistance device: A controlled interventional trial in healthy pigs. Eur J Anaesthesiol. 2018 10:35(10):736-744.

001174

Non-invasive ventilation as a strategy to reduce a demand for invasive mechanical ventilators

M. Ferreira' ; F. Kikuta, ; P. Rezende, ; D. Novaes, ${ }^{2}$; C. Dias, ${ }^{2}$; A. Almeida, ${ }^{2}$; C. Cortinhas Filho, ${ }^{2}$ : R. Lima, ${ }^{2}$

${ }^{1}$ Physical therapy department, Hospital Samaritano, Rio de Janeiro, Brazil; ${ }^{2}$ Intensive care unit, Hospital Samaritano, Rio de Janeiro, Brazil

Correspondence: M. Ferreira

Intensive Care Medicine Experimental 2020, 9(1): 001174

Introduction. Public health emergencies have the potential toput enormous pressure on the systems of health. The data available in the literature suggest that $5-20 \%$ of patients with COVID-19 develop critical illness characterized mainly by acute hypoxemic respiratory failure (AHRF) and, consequently, they can evolve to invasive mechanical ventilation (IMV), requiring planning and execution of strategies to avoid scarcity of these essential resources the maintenance of life.

Objectives. To verify the impact of non-invasive ventilation (NIV) on slowdown in demand for intensive care beds and invasive mechanical ventilation of patients in AHRF by COVID-19 and outcome analysis.

Methods. Prospective study, in a cohort unit of COVID-19, with a total sample of sixty-nine $(n=69)$ adult patients ( $>18$ years old) with AHRF and who used NIV. The statistical analysis was performed by STATA Statistical Software. Qualitative variables are expressed as frequency and percentage and quantitative variables such as median and standard deviation. Regression analysis logistics to study the association between variables and clinical outcomes were made and odds ratios 
at intervals $95 \%$ confidence intervals were calculated. The outcomes analyzed were the frequency of IMV after NIV and the mortality after ventilatory support.

Results. The mean age was 71.2 (SD 15.6) years, 61.9\% $(n=42)$ were male. The median time of NIV, until interruption due to evolutionary improvement or need for IMV, was 8 (SD 9.4) days. 44 patients $(63.8 \%$ ) did not require IMV and $25(36.2 \%)$ progressed to IMV by a median of 13 (SD 11.67) days. 51 (73.9\%) patients were discharged hospital, the overall mortality rate was $32.6 \%(n=30)$ and was lower in those treated with NIV $(26.1 \%$ vs $52.2 \%, p=0.02)$. In the multivariate analysis, NIV remained associated with lower mortality (OR $0.25[95 \% \mathrm{Cl}$ $0.08-0.84], \mathrm{p}<0.001)$, while age $\geq 75$ years was predictive of death (OR 12.2 [95\% Cl 3.4-43.1], $\mathrm{p}<0.001$ ).

Conclusion. The current pandemic has required hospitals around the world expanding its capabilities to meet the needs of critical patients' needs, requiring many the triggering of contingency plans and sorting systems due to scarcity of essential resources. NIV despite the weak recommendation in guidelines of the European Respiratory Society and the American Thoracic Society (ERS/ATS) as a strategy preventive measure to avoid orotracheal intubation, showed a lower rate of progression to invasive mechanical ventilation, contributing to the deceleration demand for these resources, avoiding their exhaustion, in addition to being significantly associated with lower mortality in COVID patients in our institution.

\section{Reference(s)}

1. Rochwerg, B., Brochard, L., Elliott, M.W., Hess, D., Hill, N.S., Nava, S., Navalesi, P. et al. Official ERS/ATS clinical practice guidelines: noninvasive ventilation for acute respiratory failure. Eur Resp J, v50, pp1-20, 2017.

2. Ranney, M.L., Griffeth, V. and Jha, A. Critical Supply Shortages - The Need for Ventilators and Personal Protective Equipment during the Covid- 19 Pandemic. NEJM, v382, n18, e41, 2020.

3. Aziz S. et al. Managing ICU surge during the COVID-19 crisis: Rapid Guidelines. Intensive Care Med, 2020.

\section{0}

Retrospective analysis of outcomes of patients declined V-V ECMO for refractory respiratory failure related to SARS-CoV-2 infection

N. Favero'; S. Patel' ${ }^{2}$ R. Loveridge ${ }^{2}$; G. Auzinger ${ }^{2}$;. T. Pirani ${ }^{2}$

${ }^{1}$ Department of critical care, King's College Hospital NHS Foundation

Trust, London, United Kingdom; ${ }^{2}$ Consultant in critical care, King's College

Hospital NHS Foundation Trust, London, United Kingdom

Correspondence: N. Favero

Intensive Care Medicine Experimental 2020, 9(1): 001180

Introduction. The UK National Health Service amongst other health systems across the globe faced unprecedented challenges due to the COVID-19 pandemic. Due to limited knowledge of the disease and lack of definitive efficacy data, the outcomes of various proposed therapeutic options were largely unknown. This is also true for invasive intensive care support modalities, such as extracorporeal membrane oxygenation (ECMO). Although recommended criteria for ECMO referral exist, these were revised during the pandemic due to the exceptional numbers of referrals. Uncertainties remain as to whether the criteria adopted for referral and outcome prediction are sufficiently accurate to predict survival of COVID-19 patients undergoing V-V ECMO.

Objectives. The primary objective of the study was to assess the clinical outcomes of patients diagnosed with refractory respiratory failure secondary to COVID-19 and were referred to the national ECMO service but were declined for V-V ECMO for a multitude of reasons.

Methods. Retrospective data collection using electronic and paper medical records. All cases referred during the first and second waves between 1st March 2020 and 31st March 2021 were evaluated. All patients at point of referral had either laboratory or clinically suspected COVID-19 and were undergoing mechanical ventilation (MV).

Results. A total of 26 (12 during the first wave and 14 in wave 2) patients referred from our centre were declined V-V ECMO during the study period. Of these, $18(69.2 \%)$ patients were male, $8(30.8 \%)$ were female, with a median age of 53.5 years (range 32,68). 12/26 (46\%) patients survived to ICU discharge and $11 / 25(44 \%)$ patients survived to hospital discharge. Median ICU LOS was 20 days $[4,113]$. Median RESP Score was $2(-1,6)$ and duration of MV at point of referral was 5.5 days $(1,33)$. 20/26 (76.9\%) patients required vasopressor therapy with a median noradrenaline dose at the point of referral of $0.14 \mathrm{mcg} /$ $\mathrm{kg} / \mathrm{min} .9 / 26$ (34.6\%) patients were established on renal replacement therapy at time of referral. The median Clinical Frailty Score was $2(1,4)$. The RESP score and median duration of MV were similar between waves, however, the ICU survival to discharge was higher in wave 1 (58\% vs 36\%). The most common documented reasons for decline were: presence of multi-organ failure, significant comorbidities, being outside the agreed national referral criteria and perceived futility.

Conclusion. Of patients referred for V-V ECMO and declined, $46 \%$ survived to ICU discharge, and $44 \%$ to hospital discharge. Survival differed between wave 1 and wave 2, with worse outcomes in wave 2 despite the more universal adoption of therapies such as steroids and biologics. The majority were declined on the grounds of being outside referral criteria and perceived futility. Further studies are necessary to identify those patients diagnosed with COVID-19 who would most benefit from V-V ECMO in order to advise future criteria.

\section{9}

\section{Utility of high-flow nasal oxygen therapy for severe COVID-19} pneumonia

R. Ghabbara' ${ }^{1}$ S. AYED ${ }^{1}$; A. Jamoussi ${ }^{1}$; E. Rachdi' ${ }^{1}$ F. Jarraya ${ }^{1}$; J. Benkhelil

${ }^{1}$ Intensive care, Hôpital Abderrahmen Mami de pneumo-phtisiologie, Ariana, Tunisia

Correspondence: S. AYED

Intensive Care Medicine Experimental 2020, 9(1): 001199

Introduction. Invasive mechanical ventilation has been associated with high mortality in coronavirus disease 2019 (COVID19). Alternative therapy with high flow nasal therapy (HFNT) has been greatly debated around the world for use in COVID-19 pandemic.

Objectives. The purpose of this study was to describe the utility of HFNT for COVID pneumonia and the outcome of patients managed in intensive care unit.

Methods. This was a prospective study carried within a medical ICU in Abderrahmen Mami Hospital between January 2020 and march 2021. Patients admitted in ICU for severe COVID pneumonia who received HFNT were included. The main indication for HFNT treatment was the failure of conventional oxygen therapy in relieving respiratory distress and/or hypoxemia (ratio of Pao2 to Fio $2<300 \mathrm{~mm} \mathrm{Hg}$ or more than $10 \mathrm{~L} / \mathrm{min}$ of supplemental oxygen required to maintain oxygen $\geq 92 \%$ ). HFNT failure was defined as upgrading respiratory support to positive pressure ventilation or invasive ventilation.

Results. During the study period, 60 patients were enrolled with a mean age of $65 \pm 10$ years. Eight patients received non invasive mechanical ventilation and HFNC simultaneously. HFNT failure was $72 \%$ (43 patients), of which 91\% (39 patients) required endotracheal intubation. Patients with HFNT success had a higher median oxygen saturation on admission (95.2\% vs 91.2\%; $\mathrm{p}=0.001$ ), higher $\mathrm{PaO} 2 /$ $\mathrm{FiO} 2$ ratio (144 vs $112 ; \mathrm{p}=0,03)$, and needed lower $\mathrm{FiO} 2$ rates $(81 \%$ vs $96 \% ; p=0.009$ ) at admission than those with HFNT failure. The mean duration of HFNO was $5 \pm 3$ days in those successfully treated versus $3 \pm 2$ days in those who failed $(p=0.007)$. Of the latter, time to intubation was $6 \pm 3$ days. HFNT failure was more likely in patients with severe impairment in the thoracic computed tomography $(81 \%$ vs $50 \% ; p=0.05$ ), with significant increase in respiratory rate and a stagnant ratio of oxygen saturation/ Fio2 to respiratory rate index (Roxindex) within 3 days of HFNT. Hospital mortality rate in patients with HFNT failure was $90,7 \%$. In multivariate logistic regression analysis model, higher $\mathrm{PaO} 2 / \mathrm{FiO} 2$ ratio at admission (cutoff 111, $\mathrm{AUC}=0,685$, sensitivity $=70 \%$, specificity $=66 \%, p=0,026$ ), on day 1 (cutoff 108 , AUC $=0,862$, sensitivity $=76 \%$, specificity $=64 \%, p<10-3)$ and on day 2 (cutoff 103, AUC $=0,810$, sensitivity $=76 \%$, specificity $=80 \%$, $p=0,007)$ were independent predictor of HFNT success. Rox index on day 1 (cutoff 3,5, AUC $=0,814$, sensitivity $=73 \%$, specificity $=90 \%$, $p=0,015$ ) on day 2 (cutoff $3,9, A U C=0,914$, sensitivity $=91 \%$, 
specificity $=88 \%, p=0,001$ ) and on day 3 (cutoff $3,8, A U C=0,936$, sensitivity $=91 \%$, specificity $=80 \%, p=0,001$ ) were predictors of HFNT success.

Conclusion. HFNT success is associated with a reduction in the rate of invasive mechanical ventilation. It may be effective for treating coronavirus disease 2019 patients with mild to moderate acute hypoxemic respiratory failure. However, HFNT failure was associated with a poor prognosis.

\section{4}

\section{What A Second Wave!}

K. Donadello'; L. Gottin ${ }^{2}$; A. Russo, ${ }^{3}$; P. Zanatta, ; V. Schweiger ' ; S. Simari ${ }^{5}$; M. Nizzero, ${ }^{1}$; E. Polati ${ }^{1}$

${ }^{1}$ Anesthesia and intensive care $b$ unit, University of Verona, AOUI-University Hospital Integrated Trust of Verona, Verona, Italy; ${ }^{2}$ Cardio-thoracic anesthesia and intensive care unit, University of Verona, AOUI-University Hospital Integrated Trust of Verona, Verona, Italy; ${ }^{3}$ Cardio-thoracic anesthesia and intensive care unit, AOUI-University Hospital Integrated Trust of Verona, Verona, Italy; ${ }^{4}$ Anesthesia and intensive care a unit, AOUI-University Hospital Integrated Trust of Verona, Verona, Italy; ${ }^{5}$ Anesthesia and intensive care b unit, AOUI-University Hospital Integrated Trust of Verona, Verona, Italy

Correspondence: K. Donadello

Intensive Care Medicine Experimental 2020, 9(1): 001204

Introduction. Since Feb 2020 Italy has been facing COVID-19 pandemic. After the tough but efficient first wave struggle, the Veneto Region in Italy faced a more difficult second surge.

Objectives. We aimed to describe the COVID-19 critically ill patients admitted to our hospital (AOUI of Verona) during the second wave.

Methods. Prospective observational study of all COVID-19-confirmed critically ill patients, treated at our ICUs between 1 October 2020 to 2 February, 2021. Date of final follow-up was May 1, 2021. Demographic and daily clinical data were collected, including data on organ failure, management and outcome.

Results. According to our local treatment protocol, all patients received dexamethasone $6 \mathrm{mg} / \mathrm{day}$, therapeutic anticoagulation with low molecular weight heparin (LMWH) and were supplemented with Vit C and D. 212 patients were included in the analysis, the median age was $65 \pm 11$ years and 161 (76\%) were male. 164 (77\%) had cardiovascular disorders, 43 (20\%) had diabetes, overall BMI was 28.7 (26-31); 105 (49.5\%) patients received pre-hospital antibiotic therapy. $112(53 \%)$ patients were admitted from an intermediate care medical ward while $18(8.5 \%)$ were transferred from another hospital ICU. Delay from symptoms onset and hospital admission was $7 \pm 4$ days. At ICU admission, APACHE II and SOFA scores were $17 \pm 8$ and $9.4 \pm 3.1$,respectively (SOFA-N was $5.9 \pm 2.8) ; 171(80.7 \%)$ patients had only respiratory failure, while $17.3 \%$ of them were on vasopressors upon ICU admission. C-Reactive Protein concentration was $105 \pm 43 \mathrm{mg} / \mathrm{L}$, Procalcitonin levels were $1.67 \pm 0.2 \mathrm{ng} / \mathrm{mL}$; total CPK, LDH and D-Dimer were $290 \pm 20 \mathrm{U} / \mathrm{L}$, $376 \pm 20 \mathrm{U} / \mathrm{L}$ and $1750 \pm 555$, respectively; arterial lactate level was 1.3 (1-1.7) $\mathrm{mmol} / \mathrm{L}$. P/F upon ICU admission was 121 (99-170), 177 (83.5\%) pts needed tracheal intubation and $15(7.1 \%)$ needed Extra-corporeal membrane oxygenation. 116 (54.7\%) patients resulted MDR positive during their hospital stay. From ICU admission, 28 day mortality was $21.2 \%$ and using log-bin regression this was related to age and BMI $(p<0.05)$; global ICU mortality was $30.2 \%(p<0.05$ for age and sex, log-bin regression) and hospital mortality was $34.4 \%(p<0.05$ for age, sex and BMI, log-bin regression). ICU and hospital LOS were (6-25) 31 (18-45) days, respectively; this latter was significantly related to BMI $(P<0.05$, linear regression). Besides severity scores, patients' outcome was significantly related to peak lymphocyte, lactate, fibrinogen and D-Dimer levels (regression).

Conclusion. According to elsewhere in Italy and Europe we faced a harder and tougher second hit compared to the first one with higher morbidity and mortality rates; detailed insights are needed.

001206

Cohort study of pneumothorax and pneumomediastinum in COVID-19 patients in intensive care

T. Ashaye'; SR. Ashok,

${ }^{1}$ Intensive Care, Croydon University Hospital, Croydon, United Kingdom Correspondence: T. Ashaye

Intensive Care Medicine Experimental 2020, 9(1): 001206

Introduction. Prior to COVID-19, pneumothorax and pneumomediastinum were rare in mechanical ventilated patients in intensive care. However, since the pandemic reports have increased.

Objectives. To establish the incidence and risk factors for the development of intra/extra pleural air leaks, i.e., pneumothorax (PTX), pneumomediastinum (PM) and surgical emphysema (SE) in COVID-ARDS patients. Secondly, we will assess the management and outcome of this event.

Methods. Retrospective cohort study of all Covid-19 admissions with ARDS to our intensive care unit over a 3-month period. Diagnosis of COVID-19 was confirmed by a positive PCR test on admission to intensive care.

The primary outcome variable was the incidence of air leaks in COVIDARDS patients in intensive care, diagnosed on CXR or CT thorax. We further characterised pneumothorax as spontaneous or iatrogenic (i.e. $<24 \mathrm{~h}$ of diagnostic or invasive procedure). We recorded mode of ventilation, invasive procedures, and ventilator settings within 24hours of the event, compared to our Trust lung protective ventilation standard in ARDS; plateau pressure $<30 \mathrm{~cm} \mathrm{H} 20$ and TV $\leq 6-8 \mathrm{ml} /$ $\mathrm{kg}$.

Secondary measures included time and settings on NIV, immunosuppressant therapy, co-morbidities, severity of ARDS and mortality.

Results. 119 patients were admitted during our study period. 98(82\%) required invasive mechanical ventilation (IMV), 16(14\%) CPAP and $5(4 \%)$ high flow oxygen.

14 patients (12\%) developed PTX:3 on CPAP, 11 on IMV. All patients on IMV were ventilated at plateau pressures $<30 \mathrm{cmH} 20$ and TV $\leq 6-8 \mathrm{ml} /$ $\mathrm{kg}$. IMV patients required immediate chest drain, whereas those on CPAP were managed conservatively. There were 7 simple, 4 small bilateral, 3 tension PTX. latrogenic pneumothorax was seen in 10 cases: mostly after CVC insertion. 
Outcomes were poor following PTX; 55\% required repeat chest drain and 12 patients $(86 \%, n=14)$ died during admission.

16 patients ( $13 \%, n=119)$ developed extra-pleural air leaks;10PM, 2 $\mathrm{SE}, 3 \mathrm{PM}$ and SE and 1 pneumopericardium. Extra-pleural air leak, preceded PTX in 12 cases and was first noted whilst on CPAP. One case of pneumopericardium occurred on CPAP in a young healthy male, with no previous lung or cardiac history.

Mean length on CPAP was 4 days at a PEEP of 8.

Severe ARDS was common, 13 patients had $\mathrm{paO} 2 /$ fio $2<100 \mathrm{mmHg}$.

Although atypical for spontaneous PTX or PM, the following factors were common in a cohort; BMI > 25, age $>40$, non-smoker, pulsed corticosteroid use and very few had pre-existing lung conditions.

Conclusion. Pneumothorax and pneumomediastinum are common in mechanically ventilated COVID patients despite lung protective ventilation.

In this study we explored ARDS severity, obesity, steroid therapy and CPAP as possible mechanisms for COVID-induced lung injury.

Uniquely, we note an increased incidence of air leaks on CPAP and postulate increase use of CPAP in the pandemic may be attributable to the development of pneumomediastinum and pneumothorax in COVID-19 patients, however further research is required.

\section{Reference(s)}

1. Martinelli AW, Ingle T, Newman J, et al. COVID-19 and Pneumothorax: A Multicentre Retrospective Case Series. European Respiratory Journal. 2020;57(5). 10.1183/13993003.02697-2020

2. Gorospe L, Ayala-Carbonero A, Ureña-Vacas A, et al. Spontaneous Pneumomediastinum in Patients With COVID-19: A Case Series of Four Patients. Archivos de Bronconeumología (English Edition). 2020;56(11):754-756. 10.1016/j.arbr.2020.06.004

3. de Lassence A, Timsit J-F, Tafflet M, et al. Pneumothorax in the Intensive Care Unit Incidence, Risk Factors, and Outcome. Anesthesiology. 2006;104(1):5-13. 10.1097/00000542-200601000-00003

4. The authors have no affiliations or external funding sources to declare for this work

\section{7}

Evaluating the response to pulse dose steroid therapy in COVID19 ARDS: a retrospective study

E. Harte ${ }^{1}$; N. Wetherall'; S. Price ${ }^{1}$; S. Ramachandran ${ }^{1}$; W. Tuckwell ${ }^{1}$;

H. Breen ${ }^{1}$; T. Szakmany

${ }^{1}$ Department of anaesthesia, intensive care and pain medicine, Cardiff

University, Cardiff, United Kingdom

Correspondence: T. Szakmany

Intensive Care Medicine Experimental 2020, 9(1): 001207

Introduction. High dose steroid therapy has been used as a rescue measure in ARDS, including in COVID-19 induced respiratory failure (1).

Objectives. We aimed to evaluate how does the COVID19 ARDS inflammatory response change in response to pulse dose steroid therapy.

Methods. We evaluated patients who were admitted to Grange University Hospital ICU department between 15/11/20 and 08/03/21 and infected with SARS-CoV-2 infection, confirmed via RT-PCR testing. We collected daily SOFA scores and details of inflammatory response. For statistical analysis chi-square test and Mann-Whitney-U test was used. Data is presented as numbers or median (IQR) as appropriate.

Results. All 121 patients received a 10 day course of Dexamethasone as per the RECOVERY trial results, with 24 patients receiving additional pulse-dose Methylprednisolone therapy at clinicians discretion (pulse dose steroid group). Age and sex distribution was similar in the two groups: 61 (52-67) vs 63 (57-70) years and 24/73 vs 7/17 females/ males in the standard care and pulse dose steroid groups, respectively. Out of the 24 patients receiving Methylprednisolone, 17 died with 7 survived. Out of the 97 patients who did not receive methylprednisolone, 40 died and 57 survived. Length of ICU stay was significantly longer in the pulse dose steroid group. There was no significant difference in CRP or PCT levels between the two groups.

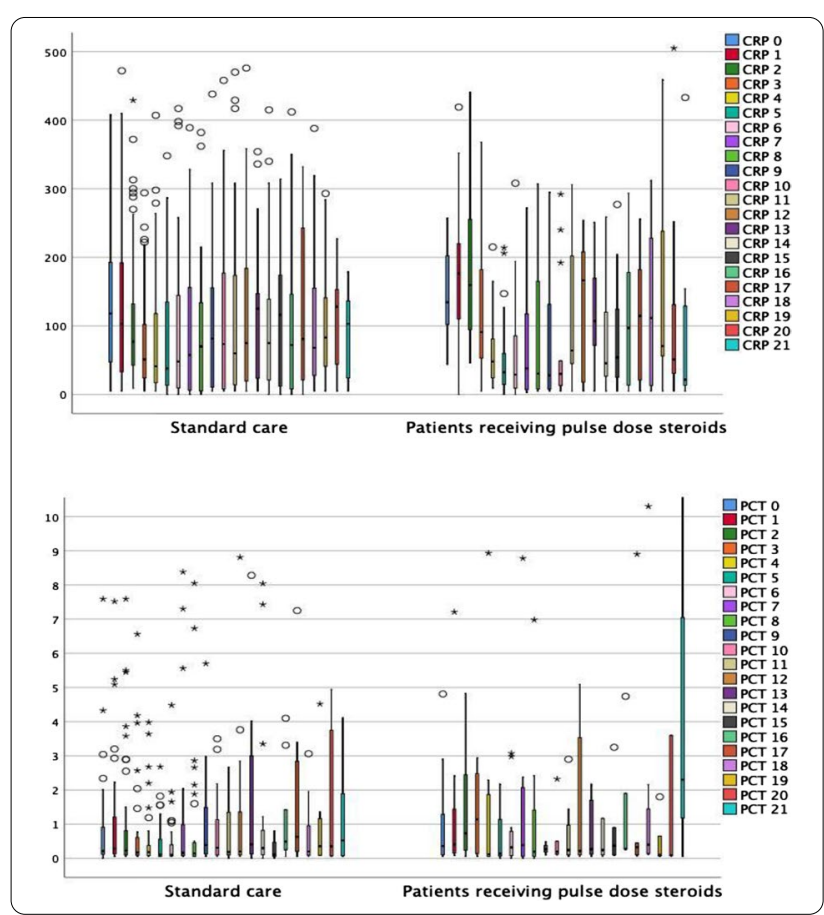

SOFA scores and Pa02/Fi02 values were also analysed in order to identify if there was a significant difference in clinical condition on admission and throughout their time on ICU. Both parameters failed to demonstrate a significant difference between the 2 cohorts, apart from a marginally higher SOFA score on Day 0 (median 8[IQR 7.5-11] in pulse dose steroid group vs median 7[IQR 5-8] in standard care group).

Conclusion. The results of this study demonstrate that the use of high-dose pulse steroids was ineffective in modulating the inflammatory response in COVID-19 ARDS. It is possible that this is due to the small sample size and non-matched nature of our cohort, however we had similar baseline characteristics in the two groups. Concerningly, there was a significant increase in mortality associated with those who received high-dose steroids, and this may be associated with a higher SOFA score on the first day of admission to ICU. Our results don't support the use of pulse-dose steroids as a rescue therapy in COVID19 ARDS outside of a randomised controlled trial.

\section{Reference(s)}

1. JAMA 1998; 280(2):159-165

001217

Ventilator-associated pneumonia in patients with SARS-CoV-2-associated acute respiratory distress syndrome (ARDS) compared to influenza-associated ARDS

D. Correia'; 'S. Lourenço ${ }^{2}$; N. Gaibino'; SM. Fernandes, ; JM. Ribeiro, ${ }^{1}$ Serviço de medicina intensiva, Centro Hospitalar Universitário Lisboa Norte, Lisbon, Portugal; ${ }^{2}$ Faculdade de medicina da universidade de lisboa, Faculdade de Medicina da Universidade de Lisboa, Lisbon, Portugal Correspondence: D. Correia

Intensive Care Medicine Experimental 2020, 9(1): 001217

Introduction. Respiratory tract involvement is the hallmark of COVID-19. Severe forms of this disease are related to pneumonia and secondary ARDS, frequently determining long periods of invasive mechanical ventilation. Apart from the risks associated with this organ support technique, infection-related immunosuppression and the use of immunomodulating agents (i.e. corticosteroids) embraces a higher 
susceptibility for coexisting bacterial complications. Influenza-associated pneumonia is also commonly seen in Intensive Care Units (ICU), associated with severe cases of lung infection, ventilatory support, secondary ARDS and, occasionally, therapy with corticosteroids.

Objectives. The authors present a retrospective cohort study to assess the incidence of ventilator-associated pneumonia (VAP), bacterial agents involved and its influence on patient outcomes, in two groups of patients presenting with secondary ARDS (COVID-19 versus Influenza associated pneumonia).

Methods. All COVID-19 associated pneumonia patients admitted in the ICU between March 2020 and January 2021 were included. Similar data was collected regarding Influenza A or B associated pneumonia patients admitted between January 2013 and March 2020. Demographic and clinical data were assembled, including invasive mechanical ventilation time, use of corticosteroids, VAP diagnosis and ICU mortality rate. A comparative analysis between both groups was performed.

Results. We included 141 patients, 87 with COVID-19 associated pneumonia and 54 with Influenza associated pneumonia. Average age in Influenza group was $56 \pm 13.2$ years compared to $64 \pm 13.7$ years in COVID-19 group. In both groups male gender was more prevalent $(63 \%$ in Influenza group, $71 \%$ in COVID-19 group). SAPS II score was higher in Influenza group (43.0 \pm 15.4 versus $39.5 \pm 13.9$ in COVID-19 group). Average mechanical ventilation time was superior in COVID-19 group (21.1 \pm 16.8 days), in comparison with Influenza group ( $16.8 \pm 12.1$ days). $96,5 \%$ of patients in the COVID-19 group were treated with systemic steroids (vs $27,7 \%) .43,6 \%(n=38)$ of patients in COVID-19 group had concurrent VAP, comparing to $27,7 \%(n=15)$ of patients in Influenza group. The most frequent isolated agents were Klebsiella pneumoniae $(n=16)$ in the COVID-19 group and Pseudomonas aeruginosa $(n=10)$ in the Influenza group. SPICE group agents were identified in $28,9 \%$ of COVID-19 patients. Among the analyzed data, corticosteroids therapy (OR 27.34, $\mathrm{p}=0.016)$ and ventilation time (OR 1.20, $\mathrm{p}=0.03$ ) were the most related to development of VAP. ICU mortality rate in COVID-19 group was $43.7 \%$ versus $27,7 \%$ in Influenza group.

Conclusion. Corticosteroid therapy and time of invasive mechanical ventilation seem to have an established association with VAP in COVID-19 patients. Comparing both groups, COVID-19 patients presented with longer ventilation periods, higher rates of corticosteroids usage and higher rates of VAP. Our study suggests that conventional risk factors associated with VAP are more relevant that SARS-CoV-2 infection per se.

\section{Reference(s)}

1. Blonz G, Kouatchet A, Chudeau N, et al. Epidemiology and microbiology of ventilator-associated pneumonia in COVID-19 patients: a multicenter retrospective study in 188 patients in an un-inundated French region.

Critical Care. 2021; 25:72. Available from: https://doi.org/10.1186/ s13054-021-03493-w

2. Wicky P, Niedermann M, Timsit J. Ventilator-associated pneumonia in the era of COVID-19 pandemic: How common and what is the impact? Critical Care. 2021;25:153. Available from: https://doi.org/10.1186/ s13054-021-03571-z

\section{0}

\section{Evaluation of the new heated wire heated humidifier F\&P 950} under varying ambient temperature conditions

F. Lellouche'; E. Rousseau, ${ }^{2}$; PA. Bouchard, ${ }^{2}$

${ }^{1}$ Reaserch center, University Institute of Cardiology and Respirology of Quebec, Québec, Canada; ${ }^{2}$ Research center, University Institute of Cardiology and Respirology of Quebec, Québec, Canada

Correspondence: F. Lellouche

Intensive Care Medicine Experimental 2020, 9(1): 001220

Introduction. It has been previously shown that heated wire heated humidification performances were influenced by ambient air temperature. With high ambient temperature, the decrease in heater plate temperature required to maintain chamber temperature stable, the humidification performances are significantly reduced, well below $30 \mathrm{mgH} 2 \mathrm{O} / \mathrm{L}$ of absolute humidity with risk of endo-tracheal tube occlusion. These performances were partially improved with compensation algorithm (1).
Objectives. The aim of the study was to evaluate the new generation heated wire HH F\&P 950 that adds several temperature measurements in its algorithm to maintain a stable humidity delivered to patients.

Methods. We measured on bench the hygrometry of inspiratory gases delivered by F\&P 950 (Fisher\&Paykel Healthcare, Auckland, New Zealand) with the psychrometric method 1 after at least $1 \mathrm{~h}$ of stability while varying the room temperature from 22 to $30^{\circ} \mathrm{C}$. We compared the data with previously measured humidity using the same method of humidity measurement, with the MR850 with low ambient temperature $\left(22-24^{\circ} \mathrm{C}\right)$ and with high ambient temperature $\left(28-30{ }^{\circ} \mathrm{C}\right)$ at different settings (37/40, 40/40, and with compensation algorithm activated).

Results. The preliminary results are shown in the figure below. With the new heated wire heated humidifier F\&P 950, the humidity delivered remained stable at $35.6 \pm 0.4 \mathrm{mgH} 2 \mathrm{O} / \mathrm{L}$ of delivered absolute humidity, despite variations of the ambient temperature from 23.1 to $28.3^{\circ} \mathrm{C}$. With previous generation of heated humidifiers, humidity delivered may fall below the safe limit of $30 \mathrm{mgH} 2 \mathrm{O} / \mathrm{L}$ when ambient temperature is high, especially if usual settings are used. With optimized settings (reduction fo the gradient temperature of compensation algorithm use), risk of underhumidification may be reduced.

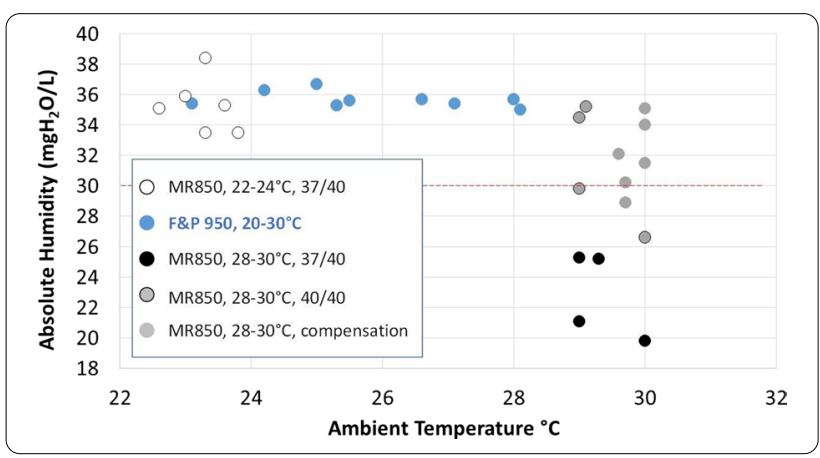

Conclusion. The new heated wire heated humidifier F\&P 950 demonstrated stable performances while varying ambient temperature.

\section{Reference(s)}

1. Lellouche L, Taillé S, Maggiore SM, Qader S, L'Her E, Deye N, et al. Influence of ambient air and ventilator output temperature on performances of heated-wire humidifiers. Am J Respir Crit Care Med 2004;170:1073-1079.

2. Fisher\&Paykel provided the humidifier F\&P 950

001223

High-Flow Nasal Oxygen therapy for severe hypoxemia in COVID-19, a possible benefit for an early start

M. Janssen ${ }^{1}$;. Türk'; Y. Aga ${ }^{3}$; D. Boer ${ }^{4}$; G. Helfrich ${ }^{5}$; W. Hanselaar 6 ; O. Hoiting ${ }^{7}$; J. Hölters ${ }^{8}$; M. Van Der Steen ${ }^{9}$; W. Jacobs ${ }^{10}$; V. Versluijs ${ }^{10}$; H. Endeman ${ }^{11}$; EJ. Wils, ${ }^{12}$

${ }^{1}$ Department of Intensive Care Medicine, Franciscus Gasthuis \& Vlietland Rotterdam, Rotterdam, Netherlands; ${ }^{2}$ Pulmonology, Franciscus Gasthuis, Rotterdam, Netherlands; ${ }^{3}$ Department of internal medicine, Franciscus Gasthuis \& Vlietland Rotterdam, Rotterdam, Netherlands; ${ }^{4}$ Department of intensive care, Maasstad Ziekenhuis, Rotterdam, Netherlands; ${ }^{5}$ Department of respiratory medicine, Maasstad Ziekenhuis, Rotterdam, Netherlands; ${ }^{6}$ Pulmonary, Franciscus Gasthuis, Rotterdam, Netherlands; ${ }^{7}$ Department of intensive care, Canisius Wilhelmina Ziekenhuis, Nijmegen, Netherlands; ${ }^{8}$ Department of respiratory medicine, Canisius Wilhelmina Ziekenhuis, Nijmegen, Netherlands; ${ }^{9}$ Department of intensive care, Martini Ziekenhuis, Groningen, Netherlands; ${ }^{10}$ Department of respiratory medicine, Martini Ziekenhuis, Groningen, Netherlands; ${ }^{11}$ Intensive care, Erasmus University Medical Center, Rotterdam, Netherlands; ${ }^{12}$ Intensive care, Franciscus Gasthuis, Rotterdam, Netherlands

Correspondence: M. Janssen

Intensive Care Medicine Experimental 2020, 9(1): 001223 
Introduction. High-flow nasal oxygen (HFNO) therapy is increasingly used to avoid invasive mechanical ventilation (iMV) in severely hypoxic COVID-19 patients. However, a large proportion of patients is eventually intubated (1-3). As evidence on the best strategy when to initiate HFNO is lacking, practice variation is significant and it may affect outcome (4).

Objectives. To determine the proportion of COVID-19 patients on HFNO requiring iMV and to assess the effect of the level of oxygen therapy prior to HFNO initiation on the intubation rate.

Methods. A prospective observational study (Netherlands Trial Register NTR-9067) is ongoing since December 2020 in 11 Dutch hospitals. All hospitalized Covid-19 patients starting HFNO are included. Demographic, clinical and treatment-related data including oxygenation parameters and data surrounding intubations are collected. This is a preliminary unadjusted analysis of patients without Donot-intubate orders. The effect of the level of oxygen support before starting HFNO on intubation rate is analyzed by comparing groups that started HFNO with prior low vs. high level of oxygen support. Starting HFNO is defined as 'low level' oxygen support when started at an $\mathrm{FiO} 2 \leq 45 \%$ (nasal cannula $6 \mathrm{~L} / \mathrm{min}$ or Venturi mask up to $10 \mathrm{~L} /$ $\mathrm{min}$ ) vs. 'high level' oxygen support that started at an $\mathrm{FiO} 2$ between $60-80 \%$ (Venturi or Non-Rebreathing Mask up to $15 \mathrm{~L} / \mathrm{min}$ ).

Results. Patients $(n=192)$ were predominantly male $(69 \%)$, median age was 63 years, comorbidities like hypertension, obesity and diabetes were common and almost all included patients were treated with dexamethasone (98,3\%). On ER admission median $\mathrm{S} / \mathrm{F}$ ratio was 249 (IQR 202-294), P/F ratio was 192 (IQR 132-248) and the ROX index was 9.8 (IQR 6.3-14.4). Seventy patients were classified as lowlevel and 122 as high-level oxygen support starters. The median S/F ratio, P/F ratio, and ROX-index at ER admission were 262 (IQR 232 -328), 205 (IQR 159-252) and 10.4 (IQR 8.5-16.0) in low-level versus 232 (IQR $132-291, \mathrm{P}=0.001), 178(\mathrm{IQR} 111-248, \mathrm{P}=0.028)$ and 8.90 (IQR 5.0-13.1, $\mathrm{P}=0.003$ ) in high-level starters. Both $\mathrm{S} / \mathrm{F}$ ratio (211 (IQR 204-231) vs. 122 (IQR 116-154, P<0.01)) and ROX index (8.4 (IQR 7.4-10.0) vs 4.8 (IQR 4.0-5.6, $\mathrm{P}<0.01)$ ) at HFNO start were higher in low-level compared to high-level starters, respectively. The median duration of illness ( 8 days) and hospital admission before start of HFNO (1 day) were similar for both groups. The intubation rate was lower in the low-level compared to the high-level oxygen support starters ( $30 \%$ vs. $57 \% ; p=0.001)$. The median ROX index prior to intubation was $3.00(2.07-3.91)$ and $3.33(2.38-4.07$; $\mathrm{P}>0.05$ ), respectively.

Conclusion. The intubation rate in this contemporary HFNO cohort was $47 \%$. Intubation rate was lower when HFNO was initiated at a low level of oxygen support, suggesting that early initiation of HFNO may be beneficial. Further recruitment is ongoing and more in-depth analysis is required to correct for baseline differences in disease severity.

\section{8}

Clinical and economic outcomes of high flow nasal cannula in patients with COVID-19: a single-center study

A. Wirgues Sousa'; IS. Rocco, ; A. Mattos ${ }^{3}$; M. Tobara ${ }^{3}$; C. Papacidero 3 ; M. Vilela, ; F. Costa, ; L. Almeida, i I. Buonso ${ }^{5}$; L. Fidelis ${ }^{5}$; R. Ralio, ${ }^{4}$; F. Paula $\mathrm{G}^{6}$; S. Almeida, ${ }^{6}$; U. Flato, ${ }^{4}$

${ }^{1}$ Physiotherapy, Hospital Samaritano, São Paulo, Brazili ${ }^{2}{ }^{2} \mathrm{cu}$, Hospital Samaritano Higienopolis, São Paulo, Brazil; ${ }^{3}$ Physiotherapy, Hospital Samaritano, São Paulo, Brazil; ${ }^{4} \mid c u$, Hospital Samaritano Higienópolis, São

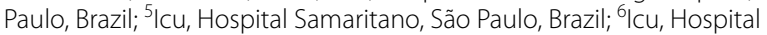
Samaritano Higienopolis, São Paulo, State of São Paulo, Brazil, Brazil Correspondence: I.S. Rocco,

Intensive Care Medicine Experimental 2020, 9(1): 001228

Introduction. At the beginning of the SARS-CoV-2 pandemic, the use of high flow nasal cannula (HFNC) was discouraged due to the high risk of bio-aerosol dispersion, hence exposing healthcare workers to contamination[1,2]. Nevertheless, advances in this research field demonstrated that HFNC is able to prevent orotracheal intubation (OTI) in a significant number of patients with hypoxemic respiratory failure due to COVID-19[3,4,5].
Objectives. We aim to compare clinical and economic outcomes of the HFNC and OTI use in patients with COVID-19.

Methods. This cross-sectional study included patients admitted in the intensive care unit (ICU) with age $>18$ years, both sex, between April and July of 2020. Patients were dichotomized in HFNC and OTI groups based on therapy of choice established by a multidisciplinary team at ICU admission. To avoid selection bias, both groups should have undergone therapy of choice for at least 4 days. Patients who underwent the 2 types of therapy were excluded. Two main outcomes were investigated: 1) clinical outcome comprised mobility at hospital discharge (analyzed by ambulation ability) and mortality; 2) economic outcome analyzed the total cost of inpatient period involving time of ICU and hospitalization stay.

Results. Thirty-nine patients composed $69 \%$ of men with a mean age of 62.4 years were included. Both groups presented similarities in anthropometric and clinical baseline characteristics such as sex, age, and Simplified Acute Physiology Score III (SAPS-III). Ambulation was achieved by $100 \%$ of patients in the HFNC group and $90 \%$ of the OTI group. The HNFC evolved with shorter ICU length of stay $(8.7 \pm 5.2$ vs. $17.1 \pm 8.9$ days, $\mathrm{p}<0.05)$ and less cost of hospital care $(R \$ 123.243,41 \pm R \$ 108.014,81$ vs. $R \$ 229.224,06 \pm 114.738,51$, $\mathrm{p}<0.05)$ compared to the OTI group. Otherwise, days of hospitalization and mortality rate were similar between the groups HFNC and OTI (19.2 \pm 9.2 vs. $25.5 \pm 11$ days and $10 \%$ vs. $15.8 \% ; p>0.05$, respectively).

Conclusion. Despite no significant reduction in mortality rate, HFNC saved costs of hospitalization in patients infected with SARS-CoV-2.

\section{Reference(s)}

1. World Health Organization. Clinical management of severe acute respiratory infection when novel coronavirus (2019-nCoV) infection is suspected: interim guidance, https://apps.who.int/iris/handle/10665/ 330893 (2020, accessed 25 January 2021).

2. Cournoyer A, Grand'Maison S, Lonergan AM, Lessard J, Chauny JM, Castonguay V, Marquis M, Frégeau A, Huard V, Garceau-Tremblay Z, Turcotte AS, Piette É, Paquet J, Cossette S, Féral-Pierssens AL, Leblanc RX, Martel V, Daoust R. Oxygen Therapy and Risk of Infection for Health Care Workers Caring for Patients With Viral Severe Acute Respiratory Infection: A Systematic Review and Meta-analysis. Ann Emerg Med. 2021 Jan;77(1):1931. https://doi.org/10.1016/j.annemergmed.2020.06.037. Epub 2020 Aug 10. PMID: 32,788,066; PMCID: PMC7415416.

3. Westafer LM, Soares WE 3rd, Salvador D, Medarametla V, Schoenfeld EM No evidence of increasing COVID-19 in health care workers after implementation of high flow nasal cannula: A safety evaluation. Am J Emerg Med. 2021 Jan;39:158-161. https://doi.org/10.1016/j.ajem.2020.09.086. Epub 2020 Oct 7. PMID: 33,059,983; PMCID: PMC7539832

4. Bonnet, N., Martin, O., Boubaya, M. et al. High flow nasal oxygen therapy to avoid invasive mechanical ventilation in SARS-CoV-2 pneumonia: a retrospective study. Ann. Intensive Care 11, 37 (2021). https://doi.org/10. 1186/s13613-021-00825-5.

5. Suhail Raoof, Stefano Nava, Charles Carpati, Nicholas S. Hill. HighFlow, Noninvasive Ventilation and Awake (Nonintubation) Proning in Patients With Coronavirus Disease 2019 With Respiratory Failure. Chest, 2020:158(5):1992-2002. https://doi.org/10.1016/j.chest.2020.07.013.

\section{1}

Clinical characteristic and survival rates during three waves in intensive care unit from Guadalajara (Spain)

R. Viejo Moreno ${ }^{1}$; N. Arriero Fernández ${ }^{1}$; B. Mariblanca-Nieves ${ }^{1}$; N. Agurto-Rivera'; A. Albaya Moreno1; JE. Romo Gonzales'; C. Benito Punzel $^{1}$; E. Yañez Parareda1; E. Quiros Oyaguez ; S. Martin-Glauce ; A. Estrella Alonso ; ;A. Silva Obregón 1; C. Marian Crespo

${ }^{1}$ Intensive care, Hospital Universitario de Guadalajara, Calle Donante de Sangre, Guadalajara, Spain, Guadalajara, Spain

Correspondence: R. Viejo Moreno

Intensive Care Medicine Experimental 2020, 9(1): 001231 
Introduction. The coronavirus disease 2019 (COVID-19) has affected more than 83.3 million people infected worldwide of which, 3.204 .531 in Spain and 20.725 in Guadalajara city. The number of deaths reported has been 1.8 million, 70.247 and 595 respectively until March 3, 2021.

Objectives. This study aims to determine the mortality and clinical characteristics between the patients from three waves of severe acute respiratory syndrome coronavirus- 2 infection.

Methods. Retrospective observational study in a single secondarycare center in Guadalajara (Spain) of patients admitted in intensive care unit (ICU) with laboratory-confirmed COVID-19 disease who had severe acute respiratory distress syndrome (ARDS). February 28, 2020 to May 03, 2020 (first infection wave), August 07, 2020 to December 30, 2020 (second infection wave) and January 01, 2021 to March 12, 2021 (three infection wave). Data was presented as number (percentage), and median and interquartile range. Chi-square and Wilcoxon rank-sum test were used to test for differences between categorical or numeric variables. Descriptive statistics were calculated, non-parametric tests were used for comparison of groups. Unadjusted mortality at 90 days and associated with three waves was calculated using the Kaplan-Meier method, differences being analysed by the log-rank test.

Results. 180 patients were included. In the first wave the patients at admission in ICU had greater tachypnea and lower oxygen saturation, as well as, higher rates of inflammatory biomarkers. Contrary to the first wave, higher proportion of patients in the second and third wave was obese or morbidly obese and received Remdesivir treatment. Nobody received Ritonavir/Lopinavir or Hydroxychloroquine in second or third wave but there were more patients who received high flow nasal oxygen (HFNO) or noninvasive mechanical ventilation (NIMV). More patients were treated with corticosteroids compared with first wave $(86,4 \%$ Vs $100,0 \%$ and $100 \% ; p<0,001)$ as well as, the time to steroids was lower $(10,0$ days $V s \quad 7,0$ and 6,0 days; $p=0,040)$ respectively. There were no differences in the rates of prone position per patient but there were more prone position cycles and duration per patient in second and third wave. The mortality was higher in the first wave $(p=0.002)$ (Fig. 1).

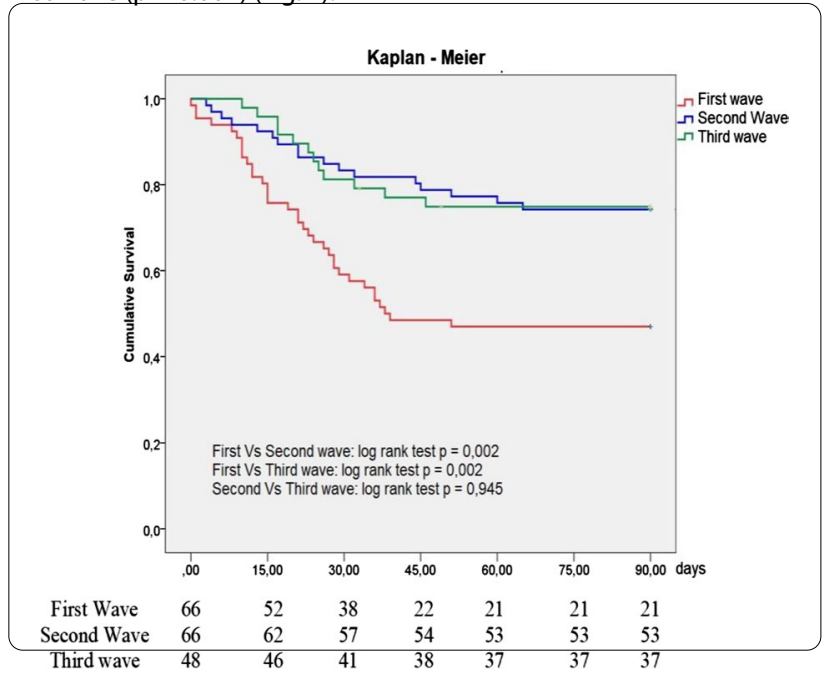

Conclusion. During second and third wave the patients have significantly lower mortality rates than those patients admitted in the first wave. The cause may be multifactorial, but the early and sustained establishment of effective treatment in ARDS could have been essential for this.
001232

An Audit of Failed Extubations in a Tertiary Hospital ICU

S. Griffin ${ }^{1}$; S. Hartigan, ${ }^{1}$; D. Connellan, ; M. Josy, ${ }_{1}^{1}$;. Hastings, ${ }^{1}$

${ }^{1}$ Critical care medicine, Mater Misericordiae University Hospital, Dublin,

Ireland

Correspondence: S. Griffin

Intensive Care Medicine Experimental 2020, 9(1): 001232

Introduction. Both prolonged mechanical ventilation (MV) and failed extubation (FE) incur significant risks. The latter is associated with increased length of MV, mortality, Ventilator Associated Pneumonia and ICU length of stay (LOS).(1).

$\mathrm{FE}$ is defined as reintubation with resumption of ventilatory support or unexpected death within $48 \mathrm{~h}$ post extubation.

Objectives. Our aim was to evaluate the rate of FE in the Mater Misericordiae University Hospital (MMUH) ICU in Dublin, Ireland, while also assessing the performance of traditional parameters of extubation readiness and the management of the patients post-extubation.

Methods. Between 1st September 2018 and 31st August 2019, 1018 patients underwent $\mathrm{MV}$ and were retrospectively analysed. 95 patients were reintubated, of whom 67 met the definition for FE. Data collected included demographics, physiological predictors of readiness to wean from MV (2), and the methods of Spontaneous Breathing Trial (SBT) used. Furthermore, data was collected on the time at which each FE took place, the indications for reintubation, the use of rescue therapy and the duration between initial extubation and reintubation. Finally, LOS, mortality rate and the rate of tracheostomy insertion were evaluated.

Results. The mean age was found to be 60.7 years (median 62; 52-73). FE was more common in males $(74.6 \%)$, in patients with respiratory (44.8\%) and cardiac (52.2\%) comorbidities and in emergency admissions to ICU (70\%). Recommended pre-extubation physiological parameters were met in our patients including Rapid Shallow Breathing Index, tidal volume, respiratory rate, FiO2, PF ratio, PEEP and blood $\mathrm{pH}$. (2) $82.1 \%$ of patients underwent SBT. Pressure Support/Continuous Positive Airway Pressure (PS/CPAP) and T-Piece trials accounted for all SBT performed; $56.4 \%$ and $43.6 \%$ respectively.

The majority (82\%) were extubated during normal working hours. A minority of patients were extubated to CPAP or Non-invasive Ventilation (NIV), each accounting for $4.47 \%$, with the majority extubated to face mask oxygen. Hypoxia (41.8\%) and hypercapnia (20.9\%) were the primary indications for reintubation. The mean time to reintubation was $27.3 \mathrm{~h}$ (median 18.75; 5.9-28). Rescue therapy consisted of CPAP, NIV and High Flow Nasal Oxygen. $60 \%$ of patients received rescue therapy in some form; $46 \%$ received multiple modalities. Of note, $68 \%$ of hypoxic patients received rescue therapy, whereas $93 \%$ of hypercapnic patients were trialled with rescue therapy.

The rate of FE was $6.6 \%$. There was no increase in mortality in those reintubated $(16.4 \%$ vs. $16.6 \%)$. Mean LOS was higher in those reintubated (ICU LOS 16.6 days, median $13 ; 9-19$ vs. 5 days, median 2.6; 1.1-6.2). The mean duration of MV was also higher in this population; $274.9 \mathrm{~h}$ (median 183; 119-346) versus $83.4 \mathrm{~h}$. A tracheostomy was inserted in $49.3 \%$ of those who were reintubated, with $66.7 \%$ of those discharged from Critical Care with the tracheostomy in-situ; the majority of whom were still requiring NIV.

Conclusion. The rate of FE was $6.6 \%$ which is below the international average (1) and may suggest some patients were exposed to excessively prolonged MV. It may, however, represent a point of good practice considering the bed demands on a busy ICU and a higher rate may have been anticipated. Higher rates were associated with male patients, emergency ICU admissions and pre-existing cardiorespiratory comorbidities. Guidelines for readiness assessment and SBT were met, however, traditional parameters to predict successful extubation performed poorly given all patients were reintubated.

The majority of FE were performed during working hours and a significant delay was noted in time to reintubation, with frequent use of rescue therapy, most noteworthy in the hypoxic cohort. FE resulted in increased LOS, duration of MV and a high rate of tracheostomy insertion. 
Reference(s)

1. MacIntyre, N., 2001. Evidence-Based Guidelines for Weaning and Discontinuing Ventilatory Support. Chest, 120(6), pp.375S-395S.

2. Thille, A., Harrois, A., Schortgen, F., Brun-Buisson, C. and Brochard, L., 2011. Outcomes of extubation failure in medical intensive care unit patients. Critical Care Medicine, 39(12), pp.2612-2618.

\section{5}

\section{Analysis of ten Scores in emergency department to predict} mortality in intensive care unit (ICU) of the patients admitted

\section{by COVID-19 ARDS}

N. Arriero Fernández ${ }^{1}$; R. Viejo Moreno ; B. Mariblanca-Nieves'; A. Albaya Moreno'; C. Benito Punzel'; N. Agurto-Rivera'; JE. Romo Gonzales'; E. Yañez Parareda'; S. Martin-Glauce'; E. Quiros Oyaguez'; A. Estrella Alonso $^{1}$; JA. Silva Obregón ${ }^{1}$; C. Marian Crespo ${ }^{1}$

${ }^{1}$ Intensive care, Hospital Universitario de Guadalajara, Calle Donante de Sangre, Guadalajara, Spain, Guadalajara, Spain

Correspondence: R. Viejo Moreno

Intensive Care Medicine Experimental 2020, 9(1): 001235

Introduction. Saturation of the intensive care unit may be associated with the fatality of COVID-19. Triage aims to identify patients in the emergency department who need priority care and admission in intensive care unit (ICU) and those who can be admitted in general ward. This process may be critical for reducing the fatality of COVID-19. Objectives. This study aimed the diagnostic ability of ten different scores to predict mortality in intensive care unit (ICU) of adult patients admitted in emergency department with acute respiratory distress syndrome secondary to COVID-19 (CARDS).

Methods. Retrospective cohort of consecutive patients with more than 18 years admitted in the ICU from Guadalajara (Spain) under CARDS diagnosis using Berlin definition between Febrery 2020 and March 2021. Scores to validate in emergency department were: 1. Shock index; 2. Modified Shock Index; 3. Age Shock index. 4. Age- systolic arterial pressure. 5. Pulse Pressure; 6 . Ratio arterial oxygen saturation and inspiratory oxygen fraction. 7 . Respiratory adjusted shock index; 8 . The Modified Early Warning System; 9 . National Early Warning Score-2; 10. National Early Warning Score-2-Lactate.

Results. 181 subjects were studied, $70.6 \%$ (127) were male, median age was 65,6 (interquartile range [IQR]: $57,4-72,3$ ) and body mass index was 28,0 (IQR 25,4-31,1). In ICU admission, lymphocyte count was $0.5 \times 109 /$ (IQR: 0.3-0.8); D-dimers was 1.86 (IQR: 0.94-10.4) $\mu \mathrm{g} / \mathrm{l}$; Ferritin was 1361,0 (IQR: $747,0-2108,0) \mu \mathrm{g} / \mathrm{l}$; C-reactive protein was 137,9 (IQR: 58,8-250,3) mg/l and LDH was 611,0 (IQR: 468.5-834.5) U/I. $88,3 \%(159)$ received invasive mechanical ventilation. Length stay in ICU was 21,0 (IQR 21,0-36,0) days and mortality rates was 36,1\% (65). National Early Warning Score-2 had the highest precisions (AUC 0.66), followed by Modified early warning score (AUC 0.63) and Age shock index (AUC 0.62).

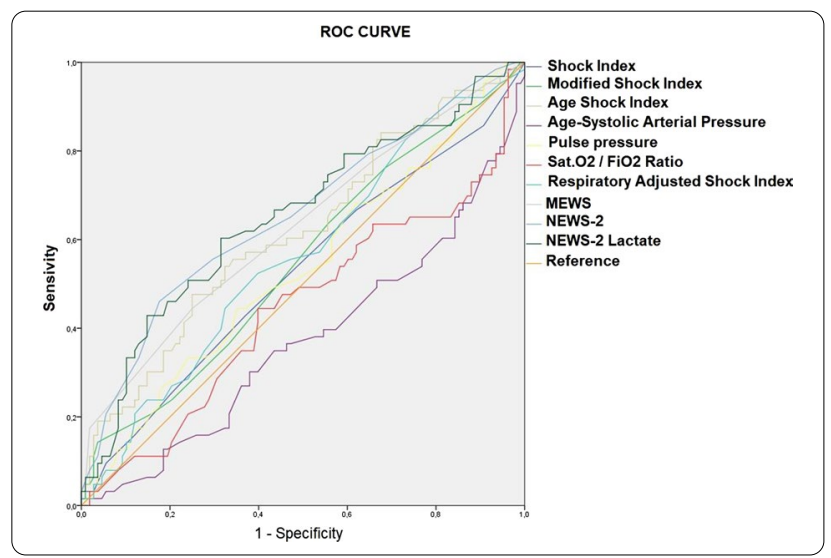

Conclusion. Overall the scores analyzed showed a poor-moderate discrimination for medium-term COVID-19 outcome. Its use in this stting is a suboptimal screening toll for that.

\section{9}

Impact of an advanced airway management training plan in prehospital emergencies: a quasi-experimental study R. Viejo Moreno ; E. Galván-Roncero²; N. Arriero Fernández³; Jl. Garrote Moreno'; B. Merchan-Sanchez ; S. Castro-Correro'; S. Parra-Soriano'; J. Rodriguez-Martín ${ }^{4}$; C. Duran-Fuentes ${ }^{1}$; JF. Jiménez-Carrascosa ${ }^{1}$;

A. Cabrejas Aparicio ${ }^{1}$; B. Mariblanca-Nieves ${ }^{3}$

${ }^{1}$ Emergency medical system (ems), Emergency Medical System (EMS) GUETS-SESCAM, Guadalajara, Spain; ${ }^{2}$ Prehospital emergency care, Gerencia de Urgencias, Emergencias y Transporte Sanitario-SESCAM;

Spain, Guadalajara, Spain; ${ }^{3}$ Intensive care, Hospital Universitario de Guadalajara, Calle Donante de Sangre, Guadalajara, Spain, Guadalajara, Spain; ${ }^{4}$ Emergency medical system (ems), SAMUR-PC; Madrid — Spain, Emergency Medical System., Spain

Correspondence: R. Viejo Moreno

Intensive Care Medicine Experimental 2020, 9(1): 001239

Introduction. Orotracheal intubation represents the gold standard for providing optimal ventilation in emergencies. However, the inherent and specific difficulties associated to prehospital medicine, make for a sub-optimal situation to carry it out.

Objectives. to evaluate the impact of a prehospital airway management training plan in terms of first-attempt intubation and survival rates.

Methods. Development of a training plan in Advanced Airway Management in prehospital emergencies for physicians of Mobile Intensive Care Unit in Guadalajara (Spain). Analysed by means of a quasi-experimental pre-post study with historical control on the impact of success in the first intubation attempt and survival in Emergency Medical Services.

Results. 219 intubations were analysed in the control group and 208 in the intervention period. The first attempt success rate was higher in the intervention period: $82.7 \%$ vs $71.7 \%(p=0.001)$; as well as survival in the mobile intensive care unit (RR: $1.61 ; p=0.02)$. Multivariate analysis showed that age $>55$ years (RR: $2.09 ; p=0.033$ ), indication for Orotracheal intubation due to Cardiac Arrest (RR: 31.1; $p<0.001)$, Cormack-Lehane grades 3 and 4 (RR: 2.66; $p=0.039)$ and the need for three or more Orotracheal Intubation attempts (RR $2.70 ; p=0.039)$, were independent predictors of mortality. Training, on the other hand, was a protective measure against mortality (RR: $0.71 ; p=0.044)$.

Conclusion. Orotracheal intubation is not a trivial technique and there is significant room for improvement in the pre-hospital emergency care setting. Simulation-based training is a useful tool to improve patient quality and safety in pre-hospital emergencies. Specific training in this area may constitute a basic tool for optimising a technique that presents important differences with respect to the hospital environment and could increase patient survival.

\section{4}

Comparison of ECMO support between patients with severe acute respiratory distress syndrome with and without SARS-CoV-2 infection

DC. Gomes ${ }^{1}$; AB. Rodrigues ${ }^{1}$; C. Candeias, ${ }^{1}$; SM. Fernandes ${ }^{2}$; JM. Ribeiro, ${ }^{3}$ ${ }^{1}$ Intensive care department, University Hospital Santa Maria, Lisbon, Portugal; ${ }^{2}$ Intensive care department, University Hospital Santa Maria, Faculdade de Medicina de Lisboa, Lisbon, Portugal; ${ }^{3}$ Ecmo referral centre director, University Hospital Santa Maria, Lisbon, Portugal

Correspondence: D.C. Gomes

Intensive Care Medicine Experimental 2020, 9(1): 001244

Introduction. The emergence of the SARS-CoV-2 associated ARDS defied the entire medical world to mount clinical strategies to deal 
with its most severe forms of respiratory failure. ELSO established recommendations to use extracorporeal membrane oxygenation (ECMO) to treat patients with refractory hypoxemia, recognizing that use of this strategy should be strictly monitored for global resource availability and clinical outcome.

Objectives. Evaluation and analyze the outcome of patients with ARDS associated with SARS-CoV-2 infection treated with ECMO support in real world conditions, in an ECMO referral center.

Methods. The authors evaluated the use of ECMO for acute respiratory failure in patients with a diagnosis of severe SARS-CoV-2 pneumonia in an ELSO recognized ECMO referral center, in a large university referral hospital. All patients with a diagnosis of COVID19 ARDS submitted to ECMO support were evaluated and outcomes compared with the population of non-COVID ARDS patients previously submitted to ECMO in our centre. Epidemiological and clinical data were reviewed. Mortality was compared using Kaplan-Meyer curves, log rank test and Cox regression analysis. T-student and Chisquare tests were used as appropriate to compare statistics between subgroups. Statistical significance was defined as a p-value $<0.05$.

Results. From March 2020 to April 2021, 53 patients with ARDS associated with SARS-CoV-2 infection were treated with ECMO support. Seven patients are still under ECMO and were excluded from this study. For matters of comparison, we included all patients with a diagnosis of ARDS $(n=166)$ treated in our centre with venovenous ECMO [patients treated with venoarterial ECMO $(n=60)$ or patients with non-ARDS conditions $(n=29)$ were excluded]. Non-COVID ARDS patients had mostly viral or bacterial pneumonia and trauma. Mortality of COVID patients was $32.4 \%$ and did not differ from nonCOVID patients (33\%), albeit having lower SAPS II (36.4 \pm 15.2 vs $43.4 \pm 15.3, p<0.05)$ and admission SOFA scores $(6 \pm 3.4$ vs $9.5 \pm 3.9$, $p<0.05)$. Patients with COVID pneumonia were older $(52 \pm 12$ vs $47 \pm 13$ years, $p<0.05)$, had more days of invasive ventilation before cannulation $(6.4 \pm 6.6$ vs $4.2 \pm 6$ days, $p<0.05)$, had longer ICU stay $(37 \pm 20$ vs $29 \pm 26$ days, $p<0.05)$ and longer ECMO run duration $(25 \pm 17$ vs $16 \pm 14$ days, $p<0.05)$. Diabetes was more prevalent in COVID patients and emerged, by Cox regression analysis, as a risk factor for mortality (HR 11.5, $p<0.05$ ).

Conclusion. Patients with severe ARDS associated with SARS-CoV-2 infection can be treated with ECMO support with highly desirable clinical outcome. Despite being a highly resource-consuming treatment, we showed that use of ECMO is feasible during the pandemic and might achieve similar survival rates when compared with the non-pandemic period. We strongly advocate that ECMO support should not be banned for patients with severe ARDS and SARSCoV-2 infection.

\section{5}

Prone position improves ventilation-perfusion mismatch in patients with severe acute respiratory distress syndrome

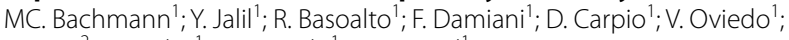
O. Diaz'; A. Bruhn'; G. Bugedo'; J. Retamal'

1Departamento de medicina intensiva, Pontificia Universidad Católica de Chile, Santiago, Chile; ${ }^{2}$ Departamento de enfermedades respiratorias, Pontificia Universidad Católica de Chile, Santiago, Chile

\section{Correspondence: M.C. Bachmann}

Intensive Care Medicine Experimental 2020, 9(1): 001245

Introduction. Acute respiratory distress syndrome (ARDS) is a frequent condition in the ICU [1]. It is clinically manifested by hypoxemic respiratory failure, airspaces flooding by inflammatory edema, and regional lung collapse due to "gas compression" of the dependent lung regions [2]. Hypoxemia is due to a ventilation/perfusion (V/Q) mismatch, especially in a heterogeneously ventilated and perfused lung [3]. Prone positioning (PP) has shown to improve oxygenation and survival in selected ARDS patients. In addition, PP has been associated with recruiting of dorsal lung regions, with more homogeneous ventilation and a reduction of pleural pressure gradient contributing to an improvement in the $\mathrm{V} / \mathrm{Q}$ ratio $[5,6]$. Electrical Impedance Tomography (EIT) is a bedside non-invasive monitoring tool of ventilation and perfusion distribution. Several studies have demonstrated EIT's benefits in establishing ventilatory parameters and improving respiratory mechanics in animal models and in the clinical settings and its role in assessing lung perfusion in ARDS [7].

Objectives. In this study, we aimed to analyze PP's effects on the distribution of pulmonary ventilation and perfusion in patients with severe ARDS connected to mechanical ventilation (MV).

Methods. An observational study was conducted in patients admitted to the ICU of the Hospital Clínico UC-Christus, between November 2019 and March 2020. Patients with moderate to severe ARDS were included. PP was indicated by the attendant physician according to ICU protocols. The distribution of pulmonary ventilation and perfusion was evaluated with EIT in supine position (SP) and then in $\mathrm{PP}, 2 \mathrm{~h}$ after the position change. Impedance Ratio (IR) and Global Inhomogeneity $(\mathrm{Gl})$ indices were obtained from the EIT data, and arterial blood gases, hemodynamic parameters, and ventilatory mechanics were registered in each position. Ventilation and perfusion maps delivered by EIT were divided into four regions of interest (quadrants) for the regional analysis of the $\mathrm{V} / \mathrm{Q}$ ratio we used the Pearson Correlation Coefficient.

Results. Eight patients with pneumonia $(57 \pm 11$ years, 50\% female) were included. Hemodynamic parameters were similar in both positions. During PP, $\mathrm{PaO} 2 / \mathrm{FiO} 2$ ratio increased (206 \pm 32 vs. $125 \pm 34 \mathrm{mmHg}, \mathrm{p}<0.005)$, driving pressure decreased $(12.25 \pm 2.5$ vs. $10.75 \pm 2.1 \mathrm{cmH} 2 \mathrm{O}, \mathrm{p}=0.04)$, and the respiratory system compliance increased $(30.25 \pm 6$ vs. $33.8 \pm 6 \mathrm{ml} / \mathrm{cmH} 2 \mathrm{O}, \mathrm{p}=0.02)$ as compared to SP. We observed a redistribution of ventilation (IR) from ventral regions in SP to dorsal regions in $\mathrm{PP}$ in all patients $(0.77 \pm 0.3$ to $2.23 \pm 0.9 \mathrm{AU}, \mathrm{p}=0.007$ ). Also, we found ventilation homogenization, as indicated by a decrease in Gl from $1.24 \pm 0.3$ to $0.82 \pm 0.1$ (AU) $(p=0.02)$. Four of the eight patients were subjected to pulmonary perfusion assessment. Perfusion was predominantly dorsal in both SP and PP. Regional distribution of ventilation and perfusion (quadrants) exhibited a positive linear correlation in PP ( $r 2=0.44$ $p=0.005)$, but not in SP $(r 2=0.056, p=0.37)$, suggesting the optimization of the V/Q mismatch with PP. We observed that those patients with a greater improvement in the $\mathrm{V} / \mathrm{Q}$ ratio presented a greater increase in $\mathrm{PaO} 2 / \mathrm{FiO} 2$, and at the same time better lung mechanics.

Conclusion. In this small series of patients, it has been shown that PP improves gas exchange and pulmonary mechanics through a change in the distribution of ventilation towards the dorsal regions of the lungs favoring $\mathrm{V} / \mathrm{Q}$ ratio and promoting a more homogeneous air distribution, this can be assessed non-invasively, in real time and at bedside. This provides the possibility to study in more detail different therapies and maneuvers that are commonly used in the ICU, such as recruitment maneuvers or position changes. New studies with a larger number of patients are necessary to validate these findings.

\section{Reference(s)}

1. Bachmann M, Morais C, Bugedo G, Bruhn A, Morales A, Borges JB, et al. Electrical Impedance Tomography in Acute Respiratory Distress Syndrome. Crit. Care. Critical Care; 2018;22:1-11.

2. . Richter T, Bellani G, Harris RS, Melo MFV, Winkler T, Venegas JG, et al. Effect of prone position on regional shunt, aeration, and perfusion in experimental acute lung injury. Am. J. Respir. Crit. Care Med. 2005;172:480-7.

3. Broccard A, Shapiro RS, Schmitz LL, Adams AB, Nahum A, Marini JJ. Prone positioning attenuates and redistributes ventilator-induced lung injury in dogs. Crit. Care Med. 2000;28:295-303.

4. Cressoni M, Cadringher P, Chiurazzi C, Amini M, Gallazzi E, Marino A, et al. Lung inhomogeneity in patients with acute respiratory distress syndrome. Am. J. Respir. Crit. Care Med. 2014;189:149-58.

5. Pelosi P, Andrea LD, Vitale G, Pesenti A, Gattinoni L. Vertical Gradient of Regional Lung Inflation in Adult Respiratory Distress Syndrome. Am J Respir Crit Care Med. 1994;149:8-13

6. Sweeney R Mac, McAuley DF. Acute respiratory distress syndrome. Lancet. Elsevier Ltd; 2016;388:2416-30.

7. Bellani G, Laffey JG, Pham T, Fan E, Brochard L, Esteban A, et al. Epidemiology, Patterns of Care, and Mortality for Patients With Acute Respiratory 
Distress Syndrome in Intensive Care Units in 50 Countries. JAMA—J. Am Med. Assoc. 2016;315:788-800.

\section{8}

\section{COVID-19: Is early mechanical ventilation the way forward?}

M. Ferraz ${ }^{1}$; C. Burgi Vieira' ${ }^{1}$ C. Pimentel' ${ }^{1}$ J. Santos-Lima ${ }^{2}$; T. Isidoro Duarte'; L. Val-Flores'; M. Cascão ${ }^{2}$; P. Faria'; N. Germano ${ }^{1}$

${ }^{1}$ Unidade de cuidados intensivos polivalente 7. Centro Hospitalar Universitário de Lisboa Central, Lisbon, Portugal; ${ }^{2}$ Unidade de urgência médica, Centro Hospitalar Universitário de Lisboa Central, Lisbon, Portugal

\section{Correspondence: C. Burgi Vieira}

Intensive Care Medicine Experimental 2020, 9(1): 001248

Introduction. COVID-19 pneumonia has brought the management of Acute Respiratory Distress Syndrome (ARDS) to the forefront of medical research. In particular, early versus delayed mechanical ventilation (MV) has been extensively discussed, with early trends showing higher mortality rates, number of days on $\mathrm{MV}$ and ventilator-associated pneumonia (VAP) in early ventilation. However, most results were reported from Intensive Care Units (ICU) that were quickly overwhelmed by large numbers of patients, implying less resources and a possible reduction in the quality of patient care.

Objectives. The objective of our analysis was to determine the effect of early versus delayed ventilation on mortality, length of ICU stay and duration of $M V$, as well as the effect on the incidence of VAP.

Methods. We retrospectively analyzed a cohort of 177 patients admitted to our ICU between March 2020 and March 2021 with COVID-19 induced ARDS. Standard of care at our ICU was to intubate and start $\mathrm{MV}$ when the $\mathrm{paO2} / \mathrm{fiO} 2$ (PF) ratio dropped below 150 Our sample was divided in two subgroups: the early ventilation (EV) group and the delayed ventilation (DV) group. The former was composed of patients intubated with a P/F ratio lower than 150 without non-invasive ventilation (NIV) or high-flow nasal oxygen (HFNO). The latter included patients intubated with a $\mathrm{P} / \mathrm{F}$ ratio lower than 150 after a $24 \mathrm{~h}$ trial of NIV, HFNO or a combination of these two modalities. The data was analysed using IBM SPSS version 26, using a significance level of 0.05 .

Results. Our sample was predominately male (68\%) with a median age of 64 [InterQuartile Range (IQR) 17] years. The overall mortality rate was $25.6 \%$ with a median length of stay of 11 (IQR 10) days and median MV duration of 9 (IQR 9) days. When comparing EV versus DV, age (64 vs 63 years; $p=0.6)$ and male predominance ( $72 \%$ vs $66 \%$; $\mathrm{p}=0.59$ ) did not differ statistically between subgroups. Mortality rate (27.3\% vs $30.6 \% ; p=0.64$ ), median ICU length of stay ( 11 vs 10 days, $\mathrm{p}=0.46$ ) and median MV duration (9 vs 9 days; $p=0.68$ ) were also not statistically different. However, VAP incidence (35.4\% vs $51.4 \%$; $\mathrm{p}=0.036$ ) was statistically lower in the EV group.

Conclusion. Our results, despite the obvious limitations of retrospectively analyzing the outcomes of a single center, seem to challenge the acquired notion that $\mathrm{EV}$ is harmful to patients.

Outcomes between the two groups were similar, with a lower incidence of VAP in the early ventilation group, suggesting that an early mechanical ventilation approach in severe COVID-19 is a sound option. Larger, multicenter trials would be needed to clarify the matter further.

\section{Reference(s)}

1. CookTM, El-Boghdadly K, McGuire B, McNarry AF, Patel A, Higgs A. Consensus guidelines for managing the airway in patients with COVID19: Guidelines from the Difficult Airway Society, the Association of Anaesthetists the Intensive Care Society, the Faculty of Intensive Care Medicine and the Royal College of Anaesthetists. Anaesth Blackwell Publ. 2020;75:785-99

2. Rola P., Farkas J, Spiegel R. et al. Rethinking the early intubation paradigm of COVID-19: time to change gears?; Clin Exp Emerg Med 2020;7:78-80

3. Zuo M, Huang Y, Ma W, Xue Z, Zhang J, Gong Y, et al. Expert recommendations for tracheal intubation in critically iii patients with novel coronavirus disease 2019. Chin Med Sci J. 2020:35:105-9

4. E. Papoutsi, V. G. Giannakoulis, E. Xourgia, C. Routsi, A. Kotanidou and I. Siempos; Effect of timing of intubation on clinical outcomes of critically ill patients with COVID-19: a systematic review and meta-analysis of nonrandomized cohort studies; Crit Care 2021;25:121

5. The authors would like to acknowledge the support from the medical and nursing staff from Unidade de Cuidados Intensivos Polivante 7

001250

The use of Tocilizumab + Methylprednisolone in the treatment of critical Covid-19 patients

I. Camerzan 1; C. Gutu-Bahov²

${ }^{1}$ Intensive Care Unit, Municipal Clinical Hospital "Sfanta Treime", Chisinau,

Moldova; ${ }^{2}$ Professor department intensive care unit, Municipal Clinical

Hospital"Sfinta Treime", Chisinau, Moldova

Correspondence: I. Camerzan

Intensive Care Medicine Experimental 2020, 9(1): 001250

Introduction. The severity of Covid-19 is correlated to the excessive immune response mediated by pro-inflammatory cytokines (IL-6, IL-12, TNF etc.), with evolution towards organ dysfunctions. Tocilizumab is an IL-6 receptor blocker proposed for the treatment of COVID-19 patients at risk of developing the "cytokine storm".

Objectives. The evaluation of the efficiency of Tocilizumab/Methylprednisolone in reducing the severity of Covid-19 patients, the ICU stay length and the mortality rate.

Methods. The retrospective study (September 2020-March 2021, the ICU of "Sf.Treime" MCH) evaluated 169 critical COVID-19 patients (mean age $56.68 \pm 3.45$ years, $P=0.204$ ) in need of high oxygen flow or non-invasive respiratory therapy, divided into 2 study groups. For study group I $(\mathrm{n}=83)$ standard treatment and Tocilizumab ( $8 \mathrm{mg} /$ $\mathrm{kg}$ single dose $(n=53)$ and in 30 patients a repeated dose after $12 \mathrm{~h}$, intravenously) + Methylprednisolone pulse therapy was applied vs. study group II $(n=86)$ with standard treatment. Clinical and laboratory data, oxygen/ventilation detachment time, ICU stay length and mortality rate were evaluated.

Results. On admission, in all the patients $\mathrm{SpO} 2$ was $<92 \%, \mathrm{PaO} 2 /$ FiO2 < 250, t-37.5-39.2 oC, Hb 8.9-14.0 g/dL, WBC 2.4-12.5*109/L, Ly $70-2500 * 106 / \mathrm{L}$, Neu 4560-10,700*106/L, PLT 125-140*109/L, ferritin $1200-5600 \mathrm{ng} / \mathrm{mL}, \mathrm{CRP} 150-460 \mathrm{mg} / \mathrm{L}, \mathrm{Fg} 220-650 \mathrm{mg} / \mathrm{dL}$, TG 0.9$3.5 \mathrm{mmol} / \mathrm{L}, \mathrm{GOT} 32-86 \mathrm{U} / \mathrm{L}$. Patient characteristics were similar in both groups, except that patients in group I were younger-38.5 \pm 2.43 compared to group II-58.45 \pm 3.24 . In group I, NIMV was necessary in $45.7 \%$ of patients (38/83), HFO2-37.3\% (31/83), oxygen cannula-13.2\% (11/83), 3.6\% (3/83) patients were intubated. In group II, NIMV was needed in $55.8 \%$ of patients $(48 / 86)$, HFO2-27.9\% (24/86), nasal cannula-10.4\% (9/86) and 5.8\% (5/86) patients were intubated. A significant evolution of white blood cells, lymphocytes and $C$ reactive protein (CRP) (a return to normal values) was seen on the fifth day of treatment with Tocilizumab + Methylprednisolone and only $10.8 \%$ of patients (9/83) maintained leukopenia, lymphopenia and PCR values of $80-95 \mathrm{mg} / \mathrm{l}$. The mortality rate in group I was $34.9 \%(29 / 83)$ vs. $39.5 \%(34 / 86)$ in group II.

Conclusion. Tocilizumab + methylprednisolone combination suggests a possible therapeutic strategy with a reduction of mortality; however, it is essential to identify the treatment stage prior to the occurrence of Covid-19 complications.

\section{5}

Impact of intermediate doses of thromboprophylaxis in 28 days mortality in patients with ARDS due to severe COVID 19 during the first pandemic surge

VD. Gumucio Sanguino1; A. Betbesé-Roig, 2; MA. Gordillo-Benitez; AL. Corrall'; J. Puentes Yáñez ${ }^{1}$; F. Di-Paolo, ; J. Sabater Riera ${ }^{1}$; JL. Pérez Fernández ${ }^{1}$

${ }^{1}$ Intensive care medicine, Bellvitge Hospital, Hospitalet de Llobregat, Spain; ${ }^{2}$ Intensive care, Hospital de la Santa Creu i Sant Pau, Barcelona,

Spain

Correspondence: V.D. Gumucio Sanguino

Intensive Care Medicine Experimental 2020, 9(1): 001255 
Introduction. Thromboprophylaxis protocols kept changing during the Covid-19 strain, some observational studies suggest that these critically ill patients could benefit from systemic anticoagulation, but recently randomized clinical trials started to give more evidence about this treatment.

Objectives. We aimed to assess the risk factors and effect of different thromboprophylaxis regimens in the outcomes of patients with Covid-19 presenting ARDS and treated Our findings suggest that an increased thromboprophylaxis regimen during the first week from ICU admission in critically ill patients with ARDS based on high d-dimer values might be beneficial in terms of survival at 28 days. with invasive MV.

Methods. We performed a regional retrospective observational study in 2 ICUs located in 2 University hospitals (Hospital de Bellvitge and Hospital de Sant Pau) of Barcelona metropolitan area (Spain) from March 01 to March 31, 2020. We included adults with ARDS receiving invasive MV within the first 3 days from ICU admission and positive SARS-Cov-2 testing by RT- PCR in respiratory fluids. Standard thromboprophylaxis regimen (STR) was subcutaneous enoxaparin $40 \mathrm{mg} /$ day or bemiparin $50 \mathrm{IU} / \mathrm{Kg} /$ day. Intermediate thromboprophylaxis regimen (ITR) was prescribed in those patients with D-dimer $\geq 1000 \mathrm{ng} /$ $\mathrm{mL}$ using subcutaneous enoxaparin $0.5 \mathrm{mg} / \mathrm{kg} / 12 \mathrm{~h}$ or bemiparin $75-80 \mathrm{lU} / \mathrm{kg} /$ day. Systemic anticoagulation was prescribed when DVT or PE was present or highly suspected.

Results. A total of 269 patients with SARS-Cov2 infection were admitted in the participating ICUs. Ninety five did not receive invasive MV. The remaining 174 patients fulfilled the Berlin definition of ARDS and received invasive MV. Among these 174 patients were identified as risk factors for death at 28 days ICU admission to be older, active smokers, had vasculopathy, a lower $\mathrm{PaO} 2 / \mathrm{FiO} 2$ ratio, and more frequently received STR. High values of creatinine (90, IQR 66,137), IL-6 $(977 \pm 1643)$, and D- dimer $(948$, IQR 527,2477$)$ at ICU admission were associated with higher mortality. Maximum thromboprophylaxis during the first week was STR in 53 (31\%) patients, ITR in 76 (45\%) patients and systemic anticoagulation in $41(24 \%)$. Mortality at 14 days was $44(25 \%)$ and during ICU stay was $90(52 \%)$. Cox regression analysis adjusted for all risk factors, showed that the use of an ITR compared to STR within the first week from ICU admission was associated with an improvement of the survival (OR $0.39,95 \% \mathrm{Cl} 0.20-0.74)$. Finally, Kaplan Meier analysis observed a benefit in survival on those patients who received ITR compared with STR $(p=0.001)$.

Conclusion. Our findings suggest that an increased thromboprophylaxis regimen during the first week from ICU admission in critically ill patients with ARDS based on high d-dimer values might be beneficial in terms of survival at 28 days.

\section{2}

Serial Diaphragm-Ultrasound as a Weaning Parameter in Invasively Ventilated Acute Exacerbated Chronic Obstructive Pulmonary Disease

N. Kongpolprom ${ }^{1}$; P. Saksripornchai, ${ }^{1}$

${ }^{1}$ Department of internal medicine, Faculty of Medicine, Chulalongkorn University, King Chulalongkorn Memorial Hospital, Bangkok, Thailand Correspondence: N. Kongpolprom

Intensive Care Medicine Experimental 2020, 9(1): 001262

Introduction. Weaning failure is common in mechanically ventilated patients with acute exacerbation of chronic obstructive pulmonary disease (AECOPD). Diaphragmatic dysfunction remains the main cause of weaning difficulty or failure. Serial ultrasonographic measurements of diaphragmatic function could be used to predict the weaning outcomes in AECOPD.

Objectives. To determine ultrasonographic parameters of the diaphragmatic function, which were associated with the weaning success in the AECOPD patients.

Methods. A multicenter prospective observational study was conducted. The invasively ventilated AECOPD patients who got ready to wean were enrolled. After a successful 30-min spontaneous breathing trial (SBT), the patients were extubated and noninvasive ventilation prophylaxis was provided for at least $4 \mathrm{~h}$. Diaphragmatic excursion (DE) and diaphragm thickness fraction (DTF) of the right diaphragm were measured at $30 \mathrm{~min}$ after SBT (before extubation) and $4 \mathrm{~h}$ after extubation. The 48-h extubation outcomes were observed. The association between these ultrasonographic parameters and the weaning outcomes was analyzed.

Results. A total of 22 patients with a mean age of 76.59(9.16) years were recruited. The majority of them were male (86.4\%). All patients were GOLD $D$ and had a rapid shallow breathing index (RSBI) of $<105$. Three of them had extubation failure at the 6th, 10th, and 13th hours. Pre-extubated DTF (DTFpre) and post-extubated DTF (DTFpost) were significantly associated with the weaning success with the cut-off values of more than $30.25 \%$ and $30.5 \%$, respectively. The odds ratios for weaning success of DTFpre and DTFpost were 65 (95\%Cl: 2.04 to $2070, p=0.018)$ and $36(95 \% \mathrm{Cl}: 1.57$ to 826.12 , $\mathrm{p}=0.025)$, respectively. In contrast, pre and post-extubated DE were not associated with the weaning outcomes.

Conclusion. DTFpre and DTFpost were the reliable parameters for the prediction of the weaning success in mechanically ventilated COPD patients. Future studies in a large population are needed to confirm our results.

\section{Reference(s)}

1. Marchioni A, Castaniere I, Tonelli R, Fantini R, Fontana M, Tabbi L, et al. Ultrasound-assessed diaphragmatic impairment is a predictor of outcomes in patients with acute exacerbation of chronic obstruc tive pulmonary disease undergoing noninvasive ventilation. Crit Care 2018;22(1):109

2. Fayed AM, Abd El Hady MA, Shaaban MS, Fikry DM. Use of ultrasound to assess diaphragmatic thickness as a weaning parameter in invasively ventilated chronic obstructive pulmonary disease patients. J Am Sci. 2016;12(6): 96-105.

3. None

001269

Higher versus lower PEEP "dose" as a risk factor for barotrauma during Non-Invasive Ventilation (NIV) in COVID-19 patients

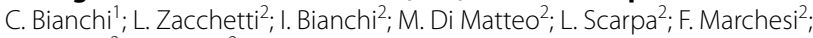
L. Grazioli²; FL. Lorini

${ }^{1}$ Department of critical care and anesthesia, University of Milan, Milano, Italy; ${ }^{2}$ Department of critical care and anesthesia, ASST Papa Giovanni XXIII, Bergamo, Italy

Correspondence: C. Bianchi

Intensive Care Medicine Experimental 2020, 9(1): 001269

Introduction. NIV [including CPAP (continuous positive airway pressure) or bilevel NIV] has been widely used as first line respiratory support therapy in patients affected by Covid-19 acute respiratory failure (1). However, while limiting tidal volume and plateau pressure have been proved to reduce mortality during invasive mechanical ventilation (2), a safe PEEP cutoff for NIV is still unknown. Concerns were recently raised about the detrimental effect on lung injury of combining positive pressure applied by NIV and negative inspiratory transthoracic pressure (3). Our hypothesis is that high PEEP dose (defined as both the level of PEEP applied $(\mathrm{cmH2O})$ and the duration of treatment) used during NIV might be associated with the occurrence of barotrauma.

Objectives. To evaluate the association between high PEEP dose during NIV and barotrauma in patients affected by acute respiratory failure COVID-related.

Methods. We retrospectively analyzed 185 consecutive patients admitted to our ICU from February 22th to May 16th 2020, affected by acute respiratory failure COVID-related, who underwent a period of NIV before intubation. Age, sex, $\mathrm{BMI}$ and $\mathrm{PaO} 2 / \mathrm{FiO} 2$ were collected at ICU admission. PEEP values during NIV were recorded daily, and the mean PEEP was calculated for each patient. Days spent on NIV were also recorded. Barotrauma occurring during NIV period was confirmed by radiological evidence of pneumothorax, pneumomediastinum or subcutaneous emphysema. Cohort was divided into four groups, based on distribution of PEEP and days spent on NIV: LP/ST, LP/LT, HP/ 
ST, HP/LT [where low PEEP (LP) and high PEEP (HP) were $\leq$ and $>$ than median PEEP; short time (ST) and long time (LT) were $\leq$ and $>$ than median days spent on NIV]. Fisher's exact test was used to compare the occurrence of barotrauma and mortality at 6 months in the four groups.

Results. Patients enrolled had 61 (54-66) years old, BMI of 28 (25-31) and $\mathrm{PaO} 2 / \mathrm{FiO}_{2}$ of 105 (84-138) at admission to ICU. Median values of Peep and days spent on NIV were $14 \mathrm{cmH} 2 \mathrm{O}$ and 3 days, respectively: HP and LP included patients with PEEP $>$ and $\leq 14 \mathrm{cmH} 2 \mathrm{O}$; LT and ST included patients who spent $>$ or $\leq 3$ days on NIV. Barotrauma occurred in $23(12 \%)$ patients; its incidence was statistically higher in patients receiving lower PEEP for longer period of time (LP/LT group) (Table 1). Mortality at 6 months was not different between groups.

\begin{tabular}{llllll}
\hline & $\begin{array}{l}\text { LP/ST } \\
(\mathbf{n = 3 7 )}\end{array}$ & $\begin{array}{l}\text { LP/LT } \\
(\mathbf{n = 5 6 )}\end{array}$ & $\begin{array}{l}\text { HP/ST } \\
(\mathbf{n = 4 3 )}\end{array}$ & $\begin{array}{l}\text { HP/LT } \\
(\mathbf{n = 4 9 )}\end{array}$ & p value \\
\hline Age (years) & $59(54-73)$ & $63(56-69)$ & $58(51-66)$ & $61(54-66)$ & 0.16 \\
Male, n (\%) & $27(73)$ & $46(82)$ & $33(77)$ & $36(73)$ & 0.68 \\
$\mathrm{BMl}$ & $29(26-31)$ & $27(24-30)$ & $30(25-35)$ & $28(26-33)$ & 0.08 \\
$\mathrm{PaO} 2 / \mathrm{FiO} 2$ & 114 & 105 & 100 & 105 & 0.42 \\
& $(90-156)$ & $(79-144)$ & $(72-132)$ & $(81-131)$ & \\
$\begin{array}{c}\text { Barotrauma, } \\
\mathrm{n}(\%)\end{array}$ & $1(3)$ & $14(25)$ & $3(7)$ & $5(10)$ & 0.005 \\
$\begin{array}{c}\text { Mortality at } \\
\text { 6 months, } \\
\mathrm{n}(\%)\end{array}$ & $13(37)$ & $21(44)$ & $17(41)$ & $22(45)$ & 0.90 \\
\hline
\end{tabular}

Conclusion. In patients affected by acute respiratory failure COVIDrelated and managed in NIV before intubation, high PEEP applied for long time is not associated to the risk of barotrauma. Further studies should investigate if a long applied low level of PEEP might be inadequate to improve patient work of breathing and gas exchange, thus contributing to excessive respiratory efforts and barotrauma.

\section{Reference(s)}

1. 3.Patient self-inflicted lung injuri and positive end-expiratory pressure for safe spontaneous breathing, Takeshi Yoshida Domenico L. Grieco, Laurent Brochard and Yuji Fuijino Curr Opin Crit Care 2020.

2. Epidemiology, patterns of care, and mortality for patients with acute respiratory distress syndrome in intensive care units in 50 countries. Bellan G., Laffey J.G., Pham T.et al., JAMA. 2016.

3. Noninvasive Ventilatory Support of COVID-19 Patients Outside the Intensive Care Units (WARd-COVID), Bellani G., Grasselli, G., Cecconi, M., Antolini, L., Borelli, M., De Giacomi, F., et al., Ann Am Thorac Soc. 2021

\section{5}

Outcome \& Safety Of Percutaneous Tracheostomy In Coronavirus Disease 2019 Patients: A Single Centre Experience

M. SHAH ${ }^{1}$; N. Bhatuka ${ }^{1}$; K. Shalia

${ }^{1}$ Critical care medicine, Sir H. N. Reliance Foundation Hospital and Research Centre, Mumbai, India; ${ }^{2}$ Medical research society, Sir H. N. Reliance Foundation Hospital and Research Centre, Mumbai, India Correspondence: M. SHAH

Intensive Care Medicine Experimental 2020, 9(1): 001275

Introduction. The COVID-19 pandemic has led to an unprecedented increase in the number of patients who are critically ill and require mechanical ventilation (MV). A shorter ICU stay and the opportunity to save operating room resources are particularly valuable when ICUs have insufficient beds. Tracheostomy is helpful in prolonged ventilatory support for liberation of patient from ventilator and decrease stay in ICU. However, in COVID pneumonia, guidelines on timing, method of tracheostomy are evolving.

Objectives. Retrospective analysis outcomes of Covid-19 pneumonia patients who required tracheostomy \& the safety with regards to the risk of transmission to the healthcare workers during the last thirteen months.

Methods. All admitted coronavirus disease 2019 (COVID 19) patients in the ICU requiring ventilatory support more than seven days were monitored in regards to admission CT severity, CORAD score, P/F ratio, duration of intubation, timing of tracheostomy, mean duration of weaning from ventilatory support (/on MV post tracheostomy), ventilator free days (VFD) and outcome at 30 days in regards to survival. We also screened the health care worker directly involved in tracheostomy care acquiring covid-19, by assessing them clinically for $\mathrm{s} / \mathrm{s}$ of fever/breathlessness and also subjected them to a rapid antigen test every alternate days and RT-PCR every 5th day. Categorical variables (Frequency [\%]) between groups were analysed by Chi-Square test and continuous data (Medians [25th/75th quartiles]) was compared by Mann Whitney $U$ test using SPSS version 21.

Results. In last thirteen months 226 patients were ventilated out of 662 admitted and a total 68 of these were ventilated for more than seven days. Of these 68 intubated patients, 42 continued with endotracheal intubation-(non-tracheostomy group), while the rest 28 underwent tracheostomy. Between tracheostomy vs. non-tracheostomy groups of patients predominately with severe COVID pneumonia, high CORADS score (median $5 / 5$ ) and low P/F ratio (median $<100$ ), we found no differences in age, gender and comorbidities. However, 30 days survival outcome was significantly higher in the tracheostomy vs. non-tracheostomy group (survivor/non survivors) $21 / 7$ vs. $11 / 31$ $(p=0.000059)$. We further analysed tracheostomy group of patients $(\mathrm{N}=15)$ of first wave and compared final outcome at 30 days with second wave (Mid-February- ongoing) $(\mathrm{N}=13)$ (Table 1). In the first wave $60 \%$ of the tracheostomies performed in $\leq 13$ days from the initiation of MV did not differ from $>13$ days with regards to age, gender, comorbidities, CORADS score and P/F ratio. Although the final outcome of survival of patients at 30 days between tracheostomy performed $<=13$ days and $>13$ days was 66.7 vs.83.3\%, (NS) while patients discharged alive from hospital were $55.6 \%$ and $33.3 \%$ respectively. Their median duration on MV post tracheostomy was 14 while VFD were 26.5. As the pandemic moved on to the second wave since mid-February 2021, we have been seeing variable presentation \& huge rise in severe acute respiratory failure (ARDS) with $\mathrm{P} / \mathrm{F}$ ratio $<100$. All these patients underwent tracheostomy before 13 days and in spite of severe disease we found an $80 \%$ survival rate at 30 days. However, data on hospital discharge alive is still awaited. During the first wave we learned the importance of tracheostomy with $70 \%: 80 \%$ survival rates in severe to moderate ARDS. During the second severe wave, tracheostomy $>13$ days of Intubation achieved 100\% 30 days survival outcome (Table 1).

$\mathrm{P} / \mathrm{F}<100 . \mathrm{P} / \mathrm{F}>100 \mathrm{P} / \mathrm{F}<100 . \mathrm{P} / \mathrm{F}>100$

Wave. 101 st wave 510 2nd Wave 3 .

Survived $7(70 \%)(\mathbf{N}=11) 4(80 \%) 8(80 \%)(\mathbf{N}=10) 2(66.7 \%)$.

$\mathbf{T}<13$ Day $3(42.9 \%)(\mathbf{N}=6) 3(75 \%) 8(100 \%)(\mathbf{N}=10) 2(66.7 \%)$.

$\mathbf{T}>13$ Day $4(57.1 \%)(\mathbf{N}=\mathbf{5}) 1(25 \%)$.

We believe in our experience of 68 COVID Pneumonia patients 77\% had severe ARDS who had a better 30 days survival (75\%).Average time to undergo tracheostomy was 12 th day after intubation.None of our health care workers were infected with Covid 19 infection.

Conclusion. We believe that Percutaneous tracheostomy is safe option and must be considered within 13 days of Intubation especially in the severe Covid pneumonia group of patients.

\section{Reference(s)}

a. Timing of Tracheostomy for Patients With COVID-19 in the ICU—Setting Precedent in Unprecedented Times: JAMA Otolaryngology-Head \& Neck Surgery October 2020 Volume 146, Number 10887.

i. Tay JK, Khoo ML-C, Loh WS. Surgical considerations for tracheostomy during the COVID-19 pandemic. JAMA Otolaryngol. Head Neck Surg. 2020;146(6):517-518. 10.1001/jamaoto. 2020.0764

4. 2) Rosano et al., -Early Percutaneous Tracheostomy in Coronavirus Disease 2019: Association With Hospital Mortality and Factors Associated With Removal of Tracheostomy Tube at ICU Discharge. A Cohort Study on 


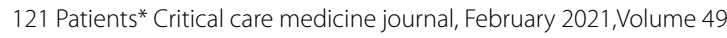
number 2, page 261,https://doi.org/10.1097/CCM.0000000000004752

5. Safety and 30-day outcomes of tracheostomy for COVID-19: a prospective observational cohort study. British Journal of Anaesthesia, 125 (6): 872e879 (2020). https://doi.org/10.1016/j.bja.2020.08.023

6. None

\section{2}

Non-invasive Respiratory Support in COVID-19 Patients with Acute Respiratory Failure

J. Thompson ${ }^{1}$; C. Leech, ${ }^{1}$; A. Morrison, ${ }^{1}$; A. Roy, ; A. Rostron,

1 Integrated critical care unit, Sunderland Royal Hospital, Sunderland,

United Kingdom

Correspondence: J. Thompson

Intensive Care Medicine Experimental 2020, 9(1): 001282

Introduction. Use of non-invasive respiratory support (NIRS) in influenza associated acute respiratory failure (ARF) is controversial[1] with a success rate of 40.7-43.2\%[2][3]. NIRS failure is associated with similar, possibly worse, mortality compared to invasive mechanical ventilation (IMV) only. Factors for success include lower APACHE II scores, haemodynamic stability, and absence of acute renal failure[2][3].

Objectives. Assess the rate of NIRS failure in those with COVID-19 ARF and its associated factors and outcomes.

Methods. Retrospective data collected from COVID-19 ICU patients admitted from 19/9/20 until 31/1/21. Data included age, gender, $\mathrm{PiO} 2 / \mathrm{FiO} 2(\mathrm{P} / \mathrm{F})$ ratios, APACHE II scores and therapy received- NIRS (CPAP and high-flow nasal cannula), IMV and NIRS to IMV. Outcomes included survival, barotrauma (pneumothorax, pneumomediastinum and surgical emphysema) and CVS/renal organ support.

Results. 122 patients were admitted with COVID-19 infection of which $91(74.5 \%)$ received initial NIRS and 58 (63.7\%) of these failed NIRS and progressed to IMV. 10 patients were lost to follow-up due to transfer to other hospitals.

Table 1-Demographics and outcomes of COVID-19 patients based on therapy received

\begin{tabular}{llll}
\hline & $\begin{array}{l}\text { NIRS only } \\
(\boldsymbol{n}=\mathbf{3 3})\end{array}$ & $\begin{array}{l}\text { NIRS to IMV } \\
(\boldsymbol{n}=\mathbf{5 0})\end{array}$ & $\begin{array}{l}\text { IMV Only } \\
(\boldsymbol{n}=\mathbf{1 8})\end{array}$ \\
\hline Alive (\%) & $29(87.9 \%)$ & $18(36 \%)$ & $4(22.2 \%)$ \\
Age (Mean (SD)) & $57.0(12.3)$ & $62.4(11.4)$ & $64.2(10.6)$ \\
Male (\%) & $20(60.6 \%)$ & $30(60.0 \%)$ & $14(77.8 \%)$ \\
APACHE II (Med (IQR)) & $12(9.5,13.5)$ & $15(13.0,18.0)$ & $16(12.0,20.3)$ \\
P/F ratios (Med (IQR)) & $12.9(9.7,15.5)$ & $9.9(8.6,12.7)$ & $9.5(8.3,13.3)$ \\
CVS Support (\%) & $0(0 \%)$ & $49(98.0 \%)$ & $16(88.9 \%)$ \\
Renal Support (\%) & $0(0 \%)$ & $18(36.0 \%)$ & $8(44.4 \%)$ \\
Barotrauma (\%) & $0(0 \%)$ & $12(24.0 \%)$ & $2(11.1 \%)$ \\
\hline
\end{tabular}

Table 2-Outcome of non-invasive respiratory support failure based on time of failure

\begin{tabular}{|c|c|c|c|}
\hline & $<24 \mathrm{~h}(n=12)$ & $24-48$ h $(n=20)$ & $>48 \mathrm{~h}(n=18)$ \\
\hline Alive (\%) & $5(41.7 \%)$ & $9(45.0 \%)$ & $5(27.8 \%)$ \\
\hline P/F (Med (IQR)) & $9.1(7.2,11.9)$ & $10.1(9.0,13.1)$ & $10.0(8.5,12.9)$ \\
\hline $\begin{array}{l}\text { APACHE II (Med } \\
\text { (IQR)) }\end{array}$ & $17(13.5,18.8)$ & $16(12.5,18.8)$ & $14.5(12.8,16.3)$ \\
\hline CVS (\%) & $12(100 \%)$ & 19 (95.0\%) & $18(100 \%)$ \\
\hline RRT (\%) & $6(50 \%)$ & $6(30.0 \%)$ & $6(33.3 \%)$ \\
\hline Barotrauma (\%) & $1(8.3 \%)$ & $3(15.0 \%)$ & $8(44.4 \%)$ \\
\hline
\end{tabular}

COVID-19 ARF patients had a higher rate of NIRS failure (63.7\%) compared to NIRS failure in other influenza associated ARF (56.8-59.3\%)[2] [3]. Those who failed NIRS had higher APACHE II scores, lower P/F ratios and increased cardiovascular/renal support needs-similar to other studies[4].

NIRS failure patients had higher rates of barotrauma and recent literature has noted an increase in NIRS and IMV associated barotrauma in COVID-19[5]. In the NIRS failure group, late failure ( $>48 \mathrm{~h}$ ) had lower APACHE II scores and an increased rates of death and barotrauma.

Conclusion. There is a high rate of NIRS failure in COVID-19 which is associated with higher APACHE II scores, lower P/F ratios and increased organ support needs. Late NIRS failure $(>48 \mathrm{~h})$ is associated with worse mortality and barotrauma.

\section{Reference(s)}

1. [1]Bellani G et al. Epidemiology, Patterns of Care and Mortality for Patients With Acute Respiratory Distress Syndrome in Intensive Care Units in 50 Countries. JAMA. 2016 Feb 26:315(8):788-800.

2. [2] Rodríguez A et al. Risk Factors for Noninvasive Ventilation Failure in Critically III Subjects with Confirmed Influenza Infection. Respiratory Care. 2017;62(10)1307-1315.

3. [3] Masclans JR et al. Early non-invasive ventilation treatment for severe influenza pneumonia. Clinical Microbiology and Infection. 2012;19(3):249-256.

4. [4] He et al. A multicentre RCT of noninvasive ventilation in pneumoniainduced early acute respiratory distress syndrome. Critical Care. 2019 Sep 4;23(1):300.

5. [5] Khan MR et al. High Incidence of Barotrauma in Patients with Severe Coronavirus Disease 2019. Journal of Intensive Care Medicine. 2021 Mar $15: 885,066,621,989,959$.

001292

Physiological response of prone positioning in intubated adults with severe COVID-19 acute respiratory distress syndrome: a retrospective study

A. Boffi'; M. Ravenel' ${ }^{2}$; E. Lupieri'; JD. Chiche'; L. Piquilloud'

${ }^{1}$ Adult intensive care unit, CHUV Centre Hospitalier Universitaire Vau-

dois, Lausanne, Switzerland; ${ }^{2}$ Faculty of biology and medicine, University of Lausanne, Lausanne, Switzerland

Correspondence: A. Boffi

Intensive Care Medicine Experimental 2020, 9(1): 001292

Introduction. Prone positioning is recommended for COVID 19 moderate to severe acute respiratory distress syndrome (ARDS) in intubated patients, but few data are available.

Objectives. The study aim was to assess the effect of proning on gas exchanges, respiratory system compliance (CRS) and ventilatory ratio (VR) and to analyze the relationship between response to proning and outcome.

Methods. Retrospective analysis of the patients intubated and proned in the Lausanne Adult ICU for COVID 19 ARDS between March 06 and May 30 2020. Patients' characteristics, blood gas analysis at admission and number and duration of the prone position sessions were recorded. Ventilator settings, gas exchanges, CRS and VR were recorded before proning and before returning to supine position. Response in term of oxygenation was defined as increase in $\mathrm{PaO} 2 /$ $\mathrm{FiO} 2>20 \%$. Treatment failure was defined as death or start of extracorporeal membrane oxygenation (ECMO). Results expressed as median (IQR).

Results. 42 patients (71\% of males) were included. Age 63 (57-62) years, SAPSII 41 (34-46) and SOFA 7 (6-8). At intubation, PaO2/FiO2 was $137 \mathrm{mmHg}(118-172)$, alveolo-arterial gradient (AaO2) $255 \mathrm{mmHg}$ (200-345), CRS $36.4 \mathrm{ml} / \mathrm{CmH} 2 \mathrm{O}$ (29.3-42.5), VR 1.7 (1.3-2.2). 191 sessions of prone positioning were performed. Time from the first $\mathrm{PaO} 2 /$ $\mathrm{FiO} 2<150 \mathrm{mmHg}$ with $\mathrm{FiO} 2>0.6$ to first proning was $16(5-36)$ hours. Number of sessions per patient was $3(2-6)$ and duration 17 (16-19) hours. For all proning sessions $\mathrm{PaO} 2 / \mathrm{FiO} 2$ increased from 107 (90-129) to $180(148-210) \mathrm{mmHg}$, FiO2 decreased from $0.6(0.5-0.7)$ to 0.4 $(0.35-0.5)(p<0.0001$ for both). $\mathrm{PaO} 2 / \mathrm{FiO} 2$ increase $>20 \%$ was found in $83 \%$ of proning. $\mathrm{AaO} 2$ decreased from 275 (212-334) to 127 (92-176) 
$(p<0.0001)$. CRS increased from $32.3(27.7-40.8)$ to $36.2(30-41.7)$ $(p=0.003)$ and VR from $2.3(1.9-2.8)$ to $2.4(2.0-2.9)(p=0.03)$ (Fig. 1). Two (5\%) patients needed ECMO support and survived. Ten patients died (24\%). Patients in treatment failure group compared to success group were older (73 (61-78) vs 60 (56-69), $p=0.009)$, had more often a $\mathrm{BMI}>30 \mathrm{~kg} / \mathrm{m} 2(47 \mathrm{vs} \%, \mathrm{p}=0.03)$ and had less frequently a positive response in term of oxygenation to the first proning session (67 vs $97 \%, p=0.02$ ).

Conclusion. Prone positioning improved $\mathrm{PaO} 2 / \mathrm{FiO} 2$ and $\mathrm{AaO} 2$ gradient. CRS and VR only slightly increased suggesting that the main effect is improvement in ventilation/perfusion ratio.

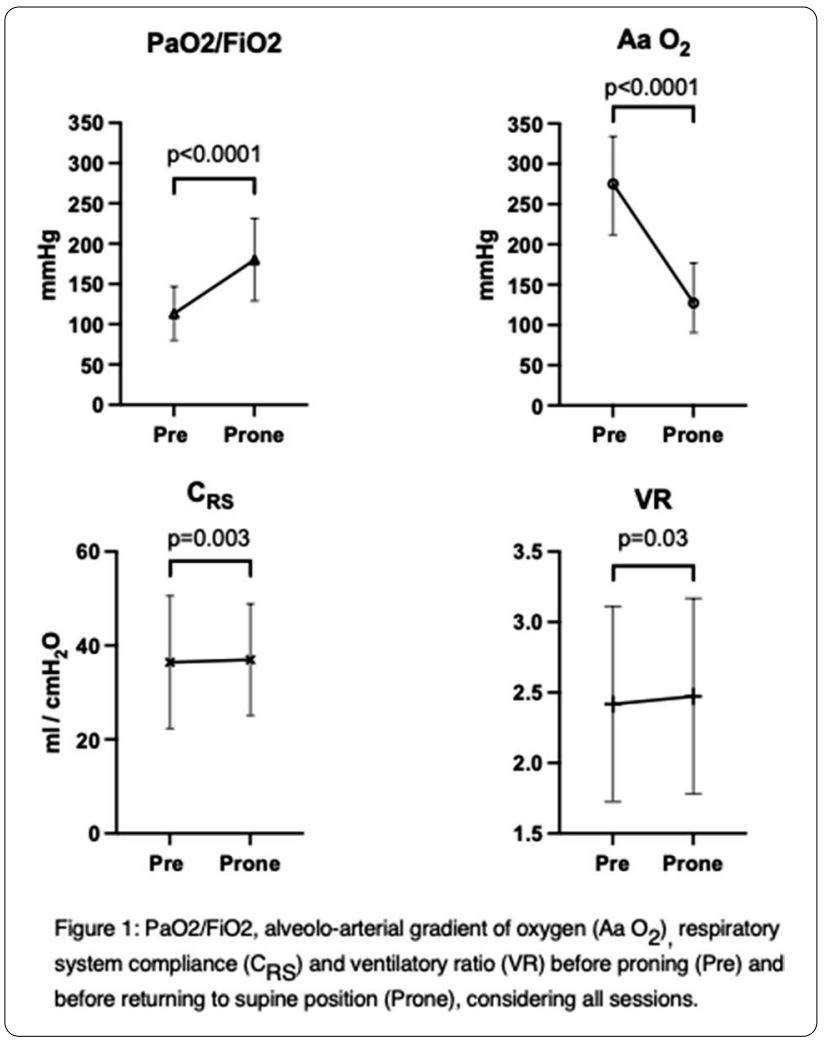

\section{Reference(s)}

1. Alhazzani W. et al., Surviving Sepsis Campaign: guidelines on the management of critically ill adults with Coronavirus Disease 2019 (COVID-19), ICM, 2020:46:854-887

2. Weiss T. et al., Prone positioning for patients intubated for severe acute respiratory distress syndrome (ARDS) secondary to COVID-19: a retrospective observational cohort study, BJA, 2021:126(1):48-55

\section{5}

Prone positioning in COVID-19: self-selected higher risk group? N. Wetherall ${ }^{1}$; S. Price ${ }^{1}$; S. Ramachandran ${ }^{1}$; W. Tuckwell ${ }^{1}$; H. Breen ; E. Harte ${ }^{1}$; T. Szakmany ${ }^{1}$

${ }^{1}$ Department of anaesthesia, intensive care and pain medicine, Cardiff University, Cardiff, United Kingdom

Correspondence: $T$. Szakmany

Intensive Care Medicine Experimental 2020, 9(1): 001295

Introduction. The use of prone position ventilation in COVID19 ARDS has been supported by prior evidence. Prone ventilation has been traditionally used as a rescue therapy in ARDS. There is conflicting evidence if the more widespread use of this modality has improved outcome in COVID19 ARDS.
Objectives. To evaluate the if prone positioning is applied to a selfselected group of patients with higher risk of death.

Methods. Single centre, retrospective study conducted during the second wave of COVID19 pandemic in Wales (15/11/2020$15 / 03 / 2021)$. Consecutive patients admitted to the Grange University Hospital intensive care unit were recruited. Demographics, ventilation and other organ support parameters and inflammatory markers were evaluated. For statistical analysis chi-square test and Mann-Whitney U test was used as appropriate. Data is presented as numbers, percentages or median [IQR] as appropriate.

Results. 121 patients were admitted during this period. 28 patients (23.1\%) did not need mechanical ventilation (MV). Out of the 93 patients on MV, 52 (55.9\%) were proned. Prone positioning was predominantly used in the first one week of treatment. There was no significant difference between the patients who were proned or not in terms of gender ( $23.1 \%$ vs $22.0 \%$ female), age (61 [51-69] vs 62 [57-71] years), comorbidity prevalence, or frequency of pulmonary embolism. Patients who were proned were ventilated with significantly higher PEEP (12 [10-14] $\mathrm{cmH} 2 \mathrm{O}$ vs 10 [8-12] $\mathrm{cmH} 2 \mathrm{O}) \mathrm{p}=0.009$, but at similar FiO2 (0.80 [0.65-0.90] vs 0.70 [0.50-0.90]) achieving similar $\mathrm{PaO} 2(9.7$ [7.5-11.5] $\mathrm{kPa}$ vs 10.5 [10.0-14.7] $\mathrm{kPa}$. Inflammatory markers such as CRP, PCT, Ferritin and Troponin levels and SOFA score was also similar at baseline.

Patients who were proned had signifcantly longer ICU stay (10.1 [7.917.0] days vs 5.8 [3.9-15.4] days), spent longer time on MV (9.0 [6.0$15.5]$ days vs 4.0 [2.0-8.0] days and vasopressor support (1.5 [0.0-5.0] days vs 0.0 [0.0-0.0] days) all $p<0.05$. Use of renal replacement therapy $(9.6 \%$ vs $14.6 \% \mathrm{p}=0.67)$ and pulse dose steroid $(30.7 \%$ vs $19.5 \%$ $\mathrm{p}=0.32$ ) was similar in the prone and not proned groups.

Hospital mortality was $61.5 \%$ vs $48.8 \% p=0.31$ in proned and not proned patients respectively.

Conclusion. In our retrospective series, prone position use was prevalent in patients with COVID19 ARDS on MV. There were no significant differences between the proned and not proned groups at baseline, however those patients who have treated with prone positioning had significantly longer ICU stay and length of MV. Despite the similar demographics, comorbidities, physiological parameters and inflammatory markers, it is plausible that patients needing prone positioning were at higher risk. However, it is also possible that using this modality necessiated more sedatives and muscle relaxants, thereby increasing the risk of critical illness polyneuropathy, weakness and delirium, leading to longer ICU stay. Further studies are required to elucidate if there are any easy to measure paramters at the bedside, which could stratify which patients would benefit from prone positioning in COVID19 ARDS.

\section{2}

Tocilizumab in critically ill patients with COVID-19: yes,

\section{with a word of caution}

M. Adao-Serrano ${ }^{1}$; M. Maneira Sousa ${ }^{1}$; R. Costa-Reis ${ }^{1}$; SM. Fernandes, ${ }^{1}$;

JL. Gouveia, '; JM. Ribeiro,

${ }^{1}$ Serviço de medicina intensiva, Centro Hospitalar Universitário Lisboa

Norte, Lisbon, Portugal

Correspondence: M. Adao-Serrano

Intensive Care Medicine Experimental 2020, 9(1): 001302

Introduction. Interleukin 6 is a potent pro-inflammatory cytokine implicated in immunologic response in infection. A hyper-inflammatory syndrome has been described in COVID-19. With this rationale, in March 2020 we approved an institutional protocol to administer tocilizumab, an IL-6 inhibitor, in critically ill patients meeting predetermined clinical and lab criteria.

Methods. We performed a retrospective matched case-control study, approved by our ethics committee comparing patients in the tocilizumab protocol with patients who were not, matched for SAPS-2 and day of illness at tocilizumab inclusion, with a ratio of 1:3. Clinical, demographic and lab data was collected using our electronic database. Data gathered for the control arm was matched at the same illness timepoints at which tocilizumab was administered for the intervention arm. Inclusion criteria for tocilizumab were COVID-19 
infection, worsening hypoxaemia, $\geq 3$ days of symptomatic disease, IL- $6 \geq 40 \mathrm{mg} / \mathrm{dl}$, ferritin $\geq 1200 \mathrm{ng} / \mathrm{ml}$ or fibrinogen $\geq 500 \mathrm{mg} / \mathrm{dl}$ or D-Dimers $\geq 1 \mathrm{ng} / \mathrm{ml}$.

Results. From April to July 2020, we administered tocilizumab to 14 patients, with mean age $59.4 \pm 12.31$ years, mainly male, with $\mathrm{PaO} 2 /$ FiO2 at ICU admission of $120 \mathrm{mmHg}(91.5 ; 136.5)$. At the time of tocilizumab infusion, 5 patients were not mechanically ventilated, mean SAPS II was $32.43 \pm 9.36$. We compared the results with a matched cohort $(n=42)$ with mean age $63.9 \pm 12.44$ years and SAPS II $34.9 \pm 8.3$, mostly male.

In the tocilizumab (T) arm, $64.3 \%$ patients were mechanically ventilated vs $73.8 \%$ in the control (C) arm. Delta $\mathrm{PaO} 2 / \mathrm{FiO} 2$ at day 0 (D0) and day 3 (D3) was not of statistical significance in both. $35.7 \%$ of patients in the $\mathrm{T}$ arm required Extracorporeal Membrane Oxygenation vs $14.3 \%(p=0.1193)$. Delta lung compliance at D0 and D3 was not statistically significant.

There was no statistically significant difference in ICU length of stay ( $T$ group $28.71 \pm 14.9 ; C$ group $28.25 \pm 23.43 p=0.95$ ), as with ventilator free days ( $T$ group $11.07 \pm 9.29 ; 12.24 \pm 11.65 \mathrm{p}=0.74$ ). Delta CRP at D0 and D3 showed a statistically significant difference in the T group $(-19.45 \pm 10.21$ vs $-2.8 \pm 13.3 ; p=0.000092)$, with a rise in delta serum IL-6 levels at the same time-point (D0 155.21 \pm 76.77 vs D3 $6807.83 \pm 10,332.48 p=0.048$ ).

During ICU stay, $78.6 \%$ of patients in the T arm had bacterial infections vs $7.1 \%$ in the control group ( $p<0,00,001), 28.6 \%$ vs $4.8 \%(p=0.0292)$ cases of septic shock respectively. Despite an increase in infection rate, there was no statistical difference in mortality between groups ( $T$ $14.3 \%$ vs. C $28.25 \%, p=0.47$ ).

Conclusion. Our tocilizumab protocol was instituted before clinical trial data was available. Relatively low mortality within the $T$ arm was observed, despite comparable SAPS-II, respiratory failure severity and increased infection rate. With caution in respect to infecton, these results open the possibility to an eventual gain in survival in selected populations, in line with recent studies. More data is necessary to personalize tocilizumab treatment in COVID-19.

\section{3}

\section{Mortality Risk in Covid-19 Veno-Venous Extracorporeal} Membrane Oxygenation-Experience of a Portuguese Tertiary Hospital

F. Sequeira, ${ }_{1}^{1}$, L. Linhares, ${ }_{1}^{1}$; A. Santos, ${ }_{1}^{1}$; C. Monteiro, ${ }^{1}$; A. Marques, ${ }^{1}$; S. Teixeira, ${ }^{1}$; E. Trigo, ; M. Simões, ; M. Miranda, ; A. Salgueiro, ; R. Freitas, ${ }^{1}$; E. Sousa, ; P. Martins,

${ }^{1}$ Department of intensive care, Centro Hospitalar e Universitário de Coimbra, Coimbra, Portugal

Correspondence: F. Sequeira

Intensive Care Medicine Experimental 2020, 9(1): 001313

Introduction. A life-threatening complication of COVID-19 is acute respiratory distress syndrome (ARDS) refractory to conventional management. Veno-venous extracorporeal membrane oxygenation (VV-ECMO) is used to support ARDS patients failing conventional management.

Objectives. The aim of the study was to determine predictors of mortality among these patients.

Methods. A retrospective review of COVID-19 VV-ECMO patients, cannulated between April 1, 2020, and April 30, 2021, at a tertiary center. The primary outcome was in-hospital mortality.

Results. 29 COVID-19 patients received VV-ECMO. Of these, 12 (41\%) died on ECMO, 8 (28\%) were discharged to another hospital and 9 $(31 \%)$ to home/acute rehabilitation center.

The median age was 54 years $(41-74)$ and median body-mass index was $34 \mathrm{~kg} / \mathrm{m}^{2}$. Twenty-three (79\%) were men, and $23(79 \%)$ had at least one pre-ECMO comorbidity (66\% hypertension, $59 \%$ obesity, $28 \%$ high cholesterol levels, $7 \%$ acute kidney injury, $3 \%$ pre-ECMO cardiac arrest, 3\% myocarditis).

Ventilator associated pneumonia (VAP) were confirmed in 18 (62\%), aspergillosis in $3(10 \%)$, Staphylococcus aureus, multidrug resistant (MDR) Pseudomonas aeruginosa, Enterococcus faecalis and
Acinetobacter baumannii were the cultured organisms for bloodstream infections $(\mathrm{n}=5 ; 17 \%)$.

Most patients (19 [66\%]) received care at another hospital before being transferred to an ECLS center.

Before ECMO initiation, median $\mathrm{PaO} 2: \mathrm{FiO} 2$ was 83 , all patients received neuromuscular blockade and prone positioning, $16(55 \%)$ patients received non-invasive ventilatory support before endotracheal intubation (EI). The median duration from El to ECMO initiation was 4 days.

After ECMO initiation, the median plateau pressure (Pplat) was reduced by $4 \mathrm{cmH} 2 \mathrm{O}(20-28)$, the median driving pressure (DP) was reduced by $2(10-16)$ and the median $\mathrm{FiO} 2$ was reduced by 0.5 (0.4$0.5)$. The median duration of ECMO support was 23 days.

Nineteen (66\%) patients had complications during ECMO support, mainly thrombotic or hemorrhagic events. Mechanical complications defined as a circuit change, cannula problems or pump failure occurred in $3(10 \%)$.

In patients who died, the median duration from El to ECMO was 4 days and the median duration of ECMO support was 24 days. After ECMO initiation, the median Pplat was $24 \mathrm{cmH} 2 \mathrm{O}$ and the median DP was $13 \mathrm{cmH} 2 \mathrm{O}$. Identified risk factors for mortality were increasing age (median age 62 years), lower $\mathrm{PaO2}: \mathrm{FiO} 2(81)$, hypertension (83\%), bleeding complications (67\%), VAP (67\%) and bloodstream infections (25\%).

Conclusion. Identified risk factors for higher mortality were age, degree of hypoxaemia, bleeding complications, VAP and bloodstream infections. Surprising, the time from El to ECMO, aspergillosis, thrombotic complications, DP and Pplat were not associated with higher mortality. Improvement strategies regarding prevention of infections and bleeding complications might therefore be the most important things to improve outcome in patients with ECMO support.

\section{Reference(s)}

1. Bartlett RH, Ogino MT, Brodie D, et al. Initial ELSO Guidance Document: ECMO for COVID-19 patients with severe cardiopulmonary failure. Asaio J 2020:66:472-4

2. Richardson S, Hirsch JS, Narasimhan M, et al. Presenting characteristics, comorbidities, and outcomes among 5700 patients hospitalized with COVID-19 in the New York City area. JAMA 2020;323:2052-9

3. Yang $X, Y u Y, X u$ J, et al. Clinical course and outcomes of critically ill patients with SARS-CoV-2 pneumonia in Wuhan, China: a singlecentered, retrospective, observational study. Lancet Respir Med 2020; 8 : $475-81$

4. Grasselli G, Zangrillo A, Zanella A, et al. Baseline characteristics and outcomes of 1591 patients infected with SARS-CoV-2 admitted to ICUs of the Lombardy region, Italy. JAMA 2020; 323: 1574-81

001322

Worsened outcome of COVID-19 patients in Intensive Care during the pandemic second surge in cases versus the first C. Murphy'; A. Mc Mahon'; P. Parvanov'; M. Nguyen ${ }^{1}$; M. Pecak'; J. Mulvihill'; R. Dwyer'; G. Curley ${ }^{1}$

${ }^{1}$ Department of anaesthesiology and intensive care, Beaumont Hospital, Dublin, Ireland

Correspondence: A. Mc Mahon

Intensive Care Medicine Experimental 2020, 9(1): 001322

Introduction. Mortality of patients admitted to the intensive care unit (ICU) during the first surge in pandemic cases in 2020 in our hospital (and in Ireland generally) was relatively low; in our hospital ICU mortality was $12 \%$. However our ICU mortality was similar to international norms[1] during the most recent surge in 2021. We present a comparison of demographics and outcomes of COVID-19 patients between patients admitted to our ICU in the first and second surges of the pandemic.

Methods. Our Unit is a 25 -bed ICU in a 820 -bed university affiliated teaching hospital. Data were collected on all COVID patients throughout the period of the pandemic. Variables included patient demographics, interventions in ICU and outcomes. Data were compared between the period of the first surge in COVID admissions (March 1st- July 31st 2020-Surge1) and the period November 22nd-April 
24th 2021 (Surge 2). Data were analysed in Graphpad Prism. The independent $\mathrm{t}$-test and chi-squared test were used to compare means and frequencies of variables.

\section{Results.}

\begin{tabular}{|c|c|c|c|}
\hline & Surge $1(\%) n=48$ & Surge $2(\%) n=73$ & $P$ value \\
\hline Readmissions & 5 & 3 & 0.65 \\
\hline Mean age (yrs) & 54.3 & 59.9 & 0.01 \\
\hline Mean weight (kg) & 99.6 & 94 & 0.31 \\
\hline Female & $7(15)$ & $25(34)$ & 0.02 \\
\hline $\mathrm{HCW}$ & $5(10)$ & $6(8)$ & 0.17 \\
\hline HA COVID & & $14(19)$ & \\
\hline ICU Mortality & $\begin{array}{l}5(12) \\
(n=43)\end{array}$ & $\begin{array}{l}24(37) \\
(n=69)\end{array}$ & $<0.01$ \\
\hline ICU LOS (days) & 19 & 15.8 & 0.29 \\
\hline Hospital Mortality & $\begin{array}{l}5(12) \\
(n=43)\end{array}$ & $\begin{array}{l}25(40) \\
(n=63)\end{array}$ & $<0.01$ \\
\hline Hospital LOS (days) & 47.5 & 33.1 & 0.24 \\
\hline \multicolumn{4}{|l|}{ ICU supports: } \\
\hline IPPV & $46(96)$ & $\begin{array}{l}58(81) \\
(n=72)\end{array}$ & 0.02 \\
\hline IPPV days (mean) & 15.72 & 14.79 & 0.76 \\
\hline Prone position & $33(69)$ & $44(60)$ & 0.34 \\
\hline Tracheostomy & $18(38)$ & $\begin{array}{l}25(36) \\
(n=70)\end{array}$ & 0.84 \\
\hline CRRT & $14(29)$ & $14(19)$ & 0.20 \\
\hline ECMO & $1(2)$ & $3(4)$ & 0.54 \\
\hline
\end{tabular}

Patients in surge 2 were older and more likely to be female. Weight, Healthcare worker (HCW) status and readmission rate was similar between the two groups. The great majority of patients required invasive ventilation in both periods. Similar numbers underwent proning, tracheostomy and transfer for ECMO in both periods. There was a trend to reduced requirement for continuous renal replacement therapy (CRRT) that was not statistically significant. The most striking feature in the data was the greater ICU and hospital mortality in surge 2. ICU and hospital length of stay (LOS) was similar in both periods.

Conclusion. There are a number of possible explanations for the higher mortality in surge 2. A more virulent strain of the virus was circulating in surge 2[2]. ICU bed occupancy was greater which is associated with increased mortality[3,4]. A significant proportion of patients in surge 2 had hospital acquired COVID-19 which meant they already had significant underlying disease and frailty. Surge 2 patients were older which is associated with increased mortality in ICU patients[5]. The routine use of dexamethasone in surge 2 , as well as improvements in care on the ward likely meant only the most severe cases of COVID19 were referred to ICU. The regular use of steroids also increased the incidence of opportunistic infections e.g. aspergillus likely contributing to mortality[6]. These potentials explanations are speculative; Further data will be required to confirm the conclusions drawn.

\section{Reference(s)}

1. Armstrong, R.A., Kane, A.D., Kursumovic, E., Oglesby, F.C. and Cook, T.M. (2021), Mortality in patients admitted to intensive care with COVID-19: an updated systematic review and meta-analysis of observational studies. Anaesthesia, 76: 537-548. https://doi.org/10.1111/anae.15425

2. Davies, N.G., Jarvis, C.I., CMMID COVID-19 Working Group. et al. Increased mortality in community-tested cases of SARS-CoV-2 lineage B.1.1.7. Nature 593, 270-274 (2021). https://doi.org/10.1038/s41586-021-03426-1

3. Bravata DM, Perkins AJ, Myers LJ, et al. Association of Intensive Care Unit Patient Load and Demand With Mortality Rates in US Department of Veterans Affairs Hospitals During the COVID-19 Pandemic. JAMA Netw Open. 2021;4(1):e2034266. 10.1001/jamanetworkopen.2020.34266
4. Dale, C.R., Starcher, R.W., Chang, S.C. et al. Surge effects and survival to hospital discharge in critical care patients with COVID-19 during the early pandemic: a cohort study. Crit Care 25, 70 (2021). https://doi.org/10.1186/ s13054-021-03504-w

5. Guillon, A., Laurent, E., Godillon, L. et al. Long-term mortality of elderly patients after intensive care unit admission for COVID-19. Intensive Care Med (2021). https://doi.org/10.1007/s00134-021-06399-x

6. Mitaka, H, Kuno, T, Takagi, H, Patrawalla, P. Incidence and mortality of COVID-19-associated pulmonary aspergillosis: A systematic review and meta-analysis. Mycoses. 2021;00: 1- 9. https://doi.org/10.1111/myc. 13292

\section{8}

Prognostic scores validation prior to venovenous ECMO implantation

J. Navarro-Rodriguez'; M. Mendoza-Prieto ; FJ. Reina-Martínez'; I. Espinosa-Rueda ${ }^{1}$; Y. Corcia-Palomo, ${ }^{1}$; L. Martin-Villen ${ }^{1}$

${ }^{1}$ Critical care unit, Hospital Universitario Virgen del Rocío, Sevilla, Spain

Correspondence: M. Mendoza-Prieto

Intensive Care Medicine Experimental 2020, 9(1): 001328

Introduction. Several studies have shown benefit among patients with severe acute respiratory distress syndrome (ARDS) who received ECMO. Despite the improvement observed in survival rates, ECMO support is still associated with high rates of complications. Therefore, it is important to assign these high-risk interventions to those patients in whom there is a greater impact on health and quality of life.

Objectives. To validate prognostic scores of survival in patients with severe ARDS who require support with veno-venous ECMO (VV-ECMO).

Methods. Retrospective, observational analytic study from 2017 to 2020 in an Intensive Care Unit (ICU). All patients with acute respiratory failure who required VV-ECMO were included. Demographic data, cardiovascular risk factors, ARDS causes, clinical management prior to device implantation, SOFA, RESP and PRESET scores and outcomes from each patient were collected. Frequencies were calculated for qualitative variables; as well as median and interquartile range (IR) for quantitative data. Chi-Squared test for qualitative variables and $U$ Mann Whitney $\mathrm{U}$ test for quantitative ones were performed.

Results. A total of 16 patients were included. Viral infection was the most frequent cause of ARDS requiring VV-ECMO (37,5\%), followed by bacterial pneumonia (25\%), fat embolism $(12,5 \%)$, alveolar hemorrhage $(6,3 \%)$ and other causes $(18,7 \%)$. Patient characteristics and prognostic scores are detailed in Table 1. Lower RESP and higher PRESET scores have shown a statistically significant association with improved ICU survival. No differences were found among deceased and survivors patients regarding the SOFA score.

Table 1. Differences between groups of surviving and deceased patients. BMI (body mass index)

\begin{tabular}{llll}
\hline Variables & $\begin{array}{l}\text { Deceased } \\
(\mathbf{n = 9})\end{array}$ & $\begin{array}{l}\text { Survived } \\
\mathbf{( 6 )}\end{array}$ & $\mathbf{p}$ \\
\hline Male; $\mathbf{n}(\%)$ & $8(88.9)$ & $4(57.1)$ & 0.2 \\
Age; median (IR) & $57(51,60)$ & $42(19,59)$ & 0.15 \\
Hypertension; $\mathbf{n ( \% )}$ & $6(66.7)$ & $1(14.3)$ & 0.06 \\
$\begin{array}{l}\text { Diabetes mellitus; } \mathbf{n ( \% )} \\
\text { BMI> 30; } \mathbf{n}(\%)\end{array}$ & $2(22.2)$ & $0(0)$ & 0.3 \\
PreECMO days on Mechanical venti- & $5(3,7)$ & $2(1,4)$ & $\mathbf{0 . 0 2}$ \\
$\quad$ lation; median (IR) & $5(55.6)$ & $4(57.1)$ & 0.5 \\
Prone sessions; $\mathbf{n}(\%)$ & $5(55.6)$ & $7(71.4)$ & 0.4 \\
$\begin{array}{l}\text { PROGNOSTIC SCORES; } \\
\text { median (IR) }\end{array}$ & & & \\
SOFA Score & $12(9,12)$ & $9(5,12)$ & 0.2 \\
RESP Score & $-1(-3,2)$ & $4(1,6)$ & $\mathbf{0 . 0 1}$ \\
PRESET Score & $7(7,10)$ & $4(4,6)$ & $\mathbf{0 . 0 0 1}$
\end{tabular}


Conclusion. Patients who received VV-ECMO support showed a significant correlation between RESP and PRESET score and the survival observed.

\section{Reference(s)}

1. Hilder M, Herbstreit F, Adamzik M, Beiderlinden M, Bürschen M, Peters , et al. Comparison of mortality prediction models in acute respiratory distress syndrome undergoing extracorporeal membrane oxygenation and development of a novel prediction score: the PREdiction of Survival on ECMO Therapy-Score (PRESET-Score). Critical Care. 2017;21(1):301.

2. Petran J, Muelly T, Dembinski R, Steuer N, Arens J, Marx G, et al. Validation of RESP and PRESERVE score for ARDS patients with pumpless extracorporeal lung assist (pECLA). BMC anesthesiology. 2020;20(1):102.

3. Schmidt M, Bailey M, Sheldrake J, Hodgson C, Aubron C, Rycus PT, et al. Predicting Survival after Extracorporeal Membrane Oxygenation for Severe Acute Respiratory Failure. The Respiratory Extracorporeal Membrane Oxygenation Survival Prediction (RESP) Score. Am J Respir Crit Care Med. 2014;189(11):1374-82.

\section{2}

COVID-19 in a Tertiary Intensive Care Unit-do corticosteroids and remdesivir make a difference?

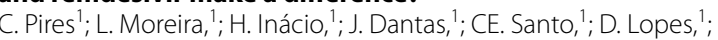
B. Ferreira, ${ }^{1}$ N. Germano,

${ }^{1}$ Ucip 7, Hospital Curry Cabral, CHULC, Lisboa, Portugal

Correspondence: C. Pires

Intensive Care Medicine Experimental 2020, 9(1): 001332

Introduction. The coronavirus disease 2019 (COVID-19) pandemic has been responsible for over 3 million deaths worldwide since its beginning. Various treatments have been evaluated to reduce its mortality. Remdesivir seems to reduce the need for mechanical ventilation without an impact on mortality(1). Conflicting results regarding the effects of corticosteroids $(2,3)$ have hindered the creation of protocols for steroid therapy.

Objectives. To evaluate the capacity of a clinically meaningful reduction in mortality and in mechanical ventilation days from remdesivir and systemic corticosteroids in severe SARS-CoV-2 infection.

Methods. This is a retrospective study in COVID-19 patients admitted to a tertiary intensive care unit (ICU) due to respiratory failure. Patients admitted between february 2020 and march 2021 were included and we compared number of days of mechanical ventilation and mortality in patients treated with systemic corticosteroid and/or remdesivir vs standard of care (SOC). Scale variable did not follow normal distribution, so non-parametric tests were performed (Mann-Whitney for comparison of means and chi-square for association between nominal variables).

Results. 195 patients were included (68.2\% male and $31.8 \%$ female) with a mean number of 12.63 days in the ICU. 153 patients received systemic corticosteroid therapy and 104 patients received therapy with remdesivir.

There was no statistically significant difference between the duration of ventilation in patients treated with corticosteroids and the SOC group $(U=2682.5, p=0.568)$, as well as when analyzing the duration of ventilation regarding the timing of beginning steroids (less than 5 days of symptoms $-U=3970.5, p=0.83 ; 5-10$ days $-U=3394$ $\mathrm{p}=0.659$; after 10 days $-\mathrm{U}=2421.5, \mathrm{p}=0.873$ ). Chi-square test showed no association between the use of corticosteroids and the occurrence of ICU $(0.241, p=0.623)$ and in-hospital mortality (2.093, $\mathrm{p}=0.148$ ).

There was no significant difference between the duration of ventilation in the group treated with remdesivir and the SOC group $(\mathrm{U}=2682.5, \mathrm{p}=0.568)$. Chi-square test also did not show any association between the use of remdesivir and the occurrence of ICU (2.029, $\mathrm{p}=0.154)$ and in-hospital mortality $(1.139, \mathrm{p}=0.286)$.

$37.9 \%$ of patients had a diagnosis of intubation-associated pneumonia (IAP). We found a borderline statically significant association between the use of corticosteroids and the occurrence of IAP (chi-square 3.143, $\mathrm{p}=0.076$ ).
Conclusion. There has been great interest in corticosteroids and remdesivir as therapeutic options for patients with severe COVID 19 disease in an effort to improve patients' outcome.

In this population of patients with severe COVID-19 disease, corticosteroid and remdesivir therapy seem to have no beneficial effect compared with SOC in reducing ventilation days as well as in reducing ICU or in-hospital mortality. Steroid therapy could be associated with IAP but a greater sample is needed.

\section{Reference(s)}

1. The RECOVERY Collaborative Group, Dexamethasone in Hospitalized Patients with Covid-19. N Engl J Med 2021; 384:693-704 https://doi.org/ 10.1056/NEJMoa2021436

2. Liu et al., Corticosteroid treatment in severe COVID-19 patients with acute respiratory distress syndrome. J Clin Invest. 2020;130(12):64176428. https://doi.org/10.1172/JCl140617

3. Siemieniuk et al., Drug treatments for covid-19: living systematic review and network meta-analysis, BMJ 2020;370:m2980 http://dx.doi.org/10. 1136/bmj.m2980

\section{3}

Percutaneous tracheostomy in COVID-19 patients

N. Koliakos ${ }^{1}$; F. Frantzeskaki ${ }^{1}$; D. Konstantonis ${ }^{1}$; M. Rizos ${ }^{1}$; V. Kitsinelis ${ }^{1}$; A. Armaganidis ${ }^{1}$; I. Tsangaris

${ }^{1}$ 2nd department of critical care, Attikon University Hospital, National and Kapodistrian University of Athens, Medical school, Chaidari, Greece Correspondence: F. Frantzeskaki

Intensive Care Medicine Experimental 2020, 9(1): 001333

Introduction. Following strict evidence-based procedure guidelines, percutaneous tracheostomy remains a safe method even in patients suffering from COVID-19 pneumonia. The most common indications for tracheostomy were acute respiratory distress syndrome and delayed weaning from mechanical ventilation.

Methods. This was a prospective observational study of mechanical-ventilated patients confirmed to have COVID-19 who required percutaneous tracheostomy at a tertiary COVID-19 referral center. Tracheostomy was classified as early or late when the procedure has been carried out within or after the first 10 days after endotracheal intubation respectively. The primary outcome measures were length of ICU stay, complications rate and 30-day morbidity and mortality.

Results. Of the 39 patients who underwent tracheostomy, the average time from endotracheal intubation to tracheostomy was 11.9 days (SD 4.5). Median length of stay was 25.1 days in those who underwent early tracheostomy versus 29.5 days in those who underwent late procedure $(p=0.13)$. Morbidity did not significantly differ between the two groups. Complications of procedure were rare and comparable between the two groups of patients. Mortality rate tended to be higher in the late-tracheostomy group ( $66 \%$ vs $26 \%, p=0.15$ ).

Conclusion. Percutaneous tracheostomy is a feasible and safe procedure in COVID-19 patients. Early tracheostomy may accelerate weaning from mechanical ventilation, while it might be associated with shorter ICU staying.

Reference(s)

1. Schoults JM et al. JAMA Otolaryngol Head Neck Surg. 2020;146(10):887-888

2. None.

001345

PNEUMOMEDIASTINUM, ANOTHER SARS-COV-2 CONSEQUENCE? A CASE SERIES

T. Marta' ; M. Cascão, ; J. Santos-Lima, ${ }^{1}$; R. Garcês, ${ }^{1}$; T. Carvalho, ${ }^{1}$; R. Cavaco ${ }^{1}$ L. Bento,

${ }^{1}$ Unidade de urgência medica (uum), Centro Hospitalar Universitário de Lisboa Central—Hospital de São José, Lisboa, Portugal

Correspondence: $\mathrm{T}$. Marta

Intensive Care Medicine Experimental 2020, 9(1): 001345 
Introduction. Pneumomediastinum is a rare, potentially life-threatening condition defined as the presence of air within the mediastinum. There have been some reports of pneumomediastinum in patients with Severe Acute Respiratory Syndrome Coronavirus 2 (SARSCoV-2) infection submitted to invasive mechanical ventilation (IMV), but also cases of spontaneous aetiology, suggesting that more than barotrauma may be causing this phenomenon.

Objectives. Characterize the cases of pneumomediastinum in patients with SARS-CoV-2 infection, admitted in a general intensive care unit (ICU).

Methods. Retrospective analysis of all patients diagnosed with SARS-CoV-2 infection and pneumomediastinum in the ICU, from March 2020 to April 2021. Patient files and diagnostic tests were reviewed. The diagnosis was clinical confirmed with chest X-ray or computed tomography imaging.

Results. From a total of 352 cases of SARS-CoV-2 infection admitted to the ICU, 7 developed pneumomediastinum. All patients were male, with a mean age of 63.57 years (49-74). 6 out of the 7 patients were submitted to IMV. From these, 3 cases of pneumomediastinum appeared related to the orotracheal intubation procedure, with the clinical features beginning a few hours to one day after intubation. 3 cases were related to barotrauma, with a minimum of 2 days of IMV and a maximum of 13 days. 3 patients were placed in prone position at least once before the diagnosis of pneumomediastinum. One particular case was considered spontaneous, as the patient was placed only on high flow oxygen with a maximum flow of $50 \mathrm{~L} / \mathrm{min}$, had no vascular catheters or nasogastric tube. This was the only patient who also had pneumothorax. None of the patients required surgical intervention, and a conservative approach was adopted. 2 of the patients were placed in extracorporeal membrane oxygenation (ECMO). The mortality was high, with 5 deaths in the ICU and only 2 patients discharged.

Conclusion. Although the number of cases described in the literature is still low, the development of pneumomediastinum in patients with SARS-CoV-2 infection may be a negative prognostic marker.

There are some suggested aetiologies such as: alveolar architectural changes, orotracheal intubation, frequent proning and barotrauma. In fact, the SARS-CoV-2 infection causes central and upper airway inflammation and oedema which increases the risk of injury from instrumentation. Furthermore, emergent intubation due to severe hypoxaemia and repositioning manoeuvres as prone positioning could be contributory factors to tracheobronchial injury. The relationship between barotrauma and pneumomediastinum is well described, especially in altered lungs as in acute respiratory distress syndrome.

This case series highlights the importance of suspecting this entity and close imaging and clinical monitoring. It also reiterates the need for following a protective ventilation setting, guarantying the lowest possible pressures, eventually initiating ECMO sooner.

\section{Reference(s)}

1. Mohan V, Tauseen RA, et al. Spontaneous pneumomediastinum in COVID-19. BMJ Case Reports CP 2020;13:e236519. http://dx.doi.org/10. 1136/bcr-2020-236519

2. Wang J, Su X, Zhang T, Zheng C. Spontaneous Pneumomediastinum: A Probable Unusual Complication of Coronavirus Disease 2019 (COVID-19) Pneumonia. Korean J Radiol. 2020;21 (5):627-628. 10.3348/kjr.2020.0281

3. Quincho-Lopez, A., Quincho-Lopez, et al. Case Report: Pneumothorax and Pneumomediastinum as Uncommon Complications of COVID19 Pneumonia-Literature Review, The American Journal of Tropical Medicine and Hygiene, 103(3), 2020, 1170-1176. https://doi.org/10.4269/ ajtmh.20-0815

4. Janssen J, Kamps M, et al. Spontaneous pneumomediastinum in a male adult with COVID-19 pneumonia. The American Journal of Emergency Medicine, Volume 40, 2021,Pages 228.e3-228.e5, https://doi.org/10. 1016/j.ajem.2020.07.066

5. Wali A, Rizzo V, et al. Pneumomediastinum following intubation in COVID-19 patients: a case series. Anaesthesia, Peri-operative medicine, critical care and pain, Volume75, Issue8, August 2020, Pages 1076-1081, https://doi.org/10.1111/anae.15113
6. Volpi S, Ali M J, et al. Pneumomediastinum in COVID-19 patients: a case series of a rare complication. European Journal of Cardio-Thoracic Surgery, Volume 58, Issue 3, September 2020, Pages 646-647, https://doi. org/10.1093/ejcts/ezaa222

\section{7}

Early tracheostomy versus late tracheostomy in ARDS caused by Sars Cov2: a mortality analysis

L. Hidalgo Manchado ; I. Guzman Adum²; DCl. De

${ }^{1}$ Intensive care unit, University Hospital Complex of Granada, Granada, Spain; ${ }^{2}$ Intensive care unit, Virgen de las Nieves Hospital, Granada, Spain; ${ }^{3}$ Icu, University Hospital Complex of Granada, Granada, Spain

Correspondence: $L$. Hidalgo

Intensive Care Medicine Experimental 2020, 9(1): 001347

Introduction. The adult respiratory distress syndrome (ARDS) caused by Sars Cov 2 infection has become the main cause of admission in ICU during the last year. Most of the patients admitted to the ICU ended up requiring mechanical ventilation (MV) and often, due to their long stay in ICU they required the performance of a tracheostomy.

Objectives. To establish a comparison of the prognosis between patients who underwent an early tracheostomy (define by the tracheostomy done between the first 14 days of MV) and patients who underwent a late tracheostomy (define by the tracheostomy done with at least 15 days of MV).

Methods. We perform a retrospective, observational study that includes 123 patients admitted in the ICU between October and November of 2020 with the diagnosis of Sars Cov2's pneumonia, who required respiratory support with high flow nasal cannula (HFNC), MV or both.

We gather information about baseline features; APACHE and SOFA score on the first day; SOFA score on the third day; the use of HFNC, MV or both; the need of tracheostomy and mortality.

Results. 123 patients were included with ages between 61.2 (SD 13.12). $25.4 \%$ were women (31) and $74.6 \%$ were men (91). $31.15 \%$ of the patients had diabetes (38), 50.82\% had hypertension (62), $6.56 \%$ had renal insufficiency (8), 9.84\% had ischemic heart disease, $6.56 \%$ had COPD (8), 9.8\% had sleep apnea (12) and 7.38 had any kind of immunosuppression (9) $21 \%$. Mortality in the ICU was $32.79 \%$, with an APACHE score at ICU admission of 11.28 (SD 5.37) and SOFA score of 4.17 (SD 1.99) at the admission and 4.36 (SD 2.27) at third day in ICU.

27 patients required only respiratory support with HFNC (23.77\%), 29 patients required MV from the beginning $(23.77 \%)$ and 65 patients required both strategies $(53,28 \%) .38 .52 \%$ (47) of the patients who required $\mathrm{MV}$, also need the performance of a tracheostomy. The mean time of MV until te performance of a tracheostomy was 15.87 days (SD 8.44), $17.08 \%$ were performed early (21) and $21.14 \%$ were performed late (26). The mortality was $38.79 \%$ between those in MV who did not undergo tracheostomy, $28,69 \%$ with an early one and $31.97 \%$ with a late one. There is a significant decrease in the mortality of those who underwent an early tracheostomy in comparison with the other 2 groups (OR 0.87 IC 0.77-0.98, p 0.04).

Conclusion. The performance of an early tracheostomy in patients with ARDS caused by Sars Cov 2 who need MV is associated with a decrease in mortality in comparison with those who did not undergo the performance of a tracheostomy and those who had a late tracheostomy.

001351

Pregnancy and SARS-CoV-2 infection: characteristics and outcomes in a chilean intensive care unit

P. Fernandez ${ }^{1}$; N. Pavez, ${ }^{1}$; C. Piderit ${ }^{2}$; A. Herrera ${ }^{2}$; P. Sanchez ${ }^{2}$; D. Ponce ${ }^{3}$; J. Lastra'; M. Hernandez ; L. Ferreira ${ }^{1}$

${ }^{1}$ Departamento de medicina interna, Universidad de Concepcion, Concepcion, Chile; ${ }^{2}$ Unidad de paciente crítico, Hospital Guillermo Grante Benavente, Concepcion, Chile; ${ }^{3}$ Unidad de paciente crítico, Hospital

Guillermo Grante Benavente, Concepcion, Chile

Correspondence: P. Fernandez

Intensive Care Medicine Experimental 2020, 9(1): 001351 
Introduction. The COVID-19 pandemic has affected different population groups and a significant number of them have required management in the intensive care units. Of these groups, pregnant women have proven to be a challenge for treatment in intensive care units. We reviewed the cases of pregnant women in our ICU from April 2020 to March 2021

Objectives. Describe the clinical evolution of pregnant women with ICU criteria of any cause infected with SARS-CoV-2. Observe the mortality in ICU and 28 days of the pregnant woman and their newborn infants.

Methods. Retrospective descriptive study. We analyzed all pregnant women hospitalized in the ICU Hospital Regional de Concepcion, Chile from April 2020 to March 2021 who matched with WHO severe to critical disease criteria. Descriptive analysis is presented using median + IQR.

Results. A total of 745 patients with severe to critical COVID-19 disease were admitted to our ICU, 9 of them were pregnant at hospital admission. Those women had a mean age of 33 years [21-41] and gestational age (GA) of 32 weeks [22-38]. None of them were vaccinated. APACHE II score was 10 [4-17]. During the ICU stay, fetal heartbeat was controlled 3 times a day and a formal OB US was performed weekly. Cesarean section had to be performed in 8 patients, 4 because of ventilatory failure 1 because of fetal fetal pain. GA at birth was 36 weeks [29-39] and birth weight was 2720 g. [1290-4190]. One patient presented with high-grade AV blockade post CPR. The other 8 patients developed severe respiratory failure and 7 required mechanical ventilation for mean 10 days, P/F ratio day 1 of 157 mean IQR [146-203], NMB [4/9], prone position [3/9] without maternal-fetal adverse events. All the patients received corticosteroid therapy. Cannulation for ECMOvv was instaured in 1 patient during pregnancy for 14 days between weeks 27 to 29 , a C-section delivery was performed 4 days after decannulation. Days of stay in ICU were 11 [5-40]. ICU mortality and mortality at 28 days was $0 \%$ in both mothers and newborns.

Conclusion. The need for critical care surveillance of COVID-19 infected pregnants is infrequent, however, they may develop severe to critical disease as shown in our study. Nonetheless, standard management was feasible and without complications for mother and fetus.

\section{Reference(s)}

1. Huntley BJF, et cols. Rates of Maternal and Perinatal Mortality and Vertical Transmission in Pregnancies Complicated by Severe Acute Respiratory Syndrome Coronavirus 2 (SARS-Co-V-2) Infection: A Systematic Review. Obstet Gynecol. 2020 Aug;136(2):303-312.

2. Andrikopoulou M, et cols. Symptoms and Critical IIIness Among Obstetric Patients With Coronavirus Disease 2019 (COVID-19) Infection. Obstet Gynecol. 2020 Aug;136(2):291-299.

3. Savasi VM, et cols. Clinical Findings and Disease Severity in Hospitalized Pregnant Women With Coronavirus Disease 2019 (COVID-19). Obstet Gynecol. 2020 Aug;136(2):252-258.

\section{7}

Mortality associated factors in patients with Acute Respiratory Difficulty Syndrome in the Intensive Care Unit of an institution in the city of Medellín, during the period 2012-2019

D. Yepes'; S. Moreno, ; JH. Arias'; A. Ramirez'; Z. Erika ${ }^{5}$

${ }^{1}$ Critical care, Clinica Ces, Clinica El Rosario, Medellin, Colombia; ${ }^{2}$ Critical care, Clinica Ces, Medellin, Colombia; ${ }^{3}$ Epidemiology, Universidad CES, Medellin, Colombia; ${ }^{4}$ Anesthesia, Universidad Ces, Medellin, Colombia; ${ }^{5}$ rgency, Clinica Ces, Medellin, Colombia

Correspondence: D. Yepes

Intensive Care Medicine Experimental 2020, 9(1): 001357

Introduction. Despite all the advances in the approach, the Acute Respiratory Distress Syndrome (ARDS) continues to be one of the main causes of mortality in Intensive Care Units (ICU). Although studies have focused on therapeutic strategies to reduce mortality, little is known about the factors that predispose patients to death.

Objectives. To identify the demographic, clinical and treatment factors associated with mortality in patients treated with ARDS in the ICU.
Methods. An observational, analytical and retrospective follow-up study was carried out on a cohort of patients diagnosed with ARDS according to the Berlin criteria and treated in the ICU of a highly complex institution in the city of Medellín (Colombia), from January 2012 to December 2019.

Results. One hundred and seventy-five patients with ARDS treated in the ICU were included in the study. The median age was 56 years (IQR $66-40)$ and the majority of the patients were men (59.4\%). The etiology of ARDS was predominantly primary $66.9 \%$, with sepsis being the main diagnosis $(90.3 \%)$. A mortality of $38.3 \%$ was observed.

No significant differences were found between the groups regarding ventilatory parameters upon admission. The performance of a tracheostomy showed a negative correlation with mortality $(\mathrm{HR} 0.14[95 \% \mathrm{Cl}$ 0.07-0.29] $\mathrm{p}=<0.001$ ); while vasopressor support stood out as a risk factor (HR $4.43[95 \% \mathrm{Cl} 1.66-11.84] \mathrm{p}=0.003$ ). A history of oncological pathology and chronic lung disease are associated with an unfavorable outcome within the ICU (HR 2.18 [95\% Cl 1.01-4.68] $p=0.040$ and HR 2.27 [95\% Cl 1.13-4.55] $\mathrm{p}=0.021$ ).

Conclusion. Mortality from ARDS in ICU continues to be high. The presence of oncological pathology, chronic lung disease, and the use of vasopressor support are associated with adverse outcomes due to this etiology.

\section{Reference(s)}

1. Bellani G, Laffey JG, Pham T, et al. Epidemiology, Patterns of Care, and Mortality for Patients With Acute Respiratory Distress Syndrome in Intensive Care Units in 50 Countries. JAMA. 2016:315(8):788.

2. Brun-Buisson C, Minelli C, Bertolini G, et al. Epidemiology and outcome of acute lung injury in European intensive care units. Results from the ALIVE study. Intensive Care Med. 2004;30(1):51-61.

3. Phua J, Badia JR, Adhikari NKJ, et al. Has mortality from acute respiratory distress syndrome decreased over time?: A systematic review. Am J Respir Crit Care Med. 2009:179(3):220-7.

4. Rezoagli E, Fumagalli R, Bellani G. Definition and epidemiology of acute respiratory distress syndrome. Ann Transl Med. julio de 2017;5(14):282-282

5. The ARDS Definition Task Force, Ranieri VM, Rubenfeld GD, Thompson BT, et al. Acute Respiratory Distress Syndrome: The Berlin Definition. JAMA. 2012;307(23):2526-33.

6. Thompson BT, Chambers RC, Liu KD. Acute Respiratory Distress Syndrome. Drazen JM, editor. N Engl J Med. 10 de agosto de 2017:377(6):562-72

7. Ashbaugh DG, Bigelow DB, Petty TL, et al. Acute respiratory distress in adults. Lancet. 1967;2(7511):319-23.

001375

Use of diaphragm ultrasound as a support tool for managing respiratory failure in COVID19

S. Oliveira'; G. Costanza'2 'L. Silva'2; PR. Costa ${ }^{2}$

${ }^{1}$ CRITICAL CARE CENTER, HOSPITAL ESTADUAL GETULIO VARGAS, RIO

DE JANEIRO, Brazil; ${ }^{2}$ Critical care center, Hospital Getúlio Vargas, Rio de Janeiro, Brazil

Correspondence: S. Oliveira

Intensive Care Medicine Experimental 2020, 9(1): 001375

Introduction. Due to the COVID19 pandemic, new measures and strategies have emerged to increase the effectiveness of oxygen therapy, preventing the patient from going to invasive mechanical ventilation.

Objectives. The aim of this study was to analyze the effectiveness of Diaphragm Ultrasound as a method of analyzing respiratory muscle fatigue and an indicator of the need for invasive mechanical ventilation or persistence in oxygen therapy.

Methods. A prospective and observational study was carried out for a period of 2 months in an adult intensive care center exclusively for COVID19. The inclusion criteria were adult patients, without previous pulmonary diseases, with pulmonary parenchyma lesions of approximately $75 \%$, time of symptoms prior to hospitalization less than 5 days, without previous use of antibiotics, corticosteroids, immunomodulators. Patients with acute and chronic kidney disease, drug 
users, patients with generalized anxiety or who progressed to delirium were excluded. As analysis were collected: age, sex, comorbidities, weight, height, onset of symptoms, diaphragm ultrasound data $2 \times$ a day, arterial blood gas $4 \times$ a day, vital signs, oxygen supply mode, mechanical ventilation mode and their respective times.

Results. For two months, 10 adult patients aged between 18 and 50 years, without pulmonary diseases prior to hospitalization, with approximately $75 \%$ of the pulmonary parenchyma were included. The average age was 46 years. Male sex in $70 \%$. Average time of symptoms prior to hospitalization was 4 days. $6(60 \%)$ of the patients had below-ideal $\mathrm{PaO} 2$ in all modes of oxygen therapy. Of these $3(30 \%)$ were for invasive mechanical ventilation (IMV) and $3(30 \%)$ remained on oxygen therapy. In the 4 patients with $\mathrm{PaO} 2$ above ideal in supplemental $\mathrm{O} 2$ and NIMV, 1 went to IMV and the other 3 remained with NIMV and supplemental O2. It was observed in the 10 patients the onset of fatigue prior to the decrease in $\mathrm{PaO} 2$, generating the decision to increase the supply of 02 . In the 6 patients who remained without $\mathrm{IMV}$, the recovery from fatigue was precocious to the increase in $\mathrm{PaO}$, allowing him to keep the patient under surveillance but without starting IMV.

Conclusion. Despite being a small study with 10 patients, 6 patients with significant involvement of the pulmonary parenchyma did not undergo mechanical ventilation. The reality at the Hospital in the previous months was that at least $70 \%$ of patients in these same conditions went to IMV where mortality is high. Another point is to be a public hospital in Rio de Janeiro where, during the study period, it was difficult to acquire supplies such as sedatives and neuromuscular blockers. The initial motivation for using the diaphragm us was, therefore, a tool to highlight the possibility of keeping the patient out of invasive ventilation, offering a greater chance of success in the treatment as well as optimization of scarce resources.

\section{Reference(s)}

1. Francis CA, Hoffer JA, Reynolds S. Ultrasonographic evaluation of diaphragm thickness during mechanical ventilation in intensive care patient. Am J Crit Care. 2016;25:e1-8

2. Lloyd T, Tang YM, Benson MD, et al. Diaphragmatic paralysis: the use of M mode ultrasound for diagnosis in adults. Spinal Cord. 2006;44:505-8.

3. Summerhill EM, El-Sameed YA, Glidden TJ, et al. Monitoring recovery from diaphragm paralysis with ultrasound. Chest. 2008;133:737-43.

4. Matamis D, Soilemezi E, Tsagourias M, et al. Sonographic evaluation of the diaphragm in critically ill patients. Technique and clinical applications. Intensive Care Med. 2013;39:801-10.

\section{6}

\section{Lung ultrasound ablity to predict outcome in COVID-19 related ARDS}

A. Jamoussi' ; S. Zarrouk' ; L. Messaoud ${ }^{1}$; E. Rachdi ${ }^{1}$; F. JARRAYA ${ }^{1}$;

M. Besbes' ${ }^{1}$; S. AYED ${ }^{1}$; J. Ben Kheliil ${ }^{1}$

'Medical icu, Hôpital Abderrahmen Mami de pneumo-phtisiologie, Ariana, Tunisia

Correspondence: A. Jamoussi

Intensive Care Medicine Experimental 2020, 9(1): 001396

Introduction. The use of lung ultrasound (LUS) is increasingly being a standard of care in critically ill patients, as a diagnostic tool or for follow up.

Objectives. The aim of this study was to describe LUS findings in COVID-19 related ARDS and to analyze association with severity.

Methods. A prospective study was carried in respiratory medical intensive care unit of Abderrahmen Mami teaching hospital between February and April 2021. It included COVID-19 related ARDS patients managed in ICU. LUS examination was performed during the first $48 \mathrm{~h}$ of ICU stay, with a low-frequency convex probe (Vivid GE) according to 6 areas per hemithorax: antero-superior quadrant (ASQ), anteroinferior quadrant (AIQ), latero-superior quadrant (LSQ), latero-inferior quadrant (LIQ), postero-superior quadrant (PSQ) and postero-inferior quadrant (PIQ). LUS findings were described and association with severity was analysed.
Results. During the study period, 98 patients were included with mean age of $61 \pm 12$ years, gender ratio of 1.7. We recorded medical history of COPD $(n=10,10 \%)$, hypertension $(n=45,46 \%)$ and diabetes $(n=36,37 \%)$.

The mean values of the Simplified Acute Physiology Score II and Acute Physiologic and Chronic Health Evaluation II were respectively $27 \pm 8.6$ and $9.3 \pm 5$.

ARDS was diagnosed in all patients divided into severe $(n=57,58 \%)$, moderate $(n=37,38 \%)$ and mild $(n=4,4 \%)$. Mean initial PaO2/FiO2 was $103 \pm 46[42-260] \mathrm{mmHg}$.

We explored 1176 areas $(98 * 12)$ : The pattern of features from the most frequent was: B2 $(n=486), C(n=401)$ and then B1 $(n=274)$. Very few quadrants $(n=15)$ were completely spared. Left hemithorax examination showed more condensations, especially left LIQ (70/98) and left AIQ (56/98).

Mean LUS score was $25.1 \pm 5.2$ [10-36]. No nificant difference was found between dead and survivors ( 25.2 vs $24.9, p=0.8$ ) or between invasive ventilation need or not ( 25.6 vs $24.5, p=0.29$ ). Nevertheless, there was a correlation between $\mathrm{PaO} 2 / \mathrm{FiO} 2$ and LUS score with $r=-0.314$ and $p=0.002$

Mean length of stay was $13.2 \pm 11$ [2-85] days and overall mortality was of $52 \%$.

Conclusion. In COVID-19 related ARDS, LUS score is correlated with initial severity as expressed by initial PaO2/FiO2. However, no association was found with mortality or need for invasive ventilation.

\section{1}

Remdesivir plus Dexamethasone in critically ill patients with COVID-19 and acute hypoxemic respiratory failure: a casecontrol study

SL. Cutuli' ${ }^{1}$; G. Pintaudi' ${ }^{1}$; ES. Tanzarella'; DL. Grieco'; M. Di Muro'; J. Vargas'; L. Cascarano'; L. Montini'; G. Bello'; S. Carelli' ; G. Paiano ${ }^{1}$; MS. Vallecoccia'; T. Rosà'; R. Xhemalaj ; G. De Pascale'; M. Antonelli ${ }^{1}$

'Dipartimento di scienze dell' emergenza, anestesiologiche e della rianimazione, Fondazione Policlinico Universitario A. Gemelli IRCCS, Rome, Italy

Correspondence: S. Cutuli,

Intensive Care Medicine Experimental 2020, 9(1): 001411

Introduction. In hospitalised patients with Coronavirus Disease 19 (COVID-19), Remdesivir (Rem) [1] shortened time to recovery and Dexamethasone (Dexa) [2] lowered 28-day mortality of whom developed acute hypoxemic respiratory failure (AHRF). However, clinical effects of concomitant Rem plus Dexa (Rem-Dexa) has never been investigated.

Objectives. To investigate clinical effects of Rem-Dexa in patients with COVID-19 and AHRF.

Methods. Monocentre, case-control study that included critically ill patients with COVID-19 and AHRF that required high-flow nasal cannula (HFNC) oxygen therapy or noninvasive ventilation (NIV). Cases received daily Rem ( $200 \mathrm{mg}$ followed by $100 \mathrm{mg}$ ) for 5 days and Dexa (6 mg) for 10 days. This group was 1:1 matched with an historical cohort (controls) that did not receive Rem-Dexa. Matching was based on the simplified acute physiology score II (SAPS II), the sequential organ failure assessment (SOFA) score and the Charlson Comorbidity Index (CCI). The primary outcome was 28-day endotracheal intubation (ETI) rate. Secondary outcomes were 7-day arterial oxygen partial pressure to fractional inspired oxygen ratio $(\mathrm{PaO} 2 / \mathrm{FiO} 2)$, clinical improvement of at least 1 point of an eight-category ordinal scale [1], ICU and hospital length of stay (LOS), 28-day and 90-day mortality. Continuous variables were presented as median [Interquartile range, IQR], categorical variables as number $(\mathrm{N})$ and percentage (\%). Predictors of primary outcome were investigated by logistic regression analysis; odds ratio (OR) and 95\% Confidence Interval (Cl) were reported. MedCalc 12.2.1 (MedCalc ${ }^{\circledR}$, MariaKerke, Belgium) was used for data analysis.

Results. Clinical characteristics of cases $(\mathrm{N}=66)$ and controls $(\mathrm{N}=66)$ were shown in Table $1 . \mathrm{PaO} 2 / \mathrm{FiO} 2$ at ICU admission was lower among cases compared with controls $(152 \mathrm{mmHg}$ [126$180]$ vs $172 \mathrm{mmHg}$ [148-205], $p=0.01)$, although the former had lower serum inflammatory markers (C-Reactive Protein: $87.2 \mathrm{mg} /$ 
$\mathrm{dL}$ [40.7-138.0] vs $155.5 \mathrm{mg} / \mathrm{dL}$ [71.0-195.6], $\mathrm{p}=0.01$; lactic dehydrogenase: $573 \mathrm{UI} / \mathrm{L}$ [370-1109] vs $1728 \mathrm{UI} / \mathrm{L}$ [772-3714], $p=0.03$; D-Dimers: $338 \mathrm{ng} / \mathrm{mL}$ [283-425] vs $405 \mathrm{ng} / \mathrm{mL}$ [315-486], $\mathrm{p}<0.03$ ). The 28-day ETI rate was lower among cases compared with controls $(19.7 \%$ vs $48.5 \%, p<0.01)$. Clinical status improved in $46(69.7 \%)$ cases and $34(51.5 \%)$ controls $(p=0.05)$, although other secondary outcomes did not differ between the two groups (Table 1). RemDexa (OR 0.22, Cl95\% [0.05-0.94], $\mathrm{p}=0.04$ ) and high $\mathrm{PaO} 2 / \mathrm{FiO} 2$ ratio (OR 0.98, Cl95\% [0.97-0.99], $\mathrm{p}=0.04$ ) were associated with reduced 28-day ETI rate while high SAPS II (OR 1.09, C195\% [1.02$1.18], \mathrm{p}=0.02$ ) and serum lactic dehydrogenase (OR 1.007, Cl95\% $[1.002-1.010], p=0.01)$ were associated with worsening of this outcome.

Table 1. Demographic characteristics and outcomes of patients included in the study

\begin{tabular}{|c|c|c|c|c|}
\hline & $\begin{array}{l}\text { Total cohort } \\
(n=132)\end{array}$ & $\begin{array}{l}\text { Controls } \\
(n=66)\end{array}$ & $\begin{array}{l}\text { Cases } \\
(n=66)\end{array}$ & $p$ value \\
\hline \multicolumn{5}{|c|}{ Demographic characteristics } \\
\hline Age, years & $64[57-72]$ & $67[57-72]$ & $64[57-72]$ & 0.7 \\
\hline Male sex & $101(76.5)$ & $51(77.3)$ & $50(75.8)$ & 1 \\
\hline SAPS II score & $30[24-36]$ & $31[24-36]$ & $29[24-34]$ & 0.45 \\
\hline SOFA score & $3[3-3]$ & $3[3-3]$ & $3[3-3]$ & 1 \\
\hline $\mathrm{CCl}$ & $2[1-4]$ & $3[1-4]$ & $2[1-4]$ & 0.85 \\
\hline \multicolumn{5}{|l|}{ Outcomes } \\
\hline $\begin{array}{l}\text { 28-Day ETI } \\
\text { rate }\end{array}$ & $45(34.1)$ & $32(48.5)$ & $13(19.7)$ & $<0.01$ \\
\hline $\begin{array}{l}\text { 7-Day } \mathrm{PaO} 2 / \\
\mathrm{FiO} 2, \mathrm{mmHg}\end{array}$ & $\begin{array}{l}178[138.5- \\
214]\end{array}$ & $171[132-214]$ & 186 [157-214] & 0.2 \\
\hline $\begin{array}{l}\text { Clinical } \\
\text { improve- } \\
\text { ment }\end{array}$ & $80(60.6)$ & $34(51.5)$ & $46(69.7)$ & 0.05 \\
\hline ICU LoS, days & $10[6-17]$ & $9[6-16]$ & $10[7-18]$ & 0.32 \\
\hline $\begin{array}{l}\text { Hospital LoS, } \\
\text { days }\end{array}$ & $23[16-34]$ & 26 [17-39] & 21 [16-29] & 0.2 \\
\hline $\begin{array}{l}\text { 28-day mor- } \\
\text { tality }\end{array}$ & $13(9.8)$ & $10(15.2)$ & $3(4.5)$ & 0.08 \\
\hline $\begin{array}{l}\text { 90-day mor- } \\
\text { tality }\end{array}$ & $18(13.6)$ & $11(16.7)$ & $7(10.6)$ & 0.45 \\
\hline
\end{tabular}

Conclusion. In critically ill patients with COVID-19 and AHRF, 28-day ETI rate was lower in patients who received Rem-Dexa compared with historical controls.

\section{Reference(s)}

1. Beigel J, Tomashek K, Dodd L, Mehta A, Zingman B, Kalil A, Hohmann E, Chu H, Luetkemeyer A, Kline S, Castilla Dd, Finberg R, Dierberg K, Tapson V, Hsieh L, Patterson T, Paredes R, Sweeney D, Short W, Touloumi G, Lye DC, Ohmagari N, Oh M, Ruiz-Palacios G, Benfield T, Fätkenheuer G, Kortepeter M, Atmar R, Creech CB, Lundgren J, Babiker A, Pett S, Neaton J, Burgess T, Bonnett T, Green M, Makowski M, Osinusi A, Nayak S, Lane H, Members A-SG, (2020) Remdesivir for the Treatment of Covid-19-Final Report. N Engl J Med 383: 1813-1826

2. RECOVERY Collaborative Group, Horby P, Lim W, Emberson J, Mafham M, Bell J, Linsell L, Staplin N, Brightling C, Ustianowski A, Elmahi E, Prudon B, Green C, Felton T, Chadwick D, Rege K, Fegan C, Chappell L, Faust S, Jaki T, Jeffery K, Montgomery A, Rowan K, Juszczak E, Baillie J, Haynes R, Landray M, (2020) Dexamethasone in Hospitalized Patients with Covid-19_Preliminary Report. N Engl J Med Online ahead of print

3. None
001414

Using anaesthetic ventilators during the COVID-19 Pandemic: a UK Surge ICU Experience

L. Shabbo'; J. Mccanny²; DC. Marshall'2;. Tixson, ${ }^{3}$

${ }^{1}$ Critical care, Guy's \& St Thomas'Trust, London, United Kingdom; ${ }^{2}$ Critical care, Guy's \& St Thomas'Trust, London, United Kingdom; ${ }^{3}$ Department of theatres, anaesthesia and perioperative care, Guy's and St Thomas' NHS

Foundation Trust, London, United Kingdom

Correspondence: $L$. Shabbo

Intensive Care Medicine Experimental 2020, 9(1): 001414

Introduction. This is an observational case-control study reporting comparative safety and efficacy outcomes for the use anaesthetic machines (AM) and ICU ventilators. We included all patients admitted between 19th March and 05 May 2020 to our surge ICU within a large NHS teaching hospital during the first wave of the COVID-19 pandemic.

Objectives. We aim to highlight key learning from our experience providing critically ill patients with prolonged mechanical ventilation (MV) using anaesthetic ventilators in such settings.

Methods. 51 patients with COVID-associated acute respiratory distress syndrome were included (25 cases: Dräger Primus anaesthetic machine; 26 controls: Dräger V500 conventional ICU ventilator), within one clinical area and staffing cohort. Patient demographics, safety, efficacy and disease severity outcomes were analysed. MV efficacy outcomes were investigated as per evidence-based lung protective ventilation $1,2,3$ by collecting ventilation parameters 4 hourly for the first $48 \mathrm{~h}$ of admission. MV safety was determined by the occurrence of clinical incidents related to $M V$ and were identified by clinician reporting, review of case-note ventilation data, and all clinical note entries.

Results. The demographics of the AM group compared to ICU ventilator group was as followed; mean age 55 versus 57 , sex $71 \%$ male versus $74 \%$ male, APACHE II score median (IQR) $7.5(4.8-11)$ versus 9.0 (7.0-11.5) and $\mathrm{PaO} 2 / \mathrm{FiO} 2$ ratio median (IQR) 130 (110-169) versus 167 (132-231). When the AM group was compared to the ICU ventilator group; the proportion of time receiving lung protective ventilation by tidal volumes was $75.3 \%$ versus $78.9 \%$, peak pressure $<30 \mathrm{~cm} \mathrm{H} 20$ : $88.3 \%$ versus $95.2 \%$, and driving pressure $<15 \mathrm{~cm} \mathrm{H} 20: 48.8 \%$ versus $57.9 \%$. When comparing the safety between the two groups the number of MV-associated clinical incidents per day was $0.04(11 / 278)$ for AM group versus 0.008 (2/365) for ICU ventilator group.

Conclusion. Overall, whilst AM ventilators can be used to provide MV across a prolonged period with comparable efficacy to ICU ventilators, the significantly higher frequency of clinical incidents reinforces that we should cautiously consider their use during further pandemic waves, depending on ventilator supply-and-demand pressure. The clinical incidents encountered were mostly ventilator changes due to deterioration or circuit issues, most commonly circuit obstruction and waterlogging. If further situations arise in future where demand for ICU ventilator provision outstrips supply, patient safety concerns associated with AM ventilators must be mitigated by careful planning and education. Imperative considerations include appropriate patient selection, equipment preparedness, staff education, training, and cohorting. Ventilator manufacturers should continue to provide updated information to reflect emerging real-world experience and data. International guidance, as well as device-specific local guidance should help standardise and improve safe care.

\section{Reference(s)}

1. Amato MB, Meade MO, Slutsky AS, Brochard L, et al. Driving pressure and survival in the acute respiratory distress syndrome. New England Journal Medicine. 2015; 372(8): 747-55.

2. Gattinoni L, Tonetti T, Cressoni $M$ et al. Ventilator-related causes of lung injury: the mechanical power. Intensive Care Medicine. 2016; 42(10): 1567-1575.

3. Bower. R, Matthay M, Morris A, et al. Ventilation with lower tidal volumes as compared with traditional tidal volumes for acute lung injury and the acute respiratory distress syndrome. New England Journal Medicine. 2000; 342(18): 1301-8. 
001449

The incidence of pneumothorax and pneumomediastinum in critically ill patients with COVID-19

O. Griffiths'; S. Mulla'; DO. Hamilton'; B. Johnston ${ }^{1}$; A. Waite, ; I. Welters, ${ }^{1}$ ${ }^{1}$ Critical care, Royal Liverpool and Broadgreen University Hospitals NHS Trust, Liverpool, United Kingdom

Correspondence: D.O. Hamilton

Intensive Care Medicine Experimental 2020, 9(1): 001449

Introduction. Lung protective ventilation reduces mortality in critically ill patients. Conversely, high pressures are associated with barotrauma and pneumothorax. There has been early evidence of an increased incidence of pneumothoraces or pneumomediastinum (hereby grouped together as PTX) in patients with COVID-19 undergoing mechanical (MV) or non-invasive ventilation (NIV) [1]. Our study aimed to determine the incidence of PTX on our Intensive Care Unit (ICU) and describe the respiratory support used.

Methods. Between 1st March 2020 and 28th February 2021, data were collected retrospectively by targeted review of the hospital coding database. Patients required a positive COVID-19 PCR test and a radiologically confirmed PTX to be included within the study. Patients were excluded from the respiratory support evaluation if the PTX was iatrogenic, associated with another surgical condition or transferred in from another hospital.

Results. Of the 338 patients with COVID-19 admitted to the ICU, 17 (5.3\%) had pneumothorax, 5 had isolated pneumomediastinum and 9 had both ( $3.3 \%$ had pneumomediastinum). Of these, 20 were included in the study on respiratory support. 16 were male, the mean age was $58.3( \pm 11.3)$ and the mean length of stay on ICU was $13.5( \pm 7.8)$ days. The mortality rate was $18 / 20(80 \%)$. The mean P/F ratio before PTX diagnosis was $13.5( \pm 5.5) \mathrm{kPa}$.

Conservative management was used in 11 cases (55\%) and chest drains were inserted in 9 cases (45\%) with 3 requiring emergency decompression. Twelve patients had PTX diagnosed whilst undergoing MV, 7 whilst on NIV (all Continuous Positive Airway Pressure [CPAP]), and one whilst on face-mask oxygen.

The mean duration from starting CPAP to confirmed radiological diagnosis was $181.3( \pm 116.3)$ hours. The mean duration of MV prior to diagnosis was $111.0( \pm 141.5)$ hours. The mean duration of ventilation (incorporating both CPAP and MV) to PTX diagnosis was 204.4 $( \pm 151.6)$ hours. In those on MV, the mean peak inspiratory pressure was $30.0( \pm 10.5) \mathrm{cmH} 2 \mathrm{O}$ and positive end-expiratory pressure was $10.7( \pm 3.5) \mathrm{cmH} 2 \mathrm{O}$. Tidal volumes were generally between $7-8 \mathrm{ml} / \mathrm{kg}$ in the days prior to PTX diagnosis. Ventilation settings did not change significantly after PTX diagnosis.

Conclusion. We describe a case series of 20 patients with COVID-19 who had PTX whilst on ICU. The total incidence of pneumothorax was $5.3 \%$ and the mortality was $80 \%$. These rates are higher than in the published literature in COVID-19 [1] although comparable to older studies on Adult Respiratory Distress Syndrome [2]. Ventilation strategies used were at the upper end of lung-protective ventilation. Further study is required to assess causality.

\section{Reference(s)}

1. Martinelli A et al. COVID-19 and Pneumothorax: A Multicentre Retrospective Case Series. Eur Respir J. 2020;56(5).

2. Terzi E et al. Acute respiratory distress syndrome and pneumothorax. J Thorac Dis. 2014;6(Suppl 4):S435-S442.

3. Nil
001451

\section{Airway pressure release ventilation vs low tidal volume} ventilation in COVID-19 associated ARDS

J. Vine ${ }^{1}$; V. Soloveichik ${ }^{2}$; E. Vinepinsky ${ }^{3}$; A. Nini ${ }^{1}$; A. Nevo ${ }^{1}$; A. Borohovitz ${ }^{4}$; N. Adi ${ }^{1}$; Y. Lichter

${ }^{1}$ Intensive care unit, Tel Aviv Sourasky Medical Center, Tel Aviv-Yafo, Israel; ${ }^{2}$ Internal medicine d, Tel Aviv Sourasky Medical Center, Tel Aviv-Yafo, Israel;

${ }^{3}$ Edmond and lily safra center for brain sciences, The Hebrew University of Jerusalem, Jerusalem, Israel; ${ }^{4}$ Cardiology, Tel Aviv Sourasky Medical

Center, Tel Aviv-Yafo, Israel

Correspondence: J. Vine

Intensive Care Medicine Experimental 2020, 9(1): 001451

Introduction. COVID-19 associated ARDS has caused significant morbidity and mortality throughout the world. Airway pressure release ventilation (APRV) has been shown to reduce alveolar strain [1], and has been hypothesized to reduce the incidence of ARDS [2] and improve clinical outcomes in patients with established ARDS [3]. It is unknown whether initiating APRV in the early stage of ventilation affects outcomes in COVID-19 associated ARDS. We hypothesized that initiating APRV during the early stage of ventilation in COVID-19 ARDS patients could improve oxygenation parameters which may have an effect on clinical outcomes.

Objectives. Comparison of oxygenation parameters in mechanically ventilated patients with COVID-19 associated ARDS in which APRV was initiated during the early stage of ventilation vs patients on conventional low tidal volume (LTV) mechanical ventilation.

Methods. A retrospective cohort of mechanically ventilated patients hospitalized in the Medical Intensive Care Unit (MICU) in our institution with COVID-19 associated ARDS was evaluated. APRV was set according to principals of time controlled adaptive ventilation (TCAV) where T-low was personalized to set a ratio of end expiratory flow to peak expiratory flow (EEF/PEF) of 75\% [4], T-high was set according to desired respiratory rate. Conventional ventilation was set according to principals of low tidal volume protective lung ventilation [5]. Mean $\mathrm{PaO} 2 \mathrm{FiO} 2$ ratios for five days post intubation was extracted from data and delta $\mathrm{PaO} 2 \mathrm{FiO} 2$ ratios were calculated and compared by t-test analysis.

Results. 37 patients with COVID-19 associated ARDS hospitalized in the MICU and mechanically ventilated were included in the study. 16 of the patients were ventilated using APRV initiated during the first $36 \mathrm{~h}$ after intubation and 21 were ventilated using conventional LTV. $70 \%$ were male, average age was $57.3( \pm 13.8)$, mean baseline SOFA score was $4.6( \pm 2)$, mean initial $\mathrm{PaO} 2: \mathrm{FiO} 2$ ratio after intubation was $118( \pm 52)$. Baseline characteristics were not significantly different between groups. Delta $\mathrm{PaO} 2 \mathrm{FiO} 2$ ratio on days $2-5$ post intubation was $43.6( \pm 8.1), 47.8( \pm 13.5), 51.6( \pm 16), 57.9( \pm 13.4)$ in the APRV group and $-5.2( \pm 13.6),-14.5( \pm 16),-15.8( \pm 18.2),-19.4( \pm 19.3)$ in the LTV ventilation group respectively ( $p<0.01$ on all days).

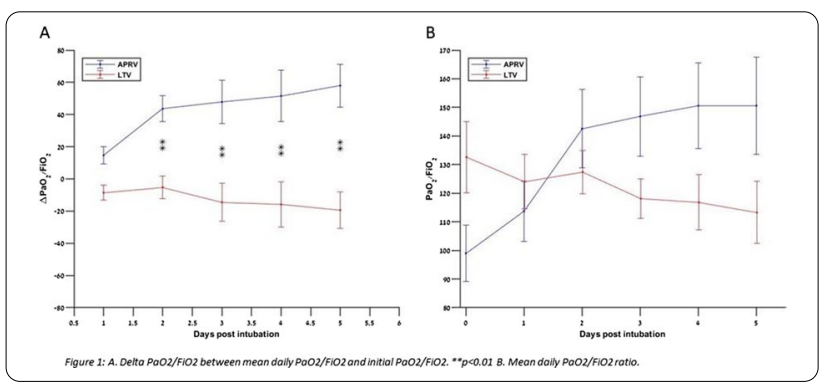

Conclusion. In our cohort of mechanically ventilated patients with COVID-19 associated ARDS, oxygenation parameters improved consistently over the course of 5 days in patients placed on APRV during the early stage of mechanical ventilation as compared to patients on LTV ventilation. Prospective randomized trials are needed in order to see if these findings translate into benefits in morbidity and mortality in these severely sick patients. 
Reference(s)

1. [5] Acute Respiratory Distress Syndrome Network. Ventilation with lower tidal volumes as compared with traditional tidal volumes for acute lung injury and the acute respiratory distress syndrome. New England Journal of Medicine. 2000 May 4;342(18):1301-8.

2. [4] Habashi NM. Other approaches to open-lung ventilation: airway pressure release ventilation. Critical care medicine. 2005 Mar 1;33(3):S228-40.

3. [3] Zhou Y, Jin X, Lv Y, Wang P, Yang Y, Liang G, Wang B, Kang Y. Early application of airway pressure release ventilation may reduce the duration of mechanical ventilation in acute respiratory distress syndrome. Intensive care medicine. 2017 Nov;43(11):1648-59.

4. [2] Andrews PL, Shiber JR, Jaruga-Killeen E, Roy S, Sadowitz B, O'Toole RV, Gatto LA, Nieman GF, Scalea T, Habashi NM. Early application of airway pressure release ventilation may reduce mortality in high-risk trauma patients: a systematic review of observational trauma ARDS literature. Journal of Trauma and Acute Care Surgery. 2013 Oct 1;75(4):635-41.

5. [1] Jain SV, Kollisch-Singule M, Sadowitz B, Dombert L, Satalin J, Andrews P, Gatto LA, Nieman GF, Habashi NM. The 30-year evolution of airway pressure release ventilation (APRV). Intensive care medicine experimental. 2016 Dec;4(1):1-8

\section{3}

Can Early Prone Positioning Decrease Mechanical Ventilation Length and Mortality in COVID-19 Patients?

N. Boyaci ${ }^{1}$; G. Koksal'; A. Yalcin ${ }^{3}$; G. Aksu²; EK. Kaya²; O. Sen

${ }^{1}$ Anesthesiology and reanimation, University of Health Sciences, Haseki

Training and Research Hospital, Istanbul, Turkey; ${ }^{2}$ Critical care, University of Health Sciences,Haseki Training and Research Hospital, Istanbul, Turkey;

${ }^{3}$ Pulmonary and critical care, University of Health Sciences, Haseki Training and Research Hospital, Istanbul, Turkey

Correspondence: N. Boyaci

Intensive Care Medicine Experimental 2020, 9(1): 001453

Introduction. Coronavirus disease 2019 (SARS CoV-2, COVID-19) can lead to severe hypoxemia by immune thrombosis, macro and micro pulmonary emboli, alveolar exudation, atelectasis, and pulmonary congestion. In patients requiring invasive mechanical ventilation due to refractory hypoxemia, rescue therapies such as prone positioning, inhaled nitric oxide, extracorporeal membrane oxygenation could be beneficial.

Objectives. In this study, we intended to investigate the effect of early prone positioning on the duration of invasive mechanical ventilation, length of hospital stay, and mortality.

Methods. We conducted this prospective clinical study in Haseki Training and Research Hospital Sultangazi, Istanbul, between 08/01/2020-04/30/2021. Patients admitted to the adult intensive care unit (ICU) with severe hypoxia (under $15 \mathrm{~L} /$ minute nonrebreather mask, Spo2 $\leq 90$ ) and whose minute respiratory rate were more than 40 and above were intubated and placed into prone position immediately after. The prone position duration was $16 \mathrm{~h}$ a day. Oxygenation and ventilation parameters were monitored with serial arterial blood gas analysis at each change of position. After collecting demographic data (age, gender), acute physiology and chronic health evaluation II (APACHE II) and sequential organ failure assessment (SOFA) scores, ferritin, D-dimer, lactate dehydrogenase (LDH) values on the first day of ICU admission, 50 patients' records were analyzed statistically. In calculating the sample size of this study, the power for each variable was determined $80 \%$ at least, and type 1 error was 5\%. Nonparametric variables were evaluated with Mann Whitney-U test. Statistical significance (a) was $5 \%$ in calculations, and SPSS (IBM SPSS for Windows, ver.24) program was used for calculations. Results were considered significant $p<0,05$.

Results. Mean values of the patients included in the study are presented in Table 1.

\begin{tabular}{cllllllll}
\hline & $\begin{array}{l}\text { Invasive } \\
\text { mechani- } \\
\text { cal } \\
\text { ventilation }\end{array}$ & $\begin{array}{l}\text { Days in } \\
\text { prone } \\
\text { posi- } \\
\text { tion }\end{array}$ & $\begin{array}{l}\text { Resp. } \\
\text { Rate } \\
\text { (min) } \\
\text { (ENT) }\end{array}$ & $\begin{array}{l}\text { Resp. } \\
\text { Rate } \\
\text { (EX) }\end{array}$ & APACHE II & SOFA & $\begin{array}{l}\text { PO2/ } \\
\text { FiO2 } \\
\text { entuba- } \\
\text { tion }\end{array}$ & $\begin{array}{l}\text { PO2/ } \\
\text { FiO2 } \\
\text { extuba- } \\
\text { tion }\end{array}$ \\
\hline mean & 15,3 & 10,55 & 39,55 & 18,9 & 19,85 & 6,1 & 83 & 196,45 \\
$\begin{array}{c}\text { Std. } \\
\text { dev }\end{array}$ & 6,2 & 2,87 & 5,14 & 4,45 & 3,18 & 1,25 & 27 & 32,56 \\
Min & 6 & 6 & 28 & 16 & 15 & 3 & 52 & 163 \\
Max & 38 & 18 & 54 & 34 & 25 & 9 & 165 & 280 \\
\hline
\end{tabular}

Conclusion. The prone position has been used as adjuvant therapy in moderate and severe ARDS patients and improved survival for many years. Since the main culprits of severe refractory hypoxemia in COVID-19 patients are atelectasis, alveolar damage, thrombotic occlusion, and ventilation-perfusion (V/Q) mismatch, prone positioning would help to improve alveolar shunt, recruitment in dorsal parts of lungs. Hence, this is a practical, low-cost treatment beneficial for respiratory improvement, survival, as well as length of hospital stay in COVID-19 patients. In the meantime, this treatment requires an experienced team to avoid undesired events, such as corneal ulceration and perforation, face injury, pressure ulcers, joint subluxation, aspiration of gastric content.

\section{Reference(s)}

1. A tricompartmental model of lung oxygenation disruption to explain pulmonary and systemic pathology in severe COVID-19 Prof Dennis McGonagle, FRCPI Charlie Bridgewood, PhD Prof James F M Meaney, FRCR Published:May 14, 2021https://doi.org/10.1016/S2213-2600(21)00213-7

2. Foti G, Giannini A, Bottino N, Castelli GP, Cecconi M, Grasselli G, et al. Management of critically ill patients with COVID-19: suggestions and instructions from the coordination of intensive care units of Lombardy. Minerva Anestesiol (Internet). 2020 Nov;86(11). https://www.minervamed ica.it/index2.php?show=R02Y2020N11A1234.

3. Chiara Robba, Denise Battaglini, Lorenzo Ball, Nicolo' Patroniti, Maurizio Loconte, lole Brunetti, Antonio Vena, Daniele Roberto Giacobbe, Matteo Bassetti, Patricia Rieken Macedo Rocco, Paolo Pelosi, Distinct phenotypes require distinct respiratory management strategies in severe COVID-19, Respiratory Physiology \& Neurobiology, Volume 279, 2020, 103,455, ISSN 1569-9048, https://doi.org/10.1016/j.resp.2020.103455.

\section{4}

Patients with COVID-19 admitted to Intensive Care Units in Sri Lanka: Experience from a resource limited setting

B. Imbulpitiya1; SWGJW. Chinthaka1; A. Galliyadda ${ }^{2}$; CV. Uduwela ${ }^{3}$;

P. Alagiyawanna ${ }^{4}$; R. Withanage ${ }^{4}$; A. Mudalige ${ }^{5}$; TGNP. Wimalasiri6.

ST. Kalhari ${ }^{7}$; U. Rodrigo ${ }^{5}$; C. Navaratne ${ }^{8}$; WDD. Priyankara ${ }^{9}$

${ }^{1}$ Critical Care, National Hospital_Kandy, Kandy, Sri Lanka; ${ }^{2}$ Emergency medicine, National Hospital—Kandy, Kandy, Sri Lanka; ${ }^{3}$ Anaesthesia and critical care, National Hospital—Kandy, Kandy, Sri Lanka; ${ }^{4}$ Anaesthesia \& critical care, Colombo East Base Hospital (CEBH) Mulleriyawa, Colombo, Sri Lanka; ${ }^{5}$ Critical care, Colombo North Teaching Hospital, Ragama, Sri Lanka; ${ }^{6}$ Critical care, Methsiri Sevana - , Anuradhapura, Sri Lanka; ${ }^{7}$ Critical care, University Hospital KDU, Boralesgamuwa, Sri Lanka; ${ }^{8} \mathrm{Critical}$ care, Colombo East Base Hospital (CEBH) Mulleriyawa, Colombo, Sri Lanka; ${ }^{9}$ Critical care, NHSL, Colombo, Sri Lanka

Correspondence: $\mathrm{B}$. Imbulpitiya

Intensive Care Medicine Experimental 2020, 9(1): 001454

Introduction. COVID-19 pandemic due to Severe acute respiratory syndrome coronavirus 2 (SARs-CoV-2) infection has made an enormous impact on lives of the world population. Sri Lanka has reported 154,786 tests positive COVID-19 patients and 1051 deaths to date(1) (2). Unavailability of data on COVID-19 related disease and intensive care management in Sri Lanka directed us to conduct this study.

Objectives. Study objectives were to describe the characteristics of admissions, organ involvement, intensity of treatment given, complications and outcomes, and associated factors on the outcome of adult COVID-19 critically ill patients. 
Methods. A cross sectional descriptive study was carried out over 6 months including adult patients (Age $>18$ years) admitted with confirmed SARs-CoV-2 infection by reverse transcription polymerase chain reaction (RT-PCR) or Rapid Antigen Test to dedicated COVID 19 ICUs/HDUs in Sri Lanka. Pregnant patients were excluded. Five centers scattered in 4 districts were included. Chi squire test was used to describe the associated factors in relation to outcome at $95 \%$ confidence interval placing the $p$ value at 0.05 .

Results. Total number of 114 patients were included in the study analysis. Majority $(80,70.2 \%)$ of the admissions to the ICUs were transfers from other hospitals. Majority (72,63.2\%) were males whilst mean age was 56.4 years (19-88). Significant proportion of patients $(92,80.7 \%)$ had one or more comorbidities. Fever was present on admission in 48 $(42 \%)$ of patients. Shortness of breath and cough was present in 70 $(61.4 \%)$ and, $53(46.5 \%)$ patients respectively. Seventy-four patients (64.9\%) had normal white blood cell count and leucopenia was observed in $7(6.1 \%)$ patients. Interestingly $23(20.2 \%)$ had normal chest $x$-rays.

All the patients required some form of oxygen therapy which included high flow nasal oxygen therapy (28,24.6\%), face mask $(49,43 \%)$, and nasal cannula $(27,23.7 \%)$. Noninvasive ventilation and invasive mechanical ventilation had been used in $33(28.6 \%)$ and $50(43.9 \%)$ at any given time during ICU stay. Sixteen patients (14\%) had circulatory shock needing cardiovascular support and $13(11.4 \%)$ had acute coronary syndrome. Acute kidney injury was present in 33 (28.9\%) patients. Significant number $(32,28.1 \%)$ of patients were already intubated at the time of admission to the ICUs. Twenty (17.5\%) patients had more than single organ failure. Ninety-four (82.4\%) patients received steroids and 92(80.7\%) received anticoagulation. Male gender ( $p-0.018)$, age more than 60 years $(\mathrm{p}-0.006)$, hypertension as a co-morbidity $(p-0.015)$, being intubated on admission ( $p-0.000)$, treated with invasive ventilation $(p-0.000)$ were significantly associated with mortality. Fifty-seven (50\%) patients survived to discharge from the ICU.

Conclusion. We observed that $50 \%$ of the patients have survived. Male gender, advanced age, hypertension, being intubated on admission and undergoing invasive ventilation have shown to be associated with poor outcome. Use of any mode of steroid had worse outcome. More studies with larger sample size are recommended for more robust data.

\section{Reference(s)}

Karunathilake K D U B, Karunadasa R M K M and Kulasiri H R

World Health Organization. COVID-19 Weekly Epidemiological Update 22.

3. Worldometer. Coronavirus Update (Live): Cases and Deaths from COVID19 Virus Pandemic [Internet]. Worldometers. 2021.

\section{8}

Effects of High Dose Vitamin C on Patient Outcomes in ARDS Patients Admitted to Intensive Care with COVID-19; Multi-Center Retrospective Study

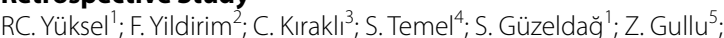
E. Eren ${ }^{6}$; M. Simsek ${ }^{2}$; H. Sipahioglu? ; K. Inci'; G. Zararsız ; K. Gundogan?; M. Sungur ${ }^{9}$

${ }^{1}$ Intensive care unit, Kayseri Sehir Hastanesi, Kayseri, Turkey; ${ }^{2}$ Intensive care unit, Ministry of Health, Diskapi Educating and Training Hospital, Ankara, Turkey; ${ }^{3}$ Intensive care, Dr. Suat Seren Chest Diseases Hospital, Izmir, Turkey; ${ }^{4}$ Internal medicine, Erciyes University, Kayseri, Turkey; ${ }^{5}$ Intensive care unit, Ministry of Health, Yenimahelle Educating and Traninh Hospital, Ankara, Turkey; ${ }^{6}$ Clinic of infectious disease, Kayseri Şehir Hastanesi, Kayseri, Turkey; ${ }^{7}$ Department of internal medicine, division of intensive care, Erciyes University, School of Medicine, Kayseri, Turkey; ${ }^{8}$ Department of biostatistics, Erciyes University, Kayseri, Turkey; ${ }^{9}$ Division of critical care medicine, department of internal medicine, Erciyes University School of Medicine, Kayseri, Turkey, Kayseri, Turkey Correspondence: R.C. Yüksel

Intensive Care Medicine Experimental 2020, 9(1): 001458

Introduction. The new type of Severe Acute Respiratory Syndrome Coronavirus 2 (Corona virus 2019- COVID-19) infection is the largest pandemic in the last decade. Acute respiratory distress syndrome (ARDS) is the complication of this infection with highest mortality and there is no medical treatment to reduce mortality with proven efficacy yet. The aim of this study was to investigate the effect of high dose vitamin C (ascorbic asid) on survival and other ICU outcomes of patients with COVID-19 associated ARDS who need invasive mechanical ventilation (IMV).

Methods. In this multicenter, non-intenventional retrospective cohort study, patients who were hospitalized in Intensive Care Units (ICU) due to confirmed COVID-19 associated ARDS and needed invasive IMV from 5 centers between March 2020 and July 2020 were included. Among these patients, those receiving vitamin $C$ treatment over $200 \mathrm{mg} / \mathrm{kg}$ for 4 days were defined as the high-dose vitamin C group, and those who did not take vitamin $\mathrm{C}$ as defined as control group by using propensity score match analysis. The primary endpoint of study was ICU mortality.

Results. A total of 86 patients with a mean age of $67.85 \pm 10.38$ were included in the study, $72.1 \%$ of whom were male. The median APACHE II score of the patients was 19.5 (15.0-26.0). 42 of them were in the high dose vitamin $\mathrm{C}$ group and 44 of them were in the control group. The mortality of the group that received high dose vitamin $C$ treatment was found to be lower than the group that did not $73.8 \%$ vs $90.9 \% ; p=0.037)$. The duration of MV [10.0 (7.0-18.0) vs $11.0(6.5-$ $17.0) ; p=0.92)$ wasn't differen and the duration of stay in the ICU (median 15 ve $13, p=0.016$ ) were longer in the high-dose vitamin $C$ group. There was no difference between the two groups in terms of other ICU clinical outcomes (acute renal failure, liver damage, thrombosis / infarction, etc.) $(p>0.05)$.

Conclusion. High dose of vitamin $C$ as an adjunctive therapy in invasively ventilated patients with COVID-19 associated ARDS can reduce mortality. Further prospective, randomised controlled trials are required to confirm these findings.

\section{Reference(s)}

1. Kumari P, Dembra S, Dembra P, et al. The Role of Vitamin C as Adjuvant Therapy in COVID-19. Cureus. 2020;12(11):e11779. Published 2020 Nov 30. $10.7759 /$ cureus. 11779

2. Cucinotta D, Vanelli M. WHO Declares COVID-19 a Pandemic. Acta Biomed. 2020;91(1):157-160. Published 2020 Mar 19. 10.23750/abm.v91i1. 9397

e-Posters: AIDS, haematologic-oncologic issues in the ICU 000081 Oncological patients who enter in ICU

T. Amat Serna'; C. Aranda Martínez²; E. Pérez Mesa; L. González Gracianteparaluceta'; RF. R. ${ }^{3}$

${ }^{1}$ Medicina intensiva, Hospital of Jaen, Jaén, Spain; ${ }^{2}$ Medicina Intensiva, Hospital of Jaen, Jaén, Spain; ${ }^{3}$ Intensive care, Hopsital Neurotraumatológico Universitario, Jaen, Spain

Correspondence: C. Aranda Martínez

Intensive Care Medicine Experimental 2020, 9(1): 000081

Introduction. We want to analyze which oncological patients presented to the Committee on Oncology and ICU of our hospital have been admitted to the ICU and what has been their evolution.

Methods. Patients with oncological pathology admitted to the Hospital Universitario de Jaén from 2019 to October 2020, presented in the Committee Oncology-ICU. Demographic data, personal history, cause of admission and evolution in ICU. We have performed a descriptive analysis with SPSS.

Results. A total of 135 patients presented, $62 \%$ male $(\mathrm{N}=83)$ and a median age of 62 years (20-79). In the Committee: $25 \%$ rejected $(\mathrm{N}=34)$ to enter en ICU, 25\% $(\mathrm{N}=34)$ candidates for non-invasive measures and $50 \%(\mathrm{~N}=67)$ candidates for invasive measures. In the follow-up, $61 \%$ of the total are still alive $(83 \%$ candidates for invasive measures)

Of the total of patients, 7 patients were admitted to the ICU (6 of them were candidates for invasive measures). Personal history: 3 hypertensive patients and one dyslipidemia. Type of tumor: 3 pancreatic tumor, 3 digestive tumors and 1 lung; administered treatment: Surgical 4/7, chemotherapy $7 / 7$ (4 active at the time of 
consultation), radiotherapy $2 / 7$ and one of them was in remission. Causes of admission to ICU: 3 due to respiratory infection, 2 after surgical intervention, one patient for septic shock and one for metabolic alterations.

Intensive measures applied: intubation 5/7 patients ( 1 tracheostomy patient), High flow nasal oxygen $2 / 7$, patients, continuos replacement renal terapy $1 / 7$ patients, vasopressors $5 / 7$. All 7 had infectious complications by germs Gram negative the most frequent. Vital support limitation was performed in 3 patients after progression of the underlying oncological disease. Of the remaining 4 , one of them died.

Conclusion. The improvement of the prognosis of cancer patients and the advances in the treatment that are currently occurring makes some of them subsidiary of admission to ICU. The creation of Oncology and ICU Committees allow to know previously those patients who do obtain a benefit upon admission to the ICU. Our results are scarce, since the number of admitted patients is small.

\section{8}

\section{Survival of patients with acute myeloid leukaemia admitted} to intensive care unit: single centre experience

S. Judickas ${ }^{1}$; R. Stasiūnaitis ${ }^{2}$; A. Zucenka ${ }^{3}$; M. Serpytis ${ }^{1}$; J. Sipylaite

${ }^{1}$ Institute of Clinical Medicine, Clinic of Anaesthesiology and Intensive

Care, Faculty of Medicine of Vilnius University, Vilnius, Lithuania; ${ }^{2}$ Faculty of medicine, Vilnius University, Vilnius, Lithuania; ${ }^{3}$ Clinic of internal diseases, family medicine and oncology, institute of clinical medicine, Faculty of Medicine of Vilnius University, Vilnius, Lithuania

Correspondence: $\mathrm{S}$. Judickas

Intensive Care Medicine Experimental 2020, 9(1): 000488

Introduction. Acute myeloid leukemia (AML) is a very aggressive haematological malignancy. Mortality of oncohaematological patients admitted to intensive care unit (ICU) is very high and it is worse compared with non-oncological patients and solid tumors.

Objectives. The aim of our study was to compare short and longterm survival of $\mathrm{AML}$ and non-AML oncohaematological patients admitted to ICU.

Methods. Prospective observational study took place in Vilnius university hospital Santaros clinics from 13072017 to 31122019. Patients with oncohaematological malignancy, age $>18$ years, arterial or central line inserted, admitted to intensive care unit (ICU) and signed informed consent form were included to the study. We grouped patients into two groups according to haematological malignancy-AML and non-AML. Statistical analysis was performed with $\mathrm{R}$ statistical package. A p-value less than 0.05 was considered to be statistically significant.

Results. Our analysis included 114 patients of which 35 (30.70\%) were diagnosed with AML. Non-AML patients included non-Hodgkin's lymphoma (24.6\%), multiple myeloma (10.53\%), chronic lymphocytic leukaemia (8.8\%), acute lymphoblastic leukaemia (7.0\%), Hodgkin's lymphoma (3.5\%). Patient characteristics is provided in Table 1. Laboratory test results on admission to ICU are summarized in Table 2. ICU, 30 days, hospital, 90 days and overall mortality did not differ between AML and non-AML patients and it was following $42.86 \%$ vs $45.57 \%, 60 \%$ vs $51.9 \%, 62.86 \%$ vs $51.9 \%, 62.86 \%$ vs $65.82 \%$ and $74.29 \%$ vs $74.68 \%, p>0.05$.

Table 1. Patient characteristics (No of patients (\%) or mean \pm SD)

\begin{tabular}{llll}
\hline Characteristics & AML $(\mathbf{n}=\mathbf{3 5})$ & Non-AML $(\mathbf{n}=\mathbf{7 9})$ & $\mathbf{p}$ value \\
\hline Male sex & $20(57.1)$ & $45(57.0)$ & 0.9856 \\
Age & $61.09 \pm 15.32$ & $59.25 \pm 15.47$ & 0.5449 \\
$\begin{array}{l}\text { Charlson's comorbidity } \\
\text { index }\end{array}$ & $4.89 \pm 2.10$ & $4.91 \pm 2.34$ & 0.9852 \\
$\begin{array}{l}\text { Intensive chemotherapy } \\
\text { regimen }\end{array}$ & $19(54.29)$ & $37(46.84)$ & 0.8286 \\
\hline
\end{tabular}

Table 1. Patient characteristics (No of patients (\%) or mean \pm SD)

\begin{tabular}{llll}
\hline Characteristics & AML $(\mathbf{n}=\mathbf{3 5})$ & Non-AML $(\mathbf{n}=\mathbf{7 9})$ & p value \\
\hline $\begin{array}{l}\text { Bone marrow transplanta- } \\
\text { tion }\end{array}$ & $12(34.29)$ & $27(34.18)$ & 0.0126 \\
-Allogenic & $12(34.29)$ & $12(15.19)$ & \\
-Autologic & $0(0)$ & $14(17.72)$ & \\
qSOFA score & $1.23 \pm 0.94$ & $1.47 \pm 0.89$ & 0.1493 \\
SAPS 3 score & $74.23 \pm 15.30$ & $75.35 \pm 12.36$ & 0.6782 \\
APACHE II score & $20.20 \pm 5.49$ & $22.39 \pm 5.67$ & 0.0557 \\
$\begin{array}{l}\text { SOFA score } \\
\text { Vasoactive drugs }\end{array}$ & $17(48.57)$ & $62(78.48)$ & 0.2569 \\
$\begin{array}{l}\text { Invasive mechanical } \\
\text { ventilaton }\end{array}$ & $18(51.43)$ & $45(56.96)$ & 0.5836 \\
$\begin{array}{l}\text { Continuous renal replace- } \\
\text { ment therapy }\end{array}$ & $5(14.29)$ & $24(30.38)$ & 0.0688 \\
& & & \\
\hline
\end{tabular}

Table 2. Laboratory test results on admission to ICU

\begin{tabular}{llll}
\hline Laboratory test & AML & Non-AML & p value \\
\hline Arterial pH & $7.41 \pm 0.18$ & $7.39 \pm 0.14$ & 0.3714 \\
Lactate & $2.65 \pm 2.72$ & $3.38 \pm 4.32$ & 0.8851 \\
Neutropenia & $16(45.71)$ & $28(35.44)$ & 0.2661 \\
Thrombocytopenia & $12(34.29)$ & $27(34.18)$ & 0.991 \\
\hline
\end{tabular}

Conclusion. Long and short-term survival between AML and non-AML oncohaematological patients admitted to ICU did not differ.

\section{9}

Time-sensitive analysis of vasopressor dose in cancer patients with septic shock

M. Chotalia'; M. Bangash, 2; D. Parekh, ; j. Patel, ${ }^{2}$

${ }^{1}$ Institute of Inflammation and Ageing, University of Birmingham, Birmingham, United Kingdom; ${ }^{2}$ Critical care, Queen Elizabeth Hospital Birmingham, Birmingham, United Kingdom; ${ }^{3}$ tu, Queen Elizabeth Hospital Birmingham, Birmingham, United Kingdom

Correspondence: M. Chotalia

Intensive Care Medicine Experimental 2020, 9(1): 000539

Introduction. It is unknown whether longitudinal assessment of vasopressor requirements can aid in the prognostication of cancer patients with septic shock [1].

Objectives. The aim of this study was to identify a threshold vasopressor dose and time associated with mortality in cancer patients with septic shock.

Methods. This is a single-centre retrospective cohort study. Patients with cancer admitted to the Queen Elizabeth Hospital, Birmingham, UK Intensive Care Unit (ICU) between April 2016 and July 2019 with septic shock were included. Daily median vasopressor dose was calculated from summation of norepinephrine-equivalent infusion rates of all administered vasopressors / inotropes and recorded for the first four days. Area under the receiver operator characteristic curve (AUROC) analysis was performed.

Results. 844 patients were admitted with septic shock, of which 294 had cancer. Of these patients, $43 \%(n=126)$ had solid loco-regional (SLR) disease, 26\% $(n=76)$ had solid metastatic (SM) disease and 31\% $(n=92)$ had haematological malignancy (HM). AUROC for vasopressor dose increased on consecutive days. The highest AUROC was obtained on day-4: $0.83(0.73-0.92)$ for SLR, $0.88(0.75-1.0)$ for SM and 0.73 (0.61-0.85) for HM. A dose threshold of $0.05 \mathrm{~g} / \mathrm{kg} /$ minute on day four elicited a sensitivity and specificity for 90 -day mortality of $80 \%, 85 \%$ and $70 \%$ for each cancer type respectively. 
Conclusion. Persistent low vasopressor requirements on the fourth day of septic shock associated closely with mortality in patients with solid organ but not haematological malignancy.

\section{Reference(s)}

1. [1] Zampieri FG, Romano TG, Salluh JIF, Taniguchi LU, Mendes PV, Nassar AP, Jr., et al. Trends in clinical profiles, organ support use and outcomes of patients with cancer requiring unplanned ICU admission: a multicenter cohort study. Intensive Care Med. 2020, 10.1007/s00134-020-06184-2.

2. None

\section{8}

Prognosis in critically ill patients with various life-threatening complications after allogeneic hematopoietic stem cell transplantation

A. Shchekina ${ }^{1} ;$ G. Galstyan ${ }^{1}$; M. Drokov ${ }^{2}$

${ }^{1}$ Intensive care unit, National Research Center for Hematology, Moscow,

Russia; ${ }^{2}$ Department for stem cell transplantation, National Research

Center for Hematology, Moscow, Russia

Correspondence: A. Shchekina

Intensive Care Medicine Experimental 2020, 9(1): 001438

Introduction. Intensive care management for a life-threatening complication is required for $10-57 \%$ of patients after allogeneic hematopoietic stem cell transplantation (allo-HSCT) [1].

Objectives. To investigate short-term and long-term outcomes of patients after allo-HSCT, who were admitted in ICU for the treatment of life-threatening complications.

Methods. In retrospective study 144 patients admitted to the ICU after allo-HSCT from 2012 to 2020 allo-HSCT were included (Table 1). The results were analyzed by IBM SPSS v.23 (USA). Overall survival (OS) was estimated using the Kaplan-Meier method with Log-rank test. A $p$-value of $<0.05$ was considered statistically significant.

\begin{tabular}{|ll}
\hline & ICU admitted patients \\
\cline { 2 - 2 } & $n=144$ \\
\hline Age (years) & $37(19-64)$ \\
\hline Underlying disease & \\
\hline AML & $70(49 \%)$ \\
\hline ALL & $37(26 \%)$ \\
\hline MDS & $13(9 \%)$ \\
\hline Lymphoma & $7(5 \%)$ \\
\hline Others & $17(11 \%)$ \\
\hline Remission & $102(71 \%)$ \\
\hline Complete remission & $42(29 \%)$ \\
\hline Non-complete remission & $32(22 \%)$ \\
\hline Donor source & $58(40 \%)$ \\
\hline Related HLA-matched & $32(22 \%)$ \\
\hline Unrelated HLA-matched & $22(15 \%)$ \\
\hline Unrelated HLA-mismatched & \\
\hline Haploidentical & $73(51 \%)$ \\
\hline Acute GvHD & $71(49 \%)$ \\
\hline Yes & \\
\hline No & \\
\hline
\end{tabular}

Results. 30 (21\%) of the 144 patients were discharged from ICU. 5-year overall survival of critically ill allo-HSCT patients admitted to the ICU was $9 \%$. The highest OS (41\%) was registered for the patients admitted in ICU for the treatment of sepsis/septic shock. The OS for patients with acute respiratory failure (ARF) was only $4 \%$. The worst OS (0\%) was observed in critically ill patients, admitted in ICU due to graft-versus-host disease (GvHD) of gastrointestinal (Gl) tract and pulmonary GvHD (Fig. 1).

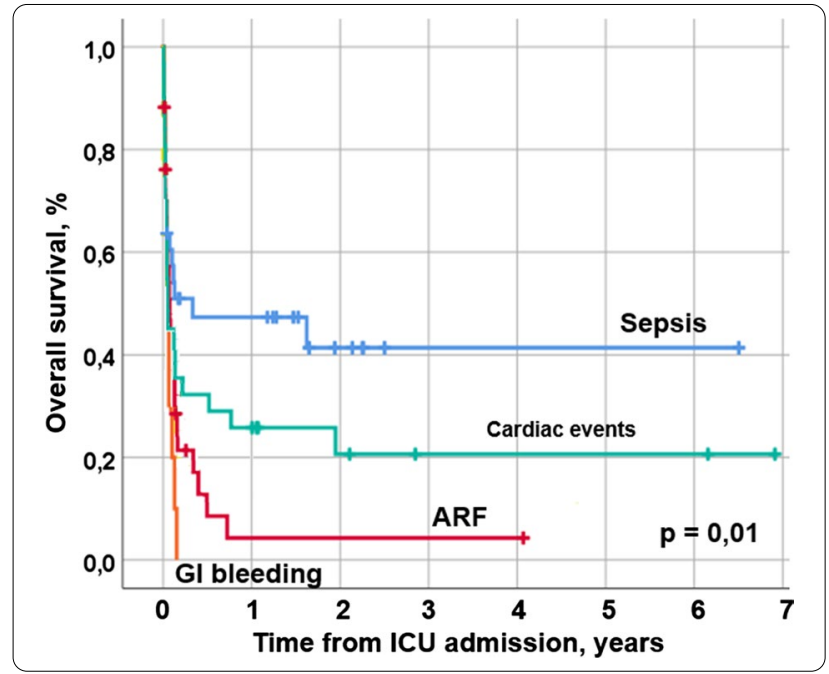

Conclusion. It was assessed that the OS rate depends on the causes of life-threatening complications. The worst prognosis among patients after transplantation was observed in steroid-refractory forms of GvHD. Infectious conditions that proceed without respiratory failure were the most curable complications.

\section{Reference(s)}

1. Saillard C., Darmon M., Bisbal M., et al. Critically ill allogenic HSCT patients in the intensive care unit: a systematic review and meta-analysis of prognostic factors of mortality. Bone Marrow Transplant. 2018;53(10):1233-

1241. 10.1038/s41409-018-0181-X.

2. National Research Center for Hematology

e-Posters: Cardiac arrest

\section{6}

Code blue between decision and delivery

A. sharma ${ }^{1}$; A. Joshi ${ }^{2}$; V. Sathe ${ }^{1}$

${ }^{1}$ ANAESTHESIOLOGY, MGM MEDICAL COLLEGE AND HOSPITAL, Navi Mumbai, India; ${ }^{2}$ Anaesthesiology, MGM MEDICAL COLLEGE AND HOSPITAL, Navi Mumbai, France

Correspondence: A. sharma

Intensive Care Medicine Experimental 2020, 9(1): 000096

Introduction. The recommendation to perform a perimortem cesarean section(PMCS) within 4 min of maternal cardiac arrest was introduced in 1986. This recommendation was based on assumptions that cardiopulmonary resuscitation is ineffective in the third trimester because of aortocaval compression, and that the fetal and perhaps the maternal outcomes would be optimized by timely delivery.

Methods. CASE 1: A 23-year female, gravida 1, parity 0 at 34-week gestation, presented in the Emergency room at $9 \mathrm{pm}$ at night with complaints of bleeding per vaginally since morning, headache \& dizziness with 2 episodes of vomiting. She was transferred to the labor room with a GCS of $11 / 15$, saturation of $90 \%$ on RA, HR of $160 \mathrm{bpm}$, and a feeble peripheral pulse with systole BP $80 \mathrm{~mm}$ hg. The decision of emergency LSCS was made. While shifting the patient to OR, she collapsed pulses absent. A CPR was commenced with a left pelvic wedge applied. Pt intubated immediately, iv access taken, intravenously epinephrine $1 \mathrm{mg}$ administered and crystalloid started. After 2 cycles of $\mathrm{CPR}$, no return of $\mathrm{CO}$, the decision of perimortem cesarean made as USG Doppler confirmed FHS. A Pfannenstiel incision made and a live female infant was delivered within 3 min with an APGAR score of 1 \& 6 at $1 \& 5$ min respectively. Advanced life support continued throughout the perimortem $\mathrm{c}$ section with a further dose of epinephrine. 
Spontaneous return of maternal CO occurred 5 min after delivery of the infant. i.e. $15 \mathrm{~min}$ after the initial arrest. An exploratory laparotomy was done to control the bleeders and the patient was given 2 PCV, 4 FFP, and 2 platelets with infusion noradrenaline and vasopressin to support the hemodynamic instability. Later, she was shifted to ICU for venti and inotropic support, a 2D echo was done which was suggestive of a global LV hypokinesia with LVEF $20 \%$. She was discharged 10 days later after the initial event with a healthy infant.

CASE 2: A 30-year-old female gravida 3, parity 1, abortion 2 at 32-week gestation, presented in the Emergency room with vague history with previous normal delivery with BD 32 weeks with Eclampsia. The patient arrived in casualty in an unconscious state with a history of 2 episodes of convulsions. On arrival, pulse $88 / \mathrm{min}$, BP $210 / 110 \mathrm{mmHg}$ FHS present, spo $280 \%$ on $6 \mathrm{~L}$ of Oxygen via AMBU, urine albumin $2+$. The patient was given injection magnesium sulfate $4 \mathrm{gm}$ IV stat, after 1 episode of convulsion at casualty, injection magnesium sulfate $5 \mathrm{gm}$ intramuscularly given on each buttock, following which patient had another episode of convulsion during Foley's catheterization, Injection Labetalol $20 \mathrm{mg}$ intravenously given and Injection Midazolam $2 \mathrm{ml}$ intravenously given. The patient was not maintaining saturation and hence, the decision of Rapid sequence intubation taken, patient tubed and was paralyzed, the vitals were BP $100 / 90 \mathrm{mmHg}$, PR feeble but carotid present, Spo2 82\% on high flow oxygen via AMBU. The obstetricians took a decision of perimortem Ceaserean section stat and patient was shifted to operation theater where patient collapsed and CPR started, alongside a Pfannenstiel incision made and a live male infant was delivered within 2 mins with an APGAR score of $2 \& 5$ at 1 $\& 5$ min respectively. ROSC was achieved after $14 \mathrm{~min}$ of CPR and the patient was shifted to ICU on supports, Noradrenaline, and vasopressin. the patient was extubated on the 4th day of the event and was discharged from the facility on the 12th day.

Results. Perimortem cesarean delivery, therefore, remains a key consideration in the management of maternal arrest from the mid-second trimester. Simulation training develops these team-orientated skills, including allocating tasks to specific team members who know and understand their individual roles. In my opinion, multidisciplinary team training in cardiorespiratory arrest leading to PMCS should be a mandatory 'hot drill' that is practiced regularly in all ER's and labor rooms.

Conclusion. The bottom line is a need for rapid action to maximize the chance of maternal survival. Therefore, the critical step is to make the decision to proceed with a PMCS keeping fetal survival as a secondary issue and not a prime driver for deciding PMCS.

\section{Reference(s)}

1. Siassakos D, Fox R, Crofts JF, Hunt LP, Winter C, Draycott TJ. The management of a simulated emergency: better teamwork, better performance. Resuscitation 2011; 82: 203- 6

2. Smith A, Edwards S, Siassakos D. Effective team training to improve outcomes in maternal collapse and perimortem caesarean section. Resuscitation 2012; 83: 1183- 4 .

3. Eldridge AJ, Ford R. Perimortem caesarean deliveries. Int J Obstet Anesth. 2016 Aug;27:46-54. https://doi.org/10.1016/j.joa.2016.02.008. Epub 2016 Mar 3. PMID: 27,103,543

4. We would like to thank our entire department of Anesthesiology and our professor and head of department Dr. R L Gogna for his constant support and suggestion.

\section{2}

Prognostic factors associated with 30-day mortality in OHCA patients with ACS in non-urban areas

J. Higny'; M. Regnier²; J. Flament ${ }^{3}$; F. Forêt ${ }^{4}$

${ }^{1}$ Cardiovascular Disease, CHU UCL Namur-Site Godinne, Yvoir, Belgium; ${ }^{2}$ Unité de support scientifique, CHU UCL Namur-Site GSsec4 odinne, Yvoir, Belgium; ${ }^{3}$ Emergency department, CHU UCL Namur-Site Godinne, Yvoir, Belgium; ${ }^{4}$ Intensive care medicine, Chu Ucl Namur-Site De Dinant, Dinant, Belgium

Correspondence: J. Higny

Intensive Care Medicine Experimental 2020, 9(1): 000172
Introduction. Pre-hospital management of acute coronary syndrome (ACS) complicated with out-of-hospital cardiac arrest (OHCA) remains challenging, particularly in non-urban areas. The implementation of appropriate treatment strategies for improving prehospital emergency care is fundamental. Most studies have been conducted in city areas. Therefore, less is known about the resuscitation performances of emergency medical service (EMS) teams in sparsely populated areas. Built at the top of a hill, the location of our institution consists of rural areas with a low population density. Established in 1903, the hospital was initially founded to treat patients suffering from tuberculosis.

Objectives. To perform a quality control assessment on the prehospital management of sudden coronary death in non-urban areas. To identify qualitative and quantitative variables influencing the 30-day outcome.

Methods. Retrospective single-center study including 40 patients resuscitated from OHCA after ACS. Baseline characteristics, resuscitation settings, time intervals, blood samples, cardiac findings and clinical outcomes at 30 days were analyzed.

Results. 30-day mortality was more likely associated with the following parameters: unwitnessed cardiac arrest, kilometers, duration of NF/LF, epinephrine dose, time to cardiac catheterization, gender, asystole, recurrent cardiac arrest, cardiogenic shock, lactate level, LVEF and LAD culprit lesion. Particularly, remoteness from hospital and prolonged pre-hospital management (duration of CPR, delayed cath lab admission) were predictors of early mortality.

Table. 30-day mortality related to patient characteristics, resuscitation settings, demographic parameters, time intervals and cardiac findings.

\begin{tabular}{lllll}
\hline Parameters & OR & Lower & Upper & P-value \\
\hline Witness status & 0.02 & 0.00 & 0.11 & 0.0003 \\
No bystander CPR & 66.00 & 9.35 & 1396.77 & 0.0003 \\
Kilometers & 5.14 & 1.35 & 22.19 & 0.02 \\
Duration of no-flow & 31.62 & 5.95 & 266.55 & 0.0002 \\
Duration of low-flow & 73.50 & 10.61 & 1546.43 & 0.0002 \\
Epinephrine dose & 10.06 & 1.97 & 77.84 & 0.01 \\
Fibrinolysis & 0.49 & 0.06 & 2.51 & 0.42 \\
Time to cath lab admission & 3.86 & 1.03 & 15.82 & 0.05 \\
Duration of cardiac catheterization & 3.50 & 0.87 & 15.24 & 0.08 \\
Time to ICU admission & 3.08 & 0.40 & 64.68 & 0.342 \\
Male & 0.13 & 0.02 & 0.68 & 0.02 \\
Asystole & 12.00 & 1.66 & 246.00 & 0.03 \\
Recurrent cardiac arrest & 16.00 & 2.31 & 325.28 & 0.01 \\
Cardiogenic shock & 17.25 & 3.41 & 135.71 & 0.001 \\
Lactate & 74.75 & 11.84 & 840.47 & 0.00004 \\
Left ventricular ejection fraction & 0.05 & 0.01 & 0.22 & 0.0003 \\
LAD culprit lesion & 4.33 & 1.07 & 22.58 & 0.05 \\
RCA/LCX culprit lesion & 0.38 & 0.07 & 1.55 & 0.199 \\
\hline
\end{tabular}

Conclusion. Beyond the usual prognostic factors, remoteness from hospital, duration of resuscitation on-site and time to cath lab admission may represent major determinants of clinical outcomes. Also, the relationship between early mortality and duration of noflow, non-shockable rhythm, hemodynamic instability and lactate level may reflect the impact of prolonged time intervals during the pre-hospital phase of management. Therefore, EMS teams should focus on improving pre-hospital response times and optimizing cardiac cath lab activation. Further RCTs are needed to evaluate the impact of the 'scoop and run' approach with direct admission to the cath lab versus the 'stay and play' approach in this setting.

\section{Reference(s)}

1. Semeraro F, Greif R, Böttiger BW, Burkart R, Cimpoesu D, Georgiou M, Yeung J, Lippert F, S Lockey A, Olasveengen TM, Ristagno G, Schlieber J, Schnaubelt S, Scapigliati A, G Monsieurs K. European Resuscitation Council Guidelines 2021: Systems saving lives. Resuscitation. 2021 Apr;161:8097. https://doi.org/10.1016/j.resuscitation.2021.02.008. Epub 2021 Mar 24. PMID: $33,773,834$ 
2. Neumann FJ, Sousa-Uva M, Ahlsson A, Alfonso F, Banning AP, Benedetto U, Byrne RA, Collet JP, FalkV, Head SJ, Jüni P, Kastrati A, Koller A, Kristensen SD, Niebauer J, Richter DJ, Seferovic PM, Sibbing D, Stefanini GG, Windecker S, Yadav R, Zembala MO; ESC Scientific Document Group. 2018 ESC/ EACTS Guidelines on myocardial revascularization. Eur Heart J. 2019 Jan 7;40(2):87-165. https://doi.org/10.1093/eurheartj/ehy394. Erratum in: Eur Heart J. 2019 Oct 1;40(37):3096. PMID: 30,165,437.

3. Chocron R, Loeb T, Lamhaut L, Jost D, Adnet F, Lecarpentier E, Bougouin W, Beganton F, Juvin P, Marijon E, Jouven X, Cariou A, Dumas F; Paris SDEC Investigators. Ambulance Density and Outcomes After Out-of-Hospital Cardiac Arrest. Circulation. 2019 Mar 5;139(10):1262-1271. https://doi. org/10.1161/CIRCULATIONAHA.118.035113. PMID: 30,586,753.

\section{5}

Optimal instructions for dispatcher-assisted cardiopulmonary resuscitation for coaching callers after out-of-hospital cardiac arrests

Y. Goto'; A. Funada 2 ; T. Maeda ${ }^{3}$; Y. Goto ${ }^{4}$

${ }^{1}$ Emergency and Critical Care Medicine, Kanazawa University Hospital, Kanazawa, Japan; ${ }^{2}$ Department of cardiology, Saiseikai Senri Hospital, Suita, Japan; ${ }^{3}$ Department of emergency and critical care medicine, Kanazawa University Hospital, Kanazawa, Japan; ${ }^{4}$ Department of cardiology, Yawata Medical Centre, Komatsu, Japan

Correspondence: $Y$. Goto

Intensive Care Medicine Experimental 2020, 9(1): 000215

Introduction. The International Liaison Committee on Resuscitation (ILCOR) recommends dispatchers provide instructions to perform compression-only cardiopulmonary resuscitation (CPR) to callers for adults with suspected out-of-hospital cardiac arrest (OHCA), despite low-quality evidence of benefits. Consistent with ILCOR, the European Resuscitation Council recommends compression-only dispatcherassisted CPR (DA-CPR) for adults with OHCA in their 2021 guidelines.

Objectives. We aimed to determine optimal DA-CPR instructions, namely compression-only or conventional CPR with rescue breaths (30 compressions to 2 ventilations), after OHCA from cardiac causes.

Methods. We analysed records of 42,767 adult patients who received bystander DA-CPR before emergency medical services' arrival after bystander-witnessed OHCA due to a presumed cardiac event. Data were obtained from a prospectively recorded Japanese nationwide Utstein-style database for a 5-year period (2013-2017). Patients were divided into compression-only CPR $(n=38,191)$ and conventional CPR $(n=4576)$ groups. Propensity score (PS) matching using 6 pre-hospital variables was applied for analyses. The primary study endpoint was 1-month neurological intact survival, defined as a Cerebral Performance Categories score of 1-2 (CPC 1-2). Secondary study endpoints were 1-month survival and pre-hospital return of spontaneous circulation (ROSC).

Results. The 1-month CPC 1-2 rate was significantly higher in the conventional CPR group than in the compression-only CPR group (before PS matching, 7.9\% [359/4576] vs. 7.0\% [2660/38,191], $p=0.03$, relative risk [RR] [95\% confidence interval $\{\mathrm{Cl}\}] 1.12$ [1.01-1.24]; after PS matching, 7.8\% [358/4572] vs. 6.2\% [283/4572], p<0.01, RR [95\% Cl] 1.26 [1.17-1.35]). The multivariable logistic regression model showed that compared with compression-only DA-CPR, conventional DA-CPR was associated with increased odds of 1-month CPC 1-2 (adjusted odds ratio $1.39,95 \% \mathrm{Cl} 1.21-1.58, \mathrm{p}<0.0001)$. However, there were no significant differences in the 1-month survival and pre-hospital ROSC analysis between the conventional CPR and compression-only CPR groups (before PS matching, 10.7\% [491/4576] vs. 10.9\% [4168/38,191] for 1 -month survival, $p=0.73,16.8 \%[767 / 4576]$ vs. $16.4 \%[6253 / 38,191]$ for pre-hospital ROSC, $\mathrm{p}=0.50$; after PS matching, $10.7 \%$ [490/4572] vs. $9.8 \%$ [449/4572] for 1-month survival, $p=0.17,16.8 \%[766 / 4572]$ vs. $15.9 \%$ [726/4572] for pre-hospital ROSC, $p=0.27)$.

Conclusion. Conventional DA-CPR with a ratio of 30 compressions to 2 ventilations was superior to compression-only DA-CPR in adults with bystander-witnessed OHCA due to a cardiac event. Conventional CPR with rescue breaths may be preferable to compression-only CPR as an optimal DA-CPR instruction for coaching callers to perform bystander CPR.

\author{
Reference(s) \\ 1. Semeraro F et al. Resuscitation 2021:161:80-97 \\ 2. This work was supported by the Japan Society for the Promotion of Sci- \\ ence (Grant Nos. 18K0999, 20H202271, and 21K10324).
}

000274

Application of a standardized EEG pattern classification in the assessment of neurological prognosis after cardiac arrest-a retrospective analysis

L. Lilja'; S. Joelsson, ${ }^{2}$; J. Nilsson ${ }^{2}$; S. Lindgren ${ }^{1}$; C. Rylander ${ }^{1}$

${ }^{1}$ Anesthesiology and intensive care medicine, Sahlgrenska University Hospital, Gothenburg, Sweden; ${ }^{2}$ Clinical neurophysiology, Sahlgrenska

University Hospital, Gothenburg, Sweden

Correspondence: L. Lilja

Intensive Care Medicine Experimental 2020, 9(1): 000274

Introduction. *Abstract from a submitted manuscript ${ }^{*}$

Electroencephalogram (EEG) is used in the neurological prognostication after cardiac arrest. "Highly malignant" EEG patterns classified according to Westhall have a high specificity for poor neurological outcome when applied within protocols of recent studies (see References). However, their predictive performance when applied in everyday clinical practice has not been investigated. We studied the prognostic accuracy and the interrater agreement when standardized EEG patterns were analysed and compared to neurological outcome in a patient cohort at a tertiary centre not involved in the original study of the standardized EEG pattern classification.

Methods. Comatose patients treated for out-of-hospital cardiac arrest were included. Poor outcome was defined as Cerebral Performance Category 3-5. Two senior consultants and one resident in clinical neurophysiology, blinded to clinical data and outcome, independently reviewed their EEG registrations and categorised the pattern as "highly malignant", "malignant" or "benign". These categories were compared to neurological outcome at hospital discharge. Interrater agreement was assessed using Cohen's Kappa.

Results. In total, 62 patients were included. The median (IQR) time to EEG was 59 (42-91) h after ROSC. An early EEG ( $<24 \mathrm{~h}$ after ROSC), the earliest $10 \mathrm{~h}$ after ROSC, was performed in six of the 62 cases $(10 \%)$ cases because of overt or suspected seizures. Three of these patients did not receive TTM treatment. In 39 (63\%) cases, EEG was performed $>48 \mathrm{~h}$ after ROSC. A "highly malignant" pattern was found 21 (34\%) patients, all of whom had died at hospital discharge. A "malignant" pattern was found in $37(60 \%)$ patients of whom ten $(27 \%)$ had a good neurological outcome.

Among the 21 patients with a "highly malignant" EEG pattern, there was agreement between two raters in five (24\%) patients and between all three raters in three (14\%) patients. Out of the 37 patients with a "malignant" EEG pattern, there was agreement between two raters in nine $(24 \%)$ patients and between all three raters in 28 (76\%) patients. In four patients, all raters considered the EEG to contain a "benign" pattern. The corresponding Cohen's kappa varied from 0.62 to 0.29 with best agreement found between the resident and one of the senior consultants.

Conclusion. The results from this retrospective study support earlier evidence of good prognostic accuracy of "highly malignant" patterns classified according to Westhall to predict poor neurological outcome in comatose survivors of cardiac arrest. However, "malignant" patterns remain indeterminate for prognosis. The interrater agreement may vary when the standardized EEG pattern classification is applied in everyday practice, even between experience EEG readers.

\section{Reference(s)}

1. Westhall et al., 2016 Apr 19;86(16):1482-90

2. Backman et al., Resuscitation 131 (2018) 24-28

3. This study was made possible due to research grants from the Gothenburg Society of Medicine and departmental funding. 
000281

Hemodynamic effect of Ketone Bodies infusion after resuscitation in an experimental model of cardiac arrest

F. Annoni ${ }^{1}$; F. Su, ${ }^{1}$; L. Peluso ${ }^{1}$; E. Bogossian ${ }^{1}$; A. Minini ${ }^{1}$; J. Creteur ${ }^{1}$;

FS. Taccone

${ }^{1}$ Department of intensive care, Erasme University Hospital, Université libre de Bruxelles, Brussels, Belgium

Correspondence: F. Annoni

Intensive Care Medicine Experimental 2020, 9(1): 000281

Introduction. Post cardiac arrest (CA) syndrome is a major determinant of poor outcome in CA survivors1,2. Ketone Bodies (KB), and in particular B-3-hydrohybutyrate (3-OHB), could be metabolized as energy source by myocardial cells and has been associated with beneficial effects on cardiac function in both healthy humans and patients with chronic heart failure 3-4 and could help reducing the post-ischemic reperfusion injury5-7. At present, no experimental study assessed the hemodynamic impact of $3-\mathrm{OHB}$ on myocardial function in a cardiac arrest model.

Objectives. The experiment was conceived to explore the hemodynamic impact of Ketone Bodies infusion in the first hours after resuscitation in an animal model of cardiac arrest.

Methods. 19 adult domestic pigs were included in the study. After sedation and preparation, including endotracheal intubation, invasive arterial pressure monitoring and a pulmonary artery catheter, a ventricular fibrillation was induced via a pace-maker wire and left untreated for $10 \mathrm{~min}$, when cardio-pulmonary resuscitation (CPR) were started at the rate of 100/minute with Lucas III device and continued for 5 more minutes. After the first minute of CPR and eventually after $7 \mathrm{~min}$, an injection of Adrenaline was provided and at the end of the $5 \mathrm{~min}$ an electric shock (200 J) was delivered and repeated every minute to achieve Retour of Spontaneous Circulation (ROSC). The animals were divided as follow: 11 as control group received crystalloids during CPR and as infusion during $12 \mathrm{~h}$ after ROSC (G0) and 8 received $\mathrm{KB}(75 \mathrm{~g} / \mathrm{L}, 1189 \mathrm{mOsm})$ both as bolus of $1.5 \mathrm{~g} / 20 \mathrm{cc}$ during CPR and as infusion $(0.18 \mathrm{~g} / \mathrm{Kg} / \mathrm{h})$ during $12 \mathrm{~h}$ after ROSC (G1).All animals were under sedation and were treated with target temperature management at $34{ }^{\circ} \mathrm{C}$ via an external temperature feedback device during the post-resuscitation phase.

Results. All the animals in the study achieved ROSC, but one animal in the $\mathrm{KB}$ group died $9 \mathrm{~h}$ after ROSC due to profound distributive and cardiogenic shock. No differences were found between groups in the distribution of sex and weight, as well as in number of shocks needed to achieve ROSC, adrenaline dose, first end tidal CO2 measurement or baseline $\mathrm{pH}$. To investigate differences on dependent variables over time between groups, a general linear mixed model was used. Arterial $\mathrm{KB}$ levels were higher in the group of animals that received 3-OHB infusion compared to controls $(p<0.001)$. To achieve the same mean arterial pressure level of $65 \mathrm{mmHg}$, there was no significantly different requirements for vasopressors between groups over the study period $(p=0.71)$. No differences in heart rate, cardiac output or troponin I were evident between groups $(p=0.12, p=0.28$ and $p=0.69$, respectively), but animals receiving study drugs required more perfusion over the study period (11.0 vs $8.31 \mathrm{~L}, \mathrm{p}=0.003)$ and had a higher PPV ( $p=0.004$ for interaction).

Conclusion. infusion of hypertonic 3-OHB in the first hours following resuscitation after CA did not ameliorates hemodynamic parameters but increase the fluid requirements.

\section{Reference(s)}

1. Jentzer JC, Chonde MD, Dezfulian C. Biomed Res Int 2015, ID 314,796.

2. Knott, EM Ryou MG, Sun J et al. Am J Physiol Heart Circ Physiol 2005; 289: $1123-1130$

Heart IJC, Koyama T. IJC Hear Vasc 2017; 15: 1-8.

4. Nielsen R, Moller N, Gormsen LC et al. Circulation 2019; 139: 2129-2141

5. Gormsen LC, Svart M, Thomsen HH et al. J Am Heart Ass 2017. e005066.

6. Lemiale V, Dumas F, Mongardon N et al. Intensive Care Med 2013; 39:1972-1980.

7. Nolan, JP et al. Resuscitation 2015; 95: e1-e31.

8. Fonds Erasme Pour la Recherche Médicale

\section{6}

Factors associated with Rebound Hyperthermia after Targeted Temperature Management in out-of-hospital cardiac arrest patients: an explorative sub-study of the TTH48 trial

A. Holm ; H. Kirkegaard ${ }^{2}$; FS. Taccone ${ }^{3}$; E. Søreide ; A. Grejs 5 ; V. Toome ;

C. Hassager ${ }^{7}$; BS. Rasmussen, ${ }^{8} ;$ T. Laitio $^{9}$; C. Storm ${ }^{10}$; J. Hästbacka ${ }^{11}$; M. Skrifvars ${ }^{12}$

${ }^{1}$ Department of emergency care and services, University of Helsinki and Helsinki University Hospital, Helsinki, Finland; ${ }^{2}$ Research center for emergency medicine, department of emergency medicine and department of clini, Aarhus University Hospital and Aarhus University, Aarhus, Denmark; ${ }^{3}$ Soins intensif, ULB Erasme, Anderlecht, Belgium; ${ }^{4}$ Critical care and anaesthesiology research group, Stavanger University Hospital, Stavanger, Norway; ${ }^{5}$ Department of intensive care, Aarhus University Hospital, Aarhus, Denmark; ${ }^{6}$ Department of intensive cardiac care, North Estonia Medical Centre, Tallinn, Estonia; ${ }^{7}$ Department of cardiology, Rigshospitalet, København, Denmark; ${ }^{8}$ Department of anaesthesia and intensive care medicine, Aalborg University Hospital, Aalborg Denmark; ${ }^{9}$ Division of perioperative services, intensive care medicine and pain management, Turku University Hospital and University of Turku, Finland, Turku, Finland; ${ }^{10}$ Department of internal medicine, nephrology and intensive care, Charité-University, Berlin, Germany; ${ }^{11}$ Intensive care units, department of anaesthesiology, intensive care and pain medicine, University of Helsinki and Helsinki University Hospital, Helsinki, Finland; ${ }^{12}$ University of helsinki and helsinki university hospital, Perioperative, intensive care and pain medicine, Helsinki, Finland

Correspondence: A. Holm

Intensive Care Medicine Experimental 2020, 9(1): 000306

Introduction. Rebound hyperthermia $(\mathrm{RH})$ is commonly seen phenomenon after targeted temperature management (TTM) in cardiac arrest patients but its causes and impact on patient outcome are not fully understood.

Objectives. To investigate $\mathrm{RH}$ following TTM after cardiac arrest and its impact on functional outcome (FO).

Methods. Post-hoc analysis from 10 European intensive care units (ICU). Patients included in the TTH48 trial treated with TTM at $33^{\circ} \mathrm{C}$ for 48 or $24 \mathrm{~h}$. Favorable FO was defined as a cerebral performance category of 1 or 2 at six months.

Results. Of 338 included patients, 103 (30\%) experienced $\mathrm{RH}$ defined as a maximum temperature after TTM and rewarming exceeding $38.5^{\circ} \mathrm{C}$ Using multivariate logistic regression analysis, increasing age (OR 0.97, $95 \% \mathrm{Cl} 0.95-0.99, p=0.02$ ) and severe acute kidney injury within $72 \mathrm{~h}$ of ICU admission (OR $0.35,95 \% \mathrm{Cl} 0.13-0.91 p=0.03$ ) were associated with less RH whereas male gender (OR 3.94, 95\% Cl 1.34-11.57 $p=0.01$ ), highest $\mathrm{C}$-reactive protein value (OR 1.04,95\% Cl 1.01-1.07, $p=0.02$ ) and use of mechanical chest compression during cardiopulmonary resuscitation (OR 2.00, 95\% Cl 1.10-3.67, $p=0.02$ ) were associated with more $\mathrm{RH}$. Patients with favorable FO spent less time after rewarming over $38.5^{\circ} \mathrm{C}$ (2.5\% vs $6.3 \%, p=0.03), 39{ }^{\circ} \mathrm{C}(0.14 \%$ vs $2.7 \%, p<0.01)$, and $39.5{ }^{\circ} \mathrm{C}$ $(0.03 \%$ vs $0.71 \%, p<0.01)$ when compared with others. Median time to $\mathrm{RH}$ was longer in the unfavorable FO group (33.2 h IQR 14.3-53.0 vs $6.5 \mathrm{~h}$ IQR 2.2-34.1, $p<0.01)$. In a predefined multivariate binary logistic regression model, RH was associated with decreased odds of favorable FO (OR $0.42,95 \% \mathrm{Cl} 0.22-0.79)$.

Conclusion. One-third of TTM patients experience $\mathrm{RH}$, and it is more common in younger male patients with an aggravated inflammatory response and those treated with a mechanical chest compression device. Later onset of $\mathrm{RH}$ and temperatures exceeding $38.5{ }^{\circ} \mathrm{C}$ associate with unfavorable FO.

\section{8}

The Role of EEG in Neuroprognostication Following Cardiac Arrest: Experience of an Irish Primary PCI Centre

E. Doyle”; J. Mcnamara, ${ }^{2}$; R. Qasem, ${ }^{3}$; M. Aldoseri, ${ }^{2} ;$ Y. Langan, ${ }^{3}$;

E. O'connor, ${ }^{2}$

${ }^{1}$ School of medicine, Trinity College Dublin, Dublin, Ireland; ${ }^{2}$ Anaesthesia and intensive care, St James hospital, Dublin, Ireland; ${ }^{3}$ Neurology, St James hospital, Dublin, Ireland

Correspondence: E. Doyle 
Intensive Care Medicine Experimental 2020, 9(1): 000318

Introduction. St. James's Hospital is the largest hospital in Ireland. It is also one of only two primary PCI centres in Dublin (population: 2 million), making it a common destination following out-of-hospital cardiac arrest (OOHCA).

EEG has a role in neuroprognostication in slow-to-wake patients. ERCESICM guidelines recommend the use of EEG as part of an algorithm for prognostication in comatose patients following cardiac arrest, nominally as a second-line investigation in situations where somatosensory evoked potentials are unavailable.

We conducted a review of all patients admitted to our ICU following cardiac arrest over a 6-month period (January-June 2020), determining how many received an EEG during their admission and why, what other investigations were correlated with EEG findings, and whether the EEG report could be shown to have impacted on the patient's disposition. We compared the demographic data from this period with the same span in 2016 to quantify what was thought to be an increasing workload.

Methods. Retrospective chart review of all patients admitted to our 22-bed General ICU between January and June of 2020 following Cardiac Arrest, using our hospital's electronic patient record (EPR). We determined which of these patients had received an EEG during their admission and whether that EEG could be deemed to have changed the patient's management or provided other clinically relevant data. We subsequently compared the demographic data to the equivalent span (Jan-Jun) in 2016.

Results. 2020: 27 patients admitted to ICU in 2020 following cardiac arrest, 7 arrested on the ward or in the ED, 2 in theatre, and 3 were transferred from other hospitals, and 15 were considered OOHCA. patients received an EEG. Of those, 12/13 also received some form of neuroimaging (5 MRI, $7 \mathrm{CT}$ ) 8/13 EEGs (in conjunction with other clinical and radiological investigations) were deemed to have changed patient management in some capacit (4 guiding de-escalation of antiepileptics, and 4 aiding with prognostication and initiation of palliative care). 4 others did not change management per se but provided other clinically relevant information (ruling out seizure; NCSE); $1 / 13$ did not alter management in any demonstrable fashion.

2016: 22 patients admitted to ICU following cardiac arrest in 2016: 10 arrested on the ward or in the ED, 1 in theatre, 1 was transferred from another hospital, and 10 were considered OOHCA. Significantly fewer patients received an EEG (13/27 vs. $3 / 22, \mathrm{p}=0.0146)$.

Difficult to tell the impact of COVID on admissions from the ward to the ICU. We theorised that a higher proportion of the Cardiac Arrest patients admitted to ICU in 2020 would be from OOHCA when compared with 2016 . This was demonstrated, but was not statistically significant ( $15 / 27$ vs. $10 / 22, p=0.57)$.

Conclusion. In our department, an increased utilisation of EEG was noted from 2016 to 2020 . The EEG played a key role in the management of cardiac arrest patients, providing useful diagnostic and therapeutic guidance (in 2020) in 12/13 patients. From our findings, we advocate the routine use of EEG in cardiac arrest patients to guide management and out rule ictus. Accordingly, neurophysiology departments should be adequately resourced to meet this clinical demand.

\section{Reference(s)}

1. Nolan JP, Soar J, Cariou A, Cronberg T, Moulaert VR, Deakin CD, Bottiger BW, Friberg H, Sunde K, Sandroni C; European Resuscitation Council; European Society of Intensive Care Medicine. European Resuscitation Council and European Society of Intensive Care Medicine 2015 guidelines for post-resuscitation care. Intensive Care Med. 2015 Dec;41(12):2039-56. https://doi.org/10.1007/s00134-015-4051-3. Erratum in: Intensive Care Med. 2016 Mar;42(3):488-9. PMID: 26,464,394.
000359

Characterization of Cardiac Arrest in the Emergency Room

F. Cardoso ${ }^{1}$; R. Passos ${ }^{1}$; D. Alves, ; L. Pereira ${ }^{1}$; A. Colmente, ${ }^{1}$; J. Abreu';

R. Corga'; $\jmath^{1}$. Caldeiro ${ }^{1}$

${ }^{1}$ Intensive Medicine, ULSAM—Hospital de Santa Luzia, Viana do Castelo,

Portugal

Correspondence: F. Cardoso

Intensive Care Medicine Experimental 2020, 9(1): 000359

Introduction. Cardiac arrest (CA) represents a public health burden with elevated mobility and mortality. Cardiopulmonary resuscitation (CPR) is a complex set of interventions that requires leadership, coordination, and best practices. Identifying and implementing systems that increase the likelihood of immediate CPR recognition and rapid defibrillation when indicated, are vital to improving survival.

This study aims to characterize the CA patients at a District Hospital Emergency Room (ER), from 2018 to 2020.

Methods. We performed a descriptive statistical analysis of all CPR's from January 2018 until December 2020 in our ER.

Results. Our ER had 1796 patients in the study period. At this number we selected the patients with CA, and ninety-two CPR's were document. The mean age of the patients was 71,9 years. $42,5 \%$ of the patients were female. Of the total number of CPRs observed, the year with the highest number was 2019 with 36 cardiorespiratory arrests in the emergency room.

In the cases observed, the arrest rhythm was shockable in 36 patients, non-shockable in 51 and 4 patients had ECG evidence of ST-segment elevation on arrival and were transferred, after stabilization, to a hospital with hemodynamic room.

Of 87 people with cardiac arrest included in our analysis, 17 recovered making a survival rate of $19,5 \%$.

Conclusion. At our center, the ER is part of the Critical Care Department and it is functional $24 \mathrm{~h}$ a day, 7 days a week, 365 days a year. Our hospital has a rapid response team implemented and an intensivist allocated to the ER, with a conscious attempted to provide early adequate management for these patients. This study aimed to study the population that suffered CA in the ER, in an attempt to understand the context for the different outcomes. Understanding our mortality, is essential to implement changes and future improvements.

Having recognized a limitation in the medical records (inherent to a retrospective analysis) we are planning a subsequent implementation for registrations rules at the ER in order to ensure the quality of medical records, and to allow future studies with more detailed information.

\section{0}

Intensive Care Unit cardiac arrest in critically ill patients with COVID-19

K. Roedl ${ }^{1}$; G. Söffker, ; W. Dominic, ${ }^{1}$; O. Boenisch, ${ }^{1}$; G. Deheer, ${ }^{1}$;

C. Burdelski, ; D. Frings, ; ;. Sensen, ; A. Nierhaus, ; ; D. Westermann, ${ }^{2}$;

S. Kluge, ${ }^{1} ;$ D. Jarczak

${ }^{1}$ Department of intensive care medicine, University Medical Center Hamburg-Eppendorf, Hamburg, Germany; ${ }^{2}$ University heart-center hamburg, department of cardiology, University Medical Center Hamburg-Eppendorf, Hamburg, Germany

Correspondence: K. Roedl

Intensive Care Medicine Experimental 2020, 9(1): 000380

Introduction. The severe acute respiratory syndrome coronavirus-2 (SARS-CoV-2) causing the coronavirus disease 2019 (COVID-19) led to an ongoing pandemic with a surge of critically ill patients. Very little is known about the occurrence and characteristics of cardiac arrest within the ICU (ICU-CA) in patients with COVID-19.

Objectives. The aim of this study was to investigate the incidence and outcome of ICU-CA in critically ill patients with COVID-19.

Methods. This was a retrospective analysis of data prospectively recorded of all consecutive adult patients with COVID-19 admitted (February 27th, 2020-February 05th, 2021) at the University Medical Centre Hamburg-Eppendorf (Germany). Clinical parameters, cardiac 
arrest (CA) characteristics, neurological outcomes and organ failure/ support were assessed.

Results. During the study period 183 critically ill patients with COVID19 were identified. $18 \%(n=33)$ of patients had an ICU-CA. The median age of the study population was $63(55-73)$ years and $66 \%(n=120)$ were male. Comorbidities, displayed by charlson comorbidity index were median $2(1-3)$ points. Demographic characteristics and comorbidities did not differ significantly between patients with and without ICU-CA. SAPS II (ICU-CA: median 44 points vs. No ICU-CA: 39 points) and SOFA score (median 12 points vs. 7 points) on admission were significantly higher in patients with ICU-CA. ARDS was present in $91 \%$ $(n=30)$ with and in $63 \%(n=94)$ without ICU-CA $(p=0.002)$. Mechanical ventilation was more common in patients with ICU-CA $(97 \%$ vs. $67 \%)$. Median stay in ICU before CA was 6 (1-17) days. The initial cardiac rhythm was non-shockable (PEA/Asystole) in $91 \%(n=30)$, presumed cause was non-cardiac in $85 \%(n=28)$ and $94 \%(n=31)$ had sustained ROSC. The median time to ROSC was $3(1-5)$ minutes. Patients with ICU-CA had significant higher ICU mortality $(61 \%, n=20)$ as compared to other patients $(37 \%, n=56)$. Multivariable logistic regression showed that presence of ARDS [OR 4.268, 95\% Cl (1.211$15.036) ; p=0.024]$ and high SAPS II [OR $1.031,95 \% \mathrm{Cl}(0.997-1.065)$; $\mathrm{p}=0.077$ ] were independently associated with occurrence of ICU-CA.

Conclusion. $18 \%$ of critically ill patients with COVID-19 suffered from a cardiac arrest within the intensive care unit. Occurrence of ICU-CA was associated with the presence of ARDS and severity of illness.

\section{8}

Cardiopulmonary resuscitation performance efficacy while wearing a powered air-purifying respirator

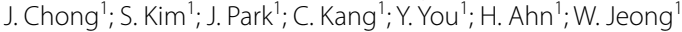

'Department of emergency medicine, Chungnam National University Hospital, Daejeon, Republic of Korea

Correspondence: J. Chong

Intensive Care Medicine Experimental 2020, 9(1): 000388

Introduction. The use of personal protective equipment for respiratory infection control in cardiopulmonary resuscitation (CPR) is a physical burden to healthcare providers. It matters how long CPR quality according to recommended guidelines can be maintained under these circumstances. We investigated whether chest compression time was appropriate for a 2-min shift and how long it was maintained in accordance with the guidelines under such conditions.

Methods. This prospective crossover simulation study was performed at a single center from September 2020 to October 2020. Five indicators of CPR quality were measured during the first and second sessions of the study period. All participants wore a Level D powered air-purifying respirator (PAPR), and the experiment was conducted using a Resusci Anne manikin, which can measure the quality of chest compressions. Each participant conducted two sessions. In session one, 2-min of chest compressions followed by a 2-min rest was repeated twice; in session two, 1-min of chest compressions followed by a 1-min rest was repeated four times.

Results. All 34 participants completed the study. The deep and sufficient compression rate was $65.9 \pm 13.1 \mathrm{~mm}$ in the 1-min shift group and $61.5 \pm 30.5 \mathrm{~mm}$ in the 2-min shift group. The mean depth was $52.8 \pm 4.3 \mathrm{~mm}$ in the 1-min shift group and $51.0 \pm 6.1 \mathrm{~mm}$ in the 2-min shift group. In these two values, there was a statistically significant difference between the two sessions. There was no statistically significant difference in the other CPR quality values.

Conclusion. We suggest that the different standard of current 2-min to 1-min cycles due to a significant reduction in the quality of chest compression in cases of CPR with PAPR.

\section{Reference(s)}

2. [1] Bergman M, Basu R, Lei Z, Niezgoda G, Zhuang Z. Development of a Manikin-Based Performance Evaluation Method for Loose-Fitting Powered Air-Purifying Respirators. J Int Soc Respir Prot. 2017;34:40-57.

3. [2] Roberts V. To PAPR or not to PAPR? Can J Respir Ther. 2014;50:87-90
4. [3] Schumacher J, Gray SA, Michel S, Alcock R, Brinker A. Respiratory protection during simulated emergency pediatric life support: a randomized, controlled, crossover study. Prehosp Disaster Med. 2013;28:33-8.

5. [4] Park SH, Hwang SY, Lee G, Park JE, Kim T, Shin TG, Sim MS, Jo IJ, Kim S, Yoon H. Are loose-fitting powered air-purifying respirators safe during chest compression? A simulation study. AJEM. 2020. 31:S07356757(20)30,196-0. https://doi.org/10.1016/j.ajem.2020.03.054.

6. [5] McDonald CH, Heggie J, Jones $\mathrm{CM}$, Thorne CJ, Hulme J. Rescuer fatigue under the 2010 ERC guidelines, and its effect on cardiopulmonary resuscitation (CPR) performance. Emerg Med J. 2013;30:623-7.

7. [6] Powell JB, Kim JH, Roberge RJ. Powered air-purifying respirator use in healthcare: Effects on thermal sensations and comfort. J Occup Environ Hyg. 2017;14:947-954.

8. [7] Roberge R, Benson S, Kim JH. Thermal burden of N95 filtering facepiece respirators. Ann Occup Hyg. 2012;56:808-14.

9. [8] Chen J, Lu KZ, Yi B, Chen Y. Chest Compression With Personal Protective Equipment During Cardiopulmonary Resuscitation: A Randomized Crossover Simulation Study. Medicine (Baltimore). 2016;95:1-5.

10. [9] Malysz M, Dabrowski M, Böttiger BW, Smereka J, Kulak K, Szarpak A Jaguszewski M, Filipiak KJ, Ladny JR, Ruetzler K, Szarpak L... Resuscitation of the patient with suspected/confirmed COVID-19 when wearing personal protective equipment: A randomized multicenter crossover simulation trial. Cardiol J. 2020;27:497-506.

11. [10] Donoghue AJ, Kou M, Good GL, Eiger C, Nash M, Henretig FM, Stacks H, Kochman A, Debski J, Chen JY, Sharma G, Hornik CP, Gosnell L, Siegel D, Krug S, Adler MD; Best Pharmaceuticals for Children Act-Pediatric Trials Network. Impact of Personal Protective Equipment on Pediatric Cardiopulmonary Resuscitation Performance: A Controlled Trial. Pediatr Emerg Care. 2020;36:267-273.

000395

Measuring global impairment of cerebral perfusion using dynamic susceptibility contrast perfusion-weighted imaging in out-of-hospital cardiac arrest survivors: A prospective preliminary study

A. changjoo ${ }^{1}$; K. Changshin ${ }^{2}$; SP. Jung ${ }^{2}$; Y. Yeonho ${ }^{2}$

${ }^{1}$ Chungnam National University Hospital, Daejeon, Republic of Korea;

2Emergency medicine, Chungnam National University Hospital, Daejeon,

Republic of Korea

Correspondence: A. changjoo

Intensive Care Medicine Experimental 2020, 9(1): 000395

Introduction. This study aimed to assess the global impairment and prognostic performance of cerebral perfusions (CP) measured by dynamic susceptibility contrast perfusion-weighted imaging (DSCPWI) in out-of-hospital cardiac arrest (OHCA) patients after sustained restoration of spontaneous circulation (ROSC).

Methods. This is a single-centre, prospective observational study. OHCA patients performed DSC-PWI within $8 \mathrm{~h}$ after ROSC were enrolled. We quantified the CP parameters, such as cerebral blood volume (CBV), cerebral blood flow (CBF), mean transit time (MTT), time to peak (TTP), and time to maximum of the residue function (Tmax) either by normalization or arterial input function (AIF). The primary and secondary outcomes were survival to discharge and comparison of prognostic performance between CP parameters and serum neuron-specific enolase (NSE) using area under the receiver operating characteristic (AUROC) and sensitivity values.

Results. Thirty-one patients were included in this study. CBV and TTP quantified by normalization, and MTT and Tmax quantified by AIF showed significantly higher $\mathrm{CP}$ values in the non-survival group $(p=0.02,0.03,0.02$, and $<0.01$, respectively). Their AUROCs and $100 \%$ specific sensitivities were $0.74 / 25.0 \%, 0.60 / 33.3 \%, 0.75 / 56.3 \%$, and $0.79 / 43.8 \%$, respectively. MTT quantified by AIF showed sensitivity in predicting mortality at an early stage of PCA care, comparable with NSE. Conclusion. Hyperaemia and delayed CP were generally observed in OHCA patients regardless of outcomes. MTT and Tmax quantified by AIF have prognostic performance in predicting mortality, comparable with NSE. Further prospective multicentre studies are required to confirm our results. 
000397

The prognostic role of serum neutrophil gelatinase associated lipocalin in out-of-hospital cardiac arrest patients who underwent targeted temperature management

K. Kim ${ }^{1}$; C. Kang ${ }^{1}$; Y. Yeonho'; J. Min ${ }^{1}$; J. Chong ${ }^{2}$

${ }^{1}$ Emergency medicine, Chungnam National University Hospital, Daejeon, Republic of Korea; ${ }^{2}$ Department of emergency medicine, Chungnam

National University Hospital, Daejeon, Republic of Korea

Correspondence: K. kim

Intensive Care Medicine Experimental 2020, 9(1): 000397

Introduction. Cardiac arrest (CA) has been reported to lead to acute kidney injury (AKI) in $12 \%-81 \%$ of patients, and AKI after CA is associated with higher mortality, a poor neurological outcome, and a prolonged hospital stay. Therefore, early and accurate diagnosis of AKI in CA patients is an important issue in post-CA care. In the last few decades, serum creatinine $(\mathrm{s} C \mathrm{Cr}$ ) and the RIFLE (Risk, Injury, Failure, Loss of kidney function, and End-stage kidney disease) criteria have been used to diagnose AKI; however, they have some inherent limitations in terms of early and accurate diagnosis.

Several studies have reported that neutrophil gelatinase-associated lipocalin (NGAL) can facilitate the diagnosis of AKI in critically ill adult patients [6-8]. NGAL has also been suggested as a potential biomarker for predicting the mortality risk and neurological outcome in CA patients.

Therefore, the first aim of this study was to investigate the association between serum NGAL levels obtained during TTM and outcomes in OHCA patients. We then investigated whether a combination of serum NGAL and NSE can improve the prognostic performance of NSE by comparing the outcomes when each biomarker was used alone and when they were used together.

Methods. This prospective observational study aimed to determine the association of serum neutrophil gelatinase-associated lipocalin (NGAL) with outcomes in patients treated with targeted temperature management (TTM) after out-of-hospital cardiac arrest (OHCA) and whether addition of NGAL measurement can improve the prognostic performance of neuron-specific enolase (NSE) in these patients. The study included comatose adult OHCA patients who underwent TTM between May 2018 and December 2020. Serum NGAL and NSE measurements were obtained at 24-h intervals until $72 \mathrm{~h}$ after OHCA (i.e., NGALinitial, 24, 48, and 72 and NSEinitial, 24, 48, and 72).

Serum NGAL and NSE levels were obtained from blood samples drawn by venipuncture at $24-\mathrm{h}$ intervals until $72 \mathrm{~h}$ after ROSC (i.e., NGALinitial, 24, 48, and 72 and NSEinitial, 24, 48, and 72). The first NGAL and NSE measurements were obtained at the time of applying an external cooling device after several procedures for TTM, including electrocardiography, echocardiography, brain imaging, or resuscitation for sustained ROSC. NGAL and NSE values were measured using a UniCel ${ }^{\circledR}$ DxC 880i chemical analyzer (Beckman Coulter Inc., Brea, CA, USA) with the NGAL Test ${ }^{\mathrm{TM}}$ kit (BioPorto Diagnostics, Gentofte, Denmark) and an electrochemiluminescence immunoassay with Elecsys NSE ${ }^{\circledR}$ (COBAS e801; Roche Diagnostics, Rotkreuz, Switzerland). The NGAL and NSE measurements were in the ranges of $25-3000 \mathrm{ng} / \mathrm{mL}$ and $0.1-300 \mathrm{ng} /$ $\mathrm{mL}$, respectively.

Results. The primary outcomes were in-hospital mortality and neurological outcome at 3 months after OHCA. Eighty-five patients were enrolled, of whom 30 (35.3\%) died in hospital and $49(57.6 \%)$ had a poor neurological outcome. NGAL levels at all time points measured were significantly higher in non-survivors and in patients with a poor neurological outcome than in survivors $(p<0.01)$ and in patients with a good neurological outcome $(p<0.01)$. NSE showed a relatively higher maximal sensitivity with acceptable specificity of at least $95 \%$ for both outcomes compared with NGAL (mortality [56.7\% vs. 26.7\%] and poor neurological outcome [69.1\% vs. $22.5 \%]$ ). Combination of NGAL with NSE showed the highest sensitivity (66.7\%) for mortality at $24 \mathrm{~h}$ and $78.6 \%$ for a poor neurological outcome at $48 \mathrm{~h}$. The prognostic performance of NGAL as a single marker was not statistically significant. However, sensitivity was better when a combination of NGAL and NSE was used than when NGAL or NSE was used alone.
Conclusion. NGAL was associated with both in-hospital mortality and the neurological outcome in OHCA patients treated with TTM. Although its prognostic performance was not significant when used as the only biomarker, when combined with NSE, NGAL could improve the sensitivity of NSE with acceptable specificity. Therefore, NGAL should be considered as an additional biomarker for prognostication in OHCA patients. Further studies with larger sample sizes and less selection bias are required to confirm our results.

\section{Reference(s)}

1. Park SO, Ahn JY, Lee YH, Kim YJ, Min YH, Ahn HC, et al. Plasma neutrophil gelatinase-associated lipocalin as an early predicting biomarker of acute kidney injury and clinical outcomes after recovery of spontaneous circulation in out-of-hospital cardiac arrest patients. Resuscitation. 2016;101:84-90. https://doi.org/10.1016/j.resuscitation.2016.01.005 PMID: $26,826,562$

2. Kaneko T, Fujita M, Ogino Y, Yamamoto T, Tsuruta R, Kasaoka S. Serum neutrophil gelatinase-associated lipocalin levels predict the neurological outcomes of out-of-hospital cardiac arrest victims. BMC Cardiovasc Disord. 2017;17(1):111. https://doi.org/10.1186/s12872-017-0545-y PMID: $28,482,803$

3. Chen X, Chen Z, Wei T, Li P, Zhang L, Fu P. The effect of serum neutrophil gelatinase-associated lipocalin on the discontinuation of continuous renal replacement therapy in critically ill patients with acute kidney injury. Blood Purification. 2019;48(1):10-7. https://doi.org/10.1159/000499026.

4. Khawaja S, Jafri L, Siddiqui I, Hashmi M, Ghani F. The utility of neutrophil gelatinase-associated lipocalin (NGAL) as a marker of acute kidney injury (AKI) in critically ill patients. Biomarker research. 2019;7:4. https://doi.org/ 10.1186/s40364-019-0155-1 PMID: 30,834,123

5. Choi YH, Lee DH, Lee JH. Serum neutrophil gelatinase-associated lipocalin at $3 \mathrm{~h}$ after return of spontaneous circulation in patients with cardiac arrest and therapeutic hypothermia: early predictor of acute kidney injury. BMC Nephrol. 2020;21(1):389. https://doi.org/10.1186/s12882-02002054-7 PMID: 32,894,077

6. Sanjeevani S, Pruthi S, Kalra S, Goel A, Kalra OP. Role of neutrophil gelatinase-associated lipocalin for early detection of acute kidney injury. International journal of critical illness and injury science. 2014;4(3):223-8. https://doi.org/10.4103/2229-5151.141420 PMID: 25,337,484

7. Thongprayoon C, Cheungpasitporn W, Kashani K. Serum creatinine level, a surrogate of muscle mass, predicts mortality in critically ill patients. Journal of thoracic disease. 2016;8(5):E305-11. https://doi.org/10.21037/ jtd.2016.03.62 PMID: 27,162,688

8. Storm C, Krannich A, Schachtner T, Engels M, Schindler R, Kahl A, et al. Impact of acute kidney injury on neurological outcome and long-term survival after cardiac arrest-A 10 year observational follow up. Journal of critical care. 2018;47:254-9. https://doi.org/10.1016/j.jcrc.2018.07.023 PMID: 30,071,447

9. De Rosa S, Antonelli M, Ronco C. Hypothermia and kidney: a focus on ischaemia-reperfusion injury. Nephrology, dialysis, transplantation: official publication of the European Dialysis and Transplant AssociationEuropean Renal Association. 2017;32(2):241-7. https://doi.org/10.1093/ ndt/gfw038 PMID: 28,186,567

10. Rundgren M, Ullén S, Morgan MPG, Glover G, Cranshaw J, Al-Subaie N, et al. Renal function after out-of-hospital cardiac arrest; the influence of temperature management and coronary angiography, a post hoc study of the target temperature management trial. Crit Care. 2019;23(1):163. https://doi.org/10.1186/s13054-019-2390-0 PMID: 31,068,215

\section{8}

Can pain pupillary index prognosticate outcome in cardiac arrest patients ?

L. Peluso ${ }^{1}$; A. Bertelli ${ }^{1}$; E. Bogossian ${ }^{1}$; F. Annoni ${ }^{1}$; E. Macchini ${ }^{1}$; A. Minini ${ }^{1}$; K. Donadello ${ }^{2}$; J. Creteur ${ }^{1}$; FS. Taccone ${ }^{1}$

${ }^{1}$ Department of intensive care, Hospital Erasme-Université Libre de Bruxelles, Bruxelles, Belgium; '2Dipartimento di scienze chirurgiche, odontostomatologiche e materno-infantili, University of Verona, Verona, Italy Correspondence: L. Peluso

Intensive Care Medicine Experimental 2020, 9(1): 000408 
Introduction. The Neurological Pupil Index (NPi), derived from automated pupillometry, showed high specificity to predict poor neurological outcome after post-anoxic brain injury [1]. Whether other indices derived from pupillometry have also a prognostic value in this setting remains unknown.

Objectives. To assess the predictive value of the Pupillary Pain Index (PPI) for unfavorable neurological outcome (UO) after post-anoxic brain injury.

Methods. Ongoing prospective study including comatose patients admitted to the Intensive Care Unit after cardiac arrest since October 2019. Two different automated pupillometers were concomitantly used and NPi (from NeurOptics NPi-200, Neuroptics) and PPI (from NeuroLight, ID-MED) were assessed at day 1 and day 2 after admission. In particular, PPI was calculated using an electrical stimulation with variable intensity (increasing from 10 to $60 \mathrm{~mA}$ ) on the left and right forearm of each patient: pupil dilation $>13 \%$ in response to such stimulation with increasing intensity and duration was converted in the PPI score, ranging from 1 (pupillary dilation $<5 \%$ to the maximal stimulation intensity) to 10 (pupillary dilation $>13 \%$ with $10 \mathrm{~mA}$ stimulus). Mean values from both eyes were used. The coefficient of agreement between PPI and NPi was computed. UO was defined as Cerebral Performance Category of 3-5 at 3 months.

Results. A total of 61 patients were included; 40 (65\%) patients had UO. Patients with UO showed a lower PPI (3 [1-5] vs. 5 [3-6]; $p=0.04$ on day 1 and 3 [1-5] vs 5 [4-7]; $p=0.01$ on day 2) than others; PPI had an area under the receiver operator characteristic (AUROC) of 0.69 [95\% Cl $0.55-0.83]$ on day 1 and 0.72 [0.59-0.85] on day 2 to predict UO. In particular, $\mathrm{PPI}=1$ on day 2 showed a sensitivity of $26 \%$ [14\%$41 \%$ ] and a specificity of $100 \%$ [ $81 \%-100 \%$ ] to predict UO, with a false positive rate (FPR) of $0 \%$. A weak correlation $(r=0.41, p<0.01)$ on day 1 and a moderate correlation $(r=0.51, p<0.01)$ on day 2 was observed between PPI and NPi. A total of 5 patients had concomitant PPI $=1$ and $\mathrm{NPi} \leq 2$ (all had $\mathrm{UO}$ ), while 6 showed $\mathrm{NPi}>2$ and $\mathrm{PPI}=1$ (all had UO). Among the remaining 50 patients, 1 had NPi $\leq 2$ with PPI $>1$ and 49 had PPI $>1$ with $\mathrm{NPi}>2$. As such, the coefficient of agreement of $\mathrm{PPI}=1$ and $\mathrm{NPi} \leq 2$ to predict $\mathrm{UO}$ was 0.53 . When at least one predictor (i.e. $\mathrm{PPI}=1$ or $\mathrm{NPI} \leq 2$ ) was present on day 2 , a sensitivity of $28 \%$ [15\%$44 \%]$, a specificity of $100 \%$ [81\%-100\%], with $0 \%$ FPR was observed. Conclusion. Pupillary pain index showed promising results in prognosticate $\mathrm{UO}$ after post-anoxic brain injury.

\section{Reference(s)}

1. Oddo M, Sandroni C, Citerio G, et al. Quantitative versus standard pupillary light reflex for early prognostication in comatose cardiac arrest patients: an international prospective multicenter double-blinded study. Intensive care medicine. 2018;44(12).

\section{3}

\section{Validity of Missmatch negativity (MMN) for predicting} neurological outcome in adults after resuscitated cardiac arrest (RCA)

I. García-González' ; M. Bringas-Bollada'; P. González-Arenas, ${ }^{1}$;

P. Balugo-Bengoechea'; P. Alonso-Martínez'; A. Gil-Conde1;

M. Sanchez-García

${ }^{1}$ Critical care, Hospital Clinico Universitario San Carlos, Madrid, Spain;

${ }^{2}$ Clinical neurophysiology, Hospital Clinico Universitario San Car-

los, Madrid, Spain

Correspondence: I. García-González

Intensive Care Medicine Experimental 2020, 9(1): 000423

Introduction. Hospital survival rates of RCA average $8 \%$. ICU mortality is mostly due to neurological injury, which most frequently leads to withdrawal of life sustaining treatment (WLST) based on predicted poor prognosis.

European guidelines recommend a multimodal strategy for neurological prognostication in comatose patients after RCA, including various neurophysiological studies. Bilaterally absence of the N20 wave in somatosensory evoked potentials (SSEP) at $24 \mathrm{~h}$ or a highly malignant EEG at $>24 \mathrm{~h}$ predicts poor outcome. However, in patients with presence of N20 wave and ambiguous EEG, who remain comatous, prognosis is uncertain and the use of $M M N$ has been proposed to aid neurological prognostication. The positive predictive value of MMN for awakening is $90-100 \%$.

Objectives. To determine the validity of MMN to predict neurological outcome according to the Cerebral Performance Categories (CPC) scale in RCA.

Methods. Prospective cohort of adult patients remaining in coma after RCA and positive for N20 wave. Under post-resuscitation care according to European Guidelines, MMN tests were performed and all patients were followed for CPC scale at ICU discharge, at hospital discharge, at 6 months and at one year.

The equipment used by the specialist in clinical neurophysiology to perform MMN in this study is a Synergy on Nicolet ${ }^{\circledR}$ EDX 20.0.

We evaluated the validity of the MMN in assessing neurological prognosis using sensitivity and specificity indices, and other probability ratios.

Results. From July 2019 to July 2020, we enrolled 7 patients, 4 men, mean age 67 years and mostly acute coronary events, with a mean duration of CPR of 26 min.

Table 1. Patient characteristics and EEG findings

\begin{tabular}{lllllll}
\hline Patient & SEX & AGE & $\begin{array}{l}\text { CPR } \\
\text { DURA- } \\
\text { TION }\end{array}$ & $\begin{array}{l}\text { ENOLASE } \\
\text { (max) }\end{array}$ & $\begin{array}{l}\text { EEG PAT- } \\
\text { TERN* }\end{array}$ & MMN \\
\hline 1 & \multicolumn{1}{c}{ F } & 53 & 16 & 62,2 & Yes & Negative \\
2 & M & 78 & 39 & 124,2 & Yes & Doubtful \\
3 & M & 76 & 14 & 36,8 & No & Positive \\
4 & F & 53 & 68 & 151,9 & - & Negative \\
5 & M & 66 & 20 & 54,7 & No & Positive \\
6 & M & 69 & 12 & 373 & Yes & Negative \\
7 & F & 74 & 15 & 83,17 & Yes & Negative \\
\hline
\end{tabular}

*Compatible with anoxic encephalophaty.

Both MMN-positive cases were CPC 1 at discharge and at 6 months. S: $100 \%$ and VVP: $100 \%$. The 4 negative and the 1 doubtful MMN died during admission, mostly under WLST according to a guideline-based multimodal approach.

Table 2Contingency table between MMN and CPC results at 6 months (one patient with doubtful MMN was excluded)

\section{CPC 1-2 CPC 3-5}

\begin{tabular}{llll}
\hline Positive MMN & 2 & 0 & 2 \\
Negative MMN & 0 & 4 & 4 \\
& 2 & 4 & 6 \\
\hline
\end{tabular}

Conclusion. Although limited by a very small sample size, our preliminary results suggest that MMN may predict neurological outcome in patients remaining in coma after RCA. Our study is currently ongoing.

\section{Reference(s)}

1. R. Näätänen, et al. The mismatch negativity (MMN)-a unique window to disturbed central auditory processing in ageing and different clinical conditions. Clin Neurophysiol. 2012 Mar;123(3):424-58.

2. A.O. Rossetti. Clinical neurophysiology for neurogical prognostication of comatose patients after cardiac arrest. Clin Neurophysiol 2017; 76-80.

3. R.A. Rodriguez, et al. Auditory-evoked potentials during coma: do they improve our prediction of awakening in comatose patients? J Crit Care 2014; 29:93-100.

4. M. Oddo, A.O. Rossetti. Predicting neurological outcome after cardiac arrest. Curr Opin Crit Care 2011; 17:254-259.

5. D. Wu, et al. Discovery of Long-Latency Somatosensory Evoked Potentials as a Marker of Cardiac Arrest Induced Brain Injury. IFMBE Proceedings 32, pp. 101-104, 2010. 
6. M.A. Koenig, P.W. Kaplan. Clinical Applications for EPs in the ICU. J Clin Neurophysiol 2015;32: 472-480.

7. J. Daltrozzo, et al. Predicting coma and other low responsive patients outcome using event-related brain potentials: A meta-analysis. Clinical Neurophysiology (2007) 606-614.

8. G.D. Perkins, et al., European Resuscitation Council Guidelines 2021: Executive summary, Resuscitation (2021), https://doi.org/10.1016/j.resus citation.2021.02.003

\section{1}

An audit of Ventilator Associated Pneumonia (VAP) rates in patients who were admitted to critical care following an out of hospital cardiac arrest and who were given antibiotics in the first $\mathbf{4 8} \mathrm{h}$

L. Dearden '; A. Martin,

${ }^{1}$ Adult critical care, Manchester Royal Infirmary, Manchester, United Kingdom

Correspondence: L. Dearden

Intensive Care Medicine Experimental 2020, 9(1): 000441

Introduction. Current guidance supports targeted temperature management (TTM) in the post-cardiac arrest patient, due to potential beneficial effects on neurological recovery(1). Yet, TTM is an independent risk factor for the development of ventilator associated pneumonia (VAP)(2). Two recent studies have assessed the use of antibiotics in the post cardiac arrest period. Ribaric et al.(3) noted that there was no change in inflammatory state, ICU outcome or mortality when prophylactic antibiotics were compared to clinician instituted antibiotics in out of hospital cardiac arrest (OOHCA) survivors who had aspiration excluded. Francois et al.(2) identified a reduction in early VAP rates when $48 \mathrm{~h}$ of antibiotics were used in OOHCA survivors, who had an initial shockable rhythm. The Advanced Life Support guidance recommends against prophylactic antibiotics in this patient group (evidence graded as weak)(4). We aimed to assess whether these findings were consistent in our population.

Methods. We assessed all patients presenting to our general critical care unit following an OOHCA between January and June 2020. Patients with documented pneumonia before their arrest were excluded. We assessed for exposure to antibiotic therapy within the first $48 \mathrm{~h}$ and then looked at whether those who did not receive antibiotics went on to be diagnosed with a VAP in the first 7 days of their stay.

Results. We identified 24 patients who met the criteria. Their mean age was 57.25 years, there was a male preponderance and most cardiac arrests were due to myocardial ischaemia. TTM was used in 19 of the patients. In the 5 who did not receive TTM, 3 were actively dying on admission and so further analysis was not performed. 20 patients had no documented concerns of aspiration on admission. 11 of the remaining 21 patients did receive antibiotic therapy within $48 \mathrm{~h}$. Of the 10 patients who received no antibiotic therapy in the first $48 \mathrm{~h}, 6$ were subsequently diagnosed with a VAP.

Conclusion. The findings of our audit are in line with the outcome of Francois et al.(2), that not being exposed to antibiotics within $48 \mathrm{~h}$ is associated with the development of VAP within the first 7 days. Limitations of our assessment are the small population size and that VAP was difficult to diagnose in those exposed to antibiotics within the first $48 \mathrm{~h}$ as they often went on to complete a full course of therapy (up to 5 days). We only performed a limited assessment of VAP rates after day 7 and could not perform a meaningful assessment on the acquisition of multi-drug resistant organisms. We are discussing within our unit about the potential benefit of introducing $48 \mathrm{~h}$ of antibiotic therapy for all out of hospital cardiac arrest patients and then plan to re-audit the rates of VAP seen.

\section{Reference(s)}

1. Couper K, Yeung K, Soar J, Berg K, Andersen LW, Böttiger BW, et al. Prophylactic antibiotics following return of spontaneous circulation in adults: Consensus on Science with Treatment Recommendations [Internet] Brussels, Belgium: International Liaison Committee on Resuscitation (ILCOR)
Advanced Life Support Task Force, 3 January 2020 Available from: http:// ilcor.org [Accessed 25/11/2020]

2. Nil

3. Neilsen N, Wetterslev J, Cronberg T, Erlinge D, Gasche Y, Hassager C, et al. Targeted temperature management at 33 oC versus 36 oC after cardiac arrest. N Engl J Med. 2013;369:2197-2206.

4. François B, Cariou A, Clere-Jehl R, Dequin PF, Renon-Carron F, Daix T, et al. Prevention of Early Ventilator-Associated Pneumonia after Cardiac Arrest. N Engl J Med. 2019;381:1831-42.

5. Ribaric SF, Turel M, Knafelj R, Gorjup V, Stanic R, Gradisek P, et al. Prophylactic versus clinically-driven antibiotics in comatose survivors or out-of-hospital cardiac arrest-A randomised pilot study. Resuscitation. 2017;11:103-109.

\section{9}

Serum Neurospecific Enolase as a Prognostic Market after Cardiorespiratory Arrest

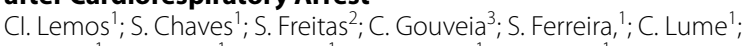
M. Mota ${ }^{1}$; S. Correia ${ }^{1}$; C. Graça ${ }^{1}$; T. Camacho ${ }^{1}$; M. Jardim¹; N. Fernandes ${ }^{1}$; G. Silva ${ }^{1}$; M. Câmara ${ }^{1}$; C. Pereira ${ }^{1}$; JJ. Nóbrega

${ }^{1}$ Serviço de Medicina Intensiva, Hospital Dr. Nélio Mendonça, Funchal, Portugal; ${ }^{2}$ Gabinete de estatística do centro dra. maria isabel mendonça, Hospital Dr. Nélio Mendonça, Funchal, Portugal; ${ }^{3}$ Serviço de medicina interna, Hospital de São Francisco Xavier, Lisboa, Portugal Correspondence: C.I. Lemos

Intensive Care Medicine Experimental 2020, 9(1): 000479

Introduction. Cardiorespiratory arrest (CPR) is a state of severe cerebral perfusion deficit and remains one of the most common causes of mortality1. Neurospecific Enolase (NSE) is a neuronal form of the glycolytic enzyme enolase which is found almost exclusively in neurons and cells of neuroendocrine origin. The identification of predictors of neurological recovery after CPR is fundamental in order to avoid useless intensive therapies. NSE has shown promise as a marker of neuronal prognosis2.

Objectives. This study aimed to determine the possibility of using serum NSE and other variables as predictors of poor prognosis after CPR.

Methods. This prospective, observational study was conducted between March 2019 and March 2021 in an intensive care unit, with the inclusion of patients with the diagnosis of CPR from any etiology $(n=101)$. We collected NSE at $24 \mathrm{~h}$ (NSE $24 \mathrm{~h})$ as well as age, gender, hypertension, diabetes, dyslipidemia, PCR rhythm, Acute Physiology and Chronic Health Evaluation II (APACHE II), Simplified Acute Physiology Score II (SAPS II), Mortality Probability Models II (MPM II), Sequential Organ Failure Assessment (SOFA), length of stay in the Intensive Care Unit (ICU), length of hospital stay, time to return of spontaneous circulation (ROSC) and lactate level at admission. The categorical variables were tested by the Chi-square test and the continuous variables were tested by the non-parametric Mann-Whitney test.

Results. The variables that were independently related to mortality were NSE $24 \mathrm{~h}(\mathrm{p}<0,0001)$, age $(p=0,033)$, hypertension $(p<0,0001)$, dyslipidemia ( $p<0,0001)$, non-defibrillable (ND) rhythm $(p<0,0001)$, length of stay in the ICU $(p=0,043)$, length of hospital stay $(p=0,0001)$, SOFA $(p<0,0001)$, time to ROSC $(p<0,0001)$ and lactate level $(p<0,0001)$. After performing logistic regression, it was determined that the statistically significant and independent variables that influenced the prognosis were NSE $24 \mathrm{~h}(\mathrm{p}=0,002)$, ND rhythm $(p=0,002)$ and APACHE II $(p=0,069)$. Each increase in the NSE level was associated with an increase of $11 \%$ in mortality, with an increase of APACHE II and ND rhythm. Patients with a non-defibrillable rhythm were 45 times more likely to die when compared to a defibrillable rhythm. APACHE II was also a risk factor since higher scores were associated with a $9,7 \%$ increase in mortality.

Conclusion. The identification of a low-cost biochemical prognostic indicator with easy application after CPR is useful to help in decisions regarding the mobilization of resources. There is evidence in favor of the role of NSE in the evaluation of neurological recovery after CPR. Although a simple, reliable and readily available test, the approach of 
clinical decisions must integrate multiple prognostic methods including NSE measurements. This strategy can offer a more accurate prognosis for these patients.

\section{Reference(s)}

1. Martinez-Losas, P.; Lopez de Sa, E.; Armada, E.; Rosillo, S.; Monedero, M.C.; Rey, J.R.; Caro-Codon, J.; Buno Soto, A.; Lopez Sendon, J.L. Neuron-specific enolase kinetics: An additional tool for neurological prognostication after cardiac arrest. Rev. Esp. Cardiol. 2019.

2. Wihersaari, L.; Tiainen, M.; Skrifvars, M.B.; Bendel, S.; Kaukonen, K.M.; Vaahersalo, J.; Romppanen, J.; Pettila, V.; Reinikainen, M.; Finnresusci study group. Usefulness of neuron specific enolase in prognostication after cardiac arrest: Impact of age and time to ROSC. Resuscitation 2019, 139, 214-221.

\section{0}

Quantitative pupillometry for prognostication in standard and prolonged targeted temperature management after out-of-hospital cardiac arrest-a post hoc analysis of the TTH48 trial

S. Paramanathan ${ }^{1}$; AM. Grejs ${ }^{1}$; E. Søreide ${ }^{2}$; CHV. Duez ; A. Jeppesen ${ }^{3}$; MB. Kvaløy, ${ }^{4}$ AJ. Reinertsen ${ }^{2} ;$ H. Kirkegaard ${ }^{3}$

'Department of intensive care medicine, Aarhus University Hospital, Aarhus N, Denmark; ${ }^{2}$ Critical care and anaesthesiology research group, Stavanger University Hospital, Stavanger, Norway; ${ }^{3}$ Research center for emergency medicine, Aarhus University Hospital, Aarhus N, Denmark; ${ }^{4}$ Department of neurophysiology, Stavanger University Hospital, Stavanger, Norway

Correspondence: S. Paramanathan

Intensive Care Medicine Experimental 2020, 9(1): 000500

Introduction. In the multi-modal prognostication model, assessment of the pupil is an essential part of the clinical neurological examination of out-of-hospital cardiac arrest (OCHA) patients. Former studies suggest that the Neurological Pupil index (NPi), measured by quantitative pupillometry, are associated with poor outcome in OCHA patients $[1,2]$. The present study is a substudy of "Targeted temperature management for 48 vs. $24 \mathrm{~h}$ and neurological outcome after out-of-hospital cardiac arrest: A randomized clinical trial (the TTH48trial)" [3].

Objectives. The aim of this substudy was to compare NPi, measured by quantitative pupillometry, in prolonged targeted temperature management at $33^{\circ} \mathrm{C}$ for $48 \mathrm{~h}$ against standard duration of $24 \mathrm{~h}$.

Methods. Pupillary assessments were achieved using a handheld device, NeurOptics ${ }^{\circledR}$ NPI100TM. NPi values were generated using the parameters, maximum and minimum size of pupil, percent change in pupil size, latency from light stimulation to movement of pupil, average and maximum constriction velocity, and average dilation velocity. NPi measurements were obtained every eight hours and graded on the NPi scale with a value from 0 to 5 for each eye. The lowest NPi value for each day was used for further analysis. Outcome was measured on the Cerebral Performance Category score (CPC) at 6 months. CPC score of 1 or 2 was considered as good neurological outcome and CPC 3-5 was considered as poor outcome.

Results. Sixty-five patients were included from Aarhus University Hospital, Denmark, and Stavanger University Hospital, Norway. The Median NPi values for day one to three and their corresponding confidence interval for both TTM and outcome groups are characterised in Table 1. NPi were not significantly different between TTM groups (all $\mathrm{p}$-values $>0.05)$. The prognostic ability of $\mathrm{NPi}$, defined by area under the receiver operating characteristic curve (AUC) was best at day three in both TTM groups, TTM24 0.98 (95\% confidence interval 0.95-1.0)) and TTM48 $0.86(0.72-1.0)$. When data were pooled, NPi was strongly associated with poor neurological outcome $(p<0.01)$ and the prognostic performance was best at day three with AUC 0.92 (0.84-1.0).

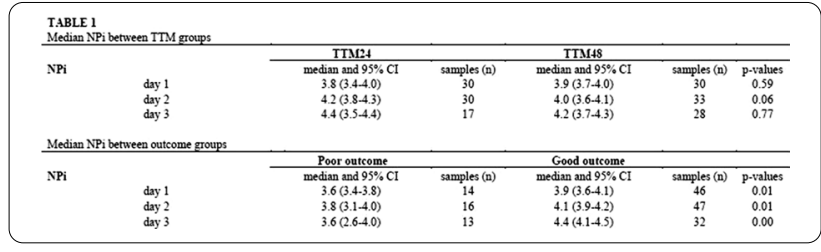

Conclusion. NPi measured by quantitative pupillometry were associated with poor outcome six months after out-of-hospital cardiac arrest but was unaffected by duration of TTM. The prognostic ability was best on day three.

\section{Reference(s)}

1. [3] Kirkegaard H, Søreide E, De Haas I, Pettilä V, Taccone FS, Arus U, et al. Targeted temperature management for 48 vs $24 \mathrm{~h}$ and neurologic outcome after out-of-hospital cardiac arrest: A randomized clinical trial. JAMA —J Am Med Assoc 2017;318:341-50. https://doi.org/10.1001/jama. 2017.8978.

2. [2] Solari D, Rossetti AO, Carteron L, Miroz JP, Novy J, Eckert P, et al. Early prediction of coma recovery after cardiac arrest with blinded pupillometry. Ann Neurol 2017:81:804-10. https://doi.org/10.1002/ana.24943.

3. [1] Oddo M, Sandroni C, Citerio G, Miroz JP, Horn J, Rundgren M, et al. Quantitative versus standard pupillary light reflex for early prognostication in comatose cardiac arrest patients: an international prospective multicenter double-blinded study. Intensive Care Med 2018;44:2102-11. https://doi.org/10.1007/s00134-018-5448-6.

4. Christophe H. V. Duez received funding for research in neuroprognostication from the private foundations the Viggo and Helene Bruun Foundation, the Lily Benthine Lunds Foundation of June 1, 1978, the Director Jacob Madsen \& Wife Olga Madsen Foundation and the Grocer A. V. Lykfeldt and Wife Foundation. The foundations had no influence on the study design, execution, analysis, or interpretation of the data.

\section{0}

Poor survival after in-hospital cardiac arrest in critically ill patients with Covid-19 pneumonia: Should we offer cardiopulmonary resuscitation?

F. Ahmad'; N. Julien²; P. Gandre²; P. Thaventhran²; T. Konstantinos²; EAA. Marwa'; J. Samuel2; C. David ${ }^{2}$; G. Pakeerathan²; M. Navaneethan ${ }^{2}$ P. Janak'2; K. Ferenc²; P. Dharam²; CST. Darren³; Y. Emma ${ }^{4}$; K. Sazzli';

C. Jeronimo ${ }^{2}$

${ }^{1}$ Department of cardiology, Hospital UiTM Sungai Buloh, Sungai Buloh, Malaysia; ${ }^{2}$ Intensive care unit, North Middlesex University Hospital, London, United Kingdom; ${ }^{3}$ Department of internal medicine, Keningau Hospital, Keningau, Sabah, Malaysia; ${ }^{4}$ Department of cardiology, Serdang Hospital, Kajang, Malaysia

Correspondence: F. Ahmad

Intensive Care Medicine Experimental 2020, 9(1): 000530

Introduction. Previously published reports showed increased risk of cardiac arrest and poor survival rates in critically ill coronavirus 2019 (COVID-19) patients $[1,2]$. Thus, the implementation and futility of DNACPR (Do Not Attempt Cardio-Pulmonary Resuscitation) order in this patient population remains controversial. More evidence is therefore needed for this informed complex decision in the midst of on going COVID-19 pandemic.

Objectives. To study the characteristics and outcomes associated with in-hospital cardiac arrest (IHCA) and cardiopulmonary resuscitation (CPR) in critically ill COVID-19 patients in a COVID-19 surge hospital in London, United Kingdom.

Methods. IHCA is defined as cases occurring within the hospital boundary. We included all cases of critically ill COVID-19 who had IHCA registered from March 2020 to 2021 . We followed patients until hospital discharge, death, or 31st March 2021(when the study database for the current analysis was locked), whichever occurred first. Inclusive 
criteria are cases whereby CPR and/or defibrillation are performed. All statistical studies were carried out using Statistical Package for Social Sciences software.

Results. Overall, 520 COVID-19 patients were to admitted to Intensive Care Unit (ICU) from March 2020 to March 2021. Of those, 401 patients (77.1\%) had IHCA with 380 patients (73.0\%) had IHCA within 21 days of admission while $11.22 \%$ of patients had IHCA within 3 days of admission to ICU, respectively. 6 patients had cardiac arrest in the Emergency Department, prior to admission to ICU. Of those 401 patients with IHCA, 68 (16.9\%) received CPR while $83.0 \%$ (333/401) of patients had a DNACPR code status at the time of cardiac arrest. Patients who received CPR were younger than those who did not [51 (41.4-68) vs. $69(60-81), P=0.03]$. Using multivariable analysis, prominent cardiovascular risk factors (smoking, diabetes mellitus, hypertension, coronary artery disease, chronic/end stage kidney disease), older age and higher mSOFA score on admission to ICU were associated with a higher risk of IHCA (Table 1). The most common initial cardiac arrest rhythm at commencement of CPR was pulseless electrical activity (PEA) $(61.2 \%, 42 / 68)$, followed by asystole $(23.5 \%, 16 / 68)$, ventricular tachycardia $(8.8 \%, 6 / 68)$ and ventricular fibrillation $(5.9 \%, 4 / 68)$. The most common cause of cardiac arrest is hypoxia $(63 \%)$ and followed by acute coronary syndrome (31\%). Altogether, 21 of the 68 patients (30.8\%) who received CPR achieved return of spontaneous circulation. 220 of 401 patients (54.8\%) with IHCA died, including 172 who died without receiving CPR and 48 who died despite CPR.

Table 1. Demographics of critically ill COVID-19 patients who had cardiac arrest

\begin{tabular}{lll}
\hline & $\begin{array}{l}\text { Cardiac Arrest } \\
\text { Receiving CPR } \\
\text { (Adjusted Odds } \\
\text { Ratio, 95\% CI) }\end{array}$ & $\begin{array}{l}\text { Cardiac Arrest } \\
\text { Not Receiving } \\
\text { CPR } \\
\text { (Adjusted Odds } \\
\text { Ratio, 95\% CI) }\end{array}$ \\
\hline Male & $1.20(0.98-1.45)$ & $0.98(0.71-1.39)$ \\
Ischaemic Heart Disease & $1.30(0.91-1.57)$ & $0.73(0.44-1.18)$ \\
Diabetes & $1.31(1.01-1.51)$ & $0.61(0.49-0.98)$ \\
Hypertension & $1.07(0.84-1.30)$ & $1.33(0.89-2.00)$ \\
Chronic/End stage renal disease & $1.23(0.91-1.49)$ & $1.12(0.73-1.55)$ \\
Smoking/Smoking history & $0.91(0.68-1.10)$ & $0.96(0.66-1.49)$ \\
mSOFA score per 2 units & $1.29(1.18-1.41)$ & $1.02(0.89-1.19)$ \\
\hline
\end{tabular}

Conclusion. This study showed that cardiac arrest is common in critically ill COVID-19 patients and is associated with extremely poor survival even when CPR is performed. The higher incidence of nonshockable cardiac arrest rhythms emphasizes the importance of monitoring and controlling if possible all reversible causes especially hypoxia. The study reflects the experience of a COVID-19 surge London Hospital, acknowledging the fact that survival improved over time as more evidence-based treatments became available. Results from this study may help in the decision making process for critically ill patients with Covid-19 pneumonia, who are at risk of IHCA.

\section{Reference(s)}

1. Kramer DB, Lo B, Dickert NW. CPR in the covid-19 era-an ethical framework. N Engl J Med2020;383

2. hao F, Xu S, Ma X, Xu Z, Lyu J, Ng M, et al. In-hospital cardiac arrest outcomes among patients with COVID-19 pneumonia in Wuhan, China. Resuscitation2020;151:18

3. No Funding Received
000593

Brain injury markers in blood associate with generalised oedema on computed tomography after cardiac arrest

A. Lagebrant'; M. Lang, ${ }^{2}$; N. Mattsson-Carlgren, ; K. Blennow, ${ }^{3}$.

J. Dankiewicz ; H. Friberg ${ }^{5}$; H. Christiann; J. Horn'; J. Kjaergaard ${ }^{8}$;

M. Kuiper, ${ }^{9} ;$ G. Lilja'; T. Pellis, ${ }^{10}$; C. Rylander ${ }^{11} ;$ R. Siemund, ${ }^{12}$; J. Undén, ${ }^{13}$.

M. Wise, ${ }^{14} ;$ H. Zetterberg, ${ }^{15}$; T. Cronberg ${ }^{16}$; M. Moseby-Knappe,

P. Stammet ${ }^{17}$

'Department of clinical sciences lund, neurology, Lund University, Skåne University Hospital, Sweden; ${ }^{2}$ Department of clinical sciences lund, radiology, Lund University, Lund, Sweden; ${ }^{3}$ Department of psychiatry and neurochemistry, institute of neuroscience and physiology, Gothenburg University, Mölndal, Sweden; ${ }^{4}$ Department of clinical sciences lund, cardiology, Lund University, Skåne University Hospital, Sweden; ${ }^{5}$ Department of clinical sciences, intensive and perioperative care, Lund University, Lund, Sweden; ${ }^{6}$ Department of cardiology, Rigshospitalet, Copenhagen, Denmark; ${ }^{7}$ Intensive care, Academic Medical Centre, Amsterdam, Netherlands; ${ }^{8}$ Department of cardiology, Rigshospitalet, København, Denmark; ${ }^{9}$ Department of intensive care, Medical Center Leeuwarden, Leeuwarden, Netherlands; ${ }^{10}$ Department of anaesthesia and intensive care, Azienda Sanitaria Friuli Occidentale, Pordenone, Italy; ${ }^{11}$ Anesthesiology and intensive care medicine, Sahlgrenska University Hospital, Institute of Clinical Sciences, Gothenburg, Sweden; ${ }^{12}$ Department of clinical sciences lund, division of neuroradiology, Lund University, Skåne University Hospital, Sweden; ${ }^{13}$ Department of clinical sciences malmö, dept. operation and intensive care, Lund University, Lund, Sweden; ${ }^{14}$ Critical care, University Hospital of Wales, Cardiff, United Kingdom;

${ }^{15}$ Department of psychiatry and neurochemistry, institute of neuroscience and physiology, Gothenburg University, Gothenburg, Sweden;

${ }^{16}$ Department of clinical sciences, neurology, Lund University, Lund,

Sweden; ${ }^{17}$ Medical and health department, National Fire and Rescue

Corps, Luxembourg, Luxembourg

Correspondence: A. Lagebrant

Intensive Care Medicine Experimental 2020, 9(1): 000593

Introduction. According to the 2021 ERC/ESICM guideline recommendations, elevated neuron-specific enolase [NSE] levels as well as diffuse and extensive anoxic damage on neuroimaging are predictors of poor neurological outcome after cardiac arrest.(1) We previously described that NSE is elevated in patients with generalised oedema on computed tomography [CT]. (2).

Objectives. In this study, we aim to examine the novel brain injury markers serum neurofilament light [NFL], glial fibrillary acidic protein [GFAP] and total-tau [tau] to predict the presence of generalised brain oedema.

Methods. Retrospective analysis of patients examined with $\mathrm{CT}$ on clinical indication within the Target Temperature Management after out-of-hospital cardiac arrest [TTM] trial. $(2,3)$ Serum samples from the biobank sub study were prospectively collected at $48 \mathrm{~h}$ post arrest and analysed after trial completion as published. (4-7) The neuronal marker NSE, the neuroaxonal injury markers NFL and tau and the astrocytic injury marker GFAP were correlated with the presence of generalised oedema on $\mathrm{CT}$, assessed by local radiologists through visual evaluation. The prognostic accuracy of NSE $\geq 60 \mathrm{ug} / \mathrm{l}$ for predicting generalised oedema was also analysed.

Results. 192 patients had data available on all four biomarkers at $48 \mathrm{~h}$ and were examined with $\mathrm{CT}<168 \mathrm{~h}$ post arrest. Brain injury markers were significantly higher in patients with generalised oedema as compared to patients without oedema on CT scans performed 24-168 h after ROSC $(p<0.001)$ (Fig. 1A-D). For CT scans performed $<24$ h, only NSE levels showed a significant correlation $(p<0.05)$. Biomarkers predicted generalised oedema with area under the receiver operating characteristics curve [AUC] $67.5-73.2 \%$ for CT scans performed $<24 \mathrm{~h}$ $(n=111)$, with no statistically significant difference between the markers ( Fig. 2A). For scans performed 24-168 h $(n=81)$ AUC for predicting generalised oedema was $78.1 \%-82.9 \%$, with no statistically significant difference between the markers. NSE $\geq 60 \mathrm{ug} / \mathrm{l}$ at $48 \mathrm{~h}$, as recommended by guidelines, predicted generalised oedema with $81 \%$ $(95 \% \mathrm{Cl} 67-90 \%)$ sensitivity and $77 \%(95 \% \mathrm{Cl} 62-87 \%)$ specificity. 


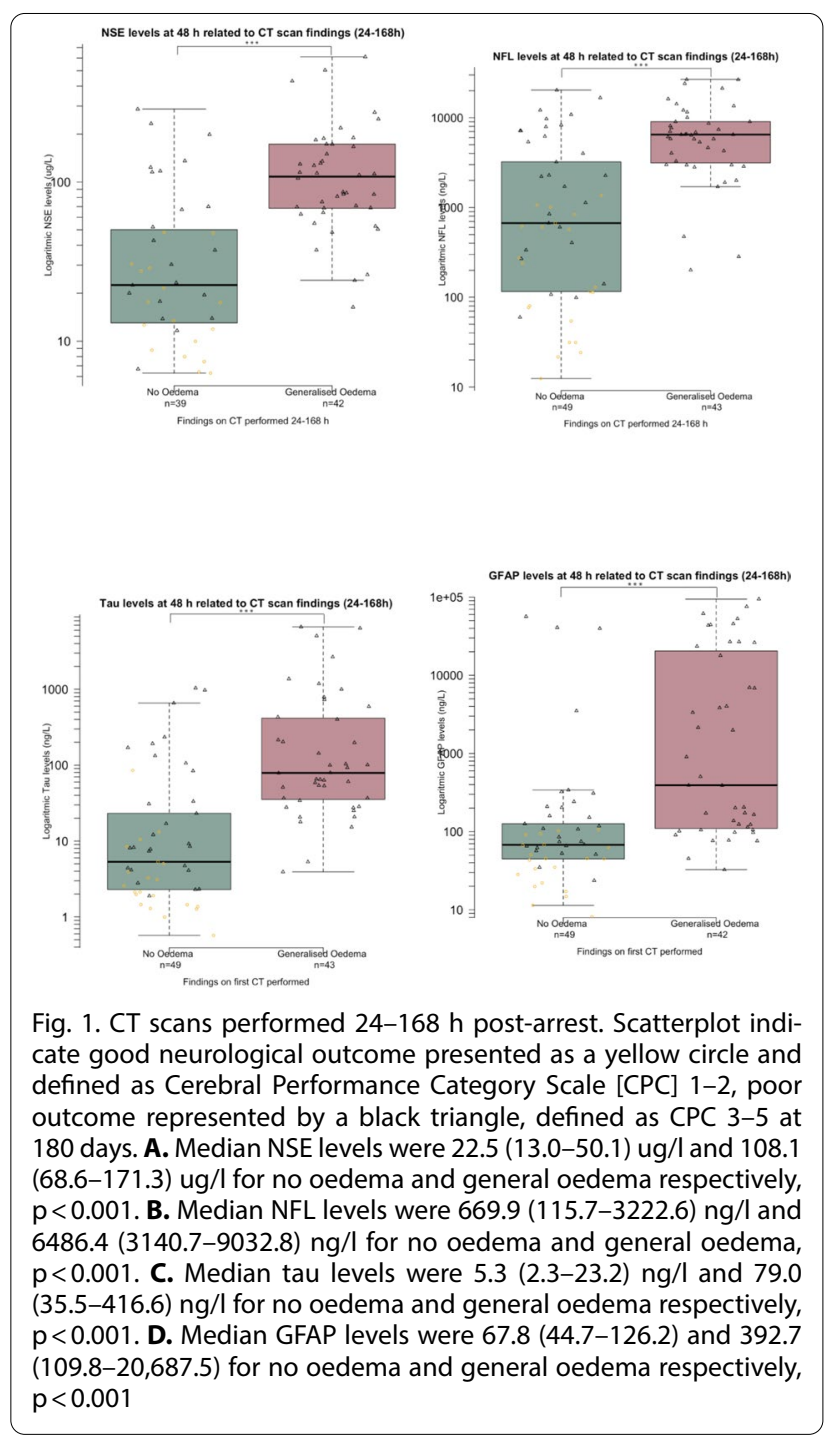

Conclusion. Concentrations of all evaluated brain injury markers were significantly higher in patients with generalised oedema on CT performed after the first $24 \mathrm{~h}$ post arrest. Biomarker concentrations indicate whether generalised oedema on CT is likely and may thus be clinically useful for deciding if a CT scan is sufficient for prognostication or if a MRI is more appropriate.

\section{Reference(s)}

1. Nolan JP, Sandroni C, Bottiger BW, et al. European Resuscitation Council and European Society of Intensive Care Medicine guidelines 2021: postresuscitation care. Intensive Care Med 2021.

2. Moseby-Knappe M, Pellis T, Dragancea I, et al. Head computed tomography for prognostication of poor outcome in comatose patients after cardiac arrest and targeted temperature management. Resuscitation 2017:119:89-94

3. Ebner F, Moseby-Knappe M, Mattsson-Carlgren N, et al. Serum GFAP and UCH-L1 for the prediction of neurological outcome in comatose cardiac arrest patients. Resuscitation 2020.

4. Mattsson N, Zetterberg H, Nielsen N, et al. Serum tau and neurological outcome in cardiac arrest. Ann Neurol 2017;82:665-675.

5. Moseby-Knappe M, Mattsson N, Nielsen N, et al. Serum Neurofilament Light Chain for Prognosis of Outcome After Cardiac Arrest. JAMA neurology 2019;76:64-71.
6. Stammet P, Collignon O, Hassager C, et al. Neuron-Specific Enolase as a Predictor of Death or Poor Neurological Outcome After Out-of-Hospital Cardiac Arrest and Targeted Temperature Management at 33 degrees $C$ and 36 degrees C. J Am Coll Cardiol 2015;65:2104-2114.

7. Role of the Funder/Sponsor The funding organizations had no role in the design and conduct of the study; collection, management, analysis, and interpretation of the data; preparation, review, or approval of the manuscript; and decision to submit the manuscript for publication.

8. Funding for the study was provided by the Swedish Research Council, Swedish Heart Lung Foundation, Arbetsmarknadens Försäkringsaktiebolag Insurance Foundation, the Skåne University Hospital Foundations, the Gyllenstierna-Krapperup Foundation, and governmental funding of clinical research within the Swedish National Health System, the County Council of Skåne; the Swedish Society of Medicine; the Koch Foundation; TrygFonden (Denmark); European Clinical Research Infrastructures Network; Thelma Zoega Foundation; Stig and Ragna Gorthon Foundation; Thure Carlsson Foundation; Hans-Gabriel and Alice Trolle-Wachtmeister Foundation for Medical Research; Lions Research fund Skåne; South Swedish Hospital Region Research Funds; the Swedish Brain Foundation; the Lundbeck Foundation; and the Torsten Söderberg foundation at the Royal Swedish Academy of Sciences. Banyan Biomarkers performed the analyses of GFAP free of charge. $\mathrm{HZ}$ is a Wallenberg Scholar. NMC is a Wallenberg Molecular Medicine Fellow.

9. Nielsen N, Wetterslev J, Cronberg T, et al. Targeted temperature management at 33 degrees $C$ versus 36 degrees $C$ after cardiac arrest. N Engl J Med 2013;369:2197-2206

\section{3}

\section{Stemi's timing during COVID pandemia}

S. Moreno Cano'; A. Garrino Fernández'; A. Estella

${ }^{1}$ University hospital of jerez., Intensive care unit, Jerez, Spain

Correspondence: S. Moreno Cano

Intensive Care Medicine Experimental 2020, 9(1): 000723

Introduction. The mainstay of treatment in patients with ST-segment elevation myocardialinfarction (STEMI) is immediate reperfusion by primary percutaneous coronaryintervention (PCl) or, if not available in atimely manner, by fibrinolytic therapy.Early coronary angiography strategy in acute coronary syndromes (ACS) isreccomended as it improves major adverse cardiac events and possibly earlysurvival. It could be modified in the SARS COV- 2 pandemic.

Objectives. The aim of this study is to clarify if timing between symptoms onset- first medicalcontact (FMC), and timing of early reperfusion strategy, were modified in pandemictime, and its impact on survival.

Methods. Patients with STEMI between seven months before pandemic (first period) andduring pandemic (second period) in our 17 beds of Intensive Care Unit (ICU) wereexplored to identify modifications on timing of invasive coronary angiography (ICA)and revascularization.Design: before-after descriptive study.We analyze our data by SSPS V18 Programme: age, sex, time from onset ofsymptoms to first medical contact, time from medical contact to $\mathrm{PCl}$, and mortalityat 30 and 90 days.

Results. A total of 157 patients were enrolled: 79 during the first period of time and 78during the second. There was no differences between age. During pandemic timethere was no statistically significant differences among timing of onset of symptomsand FMC $(63.5$ [30-157] versus 65 [23-127] p 0.3), however there was a shorteningof time regarding FMC and $\mathrm{PCl}(82$ [50-125] versus 110 [70-155] p 0.031), andprobably, because of that, the mortallity was lower in this period ( $5 \%$ versus $15 \%$ ).

Conclusion. There were no significant differences among the number of patients enrolled withSTEMI, and timing of symptoms onset$\mathrm{FMC}$, but there was a shortening of time inFMC-PCl as a reduction in mortallity. 


\section{5}

Neuron-specific enolase (NSE) predicts long-term mortality in patients after cardiac arrest: Results from a prospective trial J. Müller ${ }^{1}$; B. Bissmann, ${ }^{1}$; C. Becker, ${ }_{1}^{1}$; K. Beck, ${ }_{1}^{1}$; N. Loretz, ; SA. Amacher, ${ }_{1}^{1}$ S. Gross, ; C. Bohren, ; H. Pargger ${ }^{2}$; T. Kai ${ }^{2}$; R. Sutter, ${ }^{2}$; S. Marsch, ${ }^{2}$;

S. Hunziker,

${ }^{1}$ Medical communication and psychosomatic medicine, Universitätsspital Basel, Basel, Switzerland; ${ }^{2}$ Intensive care unit, Universitätsspital Basel, Basel, Switzerland

Correspondence: J. Müller

Intensive Care Medicine Experimental 2020, 9(1): 000775

Introduction. Neuron-specific enolase (NSE) increases in response to brain injury and is recommended for outcome prediction at short term in cardiac arrest patients. There is, however, limited understanding of NSEs influence on long term outcomes.

Objectives. Our aim was to investigate whether NSE predicts longterm mortality and poor neurological outcome in cardiac arrest patients.

Methods. Within this prospective observational study, we included consecutive adult patients after cardiac arrest admitted to the ICU. NSE was measured upon ICU-admission and on days 1, 2, 3, 5 and 7. We calculated Hazard-ratios using Cox-regression and logistic regression to study the associations of NSE levels with long term overall all-cause mortality and neurological outcome defined by Cerebral Performance Category (CPC) scale two years after cardiac arrest.

Results. From 403 patients, $176(43.7 \%)$ survived with a median follow-up of 43.7 months (IQR 14.3 to 63.0 months). NSE on day 3 showed the highest prognostic performance for mortality compared to other days of measurement, with an AUC of 0.81 and an adjusted HR of 1.55 and 1.51. Subgroup analysis indicated that predictive value of NSE for mortality was significantly higher in younger patients $<54$ years of age and showed excellent sensitivity and negative predictive value of $100 \%$. Results were similar for poor neurological outcome, however there were no significant differences in subgroup analysis.

Conclusion. NSE measured three days after cardiac arrest is associated with long-term mortality and neurological outcome and may thus provide prognostic information that improves clinical decision-making, particularly in the subgroup of younger patients $<54$ years of age, where NSE showed an excellent negative predictive value.

\section{4}

Incidence, characteristics and predictors of mortality in ICU

\section{cardiac arrest}

G. Jansen ${ }^{1}$; O. Sauzet, ${ }^{2}$; R. Borgstedt ${ }^{3}$; S. Entz, ; FO. Holland ${ }_{1}^{5}$;

S. Lamprinaki, ${ }^{4}$; KC. Thies, ; S. Scholz ; ; S. Rehberg,

${ }^{1}$ Anaesthesiology, Prostestant Hosiptal of the Bethel Foundation, Biele-

feld, Germany; ${ }^{2}$ Epidemiology and international public health, bielefeld school of public health \& center for sta, Bielefeld University, Bielefeld, Germany; ${ }^{3}$ Anesthesiology, intensive care, emergency medicine, transfusion medicine, and pain therapy, Protestant Hospital of the Bethel Foundation, University Hospital OWL, Bielefeld, Germany; ${ }^{4}$ Clinic for internal medicine and gastroenterology, The Protestant Hospital of the Bethel Foundation, Bielefeld, Germany; ${ }^{5}$ Clinic for internal medicine and nephrology, Protestant Hospital of the Bethel Foundation, Bielefeld, Germany Correspondence: $\mathrm{G}$. Jansen

Intensive Care Medicine Experimental 2020, 9(1): 000804

Introduction. Cardiac arrest in the intensive care unit (ICUCA) is a special and rarely studied type of in-hospital cardiac arrest. On the one hand, it is always observed, professional staff and all available ressources of an ICU are immediately available, resulting in the best possible conditions for a favourable outcome; on the other hand, affected patients are often critically ill with a high risk of dying. This study examines the incidence, characteristics, predictors of mortality as well as long-term prognosis in patients with ICUCA.

Objectives. To determine incidence, characteristics, predictors and marginal probabilities of ICUCA.

Methods. The ICU database of a German university hospital with 78 non-cardiac-surgical ICU beds (surgical 41, medical 37) was screened for ICUCA, defined according to Utstein criteria as need for resuscitation with chest compressions and/or defibrillation occurring for the first time during ICU stay. Primary endpoints were incidence, hospital discharge and 1-year-survival rate as well as neurological outcome quantified with cerebral performance category (CPC), secondary endpoints included predictors and marginal probabilities of no return of spontaneous circulation (ROSC), rate of ROSC, diagnostic and therapeutic measures, aetiology, comorbidities, Sequential-Organ-FailureAssessment-(SOFA)-Score, lactate and bicarbonate-levels. Predictors of No-ROSC were obtained using explorative logistic and cox regression models.

Results. From 2016-2018 a total of 114 ICUCA were observed out of 14,264 ICU-admissions with 64,809 days of occupancy (incidence: 8 per 1.000 ICU admissions; C195:6.6-9.6; 1.8 per 1,000 ICU occupancy days; C195:1.4-2.1). 45.6\% received at least one additional diagnostic test (i.e. blood gas analysis 36\%; echocardiography $19.3 \%$; chest-xray $9.9 \%$ ) with a therapeutic consequence in $52 \%$ (No-ROSC vs. ROSC $\mathrm{p}=0.023$ ). Predictors of No-ROSC were cardiac and hepatic comorbidities as well as SOFA $\geq 2$ and continuous renal replacement therapy before ICUCA. Interestingly, bicarbonate levels $>21 \mathrm{mmol} / \mathrm{I}$ in combination with cardiac comorbidities or SOFA $\geq 2$ were associated with a higher risk of dying. Hospital-mortality was $78.1 \%(n=89)$. 1-year-survival rate was $10.5 \%$ [CI95 5.5-17.7], survival with a good neurological outcome (CPC 1-2) was $6.1 \%$ (CI95 2.5-12.2) of all ICUCA patients.

Conclusion. ICUCA is a serious complication in the ICU due to its high mortality rate and low rate of good neurological outcome. Specific algorithms for ICUCA maybe required to adequately consider the complete spectrum of diagnostic and therapeutic possibilities, because based on the present study these are still underutilized. Predictors of No-ROSC as well as potential do-not-resuscitate orders need to be considered early to conserve these limited resources.

\section{5}

Incidence and risk factors for peri-operative cardiac arrest and 30-day-mortality in preterm infants requiring non-cardiac surgery

G. Jansen'; L. Irmscher, ; T. May, ${ }^{2}$; R. Borgstedt ${ }^{3}$; J. Popp, ${ }_{1}^{1}$; S. Scholz'; S. Rehberg

${ }^{1}$ Anaesthesiology, Prostestant Hosiptal of the Bethel Foundation, Bielefeld, Germany; ${ }^{2}$ Coordination office for studies in biomedicine and preclinical and clinical research, Protestant Hospital of the Bethel Foundation, Bielefeld, Germany; ${ }^{3}$ Anesthesiology, intensive care, emergency medicine, transfusion medicine, and pain therapy, Protestant Hospital of the Bethel Foundation, University Hospital OWL, Bielefeld, Germany Correspondence: $\mathrm{G}$. Jansen

Intensive Care Medicine Experimental 2020, 9(1): 000805

Introduction. Although prematurity is known to be a risk factor for peri-operative cardiac arrest and mortality, little is known about its true incidence and specific risk factors within this population.

Objectives. To determine 30-day-mortality, incidence and characteristics of peri-operative cardiac arrest as well as the respective independent risk factors in preterm infants undergoing non-cardiac surgery.

Methods. Follow-up-study of peri-operative care in preterm infants between 01/2008-12/2018 at a German University Hospital and perinatal-centre with the highest level of expertise. Only preterm infants with an age $<37$ th gestational week at time of surgery were included. Primary endpoint was overall 30-day-mortality. Secondary endpoint was occurrence of peri-operative cardiac arrest. We performed univariate and multivariate analyses and calculated odds ratios (OR) for risk factors associated with the endpoints.

Results. From January 2008 to December 2018 a total of 321 anaesthetic procedures in 229 preterm infants were performed out of 22,650 pediatric anesthetic procedures $(1.4 \% ; 95 \% \mathrm{Cl}, 1.3$ to 1.6). 30-day-mortality was $10.9 \%$ and peri-operative mortality $0.9 \%$. Univariate risk factors for 30 -day-mortality were peri-operative cardiac arrest $(\mathrm{OR}, 12.5 ; 95 \% \mathrm{Cl}, 3.1$ to 50.3$)$, comorbidities of lungs $(\mathrm{OR}, 3.7 ; 95 \% \mathrm{Cl}, 1.2$ to 11.3$)$ and gastrointestinal tract $(\mathrm{OR}, 3.5 ; 95 \% \mathrm{Cl}, 1.3$ to 9.6$)$; sepsis $(\mathrm{OR}, 3.6 ; 95 \% \mathrm{Cl}, 1.4$ to 9.5$)$; surgery between $22: 01-7: 00(\mathrm{OR}, 7.3 ; 95 \% \mathrm{Cl}, 2.4$ to 21.7$)$; emergency 
$(\mathrm{OR}, 4.5 ; 95 \% \mathrm{Cl}, 1.6$ to 12.4); pre-existing catecholamine-therapy $(\mathrm{OR}, 5.0 ; 95 \% \mathrm{Cl}, 2.1$ to 11.9$)$. Multivariate logistic regression indicated that peri-operative cardiac arrest $(\mathrm{OR}, 13.9 ; 95 \% \mathrm{Cl}, 2.7$ to 71.3$)$, low body weight (weight < $1000 \mathrm{~g}$ : OR, $26.0 ; 95 \% \mathrm{Cl}, 3.2$ to $212 ; 1000$ $1499 \mathrm{~g}:$ OR, 10.3; $95 \% \mathrm{Cl}, 1.1$ to 94.9 compared to weight $>2000 \mathrm{~g}$ ), and time of surgery $(\mathrm{OR}, 5.9 ; 95 \% \mathrm{Cl}, 1.6$ to 21.3$)$ for $22: 01-7: 00 \mathrm{com}$ pared to 7:01-15:00) are the major independent risk factors of mortality. Incidence of peri-operative cardiac arrests was $3.9 \%$ (9 of $229 ; 95 \% \mathrm{Cl}, 1.8$ to 7.3$)$. Univariate risk factors were congenital anomalies of the airways (OR, $4.7 ; 95 \% \mathrm{Cl}, 1.2$ to 20.3$)$, lungs (OR, $4.7 ; 95 \% \mathrm{Cl}, 1.2$ to 20.3$)$ and heart $(\mathrm{OR}, 8.0 ; 95 \% \mathrm{Cl}, 2$ to 32.2$)$, pre-existing catecholamine-therapy $(\mathrm{OR}, 59.5 ; 95 \% \mathrm{Cl}, 3.4$ to 1039$)$, specifically with epinephrine $(\mathrm{OR}, 432 ; 95 \% \mathrm{Cl}, 43.2$ to 4318$)$.

Conclusion. 30-day-mortality and the incidence of peri-operative cardiac arrest of preterms undergoing non-cardiac surgery are higher than previously reported. The identified independent risk factors may improve interdisciplinary peri-operative risk assessment, optimal pre-operative stabilization and scheduling of optimal surgical timing.

\section{8}

Do women have a higher mortality risk than men following an ICU-admission for out-of-hospital cardiac arrest? A retrospective cohort analysis

C. van Wees ${ }^{1}$; W. Rietdijk'; L. Mandigers ${ }^{3}$; M. Van Der Graaf ${ }^{4}$; N. Scholte

K. Adriaansens ${ }^{4}$; R. Van Den Berg ${ }^{5}$; C. Den Uil

${ }^{1}$ Erasmus University Medical Center, Rotterdam, Netherlands; ${ }^{2}$ Phar-

macy, Erasmus University Medical Center, Rotterdam, Netherlands; ${ }^{3}$ Intensive care, Erasmus University Medical Center, Rotterdam, Netherlands;

${ }^{4}$ Cardiology, Erasmus University Medical Center, Rotterdam, Netherlands; ${ }^{5}$ Cardiology, HMC Westeinde, Den Haag, Netherlands; ${ }^{6}$ Departement of intensive care, Erasmus University Medical Center, Rotterdam, Netherlands

Correspondence: C. van Wees

Intensive Care Medicine Experimental 2020, 9(1): 000948

Introduction. Previous studies showed that women have a higher mortality risk than men after out-of-hospital cardiac arrest (OHCA). This sex difference tends to disappear when analyses are adjusted for cardiac arrest characteristics. However, these studies also include patients who were not admitted to the intensive care unit (ICU). Therefore, we analysed this sex difference in mortality in OHCA patients admitted to the ICU.

Methods. A retrospective cohort analysis of OHCA patients admitted to the ICU at an academic hospital in The Netherlands. The primary outcome was 90-day mortality. As secondary outcomes we used favourable cerebral performance category (CPC) score at discharge and ICU length of stay (ICU LOS). These outcomes were analysed using a Cox proportional hazard regression, a binary logistic regression, and a linear regression, respectively.

Results. We included 1,240 OHCA patients (310 women, 25.0\%). No association was found between sex and 90-day mortality (Hazard Ratio [HR] 0.867; 95\% confidence interval [95\% Cl] 0.678-1.108). In addition, we found no statistically significant difference for ICU LOS between women and men (Beta 0.428; $95 \% \mathrm{Cl}-0.442-1.298$ ) and favourable CPC score at ICU discharge (OR 1.117; 95\% Cl 0.777-1.608).

Conclusion. We found that indeed-when adjusting for cardiac arrest characteristics-there is no difference between women and men with respect to 90-day mortality, ICU LOS, and CPC score at ICU discharge. Our results may contribute to the idea that the underlying pathophysiology of the cardiac arrest is different for women and men and this should be studied in future research.

\section{Reference(s)}

1. Mandigers L, Termorshuizen F, De Keizer N, Rietdijk W, Gommers D, Dos Reis Miranda D, et al. Higher 1-year mortality in women admitted tot he intensive care units after cardiac arrest: A nationwide overview from the Netherlands between 2010-2018. Journal of Critical Care, Forthcoming. 2021

2. Kotini-Shah P, Del Rios M, Khosla S, Pugach O, Vellano K, McNally B, et al. Sex differences in outcomes for out-of-hospital cardiac arrest in the United States. Resuscitation. 2021. PubMed PMID: 33,798,627

001012

Soluble Suppression of Tumorigenicity 2 (sST2) correlates with 6-month outcome after cardiopulmonary resuscitation: A single-center analysis

R. Rezar ${ }^{1}$; C. Seelmaier ; U. Hoppe ; B. Wernly ${ }^{2}$; M. Lichtenauer ${ }^{1}$

${ }^{1}$ Cardiology and Intensive Care, Paracelsus Medical University Salzburg, Salzburg, Austria; ${ }^{2}$ Anesthesiology and intensive care, Paracelsus

Medical University Salzburg, Salzburg, Austria

Correspondence: R. Rezar

Intensive Care Medicine Experimental 2020, 9(1): 001012

Introduction. Outcome prediction after cardiopulmonary resuscitation (CPR) is a complex process and continues to challenge intensivists. Various clinical, neurophysiological, radiographic, and laboratory parameters are used as decision-aiding tools nowadays with variable predictive performance. Multiple pro- and anti-inflammatory effects have been attributed to the novel cardiovascular biomarker named suppression of tumorigenicity 2 (ST2) and its soluble isoform SST2. Meanwhile, it has been investigated as a prognostic marker in various acute and chronic diseases with promising results. This single-center observational prospective study investigates the correlation of serum sST2 concentrations and six-month outcome after CPR.

Methods. This study included 106 consecutively admitted patients after cardiopulmonary resuscitation. Inclusion criteria were: (1) minimum age of 18 years, (2) minimum ICU length of stay of $24 \mathrm{~h}$, and (3) exclusion of traumatic cardiac arrest. Primary study endpoint was a composite endpoint of six-month mortality and bad neurological outcome (Cerebral Performance Category/CPC $>2$ ), secondary endpoint 30-day mortality. Serum sST2 levels were determined $24 \mathrm{~h}$ after ICU admission by enzyme-linked immunosorbent assay (ELISA). Patients were divided into two groups based on the cohorts median serum SST2 concentration. Univariable and multivariable logistic regression analyses were performed and adjusted for different, common confounders.

Results. The median sST2 level was $53.42 \mathrm{ng} / \mathrm{ml}$ (IQR 34.68-93.57 ng/ $\mathrm{ml})$, no statistically significant differences were shown for baseline characteristics except from history of coronary artery disease $(24.5 \%$ in the low-sST2 group vs. $9.4 \%$ in the high SST2 group, $p=0.038$ ). Regarding CPR-specific findings, significantly more patients in the low SST2 group received bystander CPR ( $49 \%$ vs. $28.3 \%, p=0.040)$. As for post resuscitation care, patients in the high SST2 group received invasive intensive care measures (mechanical ventilation: $100 \%$ vs. $88.7 \%$, $p=0.012$; targeted temperature management/TTM: $83 \%$ vs. $54.7 \%$, $\mathrm{p}=0.002$; continuous veno-venous hemodiafiltration/CVVHDF: $18.9 \%$ vs. $0 \%, p=0.001$; antibiotics: $100 \%$ vs. $84.9 \%, p=0.001$ ) more often. Furthermore, in the high SST2-group overall lower pH-values (7.151 vs. $7.257, p=0.003)$, as well as higher initial lactate values $(5.74 \mathrm{mmol} / \mathrm{l}$ vs. $3.58 \mathrm{mmol} / \mathrm{l}, \mathrm{p}=0.003)$ and leucocyte counts $(15.6 \mathrm{G} / \mathrm{l} \mathrm{vs} .12 .6 \mathrm{G} / \mathrm{l}$, $\mathrm{p}=0.002$ ) were observed. Elevated sST2-levels $24 \mathrm{~h}$ after ICU admission correlated positively with the combined endpoint of six month mortality and worse neurological outcome as a continuous (OR 1.011, $95 \% \mathrm{Cl} 1.004-1.019, \mathrm{p}=0.004)$, as well as a dichotomized variable $(21 \%$ vs. $49 \%$, OR $3.59,95 \% \mathrm{Cl} 1.53-8.45, \mathrm{p}=0.003$ ) and remained significant after adjustment for possible confounders.

Conclusion. Serum sST2 concentrations obtained $24 \mathrm{~h}$ after ICU admission correlated well with six-month and 30-day outcome after cardiopulmonary resuscitation in our single-center analysis. 


\section{7}

Neuron Specific Enolase (NSE) as a biomarker to predict neurological recovery after cardiac arrest

R. West'; R. Gray,

${ }^{1}$ Brighton and Sussex Medical School, Brighton, United Kingdom;

${ }^{2}$ Intensive care medicine, University Hospitals Sussex NHS Trust, Brighton, United Kingdom

Correspondence: R. West

Intensive Care Medicine Experimental 2020, 9(1): 001097

Introduction. Approximately two-thirds of patients admitted to the ICU after OOHCA die due to hypoxic-ischaemic brain injury (HIBI) [1]. For the majority of these patients, death occurs due to withdrawal of life sustaining treatment (WLST) [2]. Prognostication for these patients is challenging as no predictor is $100 \%$ specific [3]. Current European Resuscitation Council (ERC) and European Society of Intensive Care Medicine (ESICM) guidelines advocate a multimodal approach using neuron-specific enolase (NSE) as part of this process [4].

Objectives. This retrospective study examined adherence to the ERC-ESICM guidelines for neuroprognostication in a single centre and analysed the value of NSE within the multimodal approach.

Methods. All adult patients admitted to the Royal Sussex County Hospital ICU after OOHCA who remained comatose with a GCS Motor component of 1-2 $72 \mathrm{~h}$ post ROSC between November 2018 and January 2020 were included. Criteria audited were: clinical examination, neurophysiological studies, biochemical markers, neuroradiology, and patient outcomes (including whether death occurred due to WLST). Initial NSE analysis included all patients who remained on the ICU $\geq 72 \mathrm{~h}$ post ROSC irrespective of motor score, enabling comparison of a greater number of NSE values between survivor and non-survivors. Using this larger cohort, ROC analysis determined Yougen's statistics for 48 and 72 h NSE. These cohortspecific thresholds were then applied to those patients eligible for further neuroprognostication (GCS Motor score of 1-2 $72 \mathrm{~h}$ post $\mathrm{ROSC}$ ) to enable comparison of the relative sensitivity and specificity of 48 and $72 \mathrm{~h}$ NSE against other neuroprognostic tests used.

Results. Of 29 eligible patients, $28 \%(n=8)$ survived to hospital discharge. $81 \%(n=17)$ of non-survivors died due to WLST. Of these, $59 \%(n=10)$ fulfilled the criteria for likely poor prognosis $(\geq 1$ phase one/ $\geq 2$ phase two indicators) [4]. NSE concentrations were available for 49 patients. Differences observed between survivor and non-survivor NSE concentrations at 48 and $72 \mathrm{~h}$ were statistically significant $(p=0.002)$. ROC analysis demonstrated AUC of 0.825 and 0.872 at 48 and $72 \mathrm{~h}$ (Fig. 1). Yougen's statistic demonstrated that NSE thresholds consistent with $100 \%$ specificity for poor outcome were lower than the upper limit suggested by the UK Resuscitation Council $(37 \mu \mathrm{g} / \mathrm{L}$ vs $>60 \mu \mathrm{g} / \mathrm{L}$ and $36 \mu \mathrm{g} / \mathrm{L}$ vs $>60 \mu \mathrm{g} / \mathrm{L}$ at 48 and $72 \mathrm{~h}$ ) [5]. Application of this cohort-specific $72 \mathrm{~h} \mathrm{NSE}$ threshold was more sensitive than brain CT/MRI (70.5\% vs $75 \%)$. Both were $100 \%$ specific.

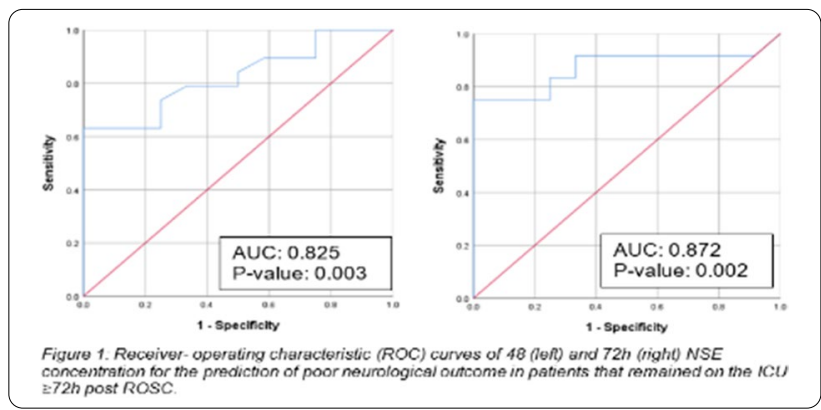

Conclusion. Adherence to the ERC-ESICM guidelines for neuroprognostication were broadly compliant. Cohort-specific NSE thresholds added value in the prediction of likely poor prognosis with $72 \mathrm{~h}$ NSE out-performing brain CT/MRI. Further prospective studies are needed to determine ideal NSE thresholds with maximum sensitivity whilst preserving specificity.

\section{Reference(s)}

1. [5] Nolan JP et al. Neuroprognostication of the comatose adult patient after resuscitation from cardiac arrest. Resucitation Council UK; 30, 2021.

2. [4] Nolan JP et al. European Resuscitation Council and European Society of Intensive Care Medicine Guidelines for Post-resuscitation Care. Resuscitation;95:202, 2015

3. [3] Sandroni $C$ et al. Prognostication after cardiac arrest. Crit Care 5;22(1):150, 2018.

4. [2] Sandroni $\mathrm{C}$ et al. Prediction of poor neurological outcome in comatose survivors of cardiac arrest: a systematic review. Intensive Care Med 46(10):1803-51, 2020

5. [1] Lemiale $V$ et al. Intensive care unit mortality after cardiac arrest: the relative contribution of shock and brain injury in a large cohort. Intensive Care Med 39(11):1972-80, 2013.

001152

Simplified Acute Physiology Score (SAPS) II vs. Sequential Organ Failure Assessment (SOFA) Score-Applicability as prognostic scores for cardiac arrest on intensive care unit

S. Entz ; FO. Holland, ; M. Krüger ${ }^{3}$; S. Lamprinaki, ; TW. May ${ }^{5}$; S. Rehberg ${ }^{6}$.

G. Jansen ${ }^{6}$

${ }^{1}$ Department of Internal Medicine and Gastroenterology, Protestant Hospital of Bethel Foundation, University Hospital of Bielefeld, Bielefeld, Germany; ${ }^{2}$ Clinic for internal medicine and nephrology, Protestant Hospital of the Bethel Foundation, Bielefeld, Germany; ${ }^{3}$ Clinic for internal medicine and gastroenterology, Prostestant Hosiptal of the Bethel Foundation, Bielefeld, Germany; ${ }^{4}$ Clinic for internal medicine and gastroenterology, The Protestant Hospital of the Bethel Foundation, Bielefeld, Germany; ${ }^{5}$ Coordination office for studies in biomedicine and preclinical and clinicalresearch, Protestant Hospital of Bethel Foundation, University Hospital of Bielefeld, Bielefeld, Germany; ${ }^{6}$ Anaesthesiology, Prostestant

Hosiptal of the Bethel Foundation, Bielefeld, Germany

Correspondence: S. Entz

Intensive Care Medicine Experimental 2020, 9(1): 001152

Introduction. Cardiac arrest on intensive care unit (ICU-CA) is a specific form of in-hospital cardiac arrest (CA) and associated with poor outcome. Although numerous scoring systems exist for prognostic assessment of critical care patients, such as Simplified Acute Physiology Score-II (SAPSII) or Sequential Organ Failure Assessment (SOFA) Score, there are no data regarding their applicability to estimate prognosis of ICU-CA.

Methods. In this retrospective study, the database summarizing ICUs (two medical, two surgical, one interdisciplinary) of a German University Hospital was screened for patients $\geq 18$ years, who suffered from ICU-CA. ICU-CA was defined according to Utstein-criteria as cessation of circulation with necessity of performing chest compression and/or defibrillation for the first time after admission on ICU. Patients were allocated to the following subgroups: death during resuscitation, return of spontaneous circulation (ROSC) but death during hospitalization, survivors being discharged and patients who were still alive one year after ICU-CA. SAPS-II and SOFA Scores before and after ICU-CA were assessed for each subgroup. Patients or family members, respectively, were interviewed by telephone and survival status one year after CA was queried.

Results. Overall, we observed 114 ICU-CA out of 14,264 ICU patients [incidence 79.9/10,000 ICU patients (Cl95 65,3-94,5)]. 29.8\% $(n=34)$ of patients died during resuscitation, $48.2 \%(n=55)$ showed ROSC, but died during hospitalization, so that a total of 89 patients died in hospital. 21.9\% ( $n=25)$ could be discharged from hospital and one year after discharge $9 \%$ of patients $(n=10)$ were still alive. Significant differences in respect to SOFA Scores were observed only for patients, post CA, who died during stay in hospital, in contrast to those who were discharged alive $(p<0.003)$. The SAPS-II-Score pre ICU-CA was significantly lower for patients, who could be discharged from hospital, than for those, who died during hospitalization (pre $(p<0.006)$ and post ICU-CA $(p<0.001))$. In addition, SAPS-II Scores post CA for patients still alive one year after CA were significantly lower (35.7) than for the non-survivors $(43.4)(p<0.025)$. SAPS-II value after ICU-CA of approximately 39.5 could 
differentiate between patients, who died in hospital after resuscitation $(\mathrm{SAPS} \geq 40$ ) and those who survived (SAPS $\leq 39)$. Table 1 shows the distribution of the SOFA and SAPS-II Scores for all subgroups pre and post CA. Table 1. SOFA and SAPS II Score Values for Subgroups of ICU-CA

\begin{tabular}{|c|c|c|c|c|}
\hline Subgroup & $\begin{array}{l}\text { no ROSC } \\
(n=34 / \\
29.8 \%)\end{array}$ & $\begin{array}{l}\text { All Patients } \\
\text { that died dur- } \\
\text { ing hospitaliza- } \\
\text { tion }(n=89 / \\
78.1 \%)\end{array}$ & $\begin{array}{l}\text { Discharged } \\
\text { survivors } \\
(n=25 / \\
21.9 \%)\end{array}$ & $\begin{array}{l}\text { Survivors one } \\
\text { year after } C A \\
(n=10 / 9 \%)\end{array}$ \\
\hline $\begin{array}{l}\text { SAPS-II pre CA } \\
\text { (Mean } \pm \text { SD) }\end{array}$ & $\begin{array}{c}43.5 \pm 14.3 \\
(n=17)\end{array}$ & $\begin{array}{c}45.3^{*} \pm 14.4 \\
(n=61)\end{array}$ & $\begin{array}{c}36 . \mathbf{9}^{*} \pm 14.8 \\
(\mathrm{n}=24)\end{array}$ & $\begin{array}{c}36.9 \pm 10.7 \\
(n=10)\end{array}$ \\
\hline $\begin{array}{l}\text { SAPS-II post } \\
\text { CA } \\
(\text { Mean } \pm \text { SD) }\end{array}$ & & $\begin{array}{l}\mathbf{4 6 . 5}+ \pm 11.8 \\
(\mathrm{n}=32)\end{array}$ & $\begin{array}{c}34.3+ \pm 9.2 \\
(n=24)\end{array}$ & $\begin{array}{c}35.7^{\prime \prime} \pm 7.7 \\
(n=10)\end{array}$ \\
\hline $\begin{array}{l}\text { SOFA pre CA } \\
(\text { Mean } \pm \text { SD) }\end{array}$ & $\begin{array}{l}8.4 \pm 5.5 \\
\quad(n=34)\end{array}$ & $8.1 \pm 4.9(n=89)$ & $\begin{array}{l}6.3 \pm 4.2 \\
\quad(n=25)\end{array}$ & $\begin{array}{l}6.4 \pm 5.0 \\
\quad(n=10)\end{array}$ \\
\hline $\begin{array}{l}\text { SOFA post CA } \\
(\text { Mean } \pm \text { SD) }\end{array}$ & & $\begin{array}{c}11.9 \# \pm 3.6 \\
(n=55)\end{array}$ & $\begin{array}{r}8.7 \# \pm 5.2 \\
(n=25)\end{array}$ & $\begin{array}{l}8.3 \pm 5.6 \\
\quad(n=10)\end{array}$ \\
\hline
\end{tabular}

Conclusion. SAPS-II Score appears to be more suitable for assessing prognosis after ICU-CA both before and after CA than SOFA Score. Higher SAPS-II Scores (pre and post CA) are associated with higher mortality with a cut of value that distinguishes better from worse outcome of SAPS-II $<39.5$

\section{6}

The effect of hyperoxia on mortality and neurological outcome in survivors after cardiac arrest: a systematic review and meta-analysis

B. Lanzafame' ${ }^{1}$ 'V. Dezio ${ }^{1}$; D. Busalacchi ${ }^{1}$; L. La Via'; M. Mascari ${ }^{1}$; F. Vasile ${ }^{2}$. C. Cassisi²; MT. Agnello'; F. Sanfilippo'; M. Astuto²

${ }^{1}$ School of anaesthesia and intensive care, University Hospital "G. Rodolico", Catania, Italy; ${ }^{2}$ Department of anesthesiology and intensive care, University Hospital "G. Rodolico", Catania, Italy

Correspondence: L. La Via

Intensive Care Medicine Experimental 2020, 9(1): 001176

Introduction. The association between hyperoxia and mortality or poor neurological outcome in survivors after cardiac arrest is under increasing scrutiny. We therefore conducted a systematic review and meta-analysis to explore the association between hyperoxia and mortality or poor neurological outcome in adult patients admitted in intensive care unit (ICU) after ROSC.

Methods. In this preliminary analysis we searched MEDLINE and EMBASE databases to identify both prospective and retrospective studies up to May 2021. We calculated Odds Ratio (OR) for survival and good neurological outcome (intended as cerebral performance category [CPC] $1-2)$, with $95 \%$ confidence interval $(\mathrm{Cl})$ using a random-effect model.

Results. We included 8 studies with a total of 24,374 adult patients. Of the 6 studies reporting data on survival, hyperoxia did not show a significantly lower mortality rate compared to normoxia (OR, 1.17; $95 \%$ $\mathrm{Cl}, 0.85-1.62$, Fig. 1). On the other hand, in the 4 studies exploring the neurological outcome of survivors after cardiac arrest, hyperoxic patients showed significative worse CPC scores compared to the normoxic ones (OR, 0.82; $95 \% \mathrm{Cl}, 0.68-0.98)$.

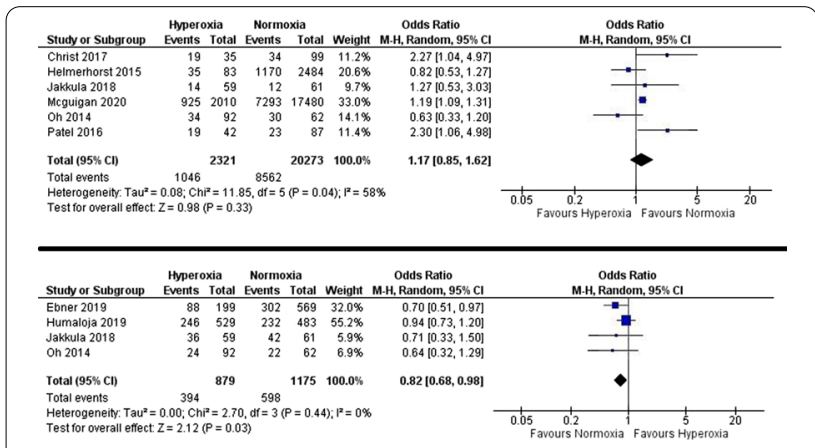

Fig. 1. Forest plot analysis on mortality and neurological outcome in hyperoxic patients who survived after CA compared to normoxic ones. Analysis performed with random effect model and Mantel-Haenszel (M-H)

Conclusion. In adult survivors after cardiac arrest, hyperoxia is associated with a worse neurological outcome. However, more studies are needed to evaluate the effect of hyperoxia on mortality in this patient population.

\section{7}

\section{A systematic review and meta-analysis of target temperature} management (TTM) in survivors after cardiac arrest (CA)

D. Busalacchi ${ }^{1}$; B. Lanzafame ${ }^{1}$; V. Dezio ${ }^{1}$; L. La Via ${ }^{1}$; C. Cutulii' ${ }^{2}$ M. Mascari'; C. Cassisi'; MT. Agnello²; F. Sanfilippo²; M. Astuto ${ }^{2}$

${ }^{1}$ School of anaesthesia and intensive care, University Hospital "G. Rodolico", Catania, Italy; ${ }^{2}$ Department of anesthesiology and intensive care, University Hospital "G. Rodolico", Catania, Italy

Correspondence: L. La Via

Intensive Care Medicine Experimental 2020, 9(1): 001,187.

Introduction. After publication of the TTM trial in 2013, several studies have shown the effect of TTM on mortality and neurological outcome in unconscious survivors after both in and out-of-hospital CA. We conducted a systematic review and meta-analysis to compare a targeted temperature of $32-34{ }^{\circ} \mathrm{C}$ versus $36-37{ }^{\circ} \mathrm{C}$ or standard of care on mortality or poor neurological outcome.

Methods. In this preliminary analysis we screened MEDLINE and EMBASE for Randomized Controlled Trials (RCTs) up to May 2021. We calculated Risk Ratio (RR) for survival and good neurological outcome (defined as cerebral performance category [CPC] 1-2), with $95 \%$ confidence interval $(\mathrm{Cl})$ using a random-effect model and Mantel-Haenszel. Analyses were performed with separation in subgroups according to the targeted temperature in the control group. Results. 5 studies were included in our analysis, with a total of 1902 adult patients. TTM at $32-34{ }^{\circ} \mathrm{C}$ did not show a significantly higher survival rate compared to the control group (RR, 1.07; 95\% $\mathrm{Cl}, 0.96-1.19$, Fig. 1). Also, neurological outcome was not statistically different between groups (RR, 1.35; $95 \% \mathrm{Cl}, 0.98-1.87)$. However, the subgroup analysis showed a significantly lower mortality when TTM at $32-34{ }^{\circ} \mathrm{C}$ was compared to standard of care (RR, $1.37 ; 95 \% \mathrm{Cl}$ $1.10-1.69)$, as well as a better neurological outcome (RR, 1.46; $95 \%$ $\mathrm{Cl}, 1.15-1.86)$. 


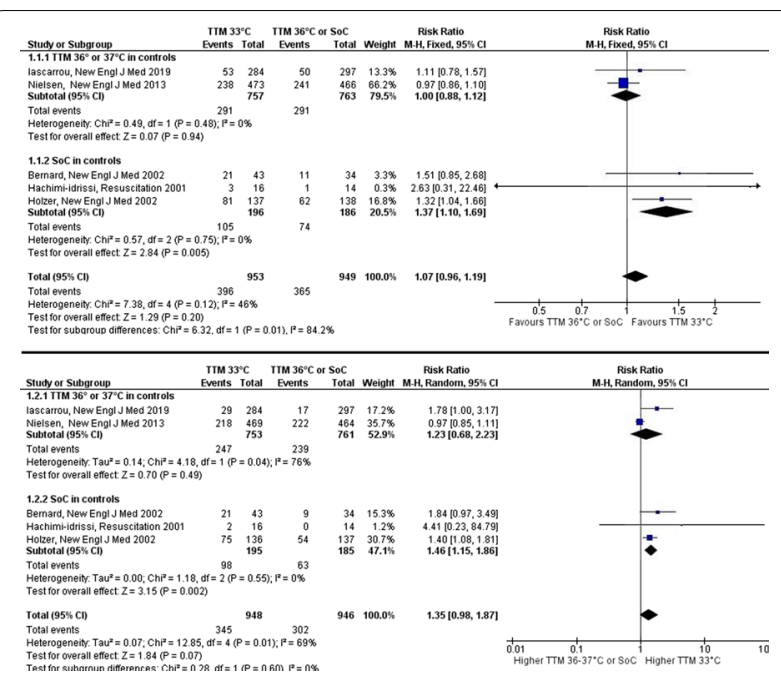

Fig. 1. Forest plot analysis on mortality and neurological outcome in survivors after $C A$ treated with a TTM at $32-34{ }^{\circ} \mathrm{C}$, compared to TTM $36-37^{\circ} \mathrm{C}$ or standard of care. Analysis performed with random effect model and Mantel-Haenszel (M-H)

Conclusion. Our meta-analysis showed that in adult survivors after CA, TTM at $32-34^{\circ} \mathrm{C}$ does not improve survival or neurological outcome compared to control group. However, when looking for subgroups, TTM at $32-34{ }^{\circ} \mathrm{C}$ is associated to a lower mortality and a better neurological outcome compared to standard of care. More studies are needed to evaluate the effect of TTM at $32-34{ }^{\circ} \mathrm{C}$ on survival and neurological outcome compared to a higher target temperature.

\section{Reference(s)}

1. Nielsen N, Wetterslev J, Cronberg T, Erlinge D, Gasche Y, Hassager C, et al.; the TTM Trial Investigators Targeted Temperature Management at $33^{\circ} \mathrm{C}$ versus $36^{\circ} \mathrm{C}$ after Cardiac Arrest. N Engl J Med 2013; 369:2197-2206

\section{4}

\section{Computational Prediction of Targeted Temperature Management} Outcomes for Personalized Cardiac Arrest Treatment

J. Guo, ${ }^{1} ;$ H. Kim² ${ }^{2}$ R. Stevens ${ }^{2}$

${ }^{1}$ Johns hopkins university, Department of Biomedical Engineering, Baltimore, United States of America; ${ }^{2}$ Anesthesiology and critical care medicine, Johns Hopkins University, Baltimore, United States of America Correspondence: R. Stevens

Intensive Care Medicine Experimental 2020, 9(1): 001194

Introduction. Targeted temperature management (TTM) has been associated with improved neurological function in comatose survivors of cardiac arrest. However, the benefits of TTM are not consistently observed and the intervention is resource intensive and may lead to adverse effects. There is consequently an unmet need to identify patients who are likely to respond to this intervention.

Objectives. The aim of this study is to predict the discharge outcome of postcardiac arrest patients receiving TTM by training a machine leaning (ML) model with physiological data and TTM treatment data. Furthermore, highly ranked features that correlate with patient outcomes will be identified. It is hypothesized that postcardiac arrest patients most likely to benefit from TTM have a specific data signature. Methods. Out of 200,859 patients in the Philips elCU database, 31,971 patients were identified as cardiac arrest patients, and 971 received TTM treatment. To identify relationships between features and clinical outcomes, three machine learning algorithms were used: GLM, random forest (RF), and gradient boosting (XGboost).
Only data from the first six hours after ICU admission were analyzed. Models were tenfold cross-validated and resampled 10 times. Models were trained to predict the probability of survival and favorable neurological outcome, measured as motor Glasgow Coma Scale (mGCS) of 6 upon discharge.

Results. For the prediction of survival, mean (SD) AUROC of the three models was $0.687(0.042)$, sensitivity $0.681(0.105)$, and specificity $0.583(0.115)$. For the prediction of favorable neurological oucome, mean (SD) AUROC was 0.654 (0.047), sensitivity 0.641 $(0.129)$ and specificity $0.569(0.109)$. The highest ranked features that predicted survival or favorable neurological outcome included male gender, ethnicity, higher mean platelet volume, and higher potassium levels.

Conclusion. ML algorithms trained with data available in the first $6 \mathrm{~h}$ after ICU admission hold the potential to predict outcomes in patients receiving TTM after cardiac arrest. Future modeling iterations will incorporate additional data modalities and external validation, enabling clinicians to optimize selection of patients for this intervention.

\section{Reference(s)}

1. Johns Hopkins University Discovery Award

\section{1}

The Effect of Pulmonary Artery Catheterisation on Physiological Parameters and Level of Haemodynamic Support in Post-Cardiac Arrest Patients

A. Ali'; M. Shaw ${ }^{2}$; P. McCall ${ }^{3}$

${ }^{1}$ School of medicine, University of Glasgow, Glasgow, United Kingdom; Intensive care unit, Glasgow Royal Infirmary, Glasgow, United Kingdom; ${ }^{3}$ Anaesthesia, critical care and perioperative medicine, University of Glasgow, Glasgow, United Kingdom

Correspondence: A. Ali

Intensive Care Medicine Experimental 2020, 9(1): 001361

Introduction. Outcome following out-of-hospital cardiac arrest (OHCA) is poor, with only $10 \%$ of patients surviving to hospital discharge in Scotland [1]. Haemodynamic instability is common, and management can be challenging. Some centres use the pulmonary artery catheter (PAC) to guide haemodynamic support, however no studies have explored the utility of the PAC in this population.

Objectives. This retrospective cohort study aimed to determine the effect of PAC insertion on level of haemodynamic support and physiological parameters following OHCA.

Methods. Retrospective review of prospectively collected, highfidelity, minute-by-minute ICU data from patients admitted to the cardiac intensive care unit (CICU) at the Golden Jubilee National Hospital following OHCA. Primary outcome was the difference in a vasoactive inotropic score (VIS) and mean arterial pressure (MAP) at two hours following PAC insertion. Secondary outcomes used generalised linear mixed effects interaction modelling to explore change in trends of vasoactive drug infusion rates, VIS and MAP following PAC insertion.Results. Between August 2015 and August 2020, 254 patients were admitted to CICU following OHCA and 32 (13\%) had a PAC inserted during admission. There was no difference in MAP at two hours following PAC insertion ( $p=0.851)$. There was a difference in VIS at this time, median $(95 \% \mathrm{Cl})$ difference $5.0(0.1,5.06[p=0.004])$. Fourteen $(44 \%)$ patients experienced a change in dose $(\geq 25 \%)$ in at least one vasoactive drug within 2-hours of PAC insertion. Thirteen (41\%) patients had an addition of at least one new vasoactive drug within 2-hours of PAC insertion. PAC insertion interacted significantly with all variables and led to a significant change in trend. Following PAC insertion; VIS (Fig. 1) and all vasoactive drug infusions trended downward, except dobutamine, and MAP trended upward relative to baseline trend. 


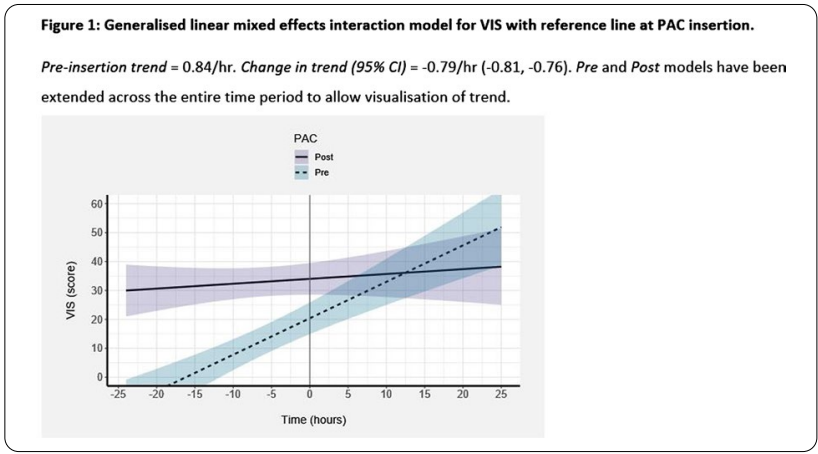

Conclusion. This is the first study to explore the impact of PAC insertion following OHCA and demonstrates it is associated with a relative decrease in trend of level of haemodynamic support and increase in trend of MAP, although the degree of change is difficult to estimate and may be clinically insignificant. Two hours is likely insufficient time to determine the true change in parameters associated with PAC insertion, and the time delay between PAC insertion and significant change in trend varies between both patients and the variable of interest Determining optimal physiological targets in post-cardiac arrest patients is a current research focus [1,2] and PAC use may allow personalised management of haemodynamic and physiological targets, which may improve outcomes for these patients.

\section{Reference(s)}

1. Social Care. Out of hospital cardiac arrest: strategy 2021 to $2026 \mathrm{gov}$. scot2021 [updated 24/03/2021. 2:[Available from: https://www.gov.scot/ publications/scotlands-out-hospital-cardiac-arrest-strategy-2021-2026/.

2. Kleinman ME, Perkins GD, Bhanji F, Billi JE, Bray JE, Callaway CW, et al. ILCOR Scientific Knowledge Gaps and Clinical Research Priorities for Cardiopulmonary Resuscitation and Emergency Cardiovascular Care: A Consensus Statement. Circulation. 2018;137(22):e802-e19.

\section{4}

Improvement of healthcare quality in tertiary hospital after implementation of a Rapid Response Team

UA. flato ${ }^{1}$; M. Vilela, ; F. Costa, ; I. Rocco ${ }^{1}$; L. Almeida'; B. Abreu'

I. Mazucatto ${ }^{1}$; A. Wirgues Sousa'; R. Ralio, ; L. Bolonha' ${ }^{1}$;. Uras ${ }^{1}$;

S. Almeida ${ }^{1}$

${ }^{1}$ Icu, Hospital Samaritano Higienópolis, São Paulo, Brazil

Correspondence: $\cup$ A flato

Intensive Care Medicine Experimental 2020, 9(1): 001464

Introduction. The success of hospitalized patient care depends on early diagnosis and adequate treatment. Improvement in healthcare is needed to reduce unfavourable outcomes and hospital costs. For this reason, health institutions must improve the ability to identify patients with clinical deterioration earlier. One of the strategies suggested in the projects to improve the quality of care, prevent cardiac arrest (CA) and reduce hospital mortality is implementing the Rapid Response Team (RRT). RRTs are composed of health professionals trained to provide care to high-risk hospitalized patients. There are substantial differences in the organizational structure and function of the RRTs between hospitals, and those that perform better usually have a dedicated team, without competing clinical responsibilities, which serves as a resource for nurses, collaborating during and after activation of the RRT and a team member can trigger that without fear of reprisals. Other methodologies collaborate in the continuous improvement of health institutions and must be implemented jointly to reduce morbidity and mortality. The "NEWS" protocol (National Early Warning Score) uses physiological parameters that signal the severity of the patient and the need for intervention in such a way as to help the early recognition of patients' clinical deterioration. The communication tool SBAR (Situation, Background, Assessment and Recommendation) recommends the systematization of information about the patient's status in real-time, facilitating interprofessional communication in a structured, transparent and standardized way.

Objectives. To evaluate the result of the implementation of the RRT in reducing the outcomes of in-hospital morbidity and mortality.

Methods. Material and Methods: Prospective study before and after conducted in a tertiary hospital, from January 2018 to January 2019, before the educational intervention and follow-up for 12 months. A verticalized RRT assistance protocol was used; that is, a team of intensivists was activated according to the yellow and blue code using the SBAR communication tool and NEWS tool for the early recognition of the patient's deterioration.

Results. There was 5 PCR / month in 24 months before implementing RRT and 1 PCR / month after implementation (RRR $80 \% ; p<0.001$ ). An increase in the attendance of yellow codes was observed, with an average of grade 9 NEWS and an average service time of the team of $8 \mathrm{~min}$ Conclusion. There was a reduction in CA incidence and in-hospital mortality after implementing the TRR protocol.

\section{Reference(s)}

1. Jones, DA; DeVita, MA; Bellomo, R (Jul 14, 2011). "Rapid-response teams" (PDF). The New England Journal of Medicine. 365 (2): 139-46. 10.1056/ NEJMra091092. PMID 21,751,906. Devita, MA; Bellomo, R; Hillman, K; Kellum, J; Rotondi, A; Teres, D; Auerbach, A; Chen, WJ; Duncan, K; Kenward, G; Bell, M; Buist, M; Chen, J; Bion, J; Kirby, A; Lighthall, G; Ovreveit, J; Braithwaite, RS; Gosbee, J; Milbrandt, E; Peberdy, M; Savitz, L; Young, L; Harvey, M; Galhotra, S (Sep 2006). "Findings of the first consensus conference on medical emergency teams". Critical Care Medicine. 34 (9): 2463-78. 10. 1097/01.CCM.0000235743.38172.6E. PMID 16,878,033. National Institute for Health and Clinical Excellence. Clinical guideline 50: "High Acuity. Response Team (HART) | Accreditation Canada". www.accreditation.ca. Retrieved 10 may 2021. Chan, PS; Jain, R; Nallmothu, BK; Berg, RA; Sasson, C (2010-01-11). "Rapid Response Teams: A Systematic Review and Metaanalysis". Archives of Internal Medicine. 170 (1): 18-26.

e-Posters: Cardiovascular issues in ICU

\section{2}

Non-invasive capnodynamic mixed venous oxygen saturation during major changes in oxygen delivery

A. Svedmyr '; M. Konrad, ; M. Wallin, ; M. Hallbäck²; PA. Lönnqvist, ${ }^{1}$; J. Karlsson,

'Physiology and Pharmacology, Karolinska Institute, Stockholm, Sweden; ${ }^{2}$ Maquet critical care, MAQUET Critical Care, Solna, Sweden

Correspondence: A. Svedmyr

Intensive Care Medicine Experimental 2020, 9(1): 000132

Introduction. Mixed venous oxygen saturation ( $\mathrm{SvO} 2)$ is an important parameter in anesthesia and intensive care but currently requires invasive pulmonary artery catheterization. Recently, however, non-invasive determination of $\mathrm{SvO} 2$ (Capno-SvO2) using capnodynamics has displayed good agreement against CO-oximetry in an animal model.

Objectives. The objective of the study was to validate CapnoSvO2 against CO-oximetry during major hemodynamic challenges and, furthermore, evaluating fiberoptic $\mathrm{SvO} 2$ for its response to the same challenges.

Methods. Eleven anesthetized mechanically ventilated pigs (median weight 29,6 kg) were exposed to hemodynamic challenges: gradually increased inhaled oxygen concentration, hemorrhage, crystalloid and blood transfusion, preload reduction and dobutamine infusion. Capno-SvO2 and fiberoptic $\mathrm{SvO} 2$ recordings were made in parallel with CO-oximetry and analyzed for agreement of absolute values and ability to detect change using Bland-Altman plots and concordance analysis. Respiratory quotient, needed for capnodynamic SvO2 determination, was measured by analysis of mixed expired gases.

Results. The interventions caused significant hemodynamic variations. Bias between Capno-SvO2 and $\mathrm{CO}$-oximetry was +3 
percentage points, $95 \%$ limits of agreements- 7 to +13 percentage points. Bias between fiberoptic $\mathrm{SvO} 2$ and $\mathrm{CO}$-oximetry was +1 percentage point, $95 \%$ limits of agreements -7 to +9 percentage points. Concordance rate for Capno-SvO2 and fiberoptic SvO2 vs CO-oximetry was $98 \%$ and $93 \%$, respectively.

Conclusion. Capno-SvO2 generates absolute values close to $\mathrm{CO}$ oximetry and shows promising trending ability during profound hemodynamic challenges. The performance of Capno-SvO2 vs COoximetry was on par with the performance of fiberoptic SvO2 vs CO-oximetry. Capno-SvO2 appears to be a promising tool for noninvasive $\mathrm{SvO} 2$ monitoring.

\section{Reference(s)}

2. 17. Critchley LA, Critchley JA: A meta-analysis of studies using bias and precision statistics to compare cardiac output measurement techniques. J Clin Monit Comput 1999; 15: 85-91

3. Bland JM, Altman DG: Statistical methods for assessing agreement between two methods of clinical measurement. Lancet 1986; 1: 307-10

4. Cecconi M, Rhodes A, Poloniecki J, Della Rocca G, Grounds RM: Benchto-bedside review: the importance of the precision of the Reference technique in method comparison studies-with specific Reference to the measurement of cardiac output. Crit Care 2009; 13: 201

5. Ferrando C, Romero C, Tusman G, Suarez-Sipmann F, Canet J, Dosdá R, Valls P, Villena A, Serralta F, Jurado A, Carrizo J, Navarro J, Parrilla C, Romero JE, Pozo N, Soro M, Villar J, Belda FJ: The accuracy of postoperative, noninvasive Air-Test to diagnose atelectasis in healthy patients after surgery: a prospective, diagnostic pilot study. BMJ Open 2017; 7: e015560

6. Karlsson J, Winberg P, Scarr B, Lönnqvist PA, Neovius E, Wallin M, Hallbäck $M$ : Validation of capnodynamic determination of cardiac output by measuring effective pulmonary blood flow: a study in anaesthetised children and piglets. Br J Anaesth 2018; 121:550-558

7. Karlsson J, Lönnqvist PA, Wallin M, Hallbäck M: A Continuous Noninvasive Method to Assess Mixed Venous Oxygen Saturation: A Proof-of-Concept Study in Pigs. Anesth Analg 2020

8. Peyton PJ, Wallin M, Hallbäck M: New generation continuous cardiac output monitoring from carbon dioxide elimination. BMC Anesthesiol 2019; 19: 28

9. Sigmundsson TS, Öhman T, Hallbäck M, Redondo E, Sipmann FS, Wallin M, Oldner A, Hällsjö Sander C, Björne H: Performance of a capnodynamic method estimating effective pulmonary blood flow during transient and sustained hypercapnia. J Clin Monit Comput 2018; 32: 311-319

10. Hällsjö Sander C, Hallbäck M, Wallin M, Emtell P, Oldner A, Björne H: Novel continuous capnodynamic method for cardiac output assessment during mechanical ventilation. Br J Anaesth 2014; 112: 824-31

11. Karlsson J, Wallin M, Hallbäck M, Lönnqvist PA: Capnodynamic determination of cardiac output in hypoxia-induced pulmonary hypertension in pigs. Br J Anaesth 2019; 122: 335-341

12. PA Lönnqvist receives an unrestricted research grant from Maquet Critical Care AB

\section{3}

Comparison Between Standard Versus High Dosing Venous Thromboembolism (VTE) Prophylaxis in COVID19 Critically III Patients: A Multicenter, Retrospective Cohort Study

O. Aljuhani ${ }^{1}$; K. Al Sulaiman ${ }^{2}$; A. Hafidh ${ }^{1}$; K. Eljaaly ${ }^{1}$; A. Alharbi ${ }^{3}$; R. Algarni ${ }^{3}$ 'Pharmacy practice department, faculty of pharmacy, King Abdulaziz University, Jeddah, Saudi Arabia; ${ }^{2}$ Pharmaceutical care department, King Abdulaziz Medical City, Riyadh, Saudi Arabia; ${ }^{3}$ Pharmaceutical care department, King Abdulaziz University Hospital, Jeddah, Saudi Arabia Correspondence: O. Aljuhani

Intensive Care Medicine Experimental 2020, 9(1): 000143

Introduction. There is uncertainty about Venous Thromboembolism (VTE) Prophylaxis appropriated dosing regimen, safety and bleeding risk as well as the predisposing factors for thrombosis/bleeding risk in critically ill COVID 19patients. The available evidence is limited and depend either on mainly observational studies or randomized trials with small sample size.
Objectives. To determine the optimal dosing of pharmacological VTE prophylaxis in critically ill patients with COVID19.

Methods. A multicenter, retrospective, cohort study for COVID19 critically ill patients who received pharmacological VTE prophylaxis during ICU stay. Conducted at two governmental tertiary hospitals in Saudi Arabi. Critically ill patients with COVID19 who received pharmacological VTE prophylaxis and were admitted to Intensive Care Units (ICUs) between March 1st to January 31st, 2021 were included.

Results. A total of 758 patients were screened; 565 patients were included in the study. We matched 352 patients using propensity score matching (1:1) according to the baseline severity scores (i.e., APACHE II score, SOFA score), history of CKD, and AKI within 24 hours of ICU admission. The differences in venous thromboembolism (OR $(95 \% \mathrm{Cl}): 0.75(0.16-3.38), \mathrm{p}$-value $=0.70)$ and any case of thrombosis $(\mathrm{OR}(95 \% \mathrm{Cl}): 1.22(0.52-2.86)$, $\mathrm{p}$-value $=0.64)$ were not statistically significant between the two groups. In contrast, minor bleeding was associated with the use of high dosing regimen of VTE prophylaxis (OR $(95 \% \mathrm{Cl})$ : $3.39(1.08-10.61), \mathrm{p}$-value $=0.04)$. There was no difference in the 30-day ICU mortality nor in-hospital mortality between the two groups $(\mathrm{OR}(95 \% \mathrm{Cl}): 1.26(0.77-2.05)$, $\mathrm{p}$-value $=0.36$ and $\mathrm{OR}(95 \% \mathrm{Cl})$ : $1.05(0.66-1.68), p$-value $=0.83$ respectively $)$.

Conclusion. Our multi-center, retrospective cohort study showed that high dosing pharmacological VTE prophylaxis in critically ill COVID-19 patients was not associated with VTE, thrombosis, or mortality benefits but led to an increased risk of minor bleeding.

\section{Reference(s)}

1. Guan W, Ni Z, Hu Y, Liang W, Ou C, He J, et al. Clinical Characteristics of Coronavirus Disease 2019 in China. N Engl J Med. 2020;382(APRIL):170820. 2. Zhou F, Yu T, Du R, Fan G, Liu Y, Liu Z, et al. Clinical course and risk factors for mortality of adult inpatients with COVID-19 in Wuhan, China: a retrospective cohort study. Lancet. 2020;395(March):1054-62. 3. Cevik M, Kuppalli K, Kindrachuk J, Peiris M. Virology, transmission, and pathogenesis of SARS-CoV-2. BMJ. 2020;371(October):1-6. 4. Malas MB, Naazie IN, Elsayed N, Mathlouthi A, Marmor R, Clary B. Thromboembolism risk of COVID-19 is high and associated with a higher risk of mortality: A systematic review and meta-analysis. EClinicalMedicine. 2020;100,639:29-30. 5. Al Sulaiman KA, Al Juhani OA, Eljaaly K, Alharbi AA, Al Shabasy AM, Alsaeedi AS, et al. Clinical Features and Outcomes of Critically III Patients with Coronavirus Disease 2019 (COVID-19): A Multicenter Cohort Study. Int J Infect Dis [Internet]. 2021;105:180-7. Available from: https://doi.org/ 10.1016/j.ijid.2021.02.037 6. Paranjpe I, Fuster V, Lala A, Russak AJ, Glicksberg BS, Levin MA, et al. Association of Treatment Dose Anticoagulation With In-Hospital Survival Among Hospitalized Patients With COVID-19. J Am Coll Cardiol [Internet]. 2020;76(1):122-4. Available from: https://doi. org/10.1016/j.jacc.2020.05.001 7. Antithrombotic Therapy to Ameliorate Complications of COVID-19 (ATTACC) [Internet]. 2021. Available from: https://clinicaltrials.gov/ct2/show/NCT04372589 8. Dosing of Thromboprophylaxis and Mortality in Critically III COVID-19 Patients [Internet]. 2020. Available from: https://clinicaltrials.gov/ct2/show/NCT04593654? term =anticoagulant\&cond $=$ Covid19+critically+ill\&draw $=2 \&$ rank $=6$ 9. Anti-thrombotics for Adults Hospitalized With COVID-19 (ACTIV-4) [Internet]. 2021. Available from: https://clinicaltrials.gov/ct2/show/NCT04 505774 10. Helms J, Tacquard C, Severac F, Leonard-Lorant I, Ohana M, Delabranche $X$, et al. High risk of thrombosis in patients with severe SARS-CoV-2 infection: a multicenter prospective cohort study. Intensive Care Med. 2020:46(6):1089-98. 11. F.A. Kloka, M.J.H.A. Kruipb, N.J.M. van der Meerc, M.S. Arbousd, D.A.M.P.J. Gommerse, K.M. Kantf, F.H.J. Kapteina, J. van Paassend, M.A.M. Stalsa, M.V. Huismana HE. Incidence of thrombotic complications in critically ill ICU patients with COVID-19. Thromb Res. 2020;191(January):145-7. 12. Ishan Paranjpe ", Valentin Fuster, Anuradha Lala, Adam J. Russak, Benjamin S. Glicksberg, Matthew A. Levin, Alexander W. Charney, Jagat Narula, Zahi A. Fayad "Emilia Bagiella, Shan Zhao GNN. Association of Treatment Dose Anticoagulation With In-Hospital Survival Among Hospitalized Patients With COVID-19. J Am Coll Cardiol. 2020;76(January):122-4. 13. Investigators I. Effect of Intermediate-Dose vs Standard-Dose Prophylactic Anticoagulation on Thrombotic Events, Extracorporeal Membrane Oxygenation Treatment, or Mortality Among Patients With COVID-19 Admitted to the Intensive Care Unit: The INSPIRATION Randomized Clinical Trial. JAMA [Internet]. 2021 Mar 18; Available 
from: https://doi.org/10.1001/jama.2021.4152 14. Barnes GD, Burnett A, Allen A, Blumenstein M, Clark NP, Cuker A, et al. Thromboembolism and anticoagulant therapy during the COVID-19 pandemic: interim clinical guidance from the anticoagulation forum. J Thromb Thrombolysis [Internet]. 2020;50(1):72-81. Available from: https://doi.org/10.1007/ s11239-020-02138-z 15. Full-dose blood thinners decreased need for life support and improved outcome in hospitalized COVID-19 patients [Internet]. National Heart, Lung, and Blood Institute (NHLBI). Available from: https://www.nih.gov/news-events/news-releases/full-dose-bloodthinners-decreased-need-life-support-improved-outcome-hospitalizedcovid-19-patients 16. Hematology AS of. ASH Releases Clinical Practice Guidelines on the Use of Preventive Anticoagulation in Patients with COVID-19. 2020. 17. Al-Samkari H, Gupta S, Leaf RK, Wang W, Rosovsky RP, Brenner SK, et al. Thrombosis, Bleeding, and the Observational Effect of Early Therapeutic Anticoagulation on Survival in Critically III Patients With COVID-19. Ann Intern Med [Internet]. 2021 Jan; Available from: https:// europepmc.org/articles/PMC7863679 18. Roomi SS, Saddique M, Ullah W, Haq S, Ashfaq A, Madara J, et al. Anticoagulation in COVID-19: a singlecenter retrospective study. J community Hosp Intern Med Perspect [Internet]. 2021 Jan 26;11(1):17-22. Available from: https://pubmed.ncbi. nlm.nih.gov/33552407

\section{5}

Outcomes of percutaneous VA-ECMO decannulation using ProGlide and Manta devices in a tertiary ECMO center in Hong Kong

SY. $\mathrm{Au}^{1}$; K. Fong ${ }_{1}^{1}$; W. $\mathrm{Ng}^{1}$; K. Leung ${ }^{1}$; SO. So

'Intensive care unit, Queen Elizabeth Ho spital, Hong Kong, Hong Kong

Correspondence: S.Y. Au

Intensive Care Medicine Experimental 2020, 9(1): 000225

Introduction. When veno-arterial extracorporeal membrane oxygenation (VA-ECMO) support can be terminated, arteriotomy wounds closure by percutaneous devices was shown to have comparable outcomes to traditional surgical open repair. Moreover, transport of the critically-ill, man-power and timeslots of operating theaters could be saved if percutaneous decannulation is performed at bedside.

Objectives. To study the outcomes and complications of bedside percutaneous VA-ECMO decannulation during 2 different closure devices, namely ProGlide and Manta.

Methods. In the Intensive Care Unit of Queen Elizabeth Hospital, bedside decannulation was the default VA-ECMO decannulation method since November 2018. Ultrasound guided ProGlide post-close technique was first employed with the ECMO arterial wound closed by 2 to 3 ProGlide stitches applied at different angles. Since March 2010, Manta, a collagen-based closure device was also employed. The success rate, procedural details and the immediate complications were compared between the 2 techniques.

Results. Between November 2018 and March 2021, Eighty-two patients received VA-ECMO and 48 survived to decannulation. All were decannulated percutaneously by bedside. Forty were decannulated using ProGlide and 8 were by Manta. Overall, forty-three $(89.6 \% \%)$ had successful bedside decannulation, with success rate of $87.5 \%$ with ProGlide and $100 \%$ for Manta respectively. For the ProGlide group, the arteriotomy wounds were of $19 \mathrm{Fr}$ for $1 ; 17 \mathrm{Fr}$ for 21 and $15 \mathrm{Fr}$ for 18 patients. For the Manta group, one patient had $17 \mathrm{Fr}$ catheter and the rest were of $15 \mathrm{Fr}$. In the ProGlide group, two ProGlide devices were used in $22(55 \%)$ patients, three were used in 17 (42.5\%) patients and 4 were used in one patient, while one Manta was used in each patient in the Manta group. There was no statistically significant difference in clinical outcomes including mortality, ventilator days and length of stay. The procedure time (49 (15-67) $\mathrm{min}$ vs $40(26-52) \mathrm{min}$ ) and the blood loss (150 (122-320) mL vs $40(20-200) \mathrm{mL}$ ) were similar between the ProGlide group and the Manta group. Minor complications were found in 4 (10\%) patients in the ProGlide group, including two arterial clot formation, one pseudoaneurysm and one wound infection. There were no other major complications.

Conclusion. The 2 bedside percutaneous techniques resulted in similar clinical outcomes and complications rate. Larger studies can further investigate the difference between the 2 techniques. Other factors which could affect the choice of devices include cost, learning curve, case load of the center, the vascular conditions, presence of septicemia, ultrasound image quality and possibility of rewiring in case of failed hemostasis. Plan B and standby support by vascular surgeon were paramount.

\section{Reference(s)}

1. Hwang, JW, Yang, JH, Sung, K, et al. Percutaneous removal using Perclose ProGlide closure devices versus surgical removal for weaning after percutaneous cannulation for venoarterial extracorporeal membrane oxygenation. J Vasc Surg 2016; 63: 998-1003.e1001.

2. None

\section{5}

Active Fixation leads for temporary pacing in TAVI procedure

MD. Pola'; A. Castillo-Rivera'; MC. Molina-De-La-Torre';

R. Vela-Colmenero'; M. Martínez-Arcos'; M. Ruiz-Bailén, ${ }^{1}$

${ }^{1}$ Intensive Care Medicine, Servicio Andaluz de Salud, Jaén, Spain

Correspondence: M.D. Pola

Intensive Care Medicine Experimental 2020, 9(1): 000255

Introduction. Conventional temporary pacing leads are unstable, with frequent loss of capture or undersensing and need for lead repositioning, especially for prolonged pacing. Active fixation leads with a reusable external generator for temporal pacing can be a safe alternative in TAVI procedure awaiting permanent pacing decision.

Objectives. To assess safety and advantages of active fixation leads, usually used in permanent pacing, for provisional prophylactic pacing or bridge to definitive pacing in TAVI procedure.

Methods. Observational retrospective study that includes all consecutive patients who underwent TAVI procedure, since November 2019 to December 2020 in an ICU of a second level hospital. All were implanted with an active fixation lead (Medtronic $507258 \mathrm{~cm}$ ) for prophylactic provisional heart pacing. The lead was connected to an external generator (St Jude Medical Endurity Core PM 1152) also used in permanent pacing. The generator was the same for all patients and exclusively used for that purpose. Pacing mode VVIR.

Active fixation lead was inserted by right subclavian vein by Seldinger procedure to right ventricle apex endocardium, and externally fixed to skin of upper thorax. Generator is fixed externally to skin.

Results. We included 16 patients who underwent TAVI procedure and previously implanted with active fixation lead provisional pacing.

Mean age was $82,00 \pm 4,77$ years, 9 women and 7 men. All of them were discharged from ICU in $48 \mathrm{~h}$ with temporary pacing and early mobilization was made, even walking.

Variables were:

- Lead complications (lead dislocation with loss of capture and need of reubication, lead infection and cardiac perforation).

- Mortality for all causes.

- Days of temporary pacing.

- Need of permanent pacing.

There were no lead complications in any patient and all patients were discharged alive.

Median days of provisional pacing were $3,00 \pm 2,98$.

Only 5 patients required permanent pacing that was implanted by left subclavian vein and later explant of temporary lead.

\begin{tabular}{lllllll}
\hline $\mathbf{n}$ & Age sex Com- & $\begin{array}{l}\text { Exitus } \\
\text { plica- } \\
\text { tions }\end{array}$ & $\begin{array}{l}\text { Perma- } \\
\text { nent } \\
\text { pacing }\end{array}$ & $\begin{array}{l}\text { Days of } \\
\text { temporary } \\
\text { pacing }\end{array}$ \\
\hline 1 & 86 & W & N & No & NO & 3 \\
2 & 84 & W & N & No & NO & 3 \\
3 & 84 & M & N & No & NO & 2 \\
4 & 85 & W & N & No & NO & 2 \\
5 & 85 & W & N & No & YES & 2 \\
\hline
\end{tabular}




\begin{tabular}{lllllll}
\hline $\mathbf{n}$ & Age sex & $\begin{array}{c}\text { Com- } \\
\text { plica- } \\
\text { tions }\end{array}$ & Exitus & $\begin{array}{l}\text { Perma- } \\
\text { nent } \\
\text { pacing }\end{array}$ & $\begin{array}{l}\text { Days of } \\
\text { temporary } \\
\text { pacing }\end{array}$ \\
\hline 6 & 81 & W & N & No & NO & 4 \\
7 & 87 & W & N & No & YES & 3 \\
8 & 84 & W & N & No & YES & 13 \\
9 & 81 & M & N & No & NO & 5 \\
10 & 80 & M & N & No & NO & 6 \\
11 & 80 & M & N & No & YES & 4 \\
12 & 78 & M & N & No & NO & 3 \\
13 & 83 & W & N & No & NO & 5 \\
14 & 73 & M & N & No & NO & 3 \\
15 & 81 & W & N & No & NO & 3 \\
16 & 69 & M & N & No & YES & 7 \\
\hline
\end{tabular}

Conclusion. Active fixation leads for prophylactic temporary pacing or brigde to permanent pacing in TAVI procedure is safe and useful for early mobilization and ICU discharge of patients with provisional pacing awaiting the decision of permanent pacing.

\section{3}

Analysis of endotoxin activity of patients with extracorporeal membrane oxygenation life support system

YC. Yeh ${ }^{1}$; CH. Wang, ${ }_{1}^{2}$; CT. Lee, ${ }_{1}^{1}$; CH. Lai, ${ }^{2}$

'Department of anesthesiology, National Taiwan University Hospital, Taipei, Taiwan; ${ }^{2}$ Department of surgery, National Taiwan University Hospital, Taipei, Taiwan

Correspondence: Y.C. Yeh

Intensive Care Medicine Experimental 2020, 9(1): 000313.

Introduction. Extra-corporeal membrane oxygenation (ECMO) life support system can support the critically ill patients with heart failure, respiratory failure, or both (1). In clinical practice with patients with ECMO, we found that some patients with venovenous ECMO (VV-ECMO) may accompany with pneumonia and some patients with venoarterial ECMO (VA-ECMO) may have fever and sepsis after initial resuscitation. Moreover, shock-related hypoperfusion may disrupt the intestinal barrier and induce bacteria or endotoxin translocation. There was no information related to endotoxin levels in these patients.

Objectives. This study aimed to investigate the serum endotoxin activity in patients with VV-ECMO and VA-ECMO within the first $96 \mathrm{~h}$.

Methods. This prospective observational study was approved by the Research Ethics Committee of National Taiwan University Hospital (approval number: 201811061RINC) and registered on the ClinicalTrials.gov protocol registration system (ID: NCT03978728). This study was conducted at National Taiwan University Hospital between August 2019 and December 2020. Critically ill patients with VV-ECMO or VAECMO within 24 hours after ECMO placement were enrolled. The blood samples were obtained at the following time points: $T 1,<24 h$; $\mathrm{T} 2,25-48 \mathrm{~h} ; \mathrm{T} 3,49-72 \mathrm{~h}$; and T4, 73-96 $h$, and serum endotoxin activity were presented as endotoxin activity analysis (EAA) level and quantified directly within $3 \mathrm{~h}$ of blood collection using the EAA ${ }^{\mathrm{TM}}$ (Spectral Medical Inc, Toronto, Canada) (2). An EAA level $\geq 0.6$ were defined as clinically significant.

Results. A total of 55 patients with ECMO were enrolled (14, VV-ECMO; 41, VA-ECMO). Patients' characteristics and endotoxin activity analysis were presented in Table 1

\begin{tabular}{|c|c|c|}
\hline \multicolumn{3}{|c|}{ Patients' characteristics and endotoxin activity analysis levels } \\
\hline & $\begin{array}{l}\text { VV-ECMO } \\
(n=14)\end{array}$ & $\begin{array}{l}\text { VA-ECMO } \\
(\mathrm{n}=41)\end{array}$ \\
\hline Age (year) & $64(51-69)$ & $64(54-71)$ \\
\hline Height (cm) & $163(158-168)$ & $168(157-173$ \\
\hline Female/male & $6 / 8$ & $7 / 33$ \\
\hline $\mathrm{E}-\mathrm{CPR} \mathrm{n}(\%)$ & $1(7 \%)$ & $16(40 \%)$ \\
\hline EAA level > 0.6 at T1 & $42 \%$ & $12 \%$ \\
\hline EAA level $>0.6$ at $\mathrm{T} 2$ & $36 \%$ & $13 \%$ \\
\hline EAA level $>0.6$ at T3 & $39 \%$ & $30 \%$ \\
\hline EAA level $>0.6$ at $\mathrm{T} 4$ & $33 \%$ & $29 \%$ \\
\hline RESP score*/SAVE score & $0(-3 \text { to } 4)^{*}$ & $-8(-10$ to -2$)$ \\
\hline
\end{tabular}

At T1, EAA levels were $\geq 0.6$ in $42 \%$ of VV-ECMO patients and $12 \%$ of VA-ECMO patients. At T3, EAA level were $\geq 0.6$ in $39 \%$ of VV-ECMO patients and $30 \%$ in VA-ECMO patients. At T4, Lactate levels were higher in the VV-ECMO patients with $E A A \geq 0.6$ than in those with EAA $<0.6$ (2.5 [2-3.1] vs. 1.6 [1.2-2.3], $\mathrm{P}=0.028)$. The median elevation of EAA level from T1 to T3 was higher in 30-day non-survivors with VA-ECMO than in 30-day survivors with VA-ECMO $(0.12$ [0.02-0.29] vs. $-0.05[-0.19-0.16], P=0.035$ ).

Conclusion. This sutdy shows that $33-42 \%$ of VV-ECMO patients had an $\mathrm{EAA} \geq 0.6$ between $\mathrm{T} 1$ to $\mathrm{T} 4$ and $12-33 \%$ VA-EMCO patients had an $E A A>0.6$ between T1 to T4. Further studies were wrranted to investigate the effect of endotoxin removal on clinical outcomes in ECMO patients with high EAA levels.

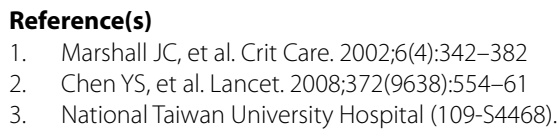

000321

Transcatheter aortic valve replacement (TAVR) in octogenarian patients

P. Padrón Espinosa'; P. Vega Ocaña ${ }^{1}$; J. Blanco López ${ }^{1}$; F. Jiménez Cabrera²; JC. Martin Gonzalez ${ }^{1}$

${ }^{1}$ Intensive care unit, Hospital Universitario Insular de Gran Canaria, Las Palmas de Gran Canaria, Spain; ${ }^{2}$ Cardiology, Hospital Universitario Insular de Gran Canaria, Las Palmas de Gran Canaria, Spain

Correspondence: P. Vega Ocaña

Intensive Care Medicine Experimental 2020, 9(1): 000321.

Introduction. Transcatheter aortic valve implantation (TAVI) has become the preferred alternative in old patients with severe aortic stenosis, inasmuch as surgical aortic valve replacement (SAVR) is associated with a high risk of morbidity and mortality.

Objectives. To assess transcatheter aortic valve replacement (TAVR) in octogenarian patients.

Methods. We carried out a prospective observational study from the beginning of the TAVR program in our hospital to the present time (2016 to 2020) in a third level Hospital with Hemodynamics and Intensive Care Unit. Two study groups were compared, patients undergoing transcatheter aortic valve implantation under 80 years-old (group 1) or aged 80 years-old and above (group 2). Data was collected prospectively from electronic hospital medical record (Drago AE and Critical Care Management) and arranged in a database designed for the purpose of this study. Epidemiological characteristics, risk factors, implantation procedure, complications and results were studied. Quantitative variables are described as mean $\pm S D$, while categorical variables are described numerically (\%). For data analysis, the Chi-square test and/or Student's T tests and the Mann-Whitney $U$ test were used. 
Results. 130 patients were included during the study period. Only one patient could not undergo TAVR. Risk factors, except for previous LVEF, did not differ between groups (Table 1). Patients $>80$ years old had a better LVEF compared to patients included in group $1(62.60 \pm 13.50$ vs. $55.07 \pm 16.18, p<0.06)$ and a lower EuroScore score $(4.29 \pm 2.73$ vs $5.38 \pm 3.77)$ (Table 2$)$. We found no differences between both groups regarding the procedure or occurrence of complications (Table 2 and 3). None of the patients had peri-procedural infarction and light bleeding was the most frequent vascular complication. Only one of the patients had a residual aortical gradient $>20 \mathrm{mmHg}$ after implantation (group 2). Hospital stay, home discharge and mortality did not show differences between the study groups (Table 4).

Conclusion. The results of the procedure and short-term survival of the TAVR in octogenarian patients in our hospital are similar to those of younger patients.

\begin{tabular}{|c|c|c|c|c|}
\hline \multicolumn{3}{|c|}{ Table 1} & $\begin{array}{c}<80 \text { years old } \\
N=50\end{array}$ & $\begin{array}{c}\geq 80 \text { years old } \\
N=80\end{array}$ \\
\hline \multirow{6}{*}{ 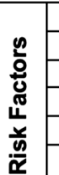 } & \multicolumn{2}{|c|}{ Age } & $74,14 \pm 4,912$ & $83,86 \pm 2,827$ \\
\hline & \multicolumn{2}{|c|}{$\operatorname{Sex}(\%)$} & $31(62 \%)$ & $39(48,1 \%)$ \\
\hline & \multicolumn{2}{|l|}{ Weight } & $79,64 \pm 14,94$ & $74,75 \pm 10,63$ \\
\hline & \multicolumn{2}{|l|}{ Height } & $167,57 \pm 7,103$ & $164,51 \pm 8,001$ \\
\hline & \multicolumn{2}{|l|}{ BMI } & $28,04 \pm 4,13$ & $28,233 \pm 4,68$ \\
\hline & \multirow{4}{*}{ NYHA } & I & 0 & $1(1,4 \%)$ \\
\hline \multirow{4}{*}{ 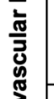 } & & II & $29(69 \%)$ & $43(59,7 \%)$ \\
\hline & & III & $15(32,6 \%)$ & $24(33,3 \%)$ \\
\hline & & IV & $2(4,3 \%)$ & $4(5,6 \%)$ \\
\hline & \multicolumn{2}{|c|}{ LVEF } & $55,07 \pm 16,180$ & $62,60 \pm 13,50$ \\
\hline \multirow{3}{*}{ 응 } & \multicolumn{2}{|c|}{ Hypertension } & $45(90 \%)$ & $77(95,1 \%)$ \\
\hline & \multicolumn{2}{|c|}{ Diabetes } & $24(48 \%)$ & $31(38,3 \%)$ \\
\hline & \multicolumn{2}{|c|}{ Dyslipidemia } & $37(74 \%)$ & $51(63 \%)$ \\
\hline \multirow{8}{*}{ 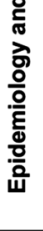 } & \multicolumn{2}{|c|}{ Previous heart disease } & $27(54 \%)$ & $41(50,6 \%)$ \\
\hline & \multicolumn{2}{|c|}{ AFibrillation } & $14(28 \%)$ & $27(33 \%)$ \\
\hline & \multicolumn{2}{|c|}{ Previous CAB } & $4(8 \%)$ & $5(6,2 \%)$ \\
\hline & \multicolumn{2}{|c|}{ ICP } & $18(36 \%)$ & $25(31,3 \%)$ \\
\hline & \multicolumn{2}{|c|}{ Previous MI } & $19(38 \%)$ & $26(32 \%)$ \\
\hline & \multicolumn{2}{|c|}{ Previous Pacemaker//CD } & $4(8 \%)$ & $8(9,9 \%)$ \\
\hline & \multicolumn{2}{|c|}{ CKD } & $12(24 \%)$ & $14(17 \%)$ \\
\hline & \multicolumn{2}{|c|}{ Peripheral Artery Disease } & $24(48 \%)$ & $34(42 \%)$ \\
\hline
\end{tabular}

\begin{tabular}{|l|l|c|c|}
\hline \multicolumn{2}{|c|}{ Table 2 } & $\begin{array}{c}<\mathbf{8 0} \text { years old } \\
\mathbf{N}=\mathbf{5 0}\end{array}$ & $\begin{array}{c}\mathbf{8} \mathbf{8 0} \text { years old } \\
\mathbf{N}=\mathbf{8 0}\end{array}$ \\
\hline \multirow{2}{*}{$\begin{array}{l}\text { Surgery Risk } \\
\text { Scores }\end{array}$} & STS & $3,85 \pm 3,09$ & $\mathbf{4 , 1 9 \pm 2 , 7 8}$ \\
\cline { 2 - 4 } $\begin{array}{l}\text { Severity } \\
\text { Indexes }\end{array}$ & EUROSCORE & $5,38 \pm 3,77$ & $4,29 \pm 2,73$ \\
\hline \multirow{4}{*}{ Procedure } & APACHE II & $10,00 \pm 4,108$ & $11,0 \pm 4,18$ \\
\cline { 2 - 4 } & I. CHARLSON & $4,24 \pm 2,385$ & $3,88 \pm 2,390$ \\
\cline { 2 - 4 } & Self-expanding (\%) & $30(76,9 \%)$ & $66(93 \%)$ \\
\cline { 2 - 4 } & Percutaneous vascular access & $47(92 \%)$ & $77(93,8 \%)$ \\
\cline { 2 - 4 } & General anesthesia & $10(20,8 \%)$ & $10(12,3 \%)$ \\
\cline { 2 - 4 } & ICU sedation & $40(79,2 \%)$ & $70(87,7 \%)$ \\
\cline { 2 - 4 } & Pacemaker stimulation & $16(33 \%)$ & $38(48 \%)$ \\
\hline
\end{tabular}

\begin{tabular}{|c|c|c|c|}
\hline \multicolumn{2}{|r|}{ Table 3} & $\begin{array}{c}<80 \text { years old } \\
N=50\end{array}$ & $\begin{array}{c}\geq 80 \text { years old } \\
N=80\end{array}$ \\
\hline \multirow{14}{*}{ 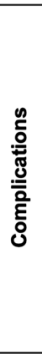 } & Stroke & $3(6 \%)$ & $2(2,5 \%)$ \\
\hline & Bleeding & $13(26 \%)$ & $22(27,2 \%)$ \\
\hline & AKI & $8(16 \%)$ & $9(11,1 \%)$ \\
\hline & Vascular complications & $12(24 \%)$ & $20(24,7 \%)$ \\
\hline & Rhythm disturbances & & \\
\hline & AVB & $11(22,9 \%)$ & $13(16,3 \%)$ \\
\hline & New onset LBBB & $13(27,1 \%)$ & $19(23,8 \%)$ \\
\hline & Transitory conduction disorders & $6(12 \%)$ & $16(20 \%)$ \\
\hline & New onset AFibrillation & $3(6,3 \%)$ & $7(8,8 \%)$ \\
\hline & Permanent pacemaker & $7(14 \%)$ & $16(20 \%)$ \\
\hline & Mechanical complications & $2(0,04 \%)$ & $2(0,025 \%)$ \\
\hline & Endocarditis & $1(2 \%)$ & 0 \\
\hline & Nosocomial infections & $13(26 \%)$ & $16(20 \%)$ \\
\hline & Mild aortic insufficiency post-TAVR & $18(36 \%)$ & $25(30,9 \%)$ \\
\hline
\end{tabular}

\begin{tabular}{|c|c|c|c|}
\hline \multicolumn{2}{|r|}{ Table 4} & $\begin{array}{c}<80 \text { years old } \\
N=50\end{array}$ & $\begin{array}{c}\geq 80 \text { years old } \\
N=80\end{array}$ \\
\hline \multirow{9}{*}{ 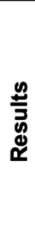 } & Pre-TAVR stay & $6,64 \pm 8,588$ & $5,41 \pm 7,379$ \\
\hline & ICU stay & $2,9 \pm 7,25$ & $1,10 \pm 1,10$ \\
\hline & Post-TAVR stay & $10,36 \pm 11,640$ & $8,89 \pm 7,375$ \\
\hline & Hospital stay & $17,66 \pm 15,910$ & $15,11 \pm 13,23$ \\
\hline & Success of procedure & $45(91,8 \%)$ & $79(99,5 \%)$ \\
\hline & Home discharge (\%) & $45(90 \%)$ & $77(96,3 \%)$ \\
\hline & ICU mortality (\%) & $3(6 \%)$ & $1(1,3 \%)$ \\
\hline & Hospital mortality & $5(10 \%)$ & $3(3,7 \%)$ \\
\hline & Mortality in $\mathbf{3 0}$ first days (\%) & $6(12 \%)$ & $3(3,8 \%)$ \\
\hline
\end{tabular}

000322

Influence of surgical risk in Trancatheter Aortic Valve Replacement (TAVR)

P. Vega Ocaña ${ }^{1}$; P. Padrón Espinosa; J J. Blanco López ; F. Jiménez Cabrera²; JC. Martin Gonzalez

${ }^{1}$ Intensive care unit, Hospital Universitario Insular de Gran Canaria, Las

Palmas de Gran Canaria, Spain; ${ }^{2}$ Cardiology, Hospital Universitario Insular de Gran Canaria, Las Palmas de Gran Canaria, Spain

Correspondence: P. Vega Ocaña

Intensive Care Medicine Experimental 2020, 9(1): 000322.

Introduction. Transcatheter aortic valve implantation (TAVI) has become the treatment of choice for inoperable and high-risk patients with severe aortic stenosis, and is getting more common in intermediate-risk patients as well.

Objectives. To assess the influence of surgical risk in transcatheter aortic valve replacement (TAVR).

Methods. We carried out a prospective observational study including patients undergoing transcatheter aortic valve replacement (TAVR) in our hospital (third level Hospital with Hemodynamics and Intensive Care Unit) from the beginning of the TAVR program to the present time (2016 to 2020). Two study groups were compared based on the adult cardiac surgery risk: low risk group (STS score $<4$ ) and intermediate-high risk group (STS score $\geq 4$ ). Data was collected prospectively from electronic hospital medical record (Drago AE and Critical Care Management) and arranged in a database designed for the purpose of this study. Epidemiological characteristics, risk factors, implantation procedure, complications and results were studied. Quantitative variables are described as mean $\pm \mathrm{SD}$, while categorical variables are described numerically (\%). For data analysis, the Chi-square test and/or Student's T tests and the Mann-Whitney $U$ test were used.

Results. 121 patients were included, 77 with low surgical risks and 44 with intermediate-high risk. The results of the anthropometric variables and risk factors are shown in Table 1. Only chronic renal failure was significant. Table 2 shows the surgical risk scores and prognostic severity indices. It is observed that patients with a higher surgical risk also have a higher APACHE II score. In the implantation procedure, between $75-90 \%$ of the procedures were performed with sedation and with the support of the ICU (Table 3). Complications, stay and mortality can be seen in Tables 4 and 5 . They show that there are no differences in the parameters studied.

Conclusion. In our series, the results obtained in the transcatheter aortic valve replacement are similar in patients with low and moderate-high surgical risk, hence TAVR should be considered and an option in patients with low surgical risk. 


\begin{tabular}{|c|c|c|c|c|c|}
\hline \multicolumn{3}{|c|}{ Table 1} & $\begin{array}{c}\text { Low surgical risk } \\
\qquad=77\end{array}$ & $\begin{array}{l}\text { High surgical risk } \\
\qquad \mathrm{N}=44\end{array}$ & $\boldsymbol{p}$ \\
\hline \multirow{9}{*}{ 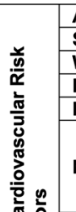 } & \multicolumn{2}{|l|}{ Age } & $79,35 \pm 6,34$ & $81,61 \pm 5,68$ & 0,052 \\
\hline & \multicolumn{2}{|c|}{ Sex (\%) } & $40(51,9 \%)$ & $23(52,3 \%)$ & $\mathrm{ns}$ \\
\hline & \multicolumn{2}{|l|}{ Weight } & $79,64 \pm 13,137$ & $72,3 \pm 10,74$ & 0,05 \\
\hline & \multicolumn{2}{|l|}{ Height } & $167,64 \pm 6,593$ & $162,84 \pm 9,06$ & 0,003 \\
\hline & \multicolumn{2}{|l|}{ BMI } & $27,94 \pm 3,75$ & $27,96 \pm 4,9$ & ns \\
\hline & \multirow{4}{*}{ NYHA } & $\mathbf{I}$ & $1(1,4 \%)$ & $0,00 \%$ & \\
\hline & & II & $49(69 \%)$ & $19(48,7 \%)$ & ns \\
\hline & & III & $18(25,4 \%)$ & $18(46,2 \%)$ & ns \\
\hline & & IV & $3(4,2 \%)$ & $2(5,1 \%)$ & \\
\hline రొ & \multicolumn{2}{|l|}{ LVEF } & $61,06 \pm 14,368$ & $58,02 \pm 15,87$ & ns \\
\hline \multirow{2}{*}{ 容 } & \multicolumn{2}{|c|}{ Hypertension } & $72(93,5 \%)$ & $41(93,2 \%)$ & ns \\
\hline & \multicolumn{2}{|c|}{ Diabetes } & $32(41,6 \%)$ & $17(38,6 \%)$ & ns \\
\hline \multirow{6}{*}{ 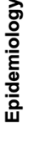 } & \multicolumn{2}{|c|}{ Dyslipidemia } & $55(71,4 \%)$ & $24(54,5 \%)$ & ns \\
\hline & \multicolumn{2}{|c|}{ Previous heart disease } & $35(45,5 \%)$ & $27(61,4 \%)$ & ns \\
\hline & \multicolumn{2}{|c|}{ AFibrillation } & $25(32,5 \%)$ & $15(34,1 \%)$ & ns \\
\hline & \multicolumn{2}{|c|}{ Previous MI } & $23(29,9 \%)$ & $17(38,6 \%)$ & ns \\
\hline & \multicolumn{2}{|c|}{ CKD } & $11(14,3 \%)$ & $14(31,8 \%)$ & 0,03 \\
\hline & \multicolumn{2}{|c|}{$\begin{array}{l}\text { Peripheral Artery } \\
\text { Disease }\end{array}$} & $30(39 \%)$ & $25(56,8 \%)$ & 0,058 \\
\hline
\end{tabular}

\begin{tabular}{|c|l|c|c|c|}
\hline \multicolumn{2}{|c|}{ Table 2 } & $\begin{array}{c}\text { Low surgical risk } \\
\mathbf{N}=\mathbf{7 7}\end{array}$ & $\begin{array}{c}\text { High surgical risk } \\
\mathbf{N}=\mathbf{4 4}\end{array}$ & $\boldsymbol{p}$ \\
\hline \multirow{2}{*}{ Surgery Risk Scores } & STS & $\mathbf{2 , 5 6 \pm 0 , 8 2}$ & $6,68 \pm 3,34$ & 0,000 \\
\cline { 2 - 5 } & EUROSCORE & $5,02 \pm 8,84$ & $5,97 \pm 4,15$ & $\mathrm{nS}$ \\
\hline \multirow{2}{*}{ Severity Indexes } & APACHE II & $\mathbf{9 , 7 9} \pm 3,484$ & $11,86 \pm 4,412$ & 0,005 \\
\cline { 2 - 5 } & CHARLSON & $3,96 \pm 2,392$ & $\mathbf{4 , 2 5} \pm 2,498$ & $\mathrm{~ns}$ \\
\hline
\end{tabular}

\begin{tabular}{|c|c|c|c|c|}
\hline \multicolumn{2}{|r|}{ Table 3} & $\begin{array}{c}\text { Low surgical risk } \\
\mathrm{N}=77\end{array}$ & $\begin{array}{l}\text { High surgical risk } \\
N=\mathbf{N 4}\end{array}$ & p \\
\hline \multirow{8}{*}{ 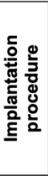 } & Self-expanding (\%) & $62(92 \%)$ & $30(83,3 \%)$ & ns \\
\hline & Percutaneous vascular access & $75(97,4 \%)$ & $41(95,4 \%$ & ns \\
\hline & General anesthesia & $9(11,5 \%)$ & $11(25,6 \%)$ & 0,04 \\
\hline & ICU sedation & $69(88,5 \%)$ & $32(74,4 \%)$ & 0,04 \\
\hline & Pacemaker stimulation & $34(43,6 \%)$ & $17(39,5 \%)$ & ns \\
\hline & Scopia time (min) & $18,4 \pm 6,5$ & $23,95 \pm 8,9$ & ns \\
\hline & Procedure time (min) & $139 \pm 33$ & $154 \pm 62$ & ns \\
\hline & Contrast volume & $155 \pm 22$ & $174 \pm 53,4$ & ns \\
\hline
\end{tabular}

\begin{tabular}{|c|c|c|c|}
\hline \multicolumn{2}{|r|}{ Table 4} & $\begin{array}{l}\text { Low surgical risk } \\
\qquad \begin{array}{l}\mathrm{N}=77\end{array}\end{array}$ & $\begin{array}{l}\text { High surgical risk } \\
\qquad N=44\end{array}$ \\
\hline \multirow{14}{*}{ 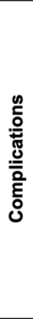 } & MI during TAVR & 0 & 0 \\
\hline & Stroke & $3(3,9 \%)$ & $2(4,5 \%)$ \\
\hline & Bleeding & $17(22,1)$ & $16(36,4 \%)$ \\
\hline & AKI & $10(12 \%)$ & $7(15,9 \%)$ \\
\hline & Vascular complications & $16(20,8 \%)$ & $15(34,1 \%)$ \\
\hline & AVB & $16(21,3 \%)$ & $8(18,6 \%)$ \\
\hline & New onset LBBB & $23(30,7 \%)$ & $8(18,6 \%)$ \\
\hline & Transitory conduction disorders & $10(13,7 \%)$ & $9(20,9 \%)$ \\
\hline & New onset AFibrillation & $5(6,8 \%)$ & $4(9,1 \%)$ \\
\hline & Permanent pacemaker & $15(20 \%)$ & $7(15,99 \%)$ \\
\hline & Mechanical complications & $2(2,6 \%)$ & $1(2,3)$ \\
\hline & Endocarditis & 0 & $1(2,2 \%)$ \\
\hline & Nosocomial infections & $18(23 \%)$ & $10(22,7 \%)$ \\
\hline & Mild aortic insufficiency post-TAVR & $22(28,6 \%)$ & $19(43,2 \%$ \\
\hline
\end{tabular}

\begin{tabular}{|c|c|c|c|}
\hline \multicolumn{2}{|r|}{ Table 5} & $\begin{array}{c}\text { Low surgical risk } \\
\mathrm{N}=77\end{array}$ & $\begin{array}{l}\text { High surgical risk } \\
\mathrm{N}=44\end{array}$ \\
\hline \multirow{9}{*}{ 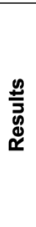 } & Pre-TAVR stay & $5,64 \pm 7,174$ & $6,70 \pm 9,461$ \\
\hline & ICU stay & $1,51 \pm 3,515$ & $2,41 \pm 6,27$ \\
\hline & Post-TAVR stay & $8,75 \pm 7,47$ & $10,66 \pm 11,477$ \\
\hline & Hospital stay & $14,83 \pm 11,323$ & $17,41 \pm 15,731$ \\
\hline & Success of procedure & $73(94,8 \%)$ & $43(95,9 \%)$ \\
\hline & Home discharge (\%) & $71(92,2 \%)$ & $40(90,4 \%)$ \\
\hline & ICU mortality (\%) & $2(2,6 \%)$ & $2(4,7 \%)$ \\
\hline & Hospital mortality & $5(6,5 \%)$ & $2(4,5 \%)$ \\
\hline & Mortality in $\mathbf{3 0}$ first days (\%) & $6(7,8 \%)$ & $3(6,8 \%)$ \\
\hline
\end{tabular}

000331

The Utility of Central Venous to Arterial Carbon Dioxide Tension Gap in Predicting Outcomes Among Postoperative Cardiac Patients: A Systematic Review and Meta-Analysis

AD. Tan' ; M. Patricio ${ }^{1}$; C. Tortosa ${ }^{1}$; C. Permejo, ${ }^{2}$; LMI. Habana, ${ }^{1}$

${ }^{1}$ Department of ambulatory, emergency and critical care, Philippine Heart Center, Quezon City, Philippines; ${ }^{2}$ Department of ambulatory, emergency, and critical care, Philippine Heart Center, Quezon City, Philippines

Correspondence: A.D. Tan

Intensive Care Medicine Experimental 2020, 9(1): 000331

Introduction. Aberrations in tissue perfusion have been associated with organ dysfunction in high-risk surgical patients. The difference between venous and arterial carbon dioxide pressures ( $\mathrm{pCO} 2$ gap) is an easily obtainable marker of tissue hypoperfusion in critically ill patients who have not been resuscitated adequately. In this review, we sought to determine the use of the pCO2 gap in predicting adverse outcomes among patients who underwent cardiac surgery.

Objectives. The general objective of our study was to evaluate the ability of the pCO2 gap to predict postoperative outcomes in terms of mortality and morbidity. Our specific objectives included determining the use of $\mathrm{pCO} 2$ gap in predicting all-cause mortality and determining the role of pCO2 gap in predicting postoperative outcomes in terms of hospital length of stay, duration of mechanical ventilation, need for and duration of vasoactive medications, other organ dysfunction, and need for renal replacement therapy.

Methods. A systematic search of electronic databases from inception to April 2021 was conducted. We searched through PubMed the Cochrane Database, and the Herdin Plus network (for local research data). Records were included if they involved adult patients (age $\geq 18 \mathrm{yr}$ ) and studied our outcomes of interest. Case reports and conference abstracts were excluded. Data extraction and study quality assessment were performed independently by three reviewers. The Newcastle-Ottawa Scale was used to assess methodological study quality.

Results. Out of 1350 records identified, only 10 qualified for systematic review. Except for 2 studies, the majority suggested that an elevated pCO2 gap taken upon ICU admission after cardiac surgery was associated with increased morbidity and mortality. However, the cut-offs of pCO2 gap and the definitions of organ morbidity varied between these studies. Only 4 of the studies qualified for meta-analysis because of their similarities in study design. The generated area under the curve (AUC) for pCO2 gap was 0.749 (95\% Cl 0.307 to 1.000 $\mathrm{P}=0.001 ; 12=0.00)$. Taken together, these results indicate that $\mathrm{PCO} 2$ is a useful hemodynamic marker for the prognostication of adverse outcomes after cardiac surgery. The Egger's test showed that the publication bias was not significant $(P=0.69)$.

\section{Gemarino 2018}

Chen 2019

Moussa 2019

Mukai 2018

Total (fixed effects)

Total (random effects)

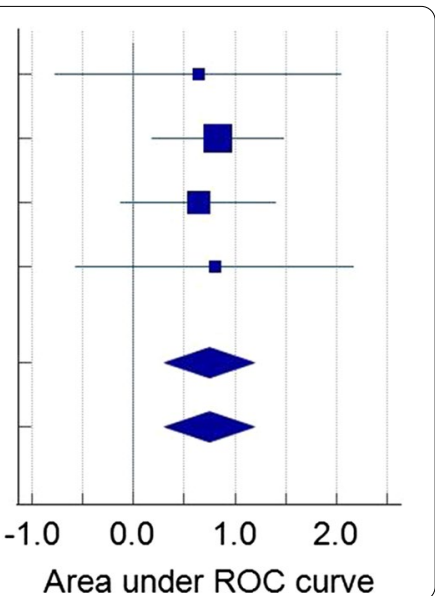


Conclusion. The findings of our study suggest that an increased pCO2 gap is a valuable predictor of postoperative complications after cardiac surgery. Measures must thus be taken to aim for a lower target of this variable. Interventions for hemodynamic optimization include adequate hydration, judicious use of vasoactive drugs, treatment of anemia, and correction of electrolytes. The $\mathrm{pCO} 2$ gap that was found to be associated with and predictive of significant postoperative morbidity was taken upon ICU admission after surgery. Thus, there may be a role for intraoperative monitoring of this parameter, whereby corrections may be instituted earlier.

\section{Reference(s)}

1. Mukai A, Suehiro K, Kimura A, Funai Y, Matsuura T, Tanaka K, Yamada T, Mori T, Nishikawa K. Comparison of the venous-arterial CO 2 to arterial-venous $\mathrm{O} 2$ content difference ratio with the venous-arterial $\mathrm{CO} 2$ gradient for the predictability of adverse outcomes after cardiac surgery. Journal of clinical monitoring and computing. 2020 Feb;34(1):41-53.

2. Habicher M, von Heymann C, Spies CD, Wernecke KD, Sander M. Central venous-arterial $\mathrm{pCO} 2$ difference identifies microcirculatory hypoperfusion in cardiac surgical patients with normal central venous oxygen saturation: a retrospective analysis. Journal of cardiothoracic and vascular anesthesia. 2015 Jun 1:29(3):646-55.

3. Cavaliere F, Martinelli L, Guarneri S, Varano C, Rossi M, Schiavello R. Arterial-venous PCO2 gradient in early postoperative hours following myocardial revascularization. The Journal of cardiovascular surgery. 1996 Oct 1;37(5):499-503.

4. Chen T, Pan T, Luo X, Wang D. High central venous-to-arterial CO2 difference is associated with poor outcomes in patients after cardiac surgery: a propensity score analysis. Shock. 2019 Dec 1;52(6):583-9.

5. Guinot PG, Badoux L, Bernard E, Abou-Arab O, Lorne E, Dupont H. Central venous-to-arterial carbon dioxide partial pressure difference in patients undergoing cardiac surgery is not related to postoperative outcomes. Journal of cardiothoracic and vascular anesthesia. 2017 Aug 1;31(4):1190-6.

6. Morel J, Grand N, Axiotis G, Bouchet JB, Faure M, Auboyer C, Vola M, Molliex S. High veno-arterial carbon dioxide gradient is not predictive of worst outcome after an elective cardiac surgery: a retrospective cohort study. Journal of clinical monitoring and computing. 2016 Dec;30(6):783-9.

7. Huette P, Beyls C, Mallat J, Martineau L, Besserve P, Haye G, Guilbart M, Dupont H, Guinot PG, Diouf M, Mahjoub Y. Central venous-to-arterial CO 2 difference is a poor tool to predict adverse outcomes after cardiac surgery: a retrospective study. Canadian Journal of Anesthesia/Journal canadien d'anesthésie. 2021:1-0

8. Moussa MD, Durand A, Leroy G, Vincent L, Lamer A, Gantois G, Joulin O, Ait-Ouarab S, Deblauwe D, Caroline B, Decoene C. Central venous-toarterial PCO2 difference, arteriovenous oxygen content and outcome after adult cardiac surgery with cardiopulmonary bypass: a prospective observational study. European Journal of Anaesthesiology (EJA). 2019 Apr 1;36(4):279-89.

9. Zante B, Reichenspurner H, Kubik M, Schefold JC, Kluge S. Increased admission central venous-arterial CO2 difference predicts ICU-mortality in adult cardiac surgery patients. Heart \& lung. 2019 Sep 1;48(5):421-7.

10. Gemarino JB, Permejo CC, Habana LI. Accuracy of mixed venous oxygen saturation (svo2) and mixed venous-to-arterial carbon dioxide difference (pco2 gap) in predicting adverse outcomes in adult patients undergoing cardiac surgery. Philippine Heart Center; 2018 (unpublished report).

\section{7}

Before and after audit of the effect of removing heparin from arterial line flushing solutions (substituting with normal saline) on the patency rates of arterial lines in the Intensive Care Unit at Mater Dei Hospital

T. Pirotta'; S. Bugeja'; B. Spiteri ${ }^{1}$;. Stafrace

${ }^{1}$ Anaesthesia \& intensive cSare, Mater Dei Hospital, MSD2090, Malta

Correspondence: S. Bugeja

Intensive Care Medicine Experimental 2020, 9(1): 000367
Introduction. The traditional approach of flushing arterial lines with pressurised heparinised saline has been challenged in recent years by a number of studies which failed to show superiority over normal saline. This is whilst also keeping in mind that exposure to heparin may result in rare but potentially life-threatening complications (Aronson, 2006) and that normal saline actually incurs a cost benefit (Robertson-Malt, Malt, Farquhar and Greer, 2014).

Objectives. The primary objective of this audit was to demonstrate that there was no statistically significant difference in the lifespan of arterial lines in our ICU if the flushing solution was changed from heparinised saline to normal saline; secondary objectives were to demonstrate that the arterial line flushing solution had no influence on complication rate, clotting values and platelet count.

Methods. All patients included were over 18 years of age, were admitted to the ICU at Mater Dei Hospital, Malta and had an arterial line in-situ. The first set of data (collected between December 2017 and January 2018) consisted of patients whose arterial lines were flushed with heparinised saline, whereas the second set (collected between September 2019 and February 2020) included only patients whose lines were flushed with normal saline.

All patients were assessed daily by a member of the data collection team; the data collected included demographics, clinical diagnosis and severity, site of line placement, any line-related complications as well as daily APTT ratio and platelet count. Furthermore, 3 separate tests were performed daily to confirm line patency:

- A 'fast-flush' test.

- Easy aspiration of blood.

- Invasive vs non-invasive blood pressure difference of less than $10 \mathrm{mmHg}$.

Results. The first set of data included a total of 114 arterial lines, each of which stayed in-situ for an average of 3.9 days with an average functional day percentage of $23.9 \%$. The second set of data included 20 lines which stayed in-situ for an average of 4.3 days with an average functional day percentage of $87 \%$.

The first and second sets of data were compared and found to be matched for all demographics with the exception of SOFA score; all numerical data was compared using a 2-sample T-Test with equal variances, whereas categorical data was compared using Pearson's chisquared test.

The percentage of days during which the lines were found functional was compared across the 2 sets of data and no statistically significant difference was found ( $p$-value $>0.05$ ).

No complications were recorded in either group and no statistically significant difference was found in average APTT ratio and platelet count between the 2 groups.

Conclusion. No statistically significant difference was found in the percentage of functional days when the arterial line flushing solution was switched from heparinised saline to normal saline. Furthermore, there was no difference in complication rate, APTT ratio and platelet count between the 2 groups.

\section{Reference(s)}

1. Robertson-Malt, S., Malt, G., Farquhar, V., \& Greer, W. (2014). Heparin versus normal saline for patency of arterial lines. Cochrane Database of Systematic Reviews, 5

2. Barret, P. J., \& Lester, R. L. (1990). Heparin versus saline flushing solutions in a small community hospital. Hosp Pharm, 25, 115-118.

3. Aronson, J. K. (2006). Meyler's Side Effects of Drugs: The International Encyclopedia of Adverse Drug Reactions and Interactions (6 Volume Set) (15th ed.). Elsevier Science. 


\section{3}

Evolving paradigms in cardiogenic shock aetiology and outcome during Coronavirus Disease 2019 pandemic

F. Ahmad'; N. Julien ${ }^{1}$; P. Gandre ${ }^{1}$;T. Konstantinos ${ }^{1}$; P. Thaventhran ${ }^{1}$; EAA. Marwa'; J. Samuel ${ }^{1}$; C. David '; G. Pakeerathan ${ }^{1}$; M. Navaneethan ${ }^{1}$. P. Janak'; K. Ferenc ${ }^{1}$; P. Dharam ${ }^{1}$; CST. Darren ${ }^{2}$;. Emma ${ }^{3}$; K. Sazzli'i; C. Jeronimo ${ }^{1}$ ${ }^{1}$ Intensive care unit, North Middlesex University Hospital, London, United Kingdom; ${ }^{2}$ Department of internal medicine, Keningau Hospital, Keningau, Sabah, Malaysia; ${ }^{3}$ Department of cardiology, Serdang Hospital, Kajang, Malaysia; ${ }^{4}$ Department of cardiology, Hospital UiTM Sungai Buloh, Sungai Buloh, Malaysia

Correspondence: F. Ahmad

Intensive Care Medicine Experimental 2020, 9(1): 000373

Introduction. Since December 2019, coronavirus disease (COVID-19) has become a global pandemic. Cardiovascular complications are becoming increasingly recognized with manifestation ranging from acute coronary syndrome (ACS) to myocarditis or complete heart block and even cardiogenic shock (CS) [1,2].

Objectives. This study investigates the outcome of critically ill COVID19 patients that developed CS and other major cardiac complications in a COVID-19 surge hospital in London, United Kingdom.

Methods. We retrospectively evaluated all critically ill COVID-19 (C19) that developed CS from March 2020 to March 2021. The results obtained were compared to critically ill non Covid-19 (nC19) CS patients from March 2019 to March 2020. Clinical variables studied included disease history, biomarkers, echocardiographic findings and mortality. All statistical studies were carried out using Statistical Package for Social Sciences software. Results. Altogether, 78 critically ill patients with CS were analyzed in this study in which 40 of them tested positive for COVID-19. There is a remarkable increase in CS in the C19 group compared to $\mathrm{nC} 19$. No differences were observed in patient age [56 (49.4-66) vs. 57 (51-65), $P=\mathrm{ns}$ ] or gender (male: $67.5 \%$ vs. $71 \%, P=$ ns). Most of the patients had hypertension ( $81 \%$ in $\mathrm{C} 19$ vs. $85 \%$ in $\mathrm{nC} 19, P=\mathrm{ns}$ ) and $51 \%$ and $74 \%$ of the C19 and nC19 patients were smokers. $22.5 \%$ of the C19 and $47 \%$ of the $\mathrm{nC} 19$ had previous percutaneous coronary intervention $(\mathrm{PCl})$ while $5 \%$ of $\mathrm{C} 19$ and $16 \%$ of $\mathrm{nC} 19$ had previous cardiac surgery, respectively. Other comorbidities such as heart failure, diabetes, dyslipidemia, chronic kidney disease, and chronic lung disease were similar in both groups. Common etiologies for CS for both groups were ACS [30\% in C19 vs $45 \%$ in $\mathrm{nC} 19, p=0.03]$,acute on chronic heart failure [20\% in C19 vs $29 \%$ in $\mathrm{nC} 19, p=0.03$ ], stress cardiomyopathy [25\% in C19 vs $5 \%$ in $\mathrm{nC} 19, p=0.03$ ] and myocarditis [17.5\% in C19 vs $2.6 \%$ in $\mathrm{nC} 19, p=0.03$ ]. We observed high incidence of myocarditis induced CS in C19 group. Cardiac markers were significantly increased for C19 group [Table 1]. Significantly depressed ejection fraction was observed in C19 $(p=0.05)$ more often as compared to nC19 group. A $32.4 \%$ increase in major complications was observed in the $\mathrm{C} 19$ group regarding cardio-pulmonary resuscitation (CPR), sudden cardiac arrest and ventricular tachycardia/Ventricular fibrillation [C19: $62.4 \%$ and $\mathrm{nC} 19: 30.0 \%, p=0.03$ ]. In hospital mortality [C19: $65 \%$ and $\mathrm{nC} 19: 29 \%, p=0.03$ ] and length of hospital stay was significantly higher in C19 group as compared nC19 group respectively.

Table 1. Demographics, aetiologies and outcome of CS between C19 and $\mathrm{nC} 19$

\begin{tabular}{lll}
\hline & C19 $(\mathbf{n}=\mathbf{4 0})$ & $\mathbf{n C 1 9}(\mathbf{n}=\mathbf{3 8})$ \\
\hline Age & $56(49.4-66)$ & $57(51-65)$ \\
Male/female $(\mathrm{n}=)$ & $27 / 13$ & $27 / 11$ \\
Previous PCI/Cardiac Surgery $(\mathrm{n}=)$ & $9 / 2$ & $18 / 6$ \\
ACS $(\mathrm{n}=)$ & 12 & 17 \\
Acute on chronic heart failure $(\mathrm{n}=)$ & 8 & 11 \\
Stress Cardiomyopathy $(\mathrm{n}=)$ & 10 & 2 \\
Myocarditis $(\mathrm{n}=)$ & 7 & 1 \\
High Sensivity Troponin, & $828 / 110-4210$ & $522 / 198-2460$ \\
Mean/range $(\mathrm{ng} / \mathrm{L})$ & & $9 \pm 1$ \\
Length of hospital stay (days) & $16 \pm 2$ & 11 \\
In-hospital mortality $(\mathrm{n}=)$ & 26 & \\
\end{tabular}

Conclusion. To conclude, COVID-19 has caused a shift in the epidemiology and a substantially increased mortality from CS. Patients with CS during COVID-19 pandemic have more extensive myocardial damage represented with higher troponin values and worsened ejection fraction on echocardiographic findings. Our data indicates that the impact of COVID-19 may cause treatment delay leading to high mortality and the outcome of CS.

\section{Reference(s)}

1. Huang CL., Wang YM., Li WX., Ren LL., Zhao JP et al. Clinical features of patients infected with 2019 novel coronavirus in Wuhan, China. The Lancet, 2020; 395:497-506

2. Gopinathannair R., Merchant F.M., Lakkireddy D.R., Etheridge S.P., Feigofsky S., Han J.K. COVID-19 and cardiac arrhythmias: a global perspective on arrhythmia characteristics and management strategies. J Interv Card Electrophysiol. 2020;3:1-8.

3. None Received

\section{0}

ABNORMAL PvaCO2 IS ASSOCIATED WITH REDUCED MICROCIRCULATORY RED BLOOD CELL VELOCITY IN NORMOXIC PATIENTS DURING RESUSCITATION AFTER CARDIAC SURGERY

J. Greenwood'; F. Talebi2 ; D. Jang 2 ; A. Spelde 3 ;Y. Augoustides 3 ; J. Gutsche 3 ; M. Acker ; J. Tonna ${ }^{5}$; N. Meyer ${ }^{6}$; T. Kilbaugh'; J. Bakker ${ }^{8}$; B. Abella ${ }^{2}$

${ }^{1}$ Departments of emergency medicine, anesthesiology \& critical care, Perelman School of Medicine at the University of Pennsylvania, Philadelphia, United States of America; ${ }^{2}$ Department of emergency medicine, Perelman School of Medicine at the University of Pennsylvania, Philadelphia, United States of America; ${ }^{3}$ Department of anesthesiology \& critical care, Perelman School of Medicine at the University of Pennsylvania, Philadelphia, United States of America; ${ }^{4}$ Department of surgery, division of cardiovascular surgery, University of Pennsylvania, Philadelphia, United States of America; ${ }^{5}$ Department of surgery, University of Utah Health, Salt Lake City, United States of America; ${ }^{6}$ Division of pulmonary \& critical care medicine, Perelman School of Medicine at the University of Pennsylvania, Philadelphia, United States of America;

${ }^{7}$ Department of anesthesiology \& critical care medicine, Children's Hospital of Philadelphia, Philadelphia, United States of America; ${ }^{8}$ Department of intensive Care Adults, Erasmus University Medical Center, Rotterdam, Netherlands Correspondence: J. Greenwood

Intensive Care Medicine Experimental 2020, 9(1): 000400

Introduction. Cardiac surgery with cardiopulmonary bypass (CPB) is associated with significant microcirculatory impairment [1]. The venoarterial $\mathrm{PCO} 2$ difference $(\mathrm{PvaCO} 2)$ has been identified as a marker of the adequacy of cardiac output relative to the metabolic demand in patients with circulatory failure [2]. It has been hypothesized that an abnormal $\mathrm{PvaCO} 2$ reflects inadequate microcirculatory blood flow, but it is unclear if convective or diffusion capacity is affected.

Objectives. To evaluate changes in microcirculatory convective blood flow and diffusive capacity associated with an abnormal PvaCO2 using sublingual incident dark field microscopy.

Methods. These are preliminary results from an ongoing prospective, single center observational study of elective CABG or valvular surgery patients. Sublingual microcirculation measurements (CytoCam, Braedius Medical BG, the Netherlands) were obtained 0-4 h post-op during resuscitation efforts in the ICU. Repeat measurements were taken when possible. Clinical hemodynamics and blood gas measurements were obtained from an existing arterial line and pulmonary artery catheter. A PvaCO2>6 was considered abnormal. Microcirculation videos were analyzed manually using ESICM 2nd consensus recommendations [3]. Red blood cell velocity (RBCv) was calculated using space-time diagrams from at least three 10-20 um diameter venules per video. Proportion of perfused vessels (PPV), total vessel density (TVD), and perfused vessel density (PVD) were manually calculated using AVA 3.1 software (Microvision Medical, the Netherlands).

Results. We enrolled 18 subjects, with an age of 63 years $( \pm 10)$ $78 \%$ were male, and EuroSCORE II was 1.56 ( \pm 1.22$)$. The RBCv of patients with a PCO2 gap $>6$ vs. $<=6 \mathrm{mmHg}$ was $314 \mu \mathrm{m} / \mathrm{s}( \pm 118)$ vs. $577 \mu \mathrm{m} / \mathrm{s}$ ( \pm 158$), p<0.0001$. There was no difference in PPV $88 \%$ 
$( \pm 4)$ vs. $85 \%( \pm 6)$, TVD $28.6 \mathrm{~mm} / \mathrm{mm} 2( \pm 5.0)$ vs. $27.4( \pm 4.8)$, and PVD $25.3 \mathrm{~mm} / \mathrm{mm} 2( \pm 4.8)$ vs. $23.3( \pm 4.2)$. PaO2 was not different between groups with a high and low PvaCO2 (110 vs. $138 \mathrm{mmHg}$ ). Capillary RBCv was correlated with PvaCO2, $\mathrm{r}=-0.61, \mathrm{r} 2=0.37, p=0.001$, but not with cardiac index, $\mathrm{r}=-0.24, p=0.25$

Conclusion. During initial resuscitation after cardiac surgery, a PvaCO2 $>6$ was associated with a decrease in sublingual microcirculatory $\mathrm{RBC}$ velocity without a difference in the density of perfused capillaries.

\section{Reference(s)}

1. National Center for Advancing Translational Sciences of the $\mathrm{NIH}$ KL2TR001879

2. Penn Center for Resuscitation Science

3. Penn Abramson Critical Care \& Resuscitation Research Fund

4. Greenwood JC, Jang DH, Hallisey SD, et al. (2021) Severe Impairment of Microcirculatory Perfused Vessel Density Is Associated With Postoperative Lactate and Acute Organ Injury After Cardiac Surgery. Journal of Cardiothoracic and Vascular Anesthesia 35:106-115. https://doi.org/10.1053/j. jvca.2020.04.045

5. Teboul J-L, Mercat A, Lenique F, et al. (1998) Value of the venous-arterial PCO2 gradient to reflect the oxygen supply to demand in humans: Effects of dobutamine. Critical Care Medicine 26:1007-1010. https://doi. org/10.1097/00003246-199806000-00017

6. Ince C, Boerma EC, Cecconi M, et al. (2018) Second consensus on the assessment of sublingual microcirculation in critically ill patients: results from a task force of the European Society of Intensive Care Medicine. Intensive Care Med 44:281-299. https://doi.org/10.1007/ s00134-018-5070-7

\section{1}

Heart Rate Variability during Lower Body Negative Pressure Test for the Non-Invasive Detection of Central Hypovolemia

L. Wiegank'; T. Tigges²; AG. Pielmus²; M. Klum²; A. Feldheiser ${ }^{3}$

${ }^{1}$ Department of anaesthesiology and operative intensive care medicine (ccm, cvk), Charité-Universitätsmedizin Berlin, Berlin, Germany;

${ }^{2}$ Department of electronics and medical signal processing, Berlin Institute of Technology, Berlin, Germany; ${ }^{3}$ Departments of anaesthesiology, intensive care medicine and pain therapy, Charité-Universitätsmedizin Berlin/ Evang. Kliniken Essen-Mitte, Berlin/Essen, Germany

Correspondence: L. Wiegank

Intensive Care Medicine Experimental 2020, 9(1): 000414

Introduction. Hemorrhagic shock is a potentially life-threatening condition. Therefore, early detection and appropriate intervention of occult bleeding is desirable. However, detection of occult hemorrhage can be difficult, as traditional vital signs like heart rate and blood pressure are not sensitive enough to blood loss. Due to physiological compensatory mechanisms, they may even be sustained stable just before cardiovascular decompensation $(1,2)$. These compensatory mechanisms are primarily driven by the autonomic nervous system (ANS). Heart rate variability (HRV) has been used to assess ANS function and thus might be useful in detecting otherwise occult bleeding.

Objectives. The aim of this prospective pilot study was to evaluate the diagnostic value of HRV regarding the non-invasive detection of progressive central hypovolemia.

Methods. Central hypovolemia was experimentally simulated using a lower body negative pressure model (LBNP). Over several progressively increased levels of negative pressure $(0 \mathrm{mmHg},-15 \mathrm{mmHg}$, $-30 \mathrm{mmHg},-45 \mathrm{mmHg}, 0 \mathrm{mmHg}$ ) heart rate, blood pressure as well as HRV in its different domains (time-domain: SDNN, RMSSD, rSDRM, pNN50, NN50; frequency-domain: VLF, rVLF, LF, rLF, HF, rHF, LF/HF, TP; non-linear: SD1, SD2, SD1/SD2, SampEn, AppEn) were measured. To assess central hypovolemia stroke volume was measured using transthoracic echocardiography (SVTTE). Parameter changes as a consequence to reduced central volume were evaluated statistically and a correlation analysis (SVTTE vs. HRV parameters) was conducted. Furthermore, a Gray Zone Approach (GZA) with receiver operating characteristic curves regarding a decline in SVTTE of $>20 \%$ was conducted.
Results. A total of 30 male, healthy volunteers were analyzed. Application of LBNP caused progressive reductions of SVTTE successfully. Nonparametric analysis of longitudinal data showed significant $p$-values $(p<0.05)$ for all HRV parameters. Furthermore, post-hoc analysis showed a significant deviation $(p<0.05)$ from baseline values for some HRV parameters at a chamber pressure of $-15 \mathrm{mmHg}$ (SDNN, RMSSD, NN50, VLF, rLF, HF, TP and SD1). Intraindividual correlation analysis showed strong correlations $(|\rho|>0.5)$ for NN50, pNN50, RMSSD, SD1 and $\mathrm{HF}$, and Repeated Measures Correlation analysis showed strong correlations $(|\rho|>0.5)$ for SD1, RMSSD, SD1/SD2, pNN50, and rSDRM. The GZA showed significant differences between groups for NN50, $\mathrm{rVLF}$, LF, and rHF. Areas under the curves were $<0.7$.

Conclusion. Progressive central hypovolemia is well reflected by HRV. Several parameters also showed a significant reaction in mild central hypovolemia already. HRV analysis could thus be helpful in the detection of otherwise occult hemorrhage. However, its diagnostic value to assess patients individually seems to be limited based on the GZA because of explicit interindividual variability.

\section{Reference(s)}

1. McGee S. Is This Patient Hypovolemic? JAMA. 1999 Mar 17;281(11):1022.

2. The authors declare no conflicts of interest. This research received no specific funding/grant from any funding agency.

3. Parks JK, Elliott AC, Gentilello LM, Shafi S. Systemic hypotension is a late marker of shock after trauma: a validation study of Advanced Trauma Life Support principles in a large national sample. The American Journal of Surgery. 2006 Dec;192(6):727-31.

\section{5}

Acute Myocardial Injury as a prognostic factor in Critically ill patients with Severe SARS-Cov-2 Pneumonia

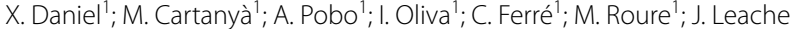
Irigoyen ${ }^{1}$; M. Bodí ${ }^{1}$ C. Villavicencio Lujan

${ }^{1}$ INTENSIVE CARE, Hospital Universitari de Tarragona Joan XXIII, Tarragona,

Spain

Correspondence: X. Daniel

Intensive Care Medicine Experimental 2020, 9(1): 000445

Introduction. Patients with severe SARS-CoV2 pneumonia admitted to intensive care units (ICUs), developed various cardiovascular complications, including: arrhythmias (16-44\%), acute myocardial injury (AMI) $(22-33 \%)$, shock (30.6\%) and acute heart failure (10\%) among others[i],[ii],[iii], probably related to the inflammatory and prothrombotic response itself against infection[iv],[v]. $67 \%$ of those patients required vasopressors and, in some cases, had a mortality as high as $67 \%[v i]$,[vii]. Characteristically these patients developed elevation of different inflammatory markers, cardiac troponin (cTn) which is an indicative of AMI, among others. [viii],[ix] For these reasons, the aim of our study was to measure the incidence of AMI and to assess it as an independent risk factor for 28-day mortality.

Methods. Observational cohort study that included consecutive patients with diagnosis of severe SARS-CoV-2 Pneumonia, admitted to the intensive care unit (ICU) at Joan XXIII University hospital in Tarragona, Spain, during 11 months. The data were collected during the first $72 \mathrm{~h}$ at ICU admission.

AMl: defined by an increase in high-sensitivity troponin (hs-cTn) $>99$ th percentile upper-Reference limit (>47 ng/L).

Results. We included 181 patients with severe SARS-CoV2 Pneumonia. The median age was 65 years and $71.3 \%$ were male. The most coexisting conditions were overweight $(50.3 \%)$, obesity (33.1\%), hypertension (47\%), diabetes (23.8\%) and cardiovascular disease (13.2\%). 85,6\% received mechanical ventilation $(\mathrm{MV})$ and $69 \%$ received norepinephrine. $89 \%$ of patients had cardiovascular complications, that included isolated hypotension (45.9\%), shock (20.4\%), arrhythmias $(59,7 \%)$ and acute kidney injury (AKI) (26\%). The 28-day mortality was $16 \%$, ICU stay median of 17 days (IQR 9-31) and median duration of MV of 15.5 days (IQR 7-28). We collected troponin levels in $77.3 \%(140 / 181)$ of patients and $21.4 \%$ (30/140) developed AMI. 
Patients with AMI had a significant association with history of cardiovascular disease (36\% vs $5 \% ; \mathrm{p}=0.03)$, protein C-reactive $(21.8 \mathrm{mg} /$ $\mathrm{dL}$ vs $12.5 \mathrm{mg} / \mathrm{dL}, \mathrm{p}=0.016)$, D-dimer (1099 ng/mL vs $606 \mathrm{ng} / \mathrm{mL}$, $\mathrm{p}=0.001), \mathrm{N}$-terminal pro-B-type natriuretic peptide $(2287 \mathrm{pg} / \mathrm{mL}$ vs $235 \mathrm{pg} / \mathrm{mL}, \mathrm{p}=0.001)$, as well as shock (43.3\% vs $16.4 \%, \mathrm{p}=0.002)$, left ventricular failure ( $31 \%$ vs $3.1 \% p=0.001)$, AKI ( $43.3 \%$ vs. $24.5 \%$, $\mathrm{p}<0.043)$, MV requirement $(100 \%$ vs. $85.5 \%, \mathrm{p}=0.026)$ and 28 -day mortality (30\% vs $12.7 \%, p=0.024)$.

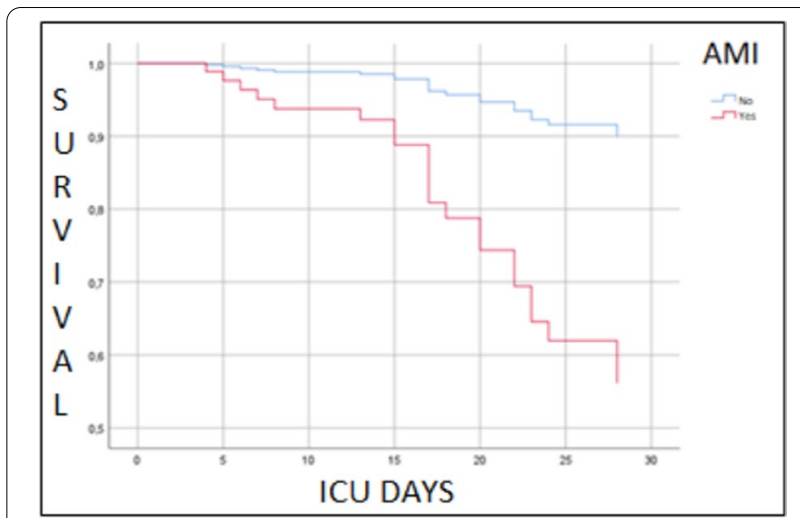

Figure 1: Cox proportional hazard regression

AMI was independently associated with 28-day UCI mortality in a survival multivariate analysis with HR 5.4 (95\%Cl 1.9-14.9). (Fig. 1). Conclusion. AMI is a common condition among patients with severe SARS-CoV-2 pneumonia, and it is associated with a severe inflammatory response as well as a higher risk of organ failure and ICU 28-day mortality.

\section{Reference(s)}

1. 10.- Thygesen K, Alpert JS, Jaffe AS, Chaitman BR, Bax JJ, Morrow DA White HD; Executive Group on behalf of the Joint European Society of Cardiology (ESC)/American College of Cardiology (ACC)/American Heart Association (AHA)/World Heart Federation (WHF) Task Force for the Universal Definition of Myocardial Infarction. Fourth Universal Definition of Myocardial Infarction (2018). J Am Coll Cardiol. 2018 Oct 30;72(18):22312264. https://doi.org/10.1016/j.jacc.2018.08.1038. Epub 2018 Aug 25. PMID: $30,153,967$

2. 9.- Xiong TY, Redwood S, Prendergast B, Chen M. Coronaviruses and the cardiovascular system: acute and long-term implications. Eur Heart J. 2020 May 14;41(19):1798-1800. https://doi.org/10.1093/eurheartj/ehaa2 31. PMID: $32,186,331 ;$ PMCID: PMC7454513.

3. Zhou F, Yu T, Du R, Fan G, Liu Y, Liu Z, Xiang J, Wang Y, Song B, Gu X, Guan L, Wei Y, Li H, Wu X, Xu J, Tu S, Zhang Y, Chen H, Cao B. Clinical course and risk factors for mortality of adult inpatients with COVID19 in Wuhan, China: a retrospective cohort study. Lancet. $2020 \mathrm{Mar}$ 28;395(10,229):1054-1062. https://doi.org/10.1016/S0140-6736(20)305663. Epub 2020 Mar 11. Erratum in: Lancet. 2020 Mar 28;395(10,229):1038. Erratum in: Lancet. 2020 Mar 28;395(10,229):1038. PMID: 32,171,076; PMCID: PMC7270627.

4. 7.- Yang X, Yu Y, Xu J, Shu H, Xia J, Liu H, Wu Y, Zhang L, Yu Z, Fang M, Yu T, Wang Y, Pan S, Zou X, Yuan S, Shang Y. Clinical course and outcomes of critically ill patients with SARS-CoV-2 pneumonia in Wuhan, China: a single-centered, retrospective, observational study. Lancet Respir Med. 2020 May;8(5):475-481. https://doi.org/10.1016/S2213-2600(20)30079-5. Epub 2020 Feb 24. Erratum in: Lancet Respir Med. 2020 Apr;8(4):e26. PMID: 32,105,632; PMCID: PMC7102538.

5. 6.- Guo T, Fan Y, Chen M, Wu X, Zhang L, He T, Wang H, Wan J, Wang X, Lu Z Cardiovascular Implications of Fatal Outcomes of Patients With
Coronavirus Disease 2019 (COVID-19). JAMA Cardiol. 2020 Jul 1;5(7):811818. https://doi.org/10.1001/jamacardio.2020.1017. Erratum in: JAMA Cardiol. 2020 Jul 1;5(7):848. PMID: 32,219,356; PMCID: PMC7101506.

6. 5.- Ganatra S, Hammond SP, Nohria A. The Novel Coronavirus Disease (COVID-19) Threat for Patients With Cardiovascular Disease and Cancer. JACC CardioOncol. 2020 Jun;2(2):350-355. https://doi.org/10.1016/j. jaccao.2020.03.001. Epub 2020 Apr 10. PMID: 32,292,919; PMCID: PMC7151392.

7. 4.- Clerkin KJ, Fried JA, Raikhelkar J, Sayer G, Griffin JM, Masoumi A, Jain SS, Burkhoff D, Kumaraiah D, Rabbani L, Schwartz A, Uriel N. COVID-19 and Cardiovascular Disease. Circulation. 2020 May 19;141(20):1648-1655. https://doi.org/10.1161/CIRCULATIONAHA.120.046941. Epub 2020 Mar 21. PMID: $32,200,663$.

8. 3.- $\mathrm{HuH}, \mathrm{MaF}$, WeiX,Fang Y.Coronavirus fulminant myocarditis saved with glucocorticoid and human immunoglobulin. Eur Heart J. 2020 Mar 16; pii:ehaa190. [Epub ahead of print].

9. 2.- Shi S, Qin M, Shen B, Cai Y, Liu T, Yang F, Gong W, Liu X, Liang J, Zhao Q, Huang H, Yang B, Huang C. Association of Cardiac Injury With Mortality in Hospitalized Patients With COVID-19 in Wuhan, China. JAMA Cardiol. 2020 Jul 1;5(7):802-810. https://doi.org/10.1001/jamacardio.2020.0950. PMID: 32,211,816; PMCID: PMC7097841.

10. 1.- Arentz M, Yim E, Klaff L, Lokhandwala S, Riedo FX, Chong M, Lee M. Characteristics and Outcomes of 21 Critically III Patients With COVID-19 in Washington State. JAMA. 2020 Apr 28;323(16):1612-1614. https://doi.org/ 10.1001/jama.2020.4326. PMID: 32,191,259; PMCID: PMC7082763.

\section{7}

Dynamic Phenotypes Preceding Hypotension in Intensive Care V. Jeanselme'; A. Dubrawski'; M. Pinsky²; G. Clermont²; JH. Yoon ${ }^{2}$ ${ }^{1}$ Auton lab, school of computer science, Carnegie Mellon University, Pittsburgh, United States of America; ${ }^{2}$ School of medicine, University of Pittsburgh, Pittsburgh, United States of America

Correspondence: $\mathrm{V}$. Jeanselme

Intensive Care Medicine Experimental 2020, 9(1): 000457

Introduction. Vital sign changes have been leveraged to model risk of hypotensive events in intensive care unit (ICU), allowing medical intervention prior to the onset of hypotension [1]. Hypotension strongly correlates with morbidity and mortality in intensive care patients [2], however its physiologic signatures are highly heterogeneous. Understanding inherent diversity in hemodynamic state evolution towards crisis could guide effective individualized medical treatment. We developed a machine learning model to identify distinct pre-hypotension hemodynamic evolution patterns.

Methods. The risk score and associated hypotension alerts were generated using a random forest model trained to predict hypotension [1] in the publicly available MIMIC-III dataset [3]. K-means clustering, an unsupervised machine learning algorithm, was performed on predicted risk trajectories from individual subjects in the hour prior to a hypotension event. The number of clusters was selected by measuring the stability of the results on a left-aside set of 718 patients (first hypotension event 1.58 days (std 1.85) after the ICU admission) over multiple bootstrapped iterations of the algorithm. Optimally stable clusters were then studied on the validation set constituted of 724 patients (first hypotension event 1.66 days (std 1.98)), then their clinical characteristics were analyzed by using Kruskal-Wallis test for continuous and $x^{2}$ test for categorical variables.

Results. Three risk trajectory clusters were identified (Fig. 1): persistent-high (203 subjects), moderate (336), and late-rising (185), each with distinctive clinical characteristics. For instance in the persistent-high group, in-hospital mortality was higher (56.2\% vs 27.4 and $24.9 \%-p$-value $<0.001$ ) and had different etiology for hypotension and outcomes (septic shock-31.0\% against 19.9 and $20.0 \%$, cardiogenic shock-15.3\% against 8.9 and $3.8 \%$, and elevated creatinine level-49.3\% against 36.7 and $51.0 \%$ - prior to the first event of hypotension) compared to the other two clusters. 


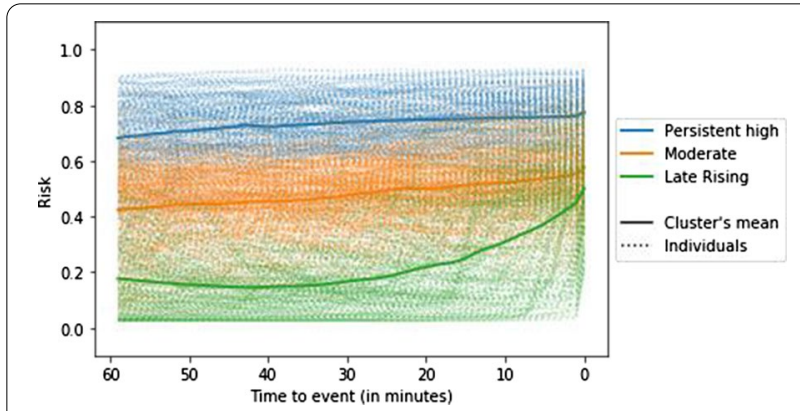

Fig. 1. Phenotypes of risk evolution prior to the first hypotension event

Conclusion. Phenotyping evolution of risk could help understand complex disease progression. Continuous phenotype-based classification would also inform individualized and timely interventions to mitigate upcoming intensive care crises, especially in late-rising patients.

\section{Reference(s)}

1. This work has been partially funded by NIH (1K23GM138984, 5R01GM117622) and DARPA (FA8750-17-2-0130).

2. [3] Johnson AE, Pollard TJ, Shen L, Li-Wei HL, Feng M, Ghassemi M, Moody B, Szolovits P, Celi LA, Mark RG. MIMIC-III, a freely accessible critical care database. Scientific data. 2016;3(1):1-9

3. [1] Yoon JH, Jeanselme V, Dubrawski AW, Hravnak M, Pinsky MR, Clermont G. Prediction of Hypotension Events with Physiologic Vital Sign Signatures in The Intensive Care Unit, Critical Care 2020:24(1):1-9

4. [2] Zenati MS, Billiar TR, Townsend RN, Peitzman AB, Harbrecht BG. A brief episode of hypotension increases mortality in critically ill trauma patients. J Trauma 2002;53(2):232-236

\section{9}

\section{Is thrombolysis for patients with intermediate-risk pulmonary} embolism safe and effective?

F. Jarraya'; A. Jamoussi'; E. Rachdi' ; S. Ayed'; J. Ben Khelil'; M. Besbes

'Intensive care, Hôpital Abderrahmen Mami de pneumo-phtisiologie, Ariana, Tunisia

Correspondence: F. JARRAYA

Intensive Care Medicine Experimental 2020, 9(1): 000509

Introduction. The benefit of thrombolysis (Th) in patients with intermediate-risk pulmonary embolism (PE) has never been strongly established. Thrombolytic therapy may reverse immediately right ventricle pressure overload. Conversly, it remains unclear whether it may improve the early and long-term clinical outcome of patients with intermediate-risk PE. Otherwise, the increased risk of major bleeding has outweighed the clinical benefit of Th according to the referring study on this subject.

Objectives. We aimed:

To assess near-term benefits and the safety of Th in comparison with conventional treatment.

To evaluate the late impact of reperfusion for survivors of acute PE.

Methods. In a retrospective single-center study, we compared thrombolytic plus heparin with heparin in normotensive patients with intermediate-risk PE.

Patient with normotensive PE was eligible if imaging (echocardiography or computed tomography) showed ventricular dysfunction and/ or myocardial injury (indicated by a positive test for cardiac troponin). The primary outcome was death within 30 days after hospitalization. The main safety outcome was major bleeding.

For survivors of acute $\mathrm{PE}$, we assessed the impact of thrombolysis on long-term symptoms, functional limitations and echocardiographic parameters.
Results. A total of 175 patients with intermediate-risk PE were enrolled in this study. Of this group, 65 patients received thrombolysis + heparin (G1) and 110 patients were treated with heparin only (G2).

The 30 -day all-cause mortality was $15,4 \%$ for G1 versus $29 \%$ for G2; $p=0.034$.

Major bleeding at 7 days was presented in $3.1 \%$ in $\mathrm{G} 1$ versus $4.5 \%$ in $\mathrm{G} 2 ; p=0.632$.

Hemorrhagic stroke occurred in one patient in Th group and 2 patients in heparin group; $p=0.514$.

At follow-up examination of survivors, persistent dyspnea was reported in $51 \%$ for $\mathrm{G} 1$ versus $44 \%$ in $\mathrm{G} 2 ; p=0.287$. Echocardiography (performed in 20 patients of $\mathrm{G} 1$ ) revealed significant decrease in pulmonary hypertension (36.5 [30-40] vs 53.5 [40-55] mmHg; $p \leq 10-3)$. Computed tomography (performed in 24 patients of $\mathrm{G} 1$ ) revealed a significant decrease in the obstruction index of Qanadli [1], thrombus had completely disappeared in 13 patients.

Conclusion. Well-conducted thrombolytic therapy is efficient in terms of mortality in patients with intermediate-risk PE with no significant risk of major bleeding. Thrombolytic treatment did not appear to reduce residual dyspnea.

\section{Reference(s)}

1. [1] Qanadli SD, El Hajiam M, Vieillard-Baron A, Joseph T, Mesurolle B, Oliva $\mathrm{VL}$, et al. New CT Index to Quantify Arterial Obstruction in Pulmonary Embolism. Am J Roentgenol. 1 juin 2001;176(6):1415-20

\section{7}

Right ventricular dysfunction and its association with mortality in COVID-19 ARDS

M. Chotalia'; M. Ali'; ;. Alderman, ${ }^{2} ;$ M. Bangash, ${ }^{2} ;$ D. Parekh, ${ }^{3} ;$ J. Patel, ${ }^{2}$ ${ }^{1}$ Institute of Inflammation and Ageing, University of Birmingham, Birmingham, United Kingdom; ${ }^{2}$ Critical care, Queen Elizabeth Hospital Birmingham, Birmingham, United Kingdom; ${ }^{3} \mid t u$, Queen Elizabeth Hospital Birmingham, Birmingham, United Kingdom

Correspondence: M. Chotalia

Intensive Care Medicine Experimental 2020, 9(1): 000537

Introduction. Right ventricular dysfunction (RVD) is common in patients with ARDS [1], but is difficult to define. European studies define RVD as RV dilation [1], whereas American Echocardiography societies use impaired RV systolic function [2]. It is unknown which of the two more closely associates with mortality in ARDS, including those with COVID-19. Objectives. To assess whether RV dilation or systolic impairment is associated with mortality in patients with COVID-19 ARDS

Methods. This is a single centre retrospective cohort study of patients with COVID-19 ARDS undergoing invasive ventilation that received a transthoracic echocardiogram (TTE) between March-December 2020. RV dilation was defined as RV:LV end-diastolic area (RV:LVEDA) $>0.6$, RV systolic impairment as fractional area change (RVFAC) $<35 \%$ or tricuspid annular plane systolic excursion (TAPSE) $<17 \mathrm{~mm}$. Correlation between TTE parameters and clinical variables was assessed. Patients were classified into those with RV dilation, RV systolic impairment and RV dilation with systolic impairment and multivariate logistic regression analysis (MVLRA) was performed.

Results. 172 patients were included, aged 59 years (IQR 49-67), with mostly moderate ARDS $(n=101 ; 59 \%)$. RVFAC correlated with blood gas and ventilatory parameters, whereas RV:LVEDA correlated with urine output (Fig. 1). Ninety-day mortality was $41 \%(n=70) ; 49 \%$ in patients with RV dilation, $53 \%$ in RV systolic impairment and $72 \%$ in RV dilation with systolic impairment. The RV dilation with systolic impairment phenotype was independently associated with mortality after MVLRA (OR 3.11 [95\% $\mathrm{Cl}, 1.15-7.60]$ ) but either disease state alone was not. 


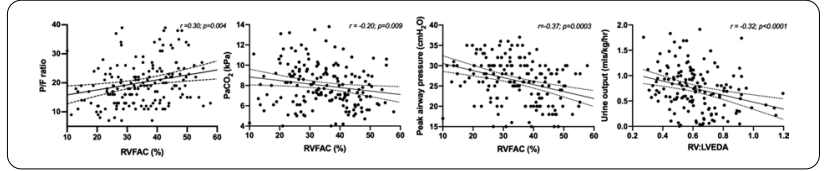

Conclusion. Combining the European and American definitions of RVD identified the RV phenotype with the strongest association with mortality. This may be because it combines pulmonary pathophysiology (associated with RV systolic impairment) with renal dysfunction (associated with RV dilation).

\section{Reference(s)}

1. [1] Mekontso Dessap A, Boissier F, Charron C, et al. Acute cor pulmonale during protective ventilation for acute respiratory distress syndrome: prevalence, predictors, and clinical impact. Intensive Care Med 2016; 42 862-870.

2. [2] Rudski LG, Lai WW, Afilalo J, et al. Guidelines for the echocardiographic assessment of the right heart in adults: a report from the American Society of Echocardiography endorsed by the European Association of Echocardiography, a registered branch of the European Society of Cardiology, and the Canadian Society of Echocardiography. J Am Soc Echocardiogr 2010; 23: 685-713; quiz 786-688.

3. None

\section{0}

High-Frequency Sampling and Signal Quality Indices Boost the Performance of Online Classification of Real Alerts versus Artifacts in Multi-Signal Vital Signs Monitoring Data G. Welter'; A. Dubrawski2 ; T. Pellathy ${ }^{3}$; S. Helman ${ }^{4}$; T. Lagattuta ${ }^{5}$; M. Hravnak ; M. Pinsky ${ }^{7}$; G. Clermont ${ }^{7}$; S. Al-Zaiti ${ }^{5}$

${ }^{1}$ AutonLab, School of Computer Science, Carnegie Mellon University, Pittsburgh, United States of America; ${ }^{2}$ Robotics institute, auton lab, Carnegie Mellon University, Pittsburgh, United States of America; ${ }^{3}$ School of nursing, University of, Pittsburgh, United States of America; ${ }^{4}$ Acute and Tertiary Care, University of Pittsburgh, Pittsburgh, United States of America; ${ }^{5}$ Acute and tertiary care nursing, University of Pittsburgh, Pittsburgh, United States of America; ${ }^{6}$ School of nursing, University of Pittsburgh, Pittsburgh, United States of America; ${ }^{7}$ School of medicine, University of Pittsburgh, Pittsburgh, United States of America Correspondence: S. Al-Zaiti

Intensive Care Medicine Experimental 2020, 9(1): 000550

Introduction. Timely recognition of cardiorespiratory instability (CRI) in monitored patients should reduce preventable morbidity and mortality. However, high rates of false alarms due to thresholdbased exceedance alerts in continuous vital signs (VS) monitoring causes alarm fatigue. 1 We previously showed that machine learning (ML) featurization, trending, and phenotyping of multi-channel VS time series data (1/20 samples/sec) using random forest (RF) classifiers distinguished real alerts from artifactual anomaly,2-3 but classification performance remained suboptimal for $\mathrm{SpO} 2$ and respiratory rate alerts.

Objectives. We evaluated the incremental gain in online classification performance of real vs. artifact VS alerts by using higher sampling frequency and adding waveform-based signal quality indicators (SQI).

Methods. We used a multi-expert, multi-tier ground truth annotated database from our prospective observational study of consecutive patients admitted to a 16-bed surgical-trauma step-down unit. High-fidelity waveform VS data $(60-250 \mathrm{~Hz})$ with corresponding VS numeric time series (1 sample/sec) were linked to electronic health record (EHR) data. VS time series were screened for potential CRI alerts of VS data based on values exceeding a predetermined threshold over a 5-min window. The alerts were then manually adjudicated by two expert clinicians as real CRI vs. artifact after reviewing available waveform and EHR data. Disagreements were resolved by committee consensus. Next, at each VS threshold exceedance, our online models featurized data over the preceding 3-min window and every 20 -s increment for $180 \mathrm{~s}$ into the exceedance using statistical and variability metrics of the multi-channel VS series, as well as several SQI of waveform data (e.g. ECG and PPG sample entropy computed every $3 \mathrm{~s}$ ). All features were fed into a RF classifier with hyperparameters optimized over a grid search of 64 combinations (CMU Auton Lab RF, C language).

Results. Our sample yielded $665 \mathrm{CRI}$ alerts from 47 unique admissions with adjudicated artifact rates of $30 \%$ and $26 \% \%$ for SpO2 and respiratory rate alerts respectively. Adding SQI features boosted the online random forest classifier performance from an area under the receiver operating characteristic curve of 0.901 to $0.943(p=0.048$, DeLong's test) for predicting real SpO2 alerts, and from 0.897 to 0.957 $(p=0.009)$ for real respiratory rate alerts using leave-one-subject-out cross-validation. Simulated online testing of our model suppressed $93 \%$ of the 32,340 nurse-set threshold-based bedside alarms observed in $12,022 \mathrm{~h}$ of continuous monitoring data.

Conclusion. Increasing sampling frequency and adding simple SQI metrics boosts online performance of ML-based classifiers for realtime discrimination of real CRI vs. artifact alerts. Embedding such models in monitors may improve alert accuracy and reduce alarm fatigue.

\section{Reference(s)}

1. Chen et al. Crit Care Med 2016:44:e456-63.

2. Hravnak et al. J Clin Monit Comput. 2016;30:875-88

3. Hravnak et al. J Electrocardiol. 2018;51(6S):S44-S48.

4. NIHT32NR008857

5. NIH R01NR013912

\section{5}

Efficacy and safety of intermediate-dose low molecular weight heparine (LMWH) in critically-ill COVID-19 patients

Z. Athanasa (Athanassa) ${ }^{1}$; A. Dimoula ${ }^{1}$; E. Ntikoudi'; A. Marathonitis';

S. Michelidou'; A. Kessaris'; V. Charizopoulou'; R. Xatzikyriakou².

A. Sakagianni ${ }^{1}$

${ }^{1}$ ICU, Sismanogleio General Hospital, Marousi, Greece; ${ }^{2}$ Laboratory, Sismanogleio General Hospital, Marousi, Greece

Correspondence: Z. Athanasa (Athanassa)

Intensive Care Medicine Experimental 2020, 9(1): 000575

Introduction. COVID-19 infection has been associated with increased incidence of deep venous thrombosis (DVT) and pulmonary embolism (PE) in critically ill patients even among those receiving thromboprophylaxis $(1,2)$, questioning the need of higher low molecular weight heparine (LMWH) doses. However, limited evidence exists regarding the efficacy and safety of this therapeutic approach.

Objectives. The aim of this study is to evaluate the efficacy and safety of intermediate dose LMWH in critically ill COVID-19 patients. The main outcomes are the incidence of DVT, PE and bleeding events during Intensive Care Unit (ICU) hospitalization.

Methods. This is an ongoing prospective, observational study started in January 2021 in a public hospital ICU. Upon admission patients were screened with intensivist-performed compression ultrasonography study (IP-CUS) for proximal lower extremity DVT. Patients with diagnosis of DVT or PE on admission were excluded. Thrombosis and bleeding risk were assessed by Padua and Improve scores respectively (3, 4). Enoxaparine was administered at an intermediate dose based on National COVID-19 recommendations (6). LMWH activity was evaluated by anti-Xa assay. Repeat IP-CUS were performed at days $7 \pm 2$ and $14 \pm 2$. Computed tomography pulmonary angiogram (CTPA) was performed in cases of high clinical suspicion of PE. Bleeding complications were evaluated by WHO bleeding scale (5).

Results. Sixty one consecutive patients ( 40 male) were eligible with a median age of 67 years. Most of the patients (93\%) had at least 1 comorbidity or were obese; $53(87 \%)$ were mechanically ventilated. The median anti-Xa activity was $0,7(0,5-1)$. In 20 patients the initial dose of LMWH was reduced due to increased $(>1,0)$ anti-Xa activity $(5$ patients), bleeding (13 patients), while in 1 patient it was replaced with fondaparinux due to heparine-induced thrombocytopenia. 
Follow-up evaluation with IP-CUS showed DVT in 2 patients. Twentynine (47\%) patients were screened with CTPA at a median of 6 days after ICU admission and 2 of them developed PE. One among them had both DVT and PE. In total 3 patients (5\%) presented thromboembolic events (DVT and/or PE).

Bleeding was observed in 28 patients (46\%), 20 of these events were classified as $\geq$ grade 2 WHO bleeding scale. Ten patients (16\%) required $\geq 1$ red blood cell transfusion and 1 died due to bronchial hemorrhage. Patients with bleeding complications had significantly $(p<0.05)$ increased mortality $(43 \%)$ compared with those without haemorrhagic events $(12 \%)$, as well as prolonged median ICU length of stay ( 15 vs 8 days, $p<0.05$ ). Severe bleeding ( $\geq$ grade 3 ) was significantly associated $(p=0.03)$ with concurrent dual antiplatelet therapy.

Conclusion. Administration of intermediate-dose LMWH may be effective for the prevention of DVT and PE. However, the increased incidence of hemorrhagic complications suggests that LMWH dose should be individualized based on anti-Xa activity and thrombosis/ bleeding risk.

\section{Reference(s)}

1. Fogarty PF, Tarantino MD, Brainsky A et al. Selective validation of the WHO Bleeding Scale in patients with chronic immune thrombocytopenia. Curr Med Res Opin. 2012;28(1):79-87

2. Rosenberg DJ, Press A, Fishbein J. et al. External validation of the IMPROVE bleeding risk assessment model in medical patients. J Thromb Haemost 2016; 116(3):530-6

3. Barbar S, Noventa F, Rossetto $V$ et al. A risk assessment model for the identification of hospitalized medical patients at risk for venous thromboembolism: the Padua Prediction Score. J Thromb Haemost 2010; 8(11):2450-57

4. Middeldorp S, Coppens M, van Haaps TF et al. Incidence of venous thromboembolism in hospitalized patients with COVID-19. J Thromb Haemost 2020: 18(8): 1995-2002

5. Helms J, Tacquard C, Severac F et al. High risk of thrombosis in patients with severe SARS-CoV-2 infection: A multicenter prospective cohort study. Intensive Care Med 2020; 46: 1089-98

\section{9}

\section{Does Amikacine increase the incidence of Acute Kidney injury} during septic shock ?

M. Benlabed'; S. Benlabed ${ }^{2}$; R. Gaudy ${ }^{3}$; S. Aissaouii ; S. Nedjari ${ }^{5}$

${ }^{1}$ Anesthesiology and intensive care, University of Lille, Lille, France; ${ }^{2}$ Saint pierre hospital, Université Libre de Bruxelles, Bruxelles, Belgium; ${ }^{3}$ Anesthesiology and intensivecare, University of Lille, Lille, France; ${ }^{4}$ Anesthesiology, Algiers university, Algiers, Algeria, Algeria; ${ }^{5}$ Anesthesiology, Algiers university, Alger Centre, Algeria, Algeria

Correspondence: M. Benlabed

Intensive Care Medicine Experimental 2020, 9(1): 000589

Introduction. Aminoglycoside have been shown to be associated in septic patients with an increased incidence of acute kidney injury (AKI)(1). However, few data exist on the renal effects of short-course adjunctive aminoglycoside therapy in patients with septic shock.

Objectives. The aim of our study was therefore to evaluate, in patients with septic shock, without previous risk factor for AKI,the effect of the combination piperacillin-tazobactam- Amikacin( PTA) vs piperacillin- tazobactam(PT) on the incidence of acute renal failure.

Methods. We carried out a prospective randomized controlled study in an ICU university hospital, from April 2018 to April 2020 and included 30 patients of average age $55+-9$ years, weighing $70+-5 \mathrm{~kg}$ and presenting post operative septic shock whose cause was of digestive origin.

We randomized 30 patients into 2 groups: one group receiving (PTA) and a second group receiving (PT) alone. SOFA score day 1 and Apache II score were not different between the 2 groups. Antibiotics were given after obtaining a mean arterial pressure(MAP) of at least $65 \mathrm{mmHg}$. Amikacin was administered during 3 days after a loading dose of $25 \mathrm{mg} / \mathrm{kg} /$ Total body weight and peak blood concentration was monitored.

All patients in each group were mechanically ventilated, and received crystalloid refill within the first $3 \mathrm{~h}$ to achieve an initial Pulse Pressure variation (PPV) of $10 \mathrm{mmHg}$. Invasive arterial pressure monitoring allowed continuous measurement of MAP and PPV.

Plasma creatinine level, urine output(UO) in $\mathrm{ml} / \mathrm{kg} /$ hour,,lactates, and hemodynamic parameters (MAP, PPV), were collected in each group at day 1 and day4 following administration of antibiotic therapy. Incidence of AKI Day14,according KDIGO criteria and day 28 mortality were evaluated in the 2 groups.

Results. Stastical analysis used Mann Whitney test and results expressed as mean + - standard deviation.

We observed, at $96 \mathrm{~h}$, that creatinine level(mg/l) was signicantly more elevated in PTA group than in PT group respectively $23+-5.76$ VS 13.7+-1.96. $\mathrm{P}<0.0001$.

Urine output in $\mathrm{ml} / \mathrm{kg} / \mathrm{h}$ during $6 \mathrm{~h}$ was also decreased significantly in PTA group compared to PT group respectively $43+-14.2$ VS $82.3+-6.22 p<0.0001$.

Amikacin peak serum concentration was in the normal range $>64 \mu \mathrm{g} / \mathrm{ml}$.

Hemodynamic parameters(MAP and PPV) and blood lactate level were not different between the 2 groups of patients,in the first $72 \mathrm{~h}$ of septic shock.

Incidence of AKI at day 14 was higher in PTA group than in PT group respectively $35 \%$ vs $15 \%$. Day 28 mortality was not different between PTA and PT groups respectively $32 \%$ vs $30 \%$.

Incidence of AKI and Mortality in PTA and PTgroups

\begin{tabular}{llll}
\hline $\begin{array}{l}\text { PARAMETERS } \\
\text { Creatinine mg/l Day 4 }\end{array}$ & $\mathbf{P T}$ & & PTA \\
\hline CRclearance ml/mn Day4 & $\mathbf{1 3 . 7 + - \mathbf { 1 . 9 5 }}$ & $\mathbf{2 3 + - 5 . 7 6}$ & $\mathbf{P}<\mathbf{0 . 0 0 0 1}$ \\
UFml/kg/h Day 4 & $82.3+-6.22$ & $43+-14.2$ & $\mathrm{P}<0.0001$ \\
Creatinine baseline mg/l & $13.1+-2.01$ & $13+-2$ & $\mathrm{P}<0.960$ \\
$\begin{array}{l}\text { Incidence of AKI } \\
\begin{array}{l}\text { Day14\% } \\
\text { Mortality Day 28\% }\end{array}\end{array}$ & $15 \%$ & $35 \%$ & \\
\hline
\end{tabular}

Conclusion. This study confirms that adjunction of aminoglycoside administered in the first $72 \mathrm{~h}$ of septic shock increase the risk of AKI without clinical benefit over antibiotic monotherapy in terms of outcome.Thus, the use of beta lactam-aminoglycoside combination therapy for sepsis should be discouraged.

\section{Reference(s)}

1. Taccone et al. Revisiting the loading dose of amikacin for patients with severe sepsis and septic shock. Critical Care 2010, 14: R53

2. Paul $\mathrm{M}$ et al. Beta lactam antibiotic monotherapy versus beta lactamaminoglycoside antibiotic combination therapy for sepsis. Cochrane Database of Systematic Reviews. 2014

3. Ong D.S.Y et al. Short-Course Adjunctive Gentamicin as Empirical Therapy in Patients With Severe Sepsis and Septic Shock: A Prospective Observational Cohort Study. Clinical Infectious Diseases. 2017;64(12):1731-6.

4. Thanks to the colleagues of Algiers University for participation to this Work.

000599

Association of Initial Hemodynamic Profiles to In-hospital Mortality of Critical COVID-19 patients in a Cardiology Referral

\section{Center}

M. Patricio ${ }^{1}$; AK. Longos, ${ }^{2}$; AD. $\operatorname{Tan}^{1}$; C. Tortosa ${ }^{1}$; JA. Cantre, ${ }^{1}$; C. Permejo, ; LMI. Habana,

'Department of Ambulatory, Emergency and Critical Care, Philippine Heart Center, Quezon City, Philippines; ${ }^{2}$ Department of adult cardiology, Philippine Heart Center, Quezon City, Philippines

Correspondence: M. Patricio 
Intensive Care Medicine Experimental 2020, 9(1): 000599

Introduction. Corona Virus Disease 2019 (COVID-19) has been declared by the World Health Organization as a global pandemic. Severe and Critical COVID-19 patients may develop shock from sepsis, cardiac injury and other mechanisms. There is limited data on their hemodynamic profile, both invasive and non-invasive. As a Cardiology Referral Center, different and multiple types of shock are anticipated.

Objectives. To determine the association of clinical and hemodynamic profiles to in-hospital Mortality of critical COVID-19 patients admitted at the ICU from March 2020 to January 2021.

Methods. COVID-19 confirmed patients admitted in the ICU from March 2020 to January 2021 were included. Clinical profile, laboratory, inflammatory and tissue perfusion indices were reviewed. Noninvasive evaluation of hemodynamics by echocardiography and pulse contour derived analysis were studied. Patients with central lines were evaluated for simultaneous testing of arterial and venous blood gases. Cardiac output (CO), cardiac index $(\mathrm{Cl})$ and systemic vascular resistance (SVR) was computed by Fick Method. Arterial-to-central venous content difference (PCO2 gap) was also computed. Associations with in-hospital mortality were determined.

Results. A total of 77 patients were included. Mean age was 61 years old with significant proportion having Acute Coronary Syndromes and Heart Failure. Acute respiratory distress syndrome was the most common complication at $63 \%$ followed by Acute Heart Failure at $48 \%$. Mortality rate was $56 \%$. Initial SOFA, qSOFA, serum lactate and inflammatory markers were predictive of mortality. Mean PCO2 gap was high at 9.9 but did not differ significantly between groups. Mean $\mathrm{Cl}$ was 2.29 and SVR was 1163, and also did not differ significantly between groups. Distributive, cardiogenic and combined shock were significantly associated with mortality. Hemodynamic Profile by Fick's Method

\begin{tabular}{|c|c|c|c|c|}
\hline \multirow[b]{3}{*}{$\begin{array}{l}\text { Cardiac Out- } \\
\text { put (L/min) }\end{array}$} & Total & Survival & Mortality & P-value \\
\hline & \multicolumn{3}{|c|}{ Frequency (\%); Mean \pm SD; Median (IQR) } & \multirow[b]{2}{*}{0.250} \\
\hline & $\begin{array}{l}3.85 \text { (3.30 to } \\
6.70)\end{array}$ & $\begin{array}{l}3.65 \text { (3.34 to } \\
3.70)\end{array}$ & $\begin{array}{l}5.09 \text { (3.16 to } \\
7.64)\end{array}$ & \\
\hline $\begin{array}{l}\text { Cardiac Index } \\
(\mathrm{L} / \mathrm{min} / \mathrm{m} 2)\end{array}$ & $\begin{array}{l}2.29(1.76 \text { to } \\
3.55)\end{array}$ & $\begin{array}{l}1.92(1.75 \text { to } \\
2.45)\end{array}$ & $\begin{array}{l}2.88(1.78 \text { to } \\
3.70)\end{array}$ & 0.329 \\
\hline $\begin{array}{l}\text { Stroke Volume } \\
\text { (mL/kg/ } \\
\text { beat) }\end{array}$ & $\begin{array}{c}48.48(28.58 \\
\text { to } 96.93)\end{array}$ & $\begin{array}{c}44(30.91 \text { to } \\
52.88)\end{array}$ & $\begin{array}{l}54.12(24.32 \\
\text { to } 127)\end{array}$ & 0.506 \\
\hline $\begin{array}{l}\text { Systemic } \\
\text { Vascular } \\
\text { Resistance } \\
\text { (dynes-s/ } \\
\text { cm5) }\end{array}$ & $\begin{array}{l}1163(770 \text { to } \\
1650)\end{array}$ & $\begin{array}{l}1606 \text { (895 to } \\
1821)\end{array}$ & $\begin{array}{l}1098 \text { (703 to } \\
1597)\end{array}$ & 0.226 \\
\hline
\end{tabular}

Initial Hemodynamic Profiles

\begin{tabular}{|c|c|c|c|c|}
\hline Initial Hemodynamic Profile & \multicolumn{3}{|c|}{ Frequency (\%) } & P-value \\
\hline Distributive Shock & 38 & 5 & 33 & $<0.001$ \\
\hline Cardiogenic Shock & 28 & 6 & 22 & 0.004 \\
\hline Obstructive Shock & 3 & 0 & 3 & 0.251 \\
\hline Multiple Types of Shock & 8 & 1 & 7 & 0.002 \\
\hline
\end{tabular}

Conclusion. Hemodynamic alternations from cardiogenic shock and distributive shock were frequently observed in COVID-19. Hemodynamic monitoring, tissue perfusion indices and inflammatory mediators were helpful in guiding management.
Reference(s)

1. World Health Organization. Director-General's remarks at the media briefing on 2019-nCoV on 11 February 2020. http://www.who.int/dg/speec hes/detail/who-director-general-s-remarks-at-the-media-briefing-on2019-ncov-on-11-february-2020 (Accessed on April 9, 2020).

\section{6}

Right Ventricle strain as acute predictor of outcome in patients undergoing mitral surgery: a pilot study

A. Russo ${ }^{1}$; E. BERGAMINI VIOLA ${ }^{1}$; A. Gambaro ${ }^{2}$; E. Bresadola'; E. Polati ${ }^{3}$;

L. Gottin ${ }^{1}$

${ }^{1}$ Cardio-thoracic anesthesia and intensive care unit, University of Verona,

AOUI-University Hospital Integrated Trust of Verona, Verona, Italy;

${ }^{2}$ Department of medicine, university of verona, Cardiology Division AOUI

Verona, Verona, Italy; ${ }^{3}$ Anesthesia and intensive care b unit, University

of Verona, AOUI-University Hospital Integrated Trust of Verona, Verona, Italy

Correspondence: A. Russo

Intensive Care Medicine Experimental 2020, 9(1): 000606

Introduction. Evaluation of right ventricular systolic function has important prognostic and treatment implications for many clinical scenarios. Its quantification should therefore be part of any routine examination, although the geometric complexity of the right ventricle makes it difficult with traditional echocardiography. The ultrasound technique of Strain and Strain Rate, developed to quantify regional myocardial function, is less dependent on the geometry of the right ventricle and its passive movement. It can provide subclinical right ventricular performance information.

Objectives. The purpose of this observational study is to evaluate the effectiveness of the longitudinal strain of the right ventricle as an early and acute predictor of postoperative right ventricular dysfunction in patients undergoing to mitral surgery. The primary endpoint was the correlation between pre-operative strain and pre-post delta strain with clinical and echocardiographic diagnosis of right ventricular dysfunction. The secondary endpoint was the correlation between strain and delta strain LOS in the ICU, ventilation days, trend of the pro-BNP and lactate in the first $48 \mathrm{~h}$, incidence of acute renal failure, ICU an 28 days mortality.

Methods. We collected the data for patients scheduled for mitral surgery. The exclusion criteria was congenital heart disease, other valvulopathies, severe coronary artery disease, age less than 18, pregnancy and absolute contraindications to TEE. All the clinical and hemodynamic parameters were collected at baseline before surgery (T1) and at the admission in ICU (T2). In T2 we evaluated clinical and echocardiographic diagnosis of right ventricular dysfunction, recorded LOS in ICU and in Hospital, mechanical ventilation duration and mortality at 28 days. TEE parameters were collected both in T1 and T2 and evaluated by a cardiologist offline.

Results. In the cohort of the 27 patients that we enrolled the mean pre- and post-operative strain value was respectively -16.44 (sd: 2.99 ) and -11.55 (sd: 3.64 ) and the mean delta-strain was 4.19 (sd: 3.27). The logistic analysis didn't showed a role of the delta-strain value in the onset of right $(p=0.889)$ or bilateral $(p=0.546)$ ventricular dysfunction. The pre-operative strain value alone was very close to statistical significance when introduced into the model. In particular, with an increase of 1 point in the pre-operative strain value the odds of right dysfunction increased by $54.81 \%(p=0.053)$ while the odds of bilateral dysfunction increased by $80.96 \%(p=0.058)$. Hypothetical predictors (such as age, sex, BMI, hypertension, etc.) have been previously tested with bivariate analysis without giving any statistical significance. No correlation between Strain-Pre or Strain Post and creatinine value, hours of ventilation or the number of days in ICU was found. We tried to identify a right ventricular strain cut-off predicting the development of right ventricular or biventricular dysfunction: a RV strain value of -14.98 had a sensitivity of $62.5 \%$ and a specificity of $100 \%$ in identifying right ventricular dysfunction and a sensitivity of $100 \%$ and specificity of $88.24 \%$ for identifying biventricular dysfunction.

Conclusion. Our study, albeit with a limited number, introduces a new parameter that could be used in the peri-operative and short-term evaluation to identify early patients at risk of postoperative cardiac 
dysfunction particularly right ventricular dysfunction. The sample we analysed seems to confirm our hypothesis on the strain: in fact, by analyzing the sample according to the presence of cardiac dysfunction (right/left or biventricular) or no dysfunction there is a significant difference, both in preoperative and postoperative analysis of the strain values. These results could, if confirmed in large numbers, support the use of this method as a parameter in evaluating the contractility of the right and biventricular sections in the immediate perioperative context as a cardiovascular risk stratification. This method, not a Point-of -care test, needs the images to be saved, and analyzed later with dedicated software; this could be a limitation of this technology.

\section{Reference(s)}

1. Francesco Maffessanti, MS, PhD, Paola Gripari, MD, Gloria Tamborini, MD, Manuela Muratori, MD, Laura Fusini, MS, Francesco Alamanni, MD, Marco Zanobini, MD, PhD, Cesare Fiorentini, MD, Enrico G. Caiani, PhD, and Mauro Pepi, MD, Milan, Italy Evaluation of Right Ventricular Systolic Function after Mitral Valve Repair: A Two-DimensionalDoppler, SpeckleTracking, and Three-Dimensional Echocardiographic Study Journal of the American Society of Echocardiography July 2012 2. Snejana Hyllen, MD, Shahab Nozohoor, MD, PhD, Annika Ingvarsson, MS, Carl Meurling, MD, PhD, Per Wierup, MD, PhD, and Johan Sjo€gren, MD, PhD Right Ventricular Performance After Valve Repair for Chronic Degenerative Mitral Regurgitation (Ann Thorac Surg 2014:98:2023-31) 3. Bonizzoli M, Cipani S, Lazzeri C, Chiostri M, Ballo P, Sarti A, Peris A. Speckle tracking echocardiography and right ventricle dysfunction in acute respiratory distress syndrome: $A$ pilot study. Echocardiography. 2018;35:1982-1987 4. Batur G, Mustafa K, Murat S, Altung C, Halil A, Alper K, Beste O. The impact of right ventricular function assessed by 2-dimensional speckle tracking echocardiography on early mortality in patients with inferior myocardial infarction. Clinical Cardiology. 2018; 41:413-418 5. Tejaswi J, Hashir K, Krishnananda N,

Umesh P, Tom D, Ramachandran P. A study of right ventricular function in pre and post-valvular surgeries. Interventional Medicine Applied Science. 2018 Sep; 10(3): 137-144. 6. Haddad F, Couture P, Tousignant C, Denault A. The Right Ventricle in cardiac Surgery, a perioperative perspective: I. Anatomy, Physiology, and Assessment. International Anesthesia Reserch Society. Vol.108, No. 2, February 2009 7. Haddad F, Couture P, Tousignant C, Denault A. The Right Ventricle in cardiac Surgery, a perioperative perspective: II. Pathophysiology, Clinical Importance, and Management. International Anesthesia Reserch Society. Vol.108, No. 2, February 2009 8. Muraru D, Onciul S, Peluso D, Soriani N, Cucchini U, Aruta P, Romeo G, Cavalli G, lliceto S, Badano L. Sex- And Method-Specific Reference Values for Right Ventricular Strain by 2 Dimensional Speckle tracking Echocardiography. Circ Cardiovasc Imaging. 2016;9:e0038669. Julious SA. Sample size of 12 per group rule of thumb for a pilot study. Pharm Stat 2005:4:287-291 10. Sim J, Lewis M. The size of a pilot study for a clinical trial should be calculated in relation to considerations of precision and efficiency. J Clin Epidemiol 2012;65:301-308 11. Lancaster GA, Dodd S, Williamson PR. Design and analysis of pilot studies: recommendations for good practice. J Eval Clin Practice 2004;10:307-312

\section{7}

\section{Cardiovascular complications in severe COVID-19 infection}

I. Sdiri' ${ }^{1}$ K. Benismail' ${ }^{1}$ S. Boutheina ${ }^{1}$; I. TALIK' ${ }^{1}$ K. Moez ${ }^{1}$;. Merhabene ${ }^{1}$ Intensive care unit, Regional HospitalZaghouan, Zaghuan, Tunisia Correspondence: I.K. Ben

Intensive Care Medicine Experimental 2020, 9(1): 000607

Introduction. The outbreak of 2019 novel coronavirus disease (COVID19) began since early December 2019 and has been quickly declared as a public health emergency by the World Health Organization. Due to the hypercoagulable state, blood stasis and endothelial injury, severe COVID-19 patients are at high risk to developeither venous or arterialthrombo-embolic events (TEE).

Objectives. to estimate incidence, risk factors and outcome of patients confirmed COVID-19with TEE or cardiovascular complications (CVC).

Methods. A retrospective analysis was conducted on 200patients with COVID-19 hospitalized in ICU Zaghouan's Hospital between 24th March 2020 and 31th January 2021.Pulmonary embolism was systematically searched in patients with persistent hypoxemia or secondary respiratory deterioration.

Results. Two hundred patients were enrolled.mean age was 68 years [34-92]. Sex-ratio was 1.8 and $44 \%$ of patients were over 65 years. Seventy-nine patients (65.3\%) were obese (IMC $\geq 30 \mathrm{~kg} / \mathrm{m} 2)$. Mean SAPS II, Charlson and APACHE II scores were 28.4 \pm 8 [5-42]; $2.8 \pm 1$ [0-7]; $7.7 \pm 3$ [1-27] respectively. Comorbidities were present in $52.3 \%$ of cases; it was mainly cadiovascular (59\%) and diabetes (35.5\%)0.182 Patients were treated with curative anticoagulation and only 18 patients had preventive one.Forty-nine patients developed TEE and/ or CVC in our study (incidence of 24.5\%).Major detectedmanifestation was pulmonaryembolism $(47,1 \%)$, it was classified at high risk mortality in $20 \%$ of cases. Severe cardiac arrythmia and acute coronary syndrome werenoted in $17,4 \%$.The other events were acute limb ischemia $(4,3 \%)$, cerebral stroke $(4,3 \%)$ and myocarditis $(4,3 \%)$. Ninety one percent of thromboembolic events occurred in previously anticoagulated patients.Patients who developed TEE and/or CVC were significantly older ( $64.9 \pm 10$ vs $61.3 \pm 12.8$ years, $P=0.038)$, more frequently active smokers $53 \%$ vs $28 \%, P=0.02)$, obese $(72 \%$ vs $63 \%, P=0.049)$, hypertensive $(57 \%$ vs $43 \%, \mathrm{P}=0.041)$ and hypoxemicon admission $(P / F=187$ vs $269 \mathrm{mmHg}, \mathrm{p}=0.036)$. TEEoccurred mainly at the eight hospital day[2-12]. TTE and/or CVC hadworse impact on patient's clinical course with longer hospital stay ( 14 days vs $8, p=0.02)$, more frequent need to invasive mechanical ventilation ( $52 \%$ vs $41 \%, p=0.03$ ) and highermortality $(57 \%$ vs $21 \%, p=0.01)$.

Conclusion. Incidence of TTE/CVC in COVID-19 treated with therapeutic anticoagulation is high. Our results suggest considering both systematic screening of thrombo-embolic complication and early therapeutic anticoagulation in severe ICU COVID-19 patients.

\section{6}

Central venous pressure-a predictor of 28-day-mortality and renal failure in patients with left and temporary right ventricular assist device?

P. Schukro'; M. Mouhieddine, ; E. Tschernko'; U. Weber

${ }^{1}$ Division of cardiac thoracic vascular anaesthesia and intensive care, Medical University of Vienna, Wien, Austria

Correspondence: P. Schukro

Intensive Care Medicine Experimental 2020, 9(1): 000676

Introduction. Patients with heart failure und left ventricular assist device therapy have been shown to have a higher mortality and higher risk of worsening of renal function when having higher central venous pressure (Tsay et al., 2020).

Acute deterioration of renal function in combination with a reduced right ventricular function is associated with a higher one-year mortality (Borgi et al., 2013).

Objectives. The aim of our retrospective study was to investigate whether central venous pressure is a risk factor of higher mortality and worsening of renal function despite the use of a temporary right ventricular assist device (temporary RVAD).

Methods. A total of 32 patients received a left ventricular assist device (Heart Mate II or HeartWare) AND a temporary right ventricular assist device between 2017 and 2020 in our tertiary care centre. Mean central venous pressure of each day and daily creatinine levels were correlated with 28-day-mortality and analyzed as primary outcome parameters during their stay in our intensive care unit. Secondary outcome parameters included length of stay, age, gender and duration of temporary RVAD therapy. We compared mean CVP levels for each day and highest daily creatinine level in the survivor and non-survivor group. For comparing the two groups, a two-way ANOVA was calculated.

Results. We included 32 patients in our retrospective cohort analysis, $5(15.6 \%)$ patients died during the first 28-days. There was no statistically significant difference between length of stay, SAPS3 score or age between the groups. Mean CVP in survivors differed significantly from mean CVP in non-survivors $(p<0,001)$. Mean creatinine levels were significantly lower in the survivor group than in the non-survivor group $(p=0,001)$. There was no significant difference between the two groups concerning time of removal of temporary RVAD 16 days in survivors vs 17 days in non-survivors. 


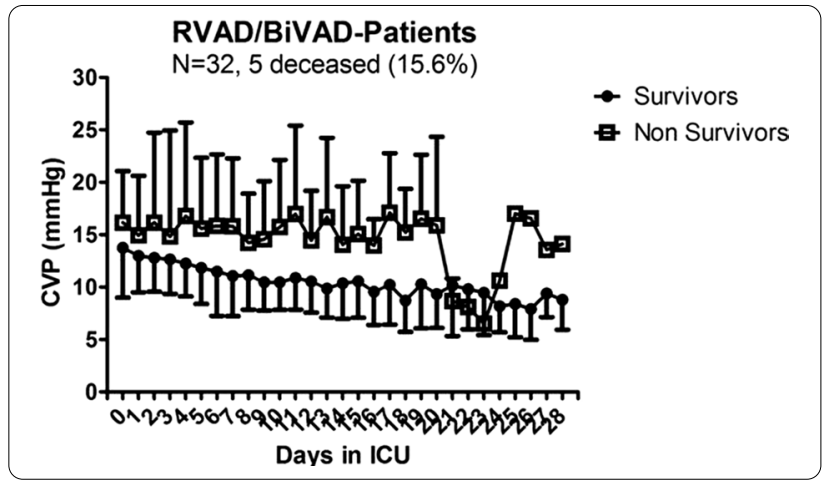

Conclusion. Survivors with left ventricular assist device and temporary right ventricular assist device have significantly lower CVP values compared to non-survivors. Furthermore, they also have significantly lower creatinine levels during ICU stay. Therefore, high CVP seems of central interest in prediction of mortality and development of renal failure in this population. Further analysis of additional parameters in patients with temporary RVAD and left ventricular assist device is needed.

\section{Reference(s)}

1. Jamil Borgi, Athanasios Tsiouris, Arielle Hodari, Chad M Cogan, Gaetano Paone, Jeffrey A Morgan;Significance of postoperative acute renal failure after continuous-flow left ventricular assist device implantation; Ann Thorac Surg. 2013 Jan:95(1):163-9.

2. Julie Tsay, MD a, Daniel Pinkhas, DO, Bryan C. Lee, MD, Aaron Guo, BS, Joel Ferrall, BS, Mohamed H. Derbala, MD, Brent C. Lampert, DO, Sitaramesh Emani, MD, Bryan A. Whitson, MD, PhD, Sakima A. Smith, MD, MPH;Worsening Renal Function in Cardiac Mechanical Support; Heart, Lung and Circulation (2020) 29, 1247-1255 1443-9506/19

\section{0}

Transthoracic Echocardiography (TTE) in Prone ventilated Patients with COVID-19 related ARDS. A Feasibility Study MY. KALLALA ${ }^{1}$; E. Bedhiafi'; K. Meddeb ${ }^{1}$; R. Toumi ${ }^{2}$;. Hassen ${ }^{1}$;

J. Mahmoud ${ }^{1}$; C. Ben Rhouma ${ }^{1}$; M. zghidi'; I. Ben Saida²; M. Boussarsar ${ }^{1}$ ${ }^{1}$ Medical intensive care unit, Farhat Hached University Hospital, Sousse, Tunisia; ${ }^{2}$ Farhat hached university hospital, medical intensive care unit, Université de Sousse, Faculté de Médecine de Sousse, UR Nº LR12SP09. Heart Failure, Sousse, Tunisia

Correspondence: M.Y. KALLALA

Intensive Care Medicine Experimental 2020, 9(1): 000700

Introduction. COVID-19 pandemic has resulted in an increase in the number of patients admitted with ARDS. Many will require prone positioning ventilation (PPV). The use of TTE, a bedside reliable, reproducible and noninvasive tool to guide hemodynamic management, could be altered within long proning sessions.

Objectives. To assess the ability to perform focused, reliable TTE examinations on COVID-19 related ARDS patients undergoing PPV.

Methods. This is a single-centre, observational, cross-sectional study of patients admitted for ARDS due to COVID-19 pneumonia, in a 12-bed MICU in Tunisia during December 2020, using VIVID T8 echocardiogram. Several frequencies and harmonics were tested. The low fundamental frequency $(2.4 \mathrm{MHz})$ was found to provide best image quality. TTEs were performed $1 \mathrm{~h}$ to $30 \mathrm{~min}$ before PPV, then repeated in PP after a stabilization period. In supine position, basic TTE views have been used.

Prone position was set in proclive $45^{\circ}$ using the "swimmer position" with a pillow at thoracic level. The operator is positioned to the left of the patient and uses his left or right hand to position the transducer. The examinations were performed as part of routine clinical assessment.

Results. Eleven patients were included. $8(72.7 \%)$ patients were male. The mean age was $67.6 \pm 9.4$ years. $5(45 \%)$ were obese. $100 \%(n=11)$ were deeply sedated and curarized. PPV ultrasound was performed 4 to $8 \mathrm{~h}$ (mean $=5.8 \pm 1.5 \mathrm{sd} \mathrm{hrs}$ ) after PPV setting. In supine position, apical four chamber (4C), parasternal long axis (PLAX) and parasternal short axis were successfully obtained for the whole cohort, subcostal $4 C$ and subcostal inferior vena cava (IVC) were obtained in $75 \%(n=8)$ of the examinations. The average duration of a scan was $20 \pm 10 \mathrm{~min}$. PPV ultrasound afforded all apical views and measures. A special view dedicated to the IVC was obtained. The dedicated RV and the $4 C$ views were obtained in $100 \%$ of patient, and the IVC view in $75 \%(n=8)$; the PLAX was not visualized in any of the patients (0\%). ASE and EACVI validated quantitative parameters were used to assess biventricular function and volemia.

Conclusion. In the present study, PPV TTE remains simple, rapid and provides the required information especially volemia and right ventricle performance.

\section{Reference(s)}

1. Ugalde, Diego; Medel, Juan Nicolás; Romero, Carlos; Cornejo, Rodrigo (2018). Transthoracic cardiac ultrasound in prone position: a technique variation description. Intensive Care Medicine

2. Marvaki A, Papachristidis A, Nakou E, Toth E, O'Gallagher K, Fisher R, Shah AM, Monaghan MJ, Innovative transthoracic echocardiographic imaging on prone ventilated patients with COVID-19 using a transesophageal probe, JACC: Cardiovascular Imaging (2020)

\section{7}

Impact of misdiagnosis in acute type $A$ aortic dissection: experience of a tertiary referral center

G. Abi Abdallah'; D. Zlotnik'; N. Polge ; C. Porte ${ }^{2}$;W. Sutter ${ }^{3}$; P. Achouh ${ }^{4}$; F. Bellenfant ${ }^{2}$; A. Godier ${ }^{1}$

'Department of anaesthesiology and critical care, European Hospital Georges Pompidou, Assistance Publique-Hôpitaux de Paris, AP-HP, and Université de Paris, INSERM UMRS-1140 Paris, France; ${ }^{2}$ Department of anaesthesiology and critical care, European Hospital Georges Pompidou, Assistance Publique-Hôpitaux de Paris, AP-HP, Paris, France; ${ }^{3}$ Department of cardiovascular surgery, vascular surgery unit, European Hospital Georges Pompidou, Assistance Publique-Hôpitaux de Paris, AP-HP, and Université de Paris, INSERM U970, Paris, France; ${ }^{4}$ Department of cardiovascular surgery, cardiac surgery unit, European Hospital Georges Pompidou, Assistance Publique-Hôpitaux de Paris, AP-HP, and Université de Paris, INSERM U970, Paris, France

Correspondence: D. Zlotnik

Intensive Care Medicine Experimental 2020, 9(1): 000727

Introduction. Acute type A aortic dissection (ATAAD) is a life-threatening condition requiring urgent aortic surgery. As ATAAD has a myriad of clinical manifestations, challenges in the diagnostic process can result in initial misdiagnosis, associated with delay in surgical management and inappropriate treatment, including antithrombotics, which may increase perioperative bleeding and jeopardize the outcome.

Objectives. To determine the rate of initial misdiagnosis in patients with ATAAD and the impact on outcome.

Methods. Retrospective study (CPP approval) including all patients who underwent urgent surgery $(<48 \mathrm{~h})$ for ATAAD in one tertiary referral center from August 2017 to March 2021. We compared patients with initially correct diagnosis of ATAAD to those with initial misdiagnosis, with or without misdiagnosis-induced treatments (MDIT). We collected demographics and clinical data, time from initial medical care to surgery, intraoperative transfusion requirement and MDIT including antiplatelets, anticoagulants, thrombolysis and coronary angiography. Primary outcome was hospital mortality. Secondary outcomes included major bleeding (BART study definition[1]), transfusion requirement and delay for surgery. Data are presented as median [interquartiles] or number (\%). Comparisons were performed 
using Wilcoxon rank-sum tests or Fisher's exact test. A p-value $<0.05$ was considered statistically significant.

Results. Among 148 patients with ATAAD (males 66\%, 68 [57-74] years), 67 patients $(45 \%)$ were initially misdiagnosed (acute coronary syndrome in 22 patients (15\%), stroke in 16 patients (11\%) and pulmonary embolism in 14 patients (9\%) (Figure)).

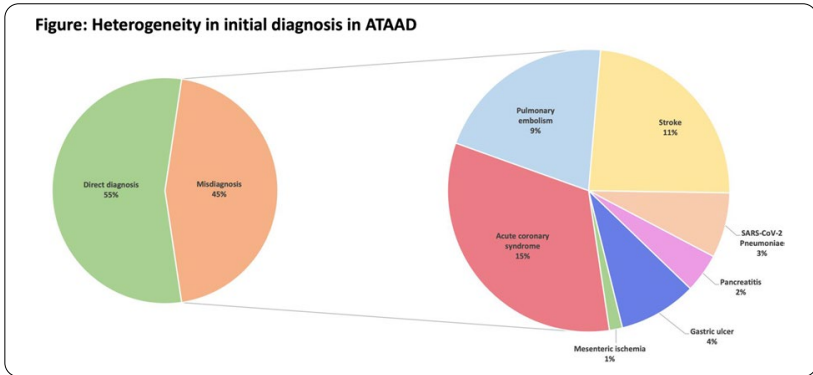

Twenty-seven patients (18\%) had MDIT before diagnosis correction: $44 \%$ of them had endovascular intervention (coronary angiography or thrombectomy) and all received treatment that interfered with haemostasis: loading dose of antiplatelets in $85 \%$ of cases, anticoagulants in $70 \%$, thrombolysis in 7\%. MDIT resulted in fewer early surgical managements $(<6 h)(p=0.012)$. All patients with MDIT had transthoracic echocardiography resulting in correction of the diagnosis in $26 / 27$ patients (96\%). MDIT was associated with more major bleeding (table) and more intra operative transfusion requirements, including red blood cells, fresh frozen plasma and platelet transfusions. Finally, MDIT was associated with higher 30-day mortality.

\begin{tabular}{|c|c|c|c|}
\hline & $\begin{array}{r}\text { Initially correct diagnosis } \\
\text { or misdiagnosis without } \\
\text { treatment }\end{array}$ & $\begin{array}{r}\text { Misdiagnosis with } \\
\text { treatment }\end{array}$ & $p$ value \\
\hline & $n=121$ & $\mathrm{n}=27$ & \\
\hline Major bleeding & 13/116 (11\%) & $11 / 26(42 \%)$ & 0.0005 \\
\hline RBC transfusion (unit) & $3[1-5]$ & $5[3-8]$ & 0.004 \\
\hline Platelet transfusion (unit) & $8[7-14]$ & $10[9-19]$ & 0.02 \\
\hline Fresh Frozen Plasma transfusion (unit) & $3[3-5]$ & $6[4-8]$ & 0.007 \\
\hline 30-Day mortality & $23(19 \%)$ & $12(44 \%)$ & 0.004 \\
\hline
\end{tabular}

Conclusion. Initial misdiagnosis of ATAAD was a frequent issue and led to inappropriate treatment for $18 \%$ of patients, resulting in increased major bleeding, transfusion requirement, and mortality. Echocardiography seems to be an interesting tool for rapid diagnosis correction. These results need to be confirmed in further studies.

\section{Reference(s)}

1. 1.Fergusson DA, Hébert PC, Mazer CD, et al. (2008) A comparison of aprotinin and lysine analogues in high-risk cardiac surgery. N Engl J Med 358:2319-2331. https://doi.org/10.1056/NEJMoa0802395

\section{7}

\section{Evaluation of Left Ventricular Microvascular Ischemia} during Vaso-Occlusive Crisis in the ICU using Global Longitudinal

\section{Strain}

G. ROGER ${ }^{1}$;T. Gobé ${ }^{1}$; JL. Teboul ${ }^{1}$; C. Lai ${ }^{1}$; A. Pavot ${ }^{1}$; N. Fage ${ }^{1}$;T. Pham¹;

X. Monnet

${ }^{1}$ Médecine intensive_réanimation, inserm umr s_999, fhu sepsis, groupe de recherche carmas, Bicetre Hospital AP-HP Université Paris-Saclay DMU CORREVE, Le Kremlin-Bicêtre, France

Correspondence: G. ROGER

Intensive Care Medicine Experimental 2020, 9(1): 000747.

Introduction. Cardiopulmonary complications are the main cause of death in adults with Sickle Cell Anaemia (SCA). The pathophysiology of cardiac dysfunction in SCA is debated. A recent experimental study in mice demonstrated that the driver of cardiac abnormalities in SCA is ischemic-scattered cardiomyocytes loss [1]. Global longitudinal strain (GLS) measured using speckle tracking echocardiography is correlated with microvascular obstruction in other settings. Our hypothesis was that repeated evaluation of GLS in patients hospitalized in the Intensive Care Unit (ICU) for Vaso-Occlusive Crisis (VOC) would be abnormal in patients with elevated troponin levels. Methods. This is an observational prospective study in a 25-bed ICU. We prospectively recruited consecutive patients presenting with VOC. Exclusion criteria were age $<18$ years, pregnancy, inability to consent and poor echogenicity. We recorded GLS and troponin Ic level at day-1, day-2, day-3 and end of the ICU stay.

Results. From January to April 2021, we recorded 20 cases of VOC in 16 different patients (age: $29( \pm 9)$ years, 50\% female, 85\% haemoglobin SS genotype). The median number of echocardiography with GLS measurement was 3 (IQR [2;3]). Patients received morphine in $17(85 \%)$ cases and ketamine in $6(30 \%)$ cases. VOC was associated with acute chest syndrome in $4(20 \%)$ cases and pulmonary embolism in $1(5 \%)$ case. The median length of stay in the ICU was 4 days (IQR [3;4]). GLS (normal value $<-16 \%$ [2]) was $-18,7( \pm 2.3) \%$, $-17,9( \pm 2.0) \%$ and $-18,8( \pm 2.8) \%$ at day-1, -2 and -3 , respectively. It was >-16\% in $2(10 \%), 2(10 \%)$ and $2(10 \%)$ patients at day-1, -2 and -3 , respectively. Troponin Ic was elevated in only two patients. In one of them, troponin Ic was elevated from day-2 to the end of the ICU stay and GLS remained normal at any time. In the second patient who suffered from pre-existing cardiomyopathy, troponin Ic was elevated from day- 1 to day- 2 and GLS was depressed from day- 1 to day-3.

Conclusion. In 20 cases of VOC hospitalized in ICU, troponin elevation was rare and was not related to GLS modification. The study is ongoing.

\section{Reference(s)}

1. Bakeer N, James J, Roy S, et al. Sickle cell anemia mice develop a unique cardiomyopathy with restrictive physiology. Proc Natl Acad Sci U S A. 2016;113(35):E5182-E5191.

2. D'Elia N, Caselli S, Kosmala W, et al. Normal Global Longitudinal Strain: An Individual Patient Meta-Analysis. JACC Cardiovasc Imaging. 2020;13(1 Pt 1):167-169.

\section{4}

Active fixation leads for prolonged temporary pacing

MD. Pola'; MC. Molina-De-La-Torre'; R. Vela-Colmenero';

M. Martínez-Arcos' ; M. Ruiz-Bailén, ; A. Castillo-Rivera

${ }^{1}$ Intensive Care Medicine, Servicio Andaluz de Salud, Jaén, Spain

Correspondence: M.D. Pola

Intensive Care Medicine Experimental 2020, 9(1): 000754

Introduction. Conventional leads for temporary heart pacing are unstable and can be complicated in up to $10 \%$ of patients, especially in long term provisional pacing. Active fixation leads usually used in permanent pacing can be an alternative in prolonged provisional pacing.

Objectives. To assess safety and usefulness of active fixation leads for prolonged temporary pacing in patients awaiting permanent pacing.

Methods. We included 6 patients who need permanent pacing and had coexisting sepsis or infection that contraindicate immediate pacemaker implant so they needed prolonged provisional pacing until infection resolution.

All patients were implanted with an active fixation lead that was connected to an external generator, both usually used in permanent pacing. The lead (Medtronic $507258 \mathrm{~cm}$ ) was inserted by right subclavian vein to right ventricle apex where are distally fixed and proximally fixed to skin. Generator (St Jude Medical Endurity Core PM 1152) was also fixed to skin on upper thorax and was reusable for all patients and exclusively used for this purpose.

Variables.

Age.

Sex.

Pacing indication. 
Prolonged temporal pacing indication.

Days of provisional pacing.

Mortality.

Lead complications.

- Lead dislocation and loss of capture.

- Cardiac perforation.

- Lead infection.

Results. Mean age of patients was $78,00 \pm 8,79$ years, 3 men and 3 women.

Pacing indication was high degree atrioventricular block in 4 patients, synus node disfunction in 1 and other patient with cardiogenic shock and sustained ventricular tachycardia that needed high rate ventricular pacing to control ventricular tachycardia awaiting DAI.

Mean days of provisional pacing was 15,50 $\pm 10,23$.

There were no lead complication in any patient. One patient died because cardiogenic shock (no attributable to pacing or lead).

Patients with active fixation leads were discharged of ICU in 24-72 h to conventional hospitalization and were early mobilizated even walking during temporal pacing.

\begin{tabular}{|c|c|c|c|c|c|c|c|}
\hline $\mathrm{N}$ & Age & Sex & $\begin{array}{l}\text { Pacing } \\
\text { indica- } \\
\text { tion }\end{array}$ & $\begin{array}{l}\text { Pro- } \\
\text { longed } \\
\text { tem- } \\
\text { porary } \\
\text { pacing }\end{array}$ & $\begin{array}{l}\text { Days } \\
\text { tem- } \\
\text { porary } \\
\text { pacing }\end{array}$ & $\begin{array}{l}\text { mortal- } \\
\text { ity }\end{array}$ & $\begin{array}{l}\text { Lead } \\
\text { complica- } \\
\text { tions }\end{array}$ \\
\hline 1 & 65 & W & $\begin{array}{l}\text { Synus } \\
\text { dis- } \\
\text { func- } \\
\text { tion }\end{array}$ & Sepsis & 33 & $\mathrm{~N}$ & $N$ \\
\hline 2 & 67 & M & AV block & Fever & 11 & $N$ & $N$ \\
\hline 3 & 85 & M & AV block & Fever & 16 & $N$ & $N$ \\
\hline 4 & 82 & M & AV block & $\begin{array}{l}\text { Pacemaker } \\
\text { pocket } \\
\text { infec- } \\
\text { tion }\end{array}$ & 15 & $N$ & $N$ \\
\hline 5 & 84 & W & AV block & Fever & 4 & $\mathrm{~N}$ & $\mathrm{~N}$ \\
\hline 6 & 74 & W & $\begin{array}{l}\text { Ven- } \\
\text { tricular } \\
\text { tachy- } \\
\text { cardia }\end{array}$ & $\begin{array}{l}\text { Cardio- } \\
\text { genic } \\
\text { shock }\end{array}$ & 22 & Y & $\mathrm{N}$ \\
\hline
\end{tabular}

Conclusion. Active fixation leads can be an alternative to conventional leads when prolonged temporary pacing is needed. Patients can be early discharged of ICU with temporary pacing as long as 15 days and benefit of early mobilization.

\section{1}

Short and long-term survival in cardiogenic shock supported by veno-arterial Extracorporeal Membrane Oxygenation A. Marcos-Morales ${ }^{1}$; JL. PerezVela'; MB. Isaías'; M. Corres Peiretti'; R. Emilio ${ }^{1}$

${ }^{1}$ Intensive Care, Hospital Doce de Octubre, Madrid, Spain Correspondence: A. Marcos-Morales

Intensive Care Medicine Experimental 2020, 9(1): 000761

Introduction. Cardiogenic shock (CS) is an often fatal condition stemming from some of the most prevalent diseases in the world(1), and veno-arterial extracorporeal membrane oxygenation (VA-ECMO) used as a rescue therapy for refractory cases is growing in popularity(2). We evaluated short-term survival and after a follow-up of 1 year in these patients, classified by cause of cardiogenic shock.

Methods. Data was collected prospectively from 2010 to 2021, patients with CS having received VA-ECMO were reviewed retrospectively. We analysed baseline demographic variables, cause of CS, ECMO characteristics, complications and global survival (at ECMO weaning, hospital discharge, and after 1 year), using SPSS software, version 26.

Results. We included 102 patients who received 109 VA-ECMO. The median age was 54 (interquartile range IQR 42-62), 64\% were male.
Causes of CS were: decompensated advanced heart failure (29\%), acute myocardial infarction (AMI) (26\%), post-cardiotomy, (25\%), post-surgical pulmonary thromboendarterectomy (PTE) or pulmonary emboslim $(12 \%)$, and acute myocarditis from infectious, toxic or metabolic causes (7\%).

A peripherally VA-ECMO was established in $86 \%$ of cases. The median length of ECMO was $192 \mathrm{~h}$ (8 days, IQR 5,5-13), median ICU stay 19 days (IQR 13-33 days).

The global survival rate during ECMO support was $68 \%$, with $45 \%$ of patients being discharged home, and all of them were alive one year later (all but one who didn't complete follow up). Patients with reversible causes of cardiogenic shock (acute myocarditis, right ventricular failure following pulmonary embolism) fared better overall, with a $100 \%$ survival rate.

In $57 \%$ of patients with CS secondary to AMI a "bridge to decision" strategy was implemented. Sixty percent were weaned from ECMO, and $44 \%$ of weaned patients survived. A heart transplantation was made in $86 \%$ of the survivors of this subgroup.

Patients with CS secondary to acute decompensation of previous cardiopathy were usually placed in the "bridge to transplant" category. Out of 32 patients, $66 \%$ were weaned from ECMO, almost all of them due to heart transplantation. Seventy-nine percent of transplanted patients were alive at 1 year follow-up. Global survival was of $50 \%$.

VA-ECMO due to right ventricule failure was required after PTE in 9 patients, and also due to congenital cardiopathy surgery and after pulmonary embolism. In two PTE cases a VV-to-VA conversion was needed. Weaning from ECMO was achieved in $70 \%$ patients, and global survival was of $42 \%$.

One year after hospital discharge, $100 \%$ out of the 42 surviving patients were still alive and out of the hospital, and 4 have been discharged but haven't completed 1 year of follow-up.

Conclusion. VA-ECMO offers an effective support tool for these patients, achieving favorable results at hospital discharge and after 1 year of follow-up.

\section{Reference(s)}

1. Jones TL, Nakamura K, McCabe JM. Cardiogenic shock: evolving definitions and future directions in managementOpen Heart 2019;6:e000960. https://doi.org/10.1136/openhrt-2018-000960

2. Makdisi G, Wang IW. Extra Corporeal Membrane Oxygenation (ECMO) review of a lifesaving technology. J Thorac Dis. 2015;7(7):E166-E176. 10. 3978/j.issn.2072-1439.2015.07.17

000821

Predicting volume responsiveness in an experimental model

\section{of aortic repair}

M. Gräßler'; C. Behem ${ }^{1}$; A. Dupree ${ }^{2}$; DA. Reuter ${ }^{3}$; E. Debus, ${ }^{4}$; SH. Wipper ${ }^{5}$; CJC. Trepte ${ }^{6}$

${ }^{1}$ Department of anesthesiology, University Medical Center Ham-

burg-Eppendorf, Hamburg, Germany; ${ }^{2}$ Departmet of general, visceral and thoracic surgery, University Medical Center Hamburg-Eppendorf, Hamburg, Germany; ${ }^{3}$ Department of anaesthesia and intensive care medicine, University Medical Center Rostock, Rostock, Germany; ${ }^{4}$ Department of vascular medicine, University Heart Center Hamburg, Hamburg, Germany; ${ }^{5}$ Vascular surgery, Medical University of Innsbruck, Innsbruck, Austria; ${ }^{6}$ Anesthesiology, University Medical Center Hamburg-Eppen-

dorf, Hamburg, Germany

Correspondence: M. Gräßler

Intensive Care Medicine Experimental 2020, 9(1): 000821

Introduction. Dynamic indicators of preload like pulse pressure variation (PPV) and stroke volume variation (SVV) have widely been shown as the best predictors of volume responsiveness in mechanically ventilated patients. Appropriate and goal directed fluid therapy is a key element of peri-operative haemodynamic management especially in patients undergoing high-risk surgery such as thoracoabdominal aortic repair. (1) In such patients maintenance of adaequate haemodynamic state aiming for optimal tissue perfusion and oxygenation is crucial for morbidity and mortality. Several studies showed that changes in cardiac afterload and systemic vascular 
resistance might influence the values of PPV and SVV and their ability to predict volume responsiveness. However the effect of changes in vascular properties on these parameters has not been systematically assessed so far. Therefore it was the aim of this study to evaluate the ability of PPV and SVV to predict volume responsiveness in a experimental model of aortic repair.

Methods. The study was designed as a prospective trial in 18 domestic pigs. Animals were anaesthetized and mechanically ventilated. A micro- tip catheter was placed in the right femoral artery for blood pressure recordings and calculation of pulse pressure variation. An ultrasonic flow-probe was placed around the descending aorta for measurement of aortic flow and calculation of stroke volume variation. 4 consecutive volume loading steps, each consisting of $6 \mathrm{ml} / \mathrm{kg}$ bodyweight hydroxyethyl starch, were performed under baseline conditions and repeated $6 \mathrm{~h}$ after hybrid aortic repair. Hybrid aortic repair was performed using a hybrid graft consisting of a thoracic stent and open re- insertion of the abdominal and iliacal branches. An increase in stroke volume $>10 \%$ was considered as a positive volume response. The area under the ROC Curve (AUC) was used as a marker of predicting ability.

Results. PPV showed a AUC of 0.854 before and 0.768 after aortic repair. The AUC for SVV was 0.846 before and 0.779 after aortic repair. For PPV the cut- off values were significantly higher after aortic repair. For SVV cut- off values no significant difference could be distinguished.

Conclusion. Pulse pressure variation and stroke volume variation are able to properly predict volume responsiveness after thoracoabdmonial aortic repair. The cut-off values for pulse pressure variation change significantly after aortic repair.

\section{Reference(s)}

1. Kubitz JC, Annecke T, Forkl S, Kemming GI, Kronas N, Goetz AE, Reuter DA. Validation of pulse contour derived stroke volume variation during modifications of cardiac afterload. Br J Anaesth. 2007 May;98(5):591-7.

2. NICE Guideline Updates Team (UK). Goal-directed therapy during repair of unruptured and ruptured abdominal aortic aneurysms: Abdominal aortic aneurysm: diagnosis and management: Evidence review $\mathrm{M}$. London: National Institute for Health and Care Excellence (UK); 2020 Mar. PMID: $32,407,033$

3. The study was supported by departmental funds of the Department of Anaesthesiology, University Medical Centre Hamburg-Eppendorf

\section{7}

\section{Cardiac output estimated by thermodilution and indirect Fick} differs: A prospective validation study in a cardiac intensive care unit

J. Grand ${ }^{1}$; C. Hassager ${ }^{2}$; L. Obling, ${ }^{3}$; B. Nyholm, ${ }^{3}$;. Josiassen ${ }^{3}$; J. Kjaergaard ${ }^{2}$ ${ }^{1}$ Department of cardiology, BørneRiget—Rigshospitalet, København, Denmark; ${ }^{2}$ Department of cardiology, Rigshospitalet, København, Denmark; ${ }^{3}$ Department of cardiology, Rigshospitalet, Copenhagen, Denmark Correspondence: J. Grand

Intensive Care Medicine Experimental 2020, 9(1): 000867

Introduction. In cardiac intensive care patients, cardiac output (CO) is often an important clinical variable to guide treatment. For determination of $\mathrm{CO}$, the thermodilution method is routinely used in many intensive care units (ICUs), but CO can also be assessed in a noninvasive approach by the indirect Fick principle.

Objectives. To describe the relationship between two methods of $\mathrm{CO}$ determination in comatose patients admitted to a cardiac ICU.

Methods. A prospective method-validation study for quality insurance was performed between April and August 2020. We included all patients admitted to the cardiac ICU, who were unconscious, mechanically ventilated and already had a pulmonary artery catheter for hemodynamic monitoring. We measured $\mathrm{CO}$ with thermodilution (PAC_CO) and indirect Fick principle (Fick_CO) simultaneously. Results were compared with linear regression and Bland-Altman plots. For Fick_CO, we used CARESCAPE Respiratory Module (GE Healthcare).

Results. We included 24 patients with a mean age of $61( \pm 11)$ years, $86 \%$ were men and $80 \%$ percent were resuscitated, comatose cardiac arrest patients. Echocardiography excluded significant intracardiac shunts and valvular disease in all patients. $50 \%$ of the patients were undergoing targeted temperature management at $36 \mathrm{C}$ at the time of measurements. We repeated the measurements in the same patients on day 2 and 3 if possible and ended up with a total of 41 paired measurements. Average PAC_CO was $4.96( \pm 1.77) \mathrm{L} / \mathrm{min}$. and Fick_CO was 7.89 ( \pm 1.97$) \mathrm{L} / \mathrm{min}$., with a mean bias (PAC_CO lower) of $-2.97 \mathrm{~L} / \mathrm{min}$ (with $95 \%$ limits of agreement of 0.29 to -5.66 ) $\mathrm{L} / \mathrm{min}$. Examining the Bland-Altman plot, accuracy seem constant in all ranges of CO (figure). A statistically significant correlation was found between Fick_CO and PAC_CO $(R 2=0.53), \mathrm{p}<0.0001)$.

Conclusion. $\mathrm{CO}$ measured with the indirect Fick principle correlated significantly with $\mathrm{CO}$ measured by thermodilution. However, there was a significant mean bias of $2.5 \mathrm{I} / \mathrm{min}$. Future studies should address this issue, and if the results can be reproduced, a correction factor can be implemented making the indirect Fick principle a possible alternative to thermodilution.

\section{2}

Mitochondrial oxygen tension to monitor circulatory shock in ICU patients after cardiac surgery: preliminary results

B. Hilderink'; RF. Crane ${ }^{1}$; B. Bogaard Van Den ${ }^{1}$; J. Pillay ${ }^{2}$; NP. Juffermans ${ }^{3}$ ${ }^{1}$ Intensive care, OLVG location East, Amsterdam, Netherlands; ${ }^{2}$ Department of critical care, University Medical Center Groningen, Groningen, Netherlands; ${ }^{3}$ Department of intensive care medicine, Amsterdam UMC, locatie AMC, Amsterdam, Netherlands

Correspondence: $\mathrm{B}$. Hilderink

Intensive Care Medicine Experimental 2020, 9(1): 000902.

Introduction. Resuscitation of circulatory shock after cardiac surgery is complicated by the absence of an absolute endpoint of tissue hypoxia, rendering patients susceptible to over- and under resuscitation. A novel non-invasive bedside measurement technique enables measurement of the oxygen tension at the mitochondrial level (mitoPO2), measuring tissue oxygenation downstream $[1,2]$. Therefore, mitoPO2 might be a more accurate marker of tissue hypoxia in postoperative cardiac surgery patients.

Objectives. To investigate the kinetics of mitoPO2 compared to lactate in critically ill postoperative cardiac surgery patients.

Methods. We prospectively obtained repeated measurements in 9 critically ill patients after cardiac surgery with cardiopulmonary bypass. During the first $6 \mathrm{~h}$ of ICU admission, hourly mitoPO2 measurements on the shoulder were obtained using the COMET monitor (Photonics Healthcare, Utrecht, Netherlands) on skin primed with an ALA containing patch (Alacare, Photonamic, Wedel, Germany). Concurrently, arterial blood gasses were drawn. Reported mitoPO2 values are the mean of 30 measurements with a frequency of $1 \mathrm{~Hz}$ at one time point. Delta lactate at each time point was calculated as the percentage increase in lactate compared to directly at ICU admission. A linear mixed model was used to assess the correlation of mitoPO2 with delta lactate and adjust for repeated measures.

Results. In 2 patients, measurements were not possible due to a technical error. Of the remaining 7 patients, median age was 70 years (IQR 69-73), 5 were male. All patients presented to the ICU on mechanical ventilation, median dose of vasopressors was $0.10 \mu \mathrm{g} / \mathrm{kg} / \mathrm{min}$ (IQR $0.03-0.17$ ). Median time on cardiopulmonary bypass was $120 \mathrm{~min}$ (IQR 75-165).

At ICU admission, median mitoPO2 was $45 \mathrm{mmHg}$ (IQR $30-80 \mathrm{mmHg}$ ) and decreased to $14 \mathrm{mmHg}$ (IQR $4-28 \mathrm{mmHg})$ after $6 \mathrm{~h}(\mathrm{p}<0.05)$. Concurrently, median lactate increased from 1.4 to $2.3 \mathrm{mmol} / \mathrm{l}$ at $6 \mathrm{~h}$ after admission $(p<0.05)$. The nadir in mitoPO2 (median $7.8 \mathrm{mmHg}$ ) occurred $36 \mathrm{~min}$ prior to the occurrence of peak lactate levels (median $2.3 \mathrm{mmol} / \mathrm{l}$ ). Furthermore, mitoPO2 showed a significant negative correlation (coefficient -1.1 with, $95 \% \mathrm{Cl}-0.3,-1.4$ ) with delta lactate when fitted using a linear mixed model ( $n=28$ measurements). When mitoPO2 of $1 \mathrm{~h}$ prior to delta lactate was fitted, the correlation coefficient of the linear mixed model improved: -1.88 ( $95 \% \mathrm{Cl}:-1.89$, $-1.87)$, signifying that approximately for every $10 \mathrm{mmHg}$ decrease in 
mitoPO2, lactate was increased with $20 \%$ compared to baseline in the next hour.

Conclusion. These preliminary results suggest that a decrease in mitoPO2 precedes increases in lactate levels and may therefore be useful for early detection of tissue hypoxia in ICU patients after cardiac surgery.

\section{2}

Occurence, risk factors and outcomes of new onset arrhythmia in surgical intensive care unit: A retrospective study

S. Hanif, ; F. Shamim²; S. Muhammad ${ }^{3}$

${ }^{1}$ Medicine, Aga Khan University Hospital, Karachi, Pakistan; ${ }^{2}$ Anaesthesiology, Aga Khan University, Karachi, Pakistan; ${ }^{3}$ Anaesthesiology, Aga Khan University Hospital, Karachi, Pakistan

Correspondence: F. Shamim

Intensive Care Medicine Experimental 2020, 9(1): 000972

Introduction. Critically ill patients are frequently subject to metabolic, ischemic, or neurohormonal stressors, and are therefore particularly at risk for cardiac arrhythmias. There is evidence of a high incidence of arrhythmias in patients with non-cardiovascular diseases, such as sepsis, chronic obstructive pulmonary disease and acute renal dysfunction. Cardiac arrhythmias in critically ill patients are most likely an indicator of severity of preexisting critical illness.

Objectives. To assess the incidence of new-onset cardiac arrhythmias among patients admitted in surgical ICU as well as associated risk factors.

Methods. A retrospective observational study conducted at Aga Khan University Hospital. Patients admitted to Surgical ICU from January 2018 till December 2019 were included. The medical record numbers of all patients admitted in surgical ICU (Jan, 2018 to Dec, 2019) were obtained from SICU case log entries and reviewed. Patients` and healthcare providers' identification were kept confidential. Data was analyzed using SPSS version 19.

Results. Only 13/1076 patients included in the study had cardiac arrhythmias during their stay in Surgical ICU. Among all patients with arrhythmias $(n=13)$, atrial fibrillation was the most common type of arrhythmia (53.8\%, $n=7)$. The study found higher occurrence of arrhythmias among the patients in the age category of 66 years or above $(4.6 \%, n=8)$ and when compared to younger patients with age categories $18-40$ years $(0.3 \%, n=2)$ and $41-65$ years $(0.3 \%, n=3)$ respectively $(p$-value $<0.05)$. Similarly, arrhythmias was higher among patients who had relatively prolong ICU stay and post-operative status as the primary cause of ICU admission.

Conclusion. Occurrence of cardiac arrhythmias is relatively low among patients admitted to surgical intensive care patient population. Patients age ( $>65$ years), prolong ICU stay, post-operative status are positively associated with development of cardiac arrhythmias among critically ill patients admitted in surgical ICU.

\section{Reference(s)}

1. Annane D, Sébille V, Duboc D, Le Heuzey JY, Sadoul N, Bouvier E, et al. Incidence and prognosis of sustained arrhythmias in critically ill patients. Am J Respir Crit Care Med. 2008 Jul 1;178(1):20-5.

2. Valderrabano RJ, Blanco A, Santiago-Rodriguez EJ, Miranda C, Rivera-Del Rio Del Rio J, Ruiz J, et al. Risk factors and clinical outcomes of arrhythmias in the medical intensive care unit. J Intensive Care. 2016 Dec;4(1):1-0

\section{3}

\section{Sex-Related Differences In Renal Edema Formation As} A Consequence Of Reduced Tie2 Expression In Mice A. van Leeuwen'; N. Dekker'; M. Van Meurs ${ }^{2}$; G. Molema, ; C. Van Den Brom ${ }^{1}$

${ }^{1}$ Anesthesiology, Amsterdam UMC, locatie VUmc, Amsterdam, Netherlands; ${ }^{2}$ Critical care, University Medical Center Groningen, Groningen, Netherlands; ${ }^{3}$ Pathology \& medical biology, medical biology section, University Medical Center Groningen, Groningen, Netherlands Correspondence: A. van Leeuwen

Intensive Care Medicine Experimental 2020, 9(1): 000972
Introduction. Endothelial hyperpermeability following hemorrhagic shock (HS) [van Leeuwen ALI, 2020] contributes to multiple organ failure, in particular acute kidney injury. An important molecular controller of endothelial permeability is the angiopoietin/Tie2 system [Parikh SM, 2017]. We have previously shown that during HS, Tie2 gene expression is reduced and that therapeutically targeting Tie2 reduced microvascular leakage following HS [Trieu M, 2018]. Interestingly, female sex is associated with a lower in-hospital mortality rate following severe HS [Pape M, 2019], however, little is known regarding potential sex-related differences in functional regulation by the angiopoietin/Tie2 system.

Objectives. To investigate whether there are differences in renal edema formation as a consequence of reduced Tie2 expression between male and female mice.

Methods. Male and female adult heterozygous Tie2 knockout [Jongman RM, 2019] (Tie2 \pm , exon 9 deletion), and healthy wild type (WT) mice were sacrificed ( $n=9-12$ per group). Renal edema formation was determined by wet/dry ratios. Renal gene expression levels and circulating levels of angiopoietin-1 (ang-1), angiopoietin-2 (ang-2) and Tie2 were quantified using RT-qPCR and commercially available ELISA kits, respectively.

Results. In healthy WT mice, plasma levels of ang- 1 were similar $(P>0.99)$, whereas ang-2 levels were $47 \%$ higher $(P<0.05)$ and levels of soluble Tie2 $20 \%$ lower $(\mathrm{P}<0.0001)$ in females compared to males. No differences were found in renal wet/dry ratio between WT males and females. However, in WT female mice, higher levels of renal gene expression levels were observed for ang- 1 ( $42 \%$ increase, $\mathrm{P}<0.0001)$, ang-2 $(336 \%$ increase, $\mathrm{P}<0.0001)$ and Tie2 $(72 \%$ increase, $\mathrm{P}<0.0001)$ compared to healthy males.

Genetic reduction of Tie2 did not affect circulating ang-1 or ang-2 levels in both sexes, whereas soluble Tie2 levels were lower in both sexes when comparing mice with reduced Tie2 expression to healthy WT mice (males: $43 \%$ lower, females: $50 \%$ lower, both $\mathrm{P}<0.0001$ ). Interestingly, in males, genetic reduction of Tie2 was associated with an increase in renal wet/dry ratio by $30 \%$ compared to healthy WT mice $(P<0.01)$, whereas in females this change was not observed $(P>0.99)$. Genetic reduction of Tie2 resulted in a $48 \%$ reduction in Tie2 gene expression levels in males and $60 \%$ reduction in females vs WT controls (both $\mathrm{P}<0.01$ ), but did not affect the expression of ang-1 or ang-2 mRNA in kidneys compared to healthy mice.

Conclusion. Gene expression and plasma protein levels of the angiopoietin/Tie2 system differ between healthy male and female mice. Genetic reduction of Tie2 expression is associated with increased renal edema in male, but not in female mice, suggesting that Tie2-mediated vascular integrity is of particular importance in males. Future research should elaborate on the role of Tie2 in sex-related responses to HS and investigate whether these differences in underlying mechanisms may be translated into sex-specific treatment strategies in HS patients.

\section{Reference(s)}

1. Jongman RM, et al. Partial Deletion of Tie2 Affects Microvascular Endothelial Responses to Critical IIIness in A Vascular Bed and Organ-Specific Way. Shock. 2019 Jun;51(6):757-769. https://doi.org/10.1097/SHK.0000000000 001226.

2. Pape $\mathrm{M}$, et al. Is there an association between female gender and outcome in severe trauma? A multi-center analysis in the Netherlands. Scand J Trauma Resusc Emerg Med. 2019 Feb 13;27(1):16. https://doi.org/10. 1186/s13049-019-0589-3.

3. Trieu M, et al. Vasculotide, an Angiopoietin-1 Mimetic, Restores Microcirculatory Perfusion and Microvascular Leakage and Decreases Fluid Resuscitation Requirements in Hemorrhagic Shock. Anesthesiology. 2018 Feb;128(2):361-374. https://doi.org/10.1097/ALN.0000000000001907.

4. Parikh SM. Angiopoietins and Tie2 in vascular inflammation, Curr Opin Hematol. 2017 Sep;24(5):432-438. https://doi.org/10.1097/MOH.00000 00000000361

5. van Leeuwen ALI, et al. In vitro endothelial hyperpermeability occurs early following traumatic hemorrhagic shock. Clin Hemorheol Microcirc. 2020;75(2):121-133. https://doi.org/10.3233/CH-190642. 
6. CEvdB is supported by the European Society of Intensive Care Medicine (Levi-Montalcini Award 2017), the Dutch Society of Anesthesiology (Young Investigator Grant 2017) and the Dutch Research Council (Veni 2019).

\section{0}

Continuous long term wireless measurement of right ventricular pressures and ePAD in patients with severe COVID-19 ARDS using a novel right ventricular pressure transducer

M. Gärtner ; F. Glocker ${ }^{2}$; HB. Hopf

${ }^{1}$ Röntgenstrasse 20, Asklepios Klinik Langen, Anaesthesia, periop. med. and interdiscipl. intensive care medicine, Langen (Hessen), Germany; ${ }^{2}$ Emka medical gmbh, emka MEDICAL GmbH, Aschaffenburg, Germany Correspondence: H.B. Hopf

Intensive Care Medicine Experimental 2020, 9(1): 000990

Introduction. In patients with severe COVID-19 ARDS development of pulmonary artery hypertension $(\mathrm{PAH})$ in combination with right ventricular hypertension might be one of the key features associated with a worse prognosis $(1,2)$. Until now, continuous long term measurement of right ventricular pressures (RVP's) was not available.

Objectives. We continuously measured RVP's and the estimated diastolic pulmonary artery pressure (ePAD) as an indicator for left heart failure (3) for up to 30 days in mechanically ventilated patients with severe COVID-19 ARDS to detect and treat right ventricular and pulmonary artery hypertension using a transcutaneous inserted right ventricular pressure probe.

Methods. We retrospectively evaluated RVP's and the ePAD measured in 30 mechanically ventilated COVID-19 ARDS patients between October 1st 2020 and March 31st 2021. Median recording time was 22 days (range 7-30). In all patients CorLog Probe 1P, (emka MEDI$\mathrm{CAL} \mathrm{GmbH}$, Aschaffenburg, Germany), a wireless high fidelity pressure measurement system with a non metallic probe of $1.1 \mathrm{~mm}$ diameter using Fluorinert ${ }^{\circledR}$ as transmission fluid, has been used for continuous RVP measurement. We retrospectively divided the patients into two groups, survivors and non survivors based on their 60 day mortality. Primary outcome variables were the values of RVP's and the ePAD over time after insertion of the RVP probe. Comparison of pressures over time within each group was done by a linear mixed effect model, pressure data at day 1 and on the last day of the probe in place, were compared between the two groups by using the Student's t-test.

Results. There was no difference in biometrical and clinical data between survivors and nonsurvivors. There was a significant decrease in systolic right ventricular pressure (RVSP) over time in the survivors (estimate: $-0,38 ; 95 \% \mathrm{Cl}:-0,59--0,17 ; \mathrm{p}<0,001)$ but not in the non survivors. $16 / 22$ survivors and $7 / 8$ non survivors received sildenafil orally, one survivor received additionally inhaled nitric oxide and one survivor and one non survivor each inhaled iloprost. RVSP was significantly lower on the first and the last measurement day in the survivors compared to the non survivors (day 1: 38 vs. $46, p=0.036$; last day: 36 vs. $51, p=0.006$ survivors vs. non survivors, respectively). On the last measurement day both right ventricular pressure amplitude (RVPAMPL, 31 vs. 38, $p=0.027$ ) and ePAD ( 22 vs. $31, p=0.043$ ) were significantly lower in the survivors compared to the non survivors.

Conclusion. RVSP and RVPAMPL both were significantly lower in the survivors compared to the non survivors with a significant decrease in RVSP over time in the survivors suggesting successful lowering by pulmonary vasodilators. The ePAD as an indicator of left heart failure was significantly higher in non survivors compared to the surviving patients. The use of continuous long-term monitoring of RVSP and ePAD might enable early therapy of right and left heart failure in patients with severe COVID-19 ARDS.

\section{Reference(s)}

a. Caravita S, Baratto C, Di Marco F, Calabrese A, Balestrieri G, Russo F, Faini A, Soranna D, Perego GB, Badano LP, Grazioli L, Lorini FL, Parati G, Senni M. Haemodynamic characteristics of COVID-19 patients with acute respiratory distress syndrome requiring mechanical ventilation. An invasive assessment using right heart catheterization. Europ J Heart Fail 2020; 22:2228-2237. b. Rath D, Petersen-Uribe A, Avdiu A, Witzel K, Jaeger P, Zdanyte M, Heinzmann D, Tavlaki E, Müller K, Gawaz MP. Impaired cardiac function is associated with mortality in patients with acute COVID-19 infection. Clin Res Cardiol 2020; 109:1491-1499.

c. Zile MR, Bennett TD, Sutton MS, Cho YK, Adamson PB, Aaron MF, Aranda Jr JM, Abraham WT, Smart FW, Warner Stevenson L, Kueffer FJ, Bourge RC. Transition from chronic compensated to acute decompensated heart failure. Pathophysiological insights obtained from continuous monitoring of intracardiac pressures. Circulation 2008; 118:1433-1441.

7. The statistical evaluation was carried out under the advice and supervision of members of the Institute for Biostatistics and Mathematical Modelling at the Department of Medicine of the Goethe University Frankfurt (Prof. Dr. Eva Herrmann).

000992

Right Ventricolo-Arterial coupling to predict fluid responsiveness: preliminary data

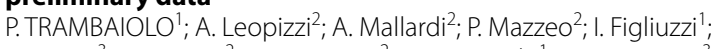
P. Bertini ${ }^{3}$; F. Santoro ${ }^{2}$; ND. Brunetti ${ }^{2}$; G. Ferraiuolo ${ }^{1}$; F. Guarracino ${ }^{3}$

${ }^{1}$ ICCU, Hospital Sandro Pertini, Roma, Italy; ${ }^{2}$ Department of medical and surgical sciences, University of Foggia, Foggia, Italy; ${ }^{3}$ Cardiothoracic and vascular anesthesia and intensive care, Azienda Ospedaliero Universitaria Pisana, Pisa, Italy

Correspondence: P. TRAMBAIOLO

Intensive Care Medicine Experimental 2020, 9(1): 000992

Introduction. The fluid challenge is the most commonly performed bedside hemodynamic intervention in functional hemodynamic monitoring. Echo parameters measured on the left side of the heart are usually used at the bedside as dynamic indexes of fluid responsiveness and are evaluated before and after leg raising: Left Ventricular Outflow Tract Velocity Time Integral (LVOT VTI), Stroke Volume Index (SVi), Cardiac Index (Cl). No parameters, from the Right Ventricle (RV), as Right Ventricular Outflow Tract Velocity Time Integral (RVOT VTI), Tricuspid Annular Plane Systolic Excursion (TAPSE), Pulmonary Artery Systolic Pressure (PASP), Right Ventricular S'Tissue Doppler Imaging (RV S'TDI), have been used so far to estimate the fluid responsiveness and no data are available to predict the positive effect of a fluid challenge.

Objectives. To compare the Right Ventricolo-Arterial Coupling (RVAC) as TAPSE/PASP and $S^{\prime} / P A S P$ ratio in responsive or not responsive patients at leg raising.

Methods. 20 consecutive medical patients (mean age $62 \pm 19$ years), hospitalized in the ICU of Sandro Pertini Hospital from March to May 2021 were enrolled. History, clinical features, echocardiographic baseline exam, and echo parameters (LVEF, LVOT VTI, SVi, RVOT VTI, Cl, TAPSE, PASP, RV S') pre-and post- leg raising were recorded. Patients were divided into two groups based on the fluid responsiveness (LVOT VTI max-LVOT VTI min/LVOT VTI mean $>15 \%$ responders; $<15 \%$, not responders).

Results. Responder patients were 9 of 20 (44\%). The cardiovascular risk factors considered in both group were the same and no difference was found between baseline echo parameters. The mean LVEF at admission was $40 \%$ in both groups. Compared to the non responder group, responder patients had a lower TAPSE $(18,4 \pm 1,4$ vs $21,9 \pm 2,98$; $\mathrm{p}<0,05)$, higher PASP $(35,7 \pm 9,2$ vs $29,6 \pm 8,2 ; \mathrm{p}=0,18)$ and RV $\mathrm{S}^{\prime}$ lower $(12,75 \pm 3,63$ vs $14,03 \pm 1,83 p=0,38)$. TAPSE/PASP and S'/PASP were significantly lower $(0,55 \pm 0,17$ vs $0,78 \pm 0,22 ; 0,36 \pm 0,39$ vs $0,50 \pm 0,14 ; p<0,05$ ). 

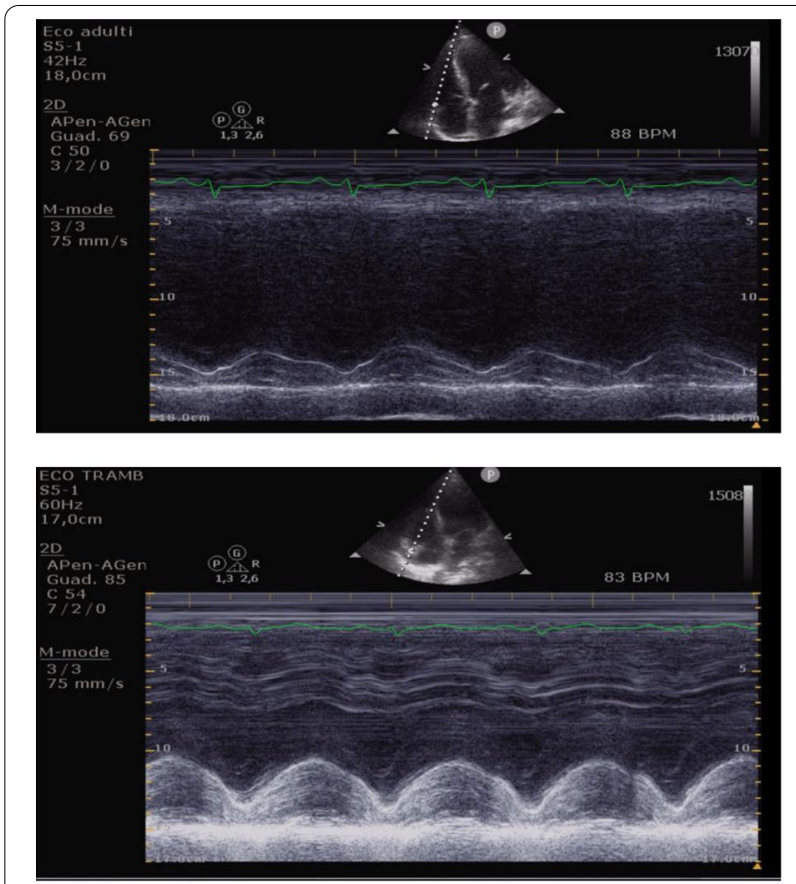

$\begin{array}{cccc}\text { Echo parameters } & \text { Responders }(\text { mean } \pm \text { SD) } & \text { Non responders (mean } \pm \text { SD) } & p \text {-value } \\ \text { TAPSE/PASP } & 0,55 \pm 0,17 & 0,78 \pm 0,22 & p<0,05 \\ \text { S'/PASP } & 0,36 \pm 0,39 & 0,50 \pm 0,14 & p<0,05 \\ \text { TAPSE } & 18,4 \pm 1,4 & 21,9 \pm 2,98 & p<0,05 \\ \text { PASP } & 35,7 \pm 9,2 & 29,6 \pm 8,2 & p=0,18 \\ \text { RV S' } & 12,75 \pm 3,63 & 14,03 \pm 1,83 & p=0,38\end{array}$

Conclusion. Right ventricolo-arterial coupling expressed as TAPSE/ PASP and $\mathrm{S}^{\prime} / \mathrm{PASP}$, is a promising predictor of fluid responsiveness at leg raising challenge maneuver.

\section{7}

The Effect of Acidaemia on Cardiac Contractility in Critically ill Adults

S. Vanapalli'; A. Spataru'; P. Eiben ${ }^{1}$; V. Recea ${ }^{1}$; C. Brathwaite-Shirley ${ }^{2}$; L. Thompson,; B. Gurung ${ }^{3}$; R. Reece-Anthony ${ }^{3}$;. Awad'; M. Toolan"; A. Collins ${ }^{3}$; J. Arévalo-Serrano ${ }^{4}$; S. Sakka ${ }^{5}$; JA. Kraut ${ }^{6}$;. Rodriguez-Villar ${ }^{1}$ ${ }^{1}$ Critical care department, King's College Hospital, London, United Kingdom; ${ }^{2}$ Critical care department, Princess Royal University Hospital, London, United Kingdom; ${ }^{3}$ Critical care department, University Hospital Lewisham, London, United Kingdom; ${ }^{4}$ Internal medicine department, Hospital Príncipe de Asturias. Alcalá de Henares, Madrid, Spain; ${ }^{5}$ Critical care department, Gemeinschaftsklinikum Mittelrhein $\mathrm{gGmbH}$. Kemperhof und Ev. Stift St. Martin, Koblenz, Germany; ${ }^{6}$ Dialysis department, VHAGLA Healthcare System, Los Angeles, United States of America Correspondence: S. Vanapalli

Intensive Care Medicine Experimental 2020, 9(1): 001017

Introduction. Low serum $\mathrm{pH}$ values have been associated with abnormal enzymatic function, hypotension, arrhythmias, and impaired oxygen delivery [1]. While it is widely recognised that acidaemia results in hemodynamic compromise, its effect on cardiac contractility have not been extensively investigated in humans [2,3].

Objectives. To establish the relationship between arterial $\mathrm{pH}$ and cardiac function measured by transpulmonary thermodilution in critically ill adults.
Methods. In this prospective cross sectional observational study, we included all mechanically ventilated adults admitted to 6 different ICUs in the UK between May 2018 and March 2019 and requiring cardiac output monitoring. Patients with arrhythmias, severe valvulopathies, or on extracorporeal circulation were excluded.

Invasive hemodynamic monitoring in these patients was initiated at the discretion of the treating physician, using transpulmonary thermodilution technique with integrated pulse contour analysis (Pulse Contour Cardiac Output technology $\mathrm{PiCCO}^{\circledR}$ ). This involved placement of a central venous catheter into superior vena cava, either via internal jugular or subclavian route, and an arterial catheter into the femoral or axillary artery. The cardiac index (CI), stroke volume index (SVI), global ejection fraction (GEF), and maximal left ventricular contractility (dPmax) were obtained using $\mathrm{PiCCO}^{\circledR}$ and selected biochemical and physiological parameters were recorded in all patients at the lowest measured serum $\mathrm{pH}$ during their ICU stay.

For the analysis, we dichotomised patients using a pH value of 7.28 , which was the median of the lowest $\mathrm{pH}$ recorded for each patient and compared the hemodynamic variables in both groups. We also have performed a regression analysis with the dichotomised $\mathrm{pH}$ as the predictor variable and the indices derived from $\mathrm{PiCCO}^{\circledR}$ as the outcome variables.

Results. We included in this study 297 patients with a median age 59 years old (IQR 49-71). Patients in the low $\mathrm{pH}$ group $(<7.28)$ had significantly lower mean arterial pressure ( 68 vs $76 \mathrm{mmHg}, p<0.001)$, higher heart rate (104 vs $88 \mathrm{~b} / \mathrm{min}, \mathrm{p}<0.001)$, and higher serum lactate ( $4.5 \mathrm{vs} 1.6 \mathrm{mmol} / \mathrm{l}, \mathrm{p}<0.001$ ). We also found a significant difference in the 28 days mortality between groups ( 60.3 vs $25.4 \%, p<0.001)$. The duration of the inotropic support, transfusion requirements, and the volume of crystalloids administered in the ICU were similar in both groups. However, the adjusted linear regression analysis did show that, with lower pH using a cut-off value of 7.28, a significantly lower stroke volume associated with an inadequately higher heart rate for maintenance of cardiac output as well as lower values for GEF, CFI and CPI.

Conclusion. Acidaemia is associated with significant impact on hemodynamic variables in critically ill patients hospitalised in the intensive care unit.

\section{Reference(s)}

1. Kraut JA, Madias NE. Metabolic acidosis: pathophysiology, diagnosis and management. Nat Rev Nephrol. 2010;6(5):274-285.

2. Wildenthal K, Mierzwiak DS, Myers RW, Mitchell JH. Effects of acute lactic acidosis on left ventricular performance. Am J Physiol. 1968 Jun;214(6):1352-9.

3. Maury E, Vassal T, Offenstadt G. Cardiac contractility during severe ketoacidosis. N Engl J Med. 1999 Dec 16;341(25):1938.

4. UCLA School of Medicine has partly aided this study with unrestricted funds.

\section{6}

Survival benefits of strict protocols in the management of patients with myocarditis requiring pVAECMO

M. Hoy ${ }^{1}$; N. Palomo-López ${ }^{2}$; R. Fernandez Garda'; N. Lees ${ }^{1}$;

A. Hurtado-Doce

${ }^{1}$ Critical care, Harefield Hospital, Harefield, United Kingdom; ${ }^{2}$ Critical care unit, Virgen del Rocío University Hospital, Sevilla, Spain

Correspondence: M. Hoy

Intensive Care Medicine Experimental 2020, 9(1): 001046

Introduction. Myocarditis defines a diverse range of pathologies causing direct damage to cardiac myocytes, including autoimmune, infective and toxic pathologies. Illness may be asymptomatic but includes a spectrum of severity ranging to include fulminant cardiogenic shock (CS) and causes approximately 600 deaths per year in the UK.

Objectives. To describe and analyse risk factors associated with mortality in patients with refractory CS secondary to myocarditis requiring peripheral veno-arterial extracorporeal membrane oxygenation ( $p$ VA ECMO) and assess change in survival after the implementation of a hospital protocol. 
Methods. Observational and retrospective study, in which patients with CS secondary to myocarditis requiring PVAECMO between 2014 and 2020 were collected. Patient data was divided into two periods (2014-2017; 2018-2020) to show the difference after implentation of a protocol including more strict inclusion criteria for the institution of pVAECMO and changes in the use of distal limb perfusion cannulae (DPC) to avoid major leg ischaemia (defined by need for fasciotomy for compartment syndrome or amputation but not including embolectomy which was used as a resuscitative technique in the event of acute limb ischaemia). Demographic variables, ECMO-related variables, prognostic scores and survival at 30,90 days and 1 year were retrospectively collected and prospectively analysed. Qualitative variables were described by median and interquartile range. $X^{2}$ test was performed for qualitative variables and $U$ Mann Whitney for quantitative variables. Survival was represented by Kaplan-Meier. Statistical significance was considered when $p<0.05$.

Results. A total of 36 patients were collected, 14 from the first period and 22 from the second. Statistically significant differences were observed between the two periods including increased use of reperfusion cannulae, lower SOFA and higher SAVE score in the second time period. Time on ECMO support and survival at 90 days were also significantly different between the two groups.

Use of implementation protocols excluded those with very high SOFA score or very low SAVE scores.

The incidence of major ischaemic complications in the cannulated leg was significantly lower in the second period ( $9 \%$ vs 50\%). During this time, patients also underwent significantly longer ECMO runs with a median of $288 \mathrm{~h}$ compared to $114 \mathrm{~h}$ in the first period.

We observed a survival at 90 days and 1 year significantly higher in the second period by comparison with the first $(63 \%$ vs $29 \%$ and $56 \%$ vs $14 \%$, respectively) which far surpasses international standards.

Conclusion. Well-constructed protocols with regards to ECMO selection criteria and its day-to-day management demonstrated the ability to improve ECMO survival and allow longer duration of therapy, inherently increasing the chance of successful weaning.

Importantly, concerted effort to distally perfuse the arterially-cannulated limbs, and fix complications as they arose appeared to be associated with a large reduction in the mortality associated with pVAECMO. Notably, the rates of other complications (haemorrhage, failure to wean, sepsis) remained stable between each period supporting that statement that the lower rate of major limb ischaemia was the principle factor in the reduced mortality seen.

Unlike in other pathologies commonly supported with pVAECMO (e.g. decompensated dilated or ischaemic cardiomyopathies), myocarditis has a much higher transplant-free survival rate and therefore the ability to sustain long ECMO runs with a view to recovery of native cardiac function and avoid other interventions or mechanical circulatory support devices is an important one.

We highly advocate the routine employment of DPC and use of aggressive limb revascularisation in PVAECMO patients. In the myocarditis population, this dramatically improved survival.

\section{4}

Venoarterial extracorporeal membrane oxygenation appears to induce higher neutrophil-derived inflammation and oxidative stress at the end of the first week of hospitalization

M. Reina-Couto ; P. Pereira-Terra ${ }^{2}$; C. Silva-Pereira, ; J. Quelhas-Santos, ${ }^{2}$; P. Serrão, ${ }^{2}$;. Afonso, ${ }^{2}$; S. Martins, ${ }^{3} ;$ R. Roncon-Albuquerque ${ }^{4}$; JA. Paiva ${ }^{5}$. A. Albino-Teixeira ${ }^{2} ;$ T. Sousa, $^{6}$

1 Intensive care department $\&$ dep. biomedicina-unid. farmacologia e terapêutica, Centro Hospitalar São João \& Faculdade de Medicina da Universidade do Porto, Porto, Portugal; ${ }^{2}$ Department of biomedicine-unit of pharmacology and therapeutics, Faculdade de Medicina da Universidade do Porto, Porto, Portugal; ${ }^{3}$ Department of clinical pathology, São João Universitary Hospital Center, Porto, Portugal; ${ }^{4}$ Intensive care department \& cirurgia e fisiologia department, Centro Hospitalar São João \& Faculdade de Medicina da Universidade do Porto, Porto, Portugal; ${ }^{5}$ Emergency and intensive care department, Centro Hospitalar de São João, Porto,
Portugal; ${ }^{6}$ Dep. biomedicina-unid. farmacologia e terapêutica, Faculdade de Medicina da Universidade do Porto, Porto, Portugal, Portugal

Correspondence: M. Reina-Couto

Intensive Care Medicine Experimental 2020, 9(1): 001064

Introduction. Venoarterial extracorporeal membrane oxygenation (VA-ECMO) provides mechanical support to patients with cardiogenic shock unresponsive to conventional medical therapy [1]. VAECMO has been suspected to affect the immune system through several mechanisms such as induction of endothelial dysfunction, activation of pro-inflammatory cascades and oxidative stress [2-5]. However, evidence on the VA-ECMO-induced immune responses is still scarce.

Objectives. This study aimed at evaluating several biomarkers of inflammation, resolution of inflammation, endothelial activation and oxidative stress in patients with cardiogenic shock (CS) with and without VA-ECMO, during the first week of hospitalization.

Methods. This study was approved by the Health Ethics Committee of our institution. Patients with the diagnosis of $C S(n=25)$ were included and blood and spot urine samples were collected at days 1-2 (admission), days 3-4 and days 5-8. Biomarkers of inflammatory status [serum IL-1 $\beta, \mathrm{IL}-6, \mathrm{IL}-10$, tumour necrosis factor-a (TNFa), myeloperoxidase, MPO; urinary cysteinyl leukotrienes, U-CysLT)], inflammation resolution mediators [serum resolvin D1 (RvD1) and resolvin E1 (RvE1)], endothelial dysfunction markers [serum intercellular adhesion molecule-1 (ICAM-1), vascular cell adhesion molecule-1 (VCAM-1), E-selectin, serum and urinary endocan] and oxidative stress markers [serum nitrotyrosine; urinary hydrogen peroxide (U-H2O2), urinary isoprostanes (U-Isop)] were determined by ELISA, multiplex immunoassays or fluorometric assays. C-reactive protein (CRP), differential leukocyte count, B-type natriuretic peptide (BNP), high-sensitivity troponin I (hs-trop I) were quantified using automated analyzers. Prognostic scores (APACHE II, SAPS II), lactate, neutrophil-to-lymphocyte ratio (NLR) and neutrophil-tomonocyte ratio (NMR) were also evaluated.

Results. At Intensive Care Unit (ICU) admission, patients in the VAECMO group (ECMO, $n=12$ ) were significantly younger than those in the conventional medical therapy group (CMT, $n=13)$, but no differences were found in prognostic scores, lactate, BNP, hs-trop I and CRP.

At days 3-4, only neutrophil count and NLR were significantly higher in the ECMO group (neutrophils $\times 109 / \mathrm{L}, \mathrm{ECMO}: 8.9 \pm 0.6$ vs CMT: $5.7 \pm 0.8, p=0.03$; NLR, ECMO: $9.1 \pm 1.7$ vs CMT: $4.0 \pm 0.7$, $\mathrm{p}=0.005$ ). At days $5-8, \mathrm{ECMO}$ patients had significantly higher values of neutrophils (neutrophils $\times 109 / \mathrm{L}$, ECMO: $7.1 \pm 0.4$ vs CMT: $5.3 \pm 0.9, \mathrm{p}=0.04)$, serum MPO $(\mathrm{ng} / \mathrm{mL})(E C M O: 451 \pm 59$ vs CMT: $268 \pm 41, \mathrm{p}=0.03)$, serum RvE1 ( $\mathrm{pg} / \mathrm{mL})(E C M O: 350 \pm 23$ vs CMT: $246 \pm 32, \mathrm{p}=0.02)$ and U-Isop ( $\mathrm{ng} / \mathrm{mg}$ creatinine) (ECMO: $4.0 \pm 0.7$ vs CMT: $2.2 \pm 0.3, p=0.03$ ). No significant differences were found for the other biomarkers analysed.

Conclusion. In our center, CS patients on VA-ECMO appear to have higher neutrophil-derived inflammation and oxidative stress at the end of the first week of ICU stay. This enhanced inflammatory and oxidative stress response is accompanied by an increase in the concentration of RvE1, a specialized proresolving mediator that inhibits vascular neutrophil infiltration, reduces cytokine production and enhances the clearance of apoptotic neutrophils [6]. Further studies are needed to explore the prognostic significance of the late rise in oxidative stress and RvE1 in VA-ECMO patients.

\section{8}

ST-Segment Elevation and Troponin Rise-Different Aetiologies in SARS-CoV-2: a Case Series

J. Santos-Lima'; R. Caetano Garcês ${ }^{1}$; M. Torre ${ }^{1}$; M. Cascão ${ }^{1}$; M. Costa ${ }^{1}$; R. Cavaco'; L. Bento ${ }^{1}$

${ }^{1}$ Unidade de Urgência Medica (UUM), Centro Hospitalar Universitário de Lisboa Central—Hospital de São José, Lisboa, Portugal

Correspondence: J. Santos-Lima

Intensive Care Medicine Experimental 2020, 9(1): 001,098 
Introduction. The Severe Acute Respiratory Syndrome Coronavirus 2 (SARS-CoV-2) first appeared in Wuhan, China, causing a pandemic of viral pneumonia. While SARS-CoV-2 primarily affects the lungs, it is recognised that there is an increased risk of thromboembolic disease, including myocardial infarction (MI) [1,2,3]. Infections increase this risk via increased levels of inflammatory cytokines, leading to increased plaque instability, associated with hypercoagulable state, endothelial dysfunction and increased platelet activation [5]. Reports suggest SARS-CoV-2 increases thromboembolic risk via similar mechanisms, as well as coronary embolism $[1-4,6-8,10,11]$. SARS-CoV-2 has also been implicated in Takotsubo cardiomyopathy $[4,9]$.

Furthermore, thromboembolic events related to SARS-CoV-2 seem to increase hospital mortality [2].

Objectives. To evaluate and characterize patients in our Intensive Care Unit (ICU) who had a clinical picture compatible with ST-segment elevation myocardial infarction (STEMI) in the context of SARS-CoV-2.

Methods. Retrospective study and data analysis of our ICU's database. Results. At data collection we had treated 352 SARS-CoV-2 patients in our ICU. $67 \%$ male, with a mean length of stay of $11.1( \pm 3.1)$ days and an all-cause mortality of $37.5 \% .76 \%$ required invasive mechanical ventilation (IMV) for a mean of 10.2 ( \pm 9.7 ) days.

We treated 6 patients, with a mean age of $54.2( \pm 10)$ years, who during the course of infection developed signs and symptoms compatible with STEMI. 5 patients presented to hospital with a STEMI-like clinical picture, all of whom were asymptomatic for SARS-CoV-2 at the time, but tested positive. 1 patient developed STEMI whilst in the ICU (day $12 / 4$ of symptoms/ICU). All patients had early percutaneous coronary angiography. Coronary artery disease (CAD) was documented in 3,2 had thrombus with no underlying CAD, and 1 had Takotsubo cardiomyopathy. All 5 patients with coronary artery occlusion underwent primary Percutaneous Coronary Intervention with thrombus aspiration and drug eluting stents. 3 patients developed severe Coronavirus Disease (COVID-19) requiring IMV, 1 of which required a 48 day venovenous extracorporeal membrane oxygenation run, currently undergoing rehabilitation as an in-patient. All other patients have been discharged from hospital, none requiring readmission.

Conclusion. STEMI-like signs and symptoms can be the first manifestation of SARS-CoV-2. It is recognized that SARS-CoV-2 increases the risk of cardiovascular thrombosis, although there is a wide variation of reported incidence $[2,12,13]$. We report an incidence of $1.4 \%(5 / 352$ patients) confirmed STEMI in our ICU population. The median age of this case series was lower than the median age of the entire SARSCoV-2 ICU population studied (54 vs 62 years). This younger age has been noted in Ml secondary to coronary embolism [7,10,11]. Unlike other reports, the thromboembolic events described were not associated with increased hospital mortality, and all 6 patients had a favourable outcome.

\section{Reference(s)}

1. Bangalore S, Sharma A, Slotwiner A, et al. ST-Segment Elevation in Patients with Covid-19_A Case Series. N Engl J Med. 2020;382(25):24782480. 10.1056/NEJMc2009020

2. Kariyanna PT, Ramalanjaona B, Al-Sadawi M, Jayarangaiah A, Hegde S, McFarlane IM. Coronary Embolism and Myocardial Infarction: A Scoping Study. Am J Med Case Rep. 2020;8(2):31-43. 10.12691/ajmcr-8-2-1. 11 Ranard LS, Engel DJ, Kirtane AJ," Masoumi A. Coronary and cerebral thrombosis in a young patient after mild COVID-19 illness: a case report, European Heart Journal-Case Reports, Volume 4, Issue 5, October 2020, Pages 1-5. 10.1093/ehjcr/ytaa270. 12. Stefanini GG, Montorfano M, Trabattoni D, et al. ST-elevation myocardial infarction in patients with COVID19: clinical and angiographic outcomes. Circulation 2020;141:2113-6. 10. 1161/CIRCULATIONAHA.120.047525. 13. Modin D, Claggett B, SindetPedersen C, et al. Acute COVID-19 and the incidence of Ischemic Stroke and Acute Myocardial Infarction. Circulation 2020; 142 (21): 2080-2082. 10.1161/CIRCULATIONAHA.120.050809

3. Giustino G, Croft LB, Oates CP, et al. Takotsubo Cardiomyopathy in COVID19. J Am Coll Cardiol. 2020;76(5):628-629. 10.1016/j.jacc.2020.05.068

4. Rey JR, Jiménez Valero S, Poveda Pinedo D, Merino JL, López-Sendón JL, Caro-Codón J. COVID-19 and simultaneous thrombosis of two coronary arteries. Rev Esp Cardiol (Engl Ed). 2020;73(8):676-677. 10.1016/j.rec.2020. 05.021

5. Shams A, et al. Coronary thrombosis in a young male with COVID-19. IDCases 2020; 21: e00923. https://doi.org/10.1016/j.idcr.2020.e00923

6. Tedeschi D, Rizzi A, Biscaglia S, Tumscitz C. Acute myocardial infarction and large coronary thrombosis in a patient with COVID-19. Catheter Cardiovasc Interv. 2021;97(2):272-277. 10.1002/ccd.29179

7. Musher DM, Abers MS, Corrales-Medina VF. Acute Infection and Myocardial Infarction. N Engl J Med. 2019;380(2):171-176. 10.1056/NEJMra1808 137

8. Giustino G, Pinney SP, Lala A, et al. Coronavirus and Cardiovascular Disease, Myocardial Injury, and Arrhythmia: JACC Focus Seminar. J Am Coll Cardiol. 2020;76(17):2011-2023. 10.1016/j.jacc.2020.08.059

9. Burkert FR, Niederreiter L, Dichtl W, et al. Case report of a COVID-19-associated myocardial infarction with no obstructive coronary arteries: the mystery of the phantom embolus or local endothelitis. Eur Heart J Case Rep. 2021;5(2):ytaa521. 10.1093/ehjcr/ytaa521

10. Bilaloglu S, Aphinyanaphongs Y, Jones S, Iturrate E, Hochman J, Berger JS. Thrombosis in Hospitalized Patients With COVID-19 in a New York City Health System. JAMA. 2020;324(8):799-801. 10.1001/jama.2020.13372

\section{8}

Early mechanical support with VA-ECMO in severe primary graft failure after heart transplantation

A. Marcos-Morales ${ }^{1}$; JL. PerezVela²; MB. Isaías² ${ }^{2}$ M. Corres Peiretti ${ }^{2}$

R. Emilio ${ }^{2}$

${ }^{1}$ Intensive Care, Hospital Doce de Octubre, Madrid, France; ${ }^{2}$ Intensive care, Hospital Doce de Octubre, Madrid, Spain

Correspondence: A. Marcos-Morales

Intensive Care Medicine Experimental 2020, 9(1): 001,188

Introduction. Severe primary graft failure (PGF) after heart transplantation is defined as the inability of the implanted graft to satisfy the circulatory demands of the body, resulting in cardiogenic shock with need of emergent mechanical support, mostly veno-arterial extracorporeal membrane oxygenation (VA-ECMO).(1-3). The purpose of this study was to evaluate the different outcomes of patients with severe PGF supported by VA-ECMO in the immediate post-transplantation setting in the intensive care unit (ICU).

Methods. Data was collected prospectively from 2012 to 2021 patients with PGF having received VA-ECMO were reviewed. We analyzed baseline demographic variables, cause of heart transplantation, risk factors for PGF, ECMO characteristics, complications and global survival (at ECMO weaning, hospital discharge, and after 1 year), using SPSS software, version 26.

Results. We included 14 patients who received VA-ECMO in the first $24 \mathrm{~h}$ after heart transplantation, due to refractory cardiogenic shock. The median age was 44 (interquartile range IQR 35-60), 57\% were male. Patients received a heart transplantation due to ischemic cardiomyopathy, anthracycline toxicity, valvular cardiopathy or congenital disease. Most transplantations procedures were pre-programmed whereas they were emergent in three patients who were already suffering from cardiogenic shock supported by VA-ECMO (after myocardial infarction or advanced heart failure). Thirty six percent of ECMO were cannulated in the ICU, the rest in the operating room, and $57 \%$ of cannulations were made peripherally. Median length of ECMO support was 192 h (8 days, IQR 5-13), median ICU stay 28 days (IQR 11-42 days). Four patients were cannulated in a state of unexpected circulatory arrest, all in the ICU, and three of them were discharged from the ICU. Ten out of fourteen patients achieved weaning from ECMO, and eight were discharged from intensive care, meaning a global ICU survival of $57 \%$. Severe ECMO complications were rare, two of them needed surgical reintervention for bleeding (intrathoracic bleeding and cardiac tamponade), no limb ischemia nor cerebrovascular accidents were reported. No mechanical complications occurred with VA-ECMO.

Conclusion. Severe PGF is a critical condition effectively managed under VA-ECMO, allowing survivors to improve mainly through supportive care. Even PGF patients falling into circulatory arrest in the 
immediate postoperative setting, have a considerable chance for survival. VA-ECMO should be considered early in this clinical picture.

\section{Reference(s)}

1- Truby LK, DeRoo S, Spellman J, et al. Management of primary graft failure after heart transplantation: Preoperative risks, perioperative events, and postoperative decisions. Clin Transplant. 2019;33(6):e13557. 10.1111/ctr. 13557

2- Iyer A, Kumarasinghe G, Hicks M, et al. Primary graft failure after heart transplantation. J Transplant. 2011;2011:175,768. 10.1155/2011/175768

3- Mihaljevic T, Jarrett CM, Gonzalez-Stawinski G, et al. Mechanical circulatory support after heart transplantation. Eur J Cardiothorac Surg. 2012;41(1):200-206. 10.1016/j.ejcts.2011.04.017

\section{9}

ECMO and acute myocarditis: a successful partnership?

M. Mendoza-Prieto' ; J. Navarro-Rodriguez ${ }^{1}$; I. Espinosa-Rueda ${ }^{1}$

FJ. Reina-Martínez ${ }^{1}$; Y. Corcia-Palomo, ${ }^{1}$;. Martin-Villen ${ }^{1}$

${ }^{1}$ Critical care unit, Hospital Universitario Virgen del Rocío, Sevilla, Spain

Correspondence: M. Mendoza-Prieto

Intensive Care Medicine Experimental 2020, 9(1): 001,259

Introduction. Myocarditis is a heterogeneous clinical entity, ranging from subclinical disease to acute fulminant disease. A percentage of patients diagnosed with myocarditis can be present with severe ventricular dysfunction and cardiogenic shock, sometimes refractory to conventional therapy. Extracorporeal membrane oxigenation (ECMO) could be a therapeutic alternative in these critical cases.

Objectives. To assess clinical outcomes of patients with acute miocarditis who need mechanical support and compare them with patients that require ECMO for other different etiologies.

Methods. Retrospective observational study from 2011 to 2020 in a third level Intensive Care Unit (ICU) with heart transplantation program. Two groups were established: first group of patients in refractory cardiogenic shock due to acute myocarditis that needed VA-ECMO and a second group with VA-ECMO implantation for other reasons. Demographic data, personal history, ICU length of stay, days on ECMO, clinical outcomes and complications were collected. Qualitative variables were described as absolute number and frecuencies; quantitative variables as median and interquartile range (IR). Chi-squared test for qualitative variables and U Mann Whitney test for quantitative ones were performed.

Results. A total of 92 patients were included, 8 (8.70\%) of whom diagnosed with acute myocarditis. Clinical characteristics, outcomes and complications are shown in Table 1. Significant differences were found between both groups in terms of history of hypertension, previous heart disease and complications.

Table 1. Main clinical characteristics, outcomes and complications. LVEF (left ventricular eyection fraction), VAP (ventilator associated pneumonia)

\begin{tabular}{llll}
\hline Demographic data & $\begin{array}{l}\text { Myocarditis } \\
\text { group } \\
(\mathbf{n = 8 )}\end{array}$ & $\begin{array}{l}\text { Other causes } \\
\text { group(n=84) }\end{array}$ & $\mathbf{P}$ \\
\hline Male; $\mathrm{n}(\%)$ & $3(37.5)$ & $62(73.8)$ & 0.031 \\
Age; median (IR) & $40.5(32.75-48)$ & $56(45-62)$ & 0.007 \\
Hypertension; $\mathrm{n}(\%)$ & $0(0)$ & $34(40.5)$ & $\mathbf{0 . 0 2 3}$ \\
$\begin{array}{l}\text { Previous } \\
\text { heartdisease;n(\%) }\end{array}$ & $0(0)$ & $48(57.1)$ & $\mathbf{0 . 0 0 8}$ \\
$\begin{array}{l}\text { LVEF preECMO; } \\
\text { Median (IR) }\end{array}$ & $10(10-15)$ & $20(15-45)$ & $\mathbf{0 . 0 0 7}$ \\
$\begin{array}{l}\text { OUTCOMES } \\
\begin{array}{l}\text { Days on ECMO; median } \\
\text { (IR) }\end{array}\end{array}$ & $7(5.25-14.50)$ & $7(3-12)$ & 0.229 \\
\hline
\end{tabular}

\begin{tabular}{llll}
\hline Demographic data & $\begin{array}{l}\text { Myocarditis } \\
\text { group } \\
(\mathbf{n = 8}\end{array}$ & $\begin{array}{l}\text { Other causes } \\
\text { group(n=84) }\end{array}$ & $\mathbf{P}$ \\
\hline $\begin{array}{l}\text { ICU lentgh of stay; } \\
\quad \text { median (IR) }\end{array}$ & $13.50(9.75-43.75)$ & $19(10-36)$ & 0.826 \\
ICU mortality; $\mathrm{n}(\%)$ & $4(50)$ & $47(56)$ & 0.746 \\
Weaning of ECMO; $\mathrm{n}(\%)$ & $6(75)$ & $49(58.3)$ & 0.358 \\
ECMO OUTCOMES; $\mathbf{n}(\%)$ & & & 0.792 \\
Recovery & $3(37.5)$ & $23(26.7)$ & \\
Exitus & $3(37.5)$ & $32(37.2)$ & \\
Heart transplant & $2(25)$ & $20(23.3)$ & \\
Second device & $1(12.5)$ & $8(9.3)$ & \\
COMPLICATIONS; $\mathbf{n}(\%)$ & & & \\
Venous thrombosis & $5(62,5)$ & $8(9.5)$ & $\mathbf{0 . 0 0 0}$ \\
Cannula Thrombosis & $2(25)$ & $6(6)$ & $\mathbf{0 . 0 5 0}$ \\
Bacteriemia & $3(37.5)$ & $16(19)$ & 0.226 \\
VAP & $4(50)$ & $31(36.9)$ & 0.976 \\
Limb ischemia & $4(50)$ & $8(9.5)$ & $\mathbf{0 . 0 0 1}$ \\
\hline
\end{tabular}

Conclusion. ECMO seems to be an useful tool in clinical management of acute myocarditis. Better ECMO succesful weaning rates are observed in patients with acute myocarditis, but with a higher thrombotic complication rate.

\section{Reference(s)}

1. Kindermann I, Barth C, Mahfoud F, Ukena C, Lenski M, Yilmaz A, et al. Update on Myocarditis. Journal of the American College of Cardiology. 2012;59(9):779-92.

2. Lorusso R, Centofanti P, Gelsomino S, Barili F, Di Mauro M, Orlando P, et al. Venoarterial Extracorporeal Membrane Oxygenation for Acute Fulminant Myocarditis in Adult Patients: A 5-Year Multi-Institutional Experience. Ann Thorac Surg. 2016;101(3):919-26.

3. Diddle JW, Almodovar MC, Rajagopal SK, Rycus PT, Thiagarajan RR. Extracorporeal membrane oxygenation for the support of adults with acute myocarditis. Crit Care Med. 2015;43(5):1016-25.

\section{2}

In-vitro analysis of thermal heat signal transduction by the water circuit of a VV-ECMO system

R. Clement'; M. Hetzer'; AJ. Fritschi' ; M. Renes, ; J. Droogh ${ }^{1}$; A. Zanella ${ }^{4}$; M. Nijsten ${ }^{1}$

'Department of critical care, University Medical Center Groningen, Groningen, Netherlands; ${ }^{2}$ Pulsion medical systems se, Getinge AB, Feld-

kirchen, Germany; ${ }^{3}$ Maquet cardiopulmonary gmbh, Getinge AB, Rastatt, Germany; ${ }^{4}$ Department of anesthesiology, Fondazione IRCCS Ca'Granda

Ospedale Maggiore Policlinico, Milano, Italy

Correspondence: M. Nijsten

Intensive Care Medicine Experimental 2020, 9(1): 001,312

Introduction. Although extracorporeal membrane oxygenation (ECMO) is known to interfere with thermodilution (TD) methods for measuring cardiac output (CO) [1], it has been demonstrated that the ECMO system can also be used to generate TD signals [2]. For instance, TD within the EMCO blood circuit can accurately quantify cannula recirculation [3].

Objectives. We explored the feasibility of using the water heater unit circuit of the ECMO system as a transducer of defined thermal pulses.

Methods. A simulated VV-ECMO system (HLS Set Advanced 7.0 \& Cardiohelp-I System, Maquet Cardiopulmonary GmbH, Rastatt, Germany) with a water circuit and a blood circuit filled with water was used. Thermistor-tipped catheters with high temperature and time resolution were used to measure the temperature at 4 locations: at the venous and arterial outlet of the ECMO (TBin and TBout) and the inlet and outlet of the ECMO water circuit (TWin and TWout). Blood and 
water flow were 3.5 and $1.55 \mathrm{~L} / \mathrm{m}$ respectively. The membrane oxygenator was connected to two heater units set at different temperatures. By rapidly switching between the heater units for $10 \mathrm{~s}$, we generated temperature pulses in the water flow. From the temperature curves, the full width at half maximum (FWHM) of pulses and the areas under the temperature curve were determined to obtain the caloric pulse in both circuits to assess the efficiency of heat-transfer in both directions. Results. The generated water thermal pulse had a max $\Delta T$ Tin of 3.76 oK, an FWHM of $10.0 \mathrm{~s}$ and an AUC of $946 \mathrm{cal}$. The resulting $\triangle$ TBout was 1.27 oK with an FWHM of $9.8 \mathrm{~s}$ and an AUC of $711 \mathrm{cal}$, corresponding with a heat transfer efficiency of $75.1 \%$. Likewise, a blood temperature pulse with $\Delta$ TBin of 0.87 oK resulted in a $\Delta$ TWout of 0.65 oK or $74.9 \%$ of the temperature difference and a heat transfer efficiency of $35.9 \%$.

Conclusion. We have demonstrated remarkably good thermal signal transfer from the water circuit to the blood circuit and vice versa in ECMO system investigated. This is the result from effective countercurrent heat exchange between water and blood. Because of this effective heat transfer in both directions, it is technically feasible to perform thermodilution measurements employing only the water circuit for both thermal pulse generation and high resolution temperature measurements. This greatly facilitates the implementation of thermodilution for measuring cannula recirculation and other parameters during ECMO.

\section{Reference(s)}

1. MN has received financial or material support from Getinge, MH \& AF work for Getinge.

2. [1] Haller et al. Thermodilution cardiac output may be incorrect in patients on venovenous extracorporeal lung assist. Am J Respir Crit Care Med 1995;

3. [2] Nijsten M, Herner A, Huber W, Konrad M. Thermal heat signal transduction by the water circuit of a VV-ECMO system. Abstract ESICM 2019 Berlin.

4. [3] Cipulli F et al. Quantification of Recirculation During Veno-Venous Extracorporeal Membrane Oxygenation: In Vitro Evaluation of a Thermodilution Technique. ASAIO Journal 2021

\section{9}

The utility of transthoracic echocardiographic measures in the prediction of postoperative pulmonary edema development

M. Karaman Ilić' ; L. ŠTefančić'; A. ŠImunić-Forićc ${ }^{1}$

${ }^{1}$ Anesthesiology, Radiochirurgia,Cancer treatment center in Strmec, Sveta Nedelja, Zagreb, Croatia

Correspondence: M. Karaman llić

Intensive Care Medicine Experimental 2020, 9(1): 001,339.

Introduction. Transthoracic ultrasound has been successfully used in intensive care units for many years. Global cardiac function, valvular apparatus, and pericardial space are observed.

This study aimed to assess the significance of changes in the Tricuspid Annular Plane Systolic Excursion (TAPSE) and Inferior Vena Cava(IVC) diameter and IVC collapsibility index (IVC Cl) as a predictor for the development of postoperative pulmonary edema.

Methods. In the period from October 2020 to May 2021.,one hundred patients scheduled for the major noncardiac surgical procedures were included in this observational study. Tricuspid Annular Plane Systolic Excursion (TAPSE) and Inferior Vena Cava diameter (IVC) collapsibility index (IVC Cl) were measured the day before and the day after the procedure. Development of pulmonary parenchymal opacity, as a sign of pulmonary edema, was monitored by lung ultrasound.

Results. Changes in preoperative vs postoperative TAPSE measurements were seen: TAPSE $25 \mathrm{~mm} 2$ vs 0 patients, TAPSE $24 \mathrm{~mm}$ 22 vs 8 patients, TAPSE $23 \mathrm{~mm} 21$ vs 6 patients, TAPSE $22 \mathrm{~mm} 28$ vs 15 patients, TAPSE $20 \mathrm{~mm} 20$ vs 28 patients, TAPSE $19 \mathrm{~mm} 7$ vs 37 patients and TAPSE $18 \mathrm{~mm} 0$ vs 6 patients. Preoperative measurements showed that 73 patients had IVC diameter $<2 \mathrm{~cm}$ with IVCCl $>50 \%$ and 27 patients had IVC diameter $\geq 2 \mathrm{~cm}$ with IVCCl $>50 \%$.
In the postoperative period, 47 had IVC diameter $\leq 2 \mathrm{~cm}$ of which 25 patients had IVCCl $>50 \%$ and $22 \mathrm{IVCCl} 35-50 \%$ while 43 patients had IVC diameter $\geq 2 \mathrm{~cm}$ of which 31 patient with IVCCl $35-50 \%$ and 12 patients with $\mathrm{IVCCl}<35 \%$. All patients with a decline in TAPSE developed a B profile on lung ultrasound.

Conclusion. A combination of TAPSE and IVCcl reduction has a strong predictive value for developing postoperative pulmonary edema due to fluid overload.

\section{Reference(s)}

1. A.McLean,S.Huang.(2012)Right heart assessment.Critical Care Ultrasound Manual. Elsevier.Australia.p 64-75.

\section{3}

Mean arterial pressure targets and patient-important outcomes in critically ill adults: a systematic review and meta-analysis of randomized trials

KL. Carayannopoulos'; A. Pidutti ${ }^{2}$; Y. Upadhyaya ${ }^{3}$; F. Alshamsi' ${ }^{4}$;. Basmaji ${ }^{5}$; W. Alhazzani6; K. Lewis,

${ }^{1}$ Critical care, McMaster University, Hamilton, Canada; ${ }^{2}$ Medicine, McMaster University, Hamilton, Canada; ${ }^{3}$ Surgery, McMaster University, Hamilton, Canada; ${ }^{4}$ Medicine, United Arab Emirates University, Al Ain, United Arab Emirates; ${ }^{5}$ Medicine, London Health Sciences Centre, London, Canada; ${ }^{6}$ Department of medicine/department of health research methods, evidence and impact, McMaster University, Hamilton, Canada; ${ }^{7}$ Critical care, St Josephs Healthcare Hamilton, Hamilton, Canada

Correspondence: K.L. Carayannopoulos

Intensive Care Medicine Experimental 2020, 9(1): 001,373.

Introduction. Hypotension is a common and potentially catastrophic complication of critical illness. Hypotension may manifest from several underlying pathologic problems in the critically ill such as hypovolemia, vasodilation, and reduced cardiac output. Persistent and severe systemic hypotension ultimately leads to tissue hypoperfusion and end organ damage, perpetuating critical illness and ischemia.

Fluids and vasoactive drugs are the mainstay therapies of undifferentiated hypotension, however, they are not without risk. Overzealous fluid administration has been associated with prolonged mechanical ventilation and intensive care unit (ICU) length of stay, increased acute kidney injury, and mortality. Vasopressor use must be balanced against the risk of arrhythmia and peripheral/gut ischemia. The 2016 Surviving Sepsis Campaign Guidelines strongly recommend targeting a MAP of $65 \mathrm{mmHg}$ through the use of fluids and vasopressors in patients presenting with septic shock, however, this recommendation was based on moderate certainty of evidence as it was driven by small, individual randomized controlled trials. Published in March of 2020, the "Effect of Reduced Exposure to Vasopressors on 90-day mortality in Older Critically III Patients With Vasodilatory Hypotension" ultimately demonstrated no significant difference in mortality in patients randomized to permissive hypotension, its results have not been examined in the context of data from previous trials. Considering this, we conducted a systematic review and meta-analysis to address the potential benefits and harms of targeting higher vs lower MAP.

Methods. We searched MEDLINE, EMBASE, and the Cochrane Library in addition to clinicaltrials.gov up to May 2021 for parallel group randomized controlled trials comparing lower versus higher MAP targets in patients with shock of any type. Outcomes included mortality (28 days and longest follow up), duration of vasopressor therapy, total volume of fluid administered, need for renal replacement therapy, ICU length of stay, hospital length of stay, peripheral or digital ischemia or necrosis, mesenteric ischemia, or cardiac arrhythmias. Screening, data extraction, and risk of bias assessment were all completed independently and in duplicate. We used the GRADE approach to assess quality of evidence. PROSPERO: CRD42020219971. 
Results. Overall, 6 studies were included, enrolling a total of 3,694 patients. Targeting a higher MAP (generally $75-85 \mathrm{mmHg}$ ) did not decrease all-cause mortality in patients with shock (RR 1.06, 95\% $\mathrm{Cl} 0.99$ to $1.15, \mathrm{I} 2=0 \%, \mathrm{P}=0.99$; high certainty). When a subgroup analysis was conducted including only studies whose control group targeted a MAP of $65 \mathrm{mmHg}$ or less, again no mortality difference was demonstrated (RR $1.07,95 \% \mathrm{Cl} 0.98$ to $1.17, \mathrm{I} 2=0 \%, \mathrm{P}=0.93$ ). Higher MAP targets increased duration of vasopressor therapy (MD $11.91 \mathrm{~h}, 95 \% \mathrm{Cl} 2.27$ to $21.55, \mathrm{I} 2=53 \%, \mathrm{P}=0.10$; moderate certainty). We found no difference in need for renal replacement therapy when targeting a higher MAP (RR $0.89,95 \% \mathrm{Cl} 0.77$ to 1.01 , $12=0 \%, P=0.63$; high certainty). The subgroup of trials with control MAP less than or equal to $65 \mathrm{mmHg}$ also demonstrated no difference with a higher MAP target (RR $0.75,95 \% \mathrm{Cl} 0.56$ to $1.01,12=0 \%$, $\mathrm{P}=0.81$ ). A further subgroup analysis of patients with a history of chronic hypertension, however, captured 2 studies $(n=1,466)$ and demonstrated a decreased need for renal replacement therapy when a higher MAP was targeted (RR $0.83,95 \% \mathrm{Cl} 0.71$ to 0.98 , $12=0 \%, P=0.40)$. We identified no difference in incidence of digital ischemia or necrosis, incidence of mesenteric ischemia, or arrhythmias with a higher MAP target. Finally, there was no difference in total fluid administered, ICU length of stay or hospital length of stay. Conclusion. In conclusion, our systematic review and meta-analysis demonstrated with high certainty that there is no difference in mortality when a higher mean arterial pressure is targeted in critically ill adult patients with shock. Further studies are needed to determine the impact of mean arterial pressure on need for renal replacement therapy in this population. Furthermore, studies to examine subgroup effects as well as permissive hypotension to a MAP of $60 \mathrm{mmHg}$ would contribute valuable knowledge to the care of this population.

\section{Reference(s)}

1. Rhodes A, Evans LE, Alhazzani W, et al. Surviving Sepsis Campaign: International Guidelines for Management of Sepsis and Septic Shock: 2016. Vol 45:2017.10.1097/CCM.0000000000002255

2. McIntyre WF, Um KJ, Alhazzani W, et al. Association of vasopressin plus catecholamine vasopressors vs catecholamines alone with atrial fibrillation in patients with distributive shock a systematic review and metaAnalysis. JAMA—J Am Med Assoc. 2018;319(18):1889-1900. 10.1001/ jama.2018.4528

3. Boyd JH, Forbes J, Nakada TA, Walley KR, Russell JA. Fluid resuscitation in septic shock: A positive fluid balance and elevated central venous pressure are associated with increased mortality. Crit Care Med. 2011:39(2):259-265. 10.1097/CCM.0b013e3181feeb15

4. Lamontagne F, Meade MO, Hébert PC, et al. Higher versus lower blood pressure targets for vasopressor therapy in shock: A multicentre pilot randomized controlled trial. Intensive Care Med. 2016;42(4):542-550. 10 1007/s00134-016-4237-3

5. Grand J, Meyer AS, Kjaergaard J, et al. A randomised double-blind pilot trial comparing a mean arterial pressure target of $65 \mathrm{~mm} \mathrm{Hg}$ versus $72 \mathrm{~mm} \mathrm{Hg}$ after out-of-hospital cardiac arrest. Eur Hear Journal Acute Cardiovasc Care. 2020;9(4_suppl):S100-S109. 10.1177/2048872619900095

6. Carrick MM, Morrison CA, Tapia NM, et al. Intraoperative hypotensive resuscitation for patients undergoing laparotomy or thoracotomy for trauma: Early termination of a randomized prospective clinical trial. J Trauma Acute Care Surg. 2016;80(6):886-896. 10.1097/TA.0000000000 001044

7. Ameloot K, Jakkula P, Hästbacka J, et al. Optimum Blood Pressure in Patients With Shock After Acute Myocardial Infarction and Cardiac Arrest. J Am Coll Cardiol. 2020;76(7):812-824. 10.1016/j.jacc.2020.06.043

8. Asfar P, Meziani F, Hamel JF, et al. High versus low blood-pressure target in patients with septic shock. N Engl J Med. 2014;370(17):1583-1593. 10 1056/NEJMoa1312173

9. Lamontagne F, Richards-Belle A, Thomas K, et al. Effect of Reduced Exposure to Vasopressors on 90-Day Mortality in Older Critically III Patients with Vasodilatory Hypotension: A Randomized Clinical Trial. JAMA—J Am Med Assoc. 2020;323(10):938-949. 10.1001/jama.2020.0930

10. None
001390

Ischemic Complications In Patients With Extracorporeal

Membrane Oxygenation

M. Gärtner ${ }^{1}$; JKM. Fichte'; HB. Hopf

${ }^{1}$ Röntgenstrasse 20, Asklepios Klinik Langen, Anaesthesia, periop. med. and interdiscipl. intensive care medicine, Langen (Hessen), Germany;

${ }^{2}$ Anaesthesia, intensive care medicine and pain medicine, University Hospital Frankfurt, Frankfurt am Main, Germany; ${ }^{3}$ Anaesthesia, perioperative and interdisciplinary intensive care medicine, Asklepios Klinik Langen, Langen, Germany

Correspondence: H.B. Hopf

Intensive Care Medicine Experimental 2020, 9(1): 001,390.

Introduction. During ECMO therapy ischemia of the limbs or internal organs are potential lethal complications.

Objectives. This study analysed incidence and type of ischemic complications during ECMO therapy, divided in limb, mesenteric, cardiac and neurological ischemia.

Methods. Single-centre retrospective observational study. 348 patients treated with V-V, V-A or V-V-A ECMO from April 1st 2011 until March 31st 2020 were screened, of whom 321 with diagnosis of ARDS, cardiogenic or septic shock were included. Primary outcome variable was the type of ischemic complication. Secondary outcome variables were serum lactate levels $24 \mathrm{~h}$ before and immediately after diagnosis of the ischemic complication, duration of ICU and hospital stay, ECMO therapy and duration of invasive ventilation. The descriptive statistics were performed with medians and interquartile ranges (IQR 25th-75th percentiles). Univariate tests were performed using the chisquare test, Mann-Whitney $U$ test, Kruskal-Wallis test, Fisher's exact test and Friedman's test. The data were processed within a multivariate logistic regression to the primary outcome variable in order to identify possible predictors, by using odds ratio and confidence intervals. The regression was carried out stepwise backwards. All tests were twosided and with at a significance level of $p<0.05$.

The study was approved by the Ethics Committee of the Medical Association of Hessen (file number 2020-1977-evBO).

Results. $62 / 321$ patients (19,3\%) were diagnosed with an ischemic complication. Most common areas were limbs $(n=32)$ and mesenteric ischemia $(n=21)$. Patients who were diagnosed with a septic shock had the highest rate of ischemic complications $(36,2 \%)$. No difference was found in survival between patients with and without ischemic complication. Using multivariate logistic regression, age $\geq 50$ years, use of haemodialysis and initial diagnosis of a septic shock could be identified as predictors for ischemic complications.

Conclusion. Ischemic complications are frequent during ECMO therapy. An age of at least 50 years, the use of haemodialysis and diagnosis of a septic shock were predictors of ischemic complications. No correlation between ECMO mode and ischemic complications was found. There was no difference in survival between patients with and without ischemic complications.

\section{Reference(s)}

1. The statistical evaluation was carried out under the advice and supervision of members of the Institute for Biostatistics and Mathematical Modelling at the Department of Medicine of the Goethe University Frankfurt (Prof. Dr. Eva Herrmann).

001434

Myocardial injury in the COVID-19 intensive care unit-an observational study of cardiac biomarkers and echocardiographic findings in critically ill COVID-19 patients

S. Jansson ${ }^{1}$; P. Johansson Blixt ${ }^{1}$; H. Andersson, ${ }^{1}$; C. Hedström, ${ }^{2}$; M. ÅStröm Aneq ${ }^{2}$; M. Chew ${ }^{1}$

${ }^{1}$ Department of anaesthesia and intensive care, biomedical and clinical sciences, Linköping University, Linköping, Sweden; ²Department of clinical physiology and department of health medicine and caring sciences, Linköping University, Linköping, Sweden

Correspondence: $\mathrm{S}$. Jansson

Intensive Care Medicine Experimental 2020, 9(1): 001,434 
Introduction. Previous studies have found an increased incidence of myocardial injury and cardiac abnormalities in patients with COVID19. It is suspected that the incidence increases with the severity of COVID-19 symptoms. This could have both short- and long-term effects, including higher mortality rates among those with myocardial injury. Patients with myocardial injury more often require critical care, however, studies on myocardial injury and echocardiographic abnormalities in critically ill patients are limited.

Objectives. The purpose of this study was to document the incidence of myocardial injury and echocardiographic abnormalities in consecutive patients treated for COVID-19 in the Intensive Care Unit at Linköping University Hospital, as well as investigate if there is any association between myocardial injury, echocardiographically defined ventricular dysfunction and 30-day mortality.

Methods. 46 patients were included in the study. All were $>18$ years of age, had laboratory confirmed SARS-CoV2 infection and an echocardiogram performed within $48 \mathrm{~h}$ of ICU admission. We extracted data regarding baseline characteristics including comorbidities, intensive care treatment, laboratory and echocardiographic variables and outcomes.

Results. $67.4 \%$ had myocardial injury (defined as high-sensitivity troponin-T (hsTnT) $\geq 45 \mathrm{ng} / \mathrm{l}$ ) at any time during the first 14 days of ICU admission. $37,9 \%$ had left ventricular dysfunction and $26.5 \%$ had right ventricular dysfunction. 30-day mortality was $17.4 \%$. Neither myocardial injury or ventricular dysfunction were associated with 30-day mortality, however there was an association between NTproBNP and ICU mortality. Patients with myocardial injury spent more days on mechanical ventilation and stayed longer in the ICU.

Conclusion. Over two thirds of our cohort suffered from myocardial injury, but we did not find an association with ventricular dysfunction or 30-day mortality.

e-Posters: Critical care organisation, quality management, outcomes

\section{1}

\section{Intensive care staff perceptions of non-verbal cues}

in the deteriorating patient

A. Wall'

${ }^{1}$ Critical Care Unit, North Middlesex University Hospital NHS Trust, Lon-

don, United Kingdom

Correspondence: A. Wall

Intensive Care Medicine Experimental 2020, 9(1): 000011.

Introduction. Identifying deteriorating patients is critical to the work of all healthcare professionals in the intensive care unit (ICU). Staff often report having a 'gut-feeling' about a patient causing them concern. This has been studied previously, predominantly in nurses[1]. In the acute setting, the visual assessment provides a great wealth of information regarding the clinical condition of a patient. Nonverbal cues, such as facial expression, may alert staff to a critically ill patient but no early warning system specifically includes their routine assessment.

Objectives. The aim of this study is to explore ICU staff perception of the importance of non-verbal cues in the ICU, their ability to raise concerns based on them, and the training received in assessing these cues.

Methods. Staff members from the Critical Care Unit in North Middlesex University Hospital (NMUH) were asked to participate in the study. Clinical staff of all grades and experience were included. A questionnaire was circulated via email and in paper form on the ward. The questionnaire contained 1 multiple choice question, and 5 7-point Likert scale questions (Strongly disagree $=1$, Neither agree nor disagree $=4$, Strongly agree $=7$ ). Data was analysed and Likert scores generated.

Results. Overall, 33 responses were received. Of these there were 11 junior doctors (33\%), 4 intensive care consultants (12\%), and 18 nurses (55\%).
33 participants (100\%) felt that assessing non-verbal cues (e.g. facial expression, posture) is an important part of identifying a deteriorating patient, and 31 participants (94\%) would feel comfortable raising their concerns about a patient based on non-verbal cues alone.

Only 14 participants (42\%) thought that non-verbal cues were routinely assessed on the unit. 33\% of participants agreed that they had received adequate training in assessing non-verbal cues. This was only $27 \%$ of the participating doctors and $50 \%$ of nurses. Overall response to this question was neutral (Likert scale average $=3.97$ ).

31 participants felt (94\%) that specific facial expressions can indicate that a patient is critically ill. The most commonly observed non-verbal cue identified by ICU staff was facial expression (79\%), followed by changes to voice $(61 \%)$, poor eye contact $(58 \%)$, and slouched posture (39\%).

Conclusion. ICU staff at NMUH unanimously believe that non-verbal cues are crucial in identifying a deteriorating patient. This shared perception is likely one of the factors allowing members of the team to raise their concerns about a patient based solely on non-verbal cues. Further to this, there is a clear perception that a patient's facial expression can indicate they are critically unwell.

Despite their perceived importance, ICU staff do not feel that these cues are routinely assessed or that they have received adequate training in identifying them. This is felt predominantly amongst the doctors on the ward. Further work is needed to ensure that staff are encouraged to identify non-verbal cues when assessing a deteriorating patient. This should apply to patients on all wards.

Routine assessment of a patient's non-verbal cues, particularly facial expression, could help ICU staff to identify the deteriorating patient. There may be reason to develop an early warning score that encourages medical staff to observe non-verbal cues.

\section{Reference(s) \\ 1. King L et al. J Adv Nurs. 2002;37(4):322-329. \\ 2. No funding or sponsorship was required to undertake this work.}

\section{4}

The Use of Serum Albumin as a Non Respiratory Predictor of Outcome of Weaning in Critically III Patients

MJ. Canillas-Amancio ${ }^{1}$; A. Guzman-Banzon²; ME. Blanco-Limpin²; E. Aventura ${ }^{3}$

${ }^{1}$ Pulmonary and Critical Care Medicine, Philippine Heart Center, Quezon City, Philippines; ${ }^{2}$ Pulmonary and critical care division, Philippine Heart Center, Quezon City, Philippines; ${ }^{3}$ Pulmonary medicine, The Medical City Hospital, Ortigas Avenue, Pasig, Metro Manila, Philippines, Pasig, Philippines

Correspondence: M.J. Canillas-Amancio

Intensive Care Medicine Experimental 2020, 9(1): 000024

Introduction. Serum albumin is the most abundant protein and is essential in maintaining the oncotic pressure needed for proper distribution of body fluids between blood vessels and body tissues. Serum albumin concentration is altered in critically ill patients especially with septic shock and after major surgery. Elevated hospital morbidity and mortality has been linked with hypoalbuminemia, which has been recognized to be a dependable prognostic indicator among critically ill patients.

Methods. This was a prospective cross sectional study done at the intensive care unit of the Philippine Heart Center. Patients' $\geq 19$ years old that require the use of invasive mechanical ventilation were included in the study. Demographic profile and clinical characteristics were recorded. Blood extraction for albumin determination was collected $24 \mathrm{~h}$ after attachment to mechanical ventilator and prior to weaning. Patients were classified according to their weaning outcomes which was categorized into: (a) Group 1, patients weaned within 24 hours; (b) Group 2, difficult weaning, weaned after $24 \mathrm{~h}$ or up to 7 days; (c) Group 3, prolonged weaning, patients weaned after $>7$ days, and (d) Group 4, no weaning, patients not weaned at all after 4 weeks or separation attempt failed. 
Results. One hundred and twenty patients were included in the study who met the selection criteria. Patients with higher albumin level had a shorter duration of weaning (group 1) while a lower albumin concentration upon recruitment was shown in groups 2,3 and 4 compared to prior to extubation ( $p$-value 0.0000 .000 and 0.029 respectively). Serum albumin of $27.9 \mathrm{~g} / \mathrm{L}$ has shown to predict the weaning outcome in mechanically ventilated patients $(82.47 \%$ sensitivity and $86.96 \%$ specificity). Those with lower albumin levels have a higher SOFA score $(r=0.4256$; $p$-value $<0.001)$ thus indicating a higher relative probability of mortality.

Conclusion. Serum albumin has been recognized as a clinical nonrespiratory marker which provides a good predictor of weaning in mechanically ventilated critically ill patients. An admitting albumin level of $>27.9 \mathrm{~g} / \mathrm{L}$ has shown to predict the weaning success among critically ill patients with a sensitivity of $82.47 \%$ and specificity of $86.96 \%$. While the association between the decrements in albumin concentration and higher SOFA score predicts a higher relative probability of mortality.

\section{Reference(s)}

1. J. P. Nicholson, M. R. Wolmarans G. R. Park, The role of albumin in critical illness: British Journal of Anaesthesia, Volume 85, Issue 4, 1 October 2000 599-610

2. Gaetan Beduneau,Tai Pham, Frederique Schotgen, et al., Epidemiology of weaning outcome according to a new definition; The WIND Study. American Journal of Respiratory and Critical Care Medicine 2017; 195:6.

3. Zhi-Yong Zhao, Ning Wu, Shiu-Xiang D., et al., Serum albumin is a predic tor for duration of weaning in patients with traumatic brain injury. Int J. Clin Exp Med 2016;9 (2):4041-4046

4. Hawkins JW, Dugaiczyl A., The human serum albumin gene: structure of a unique locus. Gene 19 (1): 55-8 PMID 6,292,049 1982

5. Plummer AL, Gracey DR. Consensus conference on artificial airways in patients receiving mechanical ventilation. Chest. 1989; 96:178-80.

\section{8}

A Review of Handovers on the Intensive Care Unit

E. Jones ${ }^{1}$; D. Hepburn,

${ }^{1}$ Cardiff medical school, Cardiff University, Cardiff, United Kingdom;

${ }^{2}$ Intensive care unit, Royal Gwent Hospital, Newport, United Kingdom

Correspondence: $\mathrm{E}$. Jones

Intensive Care Medicine Experimental 2020, 9(1): 000038

Introduction. Every new admission to the Intensive Care Unit (ICU) prompts a handover from the referring department to the ICU staff This step in the patient pathway provides an opportunity for information to be lost and for patient care to be compromised.

Mortality rates in Intensive Care have fallen over the last twenty years, however, $20 \%$ of patients admitted to an ICU will die during their admission (ICS, 2018). Communication errors contribute to approximately two-thirds of notable clinical incidents; over half of these are related to a handover (Starmer et al., 2012).

NICE have concluded that structured handovers can result in reduced mortality, reduced length of hospital stay and improvements in senior Clinical Staff and Nurse satisfaction (2018)

Methods. A checklist was created to review the information shared and to score the handover1. This checklist was created with Doctors and Nurses and is relevant for handovers between all staff members. Information was gathered prospectively by directly observing 17 handovers on the ICU.

Results. There is a notable discrepancy in the quality of handovers of new patients. This is true of handovers between Doctors, Nurses and a combination of the two. It is also true of all staff grades. Whilst a Doctor may have reviewed the patient prior to their arrival, $41 \%(n=7)$ of patients weren't handed over to a doctor. The most commonly missed pieces of information were details of the patient's weight (96\%, $n=16)$, their height $(100 \%, n=17)$, whether the patient has previously been admitted to an ICU $(78 \%, n=15)$ and whether the patient has any allergies $(71 \%, n=12)$.
Conclusion. The handover of new patients to the ICU is often unstructured and important information is missed. This can be said for all staff members and grades, and for handovers from all hospital departments.

\section{Reference(s)}

1. Chapter 32, Structured Patient Handovers, (2018).

2. Starmer AJS, Nancy D. Srivastava, Rajendu. Allen, April D. Landrigan, Christopher D. Sectish, Theodore C. I-PASS, a Mnemonic to Standardize Verbal Handoffs. Pediatrics. 2012;129(2).

3. ICS. Guidelines for the Provision of Intensive Care Services, Version 2. ICS; 2018.

\section{9}

Improving the quality of the Intensive Care Unit (ICU) microbiology ward round documentation through the introduction of a microbiology ward round proforma P. Harbham ${ }^{1}$; L. Hamer ${ }^{1}$; A. Ariff ${ }^{1}$; M. Eddula ; B. Ramamurthy

${ }^{1}$ Intensive care medicine, Sandwell and West Birmingham NHS Trust, B18

7QH, United Kingdom

Correspondence: P. Harbham

Intensive Care Medicine Experimental 2020, 9(1): 000049

Introduction. In their document Good Medical Practice, the General Medical Council sets out the standards they expect from healthcare professionals when documenting in clinical notes.

The expectation is legible documentation which must include relevant clinical findings, requested investigations and their results, treatment plans and a clear documentation of who is making the decisions (1) A failure to comply with these standards therefore has both patient safety and medico-legal implications for practitioners.

Objectives. We aimed to audit the documentation quality of the Intensive Care Unit (ICU) microbiology ward round and improve any inadequacies found through a two-step quality improvement project. Methods. A baseline audit of the ICU microbiology ward round documentation was conducted in October 2020. This looked at four areas of documentation: i) current infection issues ii) current infection treatment iii) COVID 19 result iv) advice from the ward round.

Following this, a two-step intervention comprising of an educational session for staff, and the introduction of a microbiology ward round proforma was implemented. A re-audit was performed in December 2020.

Results. Baseline data showed documentation was generally poor. Current infection issues and current infection treatment were documented in $64 \%$ and $72 \%$ of ward round entries respectively. COVID results were only documented in $36 \%$ of entries. Advice from the microbiology ward round was well documented, being present in $96 \%$ of entries.

Post-intervention, documentation of current infection issues and current infection treatment was much improved, present in $83 \%$ and $86 \%$ of entries respectively. Documentation of COVID results showed a statistically significant improvement, documented in $81 \%$ of entries, $t(19)=3.34, p=0.001691$. Advice from the microbiology ward round remained well documented at $90 \%$ of entries.

Conclusion. Our intervention showed improvement in documentation quality through a quick and efficient method that is adaptable to both in person and virtual ward rounds. The intervention is easy to replicate and has the potential to reduce the well-recognised risks of poor-quality documentation which includes patient safety and medico-legal matters. Integrating the proforma template into electronic noting systems represents the next step to explore.

\section{Reference(s)}

1. General Medical Council. Good medical practice (2013) Domain 1: Knowledge skills and performance. [online] Available at:<http://www.gmc-uk. org/guidance/good_medical_practice.asp > [Accessed 22 Feb 2021]. 


\section{9}

Does clinical frailty scale predict outcome for critically ill patients in ICUs in England?

L. Tong ; X. Watson'; S. Farnell-Ward ' ; C. Ryan ${ }^{1}$; H. Farah ${ }^{1}$; DO. Lonsdale ${ }^{2}$; M. Cecconi' ${ }^{3}$;. Jung ${ }^{4}$; F. Hans ${ }^{5}$; B. Guidet ${ }^{6}$; JM. Muessig'; S. Leaver ${ }^{1}$

'Department of critical care, St George's University Hospitals NHS Foundation Trust, London, United Kingdom; ${ }^{2}$ Department of clinical pharamcology,st george's university of london, department of critical care, St George's University Hospitals NHS Foundation Trust, London, United Kingdom; ${ }^{3}$ Anesthesia and intensive care, Humanitas Research Hospital, Milan, Italy; ${ }^{4}$ Dep. of cardiology, pulmonology and angiology, University Hospital of Düsseldorf, Düsseldorf, Germany; ${ }^{5}$ Department of anaesthesia and intensive care, Haukeland University Hospital / Health Bergen, Bergen, Norway; ${ }^{6}$ Réanimation Médicale, Hôpital Saint-Antoine, Paris, France; ${ }^{7}$ Cardiology, pulmonary diseases, vascular medicine, Universität Düsseldorf, Düsseldorf, Germany

\section{Correspondence: L. Tong}

Intensive Care Medicine Experimental 2020, 9(1): 000069

Introduction. Caring for older people is an integral part of critical care medicine and the age of patients admitted to critical care in the UK is increasing [1]. Clinical decisions for very-old patients, usually defined as over 80 [2], can be particularly challenging. The relatively shorter life-expectancy of this age group necessitates careful consideration of the potential benefits of a therapy and its risks of failure or harm. Chronological age alone is an insufficient predictor of outcome from critical illness, since there is often significant variation in physical and physiological reserve of older people. Measures of overall health in old age, such as the clinical frailty scale (CFS) are increasingly used to help guide decisions.

Objectives. Recently, two large prospective observational studies (VIP 1\&2) $[3,4]$ reported the levels of frailty and outcomes for veryold patients admitted to intensive care units (ICUs) across Europe, confirming the associations between increasing frailty and 30-day mortality. We undertook an analysis of data from patients in United Kingdom, to understand the characteristics of the very-old critically unwell population in our own healthcare system (the NHS).

Methods. Participants included in this analysis were enrolled by a critical care unit in the UK to one of two prospective observational studies of very old intensive care patients (VIP-1 and -2). Demographics and baseline characteristics were recorded, alongside ICU interventions and outcomes (ICU- and 30-day mortality). Patient characteristics, interventions and outcome measures were compared by frailty group using standard parametric and non-parametric statistical tests. Multivariable logistic regression modelling was undertaken to test association between baseline characteristics, admission type and outcome. Results.

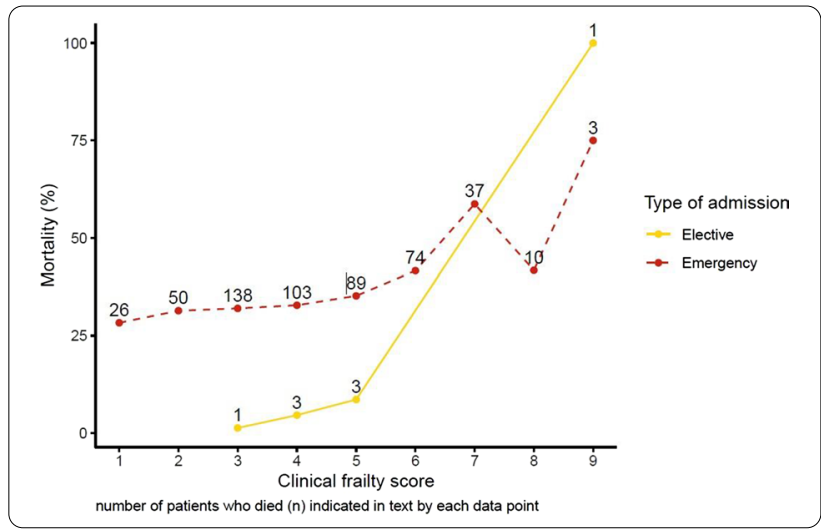

1858 participants were enrolled from 82 ICUs to VIP- $1(n=1036)$ and $-2(n=822) .53 \%$ of admissions were male. The median age was 83 . The majority of participants had a frailty score of 3-5. 30-day survival was significantly lower in the frail group (58\%) compared to vulnerable
$(66 \%)$ and fit $(68 \%)$ participants $(p<0.01)$. There was increasing mortality with increasing frailty score. At moderate frailty (CFS 5-6) there was $61 \%$ survival at 30 -days, compared with $42 \%$ survival for high frailty (CFS $>6$ ). In the multiple logistic regression analysis, SOFA score, reason for admission and frailty were independently associated with increased 30-day mortality $(p<0.01)$.

Conclusion. In the very-old cohort of patients admitted to critical care in United Kingdom, frailty is associated with an increase in mortality at 30-days. This supports the use of frailty in informing discussions about admission to intensive care for the very-old. UK national guidelines recommend CFS of 5 as the threshold at which clinicians should start to consider if harms will outweigh benefits of critical care [5]. This study suggests that the majority of patients with this level of frailty will survive an ICU admission, although we acknowledge that survival is not the only important outcome for patients, particularly the very old.

\section{Reference(s)}

1. None

2. Intensive care national audit \& research centre (ICNARC). Key statistics from the Case Mix Programme. 2019; Available from: https://www.icnarc. org/Our-Audit/Audits/Cmp/Reports/Summary-Statistics.

3. Flaatten, $\mathrm{H}$., et al., The status of intensive care medicine research and a future agenda for very old patients in the ICU. Intensive Care Med, 2017. 43(9): p. 1319-1328.

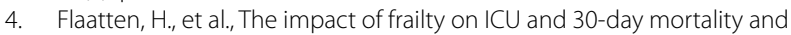
the level of care in very elderly patients ( $\geq 80$ years). Intensive Care Medicine, 2017. 43(12): p. 1820-1828.

5. Guidet, B., et al., The contribution of frailty, cognition, activity of daily life and comorbidities on outcome in acutely admitted patients over 80 years in European ICUs: the VIP2 study. Intensive care medicine, 2020. 46(1): p. 57-69.

6. National Institute for Health \& Clinical Excellence (NICE). COVID-19 rapid guideline: critical care in adults NICE guideline [NG159]. 3rd September 2020; Available from: https://www.nice.org.uk/guidance/ng159/chapt er/2-Admission-to-critical-care.

\section{0}

Establishing a Transfer Service in Aneurin Bevan University Health Board (ABUHB)

P. Collett ${ }^{1} ;$ A. Jones ${ }^{2}$

${ }^{1}$ Intensive Care, ABUHB, Cwmbran, United Kingdom; ${ }^{2}$ Intensive care, The Grange University Hospital—Ysbyty Prifysgol y Faenor, Cwmbran, United Kingdom

Correspondence: P. Collett

Intensive Care Medicine Experimental 2020, 9(1): 000090

Introduction. In 2020 ABUHB underwent a large structural change with the downgrading of two District General Hospitals (DGH) to Enhanced Local General Hospitals (ELGH) and the opening of The Grange University Hospital (GUH), an acute DGH centralising intensive care, emergency medicine, obstetrics and paediatrics. The new system would result in an increase in demand of both 'step up' and 'step down' transfers. A dedicated Transfer Service was proposed to meet this new demand. This would be an entirely new service in ABUHB with no previous model to build on. A literature search did not identify any similar articles which could guide the development of this project.

Prior to the implementation of the service the demand would need to be quantified, appropriate individuals would need to be selected and trained, a triage tool would need to be created to identify the team with the most appropriate skill set to conduct the transfer. This role would be titled 'Transfer Practitioner' (TP).

This development of this transfer service was clinically lead by the Intensive Care Department at GUH.

Objectives. Quantify the demand on the proposed Transfer Service. Select and train TPs with a background that would be conducive to performing inter-hospital transfers.

Devise a triage protocol which could be used by a non-clinical member of the team to triage transfer requests to the most appropriately skilled team. 
Methods. Multiple audits were performed to gauge the anticipated requirement of the new transfer service. These were based on current transfers, inappropriate attendances to ED and critical care outreach activity. The results of the audits suggested approximately 5 patients a day would require a 'step up' transfer to GUH. These would be the patients the TPs would be most suited to transfer.

Candidates for interview required experience in either intensive care nursing, emergency medicine nursing or operating department practitioning. They then underwent a week of intensive training including lectures and simulation.

The transfer triage tool (TTT) was developed following analysis of similar tools used in other transfer organisations. The parameters set were then discussed amongst the stake holders of the transfer service in the intensive care unit, emergency department and the Welsh Ambulance Service. This resulted in a traffic light system of triage which would determine the appropriate team to manage the transfer.

Results. The project has been very successful with the TPs taking an active role within ABUHB transferring patients within the health board and externally. The service has recently been approved to begin a $24 \mathrm{~h}$ service with the employment of 6 further transfer practitioners.

Conclusion. The proposed role of a TP was completely new to ABUHB and gauging the demand on the new system required a careful audit strategy to estimate the requirements as accurately as possible. This was an ambitious project requiring input from different departments (intensive care, emergency department, Welsh Ambulance Service and Clinical Futures-NHS Wales. The result has been very successful and is a model that could be adopted in other ares of the country.

\section{Reference(s)}

1. NWL Critical Care Network (2018). 'Transfer Triage Tool'Version V12018 2. Mueller. S (2019), 'Triaging Interhospital Transfers'PSNET

3. National Clinical Guidelines Centre (2016). 'Major trauma: service delivery [NG40]' National Institute of Health and Clinical Excellence

4. No grants

\section{9}

Improving The Diagnosis and Management of Pulmonary Embolism In A Tertiary Care Hospital, A Quality Improvement

\section{Project}

T. Aisa'; M. Abdelbaky'; A. Hussein ${ }^{1} ;$ A. Rugaan ${ }^{1}$

${ }^{1}$ Critical care department, King Abdullah Medical City Specialist Hospi-

tal, Makkah, Saudi Arabia

Correspondence: T. Aisa

Intensive Care Medicine Experimental 2020, 9(1): 000109

Introduction. The diagnosis and treatment of acute pulmonary embolism (PE) can be challenging, as it has a heterogeneous range of presentations. Before undertaking this project, we noticed that there was a significant number of negative PE studies which might put the critically ill patients at risk either from transferring unstable patients down to the radiology department or from the risk of developing contrastinduced nephropathy. Moreover, we noted that the management of PE in the department and hospital wide was variable, not unified and not based on the available evidence in some situations. A baseline audit was designed to investigate the diagnosis and management of $\mathrm{PE}$ in the hospital in addition to comparing the management of $\mathrm{PE}$ patients who were admitted in the ICU with the available latest guidelines as standard of care, (the ESC guidelines for the management of PE 2014) (1). We found that in the period from January 2018- till January 2019 at King Abdullah Medical City, a tertiary/quaternary hospital, total of 347 patients underwent CTPA studies for PE diagnosis without applying the Clinical Decision Rules (CDRs), such as Wellsc criteria and D-dimers which are proven to be safe for ruling out PE in hospitalized patients (2). Only $53(15.2 \%)$ studies were positive for $P E$, while $294(84.8 \%)$ studies were negative. Of concern, 15 (4.3\%) patients had CTPA studies repeated in spite of negative initial study.Between January 2018 and May 2019, we had 76 admissions with acute PE which represents $3.5 \%$ of our total ICU admissions in this period (total ICU admissions 2138 as per our data registry). The massive PE represented
30 (39.48\%) patients, while 46 (60.52\%) patients were presented with intermediate-high risk PE with a heterogeneity in the management decisions with unnecessary consults to different specialties such as the interventional radiology (IR) or unnecessary activation of extracorporeal membrane oxygenation (ECMO) team.

Methods. The initial intervention considered was to design a flow chart for the diagnosis and management of PE since the patient presentation till he receives the proper management in the appropriate allocation in the hospital. Thus, a task force was activated for this purpose to formulate a team and implement the best evidence based guidelines. The team included all the stakeholders involved in the management of such patients; a member from the following departments: critical care, emergency department, medicine department, pulmonology, cardiac surgery, interventional radiology, cardiology and from the quality management department. We started a series of meetings with the concerned personnel to formulate the protocol for stepwise algorithmic approach for PE management till we got the final approval of the hospital medical board. Furthermore, we initiated a series of lectures in the concerned departments to raise the awareness of the management protocol.

Results. The results from the re-audit showed an improvement in terms of reduction of the total number of CTPA requests to 246 with positive results of $50(20.32 \%)$ studies after implementation of the protocol in the period between August 2019 to August 2020, in addition to improvements in the documentation and use of the pretest score for clinical probability. Subsequently, there was a cost reduction of 151,500 Saudi Riyals per year. Improvements in the management of high risk PE admitted to the ICU based on the protocol, number of massive and intermediate-high risk PE treated with catheter directed therapy including catheter directed thrombolysis and fragmentation, catheter embolectomy and ECMO, based on the protocol was 12 patients in one year. 40 intensivists reported that they have used the protocol in their management of such patients with $84.9 \%$ agreed that it did improve their plans of management. 40 (74.07\%) physicians in the concerned departments said they are aware of the PE protocol, 37 $(68.52 \%)$ physicians have used it in the management of such patients, $45(84.91 \%)$ physicians reported that it did improve the management of PE in terms of early and rapid diagnosis. Additionally, it arranged their way of decision making and specified the indicated cases for interventions.

Conclusion. Implementation of a protocol utilizing a simple diagnostic algorithm led to reduction in the unnecessary imaging which could decrease the cost and the side effects of the procedure. This was a sustainable intervention which standardized the management of high risk PE patients using the evidence based guidelines with a multidisciplinary team approach.

\section{Reference(s)}

1. We acknowledge the committed, hard working team at critical care department at King Abdullah medical city

2. 1.Konstantinides SV, Torbicki A, Agnelli G, Danchin N, Fitzmaurice D, Galie $\mathrm{N}$, et al. 2014 ESC guidelines on the diagnosis and management of acute pulmonary embolism. Eur Heart J. 2014;35:3033-69, 69a-69 k

3. 2.Anne R Bass, Kara G Fields, Rie Goto, Gregory Turissini, Shirin Dey, Linda A Russell, Clinical Decision Rules for Pulmonary Embolism in Hospitalized Patients: A Systematic Literature Review and Meta-analysis, Thromb Haemost. 2017 Nov;117(11):2176-2185

\section{4}

The Evaluation of Maternal Critical Care Provision in a Tertiary Hospital

C. Willis'; G. Tatjana' ${ }^{1}$ ' L. Vincent ${ }^{1}$

${ }^{1}$ Intensive care, John Radcliffe Hospital, Oxford, United Kingdom

Correspondence: C. Willis

Intensive Care Medicine Experimental 2020, 9(1): 000124

Introduction. In the UK, approximately 1900 women per year are admitted to an intensive care unit (ICU) during pregnancy or the postnatal period(1). In 2018, the Royal College of Anaesthetics (RCOA) 
published new guidelines focusing on the management of critically unwell pregnant women(2). The guideline provided clear recommendations for critical care services caring for pregnant or 'recently pregnant' woman. The purpose of the recommendations is to enhance obstetric critical care outcomes by improving the organisation and collaboration between critical care and maternity services.

Objectives. This study aimed to assess the compliance of a tertiary hospital's ICU in Oxford, UK, with the published national guidance from RCOA, and to identify potential areas of improvement.

Methods. A retrospective review was conducted, analysing the medical records of all the obstetric intensive care admissions between 2017-2019. Data regarding the admission diagnosis, patient demographics, stage of pregnancy, length of ITU stay and outcome was obtained. Guideline specific data such as provision of breast-feeding support, number of days with bedside review by a consultant anaesthetist, obstetrician and midwife and number of days with infant and critical care follow up was also collected.

Results. Within the three-year period there were 45 admissions of pregnant $(13.3 \%)$ or recently pregnant $(86.7 \%)$ patients. 29 of these women weren't included in the review due to missing data. The median age was 32 (22-41), median BMI 26.2 (18.9-44.6) and median APACHE score 12 (2-30). Major obstetric haemorrhage was the most common diagnosis on admission. The median length of stay was 3 days. There were no maternal deaths, however, there were 8 fetal deaths and 4 surgical terminations (3 ectopic pregnancies plus 1 patient choice following VTE). Only one patient had a documented daily review from the recommended full team including an obstetric consultant, anaesthetic consultant, and midwife. However, an obstetric consultant or senior registrar reviewed every patient regularly. 8 patients $(27.6 \%)$ were documented to have been provided breast feeding support and only 1 patient (4.5\%) had documented maternal contact with their newborn on the ICU. 26 patients (89\%) had a documented critical care senior nurse follow up documented in the notes.

Conclusion. The study compliance with the RCOA guidance did not meet the ideal standard. There is a clear requirement to prioritise and ensure the recommended obstetric guidelines are complied with, such as providing breast-feeding support and neonatal visits whilst patients are in ICU. Unsatisfactory documentation of such outcomes may have also contributed to the effect of poor compliance. A solution is the implementation of an obstetric admission proforma, for pregnant and recently pregnant women in ICU. The proforma will contain a checklist of the recommended RCOA guidance. The overall aim is to improve the care of obstetric patients in ICU and simplify documentation.

\section{Reference(s)}

1. Jardine J, NMPA Project Team. Maternity Admissions to Intensive Care in England, Wales and Scotland in 2015/16: A Report from the National Maternity and Perinatal Audit. London: RCOG; 2019.

2. Care of the critically ill woman in childbirth; enhanced maternal care. (2018). RCOA

\section{6}

Improving tracheostomy outcomes in ICU patients during COVID pandemic-a singe Trust's annual overview

A. Marinakis ${ }^{1}$; E. Walker'; T. Markati ${ }^{2}$; RA. Smith ${ }^{3}$; Y. Ng ${ }^{4}$; J. Gordon ${ }^{4}$; K. Ratnam ${ }^{5}$; V. Dhokia ${ }^{4}$; P. Paul ${ }^{4}$

${ }^{1}$ Anaesthesia, Nottingham City Hospital, Nottingham, United Kingdom; ${ }^{2}$ Anaesthesia, School of Biological Science, University of Cambridge, Cambridge, United Kingdom; ${ }^{3}$ Anaesthesia, King's Mill Hospital, Nottingham, United Kingdom: ${ }^{4}$ Anaesthesia and intensive care medicine, King's Mill Hospital, Nottingham, United Kingdom; ${ }^{5}$ ntensive care medicine, King's Mill Hospital, Nottingham, United Kingdom

Correspondence: A. Marinakis

Intensive Care Medicine Experimental 2020, 9(1): 000126

Introduction. Our Trust reviewed all COVID patients admitted to ICU requiring tracheostomy from March to June 2020. Out of 10 tracheostomies performed, $7 / 10$ were successfully discharged (stepped down group) while 3/10 did not survive ICU stay (Deceased group). Both groups were assessed against relevant parameters of tracheostomy insertion such as indication, time of insertion, type of equipment, and mean ICU stay. Initial results revealed that most of stepped-down patients had preop checklists completed $(71 \%)$, tracheostomy tubes with subglottic suction (57\%) and earlier mean day of insertion(21 day) compared to deceases ones. These trends were audited against the revised guidance[1][2] and decision was made to adopt a more unified approach for all tracheostomy insertions for the 2 nd covid surge.

Objectives. Aim was to implement changes as per guidance and demonstrate the improvement of tracheostomy care both for COVID and non COVID patients during the 2nd wave onwards.

Methods. During the 2nd wave changes were implemented both for Covid and non Covid patients requiring tracheostomy, and relevant outcomes were assessed. Specifically, from September to December 2020, out of total 14 tracheostomies, 9 were conducted for COVID 19 patients (Covid Group) while 5 for the Non covid patients (Non Covid group). Desicion was made to prioritise a percutaneous approach of insertion for all (13/14) instead of a surgical, so as to minimise degree of intervention and associated post procedural complications. We then reviewed subsequent compliance with guidance in terms of equipment used, completion of pre op checklists and time of insertion.

Results. Results showed that Fenestrated tube with subglotic suction was prioritised both for the Covid and Non covid group respectively ( $78 \%$ vs $80 \%)$;equally, a definitive improvement was confirmed in conducting pre op checklists in both groups $(100 \%$ for Non covid and $89 \%$ for Covid group). There was significant compliance in time of insertion, with mean day of insertion being 14.2 days for the NonCovid Group instead of 19.8 for the Covid one respectively. Reviewing relavants trends only to Covid patients, it appeared that despite ICU admissions increased during the 2 nd wave (34 patients in 1st wave versus 58 patients in the 2 nd) the overall change of practice led to a substantial reduction of mean length of ICU stay and earlier discharge; 45.1 days of stay for COVID ICU survivors requiring tracheostomy during the 1st wave versus 30.3 days for the corresponding cohort during the 2 nd surge.
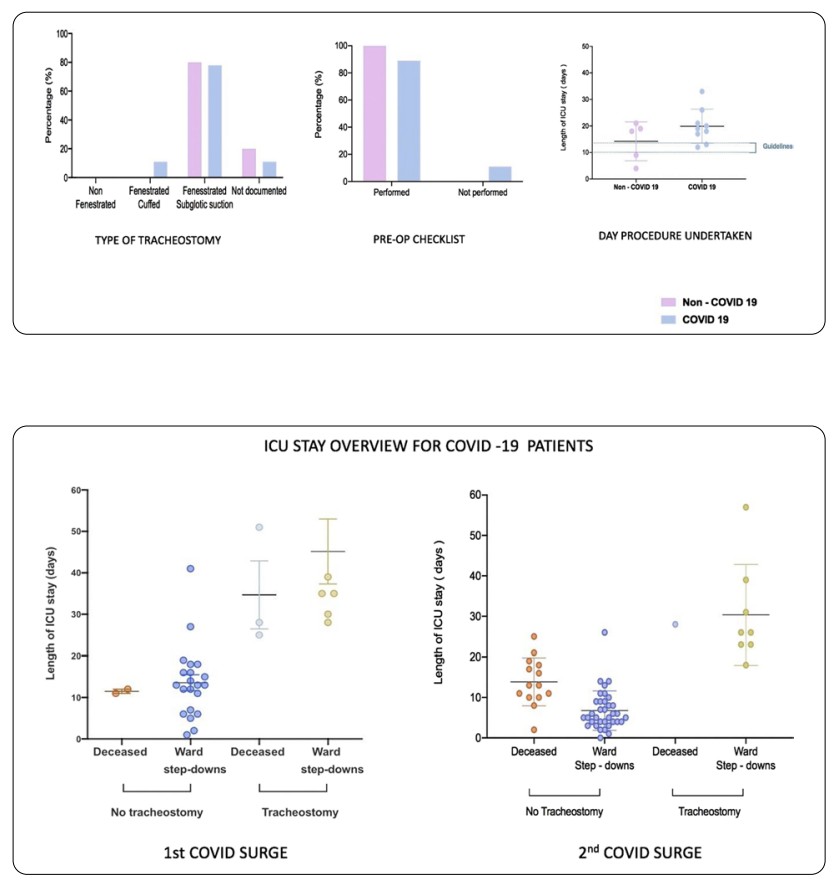

Conclusion. Putting all outcomes together we conclude that despite ICU admissions increased, earlier tracheostomy insertion and selection of appropriate equipment minimised morbidity and mortality for most tracheostomy patients and led to earlier discharge for Covid ones. Aim to continue reviewing data to confirm sustainability with optimal standards of tracheostomy care. 


\section{Reference(s)}

1. 1.Guidance for Tracheostomy Care, August 2020; Brenda AMCGrath, Sarah Wallace, Barbara Bonvento, James Lynch, Barry Coe, Dougal Atksinon; Executive Summary Key Standards and recommendations p 5-10

2. Multidisciplinary guidance for safe tracheostomy care during covid 19 pandemic; the NHS National Patient safety improvement programme(NatPa tSIP),Anaesthesia 2020, 75, 1661-1662; B.AMcGrath, N.Ashy,M. Birchall et al.

\section{0}

Serum Biomarkers and Mortality in Patients with COVID-19

Admitted to an Intensive Care Unit

R. Lima, ; R. Ferreira²; R. Lemgruber, ; J. Ramalho, ; L. Lourenço, ${ }^{2}$

I. Benchimol, ${ }^{2} ;$ G. Oliveira ${ }^{2}$; J. Rocha ${ }_{1}^{2}$; R. Beranger, ${ }^{1} ;$ L. Souza, ${ }_{1}^{1}$ C. Villela, ${ }_{1}^{3}$

J. Almeida, ${ }^{2}$; F. Albuquerque, ${ }^{2}$; C. Landsberg, ${ }^{2}$; P. Sampaio, ${ }^{2}$; C. Ferreira

Filho, ${ }^{1}$; C. Cortinhas Filho, ${ }^{1}$; M. Vaisman, ${ }^{1}$; A. Almeida, ${ }^{1}$; J. Mansur Filho, ${ }^{2}$

${ }^{1}$ Intensive care unit, Hospital Samaritano, Rio de Janeiro, Brazil; ${ }^{2}$ Cardiology, Hospital Samaritano, Rio de Janeiro, Brazil; ${ }^{3}$ Emergency depart-

ment, Hospital Samaritano, Rio de Janeiro, Brazil

Correspondence: R. Ferreira

Intensive Care Medicine Experimental 2020, 9(1): 000130

Introduction. The Coronavirus Disease-2019 (COVID-19) pandemic has demanded that healthcare professionals become increasingly efficient in identifying high risk clinical features to adequately allocate medical resources. Severe cases are defined by the presence of dyspnea, tachypnea, blood oxygen saturation $<93 \%$, or pulmonary infiltrates covering $>50 \%$ of both lungs [1]. Laboratory tests may provide additional prognostic information in patients who have already fulfilled clinical criteria for severe disease.

Objectives. This study aimed to analyse the prognostic value of serum biomarkers in hospitalized patients with severe COVID-19.

Methods. Medical records from consecutive patients with confirmed COVID-19 admitted to an intensive care unit (ICU) in Rio de Janeiro, Brazil between March 12th and May 31st, 2020 were retrospectively analysed. Clinical variables and laboratory biomarkers [lymphocyte count, C-reactive protein, D-dimer, procalcitonin, ferritin, lactate dehydrogenase, brain natriuretic peptide (BNP), cardiac troponin and interleukin-6] were collected and correlated with in-hospital mortality.

Results. Fifty four patients were identified during the study period. Median age was 73.5 years (interquartile range [IQR] 60-84) and 64.8\% were males. Previous comorbidities included hypertension (57.4\%), diabetes (27.8\%) and coronary artery disease (13\%). Mechanical ventilation was required in $61.1 \%$ of patients and total in-hospital mortality was $46.3 \%$. On admission, elevated troponin (OR 7.47; 95\% Cl 2.1-26.8), BNP (OR 4.94; 95\% Cl 1.4-17.4) and procalcitonin (OR 5.08; $95 \% \mathrm{Cl} 1.6-16.6)$ were significantly associated with subsequent in-hospital death. Median procalcitonin levels were significantly higher among non-survivors (Figure) and lymphopenia was also a predictor of mortality (OR $3.64 ; 95 \% \mathrm{Cl} 1.2-11.4)$. On multivariate analysis, only age $>75$ years $(p=0.016)$ and elevated procalcitonin levels $(p=0.04)$ remained significantly related to a worse prognosis.

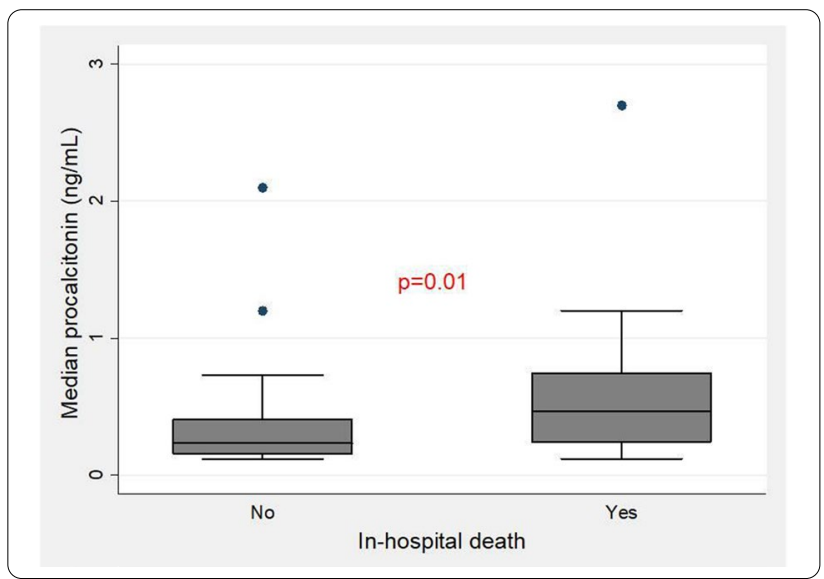

Conclusion.: In patients with clinically severe COVID-19 admitted to an ICU, serum biomarkers may be useful to provide additional prognostic information. Future studies are necessary to confirm these findings and determine the appropriate subsequent management strategies.

\section{Reference(s)}

1. Berlin D, Gulick R, Martinez F. Severe Covid-19. New England Journal of Medicine. 2020;383(25):2451-60.

\section{7}

Risk factors for the development of Post-Intensive Care Syndrome (PICS) in family members: Predict to prevent

C. Tintim Lobato ${ }^{1}$; J. Camões ${ }^{1}$; C. Vales ${ }^{1}$; D. Carvalho ${ }^{1}$; CC. Dias ${ }^{2}$; R. Araújo ${ }^{1}$; E. Gomes

${ }^{1}$ Department of Emergency Medicine and Intensive Care, Hospital Pedro Hispano, Matosinhos, Portugal; '2Department of community medicine, information and health sciences, Faculty of Medicine of the University of Porto, Porto, Portugal

Correspondence: $C$. Tintim Lobato

Intensive Care Medicine Experimental 2020, 9(1): 000147

Introduction. Family members of intensive care unit (ICU) patients play a crucial role in their recovery. Acknowledging family members' individual needs is fundamental in the modern ICU, shifting from a patient centered care to a patient and family centered care.

Coping with a situation of critical illness of a loved one, participation in treatment decisions and caretaking of patients after hospital discharge can predispose to the development of Post-Intensive Care Syndrome in Family Members (PICS-F). In order to prevent PICS-F it is crucial to understand which characteristics of family members or patients can predispose or potentiate the development of this syndrome.

Objectives. Evaluate levels of anxiety and depression in family members of patients hospitalized in the ICU, and search for the presence of factors linked to the development of PICS-F, allowing for future early identification of elements at risk and activation of adequate response services.

Methods. The study sample included 164 patients hospitalized in the ICU of a district hospital in northern Portugal and their respective family members. A survey regarding socio-demographic data was filled in by family members during the patient's hospitalization in the ICU. Three months after hospital discharge, family members were screened for anxiety and depression (emotional distress) using the Hospital Anxiety and Depression Scale (HADS).

To test for an association between family and patients' characteristics and a positive HADS score, comparison tests were used. A multivariable logistic regression model was conducted to identify independent factors associated with a positive HADS score.

Results. The majority of patients were male (53\%), whereas the majority of family members were female $(72 \%)$. The mean age of patients and family members was 60.4 and 53.9 years respectively. Emotional distress was identified in $24 \%$ of family members 3 months after patient discharge from the ICU. Only self-characteristics were shown to be associated with emotional distress in family members, namely unemployment $(p=0.008)$, smoking/drinking habits $(p=0.036)$ and a personal history of psychopathology $(p=0.045)$.

In multiple logistic regression analyses, only unemployment was found to be an independent factor associated with anxiety and depression in family members (OR $=2.74, \mathrm{Cl} 95 \%$ : 1.09-6.93). No association was found between patient characteristics and PICS-F.

Conclusion. The family members of ICU patients who experienced more anxiety and depression shared the following characteristics: were unemployed, had smoking and/or drinking habits and had a personal history of psychopathology. Unemployment was the only independent caregiver characteristic identified in this study as a risk factor for the development of anxiety and depression following ICU hospitalization. Patient characteristics (intrinsic, related to the acute illness and PICS) were not found to be associated with the development of PICS-F. 
000151

A User Engaged Iterative Design for the Graphical Interactive Display of Machine Learning-Based Intelligent Alerting Systems S. Helman ${ }^{1}$; MA. Terry ${ }^{2}$; T. Pellathy, ; A. Williams ${ }^{3}$; A. Dubrawski ${ }^{3}$; G. Clermont ${ }^{4}$; M. Pinsky ${ }^{4}$; S. Al-Zaiti'; M. Hravnak ${ }^{1}$

${ }^{1}$ Department of acute and tertiary care nursing, University of Pittsburgh, Pittsburgh, United States of America; ${ }^{2}$ Behavioral/community health sciences, University of Pittsburgh, Pittsburgh, United States of America; ${ }^{3}$ Auton lab, school of computer science, Carnegie Mellon University, Pittsburgh, United States of America; ${ }^{4}$ School of medicine, University of Pittsburgh, Pittsburgh, United States of America

Correspondence: S. Helman

Intensive Care Medicine Experimental 2020, 9(1): 000151

Introduction. Machine learning $(\mathrm{ML})$ algorithms are increasingly used to support bedside clinical decisions, and outputs must be presented in usable ways. Graphical user interfaces (GUI) are front-facing presentations for ML outputs, but clinicians are not routinely invited to participate in their design, hindering ML solution potential. We previously used $\mathrm{ML}$ to develop a dynamic cardiorespiratory instability (CRI) risk score from continuously collected step-down unit vital sign (VS) monitoring data (Chen 2017, Chen 2015, Chen 2014). Clinical researchers and computer scientists used this ML-derived CRI risk score to develop a first-pass static GUI prototype.

Objectives. We next seek feedback from clinical end-users to inform user-engaged iterative design of a bedside GUI prior to clinical testing. Methods. We conducted online qualitative focus group studies using an open-ended question guide to elicit feedback from clinical endusers on the scope and presentation of information in the GUI screen prototype (numerics/pictures/animations) they felt to be most helpful in recognizing and forming decisions about patients approaching CRI. Five online focus group sessions were held in two separate rounds, each moderated by an expert focus group methodologist. Every round consisted of 3 groups (nurses, providers [nurse practitioners and physicians], and a hybrid group). Iterative design changes were made rapidly, and the most recently modified GUI display was presented in the next round. Focus group discussions were recorded and transcribed in a de-identified manner, and the transcripts coded independently by three researchers (SH, TP, MT) to identify recurrent patterns, highlighting when thematic saturation was reached. After resolution of coding discrepancies, codes were coalesced into emergent themes. Clinicianrecommended GUI changes linked back to the emerging themes.

Results. 23 clinicians were recruited (14 nurses, 4 nurse practitioners, 5 physicians). The median age of participants was $~ 35$ years $(60 \%$ females) with median clinical experience of 8 years. Five themes emerged: trend evolution, context (risk evolution relative to vital signs and interventions), evaluation/interpretation/explanation (sub theme: holistic evaluation), clinician intuition, and clinical operations. Based on these themes, changes made to the GUI included: color and scale adjustments, integration of clinical information (medication, labs, oxygen therapy, volume), threshold personalization, numeric and graphics arrangement, ability to toggle between near/far trend views, and an information tab describing features and algorithms which produce the dynamically evolving CRI risk score.

Conclusion. User-engaged iterative design was useful in adjusting the GUI's presentation of ML output felt to more optimally facilitate clinician surveillance and decisions. Next steps include comparison, laboratory, and field tests for further GUI refinement. Clinicians should be involved early and often in clinical decision support design to optimize efficacy of ML tools.

\section{Reference(s)}

1. Chen L, Dubrawski A, Hravnak M, Clermont G, Pinsky M. 41: Forecasting cardio-respiratory instability in monitored patients: a machine learning approach Crit Care Med. 2014;42(12):A1378-A1379.

2. Chen L, Dubrawski A, Clermont G, Hravnak M, Pinsky MR. Modelling Risk of Cardio-Respiratory Instability as a Heterogeneous Process. AMIA Annual Symposium proceedings AMIA Symposium. 2015;2015:1841-1850.
3. Chen L, Ogundele O, Clermont G, Hravnak M, Pinsky MR, Dubrawski AW. Dynamic and Personalized Risk Forecast in Step-Down Units. Implications for Monitoring Paradigms. Annals of the American Thoracic Society. 2017;14(3):384-391.

4. National Institutes of Health R01NR 013,912, T32NR008857

\section{4}

Prediction of ICU mortality in COVID-19 patients with ISARIC 4C score

V. Vicka'; E. Januskeviciute ${ }^{2}$; S. Miskinyte ${ }^{2}$; D. Ringaitiene ${ }^{1}$; M. Serpytis ${ }^{1}$;

A. Klimasauskas'; 'L. Jancoriene'; ;. Sipylaite

${ }^{1}$ Clinic of anaesthesiology and intensive care, institute of clinical medicine, Faculty of Medicine of Vilnius University, Vilnius, Lithuania; ${ }^{2}$ Faculty of medicine, Vilnius University, Vilnius, Lithuania; Institute of clinical medicine, Faculty of Medicine of Vilnius University, Vilnius, Lithuania Correspondence: E. Januskeviciute

Intensive Care Medicine Experimental 2020, 9(1): 000164

Introduction. As the COVID-19 pandemic proceeds, the number of patients admitted to the intensive care unit (ICU) is still growing. ISARIC 4C Mortality Score was developed as a prognostic tool in emergency care department. The aim of this study is to determine whether 4C Mortality score can be used in ICU population.

Methods. This was a retrospective study of patients who were admitted to a tertiary referral university hospital ICU in the year of 2020 and were tested positive for SARS-Cov-19. The 4C Mortality Score was calculated upon admission to the hospital. Patients were divided into two groups-ICU survivors and non-survivors. Standardized mortality ratio (SMR), ROC-AUC and regression analysis was conducted to determine the applicability of $4 \mathrm{C}$ Mortality Score.

Results. 249 patients were included in the study, of which $63.1 \%$ were male. Overall age of all patients was $61.32 \pm 13.3$. Most of the patients were $50-70$ years old (55.4\%). The overall mortality was $41.4 \%(n=103)$. SMR for the 4C Mortality Score was calculated, revealing a good concordance to the actual mortality rate of the group $(\mathrm{SMR}=1.05)$. Mean value of mortality risk score was higher in the non-survivors group: 8 [6-11] vs 12 [9-15] $(p<0.001)$. To determine the discrimination ability of the $4 C$ Mortality Score ROC-AUC analysis was performed, with ROC-AUC value of 0.754 (95\% Cl: 0.694-0.814, $\mathrm{p}<0.001$ ). Regression analysis revealed the $4 C$ Mortality Score as a significant mortality predictor, with acceptable calibration $(x 2=7.506$, $\mathrm{df}=8, \mathrm{p}=0.483)$. Per each point increase in the score the mortality increases by $27.1 \%(\exp (\mathrm{B})=1.31195 \% \mathrm{Cl}: 1.205-1.427, \mathrm{p}<0.001)$.

Conclusion. The study demonstrated that the $4 \mathrm{C}$ Mortality Score is a good mortality predictor of the COVID-19 patients treated in the ICU. Further studies are needed to determine whether this score measures up to the conventional scores.

\section{8}

Under pressure: A massive COVID19 surge, C19_SPACE and the outcomes of a newly formed ICU

G. Karlis ${ }^{1}$; T. Katsarou' ; G. Katsagani ${ }^{1}$; V. Karaouli ${ }^{1}$; P. Spithakis ${ }^{1}$; O. Kouniaki ${ }^{1}$; P. Peppa ; M. Poulou'; M. Daganou'; D. Markantonaki ${ }^{1}$

${ }^{1}$ Intensive care unit 4, Sotiria Thoracic Diseases Hospital of Athens, Athina, Greece

Correspondence: G. Karlis

Intensive Care Medicine Experimental 2020, 9(1): 000178

Introduction. The COVID19 pandemic is constantly prompting healthcare systems around the globe to increase their ICU bed capacity. However, it is well documented that ICU strain is associated with important increases in patient mortality (1). Shortage of trained staff, such as intensivists and ICU nurses, was one of the main challenges from the beginning of this crisis (2). Use of non-ICU healthcare providers to reinforce ICU staff with training provided became a frequently employed strategy. C19 SPACE is an example of a free training course offered by ESICM to non-ICU clinicians (3). From November 2020 and on Greece is facing a severe "second wave" which rapidly increased 
the number of COVID19 related hospital admissions and the demand for ICU beds.

Objectives. To present the background, training and patient outcomes of an urgently assembled ICU team during a period of massive COVID19 surge.

Methods. We retrospectively analyzed patient data and critical care outcomes (age, sex, APACHE II score at admission, length of stay, ICU mortality, standardised mortality ratio (SMR)), from a newly formed 12-bed ICU in a tertiary COVID19 dedicated hospital, during the first four months of its operation. The background and the level of training of the ICU physicians was also taken into account. Comparisons with international COVID19 ICU registries were made.

Results. Our team of physicians consisted of 9 people. Six out of 9 had less than 5 years of ICU experience. However, all 6 had recently attended C19_SPACE. From November 20th 2020 to April 4th 2021, 75 patients were admitted to our 12-bed department. Patients' characteristics are shown in Table 1. The ICU mortality was $34.7 \%$ and the median ICU length of stay 14 days. Notably, in our cohort $88.1 \%$ of patients were supported with invasive mechanical ventilation at admission. The predicted mortality for an APACHE II score of 22 is $42.4 \%$. Therefore, our SMR is 0.82 . The readmission and reintubation rates of our unit were $5.3 \%$ and $8 \%$, respectively. Table 1.

\begin{tabular}{lll}
\hline Male sex, No (\%) & Age, median & APACHE II, median \\
\hline $57(75)$ & 65 & 22 \\
\hline
\end{tabular}

Conclusion. Observational studies have found that ICU mortality from COVID19 has plateaued since May 2020 with some geographical variation. In most regions though, including Europe, the mortality rate is $30-40 \%$ (4). Our results cannot establish a causal link between the training status of our physicians and the ICU mortality. However, we report that C19_SPACE program along with the guidance of more experienced intensivists might have positively affected our patients' outcomes during a period of crisis.

\section{Reference(s)}

1. None

2. Armstrong RA, Kane AD, Kursumovic E, Oglesby FC, Cook TM. Mortality in patients admitted to intensive care with COVID-19: an updated systematic review and meta-analysis of observational studies. Anaesthesia. 2021 Apr;76(4):537-548. https://doi.org/10.1111/anae.15425. Epub 2021 Feb 1. PMID: 33,525,063; PMCID: PMC8013495.

3. Arabi YM, Azoulay E, Al-Dorzi HM, Phua J, Salluh J, Binnie A, Hodgson C, Angus DC, Cecconi M, Du B, Fowler R, Gomersall CD, Horby P, Juffermans NP, Kesecioglu J, Kleinpell RM, Machado FR, Martin GS, Meyfroidt G, Rhodes A, Rowan K, Timsit JF, Vincent JL, Citerio G. How the COVID-19 pandemic will change the future of critical care. Intensive Care Med. 2021 Mar;47(3):282-291. https://doi.org/10.1007/s00134-021-06352-y. Epub 2021 Feb 22. PMID: 33,616,696; PMCID: PMC7898492.

4. Wahlster S, Sharma M, Lewis AK, Patel PV, Hartog CS, Jannotta G, Blissitt P, Kross EK, Kassebaum NJ, Greer DM, Curtis JR, Creutzfeldt CJ. The Coronavirus Disease 2019 Pandemic's Effect on Critical Care Resources and HealthCare Providers: A Global Survey. Chest. 2021 Feb;159(2):619-633. https:// doi.org/10.1016/j.chest.2020.09.070. Epub 2020 Sep 11. PMID: 32,926,870; PMCID: PMC7484703.

5. Gabler NB, Ratcliffe SJ, Wagner J, Asch DA, Rubenfeld GD, Angus DC, Halpern SD. Mortality among patients admitted to strained intensive care units. Am J Respir Crit Care Med. 2013 Oct 1;188(7):800-6. https://doi.org/ 10.1164/rccm.201304-0622OC. PMID: 23,992,449; PMCID: PMC3826272.
000179

Reducing the burden of bed shortages in COVID-19 pandemic by identifying modifiable risk factors that contribute to a prolonged stay in the Intensive Care Unit

D. Pérez-Torres '; JÁ. Berezo-García'; C. Cuenca-Rubio';

V. Fraile-Gutiérrez"; I. Canas-Pérez'; C. Díaz-Rodríguez ${ }^{1}$; GJ. Posadas-Pita'. J. Sánchez-Ballesteros ${ }^{1}$; P. Blanco-Schweizer ${ }^{1}$; PM. Enríquez-Giraudo ${ }^{1}$

${ }^{1}$ Servicio de medicina intensiva, Hospital Universitario Río Hortega, Valladolid, Spain

Correspondence: D. Pérez-Torres

Intensive Care Medicine Experimental 2020, 9(1): 000179

Introduction. Providing life-saving care to critically ill COVID-19 patients requires a great amount of resources. Length of stay (LOS) for these patients is longer than for other critical conditions, which may lead to a critical shortage of Intensive Care Unit (ICU) beds. Identifying modifiable risk factors that contribute to a long ICU stay might help overcome bed shortages and improve resource allocation and management for future waves.

Objectives. We aim to describe the potentially modifiable factors that contribute to ICU LOS in critically ill COVID-19 patients, so that the clinicians are aware of them and can focus their attention on an appropriate management, eventually leading to shorter LOS and better resource allocation and management.

Methods. We conducted a retrospective observational single-centre study in the ICU of a University Hospital in Spain, over 1 year. All the patients who were admitted to the ICU with PCR- or antigen testconfirmed COVID-19 were included. Potential modifiable risk factors contributing to ICU LOS were identified by a survey process. The information regarding these risk factors was obtained from the electronic medical record. Patients were classified into two groups according to their LOS. Data are expressed as mean \pm standard deviation, median (interquartile range), frequency (\%) or odds ratio (95\% confidence interval). Chi-square, Student's $T$ and logistic regression tests are applied as appropriate.

Results. We included 236 patients, $73 \%$ male, age $62 \pm 12$ years-old [survivors $60 \pm 12$ vs non-survivors $69 \pm 10, \mathrm{p}<0.01]$, BMI 29 (26-33) $\mathrm{Kg} / \mathrm{m}^{2}$, mechanical ventilation $88 \%$, ICU mortality $22 \%$, hospital mortality $29 \%$. Clinical characteristics of the patients of both groups are summarised in Table 1. Potential modifiable risk factors contributing to ICU LOS of both groups are depicted in Table 2 .

Table 1. Clinical characteristics of the patients with short and long stay

\begin{tabular}{llll}
\hline & $\begin{array}{l}<\mathbf{2 8} \text { days } \\
\mathbf{n = 1 6 2}\end{array}$ & $\begin{array}{l}\mathbf{2} \mathbf{2 8} \text { days } \\
\mathbf{n = 7 4}\end{array}$ & $\mathbf{p}$-value \\
\hline Age, years & $62 \pm 12$ & $64 \pm 11$ & 0.18 \\
Male sex, \% & 69.8 & 79.7 & 0.11 \\
APACHE-II, points & $16.5 \pm 6$ & $17.6 \pm 5.7$ & 0.22 \\
ICU mortality, \% & 22.1 & 22.4 & 0.97 \\
Hospital mortality, \% & 28.3 & 31.4 & 0.69 \\
\hline
\end{tabular}

Table 2. Potential modifiable risk factors contributing to ICU LOS

\begin{tabular}{lllll}
\hline Risk factor & $\begin{array}{c}<\mathbf{2 8} \text { days } \\
\mathbf{n = 1 6 2}\end{array}$ & $\begin{array}{l}\mathbf{2} \mathbf{2 8} \text { days } \\
\mathbf{n = 7 4}\end{array}$ & OR $(\mathbf{9 5 \%} \mathbf{C l})$ & p-value \\
\hline $\begin{array}{l}\text { Delirium, \% } \\
\begin{array}{l}\text { ICU-acquired weak- } \\
\text { ness, \% }\end{array}\end{array}$ & 26.9 & 56.8 & $3.6(1.9-6.6)$ & $<0.01$ \\
$\begin{array}{l}\text { Severe lung destruc- } \\
\text { tion, \% }\end{array}$ & 12.4 & 77 & $12.5(6.2-25.8)$ & $<0.01$ \\
$\begin{array}{l}\text { Acute kidney injury } \\
\text { (AKI), \% }\end{array}$ & 26.1 & 33.8 & $1.5(0.8-2.7)$ & 0.23 \\
\hline
\end{tabular}


Table 2. Potential modifiable risk factors contributing to ICU LOS

\begin{tabular}{lllll}
\hline Risk factor & $\begin{array}{c}<\mathbf{2 8} \text { days } \\
\mathbf{n = 1 6 2}\end{array}$ & $\begin{array}{l}\mathbf{2 2 8} \text { days } \\
\mathbf{n}=\mathbf{7 4}\end{array}$ & OR $(\mathbf{9 5 \%} \mathbf{C l})$ & p-value \\
\hline $\begin{array}{l}\text { Continuous renal } \\
\text { replacement } \\
\text { therapy, } \%\end{array}$ & 4.6 & 24.6 & $6.8(2.4-20.9)$ & $<0.01$ \\
$\begin{array}{l}\text { Fluid balance on day } \\
\quad \text { 14, litres }\end{array}$ & $3.4 \pm 5.9$ & $6.2 \pm 5$ & $1.08(1.03-1.13)$ & $<0.01$ \\
$\begin{array}{l}\text { Multidrug-resistant } \\
\text { microorganisms } \\
\text { (MDRM), } \%\end{array}$ & 8.6 & 56.8 & $13.8(6.4-30.5)$ & $<0.01$ \\
& & & & \\
\end{tabular}

Conclusion. Colonization or infection by MDRM, ICU-acquired weakness, need for CRRT, delirium, severe lung destruction and fluid balance were significantly related to prolonged ICU stay.

Shorter LOS might be achieved by implementing antimicrobial stewardship policies, early mobilization and physiotherapy, strategies to prevent AKI progression, a standardized approach to prevent, detect and treat delirium, protective mechanical ventilation and a restrictive fluid balance approach.

\section{8}

Clinical Characteristics and Risk Factors of Covid-19 Pnomonia Patients Hospitalized In A Tertiaty ICU: A Retrospective Study

H. Basar ${ }^{1}$; C. Kaymak' ; A. Ozcan ; M. Nakip ${ }^{1}$; M. Parpucu²; C. Kacan;

F. Yalcin'; M. Kaykac ${ }^{1}$

'Department of anesthesiology and reanimation, University of Health

Sciences, Ankara Health Application and Research Center, Ankara, Turkey;

${ }^{2}$ Department of anesthesiology and reanimation, University of Health

Sciences, Ankara, Turkey

Correspondence: C. Kaymak

Intensive Care Medicine Experimental 2020, 9(1): 000198

Introduction. A new virus identified, named as Covid-19 by WHO in February 2020 . The clinical spectrum of this infection is wide and ranges from asymptomatic patient to severe pneumonia and respiratory failure.

Objectives. In this presentation, demographic data and clinical characteristics of Covid-19 patients with severe pneumonia who were treated in the intensive care unit (ICU) in between March 2020 and December 2020 are presented.

Methods. After the approval of ethics committee, demographic characteristics, Apache II and SOFA scores, co-morbid diseases of 400 patients with Covid-19 pneumonia, who were taken to intensive care units.

Results. Followed 400 patients werebetween the ages of 26 and 92. The demographicdata of the patients andtheirApache II and SOFA scores are given in Table 1 . The $64.5 \%$ of the patients was female. The most comorbid disease of patients with Covid-19 were hypertension (51.6\%) and diabetes mellitus (51.5\%), respectively. \% 72.5 of patients were admitted from the emergency service. The rate of obese patients was $11.8 \%(\mathrm{BMI}>30)$. The rate of patients using ACE inhibitorswas $18.9 \%$. The mortality rate was detected $\% 68,25$ of patients with Covid-19 in ICU.

\begin{tabular}{ll}
\hline Paramethers & Mean \pm SD \\
\hline Age (year) & $67.1 \pm 11.8$ \\
\hline BMI (kg/m2) & $26.4 \pm 5.2$ \\
MV duration (day) & $4.69 \pm 8.82$ \\
SOFA Score & $4.62 \pm 3.1$ \\
APACHE II Score & $16.68 \pm 8.50$ \\
Exitus Patients & \\
APACHE II Score & $9.19 \pm 6.26^{*}$ \\
Surviving Patients & \\
ICU duration (day) & $9.79 \pm 8.39$
\end{tabular}

${ }^{*} p=0,0001$ (Surviving vs. Exitus Patients).
Conclusion. In this retrospective study, the rate of obesity and ACE inhibitor use were found to be low in our patients. Since, our patients were over 65 years of age, the rate of hypertension was relatively high. In conclusion age and APACHE II score were found to be important factors in survival in patients with Covid-19 pneumonia.

\section{9}

Relationship between ABO Phenotype and Mortality in COVID-19 Patients Followed in the Intensive Care Unit

M. ÇAkırca'; M. Nakip ; M. Engin ${ }^{1}$; C. Kaymak ${ }^{1}$; A. Ozcan ${ }^{1}$; H. Basar ${ }^{1}$

${ }^{1}$ Department of anesthesiology and reanimation, University of Health Sciences, Ankara Health Application and Research Center, Ankara, Turkey

Correspondence: C. Kaymak

Intensive Care Medicine Experimental 2020, 9(1): 000199

Introduction. Blood groups have inherited characteristics that vary between populations due to natural selection and protective effects. Blood group antigens can affect sensitivity by altering the immune response in the form of anti-ABO counterparts.

Objectives. it was aimed to investigate the susceptibility of the disease according to blood groups with $\mathrm{ABO}$ blood group analysis in cases diagnosed with COVID-19 in the Turkish population.

Methods. In order to examine the relationship between blood group and clinical outcomes in COVID-19 patients, it was aimed to examine the distribution and mortality, associated with the $A B O$ phenotype in critical patient groups diagnosed with COVID-19. After the approval of ethics committee, It was planned to compare the phenotypic characteristics with the observed distribution to statistically examine whether the blood group of COVID-19 patients is associated with critical illness.

Results. A total of 383patients were enrolled. Among critically ill patients with COVID-19, the mortality of the patients were $56.1 \%$. Blood group rates and $A B O$ phenotype of the patients were $41.3 \%, 15.7 \%, 10.9 \%$, $32.1 \%$ and $A, B, A B, O$ phenotype, respectively. The median age was $68,72 \pm 14,2$ years and $59.3 \%$ were men. The highest mortality rate of the patients was $22.19 \%$ for Type A.

Conclusion. The literature reported that patients with type A blood are at increased risk of developing COVID-19. On the other hand, it has been shown that patients with group $\mathrm{O}$ phenotypic characteristics are less likely to get the disease in SARSCoV-1 viral outbreak. We found that type A blood conferred a higher risk of severe COVID 19. This is explained by the fact that patients with type A blood are high rate to develop COVID19-associated microvascular thrombosis and endothelial dysfunction.

\section{Reference(s)}

1. Rebecca RK., Brenner SK.,Gupta S., et al. ABO phenotype and death in critically ill patients with COVID-19. Br J Haematol 2020;190(4): 204-208. 2. None

\section{0}

\section{Efficacy of Plasma Exchange In Critically III Covid-19 Patients}

A. Ozcan ${ }^{1}$; C. Kızılay ; C. Kaymak1; B. Ari ${ }^{1}$; A. Eken ${ }^{1}$; M. Bektas ${ }^{1}$

${ }^{1}$ Department of anesthesiology and reanimation, University of Health Sciences, Ankara Health Application and Research Center, Ankara, Turkey

Correspondence: C. Kaymak

Intensive Care Medicine Experimental 2020, 9(1): 000200

Introduction. The cytokine storm induces endotheliopathy and microthrombotic disease in patients with COVID-19. Severe cases can present rapid deterioration and develop acute respiratory distress syndrome, septic shock, metabolic acidosis and coagulopathy, including disseminated intravascular coagulation (DIC). The effective treatment for COVID-19 has not been defined yet.

Objectives. The aim of the study was to investigated the effect of therapeutic plasma Exchange on inflammatory markers and outcome in critically ill COVID-19 patients.

Methods. A case series of critically ill patients, with laboratoryconfirmed COVID-19, was studied during two-monthperiod. Therapeutic plasma exchange (TPE) was performed in patients admitted to the intensive care unit (ICU) with acute respiratory distress syndrome (ARDS), severe pneumonia, DIC. Demographic characteristics, 
laboratory data including D-dimer, ferritin, IL-6, CRP and PaCO2 and $\mathrm{PaO} 2$ were recorded. Wilcoxin paired samples test was used to compare pre- and post-plasma Exchange laboratory values.

Results. Case files of 10 patients with confirmed COVID-19 were assessed. All patients were male with a meanage of $61,8 \pm 11$ years. There were no significant difference between pre-and post-plasma Exchange levels of D-dimer and inflammatory parameters including IL-6, CRP, ferritin. No improvement was observed in $\mathrm{PaCO} 2$ and $\mathrm{PaO} 2$ levels of the patients. Two of the patients were discharged from intensive care unit. Mortality rate was $80 \%$.

Conclusion. Plasma Exchange may help to decrease the levels of inflammatory biomarkers and attenuate endotheliopathy and microthrombosis associated with COVID-19. In our case cohort, we did not observe any significant effect of plasma exchange on levels of inflammatory biomarkers. This may be due to the small sample size. Further studies are needed to identify the efficacy of the potential role of therapeutic plasma exchange in critically ill COVID-19 patients.

\section{Reference(s)}

1. None

\section{9}

\section{Clinical characteristics, treatment and outcomes of critically ill} Covid-19 patients in Myanmar: a single-center observational

\section{study}

C. Myei ${ }^{1}$; MH. Aung. ${ }^{2}$; LH. Aung. ${ }^{3}$; YT. Aung. ${ }^{3}$; TS. Naing. ${ }^{2}$; AZ. Phyo ${ }^{3}$;

YM. Thu ${ }^{3}$; MH. Aung ${ }^{3}$; L. Ko Ko ${ }^{3}$;. Kha ${ }^{3}$; MM. Aung ${ }^{3}$; YA. Latt ${ }^{4}$

${ }^{1}$ Anaesthesia and Intensive Care, Defence Services Medical Acad-

emy, Yangon, Myanmar; ${ }^{2}$ Anaesthesia and intensive care, Defence

Services Liver Hospital, Yangon, Myanmar; ${ }^{3}$ Anaesthesia and intensive

care, Defence Services General Hospital No. (1) (1000 bedded), Yangon,

Myanmar; ${ }^{4}$ Anaesthesia and intensive care, Defence Services Medical

Academy, Yangon, Myanmar.

Correspondence: C. Myei

Intensive Care Medicine Experimental 2020, 9(1): 000209.

Introduction. During the second wave of Covid-19 pandemic across the globe, Myanmar was one of the developing countries battling in response to the crisis with limited resources. But to date, there has not been a published report on severely ill patients with covid-19.

Objectives. To describe the clinical characteristics, treatment profiles, and outcomes of critically ill Covid-19 patients in a Covid-19 designated hospital from Myanmar.

Methods. This observational study was conducted on Covid-19 positive patients admitted to the intensive care unit from 2020 August 30 to January 30 in a single center of Yangon, Myanmar. Demographic data, clinical features, laboratory profiles, treatments, and outcomes were analyzed.

Results. Of the 163 patients included, 99 (61\%) were male with a median age of $63(56-70)$ years. $68 \%$ had at least one comorbidity in which all patients $>80$ years and $76 \%$ of patients $>60$ years had at least one comorbidity. The most common comorbidity was diabetes, found in $50 \%$ of the patients. All of the patients were treated with oxygen therapy, thromboprophylaxis, dexamethasone, and antibiotic prophylaxis. $38(23 \%)$ received convalescent plasma, 94 (58\%) remdesivir, and $16(10 \%)$ tocilizumab. $80(49 \%)$ were intubated and ventilated, 46 $(28 \%)$ required non-invasive ventilation, $62(38 \%)$ of patients underwent prone positioning. 127 (78\%) patients needed cardiovascular support, and $13(20 \%)$ required renal replacement therapy. The rate of deterioration was 6.5 days, and 23 cases showed rapid deterioration. The average length of ICU stay was 12 days. The median length of ventilator dependency was 8 days. $64 \%$ of patients died, with the majority from the $60-80$ age group and the highest mortality in above 80 years of age.

Conclusion. Understanding the clinical course and outcomes of the critically ill patients admitted to an intensive care unit in Myanmar would be indispensable in implementing clinical decision making, research, and planning for future waves of covid-19 and health security threats.

\section{Reference(s)}

1. Zhou, F., Yu, T., Du, R., Fan, G., Liu, Y., Liu, Z., ... \& Cao, B. (2020). Clinical course and risk factors for mortality of adult inpatients with COVID-19 in Wuhan, China: a retrospective cohort study. The lancet, 395(10,229), 1054-1062.

2. Richardson, S., Hirsch, J. S., Narasimhan, M., Crawford, J. M., McGinn, T., Davidson, K. W., ... \& Zanos, T. P. (2020). Presenting characteristics, comorbidities, and outcomes among 5700 patients hospitalized with COVID-19 in the New York City area. Jama, 323(20), 2052-2059.

3. National Institute of Health. Coronavirus Disease 2019 (COVID-19) Treatment Guidelines. https://www.covid19treatmentguidelines.nih.gov/. Accessed 4 June 2020.

4. Grasselli, G., Zangrillo, A., Zanella, A., Antonelli, M., Cabrini, L., Castelli, A., \& Pesenti, A. (2020). Baseline characteristics and outcomes of 1591 patients infected with SARS-CoV-2 admitted to ICUs of the Lombardy Region, Italy. Jama, 323(16), 1574-1581.

5. Chen, Y., Zhang, K., Zhu, G., Liu, L., Yan, X., Cai, Z., ... \& Hu, Z. (2020). Clinical characteristics and treatment of critically ill patients with COVID-19 in Hebei. Ann Palliat Med, 9(4), 2118-2130.

000214

Effectiveness of a 'weekday only' rapid response team (RRT) with minimal manpower

D. Kang ${ }^{1}$; SJ. Park' ; IB. Jeong ${ }^{1}$; JW. Son ${ }^{1}$; SJ. kwon

${ }^{1}$ Respiratory and critical care medicine, Konyang University Hospital, Daejeon, Republic of Korea

Correspondence: D. Kang

Intensive Care Medicine Experimental 2020, 9(1): 000214

Introduction. Previous studies have identified that various type of rapid response system (RRS) was associated with reducing cardiopulmonary arrests (CPRs) and mortality in non-intensive care units (ICU) patients $(1,2)$. In contrast to favorable outcomes of RRS, implementing it requires a lot of manpower, which is difficult for some hospitals in real world.

Objectives. We investigated whether maintaining the RRS with minimal manpower would be more effective than not maintaining it.

Methods. This study is a retrospective analysis of the effect of nurse based RRS in secondary teaching hospital, South Korea. Our RRS is a 'weekday only' system (08:30-17:30) from monday to friday and consist of a nurse and 7-8 physicians. After the nurse monitors the patient's clinical deterioration using electronic medical record based screening system and the calling from general ward (GW), nurse activates the RRS with clinical judgement and then calls the doctor on duty. We operated the RRS for hospitalized patients over 15 years of age in GW excepting ICUs, emergency department and operating room. Data were retrieved from the 12-month period before applying the RRS (July 2018 to June 2019) and compared to the 12-month period after applying the RRS (July 2019 to June 2020). The primary outcome was the incidence of CPRs of patients to be monitored. Data for 1 year before and after the start of RRS were analyzed through chi square test.

Results. The number of target patients before and after applying the RRS was 31,798 and 30,060 per year. Each number of deaths in target patients except 'Do not resuscitation (DNR)' patients was $19(0.06 \%)$ and $13(0.04 \%)$, and the $P$ value was 0.367 , showing no significant difference. The number of CPR cases was significantly different from 58 cases $(0.18 \%)$ before application of RRS and 36 cases $(0.12 \%)$ after application of RRS $(p=0.046)$. There was a significant difference in CPR cases on off-duty time between 45 cases $(0.14 \%)$ before application of RRS and 26 cases $(0.09 \%)$ after application of RRS $(p=0.043)$, as there 
was a significant difference in CPR cases on night and weekend time of off duty time between 39 cases ( $0.12 \%$ ) before application of RRS and 20 cases $(0.07 \%)$ after application of RRS $(p=0.023)$.

Conclusion. 'Weekday only and nurse based' RRS is effective to reduce the number of CPRs in GW.

\section{Reference(s)}

1. Solomon RS, Corwin GS, Barclay DC, Quddusi SF, Dannenberg MD. Effectiveness of rapid response teams on rates of in-hospital cardiopulmonary arrest and mortality: A systematic review and meta-analysis. J Hosp Med. 2016;11(6):438-45.

2. Winters BD, Weaver SJ, Pfoh ER, Yang T, Pham JC, Dy SM. Rapid-response systems as a patient safety strategy: a systematic review. Ann Intern Med. 2013;158(5 Pt 2):417-25.

\section{3}

Long term survival of very old Intensive Care patients after ICU admission: 2005-2009 vs 2015-2019

B. Vanwijnsberghe'; S. Van Biesen ${ }^{2}$; A. Koch²; E. Pannier²; K. Mignolet'; T. Sarens ${ }^{2} ; T^{\text {T. Werner }}{ }^{2} ;$ W. Swinnen ${ }^{2}$

${ }^{1}$ Anesthesiology, UZ Gent, Ghent, Belgium; ${ }^{2}$ Intensive care, az Sint-Bla-

sius, Dendermonde, Belgium

Correspondence: B. Vanwijnsberghe

Intensive Care Medicine Experimental 2020, 9(1): 000223.

Introduction. 10 years of progress in medical science and technology has had a huge impact on how we treat patients in the ICU. Obviously, the intention of this progress is to ensure better survival of patients during their stay in ICU, as well as after discharge. But does this progress benefit our most vulnerable patients, the Very old Intensive care Patients (VIP), over 80 years old?

During the VIP1 (2016) and VIP2 (2018) studies, in which our ICU actively participated, we noticed that many of our included VIP patients died shortly after discharge from ICU.

Objectives. This study was set up to investigate survival in the 12 months after ICU admission and to compare survival of 20052009 (05-09) with 2015-2019 (15-19) admissions.

Methods. Setting: az Sint-Blasius, Dendermonde, Belgium, a 438 beds general hospital with university affiliation, with a 12 beds mixed medical-surgical ICU. A query was performed in the ICU database, identifying all VIPs admitted between 05-09 and 15-19.

Data were crossmatched with data in the electronic patient file (EPF), which is linked to the Belgian national register, showing actual vital status or date of death. To avoid bias of VIPs who were repeatedly readmitted in ICU, we excluded all admissions of VIPs that were readmitted in the 12 months prior to final admission. As Kaplan-Meier survival analysis takes into account lost to follow-up, we maintained VIPs without data of actual vital status or date of death.

Statistical tests: Chi-square an Mann-Whitney U-tests for demographic analysis, Kaplan-Meier survival analysis with log-rank test; $p<0.05$ was considered statistically significant.

The study has been approved by the Committee for Medical Ethics of az Sint-Blasius ( ${ }^{\circ}$ BB012201941151). EU-GDPR requirements were met.

Results. Results are shown in Fig. 1. In 15-19 significantly more VIPs were admitted in ICU. After exclusion, 771 vs. 1093 admissions were analyzed. Exclusion was similar in both groups. No patients were lost to follow up in both groups. Median age was older in 15-19. Gender did not differ significantly. Length of stay was shorter in 15-19. ICU mortality was higher in 15-19, but after ICU, mortality did not differ significantly up to 12 months after admission.

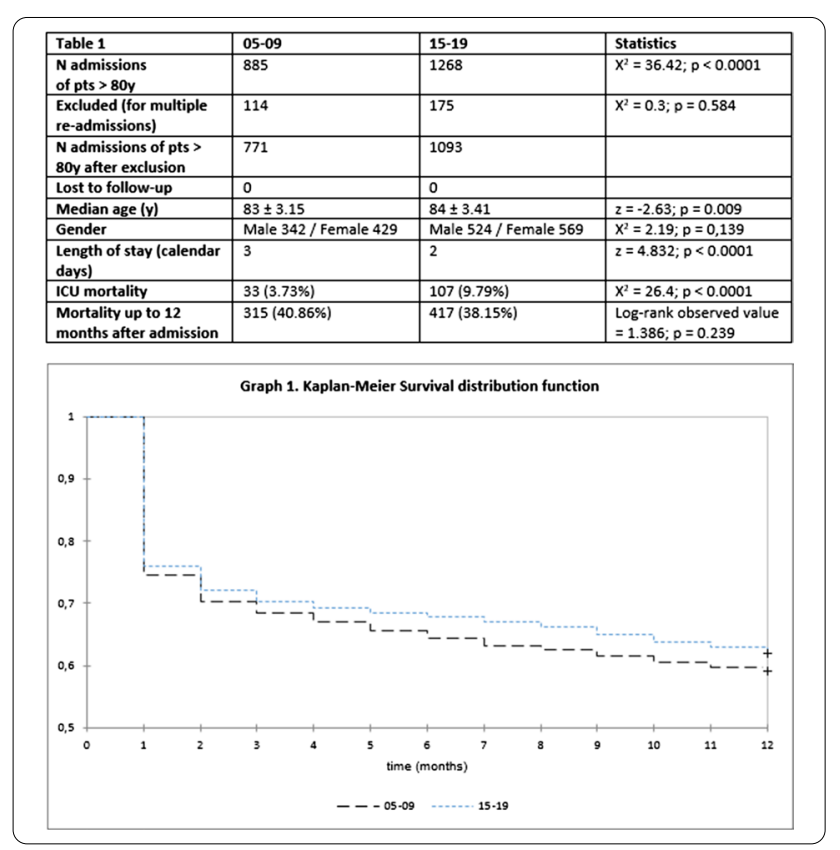

Kaplan-Meier survival analysis is shown in Graph 1.

Conclusion. As a consequence of an aging population, the number of admissions of VIPs in ICU is also increasing, as is the median age of these VIPs.

Although ICU mortality was lower in 05-09, mortality during the first year after ICU admission did not differ. In both study periods, most VIPs died in the first month after ICU admission. Our presumption that 10 years of medical advancement would make survival of this population better, was incorrect.

\section{4}

Long-term cognitive and functional status in ICU patients with COVID-19

S. Weihe ${ }^{1}$; N. Haase'2; CB. Mortensen, ${ }^{1}$; A. Perner ${ }^{2}$; O. Mathiesen, ${ }^{1}$; LM. Poulsen,

'Department of anaesthesiology, Centre for Anaesthesiological research, Zealand University Hospital, Køge, Denmark; ${ }^{2}$ Department of intensive care, Copenhagen University Hospital, Rigshospitalet, Copenhagen, Denmark

Correspondence: S. Weihe

Intensive Care Medicine Experimental 2020, 9(1): 000244.

Introduction. The COVID-19 disease manifests clinically quite differently ranging from asymptomatic or mild symptoms to multi-organ failure. It is well known that survivors after critical illness in general may experience long-term cognitive and functional impairments. Therefore, we anticipated that the critically ill COVID-19 patients in need of ICU treatment would experience similar impairments.

This study will investigate the long-term cognitive and functional outcomes of patients admitted to Danish ICUs with COVID-19.

Methods. During the initial stage of the COVID-19 pandemic a Danish national database, the Danish ICU COVID-19 Database, was established and it includes all three-hundred-twenty-three patients with COVID-19, who were admitted to Danish intensive care units (ICUs) from 10th March to 19th May 2020. Surviving patients were contacted by secure online digital mailbox and given the information of being included into a national database followed by an invitation to participate in the follow-up, which was set up as telephone interviews. The primary outcomes were cognitive and functional status 6 and 12 months after ICU admission using the Mini Montreal Cognitive Assessment (MiniMoCA) and the Clinical Frailty Score (CFS). Secondary outcomes were mortality, and assessments of health-related quality 
of life by using the 5Q-5D-5L instrument, functional status by Barthel Activities of Daily Living (ADL) and Lawton-Brody Instrumental Activities of Daily Living (IADL), and fatigue by Fatigue Assessment Scale (FAS). Furthermore, to explore the association between ventilator days and CFS and MiniMoCA, respectively.

Results. We present preliminary data from 202 patients who were still alive after 6-months (6-month mortality of 37.8\%) of whom 105 (52\%) participated in the follow-up. Baseline characteristics for interviewed vs. not interviewed correspond well on age (67 vs. 61 years), use of mechanical ventilator ( $80 \%$ vs. $79 \%$ ) and comorbidities ( $66 \%$ vs. $63 \%)$. Twenty-six percent had cognitive scores indicating impaired cognitive function, $52 \%$ had FAS scores indicating fatigue (51\% of these had scores ${ }^{3} 35$ indicating extreme fatigue) and $20 \%$ were frail (CFS $>4$ ). Pearson correlation test showed a positive correlation between the number of ventilator days and frailty, but not for cognitive function. Twenty-eight percent reported their health-related quality of life to be moderate to worst health imagined. Participants scored high in the ADL and IADL questionaries, indicating the highest level of independence.

The study is ongoing, and at the congress we present results from both the 6-and 12-month follow-up.

Conclusion. This study will describe the long-term cognitive and functional status of Danish ICU survivors after COVID-19 after 6 and 12 months. The interim conclusion based on the 6-month follow-up indicated a high degree of independency, however, some still experiences cognitive impairments and frailty, and around 50\% report fatigue. The final conclusion of the study will follow after collection and analysis of the 12-month data.

\section{Reference(s)}

1. Haase N, Plovsing R, Christensen S, Poulsen LM, Brøchner AC, Rasmussen BS, et al. Characteristics, interventions and longer-term outcomes of COVID-19 ICU patients in Denmark—a nationwide, observational study. Acta Anaesthesiol Scand. 2020 Sep;

2. None to declare

\section{7}

Comparing the outcomes of wave 1 and wave 2 of COVID-19: are we getting better over time?

TW. Hla ${ }^{1}$; J. Borkowski, ; N. sabir ${ }^{1}$; J. Gross,

${ }^{1}$ Department of anaesthesia and critical care, London North West University Healthcare NHS Trust, London, United Kingdom

Correspondence: T.W. Hla

Intensive Care Medicine Experimental 2020, 9(1): 000247

Introduction. North West London saw an early surge in COVID-19 related Intensive Care Unit (ICU) admissions preceding the UK national lockdown. Our institution (London North West University Healthcare NHS Trust) was the first in the UK to declare a critical incident on 20 March 2020 due to a significant shortage of ICU beds[1]. Many lessons were learnt with respect to service organisation and delivery together with the advent of new drug therapies shown to improve outcome[2]. The aim of this study was to explore whether or not such changes were contributory to improved outcomes for patients with COVID-19 admitted to the ICU at our institution.

Methods. For this retrospective analysis, we compared 2 six-months long temporal waves of COVID defined by the UK government[3]. Wave 1 was defined from $1 / 3 / 20-31 / 8 / 20$ and wave 2 from 1/9/20 to $28 / 2 / 21$. Our cohort is inclusive of all patients admitted to ICU at our institution during this time period. Data collected included relevant baseline characteristics and survival status at ICU and hospital discharge. For parametric data, mean, standard deviation and student- $t$ test were used to describe and compare groups respectively. Categorical variables were presented as numbers and percentages with comparison undertaken using chi-square test.

Results. A total of 1829 admissions were recorded during this period. Compared to wave 1, patients in wave 2 were older with proportionally a lower incidence of Black and Minority Ethnic (BAME) admissions.
Baseline acute illness severity was measured using a validated severity score(ICNARC score), and was comparable between the 2 groups[4]. The survival status in wave 2 was significantly lower than wave 1 at the times of ICU and hospital discharge (see Table 2)

Table 1. Characteristics of wave 1 and 2 population

\begin{tabular}{llll}
\hline Patient characteristics & 1st wave & 2nd wave & p-value \\
\hline Total admissions & 885 & 944 & \\
Age-mean (SD) & $59.3(15.7)$ & $61.1(15.1)$ & $\mathbf{0 . 0 1 9}$ \\
Male-no(\%) & $589(66.6)$ & $608(64.4)$ & 0.360 \\
Ethnicity-no(\%) & & & $\mathbf{0 . 0 1 0}$ \\
White & $289(32.7)$ & $339(36.1)$ & \\
Asian British & $351(39.7)$ & $348(37.0)$ & \\
Black & $94(10.6)$ & $69(7.3)$ & \\
Body Mass Index-mean(SD) & $26.89(6.4)$ & $26.92(5.8)$ & 0.914 \\
ICNARC score-mean(SD) & $22.21(8.11)$ & $22.78(8.72)$ & 0.270 \\
COVID admissions-no(\%) & $355(40.1)$ & $379(40.1)$ & 1 \\
ICU stay days-mean(SD) & $8.0(15.5)$ & $7.2(10.27)$ & \\
\hline
\end{tabular}

Table 2: Outcomes of wave 1 and 2 population

\begin{tabular}{lcccc}
\hline Outcomes & 1st wave & 2nd wave & p-value & $\begin{array}{l}\text { Odds Ratio(95\% } \\
\text { Confidence } \\
\text { Interval) }\end{array}$ \\
\hline $\begin{array}{c}\text { ICU survival- } \\
\text { no(\%) }\end{array}$ & $710(80.2)$ & $686(75.2)$ & $\mathbf{0 . 0 1 2}$ & $\mathbf{1 . 3 4 ( 1 . 0 6 , 1 . 6 8 )}$ \\
$\begin{array}{c}\text { Hospital survival } \\
\text {-no(\%) }\end{array}$ & $672(75.9)$ & $633(70.1)$ & $\mathbf{0 . 0 0 6}$ & $\mathbf{1 . 3 4 ( 1 . 0 8 , 1 . 6 7 )}$ \\
\hline
\end{tabular}

Conclusion. At our institution, the survival outcome was less favourable in the 2nd wave despite changes to the organisational and healthcare delivery and the introduction of new drug therapies shown to improve COVID-19 outcomes. Reasons for these unexpected findings urgently need further exploration in further studies.

\section{Reference(s)}

1. Harrison DA, Lone NI, Haddow C, MacGillivray M, Khan A, Cook B, et al. External validation of the intensive care national audit \& research centre (ICNARC) risk prediction model in critical care units in Scotland. BMC Anesthesiol [Internet]. 2014 Dec 15 [cited 2021 Apr 9];14. Available from: https://www.ncbi.nlm.nih.gov/pmc/articles/PMC4277842/

2. Prime Minister of the United Kingdom. COVID-19 Winter Plan [Internet]. HM Government; 2020 [cited 2021 Apr 9]. Available from: https://assets. publishing.service.gov.uk/government/uploads/system/uploads/attac hment_data/file/937529/COVID-19_Winter_Plan.pdf

3. Clover, Ben. London trust with most covid-19 deaths: We serve a vulnerable population. Health Service Journal [Internet]. 2020 Apr 9 [cited 2021 Apr 9]; Available from: https://www.hsj.co.uk/coronavirus/london-trustwith-most-covid-19-deaths-we-serve-a-vulnerable-population/7027369. article

4. Pett E, Leung HL, Taylor E, Chong MSF, Hla TTW, Sartori G, et al. Critical care transfers and COVID-19: Managing capacity challenges through critical care networks. Journal of the Intensive Care Society. 2020 Dec 16;175,114,372,098,027. https://journals.sagepub.com/doi/full/10.1177/ 1751143720980270

5. No conflicts of interest. No grants were received or this project. 


\section{8}

Lung sequelae in severe COVID-19 patients: outpatient assessment three months after cure of COVID-19

A. Silva-Pinto ${ }^{1}$; J. Fernandes ${ }^{2}$ ' L. Ribeiro ${ }^{1}$; T. Vieira 2 ; C. Sousa ${ }^{3}$; M. Jacob ${ }^{3}$; C. Sousa ${ }^{4}$; J. Jesus ${ }^{4}$; I. Coimbra ${ }^{2}$;V. Hespanhol ${ }^{3}$; A. Madureira ${ }^{4}$; JA. Paiva ${ }^{2}$; L. Santos,

'Infectious diseases department, Centro Hospitalar e Universitário de São João, Porto, Portugal; ${ }^{2}$ Intensive care medicine department, Centro Hospitalar e Universitário de São João, Porto, Portugal; ${ }^{3}$ Pneumology department, Centro Hospitalar e Universitário de São João, Porto, Portugal; ${ }^{4}$ Radiology department, Centro Hospitalar e Universitário de São João, Porto, Portugal

Correspondence: A. Silva-Pinto

Intensive Care Medicine Experimental 2020, 9(1): 000248

Introduction. In 2020, COVID-19 spread around the world. Severe and critical cases have multiple presentations, and knowledge about the lung sequelae of COVID-19 is still scarce.

Objectives. To study the lung sequelae of COVID-19 through radiological and pulmonary function assessment.

Methods. All patients admitted to an Intensive Care Department (ICD) of a tertiary Hospital (São João Hospital, Porto, Portugal) during the first wave of COVID-19 who survived were invited to be assessed, three to six months after discharge, in terms of lung sequelae. Lung sequelae were assessed radiologically (through a thorax CT scan) and functionally (through pulmonary function tests). We used the most suitable descriptive and inference statistics, using a significance level of 0,05 .

Results. Of the 157 patients admitted to the ICU during the first wave of COVID-19 infection, 106 survived to be discharged and 100 were assessed in the outpatient clinic. Sixty-five patients (65\%) were men, and the mean age was 63 (SD 14). The patients were in the ICU for a median of 17.5 days (IQR 6.75-33.25). The mean SAPS II and APACHE scores were 36 (SD 16) and 17 (SD 6), respectively. Sixty-seven patients $(67 \%)$ were intubated and mechanically ventilated, $26(26 \%)$ were on high flux nasal oxygen, and 7 (7\%) were on non-invasive ventilation. In the majority $(68.7 \%)$ of mechanically ventilated patients, protective ventilation was achieved (plateau pressure inferior to $30 \mathrm{cmH} 2 \mathrm{O}$ and driving pressure inferior to $15 \mathrm{cmH} 2 \mathrm{O}$ ). In thirteen patients, extracorporeal membrane oxygenation (ECMO) was used. All CT scans revealed improvement from the acute to the convalescent phases of COVID-19 infection. Twenty-four patients (26\%) had a normal CT scan. Forty patients (58\%) had ground-glass opacities and 55 patients $(59 \%)$ had some evidence of fibrosis in the CT scan: fibrotic atelectasis $(n=27)$, traction bronchiectasis $(n=21)$, bronchiolectasis $(n=18)$ and UIP-type fibrosing lung diseases $(n=5)$. Pulmonary function tests were normal in most patients (63 patients, $77 \%$ ). Two patients (2.4\%) had obstructive pattern, 11 patients (13.4\%) had restrictive pattern, and 9 patients $(10,9 \%)$ had decreased $\mathrm{CO}$ diffusion capacity. In univariate analysis, there was a statistically significant association between persistence of abnormalities in the convalescent phase CT-scan and older age $(p=0.048)$, higher SAPS II and APACHE scores $(p=0.018$ and $p<0.001)$ and having been invasively ventilated $(p=0.011)$. However, there was no statistically significant association with being on ECMO $(p=0.934)$ or having non-protective ventilation $(p=0.878)$.

Conclusion. Most patients with severe COVID-19 infection admitted to an ICD still exhibit abnormalities in CT scans three to six months after hospital discharge, such as ground-glass opacities and fibrosis, without significant abnormalities in pulmonary function tests. Persistent CT scan alteration were associated with higher disease severity, older age and invasive ventilation.

\section{9}

\section{Rapid Response System in a regional hospital: An overview}

R. Passos ${ }^{1}$; F. Cardoso, ; D. Alves, ; L. Pereira ${ }^{1}$; A. Colmente, ${ }^{1}$; R. Corga Da

Silva ${ }^{1} ;$ J. Caldeiro

${ }^{1}$ Intensive medicine, Hospital Santa Luzia_Ulsam, Viana do Castelo, Viana do Castelo, Portugal

Correspondence: R. Passos

Intensive Care Medicine Experimental 2020, 9(1): 000249
Introduction. A rapid response system (RRS) is created for prompt intervention in patients with unexpected clinical deterioration during hospitalization. The absence of an organized system to face in-hospital emergencies may result in a high risk of preventable life-threatening events to patients with an increased rate of death. In this context, RRS implementation has been increasing worldwide in the last decades and many studies have shown its efficacy in reducing the rates of inhospital mortality. Therefore, the development of precise activation criteria and risk stratification is needed for improving triage of patients and resource allocation. In our country, formal guidelines for the creation and implementation of RRS were created in 2010. At our center, the RRS was redefined in 2015, and there was a switch from a traditional code team to a RRS with other than Cardiac Arrest activation criteria. This study describes RRS presence and organization at our center from 2017 to 2020.

Methods. We performed a descriptive statistical analysis of all RRS activations from January 2017 until December 2020 since the formal implementation of our RRS registry data base.

Results. One thousand two hundred twenty-six RRS activations were performed in the study period. The mean age of the patients was 70.9 years. $54.2 \%$ of the patients were male. A decreasing pattern of activations was verified from 2018 to 2020 , with a total of 416 activations in 2018, 300 in 2019 and 235 in 2020. 25,1\% of RRS activations were performed from our Intermediate Care Units, 20.9\% from internal medicine ward and $19.9 \%$ from surgical wards. Cardiac arrest (CA) was the activation criteria in $13.5 \%$ of the cases. In $6.63 \%$ of the cases acute respiratory distress was the main motive of activation. Other causes of RSS activation were persistent hypotension, syncope, tachycardia and airway obstruction. In the cases of CA, the arrest rhythm was asystole in $66.9 \%$, pulseless electrical activity in $23.5 \%$, ventricular fibrillation in $7.23 \%$ and pulseless ventricular tachycardia in $2.41 \% .69 .3 \%$ of the patients in CA survived after RSS intervention.

Conclusion. At our center, RRS consists of a medical emergency team composed of a critical care nurse and a critical care doctor with skills in resuscitation and care of critical patients, active on a 24/7 basis. The decreasing pattern of activations observed over three years may be explained by an increasing awareness of the "afferent" limb of RSS for the exact activation criteria. In regards to CA, our data is consistent with the literature, with a predominance of nonshockable rhythms at presentation. The survival rate after CA was greater than the reported in the literature regarding in-hospital CA. However, it is important to note that the reported rate of survival was not at hospital discharge but immediately after RSS intervention. Despite the pervasive use of RSS, little is known about the best predictors of clinical outcomes of patients for whom RSS is activated. This might be explained by the heterogeneity of patients observed, with various comorbidities. In this context, it is crucial that future studies focus on the identification of outcome measures that reflect the success or failure of RSS.

\section{1}

The Bundle is Dead, Long Live the Bundle: Improving

Evidence-Based Care at a COVID-19 Surge Centre

J. McLean ${ }^{1}$; P. Tharmapoopathy, ; S. Hutchinson ${ }^{1}$

${ }^{1}$ Critical Care Complex, Norfolk and Norwich University Hospital, Norwich, United Kingdom

Correspondence: J. McLean

Intensive Care Medicine Experimental 2020, 9(1): 000271

Introduction. Care bundles are an established part of Intensive Care Unit (ICU) practice and have the potential to significantly improve patient outcome $(1,2)$. More recently however, the utility of care bundles has been questioned (3). The COVID-19 pandemic presented challenges including rapid ICU expansion, care provision by non-ICU trained staff, and a rapidly evolving, piecemeal evidence-base. These challenges have led to renewed interest in care bundles for these patients (4). We developed a care bundle for critically ill patients with COVID-19, with the aim of improving the evidence-based care of these patients.

Objectives. This abstract aims to evaluate the success of implementing a local care bundle for critically ill patients with Covid-19 infection. 
Methods. Our care bundle was based on UK national guidelines (5) and included administration of dexamethasone and tocilizumab, control of tidal volumes, enhanced thromboprophylaxis, admission investigations, and rational antibiotic usage.

The project was completed in a UK-based general ICU which is a designated regional surge centre. The bundle was introduced at the beginning of the second wave of the pandemic in the UK. We collected data for 14 weeks, which included a two-week pre-bundle baseline period. During the period of observation, tocilizumab was added based on the publication of trial data from REMAP-CAP (6).

We assessed performance of each component on a weekly basis. Each day of a patient's admission reflected one data point. A patient was considered compliant for a given day if all applicable components of the bundle were successfully implemented. We also collected performance data for each individual component. Bundle performance was regularly fed back to ICU staff at monthly clinical governance meetings. Interventions to improve performance were undertaken based on regular assessment of underperforming components.

Results. During this period 182 patients with COVID-19 were admitted, with a peak of 310 patient-days per week. Our ICU expanded to 59 beds, from a baseline of 28 , with a corresponding increase in non-ICU trained staff.

Two weeks prior to implementation, baseline bundle performance was $56 \%$. This increased to $68 \%$ for the first two weeks of bundle implementation. At the end of the 12-week observation period overall performance had increased to $90 \%$ (Fig. 1).

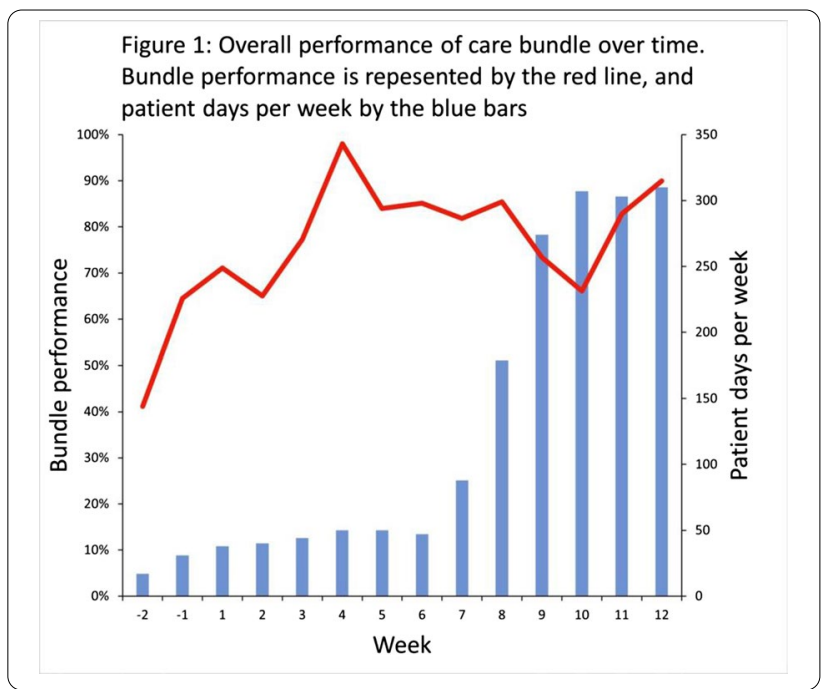

Bundle components which involved the administration of drugs demonstrated high initial performance, which was sustained throughout the observation period, for example dexamethasone (pre-bundle performance of $98 \%$ vs end performance of $100 \%$ ). In contrast, other components demonstrated lower starting performance which proved more difficult to improve.

Interventions which were associated with improvement in bundle performance included the incorporation of bundle components into daily safety briefings, the creation of an electronic order set for admissions, and the introduction of bedside weight charts.

Conclusion. The use of an evidence-based care bundle alongside regular assessment and intervention allowed our department to rapidly adopt evidence-based practices, and maintain a high standard of care despite significant pressures.

\section{Reference(s)}

1. Dellinger, R.P., Levy, M.M., Carlet, J.M. et al. Surviving Sepsis Campaign: International guidelines for management of severe sepsis and septic shock: 2008. Intensive Care Med 34, 17-60 (2008). https://doi.org/10. 1007/s00134-007-0934-2
2. Morris, Andrew Conway MB, ChB, MRCP; Hay, Alasdair W. FRCA; Swann, David G. FRCA et al. Reducing ventilator-associated pneumonia in intensive care: Impact of implementing a care bundle. Critical Care Medicine: October 2011—Volume 39—Issue 10—p 2218-2224 https://doi.org/10. 1097/CCM.0b013e3182227d52

3. Lavallée, J.F., Gray, T., Dumville, J. et al. The effects of care bundles on patient outcomes: a systematic review and meta-analysis. Implementation Sci 12, 142 (2017). https://doi.org/10.1186/s13012-017-0670-0

4. Smith V, Devane D, Nichol A, Roche D. Care bundles for improving outcomes in patients with COVID-19 or related conditions in intensive care-a rapid scoping review. Cochrane Database of Systematic Reviews 2020, Issue 12. Art. No.: CD013819. https://doi.org/10.1002/14651858. CD013819.

5. The Faculty of Intensive Care Medicine. Clinical guide for the management of critical care for adults with COVID-19 during the Coronavirus pandemic. October 2020. Available from: https://icmanaesthesiacovid-19. org/clinical-guide-for-the-management-of-critical-care-for-adults-withcovid-19-during-the-coronavirus-pandemic [Accessed 21st March 2021]

6. The REMAP-CAP Investigators. Interleukin-6 Receptor Antagonists in Critically III Patients with Covid-19-Preliminary report. medRxiv 2021.01.07.21249390; https://doi.org/10.1101/2021.01.07.21249390

\section{3}

Predicting hospital mortality for critically ill patients: a comparison of artificial neural networks with logistic regression models

S. Lau'; HP. Shum ${ }^{1}$; CYC. Chan ${ }^{1}$; MY. Man ${ }^{1}$; KB. Tang ${ }^{1}$; KKC. Chan ${ }^{2}$; WW. Yan ${ }^{1}$ ${ }^{1}$ Department of intensive care, Pamela Youde Nethersole Eastern Hospital, Hong Kong, Hong Kong; ${ }^{2}$ Department of intensive care, Tuen Mun Hospital, Hong Kong, Hong Kong

Correspondence: S. La

Intensive Care Medicine Experimental 2020, 9(1): 000283.

Introduction. Outcome prediction for critically ill patients is challenging. The Acute Physiology and Chronic Health Evaluation (APACHE) II and IV logistic regression model are the benchmark predictive tools in intensive care unit (1). Yet, it has limitation in handling non-linear relationships between variables and outcome, often subjects to loss of calibration overtime and inconsistent performance when applied outside the US due to different in case-mix and clinical practices (2). Artificial neural network (ANN) has been proposed as an alternative (3).

Objectives. To compare the performance of ANN model to APACHE II and IV-in predicting the hospital mortality in the critically ill patients in Hong Kong (HK).

Methods. This retrospective analysis included all admissions to the intensive care unit of the Pamela Youde Nethersole Eastern Hospital from Jan 2010 to Dec 2019. 10\% was randomly selected from the included samples to form the validation set. The remaining $90 \%$ was used for ANN model development. Among them, 70\% was assigned for training while $30 \%$ was assigned for testing purpose. We trained ANNs with one hidden layer with 12 units. Hyperbolic tangent and Softmax activation functions were employed for the hidden and output layers respectively. The ANN was constructed using the same parameters as in the APACHE IV score model. The performance was assessed in terms of discrimation using the area under the receiver operating characteristic curve (AUROC), and calibration using the Brier score and Hosmer-Lemeshow statistic $(H)$.

Results. A total of 14,503 admissions were included. $4.9 \%$ of the recruited cases had at least one missing data and was handled by multiple imputation method. The hospital mortality rate was $19.3 \%$. Using 53 parameters based on the APACHE IV model, the ANN model (Model "Development set", AUROC 0.886, 95\% Cl 0.879-0.92, Brier score 0.097 , Hosmer lemeshow test $p=0.3363$ ) was found to be superior to the APACHE II (AUROC $0.800,95 \% \mathrm{Cl} 0.790-0.810$, Brier score $0.1519, p<0.001$ when compared with Model "Development set") and APACHE IV (AUROC $0.841,95 \% \mathrm{Cl} 0.833-0.850$, Brier score 0.1232 $\mathrm{p}<0.001$ when compared with Model "Development set") for predicting hospital mortality. The validation set contains 1400 patients. The ANN model "validation set" (AUROC $p=0.878,95 \% \mathrm{Cl} 0.857-0.900$, Brier score 0.099 , Hosmer lemeshow test $p=0.3712$ ) was once again 
superior than APACHE II (AUROC 0.824, 95\% Cl 0.796-853, Brier score $0.1436, \mathrm{p}<0.001$ ) but similar to APACHE IV (AUROC $0.869,95 \% \mathrm{Cl}$ $0.848-0.891$, Brier score $0.1141, p=0.3432$ ). The calibration curve for the validation set showed that ANN model has better calibration than both APACHE II and APACHE IV.

Conclusion. Our ANN model, which trained with local representative data, outperformed the internationally well-validated APACHE II and IV models in local critically ill patients. External validation using data from other hospitals are recommended to confirm this finding. Future studies may explore the feasibility to reduce the number of variables while preserving the discrimination and calibration power of the ANN model. With the popularity of computerised information system in ICU and general ward settings to replace paper records, data are more readily available than before. ANN, together with other machine learning techniques, are valuable tools for real-time patients' prognostication.

\section{Reference(s)}

1. Keegan MT, Gajic O, Afessa B. Severity of illness scoring systems in the intensive care unit. Crit Care Med. 2011;39(1):163-169.

2. Zimmerman JE, Kramer AA. Outcome prediction in critical care: the Acute Physiology and Chronic Health Evaluation models. Curr Opin Crit Care. 2008;14(5):491-497

3. Xie J, Su B et.al A review of modelling methods for predicting in-hospital mortality of patients in intensive care unit. J Emerg Crit Care Med. 2017 (1): 18

\section{6}

Post-intensive care syndrome after critical COVID-19 pneumonia L. Bougard ${ }^{1}$; P. Minguet ${ }^{1}$; C. Colson ${ }^{1}$; B. Lambermont ${ }^{1}$; B. Misset ${ }^{1}$; AF. Rousseau'

'Intensive care, University Hospital of Liège, Liège, Belgium

Correspondence: A.F. Rousseau

Intensive Care Medicine Experimental 2020, 9(1): 000286

Introduction. Little is known about mid-term outcomes of critically ill Coronavirus disease-2019 (COVID-19) survivors.

Objectives. The objective of this single-center cohort study was to describe their physical, cognitive, psychological, and biological outcomes at 3 months following intensive care unit (ICU)-discharge (M3).

Methods. All COVID-19 adults who survived an ICU stay $\geq 7$ days during the first wave of the pandemic (from March 1st to July 17th2020) were included if they attended the M3 consultation at our multidisciplinary follow-up clinic. They benefited from a standardized assessment, addressing health-related quality of life (EQ-5D-3L), sleep disorders (PSQI), and the 3 principal components of post-intensive care syndrome: physical status (Barthel index, handgrip and quadriceps strength), mental health disorders (HADS detecting anxiety and depression, IES-R detecting post-traumatic stress disorder), and cognitive impairment (MoCA). Biological parameters included C-reactive protein (CRP), creatinine and cystatin C.

Results. Among the 92 patients admitted in ICU for COVID-19, 42 survived a prolonged ICU stay and $32(80 \%)$ attended the M3 follow-up visit: 62 [49-68]y, 72\% male. A majority of patients were mechanically ventilated (30/32) for 21 [12-29]days. The ICU and hospital length of stay (LOS) were respectively 23 [15-39]days and 40 [29-52]days. At M3, 87.5\% (28/32) had not return to previous level of activities, either employment for previously active patients or leisure for unemployed or retired patients. Only $6.2 \%$ (2/32) fully recovered, and had normal scores for the three MoCA, IES-R and Barthel tests. The main observed disorders were PSQI $>5$ (75\%, 24/32), MoCA $<26$ (44\%, 14/32), Barthel $<100(31 \%, 10 / 32)$ and IES-R $>33(28 \%, 9 / 32)$. Combined disorders were observed in $13 / 32(40.6 \%)$ of the patients. The EQ-5D-3L visual scale was rated at 71[61-80]. A quarter of patients (8/32) demonstrated a persistent inflammation based on CRP blood level $(9.3$
[6.8-17.7] $\mathrm{mg} / \mathrm{L})$. Measuring serum cystatin C, compared to creatinine, allowed to detect a greater number of patients presenting persistent biological kidney dysfunction $(56.2 \%(18 / 32)$ vs $28 \%(9 / 32)$, respectively). From hospital discharge until the M3 visit, 4/32 (12.5\%) patients visited an emergency department, at least one time. Nearly half of the cohort received inpatient rehabilitation between ICU discharge and M3. Their illness severity was similar but duration of mechanical ventilation and ICU LOS were prolonged, when compared to patients who did not receive rehabilitation. At $M 3$, despite rehabilitation, they were still significantly more dependent $(p=0.03)$.

Conclusion. The burden of severe COVID-19 and prolonged ICU stay was considerable in the present cohort after 3 months, affecting both functional status and biological parameters. These data are likely to apply worldwide, and are an argument on the need of closed followup for critically ill COVID-19 survivors.

000292

Quality improvement project on proning of COVID-19 patients in intensive care unit at West Middlesex University Hospital United Kingdom

S. Imtiaz' ; P. Chowdhury ${ }^{2}$; M. Popescu ${ }^{3}$

${ }^{1}$ Anaesthetics and intensive care unit, West Middlesex hospital, Isleworth, United Kingdom; ${ }^{2}$ Anaesthetics, West Middlesex University Hospital, Isleworth, United Kingdom; ${ }^{3}$ Anaesthetics and intensive care unit, West Middlesex University Hospital, Isleworth, United Kingdom

Correspondence: S. Imtiaz

Intensive Care Medicine Experimental 2020, 9(1): 000292

Introduction. Proning patients in intensive care have become an integral part of management of severe respiratory failure especially in patients with COVID-19 pneumonitis in this present pandemic.

Proning helps in improving oxygenation and ventilation in these patients by reducing ventilation perfusion mismatch. Despite proven benefits of proning, prone positioning can be challenging and involve numerous complications which include injuries to face, eyes, nerves, as well as respiratory and cardiovascular compromise.

Therefore, proning of patients safely poses significant challenges, which requires adequate training through simulation, presence of manpower and the adherence of safety checklists in intensive care units.

In our unit, we do not have a post proning care bundle and the compliance of a checklist was low.

Objectives. 1. To review complications that occurred during proning of intubated patients with COVID-19 in our intensive care unit. 2. To improve the quality of proning and to minimize complications.

Methods. We looked into a case series of 20 patients with COVID 19 undergoing invasive ventilation in the intensive care unit at West Middlesex Hospital, London, UK. Data was collected over a period of 2 weeks using a proforma during proning and from the intensive care Cerner electronic medical record of patients. The results were recorded on a spreadsheet using Microsoft Excel.

Results. Significant complications noted were facial and lip injuries in $35 \%$, pressure sores in $30 \%$ and corneal edema in $20 \%$ of patient's proned.

Other adverse events included respiratory compromise and cardiovascular compromise in $35 \%$ and $15 \%$ of patients respectively. Cuff leak and endotracheal dislodgement was recorded in $5 \%$ of patients.

Mean duration of proning in patients was recorded at 9 days and a maximum duration of proning was 20 days. Average period patients were proned at a time was $13 \mathrm{~h}$.

Conclusion. Our data analysis showed that proning and subsequent de-proning of patients in our unit with respiratory failure resulting from COVID-19 was associated with some of the well-known complications of this mode of positioning.

We also observed that compliance with proning checklist was less than satisfactory. 
We thus made the below recommendations based on current evidence, which will help to mitigate and provide better care for our patients.

Our Recommendations

1. Ensure adequate training of staff involved.

2. Use of protective pressure pads to protect pressure areas and use a grading scale for monitoring

3. Use of soft endotracheal tube ties to prevent lip injuries.

4. Daily lip and eye care and use of eye guard during proning.

5. Preparation and checking of airway trolley and immediate availability of appropriate drugs for use.

6. Documentation of proning days and complications to be recorded on the intensive care Cerner medical record.

7. Ensuring compliance with proning checklist during proning and introduction of post proning care bundle.

\section{Reference(s)}

1) Guidance for Prone Positioning in Adult Critical Care. Faculty of Intensive Care Medicine. Intensive Care Society.

2) Safe prone checklist: Construction and implementation of a tool for performing the prone maneuver, D. Piekala, D. Batista, S.Minossi. Rev Bras Ter Intensiva. 2017 Apr-Jun; 29(2): 131-141.

3) Proning during covid-19: Challenges and solutions. S. Cotton, Q. Zawayderi, S. LeBlanche, A Hussain. Heart and Lung 49:2020,686-687

4) Not applicable

\section{3}

Improving sleepiness and wellbeing during nightshifts in ED healthcare workers; evaluation of a multicomponent intervention bundle

A. Salet ${ }^{1}$; J. Van Bussel'; M. Keunigs ${ }^{2}$; P. De Jager ${ }^{1}$; R. Hut ${ }^{3}$; K. Simons ${ }^{4}$ ${ }^{1}$ Intensive care, jeroen bosch hospital, 's-hertogenbosch, Netherlands: ${ }^{2} \mathrm{cu}$, Máxima MC Veldhoven, Veldhoven, Netherlands; ${ }^{3} \mathrm{Chronobiology}$ unit, groningen institute of evolutionary life Sciences, University of Groningen, Groningen, Netherlands; ${ }^{4}$ Intensive care, Jeroen Bosch Hospital, s'Hertogenbosch, Netherlands

Correspondence: $\mathrm{G}$. salet

Intensive Care Medicine Experimental 2020, 9(1): 000303

Introduction. Shift work is associated with a multitude of short- and long-term health problems, due to a disruption of the biological clock $(1,2)$. In hospitals, care is provided $24 \mathrm{~h}$ a day and healthcare providers are therefore subject to shift work and its associated negative consequences. In this study, we evaluated a bundle of interventions, aimed at improving sleep and wellbeing during and after night shifts.

Objectives. To assess whether implementation of a bundle of specific measures improves subjective sleepiness and general well-being during nightshifts, as well as long term health effects by determining predictive markers of diabetes mellitus and cardiovascular disease.

Methods. This was a single centre interventional study with a 'before and after' design, among ED nurses and doctors working in night shifts in the Jeroen Bosch Hospital, s'-Hertogenbosch The Netherlands. The intervention was based on reducing sleepiness during the nightshift as well as the effects of external cues on the natural biorhythm. It consisted of the option of a powernap during the night shift, the option to wear glasses with blue coloured light to improve concentration during the night shift and to wear orange coloured glasses after the night shift, to reduce awakening effects of morning sunlight. Finally, a specific diet during specific times in the night was provided. Subjects were tested on the Karolinska Sleepiness Scale (KSS) to determine subjective level of sleepiness every night shift at 5:00 am. During both periods blood was taken twice in one night and a general health questionnaire was completed.

Results. Of the 67 nurses and doctors 40 participated in the study. All consumed the provided nutrition, while the option of a powernap, and wearing blue and orange glasses were used by $90,40 \& 64 \%$ respectively. We found that sleepiness at the start of a night-shift period was similar between baseline and after intervention, but that sleepiness decreased significantly during the subsequent nights after the intervention (Fig. 1).

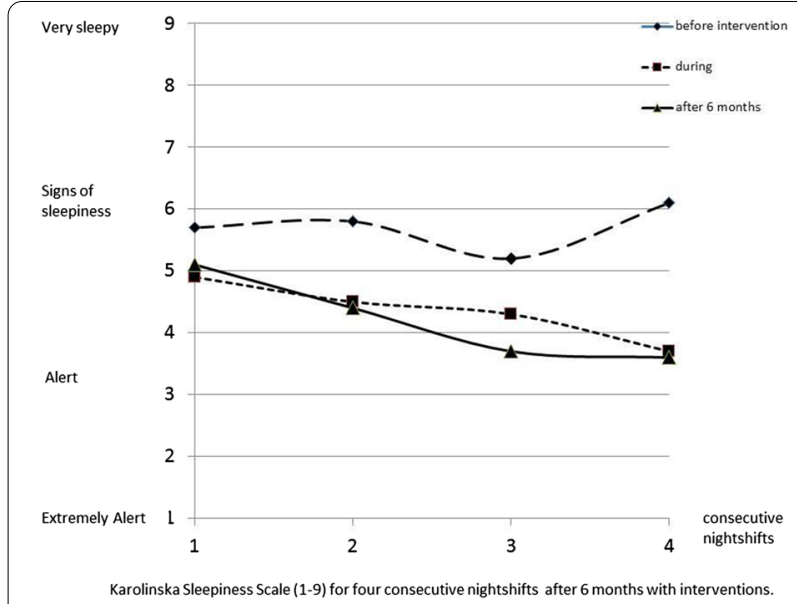

Fig. 1, made by author.

The questionnaires results showed that concentration and subjective energy levels improved significantly during the night shifts after the intervention period whereas the number of mood swings was significantly reduced. The number of participants that indicated to be able to drive home safely increased from $21 \%$ before the intervention to $37 \%$ after. Finally, insulin levels peaked after the night time meal; the magnitude of this peak was reduced from a mean 23.8 to $10.9 \mathrm{mU} / \mathrm{L}$.

Conclusion. In this study, implementation of a bundle of measure leads to a reduction in sleepiness over subsequent nights during a nightshift period. Additionally, the concentration significantly improved, as well as the ability to drive home safely after night shift. Furthermore, insulin peaks were lower after night-time meals, indicating a potential role for these measures to reduce long-term risk of cardiovascular disease.

Reference(s)

1. kerstedt T. Shiftwork and disturbed sleep/wakefulness. Occup. Med. 2003;53:89-94. 2 Spiegel K,Leproult R, Van Cauter E. Impact of sleep debt on metabolic and endocrine function. Lancet 199;345(9188):1435-9.

000307

Predictive value of serial evaluation of the SOFA score for ICU mortality in critically ill patients with COVID-19: a retrospective cohort study

L. De Donder ${ }^{1}$; I. Gruyters ${ }^{1}$; J. Hermans ${ }^{1}$; J. Dubois ${ }^{1}$; J. Dewandre ${ }^{1}$;

B. Stessel

${ }^{1}$ Anesthesiology and intensive care, Virga Jessa, Hasselt, Belgium

Correspondence: $L$. De Donder

Intensive Care Medicine Experimental 2020, 9(1): 000307

Introduction. The Sequential Organ Failure Assessment (SOFA) score has been developed to score the severity of organ dysfunction over time in critically ill sepsis patients. Although it was not initially designed to predict mortality, it has been proven that the SOFA score has high predictive value for Intensive Care Unit (ICU) mortality in severely ill patients ${ }^{2}$.

Objectives. Our goal was to evaluate if trends in SOFA score during ICU admission are also associated with outcome in patients with severe acute respiratory syndrome coronavirus 2 (SARS-CoV-2) infection.

Methods. All adult patients with a laboratory-confirmed diagnosis of COVID-19 pneumonia and admitted to ICU of JESSA hospital, Hasselt, Belgium between March 13th 2020 and October 17th 2020 were included In this retrospective, cohort study. Data were prospectively entered into a customized database covering demographic data, medical history, laboratory results and clinical outcomes. This database 
was retrospectively reviewed. The worst SOFA score was evaluated daily. Categorical variables are presented as frequency (\%) and continuous variables as median with interquartile range or mean. Mixed models for repeated measures were used to compare SOFA data of survivors and non-survivors.

Results. In total, 116 COVID-19 patients were admitted to the ICU from March 13th until October 17th 2020. Thirteen COVID-19 patients, admitted to the COVID ICU for other reasons (i.e. neurological trauma, diabetic ketoacidosis, complication after surgery) than COVID-19 pneumonia, were excluded, resulting in 103 patients for final analysis. Baseline characteristics are presented in Table 1. Mean worst SOFA score on day 1 was not different between groups $(p=0.15)$. From day 2 , difference in mean worst SOFA score gradually increases: mean worst SOFA score on day 3 is 1.76 higher in non-survivors $(p=0.0051)$ and on day $74.08(p<0.0001)$.

Table 1. Baseline demographics and medical history. Data are presented as mean \pm standard deviation or as number (frequencies)

\begin{tabular}{lll}
\hline & $\begin{array}{l}\text { ICU Survivors } \\
(\mathbf{n = 7 3 )}\end{array}$ & $\begin{array}{l}\text { ICU Deceased } \\
(\mathbf{n}=\mathbf{3 0})\end{array}$ \\
\hline Age (years) & $65.93 \pm 11.55$ & $73.73 \pm 8.66$ \\
Gender (male/female) & 17 males/ 13 females & 46 males/ 27 females \\
BMI (kg/m2) & $27.58 \pm 4.67$ & $29.15 \pm 6.26$ \\
Smoking & $2(2.74 \%)$ & $3(10.00 \%)$ \\
Arterial hypertension & $40(54.80 \%)$ & $22(73.33 \%)$ \\
Diabetes & $20(27.40 \%)$ & $13(43.33 \%)$ \\
Chronic respiratory disease & $12(16.44 \%)$ & $8(26.66 \%)$ \\
Malignancy & $5(6.85 \%)$ & $6(20.00 \%)$ \\
Chronic Kidney Disease & $10(13.70 \%)$ & $5(16.66 \%)$ \\
Chronic Liver Disease & $2(2.74 \%)$ & $1(3.33 \%)$ \\
\hline
\end{tabular}

Conclusion. Mean SOFA score on admission was not a good predictor of mortality in patients with COVID-19 pneumonia.

\section{Reference(s)}

1) Vincent JL, Moreno R, Takala J, Willatts S, Mendonca A, Bruining H. The SOFA (Sepsis -related Journal Pre-proof Journal Pre-proof Organ Failure Assessment) score to describe organ dysfunction/failure. On behalf of the Working Group on Sepsis - Related Problems of the European Society of Intensive Care Medicine. Intensive Care Med. 1996;22(7):707 -710. https://doi.org/https://doi.org/10.1007/BF01709751.

2) Ferreira FL, Bota DP, Bross A, Melot C, Vincent JL. Serial evaluation of the SOFA score to predict outcome in critically ill patients. JAMA. 2001;286:1754-1758.

\section{5}

Night-time sleep in our cardiac intensive care unit: perception and measuring

FL. PEREZ CABALLERO'. SR. Mancha'. AJ Gordillo Vacas²; MS. Pereira

Gama ${ }^{1}$; M. Diaz-Tavora Luque'; I. Peña Luna'; S. Lardelli Picallos ${ }^{1}$

1 UNIDAD DE CUIDADOS INTENSIVOS, Hospital de Mérida, Mérida, Spain;

${ }^{2}$ Medicina de familia, Centro de Salud Urbano 1, Mérida, Spain

Correspondence: F.L. PEREZ CABALLERO

Intensive Care Medicine Experimental 2020, 9(1): 000315

Introduction. The sleep of critically ill patients can be disturbed during their stay in an Intensive Care Unit, and there is evidence that sleep in the ICU is poor in quality.

The causes of this disturbance depend on a variety of patients factors and the acute nature of their illnesses (highlighting non-sedated patients, such as coronary patients), as well as factors related to the ICU environment and the treatments administered.
Acute sleep deprivation can be negatively associated with the recovery of ICU patients, being the aim of this study is to describe patients' perceptions of a night's sleep at ICU.

Objectives. To analize the night-time sleep of a group of coronary patients admitted to our Intensive Care Unit (ICU) last 10 days (1st10th April).

To determine the frequency of main causes of insomnia and degree of sleep reported by the patients using a pilot survey with 23 items about the ICU environment and characteristics of their sleep.

Methods. Observational, descriptive, prospective study in coronary patients admitted to our ICU in 10 days, using a database of 11 patients, who conducted a survey of the quality of sleep during their stay in the ICU, with the following items: sleep quality at home and ICU, general degree of daytime sleepiness, noise, light, nursing care, diagnostic tests, drugs administration, alarms, pulse oximeter, hear people talking, aspiration of secretions, nebulizers- oxygen, phone calls, room temperature and sleep medication at home and ICU.

Results. Mean and median of the items analyzed (score from 0 to 10) were:

- Sleep quality at home: 7,18 (8).

- Sleep quality at ICU: 6,60 (6).

- General degree of daytime sleepiness: 4,90 (5).

- Noise: 3,18 (2).

- Light: 2,90 (3)

- Nursing care and diagnostic tests: 1,81 (1).

- Drugs administration: 1,63 (1).

- Alarms: 4,18 (4)

- Pulse oximeter: $2,40(1)$

- Hear people talking: 3,45 (2).

- Aspiration of secretions: $1,20(0,5)$.

- Nebulizers- oxygen: 1,60 $(0,5)$.

- Phone calls: $1,63(0)$.

Percentages of the items analyzed:

- Room temperature: cold $18 \%$, hot $9 \%$, correct $72 \%$.

- Sleep medication at home: yes $36 \%$, no $64 \%$.

- Sleep medication at ICU: yes $64 \%$, no $36 \%$

We must highlight the strong positive correlation between light and hear people talking (rho $0,727, p=0,011$ ) and light and noise (rho $0,627, p=0,039 ;$ no differences between sleep quality at ICU and sleep medication were found.

Conclusion. Frequent disruptions (hear people talking), poor quality and changes in the circadian rhythm (presence of lighting intensity higher and noise), characterize the night-time sleep of critically ill patients admitted in ICUs.

Although protocols for sleep promotion in the ICUs have recently been studied, the best strategies for sleep promotion has yet to be defined. So, we create this pilot survey to determine possible causes of sleep impairment in our patients, in order to improve it, including this study in our project to humanize the intensive care unit.

\section{Reference(s)}

1. Carrera-Hernández L, Aizpitarte-Pejenaute E, Zugazagoitia-Ciarrusta N, Goñi-Viguria R. Percepción del sueño de los pacientes en una Unidad de Cuidados Intensivos. Enferm Intensiva. 2018;29:53-63.

2. Jeffs EL, Darbyshire JL. Measuring Sleep in the Intensive Care Unit: A Critical Appraisal of the Use of Subjective Methods. Journal of Intensive Care Medicine. 2019:34(9):751-760. 10.1177/0885066617712197

3. Beltrami FG, Nguyen XL, Pichereau C, Maury E, Fleury B, Fagondes S. Sleep in the intensive care unit. J Bras Pneumol. 2015:41(6):539-546. 10.1590/ S1806-37562015000000056 
000339

A Quality Improvement Initiative: The Impact

of Animation-Supported Communication in Intensive Care

FS. Ledesma ${ }^{1}$; M. Wachtl, ${ }^{2}$; H. Malcolm, ${ }^{1}$; C. Toal, ${ }^{1}$; C. Kavanagh, ${ }^{2}$; J. Hadley, ${ }_{1}^{1 .}$ J. Pennington, ${ }^{1}$; S. Shepherd, ${ }^{2}$; D. Wald ${ }^{2}$

${ }^{1}$ Intensive care-royal london hospital, Barts Health NHS Trust, London, United Kingdom; ${ }^{2}$ Intensive care — st. bartholomew's hospital, Barts

Health NHS Trust, London, United Kingdom

Correspondence: F.S. Ledesma

Intensive Care Medicine Experimental 2020, 9(1): 000339

Introduction. Communication with the families of patients admitted to intensive care units (ICUs) has proven to be particularly difficult during the CoVID-19 pandemic due to restricted visiting and limited time available for experienced staff to communicate with relatives by telephone. Families are left with increased anxiety and stress at the inability to comprehend interventions and patient progress, which are typically better communicated face-to-face.1,2 Alternative methods of communication such as animation videos may help bridge the communication gap and have previously been shown to improve patient understanding before consent for medical procedures. 3 However, this approach has yet to be evaluated as a communication aid with relatives of patients in ICU.

Objectives. To determine whether multi-language animations (www.explainmyprocedure.com) improved relatives' self-perceived understanding of mechanical ventilation-its potential benefits, risks, and any alternatives. We also evaluated the impact on reported anxiety.

Methods. This project took place between 1st of November 2020 to 31 st of January 2021 at two ICUs in Barts Health NHS Trust. We introduced animated videos in multiple languages explaining mechanical ventilation and used telephone questionnaires to evaluate relatives' reported understanding of mechanical ventilation before and after the introduction of the videos. Telephone questionnaires were administered during the first 6 weeks to 39 next-of-kin of patients admitted to ICU prior to the introduction of the animations. During the next 6 weeks, following the introduction of the animation through an initial telephone conversation with 32 next-of-kin, an email was sent with a web-link to the animation and a follow-up call was done after at least $24 \mathrm{~h}$. This was to administer the same questionnaire with the addition of whether they had watched the animation prior to the call, whether it helped their understanding, and whether the animation affected their anxiety level.

Results. There was no statistical difference between the 2 groups in terms of demographics and reason for ICU admission. All relatives in the second group reported that they had watched the animation. Prior to the animation $(n=39), 15 \%$ reported that they understood what mechanical ventilation involved, $28 \%$ understood its benefits, $0 \%$ understood its risks, and $3 \%$ knew its alternatives. After the introduction of the animation $(n=32)$, the respective results were $94 \%, 97 \%, 84 \%$, and $65 \%$ ( $p<0.0001$ for each comparison with the no animation group). Overall there was no reported increase in anxiety levels.

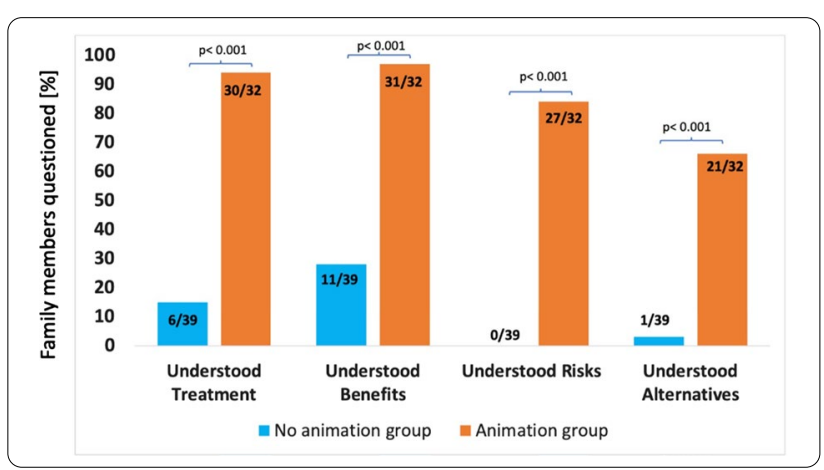

\begin{tabular}{|l|c|c|}
\hline & $\begin{array}{l}\text { Families interviewed before } \\
\text { introduction of animations } \\
\mathrm{n}=\mathbf{3 9}\end{array}$ & $\begin{array}{l}\text { Families interviewed after } \\
\text { introduction of animations } \\
\mathrm{n}=\mathbf{3 2}\end{array}$ \\
\hline Age (median/ range) & $63(19-83)$ & $58(34-77)$ \\
\hline Native English speaking family & 25 & 20 \\
\hline Non-English speaking family & 7 & 4 \\
\hline Bengali & 4 & 4 \\
\hline Hindi/Urdu & 3 & 4 \\
\hline Other* & & 19 \\
\hline Reason for patient admission & 4 & 3 \\
\hline COVID- 19** & 21 & 3 \\
\hline Cardiogenic** & 6 & 2 \\
\hline Neurogenic & 4 & 5 \\
\hline Trauma & 4 & \\
\hline Other Medical & 2other languages were Portuguese, Turkish, Arabic and Albanian. \\
p>0.1 for all comparisons apart from ** where $\mathbf{p}<0.01$ & \\
\hline
\end{tabular}

Conclusion. Web-based multi-language animations are a useful tool to support understanding of mechanical ventilation on intensive care and communication with patients' families. The approach can be extended to other intensive care treatments and has the potential to supplement communication with patients' relatives beyond the pandemic.

\section{Reference(s)}

1. Wald D, Casey-Gillman O, Comer K, Mansell JS, Teo H, Mouyis K, et al. Animation-supported consent for urgent angiography and angioplasty: a service improvement initiative. Heart. 2020; 0: 1-5.

2. Cattelan J, Castellano S, Merdji H, Audusseau J, Claude B, Feuillassier L, et al. Psychological effects of remote-only communication among Reference persons of ICU patients during COVID-19 pandemic. J intensive care. 9 Jan 2021; 9, 5. https://doi.org/10.1186/s40560-020-00520-w.

3. Newcombe V, Baker T, Burnstein R, Tasker R, Menon D. Clinical communication with families in the age of covid-19: a challenge for critical care teams. The BMJ Opinion. 11 Aug. 2020. Available from: https://blogs.bmj. com/bmj/2020/08/11/clinical-communication-with-families-in-the-ageof-covid-19-a-challenge-for-critical-care-teams/.

\section{7}

Neuropsychological outcome of patients with severe Covid-19 infection admitted to ICU

M. Della Giovampaola ${ }^{1}$; T. Tonetti ${ }^{1}$; P. Bottausci ${ }^{1}$; M. Bordini ${ }^{1}$; L. Sambati ${ }^{2}$; K. Mattarozzi ${ }^{3}$; R. Santoro ${ }^{2}$; L. Cretella ${ }^{2}$; L. Romano ${ }^{2}$; M. Ranieri ${ }^{1}$; P. Cortelli²; M. Guarino ${ }^{2}$; L. Mascia ${ }^{4}$

${ }^{1}$ Department of Medical and Surgical Sciences, Alma Mater Studiorum-Università di Bologna, Bologna, Italy; ${ }^{2}$ Irccs istituto delle scienze neurologiche di bologna, Ospedale Bellaria, Bologna, Italy; ${ }^{3}$ School of medicine-department of experimental, diagnostic and specialty medicine (dimes), Alma Mater Studiorum-Università di Bologna, Bologna, Italy; ${ }^{4}$ Department of biomedical and neuromotor sciences, Alma Mater Studiorum-Università di Bologna, Bologna, Italy

Correspondence: M. Della Giovampaola

Intensive Care Medicine Experimental 2020, 9(1): 000347

Introduction. In critically ill patients, long term cognitive and emotional impairment has been reported. In parallel caregivers are exposed to stressors that compromise their quality of life. The Covid19 pandemic has been characterized by prolonged mechanical ventilation, long ICU stay and a peculiar state of isolation that may further affect the neuropsychological outcome of patients and their caregivers.

Objectives. Our primary objective was to identify the incidence of neuropsychological disorders in patients requiring ICU admission for Covid-19 and their risk factors. Our secondary objective was to quantify the impairment of health-related quality of life in caregivers.

Methods. We performed a prospective observational study in the University teaching Hospitals Sant'Orsola and Bellaria, Bologna, Italy, from March 1 to April 30 2020. Consecutive adult patients with confirmed Covid-19 infection requiring ICU admission and invasive mechanical ventilation were recruited together with a caregiver. Patients with 
known cognitive disorders or barrier language were excluded. Demographic and clinical data at admission and during ICU stay were collected. At 3 and 12 months after ICU discharge, patients and their caregivers underwent a comprehensive neuropsychological evaluation and filled out validated questionnaires performed by well-experienced neurologists blinded to clinical data.

Results. We screened 263 patients: 22 completed the neurological assessment (142 died, 92 were not reachable or refused and 7 were lost during follow up). The median age was 58 years [38-76], 16 patients were male. The median APACHE II score and SOFA score at admission were 8 [5-15] and 4 [1-8]. The median ICU length of stay was 35 days [12-55] and the median duration of mechanical ventilation was 12,5 days [4-59]. At 3 months quality of life was mildly impaired in 19 patients, 7 had mild cognitive impairment mainly involving memory, attention and verbal fluency; 3 showed anxiety but no depression. Lower performances at MoCA were related to higher APACHE score at TO $(r-0.44 ; p<0.04)$. Working memory, short and long term memory and verbal fluency were inversely related to ICU stay $(r-0.501 p<0.03$; $r-0.69 p<0.01, r-0.69 p<0.03$ respectively) and duration of mechanical ventilation $(r-0.65 p<0.01, r-0.715 p<0.01, r-0.64 p<0.02)$. Long and short term memory were inversely related to SOFA score $(r-0.58$ $\mathrm{p}<0.01 ; \mathrm{r}-0.55 \mathrm{p}<0.02)$ and longer treatment with propofol $(\mathrm{r}-0.53$ $p<0.02 ; r-0.56 p<0.02)$ and benzodiazepines $(r>0-0.53 ; p s<0.02$; $r-0.53 p<0.02$ ). Patient's anxiety was directly related to caregiver's psychological distress $(r 0.64 ; p<0.001)$. SOFA score was inversely related to caregiver's quality of live $(r-0.53, p<0.02)$.

Conclusion. At 3 months follow up cognitive and psychological sequelae were observed in most of Covid-19 patients and their caregivers and were strictly related to severity of illness and sedative drugs; 12 months follow-up is under evaluation.

\section{Reference(s)}

Monti G, Leggieri C, Fominskiy E et al. (2021) Two months quality of life of COVID-19 invasively ventilated survivors; an Italian single-center study. Acta Anaesthesiol Scand 2:10

Rogers J, Chesney E, Oliver D et al. (2020) Psychiatric and neuropsychiatric presentations associated with severe coronavirus infections: a systematic review and meta-analysis with comparison to the COVID-19 pandemic. Lancet Psychiatry 7(7):611-627

Pun B, Badenes R et al. (2021) Prevalence and risk factors for delirium in critically ill patients with COVID-19 (COVID-D): a multicentre cohort study. Lancet Respir Med 9(3):239-50

Pandharipande PP, Girard TD, Jackson JC, et al. (2013) Long-Term Cognitive Impairment after Critical IIIness. N Engl J Med 369(14):1306-1316

Honarmand K, Lalli RS, Priestap F, et al. (2020) Natural history of cognitive impairment in critical illness survivors a systematic review. Am J Respir Crit Care Med 202(2):193-201

Herridge M, Cameron J et al. (2016) One-year outcomes in caregivers of critically ill patients. N Eng J Med 374:1831-41

Herridge M, Tansey C, Mattè A (2011) Functional Disability 5 Years after Acute Respiratory Distress Syndrome. New Engl J Med 364(14):1293-1304

Herridge M, Cheung AM, Tansey C (2003) One-Year Outcomes in Survivors of the Acute Respiratory Distress Syndrome. New Engl J Med 348(8):683-693

Azoulay E, Vincent JL, Herridge M et al. (2017) Recovery after critical illness: putting the puzzle togheter-a consensus of 29. Crit Care 21:296

Hamilton M, Chu L, Herridge M et al. (2019) Determinants of depressive symptoms at 1 year following ICU discharge in survivors of $>7$ days of mechanical ventilation. Chest 156(3):466-476

\section{2}

Crowdfunding Canadian Healthcare: A Cross Sectional Analysis of Intensive Care Unit Patient and Caregiver Needs

P. Gregoire ${ }^{1}$; E. Balcom, ${ }^{2}$; M. Douma, ${ }^{1}$; V. Lau, ${ }^{1}$; P. Brindley ${ }^{1}$

${ }^{1}$ Critical Care Medicine, University of Alberta, Edmonton, Canada; ${ }^{2}$ Neurol-

ogy, University of Alberta, Edmonton, Canada

Correspondence: P. Gregoire

Intensive Care Medicine Experimental 2020, 9(1): 000352
Introduction. Despite universal coverage for medically necessary healthcare services from jurisdictionally delivered health insurance, Canadians can still individually accrue health-related expenses outside of their coverage (1). These may be related to therapies and care needs that are not covered by their insurance, travel requirements for specialized care, or the sequelae of their illness on their functional status. In this study, we analyzed health care funding requests posted to the most popular publicly accessible crowdfunding platform to determine the unmet health care needs of Canadians with a focus on intensive care unit (ICU) patients.

Methods. A cross-sectional, descriptive analysis of a public cohort was performed on all publicly accessible funding requests in the "Medical" section of the Canadian gofundme.com website. An algorithm was developed using the python programming language to systematically extract all publicly available posts. They were reviewed for demographic and funding data including sex, age category, location, and amount of funds requested and obtained. Subsequently, we evaluated each post for the patient's underlying diagnosis and reasons for seeking financial assistance.

Results. One thousand posts were extracted on Nov. 6, 2020. Of these, 880 posts were included after excluding posts that were not related to specific individual funding requests, deleted prior to time of review, or not clearly related to someone within the Canadian health care system.

Of all posts, $15 \%(n=133)$ were related to patients who either were currently or had been in the ICU. Of this subgroup, $71 \%$ of posts were related to surgical patients and $57 \%$ had neurologic complications. At time of data extraction, all posts had accrued a total of $\$ 24.2$ million Canadian dollars (CAD), while the ICU patient subgroup had raised $\$ 4.2$ million CAD for a median of $\$ 19,945$ CAD per request. Within the ICU subgroup, $40 \%$ of posts Referenced financial hardship related to travel (e.g. routine appointment travel costs, hospital parking, transportation or relocation to distant health care settings), $40 \%$ Referenced loss of income (e.g. inability to work, family members leaving work to care for patients), $14 \%$ Referenced medication costs; $26 \%$ Referenced equipment and mobility device costs, $18 \%$ Referenced physiotherapy and rehabilitation costs, and $11 \%$ Referenced costs associated with long term care. In addition, $24 \%$ in the ICU subgroup Referenced barriers to care (financial or otherwise) related to the COVID-19 pandemic.

Conclusion. Despite publicly funded universal health insurance, some Canadian patients have significant unmet financial needs related to their illness. This study highlights financial barriers to care and potential targets for policy and resource allocation aimed at reducing the burden on ICU patients and their caregivers. Of note, travel expenses and loss of income were the most frequently reported reasons for publicly appealing for funds. The COVID-19 pandemic has added a further burden to critically ill patients. Future analysis should examine relationships between location and travel expenses as well as diagnosis-specific needs.

\section{Reference(s)}

1. Canadian Institute for Health Information. "National Health Expenditure Trends 2020." Ottawa, ON: CIHI; 2021

\section{0}

Distribution of delirium motor subtypes in the intensive care unit-a scoping review

K. la Cour ${ }^{1}$; N. Andersen-Randberg, ${ }_{1}$; S. Weihe ${ }^{1}$; C. B Mortensen'; LM. Poulsen ${ }^{1}$; MO. Collet, ${ }^{2}$; CKW. Kjerr'; S. Estrup, ${ }^{1}$; O. Mathiesen ${ }^{1}$

${ }^{1}$ Centre for anaesthesiological research, department of anesthesiology, Zealand University Hospital, Køge, Denmark; ${ }^{2}$ Department of intensive care, Copenhagen University Hospital, Rigshospitalet, København, Denmark

Correspondence: K. la Cour

Intensive Care Medicine Experimental 2020, 9(1): 000360

Introduction. Delirium is the most common cerebral dysfunction in the Intensive Care Unit (ICU) and can be subdivided into either 
a hypoactive, hyperactive or mixed motoric subtype based on the clinical manifestation. It is however unknown whether these subtypes represent different disease entities. The aim of this review is to describe the distribution, pharmacological treatment and outcomes of delirium motor subtypes in critically ill patients.

Methods. This scoping review was performed according to the Preferred Reporting Items for Systematic reviews and Meta-Analyses extension for Scoping Reviews (PRISMA-SCR) and Cochrane guidelines. We performed a systematic search in six databases to identify studies assessing distribution, pharmacological treatments and outcomes according to delirium motor subtypes. A meta-analysis was performed where pooled estimates with $95 \%$ confidence intervals were computed by a random effect model. Between-study variance was determined with the restricted maximum-likelihood estimator. Results. We included 122 studies, comprising 44,521 patients. There was a large between-study heterogeneity among included studies, such as differences in study design, delirium assessment method and outcome reporting. Hypoactive delirium was the most prevalent of the three delirium motor subtypes $(50.6 \%[95 \%$ $\mathrm{Cl}, 46.1-55.2])$, followed by mixed delirium $(26.7 \%$ [95\% Cl, $23.3-$ $30.2]$ ) and hyperactive delirium (21.4\% [95\% Cl, 17.9-24.9]). Patients with mixed delirium experienced the longest delirium duration, longer ICU and hospital stay, higher ICU and hospital mortality and recieved more medication during ICU stay compared to other delirium motor subtypes. Hyperactive delirious patients were more likely to receive delirium targeted pharmacological treatment. Older patients were more likely to develop hypoactive delirium.

Conclusion. In this review we found that hypoactive delirium was the most prevalent delirium motor subtype in critically ill patients especially among older patients. Furthermore, mixed delirium was associated with worse outcomes in terms of delirium duration, length of stay and mortality. The large between-study heterogeneity suggests that a more standardized methodology in delirium research is warranted.

\section{Reference(s)}

1. Stagno D, Gibson C, Breitbart W (2004) The delirium subtypes: a review of prevalence, phenomenology, pathophysiology, and treatment response. Palliat. Support. Care

2. Salluh JIF, Wang H, Schneider EB, et al. (2015) Outcome of delirium in critically ill patients: Systematic review and meta-analysis. BMJ Br Med J 350:

3. Girard TD, Pandharipande PP, Ely EW, et al. (2008) Delirium in the intensive care unit. Crit Care 12:S3-S3. https://doi.org/10.1186/cc6149

4. None to declare

\section{3}

Reconfiguring a critical care outreach team during Covid-19 pandemic

S. Hadfield'; D. Hadfield ${ }^{2}$; F. Harrison ${ }^{1}$; V. Metaxa ${ }^{2}$

${ }^{1}$ Critical care, Kings College Hospital NHS Foundation Trust, London, United Kingdom; ' $C$ Critical care, King's College Hospital, London, United Kingdom

Correspondence: S. Hadfield

Intensive Care Medicine Experimental 2020, 9(1): 000363

Introduction. The novel coronavirus (COVID-19) pandemic presented hospitals globally with the unique challenge of how to prepare and provide for the influx of critically ill patients. Prior to the pandemic, our hospital developed surge plans to expand the critical care (CC) footprint, including increasing capacity outside of CC to support patients requiring non-invasive respiratory (NIV) support. The CC outreach team (CCOT) played an integral role in the management of COVID-19 patients and support of ward staff outside of CC. We describe the reconfiguration of the CCOT to adapt to the demands of the pandemic, the nature and volume of work, and the effect on CCOT members.

Methods. We conducted a retrospective analysis of the CCOT workload over a five-week period (Jan-Feb 2021). We reviewed electronic records and collected data on the activity of the CCOT: the number of telephone referrals each day, number of patients reviewed, number receiving NIV and number intubated on the wards by the CCOT. Anonymised content from three debriefing sessions with CCOT nurses were analysed for repeating themes.

Results. Our hospital is a 950-bed tertiary referral centre in London, United Kingdom with a usual CC capacity of 69 beds and no dedicated respiratory high dependency unit (HDU) prior to the pandemic. CC capacity was increased to 140 beds, with expansion to wards, operating theatre and recovery areas. Two medical wards were transformed into respiratory HDUs, managed by medical teams with continuous input from the CCOT.

The CCOT members were increased from a group of six (CC consultant, 2 registrar grade CC doctors, 3 CC nurses) to eleven (CC consultant, 4 registrar grade CC doctors, 4 CC nurses, 1 theatre practitioner and 1 runner).

Compared to the same period the previous year, there were significant increases in telephone referrals (3575 [2021] versus 186 [2020]), the numbers of patients reviewed (298 [2021] versus 134 [2020]), patients receiving NIV (102 [2021] versus 15 [2020]). The CCOT intubated (71 [2021] versus 31 [2020]) patients on the wards (Fig. 1).

Recurrent themes from debriefing sessions were: 1 ) the overwhelming nature of the time-critical work; 2 ) the uncertainty of administering therapies where evidence was lacking; 3 ) feelings of guilt from having insufficient time to deliver care; 4) the increased decisionmaking responsibility; and 5) the pressure from ward teams to provide support to staff unfamiliar with NIV devices.

Conclusion. The data presented demonstrates the essential role the CCOT played in supporting the hospital during the pandemic. The activity of the team increased significantly, reflecting the immense pressure across the hospital. There was a requirement for the ward teams to care for patients receiving NIV, with minimal training and experience, and thus heavy reliance on the CCOT to guide management. This resulted in feelings of anxiety and moral distress in the CCOT.

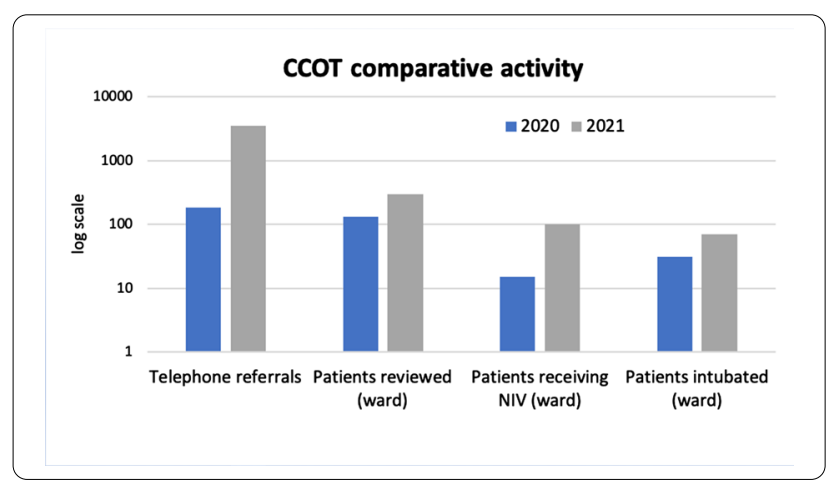

000374

A Computational Model to Predict ICU Readmission

S. Glaister ${ }^{1}$; H. Kim, ${ }^{2}$; R. Stevens ${ }^{3}$

${ }^{1}$ Biomedical Engineering, Johns Hopkins University, Baltimore, United States of America; ${ }^{2}$ Department of biomedical engineering, Johns Hopkins University, Baltimore, United States of America; ${ }^{3}$ Anesthesiology and critical care medicine, Johns Hopkins Hospital, Baltimore, United States of America

Correspondence: S. Glaister

Intensive Care Medicine Experimental 2020, 9(1): 000374

Introduction. ICU readmission is associated with adverse clinical outcomes and increased healthcare resource utilization. There is an unmet need for more accurate and reliable prediction methods to determine risk of ICU readmission.

Objectives. The aim of this study was (a) to predict the risk of ICU readmission by training a machine learning $(\mathrm{ML})$ model with clinical and physiological data extracted from a large ICU database, and (b) to 
identify specific feature patterns that are associated with high readmission risk.

Methods. We analyzed patient data from the eICU Collaborative Research Database, which contains high resolution data on $>200,000$ admissions in $>200$ hospitals across the United States. We identified cases as ICU stays of patients who were readmitted $1-72 \mathrm{~h}$ after their discharge. The control group consisted in ICU stays of patients who were discharged alive and not readmitted. Predictive features were extracted from the $24 \mathrm{~h}$ period preceding ICU discharge and included comprehensive SOFA scores, physiologic time series data (heart rate, systolic blood pressure, diastolic blood pressure), as well as patient demographics. The features from the case and control groups were used to train Generalized Linear Models (GLM), random forest and XGBoost models. The optimal weighting was tested and found for each model to account for the large class imbalance ( 25 -fold more controls than cases). Model performance was determined by area under the receiver operating characteristic curve (AUC) analysis.

Results. We identified 4,654 patients who were readmitted and 115,535 patients who were not. Hospital mortality of readmitted patients was nearly twofold higher ( $12.2 \%$ vs $6.3 \%)$. The XGBoost model had the highest discrmination with an AUC of $0.68(0.71,0.66)$, sensitivity of $0.64(0.82,0.46)$ and specificity of $0.62(0.64,0.59)$, while the random forest and GLM models had AUCs of $0.66(0.67,0.65)$ and $0.66(0.69,0.62)$ respectively. The feature rankings for the random forest model based on node impurity were as follows (node impurities in parenthesis): age (100), average respiratory rate (79.80), difference from the average non-readmitted ICU patient's discharge hour (74.48), urine output (66.63), ICU length of stay (63.30), delta of heart sign change (55.55), BMI (53.50), systolic blood pressure (51.39), minimum heart rate (50.08), IBW devine (49.60), average minimum heart rate (49.55), admission height (48.59), minimum mean heart rate (58.3), systolic blood pressure (48.13), mean blood pressure (46.40), heart rate (45.00), GCS (43.98), minimum max heart rate (43.90).

Conclusion. ML algorithms trained with data available in the $12 \mathrm{~h}$ preceding ICU discharge are able to predict the probability of ICU readmission. Ongoing modeling iterations with an expanded feature space are expected to enhance model performance and clinical impact.

\section{Reference(s)}

1. The elCU Collaborative Research Database, a freely available multi-center database for critical care research. Pollard TJ, Johnson AEW, Raffa JD, Celi LA, Mark RG and Badawi O. Scientific Data (2018). https://doi.org/10. 1038/sdata.2018.178.

2. Min X, Yu b, and Wang F. Predictive Modeling of the Hospital Readmission Risk from Patients' Claims Data Using Machine learning: A Case Study on COPD. Scientific Reports (2019) 9:2362 https://doi.org/10.1038/ s41598-019-39071-y

3. Kramer A, Kiggins T, and Zimmerman J. intensive care unit readmissions in U.S. hospitals: Patient characteristics, risk factors, and outcomes. Critical Care Medicine 40(1):3-10, January 2012. https://doi.org/10.1097/CCM 0b013e31822d751e

\section{3}

Quality Improvement Project on documentation for Nasogastric Tube insertion for ICU patients at Buckinghamshire Healthcare

\section{NHS Trust}

A. Nadeem ${ }^{1}$; S. De Soysa ${ }^{2} ;$ T. Ali $^{2}$

${ }^{1}$ Intensive care medicine, Stoke Mandeville Hospital, Aylesbury, United Kingdom; ${ }^{2}$ Intensive care unit, Stoke Mandeville Hospital, Aylesbury, United Kingdom

\section{Correspondence: A. Nadeem}

Intensive Care Medicine Experimental 2020, 9(1): 000383

Introduction. Accurate documentation is essential for good clinical practice. It is stipulated by the General Medical Council for clear accurate and legible records that detail key information for events as they happen, or soon after, as this ensures continuity and guides future care (1).
Clear documentation of the insertion and correct placement of a nasogastric tube (NGT) is particularly important when initiating its use for feeding, a common indication for ICU patients. Its absence can lead to medical and legal complications $(2,3)$. The quality of such documentation was assessed within ICU at Buckinghamshire Healthcare NHS Trust.

Objectives. To review and improve the quality of documentation for NGT insertion.

Methods. The Trust guidelines for adult enteral tube feeding include various requirements for NGT insertion and placement before initiating a patient's feed (4). These points should therefore be clearly documented in the patient's medical records. As the standard of documentation varied greatly, a Local Safety Standard for Invasive Procedures (LocSSIP) was introduced for ICU patients to ensure consistency. Following this, a retrospective assessment of the notes for patients with NGT inserted after admission was carried out in order to compare the quality of documentation using the LocSSIP versus without. Twenty patient's records were accessed using an electronic database and those without the proforma were studied according to agreed standards. Nineteen patient's records obtained either from the same database or from new admissions with a completed LocSSIP proforma were also studied. The results from the two groups were then compared to identify improvement. Results. The results are summarised in the table below:

\begin{tabular}{|c|c|c|c|}
\hline & $\begin{array}{l}\text { Without } \\
\text { proforma } \\
\text { (\% docu- } \\
\text { mented) }\end{array}$ & $\begin{array}{l}\text { With } \\
\text { pro- } \\
\text { forma } \\
\text { (\% docu- } \\
\text { mented) }\end{array}$ & $\begin{array}{l}\text { Fold of } \\
\text { improve- } \\
\text { ment }\end{array}$ \\
\hline No documentation & 15 & 0 & 15 \\
\hline \multicolumn{4}{|l|}{ Prior to insertion } \\
\hline Indication & 45 & 95 & 2.1 \\
\hline Patient consented & 0 & 26 & 26 \\
\hline Reason not consented & 25 & 68 & 2.7 \\
\hline NEX measurement & 15 & 63 & 4.2 \\
\hline Contraindications & 0 & 63 & 63 \\
\hline NGT type & 35 & 100 & 2.9 \\
\hline \multicolumn{4}{|l|}{ Procedure details } \\
\hline Depth of insertion & 50 & 89 & 1.8 \\
\hline Method of securing & 0 & 95 & 95 \\
\hline \multicolumn{4}{|c|}{ Post-procedure and placement confirmation } \\
\hline pH of aspirate & 40 & 80 & 2.0 \\
\hline CXR confirmation & 85 & 100 & 1.2 \\
\hline Indication for CXR & 30 & 84 & 2.8 \\
\hline Descends midline & 40 & 84 & 2.1 \\
\hline Bisects carina & 40 & 84 & 2.1 \\
\hline Crosses diaphragm & 35 & 84 & 2.4 \\
\hline Sits below diaphragm & 45 & 84 & 1.9 \\
\hline $\begin{array}{l}\text { Placement confirmed by } 2 \\
\text { doctors }\end{array}$ & 20 & 16 & 0.8 \\
\hline Complications & 0 & 16 & 16 \\
\hline Safe to use & 65 & 84 & 1.3 \\
\hline
\end{tabular}

Conclusion. The use of a standardised proforma for the documentation of NGT insertion and placement confirmation clearly highlights an overall improvement in the quality of documentation for this event, although there is room for improvement. Without its use, documentation was variably poor and, in some cases, had even been missed altogether. We therefore recommend for the proforma to be used for all patients in whom a nasogastrc tube is indicated. We aim 
to audit its use in the future as well as regularly re-audit the quality of documentation.

\section{Reference(s)}

1. General Medical Council. Good Medical Practice. London: GMC; 2013

2. Jones, B.J.M., 2020. A Position Paper on Nasogastric Tube Safety. Bapen. Available from: https://www.bapen.org.uk/pdfs/ngsig/a-position-paperon-nasogastric-tube-safety.pdf [Accessed 11th April 2021]

3. National Patient Safety Agency. Patient Safety Alert NPSA/2011/PSA002: Reducing the harm caused by misplaced nasogastric feeding tubes in adults, children and infants. 2011. Available from: http://www.gbuke nteral.com/pdf/NPSA-Alert-2011.pdf [Accessed 11th April 2020]

4. 298.5.1 Adult Enteral Tube Feeding Guidelines. Buckinghamshire Healthcare NHS Trust.

5. No grants were received for this project.

\section{6}

Impact of WorkAlert Medical Scheduling on Patient Safety in the Intensive Care Unit

L. Bulfin ${ }^{1}$; R. Endacott' ${ }^{1}$; D. Kevat ${ }^{2}$; S. Lockley ${ }^{3}$; M. Ponnapa Reddy ${ }^{4}$

R. Tiruvoipati ${ }^{5}$; J. Morphet ${ }^{1}$

${ }^{1}$ School of nursing and midwifery, Monash University, Frankston,

Australia; ${ }^{2}$ Monash medical centre, Monash Health, Clayton, Australia;

${ }^{3}$ Division of sleep and circadian disorders, Brigham And Women's

Hospital, Boston, United States of America; ${ }^{4}$ Intensive care unit, Pen-

insula Health, Frankston, Australia; ${ }^{5}$ Peninsula clinical school, Monash

University, Frankston, Australia

Correspondence: L. Bulfin

Intensive Care Medicine Experimental 2020, 9(1): 000386

Introduction. Current evidence suggests that a reduction in doctors shift length alone is not sufficient to improve the rates of adverse events in the Intensive Care setting (1). WorkAlert ${ }^{\circledR}$ workplace scheduling guidelines developed by the Cooperative Research Centre for Alertness, Safety and Productivity (Alertness CRC) and the Sleep Health Foundation, considers multiple factors including shift length, number of consecutive shifts, forward rotations, adequate rest periods and limiting overtime in the creating of medical rosters (2).

Objectives. The primary objective of the study was to evaluate the impact of WorkAlert ${ }^{\circledR}$ scheduling guidelines on the incidence of adverse events in a metropolitan Intensive Care Unit (ICU).

Methods. We conducted a single centre retrospective cohort study comparing traditional schedules with WorkAlert ${ }^{\circledR}$ schedules for medical officers in a 26-bed metropolitan Australian ICU during April to August 2016 (control) and April to August 2017 (intervention period). The primary outcome was all-cause adverse events associated with medical care during ICU admission and for seven days post ICU discharge. Secondary outcomes included the incidence of preventable events, ICU and hospital mortality and length of stay (LOS). Adverse event data was collected using a modified Global Trigger Tool (3) and each event was subjected to secondary review by two independent intensivists. Patient admission and demographic data were collected from the hospitals' ICU patient database.
Results. A total of 800 randomly selected adult ICU admissions from the control and intervention period were reviewed, amounting to 3,243 ICU patient days. Patient characteristics were similar between the two time periods. Of the shifts worked in the control medical schedules (2016), 8.8\% did not adhere to the WorkAlert ${ }^{\circledR}$ guidelines compared with $2.9 \%$ of the intervention shifts (2017) $(p=<0.001, \mathrm{phi}=-0.127)$. The number of all-cause adverse events related to medical care did not differ between the control and intervention period (340.9 vs 343.7 per 1000 patient days, $n=557$ vs $553, p=0.898$ ). There was no difference in any of the secondary outcomes evaluated including the number preventable events (378 vs $384, p=0.571)$, ICU mortality ( $9.8 \%$ vs $8.8 \% p=0.714)$, hospital mortality $(14 \%$ vs $16 \% p=0.428)$, ICU LOS $(2.7(1.8-4.7)$ vs $2.4(1.8-4.1)$, $p=0.266)$ or hospital LOS (10.1 (6.3-17.9) vs $11.3(6.8-19.8) p=0.181)$. Conclusion. Despite recent evidence suggesting medical schedules designed to mitigate fatigue can improve patient safety, our study found that there was no difference in key patient safety outcomes including adverse events, ICU and hospital mortality and length of stay when ICU medical officers worked schedules designed according to the WorkAlert ${ }^{\circledR}$ guidelines. This is the first study on the impact of medical scheduling on patient safety in the Intensive Care Unit in Australia.

\section{Reference(s)}

1. Landrigan C, Rahman S, Sullivan J, Vittinghoff E, Barger L, Sanderson A, et al. Effect on Patient Safety of a Resident Physician Schedule without 24-Hour Shifts. The New England Journal of Medicine. 2020;382(26):2514-23.

2. WorkAlert [Internet]. North Strathfield, NSW: Sleep Health Foundation; 2021. Workplace Scheduling Solutions 2021 [cited 2021, Mar 20]; Available from: https://www.workalert.org.au/workplace-scheduling-solut ions/what-can-i-do-as-an-employer

3. Griffin FA, Resar R. IHI Global Trigger Tool for Measuring Adverse Events (second edition). IHI Innovation Series White Paper [Internet]. Boston MA: Institute for Healthcare Improvement; 2009 [cited 2021, Mar 20]. Available from: http://www.hi.org/resources/Pages/Tools/IHIGlobalTriggerTool forMeasuringAEs.aspx

4. Lauren Bulfin received the Betty Jeffrey Award from the Australian Nurses Memorial Center, an Australian Government Research Training Program Scholarship and an Alertness CRC PhD stipend.

\section{About this supplement}

These abstracts have been published as part of Intensive Care Medicine Experimental Volume 9 Supplement 1, 2021: ESICM LIVES 2021. The full contents of the supplement are available online at https:// icm-experimental.springeropen.com/articles/supplements/volume9-supplement-1. Please note that this is part 1 of 2.

\section{Publisher's Note}

Springer Nature remains neutral with regard to jurisdictional claims in published maps and institutional affiliations. 